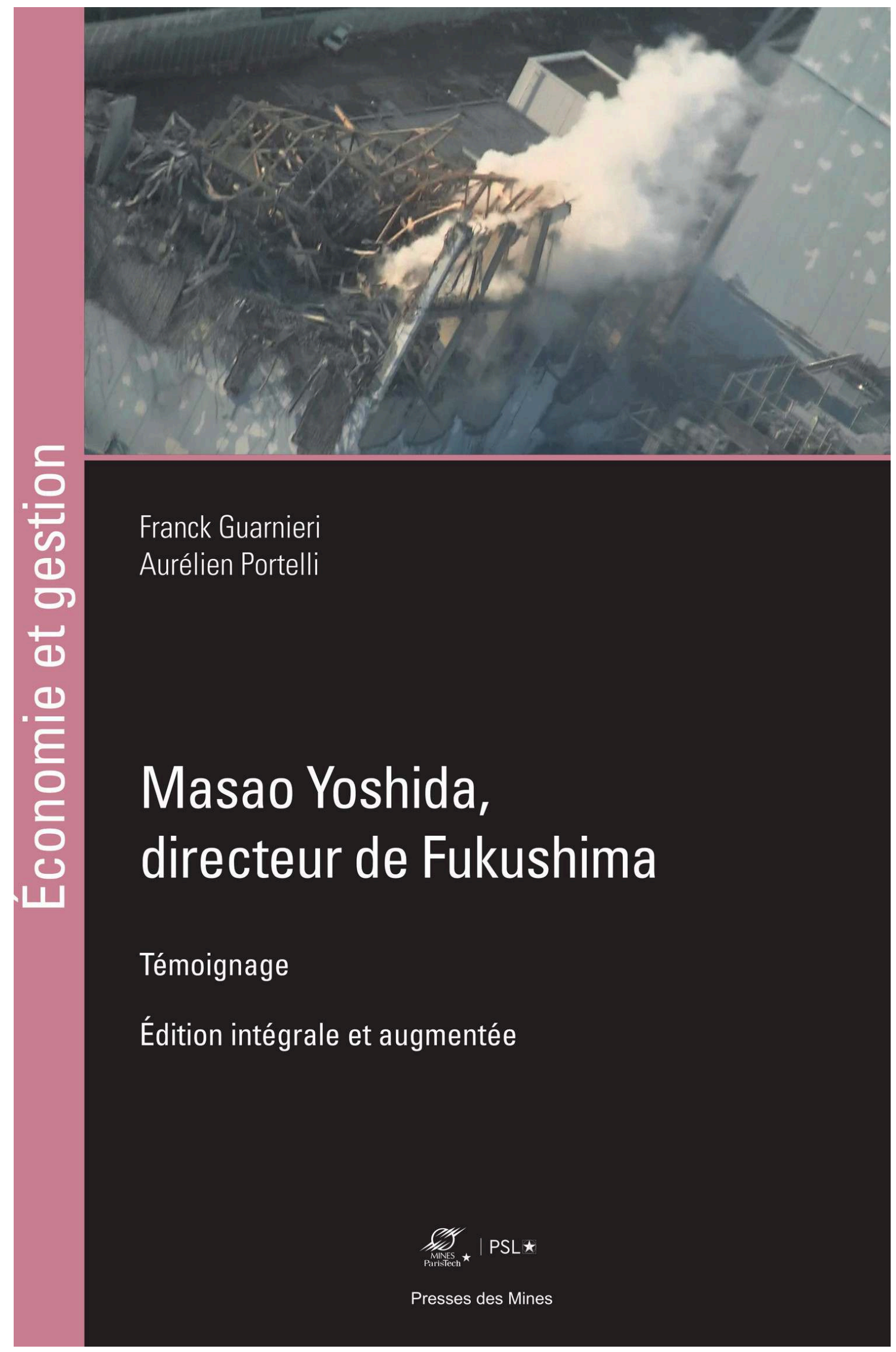




\section{Masao Yoshida, directeur de Fukushima}

Témoignage. Édition intégrale et augmentée

Franck Guarnieri et Aurélien Portelli (dir.)

Traducteur : Takesada Tomoko

DOI : 10.4000/books.pressesmines. 6217

Éditeur: Presses des Mines

Lieu d'édition : Paris

Année d'édition : 2021

Date de mise en ligne : 27 janvier 2021

Collection : Économie et gestion

ISBN électronique : 9782356716514

\section{orontation \\ Qbooks}

http://books.openedition.org

Référence électronique

GUARNIERI, Franck (dir.) ; PORTELLI, Aurélien (dir.). Masao Yoshida, directeur de Fukushima :

Témoignage. Édition intégrale et augmentée. Nouvelle édition [en ligne]. Paris : Presses des Mines, 2021

(généré le 03 mars 2021). Disponible sur Internet : <http://books.openedition.org/pressesmines/

6217>. ISBN : 9782356716514. DOI : https://doi.org/10.4000/books.pressesmines.6217.

Ce document a été généré automatiquement le 3 mars 2021

(C) Presses des Mines, 2021

Conditions d'utilisation

http://www.openedition.org/6540 
Le 11 mars 2011, le Japon est frappé par un séisme de magnitude 9, suivi d'un tsunami dévastateur. La double catastrophe fait plus de 18000 morts et disparus. Elle provoque également d'importants dégâts matériels. Cinq centrales nucléaires sont notamment touchées. L'une d'entre elles, Fukushima Daiichi, est inondée par une vague de 15 mètres, qui endommage gravement l'installation. Les jours suivants, trois réacteurs entrent en fusion et des explosions se produisent sur le site. Malgré l'ampleur de l'accident, le pire est pourtant évité. Évité grâce au courage et à l'action d'une poignée de travailleurs dirigés par Masao Yoshida, le directeur de Fukushima.

Cet ouvrage met à la disposition du lecteur l'intégralité des auditions, traduites du japonais, de Yoshida par la commission d'enquête gouvernementale sur l'accident. Au fil des entretiens, le directeur retrace une expérience humaine inédite, dévoilant l'histoire d'une équipe d'opérateurs confrontés à un scénario d'accident que nul n'avait imaginé. Une histoire qui, par-delà les termes techniques employés, peut se lire comme le roman d'une lutte acharnée menée contre une installation nucléaire libérée de ses dispositifs de contrôle. Face aux enquêteurs, Yoshida livre aussi un témoignage sans concession sur le rôle des cadres de l'exploitant TEPCO, sur celui des experts en sûreté nucléaire, des forces d'autodéfense, des pompiers et du Premier ministre Naoto Kan.

\section{FRANCK GUARNIERI}

Directeur de recherche, directeur du Centre de recherche sur les Risques et les Crises (CRC) de MINES Paris - Université Paris, Sciences \& Lettres (PSL).

\section{AURÉLIEN PORTELLI}

Enseignant-chercheur au CRC de MINES Paris - PSL. 
SOMMAIRE

Remerciements

Liminaires

Avertissement aux lecteurs

Franck Guarnieri et Aurélien Portelli

Figures

Un héros japonais

Franck Guarnieri et Aurélien Portelli

Une belle rencontre

Tomoko Takesada

Compte rendu de l'audition du 22 juillet 2011

Avertissement aux lecteurs

Compte rendu de l'audition

Compte rendu de l'audition du 29 juillet 2011

Compte rendu de l'audition du 8 août 2011

Avertissement aux lecteurs

Le « Rapport sur les dispositions pour la gestion des accidents au sein de la centrale de Fukushima Daiichi »

Compte rendu de l'audition

Compte rendu de l'audition du 9 août 2011 (I)

Compte rendu de l'audition du 9 août 2011 (II)

Compte rendu d'audition

Compte rendu de l'audition du 13 octobre 2011

Compte rendu d'audition

Compte rendu de l'audition du 6 novembre 2011 (I)

Compte rendu d'audition

Compte rendu de l'audition du 6 novembre 2011 (II)

Compte rendu d'audition

Photographies

L'accident de Fukushima Daiichi

Tableau des auditions

Glossaire des termes techniques

Glossaire des lieux, évènements et organisations

Fonctions des personnages

Références 


\section{Remerciements}

1 Nous voudrions sincèrement remercier les personnes suivantes, qui nous ont apporté leur aide et leur soutien pour préparer cette édition du témoignage de Masao Yoshida sur l'accident de Fukushima Daiichi: Bruno Adhémar, Aissame Afrouss, Matthias Braun, Yuki Kobayashi, Camille Ogawa, Tomoko Takesada, ainsi que la Fondation de MINES Paris. 


\section{Liminaires}

Franck Guarnieri, Risques et les Crises (CRC) de MINES Paris - Université Paris, Sciences \& Lettres (PSL). Aurélien Portelli, enseignant-chercheur au CRC de MINES Paris - PSL.

3 Traduction

4 Tomoko Takesada, interprète, traductrice, chargée d'enseignement à MINES Paris - PSL.

\section{Comité d'experts}

6 Bruno Adhémar, président de SUBLIME Énergie, ancien Chef de projet Actiflo Rad à Tôkyô et Fukushima Daiichi entre avril et juillet 2011.

Aissame Afrouss, docteur de l'Université PSL.

Matthias Braun, docteur, expert en atténuation et en simulation d'accidents nucléaires sévères.

Yuki Kobayashi, docteur de l'Université PSL, chercheur à l'International Peace and Security Departement de la Sasakawa Peace Foundation. 


\title{
Avertissement aux lecteurs
}

\author{
Franck Guarnieri et Aurélien Portelli
}

1 Cet ouvrage présente l'intégralité du témoignage de Masao Yoshida, le directeur de la centrale de Fukushima Daiichi, interrogé de juillet à novembre 2011 par la Commission d'enquête gouvernementale sur l'accident nucléaire survenu à la suite du séisme du Tôhoku le 11 mars 2011. Il reproduit le contenu des auditions publiées en trois volumes aux Presses des Mines entre 2015 et $2020^{1}$, en apportant de nombreuses précisions en notes.

2 Le lecteur n'étant pas forcément familier du vocabulaire du nucléaire, les termes techniques sont définis lorsqu'ils sont employés pour la première fois par Yoshida ou les enquêteurs. De la même manière, des informations complémentaires sont apportées sur les évènements, les lieux, les organisations et les personnes évoqués dans les entretiens.

3 Les expressions en anglais utilisées par Yoshida sont laissées telles quelles dans le corps du texte et traduites en notes.

4 Des confusions ou inexactitudes repérées dans la transcription des entretiens sont mentionnées.

Des extraits de témoignages d'acteurs de la gestion de crise nucléaire sont retranscrits en notes pour compléter les propos de Yoshida ou leur apporter un autre éclairage.

\section{NOTES}

1. Guarnieri F. (dir.). L'accident de Fukushima Dai Ichi - Le récit du directeur de la centrale. Volume I: L'anéantissement, Paris, Presses des Mines, 2015, 342 p.; Guarnieri F. (dir.). L'accident de Fukushima Dai Ichi - Le récit du directeur de la centrale. Volume II : Seuls, Paris, 
Presses des Mines, 2016, 395 p.; Guarnieri F. (dir.). L'accident de Fukushima Dai Ichi - Le récit du directeur de la centrale. Volume III : L'abîme, Paris, Presses des Mines, 2020, 276 p.

\section{AUTEURS}

\section{FRANCK GUARNIERI}

Directeur de recherche, directeur du Centre de recherche sur les Risques et les Crises (CRC) de MINES Paris - Université Paris, Sciences \& Lettres (PSL).

\section{AURÉLIEN PORTELLI}

Enseignant-chercheur au CRC de MINES Paris - PSL. 


\section{Figures}

Figure 1. Localisation de la centrale nucléaire de Fukushima Daiichi (NAIIC, 2012, p. 10).
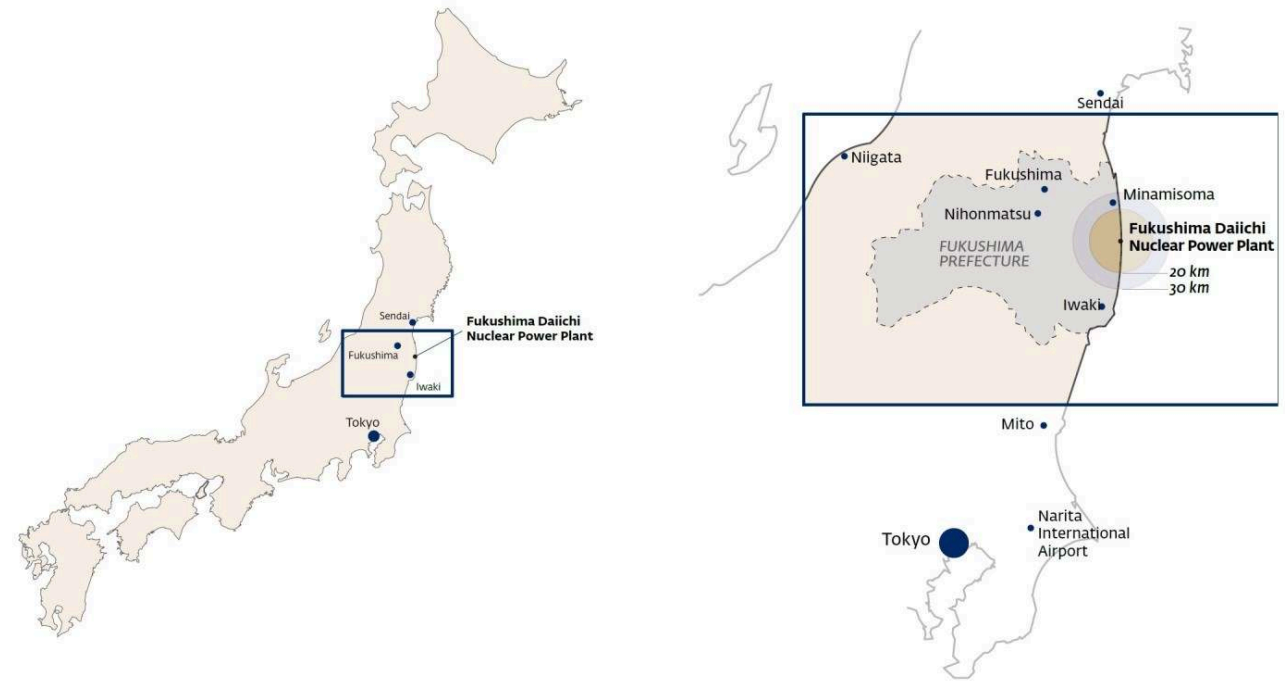
Figure 2. Hauteurs et emplacements des structures et composants de Fukushima Daiichi (AIEA, 2015a, p. 36). Définition d'O.P. : hauteur par rapport au niveau de la mer à Onahama Peil.
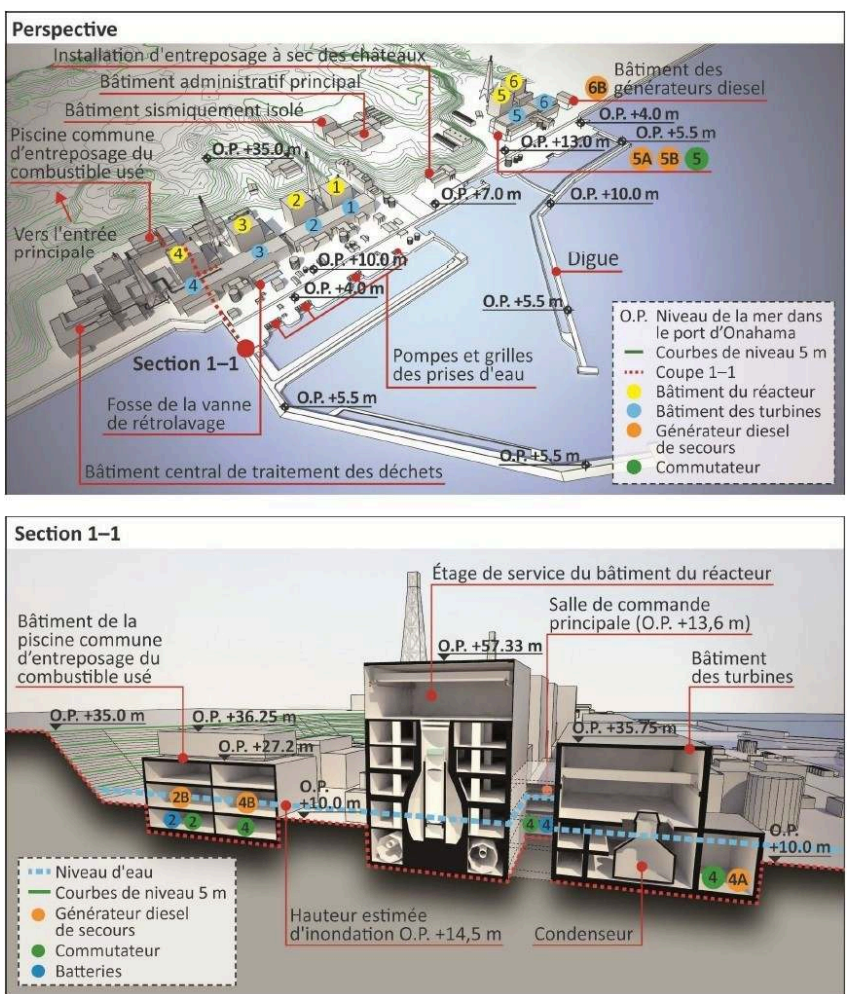

Figure 3. Schéma du fonctionnement général d'un Réacteur à Eau Bouillante (IRSN, 2012a, p. 10).

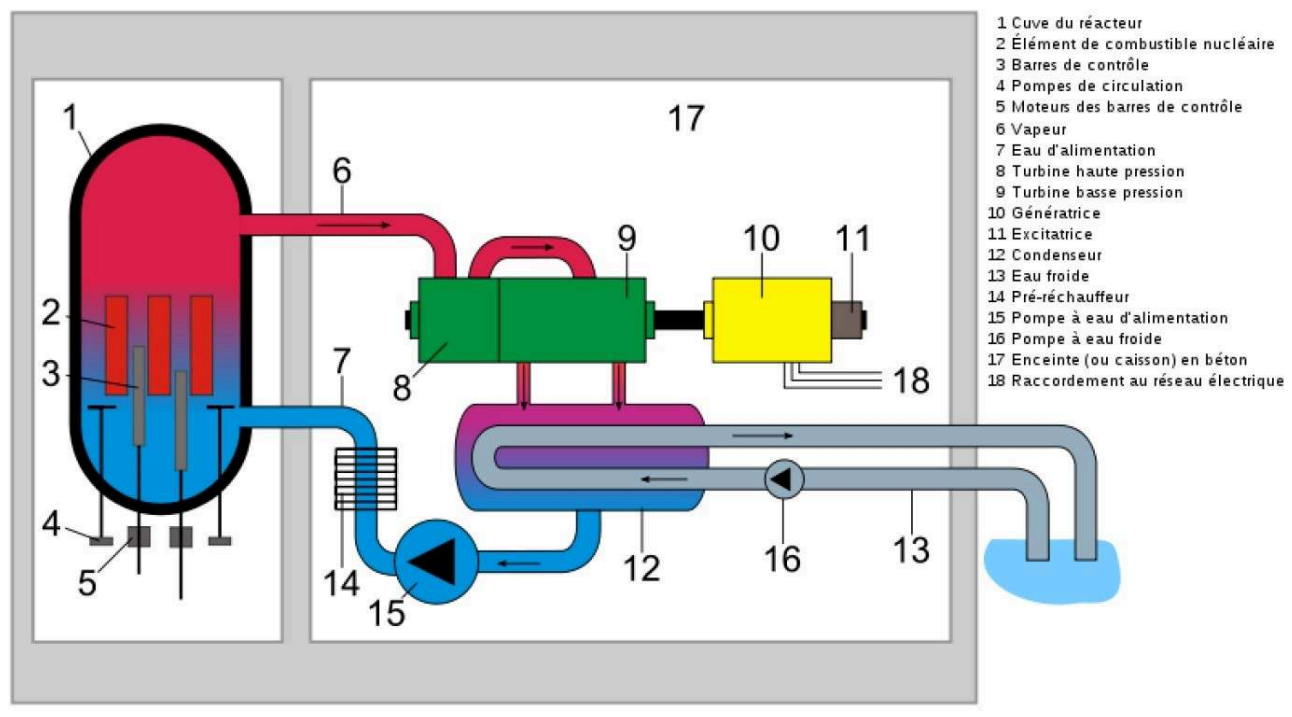


Figure 4. Enceinte de confinement de type Mark I des réacteurs 1 à 5 de Fukushima Daiichi (IRSN, 2012a, p. 11).



Figure 5. Enceinte de confinement de type Mark II du réacteur 6 de Fukushima Daiichi (IRSN, 2012a, p. 12).

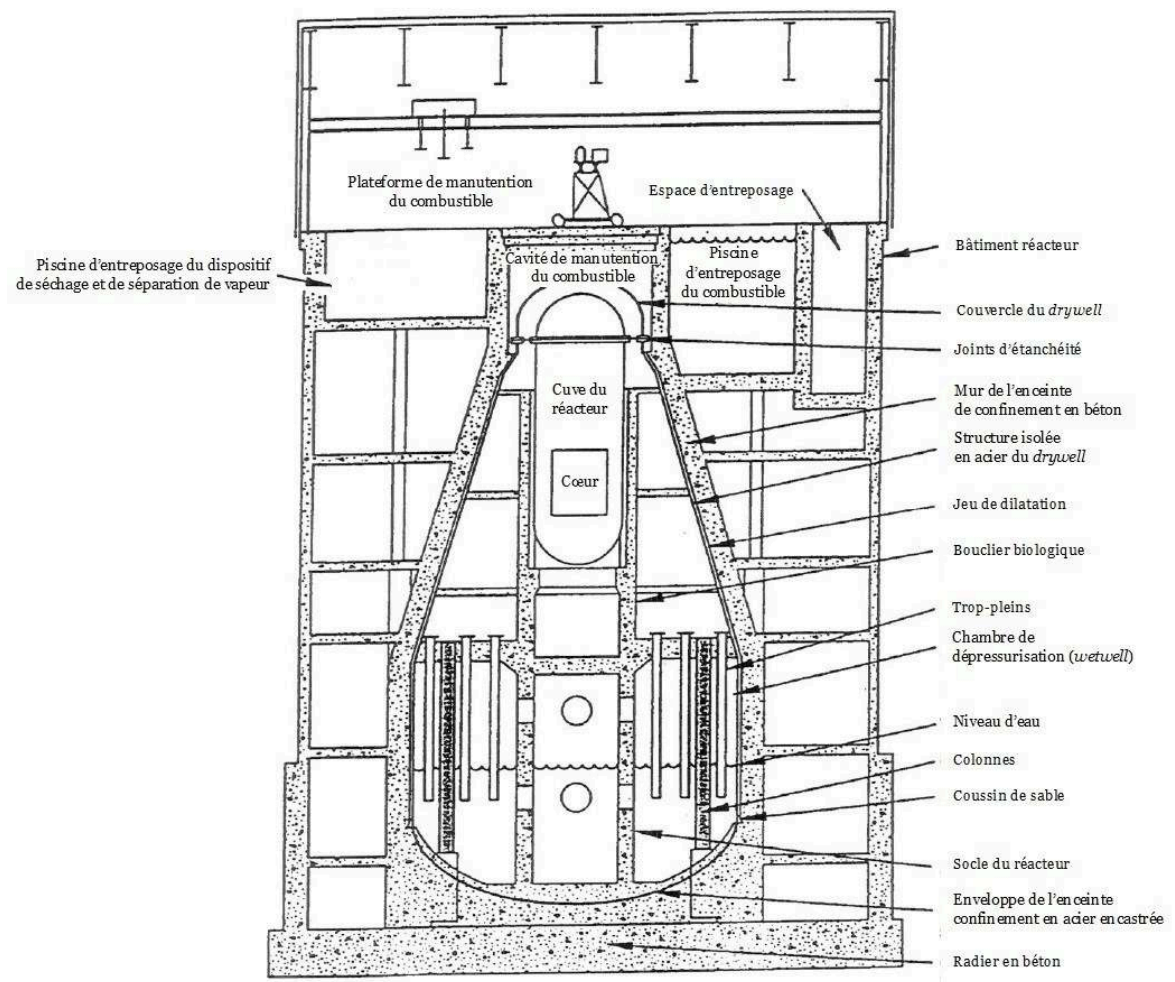




\title{
Un héros japonais
}

\author{
Franck Guarnieri et Aurélien Portelli
}

1 Le 9 juillet 2013, les médias annoncent la mort de Masao Yoshida. De nombreuses personnalités rendent alors hommage à l'ancien directeur de la centrale nucléaire de Fukushima Daiichi. L'ex-Premier ministre du Japon Naoto Kan écrit sur Twitter : "Je m'incline profondément par respect pour son leadership et sa détermination. J'aurais souhaité avoir eu la chance de lui parler longuement de la catastrophe nucléaire » (The Guardian, 2013 [notre traduction]). Tatsujiro Suzuki, vice-président de la Commission de l'Énergie Atomique Japonaise, rappelle la difficulté de l'épreuve qu'il a traversée : « Je ne peux pas imaginer à quel point cela a été dur pour lui. Il a dû décider que la plupart des travailleurs sur site devait partir car la situation empirait et il a également dû laisser une partie de son personnel travailler avec lui. Ce fut probablement la décision la plus difficile qu'il ait jamais eu à prendre " (Washington Post, 2013 [notre traduction]). Naomi Hirose, le président directeur général de l'exploitant TEPCO, déclare: "Nous apprécions profondément sa contribution et la façon dont il a géré l'accident. Nous gardons ses souhaits dans nos cœurs et ferons tout notre possible pour reconstruire Fukushima, qu'il a essayé de sauver à tout prix » (The Guardian, 2013 [notre traduction]).

Qualifié de « héros national » (Japan Times, 2013), Yoshida bénéfice de la reconnaissance de ses concitoyens, et en particulier celle des habitants de la préfecture de Fukushima : "'Je prie sincèrement pour son âme', a confié à l'AFP un ex-habitant de Tomioka, chassé de sa maison par les radiations. 'Le décès de cet homme qui s'est tant dévoué pour nous est très regrettable', dit une autre à la télévision. 'S'il n'avait pas été là, la région n'existerait plus', renchérit un troisième " (Le Temps, 2013).

3 Le dévouement de Yoshida ne prend toutefois sens qu'en le reliant à l'action des travailleurs de la centrale, que le journaliste Ryûshô Kadota a rencontrés pour préparer son ouvrage On the Brink (2014)1. Ce dernier rapporte que "Tous les ouvriers savaient que la mort était au bout. Mais quand je les ai interviewés, tous ont déclaré : 'Cela ne me faisait rien de mourir avec M. Yoshida', et 'avec un autre directeur que M. Yoshida, l'accident n'aurait pas été stoppé'. Voilà ce qu'ils répétaient tous. S'ils sont tous entrés dans le bâtiment du réacteur hautement contaminé, au péril de leur vie, c'est parce qu'ils le faisaient pour un chef en qui ils avaient une totale confiance et un grand respect. Chaque fois qu'une équipe rentrait, M. Yoshida leur serrait la main l'un après l'autre pour les remercier : 'Bien joué ! Merci d'être rentré vivant!' 
" (Kadota, 2013). Ému par l'annonce de sa disparition, un ancien salarié d'une entreprise partenaire de TEPCO exprime son admiration pour Yoshida : «Il aurait mérité une médaille [...]. La situation était si grave que, s'il n'avait pas été là, on ne s'en serait pas sorti » (Le Monde, 2013).

4 Ce statut de chef charismatique et aimé des siens se retrouve dans le surnom qui lui a été attribué : «Oyabun (親分) $»^{2}$, terme utilisé pour désigner le chef de bande chez les Yakusas ${ }^{3}$. Appeler Yoshida de la sorte ne répond nullement à un motif diffamatoire, bien au contraire. Pour le comprendre, il est nécessaire d'analyser le mot et d'en révéler le sens profond. Le premier caractère 親 (qui correspond ici au phonème oya) signifie "parent, membre de la famille». Le second, 分 (bun), signifie «minute» ou « couper ». L'ensemble désigne un chef entretenant des relations plutôt parentales et fraternelles avec ses subordonnés. En général, ces derniers appellent l'oyabun du terme affectif et respectueux d'oncle (ojisan). Puisqu'ils ont un parent commun, les subordonnés de l'oyabun sont semblables à des frères. C'est ainsi qu'il faut interpréter le surnom donné à Yoshida. Un homme qui était pour les travailleurs de Fukushima bien plus qu'un directeur d'usine.

5 Yoshida est né le 17 février 1955 à Osaka, ville située dans la région du Kansai. Il est enfant unique et ses parents possèdent une petite agence de publicité. Masao consacre une grande partie de sa jeunesse à la pratique du kendo ${ }^{4}$. Du fait de sa taille (il mesure 1,84 mètre), il ne passe probablement pas inaperçu auprès des autres kendokas du dojo. Après le lycée, il poursuit ses études en génie nucléaire dans la prestigieuse Université de technologie de Tôkyô. C'est durant sa formation académique qu'il rencontre Yoko, qu'il épouse en 1980. Le couple aura trois enfants.

6 Après l'obtention de son diplôme d'ingénieur, Yoshida est embauché par TEPCO en 1979. Sa carrière commence à Fukushima Daini, centrale nucléaire située à quelques kilomètres de Fukushima Daiichi, où il s'occupe de la construction du réacteur 2. En 1982, il est affecté au siège social au service "maintenance » des centrales nucléaires. En 1986, il est muté au service "maintenance » de Fukushima Daiichi et devient souschef du groupe des réacteurs 5 et 6 . En 1988, il retourne au siège de TEPCO. Il est souschef du service "projet énergétique » du département «technologie » jusqu'en 1991, puis sous-chef du service "maintenance » jusqu'en 1993. Il rejoint ensuite Fukushima Daiichi pour devenir chef du service «maintenance » pour les réacteurs 1 et 2. En 1995, il est envoyé à la Fédération des Compagnies d'Électricité du Japon, où il intègre le département "énergie nucléaire ». De 1999 à 2002, il est directeur du département "production » de Fukushima Daini. De 2002 à 2005, il revient au siège et devient Group manager du département "maintenance». Il quitte ce poste en 2005 pour devenir directeur d'unité chargé de l'exploitation et de la maintenance des réacteurs 1 à 4 de Fukushima Daiichi. En 2007, il est nommé directeur du département "gestion des installations nucléaires" au siège de l'exploitant. Le 28 juin 2010, il est nommé directeur de la centrale nucléaire de Fukushima Daiichi.

7 Les nombreux domaines d'expertise de Yoshida et la variété des postes qu'il a occupés durant sa carrière ont certainement été des atouts indéniables lorsque s'est produite la crise nucléaire. Au regard de son expérience professionnelle, Yoshida apparaît ainsi comme "l'homme de la situation", d'autant plus que le directeur de Fukushima est connu pour son inflexibilité et sa tendance à tenir tête à ses supérieurs, comme le confirme son entourage: "C'était un profil atypique parmi les employés de TEPCO, on le remarquait » (Le Temps, 2013), reconnaît le président de l'entreprise. Un épisode de la 
gestion de crise l'illustre particulièrement: "Le meneur d'équipe Yoshida a même eu la présence d'esprit et le courage de désobéir à des ordres jugés techniquement aberrants venus d'en haut. S'il avait cessé d'injecter de l'eau de mer dans le réacteur numéro un comme on le lui enjoignait le 12 mars, la situation aurait pu devenir incontrôlable, et le scénario du pire, celui de l'évacuation de Tôkyô, qui existait sur le papier, aurait alors dû être exécuté » (Le Temps, 2013) $)^{5}$.

8 Yoshida était décidé à aller jusqu'au-bout. Il s'était résolu à mourir, comme le souligne un journaliste, le qualifiant de «capitaine d'un Titanic de l'ère atomique " (The Independent, 2013 [notre traduction]) prêt à sombrer avec son navire. Le New York Times (2013) rappelle également que Yoshida avait proposé de mener une mission suicide avec d'autres responsables plus âgés pour empêcher la destruction des réacteurs, avant que l'exploitant ne l'en dissuadât.

9 Ingénieur, directeur paternaliste, chef insoumis, héros, sauveur. Masao Yoshida incarne toutes ces figures à la fois. Des figures renvoyant l'image d'un personnage hors normes, pris dans la tourmente d'un "évènement-monde» (Sirenelli, 2002) qui a marqué l'histoire, en particulier celle de la "jeune » industrie nucléaire née après la Seconde Guerre mondiale.

\section{Une histoire d'énergie et d'accidents}

10 Le 2 décembre 1942, le réacteur Chicago Pile-1 conçu par Enrico Fermi diverge sous les gradins du stade de l'Université de Chicago dans l'Illinois (États-Unis). Étape cruciale du projet Manhattan', cette prouesse ouvre la voie d'une nouvelle ère. Le 16 juillet 1945, un essai dénommé "Trinity" est mené dans le champ de tir d'Alamogordo, au Nouveau-Mexique (États-Unis), et aboutit à l'explosion de "Gadget ", la première bombe atomique de l'histoire. Le 6 août, à 2 h45 (heure locale), le Boeing B-29 Superfortress Enola Gay largue une bombe à l'uranium 235 sur la ville japonaise d'Hiroshima, éliminant instantanément 70000 personnes. Le 9 août, une bombe au plutonium détruit Nagasaki, faisant 40000 morts $^{7}$. Le 15 août, l'empereur Hirohito annonce la reddition du Japon et l'acte de capitulation est signé le 2 septembre. La guerre du Pacifique s'achève ainsi sur fond d'apocalypse nucléaire.

11 Le développement des premiers réacteurs électrogènes apporte bientôt une "caution morale » (Marguet, 2012) à l'atome. Le 20 décembre 1951, à 13h50, le réacteur EBR-18 est mis en service dans le laboratoire national de l'Idaho (États-Unis). Ce jour-là, il produit suffisamment de courant électrique pour éclairer quatre lampes de 200 watts. Dès le lendemain et jusqu'à son arrêt, il produit assez de courant pour alimenter le bâtiment qui l'abrite. Le 27 juin 1954, la centrale d'Obninsk ${ }^{9}$ en Russie (Union soviétique) est la première centrale du monde à être raccordée à un réseau électrique. Le 7 janvier 1956, le réacteur $\mathrm{G} 1^{10}$ diverge dans le centre du Commissariat à l'Énergie Atomique (CEA $)^{11}$ de Marcoule dans le département du Gard et commence à produire de l'électricité le 28 septembre. La centrale de Calder Hall, située sur le site de Windscale (Royaume-Uni), est connectée au réseau électrique le 17 octobre $1956^{12}$.

12 Le contexte international des années cinquante profite aux pionniers de l'industrie nucléaire. Le 8 décembre 1953, le président américain Dwight Eisenhower prononce devant l'Assemblée générale des Nations unies un discours intitulé « Atoms for Peace ", qui vise à promouvoir l'utilisation du nucléaire à des fins pacifiques. Le 8 août 1955, s'ouvre la Conférence internationale sur l'énergie atomique à Genève. Le Traité 
Euratom, signé le 25 mars 1957 à Rome en même temps que le Traité instituant la Communauté Économique Européenne (CEE), fonde la Communauté Européenne de l'Énergie Atomique. En réponse aux vœux du président Eisenhower, l'Agence Internationale de l'Énergie Atomique (AIEA) est créée le 29 juillet 1957, en tant qu'organisme mondial de «l'atome pour la paix ».

13 Le nucléaire connaît son âge d'or, mais les premiers accidents de réacteurs ne tardent pas à se produire. Le 12 décembre 1952, une explosion endommage gravement la calandre ${ }^{13}$ du réacteur expérimental $\mathrm{NRX}^{14}$, situé dans les laboratoires de Chalk River en Ontario (Canada). L'accident, qui sera classé au niveau 5 de l'échelle INES ${ }^{15}$, ne fait pas de victimes mais la calandre, hautement radioactive, doit être enterrée. L'incidence radiologique de l'évènement, mal déterminée, est considérée comme insignifiante. Le 10 octobre 1957, un incendie se déclare dans le réacteur 1 de Windscale ${ }^{16}$. La situation est maîtrisée le 12 octobre, mais une quantité importante de matières radioactives est relâchée et contamine la région. À l'instar de NRX, Windscale sera classé au niveau 5 de l'échelle INES. Le 17 octobre 1969, les opérateurs de la centrale de Saint-Laurent-desEaux dans le Loir-et-Cher procèdent au renouvellement des éléments combustibles du réacteur Saint-Laurent $\mathrm{A} 1^{17}$. $\mathrm{Au}$ cours de la manœuvre, ils font une erreur de chargement, entravant la bonne circulation du gaz carbonique qui sert de réfrigérant. Le combustible commence à surchauffer et 50 kilogrammes d'un mélange d'uranium et de magnésium entrent en fusion, libérant des produits radioactifs et des gaz rares. L'accident, classé au niveau 4 de l'échelle INES ${ }^{18}$, ne semble pas avoir engendré de contamination importante pour l'environnement et les travailleurs ${ }^{19}$.

14 Le 28 mars 1979, un accident bien plus important se produit dans la centrale américaine de Three Mile Island (TMI) ${ }^{20}$, située en Pennsylvanie (États-Unis). Il a pour origine un incident d'exploitation, suivi d'une erreur d'interprétation par les opérateurs. La situation est rétablie en fin de journée, mais le cœur du réacteur est fortement endommagé. Le 30 mars, l'exploitant Exelon effectue d'importants rejets depuis la cheminée de la centrale. La Nuclear Regulatory Commission (NRC) ${ }^{21}$ recommande au gouverneur de l'État de faire évacuer les femmes enceintes et les enfants dans un rayon de 8 kilomètres. La médiatisation de l'accident et la multiplication d'informations contradictoires suscitent la panique et 200000 personnes fuient la région. Le président Jimmy Carter finit par se rendre sur place et visite la centrale pour rassurer ses concitoyens.

15 Les conséquences radiologiques de TMI restent limitées, l'enceinte de confinement du réacteur ayant joué son rôle. L'accident, qui sera classé au niveau 5 de l'échelle INES, met cependant un terme à la certitude que le niveau de sûreté des centrales nucléaires est suffisant. Le 11 avril 1979, Carter instaure une commission d'enquête, présidée par le mathématicien et informaticien John George Kemeny. Son rapport, remis le 30 octobre 1979, réclame des changements fondamentaux pour continuer d'exploiter le parc nucléaire américain en maintenant les risques sous un seuil acceptable. Par la suite, les enseignements de TMI refaçonnent les démarches de gestion des risques dans les industries. La littérature scientifique rend en particulier saillante la question du "facteur humain" et l'étude psychologique des mécanismes d'« erreur humaine" (Reason, 2013 [1 ${ }^{\text {ère }}$ éd. 1990]) dans l'étiologie des accidents.

16 Si TMI marque profondément les esprits - l'évènement est alors considéré comme un "Vietnam » pour la technologique américaine -, l'accident nucléaire le plus grave de l'histoire survient toutefois en Ukraine (Union soviétique) dans la nuit du 26 avril 
$1986^{22}$. À $1 \mathrm{~h} 23$, le réacteur $4^{23}$ de la centrale nucléaire Lénine, située près de la ville de Pripiat à 18 kilomètres de Tchernobyl et dirigée par Viktor Brioukhanov, explose à la suite d'un essai technique au cours duquel les opérateurs transgressent les procédures de sûretée ${ }^{24}$. L'explosion diffuse une grande quantité de produits radioactifs dans l'atmosphère et contamine de vastes territoires en Europe. Le nombre de décès par cancer causés par la catastrophe varie selon les études de quelques milliers à près d'un million de morts. Entre 1986 et 1990, environ 600000 liquidateurs viennent des quatre coins de l'Union soviétique pour intervenir sur le site accidenté. Un sarcophage est fabriqué à la hâte pour confiner le réacteur détruit. Il est recouvert par une arche monumentale, finalisée en décembre 2018.

17 Tchernobyl, classé au niveau 7 sur l'échelle INES ${ }^{25}$, conduit de nouveau à évaluer la sécurité des centrales nucléaires. L'INSAG ${ }^{26}$, groupe consultatif établi par le Directeur général de l'AIEA, publie en 1987 un rapport qui mentionne pour la première fois le concept de "culture de sûreté », précisé dans deux autres rapports publiés respectivement en 1990 et en $1991^{27}$. Ce concept clé est par la suite étendu aux autres industries à haut risque.

18 L'accident soviétique, en plus de souligner comme TMI l'importance du facteur humain, révèle des défaillances organisationnelles, qu'il s'agit de résorber pour éviter une nouvelle catastrophe nucléaire. Mais vingt-cinq ans plus tard, le Japon, une grande puissance technologique disposant de l'un des parcs nucléaires les plus importants du monde, est victime à son tour d'un accident grave.

\section{L'archipel du nucléaire}

Le 19 décembre 1955, la loi fondamentale sur l'énergie nucléaire est adoptée au Japon, qui reconstruit sa puissance économique perdue depuis sa défaite contre les États-Unis. Le pays s'engage alors dans le développement d'un programme nucléaire civil pour répondre à un manque en ressources énergétiques fossiles ${ }^{28}$. Le premier réacteur nucléaire du Japon est construit dans la centrale de Tôkai (préfecture d'Ibaraki), exploitée par la Japan Atomic Power Company (JAPC). Ce réacteur britannique de type Magnox $^{29}$ est mis en service en juillet 1966. Les réacteurs suivants sont construits par des entreprises américaines : Westinghouse pour les Réacteurs à Eau Pressurisée (REP) ${ }^{30}$ et General Electric (GE) pour les Réacteurs à Eau Bouillante (REB) ${ }^{31}$. Les entreprises japonaises Mitsubishi, Tôshiba et Hitachi, d'abord associées aux Américains pour développer les premiers réacteurs expérimentaux de l'Archipel, construisent à leur tour des réacteurs commerciaux.

La construction de nouveaux réacteurs continue sans interruption. Le dernier est le réacteur 3 de la centrale de Tomari ${ }^{32}$, mis en service en 2009. À la veille du séisme du Tôhoku, le parc nucléaire du Japon se compose de cinquante-quatre réacteurs nucléaires (trente-quatre REB et vingt REP) répartis dans dix-huit centrales. Celles-ci fournissent le tiers de l'électricité du pays, plaçant le Japon au troisième rang mondial en matière de production d'énergie électronucléaire - derrières les États-Unis (centquatre réacteurs répartis dans soixante-dix centrales en 2011) et la France (cinquantehuit réacteurs répartis dans dix-neuf centrales en 2011). Ces centrales sont exploitées par neuf compagnies différentes, dont la Tôkyô Electric Power Company (TEPCO), fondée en 1951. Avant le 11 mars 2011, TEPCO exploite dix-sept réacteurs nucléaires, répartis sur les centrales de Fukushima Daiichi (6), Fukushima Daini (4) et Kashiwazaki- 
Kariwa (7). L'entreprise exploite également cent-soixante installations hydrauliques et vingt-cinq centrales thermiques.

21 TEPCO commence en 1967 la construction du réacteur 1 de la centrale nucléaire de Fukushima Daiichi, localisée dans la préfecture de Fukushima, à environ 250 kilomètres au nord de Tôkyô. Ce réacteur (conçu par GE) est mis en service en mars 1971. Le réacteur 2 (également construit par GE) est mis en service en juillet 1974, le réacteur 3 (Tôshiba) en mars 1976, le réacteur 4 (Hitachi) en octobre 1978, le réacteur 5 (Tôshiba) en avril 1978 et le réacteur 6 (GE-Tôshiba) en octobre 1979. Ces six réacteurs à eau bouillante sont répartis face à l'océan en deux ensembles : d'un côté les réacteurs 1 à 4 , de l'autre les réacteurs 5 et $6^{33}$.

\section{Face à l'abîme}

Le 11 mars 2011, environ 6400 personnes, dont 750 employés de TEPCO, se trouvent sur le site de Fukushima Daiichi. Les réacteurs 1, 2 et 3 fonctionnent alors que les réacteurs 4,5 et 6 sont en arrêt programmé. Le combustible du réacteur 4 est déchargé dans son bassin de désactivation. À $14 \mathrm{~h} 46$ (heure locale), un séisme de magnitude 9 se produit. Son épicentre se situe dans l'océan Pacifique à 370 kilomètres au nord-est de Tôkyô. La durée de la secousse enregistrée dans la capitale est de plus de deux minutes. Les systèmes d'arrêt d'urgence des réacteurs 1 à 3 sont automatiquement activés. Les employés de TEPCO qui ne sont pas chargés de piloter les réacteurs et les sous-traitants encore sur place se réfugient dans le bâtiment antisismique, qui abrite la cellule de gestion de crise, dirigée par Masao Yoshida. L'Agence Météorologique du Japon lance une série d'alertes au tsunami. Les vagues les plus hautes, de 11,5 à 15,5 mètres d'amplitude, atteignent la côte à $15 \mathrm{~h} 37$. Leur puissance ravage le site et inonde les installations. Les pompes, les panneaux électriques, les batteries et les générateurs diesel d'urgence sont endommagés. Les opérateurs sont plongés dans l'obscurité, tandis que les instruments de mesure et de commande ne fonctionnent plus. L'alimentation en courant continu du réacteur 3 est maintenue. Le générateur du réacteur 6 résiste également à l'inondation. Il est utilisé alternativement sur les réacteurs 5 et 6 pour continuer leur refroidissement. Les routes qui relient les installations sont difficilement praticables. Le site est jonché de gravats, de débris de machines, de véhicules renversés. Les épaves, impossibles à évacuer, gênent considérablement les manœuvres. À 16h36, Yoshida informe le Gouvernement de la situation, conformément au cadre législatif. La vidéoconférence reliant la cellule de gestion de crise, les autres centrales nucléaires de TEPCO et le siège de l'exploitant situé à Tôkyô, constitue le principal lien entre Fukushima Daiichi et l'extérieur.

$\mathrm{Au}$ fil des jours, les cœurs des réacteurs 1 à 3 entrent en fusion et quatre explosions se produisent. La première survient dans le bâtiment réacteur 1 le 12 mars à $15 \mathrm{~h} 36$, la deuxième dans le bâtiment réacteur 3 le 14 mars à 11h01, la troisième dans le bâtiment réacteur 2 le 15 mars à $6 \mathrm{~h} 10$ et la quatrième dans le bâtiment réacteur 4 le 15 mars à 9h38. Ces explosions font des blessés et produisent une forte pollution radioactive. Confrontés à un outil de production devenu incontrôlable, les opérateurs disposent de moyens dérisoires pour tenter de contenir la fusion des réacteurs. Ils manquent de matériel, notamment de combinaisons de protection, de masques respiratoires ou de dosimètres individuels. Ces pénuries rendent le travail plus difficile et dangereux. Soumises à des contraintes extrêmes, les équipes font preuve d'ingéniosité et recourent 
à des manœuvres non spécifiées dans les procédures de gestion d'accident. Elles utilisent des pompes à incendie à moteur diesel et des camions de pompiers pour injecter de l'eau dans le cœur des réacteurs. Elles ont également l'idée de raccorder des batteries de voiture au tableau électrique du réacteur 3. Au prix d'un combat inouï, les travailleurs parviennent à reprendre prise sur les installations et à arrêter à froid les réacteurs. Depuis lors, l'exploitant se charge de mener les opérations de décontamination et de démantèlement du site, programmées sur plusieurs décennies ${ }^{34}$.

\section{Le Tchernobyl japonais}

L'accident de Fukushima occasionne des rejets radioactifs dans l'atmosphère ${ }^{35}$, qui entraînent une contamination des territoires autour de la centrale. Selon la NRC (2014), $13000 \mathrm{~km}^{2}$ de sol sont devenus inhabitables du fait de cette contamination radioactive. L'accident provoque également une forte contamination du milieu marin, du fait du déversement, jusqu'au 8 avril 2011, d'eau contaminée depuis la centrale et des retombées dans l'océan des éléments radioactifs rejetés dans l'atmosphère entre le 12 et le 22 mars $2011^{36}$.

Les populations riveraines sont progressivement évacuées en fonction de l'évolution de la crise nucléaire. Une série d'ordres étend ainsi le périmètre d'évacuation autour de la centrale de 2 kilomètres le 11 mars à 30 kilomètres le 15 mars. Au total, environ 150000 personnes sont évacuées ${ }^{37}$. Des mesures sanitaires sont également prises par le Gouvernement, qui promulgue des directives concernant la décontamination des sols ${ }^{38}$ et interdit la commercialisation de denrées alimentaires dépassant les normes de contamination ${ }^{39}$.

$\mathrm{Au}$ Japon, l'accident nucléaire met en difficulté un modèle de gouvernance fragilisé par des années de crise. Fukushima renvoie l'image d'une démocratie minée par le manque de transparence des institutions, la collusion des élites, le pouvoir des compagnies d'électricité, et le déficit de participation citoyenne à l'élaboration des politiques énergétiques du pays. Dans les territoires contaminés, les communautés sont détruites et le tissu économique est quasiment anéanti. Le sort des réfugiés, la peur de la contamination radioactive, les incertitudes sur la fiabilité des informations fournies par les autorités, inquiètent fortement la population japonaise. La politique de gestion de crise puis de reconstruction menée par le Gouvernement de Naoto Kan et son parti politique $^{40}$ est fortement critiquée par l'opposition. De plus en plus impopulaire et discrédité, le Premier ministre est contraint de démissionner le 26 août $2011^{41}$.

27 Au plan économique, Fukushima alourdit considérablement la facture énergétique du Japon. Le parc nucléaire nippon est arrêté en avril 2012 pour inspection ${ }^{42}$. Cet arrêt prive le pays d'un tiers de sa production électrique, le conduit à recourir massivement aux hydrocarbures - en provenance des partenaires commerciaux étrangers - mais aussi à développer davantage les énergies renouvelables telles que l'hydraulique.

Fukushima, en plus d'avoir des conséquences catastrophiques au Japon, produit également une rupture d'ordre géopolitique, puisque l'accident prouve qu'une grande puissance technologique n'est pas à l'abri d'un accident nucléaire majeur, et une rupture d'ordre politique, au sens où l'évènement conduit plusieurs pays à modifier leur politique énergétique (Topçu, 2013). 
29 Au plan des représentations, Fukushima est immédiatement perçu à travers le prisme de Tchernobyl, symbole de la démesure technologique et de l'illusion du pouvoir exercé par l'homme sur la nature ${ }^{43}$. Des voix s'élèvent pour constater que les leçons de l'accident soviétique n'ont pas été apprises : «Nous sommes sur la voie de l'autodestruction. J'ai eu immédiatement des images de Tchernobyl devant les yeux, les routes goudronnées, les câbles électriques qui ne mènent nulle part. Ne restait que l'herbe, les arbres, la nature " (Alexievitch, 2011). Devenu le "Tchernobyl du Japon», Fukushima pourrait selon certains auteurs rendre définitivement obsolète l'idée d'un nucléaire pacifique (Nespoulous, 2011), d'autant plus que l'accident se produit dans le seul pays à avoir été bombardé par des armes atomiques. Kenzaburô Oé remarque pourtant que les Japonais n'établissent pas de rapport entre Fukushima et Hiroshima : «Tous les intellectuels, mais pas seulement eux, tout Japonais devrait être en train de réfléchir à cette tragédie en cours et la relier à Hiroshima (...) J'ai essayé de traiter par la littérature le cauchemar de Hiroshima, et Fukushima nous met face à un nouveau drame. Mais de même que le Gouvernement n'a rien appris de Hiroshima, rien n'est fait pour tirer les conséquences de ce que peut nous apprendre Fukushima » (Oé, 2015). La signification imaginaire de la fin du monde au Japon éclaire ce rapport ambigu à l'atome. En effet, dans la culture nippone, la catastrophe ne constitue pas une fin et s'ouvre sur une renaissance : «L'apocalypse made in Japan porte en germe un avenir qu'il revient aux hommes de faire lever. Cela est vrai même de Hiroshima, et contribue à expliquer pourquoi le nucléaire civil s'est développé au Japon sans rencontrer l'opposition farouche qu'on eût pu attendre dans un pays qui avait subi le feu atomique. L'holocauste nucléaire, si horrible qu'il ait été, a fermé un cycle d'errements guerriers et de totalitarisme oppressif, pour enfanter un Japon nouveau, pacifiste, démocratique et prospère » (Bouissou, 2011).

30 La relation entre Hiroshima et Fukushima questionne également, au plan éthique, l'autonomisation du mal dans les sociétés modernes. Dans le premier cas, le mal provient de l'intention de commettre le mal, tandis que dans le second, le mal résulte de l'intention de faire le bien. Jean-Pierre Dupuy rappelle ainsi que les plus grandes menaces qui pèsent sur nos sociétés proviennent davantage des « industriels du bien » que des "méchants »: "On doit moins redouter les mauvaises intentions que les entreprises qui, comme l'Agence Internationale pour l'Énergie Atomique, se donnent pour mission d'assurer 'la paix, la santé et la prospérité dans le monde entier' ( (Dupuy, 2012, p. 51).

31 Fukushima conduit enfin, au plan historique, à réinterroger la théorie de la postmodernité. Pour Ulrick Beck, l'accident japonais confirme sa thèse sur la « société du risque " ([1986] 2001): "Le mythe de la sécurité est en train de se consumer dans les images de catastrophes dont les exploitants nucléaires avaient catégoriquement exclu la possibilité » (Beck, 2011). Mais pour Jean-Baptiste Fressoz (2014), Fukushima n'annonce pas plus que les catastrophes technologiques précédentes l'émergence d'un monde débarrassé des illusions de la modernité. D'une part, les sociétés n'ont jamais été réflexives - la construction de centrales nucléaires en zone sismique serait sinon restée inenvisageable. D'autre part, il est peu probable qu'elles le deviennent, car «après la catastrophe, les décisions vont dans le même sens : 'continuer comme avant'» (Fressoz, 2014, p. 187). Selon Beck, la conscientisation des risques technologiques apparaît durant les dernières décennies du $\mathrm{XX}^{\mathrm{e}}$ siècle. Les historiens montrent cependant que la question de l'industrialisation et des risques qui en dépendent est débattu dès la première moitié $\mathrm{du} \mathrm{XIX}^{\mathrm{e}}$ siècle. Les sociétés intègrent toutefois ces risques à l'ensemble du monde industriel, en accordant leur confiance au progrès pour les résorber (Fressoz, 2012 ; Le 
Roux, 2016). Or, «Sommes-nous vraiment sortis de ce monde-là? » (Le Roux, 2014, p. 20). Les enseignements tirés de Fukushima tendent à démontrer l'inverse.

\section{Le cygne noir}

$\mathrm{Au}$ Japon, les principaux rapports d'enquête sur l'accident ont été rédigés par la NAIIC $(2012)^{44}$, l'ICANPS $(2012)^{45}$, le Comité indépendant constitués de civils ${ }^{46}$, le Comité nommé par TEPCO (2012) et celui de l'Atomic Energy Society of Japan (AESJ, 2015). Des organisations internationales ont également réalisé des rapports d'enquête, comme l'AIEA (2015), l'Organisation de Coopération et de Développement Économiques et l'Agence pour l'Énergie Nucléaire (OECD/NEA, 2013), ou l'Institute of Nuclear Power Operations (INPO, 2011). Ces rapports tirent une série de leçons de l'accident, soulignant le manque d'entraînement des opérateurs de TEPCO pour faire face à des évènements semblables à ceux du 11 mars 2011, l'incompétence et l'impréparation des institutions impliquées dans la gestion de l'accident, le manque d'indépendance et de transparence de l'agence de sûreté nucléaire japonaise ${ }^{47}$, ou les défaillances en matière de communication entre les cellules de crise. Ils signalent que TEPCO n'a pas procédé à la réalisation de travaux de surélévation de la digue protégeant Fukushima Daiichi. Des études, connues de l'exploitant, avaient pourtant démontré que le site pouvait être submergé par une vague d'une hauteur plus importante que celle de la digue existante. De plus, l'évolution de l'état de l'art mondial en matière de sûreté nucléaire n'a pas été suffisamment prise en compte par TEPCO et les institutions japonaises. Ces derniers auraient dû en particulier mieux appliquer les concepts de «culture de sécurité » et de «défense en profondeur " $^{48}$. À partir de ces leçons, les rapports d'enquête préconisent principalement de renforcer l'application des concepts existants, de définir des standards plus élevés, de prendre des mesures considérant la possibilité d'accidents multiples et hors-dimensionnement, et de garantir la transparence et l'indépendance des instances régulatrices.

33 À l'international, Fukushima a pour conséquence de questionner la sécurité des parcs nucléaires en exploitation. En France, le Gouvernement réclame, le 23 mars 2011, la réalisation d'un audit de la sûreté des installations nucléaires françaises (Niel, 2013). Les 24 et 25 mars 2011, le Conseil européen demande à la Commission européenne et aux autorités de sûreté nucléaire européennes d'organiser des tests de résistance pour évaluer la robustesse des centrales nucléaires face à des situations exceptionnelles. C'est dans ce double cadre que des Évaluations Complémentaires de Sûreté (ECS) sont menées en France à partir de mars 2011. Dans son rapport sur les ECS publié le 3 janvier 2012, l'Autorité de Sûreté Nucléaire (ASN) ${ }^{49}$ déclare d'une part que le niveau de sûreté est suffisant pour ne réclamer l'arrêt immédiat d'aucune des installations examinées, et d'autre part la nécessité de renforcer leur robustesse face à des situations extrêmes pour permettre la poursuite de leur exploitation. Le 26 juin 2012, l'ASN adopte une série de mesures visant à renforcer les marges de sûreté de ces installations. Les exploitants doivent ainsi réaliser d'importants travaux et mettre notamment en place un « noyau dur $»^{50}$.

34 Fukushima, malgré son ampleur, n'a donc pas donné lieu, comme ce fut le cas après TMI et Tchernobyl, à une programmation scientifique redéfinissant les bases conceptuelles de la sûreté nucléaire. Les pratiques industrielles et institutionnelles ont été réinterrogées, des améliorations techniques ont été apportées, des dispositifs ont 
été créés pour augmenter la robustesse des installations face à des évènements extrêmes. Les réflexions engagées n'apportent pourtant aucune innovation majeure en matière de sécurité. En réponse à l'accident, les préconisations vont dans le même sens : renforcer les normes et les procédures. Or, Fukushima représente un "impensé » dans l'histoire de l'industrie nucléaire. D'une part, les équipes ont dû faire face, pour la première fois, à la dégradation simultanée de plusieurs réacteurs nucléaires. D’autre part, la centrale s'est retrouvée confrontée à une perte totale d'alimentation externe doublée d'une perte de distribution électrique interne, un scénario que nulle procédure n'avait pris en compte. Dans cette perspective, Fukushima relève de la logique du " cygne noir ", évènement caractérisé par trois critères principaux : "Premièrement, il s'agit d'une aberration; de fait, il se situe en dehors du cadre de nos attentes ordinaires, car rien dans le passé n'indique de façon convaincante qu'il ait des chances de se produire. Deuxièmement, son impact est extrêmement fort. Troisièmement, en dépit de son statut d'aberration, notre nature humaine nous pousse à élaborer après-coup des explications concernant sa survenue, le rendant ainsi explicable et prévisible » (Taleb, 2012, p. 10). Dans cette logique, les choses que nous ignorons comptent plus que celles que nous connaissons et il importe davantage, du fait de l'imprédictibilité des cygnes noirs, de nous adapter à leur existence plutôt que de tenter de les prévoir.

$\mathrm{Au}$ plan d'une industrie à haut risque, l'intérêt porté à l'adaptabilité des individus et des organisations signifie non pas de renoncer à la sécurité industrielle - ce qui serait à l'évidence une aberration -, mais de sortir de l'idéalisation des modèles de sécurité. Fukushima nous révèle en effet l'utopie d'une telle approche (Guarnieri et Travadel, 2020). Les opérateurs de la centrale n'ont pas pris leur décision en appliquant une procédure ou une clause contractuelle les liant à leur employeur. Leur action montre les limites d'une sécurité définie par l'application des référentiels normatifs. L'espoir de gérer une catastrophe industrielle sans considérer la manière dont les individus qui la vivent font sens de leur situation et reconstruisent un imaginaire fédérateur relève par conséquent de l'illusion. En cela, le témoignage des acteurs de la gestion de crise constitue une source précieuse, voire indispensable, pour saisir finement la profondeur de leur expérience et tenter de décrypter les transformations de tous ordres qui se sont opérées en eux, entre eux et avec leur environnement.

\section{D'un scandale médiatique...}

Les entretiens avec les acteurs ont été menés dans le cadre de travaux journalistiques, tels que On the Brink (2014) de Kadota, roman non-fictionnel qui a inspiré le scénario du film Fukushima'5051. Ils ont également été effectués dans le cadre de projets scientifiques. Les travaux entrepris par Yuki Kobayashi pour préparer sa thèse de doctorat ${ }^{52}$ l'ont ainsi conduit à interroger vingt-et-un acteurs, dont des ministres, des experts en sûreté nucléaire, des cadres supérieurs de TEPCO et des travailleurs de Fukushima Daiichi. Ces entretiens n'ont pas été reproduits dans leur intégralité, mais des extraits ont été insérés dans le manuscrit de thèse, apportant de nouveaux éclairages sur les mécanismes de la gestion de crise. Ils complètent en outre le corpus de témoignages effectués par les différents comités d'enquête sur l'accident. Ces derniers ont parfois procédé à l'audition d'un grand nombre d'acteurs, telle l'ICANPS, la commission gouvernementale, qui a interrogé 772 individus et réalisé au total près de 1479 heures d'entretiens. Parmi les témoins, se trouve Masao Yoshida, interrogé à six 
reprises entre juillet et novembre 2011. Ses auditions ont lieu dans le J-Village, un complexe sportif situé près de Fukushima Daiichi. Les enquêteurs, treize au total, changent en fonction des jours et des thématiques abordées. L'enregistrement des entretiens, réalisé sur un appareil numérique, dure environ vingt-huit heures.

Les comités d'enquête ne communiquent pas forcément les comptes rendus de leurs entretiens. Par exemple, la NAIIC, la commission d'enquête parlementaire, rend uniquement publiques les auditions qui n'ont pas été conduits à huis clos. Les auditions de l'ICANPS sont quant à elles entièrement réalisées à huis clos. Dans un premier temps, le Gouvernement ne prévoit pas de les communiquer. L'Asahi Shimbun ${ }^{53}$ parvient toutefois à se procurer des transcriptions de l'audition de Yoshida et en publie des extraits sur son site internet le 20 mai 2014. Une révélation suscite alors un scandale médiatique. Selon l'Asahi Shimbun, $90 \%$ des cadres de Fukushima Daiichi ont désobéi à Yoshida et ont pris la fuite après l'explosion survenue dans le bâtiment réacteur 2 le 15 mars pour se réfugier dans la centrale nucléaire de Fukushima Daini (The Asahi Shimbun, 2014). Cette information contredit le discours tenu par TEPCO, affirmant que la majorité des travailleurs a été temporairement évacuée du site. La polémique fait rage dans un contexte où la question du redémarrage des réacteurs est discutée au Japon dans les arènes politiques et médiatiques.

D'autres extraits des auditions de Yoshida sont diffusés les mois suivants, notamment dans le Sankei Shimbun ${ }^{54}$. Ce dernier s'en prend à l'Asahi et l'accuse d'avoir déformé la réalité. Selon le Sankei, la centrale a été abandonnée dans un climat de confusion et non intentionnellement dans un esprit de désobéissance. Le Japan Times (2014) soutient le même point de vue. Le Gouvernement s'oppose tout d'abord à la publication intégrale de la transcription des auditions, Yoshida ayant souhaité qu'elles demeurassent confidentielles ${ }^{55}$. Mais pour clarifier l'affaire et éviter que le déshonneur ne s'abatte sur le collectif - ce que Yoshida n'aurait certainement pas permis - le Gouvernement finit par accepter, le 9 septembre 2014, de mettre en ligne sur son site internet l'intégralité des auditions du directeur ${ }^{56}$. La vérité est rétablie : le directeur a en fait ordonné un retrait temporaire pour mettre ses équipes à l'abri, ne gardant sur le site que le personnel essentiel aux opérations ${ }^{57}$. L'Asahi reconnait avoir commis une erreur et présente ses excuses dans un éditorial. Le rédacteur en chef du journal est renvoyé et un correctif est apporté à l'article publié initialement.

\section{... à un projet d'édition}

Du fait de nos travaux de recherche à l'École des Mines de Paris et de la thématique de notre laboratoire de rattachement ${ }^{58}$, l'accident de Fukushima ne pouvait que susciter notre intérêt scientifique. Dès l'annonce de la nouvelle, nous scrutons les chaînes d'information en continu, nous établissons une organisation de veille en analysant finement les déclarations des autorités japonaises, puis françaises, en collectant des articles de presse, des rapports techniques de l'ASN et de l'Institut de Radioprotection et de Sûreté Nucléaire (IRSN) ${ }^{59}$, puis dans les semaines et mois qui suivent en étudiant les rapports des commissions d'enquête et les publications académiques sur l'accident.

Dans un premier temps, notre compréhension des faits nous conduit à associer l'action des équipes sur site à une faillite de l'ingénierie. Plutôt que d'étudier la phase la plus aigüe de la gestion de crise, située entre le 11 et le 15 mars 2011, nous préférons nous intéresser à la phase suivante, consistant à "réparer » les dégâts sur les installations. 
Observant Fukushima à travers le prisme d'un "accident qui dure ", nous étudions d'abord les solutions déployées par TEPCO et des entreprises partenaires pour assurer la gestion de l'eau contaminée sur le site ${ }^{60}$. À partir des données collectées, notre groupe de chercheurs définit le concept d'« ingénierie de l'urgence $»^{61}$.

En septembre 2014, nous suivons de près la polémique suscitée par l'Asahi Shimbun. Nous apprenons ensuite que le Gouvernement est sur le point de publier la totalité des auditions de Yoshida en japonais. Dès leur mise en ligne sur internet, nous téléchargeons les fichiers. À la fin du mois de septembre, nous contactons les principaux acteurs du nucléaire français (EDF, AREVA, CEA, ASN, IRSN). Aucun d'entre eux n'a encore décidé d'en investiguer le contenu. De son côté, la presse revient sur l'affaire de l'évacuation générale et les faits sont rétablis à la lumière de l'intégralité du récit de Yoshida. Impossible désormais d'accorder le moindre crédit à la version donnée par l'Asahi Shimbun.

Disposant d'un matériau inexploité, nous décidons de procéder à un essai de traduction. Nous rencontrons Tomoko Takesada, qui enseigne le Japonais aux élèves de l'École des Mines, et nous lui proposons de traduire en français quelques dizaines de pages. En les lisant, nous sommes immédiatement saisis par l'intensité du récit de Yoshida et convaincu de l'intérêt de le traduire en totalité. Le chantier est lancé en octobre 2014. Vue l'ampleur de la tâche, nous préférons séquencer le projet éditorial pour que le public puisse prendre rapidement connaissance du témoignage. Le premier volume paraît aux Presses des Mines en mars 2015, le deuxième en mars 2016 et le troisième en mars 2020. Nous envisageons dès lors de préparer pour la dixième commémoration de Fukushima une édition intégrale et augmentée des auditions, qui fait l'objet du présent volume.

Les rapports d'enquête ont formulé des recommandations qui ont permis de clôturer le sens donné à l'accident. La lecture du témoignage de Yoshida nous conduit toutefois à modifier notre regard sur les équipes sur site et à réinterroger le cas. Notre questionnement de recherche évolue et nous choisissons de délaisser les travaux de réparation menés par l'exploitant pour étudier l'expérience vécue par les travailleurs entre le 11 et le 15 mars 2011. À cette fin, nous constituons un cadre théorique principalement fondé sur les travaux d'Ivan Illich, de René Girard, de Cornélius Castoriadis et de Jean-Pierre Dupuy. À partir de ce cadre, nous définissons le concept de " situation extrême » dans un contexte industriel ${ }^{62}$ et nous lançons un programme de recherche donnant lieu à plusieurs projets de thèse sur Fukushima ${ }^{63}$.

\section{Une source inédite}

Le témoignage de Yoshida constitue une source inédite dans l'histoire des accidents nucléaires majeurs. Viktor Brioukhanov, le directeur de Tchernobyl, n'a en effet laissé aucun récit comparable sur son expérience de crise. Un tel témoignage, s'il avait existé, aurait cependant présenté des différences par rapport à celui de Yoshida en termes d'expertise et de restitution d'expérience. Au plan de la formation académique, Brioukhanov est ingénieur en thermodynamique. Nommé directeur de la centrale de Tchernobyl au début des années 1970, il est le parfait exemple d'une génération d'ingénieurs de qualification moyenne qui accède durant l'ère Brejnev à des postes élevés de responsables (Werth, 2006). Yoshida est ingénieur en génie nucléaire et a été formé dans l'une des meilleures universités mondiales. Les deux directeurs ne 
disposent donc pas du même niveau de compétence en ingénierie nucléaire. D’autre part, le directeur de Tchernobyl n'assiste pas à l'essai technique mené dans la nuit du 26 avril 1986. L'ingénieur en chef Nikolaï Fomine est également absent. C'est l'ingénieur en chef adjoint Anatoli Dyatlov qui est chargé de le remplacer et de diriger l'expérimentation. Le réacteur explose à $1 \mathrm{~h} 23$. Brioukhanov est prévenu par téléphone à $1 \mathrm{~h} 30$. Ce dernier affirme dans un entretien paru dans Libération qu'il est arrivé sur les lieux dix minutes plus tard (Cote, 1996). D'autres témoins contestent cette version. Il ne serait en fait arrivé qu'à $2 \mathrm{~h} 30$ - et Fomine à 4 h00 (Ackerman, 2006). Quelle que soit la version retenue, Brioukhanov n'a pas « vécu » l'explosion du réacteur, ni le tout début de la gestion de l'accident. À l'inverse, Yoshida est présent à Fukushima lorsque se produit la catastrophe naturelle à l'origine de l'inondation provoquant la perte des ressources électriques, la dégradation des cœurs des réacteurs et les explosions dans les bâtiments réacteurs. Brioukhanov apparait enfin comme un acteur de second plan dans la gestion de crise. En effet, la commission gouvernementale envoyée depuis Moscou et dirigée par Boris Chtcherbina, l'un des vice-présidents du Conseil des ministres de l'Union soviétique, a rapidement pris la direction des opérations menées sur le site. Brioukhanov s'est de plus plié aux injonctions de sa hiérarchie et n'a pas été à l'origine des initiatives décisives qui ont permis de juguler la catastrophe. A contrario, Yoshida a préservé son leadership et supervisé la gestion de crise sur site dans son intégralité ${ }^{64}$. Il a par ailleurs pris des décisions capitales, n'hésitant pas à désobéir à sa hiérarchie pour continuer à injecter de l'eau de mer pour refroidir les installations. En cela, Yoshida apparaît comme l'antithèse de Brioukhanov, soumis à l'hétéronomie du système bureaucratique sur lequel reposait le régime soviétique.

Ces différences soulignent d'autant plus la valeur exceptionnelle du témoignage de Yoshida, acteur majeur de la "bataille» de Fukushima. Yotaro Hatamura, professeur émérite de l'Université de Tôkyô et président de l'ICANPS, le reconnaît explicitement : parmi les 772 entretiens menés par la commission d'enquête gouvernementale, "L'audition du directeur de Fukushima Daiichi est la plus importante» (Kobayashi, 2019, p. 63). C'est la raison pour laquelle Hatamura a tenu a diriger tous les entretiens avec Yoshida ${ }^{65}$.

Malgré sa valeur, le témoignage de Yoshida présente pourtant une série de biais et d'imperfections qu'il ne s'agit pas d'occulter. Concernant la transcription des auditions, nous ne pouvons vérifier leur fiabilité car leur enregistrement numérique n'est pas accessible. Leur traduction du japonais au français implique également des arbitrages linguistiques, qui modifient inévitablement le matériau d'origine. N'ayant pas assisté aux échanges avec le témoin, ses silences, ses intonations ou sa gestuelle ne nous sont pas connus. Yoshida étant décédé en 2013, impossible d'appliquer de dispositif correctif en réalisant des sessions supplémentaires pour compléter le témoignage ou éclairer ses zones d'ombre.

47 Les entretiens ont été réalisés dans le cadre d'une enquête sur l'accident nucléaire. Comme les enquêteurs changent selon les jours d'audition, des questions sont redondantes et Yoshida est obligé de se répéter à plusieurs reprises. Les enquêteurs interrogent de plus le témoin sur des sujets parfois éloignés de son champ de compétences. En tant que directeur, Yoshida est à l'interface de la cellule de crise de la centrale nucléaire, du siège social de TEPCO et du Gouvernement. Il est certes ingénieur en génie nucléaire et a travaillé plusieurs années à Fukushima Daiichi. Yoshida n'est cependant pas pilote de réacteur, ne sait pas manipuler tous les systèmes de contrôle 
ou de sauvegarde, et n'a pas directement participé aux opérations menées sur le terrain. Certaines questions ne sont donc pas posées au bon interlocuteur et ne peuvent aboutir à des réponses satisfaisantes.

Yoshida a également des difficultés à se remémorer précisément certains épisodes de la gestion de crise. Traumatisé par l'évènement qu'il a vécu, il confie lui-même que sa mémoire fonctionne en pointillé depuis le 11 mars $2011^{66}$. Ces pertes de mémoire ne diminuent pourtant pas la valeur de son témoignage. Premièrement, les souvenirs de Yoshida sont suffisamment précis et nombreux pour enrichir la connaissance de l'accident. Deuxièmement, l'altération de la mémoire du témoin du fait de son traumatisme n'invalide pas l'essentiel du témoignage, qui vise "le sens humain de l'évènement " (Dulong, 2000, p. 119). Enfin, la dégradation et la modification de la mémoire constituent des phénomènes inévitables: "Les souvenirs qui gisent en nous ne sont pas gravés dans la pierre; ils ont non seulement tendance à s'effacer avec les années, mais souvent ils se modifient ou même grossissent, en incorporant des éléments étrangers " (Levi, 1989, p. 23). Yoshida a été auditionné de juillet à novembre 2011, autrement dit quelques mois après l'accident. Ce bref laps de temps entre l'évènement et la constitution de la source est une aubaine, car les récits oraux sont parfois recueillis des années, voire des décennies après les faits - en témoignent les récits concentrationnaires.

De manière inéluctable, la mémoire du témoin continue de se transformer en fonction de son expérience de vie, de son regard introspectif et de ses interactions sociales. La littérature repère ainsi plusieurs "effets pervers »: la reconstruction, l'extrapolation, la hiérarchisation, le téléologisme (Peschanski, 1992), auxquels peuvent s'ajouter l'effet d'adresse (à la postérité), l'effet de reconnaissance et de transmission (Descamps, 2005). Il est néanmoins possible d'observer positivement ces effets: "Les erreurs, les réinterprétations, les approximations, les dérapages verbaux et les marques de subjectivité inhérentes au témoignage sont parfois plus révélatrices des réalités vécues par leur protagoniste que des données plus factuelles. Ce qui importe, c'est que le récit fasse sens» (Pirlot, 2007, p. 32). Or, ce qui fait sens dans le récit de Yoshida renvoie à la question de l'agir en situation extrême qui, dans le cadre d'un accident nucléaire majeur, confronte les acteurs à des enjeux industriels et sociétaux inouïs.

\section{La situation extrême}

Le témoignage de Yoshida nous procure l'extraordinaire opportunité d'étudier une organisation d'ingénierie soumise à une situation extrême, ce qui constitue une première du fait de la difficulté de « rencontrer » ce type de collectif et de recueillir des données sur ses pratiques. L'imminence du danger auquel les opérateurs sont exposés durant la crise nucléaire les confronte à l'impensable, bouleversant leurs croyances et leurs valeurs, affectant chez eux leur rapport à soi, aux autres et au monde. Le tsunami du 11 mars 2011 provoque des dégâts incommensurables sur le site de Fukushima Daiichi, et balaie l'imaginaire de maîtrise sur laquelle se fonde la sûreté nucléaire. Cet effondrement de l'imaginaire anéantit ce qui donne sens à l'action dans un contexte qui diffère radicalement d'une situation normale d'exploitation. Faire face à la situation extrême réclame alors le déploiement de diverses stratégies de résilience pour permettre au sujet de rétablir l'intégrité de son système symbolique et de reprendre le contrôle de son outil de production. 
51 En cela, le témoignage de Yoshida éclaire la fabrique de sens qui permet au directeur de dépasser le stade de la sidération, causée par la perte des ressources électriques à la suite de l'inondation de la centrale, et de reprendre prise sur le réel. Cette sortie de l'état de stupeur renvoie chez Yoshida à un renversement de conception du temps. Après l'arrivée de la vague, il réalise immédiatement l'inévitabilité de la catastrophe à venir. Cette prise de conscience d'une fin inexorable donne sens à son action, que Yoshida inscrit dans la "temporalité du projet » (Dupuy, 1994, 2004), où un point de référence est choisi sur un horizon futur pour conditionner une action dans le présent afin de déjouer la prophétie d'anéantissement. Il se fixe dès lors pour mission de mobiliser tous les moyens à sa disposition afin d'empêcher la fusion des réacteurs. Cet imaginaire temporel du projet permet à Yoshida de se libérer de la contrainte de suivre des procédures de gestion des accidents - dont il se rend très vite compte de l'inapplicabilité dans une situation aussi dégradée -, de redéfinir son rapport au monde et d'envisager des solutions entièrement novatrices.

Privés des instruments de mesure nécessaires à la surveillance de l'évolution de l'état des installations, les opérateurs ont ainsi été contraints de réinventer leur pratique pour s'attacher à des dimensions sensuelles habituellement délaissées, s'imprégnant des rythmes des machines, écoutant les bruits des tuyauteries, ressentant les vibrations des pompes pour déterminer à partir des informations recueillies l'efficacité de leur action (Travadel et Guarnieri, 2019).

53 Obligé d'improviser avec ses équipes, Yoshida adopte également une attitude de transgression à l'égard de la règle écrite en prenant des décisions pratiques. L'exemple le plus édifiant reste sa décision de continuer l'injection d'eau de mer, malgré l'ordre donné par sa hiérarchie d'interrompre l'opération. Que le directeur puisse décider et agir avec une telle autonomie est difficilement acceptable pour les autorités réglementaires ou les exploitants, qui font reposer la sûreté nucléaire sur le respect absolu des règles et des procédures. Pourtant, la réponse à la perte des ressources électriques à Daiichi n'a pas consisté à appliquer la norme définie dans les manuels, mais sur la capacité de Yoshida et de ses équipes à s'autonomiser et à faire preuve de créativité pour empêcher la destruction du Japon.

\section{Les broyés de Fukushima}

Le témoignage, lorsqu'il évoque des évènements violents telles que les situations extrêmes, permet par ailleurs de porter à connaissance la souffrance humaine, en particulier lorsqu'elle est ressentie par des groupes sociaux écartés de l'histoire "vue d'en haut». Florence Descamps qualifie cette catégorie d'acteurs de "broyés » de l'histoire : "Nous pensons là à tous les persécutés du pouvoir, à ceux qui ont subi des violences politiques ou raciales, à ceux qui ont fait l'objet de persécutions religieuses, aux déportés, aux exilés, aux dissidents, aux victimes des guerres, aux combattants pris dans l'engrenage des crimes de guerre, aux perdants ou aux vaincus des guerres civiles françaises et étrangères, aux "soldats perdus" des causes perdues, et plus largement aux victimes de ce que l'on appelle aujourd'hui la "culture de guerre" et dont la souffrance, soit ne laisse guère de trace dans les archives écrites, soit est traduite imparfaitement par l'écrit " (Descamps, 2005). Parmi cette suite de figures, nous pensons pouvoir discerner les travailleurs de Fukushima, soumis à trois modalités de broyage. 
La première correspond à la prise de conscience d'un malentendu. Pour des raisons de confort moral, les personnels travaillant dans un établissement dangereux ne doivent pas se rappeler en permanence l'existence de cette dangerosité (Zonabend, 2014). Dans le secteur nucléaire, le déni ne provient pas du refus d'admettre les risques liés au travail en milieu radioactif, car les effets des produits radioactifs sur l'homme et l'environnement sont connus depuis longtemps. Il est plutôt lié à la croyance que les moyens déployés au sein du dispositif sont suffisants pour protéger ses composantes humaines et matérielles. Or, la catastrophe survenue le 11 mars 2011 réintroduit le réel dans toute sa violence en effaçant le déni de la dangerosité. En cela, les travailleurs de Fukushima sont broyés non au titre de "persécutés ", de « déportés ", d'« exilés » ou de "dissidents", mais en tant que victimes d'un combat mené contre des installations hors de contrôle. Un combat qu'ils n'ont ni imaginé, ni voulu, et auquel ils n'ont pas été préparés. Comme figure de la souffrance, Yoshida évoque en particulier le périple d'un agent de conduite blessé à la suite de l'explosion survenue dans le bâtiment réacteur $1^{67}$. Ce dernier s'est cassé le bras gauche et a été irradié. Il ne peut être soigné dans les hôpitaux de province, et doit marcher dans le froid en sous-vêtements - ses vêtements, contaminés, lui ont été retirés - et faire de l'autostop jusqu'à Tôkyô, où il est finalement hospitalisé, après deux jours d'errance...

La deuxième modalité de broyage, reliée à la première, renvoie à la question de la finitude. Les opérateurs sont confrontés à un risque de mort imminente, en particulier lorsque se produit l'explosion dans le bâtiment réacteur 3, et à un risque de mort différée, du fait de l'exposition à une dose de radioactivité dangereuse pour la santé. Ils doivent également faire face à un enjeu considérable, puisque la crise nucléaire confronte le Japon à un risque existentiel, dont Yoshida a conscience dès l'arrivée du tsunami.

Le fait que les travailleurs aient le sentiment de représenter la seule ligne de défense pour protéger leur pays de l'anéantissement les conduit à idéaliser leur action. Cette construction de sens accentue d'autant plus les effets dévastateurs de l'outrage que leur inflige Naoto Kan, qui constitue une troisième forme de broyage. À la suite d'un quiproquo dû à une communication dysfonctionnelle, le Premier ministre pense que TEPCO veut faire évacuer la centrale, devenue trop dangereuse pour ses opérateurs. Au matin du 15 mars 2011, le Premier ministre se rend au siège de l'exploitant, et ordonne aux équipes, via le système de téléconférence, de rester à leur poste et de sacrifier leur $v^{1} e^{68}$. Son injonction relègue le collectif à une bande de lâches et d'irresponsables, prêts à abandonner le Japon à une fin annoncée. Ses paroles malheureuses bafouent l'honneur des travailleurs et sont perçues comme une agression ultime, qui s'ajoute aux remontrances formulées par le siège, incapable de comprendre la situation sur le terrain ou de considérer les efforts et les sacrifices consentis.

\section{Le jeu des représentations}

En réponse aux injustices subies, Yoshida s'arme de sa parole pour témoigner de l'engagement civique de ses travailleurs et fustiger les acteurs qui les ont dénigrés au lieu de les soutenir. Le narrateur met ainsi en tension deux groupes bien distincts : d'un côté la « société » de Fukushima, de l'autre les acteurs du " dehors ». Yoshida ne tarie pas d'éloges à l'égard de ses équipes. Au cours d'une audition, un enquêteur affirme qu'elles sont trop habituées à obéir aux ordres et ne savent pas s'adapter à une 
situation imprévue ${ }^{69}$. Yoshida le contredit et affirme que ses agents sont de remarquables professionnels. Il évoque le courage, la persévérance, la polyvalence, l'ingéniosité de ses «troupes ». En valorisant les travailleurs de la sorte, le directeur les désigne comme les héros de la crise nucléaire.

L'attachement que Yoshida ressent pour ses équipes constitue un trait majeur de son identité narrative (Ricœur, 1990), qu'il élabore au fil de ses entretiens avec les enquêteurs. Pour lui, la priorité reste la vie de ses hommes. En tant que directeur, il est responsable de la sécurité du personnel de Fukushima. Il doit mettre les travailleurs à l'abri, faire l'appel, vérifier le nombre de blessés ou de disparus. Mais son souci de l'autre dépasse le cadre de ses fonctions. Tout au long de son témoignage, Yoshida se représente comme un manager rassurant et prévenant. Il raconte ainsi la manière dont il parle à ses équipes, bouleversées par les conséquences du séisme et du tsunami. Il leur demande de retrouver leur sang-froid, d'éviter l'affolement et de procéder aux vérifications posément, tout en se préparant à de nouvelles répliques sismiques ${ }^{70}$. Le fait de devoir arbitrer entre le danger auquel il expose les travailleurs et l'urgence de la situation confronte Yoshida à des dilemmes éthiques qui continuent de le hanter durant ses auditions. Il est notamment très affecté lorsque les équipes sont prises dans l'explosion du bâtiment réacteur $3^{71}$. Il regrette profondément d'avoir cédé aux pressions du siège et d'avoir renvoyé les travailleurs sur le terrain, au péril de leur vie.

À l'extérieur de la centrale, différents acteurs interviennent dans la gestion de crise. Parmi eux, se distingue le personnage de Naoto Kan, figure honnie mais surtout figure de l'altérité radicale, qui participe en creux à l'élaboration de l'identité narrative de Yoshida. Deux épisodes mettent en exergue la dimension négative de Kan. Le premier correspond à sa visite de Fukushima Daiichi le matin du 12 mars $2011^{72}$. Venu pour s'entretenir avec Yoshida, Kan ne prend pas la peine de saluer ou d'encourager les équipes de la cellule de crise. Le second épisode relate la visite qu'il effectue au siège social de TEPCO le matin du 15 mars, durant laquelle il demande brutalement aux équipes de se sacrifier. Le personnage du Premier ministre renvoie dès lors au stéréotype du bureaucrate froid et implacable, coupé de la réalité du terrain. En cela, il est l'exact contraire de Yoshida, le chef compréhensif, proche de ses hommes et du combat qu'ils mènent. Kan n'est toutefois pas le seul à subir les foudres du directeur de Fukushima, ce dernier critiquant avec virulence les autres acteurs du «dehors». Yoshida dénonce en outre les interventions inopinées de certains membres du Gouvernement, l'inefficacité des pompiers, les discussions oiseuses avec le siège de TEPCO, ou encore les injonctions irréalisables de l'autorité de sûreté nucléaire.

61 À travers ce jeu de représentations, le narrateur se met en scène dans une dramaturgie personnelle, à la recherche d'une reconnaissance sociale (Orofiamma, 2002). En composant son personnage, Yoshida manifeste dans son récit la présence d'une forte individualité, qui s'oppose aux images stéréotypées des Japonais. Un lieu commun de longue date rapporte en effet que les Asiatiques, et les Japonais plus particulièrement, manquent d'individualité. Ce discours se cristallise dans la seconde moitié du XIX ${ }^{e}$ siècle, à une époque où les Occidentaux commencent à considérer leur société comme moderne et individualiste, contrairement aux sociétés lointaines perçues comme traditionnelles et communautaires (Lozerand, 2010, 2014, 2015). Les Japonais sont assimilés à toute sorte d'images dépréciatives, telles que le grégarisme, l'esprit d'imitation, le mépris de la vie humaine, qui font écho à un défaut d'individualité. En réponse, des auteurs japonais ont relayé, et même renforcé, ce lieu commun dès la fin 
du XIX ${ }^{e}$ siècle, en se représentant comme les membres d'une société où l'individu s'efface devant le groupe. La littérature repère pourtant dans le Japon de l'ère Meiji ${ }^{73}$ des éléments faisant état d'un processus d'individualisation, lié à une forme de modernité non occidentale (Yatabe, 2002). Ce processus évolue dans la seconde moitié $\mathrm{du} \mathrm{XX}^{\mathrm{e}}$ siècle, époque marquée par le décollage économique et la diffusion d'un mode de vie consumériste qui modifie la définition de soi. La dernière partie du $\mathrm{XX}^{\mathrm{e}}$ siècle fait apparaître de nouvelles sensibilités, et voit s'affirmer de manière plus marquée la quête et la conscience de soi, perceptible dans la sphère familiale (retard du mariage, chute de la natalité, montée du célibat) ou dans la recherche de l'originalité pour se démarquer d'autrui (Garrigue-Testard et Chevallier, 2007).

Le personnage de Yoshida ne saurait conforter, à son tour, l'absence d'individualité au Japon. Le directeur confie aux enquêteurs qu'il a menti et désobéi à sa hiérarchie concernant l'injection d'eau de mer. Même au plus fort de la crise, la priorité de Yoshida reste la sécurité de ses hommes. Son témoignage bouscule donc les lieux communs sur les Japonais, comme la grégarité ou le mépris de la vie, et invite à reconsidérer la question de leur représentation à la lumière de la littérature.

\section{L'humain comme ultime recours}

63 Huit mois après le séisme du Tôhoku, Yoshida apprend qu'il est gravement malade. Le 28 novembre 2011, il est contraint de quitter ses fonctions de directeur de Fukushima ${ }^{74}$. Ni lui, ni TEPCO ne le précise encore, mais il a un cancer de l'œsophage ${ }^{75}$. Au début du mois de décembre, Yoshida décide de retourner à Fukushima Daiichi. À l'annonce de son arrivée, des centaines de personnes se regroupent dans la cellule de crise. Yoshida prend le micro et s'adresse à l'assemblée. Il exprime sa déchirure de devoir laisser les équipes dans une situation aussi difficile. Il déclare qu'il n'oubliera jamais les jours vécus ensemble et rend hommage à leur extraordinaire travail. L'ambiance s'assombrissant, Yoshida fait alors une plaisanterie sur un collaborateur, qui déclenche immédiatement une salve de rires. Avant son départ, il réitère toute sa confiance à l'égard des travailleurs : "Je veux que vous continuiez tous ici. Il y a encore des difficultés à surmonter mais je sais que vous ferez de votre mieux pour les gérer. Les habitants de Fukushima et toute la nation ont leurs espoirs en vous. N'oubliez jamais cela. Je veux vous voir unir vos efforts sous la direction de Takahashi ${ }^{76}$. Merci pour tout. Je reviendrai un jour " (Kadota, 2014, p. 257 [notre traduction]). Yoshida sort sous un tonnerre d'applaudissements. Des employés sont en larmes. Tous le rejoignent et l'entourent, pour lui serrer la main ou lui dire un mot. L'ancien directeur les salue une dernière fois avant de quitter la centrale nucléaire. Il n'y reviendra plus.

Masao Yoshida est parti trop tôt. Sa disparition représente une perte irréparable pour la communauté des experts en sûreté nucléaire et les chercheurs de tous horizons disciplinaires, les privant d'une source importante d'informations sur Fukushima. Il reste néanmoins ce témoignage, dans lequel Yoshida expose sa version des faits, reconstituant dans la langue parfois très technique de son métier les décisions prises et les actions menées pour faire face à un accident industriel impensé. Mais par-delà la pièce versée au dossier d'enquête et le jargon du nucléaire, il y a les paroles d'un survivant qui a vu l'abîme. Il y a les difficultés, les angoisses, les frustrations, les souffrances d'un collectif qui s'est battu et a connu l'expérience de la dévastation. 
Puisse ce récit, qui touche les confins du dicible, nous rappeler que la gestion d'une crise dépend en dernier ressort de l'engagement d'êtres humains et de leur capacité à improviser lorsqu'un évènement désastreux rend inapplicables les procédures. C'est le grand enseignement que nous livre Yoshida dans son témoignage et que nous devons à notre tour transmettre.

\section{NOTES}

1. On the Brink est d'abord publié en japonais en 2012 avant d'être traduit en anglais en 2014. À ce jour, il n'a pas été publié en français.

2. Ce surnom nous a été révélé durant notre visite de Fukushima Daiichi en mars 2017 par Akio Komori, directeur de la division nucléaire de TEPCO et ancien directeur de la centrale, qui avait rencontré Yoshida sur les bancs de l'Université de Tôkyô.

3. Membres d'une organisation criminelle japonaise, implantée dans de nombreux pays.

4. Discipline du Budô (« la voie du combat »), le kendo (« la voie du sabre »), à la fois art martial et sport pratiqué en compétition, est une forme d'escrime au sabre à deux mains.

5. Une fois les réserves d'eau douce épuisées, Yoshida ordonne le 12 mars 2011 de procéder à l'injection d'eau de mer dans le réacteur 1 , manœuvre de derniers recours qui n'a jamais été expérimentée auparavant. Après des essais concluants, l'ordre est cependant donné à Yoshida d'interrompre l'injection et d'attendre l'autorisation du Premier ministre pour continuer. L'injection d'eau de mer implique en effet un certain nombre d'incertitudes, notamment la possibilité de réexploiter les réacteurs. Yoshida considère cependant que les réacteurs sont irrémédiablement perdus, et que seule compte l'issue de la crise. Il décide dès lors de mentir à sa hiérarchie et de continuer l'injection d'eau de mer.

6. Programme américain, débuté le 13 août 1942, mobilisant un grand nombre de scientifiques, militaires et industriels, dont l'objectif est de fabriquer des bombes atomiques. Son directeur militaire est le major-général Leslie Richard Groves et son directeur scientifique Robert Oppenheimer.

7. Le nombre total de victimes de ces deux bombardements reste imprécis. Certaines sources évoquent jusqu'à 250000 morts à Hiroshima et 80000 morts à Nagasaki.

8. EBR-1 (Experimental Breeder Reactor I) est un prototype de Réacteur à Neutrons Rapides (RNR), qui utilise du NaK (alliage de sodium et de potassium) comme fluide caloporteur. Il est définitivement arrêté en 1963.

9. Le réacteur AM-1 (Atom Mirny - atome pacifique) de la centrale d'Obninsk utilise le graphite comme modérateur, tandis que l'eau assure son refroidissement. Sa puissance brute est de 6 MWe. Il est le précurseur des réacteurs RBMK (Reaktor Bolshoy Moshchnosti Kanalnyi - réacteur de forte puissance à canaux). 
10. G1 est un réacteur à Uranium Naturel Graphite Gaz (UNGG). Il utilise l'uranium naturel comme combustible et le graphite comme modérateur. Il est refroidi à l'air à la pression atmosphérique. Sa puissance thermique s'élève à 46 mégawatts. G1 est le premier réacteur français à produire de l'électricité. Les réacteurs suivants, G2 et G3 qui divergent respectivement à Marcoule le 21 juillet 1958 et le 8 juin 1959 -, sont cependant les premiers de l'Hexagone à être raccordés au réseau électrique.

11. Le CEA est un Établissement Public à Caractère Industriel et Commercial (EPIC), créé en 1945 pour développer toutes les applications issues des sciences de l'atome. Depuis sa fondation, le CEA a élargi son champ de recherche et de compétences. Il intervient aujourd'hui dans quatre domaines : la défense et la sécurité, les énergies bas carbone (nucléaire et renouvelables), la recherche technologique pour l'industrie et la recherche fondamentale en sciences de la matière et sciences de la vie. En plus de son siège social, le CEA est implanté dans neuf centres (quatre centres d'études civiles et cinq centres pour les applications militaires).

12. Les quatre réacteurs de Calder Hall, d'une puissance de 60 MWe brute chacun, sont de type Magnox (Magnesium Non Oxidising). Ce type de réacteur, construit par les Britanniques de 1953 à 1971, utilise l'uranium naturel comme combustible et le graphite comme modérateur. Il est refroidi au dioxyde de carbone.

13. La calandre correspond à l'enceinte contenant les tubes de pression et le modérateur.

14. Réacteur à eau lourde (oxyde de deutérium), mis en service le 22 juillet 1947. NRX (National Research Experimental) utilise l'uranium naturel comme combustible et l'eau lourde comme modérateur. Il est refroidi à l'eau légère. Premier réacteur de recherche à grande échelle au Canada, il joue un rôle important dans le développement du réacteur CANDU (CANada Deuterium Uranium).

15. Échelle internationale de gravité des évènements nucléaires - en anglais «International Nuclear Event Scale »- graduée de 0 à 7 et utilisée à partir de 1991. Le niveau 5 correspond à un accident entraînant des rejets limités dans l'environnement.

16. L'accident se produit sur Windscale Pile 1 , réacteur à uranium naturel, modéré au graphite et refroidi par air. Le site de Windscale sera par la suite rebaptisé Sellafield.

17. Réacteur à Uranium Naturel Graphite Gaz.

18. Le niveau 4 de l'échelle INES correspond à un accident entraînant des rejets mineurs dans l'environnement. Un autre accident a lieu à Saint-Laurent-des-Eaux le 13 mars 1980, cette fois sur le réacteur Saint-Laurent A2. Il sera également classé au niveau 4 de l'échelle INES.

19. Les informations sur les rejets radioactifs liés à l'accident et à la remise en état de l'installation ne permettent pas de déterminer avec précision les conséquences radiologiques de l'évènement.

20. L'accident survient dans le réacteur 2 (TMI-2). Il s'agit d'un Réacteur à Eau Pressurisée, construit par Babcock and Wilcox.

21. Commission de réglementation nucléaire américaine.

22. Un accident s'était déjà produit en Union soviétique le 29 septembre 1957 dans le complexe nucléaire de Maïak, situé près de la ville de Kychtym en Russie. Il ne s'agit pas d'un accident de réacteur, mais d'une explosion survenue dans une usine de retraitement de combustible nucléaire. L'évènement, longtemps dissimulé par les 
Soviétiques, sera classé au niveau 6 de l'échelle INES, qui correspond à un accident grave entraînant des rejets importants dans l'environnement.

23. Réacteur RBMK à neutrons thermiques, qui utilise le graphite comme modérateur et l'eau légère bouillante comme fluide caloporteur. Le combustible est constitué d'oxyde d'uranium enrichi en uranium 235. Le réacteur 4 de la centrale de Tchernobyl, mis en service en 1983, dispose d'une puissance de 1000 MWe.

24. Le réacteur 4 de Tchernobyl doit être arrêté le 25 avril 1986 pour procéder à des opérations de maintenance. La direction de la centrale décide de profiter de cet arrêt pour mener un essai. Celui-ci consiste à déterminer si, en cas de perte de ressources électriques, la turbine d'un réacteur fonctionnant au ralenti pourrait fournir assez d'énergie pour alimenter les équipements de secours et les pompes de circulation de l'eau de refroidissement du cœur, jusqu'à ce que les groupes diesel de secours puissent en produire à leur tour (NEA / OECD, 2002). Cette expérimentation a été conçue par une équipe d'ingénieurs, dirigée par un spécialiste en énergétique de Donetsk (Ukraine). Le directeur Brioukhanov envoie le programme de l'essai au ministère de l'Énergie et au Comité d'État à la sûreté nucléaire. Si le premier accorde son autorisation, le second ne communique aucune réponse, certainement à cause des lenteurs de la bureaucratie soviétique (Ackerman, 2006). Brioukhanov décide malgré tout de passer outre ce silence et accepte que ses équipes réalisent l'essai. Le matin du 25 avril, les opérateurs débutent le test en effectuant une réduction de puissance du réacteur 4. Le lendemain après-midi, le centre de distribution électrique de Kiev leur demande de retarder l'expérience, car Tchernobyl doit fournir suffisamment d'électricité pour compenser une panne survenue dans une autre centrale électrique. Contrairement au programme initial, le réacteur est donc maintenu à mi-puissance. Le centre de distribution autorise les opérateurs à reprendre le test à 23 h00. La réduction de puissance reprend, mais l'état du réacteur n'est pas approprié pour réaliser l'essai. Les opérateurs veulent rattraper leur retard et décident tout de même de le mener. Ils procèdent à la fermeture des vannes d'alimentation en vapeur de la turbine. La température monte dans le cœur, ce qui produit une augmentation incontrôlée de la puissance du réacteur. À $1 \mathrm{~h} 23$ minutes et 40 secondes, le chef de quart actionne l'arrêt d'urgence. Les barres de contrôle commencent à descendre dans le cœur, mais n'ont pas le temps d'arrêter la réaction en chaîne. Le réacteur explose à $1 \mathrm{~h} 23$ minutes et 44 secondes, provoquant le premier accident nucléaire majeur de l'histoire. Même si la catastrophe est due à une série d'erreurs commises par les opérateurs et à la conception du réacteur RBMK, Brioukhanov en est tenu en partie responsable. En juillet 1986, il est exclu du Parti communiste. En 1987, il est condamné à l'issue d'un procès mené à huis clos à dix ans de réclusion. L'ingénieur en chef de la centrale, l'ingénieur responsable du réacteur 4 la nuit de l'accident, le chef de quart et le chef d'atelier réacteur sont également condamnés et incarcérés. Brioukhanov est libéré en 1992, sur requête de l'administration et de la police, après avoir purgé la moitié de sa peine. Brioukhanov n'a cependant jamais reconnu sa responsabilité dans l'accident nucléaire : «Ce procès était injuste, il leur fallait un coupable et ils ont déniché dans le code pénal un article qui leur permettait de me condamner. Je ne me sens pas coupable, c'est sur la conscience des inventeurs du réacteur que devrait peser cette catastrophe » (Cote, 1996).

25. Le niveau 7, le plus élevé de l'échelle INES, correspond à un accident entraînant des rejets majeurs dont les effets sont considérables sur la santé et l'environnement. 
26. International Nuclear Safety Group - en français «Groupe international pour la sûreté nucléaire ».

27. L'INSAG-4 définit la culture se sûreté comme "l'ensemble des caractéristiques et des attitudes qui, dans les organismes et chez les individus, font que les questions relatives à la sûreté des centrales nucléaires bénéficient, en priorité, de l'attention qu'elles méritent en raison de leur importance » (INSAG, 1991, p. 5).

28. Pour certains auteurs, tel l'essayiste Katayama Morihide, la politique nucléaire du Japon constitue également un moyen de prendre une revanche au plan scientifique, domaine qui a fait défaut aux Japonais face aux Américains à la fin de la guerre du Pacifique (Pelletier, 2012).

29. Il s'agit du même type de réacteur que ceux construits à Calder Hall au RoyaumeUni.

30. Ce type de réacteur utilise de l'uranium enrichi comme combustible et de l'eau légère (eau ordinaire par opposition à l'eau lourde) comme modérateur et comme caloporteur.

31. Réacteur modéré et refroidi par de l'eau légère portée à ébullition dans le cœur dans les conditions normales de fonctionnement.

32. Centrale nucléaire située dans la sous-préfecture de Shiribeshi sur l'île d'Hokkaidô et exploitée par l'entreprise Hokkaidô Electric Power Company (HEPCO). Elle comprend trois réacteurs à eau pressurisée : Tomari 1 mis en service en juin 1989, Tomari 2 mis en service en avril 1991 et Tomari 3 mis en service en décembre 2009.

33. Cf. Fig. 2 : « les installations de Fukushima Daiichi ».

34. Les travaux menés par TEPCO visent à court terme à empêcher les fuites d'eau radioactive, sécuriser le stockage d'eau contaminée sur le site et fiabiliser le refroidissement des installations. En janvier 2012, l'exploitant communique un plan à moyen et long termes. Il prévoit d'abord le retrait du combustible stocké dans les piscines et le retrait du corium situé dans les réacteurs 1 à 3 , et envisage ensuite le démantèlement intégral des installations. La finalisation des travaux est fixée vers 2040/2050. Le chantier de démantèlement et de décontamination confronte les équipes à d'immenses défis technologiques. Sur le terrain, plus de six mille personnes œuvrent quotidiennement pour maitriser les installations, toujours soumises au risque de séisme et de tsunami. L'une des réalisations les plus ambitieuses est la construction d'un mur de glace autour des réacteurs pour isoler les eaux contaminées. L'ouvrage, de 1,5 kilomètre de long et descendant jusqu'à 27 mètres de profondeur, est finalisé en août 2017.

35. Les principaux rejets radioactifs se produisent du 12 au 25 mars 2011, au cours d'une quinzaine d'épisodes dont les plus importants seraient survenus avant le 17 mars (IRSN, 2012b). Entre mars 2011 et septembre 2019, le débit de dose ambiant a été divisé par un facteur d'environ 2 (IRSN, 2020).

36. Au plan de la contamination radioactive de l'environnement marin, les niveaux de concentration de césium, dus au déversement des effluents contaminés dans l'océan Pacifique, dépassent en 2011 plusieurs dizaines de milliers de Bq/L au niveau des points de prélèvement situés à proximité de Fukushima Daiichi. En 2018, les niveaux de concentration sont compris entre 0,01 et $0,1 \mathrm{~Bq} / \mathrm{L}$. À 20 kilomètres de la côte, les niveaux actuels atteignent $0,001 \mathrm{~Bq} / \mathrm{L}$. Ils correspondent au même ordre de grandeur 
que ceux observés sur la côte avant l'accident et qui avaient principalement pour origine les retombées des essais nucléaires aériens (IRSN, 2020).

37. Durant les premiers jours de l'accident, environ 78000 personnes vivant dans un rayon de 20 kilomètres autour de la centrale et 62000 personnes vivant entre 20 et 30 kilomètres sont évacuées. En avril 2011, le Gouvernement recommande l'évacuation d'environ 10000 personnes supplémentaires vivant plus au nord-ouest de Fukushima Daiichi (NRC, 2014).

38. Fin 2011, les autorités japonaises classifient les territoires contaminés en deux grandes zones : la zone de décontamination spéciale (Special Decontamination Area, ou SDA) et la zone d'évaluation intensive de la contamination (Intensive Contamination Survey Area, ou ICSA). La SDA comprend les territoires où la dose annuelle peut atteindre ou dépasser $20 \mathrm{mSv}$. L'ICSA comprend les territoires où la dose annuelle peut quant à elle atteindre entre 1 et $20 \mathrm{mSv}$. La décontamination de la SDA est à la charge du Gouvernement, tandis que celle de l'ICSA revient aux communes situées dans cette zone, qui bénéficient de l'aide technique et financière du Gouvernement. La SDA est subdivisée en trois zones : la zone 1 (zone où les ordres d'évacuation sont prêts à être levés), la zone 2 (zone où les habitants ne sont pas autorisés à résider) et la zone 3 (zone où les résidents auront des difficultés à se réinstaller durant une longue période). Les opérations de décontamination menées dans la SDA permettent de lever les ordres d'évacuation des zones 1 et 2 entre 2014 et 2017. Dans la zone 3, les autorités créent des bases de reconstruction spécifiques et de revitalisation. En mars 2020, les ordres d'évacuation sont levés pour Futaba, Okuma et Tomioka, lieux situés dans la zone 3. Les opérations de décontamination sont terminées dans la SDA en mars 2017 (hormis dans la zone 3) et dans l'ICSA en mars 2018. 1328 sites de stockage temporaires ont été créés pour accueillir les déchets (terre, végétaux) issus de ces opérations. Ces sites ont été aménagés sur le territoire des communes soumises aux opérations de décontamination. 550 sites de stockage temporaires sont réhabilités à la fin du mois de mars 2019 du fait de l'évacuation de leurs déchets vers des centres de retraitement ou vers le site de stockage intermédiaire, d'une durée de vie de trente ans, créé par le Gouvernement autour de Fukushima Daiichi. Un site de stockage définitif, localisé hors de la préfecture de Fukushima, sera prochainement déterminé pour accueillir ces déchets après 2045 (IRSN, 2020).

39. Ces normes sont définies le 17 mars 2011 par le ministère de la Santé, du Travail et des Affaires sociales et validées par la Commission de sécurité alimentaire japonaise. De nouvelles limites de restriction sont établies en avril 2012 (IRSN, 2012c). Dix-sept préfectures effectuent à partir de cette base des mesures par sondage sur les denrées produites sur leur territoire.

40. Parti Démocrate du Japon (PDJ), fondé en 1996. Il fusionne en 2016 avec le Parti de la Restauration pour former le Parti Démocrate Progressiste. Ce dernier disparaît en 2018.

41. Yoshihiko Noda, membre du même parti politique que Naoto Kan, devient le nouveau Premier ministre du Japon. Noda quitte ses fonctions de chef du Gouvernement le 26 décembre 2012.

42. Des réacteurs sont remis en service dans les années suivantes, malgré l'opposition d'une partie de la société civile. Les réacteurs 3 et 4 de la centrale nucléaire d'Ôi (préfecture de Fukui) redémarrent en juillet 2012, avant d'être de nouveau arrêtés en septembre 2013 pour maintenance. Les réacteurs de la centrale de Sendai (préfecture 
de Kagoshima) et de Takahama (préfecture de Fukui) sont relancés en 2015. Actuellement, neuf réacteurs nucléaires sont exploités dans l'Archipel (centrales d'ôi, Takahama, Ikata, Genkai et Sendai).

43. Tchernobyl et Fukushima appartiennent à la série évènementielle des accidents nucléaires majeurs. Les deux évènements présentent cependant des différences importantes, en termes de technologies de réacteur, de séquence accidentelle et de conséquences radiologiques, comme le précise le dossier publié en octobre 2014 sur le site internet de l'IRSN: https://www.irsn.fr/FR/connaissances/Environnement/ expertises-incidents-accidents/comparaison-tchernobyl-fukushima/Pages/1-impactenvironnemental-fukushima-tchernobyl.aspx?dId=5d0cc222-c748-41eabae7-33f47b490598\&dwId=ebe35772-4442-413c-b628-068fde521abe

44. National Diet of Japan Fukushima Nuclear Accident Independent Investigation Commission: Commission d'enquête indépendante sur l'accident nucléaire de Fukushima Daiichi créée par la Diète du Japon le 7 octobre 2011. Elle dépose son rapport final en juillet 2012.

45. Investigation Committee on the Accident at Fukushima Nuclear Power Stations of TEPCO : Commission d'enquête sur l'accident des centrales nucléaires de Fukushima de TEPCO. Missionnée par le Gouvernement, la Commission remet son rapport final au Premier ministre le 23 juillet 2012.

46. Comité mis en place en juillet 2011, constitué d'ingénieurs, de chercheurs et de journalistes. À l'origine, son rapport final ne doit pas être rendu public. Des citoyens demandent néanmoins au Comité d'y accéder, et leur requête est acceptée en mars 2012 (Kobayashi, 2019).

47. Cette idée de « collusion d'intérêts » entre les acteurs du nucléaire au Japon renvoie au terme péjoratif de "Village nucléaire ». Apparu en 1997, il désigne l'ensemble des acteurs institutionnels prenant part au développement du nucléaire civil japonais (le ministère de l'Économie, de l'Industrie et du Commerce, les agences de sûreté nucléaire, les exploitants comme TEPCO, les fabricants, les sous-traitants, les universitaires et les laboratoires de recherche spécialisés dans la physique nucléaire, voire certains médias). Le terme sous-entend les connivences et les arrangements entre ces acteurs au détriment de la transparence et de la sécurité.

48. Selon l'AIEA, «L Le concept de défense en profondeur, qui concerne à la fois la protection du public et des travailleurs, est fondamental pour la sûreté des installations nucléaires. Comme il a été indiqué dans les Principes fondamentaux de sûreté pour les centrales nucléaires (INSAG-3) en ce qui concerne la sécurité des centrales nucléaires, 'Toutes les activités de sécurité, qu'elles soient liées à l'organisation, aux comportements ou aux équipements, sont soumises à des couches de disposition qui se chevauchent, de sorte que si une défaillance devait se produire, elle serait compensée ou corrigée sans causer de tort aux individus ou au grand public. Cette idée de niveaux multiples de protection est la caractéristique centrale de la défense en profondeur" »(INSAG, 1996, p. 1 [notre traduction]).

49. Autorité administrative indépendante chargée du contrôle des activités nucléaires civiles en France, créée par la loi du 13 juin 2006 relative à la transparence et à la sécurité en matière nucléaire. L'ASN assure, au nom de l'État, le contrôle de la sûreté nucléaire et de la radioprotection pour protéger les personnes et l'environnement. Elle a aussi pour mission d'informer le public et de contribuer à des choix de société éclairés. 
50. Le «noyau dur» correspond à "l'ensemble des dispositions matérielles et organisationnelles robustes visant, pour des situations extrêmes considérées dans les ECS, ̀̀ prévenir un accident avec fusion ou en limiter la progression, et permettre à l'exploitant d'assurer ses missions dans la gestion de la crise " (Coïc, 2015, p. 42). Ces dispositions impliquent entre autres la mise en place du Diesel d'Ultime Secours (DUS) sur chaque réacteur, de l'appoint ultime en eau (APU) et de dispositifs mobiles d'alimentation, de la construction d'un centre de crise local (CCL) sur chaque site nucléaire et de la création de la Force d'Action Rapide du Nucléaire (FARN). Cette unité spéciale est entraînée pour intervenir en moins de douze heures sur n'importe quelle centrale d'EDF confrontée à un évènement extrême (Renoux, 2015).

51. Film de fiction de 122 minutes, réalisé par Setsurô Wakamatsu et sorti au Japon en mars 2020. Le personnage de Yoshida est interprété par l'acteur Ken Watanabe.

52. Yuki Kobayashi (2019). "Étude de la relation entre les leaders politiques et techniques dans la gestion de l'accident de Fukushima Daiichi entre le 11 et le 15 mars 2011 », thèse de doctorat de l'Université PSL, préparée à MINES Paris, soutenue le 21 mai 2019, $263 \mathrm{p}$.

53. Grand quotidien national japonais, fondé en 1879 à Osaka. Véritable institution au Japon, il est l'un des journaux les plus lus dans le monde.

54. Grand quotidien national japonais, fondé en 1933 à Tôkyô. Les positions défendues par le Sankei Shinbun se situent à droite, tandis que celles de l'Asahi Shinbun sont plutôt considérées à gauche.

55. Yoshida refuse que son audition menée par l'ICANPS ne devienne publique. De son côté, la NAIIC demande le 15 mai 2012 à Yoshida d'accepter qu'elle l'auditionne. Yoshida, déjà très malade, décline la proposition. La NAIIC demande alors à l'ICANPS de lui communiquer son audition de Yoshida. L'ICANPS et Yoshida y consentent, mais l'ancien directeur de Fukushima Daiichi exige le 29 mai 2012 que la NAIIC ne rende pas publique son audition.

56. Les auditions de dix-huit autres acteurs, dont le Premier ministre Naoto Kan, sont publiées en même temps que celles de Yoshida. Le Gouvernement met ensuite en ligne cent vingt-sept nouvelles auditions le 25 décembre 2014.

57. Cf. la question du « retrait général » évoquée dans les auditions du 9 août 2011 (I) et du 6 novembre 2011 (II).

58. Notre laboratoire est le Centre de recherche sur les Risques et les Crises (CRC), fondé en janvier 2008 en vue de renforcer la recherche et la formation dans le domaine de la prévention des risques et de la gestion des crises de l'École des Mines de Paris. Il conduit des recherches interdisciplinaires à la croisée des sciences humaines et sociales et des sciences pour l'ingénieur. Son programme stratégique, intitulé "Énergies et transports sûrs ", recouvre les filières industrielles du nucléaire et du pétrole-gaz, auxquelles s'ajoutent les transports maritimes, terrestres et aériens.

59. Établissement public à caractère industriel et commercial (EPIC), créé en 2001 et placé sous la tutelle des ministres chargés de l'Écologie, de la Recherche, de l'Énergie, de la Santé et de la Défense. Il exerce ses missions d'expertise et de recherche dans les domaines de la surveillance radiologique de l'environnement et de l'intervention en situation d'urgence radiologique; la radioprotection de l'homme; la prévention des accidents majeurs dans les installations nucléaires; la sûreté des réacteurs; la sûreté 
des usines, des laboratoires, des transports et des déchets ; l'expertise nucléaire de défense.

60. Nos recherches portent alors sur le développement du procédé "Actiflo ${ }^{\mathrm{TM}}-\mathrm{Rad}$ ", une combinaison de technologies éprouvées pour le traitement d'effluents, mises au point par AREVA, le CEA et Veolia Environnement. Elle est proposée au Japon à la fin du mois de mars 2011. Début avril, TEPCO accepte cette solution et les premiers essais débutent sur site au mois de juin.

61. L'ingénierie de l'urgence qualifie les activités d'ingénierie dont la conduite est entravée du fait des conditions d'urgence sociétale liée à un contexte d'accident associé à une réaction en chaîne qui déclenche, depuis sa survenue, des situations de crise régulières (Guarnieri et Travadel, 2015).

62. Dans un tel contexte, "une 'situation extrême' désigne l'effondrement des significations instituées au niveau d'un groupe, après qu'il a perdu le contrôle de son outil de production et que ses membres se trouvent, de ce fait, tenus socialement responsables d'un péril irréversible. Les sujets en situation extrême sont tiraillés entre l'urgence sociétale d'agir et l'impossibilité matérielle de l'action à l'intérieur des cadres institués, les procédures de gestion de crise notamment » (Travadel et al., 2019).

63. Aissame Afrouss (2017) a ainsi analysé la notion de "coping» dans le récit de Yoshida. Yuki Kobayashi (2019) a étudié l'évolution des rapports institués entre les décideurs politiques et techniques durant la crise nucléaire. Matthieu Gaulène (2021) a quant à lui interrogé sous l'angle de la sémiotique l'interprétation de Fukushima chez les intellectuels japonais, et notamment les membres des commissions d'enquête.

64. Épuisé, il ne retourne pour la première fois chez lui qu'un mois plus tard, ne prenant que trois jours de repos avant de revenir à Fukushima pour continuer de superviser les opérations (Kadota, 2014).

65. Le récit de Yoshida n'a pourtant pas donné lieu à des développements spécifiques dans le rapport de l'ICANPS. Il en va de même pour l'ensemble des entretiens réalisés par les différents comités d'enquêtes, ces derniers se contentant le plus souvent de souligner l'engagement remarquable des travailleurs de Fukushima.

66. Cf. audition du 6 novembre 2011 (II).

67. Cf. l'audition du 9 août 2011 (II).

68. Cf. auditions du 9 août 2011 (I) et du 6 novembre 2011 (II).

69. Cf. audition du 29 juillet 2011.

70. Cf. audition du 22 juillet 2011.

71. Cf. audition du 29 juillet 2011.

72. Cf. audition du 22 juillet 2011.

73. Meiji (1852-1912) est le $122^{\mathrm{e}}$ empereur du Japon. Son nom désigne l'ère de son règne, qui s'étend du 3 février 1867 au 30 juillet 1912. C'est durant ce dernier que le Japon se transforme radicalement et se modernise en s'inspirant du modèle occidental.

74. Il garde néanmoins un poste dans la division nucléaire de TEPCO.

75. Dans sa communication, TEPCO écarte tout lien entre la maladie de Yoshida et les rayonnements auxquels il a été exposé durant la crise nucléaire.

76. Takeshi Takahashi est nommé directeur de Fukushima Daiichi le $1^{\text {er }}$ décembre 2011. Il occupait auparavant un poste de gestion des installations nucléaires à TEPCO. 


\section{AUTEURS}

FRANCK GUARNIERI

Directeur de recherche, directeur du Centre de recherche sur les Risques et les Crises (CRC) de MINES Paris - Université Paris, Sciences \& Lettres (PSL).

\section{AURÉLIEN PORTELLI}

Enseignant-chercheur au CRC de MINES Paris - PSL. 


\title{
Une belle rencontre ${ }^{1}$
}

\author{
Tomoko Takesada
}

1 J'ai été contactée très vite après la publication des présents comptes rendus d'auditions $\mathrm{du}$ directeur de la centrale de Fukushima pour en fournir une traduction française. Mais ce n'est pas sans hésitations que je me suis engagée. Mon ignorance du monde du nucléaire, le poids de la tragédie m’ont freinée. Finalement c'est la voix de mes proches qui m'a décidée. Ces auditions représentaient un moment d'Histoire, crucial, et je devais à la mémoire de cet homme, aujourd'hui disparu, de transmettre le plus fidèlement possible ses paroles au public francophone.

Et je n'ai pas regretté de m'être fait la voix française de Masao Yoshida. Yoshida a-t-il été à la hauteur des catastrophes auxquelles il a dû faire face ? A-t-il pris les bonnes décisions? Je laisse à d'autres compétences le soin de répondre à ces questions. Mais, au-delà du spécialiste du nucléaire, j'ai trouvé un homme de devoir, parfaitement conscient des enjeux, un homme capable de désobéissance aussi, pour le bien du plus grand nombre, un homme proche de ses collaborateurs, un homme dont le cœur saignait à chaque fois qu'il devait envoyer ses troupes braver les dangers de l'irradiation et, aussi, un homme révolté.

Parallèlement à cette rencontre, cette traduction a été également l'occasion pour moi d'une réflexion renouvelée sur la nature même de notre langue. Le japonais est une langue basée sur une grande connivence. On part du principe qu'on partage une large sphère de connaissances. Or le monde du nucléaire m'était inconnu. Dans une langue qui ne marque pas le nombre, comment savoir alors si, sur tel système de la tranche nucléaire, il y a une ou plusieurs vannes à ouvrir pour permettre un rejet de la vapeur, une des obsessions de Yoshida? Longtemps, on fait comme s'il n'y en avait qu'une et, tout d'un coup, au détour d'une phrase, cent pages plus loin, on découvre qu'en fait il y en avait dix-huit !

Mais cette particularité de notre langue n'a-t-elle joué des tours qu'à la seule traductrice? Lorsque Yoshida se plaint que les officiels des cellules de crise hors centrale ne comprennent pas tous les efforts déployés par ses hommes sur le terrain, ces fidèles soldats du nucléaire qui, au mépris de la radioactivité, tentent de résoudre patiemment une difficulté après l'autre, lorsqu'il se plaint, chose plus révoltante encore pour lui, que ces messieurs, confortablement installés à Tôkyô, loin de la radioactivité, 
osent prétendre que ses équipes tergiversent ou traînent des pieds, était-il conscient de cette spécificité de notre langue ? Pensez qu'il dit « je fais tout pour ouvrir la vanne » et, qu'en fait, il faut apprivoiser simultanément dix-huit vannes avec chacune ses particularités. Cette seule phrase, apparemment éloquente pour Yoshida, reflète-t-elle effectivement tous les obstacles rencontrés, les efforts engagés, les échecs subis? L'élégance de la légendaire retenue japonaise ne se serait-elle pas retournée contre ce samouraï des temps modernes?

Connivence aussi, lorsque le sujet de la phrase est passé sous silence, phénomène usuel dans notre langue, et que l'action peut être attribuée à n'importe lequel des protagonistes. Tout traducteur travaillant sur le japonais en a fait l'expérience. Il faut alors reconstituer dans sa tête la scène, la revivre de l'intérieur, pour finalement choisir l'interprétation la plus plausible.

Évidemment, rares sont les collègues qui ont eu le privilège de se nicher dans la tête d'un directeur de centrale nucléaire pour vivre quasi physiquement, minute à minute, un accident de cette ampleur. Et je dois, contrairement à mes craintes des débuts, à la personnalité de Masao Yoshida de n'avoir pas sombré dans le pessimisme. Malgré les catastrophes qui se succèdent sans pitié, malgré la pression des politiques, jusqu'au bout, il se battra et gardera le souci de ses hommes. Cette petite lueur qu'il a toujours portée en lui a empêché que tout ne bascule dans le noir. Merci, Yoshida san, d'avoir préservé cette part d'humanité dans la cellule de crise !

\section{NOTES}

1. Reproduction du texte de Tomoko Takesada publié dans F. Guarnieri (dir.). L'accident de Fukushima Daiichi. Le récit du directeur de la centrale. Volume I - L'anéantissement, op. cit., pp. 19-20. 


\section{Compte rendu de l'audition du 22 juillet 2011}

\section{Avertissement aux lecteurs}

1 Pour faciliter la lecture, les points suivants doivent être pris en considération :

- les noms propres japonais sont très difficiles à lire. Notamment les prénoms peuvent se lire de diverses manières. C'est pourquoi, en dehors des personnalités, notamment scientifiques, retrouvées à partir de moteurs de recherche sur l'Internet, il y a dans le texte livré ci-après des (?) derrière certains prénoms ;

- parfois, certains noms de personnes ont été intentionnellement escamotés dans le texte japonais ;

- le japonais est une langue qui ne marque pas le nombre (singulier ou pluriel). Cela signifie que quand le texte japonais parle, par exemple, de pompe sur le système SLC, il n'indique pas s'il y en a juste une ou plusieurs.

- Enfin, le texte qui suit est la traduction de la retranscription brute de l'enregistrement des séances d'audition. Les lecteurs y trouveront parfois certaines incohérences, voire des erreurs commises par les interlocuteurs. Celles-ci ont été respectées dans la traduction. Certaines sont signalées par des notes.

Tomoko Takesada

\section{Compte rendu de l'audition}

2 [À traiter avec la plus grande attention]

16 août 2011

Tsunemasa (?) Katô (rapporteur)

Membre du secrétariat de la Commission d'enquête sur l'accident de la centrale nucléaire de Fukushima de Tôkyô Electric Power Company (Investigation Commitee on the Accident at the Fukushima Nuclear Power Station of Tôkyô Electric Power Company). 
Ci-dessous le compte rendu de l'audition du 22 juillet 2011, menée dans le cadre de l'enquête sur l'accident de la centrale nucléaire de Fukushima de la Tôkyô Electric Power Company.

1. Auditionné, date d'audition, lieu d'audition, enquêteurs.

1. Auditionné

Masao Yoshida, Directeur de la centrale nucléaire de Fukushima Daiichi de la Tôkyô Electric Power Company.

2. Date d'audition

22 juillet 2011 de $10 \mathrm{~h} 25$ à $12 \mathrm{~h} 25$ (sans pause)

22 juillet 2011 de 13 h05 à 15 h06 (sans pause)

3. Lieu d'audition

Salle de réunion $\mathrm{A}$

Foyer féminin de la Japan Football Association Academy de Fukushima (1er étage)

J-Village

8-Utsukushimori Yamadaoka, Naraha, Futaba District, Fukushima

4. Enquêteurs

Yôtarô Hatamura, Kunio Yanagida, Masao Fuchigami, Shinji (?) Ogawa, Tsunemasa (?) Katô, Toshitaka (?) Nagata.

5. Enregistrement par enregistreur numérique

- Oui $\vdots$ Non

2. Contenu de l'audition

Situation au moment de l'accident et réponses à l'accident

Voir le compte rendu

3. Mentions spéciales

Pour faciliter les explications et aider la mémoire de l'auditionné, les documents Report regarding the Plant Data of Fukushima Daiichi Nuclear Power Station at the time of the Earthquake publié par TEPCO le 16 mai 2011, le First Response at Tôhoku Chihou Taiheiyou Oki Earthquake in Fukushima Daiichi Nuclear Power Station publié par TEPCO le 18 juin 2011 et le plan Itinéraire de la visite de la Commission d'enquête ont été utilisés.

Ce plan Itinéraire a été ajouté à la fin du compte rendu.

QUESTION : Pour commencer, je vais vous demander si on peut utiliser un enregistreur numérique. Êtes-vous d'accord pour que j'enregistre cet entretien avec un appareil numérique?

Réponse : Je vous en prie.

$\mathbf{Q}:$ : Merci. Je vais l'enclencher.

Nous allons donc commencer l'audition, mais je voudrais tout d'abord vous expliquer de manière très succincte pourquoi nous faisons cette audition aujourd'hui. Notre Commission d'enquête sur l'accident nucléaire de Fukushima vient d'être nommée et d'entamer ses activités'. Le but de cette Commission est d'éclaircir la manière dont l'accident s'est produit, comment il a évolué, d'en rechercher les différentes causes, afin que ces connaissances soient utiles aux générations futures, qu'elles puissent se baser sur cette expérience. Pour cela, nous avons besoin d'aller au-delà de ce que fait une commission d'enquête classique, savoir ce qui s'est concrètement passé, qui a fait quoi, nous pensons que nous devons rechercher les causes de l'accident dans une sphère beaucoup plus large pour que cette expérience puisse servir à ceux qui vont venir. Par 
conséquent, la recherche des responsabilités, par exemple, ne fait pas partie de notre but. Nous n'avons nullement l'intention de pousser la conversation dans cette direction.

Ce qui est extrêmement important, c'est de nous dire que nous sommes en train de vivre un énorme accident, tel que, non seulement, nous autres Japonais n'avons jamais connu, mais que le monde lui-même n'a jamais connu. Nous pensons que non seulement le Japon regarde ce que nous allons apprendre, mais que le monde attend pour étudier en détail ce que nous allons apprendre à partir de cette catastrophe.

Nous avons la conviction que nous devons étudier les faits pour ceux qui vivent aujourd'hui, mais surtout transmettre les faits que les générations futures auraient voulu que nous étudiions aujourd'hui, sous une forme qui permette des études ultérieures. Nous pensons qu'il faut relater les faits en détail pour que, si en se penchant sur l'accident dans un siècle, on puisse se dire "ah, s'ils avaient pu se rendre compte de ce fait à ce moment-là, ils auraient pu éviter la catastrophe».

J'ai encore beaucoup d'autres choses en tête, mais je vais m'arrêter, car nous gaspillons un temps précieux. De mon point de vue, et excusez l'expression peut être choquante, même si c'est un accident que nous n'avons pas voulu, maintenant qu'il est advenu, puisque nous l'avons payé si cher, il faut en tirer la moindre leçon, sinon ce serait du gâchis.

J'ai conscience que vous demander de vous soumettre à cette audition à un moment où vous avez énormément de travail sur le terrain est très difficile pour vous. Vous pensez sans doute que je vous empêche de faire ce qui doit être fait maintenant. Mais j'ai justement sollicité votre collaboration aujourd'hui car j'ai peur que, plus tard, on regrette amèrement de ne pas l'avoir fait maintenant, parce que la mémoire peut progressivement s'effacer ou changer.

Nous vous avons sollicité pour une audition aujourd'hui. Mais, de toute façon, ça va être très long, l'audition va se poursuivre la semaine prochaine. Et, suivant le développement des faits, je pense que nous serons amenés à vous solliciter de nouveau, bien plus tard. Nous vous demandons donc de collaborer maintenant pour une audition d'environ quatre heures aujourd'hui.

Je vous ai demandé au début si vous nous autorisiez à enregistrer cet entretien et comme vous avez répondu oui, je me permets d'enregistrer. Aujourd'hui, nous envisageons de vous poser toutes sortes de questions. Si vous n'avez pas envie de répondre ou que la question vous gêne parce qu'elle est posée d'un autre point de vue que le vôtre, dites-le-nous. Dans ce cas, nous arrêterons de vous poser ces questions et nous nous dirigerons vers des questions émises d'un autre point de vue.

Encore une chose. Ce que vous allez dire ici va être retranscrit et cette retranscription a des chances d'être rendue publique. On ne peut pas savoir à l'heure actuelle si tout va être rendu public et de quelle manière. Mais, suivant les circonstances, vos paroles seront peutêtre publiées telles quelles, et je voudrais que vous répondiez à nos questions en en ayant conscience. Voilà en gros ce que je voulais vous dire. Est-ce que ça va?

\section{R : Oui.}

$\mathbf{Q}$ : Alors, nous allons commencer. À vous, M. Ogawa.

Je voudrais laisser la parole à Katô pour les questions².

Si vous voulez bien, c'est moi, Katô, du secrétariat, qui vous pose les questions en premier. S'il y a des choses que vous ne savez pas ou qu'on peut retrouver plus tard en faisant des recherches, n'hésitez pas à nous le dire. Surtout qu'il y aura d'autres séances qu'aujourd'hui.

Tout d'abord, M. le directeur, je voudrais que vous nous donniez un aperçu de votre carrière depuis votre entrée chez TEPCO 3 jusqu'à aujourd'hui, dans l'ordre.

R : Je suis arrivé chez TEPCO en 1979. Comme à l'université j'avais étudié le génie nucléaire, j'ai été envoyé à la construction de Fukushima Daini ${ }^{4}$, à la construction du réacteur 2 de Fukushima Daini. Et ça, durant un peu plus de trois ans, jusqu'en 1982, où j'ai été nommé au service de maintenance des centrales nucléaires du siège social. Ce service s'occupe des différents problèmes que peuvent rencontrer les tranches ${ }^{5}$ en 
fonctionnement et des travaux de réparation ou de transformation. J'y suis resté environ quatre ans. C'était de 1982 à 1986. Et ensuite, je suis parti à Fukushima Daiichi $^{6}$, qui a des problèmes et dont je suis le directeur aujourd'hui. J'y ai été nommé de nouveau au service de maintenance. Il s'agissait de la maintenance des réacteurs 5 et 6. J'ai travaillé là deux ans comme sous-chef du groupe "réacteur ».

En 1988, j'ai été rappelé au siège social et, il ne s'agissait plus véritablement d'énergie nucléaire, j'ai été incorporé au service projet énergétique au sein du département «technologie ». Ce service est l'endroit où on élabore le programme énergétique du futur. Savoir quelle va être la proportion entre le thermique, l'hydraulique et le nucléaire ${ }^{7}$, ce genre de choses. J'y ai travaillé pendant deux ans et demi à peu près, en tant que sous-chef. Après ça, j'ai encore changé de poste au siège, toujours en tant que sous-chef, je suis retourné dans le service «maintenance » dont j'ai parlé tout à l'heure. Donc c'est en juillet 1988 que j'ai été affecté au service " projet énergétique » du siège où je suis resté deux ans et demi, puis de 1991 à 1993, j'ai été sous-chef dans le service "maintenance des centrales nucléaires ». Ensuite, de 1993 à 1995, pendant un peu plus de deux ans, j'ai été chef du service "maintenance " à la centrale de Fukushima Daiichi, pour les réacteurs 1 et 2 , ceux qui sont endommagés aujourd'hui.

Après ça, c'est en juillet 1995 que j'ai reçu l'ordre d'aller à la Federation of Electric Power Companies of Japan $(\mathrm{FEPC})^{8}$, là encore un organisme qui n'est pas complètement dédié à l'énergie atomique. J'ai été intégré au département «énergie nucléaire ", un endroit où on est en contact avec les grosses administrations, etc. Juste au moment où j'y étais, il y a eu la fuite de sodium du surgénérateur Monju et puis des histoires avec la Power Reactor and Nuclear Fuel Development Corporation (PNC) ${ }^{10}$ de l'époque, ce qui a entraîné par la suite la restructuration du PNC, dont je me suis occupé ${ }^{11}$. Habituellement, après deux ans de détachement à la Federation of Electric Power Companies, on retourne dans son entreprise d'origine, mais à cause de ces accidents dont j'ai dû m'occuper, j'y suis resté quatre ans. C'est-à-dire que tout à fait à la fin de mon détachement, le PNC a justement été restructuré en Japan Nuclear Cycle Development Institute (JNC) ${ }^{12}$ et j'ai été affecté à cette tâche.

Après ça, de 1999 à 2002, j'étais directeur du département "production» de Fukushima Daini. Ici, il s'agissait de prendre en charge le côté exploitation, mais avec des changements de structures (à l'intérieur de TEPCO), finalement je me suis retrouvé à surveiller aussi une partie de la maintenance et aussi du budget. Jusqu'en juillet 2002, pendant trois ans, ça a été ma fonction. En juillet 2002, je suis retourné au siège, je ne me rappelle plus trop bien du nom du service, oui, les noms changent souvent, en tout cas, j'ai refait encore de la maintenance avec le grade de GM. GM, c'est le « Group Manager ». Le GM n'est pas le directeur de département, il est juste endessous. Le 29 août 2002, le scandale GE (General Electric) ${ }^{13}$ a fait surface et j'ai passé pratiquement deux ans à m'occuper du problème.

$\mathbf{Q}:$ L'enveloppe $^{14}$ du cœur du réacteur ${ }^{15}$ ?

$\mathbf{R}$ : Oui, oui. Les fissures, le scandale. Il a fallu gérer tout ça pendant deux ans. Après, c'était dans le même département "gestion nucléaire ", il a fallu informatiser différentes choses et j'ai travaillé environ un an à élaborer un système concernant la maintenance. Je suis resté trois ans dans le département "gestion nucléaire », les deux premières années je me suis occupé du scandale et la dernière année de l'informatique. 
En juillet 2005, j'ai reçu l'ordre de travailler sur l'exploitation et la maintenance des réacteurs 1 à 4 de la centrale de Fukushima Daiichi, en tant que directeur d'unité. Il s'agissait de regarder l'ensemble des tranches du point de vue technique. Là, ça a été court, je n'y suis resté qu'un an et neuf mois.

Après ça, il y a eu des restructurations au siège, oui, c'est une entreprise où il y a souvent des changements, et j'ai été nommé directeur du département "gestion des installations nucléaires » le $1^{\text {er }}$ avril 2007. Pour vous expliquer ce que fait le directeur du département "gestion des installations », il faut que vous sachiez que dans notre entreprise, historiquement, il y a un département " construction », qui fabrique, qui construit les centrales nucléaires, et un département " gestion nucléaire » qui gère ces centrales, le siège social est nettement scindé en deux entités. On a développé, on a fusionné le département "construction" et le département "gestion des installations", on a fait un endroit où on s'occupait aussi de l'élaboration de l'ensemble des installations, avec des fonctions d'engineering et j'en ai été le premier directeur jusqu'au 28 juin de l'année dernière. Durant cette période, le 16 juillet 2007, ça faisait à peu près trois mois que j'occupais le poste, il y a eu le séisme au large de Chûetsu (le séisme de Niigataken Chûetsu-Oki) ${ }^{16}$. Du coup, j'ai passé à peu près trois ans à m'occuper des suites du séisme. J'ai été nommé ici, l'année dernière, comme directeur.

$\mathrm{Q}:$ Vous rappelez-vous de la date de votre nomination ici?

$\mathbf{R}$ : Les affectations sont datées du jour de l'assemblée générale des actionnaires, alors je pense que c'était le 28 juin.

$\mathbf{Q}$ : À vous entendre dérouler votre carrière en détails, on a l'impression qu'ayant pratiqué à la fois la maintenance, la sûreté, la réparation, la technique de construction et l'exploitation en elle-même, on peut penser que vous avez l'expérience de beaucoup de domaines, n'estce pas?

R : Oui.

$\mathbf{Q}$ : Quand on interroge les gens de TEPCO, on a l'impression que s'il s'agit de la conduite, on fait de la conduite, si on fait du génie civil, on fait du génie civil, et que les gens progressent toujours dans le même domaine. Mais y a-t-il aussi un certain nombre de personnes qui, comme vous, font des expériences différentes?

$\mathbf{R}$ : Oui. Oui, il y en a. Mais comme je suis issu, tout au début de ma carrière, du service de «maintenance des centrales", pour moi, la maintenance est mon pivot. Mais, bien sûr, il y a pas mal de gens qui ont ce genre de parcours.

Q : Durant les auditions que j'ai déjà menées, j'ai remarqué que les gens utilisaient des expressions typiques du monde du nucléaire, comme «nenryô-ya san» («monsieur combustible ») ou "anzen-ya san» («monsieur sûreté »)17. Cela signifie que dans chaque domaine, il y a des gens particulièrement pointus? En plus, le nucléaire, ce n'est pas quelque chose qui nous est familier. Est-ce que des notions ordinaires de construction ou le simple génie civil suffisent? Ou est-ce qu'il faut un savoir-faire un peu différent du génie civil ordinaire, des connaissances ou des expériences spéciales du nucléaire?

$\mathbf{R}$ : À la base, que ce soit du génie mécanique, du génie civil ou de l'architecture, je ne pense pas que ça diffère des autres industries. En tout cas, en ce qui concerne les connaissances. Seulement, ce qui est compliqué, ce sont les dispositions légales. Parce que c'est extrêmement spécial. S'il s'agit des autres domaines, comme le thermique ou l'hydraulique, on peut travailler uniquement dans le cadre de la loi sur l'électricité. Pour les réacteurs nucléaires, il y a la loi de réglementation, c'est-à-dire 
qu'il faut tenir compte de la double contrainte de la loi de réglementation des réacteurs nucléaires et de la loi sur l'électricité. Le savoir-faire qui en découle est assez différent des autres domaines, c'est très spécial, j'avoue. La discussion de savoir s'il y a radioactivité ${ }^{18}$ ou pas est ce qui nous concerne bien sûr en tout premier lieu dans le génie nucléaire. Mais nous avons aussi beaucoup d'autres choses en commun avec les autres domaines.

Q : Évidemment, TEPCO produit de l'électricité non seulement d'origine nucléaire, mais aussi d'autres origines, comme le thermique ${ }^{19}$. Vous nous avez dit que vous aviez été amené vous-même à vous intéresser à des projets énergétiques globaux, mais, de votre point de vue, la voie ordinaire quand on est entré chez TEPCO pour travailler dans le nucléaire est de rester dans le nucléaire jusqu'à la retraite, ou bien, est-il commun d'aller du nucléaire au thermique et à l'hydraulique, pour revenir au nucléaire ? Qu'en pensez-vous ?

$\mathbf{R}$ : Concernant les gens qui sont entrés avant moi ou un tout petit peu après moi, il était courant qu'on entre dans le nucléaire et qu'on y reste. Ces dernières années, depuis un peu plus d'une dizaine d'années, il y a tendance à faire faire différentes expériences. On peut partir au thermique, aller aussi dans des agences, par exemple à l'agence de Tochigi ${ }^{20}$, pour faire soit un travail technique ou même pour faire du commercial. Donc, à mon avis, parmi les jeunes, disons ceux qui sont là depuis un peu plus de dix ans, ceux qui ont bénéficié de cette tendance et qui ont l'expérience d'autres domaines sont de plus en plus nombreux.

$\mathbf{Q}$ : L'heure tourne. Je pense que, suivant les nécessités, nous aurons encore l'occasion de parler de TEPCO en général. Aujourd'hui, je voudrais que nous revenions sur ce qui s'est passé dans les tranches, sur le site, et les mesures qui ont été prises à partir du moment où le séisme a eu lieu, le 11 mars de cette année, à 14h4621, et le moment où le tsunami est arrivé $^{22}$. Comme je pense que vous avez dû faire face à énormément de choses depuis et qu'on pourrait rencontrer des problèmes de mémoire, j'ai fait préparer ici la chronologie publiée par TEPCO. À la page 6 , il y a la chronologie des évènements majeurs depuis le séisme jusqu'au 12 mars, concernant le réacteur $1^{23}$. Pour commencer, en nous appuyant sur cette chronologie, nous voudrions vous interroger sur ce qui s'est passé en gros autour des réacteurs 1 et 2 .

Bien entendu, comme TEPCO le note au début, ce document a été fait sur la base de renseignements et de témoignages, dont ils disposaient à ce moment-là. TEPCO insiste bien sur le fait qu'il pourrait y avoir des modifications suivant les résultats de recherches ultérieures. Donc, si vous constatez des divergences, si votre souvenir est différent, dites-lenous comme vous le sentez. Nous allons vous interroger en tenant compte du fait que les évènements décrits ici sont ceux publiés par TEPCO et qu'en ce qui concerne leur véracité, il peut y avoir des ajustements dans l'avenir, alors si vous pensez que les choses se sont passés autrement, dites-le-nous.

Donc, pour commencer, il est écrit ici, à 14h46, « séisme au large de la région du Tôhoku côté

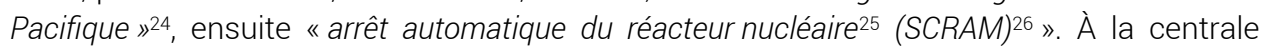
Daiichi, était-il prévu dès le départ qu'en cas de sinistre, on forme une cellule de crise?

R : Oui.

$\mathbf{Q}$ : Dans ce cas-là, est-ce le directeur de la centrale qui dirige la cellule de crise?

R : Oui.

$\mathbf{Q}$ : Ensuite, là, nous nous sommes renseignés avant de venir, la cellule de crise comprend, comme sous-directeurs, les deux directeurs d'unité, puis les trois sous-directeurs. Et, il y a encore en-dessous d'autres membres. La cellule s'installe dans cette salle avec la table 
ronde, que nous avons visitée l'autre jour, avec les gens qui travaillent derrière ${ }^{27}$. C'est bien ça?

R : Je voudrais préciser une chose. Tout d'abord, la survenue d'un séisme n'est pas un évènement relevant de la loi sur les catastrophes nucléaires ${ }^{28}$, c'est un incident ordinaire. Quand un incident ordinaire survient, du point de vue de la gestion du problème, on a à peu près le même dispositif, mais je n'ai l'appellation officielle de chef de la cellule de crise que lorsque la déclaration de l'information selon l'article 10 de la loi $^{29}$ sur les catastrophes nucléaires a été effectuée. Jusque-là, et c'est la même chose pour tous les problèmes ordinaires, étant bien sûr le responsable, je forme sous ma responsabilité une commission et nous prenons les premières décisions. Si, au fur et à mesure, l'incident s'aggrave et qu'il y a information suivant l'article 10, alors, à ce moment-là seulement, cela devient un évènement relevant de la loi sur les catastrophes nucléaires et je deviens officiellement le chef de la cellule de crise selon cette loi et les directeurs d'unité deviennent mes adjoints et m'assistent. Ensuite, comme vous l'avez évoqué tout à l'heure, nous formons divers groupes pour faire face à la crise. Du point de vue légal, ça se passe comme ça.

$\mathbf{Q}$ : Tout d'abord, lorsqu'il y a eu le séisme, où étiez-vous?

R : J'étais dans mon bureau. J'ai un bureau à moi dans le bâtiment administratif et j'étais là.

$\mathbf{Q}$ : Voici le plan que nous avons utilisé lors de notre visite sur le terrain, l'autre jour, il est un peu petit. Un peu plus grand, cela aurait été mieux, mais ce que vous appelez le bâtiment administratif est bien celui qui est au-dessus du bâtiment antisismique ${ }^{30}$ ?

R : Oui, c'est bien ça. C'est celui-là.

$\mathbf{Q}$ : Est-ce que vous voyez? C'est un peu difficile. On entre par l'entrée principale, on se dirige vers la mer et à partir du réacteur, c'est le nord, ça?

R : Oui.

$\mathbf{Q}:$ Au nord-est du réacteur. Et à quel étage des bureaux?

$\mathbf{R}:$ Au premier étage.

$\mathbf{Q}:$ Au premier.

$\mathbf{R}$ : Je vais vous faire un plan succinct. Le bâtiment antisismique que vous avez vu l'autre jour est ici. Relié par une sorte de passerelle, en fait elle est un peu plus courte, voilà le bâtiment administratif. Habituellement, je travaille ici, mais j'ai aussi mon bureau personnel dans un coin du premier étage et j'étais là.

$\mathbf{Q}$ : En ce qui concerne la position des réacteurs, les réacteurs 1, 2, 3 et 4 sont bien ici ? Et les 5 et 6 , par ici ${ }^{31}$ ?

R : Oui.

$\mathbf{Q}:$ Vous étiez seul dans votre bureau?

R : Oui, seul.

$\mathbf{Q}$ : Quand le séisme survient, que vous ressentez les secousses, qu'avez-vous pris comme mesures?

$\mathbf{R}$ : Au début, il y a eu une petite secousse. Je me suis dit, tiens un tremblement de terre. J'étais en train de lire des documents. Je me suis dit, c'est un tremblement de terre, je me suis levé. Alors les secousses sont devenues de plus en plus violentes. Je ne pouvais plus tenir debout. Les choses qui étaient sur les étagères sont tombées, le téléviseur s'est aussi renversé brutalement et je me suis dit que c'était un séisme 
assez important. J'avais la sensation qu'il avait duré assez longtemps, peut-être cinq minutes. Après, j'ai entendu qu'il avait seulement duré trois minutes. Mais j'avais vraiment la sensation que ça avait secoué longtemps. Et durant ce temps, je ne pouvais pas bouger. Je me tenais à mon bureau. Je voulais me glisser dessous, mais je ne pouvais que rester accroché, debout, à mon bureau. Ce faisant, les secousses sont devenues plus petites et j'ai pensé que c'était un séisme impressionnant.

Il y a une porte là et je suis sorti du bureau. Et là, tous les faux plafonds étaient tombés, les documents des étagères étalés partout par terre. Il y avait un brouillard blanc à cause de la poussière qui avait été soulevée au moment où le plafond a été arraché. Il y avait encore quelques personnes du service administratif. Quand je leur ai demandé où ils en étaient, ils m'ont dit que tout le monde était parti se réfugier. Je leur ai dit d'y aller eux aussi et je suis sorti. En théorie, le lieu où on se rassemble en cas d'incident se trouve devant l'immeuble antisismique. En principe le passage qui va d'ici à là est la voie officielle d'évacuation, mais à ce moment-là le volet qui est ici était fermé et on ne pouvait pas accéder à la voie d'évacuation. Il y a un espace dégagé par ici et un bon nombre de personnes étaient réfugiées là. Je leur ai donné l'ordre de se rassembler ici et je les ai toutes rassemblées ici.

$\mathbf{Q}$ : Vous me dites que vous ne pouviez pas gagner la voie d'évacuation et que le volet était fermé. Pourquoi ?

$\mathbf{R}$ : Je ne sais pas très bien. Mais c'est probablement le même principe que pour les portes anti-feu. Cela devait être prévu comme ça. Que ça devait se fermer automatiquement quand il y avait un choc. Il faudrait vérifier ce point.

$\mathbf{Q}$ : Donc, en principe, vous deviez emprunter cet itinéraire pour aller vous réfugier.

R : Oui. L'itinéraire officiel, c'est d'emprunter ce passage pour sortir de là vers là. À peu près un mois avant le séisme, on avait fait une séance d'entraînement à l'évacuation. On avait vérifié les itinéraires en cas d'incident. Mais, tout à fait par hasard, de mon bureau, je n'ai pas pu suivre cet itinéraire à cause du volet fermé, donc je suis descendu par l'escalier.

$\mathbf{Q}$ : Excusez-moi, je n'ai pas noté à quel étage vous étiez.

$\mathbf{R}:$ Au premier.

$\mathbf{Q}:$ L'immeuble compte combien d'étages?

$\mathbf{R}:$ Il a deux niveaux.

$\mathbf{Q}$ : Comme on enregistre, je voudrais vérifier. Donc, lors de l'entraînement à l'évacuation, un mois auparavant, le lieu d'évacuation, qui avait été choisi pour les gens qui travaillaient dans les bureaux, était la place qui se trouve au nord du bâtiment antisismique, pour des raisons d'itinéraire d'évacuation. Et c'était valable pour tout le monde.

$\mathbf{R}$ : C'est pour moi (qu'il y a eu des problèmes). Quand je suis arrivé ici (le premier espace dégagé $-\mathrm{a}-)^{32}$, un bon nombre de personnes étaient déjà là (place au nord du bâtiment antisismique -b-). Mais nous étions plusieurs dizaines, y compris moi, à n'avoir pas pu aller là (-b-) et à nous être retrouvés ici (-a-). Alors j'ai ordonné que tout le monde se retrouve là (-b-), même ceux qui étaient ici (-a-). Moi-même, je me suis déplacé pour aller là-bas (-b-) et je leur ai dit de faire l'appel là-bas (-b-).

$\mathbf{Q}$ : Combien de personnes se sont rassemblées sur cette place au nord du bâtiment antisismique?

$\mathbf{R}$ : Je ne me rappelle pas du chiffre exact à ce moment-là. Il y avait plusieurs groupes, c'était les gens qui travaillaient là à ce moment-là, alors, probablement, ils devaient 
être entre sept cents et huit cents. En plus, il y avait aussi le personnel des entreprises (autres que TEPCO) qui s'était réfugié là. Alors j'ai dit que chaque entreprise fasse l'appel pour son propre personnel. Je pense qu'ils étaient de l'ordre de sept cents à huit cents personnes. Nous n'avons pas noté les chiffres.

$\mathbf{Q}$ : À l'heure où ça s'est passé, combien de personnes de TEPCO et combien de personnes d'entreprises partenaires étaient présentes sur le site?

$\mathbf{R}$ : Pour ce qui est du personnel de TEPCO, à part ceux qui étaient affectés à la conduite du réacteur, qui étaient dans la salle centrale de commande et dont je ne sais pas quelle proportion est venue se réfugier avec nous, je pense qu'ils étaient grosso modo entre six cents et sept cents. Pour ce qui est des entreprises, comme le réacteur 4 était en cours de révision périodique, il devait y avoir plus d'un millier de personnes pour l'inspection. De plus, pour chaque réacteur, pour l'entretien, surtout pour les réacteurs 5 et 6 , qui étaient à l'arrêt, il y avait des gens qui étaient là pour le compte du fabricant, d'autres pour les entreprises partenaires. Je pense qu'ils devaient être de l'ordre de mille et quelques centaines.

$\mathbf{Q}$ : Donc, un millier de personnes du côté du réacteur 4.

$\mathbf{R}$ : Oui. Un millier et quelques centaines, je pense.

$\mathbf{Q}$ : Plus neuf cents ? Huit cents ? Cinq cents ? En gros, plus cinq cents ? C'est à peu près ce que vous pensez?

$\mathbf{R}$ : Oui. Je pense qu'il reste des traces de ça dans les archives, mais il me semble bien que c'était à peu près cinq cents.

$\mathbf{Q}$ : Si je comprends bien, la totalité des personnes qui étaient sur le site ne s'est pas rassemblée sur la place au nord du bâtiment antisismique. C'est bien ça?

$\mathbf{R}$ : C'est-à-dire que les agents affectés au pilotage des tranches ${ }^{33}$ se trouvent forcément dans la salle centrale de commande. Eux, ne quittent pas les lieux. Cela dépend des jours de la semaine, mais d'ordinaire, en période d'exploitation normale, dans chaque salle de commande ${ }^{34}$, il y a onze personnes en plus du chef de quart ${ }^{35}$. Ensuite, il y a le groupe de support qui, traditionnellement se trouvait dans le bâtiment administratif, mais que j'avais décidé de positionner dans une salle près de la salle de contrôle. De cette manière, il y a encore entre vingt à trente agents, le chiffre dépend des réacteurs, qui sont là en renfort. De plus, dans le cas de tranches en révision programmée, comme pour le réacteur 4, il y a du personnel pour l'entretien, mais il faut aussi des agents de conduite dans la salle de commande, car chaque manipulation dans la salle doit obligatoirement être faite par eux, même dans le cadre de l'entretien. Par exemple, s'il faut couper l'alimentation pour l'entretien, c'est aux agents de conduite de faire ce qu'il faut pour ça à partir de la salle de contrôle. Et ce genre de travail exige beaucoup de monde. Bien plus que pour une tranche en fonctionnement. Donc, (au moment du séisme) pour les réacteurs 1 et 2 qui étaient en fonctionnement, il y avait comme d'habitude une équipe de onze qui s'en occupait avec, à côté, les vingt qui étaient là pour les aider, du point de vue administratif ou autre. Mais autour des réacteurs 3 et 4 qui étaient en cours d'inspection programmée, il y avait beaucoup plus d'opérateurs de conduite que du côté des réacteurs 1 et 2 . 
$\mathbf{Q}:$ Vous êtes donc allé sur cette place, qui servait de refuge, et combien de temps y êtesvous resté?

R : Ce que j'ai fait d'abord en arrivant, c'était de chercher le chef du département "sûreté ", car c'est ce département qui centralise les commandes quand il y a des catastrophes, comme les séismes. Mais le chef n'était pas là. Aussi, j'ai donné l'ordre aux Group Managers de faire l'appel pour chaque groupe. Depuis la survenue du séisme, il s'était passé... Je ne me rappelle pas bien de l'heure, mais, pour moi, vers 15 h00 je suis parti de là pour le bâtiment antisismique.

$\mathbf{Q}$ : Avant que vous ne partiez vers le bâtiment antisismique, au moment où vous avez fait l'appel, aviez-vous déjà des informations concernant des personnes manquantes ou des blessés?

R : Oui, il y avait bien deux personnes qui s'étaient légèrement blessées à la main, pas de fractures, non, rien de très grave. Je leur ai dit de se faire soigner par le groupe médical, qui prodigue les soins médicaux. Je ne suis pas resté jusqu'à la fin des appels. Je suis parti en donnant l'ordre qu'on m'informe des résultats.

$\mathbf{Q}$ : Pour quelle raison êtes-vous allé dans la salle des crises du bâtiment antisismique?

$\mathbf{R}$ : Parce qu'il fallait diriger les tranches à partir de là. Une fois qu'on a vérifié que les personnes étaient saines et sauves, le plus important était la conduite à venir des tranches et d'être là pour diriger l'ensemble. Parce que c'était mon travail d'aller là.

$\mathbf{Q}$ : D'après ce que vous nous avez raconté tout à l'heure, la cellule de gestion de crise est constituée pour répondre aux évènements qui sont prévus dans le cadre de la loi sur les catastrophes nucléaires. C'est bien ça?

$\mathbf{R}$ : Du point de vue légal, c'est bien ça. Mais même concernant des incidents moins graves, en principe, on se réunit à la cellule de crise et on prend des mesures pour éviter l'aggravation d'un incident, etc. Pour les incidents vraiment mineurs, on peut faire ça au sein de petits groupes, comme le groupe "maintenance » ou le groupe " production ». Mais pour des incidents plus importants, on a l'habitude de prendre les mesures ici. Et parmi ces incidents, certains peuvent évoluer et donner lieu à la constitution d'une structure relevant de la loi sur les catastrophes nucléaires, comme vous l'avez dit.

$\mathbf{Q}$ : Alors ça veut dire qu'avant même qu'un incident relevant de l'article 10 de la loi sur les catastrophes nucléaires n'advienne, dans la réalité, vous constituez une structure similaire avec, par exemple le groupe "production», le groupe «sûreté », qui se rassemblent et prennent des mesures en concertation.

$\mathbf{R}$ : Oui. Seulement, quand je suis arrivé à la cellule de crise, ils étaient en train de faire l'appel, ils n'avaient pas encore fini, du coup, tous les membres de la cellule n'étaient pas là ${ }^{36}$. Au début, il y avait avec moi les chefs d'unité, les chefs de production, les chefs de maintenance, c'est-à-dire des directeurs. Puis les GM sont arrivés. Ceux qui avaient fini l'appel arrivaient les uns après les autres en renfort. Voilà la situation.

$\mathbf{Q}$ : Donc, parmi le personnel travaillant d'ordinaire sur place, tout le monde ne fait pas partie de la cellule de crise. Cela veut dire qu'il y a des personnes désignées à l'avance comme devant faire partie de la cellule?

$\mathbf{R}$ : Oui. Elles sont environ quatre cents. 
$\mathbf{Q}$ : Environ quatre cents personnes, donc. Ces personnes désignées à l'avance comme devant faire partie de la cellule de crise sont arrivées dans le bâtiment antisismique au fur et à mesure qu'elles avaient été pointées comme saines et sauves. C'est ça?

R : Oui.

$\mathbf{Q}$ : Une fois que vous vous êtes retrouvé dans le bâtiment antisismique, quelle mesure pensiez-vous prendre en premier?

$\mathbf{R}$ : Tout d'abord, quand il y a un énorme séisme comme celui-ci, il y a SCRAM de la tranche. Le SCRAM, c'est l'arrêt automatique de la tranche. C'était cette situation. Il fallait vérifier si les tranches, surtout les tranches 1,2 et 3 qui étaient en fonctionnement, s'étaient bien arrêtées automatiquement, si le SCRAM s'était fait correctement. Ensuite il fallait vérifier aussi si autour, y compris l'alimentation électrique, il n'y avait pas d'anomalie. Et puis, puisque c'était un séisme et qu'il pouvait avoir causé des destructions, vérifier la situation de ce côté aussi.

$\mathbf{Q}$ : Pour ce genre de vérification, par exemple pour vérifier le SCRAM, vous prenez contact avec le personnel de quart en passant par le groupe « production »?

R : Oui.

$\mathbf{Q}$ : Alors, je pense que cela appelle la question du moyen de communication entre la cellule et l'équipe de quart. J'ai entendu dire que vous utilisiez habituellement le PHS (Personal Handy Phone System) ${ }^{37}$. À ce moment-là, est-ce que le PHS fonctionnait ?

$\mathbf{R}$ : Je n'ai pas vérifié à chaque fois par quel moyen nous avons communiqué. Moi, j'attendais assis à la table centrale que le chef du département " production » vienne me parler de la situation des tranches. Je ne sais donc pas comment le directeur de production a communiqué concrètement avec l'équipe de quart. Seulement, d'après ce que j'ai entendu par la suite, on m'a dit qu'à ce stade-là les moyens usuels de communication étaient encore disponibles.

$\mathbf{Q}$ : Tout d'abord, il y a eu la vérification du SCRAM des réacteurs 1 à 3. Et qu'en était-il ?

R : C'était bon ${ }^{38}$.

$\mathbf{Q}$ : Vous avez eu un rapport comme quoi c'était bon. C'est un fait que vous aviez acquis avant le tsunami?

R : Oui.

$\mathbf{Q}$ : Ensuite, concernant l'électricité. Avez-vous eu des rapports signalant qu'il se passait quelque chose d'anormal ? Quel genre de rapport avez-vous reçu ?

$\mathbf{R}$ : C'est là où je ne me souviens pas très bien de la chronologie. Je ne me rappelle pas à quel moment précis est survenue la perte des sources externes d'électricité. Mais, à ce moment-là, les groupes électrogènes de secours à moteur diesel tournaient, donc je pense que c'est là que s'est passée la perte des sources externes d'électricité. Probablement, il a dû y avoir à ce moment-là des problèmes sur les câbles électriques ou des dégâts sur les machines de la station de commutation à cause du séisme. D'ordinaire, si l'alimentation électrique externe est fonctionnelle, le groupe électrogène diesel ne se met pas en marche automatiquement. Comme j'ai entendu à ce moment-là qu'il s'était mis en marche tout seul, j'en ai déduit que les sources externes étaient perdues.

$\mathbf{Q}$ : Cela signifie que vous aviez reçu un rapport du groupe «production » disant que le groupe électrogène de secours à moteur diesel était en fonction, avant l'arrivée du tsunami?

R : Oui. 
Q : Et vous en avez naturellement déduit que vous aviez perdu les sources externes d'électricité. Mais vous n'avez pas encore connaissance de faits, comme l'état des câbles électriques ou la chute d'un poteau électrique, ce genre de choses, donnant une idée concrète de la situation des dégâts, concernant cette perte de sources.

$\mathbf{R}$ : Non, pas à ce moment-là. Parce qu'on ne fait que vérifier dans la salle de commande le signal, qui indique si le courant arrive ou pas. On ne fait que vérifier sur l'écran de la salle si le courant arrive ou pas. Alors, à ce stade, on ne sait pas concrètement qu'est-ce qui est endommagé et de quelle manière.

$\mathbf{Q}$ : À part ce problème, est-ce que vous vous êtes posé la question d'autres dégâts sur les installations, alors que vous venez à peine de terminer de vérifier que le personnel était sain et sauf? Avant l'arrivée du tsunami, vous constatez que le dispositif électrique de secours s'est mis en marche, et vous en déduisez que probablement vous avez perdu vos sources externes d'alimentation, et vous savez aussi que vous avez réussi à aller sans encombre jusqu'à l'arrêt automatique des réacteurs 1 à 3 . À part tout ça, avez-vous eu des informations signalant des faits s'écartant de la norme, par exemple de l'eau qui fuirait d'une canalisation, ou une fumée blanche s'élevant d'un endroit, avant la survenue du tsunami, et dans quelle proportion ? Ou n'en avez-vous pas eu?

$\mathbf{R}$ : A priori, il n'y a presque rien eu. J'ai dit presque, mais à ce stade-là, en fait, dans la cellule de crise, je n'ai absolument rien entendu de tel, ni à propos d'une quelconque fuite de la tranche ni de dommages sur des machines.

$\mathbf{Q}$ : Alors, avez-vous personnellement donné des ordres à ce moment-là pour que les agents qui étaient sur place vérifient, par exemple, les réacteurs 1 à 3, ou même les 4 à 6 , ou bien vérifient dans quel état étaient les prises d'eau de mer ou les installations autour (des réacteurs), pour voir s'il n'y avait pas de dégâts, etc. ?

R : Non.

$\mathbf{Q}$ : Sur le fait que vous n'ayez pas donné ces ordres, quelles sont vos réflexions ?

$\mathbf{R}$ : Bien sûr, ce sont des ordres que j'aurais donnés à un moment ou à un autre. Mais à ce stade-là, le plus important était de savoir si les hommes, aussi bien nos agents que les autres, si tout le personnel était bien toujours en vie. C'était ça. C'était là ce que je devais assurer de manière sûre en priorité. Ensuite, si les réacteurs étaient en arrêt automatique et que les générateurs diesel étaient en fonctionnement, nous disposions du dispositif pour maintenir le refroidissement des réacteurs. Et donc je pouvais laisser l'équipe de conduite diriger les opérations. De plus, les agents de conduite aussi s'étaient mis à l'abri à cause du séisme. De toute façon, s'il y a un séisme, il y a toujours une possibilité de tsunami, évidemment on ne pensait pas que le tsunami serait aussi important, mais à cause de la possibilité de tsunami, on ne pouvait pas envoyer des agents inspecter. Surtout dans les parties bordant directement la mer. Donc, je leur ai logiquement dit de se mettre à l'abri dans un premier temps. Dans l'ordre des choses, a priori, on n'envoie du personnel pour des inspections qu'une fois que les choses se sont calmées. C'est pourquoi, la priorité à ce moment-là pour moi, c'était les hommes.

$\mathbf{Q}$ : Après le séisme et avant le tsunami, vous vous êtes préoccupé de savoir si tout le monde était en vie, s'ils n'étaient pas blessés. Tout à l'heure, vous nous avez raconté que vous aviez su qu'il y avait eu des blessés légers quand vous étiez encore dehors. Une fois que vous vous êtes retrouvé dans la salle de gestion de crise, avez-vous eu d'autres rapports concernant le nombre de blessés et ce genre de choses?

$\mathbf{R}$ : Les groupes continuaient à vérifier et je recevais des rapports disant, tel groupe «RAS » ou tel groupe, « tout le monde est là ». En plus, j'avais aussi des rapports des différentes salles de commande. Les agents de conduite ne pouvaient pas quitter leur 
poste, mais d'autres agents avaient quitté les lieux pour venir se réfugier avec nous. Donc ils devaient vérifier combien de personnes devaient se trouver dans la salle de commande. Ce genre de rapports s'est succédé. Parmi ces rapports, il y avait en effet quelques blessés légers. On continuait donc à vérifier la présence et l'état de santé des personnes dans la tranche ou l'unité, savoir s'il n'y avait pas de blessés, et nous étions tenus au courant.

$\mathbf{Q}$ : Vous venez de dire qu'ils ne pouvaient pas quitter leur poste. Cela veut dire qu'il y avait des gens qui ne devaient pas quitter leur poste et d'autres qui, en cas de séisme, devaient se réfugier dans un premier temps. C'est-à-dire qu'il y avait deux groupes?

R : Bien sûr.

$\mathbf{Q}$ : Des agents qui pilotent certaines installations essentielles ne doivent pas se mettre à l'abri, c'est ça?

$\mathbf{R}:$ A priori, les agents de conduite ne doivent pas quitter la salle de commande.

$\mathbf{Q}$ : Même si le bâtiment est détruit?

$\mathbf{R}$ : La destruction d'un bâtiment, vous évoquez un cas limite, mais a priori ils ne doivent pas.

Q: Si on regarde la chronologie de la page 6 , on voit qu'à 14h52, le condenseur de secours, ce qu'on appelle I'IC (Isolation Condenser) ${ }^{39}$, s'est mis automatiquement en marche. II est noté, en plus, que, concernant les réacteurs 2 et 3, le RCIC (Reactor Core Isolation Cooling system) ${ }^{40}$ a été activé manuellement. À ce moment-là, avez-vous, vous, des informations sur les différents systèmes IC ou RCIC de chaque réacteur, savoir s'ils sont en fonctionnement ou pas?

$\mathbf{R}$ : Bien sûr, concernant l'IC et les RCIC, je suis informé des opérations par les chefs de production. En plus, ces informations étaient notées sur le tableau blanc de la cellule de crise, il doit y en avoir des traces.

Q: Je suppose que le personnel de quart agissait en conséquence, si c'était un démarrage automatique, vérifier qu'il y avait bien eu démarrage, ou bien faire la manœuvre manuellement, si cela s'imposait. Et ces informations arrivaient bien jusqu'au directeur par le truchement des groupes « production »?

$\mathbf{R}$ : Jusqu'au directeur, enfin, disons que les gens font leur rapport à voix haute. Le chef de groupe "production» dit à voix haute le contenu des informations qu'il a reçues de la salle de commande. Il dit à ceux qui sont autour de la table, « rapport $d u$ groupe production, IC, réacteur 1 », etc. Les autres répondent « compris ». C'est comme ça qu'on partageait les informations.

$\mathbf{Q}$ : Donc, le chef du groupe «production » ou le responsable de la conduite, qui sont assis à la table ronde, parlent au micro et partagent l'information avec ceux qui les écoutent. C'est bien ça?

$\mathbf{R}$ : Oui, c'est ça. Et pour montrer que j'ai bien entendu l'information, je dis toujours « compris ».

$\mathbf{Q}$ : Il est noté à la page 6 que la première vague du tsunami est arrivée à 15h27. Jusque-là, d'après votre expérience, aviez-vous des inquiétudes particulières concernant certains points, à propos des réacteurs 1 à 3 , qui étaient en fonctionnement et qui étaient en cours d'arrêt à froid ${ }^{41}$ ?

R: Oui. A priori, des inquiétudes, très fortes. Puisque les générateurs diesel fonctionnaient, l'alimentation électrique était assurée pour le moment. Ça, c'était un élément rassurant. Bien sûr, on ne savait pas qu'un tsunami de cette importance 
allait venir, mais, logiquement, il y avait des possibilités pour qu'un tsunami suive le séisme. Dans ce cas-là, les pompes du circuit à l'eau de mer $^{42}$ allaient être submergées par l'eau de la vague. Mais, en fait, ce qu'on redoute le plus, c'est quand la vague se retire et qu'on ne dispose plus de suffisamment d'eau pour alimenter le circuit, qu'on ne dispose plus de la source froide pour le système à l'eau de mer. À ce moment-là, on a d'autres sources d'alimentation en eau, le réservoir du condenseur, l'eau de la chambre de suppression de pression ${ }^{43}$, par exemple. Je prévois évidemment qu'il va falloir faire des manœuvres pour injecter cette eau par le circuit de refroidissement de secours du cœur du réacteur. Dans cette situation, c'était un élément réconfortant de savoir que des systèmes, comme le RCIC et l'IC, qui marchent sans électricité, étaient en fonctionnement, puisqu'ils assurent une certaine stabilité à la tranche. Mais je pensais déjà à la manière dont il faudrait continuer le refroidissement, et ça, dès le début.

$\mathbf{Q}$ : Vous êtes donc très préoccupé par la conduite à tenir pour prendre le relais de l'IC et des RCIC. Ces inquiétudes, les avez-vous gardées pour vous, ou les avez-vous exposées aux membres autour de la table, pour discuter des mesures à prendre, les avez-vous formulées verbalement?

R : À ce moment-là, j'étais surtout occupé à recevoir les rapports. Voyez-vous, l'ambiance, ce sont des rapports qui se succèdent sans discontinuer, quid du personnel ?, etc. En plus, il y avait aussi des questions qui venaient du siège social, de la maison mère ${ }^{44}$. Si vous imaginez l'ambiance de la salle à ce moment-là, c'est la confusion. Toutes sortes de rapports verbaux, des préoccupations sur la survie des uns et des autres, on était relié par téléconférence avec le siège, qui demandait quelle était la situation. Au milieu de tout ça, tout en réagissant à tout ça, il était assez difficile de penser à la suite du refroidissement. J'ai toujours eu conscience qu'il fallait que je réfléchisse à la manière d'utiliser le ECCS (Emergency Core Cooling System $)^{45}$. Mais, à ce stade-là, je pense que, selon toute probabilité, je n'en avais pas encore parlé à voix haute. Pas avant l'arrivée du tsunami.

Il va de soi que les pilotes, dans cette situation, bien plus que moi, savaient exactement ce qu'il convenait de faire et c'est en présupposant qu'ils faisaient naturellement ce qu'il convenait que j'y réfléchissais. Mais je pense que je n'en ai pas spécialement parlé à voix haute à ce moment-là. De toute manière, tout le monde était bouleversé par le séisme, alors je me rappelle bien leur avoir dit de reprendre leur sang-froid. Ça, je le leur ai dit. Qu'il fallait qu'ils retrouvent leur sang-froid, qu'il ne fallait pas qu'ils s'affolent, qu'il fallait qu'ils procèdent aux vérifications posément. Je leur ai aussi dit qu'il allait y avoir des répliques, qu'il fallait prendre les mesures qui s'imposaient. C'est de cette manière que je leur ai parlé.

$\mathbf{Q}$ : Parmi les informations qui arrivent à la cellule de crise, on note à la page 6 la mention " arrêt automatique de la turbine principale ${ }^{46}$ du réacteur 7 » à 14 h47. D'après ce que TEPCO a rendu public et d'après le rapport que l'État a rendu à l'AIEA ${ }^{47}$ basé sur des auditions de TEPCO, il est signalé qu'à $14 \mathrm{~h} 47$ l'alimentation électrique des différents appareils de mesure a été perdue à cause de la perte des sources externes d'électricité et que cela a entraîné le fonctionnement du système de sécurité qui a entraîné à son tour la fermeture de la vanne d'isolation du circuit principal de vapeur ${ }^{48}$. Avez-vous eu l'information que la vanne d'isolation du circuit principal de vapeur s'était fermée?

$\mathbf{R}$ : Je pense qu'on ne reçoit probablement pas l'information sous la seule forme de la fermeture de la vanne d'isolation du circuit principal de vapeur. Après l'arrêt automatique du réacteur, avec le signal d'arrêt automatique, au moment où l'arrêt 
automatique se fait, suivant une séquence qui diffère de celle que vous venez d'évoquer, le système va aller isoler l'enceinte de confinement ${ }^{49}$, cela va déclencher le signal de l'isolation de l'enceinte de confinement et, très naturellement, la fermeture du MSIV (Main Steam Isolation Valve) ${ }^{50}$. A priori, c'est un déroulement naturel. Et s'il y a fermeture de la vanne d'isolation, là encore, très naturellement, ça entraîne l'arrêt de toutes les turbines. Donc, on ne se pose pas de question. On se dit, d'accord, c'est comme d'habitude. Et, du coup, il n'y a pas eu d'information séparée concernant juste le MSIV.

Q: Au moment que nous évoquons, ou même plus tard, quand on dit que la vanne du circuit principal de vapeur s'est fermée, peut-on savoir si cette fermeture est due au fonctionnement du système de sécurité, ou bien si elle est effectuée pour empêcher la fuite d'une grande quantité de vapeur à cause d'une grosse brèche dans la tuyauterie, par exemple ? TEPCO fait l'hypothèse que cette fermeture s'est faite par le système de sécurité, en arguant que, s'il y avait eu des dégâts sur la tuyauterie, cela aurait provoqué une grosse fuite de vapeur, et que, au regard des enregistrements qu'on a consultés après coup, on n'en a pas trace. Concrètement, s'il y avait eu des fuites d'un gros volume de vapeur à la suite de dommages sur la tuyauterie, est-ce que les opérateurs de quart, dans la salle de contrôle des réacteurs 1 et 2, auraient pu s'en rendre compte?

$\mathbf{R}:$ Je pense que ce serait sans doute très difficile de s'en rendre compte, quand toutes sortes d'indicateurs clignotent dans tous les sens dans la salle de commande. Vous comprenez, boum, l'arrêt automatique vient de se faire à l'instant. Puisque, s'il y a arrêt automatique, il y a forcément fermeture des MSIV, à cet instant précis, celui qui est dans la salle de commande, quand il voit le signal MSIV fermée, pense que cela s'est fermé à la suite de l'arrêt automatique. Si, comme vous venez de l'évoquer, par malheur, il y avait une rupture dans la tuyauterie vapeur et qu'il y avait fuite de vapeur, cette fois, il y aurait eu une autre alarme, par exemple s'il y avait eu rupture sur le circuit de vapeur principal, ce circuit de vapeur qui va du bâtiment réacteur ${ }^{51}$ au bâtiment turbine, à ce moment-là le détecteur de radioactivité aurait été activé et aurait donné l'alerte comme quoi la radioactivité était élevée. Mais je ne sais pas si, à ce moment-là, celui qui se trouvait dans la salle de commande a vu le signal ou pas et je n'ai pas vérifié la chose. Mais, en tout cas, on aurait pu vérifier s'il $\mathrm{y}$ avait rupture par cet autre signal.

Q: Donc, pour résumer sur ce point, vous n'aviez absolument pas connaissance d'une quelconque rupture sur le circuit principal de vapeur et vous n'aviez pas non plus reçu d'information le laissant soupçonner.

$\mathbf{R}$ : Non, absolument rien. Et donc, pour moi, je pense que l'arrêt des turbines était un arrêt automatique tout à fait normal après le SCRAM.

$\mathbf{Q}$ : Je m'écarte un peu du sujet, mais, à vous entendre, en fin de compte, vous dites que tout s'est arrêté parce que la vapeur n'arrivait plus. Mais n'arrive-t-il pas qu'on commande un arrêt d'urgence des turbines principales par d'autres systèmes, par exemple en observant une augmentation inhabituelle de la vitesse ou qu'on détecte des anomalies au niveau du rotor ${ }^{52}$ ?

$\mathbf{R}$ : Concernant les turbines, il y a bien sûr la logique de protection des turbines, comme le fait que l'arrêt s'enclenche à $17,5 \%$ de vibration axiale ${ }^{53}$. Alors, savoir si, à ce moment-là, la turbine s'est arrêtée suivant la logique de protection des turbines ou à la suite de la fermeture de la MSIV, là... 
$\mathbf{Q}:$ Vous ne le savez pas à ce moment-là.

$\mathbf{R}$ : Non. Si on ne regarde pas les enregistrements au moment où ça se passe, le SPDS (Safety Parameter Display System) ${ }^{54}$, ou les résultats détaillés enregistrés par les appareils, tant qu'on ne les regarde pas, on ne peut pas conclure.

$\mathbf{Q}$ : Vous pouvez me répondre maintenant ou plus tard, mais sait-on aujourd'hui, comment tout cela s'est arrêté?

$\mathbf{R}$ : Oui, puisque le SPDS a fonctionné et qu'on a récupéré les données. D’après ce que j'ai entendu, je pense que l'arrêt était dû au processus normal découlant du SCRAM.

$\mathbf{Q}:$ Alors, ce n'est pas parce que l'axe s'était déplacé...

$\mathbf{R}$ : Là, c'est difficile. Un séisme arrive, boum. C'était un très gros séisme et il a duré environ trois minutes. À ce moment-là, logiquement, on va vers le déplacement de l'axe. L'arrêt automatique du réacteur se déclenche à 100 gal $^{55}, 110$ gal, 120 gal. Dès qu'on dépasse 100 gal, le signal de SCRAM se déclenche et dans les trois secondes et demie le SCRAM est exécuté. Alors, bien sûr, avec le séisme l'arbre de la turbine a dû être secoué...

$\mathbf{Q}$ : Ah oui ! Si ça s'arrête au bout de trois secondes et demie, effectivement, on ne peut plus savoir.

$\mathbf{R}:$ C'est exactement ça.

$\mathbf{Q}$ : Une vérification pour savoir si la tuyauterie est endommagée ou pas, ça peut se faire en cinquante minutes?

$\mathbf{R}$ : Oui, on peut le savoir. En fait, si les détecteurs de radioactivité sont en état de fonctionner, il suffit d'y jeter un coup d'œil. Seulement, ce qui était compliqué, c'est qu'après ça, avec le tsunami, on n'arrivait plus à rien voir. Donc, on ne peut pas revenir en arrière et savoir ce qu'il en était.

$\mathbf{Q}$ : Je voudrais comprendre quel était votre état d'esprit durant les cinquante minutes avant l'arrivée du tsunami. C'est peut-être une question un peu prématurée, mais au bout des cinquante minutes, juste au moment où le tsunami s'apprête à arriver, pour vous, vous aviez la sensation que pratiquement rien d'essentiel n'était endommagé ?

$\mathbf{R}$ : Pour moi, il y avait eu SCRAM, les générateurs diesel s'étaient mis en route, je me suis demandé s'il n'y avait pas d'autres paramètres qui indiquaient des anomalies, je ne saurais vous dire lesquelles précisément, mais j'ai bien demandé à chaque salle de commande de vérifier qu'ils ne voyaient pas d'autres anomalies. Et donc, j'ai entendu de la part des différents chefs de quart qu'il n'y avait pas d'anomalie notable, de leur point de vue et au vu des différents appareils. Parmi ces anomalies notables il faut inclure des incidents du genre rupture du circuit de vapeur principal, dont on vient de parler. Si cela avait été le cas, on l'aurait su par d'autres signaux d'alarme. Dans la situation où nous nous trouvions, je n'ai bien sûr pas donné d'indications précises sur chaque point à vérifier, mais, pour moi, il était évident qu'ils vérifiaient tout cela aussi.

$\mathbf{Q}$ : Je ne sais pas si on peut parler de normalité dans un cas pareil, mais, dans la chronologie, à la page 6 , il est indiqué que vers 15h03, il y a eu: "arrêt manuel pour permettre la régulation de la pression de la cuve ${ }^{56}$ par l'IC » et ensuite, plus tard: " démarrage de la régulation de la pression de la cuve par I'IC».

$\mathbf{R}$ : Si on retourne à ce moment-là, ce qui est consigné ici ne sont pas tous des faits que j'ai pu vérifier lorsque j'étais à la cellule de crise. On y a aussi inclus des choses qui ont été repérées plus tard. D'après ce que je peux en juger, concernant l'IC, je n'ai 
pas été informé du fait, ni du moment où on l'avait arrêté manuellement, quand cela s'est passé.

Q: C'est un point que je voulais justement éclaircir avec vous: ces fréquentes manipulations d'ouverture et de fermeture de l'IC, dont parle notamment la première page de la chronologie. En règle générale, une fois que l'IC a été ouvert, est-il courant de le fermer, ouvrir, fermer, ouvrir à intervalles réguliers?

$\mathbf{R}$ : Tout d'abord, il faut que vous ayez conscience qu'il est extrêmement rare qu'on mette réellement en branle l'Isolation Condenser dans une situation d'urgence, en dehors des tests. Les pilotes ont, bien entendu, une connaissance approfondie de la manière de mettre en œuvre l'IC, comment le piloter. Mais contrôler l'IC en vrai sur le terrain est une action dont ils n'ont presque pas l'expérience jusque-là. Si on réfléchit bien, utiliser l'Isolation Condenser, c'est laisser échapper de la vapeur qui se trouve à l'intérieur de la cuve. Le niveau de l'eau à l'intérieur de la cuve descend d'autant. Il faut pourtant contrôler le niveau de l'eau de la cuve. Si on laisse passer trop de vapeur par l'IC, le niveau de l'eau à l'intérieur de la cuve va trop baisser. Je pense que c'est pour ça que le pilote a fait ces manœuvres, pour maitriser tout ça. Mais, sur le moment, je n'ai rien su de ces manipulations.

Q : C'était la première fois que vous mettiez l'IC en marche à Fukushima Daiichi ?

$\mathbf{R}$ : Non, il avait été utilisé une fois dans le passé. Je n'en ai pas de souvenirs précis, car je n'étais pas là. Mais vers 1991, ce n'était pas à cause d'un tsunami, mais il y avait eu une fuite sur la tuyauterie enterrée du circuit eau de mer du réacteur 1 de Fukushima Daiichi ${ }^{57}$. Du coup, l'électricité ne passait plus, il y a eu un phénomène approchant, et j'ai entendu dire qu'à ce moment-là ils ont mis en marche l'IC. À l'époque, moi-même, j'étais au siège social à la maintenance des réacteurs, je n'étais pas sur le terrain et je ne sais pas exactement ce qu'ils ont fait. Mais d'après les archives, ils ont mis l'IC en marche à ce moment-là.

\section{$\mathbf{Q}$ : Et en ce qui concerne les SCRAM ?}

$\mathbf{R}$ : Les SCRAM, plusieurs fois. Le recours au SCRAM, s'il s'agit du réacteur 1, il n'y en a presque pas eu ces dernières années. Mais si on remonte une vingtaine d'années en arrière, il y a eu pas mal de SCRAM. Oui, de ce point de vue, il y en a eu beaucoup. De plus, pour les pilotes, l'entraînement au pilotage qui se fait sur simulateur, comporte obligatoirement la réponse au SCRAM. Donc ils ont eu de nombreuses fois l'occasion de faire les opérations nécessaires après un SCRAM.

$\mathbf{Q}$ : Ce qui veut dire que même s'il y a SCRAM, cela n'entraîne pas obligatoirement la mise en marche de l'IC.

\section{$\mathbf{R}$ : Exactement.}

$\mathbf{Q}:$ Je pense que vous n'aviez pas reçu ces informations de l'équipe de quart, mais, selon la chronologie publiée par TEPCO, on a à la première page, vers 15h03: «la baisse de pression de la cuve du réacteur 7 étant rapide, ayant jugé qu'on ne pourrait pas rester dans la limite fixée par les normes de sûreté de $55^{\circ} \mathrm{C} / \mathrm{h}$ concernant le taux de refroidissement de la source froide (liquide de refroidissement) du réacteur, on en vient à répéter les opérations d'ouverture et de fermeture ». Donc, la pression baisse rapidement, il faut manœuvrer plusieurs fois I'IC. Vous, qui avez une expérience variée, y compris dans le domaine de la maintenance, de votre point de vue, serait-il possible que cette baisse rapide de pression soit due à une anomalie, à une fuite, par exemple, de la cuve, de la tuyauterie qui y est reliée, bref, une anomalie qui laisserait échapper la pression?

$\mathbf{R}$ : Je ne sais pas. 
$\mathbf{Q}$ : Vous ne savez pas. Ça veut dire que vous ne pouvez pas l'exclure?

$\mathbf{R}$ : Je ne peux pas l'exclure. Au moment où nous étions, avec les données que vous venez de me fournir, si vous me demandez d'en tirer des conclusions, je ne saurais pas.

Q : Par exemple, au vu des évènements qui ont suivi, je voudrais savoir s'il vous était difficile de penser à ce moment-là à une fuite ou à une rupture, compte tenu de l'état de la cuve du réacteur numéro 1 et de la situation autour. Vous voulez dire que vous ne pouvez pas forcément l'exclure?

$\mathbf{R}$ : Ce sont des faits. On ne peut pas en parler sur des impressions. S'il n'y a pas de données, je ne peux pas me prononcer clairement.

$\mathbf{Q}$ : Excusez-moi de ne pas respecter la chronologie, mais, pour vous, l'IC, par la suite, avait continué à fonctionner?

R : Oui.

Q: Donc vous pensiez que l'IC était en fonctionnement. Par la suite, ce jour-là, la nuit du 11 mars, vers $22 \mathrm{~h} 00$ ou $23 \mathrm{~h} 00$, en tenant compte de la situation du réacteur 1 , pensiez-vous que l'IC fonctionnait pleinement?

$\mathbf{R}$ : C'est-à-dire qu'à ce moment-là, je ne regardais pas que la situation du réacteur 1 . Il y avait le réacteur 1 , le réacteur 2 , le réacteur 3 , surtout le réacteur 2 , dont on ne savait pas à cette période-là si le RCIC était en marche ou pas. On ne connaissait pas le niveau de l'eau dans la cuve. On n'arrivait pas à avoir de données sur le réacteur 2 . Chaque tranche avait ses problèmes. Alors pour l'IC du réacteur 1, comme on m'avait informé que le capteur de niveau d'eau du réacteur 1 était encore opérationnel, qu'il indiquait TAF (Top of Active Fuel) $)^{58}+1370$, c'est-à-dire qu'il y avait suffisamment d'eau, en voyant ça, j'ai simplement pensé que l'IC devait être en fonctionnement. Je n'étais absolument pas au courant de chacune des manœuvres que l'équipe de quart avait entreprise.

Q: Juste pour vérification. Ces paramètres, comme les chiffres dont on vous avait informé, vous les notiez par exemple sur le tableau blanc de la cellule de crise?

$\mathbf{R}$ : Oui, on les note.

Q: Excusez-moi d'avoir anticipé. Nous allons revenir de nouveau à ce moment où le tsunami n'est pas encore arrivé. Vous nous avez dit tout à l'heure que, même si vous étiez loin d'imaginer l'envergure du tsunami qui est effectivement venu, vous aviez prévu qu'il y avait des dangers liés à la survenue d'un tsunami après le séisme que vous veniez de vivre. Étiez-vous dans une situation où vous pouviez, par exemple, voir la télévision?

R : Oui. C'est pour ça que je savais qu'une alerte au tsunami avait été lancée et, par exemple, quand le tsunami est arrivé, je ne me souviens pas vraiment des détails, mais je pense que la télé avait évoqué un tsunami de cinq mètres de haut, il me semble. Oui, on suivait heure par heure ce que disait la télé, je pense que c'était la $\mathrm{NHK}^{59}$. On avait vu qu'il y avait des alertes, oui.

Q: Vous étiez au courant de l'alerte tsunami de l'Agence Météorologique ${ }^{60}$, par exemple, par la NHK, par la télévision?

R : Oui.

$\mathbf{Q}:$ : Et, pour le personnel de quart qui est sur le terrain, comment faites-vous?

R: Il y a un téléviseur à côté du chef de quart. Je pense qu'ils ont pu avoir les informations comme ça. 
$\mathbf{Q}$ : Concrètement, si le tsunami annoncé était de cinq mètres, face à la survenue d'un tel tsunami, bien que vous ayez été pris par le temps, aviez-vous pris des mesures ou vous apprêtiez-vous à en prendre?

$\mathbf{R}$ : Je ne pense pas qu'on puisse appeler ça des mesures, mais, puisqu'en regardant la télé nous savions qu'un tsunami allait venir, j'ai fait circuler l'information comme quoi il y avait une alerte au tsunami à chaque salle de commande. Au personnel massé autour de la table, aussi. Une nouvelle fois, j'ai donné l'ordre que les personnes se trouvant côté littoral, c'est-à-dire près de la mer, se mettent à l'abri, et qu'on vérifie bien que l'ordre était exécuté. Ça, je l'ai bien ordonné. Seulement, en ce qui concerne la conduite des tranches, la réponse à un tsunami, tant qu'il n'est pas là... Bien sûr, on sait qu'on ne pourra pas utiliser le circuit eau de mer pendant un certain temps. Mais prévoir l'ampleur d'un tsunami, savoir si on peut faire quelque chose avant l'arrivée du tsunami... Des choses à faire à ce moment-là, il n'y a presque rien, enfin, carrément rien du tout. La première chose à faire, c'était mettre les gens à l'abri. Avoir conscience qu'il faudra faire des manœuvres, en prévoyant que le tsunami arrive. C'est tout. Ce qui nous fait le plus peur, dans le cas des tsunamis, ce sont les vagues qui se retirent, plus que les vagues qui arrivent. Le fait de savoir qu'avec la vague qui va se retirer on n'aura plus d'eau, ça, ça fait peur.

$\mathbf{Q}$ : Je voudrais savoir le contenu de cette peur, quand vous dites, le fait de ne plus avoir d'eau, ça fait peur. Par exemple, avec la vague du tsunami qui se retire, le niveau d'eau va terriblement descendre et on ne pourra pas alimenter en eau. Dans votre tête, c'est dix minutes que vous ne pourrez pas alimenter en eau ou une heure? Qu'avez-vous en tête? Pendant quelle durée imaginez-vous qu'il sera impossible de prendre l'eau?

$\mathbf{R}$ : C'est ça qui est difficile. Je ne sais pas quelle était l'ampleur de la vague, mais l'année dernière, il me semble, il y a eu un tsunami au Chili, n'est-ce pas ? Il y a eu un tremblement de terre au Chili et il y a eu un tsunami ${ }^{61}$. À Fukushima Daiichi, nous en avions aussi ressenti les répercussions. Il faut dire qu'il y avait déjà une marée avec un gros coefficient du côté de chez nous et les effets ce sont superposés. Donc de grosses vagues qui venaient et se retiraient. Avec cette expérience, j'avais une idée de la manière dont ça allait aller et venir.

$\mathbf{Q}$ : Et cela, avec des intervalles de trente minutes ou ... ?

$\mathbf{R}$ : Oui, oui. Quelques dizaines de minutes. Je pouvais imaginer que ça allait aller et venir avec un intervalle de quelques dizaines de minutes. Mais il m'était difficile d'imaginer concrètement l'impact que la durée pendant laquelle la vague était partie pouvait avoir sur l'affaiblissement de la capacité à refroidir.

$\mathbf{Q}$ : Si ça ne vous ennuie pas, je voudrais vous interroger un peu plus sur cette question. Par exemple, que cela soit vide pendant dix minutes ou trente minutes, cela n'a pas d'importance, puisque, même si cela se vide, l'eau va revenir. Donc, l'eau finira par revenir. Mais pendant la période où il n'y a pas d'eau, du point de vue de la pompe, elle tourne à vide, et du point de vue du refroidissement, cela chauffe aussi à vide. Si cette situation se prolonge, est-ce qu'il n'y a pas danger qu'à un endroit ou à un autre, un tuyau, par exemple, surchauffe et se détériore?

$\mathbf{R}$ : Ça, on ne le pense pas. Pourquoi? Parce que. Pour quelle raison a-t-on besoin d'eau de mer? Eh bien, la plus grosse raison, c'est le condenseur. Parce que c'est le condenseur qui utilise le plus d'eau. Pour refroidir le condenseur. C'est par ce qu'on appelle le circuit eau froide qu'on prend l'eau de mer. Or, comme il y a déjà eu SCRAM, la vapeur n'arrive plus, donc cette partie n'a pas besoin d'être fonctionnelle. Par contre, ce qui est le plus important est qu'il y ait de l'eau pour alimenter le RHR 
(Residual Heat Removal system) ${ }^{62}$ ou le circuit de refroidissement de secours. Donc, ce qui fait le plus peur, c'est de ne plus disposer d'eau pour ces circuits.

$\mathbf{Q}$ : Ce qui veut dire que tout va bien pour le circuit principal, mais on a peur de ne plus pouvoir disposer d'un système de refroidissement pour les circuits de secours?

$\mathbf{R}$ : Oui. L'enjeu, c'est de savoir pendant combien de temps la pompe d'eau de mer de secours restera indisponible. Le refroidissement de secours marche d'abord avec le RCIC ou l'IC, qui n'utilisent pas d'électricité et sont indépendants du circuit d'eau de mer. Donc ça refroidit. Même si la pompe $\mathrm{SK}^{63}$ qui procure l'eau au système RHR reste indisponible pendant un temps, comme vous le dites, elle redeviendra fonctionnelle quand l'eau reviendra. En fait, ce qui nous fait peur, en temps normal, ce n'est pas tant le fait qu'à cause d'un séisme lointain, l'arrêt automatique se fasse, mais le fait que le tsunami arrive pendant que le réacteur est en fonctionnement. Parce que quand le tsunami arrive, il faut arrêter l'eau du condenseur et faire en sorte que l'arrêt automatique se fasse correctement. Et ça, c'est ce qui nous fait peur. Mais dans le cas qui nous occupe, le réacteur était déjà arrêté avant l'arrivée du tsunami, donc il n'y avait pas de problème de ce côté. Du coup, ce à quoi il fallait réfléchir était le réseau d'eau de mer de secours. Comme je vous l'ai dit, je prévoyais que les vagues allaient venir et repartir avec des cycles de quelques dizaines de minutes et que cela n'allait pas durer $24 \mathrm{~h} 00$, je pensais bien qu'on arriverait à un moment ou à un autre à récupérer suffisamment de niveau pour faire ressusciter la pompe.

$\mathbf{Q}$ : Dans ce cas, il y a une autre question que pourraient se poser les gens qui n'y connaissent rien. Savoir, s'il n'y a plus d'eau au niveau de la pompe, que c'est vide, est-ce qu'au moment où on voudra la remettre en marche, la pompe peut fonctionner comme pompe, puisque l'eau, pour une raison ou une autre, n'arrive plus et qu'elle ne fera que brasser l'air. Concernant une pompe à eau, je crois que tout le monde peut avoir ce genre d'inquiétude. Est-ce que c'est possible dans notre cas?

$\mathbf{R}$ : Oui, c'est tout à fait possible. Évidemment, quand il n'y a plus d'eau, il y a une sorte de cavitation ${ }^{64}$ pour la pompe, y compris son moteur, et il y a des détériorations. Je pense que, probablement, il ne faut pas faire marcher la pompe au moment où la vague se retire. En ce qui concerne ce problème, je n'ai donné aucune indication à ce moment-là. Je n'ai pas dit, il y a alerte au tsunami, il faut faire cette manœuvre. Non. Quand j'y réfléchis aujourd'hui, je pense qu'au moment où la vague se retire, il faut arrêter la pompe, ou, en tout cas, faire ce genre de chose.

$\mathbf{Q}$ : Pensez-vous que l'opérateur de la pompe a pu avoir en tête le même genre de crainte que moi, même très vague?

$\mathbf{R}$ : Sans aucun doute. Pour eux, qui sont des mécaniciens, il est évident qu'il ne faut pas mettre en marche une pompe qui n'a plus d'eau. Je pense qu'ils l'ont ressentie.

$\mathbf{Q}$ : Ayant pensé que ça pouvait poser problème, est-ce que, dans la situation où vous étiez, la tendance était de se dire, j'y ai pensé, mais tant pis si je ne réfléchis pas plus loin? Ou bien de vous dire, je suis quand même inquiet, il vaut mieux approfondir encore un peu ? Ou bien, aujourd'hui, vous dites-vous que vous auriez dû y réfléchir, qu'est-ce qui correspond le mieux à cette histoire de pompe?

$\mathbf{R}$ : Comme je vous le disais, le tsunami est arrivé alors que nous étions en train de vérifier la situation des différentes tranches, de voir si tout le monde était sain et sauf, nous faisions différentes choses en même temps. La chose qui prime, dans ces conditions, ce sont les vies humaines, donc vérifier ça. Ensuite, pour les pilotes, je ne pouvais guère leur dire que d'être vigilants et de bien surveiller les données des différentes tranches. C'est une fois que le tsunami est là, mis à part le fait qu'on n'en 
attendait pas un aussi gros, même s'il n'avait atteint qu'un niveau normal, qu'on aurait commencé à donner des ordres pour vérifier le fonctionnement de la pompe, si elle marchait ou pas. Bien sûr que nous y pensions. Tous les spécialistes autour de la table de la salle de crise le savaient et les opérateurs aussi savaient qu'avec l'arrivée du tsunami, il fallait s'inquiéter de la pompe du circuit de secours, c'était une évidence. C'était une préoccupation que nous partagions tous. Je n'ai personnellement pas donné cet ordre précis, mais tout le monde en était conscient.

$\mathbf{Q}$ : D'accord. Alors, le tsunami arrive. Est-ce que vous avez su tout de suite que le tsunami était arrivé ?

$\mathbf{R}:$ Non, en tout cas pas moi.

$\mathbf{Q}$ : Comment l'avez-vous su?

$\mathbf{R}$ : En fait, on a fait la démarche inverse. J'entends qu'on a perdu toutes les sources de courant alternatif, que les générateurs diesel ne marchent plus et nous sommes surpris, incrédules. À ce moment-là, là où nous étions, nous n'avions pas de caméra surveillant la mer. On était dans une situation où les données de la caméra de surveillance n'arrivaient pas du côté de la table ronde. Et donc nous ne savons rien de ce qui se passe à l'extérieur. Nous savons tout juste par la télévision, qu'il y a une alerte tsunami et, puis, nous avons quelques rares informations venant du terrain. Donc, nous ne pouvons pas savoir quel est l'état de la mer d'où nous sommes. En ce qui concerne l'arrivée du tsunami, les gens des salles de commande, non plus, ne peuvent rien savoir. Puisqu'on ne voit pas l'extérieur. Alors, nous aurons bien la nouvelle de l'arrivée du tsunami un peu plus tard, par certains qui sont allés dehors et ont pu regarder d'un autre bâtiment, mais pour nous, la première anomalie, c'est la perte de toutes les sources de courant alternatif à 15h37. Sur ce, on apprend que les générateurs diesel ne fonctionnent plus, on se dit que ce n'est pas possible et enfin, on se dit que le tsunami a dû arriver, et c'est comme ça que petit à petit tout le monde tombe d'accord, arrive à la même conclusion. Là, à ce moment-là, c'est la stupéfaction ${ }^{65}$.

Q : Oui. On voit sur cette chronologie qu'à 15h42, jugeant la survenue d'un incident spécifique, la perte de tout courant alternatif, suivant l'alinéa 1 de l'article 10 de la loi spéciale sur les situations d'urgence en cas de catastrophe nucléaire, vous en informez les autorités compétentes.

R : Oui.

Q: Quand vous dites, à ce moment-là, que les générateurs diesel ne fonctionnent plus, c'est-à-dire la perte de tout courant alternatif, vous vouliez parler des réacteurs 1 à 3 ?

R : À ce moment-là, je n'ai pas le souvenir d'avoir su quel générateur en particulier. Ce que je savais, au moins, c'est que, sans erreur possible, en ce qui concerne les réacteurs 1 à 3 qui étaient en fonctionnement, tous les générateurs étaient hors service. En plus de ça, il y avait aussi des informations concernant les générateurs des réacteurs 4,5 et 6 . Alors, je n'arrive pas à me souvenir aujourd'hui quels étaient les générateurs concernés, lorsque j'ai fait ce rapport à propos de la perte de $15 \mathrm{~h} 37$. Je pense que j'ai dû faire une déclaration qui les englobait tous et que j'ai envoyé des rectificatifs après. De toute façon, le plus important, c'était le fonctionnement ou non des générateurs des réacteurs 1 à 3 qui étaient en exploitation, alors, pour moi, l'information les concernait en premier lieu. 
$\mathbf{Q}:$ Si je consulte les archives, je vois: «information importante concernant les réacteurs 1 à 5 ». Donc, vous aviez fait une première déclaration incluant les réacteurs 1 à 5 et vous l'aviez modifiée par la suite pour dire que c'était les réacteurs 1 à 3.

R : Oui.

$\mathbf{Q}$ : Au moment où vous recevez l'information que toute source de courant alternatif est perdue, que ce soit les réacteurs 1 à 3 , ou incluant les 4 à 6 , peu importe, vous suivez le protocole prévu et vous informez les autorités. Mais, en ce qui concerne les mesures à prendre pour les différentes tranches, à quoi avez-vous pensé en premier?

$\mathbf{R}$ : Là, pour être tout à fait franc, j'étais anéanti. Moi-même, je veux dire. Je me disais que nous étions face à une situation terrible. Mais cela ne servait à rien de se lamenter. À l'évidence, nous allions vers un accident majeur et il fallait s'y préparer. Voilà, ce que je me suis dit. Si je devais parler du ressenti, je dirais que c'était tout d'abord la constatation que quelque chose de terrible était arrivé. Est-ce que les générateurs diesel allaient repartir ? À ce moment-là, on ne savait pas s'ils avaient été noyés par le tsunami ou pas. Alors, on se demande, tout d'abord, si on ne peut pas les ressusciter. Que faire si on n'arrive pas à les récupérer ? Si on a le Condenseur d'Isolation (IC), ou le RCIC, on peut refroidir pendant quelques heures, mais que faire après $^{66}$ ? Ce genre de réflexions tournaient et tournaient dans ma tête.

$\mathbf{Q}$ : Concernant cette situation terrible, c'était terrible à quel degré ? Je pense que c'est la même chose partout, mais, par exemple, les manuels de gestion d'accidents de TEPCO, ou d'autres choses concernant la gestion d'accidents majeurs, face à la perte totale de courant alternatif, préconisent à la base des réponses qui présupposent le fonctionnement des générateurs diesel de secours. Mais, dans votre cas, vous n'aviez même pas ces générateurs. Dois-je comprendre que vous aviez le sentiment que la situation était d'autant plus terrible? Et donc vous avez d'abord réfléchi à la manière de ressusciter ces générateurs de secours. Quelles sont les mesures concrètes que vous avez prises dans ce sens-là ? Qu'avez-vous fait pour concrétiser votre pensée?

$\mathbf{R}$ : À ce stade-là, nous sommes à environ une heure après la survenue du tsunami, il y avait toutes sortes d'informations qui arrivaient. Ce qui devait être fait en priorité, c'était relayer l'information vers l'extérieur. La déclaration suivant l'article 10, informer le siège, tout cela devient le cœur de mon travail. Donc, tout en pensant que nous allions vers une situation catastrophique, je suis en contact avec l'extérieur, je relaie l'information. Je réponds aux questions, etc.

En ce qui concerne concrètement la conduite des différentes tranches, à la limite, les pilotes sont plus qualifiés que moi. Alors, je fais confiance aux chefs des groupes " production » et aux pilotes. En tout cas, en ce qui concerne les menues manœuvres à faire dans la conduite de base. Seulement, quand il s'agit de répondre à un accident majeur, je donne l'orientation, je leur dis ce qu'il faut faire. Mais pour les manœuvres à faire concrètement pour obtenir ce que je veux, je laisse les experts de la conduite trouver des solutions. Ce n'est vraiment pas au directeur de dire dans le détail, fais ceci, fais cela. Donc, au moment dont nous parlons, je suis plutôt occupé à communiquer avec l'extérieur et à tenter d'avoir une vision claire de la situation, qu'à donner des directives concernant les tranches.

$\mathbf{Q}$ : En fait, dans la réalité, avec la perte de l'alimentation électrique, sans même les générateurs de secours, je pense que les équipes de quart ont perdu aussi l'alimentation pour les différents appareils de mesure. Ensuite, même l'éclairage devenant défaillant, ils n'ont pas pu lire les paramètres. Je pense que les équipes sur le terrain étaient elles-mêmes plongées dans une situation dont elles n'avaient jamais eu l'expérience. Si elles avaient disposé d'électricité, les manœuvres auraient pu se faire en appuyant sur un bouton. Mais 
là, elles ont été précipitées dans une situation d'urgence. Alors, si en temps normal, on peut confier la conduite aux équipes de quart chevronnées, dans une situation où elles ne peuvent manœuvrer sans que l'électricité et d'autres choses soient rétablies, je ne crois pas qu'on puisse penser qu'on peut laisser les groupes « production » se débrouiller seuls. Mais je suppose qu'à ce moment-là, vous n'aviez pas encore une connaissance claire des différentes dégradations. Avez-vous eu plus tard des informations concernant la perte de l'alimentation électrique pour les appareils de mesure?

R : Oui.

$\mathbf{Q}$ : Quand on n'arrive plus à lire les différents instruments de mesure, on finit par ne plus savoir dans quel état est le réacteur. Au moment dont nous parlons, pour vous, un des grands défis est de rétablir l'alimentation de ces appareils, n'est-ce pas? Mais, parmi les différents paramètres qui sont mesurés, y avait-il pour vous un ordre de priorité ou pas?

$\mathbf{R}$ : Oui. La priorité, c'était le niveau d'eau.

$\mathbf{Q}$ : Le niveau d'eau.

$\mathbf{R}$ : La pression.

$\mathbf{Q}:$ La pression, vous voulez dire la pression dans le réacteur?

$\mathbf{R}$ : Oui, le plus important, c'est d'abord la pression à l'intérieur de la cuve. Donc, le niveau de l'eau et la pression dans la cuve. Si, à partir de là, on est bien dans les limites, après, on peut s'inquiéter de la pression dans l'enceinte. Mais il faut d'abord connaître avec précision la pression et le niveau d'eau dans la cuve, car sans ça, on ne peut pas avoir une idée juste de la situation. Il faut surtout le niveau d'eau.

$\mathbf{Q}$ : Alors concernant le rétablissement des différents instruments de mesure, avez-vous donné des indications, en suivant votre ordre de priorité, disant de rétablir en priorité la batterie de l'indicateur de niveau d'eau ou tout autre moyen pour le faire fonctionner? Ou bien les groupes « production » l'ont fait d'eux-mêmes?

$\mathbf{R}$ : Déjà, le rapport disant qu'ils n'arrivaient plus à voir les instruments n'est pas arrivé immédiatement des salles de commande. Je n'ai plus le souvenir exact du moment où j'ai su qu'ils ne voyaient plus les instruments, mais il y a eu un temps avant que je le sache. Tout d'abord, ils ont dit, les générateurs se sont arrêtés, donc, à la suite de cet état des choses, j'informe les autorités suivant l'article 10. C'est tout juste après que les informations commencent à arriver par bribes. On n'a plus d'éclairage dans la salle de commande, on n'arrive plus à voir les instruments. Il y a deux salles de commande, l'une pour les réacteurs 1 et 2, et une autre pour les réacteurs 3 et 4 . Des deux, sont arrivées des informations hachées en un laps de temps très court. "Ça s'est éteint, vérifiez dans quelle situation vous êtes, qu'est-ce que vous voyez comme instruments? " Il fallait le temps que ça vienne et que ça reparte, il y avait un décalage. Alors on n'était pas forcément au courant d'un coup dans la cellule de crise, comme c'est noté là. On a beaucoup échangé et je sais que j'ai exigé des vérifications, alors qu'ils ne pouvaient pas voir les instruments, je leur ai dit de se débrouiller pour pouvoir les voir. Et dans ces échanges, comme le niveau d'eau et la pression sont des paramètres tellement évidents, je leur ai dit qu'il fallait pouvoir voir le niveau d'eau et la pression. Donc, je ne me souviens pas en quels termes précis je leur ai donné les ordres, mais je suis sûr de leur avoir dit que si on ne rétablissait pas les instruments, on ne pourrait rien faire et qu'il fallait trouver une solution. 
$\mathbf{Q}$ : Je ne sais pas exactement quand vous avez eu le rapport, mais ici, nous avons à $16 \mathrm{~h} 36$ : "Incapacité à vérifier le niveau d'eau pour les réacteurs 1 et 2 ». Ce qui signifie qu'à ce moment-là, vous aviez l'information?

R : Oui.

$\mathbf{Q}$ : À ce moment-là, comme vous ne saviez pas quelle était la situation de l'injection d'eau, vous avez, en conformité avec l'alinéa 1 de l'article 15 de la loi spéciale sur les catastrophes nucléaires ${ }^{67}$, déclaré auprès des autorités l'incident spécial : "Incapacité à injecter l'eau dans le système de refroidissement de secours du cœur du réacteur ». C'est bien ça?

R : Oui.

$\mathbf{Q}$ : ॥ me semble que le fait de ne pas pouvoir vérifier le niveau de l'eau et le fait de ne pas pouvoir injecter l'eau, sont, à strictement parler, deux choses différentes. Mais je suppose que vous avez agi de la sorte par prudence.

$\mathbf{R}$ : Oui, c'est ça.

$\mathbf{Q}$ : Donc, vers 16h00, 17h00, vous commencez à avoir des informations, comme quoi on n'arrive pas à voir le niveau de l'eau, ce genre de choses, par bribes. Alors, qu'en était-il de l'information concernant les générateurs diesel de secours qui avaient pris l'eau, surtout pour les réacteurs 1 à 3 , qui étaient en activité ?

$\mathbf{R}$ : À ce moment-là, après avoir envoyé la déclaration à 16h36, les informations commencent à arriver des salles de commande. Informations selon lesquelles, il y a de l'eau. Ils ne sont pas allés vérifier de visu la quantité d'eau, mais ils font savoir que de l'eau est entrée et que toutes sortes de source d'alimentation électrique sont mortes. Ce qui équivaut au fait que les générateurs diesel sont morts. C'est là qu'on commence à comprendre que si ça s'est arrêté, c'est à cause de l'eau. Si je prends un cas limite, si c'est un fusible qui a sauté, il suffit de changer de fusible, ça va redémarrer. Mais là, on sait pertinemment que si ça a été noyé par l'eau, ça va être extrêmement difficile de ressusciter le système tout de suite.

$\mathbf{Q}$ : Ce qui veut dire que, juste avant le coucher du soleil, alors qu'il y avait encore du jour, vous aviez conscience qu'au moins pour les réacteurs 1 à 3 qui étaient en fonctionnement, vous ne pouviez pas avoir recours aux générateurs diesel de secours, que vous n'étiez pas dans une situation où en faisant quelque chose vous pouviez les ressusciter.

$\mathbf{R}$ : Oui, c'est ce que je pensais.

$\mathbf{Q}$ : Juste une remarque. L'un des générateurs du réacteur 6 était opérationnel en ajustant la fréquence. Ça, à quel moment l'avez-vous su?

$\mathbf{R}$ : Au moment où on a perdu le courant alternatif, qu'on a su que les générateurs diesel s'étaient arrêtés, j'avais demandé si tous les générateurs étaient arrêtés. Et on m'avait effectivement répondu que le générateur du réacteur 6 qui avait été allumé plus tard était opérationnel.

$\mathbf{Q}$ : C'est pour ça que vous avez rectifié votre déclaration de l'article 10. Perte de courant seulement sur les réacteurs 1 à 5 et pas 6 ?

\section{$\mathbf{R}:$ C'est ça.}

$\mathbf{Q}$ : En résumé, vous avez besoin de rétablir le courant pour les instruments de mesure et vous ne pouvez pas utiliser le diesel de secours, à quelle mesure avez-vous pensé ensuite?

$\mathbf{R}$ : J'étais désespéré. Au fond de moi-même. Nous étions dans une situation d'accident majeur. Pour ce qui était de l'injection d'eau, on ne pouvait utiliser aucun ECCS. Lorsque l'IC et les RCIC seraient arrêtés, il y avait bien le HPCI (High Pressure Cooling Injection system ${ }^{68}$, mais lorsque tout cela se serait arrêté, que la batterie se serait 
arrêtée, comment allait-on refroidir? J'avais donné l'ordre qu'on y réfléchisse, mais je ne voyais pas moi-même l'ombre d'une solution.

$\mathbf{Q}$ : Vous n'aviez pas de solution.

$\mathbf{R}$ : Non, pas de solution. Si on se place du point de vue des manuels de gestion de crise, il faut d'abord chercher à utiliser le réseau incendie (Fire Protection system). On savait que si on mettait en marche la pompe à incendie diesel (Diesel Drive Fire Protection), ça irait. Mais en même temps, il y avait des informations comme quoi il n'y avait plus d'eau filtrée ${ }^{69}$, qu'il y avait une fuite. Du coup, je savais que ça allait être extrêmement difficile. De toute manière, on était dans une situation où on ne pouvait faire autrement qu'utiliser le réseau incendie, la pompe diesel, la pompe à incendie pour envoyer l'eau. Alors j'ai demandé qu'on réfléchisse à la manière d'injecter l'eau. À ce moment-là, on a su que quelques commutateurs à revêtement métallique étaient encore fonctionnels dans le réacteur 2. J'ai donc ordonné qu'on regarde s'il y avait des pompes qu'on pourrait faire fonctionner en les utilisant.

Dans le cas où ça n'aurait pas fonctionné, j'ai envoyé des gens vérifier que si on baissait la pression, parce qu'il faut commencer par faire baisser la pression, que ce soit une pompe à incendie diesel ou autre, ça ne peut pas envoyer si la pression ne baisse pas, il faut, dans l'ordre, faire fonctionner la soupape de dépressurisation, laisser échapper de la pression, et agir, une fois que la pression a baissé, j'ai donc envoyer des gens vérifier que si on suivait ce protocole, la pompe à incendie diesel était suffisante. En même temps, certains disaient qu'on pourrait peut-être envoyer l'eau filtrée. Enfin, on était dans l'expectative.

Au milieu de tout ça, je ne sais plus exactement à quel moment, pendant qu'on se demandait s'il n'y avait pas d'autres moyens d'injecter l'eau, on s'est rendu compte qu'en utilisant le réseau incendie, on pouvait envoyer de l'eau avec un camion incendie. Ça, je pense qu'aucun manuel de gestion de crise n'en parle, mais puisqu'on disposait de cette possibilité, il fallait y réfléchir, même penser à faire un essai. J'ai donc demandé qu'on y réfléchisse.

Q: Dans le but d'éviter la fusion du cœur des réacteurs ${ }^{70}$, vous disposiez des systèmes RCIC et IC. D'après vous, ces systèmes pouvaient tenir pendant combien de temps, huit heures? Plusieurs jours?

$\mathbf{R}$ : Pour moi, ils devaient rendre l'âme au bout de huit heures.

$\mathbf{Q}:$ Vous pensiez qu'ils ne tiendraient que huit heures?

R : Oui.

$\mathbf{Q}:$ En fait, dans la réalité, ils ont tenu le coup assez longtemps, n'est-ce pas ?

$\mathbf{R}$ : Ça, c'est grâce à l'intelligence du pilote. Il a coupé tout ce qui était relié à la batterie. Habituellement, il y a toutes sortes de choses qui sont reliées à cette batterie. Alors, il a débranché tout pour qu'elle n'alimente plus que le RCIC. C'est pour ça que ça a tenu si longtemps.

$\mathbf{Q}$ : Donc, plus que l'eau, le point névralgique, c'était la batterie.

$\mathbf{R}$ : Oui, le point névralgique, c'était la batterie.

$\mathbf{Q}$ : Vous envisagez donc le réseau incendie et la pompe diesel à incendie. Vous pensez utiliser l'eau filtrée. Seulement, vous pensez qu'il va être difficile de disposer du réseau d'eau, parce que vous avez eu des informations sur le fait qu'il y avait peut-être des fuites sur les tuyaux. Comme solution de rechange, vous réfléchissez à injecter l'eau avec un camion incendie, et c'est ce que vous avez effectivement fait, je pense. II y a donc une 
bouche qui permet d'envoyer l'eau. En y branchant la pompe du camion incendie, on peut, de cette manière, envoyer de l'eau de l'extérieur du bâtiment réacteur. Cette bouche, c'est quelque chose qui a été rajouté après coup, n'est-ce pas? Quand a-t-elle été rajoutée et dans quelles circonstances?

$\mathbf{R}$ : Je n'ai pas été associé à la conception de cette bouche, donc je ne connais pas les détails. Comme je vous l'ai dit tout à l'heure, c'était à l'époque où j'étais à la Federation of Electric Power Companies of Japan, et puis directeur de la production à Fukushima Daini. Je n'étais donc pas impliqué dans les travaux de transformation de Fukushima Daiichi et je ne connais pas les détails, mais, quand on regarde les documents après coup, on avait prévu une nouvelle ligne pour le réseau incendie, venant de l'extérieur, dans le cadre des mesures en cas d'accidents majeurs. Je pense que ça a dû être fait vers 2001.

$\mathbf{Q}$ : C'est une question à laquelle vous pouvez répondre plus tard, mais quand on dit envoyer de l'extérieur, on envisage d'envoyer avec quoi ?

$\mathbf{R}$ : Franchement, je ne sais absolument pas ce qu'avait en vue la personne qui a conçu ça. Probablement, il a dû penser à une borne incendie. Je pense qu'il l'a faite en ayant tout simplement en tête d'injecter par une borne incendie. Mais ça n'a pas du tout marché avec la borne incendie. Le concepteur a dû penser à une borne incendie. Mais si, de toute façon, ça ne marche pas comme ça, il faut des camions incendie. Ces camions incendie, on les a achetés après le séisme au large de Chûetsu ${ }^{71}$. Ce qui veut dire que jusque-là, on n'avait pas de camions incendie. Alors, si on n'a pas de camion incendie et que la borne incendie ne fonctionne pas, cette ligne est inutilisable. Donc elle n'est absolument pas utile pour le refroidissement. Mais on avait trois camions. L'un avait été endommagé, mais on s'est ingénié à utiliser les deux camions restants, comme on a pu. Donc le concepteur a dessiné cette ligne en pensant probablement utiliser une borne incendie.

$\mathbf{Q}$ : Ce coup-ci, dans votre cas, vous avez réussi à trouver de l'eau, non pas grâce à une borne incendie, mais grâce à de l'eau stockée dans une cuve en prévision de la lutte contre le feu.

$\mathbf{R}:$ Oui, c'est ça.

$\mathbf{Q}:$ Ce n'était pas une borne incendie, mais une cuve...

$\mathbf{R}$ : Oui, c'était des cuves servant de réservoirs d'eau en cas d'incendie. Vous vous rappelez, au moment du séisme de Niigata $^{72}$, le transformateur du réacteur 3 de la centrale de Kashiwazaki qui avait brûlé ?

$\mathbf{Q}$ : Oui. J'y suis allé après, pour voir.

$\mathbf{R}$ : Il était tout noir. Comme la NHK a diffusé les images en boucle, je pense que tout le monde dans le pays a vu les fumées noires s'échapper du bâtiment. Après ça, il y a eu un tollé général contre nous. Comment ? Les centrales nucléaires n'avaient même pas de camions incendie ? On en a donc acheté. En même temps, on a décidé de revoir les mesures de lutte contre l'incendie et on a installé des cuves remplies d'eau pour avoir une source d'eau à l'intérieur des centrales. C'est donc après le séisme de Niigata qu'on a renforcé de cette manière les mesures de lutte contre le feu. Ces cuves aussi, on en a installé un certain nombre. 


\section{$\mathbf{Q}$ : Quelle est la contenance d'une cuve?}

R: Quarante tonnes. Vous pourrez demander plus tard combien de ces cuves, concrètement, étaient implantées à côté de chaque tranche. Mais puisqu'on les avait installées, on y a pris l'eau.

$\mathbf{Q}$ : C'est intéressant. J'ai bien envie qu'on en parle encore, mais j'ai peur que nous nous fourvoyions, alors.

Encore une chose. D'après les communiqués de TEPCO, vous auriez envisagé, dans le même temps, à la place de l'eau, d'envoyer de l'eau borée ${ }^{73}$.

$\mathbf{R}:$ Le SLC (Standby Liquid Control system) ${ }^{74}$ ?

$\mathbf{Q}$ : Oui. Le SLC. Dans ce cas, la source d'eau se trouve à l'intérieur du bâtiment. Seulement, pour pouvoir l'injecter, il faut de l'électricité. Donc pour pouvoir l'envisager, le souci principal va être de trouver une source électrique. Concrètement, quelles ont été vos réflexions ?

$\mathbf{R}$ : Pour ce qui est des sources électriques, à ce moment-là, on commençait enfin à savoir ce qui pouvait être opérationnel. Et là, on apprend qu'un commutateur à courant continu marche encore et on se dit qu'on va pouvoir brancher dessus. Il fallait une passerelle, mais les pompes, tout ça, on avait. Par exemple, la pompe du système des barres de contrôle ${ }^{75}$. On a, effectivement, des pompes. Mais le problème, c'est que, primo, la pompe peut elle-même être endommagée. Secundo, il n'y aura peut-être pas de source d'électricité pour la faire fonctionner. Il fallait donc vérifier tout ça un à un. Et, au bout du compte, la seule qui avait l'air d'avoir survécu était la pompe du SLC. C'est une simple pompe à piston, alors on s'est dit qu'elle était probablement indemne. Si on arrivait, à partir d'une source électrique externe, en passant par le commutateur, à faire parvenir l'électricité, on pouvait l'utiliser. Tout ça, le groupe « réhabilitation » y travaillait. Et on l'a enfin su. Oui, on a commencé par passer en revue tout ce qu'on pouvait utiliser comme source d'énergie, tout ce qu'on pouvait injecter et, en fin de compte, la seule chose qui restait, c'était le SLC. Du point de vue volume, ça ne représentait pas grand-chose. Mais, bon, ça contenait de l'acide borique $^{76}$ et, si c'était disponible pour l'injection, il fallait y aller. Voilà ce que je pensais.

$\mathbf{Q}$ : À la différence du réseau incendie, là, l'injection à haute pression était possible. Vous n'aviez donc pas à faire la manœuvre de dépressurisation. Au moment dont nous parlons, pour injecter l'eau, vous avez besoin d'électricité. Pour ce qui est des instruments de mesure aussi, vous avez besoin de disposer d'électricité. Donc, à ce moment-là, s'assurer de sources électriques devient une grande préoccupation. C'est bien ça?

R : Oui.

$\mathbf{Q}$ : Depuis un moment, on parle beaucoup de sources électriques. Mais il y a plusieurs sortes d'électricité. Et je suppose que vous aviez passé en revue toutes les sources dont vous aviez besoin.

\section{R : Bien sûr.}

$\mathbf{Q}$ : Une fois que vous aviez cerné vos besoins, à qui adressiez-vous vos demandes ? Vous commencez par vérifier si ce dont vous avez besoin est disponible à l'intérieur de la centrale?

$\mathbf{R}$ : Alors, pour les sources électriques, on ne dispose d'aucune source conséquente. En plus, nous n'avons pas les générateurs diesel. En ce qui concerne le courant alternatif, il n'y a que la solution des camions groupes électrogènes, donc on demande qu'on nous amène des camions groupes électrogènes ${ }^{77}$. Peu importe d'où ils viennent, on veut en priorité des camions électrogènes. On réfléchira à leur 
utilisation plus tard, mais on veut des camions électrogènes. Et comme on ne peut pas les trouver nous-mêmes, on fait la demande à la maison mère. Et on les a eus. Ils sont arrivés, un camion, deux camions. Donc, en ce qui concerne le courant alternatif, comme notre seule planche de salut c'était les camions groupes électrogènes, on en a fait la demande au siège, et, après une attente qui semblait interminable, ils sont arrivés par-ci, par-là̄8 .

Concernant le courant continu, on en a parlé tout à l'heure, mais c'est la même chose. Pour pouvoir utiliser les instruments de mesure, il faut du courant continu. Au moment où j'ai ordonné qu'on se débrouille pour en trouver, le groupe « réhabilitation » a eu la présence d'esprit d'aller chercher des batteries de voiture, de les monter dans les salles de commande et de les utiliser comme source d'énergie pour les instruments de contrôle. On commence donc par se débrouiller avec les moyens du bord. Par la suite, on demande au siège de nous en procurer. Pour toutes les commandes, comme on ne peut pas les faire nous-mêmes, on passe par la maison mère, avec qui nous sommes en téléconférence, et on attend.

$\mathbf{Q}$ : Donc, concrètement, le siège social et la cellule de crise sont reliés par un système de téléconférence, c'est ça? Seulement, je me demande, quand on dit juste oralement, il me manque ci, il me manque ça, est-ce qu'en face, les gens peuvent avoir une vision globale de vos demandes? Je pense que vous avez certainement des groupes « matériel ». Est-ce que c'était les groupes «matériel » entre eux, le groupe «matériel » de la maison mère et le groupe «matériel » de la centrale qui communiquaient entre eux ?

$\mathbf{R}$ : Seulement, là encore, il y avait des confusions. Si on se demande si les gens du groupe « matériel » comprennent l'usage qu'on va faire du matériel, eh bien, ils ne le savent pas. Le groupe «matériel» est là pour rassembler le matériel, mais il ne connaît pas les détails techniques d'utilisation. Alors, c'est le groupe "réhabilitation » qui doit définir l'utilisation. Par exemple, dire il faut une batterie de tant de volts et de tel genre. Pour les camions électrogènes aussi, il faut qu'il spécifie, un camion de tant de kilowatts. C'est ça qui est difficile. On dit au groupe « matériel », on veut un camion électrogène, on veut une batterie, ils disent « $O K$ ». À partir de là, il faut que le groupe « réhabilitation » définisse les caractéristiques, qu'il les communique au groupe "matériel» de la centrale, puis il y a des échanges administratifs entre le groupe «matériel » de chez nous et le groupe «matériel » de la maison mère. Alors, il faut de toute évidence les conseils du staff technique.

$\mathbf{Q}$ : Dans la réalité, le groupe « réhabilitation » a besoin de tel type de source électrique, il fait des recherches, il passe commande par le groupe "matériel», mais quand reçoit-il ce matériel? Pour les choses que vous aviez demandées dès le 11, quand ont-elles commencé à arriver?

$\mathbf{R}$ : Pour être franc, je ne me rappelle presque pas quand le matériel est arrivé. Seulement, j'ai eu la sensation que cela arrivait petit à petit. Mais, si on se met à la place des gars du groupe "réhabilitation", ils ont dû avoir la sensation qu'une éternité se passait avant de voir arriver leur commande. Comme on a passé beaucoup de commandes, les choses n'arrivaient pas forcément dans l'ordre ou dans le timing qu'on aurait voulu.

$\mathbf{Q}$ : Comme vous avez vécu beaucoup de choses, peut-être ne vous souvenez-vous pas exactement du moment où cela s'est passé, mais, par exemple, pour l'électricité, vous êtesvous retrouvé dans des situations où des choses qui auraient dû arriver ne sont pas arrivées, ou bien vous les avez réceptionnées, mais elles ne convenaient pas du tout? Vous êtes-vous dit, à un moment, que ça ne fonctionnait pas, qu'il y avait peut-être des 
problèmes dans la manière de formuler vos demandes, qu'il y avait des problèmes de communication? C'est vraiment pour que nous puissions profiter de votre expérience pour d'autres cas semblables, voyez-vous, avez-vous des anecdotes de ce genre à nous raconter?

$\mathbf{R}$ : Si j'entre dans le détail, effectivement, il y a eu quelques cas où les choses qu'on nous avait envoyées ne correspondaient pas à l'usage qu'on voulait en faire. Mais, en fait, assez vite, on nous a envoyé tout ce qui pouvait être envoyé. La maison mère a décidé de nous envoyer tout ce qui leur tombait sous la main, même s'il y avait des chances pour que ce ne soit pas le modèle attendu. Ce qui était casse-pied, c'est qu'il nous appartenait à nous d'aller voir à chaque fois les caractéristiques du matériel. En plus, le matériel était envoyé dans un premier temps à un petit centre de distribution, qui nous appartient et qui est situé à Onahama ${ }^{79}$. Ensuite, il devait être acheminé jusqu'à la centrale. Mais la radioactivité avait augmenté et on ne pouvait plus nous livrer. C'était vraiment du genre, il n'y a pas de moyen de livraison, venez vous-même chercher le matériel. Un bête problème de logistique, en fin de compte. En fait, ça nous créait plus de problème de nous envoyer des choses sans avoir réfléchi à un moyen de livraison. En plus, comme ils nous envoyaient toutes sortes de choses, il fallait faire le tri entre ce qui pouvait être utilisé et ce qui était inutile. Du coup, on mobilisait pour ça des gens du terrain qui auraient été plus utiles ailleurs. C'était un gâchis incroyable. Alors, savoir si on n'a pas pu utiliser justement ceci ou cela, je ne suis pas au courant des détails. Si vous interrogez quelqu'un du groupe " réhabilitation ", par exemple, il aura sûrement des choses à vous raconter. Pour ma part, grosso modo, ce qui m'a le plus embêté, c'était ça. Devoir envoyer quelqu'un de chez nous pour aller chercher des choses. On avait d'autres choses à faire, non? Notre désir à tous, c'était qu'on nous apporte jusqu'à nous, ce qui convenait juste à nos besoins.

$\mathbf{Q}$ : C'est tout à fait le même phénomène que les refuges ${ }^{80}$ qui recevaient tellement de choses qu'ils n'arrivaient plus à les trier pour les distribuer. Oui, celui qui envoie pense faire de son mieux, mais vous, vous n'arrivez pas à les réceptionner.

Cela fait deux heures que nous parlons. Nous allons peut-être faire une pause maintenant. Juste une dernière question avant la pause. Tout à l'heure, vous nous avez dit que dans le bâtiment antisismique vous étiez environ quatre cents personnes en charge de la gestion de crise. Je suppose qu'à un moment ou à un autre, vous vous êtes tous retrouvés là. Ensuite, avec toutes les actions que vous deviez mener, j'imagine que vous aviez besoin de beaucoup de mains. Avez-vous eu suffisamment de monde?

$\mathbf{R}$ : Tout d'abord, de base, ce sont les personnes désignées comme faisant partie de la cellule de crise qui restent et répondent à la crise. Mais, ce jour-là, comme le bâtiment administratif avait été endommagé, il y avait toutes sortes de gens dans le bâtiment antisismique. Il y avait des femmes, des administratifs, des gens qui ne faisaient pas partie de la cellule de crise. Au tout début, il y avait foule. Il y avait aussi du personnel des entreprises partenaires. Alors, on a commencé par solliciter les gens qui étaient là. Mais, au soir du séisme, on savait que la radioactivité allait augmenter à partir du lendemain. Bien sûr, on avait déjà commencé à affréter des bus le soir même pour évacuer les femmes. Et, au fur et à mesure, le nombre de personnes a commencé à diminuer. Les partenaires aussi ont jugé que ça devenait trop dangereux et ils sont tous partis. Nous aussi, parmi les quatre cents, certains étaient pris par les évacuations, et nous étions de moins en moins nombreux. Oui. Petit à petit, il y avait moins de monde. Ça, c'était jusque vers le 15. Le 15 au matin, quand on a su qu'il y avait peut-être la possibilité que l'enceinte de confinement du réacteur 2 soit 
endommagée et que j'ai donné l'ordre d'évacuation, c'est là où nous étions le moins nombreux. Bien sûr, il y a toujours la discussion de savoir ce que doivent faire dans ce cas-là les membres d'une cellule de crise, mais la priorité est de protéger la vie des hommes, donc j'ai donné l'ordre d'évacuation et ça a fait fortement baisser le nombre des présents. Mais, ensuite, on va avoir besoin de faire des manœuvres. Et, à ce moment-là, ça recommence à augmenter. Concrètement, je dirais que le premier jour, on était trop nombreux. Ensuite, à cause de la hausse de la radioactivité, on va commencer à renvoyer les gens, tous. Bien sûr, il fallait renvoyer les gens. Les partenaires aussi, les entreprises partaient les unes après les autres. Pour moi, il y a eu un pic, qu'on a atteint très rapidement. On était bien plus de quatre cents, peutêtre mille. En comptant bien sûr les gens qui s'étaient réfugiés chez nous. C'était le premier pic, ensuite ils sont tous partis en masse et revenus progressivement. Voilà comment j'ai ressenti la situation. C'est juste du ressenti. Cela vous convient-il?

$\mathbf{Q}$ : Je pense qu'entre le premier séisme et l'arrivée du tsunami, vos sentiments ont dû beaucoup changer dans la cellule de crise. Comment avez-vous vécu cette situation?

$\mathbf{R}$ : Tout d'abord, c'était le soulagement. Je suis arrivé dans la salle et j'ai su que l'arrêt automatique s'était fait. Que l'arrêt s'était fait automatiquement à la suite du séisme. De savoir que c'était à l'arrêt, c'était déjà un premier soulagement. Si ça ne s'était pas arrêté, nous allions directement à la catastrophe. Mais, pour l'heure, les réacteurs étaient arrêtés. Ensuite, j'ai l'information que les générateurs diesel sont aussi à l'arrêt automatique. Deuxième point de soulagement. Ensuite arrivent encore d'autres informations et, le premier point de stupéfaction, c'est lorsque j'apprends la perte totale des sources de courant alternatif, avec l'arrêt des générateurs diesel. Là, je change de couleur. Il n'y avait pas de doute. Nous étions face à une catastrophe.

$\mathbf{Q}$ : Comment était l'atmosphère à l'intérieur de la cellule de crise?

$\mathbf{R}$ : À l'intérieur de la cellule, à ce moment-là, il y avait des techniciens, mais il y avait aussi des administratifs. Alors je ne sais pas ce qu'ils ont bien pu penser, mais en tout cas, au moins chez les techniciens, je pense qu'il y avait un sentiment partagé de catastrophe.

$\mathbf{Q}$ : Dans une situation pareille, y a-t-il des changements dans les voix, des cris?

$\mathbf{R}$ : C'est plutôt le contraire. Nous sommes tous tellement terrassés que nous sommes sans voix. Dans l'immédiat, nous sommes calmes et nous nous attelons à des tâches administratives, comme la déclaration de la perte de tout courant alternatif, le fameux article 10. Mais, comme je vous l'ai dit tout à l'heure, tout en accomplissant ces tâches administratives, émotionnellement, nous sommes anéantis. Non, nous ne crions pas. Je pense que tout le monde était comme ça.

$\mathbf{Q}$ : Vous étiez sans voix. Si vous le voulez bien, nous allons faire une pause. Nous nous retrouvons à $13 \mathrm{~h} 00$.

(Pause)

$\mathbf{Q}$ : Je suis Katô du secrétariat et je vais continuer à vous poser les questions. Si on regarde à la page 7 de la chronologie, après 17h12, le 11 mars, il y a "début de l'examen des méthodes d'injection d'eau dans le réacteur nucléaire en utilisant le circuit incendie prévu en cas d'incident, et de l'utilisation de camions incendie ». Je suppose que cela fait allusion à ce dont nous avons parlé ce matin. D'une part, le circuit incendie, avec l'utilisation d'une pompe à incendie diesel. D'autre part, en plus de ça, vous avez ordonné qu'on commence l'examen 
d'une méthode d'injection d'eau dans le réacteur par le biais d'un camion de pompiers. C'est bien ça?

R : Oui.

$\mathbf{Q}$ : Pour que vous demandiez d'envisager en plus l'usage du camion de pompiers, si je me souviens de ce que vous nous avez dit ce matin, c'est que vous aviez des craintes qu'il y ait des fuites sur la tuyauterie du circuit incendie qui est alimenté par le réservoir d'eau filtrée?

R : Oui.

$\mathbf{Q}:$ A $17 \mathrm{~h} 30$, il y a une annotation qui dit: "Pompe diesel incendie en attente de mise en marche ». Étiez-vous au courant qu'il y avait eu mise en marche de cette pompe?

$\mathbf{R}$ : Oui. Je ne suis pas sûr du moment précis, mais je savais que j'avais donné l'ordre et que les opérateurs avaient agi en conséquence.

$\mathbf{Q}$ : Il est écrit ici qu'on est « en attente ». À quoi correspond cette situation ?

$\mathbf{R}$ : Cela signifie qu'on a la vanne qui est fermée et qu'on est en état de pouvoir injecter l'eau à tout moment. C'est-à-dire que la vanne est fermée, mais qu'on est en état de l'ouvrir pour faire entrer l'eau, dès qu'on reçoit le signal de mise en marche.

Seulement, pour pouvoir utiliser ce système, il faut déjà que la pression de la cuve baisse. Parce que sinon rien ne peut entrer. Lorsque la pression de la cuve est élevée, on est normalement à peu près à $6,9 \mathrm{MPa}$. En face, on est tout au plus à dix kilos, à environ un Méga. Alors, évidemment, ça ne peut pas entrer. Que ce soit le circuit incendie ou le réseau camion de pompiers, ce sont des réseaux qu'on ne peut utiliser qu'une fois qu'on a, comme on a dit tout à l'heure, ouvert la vanne de dépressurisation, laissé échapper la vapeur et que la pression de la cuve a baissé. Au moment dont nous parlons, la pression dans la cuve du réacteur 1 était encore élevée. On savait pertinemment que l'eau ne pouvait pas entrer. Mais il s'agissait de faire en sorte que ça devienne possible. Ça voulait dire : "préparez le terrain ».

$\mathbf{Q}:$ Vous avez dit 5,9 MPa?

$\mathbf{R}: 6,9 \mathrm{MPa}$.

$\mathbf{Q}$ : Donc, environ $70 \mathrm{~atm}$. C'est dû au fait que quand on veut optimiser le rendement, on a intérêt à augmenter à la fois la température et la pression ? C'est pour ça qu'on atteint cette pression, n'est-ce pas? Du coup, quand on veut envoyer de l'eau de l'extérieur, il y a une résistance à l'intérieur et il faut faire baisser la pression.

$\mathbf{R}$ : Oui, c'est bien ça.

$\mathbf{Q}$ : Et donc, vous devez faire baisser la pression. S'il suffisait que tout ça tourne dans le circuit de refroidissement, ça finirait par baisser. Mais, si je veux faire baisser cette pression volontairement, je pourrais me dire qu'il suffit de la laisser s'échapper. Vous n'avez pas eu ce genre de pensée?

$\mathbf{R}$ : Que voulez-vous dire par « laisser s'échapper»?

$\mathbf{Q}$ : C'est-à-dire se débarrasser de cette vapeur à 70 atm n'importe où dehors.

$\mathbf{R}$ : Dehors... Non. Cette vapeur est hautement radioactive, alors, même pour la laisser s'échapper, il n'y avait que la solution de l'enceinte de confinement. Non, le seul moyen, c'était d'ouvrir volontairement la vanne qui se trouve sur le tuyau qui sort de la cuve, que la vapeur qui s'échappe dans l'enceinte de confinement descende jusqu'à la chambre de suppression et que la vapeur condense et devienne de l'eau, comme c'est prévu structurellement. Il n'y avait que cette solution-là. De toute façon, structurellement, il n'est pas prévu qu'on sorte cette vapeur à l'extérieur. 
$\mathbf{Q}$ : Oui, mais si quelque chose vole en éclats ou se casse?

$\mathbf{R}$ : Non. Ce sont des conditions qui sont prises en compte quand on conçoit la cuve et l'enceinte de confinement.

$\mathbf{Q}$ : Et donc, vous ne pensez pas que, par exemple, la cuve va céder à la pression.

Vous pensez qu'elle résiste encore.

R : Oui. C'est ça.

$\mathbf{Q}$ : Encore une précision. L'ouverture de la soupape de dépressurisation peut-elle se faire manuellement? Ou faut-il une autre source d'énergie?

$\mathbf{R}$ : Bien entendu, il faut de l'électricité pour la manœuvre et ce qu'on appelle un accumulateur, je crois que c'en est un à azote ${ }^{81}$. Si tout cela n'est pas viable, on ne peut pas manœuvrer cette vanne.

$\mathbf{Q}$ : Alors, tout d'abord, en ce qui concerne la pompe à incendie diesel, à 17h30, elle est prête à entrer en action dès qu'on aura fait cette manœuvre pour ouvrir la vanne, ce qui fera baisser la pression à l'intérieur de la cuve. C'est ça ?

R : Oui.

$\mathbf{Q}$ : Ensuite, à 18h18, je lis « réalisation de la manœuvre d'ouverture de la vanne du réseau retour MO (Motor Operated valve) ${ }^{82} 3 \mathrm{~A}$, et de la vanne du réseau alimentation, MO2A, sur le système IC du réacteur 1, sortie de vapeur constatée ». Plus tard, à 18h25, il est noté «fermeture de la vanne du réseau retour, MO3A». Je suppose que toutes ces manœuvres pour ouvrir ou fermer ces vannes du système IC étaient faites par les opérateurs de quart, mais, dans la salle de crise, étiez-vous informés concrètement des manœuvres sur ces vannes?

$\mathbf{R}$ : Non. En tout cas, moi, je n'étais pas au courant.

$\mathbf{Q}$ : Si, à 18h18, les opérateurs sur le terrain ont manœuvré pour ouvrir les vannes 3A et 2A, cela signifie qu'avant $18 \mathrm{~h} 18$ ces vannes étaient fermées, ou qu'ils pensaient qu'il y avait des chances qu'elles soient fermées et ont manœuvré pour les ouvrir. Ce qui veut dire qu'il y avait des probabilités pour qu'à ce moment-là, avant 18h18, I'IC ne fonctionne pas. Sur ce point, aviez-vous reçu des informations comme quoi les vannes s'étaient fermées du fait du tsunami ou d'une autre circonstance quelconque?

$\mathbf{R}$ : Non. En tout cas, dans cet enchaînement de circonstances, s'agissant de l'IC, le personnel de quart était persuadé que le système fonctionnait et, nous, à la cellule de crise nous n'avions pas été informés de ce genre de manœuvres.

$\mathbf{Q}$ : Vous étiez donc convaincu que le système était viable, qu'il était même en fonctionnement, que I'IC tournait.

$\mathbf{R}$ : Oui. C'est ce que je pensais.

$\mathbf{Q}$ : Après la manœuvre de fermeture à 18h25, il semblerait qu'il y ait eu encore l'ouverture de la vanne $3 \mathrm{~A}$ à $21 \mathrm{~h} 30$. Durant toute cette période, vous pensiez que I'IC était toujours en fonctionnement.

$\mathbf{R}$ : Oui. En ce qui concerne l'IC, comme on avait réussi à vérifier le niveau d'eau, je ne sais pas aujourd'hui si on avait réussi à observer le niveau d'eau réel, mais en tout cas, à ce moment-là, nous pensions que nous avions réussi à vérifier qu'il $\mathrm{y}$ avait un niveau d'eau suffisant et nous en avions déduit, à l'inverse, que l'IC était encore en vie. Il faut reconnaître que, concernant le comportement de l'IC, la salle de crise n'était pas informée de toutes ces petites manœuvres sur les vannes, de plus, les paramètres dont nous disposions semblaient aussi confirmer le fonctionnement de l'IC. Nous étions donc persuadés de son fonctionnement ${ }^{83}$.

$\mathbf{Q}$ : Si je me mets à la place des opérateurs de quart, ils ont dû faire toutes sortes de manœuvres en pensant à la sécurité du réacteur. Mais, si on en revient aux faits, la pompe 
incendie à moteur diesel est toujours en état d'attente, puis, concernant la vanne du réseau retour de l'IC, on la ferme à 18h25. Dans ces conditions, du point de vue du refroidissement, entre $18 \mathrm{~h} 25$ et $21 \mathrm{~h} 30$, ils n'ont rien pu faire. Si, trois heures durant, on ne fait rien, il me semble que normalement on devrait commencer à s'inquiéter. Est-ce que le niveau d'eau n'est pas en train de baisser dangereusement? Est-ce que le cœur n'affleure pas ? Est-ce que ça ne va pas entraîner des détériorations ? Et qu'il serait normal qu'ils contactent la cellule de crise pour vous en informer ou vous demander conseil. N'avez-vous pas reçu des rapports, des informations, même très succincts, vous faisant part du fait que I'IC ne fonctionnait peut-être pas très bien?

$\mathbf{R}$ : Non, pas du tout. Et c'est un point que je me reproche aujourd'hui. Mais j'étais persuadé que ça fonctionnait. Et aucune information de ce genre n'est arrivée à la table ronde de la part du chef de production ${ }^{84}$. Et donc, je ne sais même pas si le chef de production lui-même avait reçu des informations allant dans ce sens de la part du chef de quart. En tout cas, s'il y avait eu quoi que ce soit, il revenait au chef de production de partager l'information avec la cellule de crise. Le protocole ne prévoit pas que le chef de quart me téléphone directement. En fait, j'aurais dû, à ce momentlà, demander si tout allait bien du côté de l'IC, j'aurais dû le faire plusieurs fois. Aujourd'hui, je me rends compte que je m'étais persuadé que tout allait bien parce qu'on avait pu vérifier qu'on avait une certaine marge pour le niveau d'eau.

$\mathbf{Q}$ : Concernant justement ce niveau d'eau, je parle de celui du réacteur 1. Dans votre déclaration suivant l'article 15, je crois que c'est dans le deuxième rapport, vous dites que vous voyez le niveau d'eau du réacteur 1. Dans un premier temps, donc, vous voyez le niveau d'eau. Mais ensuite, dans votre $3^{e}$ rapport, vous dites que vous ne le voyez plus, de nouveau. C'est signalé comme un évènement survenu à 17h07. On peut donc supposer que pendant un certain temps, après cette heure, vous n'êtes plus en état de suivre l'évolution du niveau d'eau du réacteur 1. Dans ces conditions, on ne peut savoir si le niveau d'eau baisse, s'il reste constant ou s'il augmente. L'équipe de quart est consciente que I'IC est fermé. La pompe diesel aussi est en attente. Dans des circonstances pareilles, est-ce que les opérateurs de quart n'ont pas tenté une solution? N'ont-ils pas demandé de l'électricité ou n'importe quoi ${ }^{85}$ ?

$\mathbf{R}$ : En tout cas, il n'y a pas eu de « SOS ». En tout cas, pas à moi.

$\mathbf{Q}:$ À la cellule de crise?

$\mathbf{R}$ : Pas de «SOS » à la cellule de crise. Savoir si le chef de production en a reçu un ou pas, cela dépend de ce dont il se souvient. Mais, pour nous, pas d'appel à l'aide. Si on avait reçu un "SOS », on aurait envoyé du monde ou autre chose. Il est vrai que nous, de notre côté, nous n'étions pas spécialement focalisés sur le réacteur 1 . On était préoccupé par, non seulement, le réacteur 1 , mais le 2 et le 3 , ensuite le 5 et le 6 . Il fallait surveiller tous les réacteurs où il y avait du combustible dans la cuve. Alors, il nous était difficile de penser à demander plus particulièrement des détails sur ceci ou cela.

Et puis, comme vous l'avez dit tout à l'heure, on n'a pas pu toujours voir le niveau d'eau, mais on l'avait vu une fois. Du coup, j'étais persuadé qu'il y en avait suffisamment. Mais j'aurais dû, et c'est un point que je regrette terriblement aujourd'hui, j'aurais vraiment dû demander comment ça allait. En tout cas, même s'il y avait eu l'émission d'un «SOS » du terrain, il n'est pas arrivé jusqu'à nous. En tout cas, je n'ai rien reçu de tel.

$\mathbf{Q}$ : Concrètement, à ce moment-là, disons entre le crépuscule et la nuit de ce jour, vous, vous pensez que, pour le réacteur 1, l'IC est en marche, seulement vous ne pouvez plus voir le niveau d'eau. Concernant le réacteur 2, vous ne savez pas si le RCIC fonctionne ou pas. 
Vous ne savez pas non plus quel est le niveau d'eau dans la cuve. Pour le réacteur 3, vous savez que le RCIC fonctionne. C'est bien ça?

R : Oui.

$\mathbf{Q}$ : Dans ce cas, parmi les réacteurs 1 à 3, bien sûr il fallait vous préoccuper des 3, mais, parmi eux, quel était celui qui vous semblait le plus préoccupant, ce soir-là, à la tombée de la nuit, vers 20 h00 ou 21 h00 ? C'était le 2?

$\mathbf{R}:$ Oui, tout à fait.

$\mathbf{Q}$ : Pendant ce temps, dans la chronologie à 20h50, l'ordre est donné par la préfecture de Fukushima ${ }^{86}$, aux habitants dans un périmètre de $2 \mathrm{~km}$ autour de la centrale nucléaire de Fukushima Daiichi, d'évacuer la zone ${ }^{87}$. C'est une mesure prise par la préfecture et elle est notée comme telle. Dans un cas de ce genre, est-ce que la centrale fournit des informations spécifiques au département, en dehors des déclarations de type article 15, pour la prise de décision?

R : Non.

$\mathbf{Q}:$ Vraiment? Vous ne faites rien?

$\mathbf{R}$ : Non. Nous faisons la déclaration suivant l'article 15 , puis nous continuons à communiquer à l'administration toutes les informations dont nous avons connaissance par la suite. Nous informons l'État et la préfecture de tout.

Je ne sais pas quel a été le mécanisme qui a servi au département de Fukushima pour prendre cette décision, mais habituellement, in fine, c'est dans le centre de crise hors site, où sont installées les cellules du Gouvernement, des entreprises et du département, que sont prises ces décisions d'évacuation ${ }^{88}$. À la limite, c'est une chose qui ne nous concerne pas. Nous fournissons en continu des informations sur l'état de la centrale. Mais ce sont d'autres organismes qui jugent et décident, suivant les nécessités, de l'évacuation et d'autres choses.

$\mathbf{Q}$ : Ensuite, en mettant entre parenthèses les horaires réels, il y a donc eu la notification de la pompe incendie diesel en attente de mise en marche à 17h30, de tout à l'heure, et, si on avance jusqu'à la journée du 12, on trouve à 1h48, une note signalant «arrêt de la pompe incendie diesel constaté pour problème». Ce qui veut dire que, durant cette période, il y a eu action pour faire fonctionner cette pompe incendie diesel et envoyer de l'eau?

$\mathbf{R}$ : Je vais vous expliquer. Au début, il y avait 6,9 MPa de pression dans la cuve. C'est la pression à 20h07. Cette pression tombe jusqu'à $0,8 \mathrm{MPa}$ à $2 \mathrm{~h} 45$. Pendant tout ce temps, on n'a pas eu accès aux données. On n'a absolument pas su à quel moment la pression a baissé. On ne connaît absolument pas l'état de la tranche. En fait, si la pression descend jusqu'à 0,8 , il devient possible d'enclencher la pompe diesel. Et, structurellement, elle est conçue de manière à s'enclencher toute seule, si la pression tombe. Mais, à la cellule de crise, y compris moi, nous ne savions absolument pas à quel moment elle s'était enclenchée.

$\mathbf{Q}$ : Je voudrais vérifier ce que vous m'avez dit tout à l'heure. Nous avons vu que le fait que la pompe incendie diesel soit en attente de mise en marche signifiait qu'au moment où la pression de la cuve baissait, il suffisait d'ouvrir la vanne pour la mettre en marche. Et l'action pour ouvrir cette vanne?

R: C'est un clapet anti-retour. Avec une pression de $70 \mathrm{kPa}$ dans la cuve et une pression de $10 \mathrm{kPa}$ du côté de la pompe à incendie, le clapet anti-retour fait que ça ne peut pas couler de ce côté-ci à ce côté-là. Mais si ce côté-là baisse, automatiquement, l'équilibre des pressions fait que ça coule. 
Q: Donc, pour que cette vanne s'ouvre et que l'eau entre, il n'y a pas besoin d'une intervention humaine?

$\mathbf{R}:$ Non.

$\mathbf{Q}$ : Du coup, puisqu'on note à 1h48: "arrêt de la pompe incendie diesel constaté pour problème ", c'est que l'équipe de quart ou le pilote qui était en train de conduire le réacteur, s'en est rendu compte à ce moment-là. Ce qui veut dire que la pompe s'était arrêtée avant ce moment, et qu'on l'a constaté seulement à ce moment-là ?

$\mathbf{R}$ : Encore une fois, à y réfléchir aujourd'hui, la communication entre la cellule de crise et la salle de commande des réacteurs 1 et 2 était très mauvaise à ce moment-là. Je ne veux pas dire que le chef de quart était mauvais. Mais, aussi bien concernant l'IC que la pompe diesel, les informations sur leur fonctionnement n'arrivaient pratiquement pas auprès de nous à ce stade-là. Pour être tout à fait franc, je n'ai pas eu de détails les concernant.

Et c'est en découvrant toutes les données qu'ils ont fournies sous cette forme, que je me dis aujourd'hui, c'était donc ça. Mais, sur le moment, je n'avais aucune connaissance de tout ça.

Q: Donc, au soir du 11 mars, vous pensez que, s'agissant du réacteur 1, l'IC est en fonctionnement, que la pompe diesel est en attente et que, pour que l'eau puisse entrer, la pression est encore trop haute.

R : Oui. Que l'eau ne peut pas entrer, mais qu'il fallait être prêt à envoyer l'eau.

$\mathbf{Q}:$ Que l'IC doit être en fonctionnement...

R : Oui. C'est ce qui est différent des autres réacteurs. Pour les autres, on peut volontairement ouvrir la soupape de dépressurisation et baisser la pression de la cuve. Mais on n'a pas touché à la vanne sur le réacteur 1 . Et, à un moment donné, peut-être à 20h07, je ne me souviens pas exactement de l'heure, en tout cas en fin de journée, j'avais vu qu'il y avait 7 atm de pression dans la cuve. Et ensuite, tout à coup, à un autre moment, je vois que ça a déjà chuté à $8 \mathrm{kPa}$. Je me demande ce qui s'est passé. Je me le demande encore.

$\mathbf{Q}:$ Vous ne savez pas ce qui s'est passé, mais c'est une situation qui vous arrange.

$\mathbf{R}$ : Oui, en quelque sorte. Mais il y avait aussi des choses défavorables. Parce que faire baisser la pression en ouvrant la soupape de dépressurisation, cela veut dire qu'on perd en même temps de l'eau et que le niveau d'eau va baisser. Puisque l'eau qui devrait se trouver à l'intérieur de la cuve s'en échappe. Alors, le niveau d'eau devait baisser. Mais, en fait, l'indicateur de niveau d'eau va continuer à pointer un chiffre stable pendant tout ce temps. Parfois, il va même un peu monter.

$\mathbf{Q}:$ Vous pensez qu'il était peut-être bloqué ?

$\mathbf{R}$ : Oui. Alors, nous sommes dans une situation où nous n'arrivons pas du tout à imaginer un scénario cohérent entre ce qui se passe concernant l'indicateur de niveau d'eau et ce qui se passe à l'intérieur de la cuve.

En plus, comme je viens de le dire, aucune information concernant la conduite de l'IC ou celle de la pompe diesel, ne circulait de la salle de commande des réacteurs 1 et 2 vers la cellule de crise. C'est comme si nous avions sauté toutes ces histoires. À la limite, au contraire, nous, au moment où on s'est aperçu qu'on en était à $0,8 \mathrm{MPa}$, on n'en revenait pas. On s'est demandé mais pourquoi ça a baissé dans une telle proportion? En plus, la pression de l'enceinte de confinement était à quelque chose comme $640 \mathrm{kPa}$. On s'est demandé pourquoi elle était si haute. Ça, ce sont les chiffres 
qu'on a eus la première fois qu'on a eu accès à la pression de l'enceinte. Dans ces conditions, il y avait des choses qu'on n'arrivait pas à expliquer. Des choses qu'on ne pouvait même pas imaginer dans notre pauvre tête. Pour nous, quand on a vu les indicateurs, les chiffres étaient complètement invraisemblables. C'est comme ça qu'on l'a vécu.

Q: Pour vérification. Entre $20 \mathrm{~h} 07$, où vous avez $6,9 \mathrm{MPa}$, et le lendemain à $2 \mathrm{~h} 45$, où vous avez $0,8 \mathrm{MPa}$, vous n'avez pas effectué de manœurre de dépressurisation avec la soupape. C'est bien ça?

R : Oui. Si on l'avait fait, j'aurais pu, au contraire, donner des indications pour qu'on équilibre l'injection d'eau et la dépressurisation, j'aurais pu commander des manœuvres. Mais en fait, c'est comme si la tranche dépressurisait toute seule, sans qu'on ne fasse rien, et, moi, j'étais là à me demander ce qui arrivait.

$\mathbf{Q}$ : Je pense que ce chiffre de 6,9 MPa est un chiffre qui a été obtenu en pénétrant à l'intérieur. Était-ce suivant vos ordres?

$\mathbf{R}$ : En fait, la manière d'obtenir les renseignements, où regarder et comment, est laissée au jugement de ceux qui sont de quart. De toute façon, si on n'observe pas à un endroit où on peut voir, on ne peut rien connaître. Mes ordres étaient de faire en sorte de tout observer, de surveiller tout ce qui pouvait être vérifié. Cela inclut le fait de regarder effectivement l'indicateur de pression à l'intérieur, s'il se trouve à l'intérieur.

Seulement, les conditions étaient extrêmement mauvaises et on ne pouvait pas rester longtemps. Donc, on y allait une fois pour voir et c'était fini. À la base, l'idéal, c'est de tout surveiller de manière continue avec les instruments de la salle de commande, mais si on ne le peut pas, il faut bien vérifier quelque part.

$\mathbf{Q}$ : En ce qui concerne le niveau d'eau, on voit dans ces paramètres qu'à $21 \mathrm{~h} 30$, je pense que cela concerne le circuit $A^{89}$, il y avait, dans la section combustible, $450 \mathrm{~mm}$, puis $550 \mathrm{~mm}$ à $22 \mathrm{~h} 00$. Donc, vous aviez réussi à observer ces niveaux. Au moment où vous avez vu ces chiffres, qu'avez-vous pensé?

$\mathbf{R}:$ J'ai été rassuré.

$\mathbf{Q}$ : Mais, durant le moment où vous ne pouviez plus voir le niveau d'eau, vous vous étiez demandé si le cœur n'était pas dénoyégo ?

$\mathbf{R}$ : Finalement, on ne sait pas trop quelle était la situation véritable, mais le point le plus important pour nous était de savoir s'il y avait un niveau d'eau suffisant. Pour ce qui est de savoir si le cœur fond ou ne fond pas, si le niveau d'eau est supérieur à une certaine donnée, pour nous, c'est un élément rassurant.

Q: Ensuite, vous avez été tout le temps en mesure d'observer le niveau d'eau pour le réacteur 1 ?

$\mathbf{R}$ : Oui. On le voyait, mais en même temps on avait des doutes. Parce que la pression de l'enceinte de confinement était élevée, alors que le niveau d'eau n'avait pas changé. On se demandait pourquoi la pression de la chambre sèche ${ }^{91}$ de l'enceinte avait tellement augmenté. Ensuite, on s'est aussi demandé pourquoi la pression de la cuve, elle, avait tellement baissé. Et si on mettait les deux ensemble, on finissait par se dire que ce niveau d'eau n'était pas crédible. C'est enfin à ce moment-là qu'on se l'est dit. 
$\mathbf{Q}:$ Quand, par exemple?

$\mathbf{R}$ : Le plus important, c'était le fait que la pression de la chambre sèche était de 0,8 . On se disait, mais pourquoi ça? Ça a été le point de départ. Je crois me souvenir qu'avant ça, on avait eu quelque chose comme 0,6. Oui, $600 \mathrm{kPa}$.

$\mathbf{Q}$ : Vous voulez dire la pression de la cuve?

$\mathbf{R}$ : Non. Pas la pression de la cuve, mais celle de la chambre sèche.

$\mathbf{Q}$ : La pression de la chambre sèche.

R : C'est à 0h06.

Q: Ah, oui. Un peu avant, vers 23h50, on avait en effet mesuré les $600 \mathrm{kPa}$ de la chambre sèche. Vous l'aviez eu comme information, ça?

R : Oui, j'ai eu l'information. Alors, là, nous avons eu tout d'abord un mouvement de surprise.

Et puis, s'il fallait parler d'autre chose de bizarre, c'est ce qui est écrit ici à 21h51, c'est cette radioactivité. On ne comprenait pas pourquoi la radioactivité était tellement montée. C'est quelqu'un sur le terrain qui était parti mesurer et on avait su que les chiffres étaient très élevés au sud, à l'ouest ou au nord, je ne me rappelle plus très bien, de la double porte. Et ça, c'était bizarre.

$\mathbf{Q}:$ Vous trouvez ça bizarre et vous interdisez l'accès à la zone?

$\mathbf{R}$ : Oui. La radioactivité était forte et il ne s'agissait pas de se surexposer, alors j'ai interdit l'accès à la zone ${ }^{92}$. Mais le problème, c'était que ce fait et le comportement de la tranche n'arrivaient pas à s'enchaîner logiquement dans ma tête. Parce que je n'avais aucune donnée. Pour moi, à ce moment-là, l'IC était en fonctionnement, le niveau d'eau était correct, alors, que dans ces circonstances la radioactivité grimpe n'était pas compréhensible. Il y avait forcément quelque chose qui n'allait pas. Étaitce le niveau d'eau? Et, pendant que je me posais ces questions, on apprend que la pression a l'air d'être élevée dans l'enceinte. Là, je commence à me dire qu'il y a de fortes probabilités pour que la situation soit très dégradée à l'intérieur ${ }^{93}$.

$\mathbf{Q}$ : Justement dans les 23h00, comme résultat de surveillance, on signale la hausse de la radioactivité à l'intérieur du bâtiment turbine. Concrètement, au rez-de-chaussée du bâtiment turbine, on relève $1,2 \mathrm{mSv} / \mathrm{h}^{94}$ devant la double porte nord et $0,5 \mathrm{mSv} / \mathrm{h}$ du côté sud. Ensuite, je ne sais pas si vous en avez le souvenir, un opérateur de quart au bâtiment réacteur 1 a relevé une forte radioactivité de l'ordre de 0,8 mSv en environ dix secondes avec son APD (Alarm Pocket Dosimeter) ${ }^{95}$. Avez-vous eu ce renseignement?

$\mathbf{R}$ : Je n'en ai pas le souvenir. Comme par la suite, on a continué à recevoir des rapports sur la hausse de la radioactivité, peut-être que j'avais eu à un moment l'information émanant du conducteur de la salle de commande ${ }^{96}$, mais je ne m'en souviens pas.

$\mathbf{Q}$ : Donc, vers 23h00, vous avez des informations qui signalent que la radioactivité grimpe de plus en plus.

R : Oui, ça, je l'ai su.

$\mathbf{Q}:$ Et, à ce moment-là, vous pensez que l'IC est toujours en fonctionnement.

$\mathbf{R}$ : C'est là que je commence à avoir de sérieux doutes. Si on regarde seulement le niveau d'eau, ça a l'air d'aller. Mais je commence à me dire qu'il se passe quelque chose de bizarre. Je commence à me demander si l'IC n'est pas à l'arrêt, si on n'est pas dans une situation où on aurait perdu la source froide. 
$\mathbf{Q}$ : Je voudrais qu'on précise ce «bizarre ». Avez-vous envisagé à ce moment-là que le fait que la radioactivité monte de cette manière pouvait être dû à une fuite de la cuve ou de l'enceinte?

R : Oui, j’y ai pensé.

$\mathbf{Q}$ : Vous aviez donc envisagé cette hypothèse tandis que la radioactivité augmentait.

$\mathbf{R}$ : Oui. Je me suis demandé à quoi ça pouvait être dû. Comme aucun appareil de surveillance n'était utilisable, on ne pouvait pas élaborer d'hypothèse. En temps normal, on a un moniteur de zones, qui mesure la radioactivité en continu sur différents points à l'intérieur du bâtiment réacteur. Mais à ce moment-là, il était inopérant. Si on avait eu ce moniteur de zones, on aurait su dans quelle zone la radioactivité augmentait et on aurait compris qu'il y avait une fuite d'un tuyau ou d'autre chose près de cette zone. Le moniteur de zones est là pour ça. Mais, là, il était totalement mort, on ne pouvait rien savoir. On était dans une situation où on n'avait aucun élément pour pouvoir trancher.

$\mathbf{Q}$ : Et à y penser maintenant?

$\mathbf{R}$ : À y penser maintenant, je crois que l'erreur a été de faire trop confiance à cet indicateur de niveau d'eau.

$\mathbf{Q}$ : Vous pensez que le niveau d'eau était déjà descendu...

$\mathbf{R}$ : Sans erreur possible, il était descendu. Pour en revenir à cette histoire d'IC, il aurait fallu se préoccuper plus tôt et plus sérieusement de la conduite de l'IC. Si on avait su la situation de la salle de commande, que l'IC ne fonctionnait pas, on aurait pu se rendre compte de la gravité de la situation. Pour autant, comme solutions, on n'avait, de toute façon, guère mieux que la pompe diesel, qu'injecter avec la pompe incendie et utiliser le camion de pompiers, ce que nous avons finalement fait. Est-ce qu'on aurait pu réagir plus rapidement, si on avait su? Je pense que, physiquement, on n'aurait pas pu aller plus vite. Alors, on se retrouve au même point. Savoir si on aurait pu se rendre compte de la situation plus ou moins vite, à y réfléchir maintenant...

$\mathbf{Q}$ : Le niveau de l'indicateur était farfelu...

$\mathbf{R}$ : Sans aucun doute, il était farfelu. Je ne me pardonnerai jamais d'y avoir trop cru.

$\mathbf{Q}$ : Si on continue dans la chronologie, le 12 mars vers 1 h55, l'indicateur de niveau d'eau donne les chiffres de 1300 et 500 pour $A$ et $B$. Des chiffres très différents.

Vous n'avez pas pensé que c'était bizarre ? À partir de quel moment avez-vous pensé qu'il n'était pas possible de faire confiance à l'indicateur de niveau d'eau?

$\mathbf{R}$ : Ce qui me préoccupait plus que l'indicateur de niveau d'eau, c'est, comme je vous l'ai dit tout à l'heure, le fait que la radioactivité avait augmenté. Si le refroidissement se fait normalement, le contrôle de la cuve se fait normalement. Il y a suffisamment d'eau et il n'y a, a priori, pas de risque de fuite et la radioactivité n'augmente pas. Si la radioactivité augmente, cela veut dire que la radioactivité qui, en principe, est enfermée totalement ici, en sort. Cela veut dire qu'elle s'échappe de la cuve et que ce qui s'en est échappé, s'échappe, à nouveau, de l'enceinte. On ne peut pas expliquer cela autrement. Et si, à ce moment-là, on ne peut pas savoir ce qui se passe dans le réacteur, qu'on ne connaît pas la pression, que le niveau d'eau est fantaisiste, on peut penser qu'on est peut-être allé jusqu'à la dégradation des combustibles. Dégradation des combustibles signifie que la cuve a cédé à la pression et que, s'il y a rejet d'éléments radioactifs de la cuve vers l'enceinte de confinement, c'est qu'il y a fissure 
quelque part sur la cuve. Ça, c'est le premier point qu'on peut imaginer. Ensuite, ce qui a été rejeté dans l'enceinte ne peut plus être contenu dedans et s'en échappe. C'est la seule explication possible.

$\mathbf{Q}$ : Vous voulez dire qu'étant donné que la radioactivité qui avait été mesurée dépassait largement les chiffres habituels, vous aviez pensé à ce genre de possibilité ?

R : Oui.

Q: Et, effectivement, au moment où on s'apprête à changer de date, on observe 600 de pression dans la chambre sèche. Vous avez pensé au risque qu'en effet, il y ait une fuite de la cuve vers l'enceinte de confinement, c'est ça?

$\mathbf{R}$ : Oui. Si la pression était de $600 \mathrm{kPa}$, on ne pouvait l'expliquer qu'en faisant l'hypothèse qu'il y avait fuite de la cuve vers l'enceinte de confinement.

$\mathbf{Q}$ : Est-ce que, toutes ces réflexions, vous les aviez gardées pour vous-même, ou bien en aviez-vous parlé autour de la table ? Avez-vous parlé de l'état du réacteur, tout ça?

$\mathbf{R}$ : Ce genre d'informations est annoncé par le chef du groupe "production ». Le chef du groupe "production» les énonce. On se demande ce qui se passe. Le groupe « technique » vient aussi en renfort.

Et puis, il y a le responsable du réacteur. Je me rappelle très bien qu'il avait proposé qu'on réfléchisse ensemble à tout ça.

Q : Effectivement, avant, dans la déclaration article 15, évidemment les choses ont dû changer puisqu'en fin de compte vous avez pu voir le niveau d'eau, enfin, dans la déclaration, vous dites que vous prévoyez que le TAF puisse être atteint vers 21 h00. Je suppose que, ne pouvant avoir de certitude quant au niveau d'eau, vous formez l'hypothèse que le niveau d'eau descende et qu'au pire le cœur puisse être endommagé et fondre. Vous avez en tête quelque chose comme le scénario du pire, n'est-ce pas ? Quand vous avez constaté que la radioactivité augmentait très vite, que la pression de la chambre sèche avait peut-être dépassé les 600 kPa abs ${ }^{97}$, avez-vous pensé que le niveau d'eau était encore plus bas?

$\mathbf{R}$ : Comme je vous le disais, c'est à peu près à ce moment-là que j'ai commencé à penser que le niveau indiqué était une erreur.

$\mathbf{Q}$ : Vous pensez donc que c'était une erreur. Et dans quelle situation pensiez-vous être, en réalité?

$\mathbf{R}:$ Je pensais que le niveau était encore plus bas.

$\mathbf{Q}:$ Pensiez-vous que le cœur était à découvert?

$\mathbf{R}$ : Ça, puisqu'on n'avait pas d'instrument de mesure, il était difficile de dire jusqu'à quel point c'était descendu. Peut-être que ceux qui ont fait des analyses après le savent?

$\mathbf{Q}:$ Ce que je voudrais savoir c'est ce que vous pensiez à ce moment-là.

$\mathbf{R}$ : À ce moment-là, en tout cas, je ne savais pas jusqu'à quel point le combustible était à découvert, mais j'avais commencé à penser qu'on avait peut-être atteint une zone où une certaine dégradation du combustible était possible.

$\mathbf{Q}$ : Ce qui veut dire que les mesures que vous avez prises après, ont été prises avec cette situation en tête.

$\mathbf{R}$ : Mais, cette situation, je l'ai en tête depuis le début. Comme je vous l'ai dit tout à l'heure, depuis le tout premier moment où j'ai été surpris, au moment où le RCIC ne fonctionnait plus, étant donné qu'on n'avait pas de moyen pour injecter l'eau, j'avais conscience qu'au pire on allait vers l'endommagement du cœur. 
$\mathbf{Q}$ : Oui, bien sûr. Si ça avait continué comme ça...

R : Voilà.

$\mathbf{Q}$ : Ce que je voudrais savoir c'est, entre le 11 et le 12 mars, lorsque vous avez su que la pression de la chambre sèche était de 600 kPa abs, bien sûr, j'ai conscience que vous ne pouviez pas le savoir à coup sûr, mais dans quel état pensiez-vous que le cœur se trouvait?

$\mathbf{R}$ : Pour moi, il y avait de grandes chances pour qu'on ait atteint le stade de la dégradation du cœur.

$\mathbf{Q}$ : Et cette réflexion, autour de la table, étiez-vous le seul à vous la faire?

$\mathbf{R}$ : Non, probablement, comme je viens de le dire, on a discuté avec le chef du groupe " production ", le chef du groupe "technique » et le responsable du réacteur, enfin des spécialistes de tout ce qui tourne autour du réacteur, pour savoir comment interpréter ce phénomène. On s'est demandé si l'indicateur de niveau d'eau n'était pas fiable. On s'est dit que, dans ce cas, le fait que la pression de la chambre sèche monte ne pouvait être expliqué que par le fait que quelque chose s'échappe de la cuve. On ne savait pas si la soupape de dépressurisation s'était ouverte ou pas, mais, de toute manière, ça voulait dire que la cuve avait des fissures, qu'elle était endommagée à l'intérieur de l'enceinte de confinement, qu'il y avait des dommages et que cette situation continuait à évoluer.

Tout cela n'est possible que si la pression de la cuve est tombée. Si elle tombe, ça veut dire que le niveau d'eau a baissé, puisque l'eau s'échappe sous forme de vapeur. Si le niveau d'eau baisse dans la cuve, évidemment on ne sait pas si elle couvre encore la zone du combustible ou pas, mais, de toute manière, c'est là qu'on commence à penser que le niveau doit être bas.

$\mathbf{Q}$ : Là-dessus, vous apprenez la hausse de la radioactivité, l'évolution de la pression dans la chambre sèche. Vous en discutez autour de la table. Ces discussions, étaient-elles relayées en temps réel vers la maison mère par téléconférence?

$\mathbf{R}$ : Il y a des choses qui étaient relayées et puis d'autres... Par exemple, je suis ici. Les collaborateurs viennent au rapport ici. On échange sur ce qui se passe. Ça, ça ne passe pas dans le micro.

Ensuite, dans le cadre officiel de surveillance, on fera un rapport pour dire, par exemple, qu'on a atteint les $600 \mathrm{kPa}$. Ça, ça se fait à part. On partage des informations techniques autour de la table et on en fait rapport. Encore à part.

$\mathbf{Q}$ : Ce qui veut dire que vous discutez autour de la table de la situation et, par téléconférence, vous en référez.

R : Oui.

$\mathbf{Q}:$ Après, un peu plus tard, il y aura le rapport suivant l'article 15.

R : Oui.

$\mathbf{Q}$ : Vous en êtes là de vos réflexions et la mesure que vous prenez ensuite, si je me réfère à ces notes: "face à la possibilité que la pression de la chambre sèche ait dépassé les $600 \mathrm{kPa}$ abs, ordre du directeur de la centrale d'entamer les préparatifs pour un éventuel éventage ${ }^{98}$ de l'enceinte de confinement ». Ce qui est désigné ici par éventage de l'enceinte de confinement, c'est l'éventage de la chambre humide ${ }^{99}$ ?

$\mathbf{R}$ : À ce moment-là, comme la pression a augmenté, c'est l'ordre d'éventer suivant la procédure. La procédure, c'est d'abord l'éventage de la chambre humide, et si cela ne 
suffit pas à faire baisser la pression, in fine, il faut faire l'éventage de la chambre sèche. L'ordre concernait l'ensemble de la procédure.

Q: La procédure que vous aviez en tête au moment où vous avez donné cet ordre, c'est celle du manuel en cas d'accident? Dans le cas où la pression de la chambre sèche dépasse les $853 \mathrm{kPa}$. Le manuel décrit la procédure à suivre dans ce cas-là. Était-ce cette procédure que vous aviez en tête?

$\mathbf{R}$ : Les détails du manuel, ce sont les conducteurs qui sont au courant. Ce que j'ai ordonné, je ne sais plus très bien quelle était la pression de dimensionnement du réacteur 1, parce qu'il est un peu différent des autres, mais il me semble que c'était $400 \mathrm{kPa}$, et donc nous étions déjà au-delà de la pression de dimensionnement, alors pour ne pas dégrader l'enceinte de confinement, il fallait absolument faire baisser la pression. Il n'y avait pas d'autre solution. Du point de vue d'un mécanicien, il fallait absolument éventer pour faire baisser la pression. Et dans ces circonstances, il y avait une procédure dans le manuel accident et je leur ai dit, procédez à l'éventage. Mais le détail des manœuvres est confié aux gens de la production.

$\mathbf{Q}$ : Alors, si je regarde le manuel, je vois que si on appuie sur un bouton de la salle de commande, on peut ouvrir la vanne. Mais, cette fois-là, vous ne pouviez pas le faire. II vous manquait des choses, l'alimentation électrique, un compresseur ou d'autres choses, n'estce pas?

$\mathbf{R}$ : Oui, mais à ce moment-là, pour moi aussi c'était la première fois que je me trouvais confronté à une telle situation, et, pour être tout à fait franc, moi-même, je ne l'avais pas compris. C'est qu'on ne savait pas encore les détails de la situation sur le terrain. Et en ça, nous étions encore assez proches des gens du siège. Bien sûr, sur le terrain, ils ne voyaient plus les indicateurs, dans la salle de commande ils étaient dans le noir, tous les instruments principaux étaient éteints, mais on avait l'impression que si on leur ordonnait d'éventer, cela pouvait être fait. Bien sûr, il n'y avait pas de source électrique, ni de source d'air, mais on croyait bizarrement dur comme fer que pour éventer, il suffisait d'ouvrir une vanne, que si on arrivait à ouvrir cette vanne, ça allait marcher. C'est après qu'on a compris. L'AOV (Air Operated Valve) ${ }^{100}$ n'avait pas d'air. Comme de juste, le MOV (Motor Operated Valve) ne marchait pas. On s'est demandé si on ne pouvait pas faire ça manuellement. Mais la radioactivité était trop forte pour qu'on puisse entrer. Et c'est là qu'enfin on s'est rendu compte à quel point c'était difficile. Mais on n'arrivait pas à faire passer le message au siège ou à Tôkyô, leur montrer à quel point cet éventage était difficile. Eux se contentaient de dire : "faites vite, faites vite ». C'est là où il y a un réel décalage entre ceux qui sont véritablement sur le terrain, ceux qui sont quand même proches du terrain, comme la cellule de crise, et ceux qui sont vraiment loin du terrain, par exemple le siège.

$\mathbf{Q}$ : Excusez-moi de m'immiscer, mais nous, qui sommes en dehors de tout ça, quand nous entendons dire, par exemple, qu'il faut ouvrir une vanne, la plupart des gens s'imaginent qu'il suffit de pousser sur un bouton quelque part pour que la vanne s'ouvre. Les gens ne peuvent pas s'imaginer autre chose.

Mais tout ça, ça présuppose qu'on ait de l'électricité. Si tout le monde partageait l'idée que sans électricité, on ne peut rien faire, ça nous mènerait certainement à réfléchir. Mais il est tellement évident pour nous d'avoir toujours de l'électricité qu'il ne nous est même pas possible d'imaginer ce que cela peut être d'être sans électricité. C'est pour ça qu'on a des réactions du genre : «dépêchez-vous ", "il suffit de faire telle chose », "ça devrait se faire tout de suite », etc. 
Mais ce qui devient terriblement compliqué, on l'a vu dans vos explications de tout à l'heure, c'est, par exemple, de manœuvrer manuellement. On dit manœuvrer manuellement, mais qu'est-ce que ça signifie? Est-ce que ça veut dire qu'il faut aller jusqu'au bord de la cuve et ouvrir la vanne? Pour ça, je ne sais pas s'il faut tourner le volant manuellement et que c'est ça, la manœuvre manuelle, ou s'il y a quelque chose un peu plus loin, comme un accumulateur, dont il faut pousser le bouton ou dont il faut ouvrir la vanne, ce qui serait tout de même aussi une manœuvre manuelle. S'il y a de la pression accumulée, peut-être que ça va marcher.

D'ailleurs, d'après les explications de tout à l'heure il m'a semblé que pour les installations prévues pour les situations d'urgence, qui demandent beaucoup d'énergie et au cas où toute source serait perdue, il y avait des postes où avaient été prévus des accumulateurs et d'autres postes, non.

Alors dans un cas comme celui-ci, où rien ne marche plus pour de bon, sur le terrain, vous commencez par des commandes à distance, celles qui demandent le moins de courant, puis en partant de tout ce qui peut répondre automatiquement aux ordres, petit à petit vous arrivez, in fine, à la solution de l'ouverture manuelle de la vanne. Mais là, vous êtes confrontés au problème de la radioactivité. Elle est déjà trop forte pour qu'on puisse s'approcher. Et là, vous découvrez qu'il y a des zones qu'on ne pourra plus approcher. Partager ce genre de réflexions me semble être un exercice difficile. L'avez-vous ressenti comme tel?

R : Oui.

$\mathbf{Q}:$ Comme s'il y avait une vraie faille?

R : Oui. Et dans cet ordre d'idée, le plus éloigné, c'est le Gouvernement. Ils sont persuadés que s'il y a ordre du ministre, cela va se faire dans la minute. La réalité, c'est pas ça.

$\mathbf{Q}$ : C'est ce que je veux dire. Il y a des gens qui pensent qu'il suffit qu'ils disent d'ouvrir et trouvent normal que ça s'ouvre tout de suite et ceux qui, s'ils veulent vraiment ouvrir, vont se retrouver à devoir faire des tas de choses, des gens qui vont se trouver piégés, en quelque sorte. Il y a une énorme faille entre les deux.

$\mathbf{R}$ : Nous aussi, nous pensons que c'est une bonne occasion de faire comprendre de manière concrète la distance qu'il y a entre ce que vivent ceux qui sont sur le terrain et ceux qui sont dans les bureaux. Parce que, malheureusement, je pense que c'est une situation que nous risquons de rencontrer encore et encore dans le futur. Alors, cet écart, cette distance, nous devons en parler sérieusement.

Q: Tout à fait d'accord avec vous. II ne s'agit ni de jargon technique, ni de technique spéciale. II faut bien se rendre compte qu'entre juste donner des ordres et faire fonctionner quelque chose dans la réalité, il y a une distance énorme, une différence énorme aussi du point de vue de la qualité, et que si on n'en prend pas conscience, on n'arrivera jamais à la véritable sûreté telle que nous voulons la réaliser. Quelque part, nous l'avons appris là et il faut en tirer les leçons. C'est ce que j'ai ressenti très fort en vous écoutant.

Nous pouvons revenir maintenant à la chronologie.

$\mathbf{Q}$ : Je suppose que vous avez donné l'ordre de préparer cet éventage, parce que vous avez eu des informations laissant penser que la pression de la chambre sèche risquait de dépasser les $600 \mathrm{kPa} a b s$. Mais l'éventualité d'un éventage vous était-elle venue soudainement à l'esprit à ce moment-là, ou bien, pensiez-vous depuis un certain temps qu'il conviendrait de faire cette manœuvre à un moment ou à un autre?

$\mathbf{R}$ : Je suis tout à fait confus, mais je n'y ai pensé qu'après avoir appris la pression de la chambre sèche. Même si la radioactivité était montée, on ne savait pas quelle était la pression de la chambre sèche. En fait, c'est seulement quand on a des données qu'on peut juger de la situation. Sans avoir de données réelles... On pense bien que si 
le pire arrivait, on aurait naturellement recours à l'éventage, et ça, on y pense dès le début. Mais, à ce moment précis, pour le réacteur 1, on n'imaginait même pas que l'enceinte de confinement avait dépassé la pression de dimensionnement. Alors quand on a su que la pression était de 600 , là, on a été ébahis et tout est parti de là. Cela voulait dire que la pression de dimensionnement était dépassée. Qu'allions-nous faire ? Eh bien, il n'y avait que la solution de l'éventage. Alors, j'en ai donné l'ordre.

$\mathbf{Q}$ : Savez-vous si, parmi les gens qui se trouvaient dans la cellule de crise, des gens du groupe «réhabilitation» ou du groupe «production» qui, pensant qu'il pourrait y avoir ultérieurement des éventages, avaient pris l'initiative d'étudier à l'avance les schémas de réseaux ou des plans de construction, pour savoir quel tuyau passait à quel endroit, par exemple?

R : Là, je ne sais pas. Pour ma part, j'ai émis concrètement l'hypothèse d'un éventage, je pense, probablement au moment où la pression avait dépassé les $600 \mathrm{kPa}$. Mais je ne sais pas si, eux, de leur côté, ils avaient pris de telles initiatives.

$\mathbf{Q}$ : Alors, dans la cellule de crise, n'y a-t-il pas eu des chefs de groupe « réhabilitation » ou des chefs de groupe «production» qui, avant que vous ne donniez effectivement des directives pour cette préparation, vous ont suggéré de commencer à préparer un éventage?

$\mathbf{R}$ : Il me semble que non. Mais il y a peut-être eu de telles suggestions. Je ne sais pas trop. Moi, je ne m'en souviens pas. Mais, peut-être est-ce vraiment arrivé ? En tout cas, à l'heure où je vous parle, je ne m'en souviens pas.

$\mathbf{a}$ : Je voudrais vous poser encore une question, tout en sachant très bien qu'il est plus facile d'expliquer ces phénomènes aujourd'hui que sur le coup. Cela concerne le réacteur 1. Ce jour-là, plus tard, vers 15h30, il va y avoir une explosion au réacteur 1, que nous pensons être aujourd'hui une explosion d'hydrogène, ce que vous ne pouviez sans doute pas savoir sur le moment.

Depuis, il y a eu différentes enquêtes qui se sont demandé comment cela a été possible. Et on a évoqué la possibilité qu'une grande quantité d'hydrogène ayant été produite dans la cuve du réacteur, elle se serait échappée de la cuve vers l'enceinte de confinement. II y aurait eu une fuite quelque part et elle se serait propagée dans le bâtiment. Si, par exemple, la radioactivité augmente, cela signifie que des éléments radioactifs s'échappent avec la vapeur. Alors, de l'hydrogène ne s'échapperait-il pas aussi de la même manière ? Je pense que c'est une des possibilités qui, aujourd'hui, est envisagée même à l'intérieur de TEPCO. Sur le moment, avez-vous pensé que de l'hydrogène pouvait s'échapper avec les éléments radioactifs, qu'il pouvait s'accumuler dans le bâtiment, que de l'hydrogène était produit dans la cuve et qu'il pouvait fuir?

Je veux dire, pas uniquement vous, mais les gens dans la cellule de crise ou au siège. Estce que quelqu'un avait pointé du doigt ce risque?

$\mathbf{R}$ : Non, personne. Je pense que même au niveau international, personne n'en avait parlé101.

Q: Sur le moment.

R : Oui, sur le moment.

$\mathbf{Q}$ : Donc, vous donnez des directives concernant l'éventage et, pour l'injection d'eau, vous... Ah non, c'est l'IC. Vous vous dites que l'IC n'est plus très sûr. C'est ça ?

R : Oui.

Q: Si nous continuons à suivre la chronologie, il y a le constat de l'arrêt de la pompe à incendie diesel à $1 \mathrm{~h} 48$, comme nous l'avons vu tout à l'heure. Le 12 mars vers $0 \mathrm{~h} 00$, la pression de la chambre sèche est très élevée et vous ne savez pas si vous pouvez vous fier au niveau d'eau. Dans cette situation, vous vous demandez ce qu'il en est de la pompe à 
incendie diesel. Est-ce que, par exemple, le fait de ne pas pouvoir voir la pression vous empêche d'envisager une manœuvre de dépressurisation?

$\mathbf{R}$ : Si je me remets en situation, à ce moment-là, on ne connaît pas l'état de la cuve. Mais, puisque la pression de la chambre sèche est élevée, on pense bien qu'il doit y avoir une fuite. Comme on l'a dit tout à l'heure, le niveau d'eau a baissé. On ne sait pas jusqu'à quel point le combustible est endommagé, mais on sait que le niveau est bas. Alors que faire? On ne connaît pas la pression. Dans ces conditions, on ne peut pas ouvrir les soupapes de dépressurisation. Nous sommes dans une situation où on nous dit de réfléchir à une solution sans avoir de données, sans rien pouvoir observer. Alors, ce que nous voulons, c'est pouvoir, d'une manière ou d'une autre, observer les données principales. Et, depuis le début, j'avais demandé à ce que les équipes y consacrent toute leur énergie. Malgré cela, les données tardaient à arriver et j'étais dans une situation où il était difficile de prendre des décisions de manœuvres.

$\mathbf{Q}$ : Donc, pour vous la priorité était de faire en sorte qu'on puisse avoir accès aux paramètres essentiels pour faire les manœuvres, comme la pression de la cuve, etc.

$\mathbf{R}$ : Oui, c'était la priorité des priorités.

$\mathbf{Q}$ : Excusez-moi si je repars vers des considérations parallèles. Vous dites que ce qui vous intéresse en priorité sont le niveau d'eau et la pression, que nous avons évoqués tout à l'heure. Si vous devez fonder vos actions sur ces paramètres, il faudrait, dès la conception, doubler, par exemple, ces indicateurs.

$\mathbf{R}$ : Pour être doublés, ils sont doublés.

$\mathbf{Q}$ : Ensuite, même si on a l'impression de les avoir doublés, il faut que ce soit de vrais doubles. Or, dans beaucoup de cas, on découvre au moment où il y a un incident, que les deux appareils étaient alimentés par la même source, ou que l'accumulation de pression n'avait pas été faite, ou que la pile n'était pas en état de fonctionner, etc. II me semble que, dans les technologies qui ont déjà une longue histoire, on prévoit quelque chose d'entièrement mécanique, complètement à part, quelque chose qui, même si on ne fait rien, donne toujours la valeur juste. Et je pense que plus la technologie est ancienne, plus elle a intégré ce genre d'appareil.

Mais, quand je vous écoute, en tout cas, vu de l'extérieur, on a l'impression que ce genre de philosophie n'existe pas pour les réacteurs nucléaires. Moi, je pense qu'il serait normal qu'il y ait un truc indestructible quoi qu'il arrive, un truc mécanique qui donnerait sans état d'âme une idée du niveau d'eau, de la pression et peut-être de la température, il me semble qu'elle serait utile, un truc qui donnerait forcément la valeur juste sans qu'on ait à faire quoi que ce soit, un truc absolument fiable qu'on pourrait placer quelque part à l'extérieur de la cuve ou qui dépasserait de la cuve. Mais ça n'existe pas?

$\mathbf{R}$ : En ce qui concerne la dissociation, pour les sources d'alimentation et tout ça, les parties communes ont été éliminées et c'est dissocié. Mais, comme vous le dites, il n'y a pas, in fine, d'instrument de structure extrêmement simple, quelque chose qui marcherait toujours. Ce genre de philosophie n'existe pas dans la conception. Bien sûr, ça nous amène vers des discussions de conception, en plus de conception originale, qui ne sont pas vraiment de mon domaine. Mais, à y penser maintenant, on se rend compte qu'au moment de la conception, ce genre d'évènement n'a pas été pris en compte. Et, comme vous le dites, aujourd'hui, naturellement, je regrette qu'il n'y ait pas eu ce genre d'instrument.

$\mathbf{Q}$ : Oui, j'en suis intimement convaincu. Merci pour cette digression.

Tout à l'heure vous nous avez dit que vous n'aviez pas du tout envisagé l'explosion d'hydrogène. De notre point de vue de non-spécialiste, il ne semble pas logique de penser, 
alors qu'il y a fuite d'éléments radioactifs, qu'il n'y a pas fuite d'hydrogène. De plus, il nous semble normal de penser qu'à un moment où la température est tellement élevée que le cœur est endommagé, de l'hydrogène soit produit. Malgré ça, le professeur Madarame ${ }^{102} \mathrm{a}$ déclaré à la télévision qu'il avait été totalement surpris par l'explosion d'hydrogène. C'est pas un peu bizarre, tout ça?

$\mathbf{R}$ : J'avoue que nous sommes bourrés d'a priori, mais ce qu'on craignait le plus, à ce moment-là, c'était une explosion de l'enceinte de confinement. Vu aujourd'hui, c'est totalement idiot. Alors que de l'hydrogène et de l'oxygène en quantité suffisante pour provoquer une explosion étaient produits, on n'avait pas imaginé un seul instant qu'ils pouvaient déborder de l'enceinte et aller s'accumuler dans le bâtiment. En plus, habituellement, si le SGTS (Standby Gas Treatment System) ${ }^{103}$ avait été opérationnel, une ventilation de secours aurait poussé tout ça dehors. Paradoxalement, bien que le SGTS était mort, on s'était fait quelque part l'illusion que la ventilation fonctionnait. De plus, on avait l'impression que, puisque des éléments radioactifs avaient été rejetés, l'hydrogène aussi était rejeté. Le dessus du bâtiment réacteur est couvert et des panneaux de ventilation sont disposés sur le côté. Nous n'avions même pas imaginé que ces panneaux étaient fermés et que de l'hydrogène et de l'oxygène s'étaient accumulés. Nous, nous étions focalisés sur l'enceinte de confinement. Pour nous, il fallait à tout prix sauvegarder cette enceinte. Je pense que c'est une des grandes leçons de cet accident, nous tous, spécialistes du nucléaire, y compris le professeur Madarame, nous avons été prisonniers de nos a priori ${ }^{104}$. Et c'est vrai au niveau international. Les autres pays n'ont pas pensé non plus qu'il y aurait une explosion là.

$\mathbf{Q}:$ Vous avez l'impression qu'il y a un gros trou.

$\mathbf{R}$ : Oui, tout à fait. Ça n'était absolument pas prévu dans le scénario des accidents. Et ça, c'est vrai pour tous les pays, y compris les États-Unis. Les Américains l'ont reconnu eux-mêmes. Alors, comme vous dites, c'était l'angle mort des spécialistes du nucléaire, un énorme angle mort. En tant que directeur d'une centrale, je ne peux absolument pas me justifier.

$\mathbf{Q}$ : Moi, je me demande si ce n'est pas dû au fait que la technologie (nucléaire) est encore jeune. Mais, je pense qu'il faut prendre le contre-pied et se dire que maintenant qu'on a payé un lourd tribut, il faut apprendre à penser autrement qu'on ne l'a fait normalement jusqu'ici, apprendre à imaginer ce genre de choses. Sinon, ce serait du gâchis.

$\mathbf{R}$ : Je pense qu'au fond, le plus important est de savoir comment on sera capable de refléter tous ces enseignements dans la conception à venir. Je ne sais pas si notre pays sera capable de continuer dans le nucléaire, mais s'il veut continuer, il faudra en tenir compte ${ }^{105}$.

$\mathbf{Q}$ : Je suis d'accord avec vous.

Vous donnez donc l'ordre de préparer l'éventage. À la suite de ça, les agents vont s'y mettre concrètement. Et durant ce processus, vous allez recevoir des informations vous prévenant «on ne peut pas faire ceci, on ne peut pas faire cela». Et, petit à petit, vous vous rendez compte que cette manœuvre va s'avérer extrêmement compliquée.

R : Oui.

$\mathbf{Q}$ : Et pendant tout ce temps-là, n'êtes-vous pas reliés au siège par téléconférence? Le siège n'a-t-il pas la même compréhension des faits que vous?

$\mathbf{R}$ : Bien sûr, nous sommes en téléconférence et les rapports sont faits au fur et à mesure. On leur a dit qu'on ne pouvait pas faire fonctionner les vannes MO faute de courant, ensuite qu'on ne savait pas s'il y avait encore suffisamment de pression dans 
les accumulateurs pour pouvoir ouvrir, ou qu'étant allé sur le terrain, on avait constaté que la radioactivité était élevée ${ }^{106}$, etc. Tout ça avait été rapporté.

Seulement, au siège, ils pensent qu'au final les gars sur le terrain iront ouvrir la vanne à la main. On revient à l'histoire de tout à l'heure. Même moi, qui suis tout près du terrain, je ne me suis pas déplacé auprès du chef de quart pour aller voir par moimême, puisque je ne pouvais pas quitter mon poste. Moi-même, je n'ai pas une vraie compréhension de la situation, ça n'est que de l'ouï-dire. J'ai très bien entendu ce qu'il en est des appareils de la salle de commande. Je suis quand même plus près du terrain que le siège. Mais, quelque part, je pense encore qu'ils arriveront à se débrouiller. Je suis pourtant à quelque $100 \mathrm{~m}$ du terrain, mais je ne vois pas le terrain, je ne regarde pas vers eux. Je pense qu'ils vont y arriver d'une manière ou d'une autre. Alors le siège qui est encore beaucoup plus loin... Ils vont croire qu'on peut encore faire d'autres choses et ils vont nous relancer sans arrêt. Alors qu'en fait on ne peut rien faire dans la réalité. C'était une période comme ça, où il y avait tout le temps un fossé.

Q: Là, nous sommes sur la chronologie du réacteur 1 . Mais à la page 7 , il y a une chronologie qui montre à la fois le réacteur 2 et le réacteur 1 . Et on voit que vers 1 h30, vous avez reçu l'accord du Premier ministre ${ }^{107}$, du ministre de l'Économie et de l'Industrie ${ }^{108}$ et de la NISA' 109 pour l'exécution de l'opération d'éventage. Cela signifie-t-il que vous pensiez à ce moment-là à l'exécution de l'éventage pour le réacteur 1 et le réacteur 2 ?

$\mathbf{R}$ : Concernant le réacteur 2, comme je vous l'ai dit tout à l'heure, on ne pouvait pas voir le niveau d'eau et même pour le RCIC... Pour le réacteur 1 aussi, comme on l'a vu tout à l'heure, on ne savait pas si l'IC était en fonctionnement, et comme les choses étaient très incertaines, on commençait à penser qu'il était probable qu'il ne fonctionne pas. Mais pour le réacteur 2, on avait perdu les informations sur l'état de marche du RCIC encore avant. On ne savait plus si le RCIC fonctionnait ou pas. En plus, on ne savait pas quel était le niveau d'eau. En fait, sur le réacteur 2, on n'a finalement pas observé de hausse de la radioactivité. Mais on ne pouvait pas exclure la possibilité que le niveau d'eau ait baissé. Dans ces circonstances, on pouvait penser que, tout comme le réacteur 1, le 2 pourrait avoir besoin d'une opération d'éventage. C'est pourquoi j'avais donné l'ordre de les envisager tous les deux.

Q: À la page 19 de la chronologie, concernant le réacteur 2, on voit que, quoiqu'on ne sache toujours pas l'état du RCIC, on a constaté à $22 \mathrm{~h} 00$ un TAF $+3400 \mathrm{~mm}$. Jusque-là, j'imagine qu'étant donné que vous ne connaissiez ni le niveau d'eau du réacteur, ni l'état du RCIC, vous agissiez en considérant le réacteur 2 comme plus préoccupant que le 1. Mais là, avec ce TAF +3 400 à $22 \mathrm{~h} 00$, vous avez dû vous dire que, contre toute attente, l'eau s'était maintenue à un certain niveau. Malgré ça, bien entendu le 11 mars vers 22 h00 vous ne savez toujours pas dans quel état est le RCIC, vous envisagez le 12 vers 1 h30 l'éventualité d'un éventage à la fois sur le réacteur 1 et le réacteur 2. Pourquoi ?

$\mathbf{R}$ : Là, c'est parce que la méfiance s'est installée. L'indicateur de niveau d'eau n'est pas fiable sur le réacteur 1. Celui du réacteur 2 nous dit +3400 . Mais qu'en sait-on? Dans ces conditions, on se dit que, même si le réacteur 2 n'est peut-être pas encore dans le même état que le 1, tôt ou tard il risque d'y arriver et que, face à cette éventualité, il faut être prêt pour l'éventage aussi bien pour le 1 que pour le 2. Voilà ce que j'ai pensé.

Par la suite, on a su que le RCIC marchait pour le réacteur 2 et j'ai été un peu rassuré. Du coup, je me suis dit qu'on avait encore le temps pour d'éventuels éventages ou d'autres manœuvres et je me suis recentré sur le réacteur 1. 
$\mathbf{Q}$ : Juste pour vérification. Au moment dont nous parlons, vous savez que le RCIC est en fonctionnement pour le réacteur 3. Et donc vous êtes centré sur les réacteurs 1 et 2 . C'est bien ça?

Je voudrais savoir comment se passait votre collaboration avec le siège. Dans cette chronologie, il y a à 3h06, tenue d'une conférence de presse concernant l'exécution d'un éventage. II me semble qu'il y a eu une conférence de presse avec le directeur exécutif Komori ${ }^{110}$ et le ministre de l'économie et de l'industrie. On retrouve tous les échanges qu'il y a eu à cette occasion sur le site internet. Le directeur Komori a déclaré qu'il avait donné des directives au terrain pour qu'il se tienne prêt à exécuter un éventage vers les 3h00.

Il a ajouté qu'il suffisait de donner le signal d'exécution pour que ce soit fait, que l'exécution aurait lieu dès que les habitants seraient mis au courant, y compris par l'intermédiaire de cette conférence de presse. II parle comme si l'éventage était imminent. De plus, il dit vouloir commencer par le réacteur 2 pour faire baisser la pression. Quand les journalistes lui demandent si ce n'est pas le réacteur 1, il répond qu'on lui a dit qu'on commençait par le 2. On dirait que les informations ne passent pas bien jusqu'à la personne qui fait la conférence de presse. Par exemple le fait que vous, sur le terrain, pensiez agir sur les deux réacteurs 1 et 2 , se retrouve déformé et ne concerne plus que le 2 . Comment expliquezvous ces décalages?

$\mathbf{R}:$ Je ne sais pas. Le siège a peut-être une explication. Mais il y a déjà une chose. Cela dépend du moment où Komori a quitté la cellule de crise pour aller voir la presse. Tout dépend de l'heure. Parce que la situation changeait de minute en minute. À un moment, on voyait le niveau d'eau, puis à un autre on ne le voyait pas, par exemple. Alors il faut bien réfléchir à quel moment il se prépare à cette conférence et à quoi il fait allusion. En tout cas, ce qui est sûr c'est qu'il n'est pas en train de parler de la situation réelle à 3 h06 précises. Alors, savoir quelle était la situation dont il voulait parler, savoir pourquoi l'État a voulu communiquer à ce moment-là, nous, sur le terrain, nous ne savons rien des relations avec la presse.

$\mathbf{Q}$ : Je voudrais juste vérifier une chose. Bien sûr, M. Komori ne pouvait pas suivre la situation en temps réel. Mais y avait-il eu des discussions à un moment ou à un autre pour dire que vous alliez d'abord agir sur le réacteur 2, pour qu'il en parle comme ça devant la presse?

$\mathbf{R}$ : Il est sûr qu'au siège ils avaient une image plus alarmante du réacteur 2 . Vous vous demandez pourquoi ? C'est parce qu'à un moment donné on a vu le niveau d'eau du réacteur 1 , même s'il était faux. Alors que pour le réacteur 2 , jusque vers $22 \mathrm{~h} 00$, on ne voyait pas le niveau d'eau. Pendant tout ce temps, on ne savait pas si le RCIC était en fonctionnement ou pas. Pas de niveau d'eau, aucune information sur la situation $\mathrm{du}$ RCIC, pour le siège, le réacteur 2 était plus préoccupant. Pour nous, c'est le moment où nous commençons à nous dire qu'au vu des données, la situation du réacteur 1 est plus critique. Et nous communiquons là-dessus. Mais il y avait un décalage. Alors je ne sais pas à quel moment et avec quelle connaissance de la situation Komori est parti à la conférence de presse, mais visiblement il est parti sans avoir intégré notre inquiétude.

Q: Est-ce qu'à un moment donné, vous-même, quelqu'un de la production ou un responsable quelconque, n'aurait-il pas communiqué au siège l'intention de commencer par le réacteur 2 ?

$\mathbf{R}:$ Non.

$\mathbf{Q}$ : Et, dans la nuit du 11 mars, le siège est persuadé que la situation du réacteur 2 est plus préoccupante?

$\mathbf{R}$ : Du point de vue de la surveillance. 
Q : On ne sait pas ce qui s'est vraiment passé, mais le siège, ayant cela en tête, au moment où vous demandez à agir sur les réacteurs 1 et 2 , interprète " on agit d'abord sur le 2 ".

$\mathbf{R}$ : Et là, bien que ce ne soit écrit nulle part, d'après mes souvenirs, il me semble que le siège m'a informé que le professeur Madarame trouvait la situation du réacteur 2 plus inquiétante. Parce qu'on ne pouvait pas le surveiller et qu'on ne savait pas si le RCIC fonctionnait ou pas, il pensait qu'il fallait donner la priorité au réacteur 2. Ça, je l'ai bien entendu, bien que ce ne soit pas noté ici.

Alors, c'est peut-être ça qui a été le déclencheur. Seulement, en ce qui nous concerne, comme je vous l'ai dit tout à l'heure, compte tenu de la hausse de la radioactivité et tout ça, nous étions plus préoccupés par le réacteur 1 . Et ça, c'est vrai que c'est un état d'esprit que nous n'avons pas eu en commun avec le siège. De plus, je ne sais absolument pas dans quel but le siège a voulu communiquer avec la presse à ce moment-là. Ça, il faudra vous renseigner auprès du siège.

$\mathbf{Q}$ : Ensuite, concernant le réacteur 2 . Si on regarde dans la chronologie à la page 19, juste une dizaine de minutes avant la conférence de presse, à $2 h 55$, on voit qu'on a constaté que le RCIC était en fonctionnement. Il y a plus de détails un peu avant, à la page 5. À l'endroit où on parle de l'opération de vérification de la situation du RCIC du réacteur 2 , il y a beaucoup de détails. En fait, quelqu'un était effectivement allé sur place à la salle du RCIC. Il y avait trouvé un peu d'eau et il avait entendu un bruit métallique discret, mais n'avait pas réussi à vérifier la partie rotative. II n'avait pas tout à fait réussi à vérifier le fonctionnement du RCIC et comme il n'arrivait pas à communiquer avec la salle de commande, il était retourné sur ses pas. Mais vers $2 \mathrm{hO0}$, il était encore retourné dans la salle du RCIC où le niveau d'eau avait augmenté. Une fois de plus, il n'avait pas réussi à constater le fonctionnement du système à partir de la salle. Mais il a pu vérifier la pression de la cuve et la pression de sortie de la pompe du RCIC à l'aide d'une batterie d'indicateurs qui se trouvait derrière la salle du $\mathrm{RCIC}$, apparemment à un autre étage. Comme la pression de sortie était plus élevée, il en a conclu que le RCIC fonctionnait. Cette information a été communiquée à la cellule de crise par la salle de commande à 2h55, lit-on. Vous n'étiez peut-être pas au courant de tous ces détails, mais à 2 h55, vous, à la cellule de crise, aviez bien été informé que le RCIC fonctionnait.

Étiez-vous aussi au courant de la manière dont on avait vérifié son fonctionnement, le fait d'avoir observé et comparé la pression de la cuve et la pression de sortie de la pompe du $\mathrm{RCIC}$ ?

$\mathbf{R}$ : Je ne sais pas s'ils m'avaient détaillé la méthode, mais j'ai reçu un rapport du chef du groupe « production » qui disait qu'ils avaient constaté le fonctionnement du RCIC grâce à la pression de sortie de la pompe. Oui, ils ont transmis ça, comme ça.

$\mathbf{Q}:$ : Et, en apprenant que le RCIC fonctionnait...

$\mathbf{R}:$ J'ai été soulagé.

$\mathbf{Q}$ : Vous avez été soulagé, tout au moins concernant le réacteur 2 . Finalement, comme on l'a évoqué brièvement tout à l'heure, alors qu'il n'était censé fonctionner que durant 8h00, à cause de la batterie, vous avez réussi à le faire tenir en faisant une sélection entre ce qui était nécessaire à ce moment-là et ce qui ne l'était pas et...

$\mathbf{R}$ : Oui, on avait coupé tout ce qui n'était pas nécessaire.

$\mathbf{Q}$ : Et c'était le personnel sur le terrain qui avait fait ce tri.

$\mathbf{R}$ : Oui. Ce sont ceux qui étaient sur le terrain qui ont pris ces décisions. Ce n'est pas moi qui ai donné des ordres dans les détails. Je leur ai dit d'alléger les charges, mais pour être franc, je n'avais pas idée de ce qui pouvait être coupé. En fait, ils ont extrêmement bien réagi et je leur en suis reconnaissant. 
$\mathbf{Q}$ : Excusez-moi de sauter d'un sujet à l'autre, mais vers $2 \mathrm{~h} 55$, vous êtes provisoirement un peu rassuré du côté du réacteur 2. Mais, d'un autre côté, pour le réacteur 1, vous êtes dans une situation difficile. Si on regarde les paramètres, à ce même moment, à $2 \mathrm{~h} 30$ ou à 2h45, la pression de la chambre sèche atteint $840 \mathrm{kPa}$ abs.

Si on regarde les chiffres de $2 \mathrm{~h} 45$, la pression de la cuve est à 0,8 MPa, ce qui signifie que la pression de la cuve et celle de l'enceinte de confinement sont quasi identiques. Avez-vous des commentaires sur ce fait?

$\mathbf{R}$ : Sur ce sujet, on n'avait pas arrêté d'en discuter dans la cellule de crise avec le responsable $\mathrm{du}$ réacteur, qui est le monsieur sûreté, un membre du groupe « technique ». On n'arrivait pas à savoir précisément pourquoi la pression de la cuve était descendue et pourquoi la pression de la chambre sèche était montée jusqu'à 0,8 . En théorie, on comprend qu'à l'intérieur de la cuve le combustible est endommagé, que de la pression s'en échappe. Mais on ne comprend pas le lien entre le fait que la pression ait si brutalement baissé dans la cuve et le comportement de la pression de la chambre sèche, qui a quasiment doublé et qui frôle la limite de l'explosion. Mais il ne servait à rien de discuter. On s'est dit que si la pression était à 0,8 , il n'y avait que l'éventage. Et, du coup, on a essayé d'accélérer les choses de ce côté.

$\mathbf{Q}$ : Donc, déjà à ce moment-là, vous êtes sur l'idée de l'éventage.

$\mathbf{R}:$ Oui, tout le temps.

$\mathbf{Q}$ : D'un autre côté, vous êtes aussi occupé pour réaliser l'injection d'eau. À ce moment-là, il me semble que vous étiez occupé à étudier l'injection en utilisant un camion de pompiers et le réseau de lutte contre le feu. Concernant cette opération, je suis désolé de vous balader d'une page à l'autre, mais à la page 7, on lit «début de l'étude sur la possibilité de connecter le camion de pompiers à la prise d'eau du réseau incendie à 7 h48 ». Vous avez effectivement utilisé des camions de pompiers pour injecter l'eau par ce réseau?

$\mathbf{R}$ : Oui, les pompiers.

$\mathbf{Q}$ : Ce sont les pompiers qui ont agi. Pour l'éventage et tout ça, c'est le groupe « réhabilitation » ou le groupe « production»?

$\mathbf{R}$ : C'est le groupe « production » qui opère avec l'aide du groupe « réhabilitation ».

$\mathbf{Q}$ : Et l'aide qu'ils peuvent apporter dans ces opérations, c'est pour compenser ce qui ne peut pas être fait avec ce qui est déjà là ? Par exemple, s'il n'y a pas assez d'électricité, les gens du groupe «réhabilitation » se débrouillent pour compenser ce qui manque, c'est ça ? Et puis, ceux qui s'occupent d'injecter l'eau et ceux qui s'occupent de la préparation de l'éventage, ce sont des gens qui ont des fonctions totalement différentes, c'est bien ça?

R : Oui.

$\mathbf{Q}$ : Si on continue à lire, à la page 8 , on voit « début de l'injection d'eau douce par le camion de pompiers par le réseau incendie à 5 h46 ». C'est à ce moment-là que vous avez commencé à injecter de l'eau douce.

R : Oui.

$\mathbf{Q}$ : Là, concrètement, vous aviez eu des renseignements sur la provenance de l'eau qu'on injectait?

$\mathbf{R}$ : Au début, on a commencé par mettre l'eau qui se trouvait dans la citerne du camion et, une fois la citerne vide, il fallait la remplir de nouveau. Au début, on ne pouvait que faire des injections discontinues. Ça, j’en avais été informé. Puis, je ne me souviens plus de la chronologie exacte mais, on a pensé aux $40 \mathrm{t}$ de la réserve d'eau contre l'incendie, celle qu'on avait construite après l'accident de Kashiwazaki. On s'est dit qu'on pouvait aussi l'utiliser. Au fur et à mesure, on a changé plusieurs fois de méthode et je ne me souviens pas très bien jusqu'à quel point j'ai échangé sur ces 
points avec les hommes qui étaient sur le terrain. Je n'ai pas encore réussi à remettre les choses dans l'ordre, mais dans le principe, on commençait par injecter l'eau dont on disposait, puis on se réapprovisionnait. Et dans le temps où on faisait ça, on a décidé d'utiliser l'eau de la cuve incendie, mais on a eu des problèmes de pression, puis, par hasard, d'autres camions sont arrivés de l'extérieur et, en passant d'un camion à trois, on a réussi à augmenter la pression de sortie. Et tout ça s'est fait sur le terrain en improvisant. Je ne me souviens pas à quel moment telle chose précise s'est déroulée. Il faudrait demander à ceux qui étaient sur le terrain. Pour ma part, j'avais demandé qu'on injecte l'eau de la manière la plus efficace et la plus continue.

$\mathbf{Q}$ : Je suppose que, dans l'absolu, à un moment ou à un autre, de toute façon, l'injection d'eau serait devenue indispensable aussi bien pour le réacteur 1 que le 2 ou le 3. Mais vous avez commencé par le réacteur 1.

R : Oui, le 1 .

Q : Qui décidait de savoir dans quel réacteur injecter l'eau? Le groupe "production», le groupe «réhabilitation», les pompiers ? Ou bien, est-ce que c'était la cellule de crise qui disait: " commencez par le réacteur 7 »?

$\mathbf{R}$ : Concernant les réacteurs, tout d'abord le réacteur 1. C'est moi qui l'ai décidé. Le réacteur 2 , à ce moment-là, était encore stable. Alors que les choses avaient atteint un stade irrécupérable pour le réacteur 1. Donc, la consigne était d'injecter autant d'eau qu'on pouvait dans le réacteur 1.

Ensuite, dans l'ordre, c'était le réacteur 3, puis le 2. Concernant le réacteur 3, j'avais donné des directives pour qu'on soit prêt à injecter l'eau dès qu'on aurait dépressurisé en ouvrant la soupape. J'avais précisé qu'il fallait que les préparatifs soient terminés avant que le RCIC ou le HPCI deviennent inopérants.

Pour résumer, c'est moi qui décidais sur quel réacteur il fallait travailler, mais savoir concrètement comment injecter cette eau pouvait présenter différents problèmes qui devaient être résolus sur place et, donc, les équipes étaient chargées d'analyser la situation, de trouver une solution et de m'en faire part. Voilà comment se passait l'injection d'eau.

$\mathbf{Q}$ : À propos de cette opération, vous vous êtes fait aider par des entreprises comme le Japan Nuclear Security Service System (JNSS) ${ }^{111}$, qui était en charge de la conduite des camions de pompiers, ou de Nanmei Kôsan'112?

$\mathbf{R}$ : Oui, au début, ils ont collaboré.

$\mathbf{Q}$ : Et ensuite?

R : À partir du moment où la radioactivité est montée...

$\mathbf{Q}$ : Vous voulez dire que la radioactivité était devenue trop forte pour que ces personnes interviennent ? Donc, au-delà, vous vous êtes débrouillé avec vos propres troupes?

R : Oui.

$\mathbf{Q}$ : Vous injectiez l'eau avec les seules personnes du groupe « incendie » de TEPCO ?

$\mathbf{R}$ : Oui. Mais j'ai aussi entendu dire que quelques personnes courageuses nous avaient aidés.

$\mathbf{Q}:$ C'est-à-dire?

R : Par exemple, en tant qu'entreprise, Nanmei Kôsan s'était retirée. Mais, à titre privé, si on peut dire, certaines personnes ont continué à nous aider. Oui. 
$\mathbf{Q}:$ Et, à $5 \mathrm{~h} 46$, on ne trouve pas la pression dans les paramètres, mais il est noté « début de l'injection d'eau douce ». Et donc, l'eau est entrée?

$\mathbf{R}$ : C'est un point qui était très difficile à juger pour nous aussi. Est-ce que l'eau entrait bien à l'intérieur ou pas? Tout ce qu'on pouvait voir, c'était si l'eau s'écoulait ou pas. On n'avait pas de débitmètre ni rien. Alors, on tenait le bout du tuyau et on regardait si l'eau coulait. Si elle coulait, on se disait que ça devait entrer. On n'avait pas mieux pour en juger. Comme je vous l'ai dit tout à l'heure, c'est une question d'équilibre de la pression. C'est juste une question de différence entre les pressions qui s'exercent de part et d'autre du clapet anti-retour, la pression de ce qu'on veut faire entrer et la pression de l'autre côté. Sans débitmètre, on regarde, on voit que ça coule et on se dit que ça doit être bon.

$\mathbf{Q}$ : Vous sentez les pulsations et vous avez l'impression que ça coule, c'est ça?

$\mathbf{R}$ : Oui, ce genre de sensation. On n'avait aucun instrument à disposition, alors on y allait à tâtons. Tout ce qu'on pouvait vérifier, c'était que l'eau diminuait dans le réservoir.

Q: Si on continue à suivre cette chronologie, on voit toutes les histoires autour de l'éventage et, ici, à 6h33, on lit, concernant l'évacuation des habitants : «étude en cours pour l'évacuation des habitants d'Ôkumamachil13 vers Miyakoji'14 ». Vous, est-ce que vous receviez ces informations à propos des habitants de la zone, à l'intérieur de la centrale, à la cellule de crise?

R : Oui, par le siège.

$\mathbf{Q}$ : Vous voulez dire que ces informations étaient relayées par le siège?

R : Oui.

$\mathbf{Q}$ : Je suppose qu'il fallait se coordonner de part et d'autre, qu'il fallait savoir ce qu'il en était des autorités locales, etc. Ce genre d'échanges était pris en charge par le centre hors site ou pas?

$\mathbf{R}$ : En principe, c'est le centre hors site. Mais je ne sais pas s'ils avaient échangé ou pas. De toute façon, d'après la loi sur les catastrophes nucléaires, c'est à eux de s'en occuper.

$\mathbf{Q}$ : D'accord, donc vous n'êtes pas trop au courant.

$\mathbf{R}$ : Non. Je n'étais absolument pas au courant de ce qui se passait à l'extérieur. J'avais juste les informations par le siège, une fois qu'il avait été décidé quelque chose. Je n'avais aucune information en direct, ça passait toujours par le siège.

Q : Pour continuer avec les informations venant de l'extérieur, à 6h50, le ministre de l'Économie et de l'Industrie donne l'ordre d'exécution de l'éventage, suivant le cadre légal 115. Je suppose que vous lui aviez fait des rapports exposant votre intention de procéder à un éventage, que vous en aviez obtenu l'autorisation et que, sur le terrain, vous faisiez tout votre possible en vue de cette éventualité.

Ce que je voudrais savoir, c'est pourquoi le ministre donne l'ordre à ce moment précis d'exécuter l'éventage, éventage qui est problématique et qui devra sans doute se faire manuellement ? Savez-vous dans quelles circonstances cet ordre a été émis ?

$\mathbf{R}$ : Je ne sais pas. Mais, nous, nous étions dans une colère noire. On n'avait pas arrêté de communiquer pour dire que ce n'était pas possible et il nous disait de le faire. Comme s'il suffisait de l'ordonner pour que ce soit fait. On avait envie de lui dire : «si c'est si simple, fais-le toi-même». C'était notre état d'esprit. Surtout que c'était à un moment où rien n'allait sur le terrain. 
$\mathbf{Q}$ : Et on revient à l'histoire de tout à l'heure.

$\mathbf{R}$ : Là, je ne sais pas exactement, mais, probablement, comme l'histoire de Komori tout à l'heure, ils étaient terriblement focalisés sur l'éventage. Nous aussi, bien sûr. Mais on avait beau leur expliquer qu'on n'y arrivait pas, ils ne l'intégraient pas ${ }^{116}$. Et je me demande jusqu'à quel point ils ne pensaient pas que le terrain traînait des pieds, que nous faisions volontairement traîner les choses. Alors que nous, sur le terrain, nous faisions tout pour que ça marche, mais que tout échouait, ils trouvaient qu'on y mettait de la mauvaise volonté. C'est peut-être cette colère contre TEPCO qui s'est traduite dans cet ordre d'exécution. Mais, fondamentalement, c'est une histoire entre le siège et le Gouvernement, alors je ne peux que vous répondre que je ne sais pas. Mais, croyez-moi, nous faisions tout ce qui était en notre pouvoir.

$\mathbf{Q}$ : Ensuite, à 7 h11, on lit «arrivée du Premier ministre ». Depuis quand était-il question d'un déplacement du Premier ministre?

$\mathbf{R}$ : Je n'ai pratiquement aucun souvenir du moment où on a commencé à évoquer la venue du Premier ministre ${ }^{117}$. Je pense que c'était environ une heure avant son arrivée. Parce qu'on a su que son hélicoptère était parti ${ }^{118}$. Il me semble qu'on a su qu'il allait venir un peu plus d'une heure avant son arrivée. J'en déduis qu'on a dû avoir l'information vers les 6h00. C'est ce que j'en déduis aujourd'hui. Et je pense que c'était ça.

$\mathbf{Q}$ : Et vous l'avez su par le siège, par téléconférence?

R : Oui.

$\mathbf{Q}$ : Et saviez-vous dans quel but il venait?

R : Non.

$\mathbf{Q}:$ On ne vous a rien dit de son but et on vous a annoncé brusquement qu'il venait?

$\mathbf{R}:$ On nous a juste dit qu'il venait.

$\mathbf{Q}$ : Et, concrètement, lors de cette visite, qui y avait-il en dehors du Premier ministre?

$\mathbf{R}$ : Je me souviens du Premier ministre, du directeur Madarame de la NISA ${ }^{119}$ et, comme représentant du département, j'ai reconnu le vice-gouverneur Uchibori ${ }^{120}$. Je me souviens de ces trois personnes. Il y avait d'autres personnes, mais elles étaient en combinaison et je ne les ai pas reconnues. Je pense qu'ils étaient une dizaine.

$\mathbf{Q}$ : Et, au moment de la visite, qui les a accueillis du côté de la centrale? Ils arrivent, je suppose que vous les saluez en premier et qui d'autre?

R : Moi seul.

$\mathbf{Q}:$ Vous seul?

R : Oui, moi seul. Nous étions en pleine opération. Alors j'ai donné l'ordre de continuer pour l'éventage et tout ça, j'ai dit à mes troupes de poursuivre et, si jamais il y avait du neuf, comme ni les portables, ni le PHS ne passaient, j'ai dit qu'on m'envoie carrément quelqu'un pour me prévenir. Et, c'est comme ça que je suis parti les accueillir.

$\mathbf{Q}:$ Et les visiteurs sont venus dans le bâtiment antisismique?

R : Oui.

$\mathbf{Q}$ : La cellule de crise se trouve au premier étage du bâtiment antisismique. C'est là qu'ils sont venus?

$\mathbf{R}$ : Non. Dans le bâtiment antisismique, il y a ici la grande salle avec la table ronde. Mais si on prend ce couloir, on a une autre salle où j'ai reçu les visiteurs. Il y avait le Premier ministre ici, XXXXX ici, monsieur Uchibori ici, encore quelques personnes 
que je ne connaissais pas, les gardes du corps, moi et Mutô ${ }^{121}$, nous étions ici. Le directeur d'unité et XXXXX, et puis les chefs de groupes m'avaient remplacé dans la salle de crise et devaient venir me prévenir ici, s'il y avait des choses qu'ils ne pouvaient pas trancher.

$\mathbf{Q}$ : Où était monsieur Mutô?

$\mathbf{R}:$ Il était au centre hors site.

$\mathbf{Q}$ : Alors il était venu exprès en même temps que le Premier ministre?

$\mathbf{R}$ : Oui, il était venu exprès du centre hors site.

$\mathbf{Q}$ : Et donc, le Premier ministre est venu et de quoi avez-vous parlé ?

$\mathbf{R}$ : Il était déjà installé dans la salle quand je suis arrivé par là. Tout de suite, il m'a demandé d'un ton assez sévère ce qu'il en était. Alors je lui ai expliqué que nous n'avions presque pas de source d'électricité, que nous ne pouvions rien contrôler. Il m'a demandé comment on en était arrivé là. Je ne connaissais pas la hauteur exacte du tsunami à ce moment-là, mais quand je lui ai dit que le tsunami avait submergé toutes les sources d'alimentation et qu'elles ne fonctionnaient plus, il a pris à partie le professeur Madarame en lui demandant comment un simple tsunami pouvait paralyser une centrale nucléaire. Bref, j'ai expliqué la situation où nous nous trouvions, et puis à propos de l'éventage, puisqu'il m'a demandé où on en était. Comme l'ordre du ministre de l'Économie et de l'Industrie venait d'être donné, je lui ai dit que l'ordre en avait été donné, que nous faisions notre possible mais que les choses étaient difficiles sur le terrain. D'après mes souvenirs, c'est à peu près tout. Je ne pense pas que nous ayons parlé très longtemps ${ }^{122}$.

Q: Par exemple, s'agissant de l'éventage, on a dit tout à l'heure que plus on s'éloignait du terrain, et Dieu sait si l'État en est éloigné, plus il devenait ardu de reconnaître les difficultés qu'on y rencontrait. Avez-vous profité de cette occasion pour expliquer combien la situation était difficile sur le terrain?

$\mathbf{R}$ : Nous étions dans une ambiance telle qu'il était difficile de parler. J'ai bien dit que la situation était difficile sur le terrain, mais j'ai conscience que je n'ai pas suffisamment expliqué en quoi la situation était si difficile. Mais c'est ce que je pense aujourd'hui. En fait, nous n'étions pas dans une ambiance où on pouvait parler librement. Le Premier ministre posait des questions surprenantes auxquelles on essayait simplement de répondre. Cela se résumait à ça.

$\mathbf{Q}$ : Et, pendant ce temps, dans la salle d'à côté, juste derrière ce mur, il y avait la cellule de crise où tout le monde s'affairait bruyamment, tout près, et le Premier ministre est allé les encourager?

$\mathbf{R}:$ Il est venu comme ça et il est reparti comme ça, alors...

$\mathbf{Q}$ : Vous voulez dire qu'il n'y est pas allé?

R: Non.

Q : Il n'a même pas jeté un coup d'œil ?

$\mathbf{R}$ : Pas du tout. Il est venu comme ça, il s'est assis et il est reparti.

$\mathbf{Q}:$ Et c'est vers 8 h00 qu'il est reparti'23 ?

R : Oui.

Q: Si on regarde la chronologie, il y a à 8h03: "ordre du directeur de la centrale d'opérer l'éventage avec pour objectif les 9 h00 », ensuite à 8h04: «départ du Premier ministre ». On a 
l'impression que vous avez donné l'ordre avant qu'il ne parte. Aviez-vous raccompagné le Premier ministre jusqu'au bout, jusqu'à son départ?

R : Non. Il y a un terrain de sport ici et l'hélicoptère atterrit là. De là, on passe par ici et on entre par là. Non, nous ne l'avons raccompagné que jusqu'ici. Je pense que Mutô est allé l'accueillir jusque-là et qu'il l'y a raccompagné aussi. Non, moi, je l'ai quitté ici $^{124}$.

Q: Je vois donc que vous aviez donné comme objectif les 9h00, pour l'opération d'éventage. Pourquoi?

$\mathbf{R}$ : Ce n'est pas moi qui ai commencé à parler de 9h00. Il y avait eu l'ordre du ministre de l'Économie et de l'Industrie et j'avais dit au siège que c'était très difficile. Mais on s'est quand même donné une heure, à peu près 9h00, comme limite. C'est une décision qui n'avait pas de rapport avec le Premier ministre. C'était au cours d'une discussion avec le siège qu'on avait parlé de 9h00. Je ne sais plus si c'était le Gouvernement ou le siège, mes souvenirs ne sont pas précis, mais il avait été question de décider d'un moment butoir, parce que sans ça on n'y arriverait pas. Mais 9h00, précisément, ce n'était pas une décision délibérée de ma part. Moi, je voyais ce qui se passait sur le terrain. Je pensais, ce n'est pas parce qu'ils nous disent 9h00, qu'on va y arriver. Mais, puisque l'ordre en avait été donné, il fallait bien s'y soumettre. J'ai donc demandé, enfin, ordonné à mes hommes de le faire.

$\mathbf{Q}$ : Vous nous en avez déjà un peu parlé, mais, l'ordre du ministre de l'Industrie date de 6h50. Ensuite, peu après, vingt-et-une minutes plus tard, il me semble, le Premier ministre arrive. Vous êtes dans une situation où l'ordre d'éventage a déjà été donné, le Premier ministre est déjà en route pour la centrale. Certains se demandent s'il n'y a pas eu retard de l'éventage à cause de la visite du Premier ministre, parce qu'il ne fallait pas qu'il y ait des rejets pendant que l'hélico du Premier ministre survolait la centrale. Y aviez-vous pensé ?

$\mathbf{R}$ : Pas du tout.

$\mathbf{Q}:$ Vous ne l'aviez absolument pas en tête?

$\mathbf{R}$ : Non. Ce que nous voulions, nous, c'était faire baisser la pression. Si on pouvait éventer à l'instant, on l'aurait fait, Premier ministre ou pas, qu'il soit irradié ou pas. On en était à ce point-là. Moi aussi, je n'avais qu'une chose en tête, faire baisser la pression de l'enceinte de confinement à tout prix. Et c'est normal, si on pense à la sûreté. Mais on était dans une situation extrême où on ne le pouvait pas. On se disait que, n'importe comment, il fallait trouver une solution, mais on n'y arrivait pas. Alors, vous savez, que le Premier ministre nous survole ou pas, si on pensait à la sécurité du réacteur, nous, sur le terrain, on n'avait qu'une envie, soulager l'enceinte au plus vite ${ }^{125}$.

$\mathbf{Q}$ : Vous dites que vous ne pouviez pas. Vous ne pouviez pas quoi?

$\mathbf{R}$ : C'est comme je l'ai dit tout à l'heure. Il n'y avait pas de source électrique. En plus, comme on n'avait pas d'accumulateur, il fallait bricoler. Pendant ce temps, on essayait d'accumuler la pression ou de restaurer l'électricité. Quoi qu'on fasse rien ne marchait. Il n'y avait que des mauvaises nouvelles ${ }^{126}$. À la toute fin, on finit par se dire qu'il va falloir y aller manuellement. Et on y va. La vanne $\mathrm{MO}^{127}$ qui se trouve du côté de la chambre sèche est assez lourde et pour la manipuler on se fait irradier. Mais on réussit quand même à l'ouvrir. Ensuite, on essaie d'accéder à cette vanne qui se trouve sur le circuit qui sort de la piscine de dépressurisation de la chambre sèche, mais la radioactivité est telle qu'on ne peut pas s'en approcher. Alors, on essaie de nouveau la méthode avec l'accumulateur. Sur ces entrefaites, un camion compresseur 
arrive enfin sur le site et nous rend service. Enfin, nous étions dans une situation où nous tentions des tas de choses, sans même l'outillage qu'il fallait, mais totalement en vain.

Ce que je voudrais souligner, c'est le fait que les gens qui pensaient qu'il suffisait de dire "éventage ", pour que ce soit tout de suite réalisable, n'ont rien compris aux efforts que nous avons fournis. C'est irritant. Dans la réalité, le personnel qui était sur le terrain a encore beaucoup plus souffert que moi, il y a vraiment des gens qui ont reçu là des irradiations ${ }^{128}$ proches de 100.

Q: : Au moment que nous évoquons, vous n'aviez toujours pas l'usage du PHS ?

$\mathbf{R}:$ Non.

Q: : Alors lorsque vous donnez un ordre et qu'on vous répond, comment cela se passait-il ? Pour que ça fasse un aller-retour, combien de temps fallait-il ?

$\mathbf{R}$ : Plus d'une heure.

Q : Pour un aller-retour?

$\mathbf{R}$ : Oui. Pour résumer, entre la salle de commande et la cellule de crise, il y a des moyens de communication. La cellule de crise envoie l'ordre. La salle de commande trouve le personnel pour aller sur le terrain et l'envoie. Mais, une fois qu'il est parti, on ne sait plus rien de ce qui se passe jusqu'à ce qu'il revienne. Qu'ils discutent de ce qu'il convient de faire et de quelle manière, qu'ils y aillent et qu'ils en reviennent, cela prend bien une heure. C'est pour ça que la réponse à ce que j'ai dit n'arrive qu'au bout d'une heure. Je donne les directives et, une heure plus tard, on me dit, on y est allé, mais on n'a pas pu faire la manœuvre parce que la radioactivité était trop forte.

$\mathbf{Q}$ : Dans quelles circonstances avez-vous donné cet ordre d'éventage avec pour objectif les $9 h 00$ ?

$\mathbf{R}:$ Ça ? À la cellule de crise.

$\mathbf{Q}:$ Et le Premier ministre était là aussi.

$\mathbf{R}$ : Non. Le Premier ministre était déjà parti. Oui, 7h11, c'est l'heure où l'hélicoptère a atterri ici, alors l'heure que vous avez là, c'est l'heure où l'hélico a décollé. Pour avoir le temps qu'il a passé dans le bâtiment antisismique, il faudrait enlever dix minutes à chaque bout. Donc, il vaut mieux que vous considériez que son arrivée dans le bâtiment se situe plus tard et son départ plus tôt.

$\mathbf{Q}$ : Ce n'est pas parce que le Premier ministre a su que l'heure d'exécution projetée était 9 h00 qu'il est parti?

R : Non.

Q: À la suite donc de cet ordre, à 9h04, je suppose qu'il va y avoir du mouvement, le personnel de quart qui va commencer à se rendre sur place pour l'opération, etc. Comment avez-vous vécu ce départ ? Comme quelque chose qu'on vous a obligé à faire, étant donné qu'il y avait cet objectif des 9 h00 ? Comme quelque chose qu'on vous a imposé, alors que les instruments, les accumulateurs n'étaient pas suffisants, que la radioactivité était forte et que vous ne saviez pas si vous réussiriez ou pas?

R : Là, à ce stade, nous pensions qu'il fallait y aller manuellement, puisque toutes les tentatives qu'on avait faites pour une manœuvre à distance avaient échoué. Je savais que le personnel allait être exposé à l'irradiation, mais je pensais que c'était la dernière solution. C'est le moment où j'ai donc pris ma décision et où je leur ai demandé d'exécuter l'opération à 9 h00. 
Q : Quand on suit la chronologie, on voit qu'à 8 h37 vous avisez la préfecture de Fukushima que vous commencez à vous préparer pour un éventage à 9h00. On voit qu'il y a coordination pour que l'éventage se déroule après que tous les habitants ont été évacués.

Ensuite, à 9h03, on constate l'évacuation de tous les habitants d'Ôkumamachi et à 9h04, départ du personnel de quart vers le lieu de l'opération. Y a-t-il eu des difficultés au niveau de l'évacuation des habitants qui aurait pris du temps?

$\mathbf{R}$ : Pardon, mais je ne peux pas vous répondre. Je n'avais aucune connaissance de ce qui se passait à l'extérieur, à part les informations qui nous venaient du siège. Effectivement, j'ai su par ce biais que dans certains secteurs, il y avait encore des habitants qui n'étaient pas partis.

$\mathbf{Q}$ : Ce que je voudrais savoir, c'est si, au moment où vous vous apprêtiez à procéder à l'éventage, il y a eu un ordre du siège pour vous dire d'attendre.

$\mathbf{R}$ : Attendre ? On savait qu'il fallait du temps pour cette évacuation et, bien sûr, nous étions d'accord pour dire qu'on ne procéderait à l'éventage qu'une fois tout le monde parti, mais attendre... Vous savez, comme je vous le répète depuis tout à l'heure, nous n'étions pas capables d'exécuter cet éventage. Alors surseoir ou pas, quand on n'est pas capable de réaliser l'opération... Effectivement, c'est à 9 h00 qu'on s'est décidé à faire l'opération à la main, en dernière solution. On s'est décidé parce qu'on pensait qu'on y arriverait s'il suffisait d'accepter d'être irradié. Mais, en fait, on n'a même pas pu approcher, pas une seule fois, de la vanne d'éventage du côté de la piscine de dépressurisation $^{129}$. Et, à la suite de ça, on a même dû renoncer à faire l'opération à la main. Ensuite, plus tard, on va revenir à la charge en apportant un compresseur avec une batterie au niveau de la piscine de dépressurisation pour essayer de faire monter la pression et de rendre l'ouverture à distance possible. Mais, ça, c'est plus tard.

$\mathbf{Q}$ : Jusqu'au départ à 9h04, il y a eu des préparatifs, qui sont décrits un peu plus en détails à la page 15. On note qu'après $2 \mathrm{~h} 24$, si on reste dans une zone à $300 \mathrm{mSv} / \mathrm{h}$, on peut travailler dix-sept minutes dans la limite de la dose de radiation autorisée en cas d'urgence, ce qui signifie que la radioactivité est très forte. Vers $3 \mathrm{~h} 45$ : "ouverture de la double porte pour une mesure de la radioactivité dans le bâtiment réacteur, observation d'un brouillard blanc, fermeture immédiate des portes». En fin de compte, je pense qu'on n'a pas pu mesurer la radioactivité. Mais, aviez-vous eu un rapport concernant ce brouillard blanc?

R : Oui.

$\mathbf{Q}:$ Quand vous en avez entendu parler, qu'en avez-vous pensé?

R : J'ai pensé que c'était de la vapeur.

$\mathbf{Q}$ : Vous en aviez déduit qu'il y avait une fuite quelque part?

R : Oui.

$\mathbf{Q}$ : Ensuite, vers 4h30, je lis que vous avez formulé à l'intention de la salle de commande l'interdiction d'opérer sur le terrain à cause d'un risque de tsunami lié à une réplique. II y a donc eu des répercussions du fait des répliques?

$\mathbf{R}$ : Oui. Je ne me rappelle pas des données exactes, mais ce soir-là il y a eu plusieurs répliques de force 5 à 6 . À chaque fois, j'ai donné l'ordre de retraite au personnel sur le terrain. C'était des conditions d'autant plus compliquées pour les manœuvres.

$\mathbf{Q}$ : Ensuite, je vois, vers 4h45, envoi par la cellule de crise à la salle de commande, de dosimètres ${ }^{130}$ avec l'alarme réglée à 100 mSv et de masques intégraux. Ça fait un peu kamikaze, mais je pense que cela reflète assez votre état d'esprit à ce moment-là.

Ensuite, sur le terrain, ils forment des groupes, un système de trois binômes. Et quand on regarde la constitution des groupes, on voit que ce sont des chefs de quart, des adjoints au 
chef, plutôt des vieux, excusez le terme, en tout cas plutôt des vieux que des jeunes, qui composent prioritairement les groupes ${ }^{131}$.

R : Oui.

$\mathbf{Q}$ : Pour la constitution de ces groupes, vous aviez laissé l'équipe de quart décider?

R : Oui.

$\mathbf{Q}$ : Vous avez donc commencé par tester différentes manières d'ouvrir cette vanne par une commande à distance, mais ceci sans succès. Ensuite vers les 4 à 5h00, vous vous dites qu'il faudra se résoudre à aller physiquement sur place pour la manœuvre et vous étudiez la manière de réaliser cette opération. Tout ceci, au milieu des répliques du séisme, des interruptions de manœuvres dues à ces répliques. C'est bien ça?

R : Oui.

$\mathbf{Q}$ : Concernant concrètement cette opération d'éventage, je pense qu'il s'est passé beaucoup de choses du côté de l'équipe de quart, mais, grosso modo, vous avez manœuvré pour ouvrir la vanne de rejet de l'enceinte de confinement. Pour cela, on rapporte qu'il y a eu manœuvre pour ouvrir la petite vanne, une vanne $\mathrm{AO}^{132}$, et qu'au cours de cette manœuvre, vous avez donné l'ordre d'étudier la possibilité d'installer un compresseur de secours et de le relier au réseau. Ce qui laisse penser qu'ayant rencontré des difficultés à ouvrir cette vanne $\mathrm{AO}$, vous avez d'abord étudié la possibilité d'installer un compresseur de secours et que, seulement ensuite, vous avez demandé à ce qu'on vous en prépare un, afin d'envoyer de la pression et d'ouvrir enfin cette vanne. Mais enfin, ce genre de choses, des compresseurs, etc., n'étaient pas des choses que la cellule de crise pouvait prévoir bien en amont, des choses qui deviendraient de toute façon nécessaires et que la cellule pouvait préparer à l'avance, puisque les hommes sur le terrain étaient occupés à autre chose et bien incapables de les trouver eux-mêmes? Ou bien, c'est que le compresseur avait échappé à votre liste?

$\mathbf{R}$ : Non. Ce dont on disposait dans l'immédiat était ce qu'on appelle des bébéscompresseurs, le genre de petits compresseurs qui entrent dans la composition d'autres appareils. On n'avait que ça. Alors, j'avais donné l'ordre de charger les batteries de tout ce qui pouvait être chargé, en même temps que de chercher des compresseurs plus conséquents. À force de recherche, on va finir par en trouver, mais plus tard, comme c'est indiqué à la page 17. Alors vous comprenez que la recherche d'un compresseur et les essais avec ce dont on disposait se faisaient totalement en parallèle.

$\mathbf{Q}$ : Quand on regarde la chronologie, ça a l'air d'être beaucoup plus tard, mais à la page 17, on lit que l'équipe «réhabilitation » est en train de chercher un compresseur de secours. Vous voulez dire qu'il y avait déjà bien longtemps que cette recherche était commencée?

$\mathbf{R}$ : D'après mes souvenirs, dès qu'on a évoqué la possibilité d'ouvrir cette vanne $\mathrm{AO}$, j'ai tout de suite pensé qu'il faudrait un compresseur pour ça. Je leur ai demandé avec quoi ils comptaient le faire et je me rappelle très bien que ma première réaction a été de leur dire que des bébés-compresseurs ne fourniraient sûrement pas une pression suffisante. Tout de suite, simultanément, j'ai donné l'ordre de chercher un plus gros compresseur. Et, je ne sais plus précisément à quel moment, mais j'ai aussi demandé au siège de nous fournir un gros compresseur.

$\mathbf{Q}$ : In fine, on vous informe qu'il y a un compresseur, sur le site, chez une entreprise partenaire, compresseur que vous trouvez.

R : Oui. 
$\mathbf{Q}$ : Donc pour vérifier, au moment où vous vous rendez compte, les manœuvres à distance étant difficiles, que vous allez devoir ouvrir cette vanne à la main, vous commencez à vous mettre en quête d'un compresseur?

R : Non. Avant d'en arriver là, il y a le stade où on fait des essais avec les bébéscompresseurs. Déjà à ce stade-là, je pense qu'il y a des chances que la pression ne soit pas suffisante et je donne des directives pour qu'on cherche un plus gros compresseur.

$\mathbf{Q}$ : Donc, du point de vue timing, quand le personnel de quart part à 9h04 pour la manœuvre d'éventage, vous avez déjà commencé à chercher un compresseur, puisque vous savez pertinemment, bien avant 9h04, que vous allez devoir manœuvrer cette vanne AO.

R : Oui.

$\mathbf{Q}:$ Vous ne disposiez pas de compresseur de secours, comme vous aviez des camions de pompiers?

R : Non, ça n'était pas prévu. En temps normal, nous n'avons besoin que d'un peu de pression pour utiliser les différents instruments [...] Habituellement nous disposons de gros compresseurs IDSA ${ }^{133}$ à l'intérieur [...] On en a quatre. En principe, ils sont suffisants pour fournir la pression en période d'exploitation normale, mais...

$\mathbf{Q}$ : Évidemment, rien ne fonctionne plus si on n'a pas de courant alternatif.

R: Oui. Je pense qu'à l'avenir, ce seront des choses qu'il faudra prévoir dans la gestion de crise. Si le gros compresseur IDSA est mort, que faire pour obtenir de la pression et quels outils prévoir?

$\mathbf{Q}$ : À peu près à ce moment, si on regarde les paramètres, que ces indications soient fiables ou pas, on constate que le niveau d'eau qui, quelques heures auparavant, était de 1300 mm, tombe à -500 voire -600. C'est-à-dire qu'on est carrément en zone négative.

R : Oui.

$\mathbf{Q}:$ Vous aviez conscience qu'on était en zone négative?

R : Oui.

Q: Pour en revenir à l'éventage, on lit à $14 \mathrm{~h} 30$ qu'» ayant installé vers 14 h00 un compresseur de secours pour ouvrir la grande vanne de la chambre de suppression, on constate la baisse de la pression à l'intérieur de la chambre sèche. On en déduit qu'il y a eu rejet d'éléments radioactifs à la suite de l'éventage » et vous en informez les autorités à 15h18. Avez-vous pensé sur le moment que, à la suite de la manœuvre, le disque de rupture ${ }^{134}$ avait rompu et que l'éventage s'était fait?

R: Je n'en étais pas sûr. Traditionnellement, on vérifie l'exécution ou non de l'éventage en observant le dosimètre qui se trouve en haut de la cheminée. Si la radioactivité augmente, il y a eu éventage. Mais, dans le cas présent, on n'avait pas accès aux chiffres du dosimètre. La seule chose que nous pouvions observer, c'était la pression de la chambre sèche. Elle avait l'air d'avoir baissé, nous avons donc supposé que l'éventage s'était bien effectué. Pour être tout à fait sûr de l'exécution d'un éventage, il n'y a que les chiffres du dosimètre. Mais n'y ayant pas accès, il a bien fallu se contenter de ça.

En plus, là c'est tout à fait par hasard, mais les caméras de la NHK filmaient justement le réacteur 1, quand une espèce de fumée blanche s'est échappée de la cheminée. C'était vers 14h30. Comme l'horaire correspondait assez bien et que la pression avait commencé à baisser dans la chambre sèche, on s'est dit qu'il y avait de fortes chances pour que l'éventage se soit fait. 
Comme je l'ai dit, le disque de rupture, c'est comme le dernier rempart. C'est un dispositif qui se rompt tout seul quand la pression augmente. L'homme n'y accède pas exprès pour le rompre volontairement à un moment précis. Nous sommes juste là à prier pour qu'il se rompe à un moment ou à un autre. Alors on ne sait pas au juste à quel moment, à quelle heure précise, le disque s'est rompu. C'est d'après les éléments dont je disposais que j'ai tiré mes conclusions et fait mon rapport.

$\mathbf{Q}$ : Ensuite, après ces histoires d'éventage, il y a, à 14h53, mention de la « fin de l'injection d'eau douce dans la cuve du réacteur par les camions de pompiers, avec un total cumulé de $80 t$ injectées ». Une minute plus tard, on lit «ordre du directeur de la centrale d'exécuter l'injection d'eau de mer ». Cela veut dire que jusque-là, vous aviez injecté à un bon rythme toute l'eau douce dont vous disposiez soit dans les cuves anti-incendie ou autre, ce qui correspondrait aux 80 t, et que, vous étant pratiquement retrouvé à court d'eau douce, vous avez donné l'ordre de poursuivre avec l'eau de mer. C'est bien ça?

$\mathbf{R}$ : Là, ça n'est pas vraiment «poursuivre». Si je veux être véritablement précis, nous avions commencé les préparatifs pour cette injection d'eau de mer bien avant $14 \mathrm{~h} 54$. Ce qui veut dire que l'ordre de préparer cette injection a été donné bien avant. Mais il se trouvait qu'à cette heure, les préparatifs étaient terminés et l'injection possible. C'est donc pourquoi j'ai donné cet ordre, qui est plus un ordre d'exécution qu'un ordre de préparation, si je me souviens bien. Seulement, c'est là qu'a eu lieu l'explosion. On n'a pas pu passer à exécution et on s'est retrouvé au point de départ. Ce qui est clair, c'est que l'ordre d'étudier la manière d'injecter l'eau de mer avait été donné à un stade plus précoce.

$\mathbf{Q}$ : Je pense que c'est la première fois qu'on a envisagé d'injecter de l'eau de mer. Comment en êtes-vous arrivé à cette décision? En aviez-vous parlé autour de la table de crise ou avec des gens du siège par téléconférence? Est-ce à la suite de ces conversations que vous avez décidé?

$\mathbf{R}$ : D'abord, plutôt que d'en parler avec quiconque, il était évident que nous arriverions assez vite à épuiser l'eau douce. La seule source dont nous pouvions disposer de manière illimitée, c'était la mer. Donc, il était évident pour moi qu'on en arriverait à injecter l'eau de mer, il n'y avait pas d'autre solution ${ }^{135}$. Alors, je ne sais plus si j'en avais parlé au moment des réunions générales, mais j'avais donné l'ordre au groupe " pompier » d'étudier la manière d'injecter de l'eau de mer. Parce que s'il faut aller pomper l'eau au niveau de la mer, il faut la monter de $10 \mathrm{~m}$. Avec une pompe à incendie classique, ça ne monte pas. Ici, c'est la mer. Là, c'est à $4 \mathrm{~m}$ audessus du niveau de la mer et ici, on est à $10 \mathrm{~m}$. Là, il y a le bâtiment turbine et c'est là qu'il faut apporter l'eau. Ce qui signifie qu'il faut monter l'eau de $10 \mathrm{~m}$. Avec une aspiration ordinaire, on n'y arrive pas. Alors que faire? Il fallait qu'ils trouvent une solution.

Q : S'il n'y a pas un relais à un point intermédiaire, ce n'est pas possible.

$\mathbf{R}$ : Voilà. Il fallait absolument installer un booster ${ }^{136}$. Mais comment faire ? Sur ces entrefaites, on a su que l'eau du tsunami, de l'eau de mer, était restée dans le bassin attenant à la turbine du réacteur 3 . Alors j'ai donné l'ordre d'utiliser cette eau. C'était vraiment de la débrouille. Tout en profitant de cette eau, il fallait réfléchir à un moyen de ravitailler ce bassin avec l'eau de mer. Ensuite, par chance, plusieurs camions de pompiers sont arrivés. Alors on les a mis en réseau ici, sur ce quai. On a monté l'eau en en utilisant deux comme booster, puis un autre ici comme relais. Mais ça, c'est plus tard. Au départ, pour le réacteur 1, on a commencé par utiliser l'eau 
laissée par le tsunami. Une solution très terre à terre. Mais on ne pouvait guère faire mieux.

$\mathbf{Q}$ : Vous voulez dire que vous avez utilisé l'eau de mer qui était restée dans le bassin du réacteur 3, c'est ça?

R : Oui.

Q : Si je comprends bien, c'était bien avant 14h54 que vous aviez commencé à vous dire que vous n'auriez pas d'autre solution que d'injecter de l'eau de mer et que, suivant cette conclusion, vous vous êtes mis en quête d'eau de mer, lorsqu'on vous a informé de la présence de cette eau dans le bassin et que vous avez décidé d'utiliser cette eau ?

R : Oui.

$\mathbf{Q}$ : Est-ce que le siège, par exemple, était au courant de tous ces mouvements ?

$\mathbf{R}$ : Non. Je ne les avais pas tenus au courant de ces préparatifs et des détails.

$\mathbf{Q}$ : Est-ce qu'en entendant l'expression "eau de mer", dans vos conversations, ils ne se sont pas inquiétés? Même si vous n'étiez pas en train de faire un rapport, puisque vous étiez reliés par image et son, ils devaient bien entendre des choses?

$\mathbf{R}$ : Non, parce qu'on avait coupé le son.

$\mathbf{Q}$ : Parce qu'on peut le couper?

$\mathbf{R}$ : Oui, on peut. On ne pousse sur le bouton que lorsqu'on a un rapport à faire. Ce n'est pas qu'on ne veut pas leur donner les informations. Mais c'est que nous, on est en pleine discussion pour savoir comment procéder. On étale les plans et on se dit, là, ça ne passera pas. On se demande combien de pompes on a, combien de camions de pompiers. Ah, ils sont deux. On discute de tas de choses, de possibilités autour des plans. Mais ça, le siège n'a pas besoin de savoir tout ça. De toute façon, même au bout d'un million d'années, ils ne sont absolument pas capables de nous sortir des informations du genre : "il y a de l'eau de mer dans un bassin turbine », alors on est bien obligé de chercher les solutions sur le terrain.

$\mathbf{Q}$ : Encore une chose que vous faisiez à ce moment-là, là, à 15 h18 dans la chronologie: «progression de la réhabilitation du réseau d'injection d'eau borée, mise en marche de la pompe du réseau eau borée dès la fin des préparatifs ». Là, vous vouliez relier un camion générateur au point $2 \mathrm{C}$ du réacteur 2 , puis tirer des câbles par l'intérieur pour pouvoir l'utiliser comme alimentation du SLC, c'est ça?

R : Oui, on étudiait cette possibilité en parallèle.

$\mathbf{Q}:$ Et, vous étiez assez avancés dans les préparatifs, il me semble?

$\mathbf{R}$ : Oui. En fait, ça a explosé au moment où on s'apprêtait à enclencher le système.

$\mathbf{Q}$ : Et on se trouve à $15 \mathrm{~h} 36$.

R: Oui.

$\mathbf{Q}$ : Nous allons nous arrêter là pour aujourd'hui. La prochaine fois, nous évoquerons l'explosion.

\section{$\mathbf{R}:$ Très bien.}

(Fin de l'audition) 


\section{NOTES}

1. Masao Yoshida est la deuxième personne auditionnée par la Commission d'enquête gouvernementale sur l'accident, après Nobuo Shudô le 6 juillet 2011 (cf. tableau des auditions).

2. Plusieurs enquêteurs prennent la parole successivement.

3. La Tôkyô Electric Power Company (compagnie d'électricité de Tôkyô) est fondée en 1951. Son siège social est à Tôkyô.

4. Centrale nucléaire exploitée par TEPCO, située dans la préfecture de Fukushima (région du Tôhoku). Les quatre réacteurs nucléaires de Fukushima Daini ont été mis en service entre 1982 et 1987.

5. Unité de production électrique comportant une chaudière et un groupe turboalternateur. Une tranche nucléaire se caractérise par le type du réacteur et la puissance du groupe turbo-alternateur.

6. Centrale nucléaire située dans la préfecture de Fukushima (cf. Fig. 1), entre les communes de Futaba et d'ôkuma. Fukushima Daiichi est exploitée par l'entreprise TEPCO. Le premier réacteur de la centrale est mis en service en mars 1971, le deuxième en juillet 1974, le troisième en mars 1976, le quatrième en octobre 1978, le cinquième en avril 1978 et le sixième en octobre 1979. Les six réacteurs sont répartis face à l'océan en deux ensembles, séparés de quelques centaines de mètres : d'un côté les réacteurs 1 à 4 , de l'autre le 5 et le 6 . Le site occupe une surface d'environ $3,5 \mathrm{~km}^{2}$ et s'élève entre 6 et 10 mètres au-dessus du niveau de la mer.

7. Une centrale thermique à flamme (appelée également "centrale à flamme » ou "centrale thermique classique»), produit de l'électricité à partir de combustibles fossiles (charbon, gaz ou pétrole). Une centrale hydroélectrique fabrique de l'électricité grâce à la force de l'eau, qui dépend soit de la hauteur de la chute d'eau (centrales de haute ou de moyenne chute), soit du débit des fleuves et des rivières (centrales au fil de l'eau). Dans une centrale nucléaire, une réaction nucléaire contrôlée produit de la chaleur, qui transforme de l'eau en vapeur et met en mouvement une turbine reliée à un alternateur qui produit de l'électricité. Le parc nucléaire du Japon a été mis à l'arrêt pour inspection après l'accident de Fukushima Daiichi, entraînant une modification importante de sa répartition des sources de production électrique. Le charbon est passé de $25 \%$ en 2010 à $30 \%$ en 2018, le gaz de $29 \%$ à $37 \%$, les énergies renouvelables de $1 \%$ à $8 \%$. Le pétrole est resté à $9 \%$ et l'hydraulique à $8 \%$. Le nucléaire est passé de $29 \%$ en 2010 à zéro en 2014, avant de remonter de manière progressive pour atteindre $7 \%$ en 2018 (RGN, 2019).

8. Fédération des compagnies d'électricité du Japon. Cette fédération des dix fournisseurs d'électricité du Japon a été fondée en 1952 pour faciliter leur communication et leur coopération. Elle regroupe Hokkaido Electric Power Co., Hokuriku Electric Power Co., Chubu Electric Power Co., The Chugoku Electric Power Co., Okinawa Electric Power Co., Kyushu Electric Power Co., Shikoku Electric Power Co., Kansai Electric Power Co., Tohokû Electric Power Co. et TEPCO.

9. Un surgénérateur est un réacteur nucléaire pouvant produire plus de matière fissile qu'il n'en consomme. Le surgénérateur Monju, construit dans la centrale nucléaire de 
Tsuruga (préfecture de Fukui), est mis en service en 1994. En mars 2018, la Nuclear Regulation Authority (NRA - en français " Autorité de Régulation Nucléaire », créée en septembre 2012) valide le projet de démantèlement de ce surgénérateur. L'accident mentionné par Yoshida s'est quant à lui produit le 8 décembre 1995.

10. Société pour le développement des réacteurs de puissance et des combustibles nucléaires au Japon, fondée en octobre 1967.

11. La PNC est dissoute en octobre 1998 et devient la Japan Nuclear Cycle Development Institute (JNC).

12. Institut japonais de développement du cycle du combustible nucléaire, créé en octobre 1998. Il fusionne avec le Japan Atomic Energy Research Institute (JAERI) en octobre 2005, pour devenir la Japan Atomic Energy Agency (JAEA).

13. General Electric est une entreprise fondée en 1892 à la suite de la fusion d'Edison General Electric Company et de Thomson-Houston Electric Company. Elle a supervisé la construction des réacteurs 1 et 2 de Fukushima Daiichi et co-construit le réacteur 6 . Yoshida évoque ici un scandale qui a secoué l'opinion publique en 2002. Les Japonais apprennent alors que TEPCO a falsifié une trentaine de rapports d'inspection ayant révélé des fissures et de la corrosion sur des enveloppes de réacteurs exploités par la compagnie. En conséquence, plusieurs réacteurs sont mis à l'arrêt et la direction de TEPCO doit remettre sa démission.

14. Structure en acier permettant de stabiliser les assemblages et les barres de contrôle d'un réacteur à eau bouillante, de diriger le sens d'écoulement du caloporteur et de maintenir le niveau d'eau dans la cuve.

15. Le cœur est la partie d'un réacteur nucléaire comprenant les assemblages de combustible nucléaire, agencée pour permettre une réaction en chaîne.

16. Le 16 juillet 2007, à $10 \mathrm{~h} 13$ (heure locale), un séisme de magnitude 6,6 se produit dans la préfecture de Niigata (île de Honshû, région du Chûbu). Il fait 11 morts et plus de 1000 blessés, tandis que 25000 foyers sont privés d'électricité. L'épicentre est situé à environ 10 kilomètres de la centrale nucléaire de Kashiwazaki-Kariwa, exploitée par TEPCO. Cette centrale, construite sur les communes de Kashiwazaki et de Kariwa, est l'unité de production électronucléaire la plus importante au monde (environ 8200 $\mathrm{MWe}$ ). Les trois réacteurs en fonctionnement et un réacteur en cours de démarrage sont mis à l'arrêt automatiquement durant le séisme (les trois autres réacteurs étaient à l'arrêt pour inspection). Un incendie se déclare sur un transformateur électrique à l'extérieur du bâtiment réacteur 3, une fuite d'eau est constatée sur le réacteur 6 , des centaines de fûts contenant des déchets de faible activité sont renversés et certains perdent leur couvercle. De la matière radioactive se disperse dans l'atmosphère et dans la mer du Japon.

17. Le terme « ya» (屋) signifie «monsieur » ou « spécialiste » en japonais. «D'après le dictionnaire japonais “大辞泉: DAIJISEN", la première définition du terme est: Maison; Magasin ou boutique spécialisé(e), par exemple «Pain-ya 》 (パン屋 : boulangerie); Spécialiste, quelqu'un qui connait un domaine à fond. Cette définition est souvent utilisée pour se moquer de soi-même ou des autres: "Je suis «XX-ya » (XX屋), donc je connais seulement XX à fond 》. Par conséquent, lorsqu'on utilise "ya » (屋) dans une organisation au Japon, on renvoie à la division $d u$ travail. Chaque division comme le maintien de machine et la sûreté d'équipement possède sa propre connaissance et elle n'a pas de connaissances des autres divisions. Les connaissances sont 
réciproquement exclusives comme si les membres de chaque division habitaient dans leur propre 'maison'» (Kobayashi, 2019, p. 19).

18. Émission d'une quantité d'énergie sous forme de rayonnements et/ou de particules accompagnant la désintégration d'un élément instable ou d'un noyau d'atome bombardé.

19. TEPCO exploite 17 réacteurs nucléaires, répartis sur les centrales de Fukushima Daiichi (6), de Fukushima Daini (4) et de Kashiwazaki-Kariwa (7). L'entreprise exploite également cent-soixante installations hydrauliques et vingt-cinq centrales thermiques.

20. Yoshida semble évoquer le centre de relation client de TEPCO situé à Tochigi (préfecture de Tochigi, dnas la région du Kantô).

21. Le séisme de la côte Pacifique du Tôhoku se produit le 11 mars 2011, à 14 h46 (heure locale). Son épicentre se situe dans l'océan Pacifique à 370 kilomètres au nord nord-est de Tôkyô. La durée de la secousse enregistrée à Tôkyô est de plus de deux minutes. La puissance du tremblement de terre provoque un tsunami dévastateur. Le bilan des victimes de la catastrophe s'élève à 15897 morts, 2534 disparus et 6152 blessés. Parmi les séismes les plus importants recensés dans le monde depuis le XVIII ${ }^{\mathrm{e}}$ siècle, le séisme du Tôhoku (magnitude 9) se situe au quatrième rang, après celui du 22 mai 1960 au Chili (9,5), du 27 mars 1964 en Alaska $(9,2)$, et celui du 26 décembre 2004 à Sumatra $(9,1)$.

22. La centrale de Fukushima Daiichi se situe à 145 kilomètres de l'épicentre du tremblement de terre. À $15 \mathrm{~h} 27$, soit une quarantaine de minutes après le séisme, le tsunami atteint Daiichi. Les réacteurs 1 à 4 sont construits sur un terrain situé à 10 mètres d'altitude. La digue a été édifiée pour protéger le site d'une montée des eaux de 5,7 mètres. La première vague, d'une hauteur de 4 à 5 mètres, est arrêtée par la digue. Une dizaine de minutes plus tard, une deuxième vague, de 14 à 15 mètres, passe cette fois par-dessus la digue et inonde les installations et les équipements du site. Au moment du séisme du 11 mars 2011, environ 6400 personnes travaillent à Daiichi.

23. Les réacteurs 1,2 et 3 fonctionnent à pleine puissance lors du séisme, tandis que les réacteurs 4,5 et 6 sont à l'arrêt pour maintenance. La puissance brute du réacteur 1 est de $460 \mathrm{MWe}$, celle du réacteur 2, 3, 4 et 5 de $784 \mathrm{MWe}$ chacun. La puissance brute du réacteur 6 est quant à elle de $1100 \mathrm{MWe}$.

24. La région du Tôhoku, située au nord-est de l'île de Honshû, regroupe les préfectures d'Akita, d'Aomori, d'Iwate, de Miyagi, d'Yamagata et de Fukushima.

25. L'arrêt automatique des réacteurs en service a été activé dès que les capteurs de la centrale ont détecté le mouvement du sol et déclenché les systèmes de protection des réacteurs (AIEA, 2015a). Les réacteurs de quatre centrales nucléaires (centrales d'Onagawa, de Fukushima Daiichi, de Fukushima Daini et de Tôkai; au total 11 réacteurs en fonctionnement), situées sur la côte Nord de l'île de Honshû, s'arrêtent ainsi automatiquement dès les premières secousses.

26. L'origine de ce terme n'est pas bien claire. La signification donnée varie entre Safety Control Rod Axe Man, Super-Critical Reactor Axe Man ou Start Cutting Right Away, Man. Des auteurs suggèrent encore le verbe "to scram ", soit déguerpir, en tant que réaction à avoir en cas de problème.

27. La salle où se réunit la cellule de crise se situe dans le bâtiment antisismique de Fukushima Daiichi. Cette cellule se compose de douze groupes d'opération d'urgence (groupe de surveillance, de communication publique, d'annonce, d'informations, de réhabilitation, de production électrique, de technique, de maintenance, d'aide 
médicale, de bien-être, d'affaires générales, de ressources), situés autour de la table mentionnée par l'enquêteur. À côté de Yoshida, sont assis Fukura et Yoshizawa, les vice-directeurs de la centrale, ainsi que les chefs des douze groupes. En anglais, cette cellule de crise est dénommée « Fukushima Daiichi Emergency Response Center (ERC) ».

28. Yoshida évoque la loi japonaise $n^{\circ} 156$ du 17 décembre 1999, intitulée Gensai-hô, traduite en anglais par "Act on Special Measures Concerning Nuclear Emergency Preparedness » et en français par «Loi portant sur les mesures spéciales à adopter en cas de catastrophe nucléaire ». Elle est adoptée à la suite d'un accident de criticité survenu le 30 septembre 1999. L'évènement, classé au niveau 4 sur l'échelle INES, est le premier accident important dans l'histoire du nucléaire civil au Japon. Il s'est produit dans l'usine de fabrication de combustibles nucléaires de la Japan Nuclear Fuels Conversion Company (JCO), située dans le complexe de Tôkai-mura, situé dans la préfecture d'Ibaraki. Ce complexe regroupe une centrale, une usine de traitement de combustible usagé, un centre de recherche, un accélérateur de particules et plusieurs usines). L'accident a fait deux morts parmi les employés.

29. L'article 10 de la loi précise l'obligation pour l'exploitant de signaler toute situation potentielle d'urgence nucléaire aux autorités gouvernementales et préfectorales.

30. Le bâtiment antisismique de Daiichi est mis en service en juillet 2010. Sa conception a tiré profit du retour d'expérience des effets du séisme de 2007 sur la centrale nucléaire de Kashiwazaki-Kariwa. Il est équipé d'une alimentation électrique de secours, de dispositifs de ventilation filtrée et d'un blindage assurant une protection contre la radioactivité (AIEA, 2015a).

31. Cf. Fig. 2.

32. Dans ce passage, les textes entre parenthèses sont des indications de la traductrice.

33. Le pilote de tranche est un opérateur chargé de la conduite d'un réacteur nucléaire.

34. Les six réacteurs de Fukushima Daiichi partagent une salle de commande principale par paire de tranches. Les paires sont réparties de la manière suivante : tranches 1 et 2 , tranches 3 et 4 , tranches 5 et 6 .

35. Le chef de quart dirige l'équipe de quart, qui assure l'exploitation de l'installation. Il est responsable du fonctionnement de l'installation, et veille au respect des procédures et des valeurs limites d'exploitation.

36. Yoshida rejoint la cellule de crise située dans le bâtiment antisismique le 11 mars peu avant 15 h00 (Kadota, 2014).

37. Le PHS est une norme de téléphonie mobile japonaise.

38. La vérification de l'arrêt automatique des réacteurs a été un soulagement pour les équipes de Fukushima Daiichi, comme le souligne Shiro Hikida (chef de groupe de réhabilitation d'équipement): "J'ai pensé qu'on pouvait surmonter cet accident en mobilisant les expériences des entraînements » (Kobayashi, 2019, p. 76).

39. Système de secours qui permet de refroidir le cœur de certains réacteurs à eau bouillante lorsque l'évacuation de la puissance ne peut plus se faire par le condenseur principal. Ce système condense la vapeur d'eau produite dans un échangeur thermique, puis la réinjecte par gravité dans la cuve. 
40. Système comportant une turbopompe alimentée par la vapeur produite dans la cuve. Le RCIC permet d'alimenter la cuve du réacteur en eau à partir d'un réservoir ou $\mathrm{du}$ wetwell en cas de problème de refroidissement.

41. Situation d'un réacteur nucléaire à l'arrêt dans lequel le fluide de refroidissement est soumis à une pression quasi-atmosphérique, et dont la température est inférieure à sa température d'ébullition.

42. La centrale nucléaire de Fukushima Daiichi dispose de plusieurs circuits de refroidissement à l'eau de mer, notamment pour refroidir certains générateurs diesel de secours et d'autres composants importants pour la sûreté, ainsi que pour servir de source froide aux systèmes d'évacuation de la chaleur résiduelle. Lors d'un tsunami, l'eau se retire des plages dans un premier temps avant de déferler sous la forme d'une importante vague. Ce retrait inquiète Yoshida car il pouvait causer une rotation à sec ou l'aspiration d'air dans les pompes, ce qui pourrait rendre difficile leur redémarrage. Notons que la panne simultanée de tous les circuits de refroidissement à l'eau de mer relatifs à la sécurité est désignée par un accident de perte de la source froide ultime (Loss of Ultimate Heat Sink - LUHS).

43. Désignée également en tant que chambre de condensation ou chambre humide (suppression pool ou wetwell). Cette chambre correspond à la partie basse de l'enceinte de confinement d'un réacteur à eau bouillante. Elle est généralement remplie d'eau jusqu'à la moitié de sa hauteur.

44. Yoshida évoque ici la cellule de gestion de crise située au siège de TEPCO, à Tôkyô, et créée le 11 mars 2011 à 15 h06. Elle est composée de neuf équipes d'opération d'urgence et regroupe jusqu'à deux cents personnes. Elle est dirigée par Masataka Shimizu, le Président de TEPCO. Ce dernier n'est toutefois pas présent le 11 mars, car il est en voyage d'affaires dans la région du Kansai. Le directeur exécutif de la compagnie est également absent au moment de l'accident. Les rédacteurs du rapport d'enquête de la NAIIC (2012) considèrent que cette situation est inconcevable pour un exploitant de centrales nucléaires.

45. Ce système rassemble les équipements d'un réacteur nucléaire (pompes, valves, réservoirs, échangeurs de chaleur et tuyauterie) spécifiquement conçus pour dissiper la chaleur résiduelle des combustibles en cas de défaillance du système normal de refroidissement du réacteur.

46. La turbine principale désigne la grande turbine qui entraîne le générateur pour produire de l'électricité. Hormis celle-ci, chaque tranche possède des turbopompes dans le bâtiment turbine. Celles-ci sont constituées de pompes entraînées par de petites turbines. Leur rôle est de pomper la vapeur condensée du bâtiment turbine et de la déverser dans le réacteur. À Fukushima Daiichi, ces turbopompes ont été arrêtées au moment du SCRAM.

47. Agence Internationale de l'Énergie Atomique. Fondée en juillet 1957, elle a pour objectif de promouvoir des technologies nucléaires sûres, sécurisées et pacifiques. Son siège est situé à Vienne (Autriche). L'AIEA possède également deux bureaux régionaux (Toronto, Tôkyô) et deux bureaux de liaison (New York, Genève), et administre des laboratoires spécialisés dans le nucléaire (Seibersdorf, Monaco et Trieste).

48. En cas de SCRAM, la vanne d'isolation du circuit principal de vapeur se ferme pour limiter les risques de perte de refroidissement ou de rejets radioactifs. 
49. L'enceinte de confinement renvoie à une structure étanche qui contient et isole la cuve du réacteur et qui permet de contenir ou limiter les rejets de matières radioactives en cas d'accident. Dans un REB, elle est composée du drywell et du wetwell qui sont généralement sous atmosphère inerte (cf. Fig. 4 et Fig. 5).

50. Vanne d'isolation du circuit principal de vapeur.

51. Le bâtiment réacteur est le bâtiment qui contient, entre autres, l'enceinte de confinement et la piscine de désactivation du combustible usé (cf. Fig. 4 et Fig. 5).

52. Le rotor est la partie mobile rotative (par rapport à la partie immobile, nommée stator) de l'ensemble turbine-générateur. En cas de problème affectant la turbine (vibrations fortes, rotation trop rapide, manque de lubrification, etc.), cette dernière est arrêtée grâce aux vannes d'arrêt de la turbine. Ces vannes permettent de rediriger la vapeur directement vers le condensateur.

53. Les trop fortes vibrations font partie des signaux qui peuvent déclencher l'arrêt automatique de la turbine pour éviter sa détérioration. En particulier, la vibration axiale désigne le déplacement de l'arbre du rotor par rapport à l'horizontale.

54. Système d'affichage des paramètres de sûreté.

55. Unité d'accélération $\left(=0,01 \mathrm{~m} / \mathrm{s}^{2}\right)$, exprimant l'accélération de la pesanteur en gravimétrie et en géodésie.

56. Récipient métallique étanche contenant le cœur d'un réacteur nucléaire, le modérateur et le caloporteur.

57. Yoshida mentionne un accident survenu en octobre 1991, lié à une fuite d'eau de mer provenant de la tuyauterie du circuit d'eau de mer de refroidissement au niveau B1 du bâtiment turbine de la tranche 1 de Fukushima Daiichi. À la suite de cet incident, des mesures d'étanchéité ont été mises en œuvre pour empêcher que les principaux équipements des sous-sols ne soient inondés ou endommagés par une inondation interne résultant notamment d'une rupture de tuyaux dans les installations (TEPCO, 2012).

58. Le haut du combustible est le niveau de référence dans la cuve du réacteur. La valeur qui le suit est toujours donnée au millimètre.

59. La Nippon Hôsô Kyôkai (NHK) est l'entreprise qui regroupe les stations de radio et de télévision du service public japonais. Sa première station de radio est lancée en 1925 et sa première station de télévision en 1953.

60. L'Agence Météorologique du Japon, fondée en 1875, est chargée de relever les données terrestres et maritimes du temps, de lancer et d'exploiter des satellites météorologiques, ainsi que de détecter les séismes dans le Pacifique ouest. Son siège est à Tôkyô.

61. Le 27 février 2010, un séisme de magnitude 8,8 se produit au Chili. Son épicentre se situe dans l'océan Pacifique, à plus de 6 kilomètres au large des côtes et à 325 kilomètres au sud-ouest de Santiago du Chili. Un tsunami se forme à la suite du tremblement de terre. La catastrophe fait 525 morts et disparus.

62. Ensemble des systèmes d'évacuation de la chaleur résiduelle dans un réacteur nucléaire à l'arrêt.

63. Sigle inconnu. Il pourrait s'agir d'une confusion avec le sigle SW qui désignerait dans ce cas le "Seawater System ", soit le circuit à eau de mer. 
64. Phénomène suivant lequel des cavités de gaz ou de vapeur se creusent dans une masse de liquide rapidement propulsée.

65. Yoshida précise, dans un entretien accordé à Kadota, son ressenti à la suite de la perte des alimentations électriques : «mon esprit aurait dû paniquer. Mais étrangement, alors qu'une partie de mon esprit craignait que cela ne devienne un autre Tchernobyl, l'autre moitié me disait de garder mon calme et de commencer à planifier. Toutes ces choses qui devaient être faites ont commencé à cliqueter dans mon cerveau » (Kadota, 2014, p. 34 [notre traduction]).

66. La durée de vie de l'IC est limitée par le volume d'eau stocké dans le réservoir. L'échangeur de chaleur de ce système doit être constamment noyé pour éviter sa dégradation. S'il n'est pas réalimenté en eau, l'IC reste fonctionnel pour une durée de six à huit heures successives. L'IC étant situé hors de l'enceinte de confinement, il requiert une source d'électricité pour l'ouverture des vannes d'isolation de l'enceinte. L'utilisation de l'IC est donc également limitée par la durée de vie des batteries utilisées à cet égard. Le RCIC, quant à lui, fonctionne en transférant la chaleur de la cuve vers le wetwell. Il doit être refroidi - et donc alimenté en eau - pour éviter qu'il ne chauffe plus que la température recommandée (environ $60{ }^{\circ} \mathrm{C}$ en fonctionnement normal). Dans le cadre d'une urgence, l'exploitant peut s'autoriser à aller au-delà de cette valeur. Cependant, le RCIC nécessite un système d'instrumentation et de contrôle et est ainsi également limité par la durée de vie des batteries qui alimentent ce système.

67. L'article 15 de la loi du 17 décembre 1999 concerne la déclaration d'une situation d'urgence nucléaire par le Premier ministre, l'information du public et la mise en place de procédures de protection et d'évacuation des riverains. Naoto Kan, le Premier ministre du Japon, n'est pas à sa résidence lorsque le séisme se produit le 11 mars 2011 à $14 \mathrm{~h} 46$ : «J'étais au Sénat, en train d'écouter les questions des parlementaires de l'opposition lors de délibérations en commission. Je me souviens que le chandelier au plafond de la salle de délibération s'est mis à se balancer dangereusement quand la terre a tremblé» (Kan, 2015). Le Premier ministre retourne alors à sa résidence et établit à $15 \mathrm{~h} 14$ le centre d'intervention d'urgence pour les catastrophes. Vers 15h45, il apprend que Fukushima Daiichi a perdu toutes ses sources de courant alternatif. À 16h36, le Gouvernement crée le Bureau d'intervention d'urgence pour l'accident nucléaire. À 17h42, Kan reçoit un rapport du ministre de l'Économie et de l'Industrie qui détaille les situations concernées par l'article 15 de la loi sur les accidents nucléaires. Le ministre fait alors part à Kan de la nécessité de proclamer l'état d'urgence nucléaire. À 19h03, l'état d'urgence nucléaire est décrété. Le rapport de la NAIIC (2012) considère toutefois que le Gouvernement a failli à sa tâche en déclarant trop tardivement l'état d'urgence. En même temps que sa proclamation, est créé le centre de conduite de l'intervention en cas d'urgence nucléaire (AIEA, 2015a). Cette cellule de gestion de crise du Gouvernement est dénommée en anglais «NERHQ» (Nuclear Emergency Response Headquarters).

68. Le système d'injection d'eau à haute pression est un système de refroidissement de secours indépendant qui doit permettre de maintenir l'eau dans le réacteur à un niveau suffisant en cas de problème, sans requérir sa dépressurisation au préalable. L'eau injectée par le HPCI provient du réservoir tampon ou du wetwell. Il est actionné par une turbopompe alimentée par la vapeur de la cuve.

69. L'eau filtrée est de l'eau fraîche purifiée, utilisée pour le nettoyage des systèmes et pour remplacer l'eau de refroidissement (normalement, après distillation). En cas 
d'urgence, elle peut également servir dans la lutte contre les incendies ou être injectée dans les cuves de réacteurs. La centrale de Fukushima Daiichi dispose de grands réservoirs d'eau filtrée directement reliés aux différentes tranches. Les tuyauteries qui permettent cette relation ont cependant été rompues pendant le séisme. L'eau filtrée n'est donc pas facilement accessible pour être injectée durant la lutte contre l'accident.

70. La fusion du cœur se produit lorsque le combustible du réacteur nucléaire s'échauffe puis commence à fondre. Il s'agit d'un accident très grave, puisqu'il peut potentiellement causer la dispersion de matières hautement radioactives si le cœur en fusion parvient à traverser le confinement.

71. Pour rappel, ce séisme s'est produit le 16 juillet 2007.

72. Même séisme que celui cité plus haut - le Chûetsu est le nom d'une région de la préfecture de Niigata.

73. Liquide de refroidissement à base d'eau et de bore, utilisé comme ralentisseur ou absorbeur de neutrons lents afin de contrôler la réaction en chaîne et éviter l'emballement du réacteur nucléaire.

74. Système auxiliaire de contrôle des liquides, capable d'injecter de l'eau borée dans un réacteur nucléaire à haute pression.

75. Tubes mobiles contenant une matière neutrophage dont la position permet de contrôler, par absorption de neutrons, le nombre de fissions dans le cœur.

76. Composé chimique utilisé comme substance neutrophage.

77. TEPCO demande au Gouvernement d'envoyer dans l'urgence des camions groupes électrogènes pour faire fonctionner les systèmes de refroidissement de secours des réacteurs. Les camions arrivent à Daiichi le 11 mars vers 21h00. Kenichi Shimomura (secrétaire assistant du Gouvernement), se souvient de la réaction des ministres après avoir appris la nouvelle : "Les ministres ont sauté de joie comme quand les gens poussent un cri quand l'équipe du Japon remporte un match de la coupe de monde » (Kobayashi, 2019, p. 89). Leur joie est toutefois de courte durée, car il est impossible de faire fonctionner les camions dans l'immédiat. Le Premier ministre revient sur cet épisode de la gestion de crise : «Alors que nous pensions que les choses allaient s'arranger, un peu plus tard, on nous a annoncé que les prises ne correspondaient pas et qu'on ne pouvait pas les brancher. Dire qu'un exploitant d'électricité vous demande d'envoyer des camions générateurs et que lorsque vous les envoyez, il a des problèmes de prises, j'avoue que je ne comprenais pas comment une chose pareille pouvait arriver. Finalement, à cause de ça, (bien sûr, plus tard, quand on a vu les panneaux de distribution, on s'est rendu compte qu'ils avaient eux aussi pris l'eau de mer, mais) en tout cas c'est dans un rapport que j'ai su plus tard que les camions générateurs envoyés n'avaient pas pu servir de source d'électricité, comme l'avait prévu TEPCO, et que la fonction de refroidissement n'avait pas pu être assurée par ce biais » (Kan, 2012).

78. D'autres véhicules d'alimentation électrique, envoyés par TEPCO, Tôhoku Electric Power Company et les Forces d'autodéfense, arrivent à Fukushima Daiichi dans la nuit du 11 mars. Le 12 mars, à 10h15, vingt-trois véhicules sont arrivés sur le site (AIEA, 2015a).

79. Dans la nuit du 12 mars, TEPCO décide d'utiliser le Coal Center d'Onahama, une filiale de l'exploitant, comme base locale de distribution. Ce centre est situé à moins d'une cinquantaine de kilomètres de Fukushima Daiichi et à une trentaine de kilomètres du J-Village. Il est exploité par une équipe de douze contractuels, chargés 
notamment de transporter de l'essence, du matériel de contrôle des rayonnements, des générateurs, des pompes ou des batteries à destination de Daiichi (TEPCO, 2012).

80. L'enquêteur évoque ici les refuges, tels que les écoles ou les gymnases, qui ont servi à accueillir les sinistrés de la catastrophe naturelle du 11 mars 2011 et les personnes évacuées à la suite de l'accident nucléaire de Fukushima Daiichi.

81. Les soupapes de dépressurisation des vannes de sécurité (Safety Relief Valves, SRV) restent en position fermée grâce à un ressort. Leur ouverture requiert donc l'application d'une force qui dépasse la tension de ce ressort. Ils fonctionnent selon deux modes différents: ils s'ouvrent automatiquement quand la cuve atteint une pression prédéfinie, ou alors ils sont manipulés en cas de besoin. Pour ouvrir manuellement les SRV, il faut activer une vanne solénoïde. Celle-ci laisse passer de l'azote comprimé qui soulève un piston connecté à la fermeture de la SRV, ce qui entraîne son ouverture. En cas d'accident, un accumulateur à azote est prévu pour permettre l'ouverture des vannes SRV. Il contient une quantité d'azote suffisante pour effectuer cette manœuvre entre sept et dix fois.

82. Vanne activée par un moteur.

83. L'erreur de penser que l'IC fonctionne correctement provient de la mauvaise interprétation d'une information transmise par le groupe de production électrique, qui s'est rendu devant le bâtiment du réacteur 1 pour vérifier si de la vapeur sortait de l'installation : «À 16h44, le 11 mars 2011, un membre du groupe a rapporté : 'La vapeur sort un peu'. Qu'est-ce que cet état veut dire? Malheureusement, personne n'a pu précisément répondre à cette question du fait que l'on n'a jamais démarré l'IC. Même Hikida qui a toujours travaillé à Fukushima Daiichi depuis 1973 n'a jamais vu le démarrage de l'IC. Donc la plupart des personnels n'ont pas pu comprendre ce que veut dire: 'la vapeur sort un peu'. En fait, Hikida a su que 'la vapeur sort un peu' signifie que l'IC vient de s'arrêter. Il avait entendu l'explication des anciens qui ont vu directement le démarrage de l'IC avant le lancement de Fukushima Daiichi. D'après cette explication, l'IC produit violement la vapeur lors de son fonctionnement et cela peut donner aux habitants une impression affreuse. Cependant, cette connaissance n'a pas été transmise parce que les personnels qui avaient vu directement le démarrage de l'IC avaient quitté Daiichi avec le temps » (Kobayashi, 2019, p. 144).

84. Selon Idogawa (pilote du réacteur 2), la multitude des opérations à mener n'a pas permis aux acteurs de se concentrer sur l'état de l'IC: "Dans une situation où plusieurs choses se déroulent simultanément, il est difficile de sélectionner quelle information doit être transmise en priorité à la cellule de crise » (Kobayashi, 2019, pp. 84-85).

85. Concernant l'IC, les opérateurs ont essayé à deux reprises de le redémarrer manuellement, sans y parvenir. Le rapport de l'AIEA (2015) précise qu'ils n'avaient pas reçu la formation nécessaire pour comprendre le fonctionnement des vannes dans les conditions auxquelles ils étaient confrontés.

86. Préfecture située dans la région du Tôhoku. Sa capitale est la ville de Fukushima, qui se trouve à une centaine de kilomètres de la centrale nucléaire de Fukushima Daiichi.

87. Par la suite, le Gouvernement donnera six ordres d'évacuation des populations, élargissant le périmètre selon l'évolution des évènements survenus à Fukushima Daiichi et Daini. Le premier ordre du Gouvernement est donné le 11 mars à $21 \mathrm{~h} 23$ (évacuation de la zone autour de Daiichi à 3 kilomètres), le deuxième est donné le 12 mars à $4 \mathrm{~h} 45$ (élargissement de la zone autour de Daiichi à 10 kilomètres), le troisième à 
$7 \mathrm{~h} 45$ (évacuation de la zone autour de Daini à 3 kilomètres), le quatrième à $17 \mathrm{~h} 39$ (élargissement de la zone autour de Daini à 10 kilomètres), le cinquième à $18 \mathrm{~h} 25$ (élargissement de la zone autour de Daiichi à 20 kilomètres), le sixième le 15 mars à 11 h00 (élargissement de la zone autour de Daiichi à 30 kilomètres).

88. Centre de crise situé à ôkuma à environ 5 kilomètres de Fukushima Daiichi, où sont installées la cellule du Gouvernement dénommée en anglais « Local Nuclear Emergency Response Headquarters» (NERHQ local), la cellule de TEPCO, et la cellule de la préfecture. Ce centre est notamment chargé de la gestion de l'évacuation de la population.

89. Le niveau d'eau dans la cuve du réacteur est une information primordiale. Un réacteur dispose de plusieurs systèmes de mesure du niveau d'eau avec des plages de mesure qui se chevauchent. Ces systèmes sont réalisés quasiment à l'identique en deux occurrences : le circuit A et le circuit B.

90. Le dénoyage du cœur d'un réacteur nucléaire signifie qu'il n'est plus noyé dans de l'eau. Si le cœur reste découvert, la température des crayons du combustible augmente et entraîne sa dégradation de manière irrémédiable, voire sa fusion.

91. La chambre sèche (drywell) correspond à la partie haute de l'enceinte de confinement d'un réacteur à eau bouillante.

92. Ryuta Idogawa évoque la crainte qu'il a ressentie avec ses collègues lorsque Yoshida a décidé d'interdire l'accès du bâtiment réacteur 1 à cause de l'augmentation de la radioactivité : «Le fait que nous, les pilotes des réacteurs 1 et 2, ne puissions pas avoir accès au bâtiment du réacteur 1 est inimaginable. C'était l'état d'urgence [...]. À partir de ce moment-là, il était difficile pour les jeunes pilotes de surmonter la peur de la radioactivité" (Kobayashi, 2019, p. 175). Les jeunes pilotes sont toutefois parvenus à contrôler leur peur, en particulier grâce aux relations quotidiennes de confiance qu'ils entretenaient avec Izawa (chef de la salle de contrôle des réacteurs 1 et 2 ).

93. La pression de l'enceinte de confinement du réacteur $1(600 \mathrm{kPa})$, indiquée par l'instrument de mesure, marque une étape importante dans l'appréhension de la crise dans la salle de commande du réacteur 2 , ce que confirme Ryuta Idogawa : «Je me suis attendu à ce que l'état du réacteur ne s'améliore jamais. À partir de ce moment-là, j'ai pris peur » (Kobayashi, 2019, p. 91).

94. Le sievert (Sv) est l'unité légale d'équivalent de dose qui permet de rendre compte de l'effet biologique produit par une dose absorbée donnée sur un organisme vivant. Le $\mathrm{Sv} / \mathrm{h}$ (ou par seconde) correspond à la dose équivalente absorbée par cet organisme par unité de temps.

95. Dosimètre de poche à alarme sonore. Il s'agit d'un appareil permettant de mesurer les doses radioactives absorbées lors d'une exposition à un rayonnement ionisant.

96. Le conducteur de la salle de commande est le pilote de tranche, chargé de la conduite du réacteur nucléaire.

97. Pression absolue, i.e. par rapport au vide.

98. Procédure d'urgence qui correspond au relâchement contrôlé de gaz dans l'atmosphère à partir de l'enceinte de confinement d'un réacteur nucléaire.

99. Nommée également chambre de condensation ou chambre de suppression (en anglais suppression pool ou welwell).

100. Vanne à commande pneumatique. 
101. Akira Omoto, membre de la Commission de l'énergie atomique du Japon et exdirecteur ingénieur de TEPCO et ex-directeur de la Division de l'énergie d'origine nucléaire de l'AIEA, souligne également que l'explosion d'hydrogène dans le bâtiment réacteur 1 était difficilement prévisible: "Avant l'accident de Fukushima Daiichi, un seul document de recherche (en Finlande) a fait remarquer la possibilité que de l'hydrogène produit par la réaction du zirconium avec l'eau puisse fuir de l'enceinte de confinement et risque de causer une explosion dans le bâtiment du réacteur. Pour les exploitants à ce moment-là, une explosion n'était pas facile à prévoir » (Kobayashi, 2019, p. 101).

102. Haruki Madarame est un ingénieur japonais, né en 1948. Après une carrière universitaire au sein de l'Université de Tôkyô et de la Nuclear Professional School, il est nommé Président de la Nuclear Safety Commission (en français « commission de sûreté nucléaire ») en 2010 jusqu'à son abolition en 2012.

103. Système auxiliaire de traitement de gaz, filtrant et déchargeant l'air de l'enceinte de confinement vers l'atmosphère. Il permet également de maintenir une pression basse dans l'enceinte de confinement afin de limiter les rejets radioactifs.

104. Naoto Kan précise également que Madarame n'imaginait pas la possibilité d'une explosion d'hydrogène: "Dans l'hélicoptère qui m'emmenait à Fukushima Daiichi, j'ai interrogé M. Madarame qui m'accompagnait, en lui demandant si on ne devait pas craindre une telle explosion. Il m'a répondu: "Il n'y a pas d'inquiétude à avoir sur ce sujet. L'enceinte de confinement est remplie d'azote; même si de l'hydrogène fuyait du réacteur, il n'y aura pas d'explosion puisqu'il n'y a pas d'oxygène." Et pourtant, comme vous le savez, une explosion d'hydrogène s'est produite ce jour-là, certes pas dans l'enceinte de confinement, mais elle a soufflé le bâtiment. Au moment de l'explosion, M. Madarame m'a dit: "je n'avais pas imaginé qu'une fuite d'hydrogène hors de l'enceinte de confinement puisse être à l'origine d'une explosion" »(Kan, 2015).

105. Avant l'accident de Fukushima Daiichi, cinquante-quatre réacteurs nucléaires sont exploités au Japon. Yoshida est interrogé par les enquêteurs de l'ICANPS entre les mois de juillet et de novembre 2011. Le recours à l'énergie nucléaire est alors fortement débattu au Japon. En avril 2012, le parc nucléaire japonais est mis à l'arrêt pour inspection. Des réacteurs sont remis en service dans les années suivantes, malgré l'opposition d'une partie de la société civile. Les réacteurs 3 et 4 de la centrale nucléaire d'Ôi (exploitée par KEPCO) redémarrent en juillet 2012, avant d'être de nouveau arrêtés en septembre 2013 pour maintenance. En 2015, les réacteurs de la centrale de Sendai (Kyuden) et de Takahama (KEPCO) sont redémarrés. En 2020, neuf réacteurs nucléaires sont exploités. Les autres réacteurs sont soit toujours à l'arrêt, soit en cours de démantèlement.

106. Deux chefs de la salle de commande des réacteurs 1 et 2 parviennent à ouvrir à $25 \%$ une première vanne du réacteur 1 . Une deuxième équipe est envoyée, mais ne parvient pas à atteindre la deuxième vanne car la radioactivité est trop élevée. La salle de commande tente alors plusieurs fois d'envoyer de l'air pour ouvrir la vanne. Son action échoue et à partir de ce moment, la cellule de crise et la salle de commande adoptent deux plans d'action différents. La cellule de crise veut continuer d'envoyer de l'air en grande quantité. La salle de commande choisit plutôt d'envoyer dans le bâtiment réacteur une autre équipe pour tenter d'ouvrir manuellement la vanne. La cellule de crise n'est pas informée de l'opération. Ikuo Izawa revient sur cette décision, la plus difficile qu'il ait dû prendre durant la crise nucléaire : "Bien que j'aie su qu'il y avait déjà une fuite radioactive dans le bâtiment du réacteur 1 , je me suis déterminé à y envoyer 
les deux cadres. Quand j'ai discuté avec eux après l'accident, ils m'ont dit qu'ils avaient ignoré les dosimètres qui montrent la dose à ce moment-là, car s'ils avaient vu le chiffre de la dose, ils auraient été immobilisés. Ils avaient l'intention d'ouvrir la vanne au péril de leur vie. Je ne me souviens pas d'avoir demandé la permission à la cellule de crise pour cet essai. Au contraire, même si la cellule de crise était opposée, nous serions allés jusqu'au bout » (Kobayashi, 2019, pp. 99-100).

107. Pour rappel, il s'agit de Naoto Kan, né le 10 octobre 1945. Après des études scientifiques à l'Université de technologie de Tôkyô, Kan passe l'examen d'avocat conseil en brevet. Il exerce diverses fonctions politiques avant d'être le Premier ministre du Japon du 8 juin 2010 jusqu'à sa démission le 2 septembre 2011. C'est Yoshihiko Noda, son ministre des finances, qui lui succède.

108. Banri Kaieda, né en 1949, est le ministre de l'Économie, du Commerce et de l'Industrie (METI) entre janvier et septembre 2011. Le METI est institué en 2001, en replacement du MITI (Ministry of International Trade and Industry) qui avait été fondé en 1949.

109. Créée au Japon en 2001, la Nuclear and Industrial Safety Agency (NISA - en français "Agence de sûreté nucléaire et industrielle ») est chargée de superviser le respect de la réglementation dans le domaine de la sûreté nucléaire. Elle est placée sous la tutelle du ministère de l'Économie, du Commerce et de l'Industrie. En septembre 2012, elle fusionne avec la Nuclear Safety Commission pour devenir la Nuclear Regulation Authority.

110. Akio Komori est directeur de la division nucléaire de TEPCO. Auparavant, il a été directeur de la centrale de Fukushima Daiichi.

111. Entreprise japonaise créée en 1977, dont TEPCO est actionnaire à $20 \%$. La JNSS est spécialisée dans diverses activités destinées aux installations nucléaires et aux centrales électriques (R\&D de systèmes et d'équipements de protection ; conception, construction et inspection de maintenance des systèmes et des équipements de protection ; prêt et vente de systèmes et d'équipements de protection ; surveillance des installations; sécurité des transports liés au combustible nucléaire; éducation et formation du personnel de protection).

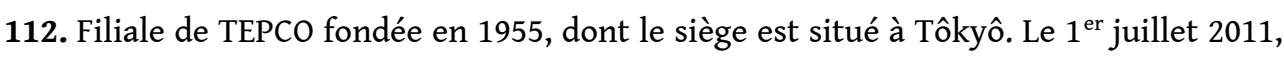
TEPCO annonce la restructuration de Nanmei Kôsan, renommée «Fuel TEPCO Limited ». Ses activités couvrent la vente et le transport de carburant, la vérification des équipements de protection incendie, les travaux de construction pour la prévention des catastrophes, les travaux de sécurité.

113. Village de la préfecture de Fukushima (district de Futaba), situé à quelques kilomètres de Daiichi. C'est à ôkuma que se trouve d'abord le centre de crise hors site, ouvert le 11 mars. Ce centre sera ensuite évacué le 15 mars 2011.

114. Miyakojimachi est un village de la préfecture de Fukushima (district de Tamura), situé à une trentaine de kilomètres de Fukushima Daiichi. Miyakojimachi est à une vingtaine de kilomètres d'ôkumamachi.

115. Naoto Kan explique sa décision d'autoriser l'éventage : «Si je reviens à l'affaire de l'éventage, on m'a dit in extremis, 'il faut faire un éventage, parce que la pression monte. C'est un éventage de la chambre humide, il n'y aura pas trop de rejet. Que faisons-nous ?'. Alors j'ai interrogé tout le monde. La NSC, la NISA, bien entendu, les intéressés eux-mêmes. Leur réponse était que si l'enceinte de confinement venait à exploser, la situation serait très grave, que même 
si l'éventage supposait un certain risque, il fallait le faire. Tout le monde était d'accord là-dessus. Alors, j'ai demandé qu'on procède de cette manière. On effectue un éventage pour dégager la pression pendant un temps. C'est pour cela qu'on le fait. Ce n'est pas parce qu'on a fait un éventage que cela va résoudre le problème. Ça n'est qu'une mesure d'urgence, comme quand on se met à l'abri pour se protéger. Je n'ai jamais pensé que l'éventage résoudrait durablement quoi que ce soit » (Kan, 2012).

116. Le Premier ministre s'est expliqué à ce sujet: "TEPCO me dit que l'éventage est nécessaire, mais il ne l'exécute pas. Comme ils me disent qu'ils ne l'ont pas encore fait, je leur demande pourquoi. Mais là, pas d'explication. Et c'est ça, le plus embêtant. Je crois que c'était M. Takekuro qui était près de moi, je lui demande pourquoi et il me répond qu'il ne sait pas. Ils avaient discuté, tout le monde était d'accord pour cet éventage. Je leur demande expressément de l'effectuer. Mais les choses n'avancent pas. Quand je leur demande pourquoi ça n'avance pas, ils me répondent qu'ils ne savent pas. En fait, c'est tout ça qui va amener la suite. Quelque part, il y a un problème de communication " (Kan, 2012).

117. Le 12 mars, vers 2 h00 du matin, Naoto Kan commence à envisager de se rendre à Fukushima Daiichi. Lorsqu'il fait part de sa décision à son entourage, certains collaborateurs tentent de le faire changer d'avis. Motohisa Ikeda, le vice-ministre du METI, trouve son idée ridicule (Kobayashi, 2019). Kan est conscient des critiques qu'il va subir, mais reste malgré tout déterminé : «J'avais jugé qu'il fallait que j'aille moi-même sur place, à cause, notamment, du séisme et du tsunami. Bien entendu, j'avais vu des images des dégâts qui avaient été causés, des images qui avaient été filmées, mais je voulais voir par moimême ce qu'il en était, même si ce n'était que vu du ciel. L'autre raison, qui rejoint ce que nous venons d'évoquer, était qu'étant donné les difficultés de communication sur la situation à la centrale de Fukushima, j'avais pensé qu'il fallait que je rencontre moi-même le responsable du terrain et que je parle avec lui » (Kan, 2012).

118. L'hélicoptère est un Super Puma des Forces d'autodéfense, construit par Eurocopter (entreprise devenue Airbus Helicopters en 2014) et réservé aux personnalités importantes (Kadota, 2014). Il décolle de la résidence du Premier ministre le 12 mars à 6 h14 et atterrit sur le terrain de sport de Fukushima Daiichi, situé derrière le bâtiment antisismique, à 7 h00.

119. Confusion soit de Yoshida, soit de la personne chargée de retranscrire l'audition : Madarame est le directeur de la Nuclear Safety Commission, et non de la NISA.

120. En mars 2011, le gouverneur de la préfecture de Fukushima est Yuhei Satô, né en 1947. Masao Uchibori, né en 1964, est le vice-gouverneur de la préfecture. Il en devient le gouverneur en 2014.

121. Né en 1950, Sakae Mutô est le vice-Président de TEPCO au moment de l'accident.

122. Les témoignages des personnes présentes lors de cette réunion à Fukushima Daiichi soulignent l'habileté dont Yoshida a fait preuve (Kobayashi, 2019). Dans la salle de réunion, Naoto Kan assaille de questions Sakae Mutô, vice-Président de TEPCO. Selon les témoins, les réponses de Mutô ne satisfont pas le Premier ministre. Yoshida montre alors le plan de Fukushima Daiichi et commence, sans être intimidé, à donner des explications claires et détaillées sur la situation. La qualité des réponses et la détermination du directeur parviennent à faire taire Kan et à le convaincre qu'il est l'homme de la situation. 
123. La réunion avec Yoshida commence à $7 \mathrm{~h} 20$. Elle dure une vingtaine de minutes. Naoto Kan quitte la salle de réunion à $7 \mathrm{~h} 42$ et repart en hélicoptère à 8h04 (Kadota, 2014).

124. Naoto Kan repart très satisfait de son entrevue avec Yoshida. "Nous ne nous étions jamais rencontrés. C'est donc le 12 mars au matin que j'ai fait sa connaissance sur le site de Fukushima Daiichi. C'était un homme franc, qui disait les choses explicitement, sans être intimidé. Il m'a expliqué clairement les raisons du retard de l'ouverture des vannes, ajoutant que, quoi qu'il arrive, il les ouvrirait, quitte à devoir envoyer une équipe prête à risquer sa vie pour ça. J'ai eu une impression très positive de lui. Je me suis dit qu'un homme de sa trempe ne ferait pas les choses à moitié en cherchant à tirer son épingle du jeu, qu'il saurait prendre les bonnes décisions. Je l'ai d'ailleurs dit à mes collaborateurs, une fois de retour à la résidence. Je sais que M. Yoshida m'a critiqué auprès de la Commission d'enquête sur les causes de l'accident mise en place par le Gouvernement. Mais je ne suis pas le seul. Il a été également très critique vis-à-vis de sa hiérarchie de TEPCO. Et ce n'est pas parce qu'il m'a critiqué que j'ai une mauvaise opinion de lui. C'est quelqu'un qui disait clairement ce qu'il pensait sans tourner autour du pot, qu'il s'agisse de ses supérieurs hiérarchiques ou d'un Ministre » (Kan, 2015). Kan considère par ailleurs que cette visite était essentielle et ne correspondait à aucune forme d'ingérence de sa part: "cette visite a été positive en ce qu'elle a permis de partager des informations fiables sur le déroulement de l'accident. Savoir également que la centrale était entre les mains de quelqu'un comme M. Yoshida m'a aussi aidé dans la gestion de la crise par la suite. Parler d'ingérence excessive de la part du Cabinet du Premier ministre est un énorme malentendu. À aucun moment, le Kantei est allé donner des ordres directs de nature opérationnelle, par exemple pour exiger l'ouverture des vannes. Par contre, TEPCO n'a aucune autorité pour décider de l'évacuation des populations. Le 12 mars, je suis allé demander pourquoi les vannes n'avaient pas été ouvertes. Parce que si l'enceinte de confinement venait à exploser, ce n'est pas une évacuation de 10 kilomètres à la ronde que je devais ordonner, mais une évacuation dans un rayon beaucoup plus large. Ce jugement est entièrement du ressort du Premier ministre. Je ne considère pas qu'aller à Fukushima Daiichi pour obtenir des informations fiables permettant d'ordonner une évacuation soit une ingérence excessive. C'est tout le contraire!» (Kan, 2015).

125. Yoshida nie dans ce passage que la visite de Naoto Kan ait pu ralentir les opérations menées sur le terrain. Le rapport de la NAIIC (2012) considère toutefois que Kan, en se rendant à Fukushima Daiichi, a détourné l'attention du personnel, lui a fait perdre du temps et a perturbé la chaîne de commandement hiérarchique.

126. Ryuta Idogawa assimile la situation où les vannes d'éventage du réacteur 1 sont restées fermées le 12 mars 2011 à « Kyokugen ", ce qui signifie en japonais « situation qui arrive à la limite, extrémité »: « Nous, les pilotes de la salle de commande des réacteurs 1 et 2 , n'avions plus du tout de ressources pour surmonter la situation donnée. C'était comme si nous étions à l'avant-garde de la bataille, devant l'ennemi, bien que nous n'avions plus de munitions » (Kobayashi, 2019, p. 176).

127. Mentionné précédemment par le sigle «MOV ».

128. Exposition externe d'un corps ou d'une substance à des rayonnements ionisants.

129. La piscine de dépressurisation correspond au wetwell, également nommé chambre de condensation.

130. Instrument désigné précédemment dans l'audition par le sigle « APD ». 
131. Le 12 mars, à 00h06, Yoshida donne l'ordre de préparer l'éventage. Dans la salle de commandes des réacteurs 1 et 2 , la tension montent chez les jeunes, qui ont de plus en plus de mal à contrôler leur émotion du fait de la dégradation de l'état du réacteur 1. Ikuo Izawa décide alors de rassembler ses équipes autour de la table du chef de salle et de leur dire : «Le directeur de la centrale nous a demandé de mettre en cuvre l'éventage. Nous devons sélectionner les membres, mais nous ne pouvons pas y envoyer les jeunes. C'est moi qui vais tout d'abord ouvrir la vanne. $Y$ en a-t-il quelques-uns qui $y$ viennent avec moi ?» (Kobayashi, 2019, p. 92). Les autres cadres présents la salle de commande, plus âgés qu'Izawa, réfutent toutefois sa proposition. Pour eux, Izawa doit rester dans la salle pour diriger les opérations. Six chefs proposent dès lors de le remplacer pour effectuer l'éventage et décident de se joindre au groupe d'intervention.

132. Vanne précédemment mentionnée par le sigle «AOV ».

133. Le sigle «IDSA » n'a pu être défini faute de référence.

134. Le disque de rupture est un dispositif à usage unique de protection contre les surpressions. Lorsque le système protégé atteint une pression prédéterminée, la membrane du disque de rupture éclate instantanément. Afin d'éviter la fuite de matériaux radioactifs de l'enceinte de confinement des réacteurs vers l'atmosphère, les lignes d'éventage sont scellées grâce à des disques de rupture. Dans les réacteurs de Fukushima Daiichi, ces disques sont conçus pour rompre à la pression nominale de l'enceinte de confinement (soit 5 bar). Cependant, si ces disques de rupture permettent d'éviter l'ouverture accidentelle des vannes d'isolation du confinement, ils limitent également la possibilité d'éventage de l'enceinte du confinement à basse pression et peuvent dès lors s'avérer contre-productifs.

135. Avant de prendre sa décision d'injecter de l'eau de mer dans le réacteur 1, Yoshida, inquiet, prend conseil auprès de Shiro Hikida. Ce dernier le rassure : «Ton inquiétude est hors de propos. N'hésite pas! Les réacteurs ont été fabriqués en tenant compte du pire et il est vrai que depuis la construction du réacteur 3, tous les réacteurs peuvent accepter de l'eau de mer. Le réacteur 1 est plus vieux que le réacteur 3, mais je t'assure que l'injection de l'eau de mer ne causera aucun problème sérieux » (Kobayashi, 2019, p. 105). Convaincu par les arguments d'Hikida, Yoshida décide dès lors de procéder à l'injection d'eau de mer.

136. Une pompe booster permet d'augmenter la pression d'arrivée d'eau dans les pompes avant l'injection. Les camions de pompiers ont alors été connectés en série pour injecter de l'eau dans la cuve du réacteur à partir du niveau de la mer. 


\section{Compte rendu de l'audition du 29 juillet 2011}

1 [À traiter avec la plus grande attention]

16 août 2011

Tsunemasa Katô (rapporteur)

Membre du secrétariat de la Commission d'enquête sur l'accident de la centrale nucléaire de Fukushima de Tôkyô Electric Power Company (Investigation Commitee on the Accident at the Fukushima Nuclear Power Station of Tôkyô Electric Power Company)

Ci-dessous le compte rendu de l'audition du 29 juillet 2011, menée dans le cadre de l'enquête sur l'accident de la centrale nucléaire de Fukushima de Tôkyô Electric Power Company.

1. Auditionné, date d'audition, lieu d'audition, enquêteurs.

1. Auditionné

Masao Yoshida, Directeur de la centrale nucléaire Fukushima Daiichi de Tôkyô Electric Power Company.

2. Date d'audition

29 juillet 2011 de $10 \mathrm{~h} 25$ à $11 \mathrm{~h} 57$ (sans pause)

29 juillet 2011 de $12 \mathrm{~h} 31$ à $14 \mathrm{~h} 50$ (sans pause)

3. Lieu d'audition

Salle de réunion $\mathrm{A}$

Foyer féminin de la Japan Football Association Academy de Fukushima ( $1^{\text {er }}$ étage)

$\mathrm{J}$-Village

8-Utsukushimori Yamadaoka, Naraha, Futaba District, Fukushima

4. Enquêteurs

Yôtarô Hatamura, Kunio Yanagida, Masao Fuchigami, Tsunemasa Katô, Atsushi Oikawa, Toshitaka Nagata.

5. Enregistrement par enregistreur numérique

- Oui Non

2. Contenu de l'audition

Situation au moment de l'accident et réponses à l'accident

Voir le compte rendu 
3. Mentions spéciales

Pour faciliter les explications et aider la mémoire de l'auditionné, les documents Report regarding the Plant Data of Fukushima Daiichi Nuclear Power Station at the time of the Earthquake publié par TEPCO le 16 mai 2011, le First Response at Tôhoku Chihou Taiheiyou Oki Earthquake in Fukushima Daiichi Nuclear Power Station publié par TEPCO le 18 juin 2011 et le plan Itinéraire de la visite du Comité d'enquête ont été utilisés.

Ce plan Itinéraire a été ajouté à la fin du compte rendu.

QUESTION : Je suis Katô, du secrétariat. C'est moi qui vais vous questionner pour commencer. La dernière fois, nous avons terminé un peu au pas de course, mais nous sommes arrivés jusqu'à l'explosion du réacteur 1, c'est-à-dire le 12 mars vers 15h36. Tout d'abord, le 12 à 15h36, lorsque l'explosion s'est produite au réacteur 1, étiez-vous dans la salle de crise du bâtiment antisismique?

Réponse : Oui.

$\mathbf{Q}$ : Comment avez-vous compris qu'il y avait eu explosion?

$\mathbf{R}$ : La situation était la suivante. Nous n'avions absolument pas envisagé la possibilité d'une explosion. Sur le terrain, les préparatifs pour mettre en marche le système d'injection d'acide borique (SLC) du réacteur 1 venaient de s'achever. Nous en étions à un stade où il suffisait de pousser sur le bouton, enfin, il suffisait de manœuvrer à partir de la salle de commande, pour que l'injection se fasse dans le réacteur.

Nous nous apprêtions donc à injecter. D’abord, il faut que vous sachiez que, du bâtiment antisismique, on ne voit pas du tout le réacteur 1. Comme la radioactivité était forte, nous ne pouvions pas sortir. Donc, personne n'était sorti pour voir ce qui se passait à l'extérieur. Nous avons ressenti une secousse pendant un laps de temps très court, comme une forte poussée du bas vers le haut. Nous avons pensé qu'il s'agissait encore d'un tremblement de terre ${ }^{1}$.

Très vite, nous avons eu des informations. D'abord un agent qui revenait du terrain nous a dit qu'il ne restait plus que les piliers dans la partie haute du bâtiment réacteur de la tranche 1 . Pendant qu'on se demandait ce qui s'était passé, un blessé est aussi arrivé et, d'après lui, les gens sur le terrain parlaient d'une explosion de la partie supérieure du bâtiment réacteur 1.

Je n'ai donc pas vu de mes propres yeux l'explosion. Je n'ai eu connaissance de la situation que par ce qu'on m'a raconté.

Sur ce, bien que la radioactivité était encore très forte, j'ai envoyé un agent sur les lieux pour qu'il constate la situation de visu. Et, effectivement, la partie supérieure n'avait plus de murs et ne restaient que les piliers.

Tout de suite après cette vérification, j'ai fait mon rapport au siège. Qu'il semblait y avoir eu une explosion et qu'on n'en connaissait pas la cause. Il y avait plusieurs hypothèses en concurrence. À l'intérieur de la centrale nucléaire, il y a ce qu'on appelle un générateur électrique principal, qui fournit l'électricité. Pour refroidir ce générateur, nous utilisons de l'hydrogène. Certains pensaient que c'était cet hydrogène qui avait pris feu. Au tout début, on ne savait pas quelle était la cause de cette explosion. Aujourd'hui, nous savons que c'est très probablement l'hydrogène qui s'est échappé de l'enceinte de confinement, qui s'est accumulé en haut et que 
c'est lui qui a explosé. Mais ce sont des choses qu'on ne comprend qu'après coup. Sur le moment, nous ne savions pas quelle en était la cause.

Q : Ce que vous venez d'évoquer en dernier, l'hypothèse que de l'hydrogène s'était échappé de l'enceinte de confinement, s'était accumulé en haut du bâtiment et que c'est lui qui a explosé. Ce genre d'hypothèse, à partir de quel moment en avez-vous parlé ?

$\mathbf{R}$ : On en a parlé le jour même, mais après un temps. Il y avait toutes sortes d'avis. Tout d'abord, un agent qui était sur le terrain a raconté qu'il y avait des étincelles qui partaient du bâtiment turbine. Un autre, qui était près du bâtiment, s'est demandé s'il ne s'agissait pas de l'hydrogène du générateur principal, puisqu'on avait parlé de turbine. Mais on s'est dit que, dans ce cas, il était étrange que le bâtiment turbine n'ait pas été détruit. On a aussi échangé avec le siège et, au milieu de tout ça, au bout de deux heures à peu près, on s'est posé la question de l'hydrogène qui se serait échappé de l'enceinte de confinement et conclu que cette possibilité était très forte. Je ne me rappelle plus exactement de l'heure, mais je suis sûr que nous en avons parlé le jour même. Le jour même, nous étions arrivés à la conclusion que l'hypothèse la plus probable était que l'explosion était due à l'hydrogène qui venait de l'enceinte de confinement.

Q : À votre avis, quelle était la situation du réacteur 1, l'état à l'intérieur de la cuve, l'état du cœur, ce 12 mars en début d'après-midi, avant l'explosion?

$\mathbf{R}$ : Comme la pression de l'enceinte de confinement était élevée, nous avons voulu faire un éventage, éventage que nous avons exécuté. Nous en avons parlé la dernière fois, mais il est difficile d'avoir l'assurance que l'éventage s'est véritablement fait ou pas. Pour ma part, aujourd'hui, je n'en suis toujours pas sûr. Je n'en ai pas la certitude. Puisqu'on ne disposait d'aucun des moniteurs qui pouvaient, seuls, le démontrer. En plus, il y a le disque de rupture. Même si on ouvre la vanne, s'il n'y a pas déséquilibre de pression, le disque ne rompt pas et l'éventage ne se fait pas. Alors savoir si le disque a rompu ou pas, il n'existe aucun instrument qui le montre. C'est une disposition mécanique toute simple. Si la pression augmente, le disque se rompt. Finalement, si le moniteur de la cheminée n'est pas opérationnel, on ne peut pas savoir si le rejet s'est véritablement fait ou pas. Pour nous, nous étions concentrés, premièrement, sur l'éventage, et deuxièmement, sur l'injection d'eau. Nous travaillions concentrés essentiellement sur ces deux axes. Oui, il y avait aussi le système acide borique, nous travaillions aussi sur le SLC.

Q: Et, pendant que vous travailliez sur l'injection, l'éventage, pensiez-vous au cœur du réacteur, au niveau d'eau du réacteur? Bref, aviez-vous conscience que le cœur devait être dénoyé et qu'il y avait des risques qu'il soit assez largement endommagé ?

$\mathbf{R}$ : Oui, j'en avais conscience.

Q: Aviez-vous conscience que dans ce cas-là, lorsque le cœur se trouve hors de l'eau dans la cuve, il y a une forte production d'hydrogène?

R : Bien sûr, je le savais.

Q: Est-ce que vous le saviez de manière théorique, parce que c'est ce qu'on déduit logiquement de la situation, ou aviez-vous conscience qu'au moment même où vous vous activiez, il y avait peut-être effectivement production d'une grande quantité d'hydrogène ?

R : Oui, j'y pensais. Seulement, ça paraît bête aujourd'hui, mais je pensais que cet hydrogène restait dans l'enceinte de confinement. Bien sûr, il pouvait y en avoir une partie qui fuyait. Mais je pensais qu'a priori l'hydrogène se maintenait à l'intérieur de l'enceinte et qu'il fallait, pour ça, baisser tout d'abord la pression de l'enceinte. Ce qui 
signifie qu'il fallait laisser sortir l'hydrogène qui se trouvait à l'intérieur. Enfin, y compris l'hydrogène, puisque la cause de l'augmentation de la pression était la vapeur qui était produite à l'intérieur de l'enceinte et qui contenait l'hydrogène. Il fallait donc faire un éventage pour laisser échapper cette vapeur. L'objectif premier était de faire baisser la pression de l'enceinte. Pour cela, il fallait une manœuvre d'éventage, puisqu'elle sert à ça. C'était la première chose à faire. Et on y travaillait depuis le matin, plus exactement depuis la nuit précédente.

Et on a enfin réussi à ouvrir la vanne et, je ne sais plus s'il était 14h00, la pression a chuté. En plus, on en a parlé la dernière fois, juste après, en regardant les images de la caméra de la NHK, on a vu une fumée blanche s'échapper de la cheminée principale. Nous avons été surpris. On s'est demandé ce qui s'était passé. Et puis, on s'est dit que l'opération d'éventage avait dû réussir. C'est là qu'on a eu la sensation qu'on avait réussi l'éventage.

Seulement, ce ne sont que des preuves par présomption. Effectivement la pression de l'enceinte de confinement avait baissé. Mais ce qu'il fallait véritablement vérifier, c'était les chiffres du monitoring. Si on avait pu avoir ces chiffres, cela aurait été la preuve irréfutable que l'éventage s'était fait. Mais en l'absence de ces chiffres, j'ai parlé de présomption de réussite de l'éventage dans mon rapport.

Q: Si j'ai bien compris, c'est ensuite que l'explosion s'est produite. Et après moult discussions concernant sa cause, vous avez fini, plus tard, par relier ensemble les faits que le cœur du réacteur était sûrement assez largement dénoyé, qu'une grosse quantité d'hydrogène avait été produite à l'intérieur de la cuve, que cet hydrogène était sorti de la cuve vers l'enceinte de confinement et qu'une partie s'en était encore échappée vers le haut du bâtiment où il s'était accumulé. C'est bien ça ?

R : Oui.

Q: Et si on remonte dans le temps, effectivement, la veille, dans la nuit du 11 mars, à l'intérieur et aux alentours du bâtiment réacteur, la radioactivité était montée de manière assez sensible et il y avait de fortes chances que l'hydrogène s'échappe à un bon rythme de l'enceinte de confinement, n'est-ce pas?

$\mathbf{R}$ : Oui, la radioactivité était élevée.

Q: En rassemblant toutes ces observations, vous en êtes arrivés à la conclusion que I'hypothèse la plus plausible était quand même que l'hydrogène s'était accumulé dans le bâtiment et que c'était lui qui avait explosé. Ai-je bien compris ?

$\mathbf{R}:$ Oui, tout à fait.

Q : Excusez-moi de revenir encore en arrière, mais, à ce moment-là, avant l'explosion, vous aviez œuvré pour pouvoir utiliser le circuit d'injection d'eau à l'acide borique, en reliant un camion générateur du côté du tableau électrique $2 \mathrm{C}$ et en tirant des câbles. Je pense que vous faisiez ça pour le réacteur 1 et aussi le réacteur 2 . Et on pouvait considérer que le système SLC était tout à fait prêt et que vous vous apprêtiez à faire l'injection, c'est bien ça?

R : Oui.

Q: : Encore une chose. Concernant le réacteur 1, le 12 mars, à la page 8 de la chronologie, on voit à 14h53: « fin de l'injection d'eau douce dans le réacteur par camion de pompiers, volume injecté $80 t$ ». Ensuite, à 14h54, il y a "ordre du directeur de la centrale d'injecter l'eau de mer dans le réacteur ». C'est la première fois que vous donnez l'ordre d'injecter de l'eau de mer, n'est-ce pas?

$\mathbf{R}$ : Enfin, c'est la première fois que c'est écrit. Parce qu'étant donné qu'on savait pertinemment qu'à un moment ou à un autre on épuiserait l'eau douce, j'avais déjà 
dit qu'il fallait se préparer à injecter de l'eau de mer dès l'après-midi, ce jour-là. Comme je vous l'ai dit la dernière fois, il fallait réfléchir à l'endroit d'où on allait pomper l'eau de mer. Une question très difficile. On ne peut pas pomper directement de la mer. Il n'y a pas de pompe directement dans la mer, nulle part. On a évoqué ce problème tout à la fin, la dernière fois. C'est là qu'on a su qu'il restait de l'eau du tsunami dans le bassin attenant au bâtiment turbine du réacteur 3, qu'il y en avait un certain volume. En puisant de là, on a besoin de moins de pression, alors je savais déjà que ce serait la meilleure solution avant même de donner l'ordre d'exécution. J'avais déjà pris ma décision. Et effectivement j'ai donné l'ordre d'injection à $14 \mathrm{~h} 54$, mais on avait commencé à étudier cette possibilité bien avant.

$\mathbf{Q}$ : Donc, cet ordre signifie qu'étant donné que les sources les plus proches d'eau douce commençaient à s'épuiser, il fallait passer à l'eau de mer. L'idée d'utiliser l'eau de mer, vous l'aviez déjà vers midi, ce jour-là?

$\mathbf{R}$ : Oui, elle était déjà là.

Q : Aviez-vous déjà entendu parler, durant votre carrière, d'injection d'eau de mer dans un réacteur?

$\mathbf{R}$ : Non, jamais. Je n'en ai pas entendu parler, parce que nulle part au monde, on ne l'avait jamais fait. Mais pour refroidir, la seule source dont nous pouvons disposer sans limite, c'est la mer. L'eau douce, comme on l'a dit tout à l'heure, est limitée. À un moment ou à un autre, forcément, elle va s'épuiser. Alors, il n'y avait pas d'autre solution que d'envoyer de l'eau de mer. On n'avait pas le choix. Il fallait refroidir à tout prix. Ce que j'avais en tête à ce moment-là, c'était tout faire pour trouver un moyen d'abaisser la pression de l'enceinte de confinement. Ensuite, injecter, injecter, injecter de l'eau dans le réacteur. Il n'y avait que ces deux points. C'était l'essentiel, tout le reste n'était qu'accessoire. Je ne pensais à rien d'autre qu'à ces deux choses. Alors, envoyer de l'eau de mer, c'était tout à fait normal. Pour dompter cette chose qui était en train de se déchaîner, il n'y avait que ça, l'eau de mer.

$\mathbf{Q}$ : Pendant que vous réfléchissiez à tout ça, au moment où vous alliez manquer d'eau douce, que vous alliez passer à l'eau de mer, avez-vous fait part de vos réflexions à quelqu'un ? À quelqu'un du siège ou à un membre de la cellule de crise ? En avez-vous parlé à quelqu'un en demandant son avis ?

$\mathbf{R}$ : Il n'y a pas de trace écrite ici, mais je me rappelle que je parlais déjà dans l'aprèsmidi de prévoir de passer à l'eau de mer quand on n'aurait plus d'eau douce.

$\mathbf{Q}:$ En téléconférence?

$\mathbf{R}$ : C'était peut-être au téléphone. Ma mémoire me joue des tours. Mais je me rappelle très bien avoir dit qu'en ce qui me concerne, moi, j'avais pris la décision d'injecter de l'eau de mer.

$\mathbf{Q}:$ : I s'agissait de l'injection d'eau de mer dans le réacteur 1 ?

$\mathbf{R}$ : Bien sûr, parce que c'était le plus inquiétant.

$\mathbf{Q}$ : Et lorsque vous avez révélé vos intentions, y a-t-il eu des avis contraires?

$\mathbf{R}:$ Non.

$\mathbf{Q}:$ Vous n'en avez pas eus?

$\mathbf{R}$ : Vous savez, pour refroidir, il fallait de l'eau. Si on n'avait plus d'eau douce, il fallait bien mettre de l'eau de mer. Il n'y avait pas d'autre solution. Alors je leur ai dit que j'allais utiliser de l'eau de mer. 
$\mathbf{Q}$ : La décision d'envoyer de l'eau en quantité à un bon débit, vous l'avez prise à cause du niveau d'eau de la cuve? De la température ? Plutôt le niveau d'eau?

$\mathbf{R}$ : Le niveau d'eau.

$\mathbf{Q}$ : II n'y avait pas assez d'eau, donc il fallait en envoyer rapidement?

$\mathbf{R}$ : Oui. On ne savait pas à quel point l'enceinte de confinement et la cuve avaient été abîmées. Alors, si le combustible était dégradé, il n'y avait que la solution d'envoyer de l'eau pour le noyer et le refroidir. C'était la solution la plus simple.

$\mathbf{Q}$ : Cela veut dire que l'indicateur de niveau d'eau était redevenu assez fiable?

$\mathbf{R}$ : Non. On ne savait pas si on pouvait lui faire plus ample confiance. On ne saura jamais, mais il semblerait qu'il se soit coincé une première fois. Il a indiqué un certain niveau, puis tout à coup ça a chuté brutalement. Alors, on ne savait pas si on pouvait lui faire plus confiance parce qu'on avait envoyé de l'eau, mais, pour nous, il fallait envoyer de l'eau. C'était la seule chose à faire.

$\mathbf{Q}$ : Est-ce qu'il y a des inconvénients à envoyer trop d'eau?

$\mathbf{R}:$ Non.

$\mathbf{Q}$ : Encore une chose. C'est sûrement une question superflue, mais n'avez-vous pas pensé qu'en mettant de l'eau de mer, vous rendiez toutes ces machines inutilisables, que ça allait coûter beaucoup d'argent? N'avez-vous pas pensé qu'il fallait tenir le plus longtemps possible avec de l'eau douce ? Je sais que c'est petit de penser ça, mais...

$\mathbf{R}$ : Absolument pas. Je savais que quand on arrive à un stade où le combustible est endommagé, un réacteur est irrécupérable. Alors, la priorité des priorités était de calmer le réacteur. Je n'ai pas pensé une seconde à une réutilisation.

$\mathbf{Q}$ : Alors, à cet instant précis, le réacteur nucléaire, c'était comme un être vivant qui se débattait dans tous les sens?

R : Oui.

$\mathbf{Q}:$ Très bien.

$\mathbf{Q}$ : Et donc, vous aviez tout préparé pour l'injection d'eau de mer et, à ce moment-là, au moment de l'explosion, je veux dire, vous en étiez au stade où vous vous apprêtiez véritablement à injecter cette eau.

$\mathbf{R}$ : Oui. Je venais d'avoir des rapports qui disaient que le circuit eau de mer était fin prêt et que du côté de l'eau à l'acide borique, aussi, tout était quasiment prêt. On avait enfin réussi à trouver des moyens d'introduire de l'eau, on se disait qu'on allait enfin pouvoir refroidir, une lueur d'espoir commençait à luire et puis, boum, cet espoir a volé en éclats.

$\mathbf{Q}$ : Après l'explosion, vous aviez donc des blessés et vous avez sans doute revérifié l'état de vos troupes. Ensuite, vous avez aussi vérifié, je suppose, l'état du terrain autour du réacteur 1 , où des hommes travaillaient à l'installation de la source électrique, le câblage, etc., pour le système SLC, et puis le circuit pour l'eau de mer, pour voir l'étendue des dégâts.

$\mathbf{R}$ : Oui. Tout d'abord, ce sont les hommes qui travaillaient sur ces opérations qui sont arrivés. Je leur ai demandé quelle était la situation. Ils m'ont répondu que ça avait explosé, que le dessus du bâtiment avait sauté, que des tas de débris avaient volé et qu'ils continuaient à tomber. Ensuite, on m'a raconté, et tous les hommes qui sont arrivés successivement du terrain me l'ont dit, même si je ne me souviens plus des termes exacts, que le camion générateur était fichu. Une fois qu'on avait fait l'appel, il fallait revérifier tout ça, alors j'ai envoyé quelqu'un faire le tour pour un état des lieux. Et résultat, on s'est retrouvé dans cette situation où on ne pouvait plus injecter 
d'eau parce que le camion de pompiers, lui-même, avait été touché et n'était provisoirement plus en état de fonctionner. Alors qu'on était sur le point d'injecter via le système SLC et que l'injection d'eau de mer devenait enfin possible, notre espoir s'est brisé d'un coup. Il fallait immédiatement trouver autre chose.

$\mathbf{Q}$ : Et, au début, vous n'avez pas tout de suite su quelle était la cause de cette explosion, n'est-ce pas?

$\mathbf{R}:$ Non.

$\mathbf{Q}$ : Malgré cette explosion d'origine inconnue, dont vous vous demandiez au départ s'il ne s'agissait pas d'un problème dans le bâtiment turbine, il me semble, est-ce que vous avez renvoyé le personnel travailler de nouveau sur le terrain, pour les travaux de remise en ordre, par exemple, ou vous avez interrompu les opérations ?

$\mathbf{R}$ : On a interrompu temporairement les travaux. J'ai demandé à tout le monde de se mettre à l'abri dans un premier temps. Je ne sais plus combien de temps ça a duré, mais, dans un premier temps, j'ai donné l'ordre que tout le monde se mette à l'abri. Ensuite on a fait l'appel. Pendant qu'on faisait l'appel, l'agent du groupe « maintenance » qui se trouvait le plus près est arrivé avec un bras cassé. C'était celui qui se trouvait le plus proche de l'explosion et le souffle de l'explosion lui avait fracturé le bras. Je lui ai demandé quelle était la situation et il m'a répondu que c'était catastrophique. Pendant qu'on échangeait comme ça sur la situation, personne n'était sur le terrain.

Ce qui nous faisait le plus peur, c'était évidemment que l'enceinte de confinement ait explosé. Mais on s'est rendu compte, en regardant les données, qu'elles n'avaient pas beaucoup bougé entre l'avant et l'après explosion. Si l'enceinte avait explosé, la pression de l'enceinte serait tombé à zéro, ou aurait, tout au moins, marqué un brusque changement. Or, les chiffres étaient stables. On en a donc déduit qu'elle était sauve. De plus, le fait que quelque chose ait explosé supposait que tout avait explosé d'un coup et qu'il ne restait probablement plus de produit inflammable. Avant toute chose, il fallait aller injecter l'eau. C'était une obligation. Alors, et c'est ce qui se passera aussi ensuite pour le réacteur 2 et le réacteur 3 , il a fallu arbitrer entre le danger auquel j'exposais mes hommes et l'urgence de la situation. Pour moi, ça a été une des choses les plus difficiles. Fallait-il les retenir à l'abri ou les envoyer œuvrer? Mais si on ne travaillait pas à la restauration de ces circuits, on ne pouvait pas avancer à l'étape suivante. Il a toujours fallu que je trouve un juste milieu entre les deux. Et c'est dans ces conditions que je leur ai demandé de retourner préparer à nouveau le circuit pour l'injection.

$\mathbf{Q}$ : Après ça, pour le réacteur 1 , vous avez préparé le circuit d'injection d'eau de mer, avec un camion de pompiers qui pompe l'eau du bassin, puis l'injecte à partir de la prise d'eau du bâtiment turbine de la tranche 1. Vous avez aussi voulu rétablir le système SLC en reliant le camion générateur et le tableau électrique $2 \mathrm{C}$...

$\mathbf{R}$ : Non. J'y ai renoncé, parce que le camion générateur ne fonctionnait plus en tant que générateur.

Q: Avez-vous demandé un autre camion générateur, pour le remplacer ?

$\mathbf{R}$ : En ce qui concerne les camions générateurs, ils ne servaient pas qu'à ça. Comme je savais qu'à un moment ou à un autre, on aurait de toute façon besoin d'une quantité astronomique d'électricité, j'avais demandé depuis le début à ce qu'on nous envoie tous les camions générateurs qui leur tomberaient sous la main. C'est pour ça que ce n'est pas parce que celui-ci était tombé en panne que j'en demandais un autre. De 
manière générale, en ce qui concerne l'alimentation électrique, les batteries, les camions de pompiers, depuis le début, j'avais donné comme consigne qu'on nous envoie tout. Alors, il n'y a pas eu de commande spéciale pour remplacer ce camion générateur. Bien sûr, faire venir des camions générateurs, ça prend du temps. J'ai bien pensé à faire réparer celui qui était tombé en panne, mais je me suis dit que la priorité, c'était l'injection d'eau de mer.

$\mathbf{Q}$ : Comme mesure après l'explosion, vous pensiez à l'injection d'eau, qui plus est de l'eau de mer, pour le réacteur 1, et vous faisiez travailler les agents dans ce sens.

R : Oui.

Q: Et dans la chronologie, vers la fin de la page 13, on lit qu'enfin à 19 h04 vous commencez à injecter de l'eau de mer dans le réacteur en utilisant le réseau incendie et les camions de pompiers. Ce qui veut dire, j'imagine, que vers le début de soirée le circuit était prêt et que vous avez commencé à injecter l'eau.

R : Oui.

$\mathbf{Q}$ : Si on regarde, par exemple, parmi les paramètres concernant le niveau d'eau et la pression du réacteur 1 dans ce document qui a été publié sur le site internet de TEPCO, à la page 4, il n'y a pas de référence à $19 \mathrm{~h} 04$ le 12 mars, mais on trouve les chiffres de 20h08, environ une heure après. La pression du réacteur est notée 0,370 MPa. Ensuite les chiffres se baladent toujours autour de 0,36 ou 0,35. Avec une pression de ce niveau dans le réacteur, l'eau peut entrer?

R : Oui.

$\mathbf{Q}:$ Je suis désolé de sauter d'un document à l'autre, mais je voudrais passer maintenant à l'information suivant l'article 15. II s'agit du vingt-sixième rapport. II s'agit du document qui porte le nombre « 27 » écrit à la main, tout en haut à droite. C'est le vingt-sixième rapport suivant l'article 15. II a été émis à 20h38. Dans le cadre prévu pour noter les évènements à signaler, il est écrit à la main : «20h20, début de l'injection d'eau de mer dans le réacteur par le réseau incendie ». Si on se fie à ce document, le début de l'injection se situe à 20h20. Or, si on regarde la chronologie publiée par TEPCO, cette injection d'eau de mer avait déjà commencé à 19h04. Comment expliquez-vous ce décalage?

R : Oui. On en a beaucoup parlé. Pour nous, nous avions obtenu l'accord pour cette injection d'eau de mer bien avant le début de l'opération. Le siège était au courant et j'avais fait un rapport pour dire qu'on avait commencé l'injection d'eau de mer à 19 h04.

On a dit beaucoup de choses à propos de ça. Mais, je vais être très franc avec vous, assez vite après le début de l'opération, j'ai reçu un coup de fil de Takekuroº ${ }^{2}$, qui était chez le Premier ministre. Et là, ça va peut-être être un peu différent de ce qui a été communiqué à la presse vers le 20 mai. Mais, si je vous rapporte fidèlement le contenu de ce que j'ai entendu au téléphone, il a dit : « le Premier ministre n'a pas encore donné son accord concernant l'injection d'eau de mer». Et il m'a ordonné d'arrêter l'injection d'eau de mer. Oui, c'est Takekuro qui me l'a ordonné. Il ne m'a donné aucune explication, ni contexte. Il m'a juste dit d'arrêter ${ }^{3}$.

Alors j'ai tout de suite contacté le siège en rapportant ma conversation avec Takekuro et je leur ai demandé s'ils étaient au courant. Je pense qu'à ce moment-là Komori n'était pas là, il devait être à une conférence de presse ou quelque chose comme ça. J'en ai donc parlé avec Takahashi ${ }^{4}$. On avait jugé qu'il était difficile de passer outre et qu'on allait arrêter. Seulement, comme nous avions déjà envoyé de l'eau dans le réacteur, nous avons décidé de présenter ça comme un essai. Un essai pour voir si le circuit était viable ou pas. D'ailleurs, il me semble que c'était le siège 
qui avait imaginé cette histoire d'essai. Parce que, voyez-vous, nous, on ne pensait absolument pas à ce genre de chose. Et donc, nous avons décidé de présenter l'injection de 19h04 comme un essai d'injection.

On avait donc fini de faire l'essai et on allait s'arrêter. On avait décidé de s'arrêter. Seulement, moi, arrivé à ce stade, je n'avais aucune intention d'arrêter l'injection d'eau. De plus, ils parlaient d'un arrêt, mais on ne savait même pas combien de temps allait durer cet arrêt. Ils auraient parlé d'un arrêt de trente minutes, passe encore. Mais un arrêt avec aucune garantie de reprise. Pour moi, il n'était pas question de me soumettre à un tel ordre. J'ai décidé de faire à ma manière. Alors, j'ai effectivement annoncé à ceux qui se trouvaient à la table de crise qu'on allait arrêter, mais j'avais discrètement pris à part le responsable du groupe "sûreté ", XXXXX, qui était en charge de l'injection et lui avais dit que j'allais annoncer à la cantonade qu'on allait arrêter l'injection, mais que lui, à aucun prix, ne devait arrêter d'envoyer l'eau. Ensuite j'ai fait un rapport au siège pour dire qu'on avait arrêtés.

Après, on se retrouve dans les discussions du vingt-septième rapport. Plus tard, je ne sais plus à quelle heure, l'autorisation d'injecter arrive du Premier ministre, sans doute juste avant le vingt-septième envoi, et, du coup, on fait un rapport annonçant, après l'essai, le début de la véritable injection à $20 \mathrm{~h} 20$, qui sera l'objet de cette vingtseptième information.

$\mathbf{Q}$ : Donc, tout d'abord, un peu après 19h04, très peu après, vous recevez un coup de fil de monsieur Takekuro vous disant de la part du Gouvernement d'attendre pour l'injection d'eau de mer, que l'accord n'est pas encore établi. Sur ce, vous vous adressez à monsieur Takahashi, qui se trouve au siège.

R: Oui, puisque nous étions en téléconférence.

$\mathbf{Q}$ : Vous lui avez donc parlé en téléconférence?

$\mathbf{R}$ : Takekuro était à la résidence du Premier ministre ${ }^{6}$ et comme la résidence ne faisait pas partie de la téléconférence, il m'a téléphoné. Je lui ai dit qu'on ne pouvait pas faire une chose pareille, qu'on venait juste de commencer l'injection, après tout le mal qu'on s'était donné. Pour parler tout à fait franc, il m'a dit de manière très autoritaire d'arrêter de discutailler et d'arrêter (l'injection). Ça m'a révolté, mais j'ai raccroché. Puis j'ai contacté le siège en les informant de ce que me disait Takekuro et en leur demandant si, de leur côté, ils avaient aussi reçu des ordres. Mais Takahashi n'a pas été très clair, je n'arrivais pas à savoir s'ils avaient eu des directives ou pas. Peut-être en avait-il reçu et tentait-il ainsi de les ignorer ? L'idée m'a effleuré. Il faudrait l'interroger en personne.

$\mathbf{Q}$ : Cet échange avec monsieur Takahashi, il s'est fait par téléconférence, c'est ça ?

R : Oui.

$\mathbf{Q}$ : En fin de compte, au siège, ils pensaient que vous ne pouviez que vous soumettre aux ordres. Vous avez donc fait semblant de l'accepter en téléconférence et vous l'avez annoncé aux membres de la cellule de crise, mais en fait vous aviez pris monsieur XXXXX à part et lui aviez dit de continuer l'injection d'eau. C'est bien ça?

R : Oui. 
Q: Par la suite, vous allez recevoir l'accord du Gouvernement. Qu'avez-vous dit, à ce moment-là, aux membres de la cellule de crise?

$\mathbf{R}$ : En fait, l'information n'était pas venue directement de la résidence du Premier ministre. Les instructions sont arrivées du siège via la téléconférence. Du coup, tout le monde autour de la table l'a su en même temps.

$\mathbf{Q}$ : Et, à partir de ce moment, puisque c'était le début officiel de l'injection, tout le monde autour de la table partageait les mêmes informations, n'est-ce pas?

R : Oui.

$\mathbf{Q}$ : Est-ce que cela signifie que parmi les membres de la cellule de crise, certains pensaient que l'injection avait été arrêtée pendant un moment?

$\mathbf{R}:$ Ah, je pense que la plupart pensaient qu'on l'avait arrêtée.

Q: Excusez-moi de bousculer encore la chronologie. Je voudrais qu'on fasse une comparaison. S'agissant du réacteur 1 , à ce moment-là, vous pensiez qu'il fallait à tout prix injecter de l'eau.

R : Oui.

$\mathbf{Q}$ : Si on fait un saut dans le temps, le 13 mars, ça concerne le réacteur 3, l'injection d'eau dans le 3, alors ça doit être quelque part à la page 30 ou 31 de la chronologie...

$\mathbf{R}$ : Tiens, ça saute de la page 30 à la page 33. On dirait qu'il manque les pages 31 et 32 . Ah, pardon. Je viens de les retrouver.

$\mathbf{Q}$ : Oui, concernant le réacteur 3 . Au réacteur 3 , aussi, il semble que vous ayez commencé à injecter de l'eau douce le 13 mars à 9h25. Ensuite, à la page 31, il y a «directives ", à 10h30, « du directeur de la centrale de préparer l'injection d'eau de mer». Plus loin, on trouve: " fin de l'injection d'eau douce à $12 h 20$ », puis "début d'injection d'eau de mer à $13 h 12$ ». Si je comprends bien, s'agissant du réacteur 3 , vous mettez de l'eau douce avant de passer à l'eau de mer. Pourquoi?

$\mathbf{R}$ : D'après mes souvenirs, pour le réacteur 1 , on a pris l'eau de mer du bassin du bâtiment turbine.

À côté du réacteur 3, comme on l'a évoqué la dernière fois, il y avait une cuve avec de l'eau pour la lutte contre l'incendie, qu'on pouvait utiliser tout de suite. Vous comprenez, j'étais dans une logique où, que ce soit de l'eau douce ou de l'eau salée, il fallait tout injecter, en commençant par ce qui se trouvait à proximité. Là, je ne faisais pas de fixation sur l'eau douce ou l'eau de mer, ça m'était égal. L'important, c'était d'envoyer de l'eau. Si la plus proche était une source d'eau douce, eh bien, on allait prendre l'eau douce.

De plus, et le raisonnement est valable aussi pour le réacteur 1 , il vaut mieux commencer par mettre de l'eau borée. C'est pour assurer une meilleure stabilité du réacteur. Pour éviter un redémarrage de réaction nucléaire ${ }^{7}$, on injecte de l'eau borée. Pour le réacteur 1 , on avait dissous de l'acide borique dans le bassin du bâtiment turbine, mais pour cette opération, il vaut mieux disposer d'une cuve. Le réservoir d'eau de lutte contre l'incendie est une vraie citerne et il est plus aisé d'y dissoudre de l'acide borique. C'est une des raisons pour lesquelles on a commencé par envoyer de l'eau douce avant de passer à l'eau de mer.

$\mathbf{Q}$ : Concernant cette injection d'eau douce dans le réacteur 3, je voudrais qu'on revienne sur la situation du réacteur à ce moment. Si je comprends bien, le $\mathrm{HPCl}$ du réacteur s'était arrêté ce jour à 2h42 du matin. Ensuite, jusqu'à 9h25, durant un laps de temps de 6h40 environ, vous aviez eu, semble-t-il, des difficultés à injecter l'eau à cause de la forte pression du réacteur. D'après ce qu'ont raconté les agents qui travaillaient à constituer le circuit 
d'injection, au début, concernant ce réacteur 3, il avait été question de relier le bassin du bâtiment turbine au réacteur à l'aide d'un camion de pompiers, et ils y travaillaient, quand on leur a demandé de changer les dispositifs pour utiliser plutôt le réservoir d'eau de lutte contre l'incendie. Plusieurs personnes ont raconté cette histoire, qu'ils avaient dû changer de dispositifs en cours de route. Savez-vous pourquoi il y a eu ce changement?

$\mathbf{R}:$ Je me rends bien compte que c'est un point qui peut donner lieu à discussion et j'essaie de me souvenir, mais je n'y arrive pas. Pour moi, je n'ai pas non plus le souvenir d'avoir rencontré de fortes réticences de la part du siège à l'utilisation d'eau de mer.

Et je sais que les hommes qui étaient sur le terrain affirment qu'ils ont eu l'ordre de quelqu'un de privilégier l'eau douce avant l'eau salée et qu'ils ont dû changer en conséquence. Mais, pour moi, c'est une histoire que j'ai entendue après coup. Je n'en ai pas le souvenir. Ce qui m'importait, c'était d'utiliser la source d'eau la plus proche, que ce soit de l'eau douce ou de l'eau salée, d'utiliser celle qui pouvait être le plus facilement injectée. C'est la seule chose qui motivait mes décisions. Et en disant ça, je n'ai l'intention de couvrir personne. Si je me remets en situation, mon idée était d'injecter de l'eau, que ce soit de l'eau douce ou de l'eau salée, injecter l'eau la plus proche, et c'était tout.

$\mathbf{Q}$ : Excusez-moi de m'immiscer dans la discussion. Je pense qu'il y avait eu des ordres, des espoirs, toutes sortes de sentiments mélangés, mais commencer l'injection d'eau de mer, l'arrêter ou continuer cette injection, pensiez-vous que vous le faisiez en raison des pouvoirs qui vous étaient conférés en tant que directeur de la centrale? Aviez-vous ce genre de réflexion en tête quand vous avez pris ces décisions? Pensiez-vous que, quelles que soient les personnes présentes, vous preniez ces décisions parce que c'étaient les décisions qu'il fallait prendre? Ou bien, le faisiez-vous parce que vous pensiez qu'il était de votre devoir de directeur de le faire? Ayant ces prérogatives, ne pensiez-vous pas qu'il n'était pas utile de prendre en compte les remarques déplacées des uns et des autres, et vous saviez qu'il y en aurait? Ce sont des nuances qu'on ne voit pas bien quand on regarde de l'extérieur et que j'aimerais savoir.

$\mathbf{R}$ : Tout d'abord, s'il s'était agi de manœuvres ordinaires, je leur aurais dit de les exécuter en suivant les procédures d'usage. Mais là, il s'agissait d'eau de mer. Une première aussi bien au Japon qu'à l'international.

$\mathbf{Q}$ : Oui, vous avez raison. C'était une première mondiale.

$\mathbf{R}$ : Pour une première, on est complètement au-delà des procédures habituelles, des manuels et compagnie. Alors, j'ai pensé qu'il fallait me fier à mon instinct, en quelque sorte.

$\mathbf{Q}:$ À ce moment-là, si certains tergiversent, raisonnent autrement, disent "arrête, attends », etc., vous percevez ça comme des bruits parasites?

R : Oui.

$\mathbf{Q}:$ C'était ce que je voulais savoir.

$\mathbf{R}$ : Oui, tout m'est apparu comme ça. Comprenez-moi, mon raisonnement était tout simple. Envoyer de l'eau. Les seules choses à faire, c'était injecter de l'eau et faire baisser la pression de l'enceinte de confinement, juste ces deux points. Et ça, pour chaque réacteur. C'est tout ce que j'ai dit et que j'ai continué à dire.

$\mathbf{Q}:$ Tout le reste, c'était des bruits parasites?

$\mathbf{R}$ : Oui, des parasites. Tous ces ordres qui disaient d'arrêter, de faire ci, de faire ça, c'étaient tous des parasites. En tout cas, pour moi. 
$\mathbf{Q}$ : Par exemple, lors des téléconférences, vous est-il arrivé de demander des suggestions sur ce que vous pouviez faire?

$\mathbf{R}$ : Pratiquement jamais.

$\mathbf{Q}$ : Alors c'était plutôt le siège qui n'arrêtait pas de vous donner des ordres, sans se préoccuper de vous?

$\mathbf{R}$ : En fait, de leur part, il y avait beaucoup de demandes d'informations. Du genre, comment est la situation? Alors, ils ne remplissaient pas du tout leur rôle de soutien. Ils n'arrêtaient pas de nous interroger pour avoir des rapports. Au bout d'un certain temps, j'en ai eu assez. Je me rappelle leur avoir dit plusieurs fois de se taire et de nous laisser tranquilles.

$\mathbf{Q}$ : Je suppose que le siège se comportait comme ça, parce qu'à leur tour, ils étaient sollicités par le Gouvernement.

$\mathbf{R}$ : Oui, tout à fait.

Q: Pour en revenir à cet ordre du Gouvernement, n'avez-vous eu aucune explication concernant cette décision?

$\mathbf{R}$ : Si je vous restitue le coup de fil, tout d'abord, on m'a dit que le Gouvernement n'avait pas encore donné l'accord pour l'injection d'eau de mer, alors j'ai répondu que j'avais déjà commencé l'injection et qu'étant donné que l'eau entrait, j'avais l'intention de la poursuivre. À ce moment-là, on m'a dit d'arrêter de discuter et de cesser l'injection. Ce coup de fil, celui-là au moins, je me le rappelle encore très bien. Et c'est là que je me suis dit qu'il y en avait marre. C'était mon ressenti. Il n'y avait eu ni argumentaire, ni explication.

$\mathbf{Q}:$ C'était donc votre plus gros parasite?

$\mathbf{R}$ : En quelque sorte. Mais, à ce moment-là, je ne pensais plus que c'était un bruit parasite. Je voulais qu'on nous laisse tranquilles. $\mathrm{Vu}$ de l'extérieur, avec ces discussions à la Diète ${ }^{8}$ et toute l'agitation qu'il y a eu autour, beaucoup de personnes pensent qu'il s'agissait d'une énorme affaire, mais il n'en était rien. C'était tout simple. Si on arrêtait l'injection maintenant, on allait à la catastrophe. J'étais donc décidé à poursuivre coûte que coûte. C'est tout ${ }^{9}$.

$\mathbf{Q}$ : J'ai lu dans un magazine, à cette époque, que vous aviez dit que vous en aviez marre. L'expression était tirée de là?

$\mathbf{R}$ : Non, à ce moment-là, je n'ai pas dit que j'en avais marre. Non, l'affaire «j'en ai marre » est sortie plus tard, à mon avis. Mais même si je ne l'ai pas dit à ce moment précis, ça a été mon ressenti pendant tout ce temps.

$\mathbf{Q}$ : Je ne sais pas d'où ça avait fuité, mais on en a parlé assez tôt.

$\mathbf{R}$ : Ah, ça doit être la téléconférence. Parce qu'on est relié à beaucoup d'endroits, à la cellule hors site, tout ça, du coup pas mal de personnes peuvent entendre ce qu'on dit. La presse est court-circuitée, mais des gens peuvent prendre en note les déclarations et les diffuser. Du coup, les téléconférences qu'on fait quotidiennement, la moitié de ce qui s'y dit est certainement divulgué. C'est le siège, le site de Fukushima Daini, Kashiwazaki, le centre hors site, le J-Village ${ }^{10}$ et puis la cellule de soutien de Fukushima ${ }^{11}$. On a été relié à tous ces centres dès le début et on échange des informations matin et soir. Alors, j'ai dû dire un jour que je n'en pouvais plus, que j'en avais marre et quelqu'un a divulgué la chose. Ils peuvent en faire ce qu'ils veulent. 


\section{Q: Merci. Nous pouvons reprendre la chronologie.}

Q: Pour en revenir à ce dont nous parlions, dans la nuit du 12 mars, à partir de 19h04, vous commencez à injecter de manière continue de l'eau de mer dans le réacteur 1 , puis, à $20 \mathrm{~h} 45$, page 9 de la chronologie, $1 \mathrm{~h} 41$ après le début de l'injection d'eau de mer, vous commencez à injecter de l'eau de mer borée dans le réacteur. Pourquoi mélanger de l'acide borique à ce moment-là ? Y a-t-il une raison précise à ça?

$\mathbf{R}$ : Depuis le début, je voulais qu'on mélange de l'acide borique à l'eau de mer et j'avais donné des directives pour qu'on prépare cette opération. Seulement, la radioactivité était très forte à cet endroit-là, et puis, il fallait diluer l'acide borique. Ce qu'on trouve dans les sacs d'acide borique, ce sont des morceaux, des cristaux, si vous voulez, c'est un truc blanc. Il ne suffit pas de mettre ça en vrac dans l'eau. Il fallait réfléchir à la manière de le diluer et il fallait y réfléchir à l'avance. C'est pour ça que j'en avais donné l'ordre beaucoup plus tôt.

Seulement, la radioactivité était vraiment très forte, de plus, les préparatifs n'avançaient pas. La priorité des priorités était d'envoyer de l'eau. Alors j'avais dit que, pour l'acide borique, on le mettrait dès qu'il serait prêt. Et, dans les faits, les préparatifs n'ont été achevés qu'à 20h45. L'ordre ne consistait pas à envoyer l'eau borée à $20 \mathrm{~h} 45$, mais à l'envoyer " as soon as possible $»^{12}$. Et c'est simplement que, dans les faits, cela s'est passé à cette heure-là.

Q: Ensuite, concernant l'explosion, vous aviez plusieurs thèses en présence. Au bout de quelques heures de discussion, vous en étiez arrivés à penser que l'hypothèse que de l'hydrogène s'était échappé de l'enceinte de confinement et, s'étant accumulé dans la partie haute du bâtiment, avait provoqué l'explosion, était la plus probable. Pensant que cette cause était la plus probable et sachant que cela pouvait encore arriver sur les réacteurs 1, 2, 3, 4, voire 6, avez-vous envisagé de faire quelque chose pour l'éviter?

R : On l'a fait en parallèle.

Q: Quel genre de discussion, par exemple, avez-vous eu à ce propos ?

$\mathbf{R}$ : Tout d'abord, il y a ce qu'on appelle le Blow Out Panel ${ }^{13}$ sur le bâtiment réacteur. Quand la pression du bâtiment réacteur atteint un certain niveau, ce Blow Out Panel est conçu pour se briser vers l'extérieur pour laisser échapper la pression.

Seulement, lors du séisme de 2007 à Kashiwazaki, la force du séisme a fait que ce panneau s'était complètement ouvert, alors que la pression n'avait pas augmenté. Du coup, on avait voulu améliorer ces panneaux, en fait ça s'est complètement retourné contre nous, mais on avait voulu les améliorer en les rendant plus difficiles à ouvrir. Donc, les Blow Out Panels de tous les réacteurs étaient devenus difficiles à ouvrir depuis cet incident.

Bien sûr, j'avais en tête que le même phénomène (une explosion due à l'hydrogène) pouvait arriver au réacteur 2 ou 3, et ce souci, c'est un souci que je partageais avec le siège. Nous nous sommes demandés que faire. Nous avons bien pensé à ouvrir ces Blow Out Panels, mais nous étions dans une situation où on ne pouvait pas pénétrer à l'intérieur du bâtiment réacteur, et nous ne pouvions pas non plus les ouvrir de l'extérieur. 
Alors nous avons réfléchi à toutes sortes de solutions. Mais l'autre souci, c'est qu'au fur et à mesure que le temps passait, on ne savait pas quelle quantité d'hydrogène s'était déjà échappée. Il devenait dangereux d'aller travailler là-bas. Suivant les travaux, on aurait pu avoir des étincelles qui auraient mis le feu à l'hydrogène. Alors on s'est dit qu'on aurait pu ouvrir les panneaux de l'extérieur avec une machine de découpe au jet d'eau, par exemple. Puisqu'on aurait ouvert avec de l'eau, on n'aurait pas eu d'étincelles. Mais on ne voyait pas de machine pouvant faire des trous en si peu de temps. Oh, on a imaginé beaucoup de choses. On s'est aussi demandé si on ne pouvait pas envoyer un homme les ouvrir de l'intérieur. Mais nous étions dans des conditions telles que nous ne pouvions rien faire à court terme. Nous y pensions donc en parallèle, mais la situation ne s'améliorait pas. En fait, nous y pensions continuellement.

Sur ces entrefaites, un homme est revenu du terrain et a fait remarquer que le Blow Out Panel du réacteur 2 était ouvert. On s'est demandé ce qui s'était passé. Je pense que, probablement, il s'était ouvert à cause du souffle de l'explosion du réacteur 1. Mais il y a une chose que je ne m'explique pas. Le panneau se trouve côté mer, l'explosion du réacteur 1 s'est faite dans cette direction. Par quelle magie ce panneau s'est-il ouvert? En tout cas, le Blow Out Panel du réacteur 2 était ouvert et permettait de réguler la pression. C'était un élément rassurant. On s'est dit qu'on allait pouvoir s'occuper plus particulièrement du réacteur 3 . Il fallait trouver un moyen d'ouvrir ce Blow Out Panel ou bien autre chose, mais il fallait absolument faire quelque chose. À la limite, on en était au point d'imaginer de demander aux forces d'autodéfense ${ }^{14}$ de venir en jet et d'arroser le panneau à la mitrailleuse pour y faire des trous.

$\mathbf{Q}$ : Vous n'étiez pas en état d'utiliser un système de ventilation d'urgence du bâtiment réacteur comme le SGTS?

R : Non. Puisqu'on n'avait pas l'alimentation électrique de secours. Si le SGTS avait fonctionné, on n'aurait pas eu d'explosion. Sûrement pas. Quand le SGTS est en marche, la ventilation se fait par ce réseau de secours, tout l'air du bâtiment réacteur est filtré et rejeté à l'extérieur via le réseau. Si le système de ventilation de secours du bâtiment réacteur avait fonctionné, il n'y aurait pas eu d'explosion d'hydrogène.

Mais c'est vrai que, quelque part, nous aussi, on avait perdu de vue que le SGTS était à l'arrêt, que le réseau habituel de ventilation aussi était arrêté. Quelque part, on avait l'illusion que ça continuait à ventiler. C'est aussi pour ça qu'on a mis tellement de temps à penser qu'il pourrait y avoir accumulation d'hydrogène et explosion.

$\mathbf{Q}$ : Vous voulez dire avant l'explosion du réacteur 1 ?

$\mathbf{R}$ : Oui. Avant cette explosion. Après, on était focalisé sur le réacteur 3 . On se demandait comment faire pour ressusciter le réseau de ventilation et puis, comme je vous l'ai dit, comment faire pour ouvrir ce panneau. On s'est même demandé si on ne pouvait pas ménager une ouverture à un angle du bâtiment, etc.

$\mathbf{Q}$ : Oui, comme d'arriver en hélicoptère avec une grosse boule de métal qu'on balancerait sur le bâtiment, ce genre de choses?

R : Oui. C'est ce genre de choses qu'on suggérait au siège. Je n'ai pas arrêté de dire, à partir de ce moment, qu'il fallait qu'eux, au siège, trouvent une solution. Pour moi, ça dépassait complètement nos capacités sur le terrain. Il fallait qu'ils réfléchissent, eux, 
à une manière de détruire partiellement, de l'extérieur, la partie supérieure du bâtiment réacteur.

Q: Ce que vous venez de nous raconter concerne donc les mesures que vous envisagiez pour le cas où, comme pour le réacteur 1, l'hydrogène s'accumulerait dans le bâtiment. En plus de cette préoccupation, vous étiez également en train de réfléchir aux mesures concernant la cuve et l'enceinte de confinement des réacteurs 2 et 3 ,

Si on continue dans la chronologie, concernant le réacteur 2 , et ce sera la même chose pour le 3 , à la page 19, ce 12 mars à 17 h30, c'est-à-dire un peu moins de deux heures après l'explosion, vous donnez l'ordre de commencer à préparer l'opération d'éventage. II y a des indications similaires pour le réacteur 3 . Cela signifie qu'à $17 \mathrm{~h} 30$, vous donnez l'ordre à la fois pour le réacteur 2 et le réacteur 3 de commencer les préparatifs pour un éventage ?

R : Oui.

Q : Pourquoi avez-vous jugé utile de préparer l'opération d'éventage pour les réacteurs 2 et 3 à ce stade?

$\mathbf{R}$ : C'est tout à fait logique. Nous avions eu beaucoup de difficultés à éventer le réacteur 1 . De toute manière, tôt ou tard, les réacteurs 3 et 2 allaient se retrouver dans la même situation, la pression de l'enceinte de confinement allait monter. Alors, j'ai pris l'initiative d'ordonner à l'avance cette préparation pour que tout soit prêt et qu'il n'y ait plus que la rupture du disque pour que l'éventage se fasse.

Q: Pour préciser la situation, vous dites que vous avez donné ces ordres à l'avance parce que vous aviez rencontré énormément de difficultés avec le réacteur 1. C'était dans la nuit du 11 au 12 mars. La radioactivité continuait à monter, il y avait des répliques. Vous vous êtes dit que vous n'arriveriez pas à vos fins en un tour de main, que cette opération d'éventage ne serait pas aussi simple que vous le croyiez au départ. Ça, vous avez dû vous le dire à l'aube du 12 mars. Pourquoi ne vous êtes-vous pas dit, déjà à ce moment-là, que, puisque vous aviez tant de difficultés avec le réacteur 1 , il fallait lancer dès maintenant les préparatifs pour les réacteurs 2 et 3 ?

$\mathbf{R}$ : À ce moment-là, l'opération d'éventage n'est pas encore terminée pour le réacteur 1 . Et tout le monde est sur le 1 . Vous comprenez, on n'avait pas de marge de manœuvre.

$\mathbf{Q}:$ Vous voulez dire, du point de vue du personnel?

$\mathbf{R}$ : Bien sûr, on n'avait pas non plus de marge de manœuvre pour le personnel. Comme je viens de vous le dire, dans l'après-midi du 12, il y a eu l'explosion. À ce stade, je n'ai pas assez de personnel pour envoyer des gens préparer l'opération d'éventage. En plus, la radioactivité était forte. Nous étions dans des conditions où il était très difficile d'envoyer du monde sur le terrain. Mais, bien entendu, il fallait faire vite.

Donc, l'ordre concernant les réacteurs 2 et 3 a été donné au moment où, après l'explosion et après avoir rappelé dans un premier temps le personnel qui était sur le terrain à l'abri, le circuit d'injection du réacteur 1 a été enfin prêt. Ma priorité, jusque-là, était de calmer coûte que coûte le réacteur 1.

$\mathbf{Q}$ : Si on revient au réacteur 2 , le 12 mars à $17 \mathrm{~h} 30$, en ce qui concerne l'injection d'eau, il est noté que le RCIC est en fonctionnement. Le saviez-vous?

R : Oui.

$\mathbf{Q}$ : Dès le départ, grâce à l'ingéniosité de votre personnel sur le terrain, tous les appareils qui étaient branchés sur la batterie du RCIC avaient été débranchés pour réserver exclusivement l'alimentation électrique au RCIC. C'est ce que vous nous aviez raconté. Cette astuce permet d'économiser l'énergie, mais vous êtes quand même dans une 
situation où vous ne savez pas pendant combien de temps cette énergie va tenir. Quels étaient les moyens de vérifier que le RCIC était bien en marche?

$\mathbf{R}$ : Comme vous pouvez le supposer, étant moi-même confiné dans la cellule de crise du bâtiment antisismique, je ne savais pas quelle était, au juste, la situation sur le terrain. J'ai demandé plusieurs fois si le RCIC était bien en marche. Sur le terrain, ils ne pouvaient plus voir les indicateurs. Malgré ça, je leur demandais de le vérifier. Et là, je ne l'ai su que plus tard, ils ont dû faire des sacrifices énormes pour me donner satisfaction. Car, pour vérifier le fonctionnement du RCIC, ils devaient pénétrer dans le bâtiment réacteur. Je n'ai pas été assez vigilant. Je savais que la vérification serait difficile. Mais je ne me rendais pas compte à quel point, à ce moment-là. Alors je leur ai demandé de vérifier le fonctionnement du RCIC. Ils ont pris des risques énormes et, à $2 \mathrm{~h} 55$, ils ont fait leur rapport. Je me rappelle avoir été soulagé.

$\mathbf{Q}$ : Donc, vous avez eu un rapport vers $2 \mathrm{~h} 55$ et, ensuite, le $\mathrm{RCIC}$ a continué à fonctionner. Dans la soirée du 12 mars, par exemple, il fallait bien que vous réfléchissiez à une solution lorsque le RCIC cesserait de fonctionner. Pour ça, vous deviez savoir si le RCIC continuait à fonctionner ou pas. Vous demandiez confirmation auprès du groupe « production »?

$\mathbf{R}$ : Bien sûr. Il était important de savoir, pour moi, si le RCIC était en marche ou pas. Car, de cela, dépendait l'étape suivante, qu'on préparait, bien entendu. Oui, j'ai demandé qu'on vérifie.

$\mathbf{Q}$ : Rappelez-moi quelle est la source d'eau du RCIC ?

$\mathbf{R}$ : Lui-même. C'est sa propre vapeur qu'il récupère du condenseur.

$\mathbf{Q}$ : Alors, on ne peut pas savoir s'il marche en observant la source.

Le RCIC est encore en marche dans la nuit du 12 mars, par exemple. Aujourd'hui, on sait que dans la réalité le RCIC a fonctionné jusqu'au 14 mars à 13h2515. Et cet arrêt, vous l'avez déduit en voyant le niveau d'eau baisser. Mais ça, vous ne pouviez pas le savoir à ce moment-là. Vous viviez donc en vous demandant quand il allait s'arrêter?

$\mathbf{R}$ : Fondamentalement, la situation du réacteur 1 était la plus préoccupante. Ensuite la situation du 3. Donc, dans l'ordre, le 1 , le 3 et le 2 . Je trouve, après coup, qu'on a eu de la chance que les choses ne soient pas toutes arrivées en même temps, que cela se soit succédé. Mais, effectivement, on était dans une situation où l'arrêt de n'importe lequel de ces systèmes pouvait survenir à tout moment. De plus, nous manquions de personnel. Pour être tout à fait franc, c'était la panique dans ma tête. Mais, comme je vous l'ai dit tout à l'heure, pour moi, la seule chose à faire pour tous les réacteurs, c'était d'injecter de l'eau, et puis d'éventer. Il n'y avait que ça. Et au milieu de tout ça, il y a eu l'explosion du réacteur 1 , à laquelle il a fallu faire face. Pendant ce temps, il fallait aussi surveiller l'évolution des réacteurs 2 et 3. C'était la confusion totale. Et c'est dans cette ambiance qu'il fallait donner des ordres. Alors, je reconnais que ça ne s'est pas fait dans un ordre logique et réfléchi. Je donnais les directives au fur à mesure qu'elles me venaient à l'esprit.

$\mathbf{Q}$ : Alors, par exemple, après l'explosion, le RCIC du réacteur 2 fonctionne encore. Vous vous demandez ce que vous allez faire quand il va s'arrêter?

R : Oui, bien sûr, j'y réfléchissais.

$\mathbf{Q}$ : Que comptiez-vous faire?

$\mathbf{R}$ : De toute façon, il n'y avait que la solution de l'injection, et même de l'injection d'eau de mer. Seulement, au moment que vous évoquez, nous étions attelés à l'injection dans le réacteur 1 au moyen de camions de pompiers, mais nous n'avions déjà pas suffisamment de camions pour le 1 . Les camions vont continuer à arriver. Je 
ne sais plus exactement à quel moment nous disposions de combien de camions. Mais au moment où nous nous apprêtions à injecter dans le réacteur 2 , il me semble qu'on en avait pas mal. Il en fallait déjà deux pour pomper l'eau de mer. On prenait l'eau au niveau du quai, puis, avec deux camions, on la montait de 0 à $10 \mathrm{~m}$. Elle arrivait comme ça, avec encore un autre camion qui l'envoyait dans le réseau. Je crois que, pour la première fois, ce circuit a été enfin complet quand on a commencé à injecter dans le réacteur 2. Avant d'en arriver là, on avait beaucoup bricolé en changeant successivement les dispositions des camions au fur et à mesure qu'ils arrivaient, puisqu'on avait commencé avec un seul camion pour le réacteur 1.

$\mathbf{Q}:$ Si je comprends bien, vous pensiez dès ce moment utiliser les camions de pompiers quand le RCIC se serait arrêté, comme vous le faisiez avec le réacteur 1 ?

$\mathbf{R}$ : Oui. Il n'y avait que cette solution.

Q: Alors, au moment de l'explosion de la tranche 1, le HPCl est en fonctionnement pour le réacteur 3. Vous pensiez que celui du réacteur 2 ne se mettrait pas en marche?

$\mathbf{R}$ : J'avais demandé qu'on fasse ce qu'il fallait pour le faire fonctionner. Mais, sur le terrain, il y avait des difficultés. Je ne sais plus si c'était la batterie ou le tableau électrique, mais le HPCI du réacteur 2 ne pouvait pas fonctionner, selon les hommes du terrain. Je leur avais demandé de continuer à essayer de régler le problème, mais le groupe «réhabilitation » était très pessimiste. Je pense que vous comprendriez si vous aviez été à ma place à ce moment-là. Vous avez trois entités déchaînées qui se débattent, vous avez des informations qui fusent de partout et vous devez trancher. Dans ces conditions, on ne sait plus où on en est. Alors, mes ordres étaient très simples, envoyer de l'eau, de quelque manière que ce soit, que ce soit de l'eau de mer ou autre chose, et faire baisser la pression de l'enceinte de confinement.

Les RCIC et HPCI, que vous avez évoqués, ne sont que des solutions pour gagner du temps. Mais il fallait tout faire pour pouvoir en profiter le plus longtemps possible.

$\mathbf{Q}$ : Vous aviez déjà en tête, à ce moment-là, que, pour une raison ou une autre, le HPCl ne serait pas disponible?

$\mathbf{R}$ : Oui. Mais j'avais quand même donné l'ordre qu'on fasse le nécessaire pour le faire fonctionner.

$\mathbf{Q}$ : À part ça, il y avait aussi le SLC, qui permet une injection à haute pression, et il me semble qu'avant l'explosion, vous étiez en train de préparer ce circuit pour le réacteur 2, aussi. Mais l'explosion a fait que vous aviez perdu la fonctionnalité des camions groupes électrogènes. Aviez-vous renoncé à cette solution, pour vous dire qu'après le RCIC, vous alliez passer à l'eau de mer?

$\mathbf{R}$ : Il fallait d'abord faire baisser la pression et puis, envoyer de l'eau de mer. Enfin, ça pouvait aussi bien être de l'eau de mer que de l'eau douce. Mais il fallait impérativement injecter de l'eau.

$\mathbf{Q}$ : Concernant le réacteur 3 , après l'explosion, de manière similaire, vous étiez en train de faire avancer les préparatifs en prévision d'un futur éventage, car ceux-ci pouvaient s'avérer très longs, et, simultanément, en ce qui concerne le point crucial de l'injection d'eau, le HPCl avait pris le relais après l'arrêt du RCIC. C'est bien ça?

R : Oui.

$\mathbf{Q}$ : Et vous pensiez déjà à ce que vous alliez faire?

R : Oui. 
Q: Vous pensiez faire la même chose pour le réacteur 3, c'est-à-dire utiliser des camions de pompiers pour injecter l'eau par le réseau de lutte contre le feu?

R : Oui.

Q: Et, concernant ce réacteur 3, aviez-vous envisagé aussi le SLC ou le CRD (Control Rod Drive) $)^{16}$ ?

$\mathbf{R}$ : Bien sûr. Nous avions tout examiné. Ce qui s'impose tout d'abord à l'esprit, grosso modo, c'est l'injection d'eau. Pour cette injection, si, dans l'état actuel, il y a un système qui permet d'injecter à haute pression, on le privilégie. Si on peut injecter sans faire tomber la pression, même si ça n'est qu'un petit volume d'eau, on privilégie cette solution, parce que, si possible, on ne veut pas perdre trop de pression. Si on baisse la pression, cela signifie qu'on perd l'eau qui s'est transformée en vapeur et qui tombe dans la chambre de dépressurisation ${ }^{17}$, et cela veut dire que le niveau d'eau baisse d'autant. Si on n'a pas besoin de baisser la pression pour injecter, on peut faire cette opération tout en continuant à préserver le niveau d'eau. En temps normal, la pression est encore haute et on commence par le HPCI de l'ECCS. De la même manière, une injection qui peut se faire tout en conservant une forte pression, c'est le SLC ou le CRD. Ce sont des réserves d'eau qu'on peut injecter à haute pression, alors j'ai fait vérifier l'état des pompes, de l'alimentation électrique de ces systèmes sur les trois tranches en parallèle. Mais, presque rien ne marchait. C'était des histoires du genre, si la pompe en elle-même était indemne et même si elle fonctionnait, le système d'huile de graissage était fichu, du coup, même si on mettait la pompe en marche, elle se serait tout de suite arrêtée. Ou bien, le réseau qui fournit l'eau au joint mécanique, qui est un circuit complètement séparé, était à l'arrêt et le système se serait coincé à ce niveau-là, etc. Je ne me rappelle plus quel incident correspondait à quel réacteur, mais des rapports négatifs se succédaient. Et, au milieu de tous ces échecs, seul le SLC avait l'air vivant. Le SLC se trouve plutôt vers le haut dans le bâtiment et il n'avait pas été submergé. On fait ainsi le choix du SLC parce qu'on se dit que si on arrive à lui fournir l'électricité, la pompe et le système en lui-même avaient l'air de fonctionner. Et, donc, on a besoin d'électricité. Seulement, le réservoir du SLC n'a qu'un petit volume. Même si on y ajoute l'eau du réservoir-test, je ne suis pas sûr que ça fasse $10 \mathrm{t}$. Même si ça marchait, on ne pouvait injecter que ce petit volume. On ne pouvait absolument pas envisager une injection continue. Mais ça permettait de gagner du temps, alors il fallait le faire.

$\mathbf{Q}$ : En résumé, est-ce que ça veut dire qu'une fois que le SLC et le RCIC sont morts, il ne reste que le réseau de lutte contre l'incendie?

$\mathbf{R}$ : Pas en temps normal. Comme je l'ai dit tout à l'heure, on a tout vérifié. Par exemple, le CRD. On peut monter la pression à plus de $70 \mathrm{kPa}$, je crois que la pression de dimensionnement est d'environ $90 \mathrm{kPa}$. Si on veut injecter de l'eau, c'est l'idéal. On envoie l'eau par le bas des barres de contrôle. Alors, on l'a bien envisagé. C'était différent selon les réacteurs, mais soit c'était la pompe qui ne marchait pas, soit c'était l'alimentation électrique, il y avait toujours quelque chose qui empêchait son fonctionnement. Le SLC, c'était bien, mais on ne pouvait pas en attendre beaucoup comme source de refroidissement, le volume d'eau injecté n'était pas conséquent. Alors, j'aurais vraiment voulu disposer du CRD.

$\mathbf{Q}$ : Ça veut dire que le SLC, par exemple, a fonctionné avec une batterie?

$\mathbf{R}$ : Le SLC marche au courant alternatif, alors on a fait venir un camion générateur. 
$\mathbf{Q}$ : L'eau, que vous continuez à envoyer en quantité aujourd'hui, passe encore par le circuit incendie?

R : Non. On l'a transférée du circuit incendie au circuit normal d'alimentation d'eau. Pour vous expliquer pourquoi, ça va faire allusion à des choses qui se sont passées après, c'est parce qu'à un moment donné, le circuit incendie du réacteur 1 s'est trouvé bouché et qu'on ne pouvait plus envoyer de l'eau par là. Je ne me rappelle plus quel jour de mars nous étions, peut-être deux semaines plus tard, on a observé que la température du réacteur avait beaucoup augmenté. Comme vous le savez, le circuit incendie a été prévu pour la lutte contre le feu et les tuyaux se séparent en plusieurs branches. Ce qui est normal pour éteindre le feu. Mais il y a différents endroits où la tuyauterie se sépare et même si on dit qu'on va fermer toutes les ramifications, il y a toujours possibilité de débordement. Du coup, on n'arrive pas à savoir si toute l'eau qu'on envoie dans les tuyaux arrive bien jusqu'au réacteur.

De plus, le fait d'utiliser de l'eau de mer fait que, comme c'est de l'eau salée, on ne sait pas quel est l'état des tuyaux, peut-être des bouchons se sont-ils formés, etc., et là encore, on n'est pas sûr que l'eau arrive bien à destination.

Aujourd'hui, après avoir opéré plusieurs modifications comme de changer de circuit, c'est-à-dire de déplacer la bouche d'entrée pour utiliser, par exemple pour le réacteur 1 , la tuyauterie d'éventage du réseau d'alimentation en eau, ou bien en construisant après coup un circuit où de l'eau peut circuler en plus grande quantité, nous améliorons sans arrêt l'installation.

$\mathbf{Q}$ : C'est après coup que vous vous êtes rendu compte que vous pouviez utiliser ces circuits?

R : Oui.

$\mathbf{Q}$ : Mais le fait que vous ayez réussi à les utiliser, dans un sens, c'était une chance dans votre malheur, non?

R : Oui, c'était une chance. S'il n'avait pas été prévu un circuit incendie permettant d'injecter de l'eau dans le réacteur en cas d'incident, je crois qu'on n'aurait rien pu faire. Pour moi, l'état où nous nous trouvons aujourd'hui ne tenait vraiment qu'à un fil.

$\mathbf{Q}:$ Un fois de plus, je vais vous dévier de vos discussions techniques. En fin de compte, l'intérieur d'une cuve est toujours maintenu à une pression élevée. Je comprends que la cuve est quelque chose qu'on fait naturellement fonctionner avec une pression élevée. Mais un non-spécialiste pourrait penser qu'on pourrait faire baisser cette pression jusqu'à la pression normale de l'atmosphère et qu'à ce moment-là, on pourrait tout simplement envoyer de l'eau par-dessus sans même avoir de pompe? Oui, je sais, c'est une idée terriblement simpliste, mais, moi, c'est une idée qui me viendrait volontiers à l'esprit. Si on voulait mettre cette idée à exécution, il faudrait faire baisser la pression. Ce faisant, toute l'eau qui se trouve à l'intérieur va se mettre à bouillir et disparaître entièrement, entraînant une hausse considérable de la température, rendant de facto impossible toute injection d'eau. Est-ce comme ça qu'il faut raisonner? Faut-il penser que vouloir faire une manœuvre en faisant baisser la pression à l'intérieur de la cuve est impossible dans la réalité ? Qu'en est-il ?

$\mathbf{R}$ : Si tout le système était viable, ce serait possible. Habituellement, quand la pression augmente à l'intérieur de la cuve, on dégage de la vapeur par cette fameuse soupape de sécurité. Cette vapeur s'en va de la soupape vers la chambre de dépressurisation où elle refroidit. Ce système d'injection d'eau qui est prévu structurellement est tout à fait adapté. 
$\mathbf{Q}$ : Alors, pensez-vous qu'il est possible d'ouvrir la cuve volontairement, comme vous venez de le décrire, et de faire baisser la pression de la cuve jusqu'à celle de l'atmosphère? Pensez-vous qu'il est possible de réaliser cette manœuvre?

R : Oui. D'après la conception d'un Réacteur à Eau Bouillante (REB ${ }^{18}$, c'est quelque chose de possible. À condition que l'ECCS soit fonctionnel. Si ces systèmes sont opérationnels, c'est une chose facile. D'ailleurs, nous l'avons déjà réalisé plusieurs fois, pendant des conduites réelles.

$\mathbf{Q}$ : Alors, c'est parce que ces systèmes n'étaient pas opérationnels que vous n'avez pas pu...

$\mathbf{R}:$ C'est ça.

$\mathbf{Q}$ : J'ai bien compris. Merci. Nous pouvons retourner au sujet principal.

$\mathbf{Q}$ : Nous en avons déjà un peu parlé tout à l'heure. Après l'explosion d'hydrogène, vous deviez vous préoccuper parallèlement des réacteurs 1,2 et 3 . Alors que vous manquiez de main-d'œuvre et de matériel, quelle a été votre priorité ? Après l'explosion, c'étaient les réacteurs 1 et 3 ?

$\mathbf{R}:$ Le 3.

Q: Pourquoi le 3 ?

$\mathbf{R}$ : Si je me remets dans la situation du moment, c'est le réacteur 3 qui a perdu en premier le RCIC.

$\mathbf{Q}:$ En effet.

$\mathbf{R}$ : Donc, le RCIC ne fonctionnait plus. Le HPCI avait bien pris le relais, mais je soupçonnais que la durée du HPCI pouvait ne pas être très longue. Vous vous demandez peut-être pourquoi j'ai donné la priorité au 3 par rapport au 2. En fait, j'ai sans doute réfléchi aux deux réacteurs en simultané. Seulement, dans les faits, c'est le HPCI du réacteur 3 qui s'est arrêté en premier, et donc, on s'est précipité sur le 3. Les deux étaient pensés en parallèle, mais comme dans les faits, c'était le 3 qui s'est arrêté en premier, on est allé vers le 3. C'est tout.

$\mathbf{Q}$ : Donc, après l'explosion, dans la nuit du 12 mars, par exemple, le RCIC fonctionne pour le réacteur 2 et le $\mathrm{HPCl}$ pour le réacteur 3. Vous ne savez pas à quel moment le RCIC va s'arrêter, vous ne le savez pas non plus pour le HPCl. Vous réfléchissez impartialement aux deux réacteurs. Seulement, le HPCl s'étant arrêté, vous pensez prioritairement au réacteur 3. C'est bien ça?

R : Oui.

$\mathbf{Q}$ : Si on retourne à la chronologie publiée par TEPCO, à la page 33, je pense que c'est quelque chose qui s'est passé après l'arrêt du $\mathrm{HPCl}$, il est noté dans la rubrique « réhabilitation de l'installation de refroidissement »: «tentative d'injection d'eau avec la pompe diesel de lutte contre l'incendie, mais échec, car augmentation de la pression du réacteur à environ $4 \mathrm{MPa}$ ». Si je comprends bien, après l'arrêt du HPCl, vous avez tout d'abord tenté d'injecter de l'eau avec une pompe à moteur diesel de lutte contre le feu. En avez-vous le souvenir?

R : Non.

$\mathbf{Q}:$ Ça non plus?

$\mathbf{R}$ : Je veux dire, ce genre de choses, c'est écrit par des gens qui rédigent de jolis rapports après coup en arrangeant les choses, mais c'est rédigé par des gens qui n'avaient aucune idée de ce qu'était le terrain à ce moment-là. Enfin, c'est évident que s'il y a $4 \mathrm{MPa}$, l'eau ne peut pas entrer. J'ai envie de dire, mais qu'est-ce qu'il raconte comme ineptie! 
$\mathbf{Q}$ : Permettez-moi d'intervenir. D'après les paramètres publiés par TEPCO, concernant le niveau d'eau et la pression du $1 \mathrm{~F}-3^{19}$, à la page $2 / 21$ des "paramètres », ils ont tous des numéros et...

Si vous nous donnez l'heure et la minute, on va trouver.

Oui ? Alors je vais vous donner l'heure.

À quelle page sur 21 ?

À la page 2. Le 13 mars 2011 à 3h44, la pression du réacteur, mesurée en A est de 4,100 MPa et, effectivement, il semble y avoir eu une tentative pour injecter l'eau avec la pompe diesel de protection incendie. Ce qui m'étonne, c'est que le HPCl s'est arrêté à 2 h42. Si on regarde les paramètres juste après, à 2h44, le 13 mars, la pression est de 0,580 MPa.

$\mathbf{R}$ : Oui. Ça, je l'ai vu après coup. Mais je n'ai pas le souvenir, aujourd'hui, d'avoir su que la pression du réacteur 3 était descendue.

$\mathbf{Q}$ : Est-ce qu'on peut supposer que le HPCl étant en fonctionnement, cela a provoqué la baisse de la pression?

$\mathbf{R}$ : Non. Ce n'est pas possible. Ce n'est pas parce que le HPCI fonctionne bien que toute la chaleur résiduelle est absorbée et que la pression baisse. Non, ça ne marche pas comme ça. Non, la pression ne peut pas chuter comme ça. C'est pour ça que, même aujourd'hui, ces chiffres me paraissent tout à fait mystérieux.

$\mathbf{Q}$ : Si on fait le parallèle entre ces paramètres et le $\mathrm{HPCl}$, le $\mathrm{HPCl}$ s'est automatiquement mis en marche le 12 mars à $12 \mathrm{~h} 35$. Du côté des paramètres, là où il y avait 7,530 MPa le 12 mars à $12 \mathrm{~h} 10$, après la mise en marche du $\mathrm{HPCl}$ à 35 , tout de suite après, à $12 \mathrm{~h} 45$, la pression est de 5,600 et ensuite elle continue à descendre. Normalement, ça ne se passe pas comme ça?

$\mathbf{R}$ : Je ne pense pas. C'est-à-dire que si on perd de la pression, le HPCI s'arrête. Parce que ça marche avec la vapeur, la pression de la vapeur. Effectivement, la pression a baissé et elle est remontée. Mais là, je ne peux rien vous dire. Je suis désolé. Je n'ai aucun souvenir de ça. Ce sont des faits qui se sont déroulés entre le matin et la nuit du 12 mars. C'était juste le moment où on était sur le réacteur 1, puis sur l'explosion du 1 , l'injection d'eau du 1, le moment où, comme on l'a dit tout à l'heure, j'essayais vaille que vaille de trouver une solution pour injecter l'eau tout en étant dérangé sans arrêt, et donc, en ce qui concerne l'évolution des paramètres du réacteur 3 , je n'ai presque aucun souvenir.

À ce moment-là, j'étais persuadé que le HPCI fonctionnait et que le réacteur 3 pouvait encore attendre. Je ne me rappelle pas très bien, mais il me semble que je considérais encore le RCIC comme l'étape suivante.

$\mathbf{Q}$ : Ces paramètres, comme la pression du réacteur, ce sont des chiffres que le groupe «production » ou les gens de quart faisaient parvenir?

$\mathbf{R}$ : Êtes-vous sûr que les chiffres que vous avez sont bien ceux qui étaient effectivement écrits sur le tableau, les données qui étaient écrites dans la salle de commande?

$\mathbf{Q}$ : Je ne sais pas trop quels sont les critères. En tout cas, ce sont des données relevées par TEPCO.

R : Moi, j'ai des doutes sur ces chiffres. Parce que, voyez-vous, ici, c'est en blanc parce que la collecte des données était impossible. Moi, je pense qu'on n'a pas pu observer ces chiffres. Je voudrais que vous gardiez en tête la possibilité que là où on était dans l'incapacité d'observer les données, on ait pu, plus tard, insérer des chiffres récupérés ailleurs. De fait, quand on observait les indicateurs dans la salle de crise, la plupart du 
temps on n'avait pas de chiffres, l'observation était impossible. Et les spécialistes des mesures s'ingéniaient à trouver une batterie pour faire marcher les instruments et observer. Mais, très vite, la batterie s'épuisait et, de nouveau, on ne voyait plus rien. Le plus important, c'est de savoir quel instrument de mesure était effectivement en fonctionnement à ce moment-là. Alors, même si on vient nous dire après coup que c'était comme ci ou comme ça, en se fondant sur des données qui avaient été mémorisées, mais étaient inaccessibles sur le moment, on ne peut que répondre qu'à ce moment-là, on n'en savait rien.

$\mathbf{Q}$ : Je voudrais savoir ce que vous pensiez de la situation au moment où ce $\mathrm{HPCl}$ était en fonctionnement? Vous ne pensiez pas que la pression était descendue en-dessous de 1 MPa?

$\mathbf{R}$ : Comme je vous le dis, le fait que le HPCI fonctionne, cela veut dire que la pression est toujours élevée, qu'il y a de la vapeur, que cette vapeur s'en va dans le circuit prévu et qu'elle se transforme en eau pour être de nouveau injectée. S'il fonctionne, nous, on pense au contraire que la pression du réacteur est stabilisée aux environs de $7 \mathrm{MPa}$.

Q: D'un autre côté, vous avez le FP qui est évoqué dans ce document. Qu'en était-il du DDFP du réacteur 3 ? Pensiez-vous y avoir recours si nécessaire?

$\mathbf{R}$ : Concernant la pompe diesel, comme je vous l'ai dit à propos du réacteur 1, c'est un système où il $\mathrm{y}$ a un clapet anti-retour qui fait qu'on ne peut pas injecter d'eau quand la pression est élevée de ce côté-ci, mais si elle tombe et que la pression de la pompe devient suffisante, l'eau peut entrer. Toutefois, je n'ai pas le souvenir d'avoir donné l'ordre d'injecter avec cette pompe. Je pense que le groupe «production » essayait des tas de choses de son côté.

$\mathbf{Q}$ : ॥ y avait eu un problème sur la pompe diesel du réacteur 1, dans la nuit du 12 mars à 1h48. Avez-vous eu des informations concernant par exemple un problème qui serait survenu sur le système ou une panne de la pompe diesel du réacteur 3 ?

$\mathbf{R}$ : J'étais au courant que la pompe diesel du réacteur 1 ne fonctionnait plus. Concernant le réacteur 3, on m'avait dit qu'elle était encore bien. Pour autant, je n'ai pas donné l'ordre d'injecter par ce réseau.

Parce que, pour moi, cela ne servait à rien de faire fonctionner la pompe diesel alors que la pression du réacteur n'était pas tombée.

$\mathbf{Q}$ : Pour continuer avec le circuit pompier, vous preniez l'eau dans le réservoir à eau filtrée. La tuyauterie part de là et, en début de soirée le 11 mars, il semblerait qu'il y avait eu des dommages du côté du réservoir d'eau filtrée et qu'il y avait des fuites quelque part sur la tuyauterie, du coup les pompiers de la centrale avaient fermé l'arrivée d'eau. Que s'est-il passé après?

$\mathbf{R}$ : C'était un moment où on avait beaucoup discuté pour savoir d'où on pouvait prendre l'eau. Aujourd'hui, on sait que finalement on a été chercher l'eau de mer, mais, à ce moment-là, on en était encore à envisager différentes sources d'eau. Dans ce contexte, le réservoir d'eau filtrée était bien sûr une source possible. Si le circuit incendie était intact, on pouvait utiliser les bouches à incendie, qui se trouvent en différents points de la centrale, pour remplir les réservoirs d'eau de lutte contre l'incendie, envoyer cette eau avec un camion de pompiers et envisager ainsi même des injections d'eau douce. Et donc nous sommes en train d'envisager cette solution, quand on nous signale que les bouches d'incendie fuient, puis que le réservoir d'eau filtrée fuit. Alors je me souviens très bien d'avoir approuvé l'action des pompiers. 
Puisqu'il y avait fuite, pour préserver notre réserve, il était logique de fermer l'arrivée d'eau. Mais de toute façon nous n'avions pas le choix. Il fallait réparer les fuites et arriver à obtenir un circuit utilisable. C'est une action que nous avons toujours menée en parallèle avec d'autres choses, mais, dans les faits, il faisait nuit, nous n'avions pas d'électricité, la radioactivité montait progressivement, c'était très difficile.

$\mathbf{Q}$ : II me semble qu'il y avait là-bas deux réservoirs à eau filtrée, et si vous fermez les robinets, du point de vue d'un non-spécialiste comme moi, structurellement, il ne reste plus pour alimenter le réseau DDFP que l'eau qui est déjà dans la tuyauterie?

$\mathbf{R}$ : Bien sûr. Mais, s'il y a des fuites, de toute façon l'eau finira par ne plus aller dans la direction voulue, alors le fait de fermer l'alimentation d'eau, ça n'est pas juste fermer pour fermer. On ferme pour pouvoir réparer les fuites. Cela nous permet de vérifier l'endroit où ça fuit et d'utiliser la réserve d'eau de manière optimale. C'est dans ce sens que j'ai donné l'ordre de fermer. Par contre, concernant le moment où s'est déroulée cette histoire de pompe et le moment où on l'a mise en marche, mes souvenirs ne sont pas nets, pour être franc.

$\mathbf{Q}$ : Par exemple, il me semble que c'est vers le 12 ou le 13 mars que le DDFP du réacteur 1 a eu des problèmes.

$\mathbf{R}$ : Permettez-moi d'être très franc. En ce qui me concerne, je ne fondais pas de grands espoirs sur la pompe diesel. Que ce soit pour le réacteur 1 ou le réacteur 3 . Franchement, plutôt que de fonder des espoirs sur un réseau qui ne possédait pas des ressources en eau considérables et qui fonctionnait avec une pompe plus qu'aléatoire, je pensais qu'il valait beaucoup mieux construire un réseau fiable avec les camions de pompiers, qui permettrait d'envoyer à coup sûr de l'eau. Tout le monde me parle du $\mathrm{DD}$, mais, pour moi, j'étais persuadé que ça ne servirait vraiment pas à grand-chose.

Q: Mais si vous voulez utiliser le $\mathrm{DD}$, après avoir isolé le réservoir, vous allez réparer les tuyaux et rouvrir le circuit. Avez-vous le souvenir d'avoir donné des ordres dans ce sens ?

$\mathbf{R}$ : Non. Comment dire, on m'a prévenu que ça fuyait, pensant qu'on pouvait encore avoir besoin de cette eau dans une circonstance ou une autre, j'ai jugé utile de faire fermer cette vanne afin de préserver le niveau d'eau du réservoir. Mais, dans les conditions où nous étions, comme je vous l'ai dit tout à l'heure, même si j'avais voulu détacher quelqu'un pour réparer les fuites, avec cette radioactivité, je ne pouvais même pas envoyer quelqu'un pour trouver la fuite. Alors vous comprendrez que, pour ma part, je n'attendais pas grand-chose du DD.

Seulement, je me suis aperçu après coup que le groupe de quart, par exemple, attendait beaucoup du DD. C'est là où il y avait un gros décalage. Moi, personnellement, je n'en espérais pas grand-chose. Je pensais que si on pouvait envoyer ne serait-ce qu'un peu d'eau par ce réseau, ce serait déjà pas mal, mais sans plus.

$\mathbf{Q}$ : Si on revient au réacteur 3 , le HPCl s'arrête. Si on regarde les paramètres, la pression est de moins d'1 MPa. Mais, déjà, vers 3h00, 4h00, elle est à $4 \mathrm{MPa}$. Alors même si vous aviez voulu envoyer de l'eau par le réseau FP (Fire Protection system) ${ }^{20}$, vous n'auriez pas pu.

Mais, malgré tout, pour l'injection d'eau, vous pensiez tout de même au réseau FP?

$\mathbf{R}$ : C'est ça que je ne peux pas comprendre depuis tout à l'heure. Vous voyez? Du 12 mars $12 \mathrm{~h} 45$ au 13 mars 5 h00, la pression du réacteur chute spectaculairement. On en arrive à moins d' $1 \mathrm{MPa}$. Ça, je n'en ai pas le moindre souvenir. Ensuite, la pression 
remonte jusqu'à 7,38 . Et ça, du point de vue de la physique, qu'une pression qui est descendue remonte, c'est tout à fait incompréhensible.

Q : Parce que, là, vous êtes dans une situation où, après l'arrêt du HPCl, vous n'avez pas injecté la moindre goutte d'eau?

$\mathbf{R}$ : Non. Au contraire, ça devrait remonter. Ça ne peut pas descendre, jamais.

$\mathbf{Q}$ : Quand vous dites que ça descend, ça veut dire?

$\mathbf{R}$ : Si on réfléchit à ce qui arrive quand le HPCI s'arrête, il y a toute la chaleur résiduelle et tant qu'on n'ouvre pas la soupape de dépressurisation, au contraire, on ne peut pas imaginer que la pression chute de cette manière.

Q: Quand vous dites que ça chute, de quel moment parlez-vous?

$\mathbf{R}$ : Là, à la page 2/21, pour la pression du réacteur, elle est en haut, vers 7,53 à 12h10, puis ça plonge tout à coup à 5,6 MPa et il n'y a même plus $1 \mathrm{MPa}$. Ensuite ça remonte jusqu'à 7,3 MPa vers 17 h00. Tout ça, je n'en ai aucun souvenir. Je ne sais pas ce que raconte le responsable de la production, mais moi, je n'en savais strictement rien. Bien que vous me montriez ces données.

$\mathbf{Q}$ : Quand on ne regarde que les paramètres, aujourd'hui, on a quand même l'impression que, pendant que le $\mathrm{HPCl}$ fonctionnait, il y a bien eu une dépressurisation, qu'il y a ensuite un rebond et que la pression remonte. Et le HPCl ?

$\mathbf{R}$ : Avec une pression aussi basse, de l'ordre de 0,8 MPa, le HPCI ne fonctionne plus.

$\mathbf{Q}$ : Oui, vers les $20 \mathrm{~h} 00$, le 12 mars?

$\mathbf{R}$ : En ce qui me concerne, ce sont ces données qui sont invraisemblables. Il faudrait savoir d'où elles sortent. Nous, au contraire, nous avons pensé qu'il y avait toujours eu au moins $7 \mathrm{MPa}$.

Q: Alors, si vous étiez dans cet état d'esprit, ça veut dire que, quand le HPCl s'est arrêté, vous pensiez qu'il fallait, au contraire, dépressuriser pour pouvoir injecter l'eau?

R : Oui.

a: C'est ce que vous pensiez, quand le HPCl s'arrête à $2 \mathrm{~h} 42$. Non, vous y pensiez naturellement avant que ça ne s'arrête, n'est-ce pas?

R : Oui.

Q: Dans ce cas-là, si on regarde la chronologie, par exemple, je trouve que pas mal de temps se passe avant que vous ne fassiez effectivement ces manœuvres de dépressurisation. Qu'en était-il ?

$\mathbf{R}$ : C'est parce que le circuit pour injecter l'eau n'était pas prêt.

$\mathbf{Q}$ : Et quelle en était la cause?

$\mathbf{R}$ : La préparation de la pompe. Et puis c'était le 13 au matin, à l'aube.

$\mathbf{Q}:$ Je lis «début de l'injection d'eau à 9 h20 ».

$\mathbf{R}$ : C'est l'histoire de tout à l'heure. On a enfin réussi à injecter de l'eau de mer dans le réacteur 1 , je pense que c'était dans la soirée du 12 , vers 19 h00, puis il y a ces bêtes histoires d'ordre et de contrordre : "arrête d'injecter l'eau de mer, on n'en a pas encore donné l'ordre", etc., pendant lesquelles, en fait, l'injection continue. Et, bien sûr, je réfléchis à la suite des opérations, et, naturellement, c'est l'élaboration d'un circuit d'injection soit pour le réacteur 3 , soit pour le réacteur 2, qui s'impose. 
En plus, tout ça dépend aussi de l'arrivée des camions de pompiers. Il me semble que c'est à peu près à ce moment-là qu'ils commencent à arriver, un par-ci, un par-là, de différents endroits. J'avais donné l'ordre de réfléchir à la manière de les déployer.

Et puis, on a beau donner l'ordre de faire ci ou ça, si on ne dispose pas des choses, on ne peut rien faire. Il fallait bien attendre que les choses arrivent avant de se lancer dans les différents déploiements.

$\mathbf{Q}$ : D'accord, vous voulez dire que pour l'injection d'eau, vous aviez besoin des camions de pompiers. En ce qui concerne l'opération de dépressurisation, vous deviez la faire avec la soupape de sécurité, je pense. À ce propos, je lis, à la page 33 de la chronologie, que la batterie n'était pas suffisamment chargée pour bouger cette vanne. Vous en rappelezvous?

$\mathbf{R}$ : Oui. Pour manœuvrer cette vanne de sécurité, il faut deux choses: il faut une alimentation électrique et puis une source de pression. Donc, ça ne marche pas, si la batterie nous lâche, ou bien si l'accumulateur n'a pas emmagasiné suffisamment de pression. Ça devait être l'une des deux causes. Je pense qu'on était en train d'y remédier.

$\mathbf{Q}$ : Quand je regarde ce document, je vois que des personnes qui étaient à la cellule de crise de la centrale ont pris l'initiative de démonter les batteries de leurs voitures personnelles pour les monter sur des instruments de mesure à la salle de commande. Le saviez-vous?

$\mathbf{R}$ : Oui. On manquait cruellement de batteries. De mon point de vue personnel, depuis le début, je réclamais au siège toutes sortes de choses et même du personnel, mais l'essentiel, c'était l'alimentation électrique, donc des camions générateurs, puis des batteries. Puis des camions de pompiers, du personnel, bien sûr, pour utiliser tout ça. Ensuite, il fallait de l'eau, de l'huile pour faire fonctionner les machines, de l'essence, tout ça en abondance. C'est tout ce que je réclamais. Mais les batteries, c'était essentiel. Alors on était très embêtés. J'ai donné l'ordre qu'on cherche sur tout le site quelque chose qui puisse remplacer les batteries. On n'est pas seuls sur le site, il y a aussi plusieurs entreprises partenaires, alors j'ai dit aux hommes de se renseigner. C'est à ce moment-là que certains parlaient de démonter les batteries de leurs voitures personnelles. Je leur ai dit que ça me convenait. C'est comme ça que j'ai su, un peu plus tard, qu'ils avaient effectivement démonté les batteries et les avaient rassemblées à la salle de commande.

$\mathbf{Q}$ : Ensuite, le jour commence à venir et, pendant ce temps, je suppose que vous êtes toujours dans les préparatifs. Si je calcule, à partir de l'arrêt du HPCl à $2 \mathrm{~h} 42$ jusqu'au début de l'injection d'eau à 9h20, il y a un laps de temps d'environ 6h40. Pendant ce temps, quel était l'état du cœur du réacteur, selon vous?

$\mathbf{R}$ : Je pensais qu'on allait mourir. Voyez-vous, on voulait injecter l'eau au plus vite, mais, dans les faits, on n'arrivait pas à établir un circuit, plusieurs conditions faisaient défaut.

$\mathbf{Q}$ : Si on regarde l'information numéro 28 suivant l'article 15, celle qui est numérotée « 29 » à la main en haut à droite, bien que l'heure imprimée de l'envoi du fax est le 13 mars à 0h53, je pense que ça correspond au moment que nous évoquons maintenant, vous rapportez que le $\mathrm{HPCl}$ du réacteur 3 s'étant arrêté, vous avez tenté une injection d'eau dans le réacteur avec le système $\mathrm{RCIC}$, mais que ce système n'avait pas fonctionné et que vous faites ce rapport car vous avez jugé que vous aviez perdu toute capacité de refroidissement du réacteur à $5 \mathrm{~h} 10$.

R : Oui. 
$\mathbf{Q}$ : D'après ce rapport, on voit qu'après l'arrêt du HPCl, comme solution de rechange, vous aviez tenté de relancer le RCIC, n'est-ce pas?

$\mathbf{R}$ : Oui, c'est ce qu'on a fait.

$\mathbf{Q}$ : Ensuite, si on regarde l'avis suivant, l'avis 29 suivant l'article 15, c'est celui qui est numéroté « 30 » à la main en haut à droite, l'heure du début d'envoi du fax est 6h19 et la fin de l'envoi 6h25. Vous écrivez, cette fois, que vous avez jugé que le niveau d'eau du réacteur 3 avait atteint son TAF à 4 h15. Effectivement, si on regarde les paramètres du 13 mars au-delà de 5h00, le niveau d'eau est de -2 000, c'est la zone A du combustible. Puis, on a tout le temps des valeurs négatives, $-2000 \mathrm{~mm},-2300 \mathrm{~mm},-2400 \mathrm{~mm}$, $-2600 \mathrm{~mm}$. On voit objectivement le niveau d'eau qui s'effondre. Vous aviez donc conscience, déjà à ce moment-là, qu'une bonne partie du combustible était hors de l'eau ?

$\mathbf{R}$ : Bien sûr. C'est pour ça, je vous le répète depuis le début, que, sachant que tôt ou tard tous les réacteurs arriveraient à cet état, je faisais l'impossible pour retarder ce moment le plus longtemps possible. C'était là ma mission. C'est pour ça que je ne pensais qu'à faire entrer de l'eau, qu'à faire baisser la pression de l'enceinte de confinement, il n'y avait que ces deux choses dans ma tête. Mais, il faut bien admettre que les préparatifs n'avançaient pas. Les gens de l'extérieur nous disaient qu'on était lents, et tout un tas d'autres choses. Mais j'ai envie de leur dire, vous n'avez qu'à essayer. Ah, ça m'énerve, ces histoires! Vous avez trois tranches nucléaires qui sont déchaînées, juste sous vos yeux, vous faites l'impossible avec le peu de personnel dont vous disposez et ils osent dire qu'on est trop lents? Je ne peux pas leur pardonner, à ces gens-là.

$\mathbf{Q}$ : Avant que l'injection d'eau douce ne commence à 9h20, on voit à la page 30 de la chronologie qu'à 7h39, c'est-à-dire deux petites heures avant l'injection d'eau douce, vous commencez l'aspersion de l'enceinte de confinement. Quel était le but de cette opération ?

$\mathbf{R}$ : Ça, c'est parce que la pression de l'enceinte de confinement était montée et il fallait la réduire d'une manière ou d'une autre. Bien sûr, il y a la solution de l'éventage, mais l'autre moyen, c'est de refroidir. Lorsque j'ai posé la question du refroidissement, l'équipe de quart a répondu que c'était possible. D'ailleurs l'équipe de conduite aussi bien que celle de quart étaient d'accord pour dire qu'il valait mieux faire fonctionner ce système au moins une fois pour faire descendre la pression. Du coup, j'ai donné mon aval.

$\mathbf{Q}$ : Où aviez-vous pris l'eau pour cette aspersion de l'enceinte?

R : Je ne me souviens pas de ce qu'on a utilisé cette fois-là. Il faudrait leur demander. Pour moi, j'ai donné mon accord, parce qu'ils ont dit que c'était possible.

$\mathbf{Q}$ : Et ensuite? Qu'est devenu ce système?

$\mathbf{R}$ : En fait, comme il faut, au contraire, une certaine pression pour la manœuvre de l'éventage, le fait de faire baisser la pression pouvait compromettre l'éventage. Du coup, le siège nous a ordonné d'arrêter l'aspersion. Je pense que ça s'est passé comme ça. Alors on s'est soumis aux ordres et on a arrêté.

Ce genre de manœuvre, c'est le genre de choses dont on ne parle nulle part. Il est difficile de savoir si c'était ce qu'il fallait faire ou ne pas faire. Si vous voulez, du point de vue de la production, voilà comment ils raisonnent: puisque la pression est trop élevée, on va asperger pour refroidir et la pression va baisser. C'est assez simple. Par contre, au siège, ils ont dû penser que si on faisait baisser la pression, ça pouvait compromettre l'éventage et ils ont jugé l'aspersion inutile. Il me semble qu'il y a eu des échanges de ce genre.

$\mathbf{Q}$ : Justement sur ce point. II me semble que l'éventage, ça consiste justement à laisser échapper de la pression pour protéger l'enceinte de confinement. C'est un avantage. Mais, 
dans le même temps, c'est une opération qui va disperser dans l'atmosphère des particules radioactives. Est-ce que ça vaut la peine de faire baisser volontairement la pression de l'enceinte par un éventage?

R : Vous savez, même si on peut asperger l'enceinte, c'est une toute petite quantité. On ne dispose pas d'une source conséquente. Ce serait un point à vérifier. Ce n'était pas un système qui permettait d'asperger l'enceinte pendant de longues heures. Il y a normalement un réseau d'aspersion de l'enceinte ${ }^{21}$. Puisqu'il s'agit du réacteur 3 , c'est le mode aspersion de l'enceinte (Containment Spray Mode) ${ }^{22}$ du système RHR. C'est un circuit qui permet d'asperger l'enceinte. Si ce circuit avait été opérationnel, il y aurait eu comme un jet d'eau d'une fontaine qui aurait arrosé l'enceinte et aurait fait baisser la pression. Seulement, ce n'était pas ce circuit qui était vivant. Il me semble que les hommes m'avaient dit qu'ils pouvaient bricoler pour emprunter un autre circuit qui aurait tout de même permis cette aspersion. Et je pense que c'est ce qu'ils avaient fait. Si on avait pu, en empruntant ce circuit de secours, refroidir durablement l'enceinte, cela aurait été formidable, mais ce n'était pas une solution aussi durable. Pour les détails, je pense que vous devriez vous adresser au responsable de la production ou aux gens qui étaient de quart. Pour ma part, je n'ai plus le souvenir de quel réseau a été employé et comment ils ont réussi à injecter de l'eau, mais j'étais partant. Si on pouvait faire baisser, ne serait-ce que d'un peu, cette pression, il fallait le faire. Mais, en même temps, ce n'est jamais resté qu'une manœuvre mineure.

$\mathbf{Q}$ : Ça n'est donc pas non plus une opération qui a duré longtemps. Ayant reçu des avis contraires du siège, vous avez arrêté en cours de route. C'est ça?

$\mathbf{R}$ : Oui, on a arrêté.

$\mathbf{Q}$ : Au moment où vous commencez à injecter de l'eau douce par le réseau FP, vous aviez déjà arrêté ?

$\mathbf{R}$ : Je pense que oui. C'est un moment où il y a eu beaucoup de choses du côté de l'éventage, alors je ne sais plus très bien. Les choses sont un peu confuses dans ma tête. Il faudrait vérifier auprès de ceux qui étaient de quart.

$\mathbf{Q}$ : Très bien. D'autre part, pour l'éventage, au moment où vous commencez l'injection d'eau douce et bien avant ça, depuis l'aube du 13 mars, vous êtes dans les préparatifs. II me semble que vous aviez eu des histoires avec la grande vanne $A 0$, que vous aviez réussi à l'ouvrir ou que c'était difficile. Je voudrais savoir si on vous tenait au courant de ce genre de manœuvres sur cette vanne?

$\mathbf{R}$ : Après toutes les difficultés qu'on avait eues la veille avec le réacteur 1, j'étais très préoccupé par l'éventage du 3 et je me rappelle avoir demandé rapport sur rapport à ce sujet. Mais ce n'est pas très clair. Je pense que le circuit était prêt. J'ai donné l'ordre à 5 h15 de faire en sorte que si la pression montait et que le disque se rompait, l'éventage puisse se faire automatiquement. Mais, après ça, je ne me rappelle plus à quel moment le circuit a été prêt. Ça ne s'est pas imprimé dans ma mémoire.

$\mathbf{Q}$ : À la page 36 de cette chronologie, on voit qu'il y a eu ordre du directeur vers 5 h15, et puis...

$\mathbf{R}$ : Vous dites à la page 36 ?

$\mathbf{Q}$ : Oui, la page 36. Cela concerne les mesures prises pour effectuer l'éventage des réacteurs 1 et 3. II y a une partie qui s'intitule «opérations pour la réalisation des préparatifs pour l'éventage ». Si on regarde une ligne plus haut, le personnel de quart, apparemment le personnel de quart se souvenait assez bien des choses, a déclaré qu'un peu avant, c'est-àdire vers 4h50, il a utilisé un petit générateur électrique pour tenter de magnétiser la vanne 
électromagnétique pour ouvrir la grande vanne $\mathrm{AO}$ de la soupape de la chambre de dépressurisation. Ils étaient donc dans les préparatifs et ils recommencent la même manœuvre vers les 5h00. Ils arrivent à grand peine à magnétiser la vanne, mais la bombe à air ne fonctionne pas bien, ils manquent donc de pression et ils doivent changer la bombe. Et ainsi de suite.

$\mathbf{R}$ : Tout ça, je l'ai su un peu par bribes. Oui, je savais que le circuit était difficile à mettre en place.

$\mathbf{Q}$ : Ensuite, ils continuent à manœuvrer tant bien que mal pour pouvoir ouvrir cette vanne AO. Ils envisagent même de l'ouvrir manuellement et, enfin, à 8h41, ils arrivent au stade où la grande vanne est ouverte et, suivant vos indications, le circuit est prêt et n'attend plus que la rupture du disque. Avez-vous été mis au courant de cette réalisation?

R : Oui.

$\mathbf{Q}$ : Et il ne restait plus qu'à attendre la rupture du disque, n'est-ce pas?

R : Oui, c'est ça.

$\mathbf{Q}$ : Et cette fois encore, comme pour le réacteur 1, vous ne savez pas si l'éventage s'est effectivement fait ? Vous l'avez juste supposé d'après l'évolution de la pression?

R : Il n'y avait que cette solution. On ne pouvait pas le savoir pour de bon. C'est que nous manœuvrions vraiment sans rien voir de la situation. Aucun des paramètres que nous aurions dû surveiller en temps normal n'était visible. Alors, quand on me demande si on a réussi l'éventage, je ne peux que répondre que je n'en sais rien. S'il fallait une réponse simple, je dirais que je n'en sais rien. Si je regarde les preuves indirectes, je pourrais dire qu'il semble que cela se soit fait à un moment ou à un autre. Je ne peux affirmer rien de plus. Alors quand on m'en parle comme s'il y avait eu éventage dans les conditions normales d'une tranche en parfait état de fonctionnement, ça m'énerve aussi.

$\mathbf{Q}$ : Si je comprends bien, vous ne savez pas si la vanne s'est ouverte ou pas, mais les gens sur le terrain pensent, par exemple, si la pression de l'enceinte de confinement baisse temporairement, qu'elle a dû probablement s'ouvrir, et si la pression recommence à monter, qu'une vanne quelque part a dû se fermer. À ce moment-là, ils vont mener des vérifications là où ils peuvent et ouvrir de nouveau, etc. C'était la répétition de ce genre de choses ?

R : Oui.

$\mathbf{Q}$ : Effectivement, si on regarde ça, on voit à la page 36 , oui, la page suivante, que la pression a baissé dans un premier temps, le 13 mars à 9h20, et que vous en avez déduit que l'éventage s'était effectué.

Ensuite, il y a eu des interventions pour resserrer certaines parties de la pompe, vous aviez constaté plusieurs fuites, c'est-à-dire que l'air fuyait et n'arrivait pas à maintenir la vanne ouverte. La vanne qui avait été ouverte dans un premier temps s'était refermée et il avait fallu la rouvrir, etc. Est-ce que vous vous rappelez de ces manœuvres où, après avoir constaté la fermeture d'une vanne ouverte, vous avez dû la rouvrir?

$\mathbf{R}$ : Oui. Enfin, j'en ai souvenir, mais ce n'est pas moi qui suis allé en personne faire ces manœuvres, alors je ne me rends pas compte à quel point tout cela a été pénible. Mais, effectivement, j'en avais eu des rapports.

Q: Dans la matinée du 13 mars, l'éventage s'était fait convenablement dans un premier temps, vous aviez constaté la baisse de la pression, ensuite vous allez découvrir que cela s'est refermé et qu'il va falloir rouvrir. Tout en faisant ça, d'un autre côté, vous avez commencé l'injection d'eau douce. À ce moment-là, que vous dites-vous ? Est-ce que vous 
vous dites que, pour le moment, vous avez fait tout ce que vous pouviez faire pour le réacteur 3 ?

R : Oui.

$\mathbf{Q}$ : Alors, dans l'ordre des priorités, vous vous dites que, pour le réacteur 3 , vous avez fait ce qu'il fallait et vous passez au réacteur 2 ?

$\mathbf{R}$ : Non. Tout d'abord, rappelez-moi à quelle heure on a réussi à injecter de l'eau dans le réacteur 3 ?

$\mathbf{Q}:$ C'était à 9h20.

$\mathbf{R}$ : Oui, 9h20. À ce moment-là, l'eau commençait à entrer, la pression a baissé et le niveau d'eau recommençait à monter. Là, j'étais heureux. Je ne sais pas si l'indicateur de niveau d'eau donnait les chiffres réels ou pas, mais on était passé de -3000 à un chiffre positif. Vous ne pouvez pas savoir à quel point j'ai été heureux. Je ne pouvais pas savoir que c'était totalement illusoire, une fois de plus. Mais j'étais vraiment rassuré. Ce qui m'a le plus rassuré, c'était le niveau d'eau. Le niveau d'eau était rassurant, mais on était encore dans une situation où on ne savait pas si l'éventage s'était vraiment fait ou pas. Et cette situation allait durer. Les rapports se succédaient et, dans cette ambiance, il fallait maintenant penser au réacteur 2 . Y penser en parallèle. Je vous assure, personne n'a jamais eu à faire face à trois tranches nucléaires à la fois et, pour être franc, je pense que cela n'arrivera probablement plus jamais. Je n'ai même pas envie d'y repenser.

$\mathbf{Q}$ : On aimerait quand même vous entendre encore un peu. À ce moment, donc, vous venez de commencer l'injection d'eau douce. Mais vous pensez que, tôt ou tard, la source d'eau douce va tarir et vous donnez des directives à 10 h30 pour préparer l'injection d'eau de mer.

$\mathbf{R}$ : Il me semble que je faisais faire les deux en parallèle.

$\mathbf{Q}:$ L'eau douce aussi ?

R : Oui, l'eau douce aussi. J'ai demandé qu'on injecte d'abord l'eau douce. Et il me semble qu'on avait fait fondre de l'acide borique dedans. Et donc, d'abord l'eau borée, puis l'eau de mer. C'est comme ça que je me souviens de la séquence. Les gens sur le terrain ont dit que j'avais d'abord donné l'ordre d'injecter l'eau de mer et qu'ensuite j'ai fait machine arrière pour injecter l'eau douce. Mais ça, je n'en ai pas souvenir. Je faisais préparer les deux injections en parallèle. De toute façon, il fallait réfléchir à deux circuits différents, un circuit pour l'eau de mer et un autre pour l'eau douce.

$\mathbf{Q}$ : Et au même moment, vous réfléchissiez de même pour le réacteur 2 ?

$\mathbf{R}$ : Oui. Seulement, à ce moment-là, on est le 12 ou le 13 au matin, il n'y avait pas encore suffisamment de voitures de pompiers, alors le réacteur 2... Il faudrait que vous vérifiiez le nombre de camions dont on disposait à ce moment-là, mais ils arrivaient un par un, de loin en loin, alors je ne me souviens pas très bien. Vous comprenez, si on ne disposait pas des camions de pompiers qui servaient de pompes, on ne pouvait pas injecter l'eau. Cette situation a duré longtemps.

Pour moi, les ordres étaient simples, après les gros problèmes du réacteur 1 , il fallait préparer son éventage et injecter l'eau, de même pour les réacteurs 2 et 3 . J'avais demandé à mes troupes de faire le maximum pour ça. De mon côté, j'avais demandé au siège de nous envoyer tous les camions de pompiers qu'ils pouvaient trouver, je leur avais demandé de les chercher partout. 
Et les camions arrivaient, les uns après les autres. Mais, pour être franc, je ne me rappelle pas de tous les rapports qui détaillaient l'avancement des travaux sur le terrain. Je me rappelle seulement qu'à un moment donné un très gros camion était arrivé et qu'on s'était dit qu'en le couplant avec un autre camion, on pourrait puiser l'eau de mer au niveau du quai et qu'on pourrait transférer cette eau de manière beaucoup plus efficace. Voyez-vous, la situation changeait de minute en minute. Pardessus ça, il fallait penser à l'injection des réacteurs 2 et 3, alors, aujourd'hui, les choses ne sont pas claires dans ma tête. De toute façon, la situation était aussi très confuse sur le terrain. Mais les ordres étaient clairs, comme je l'ai dit tout à l'heure, c'était injection et éventage, tout le temps.

$\mathbf{Q}$ : Vers 10h30, pendant que vous injectez l'eau douce, vous prévenez donc vos troupes que quand vous n'aurez plus d'eau douce, vous passez à l'eau de mer, c'est ça?

R : Oui.

Q : Finalement, l'injection d'eau douce se termine à $12 \mathrm{~h} 20$ et vous commencez l'injection d'eau de mer avec les camions de pompiers à $13 \mathrm{~h} 12$.

R : Oui.

Q : Là, je vois qu'il n'y a plus de directive. Cela signifie que, tout naturellement, vous passez d'un circuit à l'autre et vous recommencez l'injection?

R : Oui.

$\mathbf{Q}$ : J'arrive à un moment où on pourrait s'arrêter.

Alors, on va déjeuner.

Si vous êtes d'accord, nous allons nous arrêter. À quelle heure reprenons-nous?

On va s'arrêter trente minutes.

Trente minutes vous suffisent?

R : Oui.

$\mathbf{Q}:$ : Alors, tout le monde est d'accord pour que nous reprenions à $12 \mathrm{~h} 30$ ?

Q: Oui, d'accord.

\section{(Pause)}

Q : Nous sommes le 27 juillet, après-midi. C'est toujours, moi, Katô du secrétariat, qui continue à vous poser les questions.

Si nous revenons à la situation du réacteur 3 , le 13 mars, très vraisemblablement après l'arrêt du HPCl, vous avez ordonné des manœuvres en vue de l'éventage, des manœuvres qui ont été effectivement exécutées, et d'un autre côté vous avez fait réaliser un circuit permettant l'injection d'eau douce. En parallèle, concernant le réacteur 2, si on regarde à la page 20 de la chronologie, dans la matinée, on lit à $10 h 15$ "ordre d'exécution de l'éventage ». Puis, un peu plus bas, à 12h05: "ordre du directeur de la centrale de commencer les préparatifs pour l'injection d'eau de mer». Donc, concernant le réacteur 2, il y a ordre d'éventage dans la matinée. Si je me souviens bien, c'était après l'explosion du réacteur 1, le 12 en fin d'après-midi, à 17h30, que vous aviez donné l'ordre de commencer les préparatifs de cet éventage. Et ce n'est que le lendemain, 13 mars, à 10h15, que vous donnez l'ordre d'exécution de cet éventage. Pensiez-vous à ce moment-là que le RCIC était encore en marche?

$\mathbf{R}:$ Oui, il me semble bien que je le pensais.

$\mathbf{Q}$ : Le RCIC du réacteur 2 est encore en fonctionnement et on n'a constaté, finalement, son arrêt que le 14 mars un peu après midi. Et jusqu'à ce moment, l'indicateur de niveau d'eau 
du réacteur désignait constamment des valeurs aux alentours de TAF +3000 et quelques centaines de millimètres. Pour juger que le RCIC était encore en marche, vous étiez-vous basé sur ces chiffres donnés par l'indicateur de niveau d'eau ? Lui faisiez-vous confiance?

$\mathbf{R}$ : Oui, tout à fait. En plus, avant ça, le 12 vers $2 \mathrm{~h} 55$, on avait réussi, juste une fois, à vérifier la pression en sortie du RCIC. J'avais demandé qu'on continue à la vérifier périodiquement. Mais je pense que la situation sur le terrain était mauvaise et les hommes n'ont pas pu aller la vérifier. Je me rappelle avoir demandé plusieurs fois, par la suite, qu'on vérifie l'état de marche du RCIC, mais je n'ai pas eu de réponse. Donc, à défaut, je faisais confiance à l'indicateur de niveau d'eau.

$\mathbf{Q}$ : Du coup, au moment où vous donnez l'ordre d'éventage le 13 à 10h15, l'indicateur de niveau d'eau continue à donner des chiffres aux alentours de 3000 et quelques centaines de millimètres. Par exemple, le système $B^{23}$ de la zone combustible indique TAF +3700 ou quelque chose dans ces eaux-là. Pensiez-vous, à ce moment-là, que le cœur du réacteur 2 avait suffisamment d'eau et qu'il n'était pas encore dénoyé ?

$\mathbf{R}$ : C'est ce que je pensais. Seulement, je ne me rappelle pas jusqu'à quel point les informations étaient arrivées jusqu'à la cellule de crise. Toujours est-il que, depuis le 13 à l'aube, la pression de la chambre sèche était assez élevée, de l'ordre de 0,31, 0,34, par là. Sachant que la pression de dimensionnement est d'environ 0,4 , on en était très proche. Là, je ne pense pas encore à des histoires de fusion du cœur ni rien. Je ne pense pas qu'il se passe des choses tragiques à l'intérieur du réacteur, puisque le RCIC est en marche et que la pression du réacteur est élevée. Tout de même, la pression de la chambre sèche était à 0,3 et quelques, très proche des 0,4 . C'est pour ça que j'ai donné l'ordre de se préparer à l'éventage. Par contre, je ne me rappelle pas si j'en ai donné l'ordre à $10 \mathrm{~h} 15$ ou pas.

$\mathbf{Q}$ : Si on regarde à la page 4/22 de la partie "Paramètres concernant le niveau d'eau et la pression du $7 F-2^{24}$ », un peu avant 10h15... Oui, il y a les chiffres du 13 mars à 9h55, on voit que la pression du réacteur et la pression de la chambre sèche...

\section{$\mathbf{R}$ : Ont brutalement chuté.}

Q: C'est ça. Si, par exemple, quelqu'un avait été en train de mesurer la pression du réacteur à ce moment-là, ou avait été en train de mesurer la pression de la chambre sèche, celui-là n'aurait-il pas pensé qu'il se passait quelque chose? Ne se serait-il pas demandé quelle en était la cause? D'autre part, le niveau d'eau était constant à 3700 . N'était-ce pas sur la base de ce genre de réflexions que vous aviez donné l'ordre d'éventage?

$\mathbf{R}$ : Je n'en ai pas souvenir. Je suis désolé.

$\mathbf{Q}$ : Saviez-vous que le niveau d'eau était constant, mais que la pression du réacteur et celle de la chambre sèche avaient brutalement chuté ?

$\mathbf{R}$ : Aussi bien pour le réacteur 2 que pour le réacteur 3 de tout à l'heure, il y a un moment où la pression du réacteur chute rapidement. Mais ma mémoire a un trou à cet endroit-là. Alors je ne sais pas si c'est que ma mémoire a un trou ou si c'est que ces données n'ont pas été communiquées à la cellule de crise, et ça, il sera difficile de savoir aujourd'hui ce qu'il en était, mais, en ce qui me concerne, aussi bien pour le réacteur 3 que pour le 2, ma mémoire me fait défaut.

$\mathbf{Q}$ : Ensuite, à 12h05, vous donnez l'ordre de poursuivre les préparatifs en vue de l'injection d'eau de mer, mais vous n'avez pas donné cet ordre précisément parce que vous trouviez bizarre que, bien que le niveau d'eau du réacteur soit stable à 3700 ou 3800 , sa pression et celle de la chambre sèche aient chuté aussi brutalement?

$\mathbf{R}$ : Les discussions autour des paramètres et les préparatifs pour l'injection d'eau sont deux histoires différentes. Comme je vous l'ai dit tout à l'heure, je savais qu'à un 
moment ou à un autre il faudrait injecter de l'eau, qu'il faudrait aussi faire un éventage de la chambre sèche. C'est pourquoi j'ai demandé qu'on s'y prépare, qu'on fasse avancer les choses au maximum, en parallèle, aussi bien pour le réacteur 2 que pour le 3. Et je pense que ceux qui étaient sur le terrain essayaient des tas de choses pour y arriver. Il semblerait, sur ce document, que j'aie donné l'ordre précis d'éventage à ce moment-là. Mais je n'en ai pas le souvenir. Je me suis aussi demandé s'il y avait eu un paramètre qui m'avait poussé à prendre cette décision à ce moment précis, mais je n'en vois pas spécialement. Par contre, je me rappelle m'être dit que je n'aimais pas le fait que la pression de la chambre sèche soit montée, alors que la pression du réacteur n'avait pas baissé. Vous voyez, elle est à $0,35 \mathrm{MPa}$. Là, à la page 3/22. Et elle était à ce niveau depuis l'aube du 13 mars. On l'a découvert quand on a eu les données qui sont apparues tout à coup. Parce qu'on n'avait pas les chiffres de manière continue. Alors que, jusque-là, les chiffres étaient plutôt à la baisse, tout à coup, vous recevez des données, ça y est, on a réussi à prendre des mesures, et vous vous rendez compte que les chiffres ont augmenté. Et là, vous vous dites que si la courbe ne fait que commencer à monter, il y a urgence à réaliser l'éventage. C'est ce genre de raisonnement qui est à la base de mes ordres. Je ne me rappelle même plus si j'ai dit à partir de quel chiffre il fallait procéder à l'éventage.

Q: Je ne sais pas si ces chiffres arrivaient à la cellule de crise en temps réel, mais si on regarde la page 4/22, le 13 mars à 9 h55 la pression de la chambre sèche chute brutalement et, à 10h35, on n'est plus qu'à 0,01 MPa. Faut-il comprendre que c'est l'indicateur de pression lui-même qui a eu des problèmes?

$\mathbf{R}$ : C'est l'hypothèse la plus probable. En fait, cet indicateur fournissait souvent des chiffres à la baisse, et pas seulement pour le réacteur 2, mais aussi bien pour le réacteur 1,2 ou 3. (Dans le tableau des paramètres). Là où la case est vide, ça signifie qu'on n'a pas réussi à voir les chiffres. Et même les chiffres qui apparaissaient tout à coup, on ne savait pas s'ils correspondaient vraiment à la réalité. Seul l'indicateur de niveau d'eau indiquait toujours les mêmes valeurs. C'est pour ça que, pour nous, l'indicateur de niveau d'eau était l'instrument le plus fiable. Au regard de la constance de ses paramètres.

$\mathbf{Q}$ : Pour ce qui est de l'indicateur de niveau d'eau, justement, on se rend compte que vous regardez le système $A$, puis pendant un certain temps vous observez le système $B$, avant de revenir au A. Était-ce parce que vous vouliez non seulement regarder le B, mais aussi le A ?

$\mathbf{R}$ : Ça, je pense que c'était dû à des problèmes de batterie. On n'avait qu'une seule batterie. Du coup, il fallait alterner. On ne pouvait pas observer les deux à la fois. Et donc, à ce moment-là, on devait observer principalement le système $\mathrm{B}$, mais, de temps en temps, on vérifiait aussi le $\mathrm{A}$, une histoire de ce genre.

$\mathbf{Q}$ : Quand on regarde ici, la pression du réacteur chute brusquement à 9h55, puis il y a une série de cases vides, puis elle est remontée à 5,85 MPa à 15h30.

R : Là, pour moi, ce n'est pas une hausse. Je dirais plutôt que l'indicateur était détraqué.

$\mathbf{Q}$ : Vous voulez dire que ces cases sont vides, parce que, même si vous tentiez les mesures, ça n'était pas possible?

R : Oui.

$\mathbf{Q}$ : Cela ne signifie pas que vous n'aviez pas effectué les mesures?

$\mathbf{R}$ : Non. Cela veut dire qu'on a essayé, mais qu'on n'a pas obtenu de résultats. 
Q : Au moment où vous donnez ces ordres d'injection et d'éventage, pour vous, le RCIC fonctionne toujours. Vous donnez ces ordres pour prendre de l'avance dans les préparatifs, c'est ça?

R : Oui.

$\mathbf{Q}$ : Dans la matinée du 13 mars, on en a parlé au début, vous faites également étudier les mesures à prendre pour éviter l'explosion d'hydrogène.

$\mathbf{R}$ : Bien sûr. On ne voyait pas de solution de notre côté, alors on avait sollicité le siège. Bien entendu, j'avais chargé les hommes sur le terrain de faire quelque chose avec ce qu'ils avaient sous la main. Mais c'était une affaire de construction. Il fallait détruire le bâtiment. Ceux qui travaillent dans le bâtiment étaient les plus à même de réfléchir à cette question, trouver l'instrument, la méthode pour y parvenir. Alors j'avais sollicité le responsable " génie civil » de notre département "construction » au siège, en lui disant qu'il fallait absolument qu'il nous trouve une solution pour faire une brèche. Mais toutes les solutions qu'on nous a proposées paraissaient irréalisables. J'avais la sensation que le problème ne se résoudrait pas facilement. Déjà, l'accès par l'intérieur du réacteur n'était absolument plus possible, il fallait accéder par l'extérieur. Je leur ai demandé de trouver le moyen d'ouvrir d'une manière ou d'une autre ce fameux panneau de décompression, dont on a parlé tout à l'heure. Finalement, il ne restait guère que ça. Et même ça, restait très compliqué. On n'avait pas d'échelle, il aurait fallu quelque chose comme une voiture de pompiers avec une grande échelle.

$\mathbf{Q}$ : Vous réfléchissez toujours parallèlement aux mesures à prendre pour les réacteurs 2,3 et 1. Dans la matinée du 13 mars, le problème le plus urgent, c'est le réacteur 3 ?

R : Oui.

Q: Concernant la situation du réacteur 3 dans l'après-midi du 13 mars, avez-vous eu un rapport faisant état d'une très forte radioactivité autour du réacteur?

R : Oui.

Q : Vous n'avez peut-être pas les documents sous les yeux, mais d'après les dossiers de TEPCO, concrètement, on a $300 \mathrm{mSv} / \mathrm{h}$ à l'intérieur de la double porte du bâtiment réacteur. Cela se passe vers $13 \mathrm{~h} 00$ ou 14h00. On aurait observé une sorte de brouillard blanc à l'intérieur du bâtiment réacteur. En avez-vous souvenir?

R : Oui.

$\mathbf{Q}:$ : Cela se situe avant l'explosion du réacteur 3, n'est-ce pas?

R : Oui, c'était avant.

$\mathbf{Q}$ : Ayant eu connaissance de ce genre de phénomènes, que pensiez-vous qu'il se passait au réacteur 3 ?

$\mathbf{R}$ : À la base, je pensais que, comme pour le réacteur 1 , le combustible avait d'une manière ou d'une autre été endommagé, que de la vapeur - ou d'autres choses - qui s'était échappée de l'intérieur, commençait à fuir de l'enceinte de confinement dans le bâtiment lui-même.

$\mathbf{Q}$ : Dans le cas du réacteur 1, une certaine série d'anomalies s'était succédée et il y avait eu explosion d'hydrogène par la suite. Si je me souviens bien, au départ, vous ne saviez pas quelle pouvait être la cause de cette explosion, mais au fur et à mesure de vos discussions, vous en étiez venus à penser que c'était dû à l'accumulation, dans la partie haute du bâtiment, de l'hydrogène qui s'était échappé de l'enceinte. Lorsque vous avez reçu l'information qu'il y avait un brouillard blanc, que la radioactivité avait augmenté autour du 
réacteur 3, avez-vous pensé que, de nouveau, de l'hydrogène était en train de s'accumuler dans la partie haute du bâtiment réacteur?

$\mathbf{R}:$ C'est évident.

$\mathbf{Q}:$ Vous y aviez donc pensé. Et aviez-vous une idée du temps que cela tiendrait ?

$\mathbf{R}$ : Non. Je ne savais pas. Mais je pensais bien que l'hydrogène s'accumulait de plus en plus.

$\mathbf{Q}$ : Et dois-je comprendre que, tout en ayant lancé les réflexions, autour de vous et même jusqu'au siège, sur les mesures à adopter pour éviter l'explosion, vous n'aviez aucune solution réaliste en vue?

R : Oui.

Q : Ensuite, vers le 13 mars, avez-vous le souvenir d'avoir entendu parler d'une sorte de vapeur qui sortait de la piscine à combustible usé ${ }^{25}$ du réacteur 1 , où avait eu lieu l'explosion?

$\mathbf{R}$ : Quel jour ? Après l'explosion, vous dites ? Le réacteur 1 a explosé le 12.

$\mathbf{Q}$ : Le lendemain, dans la matinée.

$\mathbf{R}$ : Dans la matinée, le réacteur 1 ?

$\mathbf{Q}$ : Oui.

$\mathbf{R}$ : Tout est un peu mélangé dans ma mémoire. Parce que des histoires de vapeur qui s'échappe de quelque part, il y en a eu tellement par après, que je n'ai pas le souvenir précis de cette histoire du réacteur 1 en particulier.

$\mathbf{Q}$ : Alors, que ce soit le réacteur 1 ou 2, cela m'est égal, mais à partir de quel moment, que ce soit le 13, le 14 ou le 15, je voudrais savoir à partir de quel moment vous avez pensé qu'il faudrait intervenir au niveau des piscines de combustible.

$\mathbf{R}$ : Depuis le début.

$\mathbf{Q}$ : Depuis le début?

$\mathbf{R}$ : Oui. En gros, la plus grande préoccupation était de contrôler d'une manière quelconque les réacteurs nucléaires, mais il est évident que, les piscines n'étant plus refroidies, la température de l'eau allait monter à cause de la chaleur résiduelle des combustibles usés et que l'eau allait s'évaporer. J'ai toujours pensé qu'il fallait faire quelque chose pour ça. Et dès le début je l'ai pensé en parallèle. Toutes ces préoccupations ne se sont pas succédées en série, mais je les ai toujours eues à l'esprit en parallèle.

$\mathbf{Q}$ : D'accord. Si on regarde les données en suivant cet angle, par exemple, le quarantequatrième rapport selon l'article 15, celui qui porte la mention « 45 » à la main, comporte, au revers en tant que document joint, un tableau des paramètres des différentes tranches que vous aviez élaboré au $1 \mathrm{~F}^{26}$. Ici, à l'endroit où il est indiqué « $4 u$ » (tranche 4), on lit « à l'arrêt ». Ensuite, si vous sautez quatre pages, le quarante-septième rapport selon toujours l'article 15 , celui où on a écrit « 4 » à la main en haut à droite, possède aussi en document joint les paramètres des différentes tranches, classés de manière similaire, et à « $4 u$ », en-dessous de "à l'arrêt », on note "température de la piscine 84 à 4 h08》. Le document est daté du 14 mars 6h00, donc je suppose que vous aviez mesuré la température de la piscine le 14 mars à 4 h08. Alors que dans le document précédent, il n'y a pas d'indication concernant cette température. Est-ce parce que vous ne l'aviez pas mesurée précédemment?

$\mathbf{R}$ : C'est qu'on n'avait pas pu la mesurer. Il me semble qu'il y avait des problèmes de personnel. En plus, l'indicateur de température, lui-même, était mort. Je ne sais plus comment on a fait pour le ressusciter. De toute manière, j'avais conscience qu'il 
fallait absolument arriver à mesurer cette température, alors j'avais donné des directives pour qu'on fasse le nécessaire.

$\mathbf{Q}:$ Vous rappelez-vous quand vous avez donné ces ordres?

$\mathbf{R}$ : J'en avais donné l'ordre assez tôt. Par exemple, pour les réacteurs 5 et 6 , je pensais qu'on arriverait plus rapidement à mesurer la température des piscines. En tout cas, pour le réacteur 4 , à ce moment-là, il n'y avait aucun problème à pénétrer à l'intérieur du bâtiment réacteur. Bien sûr, la radioactivité était un peu plus forte, à cause du réacteur 3, mais il n'y avait pas de source radioactive directement dans le bâtiment, alors j'avais donné l'ordre de refroidir la piscine à combustible, en parallèle avec les autres manœuvres. Le plus important était bien entendu la température et j'avais donné l'ordre d'aller la vérifier. Dans la réalité, à ce moment-là, sur le terrain, on était effectivement très mobilisés sur le réacteur 3 et je ne sais plus combien de personnes j'ai pu détacher pour le réacteur 4. Mais il fallait simultanément vérifier la température de la piscine.

$\mathbf{Q}$ : Comment procédez-vous concrètement pour mesurer la température de la piscine?

$\mathbf{R}$ : En temps normal, si l'indicateur est en fonctionnement, on vérifie à partir de la salle de contrôle la température de l'eau qui se déverse de la piscine dans le réservoir tampon, ou bien, quand le circuit de refroidissement, le FPC (Fuel Pool Cooling system) ${ }^{27}$ qui évacue l'eau de la piscine vers des échangeurs de chaleur et réintègre de l'eau froide, fonctionne normalement, on mesure aussi la température de l'eau qui arrive à l'entrée de la pompe du système FPC, qui représente à peu près la température de la piscine. Il $\mathrm{y}$ a donc plusieurs points où on peut mesurer rapidement la température si les indicateurs fonctionnent. Mais dans le cas du réacteur 4, comme pour les autres, on avait perdu toute source d'électricité, on ne pouvait donc plus compter sur les procédés habituels. Alors je ne sais pas comment ils se sont débrouillés sur le terrain. Peut-être ont-ils bidouillé dans la salle de contrôle et réussi à ressusciter de force un des indicateurs, ou bien, se sont-ils déplacés directement sur place pour enfoncer un thermomètre quelque part, je n'en ai aucun souvenir.

$\mathbf{Q}$ : Vous aviez donc le souci, dès le départ, de surveiller l'état des piscines de combustibles usés, c'est ça?

R : Oui, bien sûr.

$\mathbf{Q}$ : Vous pensiez donc que si vous disposiez de suffisamment de personnel et de matériel, c'était quelque chose que vous deviez faire. Est-ce vrai aussi concernant les réacteurs 1, 2 et 3 ?

$\mathbf{R}$ : Tout à fait, de la même manière. Seulement, ce qui rendait les choses plus compliquées au réacteur 4, c'est que son inspection périodique venait juste de commencer. On avait donc enlevé tout le combustible. On avait mis dans cette piscine les cinq cent quarante-huit crayons ${ }^{28}$ qui avaient chauffé une année durant. On les avait mis tous ensemble dans cette piscine. Cela veut dire que cette piscine contenait les combustibles les plus chauds et que ça représentait les pires conditions qu'on pouvait imaginer pour une piscine de combustibles. En ce qui concerne les réacteurs 1,2 et 3, le fait qu'ils étaient en marche signifie que dans leur piscine ne se trouvaient que les combustibles placés là lors de la précédente inspection et, logiquement, ce sont des combustibles qui ont eu le temps de commencer à refroidir. Si on va dans ce sens, le réacteur 1 , par exemple, qui a une petite capacité du point de vue du combustible suscite moins d'inquiétude. Par rapport à la marge qui nous était laissée dans la hausse de la température, le plus préoccupant était vraiment le réacteur 4. 
Alors, je considérais le sort de la piscine de combustibles usés du réacteur 4 comme très important.

Q: Puisque vous venez de faire allusion aux réacteurs 5 et 6 , en ce qui les concerne, le 13 mars, je pense que c'est en début d'après-midi, vous réussissez à tirer l'électricité du générateur diesel de secours du réacteur 6 vers le réacteur 5 . Vers la fin de la journée, ce même jour, vous commencez même à injecter de l'eau en utilisant la pompe du réseau d'eau du condenseur et ce, aussi bien pour le réacteur 6 que pour le 5 . Concernant toutes ces opérations, en aviez-vous dirigé personnellement les étapes ?

$\mathbf{R}$ : Pas dans les détails. Concernant les réacteurs 5 et 6 , l'alimentation électrique arrivait grosso modo à circuler. Il fallait donc utiliser cette électricité pour ressusciter tout ce qui servait au refroidissement, puisque le refroidissement était la priorité numéro 1, le refroidissement de la piscine de combustibles, le refroidissement du réacteur, cela va sans dire. Bien que le réseau habituel d'électricité ne fonctionnait pas, on avait retrouvé quasiment toutes les fonctions d'avant l'arrêt de l'alimentation électrique. La cuve des réacteurs était correctement fermée. Mais, puisque le réacteur était chargé en combustible, il y avait de la chaleur qui se dégageait, bien entendu, et le rétablissement du circuit de refroidissement était la priorité des priorités. J'avais donc donné des ordres dans ce sens.

$\mathbf{Q}$ : Et, dans une situation où vous manquiez d'hommes et de matériel, combien d'hommes avez-vous pu dégager pour les réacteurs 5 et 6 ?

$\mathbf{R}$ : Pour les réacteurs 5 et 6 , il y avait les conducteurs des 5 et 6 . Géographiquement, ils étaient éloignés des réacteurs 1 à 4 et se trouvaient dans de bien meilleures conditions, du point de vue de la radioactivité, par exemple. Mais je ne leur ai pas demandé de venir en renfort des réacteurs 1 à 4 . Au contraire, je leur ai dit qu'il était de leur responsabilité d'utiliser au mieux les ressources électriques dont ils disposaient pour répondre à la priorité numéro 1 , le refroidissement, tel que je viens de l'évoquer. L'autre point critique, évidemment, était le fait que le combustible placé dans la cuve continuait à dégager de la chaleur, la température montait, de la vapeur, aussi, se dégageait, et que cela faisait monter la pression du réacteur. Alors je leur ai dit d'assurer, par n'importe quel moyen, de quoi refroidir et de ne pas laisser la pression de l'enceinte de confinement grimper, de se débrouiller si elle montait pour la faire redescendre. Pour ce qui est des mesures concrètes à appliquer, le groupe « production » s'est débrouillé seul.

Q: Excusez-moi de vous emmener à droite et à gauche, mais si on revient au réacteur 1 , vous continuez l'injection d'eau de mer. Si on regarde les chiffres du 13 mars, ici, dans le document «7F-729, Paramètres concernant le niveau d'eau et la pression", qui concernent le réacteur 1 , vers les pages $5 / 26,6 / 26$, on lit $-1700 \mathrm{~mm},-1750 \mathrm{~mm}$ pour le niveau d'eau. On dirait que les chiffres continuent à aller et à venir durablement dans ces zones-là. En aviezvous conscience?

$\mathbf{R}:$ Oui, tout à fait.

$\mathbf{Q}$ : Au moment que nous évoquons, vous êtes en train d'injecter massivement de l'eau. Alors, quelle était votre opinion concernant ce niveau d'eau qui n'arrivait pas à décoller des $-1700 \mathrm{~mm}$ ?

$\mathbf{R}$ : Pour moi, à ce moment-là, je pensais que l'indicateur de niveau d'eau était bloqué quelque part. Qu'il était bloqué quelque part dans la zone négative. Et que c'était pour ça que le niveau d'eau était inchangé. Aujourd'hui, je sais qu'il y avait des fuites importantes. Mais, sur le moment, je n'avais aucune idée du rapport entre la quantité d'eau injectée et la fuite. Pour moi, si on injectait l'eau, le niveau devait 
obligatoirement monter. Si le niveau ne montait pas, c'est que les instruments de mesure étaient défectueux. C'est ce que je pensais.

$\mathbf{Q}$ : Ça n'est qu'une hypothèse, mais si vous aviez su, à ce moment-là, qu'il y avait une fuite, est-ce que cela aurait changé quelque chose à vos réactions ?

$\mathbf{R}:$ Il n'y avait rien d'autre à faire.

$\mathbf{Q}:$ Seulement continuer à injecter l'eau?

R : Il n'y avait que ça. De plus, même si on avait voulu augmenter le débit de l'eau, il y aurait eu des problèmes avec les diamètres des tuyaux de la pompe. On était déjà au maximum de ce qu'on pouvait envoyer. On n'avait pas de chiffres concernant le volume d'eau injectée, mais j'avais donné l'ordre d'envoyer le maximum. Je me demande même si on n'en injectait pas environ 20 tonnes par heure. C'était déjà important.

$\mathbf{Q}:$ Ça, c'est pour le réacteur 1 .

$\mathbf{R}$ : Oui, pour le réacteur 1 . Je pense qu'on devrait pouvoir retrouver des chiffres quelque part. Par exemple, aujourd'hui, nous injectons 3,5t/h dans le réacteur 1. En comparaison, vous voyez que c'était une réponse très musclée.

Q : Au 13 mars, vous envoyiez l'eau à partir du bassin qui se trouvait dans le bâtiment turbine du réacteur 3. Si on regarde dans le document l'ensemble des faits concernant le 13 mars, on voit à partir de 9h20: «injection d'eau douce dans le réacteur 3 », c'est une injection que vous faites à partir du réservoir d'eau de lutte contre l'incendie, n'est-ce pas ? L'aprèsmidi, à partir de 13h10: "injection d'eau de mer", injection qui se fait à partir du fameux bassin dont nous venons de parler. Si je résume, à partir de 13h10, vous êtes dans une situation où vous alimentez à la fois le réacteur 1 et le réacteur 3 en eau à partir de ce même bassin. Si je comprends bien, même si l'eau de mer est à votre disposition de façon illimitée grâce à l'océan qui s'étale devant vos yeux, l'eau de mer qui se trouve dans ce bassin est limitée. Comment avez-vous contourné cette difficulté ?

R: J'avais demandé qu'on passe en revue toutes les solutions pour alimenter ce bassin en eau. Que ce soit de l'eau douce ou de l'eau de mer, peu importait. J'avais donné l'ordre de transporter toute l'eau qu'on avait sous la main en véhicule citerne pour alimenter le bassin. Simultanément, en parallèle, on réfléchissait à la manière de faire monter l'eau de mer jusque-là, mais le bassin est à $10 \mathrm{~m}$ au-dessus de la mer.

$\mathbf{Q}$ : Pour vous, à ce moment-là, vous n'étiez absolument pas focalisé sur le fait que l'injection se fasse à l'eau de mer ou à l'eau douce, pourvu que ce soit de l'eau. De la même manière, vous n'étiez pas spécialement attaché à ce que cela se fasse plus particulièrement à l'eau de mer, n'est-ce pas? Avez-vous émis des demandes pour qu'on vous apporte de l'eau de l'extérieur? Par exemple, par l'intermédiaire des forces d'autodéfense?

$\mathbf{R}$ : Oui, j'ai déposé des demandes.

$\mathbf{Q}$ : Vous l'avez fait vis-à-vis du siège?

$\mathbf{R}$ : Vis-à-vis du siège. Je ne me rappelle pas bien de quelle chaîne de commandement il s'agissait, mais je me rappelle très bien avoir dit au siège que je voulais de l'eau, n'importe laquelle. Nous possédions des camions citernes en propre. On les a envoyés chercher l'eau qui se trouvait dans la copie de la cuve qui nous servait de simulateur pour les sessions de formation aux manœuvres de transfert des combustibles. Cette copie, aux dimensions d'une vraie cuve, mais coupée en deux, se trouve dans le centre de formation technique qui est situé près de l'entrée principale. On a donc récupéré cette eau, ainsi que toute l'eau qui existait sur la centrale sous toutes ses 
formes, grâce à ces camions citernes qui la transportaient jusqu'au bassin. Voilà le genre de choses qu'on faisait. Et au milieu de tout ça, on nous a dit qu'il y avait des voitures des forces d'autodéfense qui allaient nous apporter de l'eau, et on a accepté avec reconnaissance. Par la suite, lorsqu'ils sont enfin arrivés avec l'eau et qu'ils s'apprêtaient à la déverser dans le bassin, le réacteur 3 a explosé et quatre personnes des forces d'autodéfense ont été blessées. Juste au moment où ils s'apprêtaient à verser l'eau, c'était de l'eau douce, il y a eu l'explosion.

$\mathbf{Q}:$ Y a-t-il eu, avant ces personnes des forces d'autodéfense qui étaient là au moment de l'explosion du réacteur 3 le 13 mars, d'autres personnes déléguées par des autorités publiques, même autres que les forces d'autodéfense, pour vous livrer de l'eau?

$\mathbf{R}:$ Non, il n'y en a pas eu.

Q: Vous voulez dire qu'elles étaient les premières?

R : Oui.

Q: Moi, en regardant tout ça de l'extérieur, je me suis demandé s'il n'y avait pas moyen de faire appel à des réseaux de pompiers des autorités locales, bien que je ne sache pas trop comment sont organisés les pompiers et comment ils fonctionnent, pour qu'ils relient les tuyaux à incendie sur 3 ou $4 \mathrm{~km}$ pour vous apporter l'eau. Vous n'y aviez pas pensé ?

$\mathbf{R}$ : Ce n'est pas que je n'y ai pas pensé. Tout ça était évidemment compris dans les demandes que j'avais adressées aux multiples interlocuteurs à l'extérieur de la centrale, mais je n'ai eu aucune réponse. Pour être plus clair, tout le monde avait fui et personne n'est venu.

$\mathbf{Q}$ : Personne ne vient parce que tout le monde a fui ?

$\mathbf{R}$ : C'est ce que je pense. Je ne sais pas quels ont été les mouvements extérieurs, puisque je n'ai pas bougé d'ici. Mais depuis le début, je n'ai pas arrêté de réclamer de l'eau, que c'était vital, que, comme je l'ai dit tout à l'heure, peu importait la manière, il me fallait de l'eau, sinon le réacteur risquait de fondre. Je n'ai pas arrêté de le clamer. Et, en face, je suppose que, si ce sont les forces d'autodéfense, elles ont dû bouger en suivant le cadre qui leur est propre. Les téléconférences sont retransmises au centre de crise hors site, alors les gens du département étaient au courant, puisqu'ils sont stationnés au centre hors site. Je pense que normalement le directeur du centre de crise hors site $^{30}$ aurait dû réfléchir à ce qu'il pouvait faire au niveau de son département et collaborer avec nous. Mais je n'ai reçu aucune réponse de sa part.

$\mathbf{Q}$ : J'entends ça pour la première fois et je suis très surpris. Parce que j'ai entendu dire que quand le transformateur de la centrale de Kashiwazaki avait brûlé, ils étaient très embêtés parce qu'ils manquaient aussi d'eau, mais que les pompiers de la commune, ou quelque chose comme ça, avaient relié plein de tuyaux à la queue leu leu pour amener l'eau. Comme j'avais entendu dire que c'était ce qui s'était passé à Kashiwazaki, je pensais que ce serait pareil pour ici. C'est ce que je pensais en regardant tout ça de l'extérieur. Mais, en fait, il ne s'est rien passé. Au fait, est-ce que ça s'est vraiment passé comme ça à Kashiwazaki ?

R : Quand il y a eu l'incendie de Kashiwazaki, j'étais affecté au siège, alors, je n'étais pas directement impliqué comme maintenant et je ne sais pas si ça s'est vraiment passé comme ça, mais il me semble qu'à Kashiwazaki aussi les pompiers s'étaient fait attendre.

$\mathbf{Q}$ : Après l'accident, je suis allé à Kashiwazaki et on a eu pas mal de discussions. Les gens de la centrale nous ont dit qu'ils avaient dû admettre que tous les scénarios qu'ils avaient 
élaborés s'étaient avérés des solutions impraticables et que, face à la réalité de l'accident, les gens de l'extérieur semblaient finalement mieux armés.

$\mathbf{R}$ : En tout cas, à Kashiwazaki, ils ne possédaient même pas un seul camion de pompiers. De plus, même les bouches à incendie n'ont pas permis de délivrer de l'eau, parce que le réseau de lutte contre le feu, qui était enterré, avait fondu. Alors, ils ont dû solliciter les pompiers sur un périmètre très vaste. Seulement, là aussi, les accès étaient en très mauvais état et les camions n'ont pas pu pénétrer. Je me demande si, finalement, l'incendie ne s'est pas éteint tout seul. Vous êtes sûr qu'on l'a arrosé ?

Q : D'après ce que j'ai entendu dire, ils auraient relié des tuyaux sur une longueur de $800 \mathrm{~m}$ ou quelque chose comme ça. Je n'en suis pas certain, mais ça m'avait amené à penser que les relations s'étaient drôlement améliorées entre la centrale et les autorités locales.

R: Il y a une chose qu'il ne faut pas oublier, à Kashiwazaki, il n'y avait pas de problème de radioactivité. C'était tout simplement un tremblement de terre et c'était tout. La centrale ne s'est pas retrouvée dans une zone d'évacuation, comme pour Fukushima Daiichi.

$\mathbf{Q}:$ Vous pensez que c'est ce qui compte le plus?

$\mathbf{R}$ : Ah, oui ! Du coup, il s'agit de savoir si les pompiers, venus d'un large périmètre, peuvent travailler dans une zone qui est soumise à évacuation.

$\mathbf{Q}$ : À y réfléchir maintenant, je me demande si le laps de temps qui s'est écoulé du tremblement de terre jusqu'à l'explosion d'hydrogène ou autre chose, on ne le savait pas à l'époque, et le moment où tout le monde va comprendre que des éléments radioactifs se sont échappés à l'extérieur du bâtiment, n'avait pas été le moment où tout le monde avait la volonté de bouger et de faire quelque chose. Si ça avait été le cas, cela aurait été justement le moment d'installer un tel réseau de tuyaux, par exemple, ou autre chose. C'est un peu ce à quoi je m'attendais, moi, dans mon coin, bien loin de la centrale. Je me disais qu'à un moment ou à un autre, vous auriez besoin d'eau et que vous aviez peut-être donné des ordres pour établir tout de suite une sorte de pipeline entre la centrale et l'extérieur, en profitant de ce moment. Mais vous n'aviez sans doute pas pensé jusque-là.

$\mathbf{R}$ : Je n'en sais rien. Comme je vous l'ai dit tout à l'heure, j'étais à l'intérieur de la centrale et je n'avais absolument aucune idée de tous les mouvements qui se déroulaient à l'extérieur. Pour moi, la seule chose que je sais, c'est que, finalement, personne n'a rien fait pour nous. Peut-être ont-ils essayé de faire des choses, mais ça je n'en ai aucune idée.

Q : D'accord. J'en ai fini.

$\mathbf{R}$ : Vous allez trouver que je souffre de la folie de la persécution. Mais, pour moi, il n'y a que cette constatation, finalement personne n'est venu nous aider. Pardonnezmoi, car je vais vous dire ce que je ressens vraiment. Eh bien, que ce soit le siège ou les autres, alors que nous nous battions de toutes nos forces avec le peu de personnel que nous étions, personne n'a su nous apporter la moindre aide concrète, efficace. Et pour ça, je leur en veux, terriblement.

$\mathbf{Q}$ : Vous avez tout à fait raison. Finalement, personne n'est venu vous aider.

$\mathbf{R}$ : On en reparlera plus tard, mais je voudrais quand même ajouter que des pompiers, des secouristes sont venus, mais leurs actions n'ont pas eu beaucoup d'effets.

Q: Le 13 mars, au niveau du réacteur 3, vous effectuez un éventage et vous poursuivez l'injection d'eau douce, pour commencer, et d'eau de mer, par la suite. Pour ce qui est du réacteur 1, après l'explosion à 12h00, vous poursuivez l'injection d'eau de mer, avec certes un temps d'arrêt, mais, grosso modo, vous la poursuivez. Quant au réacteur 2, le RCIC est en 
marche. Voilà à peu près la situation quand vous entamez la nuit du 13. Si on regarde le rapport $n^{\circ} 40$ suivant l'article 15 , celui qui porte la mention « 41 » à la main, qui a été envoyé le 13 à 19h10, sur la deuxième page, à la main, il y a un tableau intitulé "Paramètres concernant les différentes tranches ». Le tableau est daté du 13 à 18 h45 et relève les chiffres des différentes tranches. À l'avant-dernière ligne, on trouve «résultat des mesures radioactives ». Sont portés là les résultats du monitoring concernant la radioactivité. Là, vous avez "détection d'iode », suivi de la mention « en-deçà des limites de détection ». II s'agirait du résultat d'un contrôle effectué vers $17 \mathrm{~h} 16$ près de l'entrée principale. Si on regarde ensuite le rapport $n^{\circ} 41$ qui comporte aussi les paramètres des différentes tranches à la deuxième page et dont les chiffres datent du 13 à 23h30, on voit, cette fois-ci, à la ligne: «résultat des

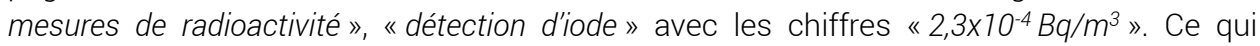
laisse supposer qu'on est au-delà des limites de détection. Que signifie le fait qu'on ait des chiffres qui se situent au-delà des limites de détection?

$\mathbf{R}$ : Je ne sais plus quel était le réacteur concerné, mais il me semble que c'était le réacteur 1 . Concernant ce réacteur, je ne sais pas si l'éventage avait été efficace ou pas, mais au moment dont nous parlons, le dessus de son bâtiment réacteur a été détruit. On est encore le 13, l'explosion du réacteur 3 n'a pas encore eu lieu. La partie supérieure du bâtiment réacteur du 1 a été soufflée par l'explosion, on peut penser qu'il y a des fuites au niveau de l'enceinte de confinement et que cela s'échappe vers l'extérieur. Et donc, j'en ai conclu qu'il y avait fuite de là vers l'extérieur. À la limite, puisque l'explosion du réacteur 1 s'est passée le 12, c'est plutôt le fait que tellement de temps se soit écoulé avant qu'on ne détecte l'iode qui m'a surpris.

Q : J'ai fait exprès de vous demander de regarder d'abord les données du 13 mars 18h45, où on n'a pas détecté d'iode de manière significative, alors que le même jour à 23h30, nous sommes au-delà des limites de détection. Comme vous le dites, concernant le réacteur 1 , l'explosion d'hydrogène a eu lieu bien avant. Même pour le réacteur 3, l'éventage a été effectué bien plus tôt dans la journée, vers 9h20 du matin. N'avez-vous pas pensé que, entre $18 \mathrm{~h} 00$ et $23 \mathrm{h00}$, il s'était encore passé autre chose qui justifierait ce phénomène? Par exemple, n'avez-vous pas pensé que, la radioactivité étant de plus en plus forte du côté du réacteur 3 , une fuite persistante du réacteur 3 aurait pu être à l'origine de ce phénomène?

$\mathbf{R}:$ J'ai pensé que c'était une éventualité. Quel était l'état du réacteur 3 à ce momentlà?

$\mathbf{Q}$ : Du côté du réacteur 3, vous injectiez vigoureusement de l'eau de mer toute la nuit mais, avant, dans l'après-midi, il y avait eu plus de $100 \mathrm{mSv} / \mathrm{h}$ autour du bâtiment et il y avait eu cette histoire de brouillard blanchâtre.

$\mathbf{R}$ : On avait déjà commencé à injecter l'eau, n'est-ce pas ?

Q: Oui.

$\mathbf{R}$ : Donc, on injectait de l'eau dans le réacteur 3, mais on savait évidemment que le niveau d'eau était en-dessous du TAF. Je pensais logiquement que, tout comme pour le réacteur 1 , de la radioactivité avait des chances de s'échapper, non, je pensais que de la radioactivité s'échappait effectivement du réacteur. Mais je dois vous mettre en garde. Vous avez évoqué tout à l'heure le fait qu'on avait détecté de l'iode près de l'entrée principale. Mais n'oubliez pas que cela dépend de la direction du vent. Selon la direction où il souffle, les chiffres vont différer. Si vous êtes complètement en amont, vous ne détecterez rien. Alors suivant la direction du vent, vous aurez des chiffres significatifs ou pas. Mais cela ne change rien au fait que j'étais conscient que de la radioactivité s'échappait vers l'extérieur, quelle que soit la quantité. 
$\mathbf{Q}$ : Comment est fixée la valeur des limites de détection de l'iode?

$\mathbf{R}$ : Il y a plusieurs façons de faire, mais c'est en général la limite de ce qu'un instrument ordinaire peut mesurer. C'est pour ça que ce qu'il indique est toujours une valeur significative. Par exemple, 2 ,x n'est pas une valeur significative, d'après cet instrument. S'il détecte une valeur plus importante, une valeur significative, alors on est au-delà des limites de détection.

$\mathbf{Q}$ : Vous voulez dire qu'en-deçà, l'instrument est conçu de telle manière qu'il ne peut pas donner de mesure?

$\mathbf{R}$ : C'est ça. La valeur ne s'affiche pas.

$\mathbf{Q}$ : La radioactivité est montée autour du réacteur 3 et c'est ainsi que vous abordez la journée du 14. La date a à peine changé que, d'après la chronologie à la page 31, à 1h10, vous arrêtez l'action des camions de pompiers, car vous n'avez presque plus d'eau de mer dans le bassin qui sert à l'injection des réacteurs. Un peu plus bas, à 3h20, vous redémarrez l'injection avec les camions. Ce qui signifie que, pendant ce temps, vous avez...

$\mathbf{R}$ : Rechargé le bassin en eau.

$\mathbf{Q}$ : C'est ça. Vous n'avez pas songé à recharger en eau le bassin par un moyen quelconque, tout en continuant à injecter dans les réacteurs?

$\mathbf{R}$ : Je ne me rappelle pas très bien de la manière dont on rechargeait le bassin, mais il me semble, comme je vous l'ai dit tout à l'heure, qu'on faisait des allers et retours sans arrêt pour pouvoir injecter. On rechargeait sans arrêt, mais si on ne recharge pas assez d'eau, il ne va plus y avoir de pression de succion, il va y avoir cavitation et on va endommager la pompe à incendie.

$\mathbf{Q}:$ Elle va tourner à vide.

R : Oui, parce qu'elle va tourner à vide. Alors c'est une question d'équilibre avec la quantité d'eau qu'on peut recharger. Je ne sais pas, à l'heure que vous évoquez, le 14 mars, où on en était des opérations de recharge du bassin. Moi, je ne disais que "rechargez, injectez », du coup, les détails ne se sont pas imprimés dans ma mémoire. Mais, fondamentalement, c'est une question d'équilibre.

$\mathbf{Q}$ : Au moment que nous évoquons, vous êtes en train d'injecter de l'eau à la fois dans le réacteur 1 et le réacteur 3 en vous approvisionnant au même bassin. L'eau de mer dans ce bassin est, par définition, limitée et presque près de se tarir. Vous vous dites que quand il n'y aura plus assez d'eau, il va falloir recharger. Et, en pensant aux situations respectives des réacteurs 1 et 3, est-ce que vous avez donné des ordres pour, par exemple, donner la priorité au réacteur 3 , ou ce genre de chose?

$\mathbf{R}:$ Je trouvais que les deux tranches étaient dans un équilibre précaire, les deux me préoccupaient terriblement, et il m'était difficile de dire d'arrêter l'injection de l'une ou de l'autre. Ce dont je me souviens à ce propos, c'est que, parallèlement aux va-etvient des véhicules d'arrosage ou des voitures citernes qui remplissaient le bassin, nous nous efforcions de construire un circuit d'eau de mer. Nous voulions pouvoir monter l'eau de la mer jusqu'au bassin en utilisant quelque chose comme une pompe submersible. Nous en avions réclamé et il me semble que nous en avions reçu une, mais, après essai, on s'est rendu compte que ça ne marchait pas. On essayait des tas de choses, tout ce que nous pouvions imaginer. Mais rien de tout ça n'apparait dans les documents. Derrière cette façade, nous nous torturions pourtant à chercher des solutions, nous nous sommes épuisés à apporter l'eau, à apporter une pompe. Nous nous sommes épuisés à injecter l'eau avec les moyens du bord. Demandez donc aux pompiers, ils vous diront... 
$\mathbf{Q}$ : Le 14 mars, de 1 h10 à 3h20, vous avez dû interrompre l'injection d'eau. Cela concerne donc à la fois le réacteur 1 et le réacteur 3 , n'est-ce pas ?

R : Oui, il me semble.

$\mathbf{Q}$ : Si on regarde les chiffres au matin du 14, c'est la page 5/21 des «Paramètres concernant le niveau d'eau et la pression du $7 F-3$ », après le redémarrage de l'injection d'eau de mer à 3h20, vous avez mesuré le niveau d'eau à la fois en zone $A$ et $B$. En A, les chiffres vont et viennent entre -1 850 et -1 800, en $B$, on passe progressivement de -2300 à -3 000, à 4h40. Ensuite, les chiffres de $A$ chutent progressivement, puis, tout à coup vers 6 h30, seul A saute à -100. B descend à -3 500 .

$\mathbf{R}$ : Oui. Je me rappelle très bien.

$\mathbf{Q}:$ Il y a un mouvement un peu bizarre, non ? Qu'en avez-vous déduit?

$\mathbf{R}$ : J'ai pensé qu'il y avait un problème au niveau des instruments de mesure, surtout en A. D'après l'évolution des choses, il me semblait normal que ça descende, comme en $\mathrm{B}$. Même si on ne connaissait pas les valeurs réelles. Mais, alors, un saut aussi brutal, de cette ampleur, je ne voyais pas d'autre explication qu'une anomalie au niveau des instruments de mesure. Un ingénieur normalement constitué ne pourrait pas même imaginer que ce saut serait dû à une arrivée massive d'eau.

$\mathbf{Q}$ : À ce moment-là, donc, vous pensez que vous ne pouvez pas vous fier entièrement aux chiffres fournis par les indicateurs, mais vous formez l'hypothèse que, d'après l'évolution des chiffres du B, le niveau d'eau baisse progressivement.

$\mathbf{R}:$ Oui, tout à fait.

$\mathbf{Q}$ : D'après vous, pourquoi le niveau d'eau baissait-il ?

$\mathbf{R}$ : Je pensais que c'était parce que l'eau ne parvenait pas jusqu'au réacteur.

$\mathbf{Q}$ : D'accord, vous pensez que l'eau n'y parvient pas. Si on regarde les chiffres concernant la pression au même moment, on a 0,137, 0,2, 0,3, on est autour de ces chiffres. Et pourquoi, d'après vous, l'eau ne parvenait-elle pas jusqu'au réacteur?

$\mathbf{R}$ : Je veux dire que c'est une question d'équilibre entre l'eau injectée et les fuites.

$\mathbf{Q}$ : Vous voulez dire que vous injectez l'eau, mais que les fuites sont plus importantes que le volume que vous injectez?

$\mathbf{R}$ : Les fuites étaient importantes, à mon avis.

$\mathbf{Q}$ : Alors quand on voit les chiffres concernant le réacteur 3 , nous sommes encore avant l'explosion, vous commencez à penser qu'il y a fuite, que ce soit de l'enceinte de confinement, de la cuve, ou que ce soit de la cuve vers l'enceinte?

$\mathbf{R}$ : Je ne commence pas à le penser, j'en suis persuadé.

$\mathbf{Q}:$ Vous pensiez déjà qu'il y avait des fuites?

R : Oui.

$\mathbf{Q}$ : Si vous injectez effectivement de l'eau en abondance dans la cuve et que le niveau d'eau ne continue pas à monter, c'est qu'il y a fuite. N'y avait-il pas des possibilités pour que vous évacuiez l'eau à dessein?

$\mathbf{R}$ : Non. Ce n'est absolument pas possible.

$\mathbf{Q}$ : Il est donc clair qu'il y a un volume important d'eau qui fuit. C'est une question très élémentaire, mais toute cette eau qui fuyait malgré vous, vous la laissiez tout simplement s'accumuler en bas du bâtiment et vous n'avez pas pensé à la récupérer?

$\mathbf{R}$ : C'est qu'il n'y a pas moyen de la récupérer. Normalement, la cuve du réacteur a une bride ici et des trous où viennent se loger les barres de contrôle. Il y a toutes sortes de tuyaux avec des vannes, tout autour de l'enceinte de confinement. En cas 
d'incident, toutes les vannes de l'enceinte se ferment. La cuve est isolée. Bien sûr, le circuit FPC, par exemple, reste accessible. C'est-à-dire que seuls les circuits permettant d'injecter de l'eau restent opérationnels. On injecte donc de l'eau par-là, mais tout le reste est fermé. Pour en revenir à votre question, si on dit que tout ça représente l'enceinte de confinement, pour qu'il y ait fuite d'ici vers ici, les endroits où on peut imaginer une fuite, puisqu'il y a une vanne de sécurité (Safety Relief Valves, $\mathrm{SRV})^{31}$ sur le réseau principal de vapeur, on peut supposer que de la vapeur s'échappe de là, ou bien, qu'il y a eu une rupture d'une manière ou d'une autre sur les tuyaux de moindre diamètre, ou bien, ce sera une hypothèse à envisager plus tard, que le combustible ait fondu et que cette bride, qui finalement n'est qu'un genre de joint torique ${ }^{32}$, ait été endommagée et qu'il y ait fuite. Je crois qu'on ne peut pas imaginer autre chose. Mais, pour ce qui est de récupérer l'eau, quand elle sort d'ici, elle arrive à la chambre de dépressurisation. Si le circuit de récupération d'eau de cette chambre était fonctionnel, on aurait pu la réutiliser, mais puisque ce circuit n'était pas utilisable, l'eau n'était pas récupérable.

$\mathbf{Q}$ : En tout cas, ce qui est sûr c'est que vous perdiez beaucoup d'eau.

$\mathbf{R}$ : Tout à fait.

Q: Ce que vous faisiez, depuis l'aube du 14 , c'était, si on se réfère aux documents, premièrement, la poursuite de l'injection d'eau de mer. Là, vous n'aviez pas d'autre choix, qu'il y ait fuite ou pas. Deuxièmement, on voit ça à la page 37 de la chronologie, l'ajout d'une ligne d'éventage supplémentaire. Ayant constaté une tendance à la hausse de la pression de la chambre sèche à partir de $14 \mathrm{~h} 00$, vous décidez d'ouvrir la vanne d'éventage de la chambre de dépressurisation (SC), la vanne AO, la petite vanne. Vers 15h40, vous tentez de magnétiser la vanne électromagnétique. Avant ça, déjà le 13 mars vers les 9 h00, vous aviez ouvert la grande vanne pour effectuer un éventage. Vous avez voulu rouvrir cette grande vanne, mais ayant eu des difficultés à la maintenir ouverte, vous aviez décidé d'ouvrir aussi la petite vanne, c'est bien ça?

R: Oui.

$\mathbf{Q}:$ :t la pression à ce moment-là?

$\mathbf{R}$ : Elle n'a pas beaucoup baissé.

$\mathbf{Q}$ : Ou bien elle avait baissé une première fois ?

$\mathbf{R}$ : Effectivement, la pression avait beaucoup de mal à descendre.

$\mathbf{Q}:$ À votre avis, pourquoi ?

$\mathbf{R}$ : À cause de la ligne. Même en ouvrant la vanne, on n'avait pas dû atteindre un rapport de pression qui permette la rupture du disque. On pensait avoir ouvert la vanne, mais il y avait de fortes probabilités pour qu'elle ne l'ait pas été.

$\mathbf{Q}:$ Si je lis ce qu'il y a à la page 37 concernant la grande vanne alors que la radioactivité montait: «toujours des difficultés à maintenir cette vanne ouverte, du fait de problèmes sur la vanne d'éventage SC, la vanne $A O$, problèmes de pression pour manœuvrer la grande vanne ou de maintien de la magnétisation sur la vanne électromagnétique de la ligne d'alimentation en air ». Du côté de la ligne supplémentaire d'éventage, on voit des annotations du même genre concernant la petite vanne: "problèmes de pression pour la manœuvre et de maintien de la magnétisation sur la vanne électromagnétique de la ligne d'alimentation en air ». II y a donc des déboires, la pression qu'on n'arrive pas à imprimer à partir des compresseurs ou des bombes ${ }^{33}$, des vannes qu'on pense avoir magnétisées qui ne le sont pas et qui restent 
fermées. Tout ça pour le réacteur 3. Ce sont les hommes du groupe «réhabilitation » qui travaillaient principalement là-dessus ?

R: Le groupe «réhabilitation» et le groupe "production». Par exemple, pour amener l'air ou prévoir des piles, ce sont des hommes du groupe " réhabilitation ", ceux des turbines ou des instruments, qui viennent en renfort, mais ceux qui opèrent directement sur les vannes, ce sont les hommes du groupe "production». On travaille en synergie.

$\mathbf{Q}$ : Et étiez-vous au courant que les choses ne marchaient pas bien?

$\mathbf{R}$ : Oui, j'avais eu des rapports.

$\mathbf{Q}$ : Si on reste à la surface de ce qui est écrit ici, vous rencontriez des problèmes de pression ou de maintien de la magnétisation sur la vanne électromagnétique de la ligne d'alimentation en air. Par exemple, il aurait suffi d'un compresseur plus performant ou d'une batterie plus puissante pour que ça marche. N'avez-vous pas pensé à réclamer un compresseur plus performant par exemple?

$\mathbf{R}: \mathrm{Si}, \mathrm{j}$ 'en ai réclamé dès le début et je n'ai pas arrêté de le faire.

$\mathbf{Q}:$ Et ? Il ne vous parvenait pas?

R : Non.

$\mathbf{Q}$ : Si je me souviens bien, vous nous aviez raconté que vous ne disposiez que de minicompresseurs et que vous vous demandiez si ça allait vraiment marcher. Vous considériez que c'était mieux que rien?

R : Il n'y avait pas d'autre solution, alors on le faisait.

$\mathbf{Q}$ : Vous voulez dire que vous étiez contraint d'agir de cette manière faute de mieux. Ce que je trouve curieux, c'est qu'un compresseur, c'est quelque chose qu'on trouve n'importe où. II y en a partout, dans la moindre petite usine.

$\mathbf{R}$ : Comme je vous l'ai dit la dernière fois, je n'arrêtais pas de formuler des demandes. Le réseau qui nous alimentait à ce moment-là était le suivant : quand le siège à Tôkyô passait commande, tout arrivait dans un premier temps à notre Coal Center d'Onahama. C'est une de nos filiales qui charge le charbon et qui nous appartient. Elle dispose d'un espace assez vaste, où étaient rassemblées les choses. De là, la marchandise transitait par le J-Village avant d'arriver à la centrale. Et la marchandise était bien arrivée à Onahama. D'après ce que j'ai entendu plus tard, il y avait des montagnes de toutes sortes de choses. Mis à part le problème du tri, la principale difficulté était que le réseau de transport entre le J-Village et la centrale n'avait pas été pensé. En plus, ceux qui apportaient la marchandise ne savaient souvent pas à quoi cela devait servir, du coup, après s'être donné du mal à apporter les choses, on se rendait compte que c'était inutilisable. On a ainsi longtemps navigué de déception en déception, dans une situation terriblement confuse.

$\mathbf{Q}$ : Parce que les gens avaient peur de la radioactivité ?

$\mathbf{R}$ : Oui, il y a de ça, aussi. Les chauffeurs ne voulaient pas venir, alors il fallait que quelqu'un de la centrale aille chercher la marchandise. Comme je l'ai dit la dernière fois, le nombre de personnel sur le site de la centrale était limité, c'était des gens qui devaient travailler sur le terrain. C'était leur devoir. Et ils nous demandaient de venir chercher le matériel jusqu'au Coal Center d'Onahama. 
Q : Si je comprends bien, la base arrière n'a pas fait son travail. Mais enfin, je suis sûr qu'on peut disposer de beaucoup de choses. Les compresseurs, par exemple, on peut en louer.

Q: Ce qui est important ici, ce n'est pas de savoir si on dispose de matériel ou pas, mais plutôt de savoir combien de personnes sont capables de concevoir les choses dans leur ensemble et si elles sont dans une situation leur permettant d'agir. Par exemple, on vous dit: "tu peux emporter tout ce que tu veux». Il faut être capable d'avoir l'idée, sur place, de ce qu'on va faire. Comment relier A à B et les positionner dans un ensemble. Il faut aussi être capable de juger tout de suite si A va bien s'emboîter dans $\mathrm{B}$, s'il ne va pas y avoir des problèmes de diamètres, par exemple. Sinon, cela ne sert à rien de transporter le matériel à grands frais ${ }^{34}$. Cela révèle de manière cruelle la véritable capacité de chacun. Et des gens comme moi ne peuvent s'empêcher de penser que des employés de TEPCO, habitués à n'obéir qu'aux ordres et aux directives, n'en sont absolument pas capables, qu'ils vont se trouver démunis face à la réalité des évènements ${ }^{35}$.

R : Excusez-moi de vous contredire, professeur, mais souvenez-vous que les gens de chez moi, ce sont des gens qui ont été capables d'aller chercher les batteries de leur voiture, certains, extrêmement ingénieux, qui ont été capables d'imaginer des solutions incroyables.

Q: Bien sûr, ils l'ont fait. Mais je voudrais savoir si vous aviez l'habitude de faire des choses qui s'inscrivent dans un mouvement d'ensemble ou même d'avoir l'occasion d'y réfléchir.

$\mathbf{R}$ : Bien sûr que oui.

Q : Parce que, sinon, tout peut s'arrêter à Onahama. Nous sommes en train de parler de quelque chose de très important. Est-ce que le système était fait en sorte d'exploiter les très grandes capacités de chacun dans un ensemble?

$\mathbf{R}$ : Je pense que c'est la même chose dans tout organisme. Mais, sincèrement, les hommes de la production ou de la réhabilitation, chez nous, sont de véritables professionnels extrêmement expérimentés. Ils sont capables de tout faire. Tout au début, quand toutes les entreprises partenaires s'étaient retirées, ils ont tout effectué eux-mêmes, des câblages à tout le reste. Ils l'ont accompli, malgré toutes les difficultés. Même pour les couplages, ils savent très bien quel est le diamètre qu'il faut. Mais le problème, c'est que le matériel n'arrivait pas. Même si tout était à Onahama, il n'y avait personne pour transporter ça d'Onahama à ici. Et c'est une situation qui a duré longtemps.

Ce n'est pas pour me vanter, mais je trouve que les hommes de la production ou de la réhabilitation de cette centrale sont remarquables. Des trois centrales ${ }^{36}$, ici, c'est celle qui a eu le plus de soucis, alors ils ont eu largement l'occasion de se frotter à tout ça. Ils ont l'expérience de manœuvres qu'ils ont faites eux-mêmes, sans forcément tout déléguer aux entreprises partenaires. Et c'est parce qu'ils avaient cette expérience qu'ils ont été capables d'accomplir tout ça. Si la même chose s'était produite à Kashiwazaki, est-ce qu'ils auraient été capables de réagir de cette façon? On en reparlera plus tard, mais à un moment donné, le circuit de lutte contre l'incendie s'est trouvé inutilisable. Pour le remplacer, ils ont eu l'idée d'exploiter le circuit d'éventage du réseau d'alimentation d'eau. Ils étaient persuadés qu'il était possible d'injecter l'eau par cette voie s'ils arrivaient à relier un tuyau à un autre, qui se trouvait à un pouce, en coupant le tuyau et en $\mathrm{y}$ ajustant tout un système de raccords. Et ils l'ont fait, même si cela leur a pris plus de six heures, y compris le temps pour recevoir le matériel nécessaire. Eh bien, ça, c'est le genre de solution qui ne peut 
même pas vous effleurer l'esprit si on n'a pas en tête le P\&ID (Piping \& Instrumentation Diagram $)^{37}$ et une véritable connaissance du terrain.

Q: Vous pensez donc que le problème était de savoir combien de ces compétences se trouvaient sur place et de savoir si elles étaient toutes en situation d'agir vraiment et en synergie?

$\mathbf{R}$ : Oui. Ce n'est peut-être pas à moi de les juger, surtout que je ne suis absolument pas capable de savoir si, moi-même, j'ai été à la hauteur en tant que directeur des opérations, mais, pour ce qui est de mes subordonnés, je peux vous affirmer que ce sont de remarquables techniciens, n'ayant pas peur de mettre les mains dans le cambouis, comme on en rencontre peu au Japon. Et, malgré ça, nous en sommes là.

$\mathbf{Q}$ : Oui, mais si on change de point de vue, c'est grâce à ça que les choses ne sont pas allées plus loin.

$\mathbf{R}$ : Oui, je le pense.

$\mathbf{Q}$ : Nous aussi, nous le pensons. Car s'ils n'avaient pas été là, il n'y avait pas de raison pour que les choses en restent là. Vous pouvez poursuivre.

Je voudrais vous interroger maintenant sur la matinée du 14 mars. Le 14, selon ce que vous nous avez déjà dit, on le voit aussi sur le schéma du tableau blanc, vous commencez à vous dire qu'il y a une fuite de l'enceinte de confinement. La radioactivité est aussi très élevée. Si on suit les données rapportées à la page 6/21 des «Paramètres concernant le niveau d'eau et la pression du 7F-2", vers 7h15, il me semble, la pression de la chambre sèche est au-delà des $500 \mathrm{kPa}$, et ça, depuis un certain temps. Je suppose que vous trouvez la situation inquiétante, avec une pression clairement anormale et une radioactivité très élevée. Vous pensiez qu'il y avait des fuites depuis l'enceinte de confinement. Est-ce que le siège partageait votre conviction?

$\mathbf{R}$ : Oui. En tout cas, je le pense, moi.

$\mathbf{Q}$ : Est-ce que vous faisiez part de ce genre de réflexions, par exemple que vous pensez qu'il y avait une fuite, quand vous étiez en téléconférence ? En aviez-vous discuté ?

$\mathbf{R}$ : Pour moi, je l'ai fait. J'ai bien dit que le réacteur 3 avait aussi son combustible endommagé et que, d'après la pression de l'enceinte de confinement, tôt ou tard, on allait vers la même situation que pour le réacteur 1 . Je n'aime pas du tout la manière dont les choses sont consignées ici. J'en avais bien parlé et j'avais même ordonné un retrait général du personnel le 14 mars vers $9 \mathrm{~h} 30$ ou $10 \mathrm{~h} 00$, à cause des risques d'explosion. Je me demande pourquoi ils ne l'ont pas écrit.

$\mathbf{Q}$ : C'était donc avant l'explosion du 14 à 11 h01 ?

R: Oui, avant. C'est agaçant. Il y a des endroits où l'heure manque. Ils auraient pourtant pu noter tout ce qui se disait. En tout cas, quand la pression du réacteur $3 \mathrm{a}$ dépassé $0,5 \mathrm{MPa}$, j'ai eu la sensation que ça devenait dangereux et j'ai ordonné le retrait général. C'était au moment où on avait commencé à remplir le bassin pour l'injection d'eau avec l'eau de mer qu'on faisait monter du quai. C'était vers 9 h20. On venait de recevoir une nouvelle pompe à incendie. Un camion de pompiers venait d'arriver. On l'avait mis en série avec celui qui était déjà là et, enfin, on s'apprêtait à alimenter ce bassin de manière continue, quand la pression était montée et que, face au danger, j'ai donné un premier ordre de retrait.

$\mathbf{Q}:$ Vous avez donné cet ordre à la table de la cellule de crise?

$\mathbf{R}$ : Oui. J'ai dit textuellement: "comme la situation devient dangereuse, j'ordonne le retrait ». Le siège était aussi au courant, tous les centres aussi. Tout le monde l'a entendu. J'ai bien donné une première fois l'ordre de retrait. Demandez-le à 
n'importe lequel de ceux qui étaient là, il vous le confirmera. J'ai ordonné le retrait dans un premier temps. Parce que c'était dangereux.

$\mathbf{Q}$ : Et quand ils reçoivent cet ordre, où vont les gens?

$\mathbf{R}$ : Dans le bâtiment antisismique.

$\mathbf{Q}:$ Vous voulez dire que tout le monde se retrouve dans le bâtiment antisismique?

$\mathbf{R}$ : Oui. Il y avait risque d'explosion. Il y avait risque d'explosion d'hydrogène, alors j'ai retiré tout le monde. Je suis absolument sûr d'avoir donné cet ordre.

\section{$\mathbf{Q}$ : Vers quelle heure, environ?}

$\mathbf{R}$ : Comme on a commencé l'alimentation du bassin vers $9 \mathrm{~h} 20$, qu'on avait fini de le remplir et que ça m'avait un peu rassuré, je pense que c'était juste après, entre $9 \mathrm{~h} 30$ et 10h00. Le retrait a duré plus d'une heure. Seulement, il fallait réaliser un circuit d'alimentation pour le réacteur 2 , entre autres. Il y avait tellement d'autres choses à faire dans l'urgence. J'ai hésité, beaucoup hésité. Pouvais-je renvoyer les hommes sur le terrain ou pas ? Pouvait-on reprendre les travaux ? Cela n'a été rapporté nulle part, mais il y a eu à ce moment-là des discussions au téléphone avec le siège. J'avais maintenu le retrait et le siège me demandait quand j'allais lever cet ordre. Moi, j'ai répondu qu'il y avait des risques d'explosion et que je ne pouvais pas envoyer d'hommes sur le terrain. Nous savions, de part et d'autre, qu'il fallait préparer l'injection du réacteur 2 et qu'il fallait reprendre les travaux à un moment ou à un autre. Nous en avons parlé. À ce moment-là, la pression qui montait s'est stabilisée vers 0,52 . J'étais en conversation avec Mutô, qui me dit que ça avait l'air d'être dans un état stable.

\section{Q: Vous parliez au téléphone?}

$\mathbf{R}$ : Oui, au téléphone. Et donc, Mutô me demande si on ne pouvait pas commencer à reprendre les travaux sur le terrain. Pour moi, c'était très difficile. Mais on ne pouvait pas négliger le réacteur 2, alors j'ai expliqué la situation au personnel. J'étais désolé. Je leur ai dit que j'avais conscience que la situation était très dangereuse mais que si on n'allait pas sur le terrain, on ne pouvait pas avancer. Je leur ai demandé d'y aller. La pression paraissait s'être un peu stabilisée. Nous n'en étions pas sûrs mais nous pensions que ça n'exploserait pas tout de suite. Nous avions jugé que la situation était un peu stabilisée, nous avons envoyé des hommes et ça a explosé ${ }^{38}$. Ceux pour qui ça a été le plus terrible ce sont les hommes de la force d'autodéfense. Parallèlement aux problèmes du réacteur 2 , j'avais en tête qu'il fallait continuer à alimenter le bassin. En plus de l'eau qu'on faisait monter de la mer, la force d'autodéfense avait pour mission de transporter de l'eau dans leur camion pour compléter l'alimentation du bassin. Ils avaient un camion prêt depuis un moment et se sont dirigés vers le bassin avec leur véhicule et, au moment de commencer à transvaser l'eau, ils ont été pris dans l'explosion.

$\mathbf{Q}:$ Vous avez donc donné l'ordre de retrait dans un premier temps, puis le personnel qui s'était mis à l'abri est reparti sur le terrain, quand ça a explosé. Combien de temps y a-t-il eu entre leur départ vers le terrain et l'explosion?

\section{$\mathbf{R}:$ C'était assez court.}

$\mathbf{Q}:$ Une heure ? Moins?

$\mathbf{R}$ : J'ai levé l'ordre de retrait, autorisé le retour sur le terrain, on s'est tous mis en branle, on a commencé à s'organiser, quand ça a explosé. J'ai entendu dire que pour 
les hommes de la force d'autodéfense, ils sont arrivés sur le terrain et avaient à peine fini les préparatifs que ça a explosé.

Q: Les hommes de la force d'autodéfense, quand ils sont venus, sont-ils passés vous voir au bâtiment antisismique?

$\mathbf{R}$ : Non, ils ne sont pas venus. Les gens de l'autodéfense ne venaient pas jusqu'au bâtiment antisismique. Il y avait quelqu'un de chez nous aux différentes entrées, notamment à l'entrée principale, qui les guidait vers le lieu où on avait besoin d'eux.

$\mathbf{Q}$ : Il y avait donc une personne du terrain qui les aiguillait à l'entrée principale.

$\mathbf{R}$ : Oui, c'était le groupe « sécurité et orientation » qui faisait ça.

Q: Ce qui signifie qu'on avait fini de les orienter, qu'ils étaient arrivés au bassin et que c'est au moment où ils s'apprêtaient à le remplir que l'explosion s'est produite?

$\mathbf{R}$ : Oui. Au début, tout juste après l'explosion, quand les tout premiers rapports sont arrivés du terrain et que j'ai su qu'il y avait une quarantaine de disparus, j'ai vraiment eu l'intention de me donner la mort. Si c'était vrai et qu'effectivement il y avait quarante morts, j'étais décidé à me faire hara-kiri. Mais, petit à petit, des informations sont arrivées et le nombre de disparus a progressivement diminué pour arriver jusqu'à zéro. Bien sûr, on nous a signalé des blessés, chez le constructeur, d'abord. Entre TEPCO et les entreprises partenaires, il y en avait quatre à cinq. Mais personne n'avait perdu la vie. Pour le groupe d'autodéfense, comme ils sont partis sur le terrain sans passer par la cellule de crise, on ne savait pas trop où ils en étaient, au début. Je pense qu'ils étaient venus à neuf. On savait qu'il y avait des blessés parmi eux, mais on ne savait pas combien. Plus tard, on a appris que quatre personnes avaient été blessées, dont une assez grièvement. J'ai fait prendre des nouvelles et je sais que personne n'y a perdu la vie et que tous ont repris du service, depuis, au sein du groupe, ce qui m'a rassuré. Nous avons eu de la chance dans notre malheur. Sous cette pluie de gravats, n'avoir aucun mort à déplorer sur le terrain, je ne peux que remercier Bouddha.

Q: Oui, je me rappelle les images impressionnantes de l'explosion. Cela n'avait rien à voir avec celle du réacteur 1 .

$\mathbf{R}$ : Comme je vous l'ai dit la dernière fois, pour le réacteur 1 , il n'y avait que des planches en bois. Alors, ça explose et c'est fini. Par contre, pour le réacteur 3, c'est du béton qui a volée ${ }^{39}$.

Q: Lorsque vous avez donné l'ordre de retrait dans un premier temps, la pression de la chambre sèche était en train de monter jusqu'à $500 \mathrm{kPa}$, c'est bien ça ? Vous avez jugé qu'il y avait danger, à ce moment-là. Quelle était l'origine de cette inquiétude ? Vous pensiez que l'enceinte de confinement pouvait être détruite?

$\mathbf{R}$ : Je pensais que ce serait la même chose que pour le réacteur 1 .

Q: La même chose que le réacteur 1, c'est-à-dire qu'avec une telle pression, les fuites allaient être encore plus abondantes, que de l'hydrogène allait s'échapper en un volume encore plus grand et vous aviez en tête les risques d'une explosion d'hydrogène, c'est ça ? Alors, dans le cas de l'hydrogène, vous ne savez pas à quel moment ça va exploser, n'est-ce pas?

$\mathbf{R}$ : Seulement, l'explosion du réacteur 1 avait eu lieu le 12 mars à 15h36, il me semble? 
$\mathbf{Q}:$ Oui, à $15 \mathrm{~h} 36$.

$\mathbf{R}$ : Cela s'est passé à peu près quand la pression était à 0,5 . Je crois que l'explosion a eu lieu, alors qu'on était aux environs de $500 \mathrm{kPa}$, vers les $15 \mathrm{~h} 00$. Depuis, pour moi, c'était une pression qui me mettait mal à l'aise. Pour le réacteur 1 , la pression était montée plus haut, puis était redescendue à 500. Mais cela n'empêche que le nombre 500 me mettait mal à l'aise. Je sais que c'est totalement irrationnel, c'était juste épidermique.

$\mathbf{Q}$ : Je me demande si ce n'est pas la pression habituelle d'un compresseur. Vous avez dû le ressentir comme ça.

$\mathbf{R}$ : Dire que j'avais eu comme un pressentiment et que j'avais donné l'ordre de retrait dans un premier temps. Vous ne pouvez pas imaginer comme je regrette d'avoir autorisé le retour sur le terrain. Puisque ça devait exploser, il aurait fallu laisser exploser. Sans renvoyer les hommes sur le terrain, bien sûr. On pouvait très bien laisser exploser et reprendre les travaux après, en commençant par déblayer le terrain. Il n'y avait rien d'autre à faire.

$\mathbf{Q}$ : Si on regarde les faits, cette inquiétude était tout à fait justifiée, mais est-ce que le siège avait bien compris que vous aviez donné l'ordre de retrait?

R : Oui.

$\mathbf{Q}$ : Avez-vous fait des rapports au Gouvernement ou à d'autres administrations ?

$\mathbf{R}$ : Je pense qu'au 14 mars, il y avait non seulement des représentants de chez nous, mais aussi des représentants du Gouvernement dans la cellule de crise du siège. Ce serait un point à vérifier ${ }^{40}$. De plus tous nos échanges avec le siège étaient relayés par téléconférence vers le centre hors site, aussi.

$\mathbf{Q}$ : Je pense que des gens de la NISA étaient aussi présents au centre hors site. Croyezvous que des gens du département de Fukushima et d'autres administrations locales avaient aussi la possibilité d'accéder à ces échanges ?

$\mathbf{R}$ : Bien entendu. Tous les sites étaient reliés par téléconférence et j'intervenais là pour dire « je vais donner l'ordre de retrait », alors.

$\mathbf{Q}$ : Dans ce cas, y a-t-il eu des discussions spécifiques pour savoir si on allait mettre la population locale ou nationale au courant de la situation ? Est-ce le genre de problème dont le siège est censé se charger?

R : Oui.

$\mathbf{Q}$ : Dans ce cas précis, votre avis n'a-t-il pas été sollicité, en tant que directeur de la centrale?

$\mathbf{R}$ : Je ne sais pas. Je ne sais absolument pas quelles ont été les discussions en central ou en centre hors site. Je ne sais même pas s'il y a eu des discussions à ce sujet.

$\mathbf{Q}$ : Après l'explosion de 11h01, vous recevez des rapports alarmants concernant au moins quarante disparus. Je pense que la situation était très confuse. Au milieu de tout ça, vous souvenez-vous s'il y a eu des informations disant qu'on avait détecté des neutrons ou des histoires de ce genre?

$\mathbf{R}$ : Le contrôle des neutrons est une opération que nous faisons périodiquement en tout temps. 
$\mathbf{Q}$ : Vous voulez dire même en temps normal ?

$\mathbf{R}$ : Oui. Il me semble bien qu'à ce moment-là on n'a pas détecté de neutron. Je me rappelle bien que cela, aussi, m'avait un peu rassuré. Le fait qu'il n'y avait pas de neutrons.

$\mathbf{Q}:$ Ça, c'est après l'explosion?

$\mathbf{R}$ : Je me rappelle très bien. Juste après l'explosion, j'avais demandé des données, j'ai fait faire un contrôle de l'ensemble. Et, parmi ces données, je m'étais particulièrement intéressé à la courbe des neutrons, mais elle était à moins de 0,01. Le rapport disait que nous étions en-dessous des limites de détection. Je n'ai plus le papier où je prenais des notes, ce papier où j'avais noté le nombre des disparus, les résultats du monitoring, etc. Mais je suis sûr qu'il y a des traces quelque part. En tout cas, en ce qui concerne les neutrons, ils étaient en-deçà des limites de détection.

$\mathbf{Q}$ : Et cette situation a perduré lors des monitorings suivants ?

$\mathbf{R}$ : Oui. Simplement, il me semble que juste après l'explosion, nous avions augmenté la fréquence des vérifications concernant les neutrons.

$\mathbf{Q}:$ Et, lors de ces vérifications, vous n'êtes jamais tombés sur un chiffre significatif?

$\mathbf{R}$ : Non. Encore une chose, tout comme pour le réacteur 1, la pression de l'enceinte de confinement elle-même n'a pas varié de manière très significative. Elle a eu tendance, par la suite, à descendre, ce que je ne m'explique pas bien. Mais, elle n'a pas chuté brutalement vers zéro. Du coup, je pensais qu'il y avait bien eu explosion du bâtiment réacteur, mais que l'enceinte en elle-même n'avait pas été endommagée.

$\mathbf{Q}$ : A posteriori, on peut dire que les hommes de la force d'autodéfense sont arrivés au mauvais endroit au moment le plus dangereux. Au moment où ils sont arrivés, vous veniez de lever à contrecœur l'ordre de retrait pour permettre l'avancée des travaux, notamment, la préparation de l'injection d'eau dans le réacteur 2. Est-ce que les hommes du groupe d'autodéfense étaient au courant du fait qu'il y avait eu ordre de retrait, puis la levée très récente de celui-ci?

R : Probablement, ils n'étaient pas au courant. Il y a deux choses. D'abord, je n'ai aucune connaissance de la chaîne de commandement du groupe d'autodéfense. Ensuite, je suis dans une situation où je ne peux pas parler directement au groupe d'autodéfense. Tant qu'il y a ordre de retrait, il y a une personne de chez nous qui arrête les gens à l'entrée du site. Mais je ne disposais d'aucun moyen de faire connaître l'état de la situation aux hommes qui étaient détachés, que ce soit en passant par l'antenne locale du groupe d'autodéfense ou par le commandement central.

$\mathbf{Q}$ : II y avait donc cinq blessés, en comptant les hommes de la force d'autodéfense. Et aussi parmi les entreprises partenaires. S'agissant de la réponse à l'explosion de 11h01, qu'avezvous fait concernant les travaux?

$\mathbf{R}:$ On a tout arrêté.

$\mathbf{Q}$ : Du coup, tout le monde a quitté le terrain. Et, ensuite, il y a bien eu un moment où vous avez repris les travaux. Quel genre d'information vous a poussé à prendre la décision de retourner sur le terrain?

$\mathbf{R}$ : Plutôt qu'une information quelconque, c'est le fait que, tout comme pour le réacteur 1 , l'explosion avait dû causer des dégâts avec de forts risques que les circuits d'injection d'eau ou d'autres réseaux soient morts. Nous étions en train d'injecter de l'eau dans les réacteurs 1 et 3 , et ces injections avaient dû s'arrêter avec l'explosion. 
En dehors de ça, il y avait des risques que de nombreux instruments aient été détruits. L'urgence était de vérifier qu'il n'y avait pas de morts et d'évacuer les blessés vers le J-Village où ils allaient recevoir les soins. Une fois ces mesures prises, il fallait réagir. Or, tout le monde était sous le choc, figé, incapable de réfléchir. Alors je les ai tous réunis pour leur parler. Je leur ai dit à quel point je regrettais de les avoir renvoyés sur le terrain alors que le danger n'était pas écarté, que j'avais pris la mauvaise décision et que je leur demandais de me pardonner. Je leur ai aussi dit qu'il y avait de fortes probabilités pour que les injections d'eau soient arrêtées, qu'il fallait préparer l'injection du réacteur 2 , que si on ne réagissait pas, on allait vers une situation encore plus catastrophique. Seulement, le terrain était sûrement jonché de gravats. Il fallait commencer par évacuer ces gravats qui étaient hautement radioactifs. Je leur ai dit d'être prudents, de mesurer scrupuleusement la radioactivité et de s'atteler au minimum requis, de changer, par exemple, juste le tuyau qu'il fallait pour pouvoir assurer l'injection d'eau, et de poursuivre, au plus vite, la préparation du réacteur 2 . Je le leur ai demandé, humblement ${ }^{41}$. C'est à ce moment-là que j'ai vécu une des plus grandes émotions de ma vie. Ils voulaient tous retourner sur le terrain, ils se bousculaient même ${ }^{42}$. J'ai dû les modérer. La précipitation aurait été dangereuse et contreproductive. On a réparti les tâches entre les groupes. Le génie civil devait sortir les tractopelles pour évacuer les gravats, etc. Une fois qu'on s'était concertés, tout le monde est parti. Et c'est là que la plupart de ces hommes ont été irradiés quasiment à la limite des valeurs tolérées, à changer des tuyaux, etc. C'est ainsi que nous avons enfin réussi à redémarrer l'injection d'eau de mer à 16h30. Mais il ne faut pas oublier que, derrière, il y a eu, entre autres, le travail de très nombreuses personnes qui ont dégagé des gravats radioactifs. Je trouve ça révoltant qu'on n'ait rien écrit entre 11 h01 et $12 \mathrm{~h} 30$, qu'ils n'aient pas noté noir sur blanc que j'avais donné l'ordre de retrait bien avant. Je ne comprends pas l'intérêt de cette chronologie.

$\mathbf{Q}$ : Ils ont omis de noter le plus important.

R: Mais, oui. Il faut être idiot pour montrer une chronologie aussi défectueuse au monde entier.

$\mathbf{Q}$ : Ce n'est pas pour défendre le siège, mais ils ont tout de même noté à la page 35 que vous avez effectué une vérification de l'état des dommages, que vous avez recommencé l'injection d'eau dans le réacteur, que vous avez redéployé les tuyaux. Je sais bien que ces quelques mots ne peuvent pas raconter tout ce que vous avez dû endurer. Vous aviez rencontré de très grandes difficultés à rendre le terrain accessible.

R : Oui, mais si on ne note pas dans une chronologie tout ce qui a effectivement été fait...

$\mathbf{Q}:$ II manque le plus important.

Sur ce point, j'ai déjà des témoignages d'autres personnes qui étaient sur le terrain.

R : Interrogez-les minutieusement, s'il vous plaît.

Q: II y a donc eu cette explosion au réacteur 3. Par la suite, vous continuez à envoyer de l'eau de mer dans le réacteur 1 et vous procédez de même pour les réacteurs 3 et 2. Après l'explosion, quelles ont été vos priorités pour le déploiement d'un personnel et de matériel forcément limités?

$\mathbf{R}$ : C'était toujours l'injection d'eau. 
$\mathbf{Q}$ : Injection d'eau dans les réacteurs 1, 2 et 3 ? Y avait-il une priorité entre les trois ?

$\mathbf{R}$ : Non, tous. Pour résumer, concernant les 1 et 3 , le combustible était dénoyé, il n'y avait pas le choix, il fallait injecter de l'eau et c'était la seule chose à faire. Il fallait veiller à ce que l'injection d'eau soit la plus continue possible. Concernant le réacteur 2 , je brûlais d'impatience d'injecter de l'eau avant que le niveau de l'eau n'atteigne le TAF. Il fallait que les préparatifs soient achevés le plus tôt possible. Je pense que c'était le point le plus important.

$\mathbf{Q}$ : Vous pensiez que le RCIC était toujours en fonctionnement pour le réacteur 2, quand l'explosion s'est produite?

R : Oui.

$\mathbf{Q}$ : Pour le réacteur 2 , si on regarde les paramètres du $1 \mathrm{~F}-2$ à la page $6 / 22$, on voit clairement qu'à partir du 14 mars midi, le niveau d'eau baisse nettement, surtout au $A$. Les chiffres du B suivent aussi le mouvement, un peu plus tard. Les chiffres partent de 3400 $\mathrm{mm}$ et baissent graduellement. C'est vers $12 \mathrm{~h} 00$ que vous commencez à recevoir des rapports vous informant de cette baisse. Sur la chronologie, il est noté que vous constatez l'arrêt du RCIC à 13 h25.

R : Oui.

$\mathbf{Q}$ : Comment avez-vous jugé de cet arrêt ? C'est en regardant les niveaux d'eau vers 13h40 ou 50 que vous vous êtes dit que c'était arrêté et que l'arrêt avait dû se produire vers $13 \mathrm{~h} 25$ ?

$\mathbf{R}$ : Je ne me rappelle pas sur quel fondement je me suis basé. Il y a eu une information de l'agent de conduite. Il m'a dit que le système s'était arrêté. J'ai dû acquiescer. Tout ça, ça n'était pas tout juste après l'explosion?

$\mathbf{Q}:$ Si.

$\mathbf{R}$ : C'était un moment où nous étions tous en pleine confusion. J'ai entendu que le système s'était arrêté. C'était très mauvais pour nous. Il fallait absolument envoyer de l'eau. J'en étais tellement obsédé que, probablement, je n'ai pas demandé pourquoi il s'était arrêté. Je pense que je n'ai pas vérifié ce point.

$\mathbf{Q}$ : Je pense que c'était une situation qui relève de l'article 15. Vous avez d'ailleurs noté à la main sur le rapport $n^{\circ} 59$, noté « 60 » à la main en haut à droite, que le niveau d'eau du réacteur 2 baissait.

$\mathbf{R}$ : Il est vrai que je ne m'étais pas effectivement penché sur le fonctionnement même $\mathrm{du}$ RCIC. Nous étions sur des suppositions. Le groupe «production » m'a informé de l'arrêt, j'en ai pris bonne note et leur ai demandé de faire le nécessaire. Nous ne nous sommes pas attachés à vérifier chaque élément.

$\mathbf{Q}$ : Après ça, vous partez du principe que le RCIC ne fonctionne plus et vous vous employez à injecter l'eau, à envoyer de l'eau de mer, c'est bien ça?

R : Oui.

$\mathbf{Q}$ : Concernant les réacteurs 1 et 3 , vous aviez constitué des circuits permettant l'injection d'eau de mer. Vous voulez faire de même pour le réacteur 2 et vous réparez les importants dommages qui ont été causés sur l'ébauche du circuit. Finalement, on voit que vous réussissez à injecter de l'eau de mer à 19h54. Avant même que vous n'interrompiez les travaux à cause de l'explosion, vous aviez déjà commencé les opérations. Vous vous remettez au travail à 13h05. Si on regarde la chronologie, il est noté la "mise en place d'un camion de pompiers à 16 h30 pour l'injection d'eau de mer », puis à 16h34: "notification aux administrations et au Premier ministre du début des manœuvres de dépressurisation et du début 
de l'injection d'eau de mer». Concernant cette injection d'eau dans le réacteur 2, quelle était votre stratégie, comment pensiez-vous procéder?

Là, vous procédez à un éventage en parallèle. Je voudrais que nous regardions les paramètres à ce moment-là. Dans les «Paramètres concernant le niveau d'eau et la pression du 7F-2 », vers $15 \mathrm{~h} 00$ ou $16 \mathrm{~h} 00$ le 14 mars, le niveau d'eau baisse rapidement, le TAF est à quelques centaines de millimètres jusque vers 16h20, la pression de la cuve est au-delà de $7 \mathrm{MPa}$ et la pression de la chambre sèche est à plus de 0,4 MPa. Si on regarde aussi la chronologie à la page 24, à la rubrique "dépressurisation de la cuve», on lit "nécessité de procéder à une dépressurisation de la cuve par l'ouverture de la valve de sécurité pour permettre l'injection d'eau au moyen d'un camion de pompiers ». Ensuite, on lit que la température et la pression de la chambre de dépressurisation sont toutes les deux élevées. Concrètement, on a $149,3^{\circ} \mathrm{C}$ de température et $486 \mathrm{kPa}$ de pression à $12 \mathrm{~h} 30$. Même en ouvrant la vanne de sécurité (SRV), il y avait le risque que la vapeur ne condense pas et que la dépressurisation ne se fasse pas bien. D'où la décision d'effectuer d'abord un éventage de l'enceinte de confinement, avant d'ouvrir la vanne de sécurité, de faire baisser la pression de la cuve et d'injecter l'eau de mer. Ensuite, vers 16h00, vous constatez que l'éventage prend du temps. Vous changez de priorité et décidez de procéder d'abord à la manœuvre de la vanne de sécurité pour faire baisser la pression de la cuve. L'éventage n'est pas abandonné pour autant, il sera effectué en parallèle. À la lecture de ces notes, on a l'impression que vous aviez d'abord envisagé un éventage suivi de la dépressurisation et de l'injection, et qu'ensuite, comme l'éventage prenait du temps, vous avez changé de programme pour tout mener de front. Votre première idée était d'effectuer l'éventage avant de dépressuriser et injecter l'eau?

$\mathbf{R}$ : Tout d'abord, l'éventage, ce n'était pas le plus important. Je veux dire que ce n'était pas le plus important à ce moment-là. Concernant le réacteur 2 , la pression, à ce moment-là, est de 0,4 environ. C'est à peu près à la limite de la pression de conception, alors on sait qu'à cette pression-là l'enceinte de sécurité ne va pas rompre. Rappelez-vous, le réacteur 1 était monté à 0,8 , jusqu'à $800 \mathrm{kPa}$. Le réacteur 3 , aussi, était monté très haut. Alors, comparé à ça, la pression est encore basse et nous ne sommes pas particulièrement pressés de faire un éventage. Bien sûr, nous savons bien qu'il arrivera sans doute un moment où il faudra le faire. Mais ce n'est pas notre priorité à ce moment-là. Ce qui est préoccupant, c'est la température de la chambre de dépressurisation qui est très élevée. En temps normal, la vapeur qui sort à $270^{\circ} \mathrm{C}$, en arrivant dans la chambre qui est habituellement à $40^{\circ} \mathrm{C}$, condense, devient tout de suite de l'eau et fait tomber la pression. Mais si la température de la chambre est élevée, même si on ouvre grand les valves de sécurité, la vapeur ne va pas condenser et ne fera pas baisser la pression. Envoyer de l'eau là où la pression n'a pas baissé ne sert à rien, l'eau ne peut pas entrer. Alors, la priorité des priorités était de faire baisser la pression pour permettre l'injection d'eau. La mission la plus pressante c'était l'injection d'eau. L'éventage n'était là que pour faciliter les choses. Ça partait du principe que si, avec l'éventage, la pression de l'enceinte et de la chambre sèche baissait, au moment où on aurait ouvert la vanne de sécurité, avec la différence de pression, la vapeur serait tombée plus rapidement. Comme on ne pouvait rien faire pour baisser la température, on a pensé que s'il y avait une différence de pression la condensation se ferait plus aisément. C'est comme ça que nous avons raisonné.

D'ailleurs, par la suite, il y a eu une autre idée à laquelle nous avons réfléchi avec l'équipe de quart, le refroidissement de la chambre de dépressurisation ou de la chambre sèche. On s'est demandé si on ne pouvait pas utiliser le système d'aspersion de la chambre sèche. Le but était de refroidir même modestement, afin de créer de meilleures conditions pour faciliter la condensation, plutôt que de faire véritablement baisser la pression de l'enceinte de confinement. C'est une chose qu'on 
a considérée en parallèle. C'est à ce moment-là que, même si ce n'est pas écrit ici, et que je ne me souviens pas de l'heure, il y a eu un coup de fil de la résidence du Premier ministre. C'était M. Madarame au bout du fil. Il m'a pressé de procéder à l'éventage et de faire baisser la pression et injecter l'eau. J'ai bien essayé de lui expliquer la situation sur le terrain, que la température de la chambre de dépressurisation était haute, etc. Mais, ça a été la même chose que précédemment. Il m'a dit de ne pas discuter, de dépressuriser et d'envoyer l'eau. Shimizu ${ }^{43}$, qui suivait tout ça par téléconférence, vociférait en disant qu'il fallait suivre les directives du directeur Madarame. Je dois dire que j'étais excédé, révolté de les entendre proférer ces ordres alors qu'ils n'avaient pas connaissance de ce qui se passait sur le terrain.

$\mathbf{Q}:$ Je pense que ce que vous dites là est très important. Vous étiez dans une situation où il ne suffisait pas de vouloir baisser la pression ou faire tomber la température, pour que ça se fasse. En fait, vous ne pouviez rien faire. Dans ces conditions, je comprends que vous ayez eu envie de leur dire: " puisque c'est si facile, t'as qu'à y aller toi-même ».

$\mathbf{R}$ : Oui. Ce serait bien que vous le leur disiez.

$\mathbf{Q}:$ Leur réaction paraît totalement hors de propos.

$\mathbf{R}$ : Mais le problème, c'est que tout le monde pensait comme eux. Moi aussi, j'étais pressé d'injecter l'eau. Bien sûr que je le voulais. Mais il y a un ordre à respecter. Sur le terrain, on essaie de faire le maximum pour que les choses se déroulent au mieux plus tard et pour cela, il y a des étapes préliminaires. Mais ils n'arrivaient pas à le comprendre. Ils pensaient que nous hésitions. Il n'y avait absolument pas matière à hésiter pour nous. C'est comme pour l'éventage du réacteur 1 , le premier jour. Ils pensaient qu'on hésitait. Je tabasserai avec plaisir toute personne qui pense que le terrain a hésité. Pour moi, que le nuage radioactif atteigne l'hélico du Premier ministre, ça m'était complètement égal. Je ne pensais qu'à faire baisser la pression au plus vite, qu'à injecter l'eau au plus vite, je n'avais que ça en tête. Et venir là, nous dire que le terrain tergiversait... Je le leur ferais payer à tous ceux qui ont pu dire une chose pareille. Vraiment. Je vous en prie, vengez-nous, à notre place. Nous en avons bien besoin.

Q: Quelle est la cause de la hausse de la température dans la chambre de dépressurisation?

R : Ça, c'est parce qu'il y a des fuites, forcément quelque chose fuit de la cuve. Même si la vanne de sécurité ne s'ouvre pas, il y a quand même une certaine quantité de vapeur qui s'échappe. C'est ce qui s'en va vers la chambre de dépressurisation. En temps normal, la chambre de dépressurisation possède des dispositifs, le Cooling $\operatorname{Line}^{44}$, le Spray Line ${ }^{45}$, qui s'enclenchent quand la température monte et permettent de la maintenir à $65^{\circ} \mathrm{C}$. Mais ces réseaux sont morts, alors la chambre est livrée à ellemême. La vapeur qui s'échappe de la cuve s'en va vers la chambre, tout en se condensant, et fait monter la température. Progressivement. Mais si cette situation se prolonge, ça monte franchement.

$\mathbf{Q}$ : Cela veut dire que les fuites sont assez importantes, non? II n'y en avait pas autant sur les réacteurs 1 et 3 .

$\mathbf{R}$ : Oui, on peut dire ça. La température n'était pas montée aussi haut. Mais on peut dire aussi que le RCIC ayant fonctionné longtemps, et même si on ne sait pas au juste ce qui se passait, l'importance des fuites, etc., la température est montée d'autant qu'elle a eu le temps de monter. Si on considère que les trois réacteurs sont partis 
d'une même ligne de départ, puisque nous intervenons sur le 2 en dernier, il est naturel que la température y soit plus élevée.

Q : Finalement, suivant l'avis du directeur de la Nuclear Safety Commission (NSC) ${ }^{46}$, suivant aussi l'avis du siège, après réflexion, concrètement, vous faites réaliser le réseau d'éventage en tant que tel et, en parallèle, vous tentez de baisser la pression pour permettre l'injection d'eau.

R : Oui.

$\mathbf{Q}$ : En effet, si on regarde la page 25 , on se rend compte qu'il vous a fallu beaucoup de temps pour baisser la pression.

$\mathbf{R}$ : Oui, on a eu vraiment du mal à ouvrir ces vannes.

$\mathbf{Q}:$ Et quelle en était la raison?

$\mathbf{R}$ : On n'a pas très bien compris, mais, tout d'abord, on n'avait pas de batterie. En plus de cette histoire de batterie, bien que ce ne soit pas consigné ici, il y a aussi eu des problèmes d'alimentation en air. En fait, on avait à la fois des problèmes de batterie et des problèmes d'accumulateur. L'accumulateur n'avait plus assez de pression et il a fallu le changer. Ensuite, il faut savoir que chaque vanne de sécurité a ses particularités. On essaie A, on échoue. On essaie B, on échoue. Il y a dix-huit vannes et on les a essayées les unes après les autres, avec cette manœuvre simplifiée d'ouverture. Au début, rien ne bougeait. Le niveau d'eau continuait à baisser à bonne allure, les vannes de sécurité refusaient de bouger. Je crois que c'est le moment où j'ai vraiment touché le fond. Je nous voyais tous morts. Je me demandais ce que j'allais faire si on échouait encore, quand, enfin, ça a cédé. Les vannes se sont ouvertes. Mais, cette fois-ci, le camion de pompiers qui était en place depuis un certain temps, pour qu'on puisse injecter l'eau à tout moment, s'est trouvé en panne sèche. La radioactivité était élevée, alors on ne pouvait pas le laisser stationner là indéfiniment. Au moment où on a voulu injecter l'eau, le moteur ne fonctionnait plus. Il a fallu faire le plein avant de pouvoir, enfin, injecter l'eau. Je crois qu'on a réussi à injecter l'eau in extremis. Nous avons vraiment marché sur une corde raide. Pour ma part, je n'avais plus l'impression d'être en vie. J'étais mort ${ }^{47}$.

$\mathbf{Q}$ : Effectivement, c'est écrit à la page 25 , où il y a les horaires. La pression de la cuve est au-delà de $6 \mathrm{MPa}$ depuis $16 \mathrm{~h} 34$ jusqu'à 18h03. Enfin, à 19h03, elle retombe à 0,63 MPa. Avec cette pression, l'injection était possible, non ? Mais vous vous êtes rendu compte que l'eau n'entrait pas et vous vous êtes aperçu à 19h20 que le système était à l'arrêt faute de carburant. Vous avez parlé de faire le plein, tout à l'heure. Comment procédiez-vous concrètement?

$\mathbf{R}$ : D'abord, il faut savoir que ce camion de pompiers ne tient qu'environ quatre heures. Donc, toutes les quatre heures, il fallait le réapprovisionner. Il y a des fois où on a transporté des réservoirs remplis d'essence en voiture, d'autres fois où on est allé en camion-citerne. Il y a eu plusieurs solutions et je ne sais pas exactement comment on avait fait cette fois-là. Il faudrait vérifier auprès des intéressés. Il y avait des hommes qui apportaient l'essence en voiture. La radioactivité était très forte, alors ils ont été irradiés. Parmi ceux qui, chez nous, ont atteint plus de cent et quelques dizaines de Sieverts, rien qu'en exposition externe, tout au début, sont des gars qui ont été irradiés en travaillant sur la réalisation du circuit d'injection d'eau et ceux qui ont alimenté le camion de pompiers en essence.

$\mathbf{Q}$ : Vous nous avez dit quatre heures. Le camion ne tient pas plus longtemps. Or, l'injection d'eau avec le camion de pompiers est une méthode que vous avez continué à utiliser longtemps après cet épisode. Ce qui veut dire que, durant tout ce temps, des employés de 
TEPCO sont allés à tour de rôle porter l'essence au camion, qu'ils ont continué à faire ça dans un environnement fortement radioactif?

$\mathbf{R}$ : Je dévie un peu du sujet, mais je voudrais vous raconter quelque chose. Il y avait une femme qui s'occupait de faire venir l'essence pour les pompiers, qui accueillait, s'occupait des pompiers et des unités de secours des pompiers de Tôkyô, qui viendront plus tard. Elle était extrêmement efficace dans son travail. On a parlé d'elle dans le journal. C'est la femme qui a dépassé le seuil de la contamination interne ${ }^{48}$. Ce n'est pas seulement une histoire d'irradiation. C'était une personne dévouée, elle se sentait investie d'une mission, c'est pour ça qu'elle travaillait ainsi. Mais quand la presse s'empare de ce genre de chose, ça devient un simple problème d'irradiation et on accuse l'entreprise de mauvaise gestion. Je voudrais dénoncer la médiocrité de la presse de notre pays. Je pense que je le ferai à une autre occasion, mais cela me met très en colère.

$\mathbf{Q}$ : Dans quelles circonstances, a-t-elle été le plus exposée ? C'était lors d'une manœuvre?

$\mathbf{R}$ : Non, pas dans son cas. En fait, même dans les bâtiments où nous nous trouvions, la radioactivité était montée. C'est pourquoi on a évacué très rapidement les femmes. Mais, elle, elle travaillait depuis longtemps dans le groupe " prévention », c'était une personne qui avait un sens du devoir très fort et on n'a pas pu lui faire quitter les lieux, je veux dire qu'elle n'a pas voulu quitter les lieux. Elle s'occupait donc de recevoir les pompiers, etc. L'entrée du bâtiment antisismique est une double porte. La fois où vous êtes venus visiter les lieux, on avait modifié ce système. On l'avait amélioré. Mais au moment de l'accident, il n'y avait que deux portes. Quand on entrait, on ouvrait une première porte, on la refermait, avant d'ouvrir l'autre pour entrer à l'intérieur.

\section{$\mathbf{Q}$ : Et cette femme s'occupait de l'ouverture et de la fermeture de la porte extérieure ?}

$\mathbf{R}$ : Non. Elle ne s'occupait pas des portes, mais elle était restée là, à côté de l'entrée avec les pompiers. Seulement, les portes n'étaient plus étanches. Elles avaient été déformées lors de l'explosion du réacteur 1. Même si on fermait, il y avait des fentes par où la radioactivité pénétrait de l'extérieur. À cause de ça, moi aussi, j'ai subi une contamination interne. Dans son cas, comme elle se trouvait à l'endroit le plus proche de l'entrée, elle a forcément subi une contamination interne. Nous, ni elle ni moi, ne sommes allés directement sur le terrain, mais rien que de nous trouver dans le bâtiment antisismique, nous avons été contaminés. Tout ça, parce qu'elle était au mauvais endroit, alors qu'elle n'était même pas allée sur le terrain. Ou peut-être y est-elle allée de temps en temps en accompagnant des pompiers? Pardon d'avoir dévié du sujet.

$\mathbf{Q}$ : Non. Merci d'avoir évoqué ce cas.

$\mathbf{Q}$ : Le 14 mars au soir, concernant le réacteur 2, vous avez constitué un circuit pour l'injection d'eau et vous réussissez à injecter. Parallèlement, vous tentez des manœuvres pour l'éventage, mais sans succès.

R : Oui, c'était difficile.

$\mathbf{Q}$ : Je pense qu'il doit y avoir des raisons similaires à ce que nous avons vu avec le réacteur 3. On voit dans la chronologie à la page 27, par-là, qu'après l'explosion, vous travaillez jusqu'au soir sur la petite vanne de la vanne AO. Vous arrivez à faire les réparations et, à partir d'environ 16h00, vous attaquez la manœuvre d'ouverture de la grande vanne, mais vous rencontrez des difficultés avec le compresseur d'air. Vous retournez donc travailler à l'ouverture de la petite vanne. Vers $21 \mathrm{~h} 00$, vous arrivez enfin à 
l'ouvrir, mais juste un peu. Cette fois-ci, vers 22h50, la pression de la chambre sèche commence à grimper, jusqu'à devenir l'objet d'une déclaration suivant l'article 15. En effet, si on regarde les paramètres du réacteur 2 , le 14 mars, vers la page 9/22, la pression de la chambre sèche monte continuellement après $22 \mathrm{~h} 00$. Simultanément, on se rend compte, si on regarde les chiffres à cheval sur les pages 9 et 10/22, que la pression de la chambre sèche et la pression de la chambre de dépressurisation amorcent des mouvements contraires. En tout cas, sur le plan des chiffres. Et on arrive à une situation où il y a un écart du simple au double entre les deux, la pression de la chambre sèche se situant aux environs de 0,74 et celle de la chambre de dépressurisation à 0,3. De plus, cette situation va se prolonger. À votre avis, comment peut-on expliquer ce phénomène?

$\mathbf{R}$ : Nous nous prenons tous la tête. Nous nous sommes tous pris la tête.

$\mathbf{Q}$ : Vous avez donc pensé sur le moment aussi que c'était bizarre. Et quelles sont les hypothèses possibles?

$\mathbf{R}$ : C'est que ça aurait dû être le contraire. La chambre de dépressurisation étant située en bas, il aurait été compréhensible que sa pression puisse un peu monter. Mais ces courbes qui s'éloignent l'une de l'autre, je n'y comprends rien. Il y a peutêtre encore eu un problème de fiabilité des instruments de mesure. D’un autre côté, nous avons tenté différentes manœuvres d'éventage, mais nous avons échoué. Enfin, échouer n'est peut-être pas la bonne expression. Disons que les choses n'allaient pas bien. C'est à ce moment-là qu'une nouvelle fois, on reçoit des pressions du siège : " arrête de discutailler», "fais-ci », "fais-ça». On entendait les anciens de la boîte vociférer derrière. Je n'avais qu'une envie, qu'ils me foutent la paix. L'éventage ? Mais bien sûr qu'on s'en occupait, bande de tarés ! ${ }^{49}$ Bref, le seul fait que la courbe puisse s'inverser était inexplicable. J'avais bien passé en revue toutes sortes de possibilités, mais pour moi, il n'était pas possible d'expliquer, de manière scientifique, pourquoi on avait $0,700 \mathrm{MPa}$ à l'intérieur de la chambre sèche et 0,400 dans la chambre de dépressurisation. D'ailleurs, je ne me l'explique toujours pas.

Q : Est-ce qu'aujourd'hui, il y a des gens chez TEPCO qui continuent à réfléchir à ce phénomène avec l'aide d'experts?

$\mathbf{R}$ : Je pense que oui. Mais je n'ai encore rien entendu. Vous avez remarqué qu'à un certain moment, la pression chute d'un coup? La pression de la chambre de dépressurisation tombe à zéro. Dès que la pression de la chambre de dépressurisation a atteint zéro, celle de la chambre sèche chute aussi d'un coup. C'est pour ça que je n'y comprends rien. Durant tout ce temps, la pression de la chambre sèche était toujours élevée, alors le siège n'arrêtait pas de nous harceler pour qu'on évente. Je leur répondais qu'on faisait ce qu'on pouvait. On était obligés de travailler en se disputant avec le siège. C'était pénible.

$\mathbf{Q}$ : Contrairement aux réacteurs 1 et 3 , pour le 2 , vous aviez décidé, à peu près à ce moment-là, d'ouvrir aussi la ligne d'éventage de la chambre sèche, en plus de celle de la chambre humide.

$\mathbf{R}$ : Indiscutablement, c'était la pression de la chambre sèche qui était élevée. Or, même si on éventait au niveau de la chambre humide, on n'avait pas de garantie que cela soit efficace sur la chambre sèche. Je n'arrivais pas à expliquer le phénomène du point de vue de la physique, mais il était certain que si la pression de la chambre sèche était élevée, on était obligés d'ouvrir les vannes de la chambre sèche.

$\mathbf{Q}:$ C'est ce que suggérait le siège ou l'idée venait de vous?

$\mathbf{R}$ : Sur ce point, nous étions d'accord. Ils intervenaient sans arrêt et nous dérangeaient, mais, fondamentalement, nous avions toujours été d'accord pour dire 
qu'il fallait procéder à un éventage avant que la pression de la chambre sèche n'atteigne 0,8 MPa. Nous étions aussi d'accord sur le fait qu'étant donné que la pression de la chambre sèche était plus élevée que celle de la chambre de dépressurisation, il fallait naturellement procéder à un éventage de la chambre sèche. Seulement, pour ma part, je n'arrivais pas à avoir la conviction que la pression de la chambre sèche était effectivement si élevée. Et j'ai donc continué à soutenir que, scientifiquement, ce phénomène était totalement bizarre. Mais, de toute manière, nous étions lancés dans les préparatifs, car il fallait être prêt à éventer la chambre sèche si la pression venait à monter encore plus.

$\mathbf{Q}$ : Il est noté dans la rubrique éventage de la chambre sèche de la chronologie à la page 27 , que vous avez réussi à ouvrir la petite vanne $\mathrm{AO}$ sur la ligne d'éventage à 0h02, que les préparatifs de l'éventage de la chambre sèche étaient achevés à part la rupture du disque. Seulement, quelques minutes après, on constate que cette vanne est fermée. Si je comprends bien, vous essayez de différentes manières d'ouvrir cette ligne d'éventage, pour finalement constater qu'elle est fermée. Mais vous vous acharnez à l'ouvrir, c'est bien ça?

R : Oui, on s'efforçait de l'ouvrir.

$\mathbf{Q}$ : Ce sera beaucoup plus tard, il y aura cette histoire, que vous avez évoquée tout à l'heure, de la pression de la chambre de dépressurisation qui chute à zéro. En fait, ça s'est passé le 15 mars entre 6 h00 et 6h10. Jusque-là, vous accumuliez les tentatives pour ouvrir la petite vanne, pour établir la ligne d'éventage de la chambre humide et de la chambre sèche, c'est bien ça?

$\mathbf{R}$ : Oui. On le faisait. Mais comme je l'ai dit tout à l'heure, finalement, on ne sait pas si le disque va rompre ou pas. Comme la pression ne descendait pas, j'avais tendance à croire que ça avait été inopérant. Mais je ne comprenais toujours pas la différence de pression entre la chambre sèche et la chambre de dépressurisation. J'en étais à me demander si la pression de la chambre sèche était effectivement à 0,75 . Dans cette situation de doute, je me suis dit qu'il fallait tout de même préparer le terrain pour une ouverture des vannes, même si ça n'avait pas l'air d'avoir d'effet. C'est pourquoi on se retrouve, une nouvelle fois, à manœuvrer pour l'ouverture des vannes. Et c'est encore une situation qui va durer longtemps.

$\mathbf{Q}$ : Je voudrais qu'on parle maintenant de vos préoccupations concernant les actions qui restaient à mener. Dans les documents que je vous ai prié de regarder tout à l'heure, on a vu que vous aviez mesuré la température de la piscine de combustibles usés du réacteur 4 à l'aube du 14 mars, vers 4 h08. Cette température était de $84^{\circ} \mathrm{C}$. D'après cette température, selon vous, était-on dans une situation où il fallait envisager des mesures pour cette piscine?

R : Il fallait ajouter de l'eau.

$\mathbf{Q}$ : Et pour cela, avez-vous fait quelque chose par rapport au réacteur 4, le 14 mars ?

$\mathbf{R}$ : J'avais confié ce problème au siège. Déjà, avec les réacteurs 1,2 et 3 , on manquait de personnel. J'avais donc demandé au siège de s'occuper de la piscine du réacteur 4 , y compris le combustible présent, le taux à venir de l'élévation de la température, tous ces calculs et la surveillance des données, y compris aussi l'injection d'eau. On ne pouvait pas tout faire. Avec le personnel dont on disposait sur la centrale, on avait déjà du mal à faire face pour les réacteurs 1,2 et 3 . Alors, concernant la piscine de combustibles usés, dès le début, j'avais chargé le siège d'y réfléchir et d'agir en faisant appel à la force d'autodéfense, ou tout autre organisme. 
$\mathbf{Q}$ : Cela veut dire que c'est le siège qui allait prendre toutes les décisions concernant le réacteur 4 ?

R : Oui. Nous ne disposions pas du matériel nécessaire non plus, alors.

$\mathbf{Q}$ : Mais, concernant la température de l'eau ou la température de l'air, par exemple, vous leur faisiez passer les informations?

R : Oui.

$\mathbf{Q}$ : Je voudrais qu'on parle de ce qui s'est passé le 15 mars entre 6 h00 et 6h10. C'est le moment où la pression de la chambre de dépressurisation du réacteur 2 chute brutalement et atteint zéro. En plus, au même moment...

R : Il y a eu un bruit d'explosion.

$\mathbf{Q}$ : Oui, on a entendu un grand bruit. Avez-vous pu l'entendre ou le sentir dans le bâtiment antisismique? Un choc ou un son?

$\mathbf{R}$ : Non, pas dans le bâtiment antisismique. Si je me remémore, ce matin-là, le Premier ministre devait venir au siège de la société. On était donc en liaison télé avec le siège. Nous étions rassemblés autour de la télévision dans le bâtiment antisismique, quand on a reçu de la salle de contrôle des informations. Je ne sais pas si cette personne était là-bas par hasard, je ne m'en souviens plus. Il faudrait demander des précisions au chef du groupe "production ». Toujours est-il qu'elle nous informe, à la cellule de crise du bâtiment antisismique, que la pression de la chambre de dépressurisation est tombée à zéro et qu'il y a eu un grand bruit. À ce moment-là, il y avait encore de la pression dans la chambre sèche. Normalement, avec de la pression dans la chambre sèche, il n'est pas possible que la pression de la chambre de dépressurisation soit nulle. Seulement, dans le pire des cas, si on suppose qu'on ne peut pas faire confiance aux chiffres de la pression de la chambre sèche et que la pression de la chambre de dépressurisation est nulle, cela signifie qu'il y a des probabilités que l'enceinte de confinement ait été endommagée. Rien n'avait pu être vérifié, mais j'ai supposé, puisqu'il y avait eu un bruit de bris, qu'il y avait eu une rupture quelconque, que cette rupture pouvait concerner l'enceinte de confinement. Pour moi, c'était une situation d'urgence et j'ai pris des mesures conservatoires. J'ai ordonné qu'hormis le personnel indispensable à la conduite et à la réhabilitation, tout le monde évacue le terrain temporairement ${ }^{50}$.

$\mathbf{Q}$ : Et au même moment, le réacteur 4...

$\mathbf{R}$ : Oui. Je vais vous expliquer. Les choses ne sont pas claires sur le papier. Ce faisant, cette fois, des hommes qui revenaient de la salle de contrôle des réacteurs 3 et 4 nous ont dit que la tranche 4 était dans un état terrible. Quand on leur a demandé des précisions, ils nous ont dit qu'au moment où ils arrivaient à la salle de contrôle ils avaient ressenti une onde de choc et qu'au moment d'en repartir, ils avaient levé les yeux et avaient constaté que le mur du réacteur 4 avait été détruit. Du coup, je ne sais toujours pas si ce bruit de bris venait du réacteur 4 ou de la chambre de dépressurisation du réacteur 2 ou d'ailleurs. Du point de vue horaire, cela s'est passé à peu près au même moment. On ne sait pas très bien si le pilote du réacteur 2 n'a pas entendu le bruit du réacteur 4. On a pourtant comparé les données des deux réacteurs, mais l'affaire est difficile à démêler. Les hommes qui allaient vers la salle de contrôle des réacteurs 3 et 4 prétendent qu'ils ont ressenti une onde de choc impressionnante, alors j'aurais tendance à penser que le bruit était bien un bruit qui venait du réacteur 4. Mais ça, c'est ce que j'en dis aujourd'hui. Parce que ces informations ne nous sont parvenues que plus tard, lorsque ces hommes reviendront 
du réacteur 4. Il y a un décalage. Mais, au moment où on l'a su, je leur ai bien demandé à quelle heure tout ça s'était passé.

Q : Que pensez-vous du fait que le conducteur situe le bruit avec autant de précision près de la chambre de dépressurisation?

$\mathbf{R}$ : Ça, c'est de l'ordre de l'intuition du spécialiste. Parce qu'on ne peut pas savoir ce qui se passe dans la chambre de dépressurisation à partir de la salle de contrôle.

$\mathbf{Q}:$ : Alors que c'était peut-être le réacteur 4.

$\mathbf{R}$ : Bien sûr, il ne pouvait pas savoir. Seulement, comme la pression de la chambre de dépressurisation était tombée à zéro juste à ce moment-là, il avait des préjugés. Alors, la manière la plus juste pour décrire notre situation à ce moment-là est de dire : « il y a eu un grand bruit, au même moment, la pression de la chambre de dépressurisation est tombée à zéro ", tout simplement.

Q : Effectivement, on note des phénomènes particuliers autour des réacteurs 2 et 4 à ce moment-là. Mais avant, vous étiez en liaison télé et le Premier ministre était en visite au siège.

R : Oui.

$\mathbf{Q}$ : Qu'était venu faire le Premier ministre?

$\mathbf{R}$ : Je n'en sais trop rien. Peut-être nous encourager ${ }^{51}$ ? Toujours est-il qu'on voyait sur l'écran le Directeur Général, le Président, tout le comité directeur au grand complet. Là, le Premier ministre est arrivé ostensiblement avec un peu de retard, un peu après 5 ho0, il me semble. Je ne sais pour quelles raisons, il avait l'air extrêmement irrité. C'était du genre: "qu'est-ce que vous foutez, tous autant que vous êtes ». Je ne sais plus ce qu'il a raconté, mais je me rappelle seulement avoir trouvé ça très désagréable. Il avait un ton désagréable. Ensuite il a vociféré qu'il n'était pas venu pour parler avec autant de monde, il a exigé qu'on lui prépare une autre salle ${ }^{52}$ et c'est à ce moment-là que les évènements sont arrivés.

$\mathbf{Q}$ : Pendant que vous assistiez à la téléconférence?

$\mathbf{R}$ : Oui. Du coup, de mon côté, je communiquais, du genre: «j'ordonne le retrait du personnel », etc.

$\mathbf{Q}:$ : Ceux qui étaient de l'autre côté pouvaient vous voir?

$\mathbf{R}$ : À ce moment-là, le Premier ministre était parti dans une autre salle. Je ne me rappelle plus très bier comment ça s'était passé, mais ce qu'on voyait sur l'écran au début n'était pas la salle habituelle de téléconférence. Comme on attendait le Premier ministre, ils avaient prévu une autre salle du siège. En cours de route, comme le Premier ministre exigeait un autre lieu, ils sont partis. Sur ces entrefaites, nous avons eu, de notre côté, des informations comme je vous l'ai dit. Les échanges que j'ai eus ensuite avec le siège, concernant le retrait, par exemple, sont passés par le réseau habituel de téléconférence. D'ailleurs à ce propos, le siège soutenait l'hypothèse que, puisqu'il y avait encore de la pression dans la chambre sèche, il ne pouvait pas y avoir explosion de l'enceinte de confinement. Mais je ne pouvais pas faire confiance aux instruments de mesure concernant la pression de la chambre sèche. Je voulais assurer la sécurité. J'ai donc opté pour le scénario le plus pessimiste et ordonné le retrait. On a même prévu des cars pour évacuer le personnel. 
Q: J'ai entendu dire qu'à cette occasion, certaines personnes du siège avaient fait remarquer qu'il y avait encore de la pression dans l'enceinte de confinement et la chambre sèche.

R : Oui. Moi aussi, sur le moment, c'est ce que j'ai pensé. Moi-même, je me suis dit que ça n'avait pas explosé malgré le zéro de la chambre de dépressurisation, puisqu'il y avait encore de la pression dans la chambre sèche. Mais il y avait eu le bruit. À ce moment-là, je n'avais pas encore eu les informations concernant le réacteur 4 . Alors le bruit, plus le zéro, c'était quand même deux points graves. Si on voulait assurer le maximum de sécurité, il fallait supposer qu'il y avait eu rupture et qu'il y avait de forts risques que de la radioactivité s'échappe. C'est pourquoi j'ai ordonné le retrait dans un premier temps. On a prévu des cars pour évacuer les gens vers le $2 \mathrm{~F}^{53}$.

$\mathbf{Q}$ : Permettez-moi de vérifier quelques points. Quelle était la position exacte de la personne qui a entendu ce grand bruit ? Où l'a-t-elle entendu?

$\mathbf{R}$ : Elle était dans la salle centrale de commande. Si je précise à propos de la pression et du bruit, la personne qui a entendu le bruit regardait l'indicateur de pression. Or, on ne pouvait voir la pression que de la salle de commande. Donc elle y était forcément. C'était la salle de commande des réacteurs 1 et 2 . C'est de là qu'elle a téléphoné et que l'information est arrivée au groupe "production ». La personne qui a entendu la première le bruit, la personne qui surveillait la pression, était sans aucun doute possible dans la salle de commande. De toute façon, il n'y avait personne sur le terrain en dehors de la salle de commande.

$\mathbf{Q}$ : Vous voulez dire que vous manquiez de personnel?

$\mathbf{R}$ : Parce qu'on ne pouvait pas aller plus loin.

$\mathbf{Q}$ : La personne qui se trouvait dans la salle de commande des tranches 1 et 2 est tout de suite revenue ici?

R : Oui.

$\mathbf{Q}$ : Lui avez-vous parlé personnellement ? Pour l'interroger sur la situation, par exemple?

$\mathbf{R}$ : Oui, je l'ai fait. On a parlé effectivement de la pression qui était à zéro et du bruit, mais je ne suis pas sûr qu'elle ait précisé que le bruit venait de la chambre de dépressurisation, comme c'est marqué ici.

$\mathbf{Q}$ : Pendant cette conversation avec cette personne qui se trouvait dans la salle de commande, est-ce que vous aviez, vous, relié ce bruit à la chambre de dépressurisation, sachant que vous n'aviez pas encore eu les informations concernant le réacteur 4, à ce moment-là?

R : Oui, j'avais fait la relation. Comme vous pouvez le voir, depuis la veille, les chiffres concernant l'équilibre des pressions du réacteur 2 étaient très bizarres. On ne savait à qui se fier, quand, tout à coup, la pression de la chambre de dépressurisation chute de $300 \mathrm{kPa}$ à zéro. Ce qui, par exemple, le 15 mars vers 16 h00 était à peu près à 0,27 tombe à zéro. Puis, le bruit. C'est sûr qu'on ne pouvait imaginer qu'une rupture quelque part. Seulement, comme vous l'avez dit, la pression de la chambre sèche est encore à 0,7 . Il n'est absolument pas possible qu'avec une rupture de la chambre de dépressurisation, on puisse encore avoir de la pression dans la chambre sèche. Mais, encore une fois, on avait été déjà tellement abusés par les instruments de mesure, qu'on ne pouvait pas écarter l'hypothèse que l'indicateur de pression de la chambre sèche soit coincé. 
$\mathbf{Q}$ : Donc, vous avez des soucis avec le réacteur 2, puis vous constatez les dégâts sur le 4. Ensuite, comme vous l'avez rapporté suivant l'article 15, il y a encore le feu qui s'est déclaré sur la tranche 4. Ce sont des personnes qui étaient à proximité qui l'ont constaté ?

$\mathbf{R}$ : Aussi bien pour les réacteurs 1 et 2 , que pour les 3 et 4 , il n'y avait pas moyen de laisser quelqu'un en permanence dans les salles de commande à cause de la radioactivité. On était obligé d'envoyer périodiquement quelqu'un pour relever les données. Et un de ceux qui étaient partis chercher les chiffres, a vu sur le chemin du retour de la vapeur qui s'élevait. À l'époque on ne pouvait pas voir les images des tranches à l'écran comme aujourd'hui, alors on se basait sur ce que racontaient les gens qui revenaient du terrain. Il prétendait donc qu'il y avait le feu. Il y a un endroit à l'arrière du bâtiment antisismique, d'où on peut voir le réacteur 4 . Alors nous y sommes allés avec des jumelles pour vérifier. J'ai fait le nécessaire pour demander de l'aide pour l'éteindre. Mais, naturellement, les pompiers ne sont pas venus, ni personne, d'ailleurs. J'avais même demandé à $\mathrm{M}$. Hosono ${ }^{54}$ si on ne pouvait pas solliciter l'armée américaine. Finalement, ils n'ont pas eu le temps d'arriver, enfin, ils ne sont pas venus.

$\mathbf{Q}$ : M. Hosono, vous voulez dire le conseiller du Premier ministre ? Vous l'aviez sollicité par téléconférence?

$\mathbf{R}$ : Non, par téléphone.

$\mathbf{Q}:$ La cellule de crise interministérielle a fonctionné à partir du 15, n'est-ce pas ?

$\mathbf{R}:$ Je ne sais pas. L'explosion du réacteur 3 était bien le 14 ?

Q : Oui, le 14 à 11 h00.

R : Si c'était le 14 à 11h00, peu avant, j'ai eu une demande de M. Hosono, relayée par un collègue du siège qui était chez le Premier ministre, que s'il arrivait quelque chose, il me téléphonerait en direct. On a échangé nos numéros de téléphone. Je l'ai donc avisé directement par téléphone de l'incendie, bien que j'avais, bien sûr, fait circuler l'information par téléconférence aussi. Je lui ai dit qu'un feu s'était déclaré, que j'avais sollicité les pompiers, mais qu'ils n'avaient pas l'air de vouloir venir. Alors, il m'a répondu qu'il y réfléchissait de son côté, avec les ministres, y compris la possibilité de solliciter l'armée américaine.

$\mathbf{Q}$ : Et qu'en est-il sorti?

$\mathbf{R}$ : Il semblerait que l'armée américaine avait commencé à bouger, mais le feu s'est éteint avant leur arrivée. Sans qu'on n'y fasse rien.

$\mathbf{Q}:$ Ça, c'est bien ce qui s'est passé le 15 ?

R : Oui, le 15.

$\mathbf{Q}$ : Chronologiquement, il y a un rapport suivant l'article 15 à 9 h38 signalant un incendie. Vous faites d'abord appel aux pompiers. Vous contactez les pompiers. Aviez-vous réussi à les joindre?

R : Oui.

$\mathbf{Q}$ : Face à votre demande d'intervention, ils ont répondu qu'ils ne pouvaient pas venir, c'est ça?

R : Oui.

$\mathbf{Q}:$ Quelle en était la raison?

$\mathbf{R}$ : Ils disaient que la radioactivité était élevée. 
$\mathbf{Q}$ : Ils ont dit que la radioactivité était élevée et que pour cette raison, ils ne pouvaient pas agir?

R : Oui.

$\mathbf{Q}$ : C'est à ce moment-là que vous avez contacté M. Hosono, en évoquant la possibilité d'une intervention de l'armée américaine ou des forces d'autodéfense?

$\mathbf{R}$ : Je lui ai demandé s'il ne pouvait pas agir au niveau de l'État.

$\mathbf{Q}$ : Et, avant même que tout ça ne soit mis à exécution, le feu s'est éteint. C'est bien ça?

R : Oui.

$\mathbf{Q}:$ Je fais un petit saut dans le temps. Le lendemain, le 16.

$\mathbf{R}$ : Il y aura encore le feu.

$\mathbf{Q}$ : II y a eu un rapport dans ce sens, je ne sais plus où. Vers 5h45, une personne se trouvant sur le terrain observe un feu. En avez-vous été informé?

R : Oui.

$\mathbf{Q}$ : Qu'avez-vous pris comme mesures, sur le moment?

R : La même chose que tout à l'heure. C'était un feu, donc suivant les procédures habituelles, je fais une demande auprès des pompiers. Mais je ne me fais pas d'illusion. De toute façon, ils ne viendront pas. Ensuite, je ne sais plus si c'était l'armée américaine ou pas. Je contacte M. Hosono. Je ne sais plus s'il était à la cellule interministérielle ou s'il était au siège.

$\mathbf{Q}$ : Le réacteur 2 montre un comportement pour le moins bizarre. Mais le réacteur 4 vous donne aussi des soucis. La partie haute du bâtiment est endommagée et il semble qu'il y ait un feu aux alentours des niveaux 3 et 4 . Ça, c'est ce qui se passe d'abord le 15 au matin. Le feu s'éteint une première fois, mais il semble qu'on voit encore des flammes vers le 16. Tous ces évènements autour du réacteur 4, quelles en ont été les causes, à votre avis?

$\mathbf{R}$ : Pour moi, la première explosion, le gros bruit, restent inexpliqués. De ce fait, l'explication la plus plausible était que la destruction était peut-être due à un trop grand échauffement des combustibles entreposés dans la piscine de combustibles usés. Ça, c'était le tout premier scénario auquel j'avais pensé. Tout ce qui suit, ce sont des réflexions que je me suis fait après coup. En fait, quand j'ai vu ces combustibles, plus tard, ils étaient assez propres, ce qui prouve que cela n'était pas dû à la surchauffe du combustible. Si on admet ce fait, il n'y a guère plus que le scénario que tout le monde évoque aujourd'hui, qui est celui de l'hydrogène qui s'échappait du réacteur 3, qui s'est accumulé du côté du 4 et qui a explosé. Je ne vois que ça. En même temps, je n'arrive pas à comprendre la manière dont le bâtiment a été détruit. Les murs ont été largement troués dans le sens nord-sud. Les murs est-ouest n'ont pas de trou. La destruction s'est faite dans le sens nord-sud. En plus, c'est dans la zone des niveaux 3 et 4 . Je ne comprends pas comment on arrive à ce résultat.

En plus, comme disent les centres de recherche en ce moment à propos de l'hydrogène qui aurait migré du réacteur 3 , je ne suis absolument pas sûr, même aujourd'hui, que l'équilibre des pressions aurait véritablement permis à l'hydrogène de s'accumuler autant du côté du réacteur 4. Mais il faut avouer que nous n'avons pas d'autre explication pour le moment. Pour moi, je ne suis toujours pas satisfait. Ce sont des phénomènes qu'on ne peut pas expliquer du point de vue de la physique, pour un ingénieur. Pour bien faire, il faudrait aller sur place constater les destructions, voir ce qui est le plus abîmé. Nous allons enfin pouvoir retourner sur le terrain. Alors c'est le moment d'ouvrir l'œil et de confronter les observations pour 
essayer de comprendre. Pour moi, la thèse de l'hydrogène n'est qu'une hypothèse. Même si la probabilité qu'elle soit la bonne est élevée.

Q: Mais ces hypothèses concernant l'hydrogène du réacteur 3 n'ont été évoquées qu'après qu'un hélicoptère ait survolé le réacteur 4 et qu'il ait observé, du haut du bâtiment, que le niveau d'eau de la piscine de combustible était encore bien suffisant, n'est-ce pas?

$\mathbf{R}$ : Oui, vous avez raison.

$\mathbf{Q}$ : Sur le moment, vous ne pensiez pas encore à tout ça.

$\mathbf{R}$ : Non. Sur le moment, le scénario le plus probable, pour nous, était celui des combustibles usés qui se seraient déchainés. L'avis le plus partagé était que de l'hydrogène s'échappait de là, qu'il y avait réaction zirconium-eau, qu'il y avait un trop grand échauffement et que ça produisait de l'hydrogène. Cet hydrogène serait descendu. Mais si cela s'était vraiment passé comme ça, ça aurait dû être l'étage de la salle d'exploitation qui aurait dû être détruit. Cela n'explique pas l'énorme trou qui a été percé au niveau $3^{55}$.

$\mathbf{Q}$ : Effectivement. Nous allons terminer là pour aujourd'hui. Je vous remercie beaucoup.

(Fin de l'audition)

\section{NOTES}

1. Des opérateurs ont néanmoins tout de suite compris que la secousse n'était pas due à un autre tremblement de terre, comme le raconte Ryuta Idogawa : "Quand j'étais allongé devant l'écran du réacteur 2 dans la salle de commande, un grand choc est arrivé du bas comme si mon corps était monté en l'air. Un pilote a crié: 'Ce choc n'est pas le séisme. Une explosion se produit quelque part!'» (Kobayashi, 2019, p. 101). Le sergent-major Hidekazu Watanabe ( $6^{\mathrm{e}}$ Régiment de la $6^{\mathrm{e}}$ Division des Forces d'autodéfense) raconte également son ressenti à la suite de l'explosion: "J'étais retourné dans le bâtiment antisismique à ce moment-là. Cinq de mes hommes venaient de partir pour la tranche 1 pour le prochain quart de travail avec certains des employés de TEPCO. Peu de temps après leur départ, il y a eu un énorme coup. Il était assez grand pour séparer les murs et les escaliers. Je suis un artilleur et ce n'était pas comme si l'un des canons partait. C'était comme si dix d'entre eux tiraient en même temps. J'étais dans la salle d'attente à droite de l'entrée et j'ai regardé par la minuscule fenêtre de trente centimètres pour voir la fumée blanche et les débris passer devant avant que tout à l'extérieur ne devienne blanc. À l'intérieur du bâtiment antisismique, il y avait des trucs qui s'écroulaient partout » (Kadota, 2014, p. 154 [notre traduction]).

2. Ichirô Takekuro est né en 1946. Il est vice-Président de TEPCO jusqu'en 2010 (ce qui lui vaut de garder le titre de Fellow de TEPCO après son départ) et depuis 2010, Président de l'International Nuclear Energy Development of Japan Co. (JINED). Ce consortium, créé en 2010, regroupe les neuf exploitants de réacteurs nucléaires japonais, trois constructeurs de centrales et un fonds d'investissement public-privé sous la supervision du METI. 
3. Dans sa version des faits, Kan tient Takekuro comme l'unique responsable de cet ordre donné à Yoshida : "Tout le monde était d'accord pour dire que si on manquait d'eau douce, il n'y avait plus qu'à utiliser de l'eau de mer. Bien entendu, moi-même j'en étais convaincu, puisqu'il fallait injecter de l'eau à tout prix, et le ministre de l'Économie et de l'Industrie donne effectivement l'ordre à 17 h55 de remplir le réacteur avec de l'eau de mer. [...] Lorsque M. Takekuro a su que l'eau de mer était déjà injectée, il a jugé, et lui seul en a jugé, qu'il fallait ordonner, directement ou indirectement, au directeur Yoshida d'arrêter, sous prétexte que le Gouvernement n'était pas encore au courant. C'est comme ça que ça s'est passé. Et là, le directeur Yoshida, qui pense qu'il ne faut pas arrêter l'injection, mais l'ordre émanant du Premier ministre, c'est-à-dire moi, déclare pour la forme qu'il va arrêter l'injection, sans n'en rien faire dans les faits. Ça, c'est sa décision à lui " (Kan, 2012). Une analyse minutieuse de l'affaire révèle en fait que des ordres contradictoires ont été donnés à différents moments (Kobayashi, 2019). Le 12 mars, à 14h50, Shimizu, le Président de TEPCO, donne son accord pour procéder à l'injection d'eau de mer. Mais l'explosion survenue à $15 \mathrm{~h} 36$ dans le bâtiment réacteur 1 oblige le terrain à interrompre la préparation de l'opération. À 17h55, Kaieda, le ministre de l'Économie, donne à TEPCO l'ordre de procéder à l'injection. À 18h00, une discussion a lieu à la résidence du Premier ministre. Ce dernier pose des questions sur les conséquences de l'opération en termes de corrosion de l'équipement du réacteur et de risque de criticité. Takekuro lui répond qu'à cause de l'explosion, il est nécessaire de s'assurer de la sûreté des tuyaux et que cette vérification devrait prendre deux heures. Kan demande à Takekuro, si le temps le permet, de répondre à ses questions avant le début de l'injection. Takekuro téléphone ensuite à Yoshida à 19h04, mais l'injection d'eau de mer a déjà commencé. Il demande alors au directeur de Fukushima Daiichi d'arrêter l'opération, en attendant que le Premier ministre donne son accord. Mais Yoshida ne comprend pas du tout la situation: d'un côté Kaieda a déjà donné l'ordre d'injecter, de l'autre Takekuro lui affirme que le Gouvernement ne s'est pas encore décidé. Finalement, Yoshida décide de continuer l'injection, comme il l'explique dans la suite de l'audition.

4. Akio Takahashi, diplômé de la Tôkyô Institute of Technology de Tôkyô en 1976 (ingénierie mécanique), rejoint TEPCO la même année. En 2005, il devient le directeur de la centrale nucléaire de Fukushima Daini. En 2007, il prend la direction de la centrale nucléaire de Kashiwazaki-Kariwa. En 2010, il devient conseiller technique de TEPCO. Il est nommé Président de la Japan Atomic Industrial Forum (JAIF) en 2015.

5. Ryûshô Kadota rapporte l'admiration que les travailleurs ont ressenti pour Yoshida lorsque celui-ci s'est dressé contre sa hiérarchie: "Les ouvriers de M. Yoshida se sont encore plus soudés autour de leur directeur quand ils le virent ronger son frein sans céder un pouce de terrain pendant une téléconférence avec la direction générale de TEPCO. La valeur de l'homme est apparue clairement quand l'un des conseillers de TEPCO, Takekuro Ichirô, s'est emporté contre lui, lui ordonnant d'arrêter les injections d'eau de mer en criant: 'Le bureau du Premier Ministre nous casse les oreilles à cause de ça! Arrête tout de suite!', à quoi M. Yoshida répliqua: 'Il n'en est pas question! Vous dites n'importe quoi!'. M. Yoshida s'attendait à ce que l'ordre de stopper les injections d'eau de mer vienne de la direction générale. Mais en prévision, il avait glissé un mot à son chef d'équipe: 'Écoute-moi. La direction générale va nous ordonner de stopper les injections d'eau de mer. Et tu me verras peut-être dire devant l'écran que nous allons arrêter. Mais si je dis ça, surtout n'obéis pas et continue de pomper! Tu as compris?' Effectivement, c'est ce qui se passa » (Kadota, 2013). 
6. La résidence et les bureaux du Premier ministre sont situés dans le quartier administratif de Nagata à Tôkyô. Le complexe immobilier qui les abrite est dénommé en japonais le «Kantei ».

7. Phénomène physique subatomique où des noyaux se transforment sous l'impulsion de particules ou de rayonnements. Cette transformation est accompagnée d'un dégagement d'énergie. Une réaction nucléaire peut être spontanée (naturelle) ou provoquée artificiellement, comme c'est le cas dans un réacteur nucléaire.

8. Parlement japonais. Créée par la Constitution Meiji de 1889, la Diète est composée de la chambre des représentants (480 membres) et de la chambre des conseillers (242 membres). Les deux chambres se réunissent dans le Bâtiment de la Diète nationale à Tôkyô.

9. A posteriori, Naoto Kan estime que Yoshida a pris la bonne décision en continuant l'injection d'eau de mer: "Je vous rappelle que le 12 mars au moment d'injecter de l'eau de mer dans les réacteurs, il avait reçu l'ordre d'arrêter l'opération. Cet ordre venait du siège de TEPCO, et plus précisément de M. Takekuro, pourtant spécialiste du nucléaire et qui savait parfaitement qu'il fallait impérativement refroidir les réacteurs, même si cela devait se faire avec de l'eau de mer. M. Yoshida a passé outre, et de son propre chef, a continué à injecter de l'eau de mer. C'est un homme entier qui a fait ce qu'il jugeait nécessaire pour éviter que les conséquences de l'accident ne s'étendent, quitte à ignorer les ordres venant d'en haut. Et même au vu de la Loi, il n'y a rien à redire sur son comportement. Je vous rappelle que le responsable ultime des opérations est le directeur de la centrale nucléaire »(Kan, 2015).

10. Le J-Village (Japan Football Association's National Training Center) est un centre de formation de footballeurs situé à une vingtaine de kilomètres au sud de Fukushima Daiichi, reconverti en base opérationnelle pour lutter contre l'accident nucléaire.

11. Bureau de TEPCO situé dans la ville de Fukushima, au siège préfectoral de réponse à la catastrophe. Cette cellule, reliée au réseau de téléconférence de l'exploitant, a pour mission de fournir des informations sur l'état des centrales nucléaires à la préfecture, qui se charge ensuite d'organiser des points de presse (TEPCO, 2012).

12. Traduction : « dès que possible».

13. Panneau de décompression.

14. Fondées en 1954, les forces japonaises d'autodéfense (en anglais «Japan SelfDefense Forces ", JSDF) constituent l'armée du Japon. Leur statut est déterminé par l'article 9 de la Constitution, votée en 1946 alors que le pays est occupé par les ÉtatsUnis, vainqueurs de la Guerre du Pacifique. Cet article stipule que le peuple japonais renonce à la guerre, à la menace ou à l'usage de la force comme moyen de règlement des conflits internationaux. Les lois sur la paix et la sécurité, adoptées par la Diète en septembre 2015, permettent désormais aux forces d'autodéfense d'intervenir militairement pour aider un pays allié dans un conflit, sans que le territoire japonais ne soit directement menacé.

15. Le rapport de la NAIIC (2012) précise que la longévité exceptionnelle du RCIC a permis d'éviter que l'état du réacteur 2 ne se détériorât davantage.

16. Mécanisme de contrôle des barres de commandes. De l'eau peut être injectée dans le réacteur à travers les tubes guides des barres.

17. Partie basse de l'enceinte de confinement d'un réacteur à eau bouillante. Elle est généralement remplie d'eau jusqu'à la moitié de sa hauteur. Dite également chambre de suppression, chambre humide, suppression pool ou wetwell. 
18. Type de réacteur nucléaire modéré et refroidi par de l'eau ordinaire, portée à ébullition dans le cœur (cf. Fig. 3). Les six réacteurs de Fukushima Daiichi sont des REB.

19. Tranche 3 de Fukushima Daiichi.

20. Système de protection incendie.

21. Série de pompes et de diffuseurs permettant d'arroser l'enceinte de confinement afin de faire baisser sa pression.

22. Mode d'aspersion de l'enceinte du confinement. Dans le cas d'un accident de perte de réfrigérant primaire (Loss of Coolant Accident - LOCA), le système RHR d'évacuation de la chaleur résiduelle des réacteurs 2 à 6 de Fukushima Daiichi peut basculer entre deux modes: le mode d'aspersion de l'enceinte de confinement (Containment Spray) et l'injection d'eau à basse pression dans le cœur du réacteur (Low Pressure Core Injection). Le mode d'aspersion du confinement permet, via un réseau de buses, de contrôler la température et la pression dans le wetwell et le drywell. Notons que dans le cas du réacteur 1 , le système d'aspersion du confinement et le système d'injection d'eau dans la cuve sont distincts.

23. Il s'agit des circuits $\mathrm{A}$ et $\mathrm{B}$, déjà mentionnés précédemment. Ils correspondent à des systèmes redondants de mesure du niveau d'eau dans la cuve, dont la plage de mesures se situe au niveau du combustible.

24. Tranche 2 de Fukushima Daiichi.

25. Bassin rempli d'eau destiné à l'entreposage de combustible nucléaire usé, permettant la protection des personnes et l'évacuation de la chaleur.

26. La centrale de Fukushima Daiichi.

27. Système de refroidissement de la piscine de désactivation du combustible usé.

28. Un crayon est constitué de pastilles de combustible nucléaire empilées dans des tubes de métal (gaines en alliage de zirconium) fermés aux extrémités.

29. Tranche 1 de Fukushima Daiichi.

30. Le directeur dont parle Yoshida est Masao Uchibori, vice-gouverneur de Fukushima et responsable de la cellule préfectorale du centre de crise hors site.

31. Système de dépressurisation de la cuve. Les soupapes s'ouvrent à haute pression et déversent la vapeur dans le drywell ou l'acheminent vers le wetwell où elle est condensée.

32. Un joint torique, ou un 0-Ring, est un joint d'étanchéité en forme de beignet fin, qui, lorsqu'il est compressé, permet d'éviter les fuites de fluides au niveau des liaisons entre deux conduits. Dans un REB, les mécanismes de déplacement des barres de contrôle se logent dans des tubes qui se trouvent en dessous de la cuve du réacteur. Ces tubes sont raccordés à la cuve. Yoshida soupçonne que les fuites se situaient au niveau de leur interface.

33. Diffuseurs (en spray) d'air comprimé.

34. Cette remarque de l'enquêteur se réfère sans doute implicitement aux camions générateurs, envoyés dans l'urgence par le Gouvernement à la demande de TEPCO et arrivés à Fukushima Daiichi le soir du 11 mars. Ces camions n'ont pu être branchés du fait de leur incompatibilité avec les prises électriques de la centrale.

35. Les propos dépréciatifs de l'enquêteur sur les employés de TEPCO rejoignent l'argumentaire culturaliste utilisé par certains experts pour expliquer l'accident, tel 
Kiyoshi Kurokawa, le Président de la Commission d'enquête mandatée par la Diète : «Ce qu'il faut admettre - très douloureusement - c'est qu'il s'agissait d'un désastre "Made in Japan ». Ses causes fondamentales sont à chercher dans les conventions enracinées dans la culture japonaise: notre obéissance réflexive; notre réticence à remettre en cause l'autorité ; notre attachement au fait de 's'en tenir au programme'; notre groupisme ; et notre insularité » (NAIIC, 2012, p. 9 [notre traduction]). Notons toutefois que ce commentaire de Kurokawa ne se trouve qu'en préambule de la version anglaise du rapport de la NAIIC et n'apparaît pas dans sa version japonaise. Yoshida, quant à lui, ne partage pas du tout l'opinion de l'enquêteur, comme en témoigne sa réponse.

36. Yoshida évoque ici les trois centrales nucléaires exploitées par TEPCO : Fukushima Daiichi, Fukushima Daini et Kashiwazaki-Kariwa.

37. Schéma tuyauterie et instrumentation - il décrit une unité de production avec tous les appareils et équipements, toute la tuyauterie et la robinetterie ainsi que toute l'instrumentation.

38. L'état du réacteur 3 se dégrade au matin du 14 mars et le personnel s'inquiète de plus en plus. Shiro Hikida, raconte ainsi que, «Pour tous les leaders, dont Yoshida, il était difficile d'envoyer les employés sur les sites. Tout d'abord, compte tenu de l'irradiation, nous ne pouvions jamais y envoyer les jeunes employés. En revanche, mêmes les personnes expérimentées ont commencé à avoir peur. J'ai pensé que le leader devait prendre l'initiative et donner l'exemple dans cette situation. Je suis allé personnellement sur le site, à l'extérieur » (Kobayashi, 2019, p. 123). De son côté, Takeyuki Inagaki (chef de groupe de réhabilitation d'équipement) affirme à ses opérateurs que la situation s'est stabilisée et exclut dans l'immédiat la possibilité d'une explosion d'hydrogène. Rassurés, ils repartent sur le terrain pour effectuer les travaux de préparation de l'éventage, lorsque l'explosion se produit dans le bâtiment réacteur 3. De ce fait, Inagaki a été appelé «chef menteur » (Kobayashi, 2019, p. 124) par ses équipes.

39. Le système de téléconférence révèle la détresse ressentie par Yoshida après l'explosion du bâtiment réacteur $3:$ :Le siège, le siège! C'est grave. Le siège, le siège! C'est grave! Le réacteur 3, peut-être, l'explosion de vapeur, je pense, elle se produit maintenant. Il est 11h01!» (Kobayashi, 2019, p. 124). Sous le coup de l'émotion, Yoshida se trompe de terme : il s'agit non pas d'une explosion de vapeur, mais d'une explosion d'hydrogène. Hikida souligne également la gravité de l'explosion comparée à celle du bâtiment réacteur 1: "J'ai pensé que cette explosion avait détruit l'enceinte de confinement du réacteur » (Kobayashi, 2019, p. 124). L'explosion du bâtiment réacteur 3 est par ailleurs vécue comme une honte par le Gouvernement, car ce dernier n'avait déjà pas pu éviter l'explosion du bâtiment réacteur 1. Osamu Maekawa, chef-ingénieur chez Tôshiba, constructeur de la tranche 3, est également affligé par l'explosion: «Nous avions de l'affection pour 'notre réacteur' et nous faisions avec soin la maintenance de ce réacteur. Nous avions confiance en la sûreté du réacteur et de la centrale nucléaire. Nous avions donc toujours dit aux familles des employés: 'Quand le grand séisme se produira, vous pourrez vous abriter dans la centrale nucléaire qui est absolument sûre'. L'explosion du bâtiment du réacteur 3 nous a beaucoup choqués " (Kobayashi, 2019, p. 121).

40. Ce n'est que le matin du 15 mars que Naoto Kan se rend au Siège de TEPCO, accompagné du ministre de l'Économie, du vice-secrétaire général du Gouvernement, de deux conseillers spéciaux et du Président de la NSC. Le Premier ministre est alors stupéfait de découvrir le système de téléconférence de l'exploitant: «le matin du 15 mars, très tôt, quand je suis allé au siège de TEPCO, dans un certain sens, je me suis dit, 'c'était 
donc ça'. C'était donc ça, dans un bon sens. Ils avaient tout. Des téléviseurs qui étaient branchés vingt-quatre heures sur vingt-quatre sur chaque site. Ce que les gens disaient là, tout était complètement accessible à tout le monde. Ce qui me laisse supposer qu'à tout le moins, entre le directeur Yoshida et le siège de TEPCO, les informations devaient circuler suffisamment. C'est en allant au siège social de TEPCO que je me suis dit qu'il fallait que nous, Hosono et tous les autres, devions venir là. Parce que tout était totalement public, que rien ne pouvait rester secret. Vous comprenez, ils ne se parlaient pas individuellement au téléphone, chacun parlait dans un micro et tout le monde entendait. Ce qui m'amène à penser, bien que je n'en sois pas sûr à cent pour cent, que TEPCO n'avait pas fourni les renseignements nécessaires à M. Takekuro, qui était chez moi » (Kan, 2012). Il ne peut donc y avoir de représentants du Gouvernement dans la cellule de crise du siège de TEPCO le 14 mars.

41. Hikida rapporte une version très proche de ce discours, lui ajoutant toutefois une coloration patriotique : "Je suis vraiment désolé de vous avoir exposé à la mort. C'est le fait de mon mauvais jugement. Cependant l'injection de l'eau dans le réacteur 2 s'est aussi arrêtée. Si nous ne prenons pas des mesures, le Japon tombera dans un état grave. Pourriez-vous travailler encore sur les sites, s'il vous plaît?» (Kobayashi, 2019, p. 125).

42. Pour Takeyuki Inagaki, ce moment reste le plus impressionnant de la gestion de l'accident nucléaire : "Ce n'était pas 'demander', mais 'supplier'. Yoshida a fait sincèrement amende honorable et a fait appel à sauver la région et le pays. Les personnels ont été très touchés par son appel. Certains ont tout de suite répondu: 'D'accord, nous y allons!'» (Kobayashi, 2019, p. 125). D'autres témoins précisent néanmoins que l'état émotionnel des opérateurs s'est considérablement détérioré à cause de l'explosion survenue dans le bâtiment réacteur 3. Les chefs ont donc dû rassurer leurs subalternes, comme le signifie Tomoyuki Arai (sous-chef du groupe de protection contre les incendies) : "Tous les employés étaient complètement découragés après l'explosion. Donc je leur ai dit 'Afin d'assurer votre sécurité, les cadres vous accompagnent. Allons-nous recommencer les travaux?" (Kobayashi, 2019, p. 126).

43. Masataka Shimizu, né en 1944, est Président de TEPCO de juin 2008 à mai 2011. Il y a fait toute sa carrière. Il a également été vice-Président du Patronat japonais, le Keidanren, jusqu'en 2011. Durant la crise nucléaire, c'est lui qui dirige la cellule de gestion de crise située au siège de TEPCO.

44. Il s'agit du circuit de refroidissement, qui correspond aux tuyaux reliant la chambre de dépressurisation à la cuve (cf. note 236 sur le Containment Spray Mode).

45. Il s'agit du circuit d'aspersion, qui correspond aux tuyaux reliant la chambre de dépressurisation au réseau de buses d'arrosage de l'enceinte de confinement (cf. note 23).

46. La Nuclear Safety Commission est fondée en 1978 à la suite d'une réforme de la législation sur le nucléaire civil, comme autorité indépendante de surveillance et de vérification des inspections menées par les agences de réglementation telle la NISA. Elle est placée sous la tutelle du Cabinet du Premier ministre. En mars 2011, la NSC est présidée par Haruki Madarame. Elle fusionne en 2012 avec la NISA pour devenir la Nuclear Regulation Authority.

47. Takeyuki Inagaki revient sur la détérioration de la situation, l'après-midi du 14 mars, tandis que les équipes tentaient par tous les moyens de manœuvrer les vannes: "Yoshida nous mettait la pression et nous faisions des efforts pour répondre à l'attente du leader, mais aucune mesure ne portait ses fruits. Même maintenant, je jette de temps en temps un regard sur cette scène dure avec les camarades du groupe: 'Nous étions sur des charbons 
ardents à ce moment-là'. Nous entrions dans la phase de 'Kyukyoku'» (Kobayashi, 2019, p. 176). En japonais, «Kyukyoku » signifie la dernière phase, une situation qui arrive à la limite.

48. Exposition interne aux particules radioactives, lorsque des éléments radioactifs ont pénétré à l'intérieur de l'organisme par inhalation ou par ingestion d'aliments contaminés.

49. Au cours de l'intervention du siège par téléconférence, un ancien cadre de TEPCO déclare: "Yoshida! S'il y a une possibilité, faites un éventage du drywell! N’hésitez pas! Le siège prendra toute la responsabilité. La destruction de l'enceinte serait pire» (Kobayashi, 2019, p. 129). Selon Takeyuki Inagaki, cette intervention ne fait que révéler la méconnaissance des hommes du siège concernant les réalités techniques du terrain : "Franchement, cette proposition était inutile, parce qu'ouvrir les vannes d'un éventage du drywell n'est pas du tout facile comparé à l'éventage normal. Peut-être ces ex-cadres ne connaissaient pas bien Fukushima Daiichi " (Kobayashi, 2019, p. 130).

50. Yoshida s'est adressé aux chefs des douze groupes de la cellule de crise et leur a demandé de procéder à la sélection des opérateurs qui devaient rester à Fukushima Daiichi. Ces travailleurs ont été appelés par les médias étrangers les « Fukushima 50 »en réalité, il ne sont pas cinquante mais soixante-neuf (Kadota, 2013). Le reste du personnel est allé se mettre à l'abri en se rendant à Fukushima Daini. Inagaki témoigne de l'atmosphère très particulière qui règne alors dans la cellule de crise de Daiichi: "Hikida et moi, nous avons tout d'abord pensé la même chose: comment mourrons-nous? Il aurait fallu mettre le masque dans le bâtiment antisismique, mais nous ne le mettions plus, parce que nous avons pensé que nous ne pouvions plus vivre puisque nous y restions. Quant à Yoshida, il nous a semblé qu'il était un peu rassuré. Nous avons imaginé qu'il se détendait du fait qu'il pouvait sauver la vie de la plupart des gens. Ceux qui sont restés étaient seulement les cadres qui avaient une longue relation avec Yoshida. Il nous a tout d'abord proposé: 'On va manger quelque chose!'. Ensuite: 'Vous pouvez faire tout ce que vous voulez!'» (Kobayashi, 2019, p. 141).

51. Naoto Kan se rend au siège le 15 mars d'une part pour assister à la première réunion de la cellule conjointe de gestion de crise entre le Gouvernement et TEPCO. Kan crée cette cellule pour résoudre les difficultés de communication avec l'exploitant. Il choisit de l'installer au siège de TEPCO et en explique la raison : "Même si on créait une telle instance, si celle-ci se trouvait à la résidence du Premier ministre ou un endroit assimilé, par rapport à l'organisation qui existait déjà, seule la forme aurait changé, mais pas le contenu. J'étais déjà le Président de la cellule de crise des accidents nucléaire et, à ce titre, j'avais déjà convoqué moult fois les responsables de TEPCO. Cela n'aurait rien changé. Comme je vous l'ai dit à plusieurs reprises, il y avait le Gouvernement, mais il y avait aussi le terrain et TEPCO. Et c'est cet endroit de la chaîne que je n'arrivais pas à saisir. Alors, à ma manière, j'avais réfléchi et pensé que c'était là qu'il fallait installer la nouvelle cellule. J'ai donc émis le souhait de l'installer chez TEPCO, les gens là-bas disent 'le siège'. J'ai demandé au PDG s'il était d'accord pour qu'on la mette au siège et il m'a répondu oui. Là-dessus, j'ai chargé le conseiller de prévoir les préparatifs pour une première réunion. Et c'est comme ça que j’y suis allé avec mes collaborateurs » (Kan, 2012). La cellule conjointe remplace donc la cellule de crise du Kantei et la cellule de crise de TEPCO. Elle est chargée d'assurer une intervention intégrée entre l'organisme public d'intervention et l'exploitant, et de veiller à ce que les informations soient communiquées en temps voulu à l'échelon national. Selon Kenichi Shimomura (secrétaire assistant au Kantei pour la communication publique), "le fait que le 
Gouvernement mette en place le quartier unifié dans une entreprise privée est exceptionnel pour un pays capitaliste et démocratique» (Kobayashi, 2019, p. 139). La première réunion de cette cellule, la seule à laquelle Kan assistera, a lieu le 15 mars à 4 h30 du matin. Kan se rend d'autre part au siège de TEPCO pour empêcher le prétendu « retrait général » du personnel de Fukushima Daiichi. L'audition du 9 août 2011 (I), dans laquelle Yoshida affirme que ses équipes et lui n'ont jamais voulu abandonner la centrale, évoque ce quiproquo.

52. Naoto Kan se rend au siège pour assister à la première réunion de la cellule conjointe et ne pense pas en effet se retrouver au milieu de tant de monde : "Quand je suis arrivé, j'ai découvert une salle avec un grand écran, tout comme au centre de gestion de crise de la résidence. Il y avait beaucoup de personnes qui s'affairaient là, avec des brassards. Moi, je m'attendais plutôt à une réunion en plus petit comité avec des personnalités de la direction. Mais c'était une vaste salle de conférence d'environ deux cents personnes » (Kan, 2012).

53. La centrale de Fukushima Daini.

54. Gôshi Hosono est un homme politique japonais né en 1971. Il est Conseiller spécial auprès du Premier ministre au moment de la crise nucléaire. Il est ensuite nommé Secrétaire d'État en charge de gestion de l'accident de Fukushima à partir de juin 2011, fonction qu'il cumule avec celle de ministre de l'Environnement de septembre 2011 à octobre 2012 dans le Gouvernement dirigé par Yoshihiko Noda (Premier ministre du 2 septembre 2011 au 26 décembre 2012). Depuis 2014, il est député à la Chambre des représentants.

55. Plusieurs éléments corroborent l'hypothèse que de l'hydrogène a migré du réacteur 3 au réacteur 4. En effet, rien ne semble indiquer que l'hydrogène qui a causé l'explosion soit originaire de la tranche 4 . En outre, les réacteurs 3 et 4 partagent des conduits d'aération. Il est donc possible que lors de l'éventage du réacteur 3 , de l'hydrogène se soit introduit dans la tranche 4 via les conduits d'aération. 


\section{Compte rendu de l'audition du 8 août 2011}

\section{Avertissement aux lecteurs}

Le texte qui suit est la traduction de la retranscription brute de l'enregistrement des séances d'audition. Les points suivants doivent être pris en considération :

- certaines interventions comportent des [...]. Il s'agit de passages où l'enregistrement n'est pas audible ;

- le nom de plusieurs acteurs a été dissimulé dans le texte d'origine, rendant parfois la lecture malaisée ;

- de même, l'audition du 9 août reproduit partiellement la bande son d'un enregistrement vidéo, sans indication des personnes qui prennent successivement la parole. Il est souvent éclairant de se reporter au résumé qu'en fait l'enquêteur Katô tout de suite après;

- enfin, les interventions ne sont pas dénuées d'incohérences, voire d'erreurs commises par les interlocuteurs. Celles-ci ont été respectées dans la traduction. Certaines sont signalées par des notes.

\section{Le « Rapport sur les dispositions pour la gestion des accidents au sein de la centrale de Fukushima Daiichi »}

Présenté en annexe des auditions des 8 et 9 août 2011, le « Rapport sur les dispositions pour la gestion des accidents au sein de la centrale de Fukushima Daiichi » est un texte rédigé en mai 2002 par TEPCO (non reproduit ici), qui décrit les nouvelles dispositions venant en renfort de celles préexistantes en cas d'accident.

3 Il s'agit, notamment, de modifications apportées aux installations nucléaires afin de proposer, par exemple, des moyens substitutifs d'injection en reliant les réseaux MUWC (Make-Up Water Condensate system) ou FP (Fire Protection system) au système RHR (Residual Heat Removal system), permettant ainsi d'atteindre le réacteur, ou de prévoir une ligne renforcée d'éventage pour aider au refroidissement de l'enceinte de confinement. 
4 En plus de ces travaux, la mise en place d'un organisme de soutien, apportant assistance aux agents de conduite en cas d'accident, est établie, avec une répartition des rôles et la désignation des responsables.

5 Enfin, le rapport signale la mise à disposition de manuels de gestion des crises et émet des recommandations quant à la formation du personnel à la gestion des accidents.

6 Tomoko Takesada

\section{Compte rendu de l'audition}

7 [À traiter avec la plus grande attention]

16 août 2011

Tsunemasa Katô

Membre du secrétariat de la Commission d'enquête sur l'accident des centrales nucléaires de Fukushima de la Tôkyô Electric Power Company (Investigation Committee on the Accident at the Fukushima Nuclear Power Stations of Tôkyô Electric Power Company).

Ci-dessous le compte rendu de l'audition des 8 et 9 août 2011, menée dans le cadre de l'enquête sur l'accident des centrales nucléaires de Fukushima de Tôkyô Electric Power Company.

1. Auditionné, date d'audition, lieu d'audition, enquêteurs.

1. Auditionné

Masao Yoshida, Directeur de la centrale nucléaire Fukushima Daiichi de Tôkyô Electric Power Company.

2. Dates d'audition

8 août 2011 de 10 h01 à 12 h02 (sans pause)

8 août 2011 de 13 h05 à 15 h00 (sans pause)

8 août 2011 de 15 h06 à $17 \mathrm{~h} 13$ (sans pause)

9 août 2011 de 9 h5 54 à 12 h00 (pause de $11 \mathrm{~h} 24$ à $11 \mathrm{~h} 35$ )

9 août 2011 de 12 h58 à $15 \mathrm{~h} 53$ (pause de $14 \mathrm{~h} 47$ à 14h59)

3. Lieu d'audition

Salle de réunion $B$

Foyer masculin de la Japan Football Association Academy de Fukushima ( $1^{\text {er }}$ étage)

J-Village

8-Utsukushimori Yamadaoka, Naraha, Futaba District, Fukushima

4. Enquêteurs

Tsunemasa Katô, Tetsu Chiba

5. Enregistrement par enregistreur numérique

- Oui ONon

2. Contenu de l'audition

Situation au moment de l'accident et réponses à l'accident

Voir le compte rendu

3. Mentions spéciales

Pas de mention spéciale. 
QUESTION : Nous allons reprendre les questions là où nous les avions laissées la dernière fois. Nous en étions arrivés, au pas de charge, au 15 mars. Nous allons donc continuer avec ce qui s'est passé après le 15.

Précédemment nous avions donc vu que le 15, vous aviez connu des incidents sur les tranches 2 et 4, et que le 16, vous aviez eu un incendie. Heureusement, ces différents feux se sont éteints spontanément.

Réponse : Oui.

$\mathbf{Q}$ : Vers les 16 et 17 mars, vous avez pris différentes décisions concernant les piscines de combustible.

Si on regarde dans la chronologie que l'unité de gestion des catastrophes nucléaires du Gouvernement a rendue publique, ce sont les forces d'autodéfense et le MPD (Metropolitan Police Department ${ }^{1}$ qui ont été chargés de l'arrosage à ce moment-là. " Et ceci, concernant plus spécifiquement la tranche $3 »$, peut-on lire au 17 mars.

Puis-je savoir, pour commencer, quel était l'ordre des priorités pour vous vers la date du 16 mars? II semblerait que, dans les transcriptions qui ont été faites par TEPCO des téléconférences à Kashiwazaki-Kariwa, tout ne soit pas tout à fait exact.

Dans la transcription, le 16 mars à 10h04, on lit donc que l'ordre des priorités pour le siège est "l'injection d'eau dans la piscine de combustibles de la tranche 4, en premier, le rétablissement des alimentations électriques externes, en deuxième, le déblaiement du terrain afin d'accueillir des camions générateurs en troisième, et, enfin, en quatrième lieu, l'injection d'eau dans les piscines de combustible des tranches 1 et 3 ». Est-ce que cela correspond à ce que vous avez en mémoire?

R : Oui, je pense que, grosso modo, c'était ça.

$\mathbf{Q}$ : Le rétablissement des alimentations électriques externes, qui est la deuxième priorité, et l'accueil des camions générateurs, en troisième position, concernent les tranches 1 à 3 , n'est-ce pas ? Pourquoi des camions générateurs?

$\mathbf{R}$ : Concernant les tranches 1,2 et 3 , nous étions toujours dans la situation où des camions de pompiers continuaient à injecter l'eau. Seulement, dans la mesure du possible, le siège voulait récupérer l'usage de toutes les installations susceptibles de fonctionner en rétablissant les alimentations externes. Et nous étions pressés. C'est pour ça que les travaux de rétablissement de l'électricité constituaient notre deuxième priorité. Mais ces travaux, nous ne pouvions pas les faire nous-mêmes, donc j'avais demandé au siège de s'en occuper. Simultanément ils réfléchissaient à tout ce qui était nécessaire, pour qu'une fois le courant rétabli, les pompes, par exemple, qui marchent avec ce réseau, soient opérationnelles. C'est le siège qui faisait ça en parallèle.

Personnellement, moi, j'étais beaucoup plus sceptique. Mais eux, à ce stade-là, pensaient encore qu'ils pourraient sauver la situation en faisant fonctionner des systèmes existants, comme le RHR. Donc, il fallait rétablir les sources externes d'électricité. Pour le cas où certains endroits resteraient hors de portée du réseau électrique externe, l'idée était de faire venir des camions générateurs, notre troisième priorité, pour alimenter en électricité et récupérer le fonctionnement de toutes les installations possibles. Ça, c'était un des grands axes du moment. Donc, plus que le retour spécifique des alimentations externes, c'était le rétablissement de l'électricité de n'importe quelle source qui était important.

Simultanément, concernant la tranche 4, il y avait toujours le problème du refroidissement des combustibles, étant donné que, comme je vous l'ai expliqué la dernière fois, cette piscine contenait des combustibles encore extrêmement chauds. 
Nous étions d'autant plus inquiets qu'à ce moment-là, nous pensions que les dégâts subis par la tranche 4 étaient peut-être dus à la détérioration de ces combustibles. Alors, ces deux sujets de préoccupation, quoique différents, se situaient au même niveau de priorité pour nous.

Q: Concernant les piscines de combustible, on voit que le plus urgent était le refroidissement de celle de la tranche 4 . Ensuite, au numéro 4 des priorités, il y a les piscines de combustible des tranches 1 et 3. II manque la tranche 2. Pourquoi ?

$\mathbf{R}$ : C'est parce que le bâtiment de la 2 n'avait pas été détruit. Si je résume, pour les tranches 1,3 et 4 , le haut des bâtiments était ouvert. Cela signifie qu'on pouvait injecter de l'eau de l'extérieur en profitant de ces ouvertures. Par contre, le bâtiment du réacteur 2 étant intact, il n'y avait pas moyen de lui injecter de l'eau de l'extérieur. Si on revient aux priorités et s'il s'agissait d'imaginer des solutions pour injecter de l'eau de l'extérieur, de facto, la tranche 2 n'était plus concernée. Pour elle, nous envisagions de lui injecter de l'eau en ressuscitant le circuit d'un des systèmes internes existants.

$\mathbf{Q}$ : Je crois que c'est ce que j'ai vu en visionnant les téléconférences. À un moment donné, M. $x x x x x^{2}$ propose d'injecter l'eau en utilisant le système FPC en passant par le bâtiment de traitement des déchets. II y a discussion autour de cette proposition entre Tôshiba ${ }^{3}$, d'autres fabricants et le siège. Effectivement, pour la tranche 2 , il fallait imaginer une solution passant par l'intérieur.

R : Voilà.

Q: Aviez-vous également pensé, de la même manière, à des solutions passant par l'intérieur pour les tranches 1, 3 et 4 ?

$\mathbf{R}$ : Oui, nous y avions réfléchi. Mais dès le départ, la radioactivité était très forte au niveau de ces circuits concernant la tranche 1. Ce qui fait qu'il n'y avait pas moyen de s'en approcher. Pour la tranche 3 , je ne me rappelle pas très bien, mais il me semble qu'il y avait aussi des problèmes de radioactivité. De toute manière, aussi bien pour la 1, la 3 que pour la 4 , il était très difficile de vérifier si ces différents circuits n'avaient pas été endommagés par les explosions.

Pour la tranche 2, puisque l'installation en elle-même n'avait pas subi d'explosion, il était assez légitime de penser qu'il y avait des chances pour que les différents réseaux soient restés intacts. De plus, à ce moment-là, la radioactivité n'était pas encore trop forte. Ça valait la peine d'essayer.

Q: En ce qui concerne les envois d'eau de l'extérieur, on voit, en effet, dans le rapport suivant l'article 15 portant la mention « 91 » à la main, envoyé le 17 mars à 7 h00, « début des préparatifs pour un arrosage au moyen de canons à eau haute pression embarqués pour le refroidissement de la piscine de combustibles de la tranche 3 \%. Le rapport précédent, celui portant la mention manuscrite « 90 », parle aussi du « début des préparatifs pour un arrosage de la partie supérieure du bâtiment réacteur 3 par hélicoptère ». On voit donc l'évocation d'une intervention par hélicoptère et canons à eau embarqués. Vous aviez sollicité pour ça les hélicoptères des forces d'autodéfense? Aviez-vous sollicité les organismes gouvernementaux?

R : Oui.

Q: : Ce genre d'ajustements avec le Gouvernement, c'était le siège qui s'en occupait?

$\mathbf{R}$ : Oui, c'était le siège ${ }^{4}$. Comme je vous l'ai dit tout à l'heure, quand l'injection d'eau était possible par l'intérieur, comme pour la tranche 2, nous réalisions nous-mêmes les circuits nécessaires. Mais pour ce qui était de l'arrosage par l'extérieur, nous 
n'étions pas du tout outillés. Du coup, on avait confié tout ce segment au siège. C'était à eux de réfléchir à ce qu'ils pouvaient faire de l'extérieur. On s'était réparti les tâches comme ça.

Q: Si on regarde le rapport portant la mention manuscrite « 89 », on lit "alors qu'on poursuivait la manœuvre d'injection d'eau dans le bâtiment réacteur 4 , observation d'une sorte de vapeur blanche s'échappant de la tranche 3. Arrêt des opérations en extérieur, retrait et mise à l'abri à l'intérieur ». La manœuvre d'injection dans le bâtiment réacteur 4 qui est évoquée ici, c'est celle dont on vient de parler?

$\mathbf{R}$ : Concernant la tranche 4 , nous, à la centrale, nous n'étions pas en état de pouvoir y toucher. Alors la manœuvre dont on parle ici doit être celle du siège.

Q: Il s'agit du 16 mars.

$\mathbf{R}$ : Ma mémoire est complètement floue concernant cette période.

$\mathbf{Q}$ : J'ai regardé la chronologie de Kashiwazaki et j'ai trouvé la mention suivante à la date du 16 mars à 10h28, dans la rubrique "siège ». "Est-il possible pour un hélicoptère de s'approcher avec de la vapeur s'échappant du $7 F-3$ ? $\rightarrow$ On peut penser que la manœuvre devienne délicate ». Puisque vous ne disposiez pas d'hélicoptère sur place, est-ce à dire que vous faisiez de votre côté des manœuvres en propre pour injecter l'eau, quand il s'est passé quelque chose?

R : Absolument pas. Non, il ne s'agit pas de ça. Nous étions bien incapables d'injecter de l'eau par nos propres moyens. De toute manière, la tranche 4 était dans un état tel que nous ne pouvions absolument rien y faire par nous-mêmes. C'est pour ça que nous avions sollicité l'aide extérieure. L'opération d'injection dont vous parlez ne peut pas concerner une de nos manœuvres à nous.

$\mathbf{Q}$ : Il y a une chose que je voudrais vérifier. Vous êtes préoccupé par l'état de la tranche 4. La température de sa piscine de combustibles est élevée. II faut donc la refroidir. Avant que vous n'ayez pu avoir les observations de l'hélicoptère, vous conjecturez que le niveau d'eau est déjà bas. Vous vous apprêtez à vous en occuper énergiquement. D'ailleurs, dans la liste des priorités dont nous avons parlé tout à l'heure, la tranche 4 est en première place. Or, le 17 mars, lorsque vous envoyez effectivement de l'eau, c'est ce qui est noté dans le rapport 91, vous écrivez que « cette opération se fait dans le but de refroidir la piscine de combustibles usés de la tranche 3 ». Comment êtes-vous passé de la tranche 4 à la tranche 3 ?

$\mathbf{R}:$ Je ne me rappelle plus très bien de l'heure, mais le 17 mars, dans la matinée, l'hélicoptère a pu voler. Pas un hélicoptère pour l'arrosage, mais un hélicoptère d'observation. Je crois bien que c'était un hélicoptère des forces d'autodéfense. Dans cet hélico se trouvait aussi un de nos employés, qui a tourné une vidéo. Et quand on a visionné le film, il nous a semblé qu'il y avait encore de l'eau dans la piscine de combustibles de la tranche 4 . En fait, on avait réussi à voir le niveau d'eau.

Techniquement, une piscine de combustibles se présente comme ceci. Il y a la piscine de combustibles ici, comme je vous l'ai déjà dit, et, par le côté, elle est reliée à la partie supérieure du réacteur. D'ordinaire, il y a l'enceinte de confinement là. Mais, dans le cas du réacteur 4 qui était en cours d'inspection, là, c'était ouvert, avec un filtre ici et, ici, c'était plein d'eau. La piscine était donc à peu près dans cet état. Ici, c'est ce qu'on appelle le bassin DS (Dryer Separator pit) ${ }^{5}$ et, ici aussi, c'était rempli d'eau. En fait, il y a une cloison là. Au début, on pensait que la piscine de combustibles usés, avec ses combustibles ici, allait voir son niveau d'eau baisser. Quand l'hélico a volé, notre homme a pu tourner une vidéo, par les brèches, puisque le bâtiment avait été détruit. $\mathrm{Et}$, en regardant cette vidéo, on a compris qu'il semblait y avoir encore suffisamment d'eau. Pourquoi? En temps normal, l'équilibre des pressions fait que 
l'eau qui se trouve ici ne peut pas circuler par là. Mais quand l'eau a commencé à baisser, l'équilibre des pressions de l'eau a dû se trouver rompu, permettant du coup à l'eau qui se trouvait de ce côté-ci de couler par-là. En fait, l'eau s'était renouvelée toute seule. Et donc, à la suite du survol de l'hélicoptère, on a su, tout à coup, qu'il y avait encore de l'eau dans la piscine du 4.

En fait, jusqu'à ce que je donne l'ordre de refroidir la tranche 3, j'étais encore en pleine discussion avec le siège pour savoir si on allait privilégier la 3 ou la 4 . Mais avec l'arrivée de ces nouvelles informations, l'urgence paraissait moindre pour la tranche 4 . Du coup, on a inversé les priorités et on a décidé de commencer par la tranche 3.

$\mathbf{Q}$ : Donc, le nouvel objectif a été la tranche 3, c'est ça ?

R: Oui.

Q: Pour la tranche 3, vous n'aviez aucun moyen de vérifier son niveau d'eau?

$\mathbf{R}$ : Non, ce n'était pas possible.

Q : Encore un point. Dans ce document qui porte le numéro « 94 » manuscrit, on voit écrit à la main «dans le but de refroidir la piscine de combustibles usés de la tranche 3, était prévu, comme en a été fait le rapport précédemment, l'arrosage au moyen de canons à eau haute pression, mais, à la suite de l'intervention du ministre à 8 h30, cette opération a été repoussée pour être effectuée après que l'arrosage par hélicoptère a été accompli. Prière de noter qu'avant l'arrosage par hélicoptère, sera effectué un vol de repérage par un hélicoptère de surveillance ». On vient de parler d'un vol de repérage à l'instant, s'agit-il du même? Ou bien, ont-ils recommencé un autre vol? Était-ce une fois que vous aviez décidé de privilégier la tranche 3 ?

$\mathbf{R}$ : Ça, c'est un point que je ne comprends pas bien moi-même. Nous, on se contentait d'écouter. On ne savait pas très bien ce qui se passait entre les forces d'autodéfense, notre siège et le Gouvernement. Ils nous annonçaient tout à coup de manière unilatérale qu'un camion chargé d'un canon à eau allait venir, ou qu'un hélicoptère allait voler. Tout ça était piloté par le siège. À la limite, dans ces documents, je me suis contenté de noter ce qu'ils disaient. Savoir pourquoi il fallait un vol de repérage avant arrosage, on n'en savait rien. Je suppose qu'ils l'ont fait parce que les forces d'autodéfense y tenaient.

$\mathbf{Q}:$ : Au début, l'arrosage devait se faire avec des canons à eau haute pression embarqués sur des camions, mais, en cours de route, le programme a changé et on a décidé de commencer avec l'hélicoptère. D'ailleurs, on voit sur le document 95, qui suit, que l'arrosage par hélicoptère a débuté à 9 h48.

$\mathbf{R}:$ On aurait dit de la pisse de cigale.

Q: Pourquoi avoir changé de programme? Y a-t-il eu des problèmes d'accessibilité par la route qui auraient retardé l'arrivée des camions, par exemple?

R : Concrètement, je ne m'en souviens presque pas. Là, c'était le siège qui avait l'initiative. Nous n'étions pas en mesure de discuter leurs décisions. Notre volonté, à nous, n'était absolument pas reflétée dans ces actions.

$\mathbf{Q}:$ Si je comprends bien, le siège vous informait des changements et, vous, vous n'aviez qu'à acquiescer?

$\mathbf{R}$ : Oui, c'est ça. Je me suis juste contenté de noter tout ça. Bien sûr, j'ai signé ici «Masao Yoshida ». Mais ce sont des choses que je n'avais même pas envie d'écrire.

Q: Après le 95, nous en arrivons au 96 et, effectivement, il semble qu'il y ait eu des arrosages par hélicoptère dans la matinée. II en reste des traces écrites au Gouvernement. 
D'après, donc, le rapport 96, on effectue l'arrosage avec les camions de pompiers le soir à 19h05. II me semble que, d'après le programme qui avait été arrêté au départ, toutes les opérations d'arrosage devaient se faire de manière regroupée pendant qu'il y avait du jour, c'est-à-dire dans la matinée et en début d'après-midi. À voir les archives, on dirait que toutes les opérations terrestres se sont trouvées décalées vers la soirée. Qu'en était-il ?

$\mathbf{R}$ : Là, ce dont je me souviens très clairement, c'est qu'aussi bien ces messieurs des forces d'autodéfense que ceux des forces de l'ordre de la police ont pris très largement leur temps pour venir. Ils arrivaient au J-Village, ensuite allaient au $2 \mathrm{~F}$ où ils se concertaient pour décider de la marche à suivre. C'est là où j'ai dû dépêcher des hommes pour leur expliquer que la radioactivité était forte, comment ils devaient en tenir compte, etc.

$\mathbf{Q}$ : Vous avez envoyé quelqu'un jusqu'au $2 \mathrm{~F}^{6}$ ?

R : Oui, jusqu'au 2F. Ensuite, ils prenaient encore du retard pour partir. Nous, nous les attendions en trépignant d'impatience. Mais, pour être franc, ils n'arrivaient pas. Je me rappelle très bien de ces circonstances.

$\mathbf{Q}$ : Vous, de votre côté, vous poursuiviez les travaux pour récupérer le réseau d'électricité du côté des réacteurs 3 et 2, n'est-ce pas?

$\mathbf{R}$ : Oui, nous y travaillions.

$\mathbf{Q}$ : Quand on arrose les installations, il faut temporairement se mettre à l'abri ?

R : Oui.

$\mathbf{Q}$ : Alors, comment vous coordonniez-vous là-dessus?

$\mathbf{R}:$ C'était horriblement casse-pied.

$\mathbf{Q}:$ Qui s'occupait de ça?

R : Moi.

$\mathbf{Q}:$ Vous faisiez ça en coordination avec le siège?

$\mathbf{R}$ : Encore une fois, c'est une histoire compliquée. Ceux qui, au siège, s'occupent habituellement de restaurer l'électricité pour nous, ce sont des gens complètement distincts des groupes de production nucléaire. Ce sont plutôt des gens qui sont proches de la distribution. Il y en a qui viennent de notre département "construction", qui s'occupent des câbles, par exemple, et puis d'autres qui s'occupent plus de la partie «distribution d'électricité ». Là, je ne parle pas des circuits qui sont à l'intérieur de la centrale. Ce sont donc eux qui s'occupaient de ce qui se trouvait à l'extérieur de la centrale et de la connexion avec ce réseau extérieur. Eux fonctionnaient en suivant leur propre programme. De l'autre côté, il y avait les différentes demandes que nous avions formulées, par le truchement de M. Kaieda, aux forces d'autodéfense, aux pompiers. Comme il y avait aussi les forces de l'ordre de la police, il y avait au moins trois organismes qui intervenaient de l'extérieur, avec chacun un fonctionnement complexe.

Nous, de notre côté, on avait donc ces collègues de TEPCO qui travaillaient d'arrachepied pour rétablir l'électricité avec un programme daté et précis. Seulement, ces équipes, le siège nous les envoyait sans aucune forme de régulation. Il y avait de plus en plus de monde qui faisait irruption chez nous. Eux ne voulaient qu'une chose, faire avancer les travaux. Mais s'il devait y avoir arrosage, il fallait les interrompre. Je me suis retrouvé à devoir faire moi-même cette régulation sur le terrain. C'était horriblement casse-pied. 
Q : Mais, pour tout ça, vous n'étiez pas vous-même en contact direct avec les forces d'autodéfense, n'est-ce pas?

$\mathbf{R}$ : Effectivement. Savoir précisément quel groupe des forces d'autodéfense allait venir, c'est le genre de choses dont discutait le siège. C'est donc le siège qui nous envoyait les informations. Mais, concernant les arrosages, il était compliqué de décider quand intervenir, puisqu'il y avait les autres travaux en cours, à terre. Il fallait mettre les gens à l'abri avant d'arroser. Surtout pour les travaux d'électricité. Vous imaginez ce qui aurait pu arriver s'ils se faisaient arroser? Il fallait donc soit les cantonner dans des lieux suffisamment éloignés ou, s'ils devaient se trouver vraiment à proximité des points arrosés, arrêter les travaux temporairement. Et ce genre de décisions ne pouvait être pris que sur le terrain. C'est comme ça que je me suis retrouvé à réguler moi-même ces choses.

Q: Finalement, si on regarde tout ça, en fait, c'est vous, sur le terrain, qui avez dû vous adapter au coup par coup aux différents changements qui intervenaient sans cesse, au retard que prenait le Gouvernement sur les projets initiaux, à l'inversion des interventions de I'hélicoptère et des canons à eau, etc.

R : Oui.

$\mathbf{Q}$ : Vous est-il arrivé concrètement de rencontrer les responsables de ces unités des forces d'autodéfense ou de police qui venaient sur le site, pour que vous vous concertiez au préalable?

$\mathbf{R}:$ Non, jamais.

$\mathbf{Q}:$ :lls étaient comment? Ils arrivaient et ensuite?

$\mathbf{R}$ : Déjà, chaque organisme se comportait différemment. Que ce soit les forces d'autodéfense, les pompiers ou la police, tout était différent. La chaîne de commandement était différente. Ce qui était compliqué c'est qu'à la cellule conjointe $^{7}$, autour de M. Kaieda, il y avait les gros bonnets des forces d'autodéfense, des pompiers, tout ça. Et ils discutaient avec le siège de ci et de ça. Quand leurs hommes arrivaient sur le site, j'envoyais quelqu'un les accueillir, mais les chefs d'unités ne venaient presque jamais à la cellule de crise. En fait, ils recevaient les explications sur l'état du terrain, etc., au J-Village ou ailleurs. J'y envoyais des personnes de chez nous pour leur expliquer la situation. Les forces d'autodéfense discutaient avec leurs supérieurs. Ils disaient " on part à telle heure ", mais, dans les faits, ils ne partaient pas ou rebroussaient chemin en cours de route. À se demander ce qu'ils foutaient.

$\mathbf{Q}:$ Et, à votre avis, pourquoi?

$\mathbf{R}:$ Je crois que personne n'a envie de s'aventurer dans des zones où la radioactivité est forte. Surtout les pompiers. Ils ne venaient pas du tout.

$\mathbf{Q}$ : Ils se rassemblaient donc au J-Village, organisaient leur intervention et chacun des trois organismes venait sur le site pour les opérations d'arrosage.

R : Oui.

$\mathbf{Q}$ : Les forces d'autodéfense ont utilisé l'hélicoptère et les canons à eau haute pression. La police, des canons à haute pression, aussi. Les pompiers ont injecté l'eau. Parmi ces 
différents moyens, y a-t-il eu des méthodes qui se sont avérées meilleures ? D'autres, moins efficaces?

$\mathbf{R}$ : Tout d'abord, la police. Ce sont ceux qui sont venus les premiers. Mais ils n'ont pas été très utiles. De plus, ils ne sont venus qu'une seule fois et sont repartis. Déjà qu'ils avaient fait plein de difficultés pour venir... Bref, ça n'a pas eu d'effet.

$\mathbf{Q}:$ Quand vous dites que ça n'a pas eu d'effet, qu'est-ce que vous voulez dire?

$\mathbf{R}$ : Cela veut dire que l'eau n'est pas entrée.

$\mathbf{Q}:$ C'était un problème du camion à canon?

$\mathbf{R}$ : Je pense que c'était un problème de hauteur manométrique ${ }^{8}$. L'eau n'arrivait pas à atteindre la piscine.

$\mathbf{Q}$ : Quelle était la source de l'alimentation d'eau?

$\mathbf{R}$ : Il y a eu plusieurs sources, mais, au début, comme il s'agissait de la tranche 3 , on avait rempli le réservoir de lutte contre l'incendie des tranches 2 et 3 . Ils prenaient l'eau là pour faire fonctionner leur canon haute pression.

Les forces d'autodéfense avaient des camions-citernes. Je ne sais pas si c'était des citernes de 20 ou $40 \mathrm{t}$. Mais ils allaient remplir ces citernes et arrosaient. Ils étaient à six ou sept camions et arrosaient à tour de rôle. Quand le camion était vide, il y en avait un qui prenait le relais, pendant que l'autre allait puiser l'eau et arrosait à nouveau.

$\mathbf{Q}$ : Où allaient-ils chercher l'eau ? Ils allaient en dehors du site?

$\mathbf{R}$ : Oui. Il me semble qu'ils puisaient dans le Kumagawa ${ }^{9}$. Je dois reconnaître qu'ils avaient réfléchi eux-mêmes à une solution pour cette question d'alimentation.

$\mathbf{Q}$ : Quelle était l'efficacité de leurs jets?

$\mathbf{R}$ : Pour vous dire la vérité, rien de tout ça n'a été significatif. On était sur des volumes de 10 ou 20 t. Comparé à la surface totale de la piscine, même si on avait réussi à tout mettre, ça ne représentait rien.

En plus, ils introduisaient le tuyau ici. Ce n'est pas comme s'ils s'étaient avancés jusque-là pour positionner le tuyau là. Ils mettaient le tuyau ici et faisaient «pschitt». On avait l'impression qu'il y avait une bonne partie de l'eau qui coulait par là ${ }^{10}$. On ne sait même pas combien arrivait vraiment à destination.

C'était surtout vrai pour les pompiers. Au début, ça allait bien. Et puis, au fur et à mesure, le bout du tuyau tombait. On le leur faisait remarquer, mais ils n'allaient pas rectifier.

$\mathbf{Q}:$ Vous parlez des pompiers?

$\mathbf{R}$ : Oui, les pompiers. Je vous le dis tout net. Les pompiers, ces héros...

$\mathbf{Q}:$ Donc, les jets des pompiers n'étaient pas très efficaces.

$\mathbf{R}$ : Pas du tout efficaces. Pour résumer, les hélicoptères n'étaient pas efficaces, les forces d'autodéfense, je suis triste de le dire, n'étaient pas non plus efficaces à cause du peu d'eau qu'ils envoyaient, les pompiers non plus n'étaient pas efficaces et la police, dès le début, n'était pas efficace.

$\mathbf{Q}$ : Le but était d'envoyer de l'eau avec des canons à haute pression en passant par les parties détruites du bâtiment, n'est-ce pas ? Est-ce que, à votre avis, si vous aviez disposé 
de suffisamment d'eau et de pression, et que vous aviez réussi à viser juste, cela aurait eu un certain effet?

$\mathbf{R}$ : Je pensais que ça en aurait. Je le pensais, et puis, c'est surtout que nous n'avions que ça, à ce moment-là. Dans l'idéal, j'aurais voulu qu'on puisse introduire le bout du tuyau franchement à l'intérieur du bâtiment, c'est le système que nous avons eu plus tard, et arroser d'importance par le haut. On n'avait pas suffisamment de hauteur manométrique, l'eau ne montait pas suffisamment, mais j'ai pensé que c'était quand même mieux que rien.

$\mathbf{Q}:$ Par la suite, vous avez eu les « girafes $»^{11}$ et les « éléphants $»^{12}$.

R : Oui.

$\mathbf{Q}:$ Qu'en pensez-vous?

$\mathbf{R}$ : Eux sont bien. Avec leur arrivée, on a enfin réussi à injecter correctement l'eau. C'est ça que je voulais dire tout à l'heure. L'extrémité du tuyau est positionnée à côté de la piscine et on introduit l'eau de cette manière, ce qui fait qu'il n'y a presque pas de perte et que toute l'eau arrive à destination. Mais ça, ça n'a été possible qu'après l'arrivée de la « girafe ». Ce qu'on a fait avant, c'était du bricolage à l'aveuglette, du n'importe quoi.

Q: Que pouvez-vous nous dire sur la manière dont vous êtes passé de cette solution bricolée à la « girafe » ? C'est le siège de TEPCO qui vous en a parlé ?

$\mathbf{R}$ : Même si l'opération "pschitt-pschitt» avait été efficace, il aurait fallu la poursuivre longtemps. C'était très compliqué. Alors j'avais demandé au siège, bien en amont, de réfléchir à une solution pour une injection continue d'eau. C'est là que le siège a commencé à parler d'utiliser une pompe à béton. Ils ont donc essayé de mettre ça sur pied pendant qu'on faisait nos opérations «pschitt-pschitt». Ils ont réussi à mettre la main sur une pompe à béton et ils ont monté une équipe " girafe ». Les hommes de l'équipe ont appris à manipuler l'engin. Parce que les opérateurs de l'engin ne voulaient pas venir, à cause de la radioactivité. Ils voulaient bien nous prêter la machine, mais ils ne voulaient pas venir. Et quand on a essayé la pompe à béton, on a enfin réussi à mettre de l'eau à peu près comme on voulait. Alors, on s'est dit qu'on allait continuer avec ce système. Après, on s'est mis en quête de plusieurs engins du genre. Grâce à quoi, on s'est débrouillé sans faire appel ni aux forces d'autodéfense ni aux pompiers.

Q: Excusez-moi d'insister, mais quand vous dites que vous avez envoyé de l'eau à l'aveuglette au début, vous l'avez fait à partir d'où?

$\mathbf{R}$ : D'ici. Entre les tranches 2 et 3 . Là, c'était un endroit où la radioactivité était particulièrement forte. Il y avait plein de gravats à $300 \mathrm{mSv} / \mathrm{h}$. Les véhicules arrivaient de l'entrée ouest, passaient par là, descendaient ce chemin, entraient ici, dirigeaient l'extrémité des tuyaux par ici. La piscine se trouvait par ici.

$\mathbf{Q}$ : Du côté de la tranche 4 ?

$\mathbf{R}$ : Oui, du côté de la tranche 4. Il y avait tellement de gravats qu'on ne pouvait accéder que par ici. On en avait enlevé le plus possible, pour dégager provisoirement un accès pour les véhicules. Mais il y avait aussi plusieurs voitures abandonnées qui ont beaucoup gêné les opérations. On faisait avancer les engins jusque-là où ils pouvaient et puis, on faisait entrer l'eau d'ici à ici. 
$\mathbf{Q}:$ Et les « girafes»?

$\mathbf{R}$ : Pour la tranche 3, les « girafes » entraient aussi dans cet espace.

$\mathbf{Q}$ : Là, entre les tranches 3 et 4 ?

$\mathbf{R}$ : Ça dépendait des moments. C'est qu'il fallait enlever les gravats. On les enlevait au fur et à mesure pour ménager un accès minimum aux « girafes ».

$\mathbf{Q}$ : Après le 16 mars, vous avait-on envoyé des engins spécifiques, des bulldozers et des choses comme ça, pour le dégagement des gravats?

$\mathbf{R}$ : On disposait déjà sur place de plusieurs tractopelles, qu'on possédait en propre. En plus, Hazama Corporation ${ }^{13}$ nous en a apportés de je ne sais où. Tout au début, pour ces travaux, c'étaient des hommes de chez Hazama qui s'étaient dévoués. Alors que la radioactivité était vraiment forte, ils se sont attelés à l'enlèvement des gravats avec beaucoup d'énergie. Vous n'avez qu'à le demander à « l'ingénierie civile ».

$\mathbf{Q}$ : Et pourquoi Hazama Corporation?

$\mathbf{R}$ : Je ne sais pas trop. La radioactivité était forte, mais les hommes de Hazama sont quand même venus.

$\mathbf{Q}:$ Et ils ont travaillé au dégagement de cette zone?

$\mathbf{R}$ : Oui. Ils ont aussi travaillé à la réfection de la route qui allait vers le réacteur 6 , qui s'était affaissée. Ce sont vraiment les seuls qui ont bien voulu venir nous aider dès le départ pour la réfection de l'infrastructure. Pourquoi était-ce Hazama Corporation? Je n'en sais rien. Tout ce que je sais, c'est que dans les faits c'étaient ces hommes-là qui étaient au travail. Je suppose que parmi toutes les entreprises contactées, c'était celle qui avait répondu le plus favorablement.

$\mathbf{Q}$ : D'accord. Mais Hazama Corporation, c'est une entreprise privée?

R : Oui.

Q: Quand vous devez échanger avec le Gouvernement ou les administrations, c'est toujours le siège qui sert de guichet?

$\mathbf{R}$ : Pour les commandes, c'est le groupe "matériel ». C'est du domaine du groupe " matériel ", chez nous. Quand il y a des travaux à commander, on le fait toujours en passant par ce groupe. Mais, par exemple dans ce cas précis, si Hazama Corporation a accepté de nous aider, c'est parce qu'on avait déjà des liens de travail avec eux. Avec notre groupe "génie civil» ou le département «construction" du siège. Le département "construction" chez nous est un véritable nid d'entrepreneurs de travaux publics. Quand il y a des travaux, notre département "construction» contacte toutes sortes de constructeurs et lance l'appel d'offre. Après examen, c'est le groupe «matériel » qui va s'occuper des précontrats, etc. En tant que directeur de centrale, je ne m'occupe pas de savoir qui on va préférer. Je laisse la décision à ces équipes.

$\mathbf{Q}$ : Dans le genre "gros travaux», je crois que c'était vers le 19 mars, vous avez fait faire des trous dans le bâtiment du réacteur 5, comme mesure préventive contre une éventuelle explosion d'hydrogène, n'est-ce pas?

R : Oui.

$\mathbf{Q}$ : Si je regarde les documents, il est mentionné que le 19 mars, vous avez "achevé de réaliser des trous en trois endroits du bâtiment réacteur 5 en prévention de la stagnation des gaz 
d'hydrogène ». Ce sont des travaux que vous avez effectués vous-mêmes ou vous aviez fait appel à des entreprises extérieures?

R : Des entreprises extérieures. Bien entendu, l'appel d'offre est passé par le groupe « construction », il me semble. Je ne m'en souviens pas.

$\mathbf{Q}$ : Vous avez effectué la même opération sur la tranche 6 ?

R : Oui, on l'a faite sur la 6 aussi.

$\mathbf{Q}$ : Et pas sur les tranches 1, 2, 3 et 4, parce que les bâtiments étaient déjà troués. C'est bien ça?

R : Oui.

$\mathbf{Q}$ : Donc, vous procédez à ces opérations. En plus, comme vous l'avez évoqué tout à l'heure, vous travaillez à la réhabilitation des sources électriques. C'est le groupe "réhabilitation » de la centrale qui en était chargé, n'est-ce pas?

R : Oui.

$\mathbf{Q}$ : On en a un peu parlé tout à l'heure. Le 16 mars un peu après 10 h00 du matin, on constate que de la fumée s'échappe de la tranche 3. Concernant la tranche 3, il y a eu explosion le 14 mars vers 11 h01 et il s'est donc passé environ deux jours depuis, quand vous observez cette fumée. Quelle est la cause qui vous est venue à l'esprit, quand vous avez reçu cette information?

$\mathbf{R}$ : Je n'en avais aucune idée.

$\mathbf{Q}:$ Vous n'en aviez aucune idée?

$\mathbf{R}$ : Vous savez, à cette époque, il faisait encore froid. J'ai donc pensé, en premier lieu, que ce qu'on voyait était de la vapeur qui s'échappait en nuage blanc du réacteur, voire de la piscine de combustibles usés. S'il y a des fuites d'un réacteur, de la vapeur fuit aussi de là. J'ai pensé que ce qui s'échappait formait un nuage blanc à cause du froid.

$\mathbf{Q}$ : Si je regarde la chronologie de Kashiwazaki, ils ont noté "possibilité que l'enceinte de confinement présente des fissures et qu'il y ait des fuites vers le bâtiment ». II y a donc de la vapeur qui s'échappe. Si elle s'échappe de l'enceinte de confinement, elle est hautement radioactive. Dans ce cas-là, pensant à cette possibilité, vous ordonnez un retrait temporaire du terrain?

R : Oui, c'est ça.

$\mathbf{Q}$ : Ce qui veut dire que, durant cette période-là, quand il y avait des phénomènes de ce genre, vous faisiez évacuer temporairement les gens qui travaillaient sur le terrain, c'est ça?

$\mathbf{R}:$ Oui, je le faisais.

$\mathbf{Q}$ : Et donc, vous attendiez un moment et vous repreniez les travaux. Tout ça s'est répété plusieurs fois.

R : Oui.

$\mathbf{Q}$ : Vous n'étiez donc pas en mesure de poursuivre durablement ces travaux, n'est-ce pas?

$\mathbf{R}:$ Non, pas du tout.

$\mathbf{Q}$ : C'est à partir de quel moment que vous êtes sortis du cycle « retrait temporaire/attente/ reprise des travaux » et que vous avez eu l'impression que vous pouviez poursuivre les travaux un peu tranquilles sans être interrompus?

$\mathbf{R}$ : Vers la fin mars. À peu près deux semaines après, il me semble. Avant, ce n'était pas possible. 
Q: Dans les différentes coopérations avec le Gouvernement, etc., je remarque qu'il y a eu un navire de l'armée américaine qui est venu apporter de l'eau. Vous en souvenez-vous?

R: Oui.

$\mathbf{Q}:$ :ll est venu vers la fin mars, c'est ça?

$\mathbf{R}$ : Oui. Je ne me rappelle pas si c'était fin mars ou début avril, mais c'était effectivement dans ces eaux-là.

Q: Quelles sont les circonstances qui ont justifié cette intervention?

$\mathbf{R}$ : Depuis le début, nous manquions d'eau. Tout au début, nous manquions d'eau à injecter. C'est d'ailleurs pour ça que nous avons injecté de l'eau de mer. Donc, nous n'avions pas d'eau. Au début, nous avons mis de l'eau de mer, mais ensuite nous avons changé pour de l'eau douce. Cette eau, on la prenait au réservoir d'eau filtrée. Mais en fait, elle venait du barrage de Sakashita ${ }^{14}$. Une fois arrivée du barrage, elle était stockée dans un premier temps dans un grand bassin qui se trouvait avant le réservoir d'eau filtrée. On prenait donc l'eau là et on utilisait toujours le même circuit que celui utilisé avec l'eau de mer, pour l'injection proprement dite. On avait juste remplacé l'eau de mer par de l'eau douce. Le problème, c'est qu'on ne savait pas quelles étaient les réserves du barrage de Sakashita. Dans notre raisonnement, de toute manière, il fallait disposer d'autant d'eau qu'on pouvait. On avait donc demandé qu'on nous fournisse toute l'eau possible. Cette demande est partie du siège jusqu'à l'armée américaine via la cellule interministérielle. L'armée disposait de barges et a proposé de transporter de l'eau par ce moyen.

$\mathbf{Q}$ : Avez-vous reçu directement des coups de fil de l'armée américaine à la cellule de crise de la centrale?

$\mathbf{R}$ : Oui, le fameux coup de fil de l'armée américaine. Ce n'était pas l'armée américaine. Enfin, je ne sais pas si c'était l'armée américaine. Ce n'était pas à propos des barges, mais des canons à haute pression. Quand on a commencé l'opération "pschitt ", ils nous ont dit qu'ils possédaient un canon à haute pression et ils nous ont proposé de nous l'envoyer. En fait, vous vous rappelez qu'on cherchait désespérément des camions de pompiers pour l'injection, au début? C'est dans ce cadre-là qu'on les avait d'abord contactés.

$\mathbf{Q}:$ L'injection dont vous parlez, c'est celle qui emprunte le circuit de lutte contre l'incendie?

$\mathbf{R}$ : Non. C'est le circuit pour injecter dans le réacteur, pour injecter dans la cuve.

$\mathbf{Q}:$ : Ah, là où vous utilisiez les camions de pompiers?

$\mathbf{R}$ : Oui. On ne savait pas à quel moment ça allait exploser, alors j'avais demandé qu'on nous envoie des camions de tout le pays. Il y en a bien eu trente. Et parmi eux, il y avait effectivement un canon à haute pression qui venait de l'armée américaine.

$\mathbf{Q}:$ Alors, il est venu?

$\mathbf{R}$ : Oui, il est venu. Il y en avait même eu un autre. Il y en a eu deux au total. On les leur a rendus maintenant. En fait, on ne pouvait pas les utiliser pour l'injection dans le réacteur.

Q: Qu'est-ce que vous voulez dire?

$\mathbf{R}$ : Pour faire court, les joints répondaient à des standards différents entre le standard japonais et le standard américain et on n'a pas pu les utiliser parce qu'on n'a pas eu le temps de rectifier ça. Mais on les avait cédés aux forces d'autodéfense et j'ai entendu dire que, plus tard, au moment de l'opération "pschitt-pschitt», ces 
canons à eau américains ont servi aussi. C'est juste de l'ouï-dire, parce que je ne les ai pas vus en action moi-même.

$\mathbf{Q}:$ Ça s'est fait au moment de l'injection?

$\mathbf{R}$ : Oui. J'ai entendu dire qu'il y avait un de ces camions qui avait servi pour l'injection dans la piscine de combustibles usés. Enfin, je n'en sais trop rien.

$\mathbf{Q}$ : Concernant l'injection d'eau dans le réacteur à l'aide des camions de pompiers, justement. Vers le 16 mars, vous la faisiez en alignant trois camions au niveau du quai et en établissant, de là, trois circuits distincts vers les réacteurs 1, 2 et 3. C'est un système que vous avez utilisé jusqu'à quand? Vous avez tout le temps utilisé des camions de pompiers?

$\mathbf{R}$ : Ma mémoire n'est pas très claire à ce propos. Je suis sûr qu'il y a des documents concernant cette période quelque part. Il faudra que vous alliez vérifier. D'après moi, jusqu'à fin mars, on a essentiellement exploité les camions de pompiers. À partir de là, on a installé des pompes provisoires, et quand on est passé à l'eau douce, on avait bien l'intention d'employer les pompes. Tout ça s'est fait simultanément. Comme c'était le siège qui s'en occupait, moi, sur le terrain, je n'en ai pas vraiment souvenir.

$\mathbf{Q}$ : Donc, d'une première solution où vous pompiez directement l'eau de mer à l'aide des camions de pompiers, eau de mer que vous envoyiez dans le réacteur via le bâtiment turbine, vous êtes passés à la solution « eau douce via le réservoir à eau filtrée ». Jusqu'au passage à l'eau douce, vous faisiez appel aux camions de pompiers, c'est bien ça ?

R : Oui.

$\mathbf{Q}:$ Y a-t-il une raison qui a motivé votre décision de passer de l'eau de mer à l'eau douce ?

$\mathbf{R}$ : Il faut avouer que l'eau salée n'est vraiment pas recommandée si on pense à la corrosion du métal. Nous avons toujours pensé que s'il y avait suffisamment d'eau douce, il fallait revenir le plus vite possible à une injection à l'eau douce. Bien sûr, ce sont des réacteurs qu'on ne réutilisera jamais, mais même dans ce cas, il fallait penser à la corrosion.

$\mathbf{Q}$ : Vous voulez dire que vous ne pouviez pas continuer indéfiniment à l'eau salée.

$\mathbf{R}:$ Non.

$\mathbf{Q}$ : Aujourd'hui, vous utilisez de l'eau douce?

R : Oui. Nous sommes complètement à l'eau douce.

$\mathbf{Q}$ : Vous dites qu'actuellement l'injection dans le réacteur se fait uniquement à l'eau douce. Concernant cette injection, il y aura demain un autre enquêteur de la Commission qui viendra vous poser des questions à propos du traitement des eaux contaminées, mais je voudrais déjà savoir ce que vous pensiez quant à ces eaux contaminées, les points auxquels vous avez particulièrement prêté attention dans cette injection, avez-vous pensé qu'il fallait restreindre, par exemple, le volume d'eau injectée ?

$\mathbf{R}:$ Il est certain que si on veut refroidir un réacteur, il faut lui mettre un maximum d'eau. Mais si on pense au problème du traitement des eaux usées, il faut réduire ce volume au minimum. Donc, aujourd'hui, nous nous restreignons et mettons le minimum requis pour refroidir le réacteur. Le contrôle du volume d'eau utilisé a une grande importance.

Q : Quand vous dites "le contrôle du volume d'eau utilisé », vous voulez dire que quelqu'un décide des limites en disant, par exemple, «x tonnes par jour » ou « $x$ tonnes à tel moment de la journée»?

R : Oui. 
$\mathbf{Q}$ : Et qui est cette personne?

$\mathbf{R}$ : À la base, la chaleur résiduelle produite par le combustible présent peut être calculée. À l'heure actuelle, c'est le groupe "technique» du siège qui calcule le minimum d'eau nécessaire en fonction de la chaleur résiduelle et de l'évaporation. On $\mathrm{y}$ ajoute une petite marge, car il peut toujours y avoir un peu de perte. Sur le terrain, nous envoyons un petit peu moins d'eau que prévu, et si la température ne monte pas, on maintient à ce débit-là. Cela veut dire que le siège et le terrain travaillent en concertation.

$\mathbf{Q}$ : Je vais complètement changer de sujet maintenant. Je voudrais que nous parlions de l'organisation de la gestion des accidents. II y a là le "Rapport sur les dispositions pour la gestion des accidents». C'est un document qui date de 2002 et, tout à la fin, il y a une description du dispositif. En cas d'accident, il y a autour des pilotes toute une organisation de soutien qui est composée, notamment, de la cellule de crise, du groupe «information», du groupe «technique», du groupe «sûreté », du groupe « réhabilitation » et du groupe «production».

R : Oui.

$\mathbf{Q}$ : Ce texte a été rédigé pour aider à la gestion des accidents et fait intervenir plusieurs groupes de soutien. Le document est daté de mai 2002. Est-ce à dire qu'avant cette date, ce genre de document n'existait pas?

$\mathbf{R}$ : Disons que c'était la première fois qu'on parlait nommément d'» organisation pour la gestion d'accidents ». Mais vous vous doutez bien que nous nous étions déjà organisés avant et qu'on savait gérer les incidents. Fondamentalement, ça n'a pas changé grand-chose. De tout temps, en cas d'incident, on s'est organisé pour aider les pilotes en les déchargeant des appréciations techniques. C'est la base. Alors, on ne peut pas dire que ce document ait beaucoup changé notre gestion des accidents. Effectivement, avant, il n'y avait pas de groupe «information" bien défini. Mais l'organisation en différents groupes existait déjà et il est logique qu'ils apparaissent dans cet organigramme. Je pense que ce document a été fait à la suite la promulgation des articles 10 et 15 de la « loi spéciale sur la préparation aux situations d'urgence en cas de catastrophe nucléaire ». On avait besoin de clarifier la situation, c'est tout. De toute manière, on a toujours connu une organisation où toute la centrale était là pour soutenir ceux de la production.

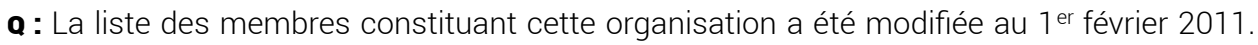
C'est sur la base de cette organisation que vous avez réagi face au tremblement de terre et au tsunami, quand vous vous êtes retrouvés en situation de SBO (Station Blackout) ${ }^{15}$, n'estce pas?

R : Oui, c'est ça.

$\mathbf{Q}:$ C'était bien cette organisation des groupes?

R : Oui.

Q: Je pense que, dans le cas actuel, vous vous êtes retrouvés dans des conditions dépassant complètement ce qu'on pouvait prévoir en matière de gestion de crise, mais y at-il eu, à votre avis, des dysfonctionnements dus à l'organisation, des choses qui n'ont pas marché, ou de nouveaux groupes dont vous auriez ressenti le besoin?

$\mathbf{R}$ : Je pense que l'organisation de base est correcte. Mais le gros problème, c'est le nombre de personnes disponibles. C'est plus une question de nombre de personnes que d'organisation à proprement parler. Par exemple, le groupe "réhabilitation ». C'est déjà lui qui était en première ligne quand on a perdu les alimentations 
électriques. Ensuite, c'est aussi lui qui s'est occupé de réhabiliter les instruments de mesure. Il y a beaucoup d'hommes qui ont été irradiés parmi ceux qui se sont occupés des instruments de mesure. Et là, c'est évident, ils n'étaient absolument pas assez nombreux.

Q: À la base, le groupe «réhabilitation» est constitué des membres des deux départements de maintien de la sûreté, n'est-ce pas?

R : Oui.

$\mathbf{Q}$ : Les gens du maintien de la sûreté sont, en fait, beaucoup plus nombreux que ce qui est écrit ici?

$\mathbf{R}$ : En février, à la centrale Daiichi, nous étions en tout environ mille quatre-vingts. Le contingent prévu en cas d'accident étant de quatre cents et quelques dizaines de personnes, cela fait moins de la moitié. Et, évidemment, la totalité du personnel du maintien de la sûreté ne fait pas partie de ce contingent.

$\mathbf{Q}$ : Justement, les personnes qui ne faisaient pas partie du contingent, que faisaient-elles, surtout les 11 et 12 mars, quand la radioactivité est montée et que vous manquiez de personnel?

R : Il y avait des gens qui ne faisaient pas partie du contingent qui sont quand même venus nous prêter main forte. Il y en a d'autres, quelques-uns, qui, bien qu'étant prévus dans le contingent, n'étaient pas là pour diverses raisons.

Le nombre de personnes présentes à la centrale n'était pas constant. Vu la radioactivité, il n'était pas possible de maintenir les gens très longtemps sur place. J'ai ordonné plusieurs retraits et, parfois, les gens ne revenaient pas forcément sur le site de la centrale. Alors le nombre de personnes sur place était très variable selon les jours. J'aimerais bien que vous vous penchiez sur la question. À mon sens, il y en avait de moins en moins.

$\mathbf{Q}$ : Grosso modo, vous avez ressenti un grand tournant au moment où il y a eu l'évacuation du 15 mars vers la centrale Daini ${ }^{16}$. Quelques Group Managers sont tout de suite revenus sur le site et, ensuite, petit à petit les autres ont commencé à revenir. C'est bien ça?

R : Oui.

Q: Ce qui veut dire que jusqu'au 15 mars, vous aviez l'impression que le nombre diminuait progressivement?

R : Oui. Pour moi, ça diminuait ${ }^{17}$.

En plus, il y a eu le coup de l'appel d'urgence. Il existe un système d'appel d'urgence qui alerte les employés quand il se passe quelque chose. Quand ils reçoivent l'appel, ils doivent venir. Or, l'administration a fait une bourde. Enfin, ce n'est peut-être pas gentil de dire ça. Toujours est-il qu'ils se sont trompés dans le signal. Ils n'ont pas envoyé le signal «venez tout de suite ». Personnellement, je ne connais pas bien ce système. Il faudrait vous renseigner auprès de l'administration. Mais comme ils avaient envoyé le signal « inutile de venir tout de suite ", j'ai l'impression que ça n'a pas suffisamment traduit l'urgence et la sévérité de la situation auprès de ceux qui étaient en congé, par exemple. Après coup, le Group Manager de l'administration est venu s'excuser. Je lui ai dit de rectifier le tir et de rappeler tout le monde tout de suite. Ce n'était pas plus grave que ça. Demandez-lui les détails. 
$\mathbf{Q}$ : D'un autre côté, y a-t-il eu du personnel d'autres entités qui a été envoyé en renfort après le 11 mars?

$\mathbf{R}$ : Oui, il y en a eu. Comme on avait atteint un haut niveau de radioactivité, il fallait du monde pour gérer la radioprotection des équipes. On n'avait pas assez de personnel pour toutes ces surveillances. Fukushima Daini était encore en pleine restauration, comme nous ${ }^{18}$. Du coup, c'est plutôt de Kashiwazaki que les renforts sont venus.

$\mathbf{Q}$ : Les gens qui venaient de cette manière étaient incorporés dans ces groupes?

$\mathbf{R}$ : Oui, s'ils venaient d'un groupe "réhabilitation », ils étaient incorporés dans le groupe "réhabilitation ", s'ils venaient d'un groupe "sûreté », ils allaient dans le groupe « sûreté ».

$\mathbf{Q}$ : Ils étaient donc incorporés dans la chaîne de commandement existante?

$\mathbf{R}:$ Oui, c'est ça.

$\mathbf{Q}$ : Ensuite, dans votre groupe autonome de pompiers, vous aviez des gens de Nanmei Corporation ${ }^{19}$, n'est-ce pas ? Ces personnes manœuvraient effectivement les camions de pompiers. Mais elles n'étaient pas membres du groupe autonome de pompiers.

$\mathbf{R}$ : Non, elles n'en étaient pas membres. C'étaient des sous-traitants. C'est aussi ça qui rend l'histoire encore une fois compliquée. Avec un contrat de sous-traitance, s'il n'y avait pas eu toute cette radioactivité, on aurait pu demander aux sous-traitants de participer activement, autant que nos propres employés, aux travaux. Seulement, vu les circonstances, si l'entreprise partenaire vous disait, "il y a vraiment trop de radioactivité, en tant qu'entreprise, nous ne pouvons pas envoyer nos hommes sur le terrain ", nous ne pouvions pas insister. Dans les faits, nous ne pouvions que les laisser partir. Dans ce sens-là, les choses n'avaient pas été bien prévues, puisque nous nous sommes retrouvés avec des choses que nous ne pouvions pas faire sans les hommes de Nanmei. Effectivement, le contrat n'avait pas prévu les incidents de ce genre.

$\mathbf{Q}$ : Il me semble que lors de l'évacuation vers Fukushima Daini, le 15 mars, les hommes de Nanmei sont aussi partis avec les vôtres jusqu'à la centrale 2.

R : Oui.

$\mathbf{Q}$ : Si je comprends bien, les personnes de Nanmei, qui vous aidaient jusque-là à injecter l'eau, sont ensuite reparties directement vers Kashiwazaki. TEPCO sous-traite de la même manière à Fukushima Daini et à Kashiwazaki?

$\mathbf{R}$ : À Nanmei Corporation?

$\mathbf{Q}:$ Oui.

$\mathbf{R}$ : Oui, en effet.

$\mathbf{Q}$ : J'ai compris, en me renseignant à droite et à gauche, qu'il n'était pas si simple d'utiliser une voiture de pompiers, qu'il ne suffisait pas de fourrer le bout du tuyau quelque part et d'ouvrir la vanne.

$\mathbf{R}:$ Non, il y a différentes manœuvres à faire.

$\mathbf{Q}$ : Ce qui veut dire que ce n'est pas quelque chose qu'on peut apprendre facilement tout de suite. II faudrait y penser à l'avenir. Je suppose que les membres du groupe autonome de pompiers ont dû apprendre sur le tas en imitant les autres?

$\mathbf{R}$ : Oui, je crois qu'ils sont capables de manœuvrer tout ça, maintenant. 
$\mathbf{Q}$ : J'ai même entendu dire que quand ils avaient des difficultés, ils se faisaient expliquer la manœuvre par téléphone. Mais il suffirait qu'il y ait quelques séances d'entraînement...

$\mathbf{R}$ : Oui, je crois que c'est ce qu'il faudrait.

$\mathbf{Q}$ : Même si, en temps normal, vous continuez à confier ça aux gens de Nanmei.

Mais, si je comprends bien, quand vous faisiez des entraînements, avant l'accident, vous n'alliez pas jusqu'à faire faire les manœuvres au groupe pompiers maison ? Est-ce que c'est tout à fait incidemment, parce que vous vous êtes retrouvés dans cette situation lors de l'accident, que les membres du groupe ont appris ces manœuvres?

$\mathbf{R}$ : C'est ce que j'aurais tendance à penser. Ceci dit, les relations étaient assez étroites et chaleureuses entre nos employés et les sous-traitants dans le domaine de la lutte contre l'incendie ou de la prévention, alors je pense que quelques-uns de nos hommes étaient sans doute capables de faire quelques manœuvres. Mais, dans l'ensemble, je pense qu'ils ont manœuvré seuls pour la première fois et que c'est là qu'ils ont appris.

$\mathbf{Q}$ : Je ne sais pas s'il y a vraiment similitude, mais cette histoire me fait penser à ce qu'on fait chez nous, quand on a une panne. Nous, quand on a une panne dans la maison, il suffit de téléphoner et on vient nous dépanner. Ça paraît très simple. Comme vous, vous êtes des employés d'une compagnie d'électricité, que vous êtes des ingénieurs, on a tendance à penser, nous, que vous n'avez aucune difficulté à brancher des batteries, à travailler sur un circuit électrique ou à réparer des instruments de mesure. Mais, en fait, ça ne veut pas dire que tous vos employés ont cette sorte d'expérience. Toutes les personnes que j'ai interrogées m'ont dit qu'elles avaient été confrontées à une cascade de problèmes en voulant en résoudre un, qu'elles n'avaient eu de répit de chercher les causes de ces difficultés qu'elles ont dû résoudre seules. Je pense que là, le grand problème a été le manque de personnel. Une manœuvre qui, en temps normal, aurait pu être faite rapidement en mobilisant cinq personnes, dans ces conditions exécrables de radioactivité, a dû demander bien plus de temps. S'il faut plus de temps, cela suppose de s'exposer plus longtemps, avec les risques d'irradiation. À un moment donné, il faut quitter le poste, se faire remplacer. De plus, les personnes qui ont été exposées ne peuvent plus œuvrer sur le terrain pendant un certain temps, n'est-ce pas?

$\mathbf{R}:$ Non. On les met au repos.

$\mathbf{Q}$ : À ce moment-là, même si on transfère la totalité du personnel affecté ordinairement à ces travaux dans les groupes d'urgence, il est évident que vous allez manquer très vite de personnel.

$\mathbf{R}$ : Comme vous dites.

$\mathbf{Q}$ : J'espère sincèrement qu'on ne revivra jamais ça, mais s'il fallait réfléchir à ce qu'il convient de prévoir en cas de catastrophe, est-ce qu'il n'y a que la solution d'un transfert du personnel d'une centrale à une autre? Si, par exemple, l'incident se produit à Kashiwazaki, que ce soit les gens de Fukushima Daiichi qui aillent les aider?

$\mathbf{R}$ : Je pense que c'est ce qu'on ferait à la base. L'autre problème, c'est celui des installations qui évoluent. Prenez juste les tableaux. Je ne veux pas dire que ceux de la centrale Daiichi soient vieux. Mais on n'a pas arrêté d'en changer. On est passé, d'un côté, à celui de Hitachi ${ }^{20}$, quand on passait à celui de Tôshiba, de l'autre. C'est une évolution normale. Mais cela veut dire aussi qu'il faut du personnel qui connaisse suffisamment cette installation pour en assurer la maintenance. Est-ce qu'il y a les mêmes installations à Fukushima Daini et à Kashiwazaki ? Je dois reconnaître que tout n'a pas systématiquement été standardisé.

En plus, Fukushima Daiichi est vraiment particulière. Nous avons une unité de $460 \mathrm{MWe}$, quatre autres de $784 \mathrm{MWe}$ et une autre unité qui produit $1000 \mathrm{MWe}^{21}$. C'est un vrai défilé de réacteurs avec des caractéristiques légèrement différentes. Côté 
constructeurs aussi, on a à la fois GE, Tôshiba et Hitachi. Ce qui fait de Fukushima Daiichi une centrale un peu atypique. Si on amène quelqu'un de Kashiwazaki, il n'est absolument pas sûr qu'il soit capable de conduire ou d'entretenir la tranche 1, chez nous, voyez-vous? C'est un souci que nous traînerons encore longtemps.

Il y a donc le problème de prévoir suffisamment de personnel, mais il y a aussi la difficulté que vous avez évoquée tout à l'heure. C'est vrai que dans la production d'électricité, il est rare qu'on opère soi-même. On sait suivre une séquence, on sait contrôler sur un plan les opérations qu'il ne faut pas faire en même temps, on sait ce qu'il convient de faire dans tel cas. Bien sûr, dans le cadre de formations, il nous arrive de faire des manipulations sur le terrain, de détacher telle partie, etc. Mais, dans l'ensemble, nous travaillons toujours de concert avec des sous-traitants qui ont le savoir-faire. Il y a donc deux problèmes. Le fait qu'il est difficile de s'adapter tout de suite à des installations qu'on ne connaît pas bien et le fait que, de par sa nature, le travail de production d'énergie aujourd'hui est un travail où on n'a pas forcément besoin d'aller soi-même sur le terrain manipuler les choses.

Je voudrais tout de même ajouter une chose. Fukushima Daiichi est une centrale qui fonctionne depuis longtemps. Elle a connu énormément de problèmes à ses débuts. Ce qui veut dire que ceux qui sont aujourd'hui chefs de groupe "sûreté » ou chefs de quart à la conduite chez nous, sont des gens qui ont une beaucoup plus grande expérience des incidents que ceux des autres sites. Ils ont eu, plus d'une fois, l'occasion d'aller sur le terrain faire des réparations, etc. Ils sont beaucoup plus expérimentés que ceux de Fukushima Daini ou Kashiwazaki et je suis persuadé que nous devons beaucoup à leur ingéniosité et à leur intelligence.

$\mathbf{Q}$ : Les chefs de groupe dont vous venez de parler sont des collaborateurs qui ont une longue expérience du travail à Fukushima Daiichi, qui ont vécu dans le passé des incidents et qui, même s'ils ne pouvaient pas calquer directement les solutions qu'ils avaient déjà utilisées, étaient capables de s'en inspirer pour proposer différentes réponses possibles. C'est bien ça?

$\mathbf{R}$ : Oui, c'est ça.

$\mathbf{Q}$ : Toujours à propos des personnes, quand on doit faire face à ce genre d'incident, il faut une organisation importante, mettre en place différentes chaînes de commandement, des systèmes de communication. Dans ce document sur l'organisation de la gestion des accidents, à partir de la page 17, il y a la description de ce que doivent être les organismes de soutien. Vers le bas de la page, en 4-1-2, il y a un paragraphe sur la répartition des rôles et les prises de décisions. Je cite. "La conduite de la tranche est dévolue à l'agent de conduite de la salle de contrôle. En règle générale, les décisions nécessaires à cette conduite seront prises par le chef de quart présent à la salle de contrôle. Cependant, face à des évènements plus complexes, les appréciations techniques auront un poids plus important dans l'estimation des circonstances de l'accident ou le choix des mesures à adopter. De nombreuses informations seront nécessaires. C'est pourquoi des organismes de soutien viendront en aide en assurant les appréciations techniques en vue d'une prise de décision ». Dans votre cas, même si, suivant cette procédure, le chef de quart avait voulu prendre des initiatives, sans électricité, il était dans l'incapacité de rassembler les informations. Du coup, il a bien fallu qu'il s'en remette à la cellule de crise, n'est-ce pas ?

R : Oui, il me semble.

Q : Ensuite, à partir du 13 mars, à peu près, il devient difficile de stationner durablement dans la salle de contrôle. Tout le monde s'était mis à l'abri et vous envoyiez quelqu'un làbas, juste le temps de relever les paramètres, et retour. C'est bien ça ?

R : Oui. 
$\mathbf{Q}$ : Dans ce sens, ce qui est préconisé ici ne peut pas forcément être mis en œuvre tel quel, quand on se trouve face à ce genre d'évènement.

\section{$\mathbf{R}$ : Vous avez raison.}

$\mathbf{Q}$ : Si on continue à regarder, il y a: «le chef de quart devra rester en contact étroit avec l'organisme de soutien, recevoir son avis, si nécessaire, pour décider de l'orientation des manœuvres». Plus loin: "lorsqu'il s'agit d'opérations exigeant des manœuvres conjointes avec une autre tranche ou d'opérations ayant une répercussion majeure sur le comportement de la tranche, le chef de quart devra solliciter l'avis ou la décision de l'organisme de soutien. Ces cas sont concrètement listés dans le manuel ». Quand on lit cette partie du texte, on sent qu'elle a été rédigée en pensant que ces cas seraient exceptionnels. Mais si on revient à l'accident de la centrale, vous étiez dans une situation où le centre de décision ne pouvait qu'être transféré à l'organisme de soutien. C'est-à-dire que la salle de contrôle devait solliciter les instructions de la cellule de crise et, suivant ces instructions, effectuer, par exemple, un éventage en se conformant aux procédures, n'est-ce pas?

R : Oui.

$\mathbf{Q}$ : Ensuite, au paragraphe 4-1-3, on parle du rassemblement des membres de la cellule de crise. C'est la procédure que vous avez suivie le 11 mars tout de suite après le séisme et le tsunami?

R : Oui.

$\mathbf{Q}$ : Si on saute un peu, on lit: "lorsque l'organisation d'urgence telle que définie dans le cadre de la prévention des accidents est ordonnée, les membres sont appelés à se rassembler suivant les voies prévues dans ce cadre. Une cellule de crise est installée, avec le directeur de la centrale à sa tête ». Je suppose que vous avez suivi ces directives.

R : Oui.

$\mathbf{Q}$ : Suit: " un système d'appel a été mis au point, au niveau de la centrale, y compris les nuits et les jours chômés. Des équipes de quart sont définies, aussi bien les jours ouvrés que les nuits, et les jours chômés. Elles se tiennent en attente dans des installations à proximité de la centrale la nuit, et à l'intérieur de la centrale de jour ». Dans le cas qui nous occupe, tout le monde est resté dans le bâtiment antisismique?

$\mathbf{R}$ : Comme les évènements se sont déroulés de jour, presque tout le monde se trouvait dans le bâtiment administratif. Ils n'ont eu qu'à se déplacer vers le bâtiment antisismique.

$\mathbf{Q}$ : Et après ? La nuit du 12 au 13, par exemple, tout le monde est resté dans le bâtiment antisismique?

\section{$\mathbf{R}$ : Oui, c'est bien ça.}

$\mathbf{Q}$ : Dans l'article «disposition des lieux et des équipements », on lit: "il est prévu une salle pour la cellule de crise dans le bâtiment administratif de la centrale pour les activités de soutien ». Mais au moment des évènements de mars, la cellule de crise se trouvait dans le bâtiment antisismique, n'est-ce pas?

R : Oui.

$\mathbf{Q}$ : «Dans la cellule de crise sont prévues des installations permettant l'observation de l'état des tranches, les appréciations techniques, l'aide à la décision concernant les mesures d'urgence, l'appréciation de la radioactivité, la communication avec l'extérieur ». Suit un descriptif des équipements dont dispose l'organisme de soutien. Tout d'abord comme moyens de communication, il est cité « un réseau téléphonique d'urgence intra-entreprise et un autre vers les autorités locales, un télécopieur, une installation intra-centrale de diffusion, une installation sans fil, un système de téléconférence intra-entreprise et un dispositif d'appel d'urgence». Je 
voudrais savoir, parmi toute cette liste, ce que vous avez utilisé. II me semble que le système de téléconférence a bien fonctionné.

R : Oui.

$\mathbf{Q}:$ Et quoi d'autre ? Quel système avez-vous utilisé ?

$\mathbf{R}$ : Le dispositif d'appel d'urgence. On s'est trompé dans l'utilisation du dispositif, mais l'installation en elle-même était en parfait état de fonctionnement.

Ensuite, le système sans fil a été coupé à un moment donné.

Je ne vois pas très bien à quoi ils font allusion quand ils parlent d'» une installation intra-centrale de diffusion ». Mais ça n'a sans doute pas fonctionné.

Pour le fax, ça dépendait des moments. Quand le réseau était coupé, on a essayé de passer par le satellite. On a aussi essayé d'autres choses. Comme il y avait des moments où ça marchait et des moments où ça ne marchait pas, il est difficile de vous dire qu'est-ce qui a marché à quel moment. Ça dépendait vraiment des cas.

$\mathbf{Q}$ : Donc, s'agissant du télécopieur, il y a eu un moment où il ne fonctionnait plus, mais vous avez réussi à le relancer.

R : Oui, c'est ça. Le plus grave, c'est qu'on ne pouvait pas envoyer de documents vers l'extérieur. En principe, il est prévu que nous envoyions simultanément des informations par fax à l'ensemble des municipalités et au département. C'est une chose que nous n'avons pas pu faire tout au début. On a essayé toutes sortes de solutions, comme de passer par la centrale de Fukushima Daini, par exemple. Mais je n'ai pas tout retenu au milieu de cette agitation.

$\mathbf{Q}$ : Concernant le réseau téléphonique, vous disposez d'un réseau spécial pour les urgences?

R : Oui.

$\mathbf{Q}$ : Et ça, ça a fonctionné ?

$\mathbf{R}$ : À côté de la cellule de crise, il y a toute une rangée de cabines téléphoniques. Dans chaque cabine, il y a un téléphone qui est relié à un lieu. Par exemple, l'un sera relié au département, l'autre à la municipalité d'ôkumamachi. En cas d'urgence, il y a une personne par cabine, qui doit téléphoner. Là, il faudrait demander les détails auprès du groupe " information » ou du groupe " communication ", mais, d'après ce que je sais, le système a fonctionné, mais les interlocuteurs n'ont pas décroché.

$\mathbf{Q}$ : Par exemple, s'il s'agit du département de Fukushima, vous êtes relié en direct avec le département?

$\mathbf{R}$ : Oui. Mais ça ne s'est pas passé de la même manière pour toutes les autorités locales. En fait, il y a eu deux problèmes, le problème de celui qui reçoit l'appel et le problème du réseau. Il y a eu des cas où le réseau fonctionnait bien mais les autorités n'ont pas décroché le téléphone et des cas où il $\mathrm{y}$ avait des problèmes sur le réseau lui-même, à ce qu'on m'a dit. Les choses sont un peu confuses. Il faudrait demander à chacun comment cela s'est passé.

$\mathbf{Q}$ : Ensuite, je voudrais vous interroger sur quelque chose qui n'est pas mentionné ici. Le site de la centrale est très vaste. Comment faisiez-vous pour communiquer à partir du bâtiment antisismique, avec ceux qui étaient partis travailler dans d'autres bâtiments ou à l'extérieur à l'autre bout du site?

$\mathbf{R}$ : Avec des talkies-walkies. Si le PHS avait fonctionné, cela aurait été l'idéal, mais tout au début il ne fonctionnait pas du tout. On a donc utilisé les talkies-walkies. Mais 
ce ne sont pas des appareils très puissants. Alors, si on est positionné à l'ombre d'un bâtiment, on ne reçoit plus rien. Pour en revenir à l'histoire de tout à l'heure, si des pompiers devaient venir sur le site, on communiquait avec les talkies-walkies pour donner l'ordre d'aller les accueillir, par exemple. Pour se concerter pour savoir où se retrouver, pour donner des instructions pour que le matériel soit transporté de tel endroit de telle manière, etc., il n'y avait que ce moyen. Mais avec des masques intégraux, la voix est très étouffée. On n'entend rien et je me rappelle m'être franchement énervé. Alors, ce point de communication, au moins, mérite sans doute d'être amélioré.

Q: Vous voulez dire que vous n'arriviez pas à communiquer, vous, de la cellule de crise, avec ceux qui travaillaient sur le terrain?

$\mathbf{R}$ : Presque pas. D'un côté, il y avait eu les communications avec les pompiers et les troupes des forces d'autodéfense, qui avaient été difficiles. Il y avait eu aussi le quai, d'où on pompait l'eau qu'on injectait. Les émissions étaient très faibles à partir du quai. Il faut dire que c'est en bas de la pente et les ondes ne passaient pas du tout. La radioactivité y était particulièrement forte. Alors les gars essayaient de se mettre à l'abri un peu plus loin pour ne pas être irradiés. Mais, à ce moment-là, on n'arrivait plus à leur envoyer les informations en temps voulu et on n'arrivait plus, non plus, à savoir où ils en étaient.

$\mathbf{Q}$ : Donc, pour ce qui est de la communication avec ceux qui étaient sur le terrain, vous utilisiez des talkies-walkies, c'est ça?

R : Oui.

$\mathbf{Q}$ : Et pour ce qui est des communications avec le siège? Vous aviez déjà le système de téléconférence, n'est-ce pas?

R : Oui.

Q : En plus de ça, vous aviez aussi des téléphones fixes? Vous aviez un circuit spécial ou vous passiez par les lignes normales? Est-ce que tout ça a bien fonctionné?

$\mathbf{R}$ : Nous avons quelques lignes intérieures avec des téléphones fixes. Pour les communications avec le siège, les numéros du siège commençant par $\mathrm{xx}$, on peut les avoir en faisant le xx et ils peuvent aussi nous appeler en formant le xxx. Ce système a fonctionné.

\section{$\mathbf{Q}:$ C'était comme d'habitude?}

$\mathbf{R}$ : Oui, on a pu communiquer comme d'habitude. Seulement, comme on ne pouvait pas utiliser les téléphones portables, si on formait le numéro xx avec un appareil PHS, qui n'est pas un téléphone fixe, on avait du mal à les avoir.

$\mathbf{Q}$ : Vous voulez dire que seuls les téléphones matériellement branchés sur le réseau fonctionnaient, c'est ça?

R : Oui.

$\mathbf{Q}$ : Cela veut dire que vous communiquiez essentiellement par téléconférence et téléphone fixe?

$\mathbf{R}$ : Oui, presque tout le temps par téléconférence.

$\mathbf{Q}$ : Alors, les coups de fil qui venaient de la résidence du Premier ministre arrivaient où ?

$\mathbf{R}$ : Sur un téléphone fixe. 
$\mathbf{Q}:$ Vous receviez ces coups de fil sur un téléphone fixe.

$\mathbf{R}$ : Oui. Au départ, je n'avais aucune intention de parler au téléphone avec le Gouvernement. Mais quelqu'un de chez nous, qui se trouvait à la résidence du Premier ministre, m'a fait savoir que des membres du Gouvernement désiraient me parler. On devait passer par un téléphone fixe, mais c'était un système très compliqué. Il fallait d'abord passer par un réseau qui se trouvait à je ne sais plus quel étage de notre siège. Bref, on n'a pas pu vraiment les appeler en direct.

$\mathbf{Q}$ : Ça veut dire que c'était le Gouvernement qui appelait votre siège et que, de là, on vous transférait l'appel?

$\mathbf{R}$ : Moi-même, je n'ai pas très bien compris sur le moment comment ça fonctionnait. On m'a dit d'appeler le numéro xxxxx, c'était le numéro d'une ligne intérieure fixe chez nous, ensuite l'appel devait être transféré et on devait me répondre. En fait, on n'arrivait pas du tout à les appeler d'ici.

$\mathbf{Q}$ : Vous voulez dire que vous n'arriviez pas jusqu'à la résidence du Premier ministre?

$\mathbf{R}$ : Ils nous appelaient pour nous dire de téléphoner au «numéro tant ». Mais, en fait, on n'arrivait pas à les joindre. Du coup, j'ai dit au siège de s'arranger pour que ce soit eux qui nous appellent et je crois me souvenir que c'est comme ça qu'on a eu notre premier contact. Par la suite, je ne sais pas si c'était parce que le réseau par satellite avait ressuscité, mais le PHS a recommencé à fonctionner correctement. En passant par le PHS, j'arrivais à téléphoner par exemple au téléphone mobile de M. Hosono, le conseiller du Premier ministre. Il fallait passer par le siège, donc on formait le xx, puis sortir du réseau interne en faisant le $\mathrm{xx}$, entrer leur numéro et on arrivait à les joindre. C'est ce qu'on faisait quand on voulait qu'ils nous rappellent. Quand c'était eux qui appelaient, comme on ne pouvait pas utiliser nos portables, les appels arrivaient soit sur le fixe ou, plus tard, sur le PHS. Mais la plupart du temps, quand ils nous appelaient, ça arrivait sur le fixe.

$\mathbf{Q}$ : Donc, c'est ce qui s'est passé au début.

R : Oui.

$\mathbf{Q}$ : C'était jusque vers quelle époque? Quand il y avait eu tous ces problèmes autour des tranches 2,3 et 4 , vers le 15 mars?

$\mathbf{R}$ : Oui, c'est à peu près ça.

$\mathbf{Q}$ : M. Hosono, par exemple, une fois que la cellule interministérielle a été constituée, est parti là-bas, n'est-ce pas?

R : Oui. Mais quand il n'y était pas, il était souvent à la résidence du Premier ministre. Dans ces moments-là, il nous appelait pour connaître la situation sur le terrain, ou bien, au contraire, c'est nous qui l'appelions quand il y avait des incidents à signaler.

$\mathbf{Q}$ : En dehors de la résidence du Premier ministre, aviez-vous d'autres réseaux de communication? Avec la NISA, par exemple?

$\mathbf{R}$ : Non.

Q: Je suppose que ça s'est passé comme ça, juste pour des raisons propres au Gouvernement. Parce qu'en cas d'urgence, en principe, le Gouvernement forme une équipe spéciale de travail. Pour pouvoir partager toutes les informations, chaque ministère délègue des hommes qui se rassemblent dans le sous-sol de la résidence du Premier ministre. C'est ce qu'on fait quand il y a des actes terroristes ou comme dans le cas qui nous occupe. II me semble que, face à un incident nucléaire, c'est la NISA qui aurait dû rassembler les différentes informations et les diffuser. Mais, visiblement, les informations ne circulaient 
pas bien au sein du Gouvernement et tout le monde se demandait ce qui se passait. Entre le $5^{e}$ étage où se trouvait le Premier ministre, qui aurait dû descendre lui-même au sous-sol pour assurer certaines choses, et les membres du Gouvernement, il a dû se passer des choses, à mon avis. Je pense que TEPCO avait été sollicité de son côté pour envoyer du monde participer à cette équipe. Mais, d'un autre côté, il y avait M. Takekuro, je ne sais pas s'il avait été appelé ou quoi, qui était là, au $5^{e}$ étage. Du point de vue de TEPCO, officiellement, tout aurait dû passer par la NISA, je suppose. La NISA demande les renseignements sur la situation, une fois qu'elle les a, elle en informe le Premier ministre. C'est la NISA qui aurait dû servir de guichet unique et cela aurait été la forme normale de fonctionnement. Qu'en pensez-vous? Ne vous êtes-vous pas demandé pourquoi c'était le Premier ministre en direct?

$\mathbf{R}$ : Ça a été mon premier sentiment. Pourquoi le Premier ministre venait-il s'informer en direct ici ${ }^{22}$ ? Je me suis demandé «que fait le siège? ». Et, bien sûr, "que fait la NISA? $»^{23}$. Je ne savais pas pourquoi, mais j'avais l'impression que le Gouvernement voulait que ça se passe comme ça. De toute façon, j'avais reçu des coups de fil, ils voulaient que je les renseigne en cas de besoin. Puisqu'il y avait demande, disons que je me suis un peu laissé faire. Mais j'ai toujours pensé que c'était bizarre.

$\mathbf{Q}$ : Vous avez toujours eu des inspecteurs de la NISA sur le site de la centrale, n'est-ce pas? Ils ont même un bureau à la centrale.

R : Oui.

$\mathbf{Q}$ : Ces personnes ont été évacuées à un moment donné. Elles s'étaient réfugiées quelque part, il me semble.

R : Oui.

$\mathbf{Q}$ : Et ensuite, sont-elles revenues?

R : Oui, assez longtemps après.

$\mathbf{Q}$ : Elles reviennent et veulent certainement connaître le niveau de radioactivité et d'autres renseignements de ce genre pour pouvoir juger de la situation. À votre avis, leur retour, ça s'est passé à quel moment? Les gens de la NISA étaient là vers le 13 ou le 14 mars?

$\mathbf{R}$ : Je ne me souviens pas très bien. Mais quand le tsunami est arrivé, les gens de la NISA sont tous venus se réfugier dans le bâtiment antisismique, comme nous. Ensuite, très peu de temps après, on a ouvert un centre hors site ${ }^{24}$, et ils sont partis là.

Comme je ne me souvenais pas bien, j'ai regardé tout à l'heure le compte rendu des DVD. À un moment donné, quand le centre hors site se trouvait encore à ôkuma, Mutô avait voulu nous envoyer les inspecteurs de la NISA à la centrale. C'était le 14 mars. Mais, en fait, ils ne sont pas venus.

$\mathbf{Q}:$ Ils ne sont pas venus?

$\mathbf{R}$ : Non. Je ne me souviens pas très bien, mais c'est un peu plus tard qu'ils ont commencé à faire des roulements $24 \mathrm{~h} / 24$, comme maintenant.

$\mathbf{Q}$ : Quand les gens de la NISA viennent ici, s'ils veulent des informations, je suppose qu'ils viennent à la cellule de crise.

R : Oui. 
$\mathbf{Q}$ : Dans ce cas-là, ils viennent vous saluer de leur propre initiative, ou bien, ils viennent se placer près de la table ronde, sans rien dire?

R : Vous savez, nous, nous n'avons nullement l'intention de les exclure. Ils viennent quand ils veulent. Comme ils sont tous en uniforme, on les repère tout de suite. S'ils sont là, ils sont tout naturellement intégrés aux discussions.

$\mathbf{Q}$ : Et y avait-il des places prévues spécialement pour la NISA quelque part autour de la table?

$\mathbf{R}:$ Au début, il n'y en avait pas. Et puis, il y a eu cette histoire avec Mutô. Puisqu'ils devaient venir, on leur avait prévu des sièges.

$\mathbf{Q}:$ Ça, c'est à quel moment?

$\mathbf{R}$ : Tout au début, quand j'ai eu le coup de fil de Mutô pour m'annoncer leur venue. Peut-être qu'ils sont effectivement venus une fois, très brièvement. Ça devait être vers le 14. Oui, il me semble bien m'en souvenir. Ils sont venus, mais très brièvement. Puis, le centre hors site a été déplacé vers Fukushima et ils sont tous partis à Fukushima $^{25}$. Il me semble qu'ils n'étaient pas là vers le 16 ou le 17 , quand les forces d'autodéfense et les pompiers faisaient leur opération « pschitt-pschitt ».

$\mathbf{Q}$ : Quand ils venaient sur le site de la centrale, ils ne restaient pas dans leur bureau?

R: Il n'y avait que le bâtiment antisismique où on pouvait stationner. Ils ne pouvaient pas aller dans leur bureau. Il y avait de la radioactivité, des risques d'irradiation, etc.

$\mathbf{Q}$ : Donc, s'ils venaient, ils restaient dans le bâtiment antisismique. C'est bien ça ?

R : Oui.

$\mathbf{Q}$ : Et vous n'avez jamais reçu de coup de fil directement de la NISA pour savoir où en était la situation, par exemple?

$\mathbf{R}$ : Non, jamais.

$\mathbf{Q}$ : Alors, c'est seulement le siège qui vous demandait où en était la situation?

R : Oui.

$\mathbf{Q}:$ En utilisant essentiellement le système de téléconférence?

R : Oui.

$\mathbf{Q}$ : Ensuite, il y a des coups de fil du Gouvernement vers le téléphone fixe, coups de fils assez nombreux au début. C'est bien ça?

$\mathbf{R}$ : Oui.

$\mathbf{Q}: Y$ a-t-il eu d'autres correspondants qui ont essayé de vous joindre?

R : Non.

$\mathbf{Q}:$ Vous n'avez pas été contacté par la Nuclear Safety Commission ou d'autres?

$\mathbf{R}$ : Non. Le directeur de la NSC m’a téléphoné juste une fois. Il m’a téléphoné de la résidence du Premier ministre, il me semble, et non pas de la NSC.

$\mathbf{Q}$ : Par exemple, quand des détachements des forces d'autodéfense, de pompiers ou de police devaient venir sur le terrain, il ne vous est jamais arrivé de recevoir un coup de fil directement d'un chef de service ou quelqu'un d'un rang équivalent pour savoir quelle était la situation à la centrale avant d'envoyer des hommes?

$\mathbf{R}$ : Non, on n'en a jamais eu. 
$\mathbf{Q}:$ Ils passaient tous par le siège?

$\mathbf{R}$ : Oui, c'est ça.

$\mathbf{Q}$ : Encore une chose. (Dans le document) on parle du SPDS. C'est M. XXXXX qui me l'a raconté. II paraît que vous avez un écran correspondant à ce système dans la cellule de crise et que vous pouvez suivre les données sur l'écran. C'est bien ça?

$\mathbf{R}$ : Oui. On peut tout afficher.

$\mathbf{Q}$ : Si vous regardez ça, vous pouvez savoir si la vanne de tel réseau est ouverte ou pas, n'est-ce pas?

$\mathbf{R}$ : Oui, on peut aussi vérifier ça.

$\mathbf{Q}:$ On peut tout connaître, alors?

R : Oui.

$\mathbf{Q}$ : Ça fonctionne au courant alternatif?

$\mathbf{R}$ : Tout à la fin, ça marche au courant continu, mais il y a un endroit où il est doublé par du courant alternatif, il me semble. Je ne connais pas très bien l'architecture du système.

$\mathbf{Q}$ : Il est écrit ici que «les paramètres concernant la sécurité du réacteur comme la pression du réacteur, son niveau d'eau, les chiffres du moniteur de la cheminée sont affichés directement » sur l'écran de la cellule de crise du bâtiment antisismique. Ces données n'étaient-elles pas affichées?

$\mathbf{R}:$ Non. Le système était cassé.

$\mathbf{Q}:$ Et maintenant ?

$\mathbf{R}:$ Il ne fonctionne toujours pas.

$\mathbf{Q}$ : Et quelle est la cause de ce dysfonctionnement?

$\mathbf{R}$ : Il y a deux choses. Le SPDS lui-même est endommagé. On ne sait pas où. Mais, de toute façon, tout a été noyé par le tsunami, alors, comme tout est relié automatiquement, il ne pouvait plus rien afficher. Et même si le système avait survécu au tsunami, s'il ne recevait plus de données, et c'est ce qui s'est passé, il ne pouvait rien afficher non plus.

$\mathbf{Q}$ : Vous voulez dire que si les instruments de mesure des pression, niveau d'eau et moniteur de cheminée ne fonctionnaient pas correctement, le SPDS ne pouvait pas réagir?

$\mathbf{R}$ : Oui, c'est tout à fait ça.

$\mathbf{Q}:$ : I semble que ce système SPDS est relié au siège et à l'État?

$\mathbf{R}$ : Oui, il est programmé pour pouvoir diffuser ces informations en simultané.

$\mathbf{Q}:$ L'État, concrètement, c'est qui ?

R : L'État, c'est la NISA. Je suppose que les systèmes diffèrent légèrement d'une centrale à l'autre, mais il existe un réseau qui permet d'afficher en temps réel toutes les données de tout le Japon.

$\mathbf{Q}$ : Ce qui signifie que si ce système avait été viable, la NISA aurait pu suivre la situation en temps réel, n'est-ce pas?

R : Oui. 
$\mathbf{Q}$ : Si on suit le document, il y a ensuite une rubrique «monitoring de la radioactivité à l'intérieur et à l'extérieur du périmètre de la centrale». Comment ça se passe ? Vous avez des dispositifs qui mesurent automatiquement la radioactivité à chaque poste?

$\mathbf{R}$ : Oui, c'est ça. Ils sont programmés pour nous envoyer directement les données. Les postes de monitoring sont au nombre de huit et encerclent la centrale, en quelque sorte.

$\mathbf{Q}:$ Ils sont positionnés à la limite du terrain, c'est ça?

$\mathbf{R}$ : Oui, à la limite de notre terrain. On arrive ainsi à savoir à quel endroit la radioactivité est forte en regardant les résultats affichés. Mais, avec le tsunami, les postes de monitoring ne pouvaient plus transmettre les données. Il a fallu envoyer des véhicules avec des moniteurs embarqués à proximité de ces postes pour faire les mesures. C'était la solution de rechange.

$\mathbf{Q}$ : Quand vous dites «faire les mesures », vous mesuriez la radioactivité, n'est-ce pas?

$\mathbf{R}$ : Oui, c'était le travail du groupe « sûreté ».

$\mathbf{Q}:$ C'était le groupe « sûreté » qui s'en occupait?

R : Oui.

$\mathbf{Q}$ : Donc, ils allaient sur place avec des véhicules équipés de détecteurs de radioactivité, mesuraient celle-ci et revenaient vous faire des rapports.

$\mathbf{R}$ : Oui. Après, on a utilisé les talkies-walkies.

$\mathbf{Q}$ : Une fois que vous receviez l'information à la cellule de crise, vous la partagiez avec tous ceux qui étaient autour de la table, n'est-ce pas?

R : Oui.

$\mathbf{Q}$ : Ensuite, il est fait référence à une installation d'observation météorologique.

Vous avez ça?

R: Oui, nous l'avons. Mais tout était mort, à ce moment-là.

$\mathbf{Q}:$ C'est quelque chose d'indépendant?

$\mathbf{R}$ : C'est un Stack Monitor ${ }^{26}$. Il mesure la direction et la vitesse du vent à un point situé à plus de $100 \mathrm{~m}$ du sol et nous envoie ces données.

$\mathbf{Q}$ : Et vous dites que c'était mort. Quelle en était la cause?

$\mathbf{R}$ : Probablement l'alimentation électrique.

$\mathbf{Q}:$ Un problème d'alimentation?

R : Oui.

$\mathbf{Q}$ : C'était la même source d'alimentation pour le monitoring de la radioactivité de tout à I'heure?

R : Oui, c'était la même source.

Q: S'il s'agit d'alimentation électrique, par exemple, quand les sources externes n'approvisionnent plus et que ça bascule sur les batteries de secours, que se passe-t-il ?

$\mathbf{R}$ : En principe, les postes de monitoring auraient dû fonctionner normalement après avoir basculé. Mais aucun des deux systèmes n'a fonctionné.

$\mathbf{Q}$ : Si je comprends bien, jusqu'à l'arrivée du tsunami, ça fonctionnait.

R : Oui. 
$\mathbf{Q}$ : C'est donc ensuite, à partir du moment où vous avez perdu toute source de courant alternatif que vous ne pouviez plus les utiliser?

$\mathbf{R}:$ C'est ça.

Il y a aussi autre chose. Je ne suis pas sûr que le dispositif d'observation météo ait été installé en pensant vraiment à l'éventualité d'un séisme. Je ne nierais pas la possibilité que le dispositif météo lui-même ait été abîmé. C'est quelque chose qui est fixé dans les hauteurs, alors on ne peut pas vraiment savoir. Même si on avait réussi à rétablir l'alimentation électrique, je ne pense pas qu'il aurait fonctionné, étant donné qu'à l'heure actuelle, il ne nous fournit toujours pas de données. À mon avis, il y a de fortes chances pour que le système lui-même ait été défectueux.

$\mathbf{Q}:$ : I est écrit qu'il «observe la direction, la vitesse du vent et la stabilité des masses d'air ». C'est ce qu'il fait habituellement?

R : Oui, de manière constante. Par exemple, nous avons procédé à des éventages. Dans des cas comme ceux-là où on rejette des éléments radioactifs, il vaut mieux un vent d'ouest, parce que les éléments rejetés vont être emportés vers la mer. C'est donc un système qui nous permet de savoir dans quelle direction se dirige le vent audessus de la centrale.

$\mathbf{Q}:$ C'est pour ça que c'est installé du côté de la cheminée?

R : Oui.

$\mathbf{Q}$ : Mais, si vous ne pouviez pas utiliser ce système, comment faisiez-vous pour savoir la direction du vent, tout ça?

$\mathbf{R}$ : Pour bien faire, il faut un monitoring en altitude pour avoir la vitesse du vent et comprendre la tendance générale des mouvements des masses d'air. Si on mesure plus près du sol, il y a des interférences. Cela occasionne beaucoup d'erreurs. Mais comme on ne pouvait pas faire autrement, on a mesuré près du sol.

Heureusement, les appareils météo de Fukushima Daini fonctionnaient. Alors on leur demandait comment était le vent chez eux et on en tenait aussi compte dans nos décisions.

$\mathbf{Q}$ : Ensuite, il y a dans la rubrique "système d'affichage des informations », " un projecteur grand modèle et un petit moniteur afin de partager les informations au sein de la cellule de crise ». Est-ce que ça a fonctionné ?

$\mathbf{R}$ : Là aussi, c'étaient les données qui manquaient. Le système d'affichage en luimême était indemne, puisqu'on l'a utilisé par la suite pour diffuser des images de la télévision. Mais puisqu'il ne disposait pas des informations qui nous auraient été fondamentalement utiles, en matière de partage, même si on avait voulu, on n'avait rien à partager. Encore un cas où le système était viable mais où on ne disposait pas de données.

$\mathbf{Q}$ : Ensuite, il y a « le système d'appréciation de l'impact environnemental ».

$\mathbf{R}$ : Je pense que c'est un système hors-ligne. Lui non plus, il me semble qu'il n'était pas opérationnel. En fait, c'était plutôt du ressort du siège.

$\mathbf{Q}$ : Ça consiste en quoi ?

$\mathbf{R}$ : Par exemple, on procède à un éventage. À un moment donné, des éléments radioactifs vont être rejetés. Ce système calcule, d'après la direction et la vitesse du vent, leur dispersion. Il y a un système qui s'appelle SPEEDI (System for Prediction of Environmental Emergency Dose Information $)^{27}$. Je ne sais plus si c'est un système du 
ministère de l'Éducation et des Sciences ou de la NSC. Notre système est assez proche de SPEEDI.

Q : Là, il y a plusieurs rapports d'après l'article 15, qui étaient joints comme documents. On y parle de la direction du vent et des conséquences. Qui a rédigé ce rapport?

$\mathbf{R}$ : C'est le siège.

$\mathbf{Q}:$ C'est le siège qui rédigeait ces rapports?

$\mathbf{R}$ : Oui. Chez nous, le système ne fonctionnait pas. Avec les données qu'on avait récoltées, le groupe "sûreté" du siège faisait les calculs et rédigeait les appréciations. Vous voyez, il y a encore plusieurs rapports suivant l'article. Tout ça, ce ne sont pas des documents que nous avons fabriqués, nous. On les recevait du siège par fax et on les joignait.

Q: Par exemple, j'ai ici un document daté du samedi 12 mars à $3 h 35$, il est écrit: «Destinataire: article 10-3». Tout en haut, on lit: «Expéditeur: TEPCO, cellule de crise de la centrale nucléaire Fukushima Daiichi ». Et à droite « 12 mars 2011, 3h00 », c'est-à-dire 35 min avant, avec la mention «TEPCO, centre de gestion de crise ». C'est le siège, n'est-ce pas ?

$\mathbf{R}$ : Oui, c'est le siège.

$\mathbf{Q}$ : Vous joigniez, comme documents, des pièces qui avaient été envoyées au préalable par le siège, c'est ça?

R : Oui. Mais, officiellement, l'expéditeur devait toujours être la cellule de crise de la centrale.

Q: C'est le genre de choses que vous étiez bien obligé de demander au siège de faire à votre place.

$\mathbf{R}$ : Nous, nous n'aurions pas pu. Le système ne fonctionnait plus, on était bien incapable de faire des évaluations.

Q : Mais si ce système d'appréciation de l'impact environnemental avait correctement fonctionné, vous auriez pu rédiger ces documents sur le site.

R : Oui.

$\mathbf{Q}$ : Les manuels, auxquels il est fait allusion ici, ce sont des documents dont vous disposez toujours à la centrale?

R : Oui.

$\mathbf{Q}$ : Ensuite, il y a les documents techniques. Qu'est-ce que « la logique du réseau maintien de la sûreté »?

$\mathbf{R}$ : Il existe différents dispositifs pour assurer la sûreté quand il y a une anomalie et qu'il faut arrêter le réacteur, ou quand il s'agit de le refroidir en toute sécurité. C'est une sorte de circuit logique qui dit d'arrêter ceci de telle manière, si tel indicateur s'allume. C'est une vue d'ensemble de ces circuits logiques. Si une vanne se ferme, c'est qu'il y a tel évènement qui a provoqué cette fermeture. À ce moment-là, le circuit indique si c'est une porte "ET» ou une porte «OU». S'il se produit tel évènement et tel autre évènement, il dit de fermer cette vanne, etc. Cela sert à comprendre pourquoi tel évènement est arrivé.

$\mathbf{Q}$ : Ces manuels et ces documents techniques, ce sont des choses que vous regardez, quand vous êtes assis à la table de la cellule de crise?

$\mathbf{R}:$ Non.

$\mathbf{Q}$ : Cela ne vous arrive jamais?

$\mathbf{R}$ : Non, jamais. 
$\mathbf{Q}$ : Et ça, depuis le début?

$\mathbf{R}:$ Oui, depuis le début.

$\mathbf{Q}$ : Et les autres membres autour de la table?

$\mathbf{R}$ : Si on prend l'exemple de la conduite, tous les manuels et autres documents qui concernent la conduite sont stockés dans la salle de contrôle. Comme je vous l'ai dit tout à l'heure, pour tout ce qui est conduite, à la base, tout est confié au jugement de l'équipe de conduite. Cela veut dire que tout ce dont ils pourraient avoir besoin pour juger de la situation est stocké à portée de main, près d'eux. C'est donc à eux de juger de la situation. Seulement, pour pouvoir leur apporter le soutien nécessaire, nous disposons aussi, dans la cellule de crise dans le bâtiment antisismique, des mêmes plans, etc. On a à peu près tous les manuels, mais pour ce qui est des plans de détails, je ne pense pas les avoir tous à la cellule de crise.

$\mathbf{Q}$ : Et ces documents, ont-ils été utiles pour élaborer les mesures à prendre lors de l'accident? Jusqu'à quel point ont-ils été utiles? J'imagine que vous n'allez pas me dire qu'ils n'ont pas servi du tout.

R : Non, je ne vous dirai pas ça. Évidemment, ils ont été utiles pour les grandes orientations de la gestion de l'accident ou pour savoir ce qu'il convenait de faire et dans quel ordre. Pour les manipulations aussi, les manuels ont été utiles pour avoir une idée de comment procéder. C'était bien. Mais, concrètement, quand on se trouve devant une situation où une vanne qui devait s'ouvrir ne s'ouvre pas, pour contourner le problème, il faut savoir d'où arrive l'électricité, à quel réseau la vanne est connectée, quelle est la structure qui commande son ouverture, etc. Si on n'arrive pas à connaître les choses jusque dans ces détails, on ne peut pas proposer de solution. Ensuite, s'il s'agit d'aller sur le terrain manipuler les choses, il faut savoir à quel type de vanne on a affaire. Or, quand on regarde ces P\&ID, c'est extrêmement schématique et on n'arrive pas à savoir quel est le type de vanne et quelle est l'énergie nécessaire pour l'ouvrir, par exemple. Pour bien faire, il faudrait disposer de quelque chose qui détaille les différentes caractéristiques des vannes. En fait, ces documents existent. Ils sont stockés dans la bibliothèque du bâtiment administratif, qui a été détruit. En temps normal, des dames d'une des entreprises de notre groupe, le Tôkyô Records Management ${ }^{28}$, gèrent ces plans à la bibliothèque. Comme vous avez pu le voir, le bâtiment a été endommagé. De plus, la radioactivité y était forte. Ça a été très compliqué. Mais nous sommes allés chercher les plans nécessaires dans le noir.

$\mathbf{Q}:$ Vous voulez dire que vous êtes allés effectivement chercher des documents là-bas?

$\mathbf{R}$ : Oui, nous y sommes allés.

$\mathbf{Q}$ : Il y avait donc à la bibliothèque des plans qui détaillaient spécialement certaines parties du réseau, qui donnaient les caractéristiques des vannes ou la structure de celles-ci?

R : Oui.

$\mathbf{Q}$ : Vous les faisiez apporter et, en vous basant sur ces documents, vous étudiiez la possibilité d'établir un circuit, par exemple.

$\mathbf{R}$ : Oui, on faisait ça, au cas par cas.

$\mathbf{Q}:$ C'était le travail du groupe « réhabilitation», ça?

R : Oui. 
$\mathbf{Q}$ : Donc, à la base, pour donner grosso modo une direction ou pour savoir que faire chronologiquement, les manuels de gestion des accidents ou autres directives vous ont quand même servi?

R : Oui.

$\mathbf{Q}$ : Mais, lorsqu'il s'est agi de mettre ces directives en pratique, il y a eu des problèmes plus concrets d'alimentation ou de compresseur, c'est ça?

R : Oui.

$\mathbf{Q}$ : En relation avec ce que nous venons d'évoquer, il y a une rubrique "Matériel et instruments nécessaires à la restauration », ici. On peut lire, "comme moyen de se procurer les pièces de rechange nécessaires, on prévoit l'utilisation de pièces prélevées sur des appareils de même type existant sur la centrale ou l'utilisation de pièces de réserve stockées sur le périmètre de la centrale». Ce qui signifie que si les appareils de même type de la centrale sont tous endommagés, on ne peut pas les utiliser pour des réparations.

\section{$\mathbf{R}$ : Effectivement.}

$\mathbf{Q}$ : Ils parlent de "l'utilisation de pièces de réserve stockées sur le périmètre de la centrale». Dans votre cas, vous avez manqué de batteries et vous ne disposiez que de compresseurs de faible puissance, ce qui a donné beaucoup de fil à retordre au personnel sur le terrain. Vous disposez de ce genre de choses à la centrale? Elles sont stockées quelque part?

$\mathbf{R}$ : Oui. Il y a ce qu'on appelle l'entrepôt des pièces de réserve. On y trouve toutes sortes d'éléments de vannes, par exemple. En fait, nous achetons et stockons tout ce qui, endommagé, pourrait contraindre la centrale à un arrêt de longue durée. Car notre mission, en tant que centrale, est d'être toujours capable de trouver tout de suite une solution de rechange quoi qu'il arrive. Nous faisons nos achats en en tenant compte.

Seulement, les batteries, tout ça, ce sont, en quelque sorte, des produits courants. Si on n'avait pas été dans la situation où nous nous sommes retrouvés, c'est quelque chose qu'on peut se procurer tout de suite. Nous ne stockons jamais ce genre de choses en nombre comme pièces de réserve. Ce sont plutôt des pièces spéciales que nous possédons en réserve.

$\mathbf{Q}$ : Ce qui veut dire qu'au moment de l'accident, vous n'aviez pas stocké de batteries ou ce genre de choses dans cet entrepôt, c'est bien ça?

R : Oui.

$\mathbf{Q}$ : Et donc, quand vous en avez eu besoin, vous avez dû aller démonter les batteries des voitures.

R : Oui.

$\mathbf{Q}$ : On lit aussi « les outils et instruments nécessaires (à la réhabilitation) sont stockés soit dans l'entrepôt à outils se situant dans le périmètre de la centrale, soit dans l'entrepôt des pièces de réserve ». Cet entrepôt, où se situe-t-il ?

$\mathbf{R}$ : L'entrepôt des pièces de réserve se trouve par ici, à côté de ça.

$\mathbf{Q}:$ C'est près du bâtiment antisismique.

$\mathbf{R}$ : Oui, c'est près.

$\mathbf{Q}$ : Ensuite, il y a "les dispositifs de protection contre la radioactivité, dispositifs de mesure de la radioactivité et autres ». Tout votre personnel a dû travailler de longues heures sur le terrain. 
Avez-vous eu suffisamment de matériel pour protéger ces hommes ? II vous a manqué des choses?

$\mathbf{R}$ : On manquait de tout. Tout d'abord, on manquait cruellement de combinaisons Tyvek $^{29}$. Même pour les masques, au départ, c'était vraiment juste. Quand on porte des masques, il faut renouveler le filtre. Au début, on n'en avait pas suffisamment. J'ai dû courir à droite à gauche pour nous faire envoyer ces dispositifs de protection de toute urgence de Fukushima Daini et de Kashiwazaki. Malgré ça, ça a pris du retard. Vous vous rappelez sans doute qu'on n'avait pas de dosimètre pour chacun ${ }^{30}$. On était pourtant déjà en avril. Il y a un journal, je ne sais plus lequel, qui l'avait dénoncé.

$\mathbf{Q}$ : À partir du moment où vous ne disposiez pas des choses sur place, à la centrale, vous étiez bien obligé de vous procurer ça ailleurs. Or, vous faisiez des demandes, mais les commandes n'arrivaient pas tout de suite, c'est ça?

$\mathbf{R}$ : C'est un peu plus compliqué que ça. Il y a des choses qui sont arrivées, d'autres pas. S'il y avait des choses qui étaient en surplus à Fukushima Daini, ou des choses susceptibles de nous être utiles, il suffisait que je donne l'ordre d'aller chercher et mes hommes revenaient tout de suite avec, puisque les deux centrales ne sont qu'à $10 \mathrm{~km}$ l'une de l'autre.

Kashiwazaki a aussi réagi très vite ${ }^{31}$. Quand j'ai dit qu'il me manquait certaines choses, ils nous ont apporté ça en voiture en pleine nuit. Il y avait un souci avec la radioactivité pour le transport, mais il suffisait de quatre ou cinq heures. Quand on passait commande par le siège, il fallait beaucoup plus de temps, puisqu'il fallait déjà trouver la chose, l'acheminer de Tôkyô au Coal Center d'Onahama, dans des conditions de transport difficiles.

En ce qui concerne les voitures de pompiers, elles sont arrivées assez vite. En plus, elles sont venues d'un peu partout.

$\mathbf{Q}$ : Justement, les camions de pompiers sont arrivés vers le 13 ou 14 mars, n'est-ce pas ?

$\mathbf{R}$ : Tout d'abord, on a eu les camions de pompiers des centrales thermiques de la compagnie $^{32}$. Ensuite, je suppose que le Gouvernement avait donné des ordres et on a eu des camions de pompiers qui sont arrivés d'un peu partout.

$\mathbf{Q}$ : Je vois qu'il y a eu beaucoup de camions qui ont été mis à disposition, mais le personnel pour les manœuvrer venait aussi?

R : Non.

$\mathbf{Q}:$ Alors, c'est le personnel de TEPCO qui a tout fait ?

R : Oui, c'était à nous de le faire.

$\mathbf{Q}:$ Les pompiers se contentaient d'apporter les camions et repartaient après, c'est ça ?

$\mathbf{R}$ : Oui, c'est ça. Ils arrivaient et nous prévenaient qu'ils avaient déposé le camion à tel endroit. Je crois qu'au début ils venaient nous apporter les camions jusqu'au JVillage et, ensuite, il fallait que, nous, nous allions les chercher jusque-là.

$\mathbf{Q}$ : Vous voulez dire qu'ils se contentaient de déposer provisoirement les camions et que, vous, vous deviez envoyer des hommes de la centrale les chercher? Après, c'était à vous de vous débrouiller pour toutes les manœuvres avec le personnel de TEPCO?

R : Oui.

$\mathbf{Q}$ : Rappelez-moi, un camion de pompiers, ça a besoin de carburant pour faire fonctionner la pompe?

R : Oui. 
Q: Vous deviez donc le réapprovisionner toutes les quatre ou cinq heures, n'est-ce pas?

R : Oui.

$\mathbf{Q}$ : C'est le personnel de TEPCO qui a toujours assuré ça?

$\mathbf{R}$ : Oui, ils l'ont fait.

$\mathbf{Q}$ : Et ce, pendant toute la période où vous avez utilisé les camions de pompiers, c'est-à-dire jusqu'à ce que vous passiez à l'eau douce?

$\mathbf{R}$ : Oui, c'est ça.

$\mathbf{Q}$ : Alors, évidemment, c'était jour et nuit.

$\mathbf{R}$ : Oui. On avait défini des roulements sur vingt-quatre heures. Je pense que c'est là, au moment où ils allaient réapprovisionner le camion, qu'ils ont été le plus irradiés.

$\mathbf{Q}$ : Parce qu'ils allaient à l'endroit le plus radioactif, n'est-ce pas?

R : Oui. Par la suite, il y a eu des gens qui sont venus nous aider, des sous-traitants, il me semble, qui ont bien voulu participer au réapprovisionnement avec nous. Ça nous a un peu soulagés. Mais, tout au début, même des gens du niveau des chefs de groupe allaient verser le carburant.

$\mathbf{Q}$ : Vous faisiez ça à partir d'un camion-citerne ou quelque chose comme ça ?

$\mathbf{R}$ : Comme je vous l'ai dit, dès le tout début, j'avais conscience qu'on allait manquer de liquides. La véritable eau douce, par exemple. Dès le tout début, j'avais demandé à ce qu'on nous envoie eau, essence et gazole à profusion, qu'on nous envoie tout ce qui leur tombait sous la main. Pour l'acheminement, ça venait, par exemple, en camionciterne. Mais le problème, c'est que si Fukushima Daini disait qu'elle avait elle aussi besoin de gazole, on le déchargeait complètement là-bas. C'était une confusion totale.

Les choses n'arrivaient pas au moment voulu. Quand on était dans le bâtiment antisismique, on était encore en situation de gestion de crise et tout marchait avec le système électrique de secours. Pour faire fonctionner tout ça, il faut du gazole. Et ça consomme pas mal. Il fallait donc du gazole pour commencer. Si on avait perdu le fonctionnement de cet endroit-là, toute la chaîne de commandement aurait été perdue. Le gazole était donc indispensable. Ensuite, comme vous l'avez fait remarquer, il en fallait pour les camions de pompiers. On transportait beaucoup de choses, de l'eau, par exemple, pour ça on utilisait de gros camions, il fallait aussi du carburant pour eux.

Je me rappelle, tout au début, avoir réclamé continuellement ces carburants, tout en donnant les directives pour les différentes tranches. On a même eu des moments où on a failli se trouver à sec. On s'est débrouillé en s'en faisant livrer par Fukushima Daini, mais mon sentiment est qu'on s'en est sorti in extremis.

$\mathbf{Q}$ : Le gazole était donc indispensable.

R: Oui.

$\mathbf{Q}$ : Concernant les carburants, puisque vous aviez vous-mêmes des camions de pompiers, vous aviez dès le départ un peu de réserve, non?

$\mathbf{R}$ : Pour le gazole, il y en avait dans le réservoir à gazole de la centrale. Mais avec le tsunami, le réservoir lui-même a été endommagé, ce qui fait qu'on ne disposait plus du tout de gazole. Il a fallu s'en procurer depuis la première goutte. 
$\mathbf{Q}:$ Le réservoir à gazole se trouve côté littoral ?

$\mathbf{R}$ : À plusieurs endroits, côté mer.

Q: Le point suivant va concerner les rapports suivant l'article 15 et des choses de ce genre. Il est dit ici que «dans une situation de crise, il est important de pouvoir échanger les informations de manière fluide, aussi bien concernant les informations à fournir à partir de la centrale que pour recevoir les conseils du Gouvernement. La gestion de ces échanges est prise en charge par un unique acteur, le groupe "information" de la cellule de crise ". Vous étiez donc organisé de cette manière.

R : Oui.

$\mathbf{Q}$ : «Une organisation assurant ces rapports, rapports au Gouvernement suivant la législation », ce qui correspond à ces rapports suivant l'article 15, je suppose, " et rapports aux autorités locales suivant les différents accords de sécurité, est mise en place de manière durable ». Quand vous faites un rapport suivant l'article 15, les autorités locales le reçoivent aussi ?

\section{R : Bien sûr.}

$\mathbf{Q}$ : Et pour ce qui est des échanges avec le département ou les municipalités, il me semble qu'il y avait des personnes de la centrale qui étaient détachées au centre hors site, n'est-ce pas?

$\mathbf{R}$ : Oui, on en a envoyées.

$\mathbf{Q}: Y$ avait-il d'autres endroits où elles avaient été envoyées ?

$\mathbf{R}:$ Non.

$\mathbf{Q}$ : Et le bureau que vous avez au département de Fukushima, par exemple ${ }^{33}$ ?

$\mathbf{R}$ : Le bureau du département de Fukushima est une entité indépendante de la centrale.

$\mathbf{Q}$ : Ah, bon. Effectivement, il ne s'occupe pas uniquement de la centrale de Fukushima Daiichi.

$\mathbf{R}:$ C'est ça.

$\mathbf{Q}:$ Mais il y avait du monde quand même là-bas?

R : Oui. Pas des gens de Fukushima Daiichi, mais la personne qui y est habituellement était là.

$\mathbf{Q}$ : Même au moment de l'accident?

R : Oui.

$\mathbf{Q}:$ Avez-vous pris contact avec cette personne?

$\mathbf{R}$ : Bien sûr, elle recevait les informations en simultané.

$\mathbf{Q}$ : Le bureau est sur le réseau de la téléconférence?

$\mathbf{R}$ : Oui, il est sur le réseau.

$\mathbf{Q}$ : Est-il arrivé que cette personne vous contacte pour recueillir des informations, qu'elle aurait eues à diffuser auprès du département, par exemple?

$\mathbf{R}$ : Je ne me rappelle pas avoir été contacté personnellement pour ça.

$\mathbf{Q}$ : Aurait-elle pu contacter des gens du groupe « information »? Qu'en pensez-vous ?

$\mathbf{R}$ : Cela s'est peut-être fait. On faisait la téléconférence et, lui, entendait toutes les informations, aussi bien que les gens du centre hors site. Je n'ai pas le souvenir qu'aucun d'entre eux ne m'ait posé de questions. Maintenant, peut-être ont-ils communiqué avec les gars de l'information par téléphone? C'est possible. 
$\mathbf{Q}$ : Au moment du rejet du réacteur 1 que vous prépariez dès l'aube du 12 mars et que vous n'avez finalement effectué que vers 9h00, il a fallu vous coordonner avec les autorités locales. Il y avait eu une partie des habitants qui n'était pas partie et on vous avait demandé d'attendre qu'ils aient tous évacué. Tous ces ajustements, vous ne les faisiez pas directement de la cellule de crise, n'est-ce pas?

R : Non.

$\mathbf{Q}:$ Alors qui devait le faire? Le bureau?

$\mathbf{R}$ : Oui. Dans le principe, c'est le travail du centre hors site. Dans l'esprit de la loi sur les catastrophes nucléaires, les responsables du département, de l'État et de l'exploitant se retrouvent dans un lieu proche du site et prennent les décisions en fonction des évènements. C'est pourquoi je pense, pour ma part, qu'il appartient au centre hors site de décider de ce qu'on fait concernant les évacuations, par exemple. C'est l'esprit de la loi. Mais dans les faits, ils n'ont rien décidé. C'est par le siège qu'on a su toute cette histoire, à la centrale. Nous étions déjà débordés. Il fallait préparer l'éventage. Pour ce qui est du moment du rejet, comme il fallait justement coordonner tout ça avec les évacuations, c'est le siège qui nous disait de faire comme ci ou comme ça. Ce sont eux qui nous ont signalé qu'il y avait du retard du côté d'ôkumamachi et nous ont demandé d'attendre. Alors, on leur a dit, " on va attendre ». Mais dans les faits, les préparatifs étaient loin d'être achevés. On n'en était pas encore à « attendre » ou « ne pas attendre ». Je me rappelle leur avoir quand même demandé que les populations évacuent au plus vite.

$\mathbf{Q}$ : Si je comprends bien, ce centre hors site a bien été constitué au départ. Des représentants de TEPCO, de l'État et du département étaient bien présents. Ils étaient là, mais n'ont pas réussi à faire fonctionner cet organe pour prendre des décisions ${ }^{34}$. C'est bien ça?

$\mathbf{R}$ : Oui. Je n'ai absolument pas compris sa raison d'être.

$\mathbf{Q}$ : Le centre hors site vous a contacté?

$\mathbf{R}$ : Non, jamais. Il y avait au centre hors site un de nos employés XXXXX. XXXXX était très impliqué dans l'acheminement des différentes choses dont nous avions besoin. Il nous servait en quelque sorte de guichet et je me rappelle avoir eu des échanges avec lui à ce propos. Mais je n'ai pas le moindre souvenir d'avoir discuté avec quelqu'un du centre hors site de sujets ayant trait au travail dont il est investi par la loi. Même pas d'évacuation. C'est quand même bizarre, non?

$\mathbf{Q}$ : À la NISA, ils nous ont dit la même chose. Ils trouvent aussi que le centre hors site n'a pas fonctionné en tant que tel. Ce qui signifie que l'esprit de la loi n'a pas été bien compris.

Si je retourne au document, on lit: "comme noté précédemment, sont installés à demeure dans la salle de crise un réseau de téléphonie ordinaire et des téléphones et télécopieurs branchés sur un réseau spécial de contact avec l'extérieur ». Vous les avez utilisés, mais personne ne vous a répondu, c'est bien ça?

R : Oui.

$\mathbf{Q}$ : «Pour communiquer largement les informations au public, lorsque l'organisme de gestion des incidents de proximité (centre hors site) est déjà constitué, en règle générale, les conférences de presse se tiendront dans la salle de presse de ce centre ». Voilà ce que dit le texte. $Y$ a-t-il eu véritablement des conférences de presse au centre hors site ? II me semble que non.

$\mathbf{R}$ : C'est-à-dire que très vite, le centre hors site d'ôkumamachi s'est trouvé en zone d'évacuation. Les journalistes ne pouvaient pas venir jusque-là et il n'y a pas eu de 
conférence de presse. Par la suite, le centre a été transféré à Fukushima et là, il me semble qu'il y a bien eu des conférences de presse.

Q: Lors de ces conférences de presse, quand ils devaient parler de la situation de Fukushima Daiichi, est-ce que quelqu'un vous contactait pour se concerter avec vous sur le contenu de la conférence, pour vous demander si le contenu vous convenait, etc. ?

$\mathbf{R}$ : Au début, on a eu quelques échanges de ce type. Mais après, la presse devenait tellement insistante qu'ils faisaient communication sur communication, sans nous consulter sur quoi que ce soit. Nous, on ne savait absolument pas ce qu'ils allaient communiquer à la presse.

$\mathbf{Q}$ : Oui, le système allait s'emballer et engendrer des situations où les communications ne correspondaient plus à la réalité.

\section{$\mathbf{R}:$ C'est ça.}

$\mathbf{Q}$ : Cet après-midi, nous verrons ce qui a bien marché dans les directives prévues dans la gestion de crise et ce qui n'a pas marché, nous parlerons des aménagements qui avaient été faits sur les installations mais qui, vu les circonstances, n'ont pas suffi ou que vous avez dû modifier pour pouvoir les utiliser. Je voudrais surtout revenir sur les dispositions que vous avez prises au niveau de l'injection d'eau. Nous allons repasser tout ça.

\section{R : D'accord.}

Q : Nous avons terminé pour la matinée. Je vous remercie beaucoup.

\section{(Pause)}

$\mathbf{Q}$ : Parmi les manuels de conduite, il y a les réponses aux évènements, les AOP (Abnormal Operating Procedures) $)^{35}$, les réponses aux symptômes, les EOP (Emergency Operating Procedures ${ }^{36}$, qui ont toujours existé, auxquelles se sont nouvellement rajoutées en 2002 les procédures en cas d'accident sévère, I'AMG (Accident Management Guideline) ${ }^{37}$ et le manuel de restauration concernant le RHR et les générateurs diesel de secours. Je suppose que vous fonctionnez toujours sur cette base, à l'heure actuelle.

Si je regarde l'exemplaire que j'ai emprunté, le «Rapport sur les dispositions pour la gestion des accidents » donne les grandes lignes de chaque document. Ce genre de documents, les manuels de conduite en cas d'accident ou l'AMG, sont des choses que l'équipe de quart possède, j'imagine. En avez-vous aussi des exemplaires à la cellule de crise?

R : Oui.

$\mathbf{Q}$ : Ces manuels de conduite, les AOP, les EOP et l'AMG, vous les avez près de vous pour pouvoir éventuellement les consulter?

$\mathbf{R}$ : Je suis sûr que dans la salle de crise du bâtiment administratif, ils y étaient tous. Mais en ce qui concerne la cellule de crise du bâtiment antisismique, je n'ai pas vérifié. Il me semble bien ne pas les y avoir vus. Peut-être n'y étaient-ils pas?

$\mathbf{Q}:$ : $i$ les choses ne s'étaient pas passées de cette manière, vous auriez dû former la cellule de crise dans le bâtiment administratif, au lieu du bâtiment antisismique, n'est-ce pas ?

R : Oui.

$\mathbf{Q}$ : Après le descriptif de chacun de ces manuels, il y a un paragraphe «critères de passage d'une procédure à l'autre ». "Le passage d'une procédure à l'autre est défini de manière claire suivant l'état de la tranche et les valeurs des paramètres de celle-ci. L'adoption des EOP sera conditionnée par des évènements comme l'arrêt automatique du réacteur ou une pression anormalement élevée de l'enceinte de confinement», peut-on lire. Concernant la gestion des accidents et la mise en œuvre de l'AMG, il est spécifié que «le passage des EOP à l'AMG est conditionné par le début de la dégradation du cœur » et que cette dégradation est appréciée 
d'après le taux de rayons gamma ${ }^{38}$. Dans le cas qui nous occupe, pendant un certain temps vous n'avez pas pu utiliser le CAMS (Containment Atmosphere Monitoring System) ${ }^{39}$ à cause de l'électricité.

$\mathbf{R}$ : Tout ça, c'est pure théorie. C'est parce qu'ils veulent théoriser tous les cas possibles qu'ils expriment ça de cette manière. Dans la réalité, concrètement, je n'ai jamais eu ce genre de préoccupation en tête, je n'ai jamais décidé de quoi que ce soit en me référant à ces discussions sur le passage d'une procédure à l'autre. On avait perdu toutes les alimentations électriques. On n'avait plus le moyen de refroidir. Dans ces conditions, il était plus qu'évident qu'on allait droit à l'accident sévère ou même encore pire. La discussion du passage d'une procédure à l'autre ne m'a absolument pas effleuré. Dès l'instant où on avait perdu toutes les sources de courant alternatif, j'avais jugé que cela correspondait à un phénomène de l'ordre de l'accident sévère. Toutes ces discussions sur les procédures, je les ai ignorées.

$\mathbf{Q}$ : Vous voulez dire que vous ne vous êtes pas préoccupé de savoir si vous alliez adopter l'AMG ou pas. Pourtant, si on regarde cette chronologie, vous perdez le courant alternatif à $15 \mathrm{~h} 37$ et, ici, à 17h12, il est écrit que vous entreprenez une action dans le cadre de l'AMG. Vous n'avez pas eu de discussion entre temps pour savoir si vous alliez passer à l'AMG ? Ou bien, vous avez jugé qu'ayant perdu toute alimentation de courant alternatif et anticipant ce qui allait suivre, il fallait agir en conséquence?

$\mathbf{R}:$ Oui, c'est ça.

$\mathbf{Q}$ : Toutes les règles sont bien explicitées sur le papier. Vous n'avez jamais pris un moment pour vous demander si votre réponse correspondait bien à ces principes?

$\mathbf{R}$ : Non. Je suppose qu'un amateur l'aurait sans doute fait. Mais pour nous, si on réfléchit aux circonstances, normalement, il n'y a pas de place pour ce genre de discussion.

$\mathbf{Q}:$ À propos, avant 2002, vous ne disposiez pas d'un AMG?

R : Non:

$\mathbf{Q}$ : Même en dehors de Fukushima Daiichi?

$\mathbf{R}$ : Non, nulle part.

$\mathbf{Q}$ : Même en dehors de TEPCO ?

$\mathbf{R}$ : Non. C'était une première au Japon.

$\mathbf{Q}$ : Et donc votre accident a été la première expérience. Vous avez été le premier à affronter un accident muni de ces procédures. Le manuel préconise, après avoir constaté le début de la dégradation du cœur, de continuer à mesurer les rayons gamma et d'en déduire l'état de dégradation. Dans la réalité, ça n'a pas été possible. Comme quoi tout ne peut pas forcément se dérouler suivant le scénario de ces manuels.

$\mathbf{R}$ : Comme vous dites.

$\mathbf{Q}$ : Plus loin, on trouve des explications concernant le manuel de restauration, qui « est à adopter dès que l'organisme de soutien juge la restauration du RHR et des générateurs diesel de secours nécessaires, qu'il y ait dégradation du cœur ou pas ». Avez-vous utilisé ce manuel à un moment ou un autre?

R : Là encore, je suppose que quand on veut mettre les choses au clair sur le papier, ça donne ça. Mais, concrètement, pour nous, sur le terrain, les générateurs ne fonctionnaient pas, le RHR ne fonctionnait pas non plus. Face à ces réalités, il n'est plus question de manuels. La situation était déjà suffisamment grave pour qu'on y apporte tout de suite une réponse adéquate. L'urgence, c'était les réponses. C'est tout. 
$\mathbf{Q}$ : Ce que je voudrais savoir, c'est comment cela s'est passé dans la réalité par rapport à ces manuels. Car, si ces AMG et autres textes ne répondent pas vraiment à ce qui se passe dans la réalité, il faudrait se demander s'il ne faudrait pas les réviser, qu'il s'agisse de TEPCO ou de tout le Japon.

Par exemple, la restauration des générateurs diesel de secours qui est citée à la page 24, on en parle dans I'AMG. Sur le moment, vous êtes-vous posé la question de savoir si vous alliez utiliser ces procédures ou pas?

$\mathbf{R}$ : Évidemment, j'ai donné l'ordre de faire en sorte qu'ils marchent. Pour résumer, il y a d'abord le tsunami. En fait, sur le moment, on ne sait pas si les DG (Diesel Generators) continuent à fonctionner ou pas. On est dans le bâtiment antisismique. On sait qu'il y a eu un tsunami. Mais on ne sait même pas de quelle ampleur il a été. Bien sûr qu'on pense qu'il va falloir rétablir les générateurs de secours. Ça, on y pense depuis le début. Mais il faut d'abord aller sur le terrain voir l'ampleur des dégâts. On ne sait même pas si on va pouvoir les réparer ou pas. On y pense et pendant qu'on y pense, arrive la nouvelle que tout a été inondé par l'eau du tsunami. Avant même de connaître la situation exacte de chaque DG, on sait, d'office, que si ça a été inondé, on ne pourra plus les utiliser. Parce que, fondamentalement, ce sont des générateurs électriques. Il est normal de penser qu'ils ne fonctionneront plus. Il fallait, au contraire, partir du principe que les DG étaient inutilisables.

Q: Quand des générateurs ont été noyés, on peut enlever l'eau et les rendre réutilisables ?

$\mathbf{R}$ : Naturellement, on y a pensé. Mais c'était l'eau du tsunami. On avait déjà eu une expérience du même genre en $1991^{40}$. C'était sur la tranche 1 . Après ça, on savait très bien qu'une fois sous l'eau, les générateurs étaient inopérants pour un bon bout de temps. Là encore, c'était de l'eau de mer. Quand un générateur a été noyé par de l'eau de mer, il faut environ six mois pour le redémarrer. Il faut le démonter entièrement, le sécher, changer des pièces. On savait pertinemment qu'un générateur inondé par de l'eau de mer ne pouvait pas être réparé instantanément.

$\mathbf{Q}$ : Et quelle avait été la cause de cette inondation en 1991 ?

$\mathbf{R}$ : La conduite du réseau " eau de mer» de la tranche 1. On avait fait un rapport détaillé à l'époque. Vous pouvez aller le consulter. Il y a le bâtiment réacteur ici et le bâtiment turbine là. Il y a là le système RHR. On prend de l'eau de mer par le RHR pour alimenter le système d'échange de chaleur. Oh, pardon. C'est le réacteur 1 , donc il n'y a pas de RHR. Le réacteur 1 a aussi un circuit d'eau de mer pour le CCS (Containment Cooling System) ${ }^{41}$, qui est l'équivalent du RHR. Vous pouvez considérer que ça fonctionne à peu près de la même manière. Cette eau, à la fin du circuit, retourne à la mer. Les tuyaux, ici, sont enterrés. L'eau de mer arrive dans le bâtiment turbine par ces tuyaux qui sont encore enterrés à l'entrée du bâtiment. C'est là qu'il y a eu des fuites et que tout a été inondé.

Le DG du réacteur 1 se trouve dans le bâtiment turbine. Au moment de l'inondation, l'eau a pénétré jusque dans la salle du DG. Je pense, même aujourd'hui, que c'était un accident très grave. Cela n'a bien sûr aucun rapport avec ce qui nous arrive aujourd'hui, mais parmi les accidents qui s'étaient produits jusque-là au Japon, je le considère comme le plus grave. Ce que nous avons fait, c'est que nous avons remplacé tous les tuyaux du circuit « eau de mer » qui étaient directement enfouis dans la terre par un réseau de tunnels qui permet de faire de la maintenance. Jusque-là, on se contentait de creuser la terre et d'enfouir les tuyaux. Là, on a fait une conduite, enfin, on a construit un tunnel dans lequel sont installés les tuyaux pour qu'on puisse les 
entretenir. Comme ça, l'eau déborde dans le tunnel. C'était une réponse possible au risque d'inondation. C'est ce que nous avons tout de suite fait. À l'époque, j'étais au siège. J'ai trouvé l'expérience de cette inondation très inquiétante. Ce que nous avons vécu en mars a quelque chose de similaire. L'eau de mer qui envahit les bâtiments turbine. Seulement, en 1991, il n'y avait pas eu de séisme. Même si le DG ne fonctionnait plus, il y avait les alimentations externes. On n'avait pas eu de souci au niveau de l'alimentation électrique. Cela nous avait permis d'envisager toutes sortes de solutions. Mais en tant qu'accident, je trouve qu'il y a des similitudes. J'étais au siège et j'ai suivi de près la construction de ces tunnels de service, alors je m'en souviens très bien.

D'après cette expérience que j'avais vécue, il était très mauvais que nous soyons inondés par de l'eau de mer.

Q: En 1991, le DG s'est trouvé inondé par de l'eau de mer. Est-ce que cela aurait été différent si cela avait été de l'eau douce?

$\mathbf{R}$ : C'est la même chose. Évidemment, l'eau de mer contient du sel et c'est mauvais du point de vue de la corrosion. Avec de l'eau douce, peut-être qu'après évacuation de l'eau et séchage, certains appareils pourraient repartir. Avec de l'eau salée, après séchage, le sel reste et provoque dans une large mesure de la corrosion, alors, de ce point de vue, l'eau douce est peut-être préférable. Mais, de toute façon, il est très difficile de faire redémarrer un appareil électrique qui a pris l'eau.

$\mathbf{Q}$ : Alors, même si au bout du compte on arrive à restaurer tout ça, il faut prévoir des mois d'attente?

$\mathbf{R}$ : Oui. C'est pour ça que j'ai pensé qu'on ne pouvait plus rien attendre des générateurs de secours.

Q: Vous avez tout naturellement pensé lors de l'accident de mars que la restauration des générateurs diesel de secours ne serait de toute manière pas possible en quelques jours.

De plus, pour les systèmes d'évacuation de la chaleur résiduelle, notamment pour le CCS du réacteur 1 , les pompes d'eau de mer n'étaient plus opérationnelles. Si vous aviez réussi à réparer ces pompes, est-ce que le reste du système aurait fonctionné ?

$\mathbf{R}$ : Non. Les pompes de chaque ECCS ont une alimentation électrique. Il y a ce qu'on appelle un tableau électrique d'urgence dans chaque bâtiment turbine. Si ce tableau a été immergé dans de l'eau de mer, il n'est plus opérationnel. Alors, même si le circuit « eau de mer » en lui-même avait été intact, cela aurait pris beaucoup de temps pour faire fonctionner les installations.

$\mathbf{Q}$ : Ce sont les P/C (Power Center) ${ }^{42}$, comme le $2 \mathrm{C}$ ou le 4D, n'est-ce pas ? Je me rappelle que vous y aviez amené par la suite des camions générateurs et tiré des câbles pour pouvoir partager l'énergie avec le réacteur 1.

Je suppose que vous aviez des priorités, mais si on s'intéresse juste au système RHR, par exemple, supposons que vous réussissiez à réparer la pompe du circuit « eau de mer », si on envoyait l'énergie des camions générateurs en passant par ces $\mathrm{P} / \mathrm{C}$, est-ce que le RHR pourrait fonctionner?

$\mathbf{R}$ : Si on partait du 2C, il n'y aurait probablement pas assez de tension. Vous savez, le RHR est un système assez lourd. Je pense que c'était au-delà des capacités du $P / C$, il n'aurait pas suffi. C'est pour ça que j'ai pensé, dès l'abord, que ce serait assez difficile en partant de là.

Franchement, je crois qu'on avait branché tout ce qui pouvait l'être sur ce P/C. Et s'il n'y a pas le RHR, c'est que ça ne suffisait pas à le mettre en marche. 
Q : Cela signifie que, dans ce cas, il faut se rabattre sur les M/C (Metal-Clad Switch Gear) ${ }^{43}$ ?

R : Oui.

$\mathbf{Q}$ : Ce qui veut dire que, au moment qui nous préoccupe, que ce soit le RHR ou le CCS pour le réacteur 1, leur utilisation n'était pas possible.

$\mathbf{R}$ : Bien sûr, leur réhabilitation fait partie des priorités, je le sais, j'en ai conscience. Mais au regard de la situation, on ne peut que penser que les chances de concrétiser ce souhait sont très minces.

Q: Si je comprends bien, vous n'en êtes probablement plus au stade de vous conformer ou non aux directives d'un manuel de restauration.

$\mathbf{R}:$ Non.

$\mathbf{Q}$ : Nous allons un peu avancer. À propos de la gestion de ces manuels...

$\mathbf{R}$ : Excusez-moi de vous interrompre. Ces manuels, à la base, ont été probablement rédigés en supposant qu'il n'y ait qu'une seule panne à la fois. Par exemple, qu'il y ait un générateur diesel sur plusieurs qui a des problèmes ou que le RHR donne du souci. Il ne tient absolument pas compte d'une situation où tout tombe en panne d'un seul coup en même temps. Disons que les données diffèrent complètement au départ.

$\mathbf{Q}$ : Effectivement, si on se penche, par exemple, sur la perte du courant alternatif, on en parle dans les EOP. Le problème apparaît tout à coup dans le document et si on regarde ce qui est préconisé, on se rend compte que la procédure consiste à aller emprunter de l'énergie auprès d'une autre tranche et de procéder au plus vite à la réparation. Or, si les autres tranches aussi sont atteintes, la procédure tombe complètement à plat. Je crois que, là encore, l'accident que vous avez connu dépassait largement tout ce qui avait été imaginé.

$\mathbf{R}$ : Ce qu'il ne faut pas oublier, c'est que, dans notre cas, nous nous sommes trouvés confrontés à plus qu'une perte totale d'alimentation électrique externe. Ce n'était pas juste une question d'alimentation électrique. Si cela avait été le cas, il aurait suffi d'aller chercher les sources ailleurs, relier les tranches à des câbles à l'extérieur de la centrale et on aurait eu l'électricité. Mais nous, nous avions en plus le problème de tout ce qui se trouvait en aval. Même si on amène l'électricité, si, en aval, les tableaux électriques et autres, qui permettent de distribuer cette électricité ne fonctionnent plus, rien ne marche. Si on avait eu un évènement, tel qu'imaginé dans ces manuels, une simple perte des alimentations externes, il aurait suffi d'aller chercher sans délai une autre source, élaborer un réseau d'urgence, on aurait pu s'en sortir d'une manière ou d'une autre. C'est ça que les auteurs des manuels ont en tête quand ils parlent de perte des alimentations électriques externes. Mais nous, ce que nous avons connu, à la limite, c'est une perte des alimentations externes doublée d'une perte de la distribution électrique interne. Alors, j'ai toujours eu le sentiment que décrire notre situation par la simple expression «perte des alimentations électriques externes » pouvait susciter des malentendus.

$\mathbf{Q}$ : Et les solutions face à ce genre de situation, la perte de la distribution électrique interne, comme vous dites, ne sont pas évoquées ici?

$\mathbf{R}:$ Non.

Q: Cela veut dire, à votre avis, que ce genre d'évènement, que vous avez connu non seulement sur la tranche 1, mais sur toutes les autres, n'a naturellement pas fait partie des 
scénarios envisagés jusqu'ici ? Puisque personne n'avait réfléchi aux solutions au préalable, il a fallu que vous, sur le terrain, le fassiez par vous-même.

$\mathbf{R}$ : Comme on l'a déjà évoqué, le siège avait placé dans les priorités le rétablissement urgent des alimentations électriques. J'avais beau leur dire, ils ne comprenaient pas. Il y avait beaucoup d'idiots qui croyaient que c'était la même chose qu'une perte d'alimentation externe habituelle, qu'il suffisait de rétablir les alimentations externes pour qu'on puisse se débrouiller d'une manière ou d'une autre à l'intérieur, alors que les dégâts étaient énormes à l'intérieur. Je n'ai pas cessé de leur dire que même si on arrivait à amener l'électricité, il y avait très peu d'endroits où on pourrait la distribuer, mais ils n'arrivaient pas à l'assimiler.

$\mathbf{Q}$ : Alors, en ce qui concerne ces manuels, il faut conclure qu'ils peuvent suggérer des solutions, mais qu'il est difficile de les appliquer telles quelles?

$\mathbf{R}$ : On ne peut pas du tout les utiliser.

$\mathbf{Q}$ : Ensuite, pour ce qui est de la gestion de ces manuels, on en a un peu parlé tout à l'heure, ils sont donc stockés dans les salles de contrôle et la salle de crise. Comme, à la suite du séisme, la cellule de crise a été constituée dans le bâtiment antisismique, vous n'êtes pas certain que les SOP (Severe Accident Operating Procedures) ${ }^{44}$ ou EOP s'y trouvaient bien.

$\mathbf{R}$ : Oui, c'est un point qu'il faudra que vous vérifiiez.

$\mathbf{Q}$ : D'accord. Le «Rapport » parle maintenant de formation. Le paragraphe 6-1 traite des personnes à former. "Tous les agents de conduite, ainsi que tous les membres des organismes de soutien de l'établissement organisant ces formations sont visés ». Suivent les indications concernant le contenu des formations. "Les organismes de soutien devant connaître l'état des tranches et étudier les solutions en cas de problème [...], leurs membres doivent avoir une connaissance globale du contenu de l'AMG, ainsi que du comportement des tranches en cas d'accident grave. Cette formation consiste à effectuer un stage en salle sur les connaissances élémentaires concernant la gestion d'accident. Les membres en charge des évaluations techniques, les responsables des équipes, tous les membres ayant besoin de posséder des connaissances plus spécifiques suivront des formations plus poussées ». On lit au dernier paragraphe: "tout membre des organismes de soutien effectuera ces formations en salle une fois durant la période où il occupe ce poste. Pour vérifier globalement l'efficacité de l'ensemble de l'organisation, on effectuera une fois par an un entraînement à la gestion d'accident ». Vous suivez donc des formations en salle.

$\mathbf{R}$ : Oui, nous en faisons.

$\mathbf{Q}$ : Et ça se passe comment?

$\mathbf{R}$ : Une personne du groupe "formation et entraînement », qui fait partie de l'équipe qui élabore ces programmes de formation et qui connaît bien le sujet, sert de prof. Il explique pendant une heure ou une heure et demie le contenu de l'AMG.

$\mathbf{Q}$ : Quand on regarde le texte, il dit que cette formation est à suivre une fois durant la période où on occupe le poste. Qu'est-ce que ça veut dire?

$\mathbf{R}$ : C'est parce qu'il y a de nouvelles nominations.

$\mathbf{Q}$ : Alors les nouveaux arrivent et, s'ils sont choisis pour faire partie des organismes de soutien, ils suivent la formation?

$\mathbf{R}$ : Oui. On leur dit de suivre la formation. Mais je ne sais pas dans quelle mesure l'équipe «formation" suivait les directives. Je suis sûr que ces stages étaient organisés. Mais je ne sais pas si, quand quelqu'un arrivait, on lui faisait la formation dès son arrivée. De toute façon, la formation était organisée une fois par an. Mais était-elle faite en fonction du moment où les nouveaux arrivaient? Je n'en ai pas idée, concrètement. 
$\mathbf{Q}$ : Qui était en charge de l'organisation de ces stages?

$\mathbf{R}$ : Le groupe «formation et entraînement ».

$\mathbf{Q}: \|$ est incorporé dans un département?

$\mathbf{R}$ : Pas un département à proprement parler. C'est un endroit qui élabore, par exemple, les programmes de formation. Je crois que c'est chapeauté par quelque chose qui s'appelle le « centre d'entraînement ». Oui, ils n'arrêtent pas de changer de nom, chez nous. À la tête de ce centre, il doit y avoir un chef du niveau d'un directeur adjoint. Ça constitue une entité en soi.

En fait, il n'y a pas que ce centre. Il y a aussi un service qui élabore l'ensemble de toutes les formations, y compris la formation des nouvelles recrues, les employés qui entrent chez nous pour la première fois. Mais, comme le contenu des formations englobe l'exploitation et les problèmes techniques de sûreté, le département «engineering » est aussi partie prenante.

Q: Vous avez donc ces formations en salle et puis, plus concrètement, des exercices de gestion d'accident.

$\mathbf{R}$ : Oui. Nous faisons un exercice une fois par an.

$\mathbf{Q}:$ Et de quand date le dernier entraînement?

$\mathbf{R}$ : Le dernier ? Janvier ou février.

$\mathbf{Q}$ : J'ai interviewé une personne du département «prévention et sûreté » qui m'a dit qu'il y en avait eu un vers la fin février. Je ne sais pas si ça correspond à ce que vous avez en tête, mais il s'agissait d'un exercice du côté de la tranche 3 ou 5 , il me semble.

$\mathbf{R}$ : Oui, c'est celui que nous faisons une fois par an.

$\mathbf{Q}:$ Vous aussi, vous y participez ?

$\mathbf{R}$ : Bien sûr, je fais le chef de la cellule de crise. On fait ça tout comme aujourd'hui.

$\mathbf{Q}$ : Vous êtes donc dans une salle de crise, vous entourez la table ronde et les chefs de groupe participent aussi tout au long de l'exercice?

$\mathbf{R}$ : Oui, tout à fait de la même manière qu'aujourd'hui. Le siège participe également.

$\mathbf{Q}:$ Et le département?

$\mathbf{R}$ : La dernière fois, en février, le département avait aussi participé.

$\mathbf{Q}$ : Et le centre hors site?

$\mathbf{R}$ : Non, on n'avait pas été jusqu'à ouvrir un centre hors site. Mais on a fait comme si on en avait ouvert un. Mes souvenirs sont un peu confus, mais, en février, il me semble que des représentants du département et de l'État sont vraiment venus au pseudo-centre hors site en hélicoptère. Il $\mathrm{y}$ a des fois où on ne fait pas jusque-là. On fait semblant, quelqu'un joue le rôle de ces représentants et téléphone à leur place, par exemple.

$\mathbf{Q}:$ Je suppose que quand vous faites ces exercices, vous avez un scénario. Qui élabore ces scénarios?

$\mathbf{R}$ : Les scénarios? Je pense que ce sont les «messieurs sûreté ». Chez nous, ce doit être le département "qualité et sûreté ». Il y a une équipe " sûreté » au département «qualité et sûreté » de la centrale. C'est cette équipe qui, en concertation avec le département « sûreté » du siège, les imagine. 
$\mathbf{Q}$ : Lors de l'exercice, des évènements s'enchaînent suivant le scénario et vous devez y répondre, c'est bien ça? Et le nombre de participants?

$\mathbf{R}$ : Presque toute la centrale participe.

$\mathbf{Q}$ : Durant cet entraînement, que font les personnes qui sont de quart et pilotent les réacteurs 2 et 3 ?

$\mathbf{R}$ : Malheureusement pour elles, c'est l'équipe du réacteur qui a été mis en scène qui va travailler en priorité.

C'est très compliqué. Vous savez que chez nous, dans cette centrale, nous travaillons par roulement. Nous avons cinq équipes qui tournent. L'équipe qui est de quart le jour de l'exercice bénéficie de l'entraînement, mais les quatre autres, malheureusement, ne font pas cette expérience. Seulement, eux, sont des agents de conduite. Les membres des organismes de soutien font un exercice par an, comme je vous l'ai dit. Mais les agents de conduite ont beaucoup plus de formations. Ils font des exercices sur des simulateurs tout au long de l'année. Sur un an, il est prévu qu'ils passent environ un cinquième de leur temps en formation.

Durant ces formations, ils étudient non seulement les EOP, mais les procédures face à un accident grave, etc.

$\mathbf{Q}:$ C'est ce qu'on appelle les quarts d'entraînement ?

$\mathbf{R}$ : Oui, c'est ça, les quarts d'entraînement.

$\mathbf{Q}$ : Et où font-ils ces entraînements ?

R : L'un des lieux est celui appelé "ancien bâtiment administratif ». Il y a là un simulateur, le simulateur sur site. On y a installé exactement le même tableau de conduite que dans la salle de contrôle de la centrale. C'est là qu'ils s'exercent.

Ensuite, il y a le BTC (B/Training Center) ${ }^{45}$, le centre d'entraînement BWR (Boiling Water Reactor $)^{46}$. Là, il me semble qu'il y a quatre simulateurs. Justement, lorsqu'ils sont de quart d'entraînement, les agents de conduite viennent s'exercer là. C'est comme ça qu'ils étudient les procédures face aux accidents graves.

$\mathbf{Q}$ : Comment ça marche? Quand une des cinq équipes se trouve en quart d'entraînement, elle est en entraînement pendant un certain temps? Pendant un mois ou deux mois d'affilée?

$\mathbf{R}$ : Non. Chez nous, les équipes font « jour, jour », ensuite « nuit, nuit », puis « repos, repos " et on tourne comme ça avec cinq équipes. Si on suit ce rythme, il y a un moment où ça libère presque une semaine. C'est là que l'équipe est en quart d'entraînement. C'est pour ça que je vous ai dit tout à l'heure que les équipes étaient en formation un cinquième du temps. On fait un roulement à cinq équipes et, dans le cycle, on arrive à ménager un temps pour la formation. Attention, toute la formation ne se fait pas obligatoirement sur les simulateurs. Il y a aussi des formations classiques en salle et bien d'autres formes d'exercices, mais les équipes s'entraînent. Et dans leur programme d'entraînement, il y a aussi la formation à la gestion des accidents graves.

Ce qu'il faut comprendre, c'est que toutes les manœuvres de conduite sont effectuées par des agents de conduite. Je vous le répète, la base, quand on est face à des incidents, c'est qu'on essaie de récupérer la situation grâce à l'action des agents de conduite. C'est pour ça que leur formation est si importante. C'est pour les autres, même si certains sont des spécialistes de la sûreté, qu'ils en savent plus long sur la 
sûreté que les agents de conduite ou qu'ils ont des connaissances bien plus vastes, qu'on organise l'exercice dont on a parlé tout à l'heure. C'est l'occasion, justement, d'apporter ces compétences en soutien de l'équipe de quart et de voir si le tout fonctionne en tant qu'ensemble.

$\mathbf{Q}$ : Alors, si je résume, les équipes de quart ont des formations à part, plus ciblées sur la conduite. Leur programme étant plus fourni, elles ont bien plus qu'une formation par an et ont l'obligation de se former au moment où elles sont de quart d'entraînement. C'est bien ça?

R : Oui.

Q: Ensuite à la rubrique "instructeurs», je lis "on accueillera comme instructeurs des personnalités, appartenant ou non à l'entreprise, qui possèdent des connaissances spécialisées dans la gestion des accidents, voire des accidents graves». Est-il arrivé que des instructeurs extérieurs soient intervenus?

$\mathbf{R}$ : Pour les instructeurs extérieurs, il y a, par exemple, les formateurs du centre d'entraînement BWR, dont on a parlé tout à l'heure. Je pense que ces derniers temps, on s'est surtout reposé sur eux et sur les spécialistes de notre entreprise.

Q: Le « Rapport » mentionne qu'il est également fait «appel aux spécialistes des fabricants des tranches ou aux membres des groupes techniques assurant la sûreté des tranches au sein de la centrale».

$\mathbf{R}$ : Je crois qu'on fait principalement appel pour les formations aux seconds, les personnes des groupes techniques qui assurent la sûreté des tranches. Les spécialistes des fabricants sont de bons connaisseurs de l'ensemble de l'architecture des systèmes, mais concernant le comportement des tranches ou leur conduite, c'est plus compliqué, parce qu'il y a l'électricité. Je ne veux pas dire que les spécialistes des fabricants ne sont pas bons. Sur tout ce qui est architecture des systèmes, ils sont imbattables. Et il arrive bien entendu qu'on les sollicite pour venir parler de ces choses-là. Mais, dans l'ensemble, on voit plus souvent les seconds.

Q: Concernant les outils de formation, sont évoqués ici, "en dehors des recueils de procédures, des textes traitant de phénomènes et de comportements des tranches pouvant survenir lors d'accidents sévères, ou des commentaires sur les procédures de gestion d'accidents ou le contenu de l'AMG ». Vous avez ce genre de documents?

R : Oui.

Q: Ensuite, "on utilisera aussi des animations commentant l'évolution des évènements, des EAO (enseignements assistés par ordinateur) sous forme d'exercices interactifs testant la compréhension du stagiaire, des vidéos permettant de comprendre les grandes lignes de la gestion d'accidents, des programmes informatiques permettant de simuler des accidents sévères ». Ces logiciels, c'est TEPCO qui les élabore lui-même?

$\mathbf{R}$ : Oui, à la base, c'est TEPCO qui les conçoit.

$\mathbf{Q}$ : C'est le siège ? Ou c'est quelqu'un d'autre?

$\mathbf{R}$ : Principalement le siège. Les outils de formation basique sont du ressort du siège. Il les distribue ensuite aux différentes centrales.

$\mathbf{Q}:$ Y a-t-il au sein du siège un département qui s'occuperait de théoriser et de former à une gestion sûre des centrales nucléaires ?

$\mathbf{R}$ : C'est le département "gestion des centrales nucléaires ». Il a dû changer de nom depuis. Vous savez, TEPCO est une entreprise stupide qui n'arrête pas de changer le nom de ses services. Bref, c'est la section qui supervise le travail des agents de 
production ou des équipes de quart. C'est elle qui gère l'ensemble de leur travail, y compris la formation.

$\mathbf{Q}$ : Tiens, par exemple, quand quelqu'un de l'équipe de quart veut passer chef d'équipe, responsable de groupe, y a-t-il des stages spécifiques?

$\mathbf{R}$ : Bien sûr. Il y a le test de chef de quart. C'est un examen qui est organisé au niveau national. Il ne dépend pas de TEPCO. L'examen comporte des questions d'un niveau assez élevé. Il y a des épreuves écrites et d'autres, orales, qu'on passe avec des spécialistes de la question. On essaie de vérifier les capacités et les qualités du candidat. Et une connaissance approfondie de la gestion d'un accident sévère fait, évidemment, partie des choses qu'on teste.

$\mathbf{Q}:$ Vous dites que c'est un examen organisé par l'État. C'est donc un examen qui donne un diplôme d'État?

$\mathbf{R}$ : En tout cas, il donne droit à un diplôme reconnu par l'État. En fait, c'est un examen dont l'organisation est déléguée par l'État à un organisme extérieur. La réussite à cet examen est requise pour devenir chef de quart, aussi bien chez TEPCO qu'ailleurs dans toutes les centrales nucléaires japonaises.

$\mathbf{Q}: \|$ arrive parfois que quelqu'un qui est chef de quart soit transféré dans une autre centrale, par exemple?

R : Oui.

$\mathbf{Q}$ : Dans ce cas, il continue à travailler en tant que chef de quart, même s'il a obtenu son habilitation ailleurs, c'est ça?

R : Oui.

$\mathbf{Q}$ : Et pour les seconds? II y a un examen spécifique?

R : Non, pour devenir sous-chef de quart, il n'y a pas d'examen. Vous savez, pour travailler dans une équipe de quart, on commence d'abord par être assistantopérateur. C'est celui qui surveille toutes sortes d'instruments dans la salle de commande. Ensuite, on devient opérateur. Mais seulement au bout de huit ans, à peu près. Là, on est enfin assis devant les tableaux de la salle de contrôle et on conduit. Pour passer d'assistant-opérateur à opérateur, d'étape en étape, on suit des formations au centre d'entraînement BWR, comme je vous l'ai dit tout à l'heure. Il faut que l'assistant atteigne un niveau qu'on estime suffisant pour passer opérateur. Après, il travaille en tant qu'opérateur pendant quelques années. On l'observe dans son travail, il suit encore des formations au centre d'entraînement et, selon les résultats, il passe second ou pas.

Il n'existe donc pas d'habilitation en tant que telle pour devenir sous-chef de quart. On regarde surtout la manière dont le candidat a accumulé l'expérience, s'il a bien suivi toutes les formations préconisées, si ses choix dans la conduite habituelle du réacteur sont appropriés. S'il satisfait à ces critères, on va le nommer sous-chef. Dans ces conditions, je crois qu'on peut dire que ce sont des hommes qui montrent des qualités suffisantes dans la conduite.

Q: : ji je suis vos explications, pour devenir sous-chef, il faut accumuler de l'expérience sur plusieurs années.

$\mathbf{R}:$ C'est exactement ça. 
Q: : Est-ce que ça veut dire qu'un candidat au poste de sous-chef doit avoir exercé toute sa carrière dans l'équipe de quart?

$\mathbf{R}$ : A priori, c'est quelqu'un qui a toujours travaillé dans une équipe de quart.

$\mathbf{Q}$ : Est-ce qu'il peut y avoir des transferts durant ce temps?

$\mathbf{R}$ : Oui, cela arrive. Il peut y avoir des transferts entre Fukushima Daini et nous, ou Kashiwazaki et nous.

$\mathbf{Q}$ : Mais il ne changera pas de métier. II sera toujours incorporé à l'équipe de quart?

$\mathbf{R}$ : La plupart du temps.

Q: Vous organisez donc des formations spécifiques pour les hommes de quart. J'imagine que vous avez des manuels spécifiques pour eux, qui sont différents de ceux utilisés par le personnel de soutien.

$\mathbf{R}$ : Oui, ils disposent de cent fois plus de documents à étudier. Ils doivent apprendre concrètement toutes les étapes des opérations, connaître aussi les principes qui commandent les différents systèmes, par exemple, et ils disposent de documents en conséquence pour pouvoir les étudier.

$\mathbf{Q}$ : Dites-moi, tout au début, comment ça se passe ? Vous entrez chez TEPCO. Vous êtes nommé au département nucléaire. Qu'est-ce qui se passe là ? On vous dit, tout à trac, vous êtes fait pour la conduite, etc. ?

R : Il y a d'abord ce que veut le candidat. La conduite, c'est quand même un métier très spécial. C'est un métier gratifiant, dans ce sens que le pilote, c'est quelqu'un qui va conduire une tranche en direct. Il y a des gens qui aiment cette idée, des gens qui entrent chez TEPCO, parce qu'ils ont envie de devenir pilote. Ceux-là, on les met tout de suite à la conduite. Après, c'est plus compliqué. Il y a les simples bacheliers ${ }^{47}$ et ceux qui ont fait l'université. C'est un point un peu délicat à évoquer. Les bacheliers, s'ils sont sérieux dans leur travail, deviennent chef de quart. Chef de quart, du point de vue statut, c'est un cadre spécial. Mais, s'il change de fonction, s'il s'en va dans une autre section, peut-il prétendre à un poste de chef de service, de cadre spécial ? Eh bien, c'est un niveau où il y a surtout des gens qui sont sortis de l'université. Et, pour les simples bacheliers, ça va être difficile. Heureusement, il y a des gens qui ont vraiment pour vocation la conduite et ce sont ceux-là qui, en devenant chef de quart et en le demeurant, soutiennent tout ce secteur.

$\mathbf{Q}$ : Vous voulez dire que ceux qui travaillent comme chef de quart chez vous, sont plutôt des bacheliers?

R : La plupart du temps. Ils sont presque tous bacheliers. À Fukushima Daiichi, jusqu'à il y a deux ans, il y avait un chef de quart qui avait fait des études universitaires. Mais aujourd'hui, il n'y en a plus un seul, à mon avis. Ce que je veux dire, c'est que quand vous avez un diplôme universitaire, on vous mute de poste en poste. À ce moment-là, vous avez forcément moins le temps d'étudier les choses que nous avons évoquées tout à l'heure ou de connaitre le terrain. Si on vous nomme second ou chef de quart dans ces conditions, vous allez vous retrouver avec des gens bien plus expérimentés. De plus, vous allez être nommé jeune. C'est un phénomène qui existe dans tous les domaines, mais, face à des gens qui en connaissent beaucoup plus long que vous sur la conduite, vous allez avoir du mal à diriger toutes ces personnes. Les postes de pilote sont des fonctions qui tendent à devenir la chasse gardée des bacheliers spécialistes de la conduite. 
$\mathbf{Q}$ : Je reviens à la formation. Dans le paragraphe "Actualisation et amélioration de la formation», on lit, "la formation au jour le jour est indispensable à l'efficacité de la gestion des accidents ». Cette formation au jour le jour, vous l'organisez régulièrement. Ensuite, «même après la mise en place des règles de gestion des accidents, parallèlement à l'organisation pérenne des formations, il est prévu d'apporter des modifications et des améliorations introduisant de meilleures méthodes de formation ou de nouvelles connaissances dans les programmes ». Je suppose que quand vous faites l'expérience d'un évènement nouveau, je pense par exemple à l'incendie qui s'est déclaré à Kashiwazaki au moment du séisme au large de Chûetsu, vous incorporez dans la formation des réflexions sur ce qui a été fait, etc.

Dans des cas comme ça, est-ce que les centrales sont sollicitées pour donner leur avis sur les modifications?

$\mathbf{R}$ : Oui, pour ce qui est des mesures concrètes. Mais c'est le siège qui se préoccupe de savoir s'il faut modifier les programmes pour refléter tel évènement. Comme ce sont des questions qui concernent les trois centrales du groupe, c'est le siège qui imprime le mouvement en disant, " on va réviser ce texte » ou « on va revoir le contenu des cours ».

$\mathbf{Q}$ : Et concrètement, qui se charge d'ajouter ceci ou cela?

$\mathbf{R}$ : Le texte de base est écrit par le siège. Seulement, quand nous voulons l'appliquer concrètement à chaque tranche, nous le réinterprétons à la centrale, puisque, comme on l'a dit tout à l'heure, chez nous, par exemple, la tranche 1 et la tranche 2 sont différentes. C'est à nous, dans chaque centrale, d'adapter le texte.

$\mathbf{Q}$ : Et dans ce cas-là, si chaque centrale doit le réadapter, qui s'en charge à la centrale? C'est le département « qualité et sûreté »?

$\mathbf{R}$ : Oui. Enfin, c'est le groupe « révision de la production ». Tous les textes concernant les pilotes sont pris en charge par le secrétariat de la production. Mais c'est le département «qualité et sûreté » qui émet les opinions et apporte le soutien technique.

Les documents que vous avez là datent de 2002 et l'organisation a changé depuis. On a parlé du groupe "technique " tout à l'heure. Ce sont les fonctions de ce groupe " technique » qui ont été, en quelque sorte, transférées au département «qualité et sûreté » d'aujourd'hui.

$\mathbf{Q}$ : Je me rappelle qu'au paragraphe des instructeurs, on a vu qu'il y avait effectivement des membres du groupe «technique ».

R : Oui, c'est ça.

$\mathbf{Q}$ : Quand ces modifications concernent donc des opérations de conduite, ce sont les membres du groupe «production» qui s'en occupent. À ce moment-là, pour refléter l'orientation que veut donner le siège, le département « qualité et sûreté » émet des avis en ce sens et la «production » effectue les modifications en tenant compte de tout ça, c'est bien ça?

$\mathbf{R}$ : Oui. C'est comme ça que les textes sont révisés.

$\mathbf{Q}$ : Dans la «production», il y a les équipes de quart qui travaillent directement sur la conduite, mais ce ne sont pas eux qui révisent eux-mêmes les textes, n'est-ce pas ? Si j'ai bien compris, il existe dans l'organigramme le "département de gestion de la conduite $n^{\circ} 1$ » et le «département de gestion de la conduite $n^{\circ} 2$ ». J'imagine que les gens qui y travaillent ne sont pas tous des gens qui travaillent dans la salle de commande.

R : Non. 
$\mathbf{Q}$ : Ce sont donc ces personnes qui ne travaillent pas directement à la conduite qui révisent ces textes?

R : Aujourd'hui vous avez XXXXX qui est chef du «département de gestion de la conduite ». Il a sous lui, le personnel de conduite de la $1^{\text {ère }}$ à la $4^{\mathrm{e}}$ tranche. Mais il a aussi sous ses ordres les groupes «production ». Là, ce sont des gens qui sont dans les bureaux. Encore en-dessous, il a des gens qui font du travail administratif. Tout ce qui concerne les révisions de procédures et autres, est sous la responsabilité de ce personnel qui n'est pas sur le terrain, mais dans les bureaux. Et par rapport à ce travail, c'est le groupe " technique », ou plutôt le département « qualité et sûreté », le plus simple serait de dire les « messieurs sûreté », qui donnent leur avis si nécessaire.

Si on remonte dans la hiérarchie, celui qui chapeaute tout ça au niveau du siège est le département "gestion de la conduite». Aujourd'hui, ils ont changé de nom, mais autrefois, il y avait là des groupes « production », avec des GM.

$\mathbf{Q}:$ À l'intérieur du siège?

R : Oui, à l'intérieur du siège. Ce sont eux qui s'occupent de l'ensemble de la conduite à Fukushima 1 ou Fukushima 2. Ce sont aussi eux qui bougent en premier quand il s'agit de réviser des procédures, par exemple. Mais la partie technique, savoir ce qu'on va introduire comme nouvelles connaissances, tout ça, est du ressort de l'équipe technique, les équipes de sûreté du siège. Ce sont eux qui décident ce qu'on va réviser, de quelle manière. C'est là que se fait, au départ, la discussion des révisions. Ils discutent aussi pour décider dans quelles procédures ou texte introduire ces modifications.

C'est après toutes ces étapes que les textes arrivent à Fukushima 1. À ce moment-là, comme on l'a vu tout à l'heure, c'est à nous, à la centrale, d'envisager des ajustements pour chaque tranche, puisque chacune est différente.

$\mathbf{Q}:$ Si je comprends bien, ça veut dire que quand il y a des révisions de procédures, le siège élabore d'abord ce qu'on pourrait appeler le cadre de base, avec le département « technique de production », et qu'ensuite le groupe «production » de chaque centrale adapte ce cadre à chaque tranche, en tenant compte des avis du département « qualité et sûreté ».

$\mathbf{R}:$ C'est bien ça.

$\mathbf{Q}$ : Concrètement, ces derniers temps, vous avez connu des révisions ?

$\mathbf{R}$ : Quand il y a des transformations et qu'on change de système, on le fait. Mais comme on n'a pas connu de changement de système, ces derniers temps... À part les conséquences du séisme au large de Chûetsu, je ne vois pas trop.

$\mathbf{Q}:$ Là, il s'agissait surtout de renforcer l'installation.

$\mathbf{R}$ : Oui. Du coup, je ne pense pas que ça ait fondamentalement changé grand-chose du point de vue des réponses aux accidents sévères.

$\mathbf{Q}:$ Vous n'avez donc pas connu de grosses révisions, ces derniers temps?

$\mathbf{R}:$ Non.

$\mathbf{Q}$ : Si vous voulez bien, nous allons revenir aux mesures concernant les installations, qui ont été prises dans les nouvelles procédures de gestion des accidents à partir de 2002. Dans ce document, à la page 4, on trouve un paragraphe sur les mesures concernant la fonction « arrêt du réacteur ». Je pense que cela concerne le réacteur 1 .

L'une des nouvelles mesures est l'installation du RPT (Recirculation Pump Trip) ${ }^{48}$ et l'autre, de l'ARI (Alternative Rods Injection) ${ }^{49}$. Ces deux systèmes ont été installés en plus des 
systèmes existants. Lors de l'accident de mars, pour l'arrêt du réacteur, cet arrêt s'est effectué automatiquement sans aucun problème, n'est-ce pas?

\section{$\mathbf{R}:$ C'est bien ça.}

$\mathbf{Q}$ : Ensuite, on parle des mesures concernant l'injection d'eau dans le réacteur et dans l'enceinte de confinement. On y note qu' « afin de pouvoir profiter au mieux des fonctionnalités existantes du MUWC (Make-Up Water Condensate system) ${ }^{50}$, du FP et du CCS, des modifications ont été apportées au raccordement de ces systèmes, pour pouvoir injecter de l'eau dans le réacteur à partir de ces systèmes en passant par le CS (Core Spray System) ${ }^{51}$ ".

«En rendant possible l'utilisation de ces systèmes en tant que solutions substitutives pour l'injection, ces aménagements contribuent à une amélioration de l'injection dans le réacteur. »

De plus, "ces mêmes aménagements rendent possibles l'aspersion de l'enceinte de confinement via le CCS, le refroidissement du corium ${ }^{52}$ amassé au fond de la cuve par la condensation de la vapeur ainsi dégagée, améliorant d'autant l'injection d'eau dans l'enceinte de confinement». Si on continue, on trouve des rubriques individuelles. Tout d'abord, «méthodes substitutives de refroidissement par le Drywell Cooler ${ }^{53}$, le CUW (Clean-Up Water system $)^{54}$ ». Ensuite, «la réhabilitation du CCS », et, enfin, « la ligne d'éventage renforcée ».

Parmi tout ça, on va peut-être commencer par revenir sur les solutions substitutives pour l'injection d'eau. Parmi les solutions évoquées, ce que vous avez effectivement utilisé lors de l'accident, c'est le système FP. C'est un système qui fonctionne avec une pompe diesel. D'ailleurs, ils ont écrit ici, «comme le système FP possède une pompe diesel, son utilisation reste possible même en cas de perte de l'alimentation en courant alternatif ». Et donc, l'idée était de rendre possible l'injection d'eau dans le réacteur en réalisant des raccords à partir du système FP qui dispose d'une pompe diesel.

Dans le cas du réacteur 1, la pompe diesel a eu des problèmes et ne marchait plus. Du coup, vous vous êtes tourné vers la solution des voitures de pompiers. Mais, ce genre de solution n'est pas évoqué dans ce document.

Je voudrais d'abord savoir où se trouve cette pompe diesel qui fait marcher le système FP.

$\mathbf{R}$ : Elle se trouve dans le sous-sol du bâtiment turbine. Selon les tranches, elle se trouve à des endroits différents, mais elle est toujours dans le bâtiment turbine. Je pense que celle de la tranche 1 se trouve au $2^{\mathrm{e}}$ sous-sol et pour les tranches 2 et 3 , au $1^{\mathrm{er}}$ sous-sol. Oui, elle se trouve à des endroits différents selon les tranches.

$\mathbf{Q}$ : Maintenant, concernant la tranche 1, la pompe diesel en elle-même a...

$\mathbf{R}$ : Non, ça n'àpas marché.

$\mathbf{Q}:$ Que voulez-vous dire?

$\mathbf{R}$ : Au début, elle a fonctionné, mais ensuite, elle n'a plus marché.

$\mathbf{Q}$ : Si on regarde la chronologie, tout au début, c'est-à-dire le 11 mars à 17h30, la pompe est en attente. Elle est prête à entrer en fonctionnement. Mais le lendemain, à 1h48, on voit: « constatation de l'arrêt de la pompe pour problème ». II semblerait qu'un peu avant, toujours selon la chronologie, vous auriez soupçonné une panne sèche. Vous réapprovisionnez la pompe et tentez de la redémarrer, mais en vain. La cause de cette panne n'est pas connue, mais, toujours est-il que la pompe en elle-même ne fonctionnait plus.

Vous ne pouvez donc plus utiliser cette pompe. Si on remonte un peu dans le temps, le 11 mars à 17h12, on note: "ordre de rechercher des méthodes d'injection dans le réacteur à l'aide de voitures de pompiers ». La ligne FP qui avait été nouvellement établie dans le cadre des mesures de gestion des accidents, telles que nous les avons évoquées tout à l'heure, est bien celle qui emprunte le réseau FP qui fonctionne avec le moteur diesel pour aller injecter l'eau dans le réacteur, n'est-ce pas?

$\mathbf{R}$ : Non, ce n'est pas tout. Dans le bâtiment, il y a un réseau FP qui peut être alimenté de l'extérieur par des tuyaux. À la base, le but du réseau FP est de faire tourner les sprinklers $^{55}$ et d'arroser avec de l'eau. Ce réseau couvre tout le bâtiment. La 
transformation évoquée dans le cadre des nouvelles réponses aux accidents sévères, consiste à raccorder le canal principal du réseau FP à cette ligne, ici, qui est le CS.

À cet endroit du réseau $\mathrm{FP}$, il y a une pompe qui va puiser l'eau dans ce réservoir d'eau filtrée. Mais il y a aussi des bouches qui permettent d'injecter de l'eau de l'extérieur du bâtiment. Si on raccorde une pompe à une de ces bouches, on peut faire vivre cette ligne. Même si on ne peut pas utiliser le réservoir d'eau filtrée, on peut injecter de l'eau directement de l'extérieur. D'ordinaire, on ne fait pas tout de suite appel aux voitures de pompiers. S'il y a encore de la pression dans les bornes à incendie, on y branche un tuyau et, une fois que c'est raccordé, l'eau passe par-là. C'est une solution qu'on peut utiliser aussi. Mais, de toute manière, il y avait la crainte qu'on ne puisse pas utiliser les pompes diesel, et puis, la source en eau dans le cas de cette pompe est le réservoir d'eau filtrée, qui est une installation de classe $\mathrm{C}^{56}$ selon les normes antisismiques, et on ne savait pas comment il avait résisté au séisme.

Évidemment, pour les bornes à incendie aussi, on avait des doutes quant à leur fonctionnement. De plus, leur source étant également le réservoir d'eau filtrée, on avait le même souci. Dans ces conditions, pour moi, si on arrivait à faire venir des voitures de pompiers, on avait nos chances. J'étais relativement optimiste quant à l'état à l'intérieur des bâtiments. Même après ce séisme. J'étais persuadé que les installations avaient tenu le coup. Rappelez-vous, après le séisme à Kashiwazaki, la tuyauterie à l'intérieur des bâtiments n'avait pratiquement pas bougé. Pour moi, on pouvait utiliser ce réseau, il suffisait d'y injecter de l'eau. C'est pour ça que j'avais donné l'ordre de chercher des camions de pompiers.

$\mathbf{Q}:$ Ces réflexions, que vous m'exposez là, les aviez-vous en tête vers $17 \mathrm{~h} 12$ ?

$\mathbf{R}$ : Oui, tout à fait.

Q: Vous commencez donc par la pompe diesel. Vous ne pouvez plus l'utiliser. Vous aviez envisagé, dès le départ, qu'elle pourrait vous lâcher?

R : Mon idée, à ce moment-là, était qu'il fallait réfléchir à toutes les manières possibles d'envoyer de l'eau. Il y avait bien sûr la possibilité de la pompe diesel, mais, suivant les circonstances, des camions de pompiers pouvaient devenir nécessaires. Voilà ce que je pensais.

$\mathbf{Q}$ : Si je reviens à ce document sur la gestion des accidents, à la page 5 , ils évoquent la transformation qui a été faite sur le réseau de tuyauterie pour permettre l'injection d'eau dans le réacteur à partir du réseau MUW en transitant par le système CS. Ça, vous ne l'avez pas utilisé.

$\mathbf{R}$ : On n'a pas pu l'utiliser. C'est ce qu'on appelle le réseau Make-Up Water. La pompe du MUW a besoin d'électricité pour fonctionner. Comme on n'en disposait pas, c'était difficile.

Je ne sais pas si cela a été noté dans les archives, mais une fois, j'ai bien eu l'idée de brancher le MUW sur le Power Center 2C. J'avais donné l'ordre d'étudier cette possibilité, mais la réponse était que, là encore, c'était difficile.

$\mathbf{Q}$ : On revient toujours au même problème de la nécessité d'avoir de l'électricité pour faire fonctionner ces pompes. Avec la différence que sur le réseau FP, vous disposiez d'une pompe à moteur diesel. 
Maintenant, concernant le système de refroidissement de l'enceinte, il y avait ces histoires de pomper l'eau de mer...

$\mathbf{R}$ : En fait, on utilise la même source pour l'eau. C'est tout simplement l'exploitation de cette même transformation qu'on va juste dévier à l'arrivée vers le réseau CCS, en plus du système CS, pour pouvoir asperger l'enceinte de confinement. La source est la même, c'est juste qu'il y a deux destinations possibles.

$\mathbf{Q}$ : Ce qui veut dire que vous n'avez pas pu l'utiliser non plus.

$\mathbf{R}$ : C'est ça. Si ce réseau permet de refroidir l'intérieur du réacteur, celui-là permet de refroidir l'enceinte de confinement. C'est juste ça.

$\mathbf{Q}$ : Cette aspersion de l'enceinte de confinement en passant par le CCS, vous l'avez utilisée sur le réacteur 3 , il me semble.

$\mathbf{R}$ : Oui, on a fait une tentative d'aspersion de l'enceinte.

$\mathbf{Q}:$ C'était à partir du réseau FP?

$\mathbf{R}$ : Il me semble que oui. Sur le moment, je n'ai pas vérifié ça dans les détails. De toute façon, c'était la seule solution.

$\mathbf{Q}$ : Là, je ne suis pas sûr de l'heure. II faudra regarder les vidéos, comme on a dit l'autre jour. Quand on interroge les gens qui étaient sur le terrain sur le réacteur 3, ils disent qu'ils étaient lancés sur l'élaboration d'un réseau « eau de mer», quand on leur a demandé de changer et de concevoir un réseau "eau douce». Et quand on leur demande quel était le réseau utilisé vers 7 h30 quand vous avez effectué l'aspersion de l'enceinte de confinement, personne ne le sait très bien. Si vous effectuiez l'aspersion de l'enceinte en passant par le réseau FP, était-ce à l'eau de mer?

$\mathbf{R}$ : Pour la tranche 3, la pompe diesel a fonctionné au début. Elle a fonctionné, puis, en cours de route, nous sommes passés à la pompe à incendie. Comme la pompe diesel avait fonctionné un temps, il devait y avoir encore de la pression dans le réseau FP. Je pense qu'au début, on a profité de cette pression. Et, à ce moment-là, c'était de l'eau douce.

$\mathbf{Q}$ : Qui provenait du réservoir d'eau filtrée?

R : Oui.

$\mathbf{Q}$ : Alors, on peut dire que cette liaison vers le système CCS, vous l'avez un peu utilisée pour la tranche 3 .

R : Oui, on l'a utilisée.

$\mathbf{Q}$ : Et pour la tranche 1 ?

R : Vous savez, je ne me souviens plus très bien. Mais la situation était telle qu'il fallait commencer tout de suite à injecter l'eau dans le réacteur. On n'avait pas le temps d'asperger l'enceinte. Dès le début, la priorité des priorités était l'injection d'eau dans le réacteur.

$\mathbf{Q}$ : Parce que vous étiez à 5 h46.

$\mathbf{R}$ : On n'avait pas le temps de refroidir l'enceinte de confinement. Pardon de sauter encore dans le temps. La priorité pour le réacteur 1 , c'était l'injection dans le réacteur, sans aspersion de l'enceinte. Dans le cas du 3, on avait pensé que c'était une bonne idée d'asperger l'enceinte pour tenter de faire baisser la pression. Pour le réacteur 2 , on avait pensé faire ce fameux éventage avant l'injection. Mais l'éventage s'est avéré très difficile. On s'est dit qu'on allait laisser échapper un peu de pression 
en ouvrant la vanne de sécurité. Avant qu'on ne procède à cette ouverture, les gars de la production ont proposé d'asperger brièvement pour refroidir.

$\mathbf{Q}:$ Vous voulez dire asperger l'enceinte de confinement?

$\mathbf{R}$ : Oui, l'enceinte de confinement. Nous l'avions envisagé, parce que cela allait aider à la condensation de l'eau. Mais, vous le comprendrez quand vous verrez la vidéo, on nous a interrompu dans notre élan, en nous obligeant à injecter l'eau illico. Du coup, cette histoire d'aspersion de l'enceinte du 2 est restée en l'air. Mais, dans les faits, nous l'avions bien envisagée.

Q: Donc, aussi bien pour les réacteurs 1,2 que 3, ce réseau était en état de fonctionner s'il y en avait eu besoin. Même si, finalement, vous ne l'avez utilisé que pour le réacteur 3 .

On lit ici: "lors de cette transformation, après l'achèvement des travaux de raccords entre le réseau FP et le réseau $M U W C$, seront installés un compteur d'eau et une vanne électrique opérable à distance entre le réseau existant et les nouveaux tuyaux ». Avez-vous pu utiliser ce compteur d'eau?

$\mathbf{R}$ : Non, on n'a pas pu. Je pense qu'il y a encore eu des histoires d'alimentation électrique. Surtout que je ne sais pas si ce compteur était une version installée sur le terrain ou pas. Toutes les données des compteurs à eau ne viennent pas forcément jusqu'à la salle de contrôle. Il y a des compteurs qui sont sur le terrain et qu'on consulte quand on fait des rondes.

Q: Quand vous dites sur le terrain, ça veut dire que c'est carrément...

$\mathbf{R}$ : Oui. Je crois que je n'ai jamais su de quel type était ce compteur. En tout cas, ce que je peux dire, c'est qu'on ne voyait pas les données de ce compteur. Alors, ça peut être dû au fait que le compteur donnait bien des chiffres, mais qu'on ne pouvait pas les afficher à la salle de contrôle, faute d'électricité, ou bien, dès le départ, il n'avait pas été prévu que ces données arrivent jusqu'à la salle de contrôle, ce qui est un scénario possible.

$\mathbf{Q}$ : De toute façon, les données indiquant quelle quantité d'eau était entrée n'arrivaient pas jusqu'à la cellule de crise.

$\mathbf{R}:$ Effectivement.

Q: Et la vanne électrique dont ils parlent ici, vous n'avez pas pu l'utiliser?

$\mathbf{R}$ : On ne l'aurait pas utilisée ? J'avoue que cette histoire de vanne du réacteur 1 n'est pas bien ancrée dans ma tête. Je ne sais même pas à quoi elle ressemble. Mais même si elle avait été fermée...

$\mathbf{Q}$ : Oui, c'est la valve qui se trouve à l'endroit où le MUWC et le CS se rejoignent.

$\mathbf{R}$ : Pardon. Je ne sais pas comment ça s'articule sur le terrain sur le réacteur 1. Là où, normalement, le réseau CS est indépendant, en effectuant ces travaux, on lui a raccordé le réseau MUWC et on nous dit d'installer une vanne et un compteur d'eau.

$\mathbf{Q}$ : L'eau venait du réseau MUWC, ici, ensuite passait par le réseau CS...

$\mathbf{R}$ : Ça veut dire que la vanne était ouverte. Comment a-t-on bien pu faire pour l'ouvrir?

Q : Mais, concrètement, elle était ouverte.

$\mathbf{R}$ : Oui, c'est une vanne qui doit être ouverte pour l'injection. Une vanne opérable à distance... 
$\mathbf{Q}$ : Les gens du groupe «réhabilitation» que j'ai interrogés m'ont dit que les vannes électromagnétiques, comme la vanne de rejet de la SC (Suppression Chamber) ${ }^{57}$, étaient manœuvrées à partir de la salle de commande en faisant passer de l'électricité.

$\mathbf{R}$ : Personnellement, je n'ai pas le souvenir qu'on ait eu du mal à ouvrir cette vanne. Si vous voulez les détails, il faudra demander à un agent de conduite.

$\mathbf{Q}$ : D'accord. On va vérifier ça. Pensez-vous que le responsable du réacteur saurait?

$\mathbf{R}$ : Le responsable du réacteur? Je ne sais pas. Peut-être XXXXX qui était là aujourd'hui.

$\mathbf{Q}:$ Ah, M. XXXXX est là aujourd'hui ?

$\mathbf{R}$ : Oui. Des gens comme XXXXX sont ceux qui connaissent le mieux ces problèmes. Il vaudrait mieux leur demander. Vous savez, moi, je n'ai jamais conduit une tranche pour de vrai, alors parfois je ne suis pas au courant des problèmes particuliers. Désolé, je ne suis pas un spécialiste.

$\mathbf{Q}$ : Plus loin, dans le document, on parle d'installer aussi un compteur d'eau et une vanne opérable à distance entre le système MUWC et le système CCS.

R : Oui.

$\mathbf{Q}$ : Ensuite, au paragraphe suivant, on évoque les «mesures concernant les fonctions de refroidissement de l'enceinte de confinement dans la gestion des accidents ». II y a d'abord le Drywell Cooler, le refroidissement par le système CUW. Cela sert, par exemple, quand la pression de l'enceinte de confinement monte très fort. Avez-vous utilisé ces systèmes en mars?

$\mathbf{R}$ : Non. Déjà, dès le départ, le système CUW ne fonctionnait pas. Ensuite, le Drywell Cooler ne peut fonctionner que si le RCW (Reactor building Cooling Water system) ${ }^{58}$ marche. De toute façon, pour ces deux systèmes, il fallait de l'électricité. De base, nous ne disposions pas d'alimentation électrique ni de source d'eau, donc nous ne pouvions pas les utiliser. Ce qui voulait dire qu'il ne nous restait plus que l'éventage.

$\mathbf{Q}$ : Au fait, quelle est la source pour alimenter le Drywell Cooler en eau?

R : Puisque le Drywell Cooler utilise le RCW, c'est l'eau de ce système. L'eau du RCW se trouve au-dessus du réacteur. C'est un réservoir qui contrôle toute l'eau qui circule par le RCW. On prend l'eau là.

Il s'agit d'asperger d'eau, de faire fonctionner le Drywell Cooler pour refroidir.

Le CUW consiste à aller chercher les composants de refroidissement dans le réacteur, à les nettoyer et les remettre en circuit. On utilise ça pour refroidir l'enceinte de confinement. De toute manière, aucun des deux systèmes n'était viable.

$\mathbf{Q}:$ À cause de l'électricité, vous voulez dire?

$\mathbf{R}:$ À cause de la pompe.

$\mathbf{Q}:$ Vous voulez dire qu'il n'y avait pas l'électricité pour faire fonctionner la pompe?

R : Oui.

$\mathbf{Q}$ : Vous ne pouviez donc pas utiliser ces systèmes, parce que vous aviez perdu le courant alternatif et que les générateurs de secours ne fonctionnaient pas non plus.

$\mathbf{R}$ : En ce qui concerne le réacteur 1 , on s'est rendu compte très vite qu'on ne pourrait presque rien utiliser. 
$\mathbf{Q}:$ Ça, c'est à quel moment ? Au soir du 11 mars ?

R : Oui. On constate qu'il n'y a plus d'électricité. On réfléchit à ce qui peut fonctionner sans électricité. Il n'y a guère que la pompe à incendie qui fonctionne au diesel et les voitures de pompiers. On n'avait pas d'autre choix que d'essayer d'élaborer un plan avec ça. Bien sûr, s'il s'avérait possible de rétablir l'électricité, nous étions prêts à donner la priorité à la réhabilitation de tous ces systèmes existants et à les utiliser.

$\mathbf{Q}$ : Un peu plus tard, vous découvrez que le $2 \mathrm{C}$ fonctionne. Vous pensez alors à y brancher le SLC ou le CRD.

$\mathbf{R}$ : Oui. Il fallait y brancher un maximum de choses.

$\mathbf{Q}$ : Incidemment, est-il possible de brancher les pompes du Drywell Cooler ou du CUW sur le 2C?

$\mathbf{R}$ : Nous avons étudié toutes ces possibilités. Mais je pense que c'était difficile pour le RCW. Quand on s'est demandé ce qu'on allait brancher sur le $2 \mathrm{C}$, il n'y a pas eu le RCW. Je ne sais pas pour quelle raison. Je me rappelle très bien avoir donné l'ordre d'y réfléchir, mais je n'ai pas forcément eu le temps de m'intéresser aux étapes intermédiaires, avant l'arrivée à la conclusion, alors...

$\mathbf{Q}$ : Si je comprends bien, concernant toutes ces mesures pour le refroidissement ou l'injection d'eau qui sont évoquées dans ce document, vous les aviez toutes envisagées, même celles qui demandaient une alimentation électrique. Quand vous avez étudié la possibilité d'utiliser l'électricité du 2C, dans la pratique, il n'est guère resté que le SLC. C'est bien ça?

R : Oui.

$\mathbf{Q}$ : Si je poursuis dans ce document, l'autre solution préconisée, concernant l'enceinte de confinement, c'est l'éventage renforcé, auquel vous avez effectivement eu recours.

R : Oui, c'est ça.

$\mathbf{Q}$ : Justement, dans le document, on parle du système AC (Atmospheric Control system) ${ }^{59}$ et du SGTS (Standby Gas Treatment System) ${ }^{60}$ comme des voies traditionnellement possibles pour un éventage. Ils signalent qu'ils préconisent, en plus, la construction d'un circuit renforcé d'éventage. Au mois de mars, avez-vous envisagé d'utiliser éventuellement le réseau SGTS pour un éventage?

$\mathbf{R}$ : Non. La pression était très élevée, trop élevée. Le système SGTS est prévu pour une pression, disons, normale. Nous en étions déjà à quelque chose comme 8,5 kilos ${ }^{61}$, $0,8 \mathrm{MPa}$. Si on avait envoyé une pression de 8 ou 9 kilos, d'un coup, en ouvrant le système, la tuyauterie n'aurait pas tenu. Ce qui signifie que quand la pression atteint cette valeur, il n'y a plus que l'éventage renforcé.

$\mathbf{Q}$ : Mais, quand on lit le document, on a l'impression qu'il est normalement prévu d'éventer en passant par le système SGTS, au cas où la pression de l'enceinte de confinement s'élèverait.

$\mathbf{R}$ : Oh, c'est qu'ils n'avaient pas prévu un évènement où la pression monterait si fort. Ce à quoi ils pensaient en rédigeant ce texte, c'est un phénomène transitoire où la pression passerait de l'habituel 1 kilo à 1,5 kilo, par exemple. C'est à ce niveau-là qu'ils pensaient. Mais dans des cas d'accidents sévères où on se trouve à $0,85 \mathrm{Mpa}$, le jour où la pression de l'enceinte monte à ce niveau, aussi bien l'AC que le SGTS n'ayant pas été structurellement conçus pour résister à une telle pression, la tuyauterie n'y résisterait pas et exploserait. Le texte n'a pas été rédigé suivant la même philosophie. 
$\mathbf{Q}$ : On doit donc comprendre qu'au moment où ils ont fait la liste des mesures à prendre en cas d'accident grave, ils avaient bien l'idée que la ligne d'éventage par le système SGTS ne suffirait pas et qu'une nouvelle mesure s'imposait, et, en conséquence, ils ont fait construire cette nouvelle ligne. Et vous, jugeant que la situation ne vous permettait pas d'utiliser l'éventage par le SGTS, avez tout de suite opté pour l'éventage renforcé. Vous avez pensé ça, quand vous avez su que la pression de la chambre sèche était à $600^{62}$ ?

$\mathbf{R}:$ Oui, c'est ça.

Q: Passons maintenant à la page 7: «Mesures concernant les fonctions support des fonctionnalités de sûreté". Là, on nous parle d'abord du "partage de l'alimentation électrique». Ça, c'est une chose que vous avez faite pour les tranches 5 et 6 , n'est-ce pas? C'est une mesure qui est rendue possible par la proximité des différentes tranches, un avantage d'avoir plusieurs tranches sur le même site. La tranche 1 partage son électricité avec la tranche 2, si elle est en difficulté. Réciproquement, si la tranche 1 a des difficultés à s'approvisionner, la tranche 2 partage avec elle. Mais tout ça n'est possible que si on dispose déjà d'électricité. Or, sans électricité, entre les tranches 1 et 2 , puis les tranches 3 et 4 , ça n'a pas été possible.

R : Voilà.

$\mathbf{Q}$ : Ensuite, on a la «restauration des générateurs diesel de secours ». On en a parlé tout à l'heure. Dans le cas présent, cette restauration aurait pris des mois, donc une restauration rapide était impossible.

R : Oui.

$\mathbf{Q}$ : Ils ont écrit ici: "quand la perte de toute alimentation en courant alternatif se produit, l'évènement évolue lentement et laisse une large marge de manœuvre ». Savez-vous pourquoi cet évènement évolue lentement ? À quoi font-ils allusion, à votre avis ?

$\mathbf{R}$ : Comme je ne suis pas un spécialiste de la sûreté, je ne sais que vous dire. Je ne comprends pas bien à quel évènement ils pensaient quand ils ont écrit ça. Ah, ces imbéciles! Une fois de plus, ils n'ont servi à rien !

$\mathbf{Q}$ : Bon. On va noter que vous n'en savez rien.

Ensuite, le texte parle de "l'attribution de générateurs diesel de secours spécifiques à chaque tranche ». Au départ, il n'y avait qu'un seul générateur de secours pour les tranches 1 et 2 ?

$\mathbf{R}$ : Non, ce n'est pas ça. Au départ, lors de la conception, chaque tranche avait un générateur rien que pour elle. Mais il y avait en plus un générateur que deux tranches se partageaient. C'est-à-dire que la tranche 1 avait son propre générateur, la tranche 2 également et qu'elles se partageaient, en plus, un autre générateur qui leur était commun.

$\mathbf{Q}:$ Vous voulez dire qu'il y avait trois générateurs ?

$\mathbf{R}$ : Oui, il y en avait trois. D'après les plans d'origine.

$\mathbf{Q}$ : Donc, trois générateurs pour la tranche 1 et la tranche 2 .

$\mathbf{R}$ : Oui. De la même manière, les tranches 3 et 4 disposaient aussi de trois générateurs. Ce qu'ils ont voulu faire de nouveau, c'était d'ajouter un générateur, pour que la tranche 1 récupère ce générateur qui était en commun avec la 2 , pour son unique usage et que la 2 ait à la fois l'ancien générateur et le nouveau, là aussi, pour elle toute seule. Comme ça, chaque tranche disposait de deux générateurs en propre.

C'est pour ça qu'on a rajouté respectivement un générateur aux tranches 2, 4 et 6. 
$\mathbf{Q}:$ On en a déjà un peu parlé, mais je voudrais revenir sur ce qui s'est véritablement passé au niveau du réacteur 1 . Au départ, on dit que l'IC s'était mis en marche. Ensuite, on prépare le DDFP et on le met en attente, prêt à fonctionner. Ce faisant, la situation se dégrade rapidement. Tout d'abord, déjà, vers 15h03, on a noté : "début du contrôle de la pression du réacteur par I'IC ». Effectivement, jusque vers 15h30, il y a plusieurs manœuvres d'ouverture et de fermeture sur I'IC. Aviez-vous déjà eu l'occasion d'utiliser le système IC pour contrôler la pression du réacteur, avant ça?

$\mathbf{R}$ : D'après mes souvenirs, j'avais entendu dire qu'ils avaient utilisé l'IC lors de la fameuse inondation à l'eau de mer. Mais je n'étais pas sur place, alors je ne connais pas le détail des manœuvres qui ont été faites ${ }^{63}$. Je crois bien que c'est la seule occasion où on a utilisé l'IC.

$\mathbf{Q}$ : Quand on utilise l'IC, je ne sais pas si c'est évoqué dans les manuels ou pas, faut-il faire de multiples manœuvres d'ouverture et de fermeture pour ajuster les choses ? Pour que la pression ne baisse pas trop, par exemple?

$\mathbf{R}$ : Là, comme je vous l'ai dit, moi-même, je n'ai pas l'expérience d'avoir jamais utilisé l'IC. Les pilotes, comme XXXXX, doivent bien connaître les manœuvres à effectuer, mais moi, je ne sais presque rien de la manière dont il faut contrôler l'IC. Bien sûr, comme j'ai travaillé par le passé comme chef de service de la maintenance des tranches 1 et 2 , j'ai des connaissances concernant sa maintenance, mais savoir par exemple la quantité de vapeur qui s'échappe lorsqu'on ouvre la vanne, puisque le principe de l'IC est de laisser s'échapper de la vapeur, qui condense et retourne dans le réacteur, je n'en ai aucune idée. Pourtant, c'est important, puisque ça va influer sur le niveau d'eau. Je suis désolé.

$\mathbf{Q}$ : J'en conclus que ce n'est pas le genre de connaissances que tout le monde possède.

$\mathbf{R}$ : Effectivement, non.

$\mathbf{Q}$ : Si je comprends bien, au tout début, il y a de fortes chances pour que I'IC ait véritablement fonctionné, puisqu'on entreprend même des manœuvres pour le régler. De plus, pour vous, en tant que directeur des opérations, vous recevez des rapports disant qu'il est en fonctionnement.

R : Oui.

$\mathbf{Q}$ : C'est une chose que nous avons déjà vérifiée, mais, au moment où vous perdez toute source de courant alternatif, ou juste après, personnellement, vous pensiez encore que l'IC était en marche?

R : Oui.

$\mathbf{Q}$ : J'ai demandé au siège de me fournir de la documentation. C'est un schéma de l'IC. On voit qu'il y a deux réseaux, le réseau $A$ et le réseau $B$. II semblerait que ce soit le réseau $A$ qui ait continué à fonctionner. Le réseau $A$ comporte donc quatre vannes et son condenseur se trouve là. Pour assurer l'alimentation en eau du condenseur, on peut visiblement ouvrir une vanne, ici, pour amener l'eau du système FP ou du système MUWC. Si je me souviens bien, le 11 mars au soir, vous, vous étiez en attente de pouvoir envoyer de l'eau dans le réacteur en utilisant le réseau FP, c'est bien ça ? Seulement, quand on regarde les différentes notes que les gens ont prises, on a l'impression que, dans la confusion, plusieurs personnes ont cru que vous étiez en attente pour envoyer de l'eau dans ce condenseur.

R : Oui, pardon, c'est très confus. Probablement, c'est comme pour la conduite de l'IC, c'était un moment où la communication avec la salle de commande était très mauvaise. Aujourd'hui, je me rends compte qu'on n'arrivait pas à bien se comprendre. Pour moi, « envoyer de l'eau », c'était forcément « envoyer de l'eau dans le réacteur", et rien d'autre. Mais peut-être y a-t-il eu des gars qui, en recevant 
l'ordre, ont cru qu'il s'agissait d'envoyer de l'eau dans le condenseur de l'ic. Tout dépend aussi de la chaîne de commandement. Comment les ordres ont-ils été transmis ? On n'en sait rien. Peut-être que XXXXX pensait qu'il fallait mettre de l'eau dans le condenseur. C'est possible. Mais ce n'est qu'une hypothèse. En tout cas, pour moi, je ne pensais qu'à envoyer de l'eau dans le réacteur.

$\mathbf{Q}$ : Avec le réseau FP, si on ne passe pas par-là, mais par ici, on peut envoyer de l'eau à l'intérieur de la cuve du réacteur?

$\mathbf{R}$ : Oui, à l'intérieur de la cuve du réacteur. Mais, si on y réfléchit après coup, bien sûr, il fallait aussi réapprovisionner l'eau de l'IC, parce qu'à la longue l'eau s'épuise. Mais l'eau qui se trouve ici est censée durer au moins quatre heures. Alors, décidément pour moi, plus que l'approvisionnement de l'IC, il fallait envoyer de l'eau dans le réacteur. Seulement, le temps que les ordres arrivent sur le terrain, c'est comme le jeu du téléphone arabe, les mots étaient transformés, les choses devenaient ambiguës, c'était des phrases remplies de "devrait", "semblerait». On en a fait plusieurs fois l'expérience. C'était la même chose pour les informations qui arrivaient du terrain. Le temps que ça arrive à la cellule de crise, les choses avaient changé ou n'étaient plus sûres. Je recevais des rapports avec des "il semblerait ", alors j'étais obligé de demander des précisions, "où exactement?", " comment avez-vous vérifié ce fait?" ", etc. C'est comme pour le RCIC de tout à l'heure. J'avais demandé si le RCIC du réacteur 2 fonctionnait. Ils me répondent oui. Je leur demande comment ils l'ont vu, sur quel appareil ils l'ont vérifié. Alors, ils me répondent qu'ils ne l'ont pas vérifié. J'insiste pour leur demander ce qu'ils ont regardé, et ainsi de suite. En voyant les rapports qui arrivaient du terrain, je me posais des questions sur la manière dont les ordres que j'avais donnés avaient été transmis au chef de quart ou autre responsable. C'était vraiment un moment où il a été difficile de se comprendre mutuellement.

$\mathbf{Q}$ : L'autre jour, j'ai eu l'occasion de jeter un coup d'œil dans le carnet du responsable du réacteur. Non, pardon, il me semble que c'était plutôt une personne du groupe « production », une personne qui était en contact avec l'équipe de quart, qui avait laissé des notes qui faisaient penser qu'il s'agissait d'envoyer de l'eau dans I'IC. J'ai eu un entretien avec $M . X X X X X$, responsable du réacteur. II avait l'air de penser la même chose que vous. M. XXXXX pensait, comme vous, que l'IC n'était plus opérationnel et qu'il fallait envoyer de l'eau vers la cuve. II pensait qu'il fallait envoyer de l'eau en passant par le réseau FP et il avait commencé à constituer une ligne pour ça.

Seulement, du côté de ceux qui ont reçu rapport de tout ça, des gens qui étaient au siège, ils donnent l'impression d'avoir pris ça de travers. D'après les notes qu'ils ont laissées, bien sûr on ne sait pas vraiment ce qui s'est passé et le siège a l'air de s'intéresser aussi à la chose, on dirait qu'il y a eu malentendu. Les gens du groupe «information » ont, notamment, écrit des choses comme « on n'aura plus d'eau dans le réservoir IC », » possibilité d'injecter l'eau par le FP», "I'IC est en marche, mais impossibilité de vérifier si le réservoir est plein », "vérification de l'injection d'eau en faisant tourner la FP». Par exemple, pendant que la DDFP marchait, la personne du groupe «production » qui a reçu ces messages a noté, de son côté, «fermeture de la vanne d'arrivée du réseau FP, le système FP du réacteur 1 en marche, pour alimentation en eau de I'IC». Quand on voit dans ce contexte "alimentation en eau de I'IC», on peut se demander ce que ça désigne. Vous ne trouvez pas qu'il y a comme un décalage?

$\mathbf{R}$ : Effectivement.

$\mathbf{Q}$ : Mais vous, quand vous dites FP, vous partez de l'idée qu'il faut envoyer de l'eau dans le réacteur, n'est-ce pas?

$\mathbf{R}$ : Oui, tout à fait. 
$\mathbf{Q}$ : Alors, je voudrais qu'on revienne sur quelque chose dont on a déjà un peu parlé. Ça n'est qu'une hypothèse, une fois de plus. Vous avez ouvert l'IC à 18h18, puis vous l'avez fermé à 18h25. Si vous dites que vous l'avez fermé à 25, ça signifie qu'il n'est plus ouvert. Vous êtes bien d'accord ? Ce M. XXXXX du groupe « production »...

\section{$\mathbf{R}:$ XXXXX?}

$\mathbf{Q}:$ Oui. Je ne connais pas son prénom.

\section{$\mathbf{R}$ : Vous voulez dire XXXXX qui s'écrit XXXXX?}

$\mathbf{Q}$ : ॥ était resté à côté de $M . X X X X X$. Et M. XXXXX reçoit un rapport signalant, à 18h20 à sa montre, mais je pense que c'est la même chose que ce qui s'est passé à 18h18, l'« ouverture totale des vannes M03A et MO2A, pour le réacteur 7 ». Mais ensuite, il n'y a pas de rapport signalant leur fermeture. En tout cas, quand on regarde leurs carnets, rien de tel n'est écrit. Ce qui est signalé à peu près au même moment, c'est la « vérification du fonctionnement du réseau A de I'IC du réacteur 1 à $78 h 24$ ». Et il n'y a rien d'autre. Ensuite, il n'y a rien pendant assez longtemps, jusqu'à ce qu'on apprenne de nouveau à 21h30, "ouverture de la vanne MO3A de I'IC». II n'y a pas l'étape où elle aurait été fermée. Alors, il est diffıcile de savoir si c'est l'équipe de quart qui ne l'avait pas signalé, ou bien, qu'elle l'avait bien signalé, mais que, dans la confusion, l'information qui est arrivée jusqu'au groupe «production » ait pu être tronquée. Quand on regarde la manière dont les rapports sont rédigés, on peut aussi se poser des questions. Les informations arrivent isolées les unes des autres. On dirait que les manœuvres sont faites isolément les unes des autres, en rang dispersé. On n'arrive pas à savoir, au moment où ils ferment ces vannes, jusqu'à quel point ils se rendent compte que le réacteur 1 va se trouver pendant un moment sans aucun apport en eau. On se demande jusqu'à quel point les gens en avaient conscience et s'ils partageaient cette inquiétude.

Pour en revenir au malentendu, c'est comme si, de fil en aiguille, à force de voir arriver des rapports sur la DDFP, en entendant parler d'» $I C$ en marche » et de "passer par la DDFP », les gens s'étaient persuadés tout seuls que c'était dans l'IC qu'il fallait envoyer l'eau. Peut-être que la personne qui a pris ces notes, à partir de faits avérés, s'était échafaudé une thèse, tout à fait hypothétique, et avait compris qu'il fallait passer par le réseau FP pour alimenter I'IC.

Pour en revenir à vous, vous, vous pensiez que l'IC était toujours en marche?

R : Oui.

$\mathbf{Q}$ : Vous étiez persuadé que l'IC fonctionnait. C'est à partir de quel moment que vous avez commencé à avoir des soupçons quant au fonctionnement de l'IC ?

$\mathbf{R}$ : Comme je vous l'ai dit l'autre jour, parce que la radioactivité était montée. Tout ce qu'on pouvait surveiller, à ce moment-là, c'était le niveau d'eau. Et il était stable. Si le niveau d'eau avait commencé à baisser, on se serait douté qu'il y avait peut-être quelque chose d'anormal au niveau de l'IC. Mais l'IC était en fonctionnement et, par la suite, il n'y avait eu aucun signalement concernant des changements dans le comportement de l'IC. Vous voyez? Rien n'a été noté. Du coup, on pense que l'IC fonctionne. Puisqu'il n'y a de visible que le niveau d'eau, on le surveille. On pense qu'il est suffisant. Puis, la radioactivité commence à monter. Ce sont les premières anomalies. C'est là que je commence à me dire que c'est bizarre. Je commence à me demander si l'IC fonctionne véritablement, si le niveau d'eau est véritablement suffisant.

$\mathbf{Q}$ : Là, c'est une chose qui n'a pas été notée noir sur blanc, mais quel est le moment où vous vous êtes dit que vous ne pouviez plus compter sur l'IC?

$\mathbf{R}:$ J'ai presque tout oublié.

$\mathbf{Q}$ : Par exemple, au moment où la DDFP s'arrête, vous en recevez l'information. Est-ce qu'à ce moment, vous vous dites, "c'est mauvais, heureusement que l'IC fonctionne encore, mais il 
va falloir faire quelque chose avant que I'IC ne s'arrête»? Ou bien, vous pensez que I'IC, non plus, ne fonctionne plus?

$\mathbf{R}$ : C'est que les images sont plus que floues, pour ce qui est des rapports concernant la DD et son fonctionnement. Pour moi, je ne réfléchissais pas à la DD et à l'IC en corrélation. Les deux n'étaient pas liés. Dans ma tête, la DD n'occupait pas une place prépondérante. Pour moi, ce qui était préoccupant, c'était l'état du réacteur. Quand j'ai su que la radioactivité avait grimpé, ce qui m'a inquiété, ce n'est pas tant ces histoires d'IC, mais l'état du réacteur. Que la radioactivité monte, c'est qu'il se passe des choses anormales au niveau du réacteur.

$\mathbf{Q}$ : Mais vous avez quand même pensé que l'IC n'était plus fiable, qu'il ne fonctionnait plus beaucoup?

$\mathbf{R}:$ Non, je ne pensais pas jusque-là.

$\mathbf{Q}$ : D'accord. Ensuite, vous vous mettez à envoyer de l'eau avec les voitures de pompiers.

R : Oui. Ça, c'est le matin.

$\mathbf{Q}$ : Oui. Et au matin ? Qu'en pensiez-vous ?

$\mathbf{R}$ : Là, je pensais que l'IC était totalement fichu.

$\mathbf{Q}$ : Vous ne comptiez plus du tout dessus.

R : Non.

$\mathbf{Q}$ : Alors, entre ces deux moments, il s'est passé quelque chose qui vous a fait penser que I'IC était fichu? Vous aviez peut-être demandé qu'on vérifie son état de fonctionnement, non?

$\mathbf{R}$ : Non. Tout ce qui concerne les ouvertures ou les fermetures de vannes, tout ça, je ne m'en faisais pas informer systématiquement, à ce moment-là. Mes souvenirs sont vraiment flous, surtout en ce qui concerne le premier jour. Je ne m'en souviens vraiment pas.

$\mathbf{Q}$ : Alors, lorsque vous avez commencé à préparer l'éventage, vous en avez donné l'ordre vers 0h06, qu'en pensiez-vous?

$\mathbf{R}$ : Là, je pensais que l'IC ne fonctionnait pas. Il me semble que c'était vers $22 \mathrm{~h}$ que la radioactivité était montée. À ce moment-là, je n'attendais presque plus rien de l'IC. Ce n'était même pas la question de savoir s'il était en marche ou pas. Ce que je savais, c'est que l'IC n'arrivait pas à refroidir comme je l'aurais voulu. C'est pour ça qu'il fallait envoyer de l'eau et qu'il fallait éventer. Seulement, à ce moment-là, on n'avait aucune idée de la pression de la chambre sèche. On avait bien commencé à préparer son éventage, mais on n'y voyait rien. Seule la radioactivité augmentait. Alors je voudrais bien savoir ce qu'il fallait en penser. Si le vieux Madarame avait été là, ou qui que ce soit d'autre, j'aurais bien voulu qu'ils me disent sur quoi je pouvais me fonder pour juger de la situation.

$\mathbf{Q}$ : Vous avez donc pensé que c'était parce que, que l'IC soit en fonctionnement ou pas, et que même s'il avait été en fonctionnement, il ne donnait pas de résultat satisfaisant, que vous étiez dans cette situation critique. Ce qui veut dire, je suppose, qu'au moment où vous vous apprêtez à éventer, vous n'attendiez plus rien de l'IC.

Ensuite la situation évolue, puisque finalement la DDFP ne fonctionne pas. Je vois que, avant ça, le 11 mars à $17 \mathrm{~h} 12$ précisément, vous donnez des ordres pour qu'on commence à étudier le moyen d'injecter l'eau avec les voitures de pompiers. Concrètement, pour cette injection, il va vous falloir un peu de temps. J'ai interrogé à ce propos une personne de Nanmei Corporation qui m'a dit que c'était vers 3h00 du matin, ou un peu avant, qu'ils avaient commencé à chercher des bouches d'incendie, etc. Ensuite, le personnel de 
Nanmei faisait des allers et retours avec leur camion. II semblerait qu'au début l'opération n'était pas encore très rationnalisée. Visiblement, ça a duré comme ça pendant un certain temps. Si je résume, vous avez donc commencé les différentes manœuvres vers les 3h00 du matin, mais, jusque-là, on ne signale aucun mouvement. $Y$ avait-il une raison particulière à ça?

$\mathbf{R}$ : C'est parce qu'on ne connaissait pas la pression du réacteur. Au début, on a une pression d'environ 6,9. Ça, c'est une pression normale de conduite et c'est la pression à $20 \mathrm{~h}$. Ensuite, on n'arrive plus à voir la pression du réacteur. Pendant ce temps, la situation se dégrade. La radioactivité grimpe. On voit enfin la pression pour la première fois à $2 \mathrm{~h} 45$. On a 0,8 . C'est une pression qui permet d'injecter l'eau. Plus élevée, on n'aurait pas pu. C'est donc à ce moment-là que j'ai jugé qu'on pouvait commencer effectivement l'injection.

$\mathbf{Q}$ : Et c'est alors que vous rencontrez quelques difficultés. Pour reprendre les choses, vous vous dites que la pression a suffisamment baissé pour qu'on puisse injecter l'eau. Vous donnez le signal de départ. Mais c'est à ce moment-là qu'on s'aperçoit que les bouches ne sont pas forcément accessibles. Là, c'était à cause du tsunami. J'ai entendu dire qu'il y avait des voitures qui avaient été renversées, etc.

$\mathbf{R}$ : Oui, on m'a dit que l'accès était très difficile.

$\mathbf{Q}$ : Vous commencez donc par déblayer un peu et, vers les 3 ou 4h00, vous êtes en train d'injecter l'eau avec les voitures de pompiers.

Je voudrais qu'on parle encore des fonctionnalités du réacteur 1. C'est quelque chose dont on ne parle pas dans le déroulement des évènements. À la page 31 de ce document, il y a un résumé des nouvelles mesures prises dans le cadre de la gestion des accidents pour le réacteur 1. II y a une énumération des différents moyens d'injecter l'eau dans le réacteur ou l'enceinte de confinement.

D'autre part, à la page 32, il y a aussi un résumé similaire concernant les réacteurs 2 à 5 . On remarque que pour les 2 à 5, on note l'« automatisation de la dépressurisation du réacteur, par la vanne de sécurité ». Et, pour le réacteur 1 ?

$\mathbf{R}$ : Vous faites allusion à la fonction ADS (Automatic Depressurization System) ${ }^{64}$ ?

$\mathbf{Q}$ : Cette fonctionnalité n'existe pas sur le réacteur 1 ?

R : Mais si, elle existe.

$\mathbf{Q}$ : Vous voulez dire qu'on l'a rajoutée avant ou après la parution du document ?

$\mathbf{R}$ : Elle existe dès le départ.

$\mathbf{Q}:$ Le réacteur a été livré avec cette fonctionnalité ?

R : Oui.

$\mathbf{Q}$ : Ah, et du coup, on n'a pas eu besoin de la rajouter plus tard!

$\mathbf{R}$ : Tous les réacteurs possèdent l'ADS. Je ne comprends vraiment pas où veut en venir ce document.

$\mathbf{Q}:$ Et ça ? Ici?

$\mathbf{R}:$ Il s'agit de la SRV?

$\mathbf{Q}:$ Oui, il semble bien que ce soit la SRV, quand on voit ça.

$\mathbf{R}$ : Je pense qu'ils parlent de l'ouverture manuelle de la SRV. À moins que ce ne soit l'ouverture automatique?

$\mathbf{Q}:$ Moi, j'ai cru qu'il s'agissait de l'ouverture automatique.

$\mathbf{R}$ : De toute façon, ça existait déjà avant qu'on ne mette en place les nouvelles dispositions. 
Q: Ils ont écrit: "on a fait en sorte que, grâce à la dépressurisation automatique du réacteur nucléaire par la SRV, même dans ces circonstances, l'injection d'eau dans le cœur du réacteur soit possible par des systèmes à basse pression comme le ECCS ». Jusque-là, ça n'était pas possible?

$\mathbf{R}$ : Excusez-moi. Je vais vous expliquer. L'ADS ne fonctionne pas quand la pression est basse. Dans ce cas-là, comme le signal " pression haute dans la chambre sèche " ne s'allume pas, l'ADS ne s'enclenche pas. Jusque-là, seul le signal « pression haute dans la chambre sèche " pouvait déclencher l'ADS. Mais on a fait en sorte que même sans ce signal «pression haute dans la chambre sèche ", l'ADS par la vanne SRV puisse se faire, si le signal « niveau d'eau bas dans le réacteur » s'allumait. C'est-à-dire qu'on a fait des aménagements pour que si le niveau d'eau baisse, la dépressurisation du réacteur se fasse automatiquement pour permettre l'injection d'eau à basse pression. C'est un changement dans la logique du déclenchement de l'ADS, mais juste une histoire de logique.

$\mathbf{Q}:$ La fonctionnalité, en soi, a toujours existé.

$\mathbf{R}$ : Oui, c'est ça.

$\mathbf{Q}:$ Et ça, pour le réacteur 1 ?

$\mathbf{R}$ : Probablement, ça a toujours existé.

$\mathbf{Q}$ : Vous voulez dire que le réacteur 1 possédait bien une fonction qui permettait, si le niveau d'eau baissait, de le signaler et que ce signal déclenchait une dépressurisation en laissant échapper automatiquement de la vapeur par la vanne SRV ?

$\mathbf{R}:$ Oui, c'est ça.

$\mathbf{Q}$ : Cette fonction qui permettait d'envoyer ces signaux était bien vivante sur les réacteurs 1, 2 et 3, lors de l'accident?

$\mathbf{R}$ : Non, pas du tout. Cette fonction fait partie du système RPS (Reactor Protection System $)^{65}$. Or, aucun signal de ce système n'était détectable, faute de courant. Alors, on peut considérer qu'aucun de ces automatismes ne pouvait fonctionner après l'arrivée du tsunami.

$\mathbf{Q}$ : Alors si, par exemple, de la vapeur est subitement produite en grosse quantité à l'intérieur du réacteur, que le réacteur est envahi de vapeur, que la pression monte à un niveau élevé, y a-t-il un mécanisme qui, sans passer par ces signaux, s'ouvrirait tout seul?

$\mathbf{R}$ : Oui, ça existe. C'est ce qu'on appelle la vanne de sécurité. La vanne de sécurité est prévue pour s'ouvrir mécaniquement quand la pression du réacteur atteint sa valeur de dimensionnement, 87,2 kilos, comme on disait autrefois, c'est-à-dire environ 0,87 mégas. Mais la pression dont on parle maintenant n'atteint pas ce niveau. La fonction de la vanne de sécurité est d'assurer la protection de la cuve. Pour ça, si la pression atteint la valeur de dimensionnement, elle laisse automatiquement s'échapper la vapeur.

Mais avant d'atteindre ce stade, quand on est encore à 73 ou 75 kilos, ce qui permet de baisser la pression par étape est la fonction «vanne de rejet ». C'est pour ça qu'on dit la vanne de rejet de sécurité. La fonction « vanne de sécurité » va laisser échapper la pression pour protéger la cuve quand celle-ci atteint une pression vraiment proche de la valeur de dimensionnement. La fonction » vanne de rejet ", elle, à un niveau de pression moins élevé, permet de rejeter de la vapeur suivant les besoins. 
$\mathbf{Q}$ : Alors, si on est à 7 mégas, comme ici, la fonction «vanne de sécurité » ne va pas s'enclencher?

$\mathbf{R}$ : Non, pas la fonction « sécurité ». C'est justement vers les 0,73 de pression que la fonction « rejet » va se mettre en marche pour la première fois.

$\mathbf{Q}$ : Dans ce cas, si on veut injecter de l'eau à basse pression, il faut dépressuriser en utilisant la fonction « rejet », c'est bien ça?

R : Oui.

$\mathbf{Q}$ : Quand on a parlé du réacteur 3, on avait vu que quand le HPCl s'était arrêté le 13 mars à $2 \mathrm{~h} 42$, en fait la pression était à quelque chose comme 0,5 . Et je vous avais fait remarquer que, dans ces conditions, vous auriez pu injecter de l'eau, avec la DDPF ou les voitures de pompiers. Avec cette pression, l'eau serait entrée. Bien sûr, c'est une réflexion que je vous avais faite après coup. J'ai visionné les échanges de la conférence TV à ce sujet et je voudrais savoir s'il vous était arrivé d'effectuer cette manœuvre de dépressurisation dans la réalité.

\section{$\mathbf{R}$ : Oui, on l'a faite sur le réacteur 3.}

$\mathbf{Q}$ : Et avant ? Je veux dire, vous était-il arrivé de faire cette manœuvre avant le séisme et le tsunami de mars?

$\mathbf{R}$ : Pour ma part, je n'avais jamais effectué cette manœuvre pour de vrai.

$\mathbf{Q}$ : Mais il existe des exemples de cette manœuvre?

$\mathbf{R}:$ Oui, il y en a.

$\mathbf{Q}$ : Et sait-on dans quelle mesure la pression va baisser si on effectue cette manœuvre dans la réalité?

$\mathbf{R}$ : Je pense que oui.

$\mathbf{Q}$ : Au moment où vous avez effectué la manœuvre en mars, le saviez-vous ?

R : Oui.

$\mathbf{Q}$ : Et peut-on contrôler cette baisse?

$\mathbf{R}$ : Non, on ne peut pas. Ça descend pas mal.

$\mathbf{Q}$ : En fait, la discussion que j'ai vue sur la vidéo de la téléconférence a lieu beaucoup plus tard, vers 4 ou 5 h00 du matin. À un moment donné, la pression est tombée à 4,1 MPa et vous constatez que l'eau n'entre pas avec la DDFP. Ce qui est naturel avec la pression qu'il y a encore. Pour faire baisser la pression, il faudrait manœuvrer la vanne de rejet. Mais la discussion porte sur l'éventualité, par exemple, que la pression ne descende qu'à 1,1 MPa, ou que, même si elle descendait à 0,9 ou 0,8, vous ne réussissiez pas à injecter l'eau. Premièrement, vous ne savez pas jusqu'où la pression va descendre. Deuxièmement, vous n'êtes pas sûrs que l'eau entre avec un véhicule de pompiers. Là, il y a discussion car, si l'eau pouvait entrer de manière certaine d'après les chiffres que vous avez calculés objectivement, ce serait rassurant, mais si, par malheur, elle n'entrait pas, comme vous auriez fait baisser la pression en laissant s'échapper la vapeur du réacteur, le cœur du réacteur risquait de rester dénoyé. Et vous vous demandez de nouveau quelle est la pression de la DDFP. Si j'ai bien compris, la DDFP est assez faible, de l'ordre de 0,5 à 0,6. Ce qui veut dire qu'il ne suffit pas que la pression du réacteur descende à un peu moins de $1 \mathrm{MPa}$. Du coup, même si avec le HPCl, on est arrivé à 0,5, vous n'êtes pas sûrs que ça va marcher. Par contre, avec les voitures de pompiers...

$\mathbf{R}$ : Oui, les voitures de pompiers disposent d'une pression de 1 méga, alors.

$\mathbf{Q}$ : Là, vous pensez que ça pourrait marcher. Ce genre d'échanges dure assez longtemps. 
Comme il y avait eu toutes ces discussions autour du réacteur 3 , je me suis dit qu'il était compréhensible, quelque part, que vous n'ayez pas tout de suite tenté d'injecter l'eau à l'arrêt du HPCl.

Pour en revenir au réacteur 1, avez-vous aussi eu des discussions pour savoir s'il fallait faire ce genre de manœuvres de dépressurisation?

R : Non, pour lui, on n'a pas discuté pour savoir s'il fallait faire des manœuvres de dépressurisation. Je me répète, mais, vous comprenez, dépressuriser, c'est la dernière solution. Puisque ça consiste à rejeter de l'eau du réacteur vers l'extérieur, ça va faire baisser le niveau d'eau à l'intérieur. Ce qu'on voudrait, ce à quoi on réfléchit, c'est de trouver un moyen de faire entrer de l'eau tout en gardant une pression élevée. Comprenez-moi, le réacteur 1 , c'était le premier parmi tous les autres. C'était le premier pour lequel il a fallu réfléchir et on en a brassé, des idées. Bien évidemment, dépressuriser et injecter l'eau, c'était une solution possible et, d'ailleurs, j'avais donné des ordres dans ce sens. Puisqu'on n'avait pas d'alimentation électrique, j'avais ordonné qu'on prépare bien en amont l'utilisation de la DD et des voitures de pompiers. J'avais tout ça en tête, c'est sûr. Mais c'était la dernière solution à laquelle j'avais envie de recourir. S'il y avait eu moyen de rétablir l'électricité, j'aurais choisi en premier d'injecter l'eau en gardant une pression élevée. Cela aurait été la meilleure solution. J'y ai pensé à tout ça. En plus, j'étais persuadé que l'IC tournait. Et puis, au milieu de tout ça, il commence à y avoir des indices inquiétants. Seulement, on ne connaît pas la pression du réacteur. On continue à vérifier l'alimentation de tous les moyens substitutifs d'injection d'eau. Ce faisant, la situation se dégrade de plus en plus. J'avais donné des ordres pour l'injection d'eau, mais nous ne sommes pas capables de la réaliser tout de suite. On commence à envisager la possibilité d'un éventage. Et, en fait, avant même qu'on ne commence à juger de la nécessité d'une dépressurisation, pendant qu'on courait à droite et à gauche, à un moment donné, on a vu que la pression était déjà descendue. Alors, bien sûr, pendant tout ce temps, j'avais bien en tête qu'il fallait dépressuriser à un moment ou un autre pour pouvoir injecter l'eau, cette idée je l'avais en tête depuis le début, mais, pour moi, ça a toujours été la dernière des solutions. C'était la toute première tranche. J'ai vraiment cherché tous les moyens d'injecter l'eau.

$\mathbf{Q}:$ Si je me souviens bien, à ce moment-là, vous ne pouviez pas lire les instruments qui donnaient la pression. Est-il concevable d'entreprendre une manœuvre de dépressurisation sans pouvoir vérifier la pression?

$\mathbf{R}$ : Non, ce n'est pas possible.

$\mathbf{Q}$ : Quand vous ne pouvez pas connaître la pression, je veux dire.

R : Non.

$\mathbf{Q}$ : Vous êtes donc dans cette situation, quand, tout à coup, vous avez pu voir la pression et elle était déjà en-dessous de 1. Quand on regarde la manière dont les évènements se sont enchaînés au niveau du réacteur 1 , ne trouvez-vous pas que leur évolution est extrêmement rapide comparée aux autres réacteurs ? Avez-vous une idée pour expliquer ça?

$\mathbf{R}:$ Je' ne sais pas.

$\mathbf{Q}$ : Supposons que l'IC n'ait pas bien fonctionné, est-ce que cela peut expliquer cette vitesse de dégradation?

$\mathbf{R}$ : Je ne sais pas. Les analystes, qui ont étudié ça, ont conclu que c'était possible. D’après eux, l'IC n'avait pas fonctionné et ils ont publié des données qui montrent que, dès ce moment-là, le combustible était totalement à l'air. Ils le disent après coup. 
Puisqu'ils le disent, il ne me reste qu'à le croire. Mais, pour moi, c'était la première fois que je vivais ce genre d'expérience sur le terrain et il m'est impossible d'imaginer ce qui s'est passé. C'est une zone d'ombre.

Q: Évidemment, aujourd'hui, après coup, on peut dire toutes sortes de choses en remontant des résultats. Mais, sur le moment, vous ne pouviez pas voir la pression, vous cherchiez désespérément une solution, des anomalies surviennent coup sur coup sur l'IC ou d'autres moyens d'injection, même si vous ne comptiez pas trop dessus. Vous prévoyiez d'utiliser la DDFP. Si ça ne marchait pas, vous aviez encore la solution des camions de pompiers. Et, sur ces entrefaites, vous vous apercevez tout à coup que la pression est tombée à 0,800 . Là, l'injection devient possible et les pompiers maison se précipitent à plusieurs pour commencer la manœuvre. C'est bien comme ça que ça s'est déroulé?

R : Oui.

Q: Dans cette succession d'évènements, vous aviez envisagé l'utilisation de la vanne de rejet, mais comme, fondamentalement, le fait de rejeter de la vapeur revient à faire baisser le niveau d'eau et que, pour y remédier, il faut pouvoir injecter tout de suite de l'eau, cette solution était véritablement pour vous le dernier choix. Mais, avant même que vous ne soyez confronté à une situation exigeant ce choix, la pression avait baissé.

Que signifie le fait que la pression ait baissé sans qu'on n'y fasse rien?

$\mathbf{R}$ : Je n'en sais rien. Ça me pose vraiment problème, vous savez. Une des hypothèses possibles est que la vanne se soit ouverte en grand et qu'elle soit restée bloquée dans cette position. C'est un système mécanique simple. La vanne s'ouvre quand la pression monte et, quand la pression retombe, en principe, elle se referme toute seule. Mais si elle reste bloquée en position » ouvert ", elle va laisser s'échapper toute la vapeur. C'est donc l'une des hypothèses auxquelles on pourrait penser.

$\mathbf{Q}$ : Ça veut dire que la pression a pu monter un peu au-delà des 6,9 que vous aviez là, que la vanne de rejet s'est ouverte et qu'elle est restée ouverte, provoquant la chute de la pression?

$\mathbf{R}$ : Oui. Mais quand on regarde les conclusions de cet idiot d'analyste, il prétend que c'est arrivé bien plus tôt. C'est ça que je ne comprends pas. Je n'arrive pas à comprendre ce que l'analyste veut dire.

$\mathbf{Q}$ : Ce sont des analyses entreprises par le siège, n'est-ce pas?

$\mathbf{R}$ : Oui, ce sont les analyses du siège.

$\mathbf{Q}$ : Les analyses sont des analyses, ce ne sont que des hypothèses. Moi, ce que je voudrais savoir c'est ce que vous pensiez être la réalité à ce moment-là. De toute façon, vers 20 h00, $21 \mathrm{~h} 00$, vous croyiez que l'IC était en marche, n'est-ce pas?

R : Oui.

$\mathbf{Q}$ : À un moment donné, vous réussissez à voir le niveau d'eau. Vous êtes encore dans la zone positive du TAF. Le niveau est relativement bas mais semble stable. C'est alors que, à partir de 22h, différents évènements montrant la hausse de la radioactivité se succèdent, vous donnant des soupçons. Sur ces entrefaites, vous apprenez que la pression de la chambre sèche est montée à 600 . Faute de mieux, vous envisagez l'éventualité d'un éventage pour protéger l'enceinte de confinement. D'un autre côté, vous êtes aussi occupé à la préparation de l'injection d'eau, quand, à $2 \mathrm{~h} 45$, la pression est descendue à 0,8 . Les évènements avaient évolué tout seuls dans ce sens sans que vous ayez eu besoin de faire des manœuvres de dépressurisation.

$\mathbf{R}$ : C'est ça. 
$\mathbf{Q}$ : Nous devons aborder maintenant l'histoire du réacteur 2. Mais cela fait déjà deux heures que nous parlons. Nous allons faire une petite pause avant d'enchaîner sur le réacteur 2.

(Pause)

$\mathbf{Q}$ : Je voudrais maintenant vérifier les faits pour le réacteur 2 . On lit à peu près la même chose que pour le réacteur 1. Tout d'abord, concernant l'injection d'eau, là encore, pour la même raison, vous ne pouvez pas utiliser le système MUWC.

$\mathbf{R}:$ C'est ça.

$\mathbf{Q}$ : Ensuite, pour le réseau FP, la tuyauterie en elle-même a l'air indemne, mais, dès le début, la pompe DDFP est inutilisable.

R : Oui.

$\mathbf{Q}$ : Pourquoi ne pouviez-vous pas l'utiliser?

$\mathbf{R}$ : Probablement, à cause de l'eau. Elle avait été inondée.

$\mathbf{Q}$ : Vous faites donc très vite une croix dessus et il ne vous reste plus que la solution de l'injection par les camions de pompiers, c'est bien ça?

R : Oui.

$\mathbf{Q}$ : Je continue à lire. II y a le descriptif des moyens d'injection dans les réacteurs 2 à 5 . En fait, ce que vous avez fini par faire lors de l'accident, c'est-à-dire l'injection par le réseau de lutte contre l'incendie au moyen des véhicules de pompiers, puisque la pompe diesel du système FP, elle-même, ne fonctionnait pas à cause, sans doute, de l'eau, n'est pas décrit en tant que tel dans cette liste. Mais il est fait allusion au réseau que vous avez utilisé, puisqu'ils signalent la modification qui permet de passer par le réseau RHR pour une injection dans le réacteur.

II y a un schéma du réacteur 2 à la page 41. Là, c'est la ligne qui va vers le réseau FP avec la pompe diesel. Ensuite, ici, les nouveaux raccords qui permettent de partir vers le réseau RHR. C'est par ici que vous êtes passé, n'est-ce pas?

R: Oui, la ligne d'injection à basse pression LPCI (Low Pressure Coolant Injection system $)^{66}$.

Q: Vous voulez dire que vous avez envoyé l'eau à l'intérieur du réacteur par cette ligne d'injection?

R : Oui. La ligne d'injection à basse pression n'y est pas reliée directement. Il faut d'abord enter dans le système de recirculation du réacteur.

$\mathbf{Q}$ : Quand vous dites que vous injectez à basse pression, que faut-il imaginer? Ça ressemble à une aspersion?

$\mathbf{R}$ : Dans le cas spécifique du réacteur 2, ça n'entre pas dans le système Core Spray. Le réacteur 1 de tout à l'heure était prévu pour que l'eau entre dans le Core Spray. Mais, pour le réacteur 2, comme l'eau ne va pas dans le système Core Spray, elle entre par le bas. Elle coule tout simplement d'un tuyau.

$\mathbf{Q}$ : Pouvez-vous m'expliquer comment ça se passe pour le réacteur 1 ?

R: L'eau entre dans le système Core Spray. Du coup, elle arrive par le haut, en aspersion.

$\mathbf{Q}$ : Et ici, pour le 2, c'est différent?

$\mathbf{R}$ : Oui, c'est différent.

$\mathbf{Q}$ : Ça veut dire qu'il y a plusieurs modèles?

$\mathbf{R}$ : À la base, la conception « système » diffère. Le réacteur 1 ne dispose pas du RHR. 
Q: Quand je regarde ça, ils font allusion à un système de Containment Head Spray67. C'est différent du Core Spray?

R: Le Containment Head Spray est un système qu'on utilise lorsqu'on veut baisser rapidement la température de l'enceinte quand on arrête le réacteur. On lui envoie de l'eau par le haut. Mais ce n'est pas un système prévu pour être utilisé en cas d'incident.

$\mathbf{Q}$ : Ça permet d'envoyer de l'eau à l'intérieur de l'enceinte ?

R : Oui.

$\mathbf{Q}:$ Ce n'est donc pas le système que vous avez utilisé lors de l'accident. L'eau est en fait passée par le réseau d'injection à basse pression, c'est ça ?

R : Oui.

Q: Ce qu'on voit ici, c'est un système d'aspersion pour refroidir l'enceinte, n'est-ce pas ? Ce système existe aussi bien pour le réacteur 2 que le réacteur 3 ?

R : Oui.

$\mathbf{Q}:$ Et ça, qu'est-ce que c'est?

R : C'est le Suppression Chamber Spray ${ }^{68}$ du Containment Spray System ${ }^{69}$. C'est la même chose que le Drywell Spray ${ }^{70}$. C'est juste qu'il est placé à un endroit différent.

$\mathbf{Q}:$ : I y a donc des lignes des deux côtés. C'est commandé par des vannes MO ?

R : Oui.

$\mathbf{Q}$ : Alors, est-il possible de les ouvrir à la main?

R : Oui, là, on peut ouvrir à la main.

$\mathbf{Q}:$ : Et elles se trouvent où, géographiquement?

$\mathbf{R}$ : Comme c'est au niveau des chambres de dépressurisation, c'est assez bas.

$\mathbf{Q}$ : C'est à l'intérieur des tores ${ }^{71}$ ?

$\mathbf{R}$ : Oui, c'est dans les tores. À l'intérieur. Il me semble que c'est au $2^{\mathrm{e}}$ niveau.

$\mathbf{Q}:$ Le CCS?

$\mathbf{R}$ : Il me semble bien que c'est au $2^{\mathrm{e}}$ niveau du bâtiment réacteur.

$\mathbf{Q}$ : Et vous sauriez où se situe la vanne $\mathrm{MO}$ du $\mathrm{LPCl}$ ?

$\mathbf{R}$ : Je crois qu'elle aussi, elle est au $2^{\mathrm{e}}$ niveau.

$\mathbf{Q}$ : Dans les deux cas, c'est à l'intérieur du bâtiment réacteur?

R: Oui, c'est à l'intérieur du bâtiment. À l'extérieur de l'enceinte de confinement et à l'intérieur du bâtiment réacteur.

$\mathbf{a}:$ Si on veut ouvrir ces vannes, je suppose que ça soulève des problèmes d'irradiation.

$\mathbf{R}$ : Oui. Je n'ai pas le souvenir ni du moment ni de la manière dont on est allé ouvrir cette vanne. Je suppose qu'au moment où j'ai donné l'ordre de réaliser la ligne pour l'injection, les gars de la production ont dû s'en occuper, mais on n'a pas eu écho de vannes difficiles à ouvrir, comme quand on avait voulu manœuvrer la vanne MO au moment de l'éventage de la chambre sèche.

$\mathbf{Q}$ : D'après ce que m'a raconté $M . X X X X X$, pour les réacteurs 1 et 2 , la manœuvre s'est faite très tôt, le 11 vers 18h00, au moment où vous aviez renoncé à l'IC. C'était encore une période où la radioactivité n'était pas très forte. Ils ont été gênés par le manque de lumière, mais apparemment, cela s'est fait sans trop de problème. 
Pour les réacteurs 3 et 4 , on n'arrive pas à bien comprendre ce qui s'est passé. Même M. XXXXX n'a plus de souvenir clair. Vous vous rappelez que, dans le cas du réacteur 3, vous aviez utilisé le réseau du CCS le 13 mars ? Pour faire ça, il me semble qu'il fallait ouvrir la vanne, non?

R : Oui.

$\mathbf{Q}:$ Et, à ce moment-là, comment était la radioactivité ?

$\mathbf{R}$ : Elle était déjà forte. Les souvenirs m'échappent. Surtout que je suis resté confiné dans le bâtiment antisismique... J'avais donné l'ordre qu'on réalise cette ligne, j'avais beaucoup insisté. Sur ces entrefaites, on m'a dit que l'aspersion de l'enceinte de confinement était possible. Si elle était réalisable, cela nous permettait de refroidir l'enceinte. Je venais de dire "d'accord, allons-y", quand le siège m'a ordonné d'arrêter, parce qu'ils craignaient que, si on refroidissait l'enceinte, la vapeur ait du mal à sortir. Tout ça, je m'en souviens très bien. Mais, s'agissant de l'ouverture de la vanne, je crois que personne ne m'a demandé mon avis.

$\mathbf{Q}$ : II n'y a pas eu de problème pour entrer dans le bâtiment à cause de la radioactivité ?

$\mathbf{R}$ : Non, je n'ai rien entendu de tel. Je ne sais même pas s'ils ont ouvert manuellement ou pas. Le souvenir s'est volatilisé.

$\mathbf{Q}$ : Il me semble qu'au réacteur 3 , il y avait pas mal de choses qui disposaient de batterie et que vous pouviez commander de la salle de contrôle, n'est-ce pas?

$\mathbf{R}$ : Oui, c'est vrai. Peut-être l'ont-ils ouverte comme ça. Seuls ceux qui étaient sur place pourraient vous le confirmer.

$\mathbf{Q}$ : Si je résume, lorsqu'il s'est agi de réaliser un réseau de tuyaux à l'intérieur du bâtiment réacteur, personne ne vous a parlé de vanne qui ne s'ouvre pas ou d'autres problèmes de ce genre.

$\mathbf{R}$ : Vous savez, le principal souci du réseau de lutte contre l'incendie, c'est qu'il se scinde en plusieurs branches à l'intérieur des bâtiments. Il y a des branches qui partent vers le bâtiment turbine, d'autres, ailleurs. Même si on arrivait à injecter l'eau, celle-ci risquait de partir ailleurs. Je n'ai aucun souvenir de propos autour des vannes de la ligne d'injection, mais je me rappelle avoir dit, au contraire, de fermer les vannes commandant l'accès aux autres branches. Il y a énormément de ramifications. Il fallait interdire l'accès vers ces branches inutiles en fermant les robinets d'arrêt pour que l'eau soit acheminée le plus efficacement possible vers le réacteur. Ça, je me rappelle en avoir parlé plusieurs fois. Par contre, je suis désolé, mais je n'ai aucun souvenir de discussion autour de l'ouverture des vannes de cette ligne d'injection. Pour moi, les choses s'étaient bien passées et je n'étais pas plus au courant que ça de ce qu'il en était sur le terrain.

$\mathbf{Q}$ : D'accord. Si on revient au schéma, ici, c'est le réseau FP, et là, le réseau MUWC, n'est-ce pas?

Ensuite, qu'en est-il de l'automatisation de la dépressurisation du réacteur ? Vous savez, ce dont on a parlé tout à l'heure, la modification qui permet le déclenchement de cette dépressurisation automatique, lorsque le signal «niveau d'eau réacteur bas » est émis. L'avez-vous utilisé ?

$\mathbf{R}$ : Non, ça n'a pas fonctionné.

$\mathbf{Q}$ : Ah, oui. Le signal ne pouvait pas être émis, en effet. Au niveau du réacteur 2 , vous avez finalement effectué une manœuvre de dépressurisation. Vous l'aviez faite intentionnellement, à la main ?

R : Oui. 
$\mathbf{Q}$ : Donc, le signal n'a pas pu être émis faute d'électricité.

$\mathbf{R}:$ C'est ça.

$\mathbf{Q}$ : Mais ça veut dire aussi que, si vous aviez disposé d'électricité, après l'arrêt du RCIC, quand le niveau d'eau s'est mis à descendre rapidement, le signal aurait été émis et l'ensemble du dispositif amenant à la dépressurisation automatique du réacteur aurait pu fonctionner. Si vous aviez eu de l'électricité, bien sûr.

R : Oui.

$\mathbf{Q}$ : Ensuite, il y a les nouveaux dispositifs concernant le refroidissement de l'enceinte de confinement, le Drywell Cooler et le système utilisant le réseau CUW, dont on a parlé tout à l'heure. Les avez-vous utilisés ?

$\mathbf{R}$ : Non, on n'a pas pu.

$\mathbf{Q}$ : Vous n'avez pas pu les utiliser.

R : Non.

$\mathbf{Q}$ : Après, la restauration du RHR étant également difficile, il ne vous restait guère plus que la solution de l'éventage renforcé, pour le réacteur 2 aussi. C'est bien ça?

R : Oui.

$\mathbf{Q}$ : L'aspersion de l'enceinte de confinement aurait été réalisable, si vous l'aviez voulu?

$\mathbf{R}$ : On en a parlé tout à l'heure. C'est la fameuse histoire avec le siège. Le RCIC avait fonctionné assez longtemps. La température de la chambre de dépressurisation était très élevée. Dans ces conditions, même si on avait ouvert la vanne $\mathrm{SR}^{72}$, la condensation se serait faite difficilement à cause de la température. Du coup, on pouvait craindre que la dépressurisation du réacteur ne se fasse pas aisément. En tout cas, c'est ce que je craignais. J'ai donc pensé que, pour faire un peu baisser la pression, on aurait pu tenter une aspersion de l'enceinte de confinement. Mais pendant ce temps, le niveau d'eau avait commencé à chuter. Vous l'avez sûrement vu sur la vidéo, pendant qu'on s'activait à différentes choses, il y a eu un coup de fil du directeur Madarame, qui nous téléphonait de la résidence du Premier ministre, pour nous dire d'ouvrir au plus vite la vanne de sécurité et de dépressuriser. J'ai bien essayé de lui dire que la température étant élevée, la dépressurisation risquait d'être difficile. Mais il m'a dit de dépressuriser à tout prix. Pour la première fois, il y a même eu ordre officiel du directeur du centre de gestion de crise du siège. Le directeur du centre de gestion du siège est le Directeur général. Ils m'ont donc ordonné d'ouvrir, de dépressuriser. Finalement, je me suis rallié à leur idée. Et, du coup, on n'a pas utilisé l'aspersion de l'enceinte de confinement.

$\mathbf{Q}$ : Je voudrais revenir à la page $41 \mathrm{du}$ «Rapport sur les dispositions pour la gestion des accidents ». Est-il possible d'ouvrir à la fois la vanne MO du système LPCl et les deux vannes MO du système CCS pour faire fonctionner l'aspersion de l'enceinte, tout en injectant à basse pression?

$\mathbf{R}$ : Je pense qu'on n'aura pas de volume suffisant pour alimenter les deux. Il faudra partager le volume d'eau.

$\mathbf{Q}$ : Ce qui signifie que le volume d'eau dont on disposera pour l'injection à basse pression va diminuer?

$\mathbf{R}:$ Oui, c'est ça. 
$\mathbf{Q}$ : Le fait que le volume d'eau diminue quand on partage la même alimentation est lié à la pression? Je crois que c'est ce qu'on appelle la « pression à la sortie».

$\mathbf{R}$ : La pression à la sortie va rester constante, mais les deux systèmes vont se partager l'eau.

$\mathbf{Q}:$ Le volume d'eau qui va entrer dans la cuve du réacteur va diminuer.

$\mathbf{R}$ : Oui, ça va diminuer. Seulement, si avant d'envoyer l'eau, la vapeur ne condense pas, la dépressurisation ne se fera pas. Et, bien évidemment, si la pression ne baisse pas, l'eau n'entrera pas. C'est pour ça qu'il fallait à tout prix baisser la température pour faciliter la condensation, afin de faire tomber la pression. On en était là de nos discussions, quand on nous a ordonné de sauter cette étape et d'envoyer de l'eau par là.

$\mathbf{Q}$ : Si la température de la piscine de dépressurisation était élevée, en effet, cela aurait pu être efficace d'envoyer de l'eau ici.

$\mathbf{R}$ : En fait, je ne sais plus si, quand on a évoqué la possibilité d'utiliser l'aspersion de l'enceinte de confinement, on s'apprêtait à asperger la chambre sèche ou la chambre humide, les deux sont des aspersions de l'enceinte. Je pense qu'il s'agissait de la chambre humide. En tout cas, on en a discuté, mais on n'a pas eu l'occasion de passer à l'acte.

$\mathbf{Q}$ : Sur ces entrefaites, vous recevez le coup de fil du directeur de la Nuclear and Industrial Safety Agency ${ }^{73}$ vous ordonnant, de la part du Directeur général Shimizu ${ }^{74}$, de procéder à la décompression et vous vous dirigez vers la solution de l'ouverture volontaire de la vanne SR.

R : Oui.

$\mathbf{Q}$ : Mais, quand on regarde la chronologie, cette décompression ne s'est pas faite tout de suite. Vous avez dû manœuvrer jusqu'au soir, vers 18h00, n'est-ce pas?

R : Oui.

$\mathbf{Q}$ : Pourquoi la pression a-t-elle mis si longtemps à baisser?

R : Il y avait deux choses. Pour manœuvrer la vanne SR, il faut à la fois de l'électricité et de l'air. On avait réussi à amener l'air. Mais on avait beau relier la vanne à la batterie, elle ne voulait pas s'ouvrir. Il y a plusieurs vannes, alors on les a essayées l'une après l'autre, mais aucune ne voulait s'ouvrir. Je ne sais vraiment qu'en penser. Si je me souviens bien, on leur avait même donné des noms. Il y avait la vanne " $A$ ", la « B », la « $C$ », la « D», et on essayait donc la batterie sur chacune dans l'ordre et c'est, enfin, l'une des dernières qu'on a réussi à ouvrir.

$\mathbf{Q}$ : Vous aviez donc plusieurs vannes $S R$, la « $A »$, la « $B »$, la « $C$ », la « D», et comme aucune ne réagissait, vous les avez essayées l'une après l'autre. En fait, il suffit qu'une vanne s'ouvre?

R : Oui, à la base, si l'une s'ouvre, cela suffit à faire baisser la pression de manière conséquente.

$\mathbf{Q}$ : Ce qui signifie que pour pouvoir en ouvrir une correctement, il vous a fallu batailler jusqu'à un peu plus de 18 h00.

R : Oui.

$\mathbf{Q}$ : Vous ouvrez cette vanne et, en même temps, il vous faut aussi refaire la manœuvre pour ouvrir la ligne d'éventage.

$\mathbf{R}:$ C'est ça. 
$\mathbf{Q}$ : Si je comprends bien, en ce qui concerne le réacteur 2 , vous avez eu beaucoup de difficultés à, non seulement, ouvrir cette ligne d'éventage, mais également à la maintenir ouverte. Ensuite arrive le problème de la hausse de la pression dans la chambre sèche et la chute de la pression du réacteur.

$\mathbf{R}$ : Oui, l'équilibre était pour le moins bizarre.

$\mathbf{Q}:$ Vous n'en connaissiez pas la cause.

R : Non.

$\mathbf{Q}$ : À propos, après cet épisode, le 15 mars, la pression de la chambre de dépressurisation du réacteur 2 est tombée à 0 . J'ai regardé les paramètres et je me suis rendu compte qu'il y avait eu quelques moments où il y avait eu un tout petit peu de pression.

$\mathbf{R}:$ En effet.

$\mathbf{Q}:$ Quelle en était la raison, à votre avis ?

$\mathbf{R}$ : Je n'en sais strictement rien. Il faudrait voir sur place. Il faudrait observer ce qui a été détruit et comment. Sinon, on ne peut pas savoir.

$\mathbf{Q}$ : Depuis, on a eu des images à la télévision. Vous savez, ces caméras, ces robots qui entrent à l'intérieur des bâtiments et qui filment ${ }^{75}$. On en a vu des images. A-t-on des images de l'intérieur des tores du réacteur 2 ?

$\mathbf{R}:$ On ne peut pas encore accéder aux tores.

$\mathbf{Q}:$ Ah, ça n'est pas encore possible?

$\mathbf{R}:$ La radioactivité y est très forte.

$\mathbf{Q}:$ Vous voulez dire par ici?

R : Oui.

$\mathbf{Q}$ : Alors, pour le moment, vous ne savez pas du tout quel est l'état de destruction de cet endroit?

$\mathbf{R}$ : On n'en a aucune idée. Les tores sont inondés d'eau. C'est l'eau qu'on a injectée. Vous voyez? Ici, ce sont les tores. L'eau s'écoule des tores vers l'extérieur. En fait, cette eau fortement radioactive en provenance des tores les recouvre à l'extérieur. Il n'y a pas moyen d'en approcher.

$\mathbf{Q}$ : Vous voulez dire que toute cette partie en forme de « doughnut » est immergée?

$\mathbf{R}$ : Oui, elle est complètement immergée.

$\mathbf{Q}$ : Et qu'allez-vous faire de cette eau ?

R : Quand il y a beaucoup d'eau là, elle s'écoule vers le bâtiment turbine. Du coup, la radioactivité de l'eau du bâtiment turbine grimpe. Cette eau contaminée est traitée et elle est réinjectée par ici. Et on recommence. Je pense que le niveau d'eau dans les tores ne baissera pas de sitôt. Ce qui veut dire que, pour le moment, on ne peut pas savoir ce qui s'y est passé.

$\mathbf{Q}:$ Oui, ça risque de durer un moment.

$\mathbf{R}$ : On ne saura pas de sitôt.

$\mathbf{Q}$ : Si je reviens aux solutions préconisées lors des accidents, concernant le réacteur 2, ce que vous avez utilisé c'est l'éventage renforcé.

R : Oui. 
$\mathbf{Q}$ : C'est mentionné dans les procédures à utiliser pour refroidir l'enceinte de confinement. Vous aviez aussi envisagé de procéder à une aspersion de l'enceinte en passant par le réseau FP, mais vous ne l'avez pas fait.

Ensuite, toujours pour le réacteur 2, concernant le réseau de lutte contre l'incendie, vous n'avez pas pu utiliser la pompe diesel, mais, en vous servant du réseau lui-même, vous avez réussi à envoyer de l'eau avec les voitures de pompiers. En passant à la fois par le réseau FP et le réseau de l'injection à basse pression, vous avez injecté de l'eau dans la cuve du réacteur. Ça, c'est vraiment une ligne d'injection qui a été rendue possible par les travaux de raccordement qui avaient été faits au moment de la rédaction de ce rapport.

\section{$\mathbf{R}:$ Tout à fait.}

$\mathbf{Q}$ : Je voudrais vérifier une chose. Pour le réacteur 3 , le RCIC avait fonctionné dès le début, relayé ensuite par le HPCl qui, lui aussi, a fonctionné. Si on fait une comparaison, les HPCl des réacteurs 1 et 2 n'ont pas fonctionné. Vous vous doutiez dès le début que les HPCl ne fonctionneraient pas?

$\mathbf{R}$ : Pour le réacteur 1 , dès le début, l'équipe de quart m'avait fait savoir qu'il ne fonctionnait pas. Je ne sais plus si c'était une question de batterie ou autre chose. Toujours est-il que le message était clair. Ils me disaient qu'il ne fallait surtout pas compter dessus.

Pour le réacteur 2, on a fait pas mal de tentatives. Puisqu'on soupçonnait la batterie de s'être vidée, il fallait changer de batterie. Pour ça, il a fallu en chercher une. On en a trouvé une, mais, finalement, le HPCI du réacteur 2 ne répondait pas. On a fait une croix dessus et on a compris qu'il fallait essayer de tirer le RCIC le plus longtemps possible.

Pour le 3, j'avais su d'emblée qu'on pouvait utiliser à la fois le RCIC et le HPCI. J'ai donc décidé de commencer par le RCIC. À un moment, il me semble, la batterie du RCIC s'est épuisée et on est passé au HPCI.

$\mathbf{Q}$ : Excusez-moi, on va revenir au réacteur 2. Lorsque vous dites que le $\mathrm{RCIC}$ ou le $\mathrm{HPCl}$ fonctionnait ou ne fonctionnait pas, quel était le moyen pour vérifier leur fonctionnement? II y a bien un endroit, une pièce destinée au $\mathrm{HPCl}$ à l'intérieur du bâtiment réacteur, il me semble?

R : Oui.

$\mathbf{Q}:$ De même pour le RCIC, n'est-ce pas ?

R : Oui, c'est au même endroit.

$\mathbf{Q}:$ Ces deux emplacements sont donc au même niveau?

R : Oui.

$\mathbf{Q}$ : Les vérifications concernant l'état de marche du $\mathrm{HPCl}$, pour savoir si le système fonctionnait ou pas, ont été faites pendant que le RCIC était en fonctionnement?

$\mathbf{R}$ : Là encore, il faudrait interroger les gens de la salle de contrôle pour savoir comment ils ont procédé. Pour ma part, je leur ai demandé de vérifier si on pouvait utiliser le HPCI et le RCIC. Pour le HPCI, il me semble que c'était la batterie. On m'a répondu que la batterie avait été inondée, ou quelque chose de ce genre, et qu'il risquait de ne pas fonctionner. Comme je ne l'ai pas constaté de visu sur le terrain, je ne sais pas concrètement ce qui a fait que cela n'a pas fonctionné.

$\mathbf{Q}:$ Le RCIC était, lui, en fonctionnement.

$\mathbf{R}$ : Au début, on m'a dit qu'il fonctionnait. Puis, on n'y a plus eu accès et on n'a pas pu savoir s'il marchait ou pas. Ce faisant, on ne pouvait plus voir le niveau d'eau, non 
plus. Si le RCIC ne marchait plus et que le HPCI était inopérant, cela équivalait à une situation terrible, une situation aussi inquiétante que celle du réacteur 1, qui aurait réclamé une déclaration suivant l'article 15 . Aussi il était urgent de vérifier l'état du RCIC et je me rappelle avoir lourdement insisté auprès de mes hommes pour qu'ils aillent vérifier ça.

Et là, j'ai été dur avec ceux qui étaient de quart. Comprenez-moi, j'étais dans le bâtiment antisismique, je n'avais pas véritablement conscience des conditions concrètes sur le terrain. Ce n'est que plus tard qu'ils m'ont raconté comment ils avaient dû s'équiper, se protéger pour aller faire cette vérification. Mais sur le moment, je me demandais pourquoi ils n'arrivaient pas à faire une chose aussi simple. À la limite, pour moi, il suffisait qu'ils sortent de la salle de contrôle, qu'ils descendent vite fait dans la salle du RCIC, qu'ils y jettent un coup d'œil rapide et qu'ils remontent. Quand j'y pense aujourd'hui, je ne sais comment me faire pardonner. Mais, c'est sûr, j'ai insisté pour qu'ils vérifient l'état de fonctionnement du RCIC.

Q: C'est donc ainsi que vous avez vécu la nuit du 11 et toute la période où vous ne voyiez plus le niveau d'eau.

R : Oui.

$\mathbf{Q}:$ Si je me réfère à la chronologie, c'était vers 2 h00 du matin, n'est-ce pas?

R : Oui.

Q: C'est là que vous apprenez que le RCIC est en marche. Le niveau d'eau aussi est stable autour des trois mille et quelques centaines de millimètres. Pensiez-vous tout ce temps que le réacteur 2 réagissait correctement?

$\mathbf{R}$ : Oui, je le pensais. Du coup, la priorité était toujours le réacteur 1 , puisqu'à ce moment-là, il y était question d'éventage et d'explosion. Ensuite le réacteur 3, dont le RCIC s'était arrêté. Bien sûr, j'étais préoccupé par le réacteur 2 aussi. Mais, étant donné que le RCIC marchait toujours et qu'on pouvait vérifier le niveau d'eau, qui était acceptable, pour moi, du point de vue des priorités, le réacteur 2 était en dernière position.

Q: : Aujourd'hui nous savons jusqu'à quel moment le RCIC a tenu, mais, sur le coup, vous ne pouviez pas le savoir. D'ailleurs, si vous aviez su qu'il allait tourner jusqu'au 14 mars à $13 \mathrm{~h} 25$, vous auriez peut-être pu élaborer d'autres plans. Mais étant donné que vous ne pouviez pas le deviner, au fur et à mesure que le temps passait du 11 au 12 et du 12 au 13 mars, n'avez-vous pas eu de doutes? Ne vous êtes-vous pas demandé s'il fonctionnait vraiment?

$\mathbf{R}$ : Si, bien sûr. Tout le monde en était préoccupé. Mais il n'y avait rien à faire.

Q: Si je me souviens bien, ce qui a étayé, à la base, le fonctionnement du RCIC du réacteur 2 était la pression de sortie. Avez-vous pu vérifier plus tard cette pression?

$\mathbf{R}$ : C'est qu'entre temps, on avait réussi à voir le niveau d'eau. L'indicateur de niveau d'eau avait ressuscité et fournissait des données montrant que le niveau d'eau était suffisant. Alors nous nous sommes basés dessus. Mais, évidemment, on ne savait pas jusqu'à quand le RCIC allait tenir. C'est pourquoi j'avais donné l'ordre de préparer le terrain pour l'injection d'eau. Ces préparatifs ont été menés en parallèle avec ceux du réacteur 1 et du réacteur 3. 
$\mathbf{Q}$ : Effectivement, il y a eu des préparatifs pour l'injection. C'est à partir du moment où, le 14 mars vers $12 \mathrm{hO0}$, le niveau d'eau du réacteur commence à chuter que vous vous dites que le RCIC arrive au bout?

$\mathbf{R}$ : Oui. Pour le réacteur 2, je voulais dépressuriser et injecter sans tarder, pendant qu'il y avait suffisamment d'eau, contrairement aux réacteurs 3 ou 1, pour qui on avait agi dans l'urgence. C'est à ce moment-là que survient l'explosion de la tranche 3 qui détruit la ligne d'injection du réacteur 2 qu'on s'était donné tant de peine à construire. Il a fallu tout recommencer, une vraie perte de temps. L'injection ne sera possible qu'en fin de journée. S'il n'y avait pas eu l'explosion, j'avais bien l'intention de dépressuriser et injecter dès midi.

$\mathbf{Q}$ : C'est l'expérience que vous aviez vécue avec le réacteur 1 qui vous avait poussé à penser de cette manière?

$\mathbf{R}$ : J'avais fait avancer les travaux parce que je pensais que si j'arrivais à injecter de l'eau assez tôt, pendant qu'il y en avait encore un certain niveau dans le réacteur, le dénoyage aurait pu être limité au minimum, que j'arriverais à protéger le combustible.

$\mathbf{Q}:$ L'injection dans le réacteur 2, c'était bien le 14 ?

$\mathbf{R}$ : Le 14, avant que la tranche 3 n'explose, la ligne d'injection était déjà prête.

$\mathbf{Q}$ : L'explosion s'est produite vers 11h00, n'est-ce pas?

R : Oui.

$\mathbf{Q}$ : Et c'est passé $12 \mathrm{~h} 00$ que le niveau d'eau commence à baisser?

R : Oui.

$\mathbf{Q}$ : Vers $12 \mathrm{~h} 30$, les pressions de la chambre sèche et de la chambre de dépressurisation sont respectivement de 0,46 et 0,486. C'est un peu élevé, mais tout à fait normal. Pas du tout du genre 0,7 ou 0,3 .

$\mathbf{R}:$ C'est ça.

$\mathbf{Q}$ : Dans ces conditions, vous vous dites, avant l'explosion, que si vous procédez normalement, si je puis dire, à une opération de dépressurisation, vous pourriez injecter et que, suivant votre expérience, cela pourrait passer.

Vers 13h00, évidemment, le RCIC finit aussi par s'arrêter. Si on revient sur le fonctionnement de ce RCIC qui a tourné si longtemps, il me semble que, bien que ce soit un système de turbine, au moment où on le met en branle, au début, il faut faire marcher des pompes à huile et d'autres choses pour assurer le graissage de la turbine, et pour cela il faut de l'électricité. La presse a insisté sur le fait que le système RCIC fonctionnait sans électricité. Elle a même laissé entendre que s'il avait cessé de fonctionner c'est qu'il devait y avoir d'autres raisons que le défaut d'électricité. Je comprends que le RCIC a besoin d'électricité, mais pour quoi?

$\mathbf{R}:$ Vous voulez parler des batteries?

$\mathbf{Q}:$ Oui, les batteries.

$\mathbf{R}$ : Comme vous venez de le dire, il y a des sortes de pompes miniatures sur la ligne de graissage qui marchent avec des batteries. Ensuite vous voulez savoir à quoi sert l'électricité dans le RCIC?

$\mathbf{Q}:$ Oui, comme ouvrir ou fermer des vannes...

R : Il y a ça aussi, puisqu'il y a des vannes de contrôle. C'est la même chose que pour d'autres turbines ordinaires, il y a un système de contrôle. On régule le nombre de rotations. À la base, on compte le nombre de rotations et un mini système de 
" régulation turbine » contrôle la quantité de vapeur. Là, il faut une alimentation électrique.

À part ça, comme vous l'avez dit, pour mettre le RCIC en marche il faut de l'huile, qui est amenée par une mini-pompe, je crois. De toute manière, c'est un dispositif rotatif, il lui faut du lubrifiant pour ses roulements. C'est évidemment quelque chose d'indispensable.

$\mathbf{Q}$ : Dans un cas limite, que se passerait-il si la batterie s'épuisait à un moment où la vanne serait en position «ouvert»? On ne peut plus réguler et la vapeur continue à arriver. La vapeur continue à faire fonctionner le système. Seulement, on ne peut pas réguler. Est-il possible qu'à un moment donné, la vapeur devenant insuffisante, le système s'arrête de luimême?

$\mathbf{R}:$ C'est probablement impossible.

Q: Vous croyez?

$\mathbf{R}$ : Si on laissait faire sans intervenir, il y aurait trop de vapeur dans le réseau, la turbine s'emballerait et il y aurait arrêt. Voyez-vous, c'est une turbine. Au-delà d'un certain nombre de rotations, la machine se trouve en danger. Pour se protéger, elle possède une fonction qui l'arrête automatiquement dans ce cas. Si on perdait la possibilité de contrôle, le système s'arrêterait de lui-même.

$\mathbf{Q}$ : Alors, au regard de ce que vous venez de nous raconter, il n'y a pas de probabilité que le $\mathrm{RCIC}$ du réacteur 2 ait continué à fonctionner longtemps par la seule force de la vapeur sans contrôle et qu'il se soit finalement arrêté parce que la quantité de vapeur avait diminué?

$\mathbf{R}:$ Non, je ne pense pas.

$\mathbf{Q}$ : Cela veut dire qu'un certain contrôle est indispensable?

R : Oui.

$\mathbf{Q}:$ Le fait que ce système ait fonctionné si longtemps, c'était une vraie chance, non?

$\mathbf{R}$ : Oui. Tout à fait. C'est un miracle. Je m'attendais à ce qu'il s'arrête d'un moment à l'autre. Je n'ai jamais été tranquille.

Q : Évidemment, aujourd'hui, on sait qu'il a tenu, mais vous ne pouviez pas deviner à l'époque qu'il tiendrait si longtemps.

$\mathbf{R}:$ Effectivement.

Q: Peut-être que vous aviez encore confiance le 11 ou le 12 jusqu'en milieu de journée, mais au fur et à mesure que le temps passait, vous avez dû commencer à vous inquiéter. Vous ne pouviez pas savoir s'il allait s'arrêter le 12, le 13 ou le 14.

$\mathbf{R}$ : De toute façon, mon objectif était d'être prêt le plus tôt possible pour la suite. Parce qu'on avait eu l'explosion du 1 et l'explosion du 3, entre temps...

Il y avait un autre facteur, c'était la disponibilité des camions de pompiers. Ils venaient, mais vraiment au compte-gouttes. Pour construire un système d'injection, il fallait que les camions soient là physiquement et il a fallu les attendre.

$\mathbf{Q}$ : C'est aussi un point sur lequel je voulais justement vous interroger. Sans trop entrer dans les détails, comment avez-vous ressenti la chose? Au départ, pour le réacteur 1, vous ne disposiez que du camion que la centrale possédait en propre. Vous avez effectué des injections avec lui. Ensuite, vous avez aussi injecté de l'eau dans le réacteur 3.

$\mathbf{R}$ : Il y a d'abord eu un camion qui faisait des allers et retours pour le réacteur 1. Ensuite, on a réussi à remettre sur pied un camion qui avait été sinistré du côté des 
réacteurs 5 et 6 et qu'on a amené vers le réacteur 1. Et puis, un camion que la centrale de Kashiwazaki avait prêté à Fukushima Daini est arrivé. Cela nous a d'abord fait trois camions, qu'on a installés en ligne pour l'injection du 1.

Après, c'est au moment où on s'apprêtait à injecter dans le réacteur 3 que les camions ont commencé à arriver de manière significative. On a construit une ligne à partir du quai, au niveau de la mer. Cette ligne était installée devant le réacteur 2. L'arrivée des camions de très grosse capacité nous a permis d'envisager d'aspirer l'eau directement dans la mer et de la répartir entre les différents réacteurs. Du coup, on a changé de disposition.

Q: L'idéal serait que vous vous souveniez d'une ligne en particulier. Avez-vous eu le sentiment, à un moment ou un autre, qu'ayant une idée en tête, par exemple pour l'injection dans le réacteur 2 ou le réacteur 3 , vous n'avez pas pu passer à l'acte, parce que les camions n'arrivaient pas? Bref, vous êtes-vous retrouvé dans une situation où vous ne pouviez pas passer à l'action faute de camions?

$\mathbf{R}$ : À vrai dire, les évènements se sont enchainés un peu moins vite que je ne le craignais. Je crois que, de ce point de vue, nous avons eu beaucoup de chance.

$\mathbf{Q}$ : De toute évidence, dès le début, vous réclamiez des camions de pompiers en nombre. Je suppose que ces véhicules devaient venir par la route. Ils vont arriver petit à petit de toute la région du Kantồ ${ }^{7}$. Mais, vers le 12 ou le 13, vous ne pouviez pas espérer qu'ils arrivent comme ça, d'un claquement de doigts, comme par magie. II a bien fallu un ou deux jours de délai. D'ailleurs, quand ils ont commencé à arriver, ils ont même dû arriver d'un coup en quelques heures, de différentes provenances. Les camions de pompiers que vous receviez, où les mettiez-vous ? Vous les aviez stationnés près du bâtiment antisismique?

R : Il y a le bâtiment antisismique ici. Là, aujourd'hui, il y a un parking. Il y avait un autre parking un peu plus loin. Il y avait aussi un endroit dégagé où on pouvait garer des véhicules devant l'entrée ouest. On avait réparti les camions dans ces deux endroits.

Q: Vous avez injecté de l'eau dans les réacteurs 1, 2 et 3. Vous utilisiez des camions de pompiers. Au moment où la tranche 3 a explosé, il y a eu beaucoup de dégâts du côté de ces véhicules, si j'ai bien compris. Les véhicules qui avaient été endommagés sont restés sur place pendant un certain temps, n'est-ce pas?

R : Oui. Nous n'avons pas pu les évacuer sur le moment. Du coup, ces épaves ont beaucoup gêné les manœuvres.

$\mathbf{Q}$ : Vous avez reconstruit une ligne pour l'injection avec de nouveaux camions de pompiers. Ces camions ont servi de pompe. Par exemple, le véhicule de pompiers qui se trouvait sur le quai, au niveau de la mer, a fonctionné sans interruption pendant des heures. Avez-vous eu des soucis avec ces véhicules du fait qu'ils fonctionnaient sans discontinuer? Avezvous dû les remplacer par de nouveaux véhicules à cause de ça?

$\mathbf{R}:$ Oui, tout à fait.

$\mathbf{Q}:$ Ce sont des choses qui arrivent.

$\mathbf{R}$ : Oui, c'est arrivé.

$\mathbf{Q}$ : Alors, plus vous aviez de camions à disposition, mieux c'était.

$\mathbf{R}$ : Bien entendu. Il nous fallait de la réserve. De base, les pompes des camions de pompiers ne sont pas conçues pour fonctionner des heures sans discontinuer. Habituellement, les pompiers arrosent le feu pendant une heure et c'est fini. Les pompes n'ont pas été conçues pour tourner vingt-quatre heures durant. C'était, bien 
sûr, un grand sujet d'inquiétude pour moi. C'est pour ça que j'avais réclamé tout ce qu'on pouvait trouver en matière de véhicules incendie. Il nous fallait de la réserve.

Q: Je voudrais maintenant revenir à des questions concernant la gestion du réacteur 3. Pour ce réacteur, comme pour le réacteur 2, le défaut d'électricité a fait que vous ne pouviez plus utiliser le système MUWC.

$\mathbf{R}:$ C'est ça.

$\mathbf{Q}$ : Ensuite, concernant le système FP, la pompe diesel était encore vivante.

$\mathbf{R}$ : Oui, elle était encore en vie.

$\mathbf{Q}:$ Et comment s'est-elle comportée?

$\mathbf{R}$ : Elle a fonctionné.

$\mathbf{Q}$ : Dans cette chronologie du réacteur 3 , concrètement, à quel moment parle-t-on en premier de cette pompe?

R : Probablement, le 13 au matin.

Q: Oui, le 13 au matin, on a noté que la pression étant à 4,1 MPa, l'eau n'est pas entrée. C'est à la page 33. Le 13 à l'aube, ils ont écrit: «la pression du réacteur étant montée jusqu'à $4,1 \mathrm{MPa}$, impossibilité d'injecter ». Je me rappelle que vous aviez commencé très tôt à vous préoccuper de cette pompe sur les réacteurs $1^{\circ}$ et 2 . Vous aviez donné l'ordre à $17 \mathrm{~h} 12$ de commencer à étudier la possibilité d'une injection par le réseau incendie pour ces deux réacteurs. C'est écrit là. Et pour ce qui est du réacteur 3 ? C'est peut-être un oubli de celui qui a rédigé cette chronologie, mais on ne voit pas de mention concernant le réacteur 3.

Si je reviens à la fin de l'après-midi du 11 mars, vers $17 \mathrm{~h} 00$ justement, vous aviez perdu toutes les alimentations en courant alternatif. Vous ne pouviez pas non plus utiliser les générateurs diesel de secours. Seulement, par rapport aux réacteurs 1 ou 2, le 3 était relativement en meilleure posture. La batterie pour l'injection fonctionnait. Le RCIC avait l'air de s'être mis en route. Je comprends que, dans ces conditions, vous ayez donné la priorité aux réacteurs 1 et 2. Mais aviez-vous déjà dans l'idée d'utiliser la ligne incendie pour le 3 ?

$\mathbf{R}$ : J'ai raisonné de la même manière pour tous les réacteurs.

$\mathbf{Q}$ : Vous raisonniez de la même manière. Mais, au moment que nous évoquons, le RCIC était en marche. II s'est arrêté le lendemain, le 12, à 11h36. Environ une heure après, à 12h35, cette fois, c'est le HPCl qui s'est mis en branle. Je suppose qu'à ce moment-là vous n'aviez pas encore mis la DDFP en marche.

$\mathbf{R}$ : D'après les procédures habituelles, dans des cas où on n'utilise pas les voitures de pompiers, la priorité pour l'injection, c'est la pompe diesel. Puisque la pompe diesel était vivante, il était naturel de donner l'ordre pour qu'on se prépare à l'utiliser. C'est le genre de discussion que nous avions eu autour du réacteur 1 . Je ne sais pas si j'ai donné des instructions claires concernant la préparation de cette pompe à chacune des équipes d'injection, mais c'était tout à fait dans l'ordre des choses de préparer en parallèle, pour tous les réacteurs, la pompe diesel.

$\mathbf{Q}$ : De toute façon, sur le terrain, le RCIC du réacteur 3 s'arrête le 12 mars à 11h36. À ce moment-là, vous pouviez voir les données, n'est-ce pas ? Le RCIC s'arrête à 11h36. Un tout petit peu avant, à $11 \mathrm{~h} 23$, la pression est de $7,360 \mathrm{MPa}$. À $12 \mathrm{~h} 10$, elle est à 7,53 . Ce qui veut dire qu'elle est toujours aux environs des $7 \mathrm{MPa}$. Dans ces conditions, la DDFP ne pouvait pas envoyer d'eau.

$\mathbf{R}$ : Comme vous dites.

Q : L'organisation sur la centrale Fukushima Daiichi fait que c'est la même équipe de production qui s'occupe des réacteurs 1 et 2 et une autre qui s'occupe des réacteurs 3 et 4 . Ce serait quand même un peu contradictoire que ce que vous faisiez sur les réacteurs $1^{\circ} \mathrm{et}$ 2 , n'ait pas du tout été fait sur les réacteurs 3 et 4 . Si l'équipe de M. XXXXX était occupée à 
préparer les pompes pour les réacteurs 1 et 2, il est naturel que vous ayez pensé à une solution similaire pour les réacteurs 3 et 4 .

R : Oui.

$\mathbf{Q}$ : Mais, avec une pression si élevée, de plus avec un HPCl capable de fonctionner, vous choisissez naturellement d'utiliser d'abord le HPCl. Pendant un certain temps vous faites tourner le HPCI. Mais celui-ci s'arrête le 13 à l'aube. Vers 2h42 ? Donc, une fois que le HPCI est à l'arrêt à 2h42, vous faites des tentatives pour utiliser la DDFP.

R : Oui.

$\mathbf{Q}$ : Concernant l'injection dans le réacteur 3, une fois le HPCI à l'arrêt, vous pensiez d'abord à la DDFP? Puis l'injection avec les voitures de pompiers?

$\mathbf{R}$ : D’abord, il fallait que la pression baisse.

Q : D'accord, il fallait dépressuriser. Ce jour-là, à l'aube, vers 3h53 ou dans ces environs, vous tentez d'injecter l'eau en utilisant le réseau d'incendie, mais, à ce moment-là, vous n'aviez évidemment pas exécuté d'opération de dépressurisation.

$\mathbf{R}$ : Non, on ne l'avait pas faite.

$\mathbf{Q}$ : Si je regarde cette chronologie, il semblerait que vous ne l'ayez pas faite parce que vous cherchiez une batterie.

$\mathbf{R}$ : La batterie du HPCI.

$\mathbf{Q}:$ Non, après l'arrêt du HPCl.

$\mathbf{R}$ : Vous dites batterie, mais une batterie pour quoi ?

$\mathbf{Q}$ : Je pense qu'il y avait des problèmes de batterie pour le $\mathrm{HPCl}$, mais ce qui est écrit ici, pour le réacteur 3, c'est...

$\mathbf{R}$ : À quelle page?

$\mathbf{Q}$ : D'abord, à la page 33, on lit: "tentative pour redémarrer le $\mathrm{HPCl}$, mais échec faute de batterie». Ensuite, il y a des notes concernant l'opération de dépressurisation. Sur cette même page 33, dans la rubrique "exécution de l'injection par les véhicules de pompiers », au troisième point, on voit que vous n'arrivez pas à actionner la vanne SR faute de batterie et que vous collectez les batteries des voitures personnelles de vos collaborateurs, etc. Vous m'avez dit tout à l'heure que lorsque vous avez fait la tentative d'injecter l'eau avec la DDFP, vous n'aviez pas fait de manœuvre de dépressurisation avant. Cela veut dire que vous étiez dans des conditions telles que vous ne pouviez pas la faire même si vous l'aviez voulu?

$\mathbf{R}$ : Il y a deux choses. C'était la même chose pour le réacteur 1. J'avais toujours eu une confiance très limitée dans la pompe diesel. Est-ce que l'équilibre des pressions allait faire que le clapet allait s'ouvrir? Ensuite, je me demandais si les $800 \mathrm{t}$ d'eau qui étaient censées se trouver dans le réservoir à eau filtrée allaient vraiment se déverser en totalité dans le réacteur. Là, j'étais inquiet.

$\mathbf{Q}$ : «Se déverser en totalité », vous voulez dire que vous aviez des doutes quant à la capacité du réservoir à résister au séisme?

R : C'est tout à fait ça. Donc, primo, je n'étais pas sûr que toute l'eau censée se trouver dans le réservoir allait réellement entrer dans le réacteur. Secundo, le gros souci, c'était l'équilibre des pressions. J'avais trop peur de dépressuriser sans avoir assuré la viabilité de l'injection d'eau de mer par les camions de pompiers. Je ne voulais pas du scénario, "j'ai dépressurisé, finalement la pompe diesel n'a pas fonctionné ». Parce que cela veut dire qu'on a vidé le réacteur, sans solution de rechange. Il m'était impossible de faire confiance à la seule pompe diesel. Avant dépressurisation, il fallait absolument 
que la ligne des camions de pompiers soit prête. Je ne pouvais décider du moment de la dépressurisation qu'en fonction de l'avancement des préparatifs côté camions.

$\mathbf{Q}$ : Donc, vous attendez que la ligne des camions soit prête pour le réacteur 3.

$\mathbf{R}:$ Je crois qu'ils ont commencé à envoyer de l'eau douce vers 10 h00.

Q: Oui, tout au début.

$\mathbf{R}$ : On a eu, avec vous, la discussion de savoir pourquoi on avait commencé par l'eau douce. Des souvenirs me sont revenus. Il me semble bien qu'il y avait eu des directives du Gouvernement pour qu'on injecte de l'eau douce. Je pense, aujourd'hui, que je me suis laissé influencer par ça. C'est un épisode qui s'était complètement effacé de ma mémoire.

$\mathbf{Q}$ : Vous vouliez donc éviter à tout prix le scénario, « je ne dispose que de la pompe diesel, j'ai dépressurisé, mais l'eau n'est pas entrée». Pour ça, vous teniez absolument à attendre l'arrivée des camions de pompiers pour pouvoir les utiliser pour injecter l'eau, avant de dépressuriser. Vous n'aviez donc pas encore franchi le pas, à ce moment-là.

$\mathbf{R}:$ C'est ça.

$\mathbf{Q}$ : De plus, même pour cette opération de dépressurisation, vous ne disposiez pas de batterie. En fait, vous étiez à la recherche d'une batterie.

À part ça, vous aviez aussi essayé de redémarrer le HPCl. On lit ici : « vérification sur place de l'état du RCIC en passant par la salle HPCl, tentative de démarrage du RCIC pour une injection dans le réacteur, échec ». Vous aviez donc essayé de ressusciter un système qui s'était arrêté?

R : Oui.

Q: Tout en menant ces tentatives, d'un autre côté, vous vous préparez à injecter l'eau au moyen des véhicules de pompiers. Ensuite, le document évoque le SLC.

$\mathbf{R}:$ Oui, tout comme pour le réacteur 1.

$\mathbf{Q}:$ C'est ça. Vous travailliez aussi à sa restauration, n'est-ce pas ?

R : Oui.

$\mathbf{Q}$ : Votre situation correspond tout à fait aux conditions pour mettre en œuvre toutes les solutions substitutives des moyens d'injection dont parlent les manuels de gestion de crise. Mais, comme vous n'avez pas d'électricité, vous ne pouvez pas utiliser le MUWC. II vous reste le réseau FP.

Ensuite, comme on en a parlé à propos de l'automatisation de la dépressurisation du réacteur, la vanne SRV n'est pas opérationnelle pour une dépressurisation en vue de l'injection, puisque le signal «niveau d'eau bas dans le réacteur », lui-même, ne fonctionne plus.

$\mathbf{R}:$ C'est ça.

$\mathbf{Q}: \|$ reste les fonctions «refroidissement» de l'enceinte de confinement. Ni le Drywell Cooler, ni le Reactor Water Cleanup System ne sont possibles faute d'électricité.

\section{$\mathbf{R}:$ C'est bien ça.}

Q : Il y a bien la restauration du RHR, mais sans électricité, là aussi, cela semble difficile. Je vois que vous avez quand même un peu utilisé l'aspersion de l'enceinte de confinement, mais, globalement pour le réacteur 3 , il ne vous restait guère, une fois de plus, que la solution de l'éventage renforcé. Je suppose que c'est à peu près ce que vous avez pensé.

R : Oui.

$\mathbf{Q}$ : La solution du partage de l'électricité était aussi assez difficile. 
Nous allons laisser de côté la discussion autour des circonstances du passage de l'eau douce à l'eau de mer pour le réacteur 3 et nous intéresser à autre chose.

Il y a un certain nombre de faits que je voudrais vérifier rapidement, sans trop entrer dans les détails. II y a assez longtemps, quand vous étiez au siège, vers 2006 ou 2007, peut-être était-ce déjà en 2008, vous aviez des réunions de travail entre exploitants à propos des tsunamis, savoir comment se protéger contre les tsunamis.

À l'époque, on commençait tout juste à parler du tsunami de l'ère Jôgan ${ }^{77}$. On savait depuis longtemps qu'il y avait eu un gros tsunami, mais il avait fallu du temps pour arriver à obtenir des données chiffrées. Vous deviez vous ajuster à ces chiffres pour prendre les mesures de protection et vous aviez sollicité pour ça la Japan Society of Civil Engineers $\left(\right.$ JSCE) ${ }^{78}$. Il existe des documents de sollicitation de l'époque qui portent votre nom. Vous en souvenez-vous?

$\mathbf{R}:$ Je ne me rappelle pas exactement quel était l'organisme qui avait été sollicité. Mais, que ce soit le tsunami de Jôgan ou un autre, les secousses dues aux séismes tectoniques et tous les phénomènes qui en découlent, ont toujours été un sujet d'inquiétude pour tous les exploitants de l'est du Japon ayant des implantations sur la côte Pacifique, comme Tôhoku Electric Power ${ }^{79}$, nous, la centrale Tôkai de la Japan Atomic Power Company ${ }^{80}$. C'est une préoccupation commune à tous les exploitants de l'est du Japon. C'est pourquoi nous tenions des réunions ensemble. C'est de ça que vous voulez parler? Je ne vois pas très bien quelle réunion est visée?

$\mathbf{Q}$ : Parmi les documents qui nous ont été fournis par TEPCO, il y avait des demandes de votre part à la JSCE.

$\mathbf{R}$ : Effectivement, j'ai fait des demandes à la JSCE. Personnellement, je n'y connaissais strictement rien en matière de tsunami ou de séisme. "Qu'est-ce qui provoque les secousses sismiques? " Je ne savais rien de ce genre de choses. Jusque-là, j'étais plutôt du côté de ceux qui, recevant les informations sur l'importance de ces secousses, devaient prévoir des constructions qui puissent y résister. Ce qui fait que je n'y connaissais rien en matière de secousses.

Le $1^{\text {er }}$ avril 2007, je suis devenu directeur du département "gestion des installations nucléaires » au siège. J'avais sous mes ordres des ingénieurs "génie civil » et des ingénieurs " construction ». Le département devait notamment étudier les secousses sismiques et les tsunamis pour les nouvelles installations nucléaires. Il fallait aussi intervenir dans la mise aux normes des centrales déjà édifiées. Ces gens-là étaient là pour ça. Je suis donc arrivé en avril dans ce département. Mais je n'avais aucune culture concernant les séismes et les tsunamis. Du coup, à la base, je me suis reposé sur le travail de nos ingénieurs.

Assez vite, le 16 juillet 2007, il y a eu le séisme au large de Chûetsu et il a fallu que je gère complètement les suites du séisme au siège. Sur place, il y avait bien sûr le directeur de la centrale. Mais j'étais chargé, avec mon département, au siège, de toute la partie technique. Nous avons donc créé une structure, le "Centre de gestion du séisme au large de Chûetsu ", où nous nous efforcions d'analyser les secousses sismiques et d'apprécier les tsunamis. Simultanément, nous devions réviser les mesures antisismiques des différentes tranches pour mettre en application les nouvelles directives antisismiques qui venaient d'être promulguées en 2006. Nous menions ces deux opérations de front. Le séisme du 16 juillet 2007 nous a fait prendre conscience de l'urgence de nous mettre aux nouvelles normes, bien qu'il y ait eu, par ailleurs, des discussions pour savoir si elles étaient appropriées ou pas. 
C'est là que nous avons commencé les études pour Fukushima Daiichi et Fukushima Daini, aussi. Ne connaissant rien en matière de tsunami, j'avais questionné quelques collaborateurs » génie civil». D'ailleurs je n'avais aucune idée de «qui » devait légitimement décider de ça et comment. Ces collaborateurs m'ont dit qu'ils appréciaient l'importance des tsunamis en se référant à une sorte de guide élaboré par la JSCE, exposant la manière de les évaluer. C'est à ce moment-là que j'ai entendu parler du fameux tsunami de Jôgan. Mais il ne faut pas oublier, quand on parle de ce tsunami, que, dans leurs raisonnements, les savants situent la zone de formation du tsunami au large de la côte Sanriku ${ }^{81}$, dans le département de Miyagi ${ }^{82}$. Il n'était pas possible de savoir si un tel tsunami pouvait survenir au large du département de Fukushima. Pour toutes ces raisons, il paraissait urgent de demander à la JSCE de nous livrer des directives pour que nous puissions calculer les données pour les centrales de Fukushima Daiichi et Daini, qui se trouvaient dans le département de Fukushima, et je me rappelle avoir donné mon accord pour les solliciter.

$\mathbf{Q}:$ Les études sur les sédiments datent de votre époque?

$\mathbf{R}$ : Oui, j'avais donné l'ordre de les faire.

Q: Je simplifie un peu. Si vous voulez savoir la hauteur d'un tsunami qui viendrait sur la centrale de Fukushima Daiichi, selon la zone de formation du tsunami et son ampleur, les données vont naturellement changer. Avez-vous eu vent d'une vague de 9 ou $10 \mathrm{~m}$ de haut, à partir du calcul d'un tsunami d'une certaine ampleur fixe, en changeant juste sa zone de formation?

$\mathbf{R}:$ Oui, j'en ai entendu parler.

$\mathbf{Q}$ : Dans un cas de ce genre, vous êtes amené à faire des vérifications pour savoir si les chiffres sont légitimes, n'est-ce pas?

R : Oui.

$\mathbf{Q}$ : Du temps où vous étiez à la tête de ce département, vous est-il arrivé de recevoir ce genre de rapports de la part de votre personnel chargé du génie civil ?

R : Oui.

Q: Quand vous receviez ces rapports, c'étaient encore des études internes à votre département?

$\mathbf{R}$ : Oui, puisque ça concernait les révisions antisismiques. Ce qui se passait à cette époque, c'est qu'avec ce tremblement de terre au large de Chûetsu, l'attention du public était focalisée sur les séismes. Le tsunami qui avait suivi n'avait pas été d'une grande ampleur. Mais dans le cadre des révisions antisismiques, on nous demandait une évaluation des tsunamis. Il fallait donc décider de la puissance du tsunami dont on allait tenir compte pour les centrales de Fukushima Daiichi et Daini. Sinon, on ne pouvait pas décider des travaux à entreprendre. Or les choses étaient très floues, comme je viens de vous l'expliquer.

Il y avait bien eu ce tsunami de Jôgan, en l'an 869, mais on ne savait pas quelle influence il avait eue sur le département de Fukushima. Celui qui avait rédigé la thèse se contentait d'affirmer qu'il y avait bien eu un tsunami et qu'il avait était de telle ampleur. Mais les conséquences qu'il aurait pu avoir sur le département, il fallait faire des recherches pour le savoir.

Ensuite, les histoires de "chercher les conséquences en déplaçant la zone de formation du tsunami ", ce ne sont que pures hypothèses. On n'arrive pas à savoir sur quels critères ces gens se basent pour décider de l'endroit où se forme le tsunami. 
Alors j'ai dit à mes troupes de consulter la JSCE. D'autre part, dans le cas où tout cela aurait abouti à des normes très sévères, il fallait que nous nous organisions. C'est pour ça que dans le cadre de la gestion du séisme au large de Chûetsu, nous nous réunissions une fois par mois, le samedi ou le dimanche, je ne sais plus, avec le Directeur général et le Président. Lors de ces réunions, on n'évoquait pas seulement le sort de la centrale qui se trouvait effectivement sur la côte du Chûetsu, dans le département de Niigata, mais aussi celui des centrales de Fukushima. Ces révisions des mesures antisismiques demandaient de l'argent. Il fallait que nous y pensions. Les études pour l'appréciation de l'importance des tsunamis étaient en cours, mais à ce moment-là nous n'avions pas de réponse nette.

Il me semble que la hauteur calculée d'après les préconisations de la JSCE de 2002 était de $5,7 \mathrm{~m}$ ou quelque chose comme ça. Mais il y avait eu des progrès techniques depuis, même en dehors de l'histoire du tsunami de Jôgan. On s'est posé des questions par rapport aux chiffres de 2002. Étaient-ils corrects? Le relief à l'intérieur de la baie avait changé. En maintenant le même point de formation du tsunami, avec la même méthode de calcul qu'en 2002, il semblait déjà que le tsunami devait être encore plus haut, de l'ordre de 6,1 m, il me semble. Il s'est avéré du coup que les installations des pompes des réacteurs 5 et 6 n'étaient pas adaptées. Nous avons effectué des travaux pour les transformer et les surélever. L'étape suivante était de prévoir des travaux pour faire face à un encore plus gros séisme. C'est le genre de discussions que nous avions toujours avec le Directeur général et le Président, et je faisais des rapports en ce sens au grand responsable de l'activité nucléaire ainsi qu'à ses troupes.

$\mathbf{Q}$ : Donc, votre attitude était que, si la JSCE produisait de nouvelles directives, vous les auriez considérées comme ayant une objectivité suffisante pour servir de fondement à vos décisions. Dans ces conditions, il fallait que vous vous teniez prêts à les mettre en application, c'est bien ça?

R : Oui.

$\mathbf{Q}$ : Cela signifie que s'ils vous avaient dit qu'il allait y avoir un tsunami de $8 \mathrm{~m}$ ou un tsunami de $10 \mathrm{~m}$, il fallait que vous preniez tout de suite les mesures pour y faire face?

$\mathbf{R}:$ C'est ça.

$\mathbf{Q}$ : J'ai un peu de mal à cerner la situation. Est-ce qu'il aurait été irréaliste que, par exemple, sans attendre les conclusions de la JSCE, TEPCO fasse ses propres calculs et rehausse ses digues?

$\mathbf{R}$ : Lorsqu'on effectue des améliorations et qu'on en fait rapport à l'État, il faut justifier. Il faut notamment expliquer sur quel critère on a agi de la sorte. Tout est comme ça. On remet des documents à l'État. L'État, dans ce cas, c'est la NISA. Lorsqu'on lui remet des documents, il faut toujours justifier le fondement des décisions. Si, à ce moment, on n'a pas de critères à invoquer, cela ne peut pas passer. C'est que ces appréciations ou ces critères, nous les partageons avec tous les autres exploitants, c'est une pensée commune à tout le Japon. Bien sûr, il y a des particularismes locaux. Mais, concernant les séismes ou les tsunamis, nous avons une manière commune de raisonner. Nous évaluons les secousses suivant ce raisonnement commun, nous calculons la hauteur des tsunamis en fonction de ce même raisonnement. C'est ainsi que nous démontrons pourquoi il faut construire ou consolider de cette manière, ou bien que nous affirmons que les installations tiendront très probablement le coup sans qu'on n'y fasse rien. S'il n'y a pas de critère, 
il est urgent d'en définir clairement. C'est par là qu'il faut commencer. Voilà ce que je pensais.

Q : Si, par exemple, on prend la zone de formation du tsunami de Jôgan, cela aurait eu des répercussions différentes sur la centrale d'Onagawa ${ }^{83}$, par rapport à Fukushima?

R : Oui, différentes.

$\mathbf{Q}$ : Cela aurait été à cause de la position?

$\mathbf{R}$ : Onagawa aurait été plus proche.

$\mathbf{Q}$ : Est-ce qu'il peut arriver qu'on tienne compte de certaines choses à Onagawa et pas à Fukushima Daiichi?

$\mathbf{R}$ : Bien sûr. Si on suppose que le point de formation du tsunami est là et qu'il y ait par exemple un tsunami de $10 \mathrm{~m}$ à Onagawa, la vague qui viendrait à Fukushima ne serait que de 3 ou $4 \mathrm{~m}$. Ça, c'est le genre de choses qu'on peut calculer.

$\mathbf{Q}$ : Est-ce qu'il pourrait arriver, par exemple, que, puisqu'on tient compte du tsunami de Jôgan à Onagawa, on suppose qu'une vague de même hauteur puisse arriver à Fukushima Daiichi et qu'on construise des protections en conséquence?

R : Jusqu'à présent, il n'y avait jamais eu de zone de formation de tsunami au large de Fukushima. Alors, dire qu'on va tout à coup en tenir compte, ça ne sort pas du domaine des suppositions. Mais dans le secteur du nucléaire, il y a toujours le rapport investissement/rentabilité. Pour investir de l'argent, il faut des arguments fondés. À la limite, si on supposait qu'il y ait un séisme de magnitude 9 au large de Fukushima, il pourrait y avoir une vague de $20 \mathrm{~m}$. On peut toujours faire des suppositions avec des valeurs de plus en plus grandes, les calculs donneraient toujours des chiffres encore plus importants. On ne peut pas prendre des mesures de protection sans fondement.

$\mathbf{Q}$ : Lorsque vous réfléchissez aux mesures de protection, le fondement, c'est quoi? Les explications à fournir à la NISA ?

R : Il y a ça aussi. Mais il y a surtout l'avis des spécialistes. Par exemple, le professeur qui a parlé du tsunami de Jôgan a soutenu qu'il pourrait y avoir, de nouveau, formation d'un tsunami à cet endroit-là. C'était l'avis fondé d'un spécialiste. Nous avons donc fait faire des études pour en connaître les effets sur Fukushima. Mais les autres chercheurs se contentaient de déplacer de manière aléatoire la zone de formation en se demandant ce qui arriverait «si le tsunami se formait là ». Avaient-ils des arguments pour justifier que cela puisse se passer là ? Ces thèses faisaient-elles consensus parmi les experts? La plupart les trouvaient farfelues et sans fondement. Au milieu de tout ça, on se demandait comment prendre les décisions. Nous, nous ne sommes que des exploitants. Il fallait qu'un groupe de spécialistes décide des règles et nous nous serions conformés à ces règles.

Tout à l'heure, on a évoqué la NISA. Ce que je voulais dire c'est que si on informe la NISA que nous avons pris telle décision en fonction de telle ou telle règle, l'Agence va l'accepter. Ce qui compte, ce n'est pas ce que la NISA pourrait en penser, mais c'est l'avis des spécialistes.

$\mathbf{Q}$ : Vous venez de parler de rapport investissement/rentabilité. Si, en raisonnant sur tout de manière ultraconservatrice, en tenant compte de phénomènes qui ont une chance quasi nulle d'advenir, on vous disait qu'à la centrale nucléaire, il faudrait élever non seulement les digues jusqu'à 20 m, mais tout relever en conséquence, que les installations pour pomper 
l'eau de mer devraient aussi se situer plus haut, qu'il faille, de là, prévoir tout un réseau de tuyaux, par exemple, qui descendent tout en bas, cela ferait exploser les investissements.

$\mathbf{R}$ : Si on devait parler d'un tsunami de $20 \mathrm{~m}$, de toute façon, il faudrait démanteler les réacteurs. Avec cette localisation, c'est fichu d'avance.

$\mathbf{Q}$ : Vous voulez dire que l'existence même de la centrale Fukushima Daiichi serait mise en cause?

R : Oui.

$\mathbf{Q}$ : Et pour résister à une vague de $15 \mathrm{~m}$, comme cette fois-ci ?

$\mathbf{R}$ : En supposant que le tsunami se forme au large de Fukushima, on avait obtenu une hauteur d'environ $10 \mathrm{~m}$.

$\mathbf{Q}$ : Oui ?

$\mathbf{R}$ : Si on avait eu une vague de $10 \mathrm{~m}$, avec une digue conséquente, je pense qu'on aurait pu répartir sa force, mais, une vague de $15 \mathrm{~m}$, je ne suis pas sûr qu'on aurait réellement pu.

$\mathbf{Q}$ : Bien sûr, l'état de Fukushima Daiichi est tel qu'il n'est même pas question de la remettre en marche, mais pour les autres centrales ? À Onagawa ou à Genkai, la centrale de Kyushu Electric Power ${ }^{84}$ ? S'il y avait une vague de $10 \mathrm{~m}$, là ?

R : Là, c'est un peu la caricature. C'est comme si vous disiez que, partout au Japon, il peut y avoir un séisme de magnitude 9. À ce rythme-là, il n'y aurait plus aucun bâtiment debout par ici. Il n'y a pas que les centrales nucléaires. Si on fait l'hypothèse que l'épicentre peut se trouver juste en-dessous, on ne pourra jamais rien entreprendre. C'est pour ça que, pour chaque tranche, pour chaque centrale, on a modulé l'appréciation des tsunamis et des séismes en fonction de sa localisation et des failles. C'était notre attitude jusqu'ici.

$\mathbf{Q}:$ On vous a reproché des choses du genre, puisque telle centrale tenait compte du tsunami de Jôgan, il n'est pas normal que Fukushima Daiichi n'en ait pas fait autant.

$\mathbf{R}$ : Là, le raisonnement est faussé. Au contraire, quand on a fait faire des calculs sur le modèle du tsunami de Jôgan, la vague n'atteignait que $4 \mathrm{~m}$, environ. Si un tsunami se formait au même endroit qu'à l'ère Jôgan, nous étions parés, puisque la vague n'aurait pas dépassé ce niveau. Seulement, cette fois-ci, il y a eu un séisme plus important que celui de Jôgan. Un séisme de magnitude 9. Quel sismologue, quel expert en tsunami au Japon avait-il prédit la survenue d'un séisme de magnitude 9 à cet endroit-là ? Même les chercheurs qui s'étaient penchés sur le tsunami de Jôgan n'avaient pas envisagé un séisme de magnitude 9. J'ai envie de dire à tous ces détracteurs qu'il est facile de critiquer une fois que c'est fait.

$\mathbf{Q}$ : Ce que je vais vous dire est plus une opinion personnelle que l'avis de la Commission d'enquête. Je pense que quand quelqu'un du génie civil vous sort des chiffres comme «10 m de haut», pour vous, c'est une hypothèse aussi farfelue que quand je vous demandais d'envisager ce qui arriverait s'il y avait une vague de $15 \mathrm{~m}$ à la centrale de Genkai.

De toute façon, tout au début, au moment où vous sélectionnez des lieux pour l'implantation d'une centrale nucléaire, vous menez des études pour savoir si le relief est suffisamment stable, quelle est I'histoire des failles par le passé, si le site risque d'être affecté par les conditions météorologiques et dans quelle mesure, n'est-ce pas?

$\mathbf{R}$ : Oui, nous pensons à tout. 
$\mathbf{Q}$ : Du coup, même si on vous fait remarquer qu'à telle autre centrale, c'est comme ci ou comme ça, si vous estimez que l'endroit offre un niveau de sécurité suffisant, vous le choisissez comme lieu d'implantation.

$\mathbf{R}$ : Oui. C'est comme ça que nous procédons, depuis le début. Ici, la décision a été prise il y a quarante ans. À l'époque, la référence pour le calcul des tsunamis était les tsunamis du Chili ${ }^{85}$. On avait calculé que s'il se produisait un gros séisme au Chili et que le tsunami traversait le Pacifique, il ferait environ $3,1 \mathrm{~m}$ à son arrivée à Fukushima. À cette époque-là, les recherches sur le tsunami de Jôgan ou sur le calcul de l'ampleur des tsunamis selon sa zone de formation le long de la faille, étaient immatures. De nouvelles découvertes ont été faites, depuis. Lorsque la JSCE a sorti ses critères, ils reflétaient les découvertes récentes. Nous, de notre côté, nous les avons appliqués. Nous avons pris les chiffres de «5,7 $\mathrm{m} »$. Nous avons fait les transformations nécessaires. Comme étape suivante, nous avons pensé que s'il fallait tenir compte de la forme du port, avec les mêmes conditions de calcul, la vague risquait d'être un peu plus haute. Nous avons donc décidé d'adopter les chiffres de «6,1 $\mathrm{m} »$. Pour nous, nous nous sommes adaptés à chaque fois aux exigences du moment.

C'est une chose que je voudrais dire plus spécialement à toutes les personnes qui nous parlent du tsunami de Jôgan. S'il s'était formé un tsunami de cette ampleur au même endroit, à l'arrivée, chez nous, il aurait fait 3 ou $4 \mathrm{~m}$. Notre digue aurait largement suffi à le contenir. C'est le jugement que nous avions porté. Personne, avant le 11 mars, n'avait parlé d'un séisme de magnitude 9 à l'endroit même du tsunami de Jôgan. Alors, nous reprocher de ne pas l'avoir envisagé, je trouve ça offensant. Nous reprocher ça, c'est comme si on nous disait de reconstruire toutes les centrales nucléaires du Japon, sans exception, pour qu'elles puissent résister à un tsunami de $15 \mathrm{~m}$, sans tenir compte du relief ou autre donnée.

$\mathbf{Q}:$ Vous ne faites pas ça?

R : Non.

$\mathbf{Q}$ : S'il fallait le faire, il suffirait de le faire de manière standardisée, on n'aurait plus besoin de réviser les chiffres pour chaque localité.

$\mathbf{R}$ : À la limite, il n'y aurait même plus besoin de directives. Les directives disent de décider de la secousse sismique ou de la hauteur du tsunami de référence, en tenant compte $\mathrm{du}$ relief et d'autres conditions. Pour prendre cette décision, elles recommandent de ne pas se limiter à un seul mode de pensée, mais de les diversifier. C'est tout.

$\mathbf{Q}$ : Actuellement, y a-t-il, par exemple au sein de la JSCE, des études pour calculer les effets d'un tsunami comme celui de Jôgan, en amenant tout simplement sa zone de formation au large de Fukushima? Y a-t-il des mouvements parmi les exploitants pour en tenir compte?

$\mathbf{R}:$ Non.

$\mathbf{Q}:$ Si, au lieu de transférer cette zone de formation de manière arbitraire, la JSCE fournissait des arguments rationnels pour la bouger, vous en tiendriez compte?

$\mathbf{R}:$ Tout à fait.

$\mathbf{Q}$ : Ce qui veut dire qu'à l'heure actuelle, les études n'étant pas suffisamment avancées, vous n'êtes pas encore arrivés à ce stade.

$\mathbf{R}$ : Non, nous n'y sommes pas encore. 
$\mathbf{Q}$ : Est-ce que pour vous, TEPCO ou Tôhoku Electric Power, les directives ou les critères de la JSCE font autorité ? Leur accordez-vous une objectivité certaine?

$\mathbf{R}$ : Oui. Ce sont des directives qui sont non seulement valables pour nous, mais pour l'ensemble du Japon. De plus, elles ne concernent pas que les centrales nucléaires. C'est une chose que je voudrais clamer haut et fort. Il ne s'agit pas que de la sûreté des centrales nucléaires. Le tsunami de mars a fait 23000 victimes. Qui les a tuées ? C'est un séisme de magnitude 9 qui les a tuées. On nous reproche notre responsabilité. Mais pourquoi n'avait-on pas pris les dispositions pour que ces personnes ne meurent pas? Puisque les gens évoquent le tsunami de Jôgan, pourquoi n'avait-on pas mis les personnes les plus proches de son point d'impact à l'abri ? Au lieu de se poser ces questions, la discussion fait un bond et se concentre sur le seul point de la responsabilité de TEPCO. Je ne trouve pas ça normal. S'il s'agit de mesures fondamentales pour protéger la vie et les biens des Japonais, il faudrait que la cellule de gestion de crise du Premier ministre s'empare du sujet et prenne les mesures qui s'imposent avec les autorités locales, communes comprises. Mais l'État ne fait rien. Il se contente de remettre en question l'organisation des centrales nucléaires.

Si je vais plus loin, de tout le Japon, les cartes les plus sérieuses de l'activité des failles sont celles concernant l'environnement proche des centrales et ce sont quasiment les seules. En dehors de ça, ni l'Agence nationale de météorologie, ni le Geospatial Information Authority of Japan ${ }^{86}$ ne finançant jamais les études avec leurs propres deniers, il n'y en a pas. Les seuls endroits dont on connaisse l'activité des failles sont les environs des centrales nucléaires. Sachant cela, nous aurions bien envie de demander à l'État ce qu'il en pense et exiger qu'il commence par s'expliquer clairement là-dessus.

Si la JSCE nous donnait des indications, nous étions prêts à construire une digue ou toute autre chose pour nous protéger de la vague. Mais si on ne fait rien alentour, même si la centrale peut être protégée, les villes, les villages qui sont à côté vont se retrouver complètement sous l'eau. Bien sûr, protéger une centrale nucléaire est important, mais si on n'a pas de plan d'ensemble, on ne peut pas parler de véritables mesures de protection. Je trouve que l'État a une vision biaisée, concernant les séismes et les tsunamis.

$\mathbf{Q}$ : Le département de Fukushima avait pris des mesures.

R : Oui.

$\mathbf{Q}:$ Le département $\mathrm{d}^{\prime}$ Ibaraki ${ }^{87}$ aussi, il me semble.

R : Oui.

$\mathbf{Q}:$ Ces mesures vous servaient-elles aussi de références?

$\mathbf{R}$ : Bien sûr. Mais nos critères étaient encore plus sévères.

$\mathbf{Q}$ : Vous preniez plus de marges?

R : Oui.

$\mathbf{Q}$ : Si on vous reproche de ne pas avoir tenu compte du tsunami de Jôgan, la remarque est aussi valable pour le département de Fukushima.

$\mathbf{R}$ : En effet. Et d'autant plus, pour les départements de Miyagi et d'Iwate ${ }^{88}$.

$\mathbf{Q}$ : Oui, puisque le tsunami a fait tellement de victimes là-bas ${ }^{89}$.

$\mathbf{R}$ : Si vous permettez, je voudrais dire encore une chose. Le séisme de mars était un tremblement de terre encore plus important que celui qu'on avait calculé pour le 
tsunami de Jôgan. Il y a fondamentalement deux choses qui diffèrent entre ce qui s'est passé en mars et l'épisode de Jôgan.

La première est l'importance du séisme. C'était un séisme de magnitude 9. Aucun sismologue, aucun spécialiste des tsunamis n'en avait fait l'hypothèse jusque-là.

La seconde est le fait que trois plaques tectoniques ont bougé quasi simultanément. Ça non plus, personne ne l'avait jamais supposé. «Si une plaque bouge, les autres restent en sommeil ». Telle était la théorie en vigueur chez les spécialistes. Or, les plaques ont bougé en même temps. C'est un saut dans l'inconnu. Je ne comprends pas ce Gouvernement pourri qui voudrait que nous admettions que ce n'était pas en dehors de ce qui était prévisible. C'est un point sur lequel je ne céderai jamais.

$\mathbf{Q}$ : Je pense qu'il n'aurait pas été possible à un exploitant, en l'occurrence TEPCO, de présupposer ce genre de chose, alors qu'alentour personne n'en parlait. C'est quand même le genre de sujet sur lequel un exploitant isolé ne peut pas faire cavalier seul. II faut, naturellement, que l'État, les autorités locales, les cercles savants, bref, que tout le monde soit au diapason. II me semble que l'État et les autorités locales ne sont pas très mobiles. Peut-être que les chercheurs étaient les plus en pointe. Savez-vous s'il y avait des chercheurs qui parlaient de plaques qui bougent simultanément, ou qui envisageaient la possibilité d'un séisme de magnitude 9 , au lieu de 8, au large, côté Pacifique ?

$\mathbf{R}:$ Je ne pense pas qu'il y en ait eu.

$\mathbf{Q}$ : Vous n'en avez pas entendu parler?

$\mathbf{R}$ : Moi, personnellement, non. Mais comme je vous l'ai dit tout à l'heure, je ne suis pas un spécialiste du domaine.

$\mathbf{Q}$ : Bien sûr, mais on peut tout de même dire que cela n'était pas devenu un sujet suffisamment important pour arriver à l'oreille de quelqu'un comme vous qui, chez un exploitant comme TEPCO, étiez en charge de vous tenir au courant de ce genre de choses.

$\mathbf{R}$ : On peut le dire.

$\mathbf{Q}$ : Peut-être un ou deux experts en ont-ils parlé. Peut-être en avaient-ils parlé lors de conférences en région, par exemple. Si cette thèse avait été retenue, fait l'objet d'études sérieuses, qu'elle ait été reconnue comme juste ou pas, vous en auriez eu vent chez TEPCO, n'est-ce pas?

R : Oui.

Q: Vous auriez surveillé l'état d'avancement des études.

R : Oui.

$\mathbf{Q}$ : Ce qui veut dire que cette thèse n'était même pas arrivée à un stade où on s'intéresse à elle.

R : C'est ça.

$\mathbf{Q}$ : Concernant le tsunami de Jôgan, il vous était difficile de décider comment en tenir compte. Comme vous n'étiez pas en état d'en tirer les conclusions, vous aviez chargé la JSCE de mener les études nécessaires.

$\mathbf{R}$ : Oui. Nous avons aussi mené des recherches de notre côté.

$\mathbf{Q}$ : En effet, pour savoir objectivement jusqu'à quel point de la côte le tsunami de Jôgan avait pénétré, vous avez mené des recherches sur les sédiments, suivant les conseils des experts.

R : Oui. 
$\mathbf{Q}:$ Vous étiez prêts à suivre les critères indiqués par la JSCE.

$\mathbf{R}$ : S'ils avaient été sévères, nous étions prêts à agir en conséquence.

$\mathbf{Q}$ : En fait, vous aviez obtenu les chiffres de «6,1 $\mathrm{m}$ » et vous aviez remonté la digue en proportion pour les réacteurs 5 et 6 , quand le séisme et le tsunami de mars sont survenus.

R : Oui.

$\mathbf{Q}$ : J'ai là des documents qu'on m'a donnés chez TEPCO. Si j'y jette un rapide coup d'œil, on a l'impression que vous êtes encore dans l'expectative, que vous êtes encore dans les études. La NISA ne vous dit pas de faire comme ci ou comme ça? D'abord, aviez-vous avisé la NISA des chiffres comme «10 $\mathrm{m}$ » ou « $9 \mathrm{~m}$ », que vous aviez obtenus en faisant vos calculs?

$\mathbf{R}$ : Je pense qu'au moment de l'accident, l'agence n'en avait pas encore été informée.

$\mathbf{Q}$ : Par exemple, lorsque vous aviez obtenu «6,1 m», vous l'en aviez dûment informée, je suppose.

R : Oui.

$\mathbf{Q}$ : Et à ce moment-là, on ne vous a pas dit que cela n'allait pas, n'est-ce pas?

$\mathbf{R}:$ Non.

$\mathbf{Q}$ : Parmi les gens de la JSCE, ces spécialistes qui définissent les normes, il y a des personnes de TEPCO, il me semble.

$\mathbf{R}$ : Oui, il y a aussi des gens de chez TEPCO à la JSCE.

$\mathbf{Q}$ : Vous surveillez tous les mouvements de la JSCE, pour pouvoir réagir rapidement?

R : Oui.

$\mathbf{Q}$ : Vous est-il arrivé, lorsque vous étiez à la tête de ce département au siège, de rencontrer des membres de la JSCE pour connaître les sujets de discussion, par exemple?

$\mathbf{R}$ : Non, jamais. Vous savez, j'étais un vrai amateur en la matière, alors j'avais laissé au responsable du génie civil le soin de suivre tout ça. Je lui faisais totalement confiance. C'était quelqu'un qui s'était toujours occupé de ça, il était très expérimenté.

$\mathbf{Q}$ : ॥ ne vous est donc jamais arrivé de vous déplacer sur place pour assister à ces réunions.

R : Non.

Q: Après avoir occupé cette fonction au siège, vous devenez directeur de la centrale nucléaire de Fukushima Daiichi.

R : Oui.

$\mathbf{Q}$ : Une fois que vous étiez devenu directeur de la centrale, avez-vous eu, de la part du siège, des informations concernant les mesures de protection contre le séisme ou ses conséquences, comme le tsunami?

R : Oui. Puisqu'il s'agissait de nos tranches, je devais participer, en tant que directeur, à la prise des dernières décisions concernant les travaux à entreprendre, donc j'étais informé de la situation en conséquence.

$\mathbf{Q}:$ Y a-t-il eu des mouvements concrets au niveau de la JSCE, les derniers temps ? Ou des signes qui laissaient entrevoir la direction qu'ils allaient prendre, par exemple?

$\mathbf{R}$ : Depuis que j'occupais le poste de directeur, je n'avais pas entendu dire que les chiffres allaient faire un bond à cause des décisions de la JSCE. 
$\mathbf{Q}:$ Pour les réacteurs 5 et 6 , vous avez pris des dispositions pour faire face à une vague de $6,1 \mathrm{~m}$.

R : Oui.

$\mathbf{Q}$ : Vous n'avez pas entendu dire qu'il faudrait peut-être renchérir encore un peu ? Vous n'en étiez pas encore à ce stade?

$\mathbf{R}$ : Depuis que j'étais devenu directeur de la centrale, il n'y a jamais eu de grands changements concernant l'appréciation des tsunamis de référence, ni d'exigence officielle pour que nous prenions en compte des tsunamis plus importants.

$\mathbf{Q}$ : Ce qui veut dire qu'il y en a eu par le passé, de non officielle?

R : Non, ce n'est pas tout à fait ça. Personnellement, j'ai toujours pensé qu'il y avait des possibilités pour qu'un jour ou l'autre, il faille envisager une vague plus haute, suivant certaines méthodes de calcul. Mais je ne pensais pas à une vague de plus de $10 \mathrm{~m}$. Même s'il s'agissait d'une vague d'un peu plus de 6,1 m, il fallait tout de même songer à la capacité des pompes, par exemple. Je savais qu'il fallait y songer à l'avance. Bien entendu, l'idée qu'une vague de $15 \mathrm{~m}$ puisse venir ne m'avait jamais effleuré l'esprit. Mais je pensais, et je l'ai toujours pensé, qu'on ne pouvait pas écarter la thèse qu'une vague qui dépasse les $6,1 \mathrm{~m}$ puisse se former.

$\mathbf{Q}:$ Vous le pensiez déjà, dès avant votre prise de fonction?

R : Oui, je l'ai toujours pensé.

$\mathbf{Q}$ : TEPCO a donc sollicité la JSCE sur ce sujet. Savez-vous si elle en a débattu depuis et dans quelle mesure?

$\mathbf{R}$ : Je n'en sais strictement rien.

$\mathbf{Q}:$ Vous ne le savez pas?

R : Non.

$\mathbf{Q}:$ Y a-t-il eu des suggestions de la NISA concernant le sujet? Du genre, « ce serait bien de revoir vos chiffres à la hausse»?

$\mathbf{R}$ : Vous voulez dire quand j'étais directeur du département «gestion des installations nucléaires »?

$\mathbf{Q}$ : Oui, à ce moment-là.

$\mathbf{R}$ : Non, rien.

$\mathbf{Q}:$ : I n'y a rien eu ?

$\mathbf{R}$ : Ces messieurs de la NISA ont un côté "dégonflés ». Ils arrivent toujours à la conclusion " on va demander l'avis des grands spécialistes ». Qu'il s'agisse des remises aux normes ou autre chose, la NISA ne décide jamais rien. Parce que ce sont des dégonflés. Ils finissent toujours par nommer un comité d'appréciation des normes antisismiques, par exemple, aligner les grands professeurs, faire rédiger de la documentation aux exploitants, faire faire des rapports, chercher la petite bête, exiger que les exploitants fassent quelque chose sur les seules parties où il y a eu des commentaires ou exiger qu'on fasse la lumière sur ces seules parties, et c'est tout. En fait, ils ne décident jamais des normes en tant que NISA. Ça, ils ne le font jamais. Parce que ces gens-là ne veulent pas prendre leurs responsabilités. 
$\mathbf{Q}$ : Si je comprends bien, ils se comportent un peu comme un pôle administratif. Ils alignent des sachants, leur demandent de plancher et, si un quelconque avis en sort, ils vous ordonnent de vous y conformer. C'est ça, leur attitude?

$\mathbf{R}:$ Tout à fait.

$\mathbf{Q}$ : Si vous alliez demander des conseils, la NISA, en tant que NISA, ne vous suggérerait jamais de faire comme ci ou comme ça.

$\mathbf{R}:$ Non.

$\mathbf{Q}$ : C'est seulement concernant les tsunamis ou c'est la même chose pour tout?

$\mathbf{R}$ : Pour tout. Ils sont totalement conservateurs. Conservateurs, je veux dire dans le sens où ils ne veulent pas changer la manière de faire qui est en vigueur. Il ne leur arrive pas de changer les choses en suivant de manière ferme l'avis de quelqu'un. De plus, pour ne pas être pris en faute, ils consultent les grands professeurs dans une sphère très élargie et présentent toujours les décisions prises comme découlant de l'accord de la totalité des consultants.

Q: Lorsque vous étiez au département »gestion des installations nucléaires », vous n'agissiez pas tellement parce que la NISA vous avait dit de faire ci ou faire ça. Pour vous, vous considériez ces préconisations comme celles des diverses commissions, plutôt que celles de la NISA en elle-même, je suppose.

$\mathbf{R}$ : Oui. Tout était comme ça. C'est vrai que concernant les séismes, ça l'était d'autant plus. La NISA, c'est un ramassis de théoriciens. Les ingénieurs font des calculs pour dire ce à quoi peut, par exemple, résister telle construction. Ils réfléchissent ensuite à la marge qu'il faut prévoir pour assurer sa conservation. C'est une histoire concrète. Mais les discussions des théoriciens sont tout autres. Certains prétendent, là où il n'y a aucune faille, qu'il vaut mieux penser qu'il y en a une, parce que quand on regarde les cartes de la surface, tel endroit est rétréci. Il y a des gens qui s'appellent des géomorphologues. Ce sont eux qui disent ça. Si on suivait leur théorie et qu'on considérait que toute cette partie, ici, est une faille en activité, on arriverait à des séismes de magnitude 9. Seulement, si on effectue une étude géologique, ce n'est pas une faille. Ces réunions de la NISA ont des allures de lieu d'ajustement entre les géologues et les différents types de théoriciens. Ce sont les idées qui ont réussi à réunir la quasi-totalité de l'assemblée qui servent en fait de référence à la conception des différentes installations. Mais est-ce suffisant? Je me pose des questions. À titre personnel, je pense qu'il existe toujours un seuil où, à force de concessions, les gens finissent nécessairement par tomber d'accord. Mais je ne suis pas sûr que ce soit vraiment la solution.

Au moment des évènements de Chûetsu, il y a eu un séisme de magnitude 6,8. Déjà, on ne pensait pas qu'il pourrait y avoir un séisme dans cette zone, côté mer. De plus, personne ne s'attendait à un mouvement aussi fort. C'est vous dire si, ces derniers temps, des évènements remettant en cause tout ce qui était normalement admis en géologie ou en sismologie se succèdent. Cette fois aussi, tout le monde disait qu'il n'y aurait pas de séisme de magnitude 9 et il y en a eu un. Enfin, ils ne disaient pas qu'il n'y en aurait pas, mais personne n'en avait évoqué la possibilité et il y en a quand même eu un. Pour être honnête, personnellement, mon sentiment est que je ne sais plus à quoi me fier pour prendre les décisions à venir. 
$\mathbf{Q}$ : Effectivement, nous avons connu un séisme et un tsunami hors normes. Est-ce que, depuis lors, la NISA a, par exemple, réuni des experts pour former une commission qui donnerait des directives aux exploitants pour faire ci ou ça?

$\mathbf{R}$ : Cela ne concerne pas la valeur des séismes ou des tsunamis de référence, mais d'après les dommages que nous avons eus sur la centrale de Fukushima et en en tirant les leçons, il y a eu des directives, qui émanaient du ministre de l'Économie et de l'Industrie ou du directeur de la NISA, je ne sais plus, concernant, par exemple, la multiplication des alimentations électriques, le matériel pour raccorder les camions de pompiers, etc. Tous les exploitants s'y sont mis. Disons qu'il s'agit de prévoir de quoi traiter les symptômes, mais la discussion de fond sur les mouvements sismiques et tout ça n'a pas encore abouti. Je pense qu'ils sont encore en train de débattre sur la manière de réapprécier tout ça en tirant les leçons de ce qui est arrivé. Mais je ne suis pas ça de près. Je ne suis donc pas au courant pour les détails.

$\mathbf{Q}:$ Vous n'avez pas été spécialement sollicité pour assister à ces réunions ?

$\mathbf{R}:$ Non. Et je pense que je n'y ai pas ma place.

$\mathbf{Q}:$ Vous croyez?

$\mathbf{R}$ : Ce sont des réunions de savants.

$\mathbf{Q}$ : Quelques jours avant le séisme du 11 mars à 14h46, il y avait eu des secousses. Vous en rappelez-vous?

R : Oui.

$\mathbf{Q}:$ La plus grosse a été observée du côté de Miyagiji ${ }^{90}$ il me semble.

R : Oui.

$\mathbf{Q}:$ Ici, qu'aviez-vous enregistré au même moment?

$\mathbf{R}$ : Il y avait quelque chose comme 4 . Ça avait secoué assez fort.

$\mathbf{Q}$ : En vous remémorant cette époque, vous diriez que c'était une période où il y avait des séismes sans arrêt?

$\mathbf{R}$ : Non, pas vraiment.

$\mathbf{Q}$ : S'il y avait eu ce genre de prémices, on aurait pu dire que cela pourrait servir de sonnette d'alarme à l'avenir.

$\mathbf{R}$ : Non. Au contraire, ici, nous sommes à un endroit où il $\mathrm{y}$ a souvent des séismes. C'est parce que la plaque, là-bas, plonge régulièrement. Et donc, nous avons des séismes. Jusqu'à présent, nous avons connu pas mal de secousses de degré 3 ou 4.

$\mathbf{Q}:$ Ah, bon.

$\mathbf{R}$ : C'est pour ça que le séisme du 8 mars, à y réfléchir, n'avait rien de spécial. Vous savez, il est déjà arrivé au moins trois fois que les réacteurs se mettent en arrêt automatique à cause de séismes, ici, à Fukushima Daiichi. Des séismes de cette ampleur "pouvaient» survenir. Seulement, sur cette zone, on pensait que ça resterait des séismes de ce niveau, c'est tout.

$\mathbf{Q}$ : Vous, personnellement, vous avez occupé plusieurs postes sur la centrale Daiichi.

R : Oui. 
$\mathbf{Q}$ : Durant ces années, le plus gros séisme que vous ayez vécu faisait quoi ? Plus de « 4 »? « $5 » ? \ll 6 » ?$

R : Vers 2006, quand j'étais chef d'unité, il y avait eu un assez gros tremblement de terre. Je travaillais dans le bâtiment administratif. Le tableau qui était accroché derrière moi est tombé ${ }^{91}$.

$\mathbf{Q}:$ : était assez gros, alors.

$\mathbf{R}$ : Je pense que ce jour-là, on a dû atteindre « 4 » ou « 5 faible ». Oui, on a connu ça.

$\mathbf{Q}$ : Et ce jour-là, les réacteurs fonctionnaient normalement?

R : Oui. Il n'y a pas eu de problème. On n'avait pas atteint le niveau du SCRAM. Il n'y a donc pas eu SCRAM. Ce qui signifie que l'accélération du terrain était en-dessous de 110 gal.

Q: Aviez-vous remarqué des détériorations particulières dues à la secousse sur la tuyauterie externe, par exemple?

R : Non.

C'est une question qu'on me pose souvent, mais les bâtiments sont très résistants visà-vis des séismes. Cette fois encore, on m'a dit des tas de choses les concernant, mais la plupart des tuyaux, des machines n'ont pas subi de dommages du fait du tremblement de terre. Concrètement, lors du séisme au large de Chûetsu, la tranche 1 de Kashiwazaki a atteint 680 gal. Je pense qu'en mars la plus grosse accélération, ici, à Fukushima Daiichi, a été de l'ordre de 300 à 400 gal. Contre 680 gal à Kashiwazaki. De fait, nous avons organisé des tours d'inspection juste après la secousse. Bien sûr, avec le déplacement relatif, les bâtiments étaient déformés, mais les tuyaux, à l'intérieur, n'avaient pas rompu. Pourtant, il y a des tuyaux qui ne sont pas de catégorie très élevée par rapport aux normes antisismiques, mais tous avaient tenu le coup. Alors j'ai toujours pensé que les installations étaient relativement résistantes face aux séismes.

En 2006 et 2007 aussi, nous avions procédé à des inspections, mais avec moins de 100 gal, rien n'avait bougé.

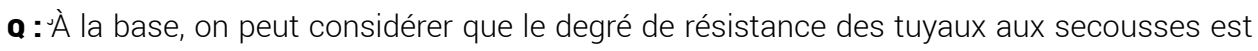
équivalent à Kashiwazaki et à Fukushima Daiichi ?

$\mathbf{R}$ : La manière de concevoir toute cette architecture a beaucoup évolué depuis les origines. Je veux dire, lorsque le réacteur 1 a été construit ici, il n'y avait pas d'ordinateur. Donc quand ils analysaient, ils ne disposaient pas d'un modèle sur ordinateur. Tout le calcul était fait à la main, si j'ose dire. Ce qui se passe, dans ce caslà, c'est qu'on prend beaucoup plus de marges de sécurité. Plus les tranches sont récentes, plus la conception est rationalisée. On analyse les mouvements lorsqu'on applique une secousse tellurique et on calcule en fonction, par exemple. Mais, à la limite, quand on regarde les conceptions d'origine...

$\mathbf{Q}$ : Paradoxalement, elles sont parfois plus costaudes?

$\mathbf{R}$ : À certains endroits, oui. Dans ce sens, je ne me faisais pas particulièrement de souci vis-à-vis des secousses sismiques. Si le SCRAM se faisait correctement, mis à part la question du moment du redémarrage, en tant qu'ingénieur, j'avais la conviction qu'il ne se passerait rien de terrible.

$\mathbf{Q}$ : Ça, c'est le 11 mars avant l'arrivée du tsunami.

R : Oui. 
$\mathbf{Q}$ : Lorsqu'il y a eu le tremblement de terre de magnitude 7 du côté de Miyagi, le 8 mars, avez-vous observé un tsunami à Fukushima Daiichi?

$\mathbf{R}$ : Il me semble qu'il y en a eu un tout petit.

$\mathbf{Q}$ : De l'ordre de quelques mètres ?

$\mathbf{R}$ : Non, ça ne se mesurait pas en mètres, mais en centimètres, je crois.

$\mathbf{Q}:$ Il n'y a donc pas eu de grosses répercussions.

$\mathbf{R}$ : Il me semble que c'était un tsunami de $50 \mathrm{~cm}$, quelque chose comme ça.

$\mathbf{Q}$ : Le 11 mars, l'Agence nationale de météorologie avait émis des alertes aux tsunamis, avec une estimation croissante de la hauteur des tsunamis. II y en a eu trois au total. Par exemple, à 14h49, il y a eu une alerte avec une prévision d'une vague de $3 \mathrm{~m}$ pour la côte du département de Fukushima. À 15h15, l'estimation passe à $6 \mathrm{~m}$. À 15h30, plus de $10 \mathrm{~m}$. En fait, la première vague est arrivée juste avant cette alerte, à 15h27. Vous nous avez dit que quand le tsunami est arrivé, vous ne vous en étiez pas rendu compte tout de suite. Ça n'apparaissait pas sur les écrans de surveillance, par exemple?

$\mathbf{R}:$ Non.

$\mathbf{Q}$ : Lorsque vous avez entendu la première alerte à $14 \mathrm{~h} 49$ et que vous avez su que la hauteur de la vague serait de $3 \mathrm{~m}$, vous étiez en mesure de résister.

$\mathbf{R}$ : Oui, on pouvait résister.

$\mathbf{Q}$ : Quand vous dites que vous pouvez résister à une vague de 5,7 $\mathrm{m}$, en tout cas une vague de plus de $5 \mathrm{~m}$, c'est au niveau de la digue que toute la force est absorbée?

$\mathbf{R}$ : On prend en compte la digue et c'est conçu pour que lorsque la vague arrive à cette partie, à la bouche de prise d'eau, elle ne dépasse pas $5 \mathrm{~m}$.

$\mathbf{Q}$ : D'accord. Donc, si la vague ne fait pas plus de $3 \mathrm{~m}$, par exemple, l'ensemble de l'installation est opérationnelle, y compris les pompes à eau de mer?

$\mathbf{R}$ : Il y a une chose dont nous devons nous préoccuper en priorité. Dans la conduite normale, pour refroidir la turbine, nous alimentons le condenseur en eau de mer. Or, si un gros tsunami survenait au moment où la tranche est en fonctionnement, non pas le réseau d'eau de mer de secours, mais le réseau normal du condenseur ne pourrait plus aspirer l'eau de mer, le vide du condenseur serait rompu et il s'arrêterait. C'est là une chose à laquelle nous devons absolument faire attention quand une tranche est en état de fonctionnement. Mais, au mois de mars, il y avait eu SCRAM, toutes les tranches étaient à l'arrêt, donc nous n'avions pas à nous préoccuper de ce problème.

Le souci suivant, c'est que, lorsque la vague arrive, elle inonde la pompe de secours, que l'isolation ne soit plus assurée et qu'elle tombe en panne. C'est ce qui nous est arrivé.

L'autre souci, c'est lorsque la vague se retire. Lorsqu'elle s'en va, le fond de la mer est complètement dénudé. Si on ne peut pas approvisionner en eau, la pompe ne va plus fonctionner. Ou plutôt, elle va tourner dans le vide et se détériorer. Il faut donc se préoccuper de cette pompe. Mais, s'il s'agit d'une vague de $3 \mathrm{~m}$, voire $6 \mathrm{~m}$, c'était le chiffre de la JSCE, on peut tenir sans que la pompe ne casse. Car, avec le temps, même si la vague se retire, après la première vague, puis la deuxième, la mer va finir par retrouver un niveau normal. Si on arrive à continuer à refroidir jusque-là, avec le retour de la mer, on peut reprendre le refroidissement à l'eau de mer. 
Q: Cela veut dire que, malgré les prévisions à la hausse des alertes au tsunami successives, même si la vague devait atteindre les $6 \mathrm{~m}$, vous pensiez que vous arriveriez à vous en sortir in extremis?

$\mathbf{R}$ : Oui, je pensais que ça pouvait passer.

$\mathbf{Q}$ : Finalement, c'est une vague de plus de $10 \mathrm{~m}$ qui est arrivée. Avez-vous le souvenir d'avoir su que c'était une vague de plus de $10 \mathrm{~m}$ ?

$\mathbf{R}$ : Non. À ce moment-là ont commencé à affluer les informations comme quoi il n'y avait plus d'électricité.

$\mathbf{Q}$ : Effectivement, avec la première vague qui est arrivée à 15h27, différents évènements ont dû se produire. Les rapports devaient se succéder auprès des groupes «production ». Oui, les prévisions initiales de l'Agence météorologique ont été largement dépassées.

Au moment où arrive le tsunami, le personnel non spécialisé, je veux dire les personnes qui ne faisaient pas partie des équipes de soutien en cas d'incident, étaient aussi dans le bâtiment antisismique? Elles n'étaient pas restées sur le parking?

R: D'abord, quand il y a eu le séisme, j'ai donné l'ordre que tous, y compris le personnel des entreprises partenaires et le personnel féminin du bâtiment administratif, se rassemblent une première fois ici. Bien sûr, les pilotes étaient à part. Là, on a fait l'appel et j'ai demandé à ceux qui pouvaient le faire de rentrer directement chez eux.

$\mathbf{Q}$ : Sans rentrer dans les bâtiments?

$\mathbf{R}$ : Oui, sans rentrer dans les bâtiments.

Il y avait des répliques. Il y avait des gens qui avaient peur et voulaient se mettre à l'abri pendant un moment. Ceux-là sont entrés dans le bâtiment antisismique. Les autres bâtiments étaient plus ou moins effondrés, alors le seul endroit où on pouvait se mettre à l'abri, c'était le bâtiment antisismique. Dans ce sens, il y avait des gens qui s'étaient mis là comme dans un refuge, alors ça grouillait de monde le premier jour.

$\mathbf{Q}:$ À quoi utilisiez-vous le bâtiment antisismique en temps normal ?

$\mathbf{R}$ : En temps normal, on ne peut pas l'utiliser.

$\mathbf{Q}:$ Ah, vous ne l'utilisez pas?

$\mathbf{R}$ : Même si ce n'est pas un séisme, à chaque fois qu'il y a des incidents, on l'utilise comme salle de gestion des incidents, donc on le laisse libre. Seul du personnel de la catégorie des Group Managers vient y monter la garde à tour de rôle, de nuit et de jour, pour pouvoir donner l'alerte et que tout le monde puisse s'y rassembler au moindre incident. Mais la salle de réunion, en soi, n'est pas utilisée habituellement.

$\mathbf{Q}$ : Aviez-vous fait récemment un entraînement à l'évacuation?

$\mathbf{R}$ : Oui, comme je vous l'ai dit, on l'avait fait en même temps que l'entraînement à la gestion d'accident.

$\mathbf{Q}$ : Ah, c'est ce qu'ils voulaient dire. Certaines personnes m'avaient parlé d'un entraînement à l'évacuation qui avait eu lieu environ une semaine avant l'accident. C'était I'histoire de cet entraînement de la fin février.

$\mathbf{R}$ : Oui, c'est ça. 
Q : Au moment de cet entraînement, où vous deviez faire face à un supposé accident grave, vous aviez aussi effectué l'évacuation du personnel.

$\mathbf{R}$ : Oui. Le scénario avait prévu un état d'urgence dû à un séisme et il devait y avoir quelque chose de cassé au niveau du réacteur 5 . Du coup, on avait eu un rassemblement, fait l'appel. Tout le monde avait vérifié son itinéraire d'évacuation.

$\mathbf{Q}$ : Cet entraînement à l'évacuation, les entreprises partenaires y participaient aussi ?

R : Oui.

$\mathbf{Q}$ : Alors ça devait concerner beaucoup de monde.

$\mathbf{R}$ : Les entreprises partenaires participent. Mais ce sont surtout les quelques entreprises avec qui nous avons beaucoup de liens. Ces entraînements, à la base, ne concernaient que nos propres employés. Mais leur personnel participe aussi.

$\mathbf{Q}$ : «Des entreprises avec qui vous avez des liens », c'est qui, par exemple?

R : Par exemple, Japan Nuclear Security Service System, ou Nanmei, des entreprises comme ça. Il y a aussi, pour les analyses, des gens de Tôshiba, de Hitachi qui participent.

$\mathbf{Q}$ : Évidemment, en mars, ce n'était pas un entraînement. II devait aussi y avoir des gens d'entreprises partenaires qui n'avaient jamais participé à ces entraînements, je suppose.

$\mathbf{R}:$ Oui, il y en avait.

$\mathbf{Q}:$ : I me semble qu'il y avait beaucoup de monde du côté de la tranche 4 .

$\mathbf{R}$ : Oui, elle était en révision programmée. Il devait y avoir un millier de personnes.

$\mathbf{Q}$ : Tous ces gens sont aussi venus se réfugier par ici?

R: Oui. Je pense qu'il y a des gens qui sont venus ici et d'autres qui sont probablement allés se réfugier dans leurs propres bureaux. Ce qui veut dire que tout le monde, absolument tous, n'est pas venu ici. Par contre, tous nos employés à nous ont bien été rassemblés ici. Comme j'avais vu que du personnel des entreprises partenaires était là, j'avais demandé à chaque entreprise de vérifier elle-même l'état de son personnel.

$\mathbf{Q}:$ : I n'y avait pas trop de monde du côté des tranches 5 et 6 ?

$\mathbf{R}$ : À ce moment-là, on était en train de tester la résistance à la pression de la cuve et je pense qu'il devait quand même y avoir du monde. Mais comme les travaux de démontage-remontage étaient terminés, ce devait être un moment où le nombre de présents commençait à diminuer sensiblement. Peut-être y avait-il quelques centaines de personnes pour les deux tranches?

$\mathbf{Q}$ : Lors de l'entraînement à l'évacuation de fin février, environ combien de personnes, au total, avaient-elles participé ?

$\mathbf{R}$ : À la base, c'est le personnel de chez nous qui se trouve sur place au moment de l'entraînement, donc cela doit faire huit cents personnes. Évidemment, les équipes de quart ne sont pas toutes présentes, certaines sont au repos. Donc, environ huit cents personnes participent. Les huit cents personnes font le volet « évacuation ». Ensuite, seuls ceux qui ont été désignés à l'avance comme faisant partie des équipes de soutien se réunissent dans le bâtiment antisismique.

$\mathbf{Q}:$ : l y a donc des gens pour qui l'entraînement se termine avec l'évacuation.

R : Oui. 
$\mathbf{Q}$ : Ensuite, les personnes désignées comme équipe de soutien continuent l'entraînement avec le volet « réaction à l'accident », c'est ça?

R : Oui.

$\mathbf{Q}$ : Pour ce qui est des relations avec le département, il y a des gens du département qui participent à l'entraînement à la préfecture?

$\mathbf{R}$ : Ils sont au centre hors site.

$\mathbf{Q}:$ Vous l'aviez effectivement fait, fin février?

R : Oui.

$\mathbf{Q}$ : Vous voulez dire que, par exemple, M. XXXXX, ou quelqu'un d'autre, était effectivement au centre hors site?

$\mathbf{R}$ : Oui, il y était.

$\mathbf{Q}$ : Ce qui veut dire que vous vous étiez entraînés à prendre contact avec eux, etc. ?

R : Oui, nous l'avons fait.

$\mathbf{Q}:$ La NISA avait aussi participé ?

R : Oui, la NISA était là aussi.

$\mathbf{Q}$ : C'est le chef du bureau de la NISA à la centrale, ou quelqu'un d'équivalent, qui était parti au centre hors site?

R : Oui.

$\mathbf{Q}:$ : y avait aussi des gens de la NISA au bâtiment antisismique?

$\mathbf{R}$ : Oui, ils y étaient aussi.

$\mathbf{Q}$ : Accordez-moi encore cinq minutes.

Quand on regarde l'organisation de la centrale de Fukushima Daiichi, tout a l'air d'avoir été conçu par paire, réacteurs 1 et 2,3 et 4, puis 5 et 6 . J'imagine qu'il y a des avantages à ça, comme de pouvoir s'échanger l'alimentation électrique, chose que nous avons déjà évoquée. Mais s'il existe une solution commune pour un problème et que cette solution n'est plus disponible, c'est fichu à la fois pour les deux réacteurs, que ce soient les 1 et 2, 3 et 4 ou 5 et 6 . Quelle était la philosophie qui sous-tendait cette conception particulière par paire? Cela paraissait la meilleure solution?

$\mathbf{R}$ : Je ne sais pas comment ils ont décidé des choses il y a quarante ans. La conception d'origine, c'est une histoire que je ne connais pas. Mais si je résume, puisqu'il n'y a qu'une salle de contrôle pour deux réacteurs, du point de vue de la gestion, il faut moins de monde que si chaque tranche était indépendante.

De plus, si on peut disposer d'équipements en commun, on peut dire que c'est une conception rationnelle.

Dès le début, il était prévu que TEPCO construise un assez grand nombre de centrales nucléaires. Je pense donc qu'ils ont voulu les construire à la chaîne.

$\mathbf{Q}$ : Comment est organisé Kashiwazaki ?

$\mathbf{R}$ : Les tranches 6 et 7 de Kashiwazaki sont comme ici. Mais les autres tranches sont indépendantes. C'est parce que la roche est très profonde, là-bas. Quand on creusait, il fallait aller tellement profond qu'il était très difficile de creuser tout à côté pour construire. Les conditions géologiques ont fait qu'il fallait laisser une certaine distance entre chaque tranche.

Sur la centrale de Fukushima Daiichi, on a construit six tranches en huit ans entre 1971 et 1979. Cela fait quasiment une tranche par an. C'est un rythme très rapide. Ce 
qui veut dire qu'on creusait et construisait quasi en même temps. Par contre à Kashiwazaki, il y a eu du temps entre deux tranches. Il fallait creuser profond et chaque tranche a été construite séparément. On a tendance à considérer Fukushima comme l'exemple d'une conception rationnelle.

Je ne sais pas trop quelle est la norme dans tout le Japon, mais il me semble qu'il y a plus de cas de tranches séparées avec chacune une salle de contrôle. La norme doit être de construire une tranche et de prévoir une salle de commande pour chacune.

$\mathbf{Q}$ : Même dans le cas d'une tranche indépendante avec sa salle de contrôle en propre, faiton en sorte que deux tranches ne soient pas trop éloignées l'une de l'autre pour qu'elles puissent s'échanger l'alimentation électrique, par exemple?

$\mathbf{R}$ : Oui. Comme je vous l'ai dit tout à l'heure, il était prévu qu'un maximum d'équipements soit utilisé en commun par les deux tranches. Je vous ai expliqué que, sur les plans d'origine, il y avait un des générateurs diesel en commun pour les deux tranches. C'était tout à fait le reflet de la philosophie de l'époque, l'idée que tout ce qui pouvait être utilisé en commun devait l'être de manière rationnelle.

$\mathbf{Q}$ : Lors de l'accident de mars, il me semble qu'on a pu vérifier ce côté rationnel, mais il y a eu aussi des épisodes où la localisation très proche des différents réacteurs a rendu les opérations très compliquées. Une chose qui me vient tout de suite à l'esprit, c'est le cas particulier de la tranche 2 . En sandwich entre les tranches 1 et 3 , après l'explosion de la tranche 1, il a fallu reprendre à zéro les travaux de la tranche 2, même chose après l'explosion de la tranche 3. N'y a-t-il pas des inconvénients de ce genre, «être vulnérable aux incidents qui surviennent chez les voisines »?

$\mathbf{R}: \mathrm{Si}$.

$\mathbf{Q}$ : Voyez-vous d'autres avantages ou inconvénients à cette manière de construire les tranches?

$\mathbf{R}$ : Si je commence par les avantages, je pense qu'il y a le moindre coût de développement.

Si je passe aux inconvénients, il y en a plus. Si on regarde les autres centrales nucléaires, il y a tout au plus quatre tranches sur un site. Depuis toujours, j'ai détesté les constructions concentrées. Mais mon entreprise pratiquait la concentration depuis longtemps. En fait, du point de vue d'un directeur de centrale, un site comme Fukushima Daini, où il y a tout juste quatre tranches, est l'idéal du point de vue gestion. C'est un champ suffisamment restreint qu'un directeur peut maîtriser. Quand on en arrive à six ou sept tranches, regardez ce qui est aussi arrivé à Kashiwazaki, la confusion est totale. En plus, boum, d'un coup toute la production électrique s'arrête. Qu'on ne puisse plus alimenter le réseau de cette façon est dramatique pour un exploitant. Regardez ce qui s'est passé en mars, non seulement il y avait les problèmes à Fukushima Daiichi, mais même Fukushima Daini ne pouvait plus fournir. J'ai toujours pensé que c'était loin d'être l'idéal du point de vue de la répartition des risques. Mais quand je suis arrivé chez TEPCO, la construction de Kashiwazaki était déjà lancée, donc il a bien fallu faire avec. Quand on commence à parler de ce problème, beaucoup sont très critiques, mais j'ai un peu envie de leur dire d'adresser leurs reproches à ceux qui ont conçu la centrale il y a quarante ans.

$\mathbf{Q}$ : Si on remonte un peu l'histoire de la centrale, pour la tranche 1, vous avez installé un réacteur GE, puis petit à petit, vous êtes passés aux modèles des constructeurs japonais. Y 
avait-il une raison à ça ? Y avait-il des avantages, comme une meilleure maintenance, pour passer aux modèles japonais ?

$\mathbf{R}$ : C'était le premier réacteur nucléaire de tout le Japon ${ }^{92}$. On a donc commencé par introduire la technologie étrangère. Comme le fait la Chine aujourd'hui. Le premier réacteur était un cas modèle pour pouvoir produire ensuite des installations similaires par nos propres moyens. On a donc construit la tranche 1 et la tranche 2 avec un réacteur GE. Dans le cas du réacteur 2, Tôshiba et Hitachi avaient été associés pour études. Après le développement des tranches 1 et 2, la tranche 3 a été construite entièrement par Tôshiba et la 4 , là encore entièrement, par Hitachi. Dans un certain sens, ce site de Fukushima Daiichi était un véritable terrain d'entraînement.

$\mathbf{Q}$ : Concrètement, y a-t-il des différences dans l'utilisation de ces quatre tranches ? Y a-t-il eu des réponses différentes lors de l'accident de mars, selon les modèles ?

$\mathbf{R}:$ Non.

$\mathbf{Q}:$ Rien de spécial ?

$\mathbf{R}$ : Les réacteurs $2,3,4$ et 5 sont les mêmes à la base.

$\mathbf{Q}$ : Vous voulez dire que ce soit un réacteur GE ou un autre, à la base, c'est la même chose ?

$\mathbf{R}$ : Oui, puisque ce sont des copies du réacteur GE, la manière dont ils ont été conçus est tout à fait similaire.

$\mathbf{Q}:$ Le réacteur 1 est le seul différent?

R : Il n'a pas la même capacité.

$\mathbf{Q}$ : Je suppose que, ne disposant pas des mêmes fonctions, les réponses ont été différentes par rapport aux réacteurs 2, 3 ou 4, mais la manière de concevoir le refroidissement, par exemple, ne diffère pas tellement d'un réacteur à l'autre.

$\mathbf{R}$ : Non. Le RHR, dont nous avons parlé tout à l'heure, qui est un système tout à fait caractéristique des réacteurs à eau bouillante, n'existe qu'à partir du réacteur 2 . Je pense qu'il continue à être prévu sur les réacteurs 6 et 7 de Kashiwazaki. Comme le réacteur 1 , chez nous, ne dispose pas de ce système RHR, il utilise la pression. Il a un système d'évacuation de la chaleur résiduelle utilisant la pression. C'est juste là la différence avec les autres réacteurs.

$\mathbf{Q}$ : Concernant justement ce réacteur 1 , comment se situe-t-il au Japon ? Il est assez ancien.

$\mathbf{R}$ : C'est le plus vieux. C'est le plus ancien modèle de GE.

$\mathbf{Q}$ : Même du point de vue mondial, il est vieux?

R: Oui. GE a d'abord uniquement fabriqué pour les États-Unis. Le BWR-3 ${ }^{93}$ est le premier modèle qu'il a proposé à l'étranger.

Il y a une centrale, en Espagne, qui a exactement le même réacteur. C'est la centrale de Santa Maria de Garona ${ }^{94}$. Elle a été construite sur le même plan au même moment.

Si on cherche aux États-Unis, il me semble que Browns Ferry-1 ${ }^{95}$ et Nine Mile Point- $1^{96}$ sont aussi sur le même plan que notre réacteur 1 . Peut-être avec quelques différences, mais ils ont été construits à la même époque.

$\mathbf{Q}$ : Des réacteurs frères, donc. Ces réacteurs fonctionnent toujours ?

$\mathbf{R}$ : Oui, ils fonctionnent toujours. 
$\mathbf{Q}$ : Je crains que nous ne nous fassions chasser de la salle. Nous allons finir pour aujourd'hui.

$\mathbf{R}:$ D’accord.

$\mathbf{Q}$ : Demain, je vous demanderai de regarder des passages de DVD et je vous poserai des questions plus approfondies concernant la période du 11 au 14 mars, si vous le voulez bien.

$\mathbf{R}:$ Très bien.

$\mathbf{Q}$ : Eh bien, nous en avons terminé.

(Fin de l'audition)

\section{NOTES}

1. Département de la Police Métropolitaine de Tôkyô (Keishichô en japonais). Créé en janvier 1874, le MPD est dirigé par un surintendant-général, nommé par la Commission Nationale de la Sécurité Publique, avec l'approbation de la Commission Métropolitaine de Sécurité Publique et l'approbation du Premier ministre.

2. Certains noms propres et numéros de téléphone ont été remplacés par des XXXXX.

3. Entreprise japonaise fondée en 1875 , dont le siège est situé à Tôkyô. Ses activités couvrent le secteur de l'électronique, de la téléphonie, de l'informatique, de l'audiovisuel et du nucléaire. Tôshiba est le constructeur des réacteurs 3 et 5 de Fukushima Daiichi, et le co-constructeur avec GE du réacteur 6.

4. Le siège de TEPCO demande au Gouvernement de faire intervenir les forces d'autodéfense. Le 16 mars après-midi, Toshimi Kitazawa, le ministre de la Défense, transmet ses instructions au général Ryôchi Oriki, son chef d'état-major, pour envoyer des hélicoptères et larguer de l'eau sur les installations. Les hélicoptères utilisés sont des $\mathrm{CH}-47$ Chinook, conçus par Boeing et munis chacun d'un double rotor (Kadota, 2014). Les largages d'eau commencent le matin du 17 mars.

5. Le bassin DS correspond à la piscine du sécheur-séparateur. Le sécheur et le séparateur se situent au-dessus du cœur et filtrent la vapeur qui s'en dégage avant qu'elle ne soit dirigée vers la turbine. Étant donné que ces dispositifs sont hautement contaminés par la radioactivité, ils doivent être stockés sous un important volume d'eau pendant les opérations qui nécessitent d'accéder au reste de la cuve. Pour cette raison, il existe une piscine située à côté du puits de cuve du réacteur, destinée à l'entreposage des composants internes de la cuve du réacteur, et souvent désignée comme la piscine du dryer separator (cf. la «piscine d'entreposage du dispositif de séchage et de séparation de vapeur » de la Fig. 5). Lors du fonctionnement du réacteur, cette piscine est séchée et couverte de briques en béton.

6. La centrale nucléaire de Fukushima Daini se trouve à une douzaine de kilomètres au sud de Fukushima Daiichi. 
7. Pour rappel, il s'agit de la cellule conjointe de gestion de crise entre le Gouvernement et TEPCO, créée le 15 mars et située au siège de l'exploitant.

8. La hauteur manométrique totale désigne la pression totale qu'une pompe doit fournir pour acheminer un liquide d'un point $\mathrm{A}$ à un point $\mathrm{B}$. Elle prend en compte la différence de hauteur entre les deux points, la structure des canalisations entre eux, ainsi que la pression souhaitée à la sortie du point B. Yoshida indique ainsi dans sa réponse que les canons n'arrivent pas à fournir assez de pression pour que l'eau, puisée dans le réservoir de lutte contre les incendies, atteigne la piscine de la tranche 3.

9. Fleuve Kuma-gawa, d'une longueur de 239 kilomètres, qui traverse les préfectures de Fukushima et de Miyagi.

10. À l'extérieur.

11. Pompes à béton embarquées.

12. Robots «Arounder » (construits par Hitachi).

13. La Hazama Corporation est une entreprise japonaise de construction créée en 1889, dont le siège est situé à Tôkyô. C'est l'une des dix plus grandes sociétés de construction du Japon.

14. Le barrage de Sakashita est construit en 1973 dans la préfecture de Fukushima. Il est utilisé pour la production hydroélectrique et l'alimentation en eau de la région. D'une hauteur de 43 mètres et d'une longueur de crête de 231 mètres, son volume de retenue est de $2840000 \mathrm{~m}^{3}$.

15. Perte des alimentations électriques de la centrale nucléaire.

16. Environ 650 personnes sont évacuées le matin du 15 mars en raison de l'aggravation de la situation. Seuls les « Fukushima 50 » restent sur le site. Les travailleurs évacués se rendent temporairement en bus ou en voitures à Fukushima Daini. Ils commencent à revenir à Daiichi à partir de midi le même jour (AIEA, 2015a).

17. Yoishi Funabashi (organisateur du comité civil et indépendant sur l'accident de Fukushima Daiichi), s'appuyant sur les travaux menés par Hando, un historien japonais, compare la gestion de crise nucléaire et l'armée japonaise durant la Seconde Guerre mondiale. Dans les deux cas, la rotation des soldats comme des travailleurs est restée insuffisante: «On a poussé les gens qui étaient sur les sites à combattre jusqu'à mort. Il est impossible d'imaginer que seuls cinquante personnes y restent sans remplacement pour la gestion de la crise alors que six réacteurs ont des problèmes » (Kobayashi, 2019, p. 119).

18. La situation est toutefois très différente entre Fukushima Daiichi et Daini. Si le tsunami a également submergé les installations de Daini, une ligne électrique et trois générateurs diesel ont continué de fournir du courant, permettant le contrôle et le refroidissement des quatre réacteurs de la centrale nucléaire - ils sont arrêtés à froid le 15 mars.

19. Il s'agit de la Nanmei Kôsan, déjà évoquée dans l'audition du 22 juillet 2011.

20. Entreprise japonaise fondée en 1910, dont le siège est situé à Chiyoda-ku (Tôkyô). Elle fabrique des équipements industriels, des machines de construction, de l'équipement médical, des matériaux et composants hautement fonctionnels, et des produits électroniques. Elle fournit également des produits et des services dans le domaine de l'informatique, des télécommunications, des transports et de la mobilité. Hitachi a supervisé la construction du réacteur 4 de Fukushima Daiichi. 
21. Yoshida évoque respectivement le réacteur 1 (460 MWe), les réacteurs 2 à 5 (784 MWe chacun), et le réacteur 6 (qui produit $1100 \mathrm{MWe}$ ).

22. Pour rappel, Kan estime que TEPCO ne lui transmet pas de données précises sur l'évolution de la situation à Fukushima Daiichi. Il se rend ainsi au siège pour obtenir davantage d'informations et améliorer la communication entre l'exploitant et le Gouvernement (cf. audition du 29 juillet 2011).

23. La NISA, censée jouer un des rôles principaux dans la gestion de crise nucléaire, a été vivement critiquée par la suite. La NAIIC (2012) estime ainsi que l'agence était insuffisamment préparée à un accident de cette ampleur et qu'elle a échoué dans sa fonction.

24. Pour rappel, centre de crise situé au niveau local ouvert dans l'après-midi du 11 mars 2011.

25. À cause de l'augmentation de la radioactivité, le centre de crise hors site, d'abord situé à ôkuma, est évacué le 15 mars 2011. Il est transféré dans la salle publique de la préfecture de Fukushima. La mise en commun des informations en temps réel entre les autorités concernées est difficile car le nouveau centre ne dispose pas des mêmes moyens que celui d'ôkuma.

26. Système de contrôle des cheminées. Il s'agit d'une station d'observation qui permet de mesurer la direction et la vitesse du vent, les précipitations, etc. En situation d'urgence, ces mesures permettent de déterminer le meilleur moment de réaliser un éventage, en fonction de l'impact potentiel des rejets radioactifs.

27. Réseau de détecteurs et de calculateurs développé au Japon dans le but de prédire en temps réel les conséquences potentielles d'une urgence nucléaire sur l'environnement.

28. Tôkyô Records Management Corporation est une filiale de TEPCO, spécialisée dans la production, le stockage et la gestion de documents d'archives.

29. Tenue de protection pour les agents. Le Tyvek est une marque déposée de l'entreprise américaine du Pont de Nemours.

30. Le rapport de la NAIIC (2012) pointe la pénurie de dosimètres causée par les dégâts du tsunami. Il estime que $5 \%$ des travailleurs - à la fois ceux de TEPCO et des entreprises partenaires - n'ont pas du tout eu accès à un dosimètre.

31. La centrale nucléaire de Kashiwazaki-Kariwa se trouve à environ 330 kilomètres de Fukushima Daiichi.

32. Des vingt-cinq centrales thermiques exploitées par TEPCO, les plus proches de Daiichi sont les centrales de Hirono (préfecture de Fukushima, environ 30 kilomètres), de Hitachinaka (préfecture d'Ibaraki, 130 kilomètres), de Higashi-Ogishima (préfecture de Kanagawa, 140 kilomètres) et de Kashima (préfecture d'Ibaraki, 206 kilomètres).

33. Pour rappel, il s'agit du bureau de TEPCO situé dans la ville de Fukushima, au siège préfectoral de réponse à la catastrophe. Cette cellule de soutien est déjà mentionnée dans l'audition du 29 juillet 2011.

34. Ce centre hors site connaît des dysfonctionnements dès le début de la crise. Selon Naoto Kan, "j'y ai tout de suite envoyé le vice-ministre de l'Industrie. Mais à cause des embouteillages et de la situation sur place, il n'est arrivé que très tard dans la nuit du 11 mars. En plus, le centre était coupé d'électricité, et les fonctionnaires des collectivités locales qui auraient dû s'y rassembler étaient débordés par la gestion post-séisme» (Kan, 2015). En effet, 
le vice-ministre Ikeda, nommé directeur de la cellule du Gouvernement située dans le centre de crise hors site d'ôkuma, se retrouve dans l'obscurité en arrivant dans les lieux, car les générateurs diesel sont tombés en panne. Ils ne sont réparés que le lendemain, le 12 mars (Kadota, 2014).

35. Procédures d'exploitation en cas de situation anormale.

36. Procédures d'exploitation en cas d'urgence.

37. Guide pour la gestion des accidents.

38. Rayonnement ionisant composé de photons de haute énergie. Bien qu'il soit moins ionisant, le rayonnement gamma $(\gamma)$ pénètre davantage dans l'organisme que les rayonnements alpha $(\alpha)$ et bêta $(\beta)$. Il est donc très dangereux et peut endommager la moelle osseuse et les organes internes.

39. Le système de contrôle de l'atmosphère dans le confinement (CAMS) est un système qui permet de déterminer la composition du gaz (et notamment la concentration d'hydrogène et d'oxygène), ainsi que de mesurer le rayonnement gamma dans l'enceinte de confinement (wetwell et drywell). À Fukushima Daiichi, les dispositifs de mesure d'oxygène et d'hydrogène (qui sont particulièrement énergivores) n'ont pas pu être utilisés après la perte des ressources électriques.

40. Pour rappel, il s'agit de l'accident survenu à Fukushima Daiichi en octobre 1991, lié à une fuite d'eau de mer. L'évènement est précédemment mentionné dans l'audition du 22 juillet 2011.

41. Série de pompes et de diffuseurs permettant d'arroser l'enceinte de confinement afin de faire baisser sa pression.

42. Les Power Centers sont des jeux de barres, c'est-à-dire des installations qui permettent de conduire l'électricité depuis une ligne d'alimentation, vers un ou plusieurs circuit(s) électrique(s).

43. Appareillages blindés de commutation électrique. Il s'agit d'appareillages électriques (interrupteurs, fusibles, branchements, transformateurs de mesure, câblage de commande, etc.) installés en intérieur, dans des enveloppes métalliques séparées et adéquatement mises à la terre. Ils ont pour objectif de préserver les composants électriques d'agressions externes (éclairs, saleté, animaux...), ainsi que de protéger chaque armoire de l'autre, notamment en cas de défaillance ou d'incendie. Dans les centrales nucléaires, chaque système est opéré grâce à un switch gear, qui peut inclure une ou plusieurs armoires métalliques.

44. Procédures d'exploitation dans le cadre d'un accident sévère.

45. Le BTC correspond certainement à l'entreprise BWR Operator Training Center corporation, spécialisée dans la formation et la certification du personnel intervenant dans les centrales nucléaires au Japon. Co-détenue par TEPCO, cette entreprise propose des entraînements aux exploitants de REB, notamment grâce à des simulateurs qui reproduisent des salles de commandes de réacteurs japonais.

46. Réacteur à Eau Bouillante (REB).

47. En France, un élève passe cinq années dans l'enseignement primaire, quatre années au collège et trois années au lycée. Au Japon, un élève passe six années dans l'enseignement primaire, trois années au collège et trois années au lycée. Le secondaire se termine par le test du Centre National des Admissions à l'Université. Il est composé de vingt-neuf tests organisés sur deux jours. Ce test est utilisé par les universités 
nationales et par certaines universités privées comme critère d'admissibilité. La plupart des universités privées utilisent cependant un test organisé par l'institution où candidate l'étudiant. Les juku, établissements d'enseignement privé, préparent le lycéen à passer ce type de test.

48. Arrêt des pompes de recirculation. Lors du fonctionnement normal des REB, les pompes de recirculation permettent de contrôler la puissance du réacteur à travers le débit d'eau à l'intérieur de la cuve. En effet, à l'arrêt des pompes de recirculation, des bulles d'eau se forment dans le cœur du réacteur et affectent la densité du modérateur. Ainsi, l'arrêt de ces pompes suffit pour baisser la puissance du réacteur jusqu'à $70 \%$ de sa valeur nominale.

49. Injection alternative des barres. Le mécanisme d'insertion des barres de contrôle des réacteurs de Fukushima Daiichi est à commande hydraulique. L'injection ou le pompage d'eau exerce une pression sur le mécanisme qui permet d'ajuster la position des barres de contrôle dans le cœur du réacteur. En cas d'urgence, c'est grâce à un accumulateur à azote que l'insertion est effectuée. Si ce dernier dysfonctionne, le mécanisme d'injection alternative des barres permet leur insertion grâce à de l'eau pressurisée qui provient directement de la cuve du réacteur. Ce mécanisme n'est efficace que si la pression dans la cuve est suffisamment élevée.

50. Système d'appoint en eau à partir d'un réservoir de condensation. En général, cette eau provient initialement du réacteur et est filtrée.

51. Système d'aspersion du cœur.

52. Amas de combustibles et d'éléments de structure du cœur d'un réacteur nucléaire fondus et mélangés, pouvant se former en cas d'accident grave.

53. Le drywell contient la cuve du réacteur et l'essentiel des conduits de réfrigération. Bien qu'ils soient isolés thermiquement, ces composants peuvent transmettre de la chaleur au drywell. Le refroidisseur du drywell est un système dont la fonction est d'éviter que l'air dans l'enceinte de confinement ne chauffe trop. Il consiste en de grands ventilateurs qui poussent l'air vers des échangeurs de chaleur refroidis à l'eau.

54. Système de nettoyage de l'eau. Dans un REB en fonctionnement, l'eau est introduite directement dans la cuve du réacteur dans un état liquide, puis elle s'évapore vers la turbine. Les impuretés peuvent alors s'accumuler dans la cuve du réacteur. Le système CUW permet d'extraire l'eau de la cuve, de la filtrer et de la réinjecter ailleurs, le plus souvent directement dans la cuve. Plusieurs manuels de gestion des accidents prévoient l'utilisation de ce système pour injecter de l'eau et refroidir la cuve en dernier recours.

55. Le sprinkler est un arroseur, utilisé notamment en cas d'incendie. Le bâtiment réacteur contient des sprinklers alimentés par des pompes, afin de permettre d'éteindre des incendies et limiter les dégâts. Le réseau de sprinklers n'atteint cependant pas l'enceinte de confinement du réacteur, qui est généralement inerte et contient peu de matériels inflammables.

56. Dans une centrale nucléaire, plus une installation est primordiale pour la sûreté, plus elle est conçue pour résister aux différents chocs, y compris les tremblements de terre. Au Japon, les installations sont classées « $\mathrm{S}$ ", «B " ou " $\mathrm{C}$ », dans un ordre décroissant, selon leur robustesse. Les installations de classe $S$ contiennent ou sont directement reliées à des installations contenant des matières radioactives, dont la détérioration occasionnerait une pollution radioactive importante. La classe $\mathrm{S}$ concerne également les installations qui peuvent éviter la diffusion de la radioactivité dans 
l'atmosphère ou en atténuer la portée. Les installations de classe B ont les mêmes fonctions, mais elles contiennent des volumes peu significatifs de matières radioactives, ou sont des installations dont la perte n'occasionnerait pas une pollution radioactive importante. Les installations de classe $\mathrm{C}$ sont celles qui ne sont pas classées $\mathrm{S}$ ou B. Ainsi, la cuve du réacteur, le système de SCRAM et les systèmes de refroidissement d'urgence du cœur sont, en théorie, les systèmes qui résistent le mieux aux séismes. Le réservoir d'eau filtrée, n'étant pas considéré comme primordial pour la sûreté nucléaire, est classé $C$. Il est donc théoriquement moins résistant aux séismes.

57. Chambre de suppression, ou wetwell.

58. Système d'eau de refroidissement du bâtiment réacteur. Il s'agit de conduits qui fournissent de l'eau pour le refroidissement d'équipements auxiliaires du bâtiment réacteur en temps normal, ainsi que pour les systèmes importants pour la sûreté en cas de besoin.

59. Système de contrôle atmosphérique. Lors du fonctionnement du REB, l'enceinte de confinement est remplie d'azote, notamment pour éviter les combustions d'hydrogène. Il est cependant possible de remplir l'enceinte d'air normal, notamment en cas de nécessité d'interruption du REB et d'introduction de personnel. Le système de contrôle atmosphérique permet de remplir l'enceinte de confinement du gaz souhaité et de le maintenir à la composition désirée. Il permet également de filtrer le gaz évacué lors des interruptions planifiées. À Fukushima Daiichi, un conduit reliant le système de contrôle atmosphérique à la cheminée (via le SGTS) a été conçu pour offrir un circuit d'éventage renforcé. Toutefois, le système AC n'est pas disponible en cas d'accident et le conduit d'éventage n'a donc pas pu être utilisé.

60. Système auxiliaire de traitement de gaz, filtrant et déchargeant l'air de l'enceinte de confinement vers l'atmosphère. Il permet également de maintenir une pression basse dans l'enceinte de confinement afin de limiter les rejets radioactifs.

61. Ces mesures en «kilos" correspondent probablement à une mesure en millibars (ou en hectopascals), unités utilisées pour exprimer la pression atmosphérique. On a donc $« 8,5$ kilos » $=8,5$ bars $=0,8 \mathrm{MPa}$.

62. Ici, la mesure est en $\mathrm{kPa}$.

63. En 1991, Yoshida est au siège de TEPCO, où il occupe le poste de sous-chef dans le service "maintenance des centrales nucléaires». Il ne retourne à Fukushima Daiichi qu'en 1993, où il avait déjà travaillé de 1982 à 1986.

64. Système de dépressurisation automatique du réacteur.

65. En cas de problème significatif sur un REB, ce dernier possède un système de protection qui déclenche une réponse automatisée pour protéger l'intégrité du réacteur : celle-ci consiste dans l'initiation du SCRAM et la dépressurisation de la cuve du réacteur (via les vannes SRV).

66. Système de refroidissement de secours permettant l'injection d'eau à basse pression en cas d'incident. Il s'agit d'un mode du système d'évacuation de la chaleur résiduelle RHR.

67. Système d'arrosage du drywell, similaire au système d'arrosage du wetwell (cf. le Containment Spray Mode). Il s'agit cependant d'un système moins important pour la sûreté du réacteur, puisque sa fonction est uniquement de normaliser rapidement la situation dans le réacteur après son arrêt d'urgence.

68. Mode d'aspersion de la chambre de condensation, ou wetwell. 
69. Il s'agit du Containment Head spray, évoqué plus haut.

70. Il s'agit du système d'arrosage de la chambre sèche, ou drywell.

71. Le tore correspond à la chambre de condensation, ou wetwell dans les REB qui possèdent une enceinte de confinement de type Mark I (tranches 1 à 5 de Fukushima Daiichi).

72. Également mentionnée dans les auditions par le sigle «SRV » (Safety Relief Valves).

73. En mars 2011, le directeur de la NISA est Nobuaki Terasaka. Licencié le 12 août 2011 (soit quatre jours après cette audition de Yoshida), il est remplacé par Hiroyuki Fukano.

74. En mars 2011, Shimizu est en fait le Président de TEPCO et son Directeur général est Tsunehisa Katsumata.

75. Le 17 avril 2011, des robots téléguidés sont envoyés dans les bâtiments des réacteurs 1 et 3 pour y mesurer le niveau de rayonnement. Équipés de caméras, ils ont pu prendre des images montrant pour la première fois depuis le 11 mars l'intérieur des installations.

76. La région du Kantô, située sur l'île de Honshû au sud du Tôhoku, regroupe les préfectures de Chiba, Gunma, Ibaraki, Kanagawa, Saitama, Tochigi et Tôkyô.

77. Le 13 juillet 869, durant l'ère Jôgan (859-877), un séisme dévastateur se produit au large de l'actuelle préfecture d'Iwate. Il provoque un tsunami détruisant la ville de Tagajo (dans l'actuelle préfecture de Miyagi). La catastrophe fait un millier de morts.

78. Société japonaise des ingénieurs civils, fondée en 1914. Elle est chargée de contribuer à l'avancement de la culture scientifique en promouvant le domaine du génie civil et l'expansion de ses activités. Au fil des années, les membres de la JSCE ont considérablement augmenté, passant des 443 membres initiaux à environ 39000 membres actuellement.

79. La Tôhoku Electric Power Company est une compagnie d'électricité japonaise, dont le siège social se situe à Sendai (préfecture de Miyagi). Créée en 1951, elle exploite deux-cents-neuf centrales hydroélectriques, douze centrales thermiques, quatre centrales géothermiques, quatre centrales solaires, et deux centrales nucléaires (centrale d'Onagawa mise en service en 1984, et centrale d'Higashidori mise en service en 2005).

80. La Japan Atomic Power Company (JAPC) est une compagnie d'électricité japonaise, dont le siège social se situe à Chiyoda (arrondissement de Tôkyô). Créée en 1957, elle exploite deux centrales nucléaires : la centrale de Tôkai (préfecture d'Ibaraki) depuis 1966, et la centrale de Tsuruga (préfecture de Fukui) depuis 1970.

81. Située au nord-est de l'île de Honshû, la côte Sanriku traverse les préfectures de Miyagi, d'Iwate et d'Aomari.

82. Préfecture située dans la région du Tôhoku. Sa capitale est Sendai.

83. La centrale nucléaire d'Onagawa (préfecture de Miyagi, région du Tôhoku), exploitée par la Tôhoku Electric Power Company, est composée de trois réacteurs à eau bouillante, mis en service entre 1984 et 2002. Il s'agit de la centrale la plus proche (80 kilomètres) de l'épicentre du séisme du 11 mars 2011. Le site est submergé par des vagues de 13 mètres de hauteur, mais les principaux bâtiments, construits à 14,8 mètres au-dessus de la mer, ne sont pas inondés. Si le tsunami endommage malgré tout de nombreux équipements, une ligne électrique et six générateurs diesel continuent de 
fonctionner. Les opérateurs ont donc pu garder le contrôle des trois réacteurs et les arrêter à froid.

84. La Kyushu Electric Power est une compagnie d'électricité japonaise, dont le siège se situe à Fukuoka (préfecture de Fukuoka). Créée en 1951, elle exploite de nombreuses centrales de production électrique, dont la centrale nucléaire de Genkai (préfecture de Saga, située sur l'île de Kyûshû). Les quatre réacteurs à eau pressurisée de Genkai ont été mis en service entre 1975 et 1997.

85. Yoshida se réfère ici aux conséquences du séisme de Valdivia. Le 22 mai 1960, un séisme de magnitude 9,5 - la plus élevée à avoir été enregistrée - se produit au Chili. Son épicentre se situe dans le sud du pays, près de la ville de Valdivia, à environ 700 kilomètres de Santiago du Chili. Selon l'Institut d'Études Géologiques des États-Unis (United States Geological Survey, USGS), le tremblement de terre cause la mort d'environ 1655 personnes, et le tsunami qu'il provoque fait 61 victimes à Hawaï, 138 au Japon et 32 aux Philippines (cf. https://earthquake.usgs.gov/earthquakes/eventpage/ official19600522191120_30/executive).

86. The Geospatial Information Authority of Japan (GSI) est une organisation nationale chargée de l'étude et de la cartographie du territoire japonais. Elle remonte historiquement à 1869. Elle est réorganisée à plusieurs reprises et obtient son nom actuel en avril 2010. La GSI dépend du ministère du Territoire, des Infrastructures, des Transports et du Tourisme. Son siège est situé à Tsukuba (préfecture d'Ibaraki).

87. Préfecture située dans la région du Kantô. Sa capitale est Mito.

88. Préfecture située dans la région du Tôhoku. Sa capitale est Morioka.

89. La catastrophe fait 9781 morts dans la préfecture de Miyagi et 4150 morts dans la préfecture d'Iwate, nombres rendus publics le 11 novembre 2011 (Nakahara et Ichikawa, 2013).

90. La préfecture de Miyagi se trouve au nord-est de la préfecture de Fukushima. L'enquêteur et Yoshida évoquent un séisme de magnitude 7, survenu le 8 mars 2011.

91. Yoshida fait référence à un séisme de magnitude 6 qui a frappé la côte de l'île de Honshû le 10 octobre 2006.

92. Imprécision de Yoshida. Le premier réacteur nucléaire du Japon est construit dans la centrale de Tôkai (préfecture d'Ibaraki, à 150 kilomètres au nord-est de Tôkyô), exploitée par la Japan Atomic Power Company (JAPC). Ce réacteur, de type Magnox, est de conception britannique et mis en service en juillet 1966. Le réacteur 1 de Tsuruga, mis en service en mars 1970, est quant à lui le premier REB exploité au Japon. Il a été construit par GE. Le réacteur 1 de Fukushima Daiichi, REB également construit par GE, n'a été mis en service qu'en mars 1971.

93. Le VBWR, réacteur à eau bouillante expérimental conçu par GE en 1955, est mis en service dans la centrale de Vallecitos (États-Unis, Californie) en octobre 1957. Il est l'ancêtre du BWR-1, dont le premier est mis en service à Dresden Generating Station (Illinois) en juillet 1960. Le modèle BWR-2 est mis en service pour la première fois à Oyster Creek Nuclear Generating Station (New Jersey) et à Nine Mile Point Nuclear Generating Station (État de New York) en décembre 1969. Le BWR-3 est quant à lui mis en service pour la première fois à Dresden Generating Station en juin 1970.

94. La centrale nucléaire de Santa Maria de Garona, située dans la province de Burgos (Espagne, Castille-et-Leon), est mise en service en 1971. Elle se compose d'un seul 
réacteur à eau bouillante, de conception GE comme le mentionne Yoshida. Ce réacteur est arrêté en décembre 2012.

95. La centrale nucléaire de Browns Ferry (États-Unis, Alabama), est exploitée par l'entreprise américaine Tenessee Valley Authority. Elle se compose de trois REB, construits par GE. Le réacteur Browns Ferry-1 est mis en service en 1973, Browns Ferry-2 en 1974 et Browns Ferry-3 en 1976. La centrale est connue pour avoir subi un grave incendie le 22 mars 1975.

96. En 2011, la centrale nucléaire de Nine Mile Point (États-Unis, État de New York), est exploitée par l'entreprise américaine Constellation Energy. Elle partage le site qu'elle occupe avec la centrale nucléaire de Fitzpatrick, alors exploitée par Entergy. Ces deux centrales sont désormais exploitées par Exelon(Nine Mile Point depuis 2012 et Fitzpatrick depuis 2017). Nine Mile Point se compose de deux REB, construits par GE. Le réacteur 1 est mis en service en 1969 et le 2 en 1987. Le réacteur 1 est le plus ancien qui soit encore en service aux États-Unis. 


\section{Compte rendu de l'audition du 9 août $2011(\mathrm{I})^{1}$}

QUESTION : Je voudrais que nous regardions ensemble le contenu des téléconférences. II s'agit du 12 mars à $22 \mathrm{~h} 50 . .$.

\section{Réponse : Le 12 mars à 22 h00 ? Ça doit concerner le réacteur 1.}

$\mathbf{Q}$ : Nous avons fait un montage de la vidéo. Ça commence à partir de 22h59. II y a des moments où le son a été enregistré et d'autres, non. J'ai choisi des passages qui concernaient plus particulièrement les prises de décision. En fait, il y a des moments où la décision est influencée par ce qui se dit et d'autres moments, non. Grosso modo, j'ai sélectionné les échanges que vous avez pu avoir avec différentes personnes à divers moments où vous vous êtes trouvé dans une situation délicate où vous deviez prendre des décisions. Je voudrais qu'on les regarde dans l'ordre, histoire de vous remettre ça en mémoire.

Tout d'abord, c'est un évènement qui s'est passé dans la nuit du 12 au 13 mars. Le HPCl du réacteur 3 s'arrête le 13 mars à 2 h42. Du temps passe et on est vers 3 h50. Vous tentez d'activer la DDFP, mais la pression de la cuve étant à environ 4,1 MPa, l'eau n'entre pas. J'ai choisi ce moment car on y voit un exemple des discussions récurrentes concernant le moment d'actionner la DDFP après l'arrêt du HPCl, d'autres sur la pression qui permettrait à cette pompe d'être efficace ou bien aux camions de pompiers d'entrer en action. J'aimerais que nous regardions ça pour que vous puissiez vous rappeler ces circonstances. ( $A u$ technicien) Allez-y, s'il vous plaît.

(Vidéo)

« Je voudrais intervenir pour vous signaler quelque chose sur le réacteur 3. »

«Oui, le réacteur 3?»

"Le HPCl s'est arrêté provisoirement à 2h44. »

« D'accord, arrêt provisoire à 2h44. »

"Comme la pression de la cuve était basse, on n'a pas actionné le RCIC et on a tenté d'injecter l'eau en activant la pompe diesel incendie. Mais, à ce moment-là, la pompe n'avait que 0,61 MPa et l'eau n'a pas pu entrer. Quand on regarde les données de 3h44, après l'arrêt du $\mathrm{HPCl}$, la pression de la cuve, qui était avant à 0,7 MPa, est montée à 4,1 MPa. Ça fait plus de cinq fois plus. De 7 kilos à 41 kilos. On est en train de vérifier ce que ça ferait si elle dépasse ces chiffres. Mais, c'est sûr, la pression de la cuve augmente. Du coup, on essaie de voir si on ne pourrait pas remettre en marche le HPCI ou si on ne pourrait pas utiliser un des moyens substitutifs d'injection. Le réacteur 3 est donc dans un état fluctuant. » 
« D'accord, compris. »

$\mathbf{T}^{2}$ : Je peux peut-être continuer?

$\mathbf{Q}:$ Oui, continuez.

(Vidéo)

«Ici, le groupe «sûreté ». Je voudrais vous communiquer les données des postes de monitoring de ce matin. Je suis près du poste de monitoring 8. Les données de [...], 4,5 $\mu \mathrm{Sv} / \mathrm{h}$ à 2h50. Entrée principale, 3,14 $\mu \mathrm{Sv} / \mathrm{h}$ à 2h50. II n'y a pas de changement. Pour ce qui est de la poussière à l'entrée principale, on a fait des vérifications entre 1 h45 et 2h05, la poussière est à $6,4.10^{-5} \mathrm{~Bq} / \mathrm{m}^{3}$. Pour les gaz nobles ${ }^{3}$ et l'iode ${ }^{4}$, on est en-deçà des limites détectables. Au poste de monitoring 4, on est à 39,6 $\mu \mathrm{Sv} / \mathrm{h}$. II n'y a pas non plus de grands changements. C'est tout. »

$\mathbf{Q}$ : Vous pouvez nous passer directement la suite.

$\mathbf{T}$ : Oui. C'est environ trois minutes plus tard.

$\mathbf{Q}:$ Ça se passe à $3 \mathrm{~h} 59$

(Vidéo)

«Tu veux utiliser ça pour quoi? Tu veux raccorder ça à l'alimentation du réacteur 3 ? Ce serait à l'alimentation?»

«La DDFP est encore vivante au réacteur 3. Elle a une pression de 5 à 6 kilos. À côté de ça, la pompe des camions fait plus de 10 kilos. Quand la pression de la cuve aura chuté, ce serait mieux de disposer d'une solution avec plus de pression et plus de volume d'eau [...]. »

« II ne s'agit pas d'envoyer avec la pompe incendie dans chaque réseau, il me semble. Tu veux bien vérifier ce point de toute urgence avec XXXXX?»

$\mathbf{Q}$ : On va arrêter la vidéo ici. Ce que nous venons de voir se passe entre 3 h50 et un peu avant 4h00. Une dizaine de minutes, jusqu'à l'intervention de M. XXXXX, avec, au milieu, les informations fournies par le groupe "sûreté ». Si je résume, après l'arrêt du HPCl, à cause de la pression de la cuve, l'eau n'est pas entrée avec la DDFP. M. XXXXX, lui-même, le fait remarquer à la fin, la DDFP n'a qu'une pression de 0,5 ou 0,6 MPa. Avec cette puissance, par rapport à la pression de la cuve, l'eau, bien sûr, ne peut plus entrer. Mais puisque cette pompe était vivante depuis le début, pourquoi, sur le terrain, n'avez-vous pas tenté d'injecter l'eau plus tôt, avant que la pression de la cuve ne grimpe de manière si spectaculaire?

$\mathbf{R}$ : Comme on en a parlé précédemment, l'histoire de la pompe diesel, c'est une question d'équilibre des pressions. On la met en marche et si l'équilibre lui est favorable, elle envoie l'eau, si elle est trop faible, l'eau n'entre pas. C'est un système très simple. On la met en marche et, le reste, c'est une histoire de vanne à clapet. Par contre, j'ai complètement oublié à quel moment j'en avais parlé. Mais une partie de l'équipe de pilotage avait préparé cette pompe pour que dès que l'équilibre serait favorable, elle puisse envoyer l'eau. Seulement, et c'est ce constat dans la vidéo, on se rend compte que, bien que prête, finalement l'eau n'est pas entrée, alors même que la pression de la cuve avait chuté. Voilà ce qu'il dit dans cette vidéo. Ce qu'il faut comprendre, c'est que ce n'est pas parce que la pression de la cuve a chuté à 0,8 que nous nous sommes empressés d'actionner cette pompe, mais qu'elle était prête bien avant. Seulement, du point de vue de l'équilibre des pressions, elle n'était pas suffisante et, finalement, l'eau n'était pas entrée. C'est ce que XXXXX est en train d'expliquer ici.

$\mathbf{Q}$ : La pression de sortie de la pompe DDFP, vous la mesurez où ? À quel endroit de la pompe?

R : Là. Oui, à [...] 
$\mathbf{Q}$ : Est-ce que ça veut dire que le temps que ça aille de là jusqu'à la cuve du réacteur, il y a des risques que la pression chute?

$\mathbf{R}$ : C'est pour ça qu'il y a une vanne à clapet. Si la pression est plus forte de ce côté-ci, le liquide va entrer, si la pression est plus forte de l'autre côté, le clapet va fermer le réseau et empêcher que ça reflue.

$\mathbf{Q}$ : Éclairez ma lanterne. Si on mesure une puissance de 5 ou 6 kilos de pression à la pompe, ça veut dire qu'on va retrouver une pression de 6 kilos à l'endroit où l'eau va pénétrer dans la cuve?

$\mathbf{R}$ : Oui. La pompe diesel n'a pas de manomètre en propre. D'après la capacité d'une pompe ordinaire, on se dit que la pression à la sortie doit être à peu près de cet ordre. Et si, au niveau du clapet, il n'y a pas de courant, la pression reste la même. Dès que le liquide va couler, il va y avoir une perte de charge maximale qui va poser des problèmes d'équilibre. Mais, de toute manière, c'est une simple question de pression au niveau du clapet, savoir de quel côté la pression est la plus forte. Quand ils parlent de la pression de la pompe dans la vidéo, je ne sais pas trop de quoi ils parlent, puisqu'ils ne disposent pas de manomètre. Mais c'est vrai qu'ordinairement on estime la puissance de cette pompe diesel à quelque chose ne dépassant pas 5 à 6 kilos. Alors, vous savez, cette histoire d'injection, elle se fait un peu au petit bonheur la chance, concernant l'équilibre des pressions.

$\mathbf{Q}$ : Si je comprends bien, que ce soit du point de vue de la quantité d'eau injectée ou que ce soit du point de vue de la pression, comme vous nous l'avez déjà dit, vous ne faisiez pas grande confiance à cette solution avec la DDFP. En tout cas, il ne vous était pas possible de la considérer comme votre va-tout.

R : C'est ça.

$\mathbf{Q}$ : Effectivement, vers la fin du passage que nous venons de visionner, la pression de la cuve grimpe de 7 à 41 kilos et, d'après ce que raconte $M$. XXXXX, puisque les pompes des camions ont une puissance de plus de 10 kilos, vous vous demandez ensemble s'il ne serait pas plus judicieux de passer à une solution avec les camions, ou bien de procéder à une dépressurisation pour faire entrer l'eau. Et c'est comme ça que vous chargez M. XXXXX, qui supervise les pompiers, de voir ce qu'il peut faire.

$\mathbf{R}:$ En effet.

$\mathbf{Q}$ : Maintenant, nous allons passer à un extrait qui se situe une quarantaine de minutes plus tard, vers 5h44. C'est le moment où vous discutez de bornes et de pompes à incendie, puis d'injection pour savoir si vous allez injecter de l'eau de mer ou de l'eau douce. On va commencer par l'extrait de 5h44. (Au technicien) Vous voulez bien lancer la vidéo, s'il vous plaît?

(Vidéo)

«Pour résumer, tous les gros réservoirs des bornes à incendie sont vides. II ne reste plus que des petites réserves de 2 à $3 \mathrm{~m}^{3}$. Par contre, on a des réservoirs entiers d'eau de mer. Qu'est-ce qu'on fait?»

« II ne nous reste plus qu'à envoyer l'eau de mer. »

\section{R : Là, c'est XXXXX.}

$\mathbf{Q}$ : Ah? C'est M. XXXXX? (Au technicien) Vous voulez arrêter ici, s'il vous plaît? (À Yoshida) Je n'avais pas remarqué que $M . X X X X X$ était assis à côté de vous. Là, visiblement vous disposez de bornes ou de pompes à incendie, mais vous manquez de source d'eau. Quand il dit «tous les gros réservoirs des bornes à incendie sont vides», que désignent ces "gros réservoirs »?

$\mathbf{R}$ : Ce sont probablement les réservoirs d'eau pour la lutte contre l'incendie. 
$\mathbf{Q}$ : Pas les réservoirs d'eau filtrée, donc, mais les réservoirs pour la lutte contre le feu.

R : Oui.

$\mathbf{Q}$ : Et il ne vous reste plus que 2 ou $3 \mathrm{~m}^{3}$ d'eau douce. C'est là que vous dites, "puisqu'il n'y a plus d'eau douce, il ne reste plus que la solution de l'eau de mer ».

R : Oui.

$\mathbf{Q}$ : C'était une discussion portant sur l'injection dans le réacteur 3, n'est-ce pas ?

R : Oui.

$\mathbf{Q}:$ (Au technicien) On va un peu avancer et projeter le « 05 », là où c'est écrit à la main «6h14, le 13 mars ».

(Vidéo)

«Vous m'avez dit que le TAF allait être atteint vers 5h30, mais ce que je voudrais savoir c'est s'il a déjà été atteint au réacteur 3. »

$\mathbf{Q}$ : (Au technicien) Ah, c'est un peu plus loin. Vers 6h43. Vous pouvez commencer là ?

(Vidéo)

«M. Yoshida, je vais revoir la pression de la chambre sèche en fonction des données actuelles et recalculer l'heure de l'éventage. »

«Urgence, urgence. XXXXX, XXXXX. Directeur Yoshida, directeur Yoshida, m'entendezvous?»

«M. Yoshida. Directeur Yoshida. »

«Oui, c'est Yoshida. Je vous écoute. »

« J'ai actuellement le bureau du Premier ministre au téléphone. Je vous fais passer la communication. »

«Oui. Vous me la passez où?»

«Sur votre PHS. »

«Passez-la-moi plutôt sur le xxX des lignes intérieures. XXXXX. XXXXX. »

«Centrale, centrale de Fukushima Daiichi, m'entendez-vous? Centrale, c'est le siège. Je vous téléphone de la salle de crise de la NISA. II s'agit du disque de rupture. Vous m'entendez?»

«Oui, allez-y. »

«Si on laisse faire le disque de rupture, l'éventage risque d'être retardé. On va aller vers un éventage avec une plus grande dégradation du combustible. Dans l'état actuel des choses, vous serait-il possible de rompre dès à présent le disque, avant l'ouverture de la vanne? »

$\mathbf{R}$ : Quelle question idiote ! Il n'a vraiment rien compris à la technique, cet imbécile !

$\mathbf{Q}$ : Est-il possible de rompre volontairement le disque, comme il le préconise?

$\mathbf{R}$ : Mais, non. Ce n'est pas possible. Pas de l'extérieur. Quel taré !

(Vidéo)

«Excusez-nous. On fait tout notre possible pour ouvrir la vanne AO en ce moment. Je pense qu'il nous faut encore trente minutes. »

"Même si tu gagnes quelques minutes à l'éventage, ça ne changera pas grand-chose à la quantité de combustible qui va se dégrader. »

« Ici, le groupe « technique ». D'après l'évolution de la pression de la chambre sèche, nous prévoyons que la pression maximale de service sera atteinte à $7 \mathrm{~h} 50$ et la pression maximale admissible à 13h30. L'augmentation de la pression est très rapide. " 
«Excusez-moi, permettez-moi de vérifier. L'heure d'atteinte de la pression maximale de service pour le réacteur 1F-3 doit être modifiée de 8 h30 à 7h50, c'est bien ça ? »

"C'est bien ça. Modification à 7h50. Pour l'atteinte de la pression maximale de service du 1F-3. »

"Je répète. Pression maximale de service à $7 \mathrm{~h} 50$ et pression maximale admissible à 13h30. C'est bien ça?»

"C'est ça.»

«Pour l'éventage, il va encore falloir trente minutes. Ça va aller?»

«Je n'entends pas. Qu'est-ce que tu dis?»

«C'est OK dans trente minutes. »

«Compris.»

"Je viens de recevoir un commentaire du Premier ministre. Il se demande si la décision d'employer de l'eau de mer n'est pas prématurée. Il pense que si on utilise l'eau de mer, ça signifie qu'on devra démanteler le réacteur. Il voudrait que vous réfléchissiez le plus possible à une solution avec de l'eau filtrée ou de l'eau douce. »

«Nous ałlons essayer de nous conformer le plus possible à sa directive. On va commencer par les endroits où on peut utiliser uniquement de l'eau filtrée. Ça va un peu nous retarder, mais nous allons procéder de cette manière. "

$\mathbf{Q}$ : Nous allons déjà nous arrêter là. On voit que les informations concernant injection et éventage s'entremêlent. On comprend que la situation requiert l'urgence. Je remarque que vers la fin de l'extrait, c'est M. XXXXX qui échange avec le siège. À l'image, on vous voit à gauche, dans l'écran incrusté tout à fait à droite. Vous êtes en train de parler au téléphone. Qui vous avait téléphoné?

$\mathbf{R}$ : Probablement, au début, c'était XXXXX.

Q: De TEPCO ?

$\mathbf{R}$ : Oui. Il était chez le Premier ministre. Il y a eu un coup de fil de la résidence au siège et on a décidé de le transférer sur XXXXX. En fait, après avoir parlé avec XXXXX, j'ai un trou de mémoire. Vraiment. J'ai l'impression que plutôt que d'avoir reçu des directives de XXXXX, quelqu'un d'autre est venu au téléphone. Mais je ne me rappelle toujours pas qui est venu me parler au téléphone.

\section{$\mathbf{Q}:$ C'était quelqu'un de chez TEPCO?}

$\mathbf{R}$ : Il me semble que XXXXX a passé le téléphone à Takekuro. À cette époque-là, on n'avait pas encore établi la hot line avec M. Hosono.

$\mathbf{Q}$ : Ces contacts avec M. Hosono, vous les avez eus à partir de quand?

$\mathbf{R}$ : Là aussi, ma mémoire est ... La vidéo, elle date bien du 13 ?

Q : Est-ce que vous vous souvenez à quel moment, au plus tard, cette ligne a été établie?

$\mathbf{R}$ : La tranche 3 explose le 13, vous vous souvenez. À ce moment-là, je me rappelle que M. Hosono m'avait déjà dit de prendre directement contact avec lui. Et, effectivement, je l'ai informé personnellement de l'explosion. Tout de suite après l'explosion. Bien sûr, auparavant, j'en avais parlé en téléconférence. Mais j'ai aussi téléphoné directement à la résidence du Premier ministre, j'ai parlé à M. Hosono et je l'ai informé que ça venait d'exploser. Ça, je m'en souviens très bien. 
Q : Et avant? Aviez-vous déjà téléphoné à M. Hosono avant cet épisode ? Ou bien aviezvous été contacté par lui?

$\mathbf{R}$ : Oui. Il m'a téléphoné. Quand la tranche 1 a explosé ${ }^{5}$, les informations n'arrivaient pas du tout au bureau du Premier ministre. Ça avait pris du retard. Que ce soit de la part de TEPCO ou de la NISA. Il avait fallu près de deux heures pour qu'elles arrivent. En fin de compte, les images de l'explosion de la NHK étaient sorties à l'écran avant qu'on ne contacte la presse. Alors, à la résidence, ils se plaignaient de la lenteur des communications. Ils voulaient qu'on les appelle pour confirmer la situation sur le terrain suivant les besoins. Ils voulaient aussi savoir comment ils pouvaient faire pour nous contacter. Tout ça est arrivé par l'intermédiaire d'une personne de TEPCO qui était en charge des liaisons avec le bureau du Premier ministre. En fait, j'ai changé plusieurs fois d'interlocuteurs et, tout à coup, j'ai eu M. Hosono au bout du fil. Il s'est présenté et m'a dit de l'appeler au téléphone s'il y avait quelque chose sur le terrain. Ensuite, on m'a donné son numéro de portable, puis le numéro de son secrétaire, en me disant de téléphoner là. Après, ils m’ont demandé comment ils pouvaient faire pour nous joindre. Mais, nous, nos portables ne marchaient pas. Alors, je leur ai dit, soit de passer par le siège, soit de voir avec l'homme de TEPCO à la résidence ce qu'ils pouvaient faire pour nous joindre. Ça, je m'en souviens bien. Mais savoir si ça se situe avant l'épisode qu'on vient de voir ou pas, mes souvenirs sont flous.

\section{$\mathbf{Q}$ : Mais c'est bien après l'explosion d'hydrogène de la tranche 1, le 12 ?}

R : Oui, après. Ça, c'est sûr. Je suis sûr que c'était après l'explosion d'hydrogène et également sûr que c'était avant l'explosion de la tranche 3. C'est entre les deux qu'il y a eu cette histoire d'établir une hot line avec la résidence.

$\mathbf{Q}$ : À propos, vous souvenez-vous du nom du secrétaire avec qui vous avez parlé ?

$\mathbf{R}$ : Oui, le secrétaire... Il m'avait rappelé, d'ailleurs. Comment s'appelait-il déjà ?

$\mathbf{Q}:$ C'était bien le secrétaire de M. Hosono.

R : Oui, le secrétaire de M. Hosono.

$\mathbf{Q}:$ Pas le secrétaire du Premier ministre.

R : Non, non. M. Hosono devait se charger personnellement de faire le lien avec le Premier ministre. Après, j'ai eu aussi affaire à d'autres secrétaires, même plusieurs. Mais c'est vrai que j'ai effectivement téléphoné à ce secrétaire, en particulier. Je ne sais pas si c'est que j'ai envie d'oublier tout ça, mais il ne me reste presque aucun souvenir. M. Naitô ${ }^{6}$, peut-être ? Puisque j'ai noté « Naitô, secrétaire, conseiller ». Oui, c'est le secrétaire du temps où M. Hosono était conseiller du Premier ministre. Peutêtre bien M. Naitô.

$\mathbf{Q}:$ À cette période, M. Takekuro était basé à la résidence du Premier ministre ?

$\mathbf{R}$ : Il me semble que oui.

$\mathbf{Q}$ : Dans ce cas, (la personne à qui vous avez parlé) en plus de M. Takekuro, comme autre alternative, ça pourrait être M. Hosono?

$\mathbf{R}$ : Oui, c'est possible. Mais je n'ai jamais discuté de savoir si on injectait de l'eau de mer ou pas, ni même discuté eau de mer/eau douce, avec M. Hosono. Je n'en ai absolument pas souvenir, quand j'essaie de me remémorer mes conversations avec lui. 
$\mathbf{Q}$ : Alors, en dehors de lui, qui cela pourrait être ? Vu l'orientation que prend la conversation, en parlant de démantèlement du réacteur, etc., est-ce que cela pourrait être quelqu'un de la NSC?

$\mathbf{R}:$ Non.

$\mathbf{Q}:$ Ce n'est pas possible?

$\mathbf{R}$ : Quand on téléphonait à M. Hosono, il y avait plusieurs schémas possibles. Des fois, il était à côté du Premier ministre et il y avait aussi le directeur de la NSC ${ }^{7}$. D'autres fois, il était seul. Je ne sais pas dans quelles circonstances il se trouvait lors de ce coup de fil. De toute façon, ce n'est pas moi qui ai appelé. Ce sont eux qui m'ont appelé. Probablement, Takekuro a dû leur dire qu'on ne pouvait pas utiliser les portables. Enfin, je ne sais pas exactement où se trouvait Takekuro à ce moment-là. Mais, au début, c'était soit XXXXX, soit Takekuro. Je pense que c'étaient eux qui s'occupaient du téléphone. Ensuite, je ne sais plus si on m'a passé quelqu'un d'autre ou si c'étaient les directives de Takekuro. Je ne m'en souviens pas.

$\mathbf{Q}:$ Alors, ça veut dire que M. XXXXX a passé le téléphone à quelqu'un d'autre?

$\mathbf{R}$ : Probablement, parce que XXXXX ne pouvait guère faire que le lien. Il n'était pas en position de donner des ordres du type " passe de l'eau de mer à l'eau douce ", etc. La seule personne qui aurait pu dire ce genre de choses, parmi ceux qui étaient détachés de chez nous, ça ne peut être que Takekuro. Si ça n'était pas le cas, il n'y a guère que la possibilité de M. Hosono. Je n'ai absolument pas le souvenir d'avoir parlé à quelqu'un de la NSC à ce moment-là. S'il fallait choisir entre les deux, je dirais plutôt Takekuro.

$\mathbf{Q}$ : Vous avez l'impression que c'était plutôt lui. Et la NISA ? Y a-t-il une possibilité de ce côté ?

$\mathbf{R}$ : Non. Je n'avais pas de contact téléphonique avec les gens de la NISA.

$\mathbf{Q}$ : Vous n'avez pas le souvenir d'avoir été en contact direct avec eux, c'est ça ?

$\mathbf{R}$ : Attendez un peu. J'ai un vague souvenir qu'une fois il y a eu M. Yasui ${ }^{8}$ au téléphone. Je ne sais plus si c'était à cette occasion-là ou pas. Dans mon souvenir, celui avec qui j'ai parlé au bureau du Premier ministre pour des arbitrages, c'était Takekuro. Ensuite, pour des rapports de situation et des questions concernant différentes choses, c'était M. Hosono. Il arrivait même que, quand j'étais au téléphone avec lui, il me passe le Premier ministre Kan, du genre «pouvez-vous patienter un peu, Kan voudrait vous parler ». Il me semble que j'ai aussi eu M. Edano ${ }^{9}$, une fois. Un peu plus tard, quand on a eu ces histoires autour de la dépressurisation et de l'injection du réacteur $2^{10}$, il y a bien sûr eu le professeur Madarame, et pas qu'une fois. Au milieu de tout ça, il me semble bien qu'une fois j'ai eu M. Yasui, mais je ne sais pas si ça se situe ici ou pas.

$\mathbf{Q}:$ Alors, vous ne vous souvenez pas non plus de quoi vous avez parlé.

R : Non. Aucun souvenir.

$\mathbf{Q}:$ Et avec M. Edano?

$\mathbf{R}$ : La conversation avec $M$. Edano ne se situe pas ici. Je ne sais pas très bien pourquoi, mais il m'a posé des questions. Par l'intermédiaire de M. Hosono, qui m'a dit «M. Edano voudrait vous poser des questions ». Ce n'étaient pas des questions très importantes, plutôt des vérifications sur la situation sur le terrain. Il voulait avoir confirmation sur certains points. Je suppose qu'il avait voulu vérifier certaines choses 
avant un point presse. Ce n'étaient pas du tout des directives pour nous dire de faire ci ou ça.

$\mathbf{Q}:$ Vérification des faits, alors?

$\mathbf{R}$ : Oui. Une seule fois, une vérification par téléphone.

Q : Et M. Kan?

$\mathbf{R}$ : Lui, c'était plutôt des questions. "Quel est le mécanisme qui provoque une explosion d'hydrogène? ", " est-ce différent d'une explosion de vapeur? ", c'est le genre de questions qu'il m'a posées. Ça, c'est la première chose.

Il y a eu aussi des fois où il y avait $\mathrm{M}$. Hibino ${ }^{11}$, un conseiller, ou le directeur adjoint de l'Université de Fukui ${ }^{12}$, à côté de lui. Le Premier ministre m'a demandé si, d'après la structure des REB, on pouvait envisager de refroidir le réacteur en envoyant la vapeur vers la turbine. Une question relativement élémentaire. Quand j'ai commencé mes explications, le ministre m'a arrêté et demandé de répondre directement à M. Hibino, car la question émanait de lui. J'avais pourtant d'autres chats à fouetter. Il a passé le téléphone à $\mathrm{M}$. Hibino et c'est à lui que j'ai expliqué que d'après la structure des REB, en cas d'accident de ce genre, il n'était pas prévu que la vapeur soit refroidie par la turbine, que, déjà, cette vapeur était de la vapeur contaminée et que la logique de base était de l'isoler dans l'enceinte de confinement et de l'y retenir. Qu'il n'était donc pas question d'utiliser la turbine pour ce refroidissement, qu'il n'était pas possible d'utiliser un condenseur au niveau de la turbine, etc. Là j'avais donc parlé au conseiller du Premier ministre via le téléphone du Premier ministre. C'était la deuxième chose.

J'ai parlé à M. Kan par quatre fois, il me semble. Il y a eu aussi la fois où il m'a demandé ce que je pensais de la définition des zones d'évacuation et d'alerte des habitants à respectivement 20 et $30 \mathrm{~km}$. Je lui ai répondu que je n'en pensais rien. Je lui ai expliqué que nous, nous étions chargés de gérer l'accident, que ce n'était pas nous qui calculions la dispersion de la radioactivité et que, par conséquent, malheureusement nous n'en savions rien. J'ai bien insisté sur le fait que c'était le siège, les analystes qui émettaient ces avis, et que ces préconisations n'étaient pas le fait du terrain.

Q : Quand vous receviez ces coups de fil, ils passaient d'abord par le siège qui vous les relayait?

R: Oui, c'est ça. En général, ils arrivaient par cette route. Des coups de fils qui arrivaient sur nos téléphones fixes par l'intermédiaire du siège.

Q: Vous avez dit que les coups de fil de M. Kan étaient plutôt des questions que des directives pour vous dire de faire ci ou ça, n'est-ce pas?

R : Oui. Je n'ai jamais reçu d'ordre direct de M. Kan pour faire ci ou ça. C'était plutôt des questions. "Je pense ci ou ça, est-ce juste?", "que faut-il penser de tel phénomène à la suite de l'explosion d'hydrogène? ?, etc. En général, des questions.

J'ai eu aussi pas mal d'échanges avec M. Hosono. Quand c'était moi qui l'appelais, c'était pour lui décrire la situation sur le terrain. Comme pour l'explosion de la tranche 3 , tout à l'heure. Je l'appelais pour lui dire « voilà où nous en sommes ». Même chose quand il y avait un souci. Bien sûr, j'en parlais aussi à la téléconférence. Mais après l'avoir fait, je prenais mon téléphone pour lui en parler directement. Souvent, il avait aussi des questions. "On est en train de discuter de telle chose, qu'en est-il sur le 
terrain? ", ou bien, "de combien de marge disposons-nous? ", etc. Il y avait beaucoup de questions. Ces échanges étaient assez fréquents. Du coup, je ne me rappelle plus à quel moment on a eu tel échange précis. Mais, ce qui est sûr, c'est que je n'ai jamais reçu d'ordre de M. Hosono, non plus.

$\mathbf{Q}$ : Alors, vu le contenu de la discussion...

$\mathbf{R}$ : Je pense que, très probablement, c'était un ordre de Takekuro. Comme possibilité, il y a d'abord Takekuro. On ne peut pas non plus écarter l'hypothèse que M. Yasui, ou quelqu'un qui était à côté de Takekuro ait pu émettre cette opinion. De toute manière, il ne peut guère y avoir que Takekuro ou $\mathrm{M}$. Yasui qui était à côté de lui.

$\mathbf{Q}$ : Si je résume, le 13 mars vers 6h00, il y a un coup de fil du bureau du Premier ministre au siège, coup de fil qui arrive jusqu'à vous et qui vous fait remarquer qu'il est peut-être prématuré d'utiliser l'eau de mer, car cela implique qu'on ne pourra plus récupérer le réacteur, et vous demande de réfléchir à la possibilité d'utiliser l'eau filtrée ou l'eau douce.

$\mathbf{R}$ : Là, comme je vous l'ai déjà dit, ma mémoire me fait totalement défaut. C'est en regardant la vidéo que je me suis dit qu'il y avait effectivement eu une scène de ce genre, voyez-vous. Mais je ne me souviens toujours pas avec qui j'ai vraiment parlé au téléphone. Alors, tout ça reste du domaine des hypothèses.

$\mathbf{Q}$ : À voir la vidéo, un peu auparavant, vous étiez prêts à injecter de l'eau de mer, faute d'eau douce, puis le coup de fil arrive de la résidence du Premier ministre, vous demandant d'envisager d'utiliser autant que possible de l'eau douce. $M . X X X X X$ répond qu'il va injecter en priorité de l'eau filtrée. Du coup, sur le terrain, suivant sans doute l'ordre de $M . X X X X X$, vos hommes doivent tout à coup changer leur fusil d'épaule. Ils m'ont raconté qu'ils avaient dû se mettre en quête des réservoirs d'eau prévus pour la lutte contre l'incendie. Ils ont dû s'y mettre tous pour les trouver, car les accès étaient cachés par les différents débris et les épaves. Ils ont eu du mal à déterminer les accès utilisables. Comme ils ont dû changer la ligne qu'ils avaient initialement prévue pour l'injection, ils s'en souviennent tous très bien. Pour ce qui est du donneur d'ordre, je pense que M. Takekuro doit être au courant. Je lui en parlerai. Je voulais juste vous rappeler qu'il s'était passé ce genre de choses vers 6h47.

On va revenir à l'injection. Nous allons regarder l'extrait 8. II s'agit d'un moment où vous injectiez encore de l'eau douce. Ça se passe vers 9 h10.

(Vidéo)

« Je pense que l'éventage s'est fait. »

«Ce qui est important dans cette opération, comme nous ne disposons que de 80 t d'eau, il faut qu'une solution, que ce soit le SLC ou autre chose, soit prête avant qu'on ait injecté les 80 t. L'autre priorité est d'alimenter et d'augmenter ces 80 t. Prenez tous les bras dont vous disposez. Allez chercher toute l'eau que vous pourrez trouver et alimentez ce réservoir. Exécution immédiate. Oui ? C'est bon? Je viens de donner l'ordre à l'équipe de soutien du réacteur 3 de mettre de l'acide borique. Oui, dans les 80 t [...]. »

« 400 kilos?»

« 400 kilos. »

«Est-ce que le SLC est prêt?»

«Pour le SLC, il va falloir encore du temps. On a exécuté l'opération de dépressurisation. II va falloir du temps. »

«Alors, travaillez en parallèle. Faites en sorte qu'on puisse y recourir quand on veut. »

«Je pense que dans l'état actuel des choses, il serait plus réaliste d'assurer le réapprovisionnement de l'eau. »

« D’accord. Priorité absolue, le réapprovisionnement en eau. » 
«Centrale, excusez-moi, c'est le siège. Pour faire le rapport à la NISA, j'ai besoin de savoir à quelle heure a commencé l'injection FP. Pouvez-vous me donner l'heure ? »

«L'injection FP, c'était 9h00 combien?»

« Je vous communique ça tout de suite.»

« D'accord. »

«Est-ce qu'il ne faudrait pas songer à l'eau de mer? II faut en parler avec le Premier ministre?»

« Excusez-moi. Parmi les informations concernant le réacteur, j'ai dit tout à l'heure [...]. »

«Excusez-moi. Pour le réacteur 3, on a commencé à envoyer de l'eau dans le SLC. On a évité l'eau de mer. On a rassemblé de l'eau douce et on l'injecte du réservoir par le haut. On réfléchit à la suite. »

«Directeur Yoshida, avez-vous une idée de la manière dont vous allez vous procurer de l'eau douce?»

«Par exemple, on a de l'eau dans la piscine d'entraînement ${ }^{13}$, vous vous souvenez ? Mon idée est d'aller chercher cette eau en déployant du personnel. II y a aussi d'autres petits réservoirs [...] d'une manière ou d'une autre. II faudrait récupérer toute l'eau possible sur le site. »

"Hier, il y a eu trois camions des forces d'autodéfense qui sont venus. Voulez-vous qu'on leur demande de revenir? Centre hors site, m'entendez-vous ?»

"Je le leur demande depuis le matin, mais ça ne réagit pas. Depuis hier, on a presque épuisé tout ce qui est liquide. S'ils pouvaient nous fournir quoi que ce soit... »

«Que peut-on faire au centre hors site?»

«Excusez-moi, c'est le centre hors site. Hier, les hommes des forces d'autodéfense sont venus pour apporter de l'eau. Ils étaient là au moment de l'explosion. Et il semblerait qu'ils aient été assez sévèrement irradiés. Du coup, il va peut-être être difficile de leur demander de venir de nouveau. »

«Ici, le groupe « administration » du siège. Concernant les forces d'autodéfense, nous les avons sollicitées de nouveau. Je vous demande de patienter encore un peu. Le centre hors site servira de guichet. »

$\mathbf{a}$ : On va s'arrêter là. II s'agit donc de ce qui se passe vers 9h10. Vous veniez de dépressuriser et c'est juste avant que vous n'injectiez de l'eau à 9h20. Finalement, en rassemblant ce que vous pouviez, vous disposiez d'environ $80 \mathrm{t}$ d'eau. De votre point de vue, il fallait prévoir l'étape suivante, que ce soit le SLC ou autre chose. II fallait donc récupérer tout ce qui était "eau » sur le site de la centrale. II fallait aussi envisager de s'approvisionner en eau de l'extérieur. Vers les 9h00, votre priorité est d'amasser toute l'eau douce possible, à la suite de la demande du Premier ministre. Si l'eau douce venait à s'épuiser...

$\mathbf{R}$ : De toute manière, comme je vous l'ai dit au début, ma position n'avait pas fondamentalement changé : il ne nous restait que l'eau de mer. Seulement, comme on m'a dit de privilégier l'eau douce, je l'ai fait dans la mesure du possible. En plus, j'avais aussi dans l'idée que je voulais mettre de l'acide borique. Pour diluer l'acide borique, il est beaucoup plus facile de le faire dans de l'eau douce et dans un réservoir. C'est plus facile à contrôler. Mais, si l'eau douce venait à manquer, j'ai toujours pensé qu'il fallait passer à l'eau de mer. Tout en sachant ça, puisqu'il fallait privilégier l'eau douce, je l'ai fait dans la mesure du possible.

$\mathbf{Q}$ : Dans la conversation, à un moment donné, M. Mutô qui était au centre hors site, évoque la nécessité de penser à l'eau de mer. II se demande même s'il ne faut pas prendre l'avis du 
Premier ministre. Est-ce que M. Mutô était au courant de tout ce qui s'était passé depuis 6h00?

R: Je suppose que oui. Mutô a l'air de penser qu'on pouvait directement passer à l'eau de mer. On ne sait toujours pas qui a décidé de rester à l'eau douce. Toujours cette histoire du coup de fil qui vient de la résidence du Premier ministre. Comme Mutô était au centre hors site, il ne connaissait pas la volonté du Gouvernement. Du coup, ça donne lieu au commentaire " est-ce qu'il faut en parler au Premier ministre?». C'est typique d'une situation où on ne sait pas qui décide.

$\mathbf{Q}$ : Vous, vous vouliez utiliser de l'eau de mer d'emblée. Mais il y a eu l'ordre émanant de la résidence du Premier ministre, qu'on peut considérer comme l'ordre du Premier ministre. Mais l'homme qui vous a parlé au téléphone était présent, physiquement, à la résidence. II n'y a pas de chance que vous ayez pu lui parler face à face.

\section{$\mathbf{R}$ : Non, ce n'est pas possible.}

$\mathbf{Q}$ : ॥ y a aussi dans ce passage l'histoire des hommes des forces d'autodéfense. Ils arrivaient sur le site, quand il y a eu l'explosion de la tranche 1. Ils ont assisté à l'explosion et sont repartis au centre hors site. Comme le dit M. XXXXX du centre hors site dans l'extrait, ils ont été irradiés et il semblait difficile d'envisager qu'ils reviennent à Fukushima Daiichi. En fait, ils étaient venus dans le cadre d'une opération de ravitaillement en eau. Et l'opération n'avait pas pu aboutir. Mais $M$. XXXXX du groupe «administration » du siège avait tout de même sollicité de nouveau les forces d'autodéfense en passant par le siège. II vous demande de patienter et vous rappelle que le centre hors site vous servira de guichet. Ce qui veut dire que vous comptiez sur l'eau apportée par les forces d'autodéfense?

\section{R : Bien sûr.}

$\mathbf{Q}$ : De fait, juste avant que la tranche 3 n'explose, vers 10h53, n'est-ce pas, il y aura sept camions de 5 t qui vont arriver. Ils transportaient de l'eau douce, c'est ça?

R : Oui.

$\mathbf{Q}$ : Nous allons maintenant visionner l'extrait 9. Cette fois-ci, nous sommes à 10 h08.

(Vidéo)

«Ici, le groupe «incendie». Concernant l'injection dans le réacteur 3 par les camions incendie, nous en sommes à $750 \mathrm{l} / \mathrm{min}$ avec une pression de 0,75 $\mathrm{MPa}$. »

«Excusez-moi. Je sais que vous êtes occupés avec le réacteur 3 et je suis navré de vous déranger, mais il faut qu'on commence à préparer les choses pour le réacteur 2. II faudrait que vous prévoyiez de l'eau, et puis des véhicules, des véhicules avec des pompes. »

"Et puis, il faudrait que vous nous disiez ce qu'on va injecter. Selon que c'est de l'eau de mer ou de l'eau douce, ce ne sont pas du tout les mêmes lignes. »

« Je pense qu'il y aura des changements, mais puisque, en dernier lieu, il faudra envoyer de l'eau de mer [...]. Pour l'eau, ce serait mieux d'utiliser l'eau de mer. J'ai envoyé quelqu'un voir l'état du bassin. »

«Non, pour le moment, on n'utilise pas d'eau de mer. On est en train de rassembler toute l'eau douce qu'on peut. II est question qu'il y ait des camions qui viennent de l'agence nationale de pompiers ${ }^{14}$, de l'agence de Sendai ${ }^{15}$, d'un peu partout. Mais, concrètement, à I'heure qu'il est, il n'y a qu'un camion de notre succursale de Chiba ${ }^{16}$ qui se dirige vers la centrale. Je pense qu'il devrait arriver sous peu. Mais cela n'en fait qu'un. »

«On va donc commencer par rassembler toute l'eau dont on dispose sur la centrale, l'eau de [...], tout ça. Depuis le temps qu'on les réclame, les camions devraient arriver en masse aujourd'hui ou demain. On va donc commencer avec de l'eau douce. S'il n'y en a vraiment plus, quand on ne pourra plus faire autrement, on passera à l'eau de mer. Mais, tant qu'on pourra, on injectera de l'eau douce. Voilà comment je vois les choses. »

« Compris. » 
«Permettez-moi d'intervenir. On recommence à voir les paramètres du réacteur 2 . Pour le moment, on ne voit qu'un appareil pour le niveau d'eau et on dirait qu'il y a de l'eau jusqu'à [...]. La pression de la cuve est en nette baisse. Du fait que le RCIC a marché trop longtemps, il y a des risques qu'on arrive à un état critique pour trois [...]. »

"C'est pour ça qu'il faut réfléchir bien en amont au moyen de refroidissement qu'on va adopter pour la suite. II faudrait, comme on l'a dit tout à l'heure, écrire sur une feuille ce que vous avez déjà fait, les prévisions pour le redémarrage, noter qui fait quoi, quels sont les problèmes, pour régler les problèmes un à un et pour que tout le monde soit au courant. »

«Et donc, pour en revenir à cette histoire d'eau douce, il va nous falloir beaucoup d'eau, en faire venir de différents endroits, il va falloir que vous vous en occupiez. »

«Je crois que cela fait bien une petite heure que le camion est parti. Je pensais qu'on pourrait mettre ce camion de pompiers de Chiba en attente au réacteur 2. »

$\mathbf{Q}$ : On va couper provisoirement ici. II s'agit de ce qui se passait vers 10h08. Si on fait le parallèle avec la chronologie de TEPCO, le 13 mars à 10h30, on signale la « décision du directeur de la centrale de commencer à préparer l'injection du réacteur 3 en vue d'utiliser l'eau de mer ». La vidéo montre donc ce qui se passait juste avant cette décision. Vous aviez déjà commencé l'injection d'eau douce dans le réacteur 3. Vous aviez également effectué un éventage à 9 h20. A priori, cet éventage avait dû être efficace, puisque, si on regarde les paramètres, la pression est descendue. Ce que suggère $M . X X X X X$, c'est de commencer à s'inquiéter des préparatifs pour le réacteur 2. Il se demande notamment si vous allez continuer à utiliser l'eau douce, comme pour le réacteur 3, ou si vous allez passer directement à l'eau de mer. S'agissant d'eau de mer, vous en aviez devant vous à perte de vue, mais s'il fallait continuer à utiliser l'eau douce et qu'il faille la partager entre le réacteur 2 et le 3 , il y avait des risques que vous manquiez très vite d'eau. Dans cette hypothèse, il se demande s'il ne faudrait pas d'ores et déjà commencer à se préoccuper de commander de l'eau un peu partout, sans trop y croire. Et là, vous, vous dites que vous n'allez pas utiliser l'eau de mer, que votre objectif, à ce moment-là, est d'injecter de l'eau douce en priorité. Que si vous arriviez à manquer d'eau douce, vous vous résigneriez à injecter de l'eau de mer, mais que vous envisagez de donner la priorité à l'eau douce. Quelle est la raison qui vous a poussé à faire ce genre de déclaration?

\section{$\mathbf{R}:$ La réaction du Gouvernement.}

$\mathbf{Q}:$ C'était la grande raison de cette déclaration?

$\mathbf{R}$ : Oui. Comme je vous l'ai dit, j'avais toujours pensé au fond de moi que, si cela s'avérait nécessaire, on allait injecter de l'eau de mer. J'avais donc commencé par dire qu'on allait injecter de l'eau de mer dès le début. C'est par après, que j'ai reçu ce coup de fil de la résidence du Premier ministre qui me demandait d'essayer d'en rester à l'eau douce. J'ai donc décidé d'essayer de tenir le plus longtemps possible avec l'eau douce. Seulement, nous n'étions pas capables, seuls, de rassembler autant d'eau. C'est pour ça que nous avions sollicité toutes sortes d'organismes, y compris les forces d'autodéfense. Je pensais encore à ce moment-là que si les forces d'autodéfense travaillaient efficacement, on arriverait à alimenter suffisamment en eau douce. J'étais encore plein d'espoir. Il me semble que je ne pensais pas encore à passer à l'eau de mer. C'était un moment assez délicat.

$\mathbf{Q}$ : Du point de vue de $M . X X X X X$, on a l'impression qu'il est déjà totalement pour l'injection d'eau de mer. Son argument est que toutes sortes d'organismes, que ce soit l'agence nationale des pompiers ou des agences locales, vous promettent de vous envoyer des camions, mais que dans les faits, seul un camion en provenance de votre succursale de Chiba est effectivement en route et qu'il n'y en a pas d'autres. Dans ces conditions, il pense qu'il est illusoire de vouloir alimenter à la fois les réacteurs 2 et 3 et il penche sans hésiter pour l'eau de mer. Mais vous, vous lui répondez que vous envisagez d'approvisionner le réacteur 2 avec l'eau du camion de Chiba. Malgré tout, au fond de vous-même, pensiez- 
vous qu'en cas de nécessité, si vous ne disposiez plus d'eau douce, vous injecteriez de l'eau de mer?

R : Oui.

$\mathbf{Q}$ : Mais, rappelez-vous, il y avait eu précédemment l'histoire de l'injection d'eau de mer dans le réacteur 1. On vous avait donné l'ordre d'arrêter l'injection d'eau de mer, mais vous aviez continué.

$\mathbf{R}$ : Oui, l'injection continue d'eau de mer.

Q: C'était vers 19h04. Est-ce que vous avez été tenté de feindre, au moment de cet enregistrement? De dire que vous injectiez de l'eau douce, alors qu'en fait vous injectiez de l'eau de mer, un peu comme vous l'aviez fait pour le réacteur 1 ?

$\mathbf{R}$ : Je sais que tout le monde voit les choses de cette manière-là. Mais, franchement, à ce moment-là, j'étais persuadé qu'on arriverait encore à approvisionner en eau douce à un rythme suffisant. Je pensais qu'on arriverait encore à assurer une injection continue en eau douce, que je n'utiliserais l'eau de mer qu'en dernier ressort, quand on n'aurait plus aucun moyen d'approvisionner en eau douce. Ça a été ma première position. Quoi que dise le Gouvernement, d'ailleurs. Précédemment, on avait recensé toute l'eau douce qui était encore disponible sur le site de la centrale. En discutant sur les possibilités, on était tombé sur l'eau de cette piscine d'entraînement qui représentait quelques centaines de tonnes. Je m'étais dit qu'il était sans doute possible de transférer cette eau pour l'injection. Ensuite, il y avait également la possibilité de se faire approvisionner de l'extérieur, y compris par les forces d'autodéfense. Cela faisait tellement longtemps qu'on réclamait de l'eau que je m'étais dit que si celle-ci finissait par arriver, on pourrait continuer à injecter de l'eau douce. Je comptais donc sur deux sources d'approvisionnement possibles. Et je pense en avoir parlé au Gouvernement. Je ne me souviens plus des détails de ce que j'ai raconté, mais grosso modo j'ai dit que si l'approvisionnement en eau douce était suffisant, on pouvait aussi envisager une injection d'eau douce. Sur le site, on n'avait guère que l'eau de cette piscine, mais je pensais que, si on arrivait à nous approvisionner de l'extérieur, avec les forces d'autodéfense, on pouvait tenter le coup.

Par contre, la veille, pour le réacteur 1, il n'y avait pas alors la possibilité de réapprovisionner en eau douce, c'est pour ça que j'avais décidé de commencer à injecter l'eau de mer. Pour moi, il n'y avait pas à tergiverser, réutilisation du réacteur ou pas. De toute façon, ce réacteur n'était plus réutilisable. Nous étions effectivement en train d'injecter de l'eau. Il n'y avait aucune raison d'arrêter cette injection, alors qu'elle venait juste de commencer. Si on l'avait arrêtée, la situation se serait dégradée rapidement.

En plus, il y avait une autre chose qui me tracassait. Il n'y avait pas eu d'indication de durée concernant l'arrêt de l'injection. On nous donnait l'ordre d'arrêter l'injection. Mais on ne savait pas au moment où on a reçu l'ordre pendant combien de temps on allait l'arrêter. Or, laisser le réacteur sans injecter l'eau était extrêmement dangereux. Alors, naturellement, j'ai réagi violemment. "Qu'ils me foutent la paix ». J'ai décidé d'ignorer l'ordre et j'ai continué l'injection. Dans la vidéo qu'on vient de voir, on est encore dans un moment où il $\mathrm{y}$ a des possibilités éventuelles d'approvisionnement. Du coup, ma position est de continuer à injecter de l'eau douce, tant qu'on arrivera à alimenter la source. On n'est pas encore au point où il faut 
envoyer, envoyer, envoyer de l'eau de mer. On n'est pas du tout dans la même situation que la veille.

$\mathbf{Q}$ : Pour résumer, si je restitue tout ça de manière simple, pour réapprovisionner les bassins, il faut des véhicules de pompiers et ils vont arriver. Après la scène de la vidéo, le nombre des voitures de pompiers disponibles va aller croissant. Au 13 mars, par exemple, des camions vont arriver de Kashiwazaki en soutien. Ce qui est très différent de la situation que vous aviez connue pour l'injection du réacteur 1. Pour le réacteur 1, les moyens étaient extrêmement réduits. Vous n'aviez guère qu'un seul camion à votre disposition. Par exemple, même si vous aviez voulu aller chercher l'eau des bassins des bâtiments réacteurs 2 ou 3 pour injecter dans le 1, cela aurait posé des problèmes et, surtout, cela n'aurait pas permis d'assurer une injection en continu. Au moment où vous vous occupez de l'injection dans le réacteur 3, vous disposez déjà de plus de matériel. Vous vous dites qu'en utilisant tous les moyens dont vous disposez, vous pourriez éventuellement rassembler tout ce qui reste d'eau douce sur le site de la centrale pour une injection à l'eau douce. En contrepartie, ces opérations vont obliger les hommes du groupe « incendie » ou de Nanmei Corporation à travailler dans un environnement hautement radioactif. Malgré tout, si besoin il y avait, vous vous sentiez capable de construire des réseaux permettant ce genre d'injection. Sur ces entrefaites, le Gouvernement vous sollicite pour assurer, si possible, une injection à l'eau douce. Du coup, vous décidez de continuer une injection à l'eau douce le plus longtemps possible.

\section{R : C'est ça.}

$\mathbf{Q}$ : Eh bien, nous allons regarder l'extrait suivant. Ça se passe vers $10 \mathrm{~h} 30$.

(Vidéo)

«Centrale 1F, vous m'entendez ? C'est XXXXX du siège. Centrale 1F, vous m'entendez ?»

«Oui, je vous entends. »

« Je ne sais pas si tu as aussi eu le coup de fil, Yoshida, mais je voudrais faire un point de la situation avec toi. D'abord, concernant cette histoire avec M. Takekuro et la résidence du Premier ministre. On pense que vous devez agir en gardant naturellement à l'esprit l'idée que vous finirez par utiliser l'eau de mer. D'ailleurs, on pensait que vous aviez déjà envoyé de l'eau de mer. Mais si vous pouvez, même pour un temps, envoyer de l'eau douce, on pense que ce n'est pas une mauvaise solution. En tout cas, vous avez d'ores et déjà l'autorisation d'utiliser de l'eau de mer. Premier point.

Ensuite, M. Takekuro a besoin de savoir de manière urgente le volume d'eau que vous injectez par le réseau DD, l'eau dont vous disposez actuellement, et puis ce que vous injectez par le réseau FP. Là, c'est de l'eau filtrée, c'est bien ça ? Combien en avez-vous ? »

«Concernant la DD, pour le moment, nous avons deux fois $40 \mathrm{t}$, ce qui fait $80 \mathrm{t}$. »

« $80 \mathrm{t}$, donc. Ça va tenir combien de temps, grosso modo ? »

« En gros, c'est un débit de $800 \mathrm{l} / \mathrm{min}, 0,8 \mathrm{t} / \mathrm{min}$. Puisqu'on a 80 t, cela fait... Oh, je n'y arrive plus. »

«Demande à quelqu'un de faire le calcul et tiens-moi au courant. »

«Je vous donne juste un ordre d'idée. Le gros camion chimique a deux réservoirs de $40 \mathrm{t}$ chacun, ce qui fait 80 t. Ensuite, la pompe diesel est alimentée en eau filtrée. On me dit qu'il y en a environ 800 t. »

« 800 t d'eau filtrée, donc. »

«Comme la pression de la pompe diesel est plus faible, pour le moment, c'est le camion chimique qui travaille. Le débit est de $800 \mathrm{l} / \mathrm{min}$ et puisqu'on dispose de $800 \mathrm{t}, 800 \div 0,8$, cela va faire cent minutes ${ }^{17}$, en gros. Pour le moment, on est parti chercher l'eau. »

"Si on tient compte de l'eau qu'il y a dans la piscine d'entraînement, on peut considérer qu'on a encore 800 t, c'est ça?»

«Oui. » 
«Et, en ce qui concerne les préparatifs pour l'injection d'eau salée, où en êtes-vous ? »

«On continue. »

«Quand est-ce que vous allez passer à l'eau de mer? »

«Dans deux heures, environ. De toute façon, il suffit de réutiliser le tuyau qui est raccordé du camion de pompiers au bassin [...], il suffira d'une dizaine de minutes pour faire le raccord. »

«On peut donc considérer que vous pouvez faire la manœuvre à tout moment, alors. Continuez pour le moment à envoyer l'eau douce. Je vais faire mon rapport à M. Takekuro. »

«Excusez-moi, M. Yoshida. Je viens de faire le calcul. Ce n'est pas « deux heures », mais «vingt heures ». Avant qu'on ait épuisé notre réserve d'eau. »

« Non, non. Là, tu tiens compte de la réserve d'eau filtrée. »

«Oui. »

«Ce que je veux savoir c'est juste avec ce qu'il y a dans les réservoirs du camion. »

«Ah, d'accord. »

«Oui, je voudrais que tu calcules exclusivement avec ce qu'il y a dans le camion. »

« D'accord. Je voudrais vérifier de nouveau, alors. Les deux fois 40 t, qui font les 80 t qu'on envoie en ce moment. Plus, les deux réserves de 40 t. »

"C'est tout à fait ça. C'est ce qu'on va envoyer en premier. »

«Ensuite, vous avez les 800 t d'eau filtrée. De ce côté, la pression est plus faible, mais, si ça se trouve, vous pourrez tenir vingt heures. »

«Oui, c'est ça. »

«Pour l'eau de mer, si tout va bien, vous pourrez raccorder le tuyau à la mer en dix à quinze minutes et injecter via le camion de pompiers. »

«C'est bien ça. »

«Bon. Je crois que nous sommes d'accord. Je vais donc transmettre ça. Excusez-moi de vous avoir interrompu dans vos travaux. »

$\mathbf{Q}$ : (Au technicien) On peut arrêter ici.

(À Yoshida) Nous venons de voir M. Komori vous poser toutes sortes de questions en passant par la visioconférence. Au début, il dit qu'il veut faire un point de la situation avec vous, concernant I'histoire de M. Takekuro et la résidence du Premier ministre. II dit en l'occurrence, " on pense que vous devez agir en gardant naturellement à l'esprit l'idée que vous finirez par utiliser l'eau de mer. D'ailleurs, on pensait que vous aviez déjà envoyé de l'eau de mer ». Cette dernière remarque, sur le fait qu'il pensait que vous aviez déjà envoyé de l'eau de mer, c'est M. Komori, personnellement, qui pensait ça?

\section{$\mathbf{R}:$ Je n'en sais trop rien.}

$\mathbf{Q}$ : Parce que si c'était M. Takekuro, on pourrait lui retourner que c'était lui qui en avait donné l'ordre initialement. Est-ce que, plus tard, vous avez eu des échanges téléphoniques à ce sujet avec la résidence du Premier ministre?

\section{$\mathbf{R}$ : Non, il n'y en a pas eu.}

$\mathbf{Q}$ : Vous souvenez-vous d'avoir repris contact de vous-même avec M. Takekuro vers 9h30 ou 10h00, pour mettre le Gouvernement au courant de vos opérations?

$\mathbf{R}$ : Non. Mais je vous avouerais que c'est un moment qui s'est complètement effacé de ma mémoire.

$\mathbf{Q}$ : D'après ce que raconte M. Komori, c'est lui qui envoyait les informations à M. Takekuro à la résidence du Premier ministre?

$\mathbf{R}$ : Ce que je veux dire, c'est qu'il y avait deux filières. Il y avait d'abord la filière qui reliait la cellule de crise du siège à la résidence du Premier ministre. C'est celle qui 
était officielle. Mais en plus de celle-là, je ne sais pas pourquoi, il s'est formé une autre filière directe entre la résidence et le terrain. C'est comme ça que je me suis retrouvé à recevoir des ordres directement. Des histoires qui auraient dû passer par la cellule de crise du siège et la NISA passaient par l'autre filière. En fait, ça dépendait des moments. Il y avait des moments où ça passait par la ligne officielle, et d'autres où ça arrivait directement. Je suppose qu'il devait y avoir aussi de multiples discussions au sein du Gouvernement. Mais, vu d'ici, on n'y comprenait rien.

$\mathbf{Q}$ : Quand il y avait des directives à faire passer, les ordres passaient soit par le siège, soit par la voie directe. Est-ce qu'il n'y avait pas une catégorie d'ordres qui passait systématiquement par une route ou une autre?

R : Non. Pour l'histoire de l'injection d'eau de mer à remplacer par l'eau douce, par exemple, au lieu de me téléphoner directement, il aurait fallu, officiellement, poser la question au siège, à qui j'aurais expliqué la situation. Mais au lieu de ça, on a reçu directement le coup de fil. Du coup, je crois qu'on a eu tendance à donner plus d'importance aux directives qu'on recevait. Puisqu'on n'en était pas encore au point de ne plus avoir une goutte d'eau douce, j'ai répondu qu'on allait faire notre possible pour nous y conformer. Mais, après, il fallait que, moi-même, j'informe à mon tour le siège que la résidence du Premier ministre m'avait contacté pour que je donne la priorité à l'injection d'eau douce et que j'allais me conformer à cette directive. C'était le monde à l'envers.

$\mathbf{Q}$ : J'ai remarqué que, concernant l'injection du réacteur 3 , tout n'est pas non plus passé en direct entre le terrain et la résidence du Premier ministre. Au tout début, comme on l'a vu, les choses se passaient en direct, mais, par la suite, il y a eu des moments où c'était le siège qui fournissait les informations. De toute façon, les politiques ne savaient pas quelle était la situation, ils avaient besoin de poser des questions pour décider. Seulement, la manière officielle de procéder aurait été de passer par le siège.

$\mathbf{R}$ : Exactement. Ça n'est pas concevable. Avouez que le fait même que la résidence du Premier ministre et le terrain soient directement reliés n'est absolument pas concevable.

$\mathbf{Q}$ : Ce qui signifie que la résidence du Premier ministre doit aussi revoir sa manière de répondre au terrain.

Passons, maintenant à la suite. II est 10h43.

(Vidéo)

« Je voudrais vérifier quelque chose. »

«Oui. »

«Centrale 1F, je peux intervenir? Nous venons de recevoir une directive de la NISA. La NISA pense qu'étant donné que la radioactivité était en hausse une heure avant l'éventage de l'enceinte de confinement, il y a eu des fuites. Ce qui veut dire qu'il y a possibilité d'une explosion comme pour le 1F-1. La NISA voudrait qu'on étudie des solutions, comme d'ouvrir le Blow Out Panel. »

« D'accord. »

«Oui, réfléchissez-y. Nous aussi, de notre côté, on va y penser. »

«On va y réfléchir tous ensemble, comme on avait dit tout à l'heure. »

"C'est XXXXX, du siège, qui doit faire les évaluations, alors je lui ai dit tout à I'heure de discuter avec XXXXX. Mais ça, c'est sur le papier. Dans le concret, il va falloir qu'on y réfléchisse tous ensemble.»

« Oui. » 
«Alors, vous avez réussi à lui parler? Dis donc, Yoshida, il faudrait que tu te fasses présenter XXXXX qui va discuter avec lui. Je n'arrive pas à joindre XXXXX par téléphone. À qui pourrais-je téléphoner?»

" Le mieuX serait que XXXXX décide et qu'il prenne contact avec XXXXX. »

«OK. »

$\mathbf{Q}$ : Là, dans ce passage, la NISA vous demande de réfléchir à des solutions, comme d'ouvrir le Blow Out Panel, parce que la radioactivité autour du réacteur 3 est en train de monter. La NISA a peur qu'il y ait encore une explosion. Je suppose que, sur le terrain, vous aviez déjà le même genre d'inquiétude et ce, depuis longtemps. On vous demande donc d'étudier la question et, à vous entendre, on voit que le siège et le terrain sont d'accord pour que M. XXXXX décide d'un correspondant et que celui-ci serve de guichet, avec XXXXX, pour faire avancer les choses. $Y$ avait-il, à ce moment précis, quelqu'un qui réfléchissait concrètement à la manière d'ouvrir ce panneau?

$\mathbf{R}$ : Oui, effectivement. J'en ai déjà parlé la dernière fois, quand les professeurs sont venus $^{18}$. Depuis l'explosion de la tranche 1 , on ne pouvait pas écarter la possibilité d'une autre explosion d'hydrogène. Il y avait de l'hydrogène qui s'était accumulé, il fallait l'évacuer. Il fallait ouvrir ce panneau de décompression. Seulement, ce panneau avait été rendu plus difficile à ouvrir, à la suite de ce qui s'était passé lors du séisme au large de Chûetsu, où le panneau était tombé et s'était ouvert tout seul. On était donc intervenu pour le rendre plus difficile à ouvrir. Et ça s'est complètement retourné contre nous. Le panneau, tel qu'il était, était quasi impossible à ouvrir avec la force de l'homme. Ouvrir ce panneau, il fallait y réfléchir en adoptant le point de vue d'un constructeur. C'est pour ça qu'on avait sollicité le département «construction » du siège pour réfléchir avec nous. Mais il était difficile de trouver une solution praticable. À la fin, on envisageait même de faire venir un camion échelle et de découper le panneau de l'extérieur. Mais si on avait opté pour cette solution, les préparatifs auraient pris énormément de temps. On en était là de nos réflexions, quand Takahashi nous a appelés. Bien sûr qu'on y réfléchissait, mais on n'avait pas de solution. Pour être tout à fait franc, j'avais envie de dire à ces imbéciles de la NISA, "vous n'avez qu'à venir faire le boulot vous-mêmes, bande de crétins! ». Ils ne donnaient que des ordres pourris. Quand j'y repense, j'en ai encore des bouffées de colère. C'était toujours la même chose. Ils se contentaient de dire de faire ci ou ça. Comme si c'était facile!

$\mathbf{Q}:$ Comme de rompre volontairement le disque...

R : Oui, comme si c'était possible! Honnêtement, à chaque fois que la NISA nous contactait, c'était toujours pour nous dire des choses qui nous hérissaient.

$\mathbf{Q}$ : Dans cet épisode, l'ordre de la NISA vous arrive via le siège. Etait-ce toujours le cas?

R : Oui:

$\mathbf{Q}:$ Vous n'avez jamais reçu d'ordre de la NISA en direct?

R : Non.

$\mathbf{Q}$ : Et le bureau de la NISA qui se trouve sur la centrale?

R : À ce moment-là, il n'y avait plus personne.

$\mathbf{Q}:$ Plus personne?

$\mathbf{R}$ : Non, il n'y avait physiquement plus personne de la NISA présent sur le site. Peutêtre que les personnes normalement affectées ici ont fait des rapports, mais on n'a jamais reçu d'ordre par leur intermédiaire. 
$\mathbf{Q}$ : Pour en revenir à ce Blow Out Panel, vous m'avez dit que, à la suite du séisme au large de Chûetsu, dans le cadre des mesures antisismiques, il avait été rendu plus difficile à ouvrir. Avant cet « aménagement », on pouvait l'ouvrir à la main ?

$\mathbf{R}$ : Ça n'a jamais été quelque chose de léger. Avant "aménagement ", ça n’était déjà pas aisé, mais on pouvait l'ouvrir tant bien que mal avec des outils. Je ne sais pas concrètement à quel point l'opération était devenue difficile après aménagement. Je ne l'ai pas testé moi-même. Mais le département "construction » affirmait que l'opération n'était vraiment pas facile. Je leur ai demandé pourquoi c'était devenu si difficile. Et c'est là qu'ils m'ont dit qu'ils avaient eu pour consigne de renforcer ce panneau après le séisme de Chûetsu. Mais nous, nous devions l'ouvrir coûte que coûte. Alors nous étions en discussion avec le siège pour trouver une solution.

$\mathbf{Q}$ : Ce n'est pas, par exemple, le genre de panneau qu'on ouvre en tirant sur un levier qui donnerait du mou, j'imagine?

R : Non, malheureusement.

$\mathbf{Q}$ : Alors, si on veut l'ouvrir, il faut chauffer le tour, ou quelque chose de ce genre?

R : Oui. En fait, il y a deux histoires qui se mélangent ici. D'un côté, il y a la discussion autour de l'ouverture du panneau. Ça, c'est l'histoire de la NISA. Mais il y a aussi l'histoire autour de XXXXX et XXXXX. Ce que fait XXXXX, ce n'est pas trouver une solution pour l'ouverture du panneau. Il calcule le volume d'hydrogène qui s'échappe. En fait, il essaie de prévoir la limite où l'hydrogène risque de s'enflammer. Et ce que veut savoir XXXXX du siège, c'est ça. Il fallait que cette question soit aussi suivie par quelqu'un sur le terrain. C'est pour ça qu'il dit à XXXXX, qui est sur le site, de prendre contact avec XXXXX pour qu'il le mette bien au courant de ses conclusions. Ce n'est pas tout à fait l'histoire de l'ouverture du panneau de décompression.

$\mathbf{Q}$ : Ah, oui ! C'était une autre histoire ! I s'agissait du calcul de l'hydrogène qui s'échappe.

$\mathbf{R}$ : Oui, savoir le volume qui est produit et le volume qui fuit.

$\mathbf{Q}$ : Oui, savoir combien d'hydrogène est en train de s'accumuler dans le bâtiment. Ce genre de calcul, c'est le groupe « technique » qui l'effectue?

$\mathbf{R}$ : Oui, le groupe « technique ».

$\mathbf{Q}$ : Et ils doivent communiquer avec le siège, échanger les informations.

$\mathbf{R}$ : Là, les calculs étant assez pointus, c'était XXXXX, du siège, qui était en train de les faire. Le groupe « technique » de la centrale avait du mal à suivre tout. Du coup, dans cet extrait, on est en train de donner l'ordre au chef d'unité de désigner XXXXX, sur le site, pour suivre plus spécialement ces calculs.

$\mathbf{Q}$ : À la suite de ça, avez-vous eu des retours du siège vous informant, par exemple, d'un danger dans « $\mathrm{X}$ » heures?

$\mathbf{R}$ : Je n'en ai pas le souvenir.

$\mathbf{Q}:$ Parce que ça a explosé avant?

R: On a encore une journée, après ça. Ce film date du 13 mars. On a encore une journée avant l'explosion ${ }^{19}$. On a eu divers échanges. On avait encore le temps.

$\mathbf{Q}:$ Vous aviez encore le temps.

$\mathbf{R}$ : C'est pour ça que même pour l'histoire de ce panneau, on en était encore à se dire que c'était dur à ouvrir, j'avais donné l'ordre de réfléchir aux solutions, mais je 


\section{n'avais pas encore eu de retour. C'est dans la nuit qui a suivi qu'on a beaucoup discuté au sujet de ce qu'on allait faire.}

$\mathbf{Q}$ : Ce qui veut dire qu'après cet épisode, pendant un certain temps, ce sont $M M . X X X X X$ et $X X X X X$ qui vont travailler de concert tout en continuant à communiquer avec le siège.

\section{R : Oui.}

$\mathbf{Q}$ : Passons maintenant à l'extrait 20.

(Vidéo)

«Oui, on utilise le réseau [...]. »

«À propos de l'injection d'eau de mer dans le réacteur 2 qui nous avait donné du souci cet après-midi, où en êtes-vous?»

«Nous allons commencer dans trente ou quarante minutes. »

«Attendez une seconde. Concernant le réacteur 2, je voudrais commencer assez vite. Mais, pour le moment, les données sont stables. De plus, je voudrais qu'on assure d'abord l'alimentation du réacteur 3 et du réacteur 1 . Je préfèrerais qu'on prenne les décisions pour le réacteur 2, une fois qu'on aura bien assuré tout ça. Si vous permettez, je vous recontacterai pour donner l'heure. »

«Ce qui veut dire que, contrairement à hier où les données te donnaient des inquiétudes pour le réacteur 2, tu estimes que les données que tu as actuellement sont fiables et qu'il y a moins d'urgence?»

«On n'avait que le niveau d'eau de la cuve, ce matin. Mais à l'heure actuelle, nous avons des données un peu plus fines concernant la pression de la cuve, la pression de la chambre sèche et, à voir les tendances, les deux indicateurs de niveau d'eau donnent à peu près les mêmes chiffres. Ce matin, on n'avait qu'un indicateur opérationnel sur les deux. En tenant compte de l'ensemble de ces données, je dirais que nous sommes dans une situation qui laisse encore un peu de marge. »

« D'accord. »

« Je voudrais donc que vous nous laissiez juger globalement de la situation à la centrale. »

«Tu veux dire que tu veux continuer à donner la priorité au réacteur 3 et au réacteur 4, c'est ça?»

«Oui. »

« D'accord. »

«À ce propos, ici, le siège. Nous avons bien conscience qu'il n'y a pas suffisamment d'eau pour le réacteur 2, mais j'ai cru comprendre que vous alliez commencer d'emblée l'injection avec de l'eau de mer, est-ce exact?»

"C'est bien ce que nous allons faire. Nous allons injecter directement de l'eau de mer. Je crois qu'il y a quelques confusions. Pour le réacteur 1, comme on n'avait pas du tout d'eau douce, on a injecté de l'eau de mer. Pour le réacteur 3, on disposait d'une assez grande quantité d'eau douce, on a donc commencé avec de l'eau douce. Une fois qu'elle a été épuisée, on est passé à l'eau de mer. Ça, c'est pour le réacteur 3. Pour le réacteur 2 aussi, on voulait initialement injecter de l'eau douce et on avait passé commande pour qu'on nous en apporte. Mais, aujourd'hui à midi, il y a eu un moment où on s'est dit qu'on pouvait être confronté à une situation où on aurait besoin d'injecter l'eau de manière urgente dans le réacteur 2 et, du coup, on a commencé à lui prévoir une ligne d'injection avec l'eau de mer. C'est à ce moment précis que le réacteur 3 a commencé à donner de réelles inquiétudes. On a pensé qu'il fallait donner la priorité au réacteur 3 par rapport au 2. Et nous en sommes toujours là, à l'heure où je vous parle. Pendant qu'on s'occupait du réacteur 3, on n'a pas pu transporter l'eau douce pour le réacteur 2. C'est pour ça qu'on continue à préparer la ligne d'injection d'eau de mer. Voilà le résumé. » 
«D'accord, j'ai compris. Mais si je puis me permettre, si on envoie directement de l'eau de mer, ça veut dire que le matériel va «pourrir ». Ce ne serait pas du gâchis ? Est-ce qu'il n'y aurait pas une possibilité d'attendre autant que possible l'arrivée de l'eau douce? »

«Non, ce choix n'existe pas. De toute façon, la ligne que nous avons construite a pour source d'alimentation l'eau de mer. II n'y aura pas d'injection d'eau douce. II est trop tard. »

«On avait bien décidé de la sorte dans la journée. Mais ce serait peut-être bien que XXXXX t'explique ce qu'il veut dire. »

«Ce qu'il veut dire, c'est que ce serait mieux d'utiliser l'eau douce, parce qu'il n'y aurait pas le problème du sel et qu'on pourrait réutiliser le réacteur, c'est ça ? »

«Oui, c'est ça. »

«Moi aussi, j'ai longtemps réfléchi à la question. Mais quand on a un besoin énorme d'eau, comme nous en ce moment, on ne peut pas faire une fixation sur l'eau douce. Si la situation continue à évoluer comme ça, je pense qu'on ne pourra pas faire autrement que de recourir à l'eau de mer. »

« J'ai bien compris les arguments du terrain. »

«Si nous en avons fini avec les histoires d'alimentation en eau, on va passer à la suite. »

$\mathbf{Q}$ : On va s'arrêter ici pour le moment. La scène se situe le 13 mars vers 20h20, c'est-à-dire en soirée. C'est un moment où vous êtes très pris par le réacteur 3 . Concernant le réacteur 2, on voit, à la page 20 de la chronologie que vous aviez donné l'ordre à 12 h05 de commencer à préparer l'injection d'eau de mer. II s'agit du 13 mars à 12 h05.

\section{$\mathbf{R}$ : Vous dites la page 20 ?}

$\mathbf{Q}:$ Oui, ici. Le 13.

\section{$\mathbf{R}:$ Ça concerne le réacteur 2.}

$\mathbf{Q}$ : Oui, le réacteur 2. Quelle était la situation au réacteur 3, à ce moment-là ?

\section{$\mathbf{R}$ : Juste avant qu'on ne passe à l'eau de mer?}

$\mathbf{Q}$ : C'est ça. L'injection d'eau douce s'était terminée le 13 mars à 12h20. Vous vous apprêtiez à passer à la ligne d'eau de mer. C'était juste avant. Comme vous le disiez dans la vidéo, le réacteur 1 ne disposant pas d'eau douce, vous avez tout de suite utilisé l'eau de mer. Le réacteur 3 avait de l'eau douce. Cette eau douce, c'est celle que vous aviez rassemblée de toute la centrale et celle que vous espériez voir arriver de l'extérieur, n'est-ce pas ? Vous aviez donc commencé avec de l'eau douce, mais, finalement, vous avez manqué d'eau et vous êtes passé à l'eau de mer. L'eau douce était épuisée à $12 \mathrm{~h} 20$ et vous avez commencé l'injection à l'eau de mer à $13 \mathrm{~h} 12$. Pour le réacteur 2, également, vous auriez aimé faire de même, avec de l'eau douce, et vous aviez effectivement passé commande. Mais vers midi, vous vous êtes dit que les circonstances pourraient vous forcer à commencer l'injection en urgence et vous avez pensé qu'il était temps de prévoir une injection avec de l'eau de mer. Ce qui va se traduire par cet «ordre du directeur de la centrale de commencer la préparation de l'injection à l'eau de mer », émis à 12 h05.

Par la suite, la situation va se dégrader du côté du réacteur 3 et l'eau qui était dans les camions de pompiers et que vous aviez prévue pour le réacteur 2 va se retrouver mobilisée pour le réacteur 3, qui aura priorité. Du coup, vous n'avez plus de provision pour le réacteur 2. Ce qui va vous obliger à effectuer directement l'injection à l'eau de mer. On ne voit rien dans cette séquence, mais y a-t-il eu des réactions de la résidence du Premier ministre à ce propos ?

\section{$\mathbf{R}$ : Non, rien. Je ne m'en souviens pas.}

$\mathbf{Q}$ : Ensuite, ce M. XXXXX, qui est-il ?

$\mathbf{R}:$ XXXXX est le chef du groupe "réhabilitation » du siège. Le siège a aussi sa cellule de crise, qui est présidée par le Directeur général. Cette cellule compte aussi différents groupes et XXXXX était le chef du groupe « réhabilitation ». 
$\mathbf{Q}: M . X X X X X$ a l'air réticent à l'idée d'envoyer de l'eau de mer. Il dit que ça va faire » pourrir » le matériel, que ce serait du gâchis, il demande si vous ne pourriez pas attendre l'arrivée de l'eau douce. J'imagine que vous l'auriez évidemment fait, si vous aviez eu un espoir raisonnable de la voir arriver. Mais je suppose que vous n'en étiez plus au stade de rêver à l'impossible.

\section{$\mathbf{R}$ : Tout à fait.}

$\mathbf{Q}$ : Vous donnez cet ordre et vous faites en sorte que tout soit prêt au moment où le RCIC va s'arrêter.

R : Oui.

(Pause)

$\mathbf{Q}$ : Vous n'avez jamais reçu de coup de fil en direct de M. Ikeda?

$\mathbf{R}$ : Non, je n'en ai pas le souvenir. C'était au moment où on préparait l'éventage, le terrain était débordé. Là-dessus, on nous a dit que le Premier ministre allait venir ${ }^{20}$. On s'est dit qu'on ne pouvait pas faire autrement que de le recevoir. Le siège nous a dit de préparer sa réception. J'ai un trou de mémoire, peut-être y a-t-il eu une histoire de ce genre avec l'éventage. De toute façon, malgré tous les efforts déployés sur le terrain, on n'y arrivait pas. Alors, nous demander de retarder l'éventage parce que le Premier ministre venait, n'avait pas vraiment de sens pour nous. Imaginez, sur place, on n'arrivait pas à faire bouger les vannes d'un pouce. On faisait tentative sur tentative et on pensait bien qu'on en avait encore pour un certain temps. Non, décidément, je n'en ai pas gardé souvenir.

$\mathbf{Q}$ : Par exemple, n'avez-vous pas reçu un coup de fil de M. Mutô vous faisant part de la demande du vice-ministre Ikeda et vous demandant l'heure approximative de l'éventage?

$\mathbf{R}$ : Peut-être y en a-t-il eu...

$\mathbf{Q}$ : Mais vous ne vous en souvenez pas.

$\mathbf{R}$ : Non, presque pas.

$\mathbf{Q}$ : Est-ce que ça signifie que, comme vous le dites vous-même, ça ne s'est pas imprimé dans votre mémoire parce que cette demande n'a eu aucune influence sur la décision que vous deviez prendre concernant le moment de l'éventage?

$\mathbf{R}$ : Oui. De toute façon, je n'ai jamais pensé que l'éventage serait possible à l'heure où M. Kan s'était annoncé.

$\mathbf{Q}$ : Comme le ministre Kaieda, ministre de l'Économie et de l'Industrie, avait donné l'ordre d'exécution de l'éventage vers les 6h00, le Gouvernement pensait sans doute qu'il pouvait être imminent, étant donné qu'ils ne connaissaient pas la situation sur le terrain. Dans ce cas-là, ils ont dû croire qu'il pouvait être possible que l'éventage se fasse juste au moment où M. Kan allait arriver. En tant que vice-ministre, il s'est sûrement cru obligé de gérer la chose, d'où sa demande pour retarder l'heure de l'éventage.

R : Oui.

$\mathbf{Q}$ : Mais, concrètement, sur le terrain, vous n'étiez pas en mesure d'éventer à ce moment-là. Cette demande n'a donc eu aucune incidence.

$\mathbf{R}$ : Et elle ne m'est même pas restée en mémoire.

$\mathbf{Q}:$ D'accord.

Alors, après $M . X X X X X$, on va passer à l'extrait 26. On vient de changer de date, nous sommes le 14 mars, vers 1 h00. Pour vous situer, c'est un peu avant qu'on ne constate à 
2h20 que la radioactivité était montée vers l'entrée principale et que vous ne fassiez une déclaration suivant l'article 15.

(Vidéo)

«Centrale 1F, m'entendez-vous?»

« Oui, je vous entends. »

" J'ai une affaire urgente. C'est quelque chose dont on a déjà parlé, mais la NISA me demande que vous preniez une décision concernant le moment de passer au réseau FP pour le réacteur 2. Étant donné les problèmes qu'on a eu sur le réacteur 3 , le $\mathrm{HPCl}$ qui était en marche mais qui s'est arrêté tout à coup et la dégradation du combustible qu'on y a connu, la NISA me fait remarquer que, bien que le RCIC soit en fonctionnement pour le moment, elle ne comprend pas pourquoi nous ne passons pas à la pompe FP. Elle nous presse de prendre une décision au plus vite. Et donc, je voudrais que nous prenions une décision maintenant. Quel est votre point de vue à la centrale, concernant ce problème? Pour nous, au siège, si on arrive à vérifier le bon fonctionnement de la vanne SR et si le réseau FP est prêt, nous pensons que la proposition de la NISA présente des avantages. Nous voudrions votre avis, y compris sur les prérequis de cette proposition, et prendre une décision maintenant. Qu'en pensez-vous?»

«Comme je vous l'ai expliqué cette nuit, les préparatifs pour basculer sur le réseau FP sont achevés pour le réacteur 2 . Seulement, le bassin d'eau de mer est vide pour l'instant. Cela veut dire que l'injection dans les réseaux FP du réacteur 1 et du réacteur 2 est aussi suspendue pour le moment. Nous faisons ce qu'il faut. C'est-à-dire que nous sommes en train de renouveler l'eau de mer du bassin. Nous y travaillons, mais l'injection dans les réacteurs 1 et 3 est arrêtée. Quand on aura fini de remplir le bassin avec de l'eau de mer, on pourra de nouveau poursuivre l'injection dans les réacteurs 1 et 3 , et on pourra considérer que les préparatifs seront achevés pour l'injection du réacteur 2 aussi. Toutefois il y a un bémol pour le réacteur 2. Ça aussi, je vous en ai parlé hier soir, il faut qu'on installe encore un compresseur, un compresseur avec un moteur puissant, sur la ligne d'éventage de l'enceinte. Et ça, ça n'est pas encore fait. »

«Excusez-moi. L'engin vient d'arriver de Fukushima Daini. Nous allons le réceptionner, l'emporter sur le terrain et l'installer. »

«Dans ce cas, pour basculer vers le réseau FP, il nous reste le problème de la pression de la cuve. C'est le seul des paramètres essentiels qu'on n'arrive pas à voir. On travaille actuellement à y remédier. On est en train de relier une batterie pour pouvoir lire les chiffres. Dès qu'on aura ces chiffres, on pourra considérer que les conditions sont remplies pour une injection par le réseau « eau de mer ». »

«Bon. On va répondre à la NISA qu'à la base, nous allons dans la même direction qu'elle, mais qu'actuellement les conditions ne sont pas encore remplies, que nous travaillons activement à les remplir et que, dès que tout sera prêt, nous basculerons. Vous êtes d'accord?»

« Oui, d'accord. »

«M. Komori, vous êtes d'accord pour qu'on lui réponde ça? »

«Oui. »

$\mathbf{Q}$ : On va s'arrêter ici. Au début de la séquence, la NISA se manifeste en demandant s'il ne serait pas judicieux d'envoyer de l'eau de mer avec la pompe à incendie à un stade précoce, pendant que le RCIC du réacteur 2 continue à fonctionner. Le siège est d'accord, si toutes les conditions sont remplies pour la manœuvre, et demande l'avis du terrain. Là, on évoque votre nom, mais en fait c'était M. XXXXX. L'autre personne qui parle, qui est-ce?

\section{R : Lui ? C'est XXXXX. Celui qui fournissait les explications à partir de la centrale, c'était XXXXX.}

$\mathbf{Q}$ : D'après ce que raconte $M . X X X X X$, de 1 h10 à 3h20, le 14 mars, c'est ce qui est rapporté dans la chronologie concernant le réacteur 3 , à la page 31, le niveau d'eau du bassin qui se trouve devant le réacteur 3 baisse et vous n'arrivez plus à y puiser l'eau. Le renouvellement 
de l'eau devient indispensable. Et ça n'est qu'à 3h20 qu'on note le redémarrage de l'injection d'eau de mer par les voitures de pompiers. Ce qui veut dire que durant ce temps, l'eau n'était plus injectée.

3h20. On a des dessins de TEPCO qui permettent de voir comment était organisée cette injection par les voitures de pompiers. Ce sont des schémas qui ont été faits à partir du témoignage de différentes personnes présentes. Bien qu'on y ait déjà détecté des erreurs, je pense que le schéma concernant ce moment est à peu près exact. À la page 11, il est noté que la réserve du bassin est épuisée et ce depuis 1h10. La situation, à ce moment précis, est la suivante. Aussi bien pour les réacteurs 1, 2 que 3, un ou plusieurs véhicules de pompiers sont reliés du bassin du réacteur 3 à la bouche de chaque bâtiment turbine, pour envoyer l'eau de ce bassin. L'injection est effective pour les réacteurs 1 et 3 . Et la NISA se demande s'il ne serait pas le moment de faire de même pour le réacteur 2 . Cette suggestion arrive après 1h10. Mais, en fait, à ce moment-là, l'injection dans les réacteurs 1 et 3 est aussi arrêtée, faute d'eau. Vous vous démenez pour réapprovisionner le bassin et, effectivement, vers 3h20, vous redémarrez d'abord par l'injection du réacteur 3. Ici, il est noté que l'eau était «épuisée ». Mais cela ne voulait pas dire qu'il n'y avait plus du tout d'eau, n'est-ce pas?

\section{$\mathbf{R}:$ Non, ce n'était pas tout à fait ça.}

$\mathbf{Q}$ : En fait, il y avait des endroits, dans le bassin, où il y avait plus d'eau. En changeant les points d'aspiration, vous aviez réussi à pomper l'eau restante et vous aviez décidé de l'injecter dans le réacteur 3. Dans les faits, peu après, dans la nuit et jusqu'à l'aube, de nombreux camions de pompiers sont arrivés sur le site. En reliant les camions les uns aux autres, vous aviez là encore réussi à monter l'eau du quai jusqu'au bassin du réacteur 3 . Tout au début du 14 mars, au moment où la date venait de changer, quel était justement pour vous l'ordre des priorités ? Était-ce le réacteur 3 qui vous donnait le plus de soucis ? Si on regarde les situations respectives, le réacteur 2 a son $\mathrm{RCIC}$ qui continue à fonctionner. Le réacteur 1 avait déjà reçu de l'eau de mer, mais l'injection, à ce moment-là, est arrêtée à cause de l'épuisement de l'eau dans le fameux bassin. Pour vous, qu'est-ce qui était le plus préoccupant ? Le réacteur 1 ou le réacteur 3 ?

$\mathbf{R}$ : Concernant le réacteur 1 , il avait déjà reçu pas mal d'eau. Bien sûr, on ne savait pas quelle était l'ampleur des fuites, mais si on considérait le volume d'eau injectée, le réacteur 1 en avait reçu une quantité nettement plus importante que le réacteur 3. La durée d'injection avait été longue. Il était légitime de penser qu'il y avait pas mal d'eau dans le réacteur, eau qui aurait contribué au refroidissement. Par contre, pour le réacteur 3, cela ne faisait pas très longtemps qu'on avait commencé à injecter l'eau. Alors j'ai donné la priorité au réacteur 3. De toute manière, les deux avaient besoin d'eau. Mais si on réfléchissait au volume d'eau déjà injectée, la situation du réacteur 3 était plus critique.

$\mathbf{Q}$ : Et donc, pour vous, l'ordre d'intervention était : d'abord le réacteur 3, avant les autres.

R : Oui.

$\mathbf{Q}$ : En fait, vous vous dites que si le bassin d'eau de mer pouvait à nouveau être rempli, vous pourriez ressusciter l'injection aussi bien du réacteur 1 que du réacteur 3, injection qui pouvait être continue. Vous avez toujours eu le souci de l'alimentation de ce bassin. La mer s'étend en face de vous de manière illimitée, mais le bassin, lui, est limité. Il fallait le remplir d'une manière ou d'une autre. En définitive, des camions de pompiers commencent à arriver par vagues et, puisque vous disposiez à présent d'un moyen pour faire monter l'eau directement de la mer, vous vous dirigez naturellement vers cette solution. Je voudrais savoir si, lorsque vos troupes ont commencé à aligner les camions et les relier les uns aux autres, vous étiez concrètement averti à chaque étape de ce montage?

$\mathbf{R}$ : Oui, j'en étais averti. 
$\mathbf{Q}:$ Ah, vous étiez au courant de chaque étape !

$\mathbf{R}$ : Vous savez, toutes ces informations, nous les partagions avec le siège. On leur disait, "tant de camions de pompiers sont arrivés, nous les alignons de telle manière ».

$\mathbf{Q}$ : Vous receviez donc des rapports vous disant « on va pomper l'eau au niveau du quai et l'envoyer dans le bassin du réacteur 3 ».

R : Bien sûr.

$\mathbf{Q}$ : Non seulement vous receviez ces rapports, mais est-ce qu'autour de la table ronde, vous discutiez de savoir si tel endroit était plus favorable que tel autre?

$\mathbf{R}$ : Vous ne voulez pas dire si on envoyait dans le réacteur 3 ou le réacteur 1 ?

$\mathbf{Q}$ : Non, pas ça. Mais par exemple, si tel endroit du quai était plus favorable pour pomper l'eau.

R : La question de savoir d'où on allait pomper l'eau était une question qui avait déjà été longuement débattue, dès le début. On s'était demandé, par exemple, si on ne pouvait pas pomper l'eau par ici, du côté du réacteur 4. Pour être tout à fait précis, dans le sous-sol du bâtiment turbine du réacteur 4 , il y avait deux personnes qui étaient décédées ${ }^{21}$. Les deux corps étaient encore là et il y avait de l'eau. On avait essayé de récupérer cette eau, car cela nous aurait aussi permis de sortir les corps de ces deux personnes. On avait fait plusieurs tentatives. Il y a ici une entrée pour les encombrants. On avait pulvérisé la porte d'entrée avec un tractopelle, on y avait fait entrer un camion de pompiers pour essayer de pomper l'eau. On a réussi à pomper un peu, mais le niveau d'eau était trop bas pour que ça fonctionne de manière efficace. Comme vous voyez, on avait déjà essayé toutes sortes de choses.

$\mathbf{Q}$ : Vous voulez dire que vous aviez déjà effectué ces essais avant ce moment.

$\mathbf{R}$ : Oui, on l'avait déjà fait. Tout ça n'apparaît pas dans les différents documents, mais, par exemple, à côté du réacteur 4 , il y a un endroit d'où on peut atteindre directement la mer, dix mètres plus bas, sans passer par le palier des quatre mètres. On s'est demandé si on ne pouvait pas pomper l'eau directement de là. Mais l'endroit était couvert de gravats, il y avait très peu de place. Nous avons tout de même descendu un tuyau de là, mais on a pu vérifier qu'il n'était pas possible de pomper l'eau à partir de là. Les hommes sur le terrain avaient déjà procédé à ces vérifications, ce qui a fait que, lorsque les camions de pompiers sont enfin arrivés, nous savions déjà d'où on pouvait pomper l'eau de la manière la plus efficace. Ce sont des informations que nous partagions avec tout le monde.

$\mathbf{Q}$ : Vous venez d'évoquer le cas de ces deux personnes qui ont perdu la vie. Quand avezvous pu procéder à la récupération des corps?

$\mathbf{R}$ : Leur cas me préoccupait tellement que, dès le premier jour, je voulais que des plongeurs aillent à leur recherche. De fait, on a fait venir des personnes compétentes de Kashiwazaki, mais la radioactivité était trop forte. Elles n'ont pas pu plonger. Pendant tout ce temps où on recherchait l'eau, je ne pouvais me défaire de leur image. C'est pour ça que je tenais tellement à ce qu'on fasse des tentatives pour récupérer l'eau du réacteur 4. J'espérais qu'au cours de l'opération, on puisse les retrouver. Mais mes vœux n'ont pas été exaucés.

$\mathbf{Q}$ : Finalement, quand avez-vous réussi à sortir les corps?

$\mathbf{R}$ : Peut-être deux à trois semaines plus tard. Ça n'est qu'une fois que l'état des réacteurs s'était stabilisé, qu'on avait pu procéder à l'évacuation de l'eau du 
réacteur 4 et que le niveau était suffisamment descendu. Je pense qu'il doit y avoir des notes quelque part. Je crois qu'il s'était bien passé deux semaines. On était peutêtre déjà en avril. Quelque part par là̀22.

$\mathbf{Q}$ : Pour ça, il fallait enlever toute l'eau du réacteur 4 ?

$\mathbf{R}$ : Oui, il fallait la retirer entièrement. Pour en revenir à la vidéo, je n'ai pas grand souvenir de cette séquence. D'ailleurs, je n'apparais pas sur la vidéo. Celui qu'on voit là, c'est Takahashi, du siège. Et pour lui répondre, c'est XXXXX qui parle. Moi, je devais certainement être au téléphone.

Q: Oui, il dit ce que vous auriez dû expliquer.

$\mathbf{R}$ : Oui, XXXXX raconte ce que j'aurais dû expliquer. Je devais attendre un coup de fil du bureau du Premier ministre ou quelque chose de la sorte. Je ne me rappelle pas précisément si c'était à ce moment-là, mais pour les rapports de situation, tout ça, on utilisait le fixe en parallèle. Je pense que ce devait être le bureau du Premier ministre.

$\mathbf{Q}$ : Ça se passe le 14 mars, n'est-ce pas?

R : Oui, à l'aube.

Q: Une fois que M. Hosono avait établi une ligne directe avec vous, il vous téléphonait tous les jours?

$\mathbf{R}$ : Il téléphonait assez souvent. Il me demandait comment ça allait, par exemple.

Q : Et avec le siège, aviez-vous des conversations téléphoniques, avec M. Mutô, par exemple?

R : Avec Mutô? Mutô était plutôt au centre hors site, pas au siège. J'ai eu quelques coups de fil du centre hors site.

Q: M. Mutô vous téléphonait quand il voulait vous parler en privé plutôt qu'en passant par la visioconférence?

R : Oui.

$\mathbf{Q}$ : Bon, on va passer un dernier extrait pour la matinée. C'est le numéro 31, ça se passe à l'aube du 14 mars. II fait tout juste jour. Ça doit se passer vers 6 h00.

(Vidéo)

«Là, il faut vraiment faire attention. Entre 6 h00 et 6h20... »

"Pour le moment, la possibilité que [...] est faible. »

«La pression est montée de $50 \mathrm{kPa}$. On va dépasser [...] avant 7h00. »

"Concernant [...], nous avons les prévisions. Pour les zones concernées, entre 6h00 et 6 h20

[...]. »

«M. Komori, le niveau d'eau est coincé tout en bas. »

"C'est dangereux, ça. »

«Tu dis que le niveau d'eau est coincé en bas ? Il faut prévenir la NISA et le bureau du Premier ministre que la pression de la chambre sèche a augmenté... »

«Oui, que la pression de la chambre sèche est montée en flèche... »

«Et que le niveau d'eau... Quoi ? Appelle-les vite. »

«L'indicateur est coincé tout en bas depuis 6h10. Ça a baissé. »

"C'est bon pour le groupe 1. »

« Ça a baissé et ça s'est coincé à 6 h10 [...]. »

"Ça va un peu se superposer. » 
«Tu peux nous trouver un téléphone qui nous relierait en continu avec la NISA et le Premier ministre?»

«Pour les zones où ça va se superposer, on va arrêter (l'électricité) à partir de [...]. On fait comme on avait prévu. »

«Vous voulez dire la NISA et le Premier ministre en simultané ? On a donné des ordres dans ce sens.»

« Je pense qu'il faudrait ouvrir la vanne d'éventage du haut assez vite. Est-ce que la batterie est en place?

$\mathbf{a}$ : On va arrêter ici. Nous sommes entre 6 h00 et 6 h20 et c'est assez confus, avec les histoires de délestage qui viennent empiéter.

$\mathbf{R}$ : Il s'agit du réacteur 3.

$\mathbf{Q}$ : Oui, le réacteur 3. C'est au moment où la pression de la chambre sèche augmente d'environ $50 \mathrm{kPa}$. C'est vers $6 \mathrm{h00}$, le 14. Ce qui était à $425 \mathrm{kPa}$ à 6 h00 est monté à 470 . Une hausse d'environ $50 \mathrm{kPa}$. D'autre part, le niveau d'eau continuait à descendre. Vous étiez déjà dans des zones en-dessous du TAF. En fait, le 14 mars vers 6h20, vous n'aviez pas pu lire les chiffres. Que ce soit sur le système A ou le système B. Ensuite, vers 6h30, vous avez - 100 en A et - 3500 en B. Vous alertez personnellement le siège du danger. Quand nous nous sommes vus la dernière fois et que nous avons parlé du réacteur 3 , vous m'aviez dit que cette situation où la pression de la chambre sèche se baladait autour de $500 \mathrm{kPa}$ ne vous plaisait pas du tout, que c'était dû à l'expérience que vous aviez faite de la tranche 1 où l'explosion d'hydrogène s'était produite vers cette pression-là et que, physiquement, cela vous mettait mal à l'aise. En fait, dans cette séquence, nous sommes en plein dans cette situation. La personne à qui vous parlez au siège, c'est bien M. Komori ? Dans la vidéo, vous réclamez une ligne téléphonique qui vous mette simultanément et continuellement en relation avec la NISA et le bureau du Premier ministre. Vous, à la centrale, vous ne saviez pas à ce moment-là comment étaient organisées les liaisons entre le siège et le bureau du Premier ministre ou la NISA?

$\mathbf{R}$ : Non, pas du tout. Je ne savais pas du tout qui était où et comment la cellule de crise du siège communiquait avec les autres.

$\mathbf{Q}$ : Ils devaient communiquer par téléphone. À ce moment-là, il n'y avait pas de système de téléconférence, il me semble.

$\mathbf{R}:$ Non.

$\mathbf{a}$ : En dehors de ça, il n'y avait pas de système de téléconférence qui permette de voir ce qui se passait dans la centrale, à ce moment-là?

$\mathbf{R}$ : Je pense que le système ne couvrait pas le bureau du Premier ministre. En fait, je n'avais aucune idée de la manière dont le siège communiquait avec les autres instances.

$\mathbf{Q}$ : Donc, vous n'en saviez rien. En tout cas, face à cette situation d'urgence, vous aviez directement prévenu M. Komori en passant par la téléconférence.

$\mathbf{R}$ : Je me rappelle. Ces histoires de délestage venaient nous perturber. Ça m'a terriblement énervé. J'avais demandé qu'ils arrêtent, mais ils revenaient toujours à la charge.

$\mathbf{Q}:$ Le délestage, c'était une histoire d'économie d'énergie?

R : Oui. On ne produisait plus d'électricité à Fukushima Daiichi et Daini, alors que Daini tournait à plein régime et que nous, à Daiichi, nous avions trois réacteurs en service. En plus, la centrale thermique de Hirono ${ }^{23}$ ne produisait plus non plus. On n'avait plus d'électricité et on prévoyait qu'on aurait des difficultés à fournir l'électricité pour le lendemain. Alors on avait décidé d'adopter des arrêts programmés de distribution. En fait, il y avait deux problèmes. Avant même que la 
presse et le public ne se focalisent sur le problème des réacteurs nucléaires, ils avaient fait tout un foin autour des délestages de TEPCO. C'est pour ça que les deux s'entremêlent dans cette séquence.

$\mathbf{Q}$ : Oui, on voit que tout se mélange.

Nous allons en rester là pour ce matin. Nous reprendrons cet après-midi. Nous allons faire une pause maintenant et reprendre à partir de 13 h00.

\section{(Pause)}

Q : Nous allons reprendre maintenant.

Nous allons attaquer l'après-midi avec cet extrait du 14 mars. Le jour vient de se lever, ça se passe entre 6 h00 et $7 \mathrm{~h} 00$. On revient à l'histoire du réacteur 3 . La pression de la chambre sèche monte anormalement. La dernière fois, je crois que c'était la dernière fois que nous nous sommes vus, vous m'avez fait remarquer que vous aviez ordonné un retrait des hommes du terrain parce que ces chiffres vous mettaient mal à l'aise et que cet ordre n'apparaissait pas dans les différentes chronologies. On retrouve effectivement des échanges qui correspondent à cet ordre de retrait dans cet extrait. Nous allons le vérifier ensemble.

(Vidéo)

«Yoshida, tu m'entends ? Yoshida! »

«Oui. »

« II me semble que d'après le protocole on n'exécute l'éventage de la chambre sèche que Iorsqu'on atteint à peu près le double de la pression de conception. C'est ce que vous allez faire?»

«Ce qui veut dire $800 \mathrm{kPa}$. »

«Oui, oui. »

« Oui, théoriquement c'est bon. Mais la montée de la pression est tellement brutale... »

«Ah, oui ?»

"Cela me préoccupe beaucoup. Et puis le niveau d'eau dans la zone du combustible est au plus bas depuis 6h20. Là aussi, la descente a été rapide. Ce qui veut dire que ce doit être bien découvert. En forçant un peu, on pourrait dire qu'on a déjà atteint le pire des scénarios. »

« D'accord. »

"Vu la situation, je me demande s'il ne faudrait pas réfléchir en priorité au sort du personnel, parce qu'ils sont assez nombreux à travailler devant la tranche. Ici aussi, dans le bâtiment antisismique, il y a beaucoup de monde. »

« D'accord. De toute façon, il faudra éventer du côté de la chambre sèche quand la pression atteindra le double. Je prévois que ça puisse arriver assez vite. On va demander qu'on nous calcule la quantité de particules qui va être relâchée. »

«La pression de la chambre sèche est de $520 \mathrm{kPa}$ à 6h35. »

"Quoi ? Je n'ai pas entendu. »

« 500 ? Ah, la chambre sèche à 520 ! »

"La situation est vraiment préoccupante. Dis, tu peux nous sortir au plus vite les chiffres pour qu'on ait une idée des particules qu'on va lâcher? Tu comprends, ça va nous mener à des histoires d'évacuation de la population. Savoir s'il faut étendre la zone d'évacuation ou pas. Il faut qu'on puisse évaluer tout ça. Demande à ce qu'on se dépêche de nous donner les chiffres, tu veux bien?»

«On est en train de faire les calculs. Donnez-nous encore cinq minutes. » 
«Ensuite, du point de vue de la protection des ouvriers, il y a le problème de l'orientation du vent. Pour le moment, il souffle un peu vers Ôkuma. S'il y a des gens dehors, ça va être un peu dangereux devant le réacteur 3. »

« M. Mutô, M. Mutô, je suis conscient que les travaux vont s'arrêter si on fait ça, mais est-ce que vous me permettez de rappeler le personnel qui est sur le terrain pour les mettre à l'abri ici?»

« D'accord. »

«Ordre de retrait. «Toutes les personnes, personnel de TEPCO et personnel des entreprises partenaires, qui sont sur le terrain, veuillez quitter le terrain et venir vous réfugier dans le bâtiment antisismique. » II y a aussi du personnel dans les salles de contrôle. Qu'est-ce qu'on va en faire? II vaut mieux qu'ils viennent aussi se mettre à l'abri ici. »

«Décidez en fonction de la radioactivité dans les salles de contrôle. Tu peux connaître la radioactivité dans les salles de contrôle?»

«Oui, la radioactivité dans les salles de contrôle. Où en êtes-vous de la radioactivité dans les salles de contrôle? On va vérifier ça. »

«Écoutez tout le monde. Nous sommes tous reliés par téléphone. La NISA et le bureau du Premier ministre sont aussi reliés avec nous. Ne coupez pas, laissez le volume à ce niveau qu'ils entendent aussi ce que nous disons. Nous devons maintenant discuter de ce que nous allons faire, car si on retire le personnel de la salle de contrôle, on ne pourra pas procéder à l'éventage. »

" Je vous l'ai dit, ce sera en fonction de la radioactivité dans la salle de contrôle. »

"Yoshida, mesure bien la radioactivité, y compris là où tu es. »

«Oui. »

«XXXXX, mesurez avec soin tout alentour. »

«Nous en sommes à $28 \mu \mathrm{Sv} / \mathrm{h}$ au maximum ici, dans cette pièce. Pour les postes de monitoring, la radioactivité augmente sur une grande zone. On va mesurer ça soigneusement. »

«Si vous en êtes à 28 dans la cellule de crise, je pense qu'il n'y a pas de problème pour vous. »

«Voici les données les plus récentes des postes de monitoring. Près du poste 2 , vers 6 h00, on en était à $400 \mu \mathrm{Sv} / \mathrm{h}$. Près de l'entrée principale, 6,65 $\mu \mathrm{Sv} / \mathrm{h}$ à 5h00. »

«Ce qui veut dire, Yoshida, que les chiffres n'ont pas beaucoup bougé aux alentours. »

$\mathbf{Q}$ : On va couper ici. La pression de la chambre sèche du réacteur 3 est très élevée. Au milieu de l'extrait, vous avez un échange avec M. Mutô. Bien que conscient que les travaux ne pourront plus avancer, vous demandez la permission de retirer le personnel du terrain. II accepte. L'ordre de retrait est donné. Vous dites textuellement: "personnel de TEPCO, personnel des entreprises partenaires, veuillez quitter le terrain et venir vous réfugier dans le bâtiment antisismique ». Aviez-vous un moyen de joindre les hommes sur le terrain pour leur transmettre l'ordre?

R : Oui. À ce moment-là, nous disposions de talkies-walkies pour communiquer avec le personnel sur le terrain. C'est d'ailleurs le moyen que nous avons utilisé pour prévenir les gens qui étaient à l'injection avec les véhicules de pompiers ou les gens qui réapprovisionnaient en eau. Ils n'étaient pas très dispersés. On a pu les joindre par talkies-walkies.

$\mathbf{Q}$ : Ce qui fait que les hommes qui étaient à la préparation de l'éventage ou à l'injection, vont se retirer un temps pour se mettre à l'abri. Ce retrait, il va durer combien de temps?

\section{$\mathbf{R}:$ L'extrait était de quelle heure?}

$\mathbf{Q}$ : Assez tôt dans la journée, il y avait des échanges de manière continue à partir de 6h30. Ça doit se passer vers $6 \mathrm{~h} 30,6 \mathrm{~h} 40$, je pense. Vous faites revenir tout le monde et vous vous demandez ce que vous allez faire du personnel qui est dans les salles de contrôle. On vous 
conseille de mesurer la radioactivité des salles. Une fois que le personnel sur le terrain s'est mis à l'abri, je suppose qu'à un moment ou un autre, vous donnez l'ordre de retourner vers le terrain. D'après les documents, l'explosion se produit ce jour à $11 \mathrm{~h} 01$. Par rapport à cet évènement, ça se situe quand?

\section{$\mathbf{R}$ : Environ deux heures.}

$\mathbf{Q}$ : Le retrait va durer environ deux heures? Au bout de ce temps, vous autorisez les troupes à retourner travailler sur le terrain, quand l'explosion se produit. C'est bien ça? L'ordre de retrait, il n'y en a eu qu'un ce jour-là ?

R : Oui, un seul, ce jour-là.

$\mathbf{Q}:$ : n'y a pas eu d'autres retraits?

R : Non. J'ai donné une seule fois l'ordre de retrait, pour voir comment allait se comporter l'enceinte de confinement.

$\mathbf{Q}$ : Justement, l'enceinte tournait autour de 500 de pression. Elle ne donnait pas signe d'une augmentation brutale. II fallait avancer les préparatifs pour l'éventage. II fallait aussi travailler à l'injection. Vous étiez tiraillé entre l'impératif d'avancer ces travaux et la sécurité de vos hommes, mais il fallait se décider à un moment ou un autre. Vous avez surveillé l'évolution de la pression de l'enceinte de confinement et décidé de recommencer les travaux.

Quand on regarde l'extrait, on entend: "nous sommes tous reliés par téléphone. La NISA et le bureau du Premier ministre sont aussi reliés avec nous. Ne coupez pas, laissez le volume à ce niveau qu'ils entendent aussi ce que nous disons. Nous devons maintenant discuter de ce que nous allons faire, car si on retire le personnel de la salle de contrôle, on ne pourra pas procéder à l'éventage ». Vous êtes donc tous reliés par téléphone, y compris la NISA et le bureau du Premier ministre. Du coup, il y a de fortes chances qu'ils aient partagé avec vous ces informations. La NISA ou le bureau du Premier ministre ont-ils pris contact avec vous à cette occasion?

$\mathbf{R}:$ Pas à ce moment-là.

$\mathbf{Q}:$ Pas spécialement à ce moment-là.

$\mathbf{R}$ : Non, on n'a pas eu de contact.

$\mathbf{Q}$ : Bon. Pour résumer grossièrement, entre 6 h30 et 40, vous donnez l'ordre de retrait. Le retrait dure environ deux heures. Pendant ce temps, vous surveillez le comportement de l'enceinte de confinement, qui ne bouge pas. Vers 8h30, 9h00, vous renvoyez les hommes sur le terrain pour faire avancer les travaux, quand, vers 11h00, vous subissez l'explosion. C'est à peu près ça?

R : Oui.

$\mathbf{Q}$ : Alors, passons à la suite.

(Vidéo)

"La radioactivité au niveau du siège du chef d'équipe dans la salle de contrôle des réacteurs 3 et 4 est de 3,5 mSv. Elle est plus faible qu'hier. Nous en sommes à 15. Si on fait la moyenne dans la salle de contrôle des réacteurs 3 et 4, près de [...] nous en sommes à 15. »

«15 mSv?»

«Oui, $15 \mathrm{mSv}$. »

«Ces données datent de quand? De maintenant? Ce sont les chiffres actuels? »

"Concernant les 3,5, ce sont les chiffres actuels. 3,5 mSv/h, au siège du chef d'équipe, ce sont les chiffres de maintenant. »

«À peu près 6h45, donc. » 
« Là non plus, il n'y a pas grande différence par rapport à hier. »

«Non, pas grande différence. Continuez à relever la radioactivité avec soin. »

«Pour en revenir à la pression de la chambre sèche, à 6h35, 6h40,6h45, on est toujours à $520 \mathrm{kPa} . »$

«La situation a l'air d'être assez stable. Est-ce qu'on ne réfléchirait pas de nouveau à ce que nous allons faire, y compris la reprise des travaux sur place?»

"Oui. Bien sûr, l'enceinte de confinement a l'air stable, mais il y a encore beaucoup de risque qu'on subisse la même chose que pour le réacteur 1. Avec cette pression dans l'enceinte, il y a de l'hydrogène. Et dans ce sens-là, il me semble très difficile d'envoyer des gens sur le terrain. »

«Où en est-on finalement pour la préparation de l'éventage? »

"Comme il s'agit du même panneau de la salle de contrôle, il suffit de déconnecter le soutien de la chambre de dépressurisation et de relier du côté de la chambre sèche. C'est une opération qu'on peut faire sans temps mort. »

«OK. Et pour l'injection? II n'y a pas d'eau en ce moment. »

« $\mathrm{Si}$, on continue à envoyer de l'eau. On envoie $900 \mathrm{l} / \mathrm{min}$ d'eau de mer. »

"Ah, vous continuez à envoyer de l'eau. »

«Oui. »

«Vous arrivez à réapprovisionner?»

"D'après ce que j'ai entendu tout à l'heure, pour le moment, il y a tout juste de quoi alimenter le réacteur 3. Évidemment, on ne peut plus réapprovisionner à nouveau, puisqu'on n'a plus personne sur le terrain. C'est le point le plus préoccupant. »

«Effectivement, c'est préoccupant. Je suis d'accord pour qu'on procède en donnant la priorité à la sécurité des hommes, mais est-ce qu'on ne pourrait pas réfléchir à un moyen d'assurer l'alimentation en eau ? »

«Ça va être difficile. Pour le moment, nous comptons uniquement sur les hommes pour la transporter. »

"Comme moyen de faire baisser la pression de la cuve, est-ce qu'on ne pourrait pas envisager de tirer de l'électricité jusqu'aux vannes L 005 et L 006 sur la ligne d'évacuation de la ligne MS (Main Steam) ${ }^{24}$ et laisser la pression s'échapper vers le condenseur? Excusez-moi, je me suis trompé dans le numéro des vannes MO. »

«Oui, mais l'alimentation électrique des vannes MO est totalement morte. En plus, pour le moment, on ne peut pas approcher de la chambre sèche, ni pénétrer dans le bâtiment réacteur. C'est totalement impossible. »

«D'accord. »

«Nous venons de recevoir les prévisions d'irradiation. Des chiffres encore très grossiers. Je vous les communique. En supposant que la quantité relâchée dans le scénario du pire soit $100 \%$, nous avons fait des calculs avec $25 \%$, ce qui nous fait à peu près le quart [...] des résultats que nous obtenons avec le pire des cas. De toute manière, il y aura en majorité de l'iode. On a calculé les chiffres, au bout de trois heures, avec un vent de $2 \mathrm{~m}$ soufflant au SSE. Le point le plus irradié se situerait à $2,2 \mathrm{~km}$, juste à la limite du terrain de la centrale, il y aurait $5700 \mathrm{mSv}$. Si on prend le quart, cela ferait $1600 \mathrm{mSv}$. Ce serait de l'iode. Ensuite, puisque cette ligne matérialise la zone des 1000 mSv, la zone des 250 mSv s'étendrait vers la commune de Sôma ${ }^{25}$ en moins de trois heures. »

« $250 \mathrm{mSv}$ vers le nord, c'est bien ça?»

«Oui. Nous surveillons les vents, mais il est difficile de dire si c'est le vent d'est qui l'emporte ou le vent d'ouest. Selon qu'on privilégie l'un ou l'autre, les résultats sont bien sûr très différents. Mais pour le moment, nous n'arrivons pas à déterminer quel est le vent dominant. Désolé. » 
«Continuez à nous décrire la situation au fur et à mesure et à discuter avec le bureau du Premier ministre. »

«Les $20 \mathrm{~km}$, ça se situe où? »

«Là, c'est la zone des $5 \mathrm{~km}$. À peu près $5 \mathrm{~km}$. Ensuite, on progresse, 10 km, 20 km. Là, c'est à peu près la zone des $20 \mathrm{~km}$. »

«Et quelle est la dose à $20 \mathrm{~km}$ ?»

«À $20 \mathrm{~km}$, il y aurait $250 \mathrm{mSv}$. »

« $250 \mathrm{mSv} / \mathrm{h} ? »$

« Non, pas par heure. Ce serait 250 mSv en dose cumulée. Là, ce sont les prévisions trois heures après le lâcher, donc, avec les heures qui passent, la radioactivité va encore un peu augmenter. »

«Y a-t-il un organisme pour s'occuper de ce genre de chose au centre hors site ? Une commission d'évaluation ou quelque chose de la sorte. Normalement, il devrait y avoir ça, non?»

"Ici, le groupe «énergie». Comme on ne peut pas utiliser les postes fixes de monitoring depuis le séisme, nous avons positionné une des voitures de monitoring près du poste 4 , juste à l'ouest de la centrale. [...] On vient de retirer celle qui était à proximité du poste 2, elle est en train de se déplacer. Quand on commencera à lâcher la vapeur, on mesurera la radioactivité à partir de la voiture et on se déplacera vers le sud. On va déjà positionner la voiture de façon à attendre l'arrivée du nuage et, ensuite, on se déplacera avec lui. »

«Je vous communique la radioactivité de la cellule de crise. Concernant cette pièce, les chiffres du moniteur et des rayons gamma sont de 0,014 $\mathrm{Sv} / \mathrm{h}$. II n'y a pas de changement. Nous n'avons pas non plus détecté de neutron. »

« M. Komori, la NISA et le bureau du Premier ministre sont au courant de la situation?»

« Il y a notre correspondant là-bas et ils entendent tout ce qu'on raconte par téléphone. »

«Très bien. »

$\mathbf{Q}$ : On va s'arrêter ici. Là, les hommes ont été mis à l'abri et vous avez un échange avec M. Mutô. La pression de la chambre sèche est d'environ $520 \mathrm{kPa}$. Elle n'a pas l'air d'augmenter et paraît relativement stable. M. Mutô propose de réfléchir avec vous à une éventuelle reprise des travaux, qui paraît indispensable. Vous, vous êtes préoccupé par, non seulement, la pression de l'enceinte, mais encore plus par la fuite d'hydrogène. Avant même la discussion de savoir si l'enceinte de confinement tient le coup ou pas, la fuite et l'accumulation d'hydrogène est un phénomène qui ne se mesure pas à la pression de l'enceinte. Je pense que vous serez d'accord pour dire que, même aujourd'hui, vous ne pouvez pas prévoir la production d'hydrogène en vous basant sur la pression. Raison de plus à ce moment-là: vous n'avez aucun moyen de mesurer ça. En comparant cette situation à celle du réacteur 1, vous estimez que le réacteur 3 s'est installé durablement dans un état dangereux et vous exprimez votre très grande réticence à envoyer du monde travailler sur le terrain.

\section{$\mathbf{R}:$ C'est ça.}

$\mathbf{Q}$ : Par rapport à votre position, M. Mutô n'a pas opposé d'arguments contraires, il me semble. Après cette discussion, vous continuez à effectuer des mesures de radioactivité, etc. II me semble que nous l'avons eu à un moment donné à l'écran, il a été question d'un éventage au niveau de la chambre humide. N'avez-vous pas envisagé un éventage du côté de la chambre sèche?

\section{$\mathbf{R}:$ Si, bien sûr, nous l'avons envisagé.}

Q: Vous l'avez donc envisagé. Mais, dans les faits, vous ne l'avez pas exécuté pour le réacteur 3 , n'est-ce pas?

$\mathbf{R}$ : C'est parce que l'éventage de la chambre humide a été exécuté avant. Pendant qu'on procédait à cet éventage, l'explosion s'est produite et, on ne sait pas bien pourquoi, la pression était descendue. Comme la baisse de la pression a été 
importante, à la limite, on a perdu l'occasion d'exécuter l'éventage de la chambre sèche. En fait, on l'avait envisagé, mais on n'a pas eu le temps de l'exécuter.

Q: Vous voulez dire que vous avez exécuté le seul éventage de la chambre humide sur le réacteur 1 et que, pour le réacteur 2, vous aviez envisagé, en plus de l'éventage de la chambre humide, l'éventage de la chambre sèche. La possibilité d'un éventage de la chambre sèche avait bien été évoquée.

\section{$\mathbf{R}$ : Pour le réacteur 3 .}

Q: Oui, pour le réacteur 3. Seulement, vous n'avez pas eu l'occasion d'en arriver concrètement à l'ouverture de la vanne ou ce type d'opération. C'est bien ça ?

$\mathbf{R}$ : Tout à fait. On a vu dans l'extrait que le siège se préoccupe de l'évaluation de la radioactivité. Je pense que c'était en vue d'un éventage de la chambre sèche.

$\mathbf{Q}$ : Vous avez raison. De la manière dont ils en parlent, ce doit être ça.

$\mathbf{R}$ : Donc, je pense bien qu'à ce moment-là, XXXXX du siège était en train de faire des calculs dans l'hypothèse d'un éventage de la chambre sèche.

$\mathbf{Q}$ : D'accord. Alors, lorsque l'explosion de la tranche 3 s'est produite, vous étiez déjà en plein milieu des préparatifs pour cet éventage.

\section{R : Oui.}

$\mathbf{Q}$ : On va regarder l'extrait 33, maintenant. C'est juste pour vérification. Les travaux se poursuivent autour du réacteur 3 , mais la situation étant devenue dangereuse, vous donnez l'ordre de retrait. Naturellement, la NISA et le bureau du Premier ministre sont au courant, puisqu'ils sont reliés par téléphone et entendent tous les échanges que vous avez. L'extrait que je voudrais vous montrer maintenant évoque les relations avec la presse. Savoir si on va communiquer à la presse ou pas.

\section{$\mathbf{R}$ : Les communications à la presse ?}

Q: Oui. Je voudrais que vous regardiez cet extrait et que vous me disiez si vous vous souvenez de cette scène. On entend des déclarations du genre "on a arrêté les communications à la presse » et ça a l'air de concerner les informations concernant l'état de la tranche 3.

$\mathbf{R}$ : C'est la première fois que j'entends parler de ça. Vous dites l'extrait 33 ?

$\mathbf{Q}$ : Oui. À la page 9 . On va vous passer une séquence où le problème est évoqué. Si vous vous souvenez des circonstances, je voudrais vous poser quelques questions. ( $A u$ technicien) Allez-y.

(Vidéo)

«Centrale Daiichi, m'entendez-vous ? Centrale Daiichi. »

«Oui, c'est Yoshida. »

«La NISA nous fait savoir qu'elle est en train de régler deux, trois choses en concertation avec le bureau du Premier ministre. Elle nous informe qu'elle a arrêté de communiquer avec la presse, pour le moment. Elle dit qu'elle ne donne plus d'information. Elle veut attendre que la réalimentation en eau reprenne. Concernant [...] de l'eau, elle partage toujours les informations avec le bureau du Premier ministre. »

«D'accord. »

« Je n'ai pas écouté. Pardon. Vous avez dit qu'on faisait quoi avec le bureau du Premier ministre?»

"Le bureau du Premier ministre et la NISA ont arrêté provisoirement de communiquer avec la presse.»

«Qu'est-ce qu'ils ont arrêté ?» 
«De communiquer avec la presse. »

«Communiquer quoi?»

«Les informations. Les informations concernant l'état actuel. »

« L'état de la tranche 3 ? »

«Oui. Ils ont décidé d'attendre, de se donner un temps d'observation et ils voudraient que vous les teniez au courant de l'évolution de la situation. »

« D'accord. »

«Excusez-moi de vous interrompre. Je vous informe que le camion-citerne que nous fournissent les forces d'autodéfense est plein. »

«Écoutez-moi tous, ce qui va être le plus important maintenant, c'est de surveiller soigneusement les paramètres de chaque réacteur. Ça, ça va être le point le plus important. Ensuite, surveiller minute à minute la radioactivité, bien suivre l'évolution des chiffres. Ces deux choses doivent être assurées en priorité. Après, encore une chose très importante actuellement, l'eau. Je voudrais qu'on nous informe de l'état d'avancement des travaux de réalimentation en eau. Je remarque que les membres de l'équipe "eau » sont amenés à s'absenter assez souvent. Lorsque vous partez pour une opération extérieure, désignez quelqu'un comme agent de liaison, qu'il puisse répondre tout de suite aux questions. D'ailleurs, je vais le faire tout de suite. Dites-moi combien de temps il va falloir pour organiser une ligne en utilisant le réseau d'eau filtrée et combien de temps il va falloir prévoir pour le réapprovisionnement. Quelqu'un peut répondre ? À qui dois-je m'adresser ? »

$\mathbf{Q}$ : (Au technicien) Vous pouvez arrêter momentanément ici. (À Yoshida) Ce n'est peut-être pas très parlant si on ne voit que ce bout d'extrait. Mais si on regarde à la page 6 du numéro 36, c'est un peu la même chose.

\section{$\mathbf{R}:$ L'extrait qui commence à 9 h52 ?}

$\mathbf{Q}:$ Oui, celui-là.

\section{$\mathbf{R}:$ Je vois.}

$\mathbf{T}$ : Je crois que c'est une autre version.

$\mathbf{Q}$ : C'est une version différente ? C'est intitulé $1,1 / 3$, c'est l'extrait où on voit le Directeur général Shimizu. Le groupe «communication » du siège parle de l'information donnée par la NHK.

T: Ah, oui. Il faut le Directeur général Shimizu. C'est ici ? Non, ça n'a pas l'air.

$\mathbf{Q}$ : On le voit déjà dans un extrait précédent. L'extrait qu'on cherche se situe un peu après. Vous voyez? Tout en bas le groupe "communication» du siège est cité. C'est cette fameuse histoire de l'arrêt de la communication avec la presse. "Tout à l'heure la NISA nous a demandé de ne plus communiquer avec la presse. " «Concernant le réacteur 3 de la centrale de Fukushima Daiichi et la hausse anormale de la pression dans l'enceinte de confinement, tout à l'heure la NHK a rendu publique l'information du retrait provisoire des ouvriers du terrain à la suite de cette hausse de la pression de l'enceinte de confinement. Il est à prévoir que la presse nous questionne. Pour le moment, si on peut, on fera un point presse. Même si celui-ci n'est pas possible, nous répondrons oralement aux questions », peut-on lire. Quand on regarde ça, on ne sait pas trop si c'est la volonté de la NISA ou celle du bureau du Premier ministre, mais on sent que l'État a une attitude très hésitante quant à cette situation de l'enceinte de confinement du réacteur 3.

\section{$\mathbf{R}$ : Oui, visiblement, ils n'arrivent pas à se décider.}

$\mathbf{Q}$ : On a l'impression qu'ils ont peur que, s'ils révèlent la situation dangereuse de l'enceinte sans que l'injection d'eau ne soit rétablie, cela ne crée la confusion, que cela ne fasse monter inutilement l'inquiétude dans la population et que, du coup, ils ont voulu retenir 
l'information pendant un certain temps, au lieu de la livrer tout de suite. Tous ces problèmes de communication, pour vous, ça passait toujours par le siège, n'est-ce pas?

R: Oui. Je n'ai presque pas de souvenir de ces discussions. Pour moi, qu'ils communiquent, qu'ils ne communiquent pas, qu'ils fassent un point presse ou pas, je ne me sentais pas concerné. Qu'ils fassent ce qu'ils veulent! Nous, sur le terrain, on avait d'autres chats à fouetter. Ça a toujours été ma position. Donc, de tout ce qu'ils se disaient, je n'entendais rien.

$\mathbf{Q}:$ Ah, c'est pour ça qu'au début de l'extrait, vous aviez l'air si étonné?

$\mathbf{R}$ : Je suppose que oui.

$\mathbf{Q}:$ À la base, donc, c'est le groupe «communication » du siège qui s'occupait des relations avec la presse, etc.

R : C'était leur boulot. Après, il y avait aussi XXXXX. C'est celui à qui on a interdit de communiquer. Celui qui lui dit que la NISA ne communique plus, c'est XXXXX, du siège. Lui, c'était le chef du groupe «liaison avec les ministères ». En fait, il y a le groupe « communication », qui parle de faire un point presse ou non, et puis celui qui maintient la liaison avec les ministères. De toute manière, que ce soit l'un ou l'autre, c'étaient des problèmes qui dépendaient du siège. Alors la centrale n'avait rien à voir avec ça. Ils n'avaient qu'à se débrouiller tout seuls.

$\mathbf{Q}$ : Je suppose que c'est parce que l'État voulait être avisé dès que l'injection aurait repris, qu'il demandait à être tenu informé de l'évolution de la situation heure par heure. En fait il attendait le moment propice pour communiquer avec la presse. Et pendant que le groupe «communication » du siège et l'homme de liaison avec le Gouvernement se coordonnaient, l'information du retrait provisoire avait filtré par la chaîne NHK. Ensuite, il a bien fallu gérer la situation face à la presse en tenant compte de la fuite. Mais tout ça, pour vous à la centrale, c'était l'affaire du siège.

$\mathbf{R}$ : Tout à fait. Tout ce qui concernait les relations avec l'extérieur, c'était leur domaine. Nous, nous étions suffisamment absorbés par nos soucis. La pression qui augmentait, l'eau qu'il fallait aller réapprovisionner pour pouvoir injecter. Une fois que j'avais donné l'ordre de retrait, c'est tout ce qui occupait mon esprit. Alors la communication... C'était le dernier de mes soucis.

$\mathbf{Q}$ : Tout ce dont nous venons de parler se passe dans la matinée du 14 mars. Nous allons sauter la fin de l'extrait et passer directement au numéro 36. II y est question de la priorité à donner dans les injections. La séquence se situe vers 10h30, le 14 mars.

(Vidéo)

«Si vous êtes d'accord [...]. Je vous en charge. »

« Yoshida, je viens d'avoir un coup de fil de M. Takekuro. Le bureau du Premier ministre [...]. Il est au téléphone. »

«Le bureau du Premier ministre [...]. »

« J'ai bien reçu un ordre émanant du bureau du Premier ministre, par l'intermédiaire de M. Takekuro. II s'agit de continuer le refroidissement du réacteur 3 avec 10 t (d'eau) et d'utiliser prioritairement le restant pour refroidir le réacteur 1. »

«Bien compris. »

«Très bien. »

«XXXXX, XXXXX, concernant ce dont on vient de parler, étudie vite comment on peut faire ça. Tu baisses le refroidissement du réacteur 3 à $160 \mathrm{l} / \mathrm{min}$. Tu mets le reste dans le réacteur 1. Étudie ça d'urgence. Qui peut faire ça? XXXXX et XXXXX? Étudiez bien la question et tenez-moi au courant du moment où vous allez commencer. » 
$\mathbf{Q}$ : Stop. Ici, il est question d'un ordre émanant du bureau du Premier ministre. Vous êtes en train de parler avec M. XXXXX?

$\mathbf{R}$ : Non. Je parle avec Takahashi.

$\mathbf{Q}:$ Ah, c'est quelqu'un d'autre?

$\mathbf{R}$ : Oui, c'est Takahashi, le «fellow ${ }^{26} »$. Quand Komori n'était pas là, il le remplaçait. Vous vous souvenez, le directeur du centre de gestion était au centre hors site. Je veux dire, le directeur du centre de gestion de crise du Siège était Mutô ${ }^{27}$. Celui qui le remplaçait au siège, lorsqu'il s'absentait, était le directeur adjoint Komori. Quand Komori, lui aussi, s'absentait pour un point presse ou autre chose, c'était Takahashi qui le remplaçait. Celui dont on a parlé tout à l'heure, XXXXX, a toujours été affecté à la liaison avec les ministères. Il échangeait des informations avec les ministères. Celui avec qui je parle du coup de fil de Takekuro, qui était au bureau du Premier ministre, c'est Takahashi.

$\mathbf{Q}$ : Et concrètement, M. Takahashi et M. Takekuro se sont parlés.

$\mathbf{R}$ : Oui, je suppose que d'une manière ou d'une autre ils ont été en contact.

$\mathbf{Q}$ : L'ordre consistait à continuer le refroidissement du réacteur 3 avec 10 t d'eau.

R : Oui, 10 t à l'heure.

$\mathbf{Q}: 10$ t par heure dans le réacteur 3 et le reste, prioritairement, dans le réacteur 1.

$\mathbf{R}$ : En fait, à ce moment-là, on n'injectait que dans le réacteur 3 . Et donc, on me donnait l'ordre d'injecter aussi dans le réacteur 1. Et l'eau dont on disposait à ce moment-là devait être répartie de la manière suivante: tout d'abord, $10 \mathrm{t}$ pour le réacteur 3 , le reste, ensuite, pour le réacteur 1 . L'ordre était de refroidir le réacteur 1 en parallèle.

$\mathbf{Q}$ : Est-ce qu'il vous est arrivé de recevoir des directives assez précises, comme celle-ci, de la part du bureau du Premier ministre, concernant le débit de l'injection ou la priorité à donner aux réacteurs sur le terrain?

$\mathbf{R}$ : Oui, il y en a eu quelques-unes. Je pense qu'il devait y avoir des analystes ou d'autres personnes au bureau du Premier ministre, qui devaient faire des calculs. Il faudrait demander confirmation à quelqu'un qui y était. Je ne sais pas si c'étaient des hommes de la NISA ou de la NSC. Je ne leur ai jamais posé la question, mais je suppose que c'étaient des gens comme ça. Ils ont dû faire des calculs pour savoir quel serait le danger si on arrêtait l'injection dans le réacteur 1 pendant «x heures ». Et ces résultats ont dû atterrir quelque part au bureau du Premier ministre, et, je ne sais trop par quel chemin, arriver à Takekuro qui nous les a fait suivre. Tout ça est assez opaque pour nous.

$\mathbf{Q}$ : Ils vous demandaient donc de refroidir le réacteur 1 en priorité avec le reste d'eau. Et, de fait, vous étiez déjà en train de vous orienter dans cette direction, n'est-ce pas?

R : Oui.

$\mathbf{Q}$ : La scène se situe bien avant l'explosion. La situation, à ce moment-là, était la suivante. Des camions de pompiers étaient arrivés. Vous les aviez reliés les uns aux autres pour monter l'eau à partir du quai et remplir le Reversing Valve Pit ${ }^{28}$ du réacteur 3 . Vous aviez construit des lignes qui permettaient, à partir de ce bassin, d'injecter de l'eau dans les réacteurs 1, 2 et 3, en utilisant, un, deux ou trois camions. Au moment de l'explosion de la tranche 3, il n'y avait que la ligne d'injection du réacteur 1 qui était en fonctionnement, les autres étaient encore en attente. C'est bien ça?

R : Oui. 
$\mathbf{Q}$ : C'est vers 9h20, une fois que vous aviez renvoyé les hommes travailler sur le terrain, que le réseau d'approvisionnement en eau à partir du quai est achevé, bien avant l'explosion de la tranche 3. Un amateur comme moi pourrait penser qu'à partir de ce moment, vous disposiez d'autant d'eau de mer que vous vouliez et que, même si une injection aurait été un peu prématurée pour le réacteur 2 , vous auriez très bien pu injecter à la fois dans le réacteur 1 et le réacteur 3. Ou bien, le volume d'eau était-il encore insuffisant?

\section{$\mathbf{R}$ : Rappelez-moi quel avait été le moment du retrait provisoire.}

Q : Probablement, vers 6h40, le 14 mars. C'est à ce moment-là que vous aviez ordonné le retrait.

$\mathbf{R}$ : C'est ça. Je donne l'ordre de retrait. Ensuite quand est-ce que les hommes sont retournés sur le terrain?

Q: La situation est un peu floue. Quand je regarde les documents, on n'a pas noté le moment précis où vous retirez cet ordre. Tout à coup, il est question d'hommes qui retournent sur le terrain.

$\mathbf{R}$ : Oui, il me semble avoir dit que j'allais renvoyer les hommes travailler. Je pense que j'avais envoyé des hommes en petit nombre, juste pour faire avancer le ravitaillement en eau. C'est d'ailleurs comme ça que le réseau jusqu'au bassin a pu être terminé. Mais là, j'ai un gros trou de mémoire. C'était, bien sûr, avant l'explosion...

$\mathbf{Q}$ : Concernant les différents réacteurs, qu'en pensiez-vous à ce moment-là ? Vous pensiez qu'il n'y avait pas encore urgence pour le réacteur 2 ?

\section{$\mathbf{R}$ : Non, pas encore pour le 2.}

$\mathbf{Q}$ : Vous étiez tout à fait conscient qu'il fallait aussi injecter de l'eau dans le réacteur 1.

$\mathbf{R}$ : Oui, bien sûr, j'en suis conscient. Mais à ce moment-là le bassin est en train de se vider. On n'a plus assez d'eau. La priorité a été d'aller chercher l'eau. On commence par envoyer l'eau dans le réacteur 3, car il y a déjà un certain temps que l'injection a été arrêtée pour lui. Il fallait continuer à envoyer l'eau dans le réacteur 3. Comme je vous l'ai dit tout à l'heure, pour moi, le réacteur 1 avait encore de la marge, parce qu'on lui avait déjà injecté un volume important d'eau.

$\mathbf{Q}$ : Si vous avez jugé de la sorte, ça veut aussi dire que plus le temps passait, deux heures, trois heures, peut-être six heures, plus il devenait impératif de reprendre aussi l'injection du réacteur 1.

$\mathbf{R}$ : Bien sûr, c'est ce que je pensais.

$\mathbf{Q}$ : En fait, qu'il y ait eu ordre du bureau du Premier ministre ou pas, de toute manière, si le réacteur 2 se maintenait dans sa situation actuelle, vous aviez déjà tout naturellement dans l'esprit de commencer par le réacteur 3, puis, à un certain moment, de reprendre l'injection du réacteur 1, n'est-ce pas?

$\mathbf{R}:$ Oui, c'est ça.

$\mathbf{Q}$ : L'extrait suivant se situe après l'explosion, vers la fin de l'après-midi.

$\mathbf{R}:$ C'est l'extrait 42 ?

$\mathbf{Q}$ : Oui, le 42. Cette fois, c'est encore le 14 mars, après l'explosion. Le RCIC du réacteur 2 s'est arrêté. La température et la pression sont très élevées dans la chambre de dépressurisation du réacteur 2. Vous prenez la décision d'établir une ligne d'éventage pour une manœuvre de dépressurisation. Mais avant, vous dites, je vous cite: «Écoutez-moi tous à la centrale, c'est Yoshida qui vous parle. Avant d'aller plus avant, je voudrais qu'on fasse un point de la situation. Actuellement, le groupe «instruments» finit de constituer une ligne d'éventage pour permettre une dépressurisation et une injection du réacteur 2. Les dernières 
prévisions donnent 17h30, comme heure où on atteindra le TAF. L'objectif, maintenant, est de dépressuriser à 77 h00 en manœuvrant la vanne SR et de passer à l'opération d'injection ». Au moment où vous parlez, il est question de finaliser la ligne d'éventage pour permettre de dépressuriser et d'injecter l'eau. Puisque le TAF devrait être atteint à 17h30, il s'agit de manœuvrer la vanne SR, trente minutes avant, c'est-à-dire à 17h00, et de commencer à injecter l'eau. L'idée est d'injecter le plus d'eau possible pour noyer le combustible avant que le niveau d'eau n'arrive à zéro. La séquence que nous allons voir se situe juste après ces explications.

(Vidéo)

« M. Akio Takahashi, du siège, aimerait parler au directeur Yoshida [...] »

«M. Yoshida est actuellement au téléphone. »

«Le bureau du Premier ministre [...] voudrait qu'on active le réacteur 2. II ordonne de commencer l'injection. Je suis sûr que vous avez dû recevoir aussi des instructions dans ce sens. Je voulais juste vous prévenir. Je me demande si Yoshida n'est pas en train de parler de ça avec M. Takekuro. »

«Compris. »

« M. Takahashi, le directeur est encore au téléphone. »

«Excusez-moi d'interrompre. Je viens d'en référer au siège. On m'a dit tout à l'heure de réceptionner le camion de pompiers à Fukushima Daini et de le convoyer jusqu'à Fukushima Daiichi. J'ai bien quatre pompiers qui sont arrivés, mais je n'ai pas de camion de pompiers. Je peux y aller comme ça?»

«Oui, allez-y comme ça. »

« II suffit que j'emmène les pompiers, c'est ça? »

«Tout à fait. Si vous voulez bien emmener les pompiers qui sont arrivés avec la Toyota Estima... »

« D'accord. Nous y allons, alors. »

«Merci beaucoup.»

«Directeur Masuda29?»

«Oui, c'est moi, Masuda. »

«Écoutez, tout le monde. Au siège aussi, écoutez, s'il vous plaît. Je viens de recevoir à l'instant un coup de fil du directeur de la NSC, le professeur Madarame. II se demande s'il ne faudrait pas injecter d'abord, sans attendre l'éventage de l'enceinte de confinement. Le directeur de la NSC suggère que, puisque si on dépressurise, l'eau va entrer, on envoie l'eau sans perdre une minute. Le groupe "sûreté », que pensez-vous de cette suggestion, en tenant compte de la situation?»

«La température de l'eau de la chambre de dépressurisation est à plus de $130{ }^{\circ} \mathrm{C}$. Même si la vapeur arrivait dans la chambre, elle risque de ne pas condenser. À ce moment-là, on ne pourra pas espérer une baisse de la pression. Même si l'eau [...], j'ai vraiment peur qu'on ne puisse pas dépressuriser. »

«Vous avez entendu? La température de l'eau dans la chambre de dépressurisation est à plus de $100^{\circ} \mathrm{C}$. »

«Cette histoire, le professeur Madarame [...]. »

«Excusez-moi. Je suis XXXXX du siège. Directeur Yoshida. »

«Le bureau du Premier ministre dit qu'il y a des risques que l'eau n'entre pas [...]. »

«Directeur Yoshida. M. XXXXX.»

«Oui. »

" Je suis XXXXX du siège. Je sais que l'eau est à $138^{\circ} \mathrm{C}$. Mais la vapeur est à plus de 200

${ }^{\circ} \mathrm{C}$. Si elle arrive dans une ambiance à $138^{\circ} \mathrm{C}$, elle va tomber. Si elle condense, elle va tomber et cela aura pour conséquence d'élever légèrement la pression à l'intérieur de la cuve. Mais si elle condense, cela signifie qu'il n'y aura pas de perte (d'eau). » 
«Alors, vous êtes d'accord?»

«Avec ce que vient de raconter XXXXX?»

« Je veux dire qu'on peut (injecter) avant de procéder à l'éventage, qu'il faut penser d'abord à refroidir. »

«Mais non, ça ne concerne pas le refroidissement. Je veux dire qu'il n'y a pas de problème à éventer. »

"Mais non, ce n'est pas ça. Il s'agit de refroidir. »

«Ce que dit le professeur Madarame, c'est qu'il faut dépressuriser et envoyer de l'eau sans attendre que la ligne d'éventage soit prête. »

"Si on fait ça, le niveau d'eau de la chambre de dépressurisation va continuer à monter, bien que le niveau exact actuel ne soit pas précisément connu, et cette eau risque d'envahir la ligne d'éventage de la chambre humide. Même maintenant, le risque qu'elle soit déjà noyée n'est pas à exclure. Si on ne procède pas au plus vite à l'éventage, il y a des craintes qu'on n'ait plus l'occasion d'utiliser cette ligne de la chambre humide. »

«Si je comprends bien, le terrain est d'accord avec ce que propose le professeur Madarame?»

«Non, non. Ce n'est pas du tout ça.»

« Nous voulons essayer l'éventage par la chambre de dépressurisation. »

«C'est ce que nous essayons de faire en ce moment même. »

«Ce que je voudrais dire, c'est que, plutôt que de continuer à injecter dans le réacteur, il faudrait le faire après avoir baissé la pression en procédant à un éventage. »

"Je ne sais pas trop ce qui se passe sur le terrain, mais qu'est-ce qui diffère entre le professeur Madarame et toi, Yoshida? II me semblait bien que nous étions dans l'éventage, non?»

«Bien sûr. »

« Nous, on voudrait d'abord refroidir la vapeur pour faire baisser la pression avant d'injecter. Mais le professeur Madarame pense qu'il n'est pas nécessaire d'utiliser la ligne d'éventage pour faire baisser la pression dans la chambre de dépressurisation. II voudrait qu'on injecte d'abord. »

« II n'a peut-être pas totalement tort, mais, nous, nous pensons qu'il vaut mieux commencer par l'éventage de la chambre de dépressurisation. Parce que, finalement, si on réfléchit à ce qui se passera quand le cœur sera dégradé, il risque de ne plus y avoir que la solution de l'éventage de la chambre sèche. »

«C'est gentil de « penser ». Mais on n'a plus le temps. »

«Heu, excusez-moi. »

« J'ai ordre du directeur de la NSC, du directeur de la NISA et du bureau du Premier ministre de procéder très bientôt à l'opération. Ce que je voudrais savoir, c'est si vous êtes d'accord pour ça.»

«Si je vous dis qu'actuellement il reste, allez, $100 \mathrm{~mm}$ [...] plus $1000 \mathrm{~mm}, 1100 \mathrm{~mm}$, je peux penser que je vous ai convaincus?»

«Oui. »

«Donc, vous êtes d'accord avec notre manière de procéder?»

«Oui, c'est ça. »

«À propos, Yoshida, d'après ce qu'on avait dit tout à l'heure, la vanne d'éventage devrait être ouverte maintenant, non?»

«Oui. C'est pour ça que j'ai fait vérifier ça. On avait parlé de 17h00. Mais si on pouvait ouvrir la vanne avant [...]. Dites, où en êtes-vous avec ça?»

«On est en train de faire les raccordements. »

«Quoi ?» 
«Ce n'est pas encore fini. Vous pouvez attendre un peu?»

«Arrête de parler d'« attendre un peu ». Sois plus précis. »

« Nous allons procéder au plus vite, avec en tête l'idée qu'on puisse démarrer l'opération sans attendre 17h00. Est-ce que cela vous convient?»

«Oui. Faites ça. »

«Compris. »

«Une demande de la part du groupe « sûreté » de Fukushima Daiichi. Il y a des membres de Kashiwazaki qui sont venus nous aider à Daiichi. Ils sont actuellement devant la salle des visiteurs de Fukushima Daini. On leur refuse l'entrée du fait qu'ils viennent de Daiichi. »

$\mathbf{R}$ : Cette histoire n'a rien à voir.

$\mathbf{Q}$ : (Au technicien) Vous pouvez arrêter là ? Un petit peu plus loin, on voit le Directeur général Shimizu, au siège. Vous pourriez reprendre par là?

(Vidéo)

«[...] S'agissant des 17h00, si la ligne d'éventage est prête, êtes-vous d'accord pour que nous procédions à l'éventage sans attendre 17h00?»

«Oui, allez-y. »

« D'accord. »

« Je voudrais une autorisation. »

«C'est bon. »

«Excusez-moi. Je vous explique l'état actuel de la ligne d'éventage [...] »

« Nous venons d'envoyer l'électricité, mais il n'y a pas eu de mouvement. Du point de vue de l'air, le compresseur fonctionne, mais il y a possibilité que la pression ne soit pas uniforme. Nous allons vérifier ça. »

«Et ça, à quelle vitesse?»

«On ne voit pas la pression. On est donc obligé d'attendre que ça bouge. »

«Directeur Yoshida, c'est Shimizu. Je voudrais que vous procédiez en suivant la méthode du professeur Madarame. »

« Oui. D'accord. »

«Suivez sa consigne. »

«XXXXX, XXXXX, tu as entendu ce qu'on vient de dire? La préparation de la ligne d'éventage prend du temps. Mais continue à y travailler, de ton côté. [...] C'est absolument vital. Toi, tu continues de ton côté. Seulement, si on attend l'achèvement de cette ligne, il y a des risques que le combustible se retrouve dans une situation de plus en plus dangereuse, alors nous allons procéder à l'opération qui nous est demandée. »

«D'accord. Procédez comme ça. »

« II y a eu ordre du Directeur général, mais, directeur Mutô, est-ce que ça va aller du point de vue technique?»

«Oui, ça va aller. »

«Écoutez. Je résume encore une fois. »

$\mathbf{R}$ : Ça, ce n'est pas moi. Ce n'est pas la centrale Daiichi. C'est Takahashi. Il n'y a personne de Daiichi. Ça, c'est Takahashi. C'est le siège.

Vous voyez qu'il demande plusieurs fois à Mutô si ça va aller, une fois qu'on a reçu l'ordre du Directeur général. Ça, c'est parce qu'il n'a pas confiance. Il n'a pas confiance dans le DG, parce que ce n'est pas un technicien. Alors, quand il demande à Mutô si ça va aller, c'est qu'il a besoin d'être conforté techniquement. En fait, on n'a pas eu de réponse de Mutô, parce qu'il n'était pas au centre hors site, il me semble. Je ne sais plus s'il n'était pas là, s'il était parti quelque part. 
$\mathbf{Q}:$ : I était bien quelque part dans le département de Fukushima, non?

$\mathbf{R}$ : Bien sûr. Je ne pense pas qu'il ait été en déplacement. Il était sans doute dans le centre hors site, mais là où il n'y avait pas la téléconférence.

(Vidéo)

«Vous êtes sûr qu'on va procéder selon la méthode du professeur Madarame?»

"OK. Écoutez-moi tous. Vous allez continuer à travailler à la préparation de la ligne d'éventage en parallèle. En plus de ça, vous sentez-vous capables de préparer le terrain pour une opération de dépressurisation que nous commencerions à partir de $16 \mathrm{~h} 30$ ? Vous en sentez-vous capables? Si ça ne va pas, dites-le-moi simplement. Nous allons entamer les préparatifs en gardant en tête que le début de la manœuvre est pour 16h30. Décidez [...].»

«[...] Je demanderai l'accord final à M. Mutô, mais je voudrais simplement vérifier l'ordre suivant lequel nous allons procéder. C'est écrit sur le tableau à l'extrême droite. Tout d'abord, vérifier que l'eau de mer monte bien jusqu'au bassin Reversing Valve Pit. Je pense que cette partie fonctionne déjà. Ensuite, une fois que la ligne allant du bassin au cœur du réacteur 2 sera achevée, commencer la manœuvre d'ouverture de la vanne SR du 4. »

«Le professeur Madarame dit qu'on n'a plus le temps pour tout ça. [...] Il faut envoyer. »

«[...] On envoie alors. »

« Je vais [...] et procéder à l'opération de dépressurisation. »

«Quelle heure, la dépressurisation?»

«Pardon, Yoshida. Il n'y a pas de danger pour les hommes qui travaillent à la ligne d'éventage ? À l'endroit où ils travaillent? »

« Non, il n'y a pas de danger pour ceux qui travaillent à la ligne d'éventage. »

« Non? Tant mieux.»

«[...] vers le bassin. »

«On n'a pas le temps. Ne viens pas perturber.»

«Celui qui guide l'opération, donnez les ordres en criant fort. 16h28, exécution de l'ordre de dépressurisation. »

«Manœuvre de dépressurisation, première tentative, 16h28. Il faut peut-être informer les autorités? Vous pouvez vous en charger?»

«S'il vous plaît, veuillez entamer les procédures pour informer les autorités. "

«C'est déjà fait. »

$\mathbf{Q}$ : Dans cette séquence, il y a les recommandations du professeur Madarame, avec quelques échanges téléphoniques, il me semble. Tout d'abord, lorsque vous êtes au téléphone, à l'extrait 42, la personne qui parle à la téléconférence, c'est M. Akio Takahashi, du siège. Quel est son statut ? II est «fellow »?

R : Oui. 
Q: Quand le fellow Takahashi demande à vous parler, on lui répond que vous êtes au téléphone. Vous étiez déjà en train de parler avec M. Madarame?

R : Oui.

$\mathbf{Q}$ : Quand il vous téléphonait, M. Madarame vous téléphonait directement?

$\mathbf{R}$ : Ah, là, j'ai été surpris. Tout à coup, il était au téléphone. En plus, il ne s'est même pas présenté, le vieux. Il vociférait dans le combiné, une vraie tornade. J’ai compris, à la voix, que c'était lui.

$\mathbf{Q}:$ Vous parlez de M. Madarame, là?

R : Oui, de Madarame.

$\mathbf{Q}:$ Ah, bon? Il est comme ça?

$\mathbf{R}$ : Il était complètement paniqué. Il déversait toute son histoire sans même reprendre son souffle. Je me suis d'abord demandé qui était ce mec. Et puis, au bout d'un moment, je me suis rendu compte que ça avait l'air d'être le professeur Madarame. Je lui ai demandé ce qu'il voulait. Alors, il m'a dit d'envoyer l'eau le plus vite possible, qu'on n'avait plus le temps. Il ne faisait que répéter ça. Je lui ai expliqué qu'on était en train de préparer l'éventage, mais il m'a dit qu'on n'avait plus le temps pour un éventage et qu'il fallait envoyer l'eau tout de suite. À partir de là, il y a eu des échanges entre le professeur Madarame et le directeur de la NISA, qui devait probablement être à côté de lui.

$\mathbf{Q}$ : Est-ce que d'autres personnes, comme le directeur de la NISA, ont voulu vous parler?

R: Avant que le professeur Madarame ne monopolise la parole, parce que c'est surtout avec lui que j'ai parlé, il me semble qu'il y avait eu quelqu'un d'autre au bout du fil, au tout début. Je ne me rappelle plus si c'était le directeur de la NISA ou quelqu'un d'autre. En tout cas, j'étais en train de parler avec cette personne quand le professeur Madarame lui a arraché le combiné des mains pour me donner l'ordre de faire ci et ça, sans même se présenter et que je me suis demandé qui pouvait être cet excité. C'est au son de sa voix que je me suis dit que ça ne pouvait être que le directeur de la NSC qui avait perdu les pédales.

$\mathbf{Q}$ : Ce qui veut dire que toutes les personnes qui étaient de l'autre côté du téléphone pensaient que la situation du réacteur 2 était extrêmement dangereuse.

$\mathbf{R}:$ C'est ce que je pense.

$\mathbf{Q}:$ Pour eux, si vous n'envoyiez pas l'eau tout de suite, il y avait danger.

$\mathbf{R}$ : Comprenez-moi. Depuis le début, on avait donné la priorité aux réacteurs 1 et 3. Comme on manquait d'eau dans le bassin, on avait dit qu'on l'utilisait d'abord pour les réacteurs 3 et 1 . Mais, déjà, à ce moment-là, le bureau du Premier ministre voulait qu'on envoie l'eau dans le réacteur 2. J'imagine qu'ils devaient être plusieurs à la résidence du Premier ministre. Ils avaient vu les données et avaient dû se dire que ça devenait dangereux. Ils n'arrêtaient pas d'insister ${ }^{30}$. J'avais envie de leur dire de nous foutre la paix. Bien sûr que, nous aussi, naturellement, on voulait injecter au plus tôt. Mais les conditions n'étaient pas réunies. C'était évident. Il y avait l'histoire de l'éventage. Même pour la condensation, comme je vous l'ai dit tout à l'heure, on n'était pas véritablement sûr que la vapeur allait se condenser dans une chambre de dépressurisation à plus de $100^{\circ} \mathrm{C}$. Est-ce que la pression de la cuve allait descendre? Est-ce que la baisse de la pression de l'enceinte de confinement n'allait pas naturellement entraîner une hausse de la pression dans la cuve? Tout ça, on ne 
pouvait pas le savoir à l'avance, on avançait sans trop savoir quelles allaient être les conséquences, parce que c'était une première. Personne n'était passé par là avant nous.

$\mathbf{Q}:$ Si je me rappelle la séquence, $M$. Madarame est très pressé. II voudrait presque que vous commenciez l'injection tout de suite. Ce dont parle M. XXXXX, c'est ce que vous faisiez effectivement sur le terrain.

$\mathbf{R}$ : Oui. Nous, on voulait éventer au plus vite. Alors quand on entendait le directeur Madarame, on avait envie de lui dire, "mais qu'est-ce que tu racontes?", " on ne parle pas de la même chose ", "ce que je veux savoir, c'est si l'éventage va marcher ou pas ", enfin, là, c'était la confusion totale. Il était complètement hors de lui.

$\mathbf{Q}:$ En fin de compte, bien que le professeur Madarame ait été très insistant, le siège et le terrain pensaient toujours, au départ, que l'établissement de la ligne d'éventage était prioritaire et, dans un premier temps, vous bougiez même dans ce sens.

R: Oui, nous avons essayé de continuer dans ce sens-là. Mais on avait des soucis avec l'éventage. C'est là où on retrouve XXXXX. Quand je lui ai demandé où il en était, il m'a dit qu'il rencontrait des difficultés. Les nouvelles n'étaient pas bonnes. C'est là qu'on commence à se dire que le scénario qu'on avait prévu ne se déroule pas comme on aurait voulu. On commence, nous aussi, à se demander s'il n'y a plus que la dépressurisation en dernier recours, quand le directeur général Shimizu, je ne sais pas jusqu'à quel point il avait compris la portée technique de la manœuvre, nous commande de l'exécuter.

$\mathbf{Q}$ : Est-ce que le directeur général est intervenu plusieurs fois pour vous ordonner de faire ci ou ça sur le terrain?

R : Non. C'était la première fois qu'il intervenait.

Q : Et il vous a dit d'agir en suivant la méthode du professeur Madarame. Vous vous y attelez avec l'objectif de commencer la manœuvre de dépressurisation vers 16h30. Dans les faits, il vous a fallu bien plus de temps pour cette manœuvre.

$\mathbf{R}$ : Oui, la vanne ne s'ouvrait pas.

Q: Par exemple pour le réacteur 3 , vous aviez réussi à dépressuriser entre 9h08 et 9h20. Mais ce coup-ci, avec le réacteur 2, quand on regarde la chronologie, vers 18h00, vous êtes encore à 5,4 MPa, puis il y a une chute assez brusque jusqu'à 0,63 MPa à 19h03. Ce qui signifie qu'entre $16 \mathrm{~h} 30$ et $19 \mathrm{~h} 00$, pendant environ deux heures et demie, vous avez dû vous escrimer à effectuer cette manœuvre de dépressurisation. Pendant ce temps, vous n'avez pas eu de coup de fil de l'extérieur? Des réclamations du genre, "qu'est-ce que vous foutez?»?

$\mathbf{R}$ : Non, on n'a pas eu ça. Enfin. Un tout petit peu. Vous n'avez pas vu ça sur la vidéo ?

$\mathbf{Q}:$ Oh, je suppose que ça a dû être enregistré aussi.

$\mathbf{R}$ : Je n'en ai ${ }^{\top}$ pas le souvenir, parce que, moi-même, j'étais dans un état d'énervement incroyable. Vite, il fallait dépressuriser. Là-dessus, la vanne S3 qui ne s'ouvre pas! Je vous dis, moi aussi, j'étais en proie à la panique.

Q: Quand je regarde les archives, on voit pas mal d'annotations concernant cette vanne SR. Vous avez eu quelques petits soucis au début. Par exemple, vous aviez manqué de puissance...

$\mathbf{R}:$ Ah, oui. La batterie. 
$\mathbf{Q}:$ Oui. Il y a des soucis de ce type tout du long.

$\mathbf{R}$ : On avait besoin d'une nouvelle batterie, mais il fallait savoir où trouver une batterie de réserve, etc. D'après mes souvenirs, tout le monde était là à harceler le terrain, à les presser sans arrêt. C'était surtout le chef de quart qui était leur cible. Oui, parce que, là-dessus, ils ont commencé à discuter pour savoir si c'était l'équipe de quart ou le groupe "réhabilitation" qui devait opérer la manœuvre. Je me rappelle les avoir houspillés en disant que ce n'était pas le moment pour des discussions inutiles, que ce qui devait être fait, devait être fait. Ce n'était vraiment pas le moment pour des discussions de répartition des rôles.

$\mathbf{Q}$ : Finalement, l'équipe de quart et le groupe «réhabilitation» ne travaillaient pas de concert?

R : Si. C'était nécessaire, parce qu'un pilote est capable de faire les manœuvres quand toute l'installation est dans un état normal de fonctionnement, mais s'il faut brancher une batterie, c'est plutôt le travail du groupe « réhabilitation ». Il faut qu'il y ait un spécialiste des instruments qui suive le travail. Ce qui veut dire qu'ils doivent impérativement travailler ensemble. J'étais littéralement hors de moi quand j'ai compris qu'ils étaient en train de discuter sur une question aussi futile que de savoir qui devait faire la manœuvre.

$\mathbf{Q}$ : Juste après cet extrait, quand vous vous êtes rendu compte que vous aviez de réelles difficultés à ouvrir la vanne SR, vers la fin de la journée, bien que je ne sache pas si le siège se sentait aussi concerné, avez-vous eu des discussions pour savoir s'il ne faudrait pas organiser un retrait? Je sais que, dans les faits, il y a eu une évacuation vers Fukushima Daini ${ }^{31}$. Aviez-vous commencé à y réfléchir?

$\mathbf{R}$ : Oui. C'était une préoccupation importante. Mais je ne voulais pas que cela fasse trop de bruit. Ce n'est pas que le siège m'en ait parlé, mais j'y pensais. Le problème, c'est qu'il y avait des gens des entreprises partenaires, même dans les couloirs du bâtiment antisismique. Si j'en parlais à la table de la cellule de crise, ils risquaient d'entendre. Or, nous savions que le combustible était complètement découvert, que nous n'arrivions pas à dépressuriser, que l'eau n'entrait pas. C'est vraiment un moment que je n'ai pas envie de me remémorer. Il y a eu plusieurs occasions, durant ces journées, où j'ai cru que j'allais mourir, mais cette fois-là j'ai vraiment cru que j'y passerais.

Je pensais que, sans injection d'eau, le combustible du réacteur 2 allait fondre, que la pression de l'enceinte de confinement allait la faire exploser et que tout le combustible allait fuir. Si ce scénario se produisait, cela signifiait que toute la radioactivité allait être dispersée à l'extérieur. C'était le pire accident imaginable. Ce n'était pas encore Tchernobyl $^{32}$, ni le syndrome chinois ${ }^{33}$, mais on n'en était pas loin. Cela nous aurait obligé à arrêter aussi l'injection d'eau dans les réacteurs 1 et 3. Tôt ou tard, on allait vers la catastrophe.

Si on supposait que cela allait arriver, il fallait quitter les lieux. Il y aurait eu beaucoup de victimes. Bien sûr, cela allait disperser des éléments radioactifs sur une plus grande surface et en plus forte concentration. Mais ce qui me préoccupait le plus, c'était la survie de tous ceux qui étaient là, tous ceux qui étaient dans le bâtiment antisismique et à proximité. Si j'avais parlé de mes préoccupations ouvertement, à la table de la cellule de crise, j'aurais semé la panique parmi eux. J'en ai parlé à Mutô, au téléphone, je crois. Je me rappelle lui avoir expliqué que la situation était extrêmement dangereuse, que je me demandais si, hormis le personnel 
minimum nécessaire à la conduite et à la réhabilitation, il ne convenait pas de penser sérieusement à mettre toutes ces personnes à l'abri.

J'ai également référé de la situation à $M$. Hosono. Je ne lui ai pas parlé tout de suite de retrait, mais je hui ai expliqué que l'état du réacteur 2 était extrêmement préoccupant, que, si on ne réussissait pas à injecter d'eau, nous allions vers la catastrophe. Je crois que je lui ai dit, à ce moment-là seulement, que dans ce cas il faudrait évacuer le personnel, hormis le minimum nécessaire sur le terrain. Je le lui ai dit au téléphone. Je me rappelle m'être isolé pour passer ce coup de fil. Il y avait des oreilles partout et je ne voulais surtout pas semer la panique. C'est vraiment l'épisode dont j'ai le moins envie de me souvenir, franchement.

$\mathbf{a}$ : MM. Mutô et Hosono n'étaient pas ensemble au même endroit, n'est-ce pas ?

$\mathbf{R}$ : Non, ils étaient dans des endroits tout à fait différents. Je pense que j'ai téléphoné au siège. Je crois bien que c'était Mutô. Vous savez, il n'y avait guère qu'à Mutô que je pouvais parler de ce genre de choses, dans ces moments-là.

$\mathbf{Q}$ : Et quelles ont été leurs réactions, quand vous avez téléphoné, que ce soit M. Mutô, M. Hosono ou quelqu'un du siège?

$\mathbf{R}$ : Pas vraiment de réaction notoire. Je faisais le point de la situation. Ils ne me disaient ni «OK», ni rien. Je leur disais, « il y a ce risque », ils répondaient "d'accord ». C'étaient des échanges tout simples. Ce que j'ai fait ensuite, c'est que je suis allé voir le personnel des entreprises partenaires qui était dans le couloir. Ils étaient tous là, le regard un peu dans le vague. Je crois qu'ils ne comprenaient pas très bien ce qui leur arrivait. J'ai pensé très fort que je ne pouvais pas entraîner ces gens-là dans ce qui pouvait suivre. Je leur ai dit: "nous avons fait tout ce qui était en notre pouvoir, mais la situation devient très dangereuse. S'il vous plaît, rentrez chez vous ». Je ne leur ai pas dit que c'était un retrait, une évacuation. Je leur ai juste dit de rentrer chez eux. [...] Après ça, je suis revenu dans la salle. Je ne pouvais plus articuler un mot. Il ne restait plus qu'à attendre. Soit l'eau entrait, soit elle n'entrait pas. C'était comme un pari. Une fois que j'avais parlé aux gens dans le couloir, je n'ai presque plus rien dit. J'ai dormi. Enfin, disons que j'étais dans un état second.

\section{$\mathbf{Q}$ : Tout ça parce que la vanne SR avait du mal à s'ouvrir?}

$\mathbf{R}$ : Non. Elle était ouverte. Elle était ouverte, mais la pression n'arrivait pas à descendre. Encore, quand il y a une vanne à ouvrir, ça va, parce qu'on est dans l'action, on fait quelque chose, on peut se demander que faire pour faire avancer les choses. Mais quand la vanne est ouverte et que, malgré ça, la pression ne tombe pas, on se dit, ce qui devait arriver est arrivé. On se demande si, finalement, la pression ne descend pas parce qu'elle est plus élevée dans la chambre de dépressurisation. On se dit que, la pression ne baissant pas, le niveau d'eau doit être de plus en plus bas dans la zone du combustible.

Il y avait aussi autre chose. Comme on avait manqué de temps, on s'est aperçu que le réservoir du camion de pompiers dont on devait utiliser la pompe pour l'injection n'avait pas été approvisionné. Au moment où la pression de la cuve avait baissé et où on aurait pu injecter l'eau, on était en panne sèche et on n'a pas pu exécuter l'opération. Là aussi, ça a été un coup très dur. Bien sûr, j'ai donné tout de suite l'ordre de ravitailler le camion. Mais j'avais l'impression que les choses étaient contre nous. J'ai bien cru que c'était la fin, que mon heure était venue. 
$\mathbf{Q}$ : Si on étudie les paramètres, on voit que le 14 mars, vers la fin de l'après-midi, la pression tombe dans les environs de 0,8 ou 0,7, comme si l'opération de dépressurisation avait réussi. Ça descend même jusqu'à 0,5. Mais un peu plus tard, vers 21h00, à 20h54, par exemple, elle remonte au-delà de 1 , puis elle redescend à 0,4 , pour remonter encore à 1, 2 ou 3. Bref, la pression est totalement instable. Mais, du coup, avec cette remontée, l'injection devient de nouveau impossible. Dans un cas comme ça, vous devez recommencer l'opération de dépressurisation avec la vanne SR ?

$\mathbf{R}$ : Là, on s'est demandé pourquoi la pression remontait. J'ai fait vérifier que la vanne était bien ouverte. Je pense que c'est ce que j'ai fait faire. Vous savez, c'est une période que j'ai tellement envie d'effacer de ma mémoire. C'est traumatisant de se remémorer ces moments.

$\mathbf{Q}$ : Ce que vous me racontez là se situe dans la nuit du 14 mars, n'est-ce pas ?

R : À partir de 19h00, environ, dans les faits.

$\mathbf{Q}$ : Si on regarde les paramètres, à 20h54, la pression remonte à 1,170 , puis 1,3, puis encore 1,4 . À $21 \mathrm{~h} 20$, elle redescend pour un temps à $0,8,0,6,0,5$. Ça reste stable pendant un petit moment, mais à $22 \mathrm{~h} 50$, elle est à 1,8 , puis à 2,07 à 23 h00 et ensuite à 2,65 , puis 3,15 . Ensuite, elle semble redescendre à partir de 23h30. Provisoirement, elle descend. Mais si on regarde un peu plus tard, elle est encore repartie pour dépasser 1 et atteindre 2 . Ça, c'est ce qui se passe entre minuit et $1 \mathrm{~h} 00$. Tout ça se répète indéfıniment.

$\mathbf{R}$ : Juste avant ça, il y a eu un moment où la pression était descendue. Ce qui était bien. Ce qui aurait été encore mieux, c'est si, à ce moment-là, vers 21 h00, je suppose, on avait pu injecter de l'eau dans le réacteur. Mais là, on n'avait plus d'essence, la pompe ne marchait pas et on n'a pas pu injecter. C'est là que j'ai méchamment accusé le coup. "Quoi ? L'eau n'est pas entrée? » "Ça n'entre pas, on n'a plus d'essence. » Là, j'ai pété les plombs. J'ai injurié XXXXX et je lui ai envoyé un coup de poing dans la figure ${ }^{34}$.

$\mathbf{Q}$ : La pression était donc enfin descendue à moins de 1 , vers $21 \mathrm{~h} 00,21 \mathrm{~h} 20$, mais vous vous êtes retrouvés malencontreusement en panne sèche à ce moment-là. Comme vous n'injectez pas d'eau, la pression recommence à monter. C'est bien ça ?

$\mathbf{R}$ : C'est ça. Là encore, je ne me souviens pas exactement du moment où on a fini de ravitailler la pompe et où l'eau est entrée. Mais, étant donné que la pression a baissé à certains moments, j'aurais tendance à penser que l'eau était bien entrée. Seulement, quand l'eau pénètre et entre en contact avec le combustible, qui est très chaud, cela provoque un phénomène de flash et la pression remonte brutalement. Du coup, l'eau ne peut plus entrer. Je crois que c'est ce phénomène que nous avons connu. Bien sûr, on n'aura confirmation que quand on aura analysé tout ça. De toute façon, je pensais, personnellement, que les dégradations étaient importantes et que nous étions proches de la fusion.

$\mathbf{Q}:$ Ça, c'était dans la nuit du 14 au 15 mars, n'est-ce pas?

R : Oui.

$\mathbf{Q}$ : Est-ce que les entreprises partenaires étaient réellement parties ?

$\mathbf{R}$ : Tout d'abord, les gens qui stationnaient dans le couloir sont presque tous partis.

$\mathbf{Q}$ : Si je me remets en situation, il y avait aussi des employés de TEPCO à la cellule de crise. Et ces gens-là?

$\mathbf{R}$ : Plutôt que la cellule de crise, vous voulez dire les gens qui étaient sur le site, dans le bâtiment antisismique. En fait, j'ai commencé par appeler très discrètement XXXXX qui s'occupait de l'administration. Je lui ai donné l'ordre de vérifier le 
nombre des employés présents. Le personnel des entreprises partenaires avait des moyens de locomotion, alors [...]. Je lui ai demandé de vérifier le nombre de nos employés à nous. Surtout le nombre de personnes qui n'avaient spécialement rien à voir avec la conduite ou la réhabilitation des installations. Ceux qui étaient affectés à la cellule de crise n'étaient évidemment pas concernés. Je voulais aussi qu'il vérifie le nombre de cars dont on disposait. Il me semblait bien qu'il y en avait deux ou trois. Il fallait voir si on avait des chauffeurs, s'il y avait de l'essence dans les véhicules. Je lui ai ordonné de les mettre en attente devant le bâtiment, prêts à démarrer s'il arrivait quoi que ce soit. Tout ça n'apparaît pas dans les documents, mais j'en avais donné l'ordre.

Q: Vous avez donné ces ordres à quel moment? Le 15 mars vers 6h00, vous avez eu d'autres soucis, non seulement avec le réacteur 2, mais avec le réacteur 4. Était-ce bien avant, que vous aviez donné ces ordres?

R : Oh, bien, bien avant. Honnêtement, il y a eu un moment où j'ai cru que le réacteur 2 était foutu.

$\mathbf{Q}:$ Le réacteur 2 , plutôt que le réacteur 3 ?

$\mathbf{R}$ : Vous vous rappelez, on injectait de l'eau dans le réacteur 3. Même chose pour le réacteur 1. Alors que l'eau n'entrait pas dans le réacteur 2. Si l'eau n'entre pas, ça signifie qu'au bout il n'y a que la fusion du combustible. Si le combustible fond et atteint $1200{ }^{\circ} \mathrm{C}$, et qu'on ne refroidit pas, il va percer le fond de la cuve, avant de percer, à leur tour, les parois de l'enceinte de confinement. Après, c'est le syndrome chinois. On avait beau dire, même si tout ce que nous avions entrepris n'était pas aussi efficace que nous l'aurions voulu, il y avait encore une enceinte de confinement, une cuve, qui représentaient des barrières de protection. Si tout ça devait disparaître complètement, tout le combustible se serait trouvé à l'extérieur. Que ce soit du plutonium ou autre chose, cela aurait été une situation autrement plus grave que le césium dont on parle aujourd'hui. Toute la matière radioactive se serait trouvée dehors, rejetée. Pour nous, c'était la destruction de tout l'est du Japon ${ }^{35}$.

Q: Pour l'évacuation, vous aviez prévu de ne garder que le minimum de personnel nécessaire. Vous aviez fait faire des préparatifs pour les autres. Mais, en fait, vous ne les avez pas évacués tout de suite. Pourquoi?

$\mathbf{R}$ : Ça, c'est parce que nous sommes allés une nouvelle fois injecter l'eau et que, cette fois, elle est entrée. On a eu des indices nous permettant de penser qu'elle était entrée. On était contents. Je pensais qu'il ne restait plus qu'à continuer à injecter l'eau et j'ai oublié l'évacuation. Franchement, ce sont des heures que je voudrais oublier. Il y a plein de souvenirs qui manquent, mais, assurément, il y a eu un moment où je me suis dit que nous étions enfin sauvés.

$\mathbf{Q}$ : Et ensuite?

$\mathbf{R}$ : Il fallait continuer à envoyer l'eau. C'était la seule solution. J'ai donc demandé à mes troupes de continuer à veiller à l'approvisionnement en essence, bien que j'avais conscience que c'était une opération qui les exposait.

$\mathbf{Q}$ : Les niveaux d'eau n'apparaissent pas ici, mais le 15 mars entre $1 \mathrm{~h} 10$ et $1 \mathrm{~h} 20$, puis après, la pression se balade durablement autour de 0,63 ou 0,65. Là, vous avez l'impression que l'eau est entrée?

R : Oui, je pense, à ce moment-là, que l'eau entre, qu'elle entre de manière sûre. 
$\mathbf{Q}$ : Et c'est pour ça qu'au lieu d'évacuer, vous êtes tous restés sur place pour continuer à œuvrer.

R : Oui.

$\mathbf{Q}$ : Après une nuit tendue, passée à vous demander si vous alliez mourir ou pas, il y a eu la visite du Premier ministre. J'ai eu accès à des notes assez détaillées qu'avait prises une personne qui se trouvait, non pas au siège, mais sur le site de la centrale, au moment où M. Kan arrive, vers les 5 h00.

$\mathbf{R}$ : C'est quand il est allé au siège.

$\mathbf{Q}$ : Oui, au siège, à un endroit qu'on pouvait voir avec la caméra de la téléconférence.

R: Je ne sais pas si c'était la salle de téléconférence, elle-même, ou ailleurs. Mais, effectivement, on avait vu sur l'écran qu'à l'annonce de sa visite, on avait aménagé une salle avec un siège pour $\mathrm{M}$. Kan et, de l'autre côté, des sièges pour les administrateurs. C'était sur la vidéo du siège. Non, plutôt l'écran de la téléconférence. Il me semble qu'on est resté longtemps sur cette image, en attendant 5h00. En fait, ce n'était pas 5h00. C'était un peu après que M. Kan est apparu sur l'écran. Il y avait aussi le Directeur général Shimizu, bien sûr, le Président Katsumata ${ }^{36}$, des administrateurs, peut-être aussi des directeurs. Je ne les ai pas vus moi-même. Il faudrait que vous demandiez à quelqu'un du siège, ce sera plus exact. Bref, il y avait du monde et M. Kan a commencé à demander pourquoi il y avait autant de monde ${ }^{37}$. J'ai oublié les détails, mais je me souviens qu'il avait une attitude extrêmement désagréable, qu'il était dans une colère noire et qu'il vociférait sur tout le monde ${ }^{38}$.

$\mathbf{Q}$ : Je suppose qu'il n'y a pas tout, mais cette personne a noté quelques expressions qui l'avaient marquée. Je voudrais savoir si vous les reconnaissez, vous aussi. Moi, ce qui m'a frappé, ce sont des expressions comme «il n'y aura pas de retrait », "je vous demande de sacrifier vos vies $»^{39}$.

R : Oui, il l'a dit.

$\mathbf{Q}$ : Eh, oui. Il l'a dit. Mais avant qu'on ne parle de ça, je voudrais vérifier quelque chose. Vous m'avez dit tout à l'heure que vous aviez contacté M. Hosono pour lui expliquer que la situation devenait dangereuse et qu'il pourrait éventuellement y avoir un retrait.

R : J'ai parlé de retrait, mais je n'ai pas parlé d'un retrait général de toutes les troupes. Moi-même, je serais resté. J'aurais maintenu les hommes nécessaires aux différentes manœuvres, naturellement. Ce que je lui ai dit, c'est qu'il fallait qu'il prenne les décisions à venir en sachant que le pire pouvait arriver et que j'allais mettre à l'abri les gens qui n'avaient rien à faire à la centrale. C'est tout.

$\mathbf{Q}$ : Je suppose qu'à partir de là, c'était un peu le jeu du téléphone arabe. M. Kan, qui était le dernier à recevoir le message, n'a pas perçu les nuances.

$\mathbf{R}$ : Je ne sais pas s'il y a eu des pertes de contenu concernant mon message, mais j'ai aussi entendu parler d'un épisode où le directeur général Shimizu avait demandé à M. Kan l'autorisation d'évacuer. Il semblerait que ce que j'avais dit à quelqu'un du siège s'était transmis jusqu'à Shimizu. Du coup, c'était comme s'il y avait eu deux sources, deux voies de transmission. Ce qui est arrivé à l'oreille de Shimizu et ce que j'ai raconté à M. Hosono. Je ne sais pas comment ces deux lignes se sont trouvées reliées, mais il y avait double ligne d'information, en quelque sorte ${ }^{40}$.

$\mathbf{Q}$ : Je crois comprendre que vous, votre position a toujours été d'évacuer, mais en maintenant sur place les membres de la cellule de crise et le minimum de personnel 
nécessaire à la conduite et à d'autres opérations sur le terrain. On le voit dans les divers échanges que vous avez autour du réacteur 2 , par exemple.

$\mathbf{R}:$ Oui, c'est ça.

$\mathbf{Q}:$ M. Kan était peut-être même opposé à cette évacuation partielle. Toujours est-il que votre collègue a noté des phrases comme "il n'y aura pas de retrait », "je vous demande de sacrifier vos vies", "vous êtes lents», "pas assez rigoureux», "vous faites des erreurs », ou bien des choses comme «ce n'est pas juste le problème du Japon, c'est un problème qui concerne le monde. Il faut donner le maximum car c'est la survie du Japon qui est en jeu », etc., etc. Est-ce que vous retrouvez la tonalité de ce que vous avez entendu?

$\mathbf{R}$ : Oui, il a dit des choses de ce genre.

$\mathbf{Q}$ : Qui l'accompagnait parmi les ministres ? Est-ce que M. Kaieda était venu ?

$\mathbf{R}$ : Est-ce que son chef de cabinet était avec lui ? Est-ce que M. Kaieda était venu ? Je ne m'en souviens pas bien. Je crois que la mémoire de quelqu'un qui était au siège à ce moment-là serait bien plus fiable que la mienne.

$\mathbf{Q}$ : Évidemment, le mieux serait que quelqu'un du siège ait le courage de me raconter précisément ce qui s'est passé.

$\mathbf{R}:$ Oh, ils ne diront rien.

$\mathbf{Q}$ : J'aimerais qu'ils me fassent plus confiance.

$\mathbf{R}$ : Concernant ces notes, vous pouvez considérer que les choses ont été dites grosso modo de cette manière. Mais c'est justement à ce moment que, nous, à la centrale, nous vivons l'épisode de la pression zéro de la chambre de dépressurisation du réacteur 2 et du grand bruit. Juste ce matin où $M$. Kan était au siège. Face à ces nouveaux évènements, j'ordonne le retrait. Le retrait dans le sens de tout à l'heure, retrait de toute personne non indispensable sur le terrain.

$\mathbf{Q}$ : Au moment où vous formulez vos décisions, face aux anomalies du réacteur 2, est-ce que M. Kan vous a demandé l'état de la situation en passant, par exemple, par la téléconférence?

R : Non. Il n'y a rien eu de plus. Mais, à M. Hosono, j'avais déjà fait part de la précarité de la situation. C'était même avant que l'eau ne réussisse à entrer dans le réacteur. Je lui avais dit que si l'eau n'entrait pas, le cœur du réacteur allait fondre et qu'on atteindrait le syndrome chinois. Je l'avais mis en garde notamment sur le fait que, si nous en arrivions à cette situation, on ne pourrait plus accéder au terrain et qu'on ne pourrait plus non plus s'occuper des réacteurs 1 et 3, qui risquaient eux aussi de se trouver sans eau, que nous nous retrouverions alors avec trois tranches incontrôlables et que ce serait une terrible catastrophe.

$\mathbf{Q}:$ Ça, c'est ce que vous avez dit à M. Hosono. Le lui avez-vous dit par téléphone?

R : Oui, je l'avais appelé.

$\mathbf{Q}$ : Et vous aviez utilisé le portable ou le PHS ?

$\mathbf{R}$ : J'ai appelé son portable en passant par le siège. On appelle le numéro XXXXX au siège avec notre PHS et, ensuite, on fait le numéro du portable de la personne qu'on veut appeler. J'ai utilisé ce moyen, parce que c'était la manière la plus confortable d'appeler quelqu'un.

$\mathbf{Q}$ : Donc, vous, vous aviez utilisé votre PHS.

$\mathbf{R}$ : Oui, c'est ça. 
$\mathbf{Q}$ : Et, en passant par le siège, vous obteniez M. Hosono.

$\mathbf{R}$ : Là, on l'a en direct. On dit qu'on passe par le siège, mais en fait on se branche automatiquement sur les lignes du siège et on obtient directement le correspondant. J'ai directement eu M. Hosono sur son portable.

$\mathbf{Q}:$ : Ah. Vous avez tout de suite eu M. Hosono.

$\mathbf{R}:$ Oui, je lui ai parlé directement.

Q: Ce que vous lui annonciez, c'était le scénario du pire. Comment a-t-il réagi ?

$\mathbf{R}$ : Il m'a dit, " je comprends bien la gravité de la situation que vous me décrivez. Seulement, je vous demande de ne pas renoncer et de faire tout votre possible ».

Q: Je pense que vous avez eu de nombreux échanges au téléphone avec M. Hosono. Dans ces occasions, comment l'avez-vous trouvé ? Comparé à M. Kan, par exemple.

$\mathbf{R}:$ J'ai trouvé que $M$. Hosono était très calme en toutes circonstances. Il n'a jamais élevé la voix. Il demandait toujours posément quelle était la situation. Une fois que je lui avais communiqué les informations, il vérifiait qu'il les avait bien comprises. Il nous encourageait en disant, "je devine que la situation est très dure, mais faites votre possible $»^{41}$.

Q: D'accord. Ce qui fait que, même dans des situations qui demandaient des décisions ou dans des circonstances compliquées sur le terrain lors de manœuvres, il n'amenait pas de confusion.

Si on revient aux évènements de ce 15 mars, vers 6h00, il y a eu cette chute spectaculaire de la pression au réacteur 2, suivie d'une déflagration. Vous recevez d'abord un rapport laissant penser que ce bruit provenait du réacteur 2. Ensuite, vous recevez un autre rapport liant, cette fois, ce bruit à la tranche 4. Et là, vous allez perdre du personnel.

R : Oui, je les ai fait mettre à l'abri en car. Ils sont partis du côté de Fukushima Daini.

$\mathbf{Q}$ : Quelles ont été vos réactions à ce moment-là ? Vous avez eu ce bruit d'explosion, mais vous ne savez pas ce qui s'est passé. Dans ces conditions, vous ne pouvez plus faire de manœuvre sur le terrain. Du moins, pour un temps. Et l'injection d'eau, par exemple?

$\mathbf{R}$ : On l'a arrêtée. Ce que nous avons fait, c'était de nous préoccuper de la radioactivité alentour. Là, j'ai dit à mes hommes de commencer par mesurer ça, le plus sérieusement possible. J'ai dit aux autres de revenir au bâtiment antisismique, puisqu'il était évident qu'il s'était passé quelque chose. Mais le plus important était de savoir si la radioactivité grimpait anormalement. Si l'enceinte de confinement avait été détruite, la radioactivité serait montée en flèche. Il fallait donc surveiller de manière certaine l'évolution de la radioactivité. Ça n'est qu'une fois qu'on aurait eu ces données qu'on pouvait décider de la conduite à tenir. Il était donc vital de les avoir.

Q: Ensuite, vous vous êtes occupé des paramètres? Avez-vous essayé de savoir ce qui s'était passé?

$\mathbf{R}$ : Comme j'avais fait aussi évacuer les salles centrales de commande, pendant un certain temps, on n'a pas eu accès à ces paramètres. De toute manière, le plus important était de connaître l'évolution de la radioactivité. S'il y avait eu de grands changements, il aurait fallu réfléchir aux actions à mener. Le plus urgent était d'observer la radioactivité et de connaître son évolution de manière sûre. 
$\mathbf{Q}$ : Je ne suis pas sûr que vous ayez eu l'information à ce moment-là, mais que signifient ces pressions négatives de la cuve du réacteur au 16 mars?

$\mathbf{R}$ : Ce sont les données du 16 mars à 1h00. On n'arrive plus à comprendre les paramètres du réacteur 2 . La pression de la chambre de dépressurisation, qui était à zéro, chute encore. D'une situation où la pression de la chambre sèche est élevée et celle de la chambre de dépressurisation basse, on passe à une situation où tout en gardant la même pression à la chambre sèche, la chambre de dépressurisation chute à zéro. Ensuite, la pression de la chambre sèche chute également. Pendant tout ce temps, le niveau d'eau est négatif. Comme la pression de la cuve évoluait bizarrement entre temps, on s'était dit, à la limite, que c'étaient les instruments de mesure qui avaient été abîmés par l'accident, et qu'ils se comportaient de manière anormale à cause de ça.

$\mathbf{Q}$ : Si on fait l'hypothèse que les instruments n'étaient pas en panne, une pression négative est une situation difficile à imaginer, je suppose. Vous avez donc pensé assez naturellement que c'étaient les instruments qui étaient en panne à cause de l'explosion.

Pour ce qui est du personnel qui était parti se mettre à l'abri, j'ai entendu dire que le 15 mars vers 10h00, en tout cas dans la matinée, la plupart des personnes de niveau GM avaient commencé à revenir sur le site de la centrale. Ce sont d'ailleurs eux qui m'en ont parlé. Vous, de votre côté, aviez-vous donné des consignes précises de retour? Par exemple, « rappel en priorité des GM », etc. ?

$\mathbf{R}$ : En fait, je ne leur avais pas dit d'aller à Fukushima Daini. Encore une fois, c'était un peu le jeu du téléphone arabe. Effectivement, en discutant des endroits où on pouvait mettre les gens à l'abri, j'avais évoqué, entre autres, la possibilité de Fukushima Daini. À un moment donné, j'ai donc donné l'ordre de retrait et l'ordre de préparer les véhicules. La personne à qui j'ai donné ces ordres a transmis comme consigne aux chauffeurs d'aller à Fukushima Daini. Moi, j'avais eu l'intention de dire à cette personne d'aller mettre provisoirement les gens à l'abri à proximité de la centrale Daiichi, à un endroit où la radioactivité aurait été faible, que ce soit à l'intérieur du périmètre de la centrale ou pas, et d'attendre les consignes à venir. Mais on m'a annoncé qu'ils étaient tous partis à Fukushima Daini. Qu'est-ce que je pouvais y faire? On a pris contact avec eux à leur arrivée à Fukushima Daini et j'ai demandé aux GM de revenir. C'est comme ça qu'ils ont été les premiers à revenir.

$\mathbf{Q}$ : Ah, bon ? Alors, dans votre tête, ils étaient censés attendre à un endroit proche de la centrale, où la radioactivité n'aurait pas été trop forte. Dans les cars, par exemple?

$\mathbf{R}$ : Si on se remet dans le contexte, il y avait le réacteur 2 qui donnait des signes d'inquiétude. C'était certainement le plus exposé. Si on réfléchissait à la radioactivité, le bâtiment antisismique n'était pas la solution. Il est très près du réacteur 2 . Il fallait impérativement s'en éloigner, que ce soit vers le sud ou le nord, il fallait s'en éloigner et se réfugier là où la radioactivité aurait été stable. C'est ce à quoi je pensais. C'est ce que j'avais pensé avoir dit. Mais à bien y réfléchir... Vous comprenez, ils portaient tous le masque intégral. Continuer à porter ça des heures, pendant qu'ils étaient réfugiés... Il y avait de quoi mourir. À bien y réfléchir, je me suis dit qu'ils étaient aussi bien à Fukushima Daini, que c'était la bonne solution. Ils sont allés à Fukushima Daini, ils ont pu enlever les masques et je pense que ça leur a été profitable. 
$\mathbf{Q}$ : Vous avez rappelé les GM en premier. Ensuite, les gens vont se suivre les uns après les autres. Est-ce vous qui désigniez les personnes que vous souhaitiez voir revenir?

$\mathbf{R}$ : La radioactivité était certes montée, mais pas dans des proportions alarmantes. Alors j'avais demandé à chaque chef de groupe de faire revenir les personnes qui étaient susceptibles d'exécuter des manœuvres ou pouvaient venir en soutien.

(Pause)

$\mathbf{R}$ : Vous m'aviez demandé, à un moment, si l'IC du réacteur 1 avait déjà fonctionné par le passé ${ }^{42}$. Je vous avais répondu qu'il y avait peut-être eu possibilité qu'il ait fonctionné en 1991, lorsqu'il y avait eu une fuite, mais que je n'en étais pas certain parce qu'à l'époque j'étais au siège. J'en ai reparlé, depuis, avec des gens qui étaient à la centrale à ce moment-là, comme XXXXX. Ils m'ont dit qu'ils n'avaient pas mis l'IC en marche et que l'accident de mars était la première occasion où ils l'avaient fait fonctionner pour de vrai. Parce qu'ils l'avaient déjà fait fonctionner pour des tests ${ }^{43}$.

$\mathbf{Q}$ : Et si on regarde les autres centrales au Japon?

$\mathbf{R}$ : Les seuls réacteurs qui sont équipés de l'IC, ce sont notre réacteur 1 et le réacteur 1 de la centrale de Tsuruga ${ }^{44}$. Je pense qu'à Tsuruga, non plus, ils ne l'ont jamais mis en marche.

$\mathbf{Q}$ : Vous n'en avez pas entendu parler. Et, évidemment, vous n'aviez pas entendu parler de la manière dont un réacteur peut réagir quand on met l'IC en marche.

$\mathbf{R}$ : Effectivement, nous n'avions pas eu de retour d'expérience.

$\mathbf{Q}$ : Depuis ce matin, nous avons visionné les vidéos, notamment les passages où on voit le directeur de la NSC et les dirigeants de TEPCO, et je vous ai posé des questions pour vérifications. Pour en revenir aux grandes lignes, tout d'abord, concernant les systèmes $\mathrm{HPCl}, \mathrm{RCIC}$ et IC, vous pensez que l'IC pour le réacteur 1, le $\mathrm{RCIC}$ pour les réacteurs 2 et 3 sont en fonction, dès la survenue du séisme. Ensuite, il y a le tsunami. Pour vous, la situation ne change pas pour le fonctionnement de ces systèmes. Le RCIC est toujours en marche pour les réacteurs 2 et 3, I'IC également pour le réacteur 1. Concernant l'HPCI, vous constatez qu'il peut être utilisé sur le réacteur 3, mais pas sur les réacteurs 2 et 1 . Votre but est de préparer la suite, c'est-à-dire l'injection d'eau, tant que l'IC, le HPCl et le RCIC pour le 3 , et le RCIC pour le 2 sont encore opérationnels. Parallèlement, vous commencez à œuvrer pour l'établissement d'une ligne d'éventage pour protéger l'enceinte de confinement, et ce, à un stade précoce de l'accident.

Ensuite, nous avons parlé de ce qu'on trouve dans les manuels de gestion des accidents. Nous avons passé en revue, hier, un certain nombre de nouvelles fonctions qui avaient été ajoutées récemment. Mais, à la base, sans alimentation électrique, il y avait finalement peu de choses utiles. II y avait la DDFP, qui ne demandait pas de source électrique, le SLC ou le CRD, qui s'étaient avérés disponibles lorsque vous aviez mené des recherches pour savoir ce que vous pourriez utiliser en restaurant l'électricité du 2C45. Vous avez travaillé à les rendre opérationnels.

Je voudrais maintenant que vous m'éclairiez sur le réacteur 1, parce que les réponses sont confuses et différentes selon les gens que j'interroge. Concernant le réacteur 1, vous n'avez pas fait d'opération de dépressurisation en ouvrant la vanne SR, n'est-ce pas ?

$\mathbf{R}$ : En tout cas, je n'en ai pas donné l'ordre.

Q: C'est une opération que vous avez uniquement exécutée sur le réacteur 2 et le réacteur 3.

$\mathbf{R}:$ C'est ça.

$\mathbf{Q}$ : Ensuite, on va revenir sur ce qui s'est dit aujourd'hui. Je pense que la première fois que vous avez été contacté directement par le bureau du Premier ministre, ça a été par 
l'entremise de M. Takekuro. Mais en dehors de lui, je veux dire, en dehors des gens de TEPCO, le premier qui vous a contacté est M. Hosono?

R : Oui, c'était M. Hosono.

$\mathbf{Q}$ : C'était après l'explosion de la tranche 1 ?

$\mathbf{R}$ : Oui, je suis sûr que c'était après l'explosion de la tranche 1.

$\mathbf{Q}$ : Mais vous ne savez plus à quel moment précis ça se situe dans le laps de temps qui s'est écoulé jusqu'au 14 mars, avant l'explosion de la tranche 3. C'est ça?

$\mathbf{R}$ : Malheureusement, ça reste flou.

$\mathbf{Q}$ : Vous rappelez-vous de ce dont vous avez parlé cette première fois?

$\mathbf{R}$ : Ça, je m'en souviens assez bien. Une fois de plus, cela avait été compliqué. Une personne de chez nous avait servi d'intermédiaire et m'a passé le secrétaire de M. Hosono. Ensuite, le secrétaire a porté le téléphone à M. Hosono pour qu'il puisse me parler. Il s'est d'abord présenté : «Je suis Hosono. Je sers de conseiller au Premier ministre concernant l'accident de Fukushima». Il m'a tout de suite dit qu'il aurait certainement besoin de me contacter de nombreuses fois pour connaître directement la situation sur le terrain. Il m'a expliqué que le bureau du Premier ministre avait été informé très tardivement de l'explosion de la tranche 1, que TEPCO, ainsi que la NISA avaient tardé à les informer. Il m'a même laissé entendre que, quelque part, il ne faisait pas confiance à cette voie de transmission des informations. En l'occurrence, il désirait établir une liaison directe avec moi pour pouvoir avoir les informations du terrain sans passer par des intermédiaires. Il m'a dit de le contacter s'il y avait quelque chose, que, lui-même, s'il avait quelque chose à vérifier, me contacterait pour m'en parler. Je ne sais pas si je dois vous le dire, mais il m'a demandé de garder la chose secrète. Je pense qu'il ne voulait pas que je l'ébruite trop. Ensuite, on m'a donné le numéro de son portable, ainsi que celui du secrétaire. Ils ont bien insisté pour que je les appelle si nécessaire. Ça, c'est ce que nous nous sommes dit lors de ce premier coup de fil.

$\mathbf{Q}$ : Justement, lors de ce coup de fil, avez-vous évoqué, pour chaque réacteur, ce que vous comptiez faire concrètement?

R : Non, pas cette fois-là. On a juste établi le contact et on s'est promis de se téléphoner en cas de besoin.

Q: Par exemple, vers le 14 mars, au petit matin, la pression de la chambre sèche du réacteur 3 est à 500, vous donnez l'ordre de retrait et les gens qui travaillaient sur le terrain partent se mettre à l'abri. On en a parlé tout à l'heure. Dans une situation de cette sorte, où les informations qui n'étaient plus communiquées à la presse passent quand même par le canal de la NHK, vous n'en parlez pas avec le conseiller?

$\mathbf{R}$ : Nous n'avons pas communiqué à ce moment précis. Mais je pense qu'il y avait eu contact avant. Au moment où j'ai donné l'ordre de retrait. Je ne me rappelle pas si c'était lui qui nous avait contactés ou si c'est moi qui l'avais appelé, mais j'ai le souvenir de l'avoir informé de la situation.

$\mathbf{Q}:$ L'informer de la situation sur le terrain?

$\mathbf{R}$ : Oui, de la situation sur le terrain. Dans la vidéo de tout à l'heure, on m'a vu en train de dire que la situation était très inquiétante. Je pense qu'avant ou après, je l'ai contacté pour le prévenir que la situation était préoccupante, que le réacteur 3 donnait des signes d'inquiétude. À y réfléchir maintenant, il me semble bien l'avoir fait. Évidemment, je n'ai pas de preuves pour étayer ça. 
$\mathbf{Q}$ : Donc, si j'ai bien compris, au début de l'accident, quand les évènements s'enchaînaient très vite, à chaque fois qu'il y avait une évolution notable dans la situation, soit M. Hosono vous appelait, soit vous l'appeliez.

$\mathbf{R}$ : Je ne me rappelle plus très bien quand ça a commencé, mais, effectivement, quand il y avait évolution dans la situation, après l'avoir évoquée en téléconférence, je l'appelais à part pour l'en informer.

$\mathbf{Q}$ : Si vous étiez passé par la ligne officielle, comment cela aurait dû se passer?

$\mathbf{R}$ : Dans ce cas, évidemment, le centre de crise du siège, après avoir reçu les nouvelles du terrain, aurait dû en informer directement le correspondant au bureau du Premier ministre et celui de la NISA, par téléphone, comme on l'a vu tout à l'heure sur la vidéo, pour que tout le monde partage l'information. C'est ce qui aurait été la voie normale. Je pense que ce système n'était pas encore bien rôdé, au moment de l'explosion de la tranche 1 , et que, pour une raison ou une autre, l'information qui était passée de la centrale au siège n'a pas été transmise tout de suite, qu'on a perdu du temps quelque part.

$\mathbf{Q}$ : Je viens de m'en souvenir à l'instant. Tout au début des vidéos de la téléconférence, le 12 mars à 22h59, oui, celui qui est catalogué 23h00, c'est tout au début, vous le trouvez? Non? (Au technicien) Vous voulez nous montrer le tout début? ( $\grave{A}$ Yoshida) On voit M. Takekuro parler longuement. II commence par se plaindre, puis il se lance dans un grand discours. Ça vous dit quelque chose?

$\mathbf{R}:$ Non, je ne m'en souviens presque pas.

$\mathbf{Q}$ : Vous ne vous en souvenez pas? Alors vous ne vous souvenez pas pourquoi il s'est lancé tout à coup dans ce discours exalté.

$\mathbf{R}$ : Non, je ne sais pas. Vous dites le 12 à 22h00 ? C'est après l'explosion de la tranche 1 , alors.

$\mathbf{Q}:$ Allons-y.

(Vidéo)

«En règle générale, il y a des conseillers du Premier ministre, le vice-chef de cabinet, des gens comme ça, qui arrangent les choses au préalable. Comme vous savez, au sein du Gouvernement démocrate, ce sont des personnalités extrêmement jeunes qui remplissent la plupart de ces rôles. Je pense que ce doit être des personnes brillantes, ayant, bien entendu, une certaine largeur de vue. Mais je dois admettre que j'émettrai quelques réserves quant à leur profondeur, leur capacité à encaisser. C'est peut-être ce qui explique cette ambiance très spéciale. Vous connaissez l'expression «Ira Kan», Kan l'irascible ${ }^{46}$. Je peux vous confirmer qu'il se met en colère à tout bout de champ. Moi-même, je me suis fait enguirlander six ou sept fois. À côté de ça, les colères de Yoshida sont gentillettes.

Pour vous expliquer comment se prennent les décisions, je vais vous relater l'exemple d'hier, quand on a déterminé les zones d'évacuation. Pour commencer, M. Kan arrive et nous presse pour savoir ce qu'on va faire. Avec M. Madarame, on essaie de lui expliquer. À ce moment-là, il fait une fixation sur deux points: "Quel est le fondement de cette décision?»; "Peut-on être sûr que tout ira bien quoi qu'il arrive? ». II revient infatigablement sur ces deux points en vociférant. Malgré quelques hésitations, il décide d'évacuer la zone des $3 \mathrm{~km}$, en se disant que ce sera mieux que de ne rien faire. Pendant la nuit, le périmètre s'élargit à $10 \mathrm{~km}$, puis à $20 \mathrm{~km}$ aujourd'hui. Comment vous dire ? II ne raisonne pas tout à fait comme nous, son axe de raisonnement est légèrement décalé. Face à un raisonnement rationnel qui mène à une certaine conclusion, il est extrêmement sensible à ce qu'elle contient d'incertitude ou de risque, il est taraudé par la question de ce qu'il va faire s'il y a eu erreur. C'est à peu près comme ça [...], que les choses se décident.

C'est pourquoi je pense que si j'ai réussi jusqu'à maintenant à faire approuver les différentes orientations que vous avez prises concernant l'injection d'eau de mer, l'éventage 
d'hier ou la détermination des zones d'évacuation, tout en tenant compte de l'état du bureau du Premier ministre, de la marge de manœuvre dont je dispose et de mes concertations avec M. Madarame, c'est que votre travail, en amont, était bien réfléchi, étayé et que vous êtes restés extrêmement souples et ouverts.

À mon avis, la décision audacieuse, in fine, d'injecter de l'eau de mer et la malheureuse explosion d'hydrogène, toutes deux advenues aujourd'hui, vont lourdement marquer les évènements que nous vivons actuellement. Dans un petit moment, lorsque le temps viendra de revenir à moyen ou long terme sur ces évènements, que ce soit dans le cadre d'une enquête officielle ou pas, il vous appartiendra de débattre à nouveau sur ces faits et d'en tirer les leçons pour une prévention bien pensée des accidents, une gestion des risques au quotidien ou un engineering plus efficace.

De manière inattendue, j'ai eu l'occasion de côtoyer, au sein du bureau du Premier ministre, différentes personnalités politiques dans un contexte sortant de l'ordinaire. II a été très délicat de présenter les choses de manière à ce que ces messieurs du Gouvernement puissent comprendre, accepter, c'est-à-dire puissent se laisser convaincre, par le point de vue d'un exploitant. II ne faut pas oublier que le bureau du Premier ministre est un lieu extrêmement fermé. Je crois avoir eu beaucoup de chance que ce que les politiques pensaient, dans ce lieu où les informations ne se transmettent pas suffisamment, et ce que vous pensiez, vous, ici, n'aient pas été trop éloignés. Je vous suis aussi reconnaissant d'avoir accepté différentes suggestions qui venaient de là-bas.

En même temps, ce lieu ne peut pas rester isolé de la sorte, sans presque recevoir d'information. Moi-même, j'ai appris l'explosion de 15h30 à 17h30 en regardant la télévision dans le cabinet de travail de M. Kan. Je ne vous dis pas ma surprise. Si on réfléchit un tant soit peu à l'avenir, il est indispensable d'améliorer la communication. On ne peut pas laisser les choses en l'état, c'en est gênant.

Je pense que c'est une chose que j'aurai encore l'occasion de demander à chacun de vous, mais il me semble [...] que, concernant les réponses aux séismes, il faudra se poser la question de savoir jusqu'à quel point cette préoccupation se reflète dans la conscience de chacun, car cela influencera la compréhension, le partage d'information et la réponse [...]. Excusez-moi d'avoir été long et confus. Cet énorme séisme représente, aussi bien pour vous que pour notre entreprise, un grand fardeau, dont il faudra tenir compte dorénavant. Je souhaite que vous ne vous laissiez pas écraser par le poids de ce fardeau et que vous agissiez toujours en réfléchissant à ce qui doit être. Je fonde beaucoup d'espoir en vous tous. Merci beaucoup. »

«Je pense que nous n'avons pas été à la hauteur dans notre communication avec le bureau du Premier ministre. Le directeur de la NISA m'a également fait savoir tout à l'heure qu'il ne recevait pas suffisamment d'informations. Nous allons améliorer tout ça à partir de demain. »

«Puis-je évoquer un autre problème? »

«Bien sûr, directeur Yoshida. Je vous en prie. »

"C'est une chose que j'ai entendue dire ici, à la centrale. [...] II semblerait qu'il y ait un très gros mouvement de colère parmi les personnes qui ont été évacuées de chez elles. Elles nous reprochent de ne pas venir nous expliquer en tant que TEPCO. Elles se demandent, par exemple, jusqu'à quand va durer cette évacuation. Je trouve qu'il y a beaucoup de questions et de sujets de mécontentement auxquels nous n'avons pas vraiment répondu. II est sûr que les gens vont nous haïr à la suite de cet accident. Mais si on ne fait rien à ce stade, ça va être encore pire. Nous, notre gros souci à la centrale, c'est que, à l'heure actuelle, nous ne disposons pas de suffisamment de personnel pour nous occuper sérieusement de ce problème. »

« Nous y pensons. Je voudrais qu'on prenne l'avis du département « implantation » et puis du groupe «communication » de la centrale avant de prendre des décisions. » 
«Tu sais, le groupe «communication» de la centrale est déjà débordé par ses relations avec le département, les municipalités, sans parler de la presse. II va avoir du mal à trouver le temps de se déplacer auprès des habitants. »

«On peut envoyer des gens du siège. Mais il faut qu'on sache où aller. Là, on a besoin des conseils du terrain. »

«Bien sûr, c'est le moins qu'on puisse faire. Mais, s'il vous plaît, gardez tous à l'esprit qu'avec les membres dont nous disposons actuellement, il y a des choses qu'il nous est difficile de faire. »

" Je vais en discuter avec le département « implantation ». »

«Ce problème me rappelle ce dont nous avons parlé, à nos moments perdus, avec M. Madarame, ces deux derniers jours. Ce séisme, ce tsunami et, bien sûr, l'accident de la centrale de TEPCO ont obligé de très nombreuses personnes à mener une vie de réfugié, dans des conditions très difficiles. Pour nous, dorénavant, il est indispensable de ne pas perdre de vue le sort de ces personnes. Nous devons agir en réfléchissant à ce qui pourrait raccourcir autant que possible cette vie de réfugié que nous leur avons imposée. Nous devons, dans les meilleurs délais, reconstruire un environnement, remplir les conditions qui permettent un retour à la normale pour ces personnes. »

$\mathbf{Q}$ : On peut couper ici. Voici donc un extrait qui date des débuts de l'enregistrement vidéo, bien qu'il se situe déjà après l'explosion de la tranche 1. On y voit M. Takekuro raconter toutes sortes de choses qui lui passent par la tête.

\section{$\mathbf{R}$ : Il est « long et confus».}

$\mathbf{Q}$ : Oui, il parle très longuement. Avez-vous une idée de ce qui a occasionné un discours aussi exalté?

$\mathbf{R}$ : Je vais vous raconter ce qu'il m'a dit après. En fait, moi-même, je n'ai pas un très grand souvenir de ce discours. D'après ce qu'il m'a dit après coup, il avait été envoyé auprès du Premier ministre sur ordre du Président ou du Directeur général, je ne sais plus, parce qu'il fallait que quelqu'un de TEPCO y aille. Or, Mutô, qui aurait pu y aller, était parti au centre hors site. Du coup, c'est Takekuro qui y est allé. Il pensait n'y rester que quelques heures, histoire de savoir ce que voulait le Gouvernement. Mais quand il a voulu repartir, on l'a retenu et, en fin de compte, il est resté enfermé à la résidence du Premier ministre pendant deux jours, les 11 et 12 mars.

$\mathbf{Q}:$ II n'était pas prévu, au départ, qu'il y reste si longtemps?

$\mathbf{R}$ : Non. À un moment il a voulu partir, mais on l'a retenu. C'est ce que l'intéressé m'a dit en personne. Pendant tout ce temps, il a dû répondre aux questions du Premier ministre et, peut-être de M. Hosono, ça je ne sais pas trop. Toujours est-il que la séquence qu'on voit sur la vidéo est juste le moment où, les choses s'étant un peu tassées chez le Premier ministre, il a enfin réussi à revenir au bureau. Au siège, au centre de crise. C'est pour ça qu'il s'étend longuement sur les problèmes de communication avec le bureau du Premier ministre ou ses impressions personnelles.

$\mathbf{Q}$ : Je vous explique pourquoi j'ai choisi de vous faire passer cet extrait. M. Takekuro dit qu'il n'a appris l'explosion de $15 \mathrm{~h} 30$ qu'à 17 h30 en regardant la télévision. Ça m'a semblé une illustration parfaite de ce dont parlait tout à l'heure M. Hosono, la lenteur des informations. On y évoque aussi la réclamation de la NISA concernant le manque d'informations, encore une fois. Je pense que lors d'un accident, tout le monde est focalisé sur la réponse à apporter sur le terrain. "Qu'allons-nous faire? ». C'est certainement d'autant plus vrai et naturel pour ceux qui sont sur le terrain. Mais, même pour ceux qui sont au siège, il s'agit d'une première, d'une expérience qu'ils n'ont jamais vécue. Alors, tous les regards sont tournés vers le terrain et on oublie. On oublie ci, on oublie ça, et on a toujours un temps de retard. Ce n'est qu'une fois qu'on a une réclamation, "mais qu'est-ce qui se passe donc ici ?", qu'on se rappelle qu'il faut en rendre compte et qu'on le fait. Je suis persuadé que tout n'a 
pas pu être assumé de manière impeccable sur le plan de la communication et ça me semble naturel. Mais à force d'être victimes des retards, vos correspondants en ont eu assez, d'où ces réclamations. Mais, si je comprends bien, il revenait au siège d'entretenir les relations avec les différents ministères et le bureau du Premier ministre. Ils parlent d'ailleurs dans l'extrait d'améliorer leur communication. II me semble que c'était leur problème à eux et que, vous, sur le terrain, n'étiez pas vraiment concernés par le problème. Si la relation est étroite entre le terrain et le siège, vous, vous avez rempli votre part du travail, n'est-ce pas ?

$\mathbf{R}$ : C'est pour ça que nous sommes connectés par vidéoconférence.

$\mathbf{Q}$ : Et cette vidéoconférence a toujours bien fonctionné durant l'accident.

L'autre problème, que vous évoquez là, concerne les habitants qui ont été évacués. Forcés de se mettre à l'abri, le mécontentement gronde parmi eux. Du fait que TEPCO ne se manifeste pas, leur colère a tendance à décupler. Si TEPCO ne fait rien, l'entreprise va se trouver de plus en plus isolée. N'est-il pas temps de répondre sérieusement aux attentes de la population afın d'obtenir un minimum d'adhésion de sa part? Voilà ce que vous suggérez. Depuis quand pensiez-vous de cette façon?

$\mathbf{R}$ : Les évacués étaient regroupés en différents endroits. Bien sûr, il y avait parmi eux des employés de chez nous aussi. Par hasard, un de ceux qui étaient partis donner un coup de main dans un de ces refuges est passé par le bâtiment antisismique.

$\mathbf{Q}:$ Un employé de chez TEPCO ?

$\mathbf{R}$ : Oui, un employé de chez TEPCO. Il m'a pris à part et m'a raconté que les réfugiés étaient très mécontents, surtout du fait qu'on ne leur donnait aucune information sur ce qui se passait. J'ai tout de suite pensé que c'était mauvais. J'ai demandé à la «communication » de la centrale ce qu'il en était, s'ils avaient communiqué de manière suffisamment dense. Mais même ceux de la "communication " avaient été embrigadés dans la décontamination, du fait de l'application des règles de prévention en cas d'accident. Il n'y avait personne de disponible. Les rares qui étaient là étaient occupés à contacter les organes officiels, les ministères. De fait, personne, ici, n'était disponible pour sortir de la centrale et aller au-devant de la population pour fournir des explications. Nous ne pouvions pas résoudre le problème à la centrale, il fallait demander de l'aide extérieure, c'est pour ça que j'ai sollicité le siège.

$\mathbf{Q}$ : Je pense, en effet, que c'est une question très importante. Que s'est-il passé ensuite?

$\mathbf{R}$ : Finalement, on a créé un «service de soutien local». Mais avant ça, le siège a recruté des gens auprès du département " communication » du siège, de la centrale de Fukushima Daini et d'autres services du siège, pour former des groupes qui devaient s'occuper des différents refuges. Ça nous a pris un peu de temps, mais on a réussi à élaborer un système d'information vis-à-vis de la population.

$\mathbf{Q}:$ Vous dites que ça a pris du temps. C'est après tous les évènements qui sont survenus sur les tranches 1, 2 et 3 ?

$\mathbf{R}$ : Je crois qu'on n'avait pas encore eu le temps de s'en occuper, à ce moment-là.

$\mathbf{Q}:$ C'est donc un peu après.

$\mathbf{R}$ : Mettez-vous à la place des évacués. On commence par vous dire de partir à $3 \mathrm{~km}$, ensuite, au fur et à mesure que le temps passe, on vous trimballe d'un refuge à l'autre. Je crois que la première semaine a été vraiment mouvementée pour eux. Et, pour nous aussi, les évènements ont fait qu'on n'a pas pu répondre correctement à leur attente. Moi-même, je ne suis pas sorti du bâtiment antisismique, qui était notre refuge. 
$\mathbf{Q}$ : Quand vous parlez des membres de la cellule de crise, est-ce que cela comprend aussi des femmes?

$\mathbf{R}$ : En principe, oui. Mais quand il y a des risques de radioactivité, comme cette foisci, on peut difficilement garder des femmes. Dans les faits, il y a eu quelques volontaires. Malheureusement, elles ont été contaminées (contamination interne) et les journaux ne se sont pas gênés pour nous taper dessus. Les journaux, mais aussi le ministère de la Santé et du Travail. Pourtant elles avaient un rôle très important dans le bâtiment antisismique. Mais, une fois qu'on a su qu'elles avaient été contaminées, on a décidé d'évacuer toutes les femmes.

$\mathbf{Q}$ : En temps normal, il y a des femmes qui travaillent à la centrale. Que sont-elles devenues ? Ont-elles été toutes mutées?

$\mathbf{R}$ : Cela dépend des personnes, mais la plupart ont été envoyées, sous forme de détachement, dans une nouvelle structure qui a été créée, le «service de soutien local ».

$\mathbf{Q}:$ C'est une structure qui a été créée au sein de TEPCO ?

$\mathbf{R}$ : Oui, au sein de TEPCO.

$\mathbf{Q}:$ Au siège?

$\mathbf{R}$ : Structurellement, le service dépend du siège. Elles y sont d'abord allées en détachement, puis, en juillet, il y a eu les nominations officielles.

$\mathbf{Q}$ : Cela veut dire qu'elles ne reviendront pas sur le site pour occuper les fonctions qu'elles y exerçaient.

$\mathbf{R}:$ Non, pas les femmes.

$\mathbf{Q}$ : Prenons l'exemple d'une personne qui aurait travaillé au département «prévention ». Je pense que ce genre de personne est appelé à beaucoup travailler sur le terrain. Les femmes qui travaillaient dans le département «prévention » ont dû évacuer le site, à la suite du séisme et du tsunami. Est-ce qu'elles continuent à travailler dans le même secteur, là où elles ont été évacuées?

$\mathbf{R}$ : Il y a plusieurs scénarios possibles. Il y en a quelques-unes qui ont été affectées au " centre de stabilisation ", nouvellement créé à Fukushima Daini, et qui y fournissent un travail assez technique. D'autres sont plus dans l'infrastructure et s'occupent des repas et d'autres choses de ce genre. Cela dépend beaucoup des personnes. Certaines ont été distribuées au « service de soutien local » et s'occupent des réfugiés.

$\mathbf{Q}$ : Dans ce dernier cas, elles travaillent en coordination avec l'État ou les autorités locales, je suppose. TEPCO ne fait pas ça seul, n'est-ce pas?

$\mathbf{R}$ : Non. Comme je suis bloqué ici, je ne sais pas au juste ce qu'elles font comme travail, mais TEPCO ne fait pas ça seul.

$\mathbf{Q}$ : Au moment de l'extrait qu'on vient de voir, vous ne savez évidemment pas ce qui va se passer par la suite. La tranche 1 vient d'exploser, peut-être les tranches 2 et 3 vont connaître le même sort. Vous ne savez pas non plus au bout de combien de jours les choses vont se stabiliser. L'expérience de la tranche 1 vous a appris, en tout cas, qu'il faut énormément de monde quand la radioactivité augmente. Je veux dire que, ne serait-ce que pour l'éventage, il a fallu former trois équipes de deux personnes pour tenter d'ouvrir les vannes: une équipe ne pouvant s'attarder longtemps sur place, était remplacée par la suivante, et ainsi de suite, pour un résultat décevant, puisque vous n'avez pas réussir à ouvrir les vannes. Je pense qu'au soir du 12 mars, vous accusez le coup de cette expérience malheureuse. Vous savez que si une autre situation d'urgence se présentait, vous allez manquer de personnel. De même, vous manquez cruellement de matériel. Aviezvous une idée, au stade de la soirée du 12, de la manière dont vous alliez trouver le 
personnel nécessaire ou la manière de vous procurer le matériel manquant? En aviez-vous discuté autour de vous?

$\mathbf{R}$ : Bien sûr. Et depuis longtemps, déjà.

Q : Déjà, au soir du 11, vous manquiez de personnel ?

$\mathbf{R}$ : Je savais qu'une situation d'extrême urgence allait continuer à nous accaparer. La centrale ne disposait que de moyens limités. La radioactivité avait déjà commencé à grimper la nuit du 11 mars. Effectivement, nous avions vécu l'expérience très pénible de la tranche 1. Il était déjà évident, pour commencer, que nous avions totalement explosé les effectifs des surveillants des doses radioactives. Puisqu'on n'arrêtait pas d'entrer et de sortir des zones contaminées, il nous fallait du personnel pour surveiller les doses reçues. Les surveillants constituaient un personnel de première nécessité. D'autre part, sur le terrain, ce sont les agents de conduite qui effectuaient les manœuvres. Tous n'avaient pas encore été rappelés à la centrale. Là, nous disposions encore d'une certaine réserve. Pour ce qui était du matériel, comme on l'a déjà évoqué, depuis le début, j'avais réclamé de l'eau et des carburants, de l'essence, de l'huile, en priorité et le plus rapidement possible. Il nous fallait aussi des véhicules de pompiers.

$\mathbf{Q}$ : L'alimentation électrique aussi?

$\mathbf{R}$ : Bien sûr, des groupes électrogènes, des batteries.

$\mathbf{Q}$ : Du côté de ceux qui recevaient vos demandes, ils devaient sans doute passer euxmêmes commande à d'autres qui, même en ayant la volonté de vous apporter ça au plus vite, se sont trouvés confrontés à encore d'autres sortes de difficultés, du genre problèmes de circulation. C'est une histoire que j'ai entendue de la bouche d'un collaborateur de Nanmei Corporation. II devait faire le trajet Kashiwazaki - Fukushima Daiichi. À l'instant où il est parti, il s'est mis à neiger violemment. Quand il a rejoint la route, il y avait une file ininterrompue de voitures de pompiers. Les pompiers étaient sollicités partout. II n'y avait pas que Fukushima Daiichi. II y avait des zones sinistrées un peu partout et, de tout le pays, les voitures roulaient vers le Tôhoku, créant des embouteillages monstres. Vous, de votre côté, sur le terrain, vous rongiez votre frein. Peut-être aviez-vous eu vent de ces histoires, mais les mots n'ont pas l'impact de ce qu'on peut voir en vrai. Toujours est-il que je me suis posé la question des soins médicaux. Aviez-vous demandé l'assistance de médecins?

$\mathbf{R}$ : Oui, je l'avais fait. Au départ nous nous sommes arrangés avec le système habituel. Il y a un médecin détaché à Fukushima Daini. Quand il arrivait quelque chose, nous faisions le déplacement jusque-là pour le consulter. C'est ce que nous avons continué à faire au début. À un stade relativement précoce, après l'accident, on a installé un médecin à demeure au J-Village, où il y avait déjà des locaux médicaux. En plus, comme cela constitue l'une des missions du centre hors site, il me semble qu'au tout début, un médecin s'y trouvait aussi.

$\mathbf{Q}$ : Ah, il y avait un médecin à Fukushima Daini. II me semble qu'il y a eu des blessés lors de l'explosion de la tranche 3. II y avait sans doute des problèmes d'irradiation, mais n'y avait-il pas aussi des soins chirurgicaux à prodiguer?

$\mathbf{R}$ : Le médecin de Fukushima Daini n'était pas qualifié pour de la chirurgie. Il examinait les blessés et on les évacuait vers le J-Village ou vers les hôpitaux ${ }^{47}$. Aujourd'hui, le système est bien rôdé avec le J-Village qui fait le tri et envoie les blessés vers l'hôpital municipal d'Iwaki ${ }^{48}$, par exemple. Mais au début, c'était terrible. Je ne sais pas si vous avez eu l'occasion de vous entretenir avec un de nos agents de conduite, XXXXX. Il s'est cassé le bras gauche lors de l'explosion de la tranche 1. Il a éprouvé les pires difficultés pour rentrer sur Tôkyô ${ }^{49}$. Il lui a fallu près de 48 heures, 
en faisant de l'auto-stop. Comme il avait été irradié, on lui avait arraché tous ses vêtements. Il a dû marcher en sous-vêtements dans le froid qui sévissait. C'était tellement rocambolesque que je lui ai dit de tout mettre par écrit.

$\mathbf{Q}:$ : Ce que vous me racontez là, c'est ce qui s'est passé après l'hôpital ?

$\mathbf{R}$ : C'est que personne ne voulait de lui. On l'a d'abord transporté de la centrale vers le centre hors site, il me semble. Là, on lui dit d'aller à l'hôpital départemental Ôno ${ }^{50}$. Mais, à l'hôpital Ôno, les médecins ne sont pas là. En fait, il n'y a personne. L'hôpital est vide. On lui dit d'aller cette fois à Kôriyama ${ }^{51}$. (...) aurait dû l'accompagner, mais, je ne sais pour quelle raison, il a été abandonné à lui-même. Il arrive tout de même par ses propres moyens à atteindre Kôriyama, où on lui prodigue les premiers soins. Mais on lui dit qu'on ne peut pas l'admettre à l'hôpital, car il a été irradié, donc pollué. C'est à ce moment-là qu'on le dépouille de ses vêtements. La nuit tombe et il se balade en sous-vêtements, quand le patron du magasin Shimamura ${ }^{52}$ le prend en pitié et lui donne un blouson. Il arrive enfin à entrer en contact avec sa famille qui lui dégote un billet d'avion de Fukushima à Tôkyô. Il rentre enfin à Tôkyô et se présente à l'hôpital TEPCO ${ }^{53}$, où on lui dit d'aller à l'Institut national de sciences radiologiques (National Institute of Radiological Sciences - NIRS) ${ }^{54}$. J'ai vraiment trouvé qu'ils n'avaient pas de cœur à l'hôpital TEPCO. Voilà tout ce qu'il a subi, le tout toujours avec un bras cassé. Moi-même, je n'en savais rien à ce moment-là.

Q: Il a fini par être soigné ?

$\mathbf{R}$ : Comme il a attendu longtemps avant de recevoir les premiers soins, il a perdu du sang. Vous vous imaginez? Le bras cassé, perdant du sang... Si vous l'interrogez, il vous racontera dans quel état pitoyable il se trouvait.

Q : Et qu'est-il devenu depuis ? Est-il revenu sur le terrain ?

$\mathbf{R}$ : Il a été soigné à Tôkyô. Pendant un temps, il a travaillé là-bas, mais maintenant il est revenu ici, au centre de «stabilisation ».

$\mathbf{Q}: \|$ a recouvré la santé?

$\mathbf{R}$ : Oui, son bras a l'air d'être revenu à la normale. Il se comporte comme avant, mais je soupçonne que, mentalement, il a vécu quelque chose d'assez traumatisant.

$\mathbf{Q}$ : Évidemment, lorsque toute une zone est évacuée, les médecins aussi sont compris dans l'évacuation. Du coup, il n'y a plus un seul médecin sur la zone.

R : Eh, oui !

Q : Plus tard, quand la tranche 3 a explosé, vous aviez encore eu des blessés, il me semble. Qu'en avez-vous fait?

R : Au bout 'de trois jours, c'est-à-dire vers le 14 mars, on avait enfin établi un circuit qui envoyait d'abord les blessés vers Fukushima Daini, ensuite au J-Village, qui faisait le tri vers l'hôpital. Mais il avait fallu trois jours pour en arriver là. Avant, a fortiori vers le 12 , rien n'avait été établi de manière claire. Personne ne savait au juste ce qui relevait de sa responsabilité. Je pense que les premiers blessés ont été, dans un certain sens, de grandes victimes.

Q: Oui, comme ce chef du groupe « incendie».

L'heure tourne. Cela fait donc la quatrième séance d'audition aujourd'hui. Cela représente environ vingt heures d'enregistrement, qui nous ont permis de faire un tour rapide des évènements. Je vous remercie de vous être plié à cet exercice, en vous remémorant des scènes que vous aviez envie d'oublier et en vous confiant à moi. Maintenant, la Commission, il s'agit surtout de moi, va se tourner vers les personnes du siège ou des 
personnalités représentant l'État. Dans ce contexte, il m'arrivera peut-être de vous solliciter de nouveau pour étayer tel ou tel fait, pour savoir si ces personnalités me racontent bien la vérité. Si l'occasion devait se présenter, je formulerai ma demande auprès du siège de TEPCO.

$\mathbf{R}$ : Je vous en prie. Je suis à votre disposition.

$\mathbf{Q}$ : Merci. Je compte encore sur votre coopération. Je dois également vous remercier d'avoir incité nombre de vos collègues et subordonnés à collaborer à cette enquête. Cela m'a été d'une grande aide. Cette partie de l'enquête va se poursuivre, mais soyez assuré que je ferai mon possible pour que ces séances ne viennent pas perturber les travaux dans lesquels vous êtes engagés.

$\mathbf{R}$ : Je suis heureux de pouvoir coopérer.

$\mathbf{Q}:$ Eh, bien. Nous allons terminer maintenant.

R : D'accord.

$\mathbf{Q}$ : Merci beaucoup.

R : Merci beaucoup.

(Fin de l'audition)

\section{NOTES}

1. Deux auditions sont organisées le 9 août 2011. La première se déroule de 9 h54 à $12 \mathrm{~h} 00$ (pause de 11h24 à 11h35), puis de 12h58 à 15h53 (pause de 14h47 à 14h59). Les deux enquêteurs chargés d'interroger Yoshida sont Tsunemasa Katô et Tetsu Chiba les mêmes que pour l'audition du 8 août 2011. La seconde audition du 9 août 2011, conduite cette fois par l'enquêteur Kôta Okada, a lieu de 16 h00 à 17h00. Son contenu est présenté dans le compte rendu suivant.

2. Le technicien.

3. Les gaz nobles, ou gaz rares, sont des éléments chimiques qui appartiennent au groupe 18 du tableau périodique des éléments. Ce groupe de gaz comprend l'hélium, le néon, l'argon, le krypton, le xénon, le radon.

4. Élément chimique qui appartient au groupe 17 (famille des halogènes) du tableau périodique des éléments.

5. Pour rappel, l'explosion dans le bâtiment du réacteur 1 a lieu le 12 mars à 15 h36.

6. Confusion de Yoshida : aucune personne du nom de Naitô ne fait partie du cabinet du Premier ministre.

7. Pour rappel, il s'agit du professeur Haruki Madarame.

8. Masaya Yasui, né en 1958, rejoint le METI en 1982 après des études en génie nucléaire à l'Université de Kyoto. Au moment de l'accident de Fukushima Daiichi, il est directeur de l'Agency for Natural Resources and Energy. Il est ensuite chargé de la réforme de la 
sûreté nucléaire, avant de devenir le $\mathrm{n}^{\circ} 3$ de la NRA (en charge de la supervision des mesures d'urgence).

9. Yukio Edano, né en 1964, est Secrétaire général du Cabinet de Naoto Kan entre janvier et septembre 2011. Ce poste correspond grosso modo à celui de "Secrétaire général du Gouvernement » en France, mais il a un statut de ministre d'État et assure le rôle aussi de directeur de Cabinet et de porte-parole du Gouvernement. La traduction officielle en anglais est "Chief Cabinet Secretary ». Il est assisté de trois Secrétaires généraux adjoints. Edano est député (Chambre des représentants) depuis 1993 et secrétaire général du Parti démocrate japonais de 2014 à sa disparition en 2016. Il préside le Parti démocrate constitutionnel, qu'il lance le 3 octobre 2017.

10. Ces échanges avec le professeur Madarame sont mentionnés dans les auditions du 22 et du 29 juillet 2011.

11. Yasushi Hibino est ingénieur en électricité et a été camarade de classe de Naoto Kan à l'Université de technologie de Tôkyô (Kan, 2017). Il travaille au Nippon Telegraph and Telephone Corporation (NTT) Research Laboratory, avant de devenir vice-président de la Japan Advanced Institute of Science and Technology (JAIST). Il est nommé conseiller spécial du Gouvernement durant la crise nucléaire.

12. Université nationale située dans la ville de Fukui (préfecture de Fukui, région de Chûbu). Elle est fondée en 1949.

13. Les centrales nucléaires disposent souvent de centres d'entraînement pour leurs employés. Certaines disposent même de piscines pour reproduire les piscines d'entreposage de combustible irradié. Nous ne pouvons néanmoins certifier que Fukushima Daiichi possédait bien une de ces piscines d'entraînement.

14. L'Agence nationale de pompiers (National Fire Agency) est sous la tutelle du ministère de l'Intérieur japonais. Elle est fondée le $1^{\mathrm{er}}$ mars 1995 afin d'améliorer la lutte contre les incendies, l'efficacité des secours et des services médicaux en cas de catastrophe.

15. Yoshida évoque ici la Tôhoku Electric Power Company (dont le siège est à Sendai) et qui fournit de l'électricité dans le nord du Japon.

16. TEPCO exploite la centrale thermique de Futtsu à Chiba (préfecture de Chiba, région du Kantô).

17. $800 \div 0,8=1000$. Pourtant la transcription de l'audition note bien « cent minutes ».

18. Allusion aux auditions des 22 et 29 juillet 2011, auxquelles ont participé les professeurs Yôtarô Hatamura et Masao Fuchigami.

19. Yoshida fait référence à l'explosion survenue dans le bâtiment réacteur 3 le 14 mars à 11 h01 (cf. audition du 29 juillet 2011).

20. Pour rappel, Yoshida évoque la visite de Naoto Kan, qui s'est rendu à Fukushima Daiichi le matin du 12 mars (cf. audition du 22 juillet 2011).

21. Après le séisme, deux travailleurs, qui étaient en train d'inspecter le matériel dans le sous-sol du bâtiment de la turbine de la tranche 4, ont été emportés par l'inondation causée par le tsunami. Ils s'appelaient Yoshiki Terashima et Kazuhito Kokubo (Kadota, 2014).

22. Les deux corps ont été retrouvés dans le sous-sol de la tranche 4 le 30 mars 2011.

23. La centrale de Hirono, exploitée par TEPCO, est une centrale thermique au fioul située à une trentaine de kilomètres de Fukushima Daiichi. 
24. Référence au circuit principal de vapeur.

25. Ville située dans la préfecture de Fukushima à une cinquantaine de kilomètres de Fukushima Daiichi.

26. Fellow: conseiller technique de la direction de TEPCO.

27. Selon le règlement établi par TEPCO, le directeur de la cellule en charge des régions doit se rendre au centre hors site d'ôkuma. Comme le précise Yoshida, Sakae Mutô (vice-Président de TEPCO) est le directeur de cette cellule et Akio Komori (directeur de la division nucléaire de TEPCO) en est le directeur adjoint.

28. Fosse du robinet inverseur.

29. Naohiro Masuda est né en 1958. Il étudie à l'Université nationale de Yokohama, où il est diplômé en ingénierie électrique. En 1982, il est engagé à TEPCO. Il occupe son premier poste à Fukushima Daini, alors que la centrale nucléaire est en construction. En 1989, il devient chef d'équipe à Kashiwazaki-Kariwa. Il est ensuite envoyé au siège de TEPCO, puis revient travailler à Daini en 2005 comme chef de l'exploitation et de la maintenance. Il est de nouveau appelé au siège, avant d'être nommé directeur de Daini en juillet 2011. En 2014, il devient Directeur général en charge de la décontamination et du démantèlement de Fukushima Daiichi. Le 14 mars 2018, l'ambassadeur de France au Japon lui remet les insignes de chevalier de l'Ordre national du Mérite.

30. La NAIIC considère que les interventions inopinées du Gouvernement dans la gestion de crise nucléaire relève d'une faillite de la gouvernance politique : «À tout moment, la priorité du Gouvernement doit être sa préoccupation en matière de santé et de bienêtre publics. Mais parce que l'attention du Kantei était concentrée sur les problèmes en cours à la centrale - ce qui aurait dû être de la responsabilité de l'opérateur - le Gouvernement a échoué dans sa responsabilité envers le public » (NAIIC, 2012, p. 35 [notre traduction]).

31. Pour rappel, les travailleurs se réfugient à Fukushima Daini le matin du 15 mars et commencent à revenir à Daiichi à partir de midi (cf. audition du 8 août 2011).

32. Le réacteur $\mathrm{n}^{\circ} 4$ de la centrale nucléaire de Tchernobyl (Union soviétique, Ukraine) est détruit le 26 avril 1986, à 1h23, à la suite d'un essai technique durant lequel les opérateurs transgressent les procédures de sûreté. L'explosion du réacteur entraîne l'émission dans l'atmosphère d'une grande quantité de produits radioactifs qui contaminent de vastes territoires en Europe. L'accident de Tchernobyl est classé au niveau 7 sur l'échelle INES. Il s'agit du niveau le plus élevé, qui correspond à un accident entraînant des rejets majeurs dont les effets sont considérables sur la santé et l'environnement.

33. Expression utilisée pour la première fois en 1971 par Ralph Lapp (1971, p. 11). Elle renvoie à l'hypothèse selon laquelle les éléments en fusion d'un réacteur nucléaire situé en Amérique du Nord pourraient s'enfoncer dans la terre jusqu'en Chine. Elle est popularisée par Le syndrome chinois (The China Syndrome), réalisé en 1979 par James Bridges (Portelli, 2018). Il s'agit du film le plus célèbre sur l'industrie nucléaire. Une journaliste (Jane Fonda) et son cadreur (Michael Douglas) réalisent un reportage dans la centrale de Ventana aux États-Unis. Une secousse sismique cause un incident, filmé clandestinement par le cadreur. La fusion du cœur du réacteur est évitée, mais l'exploitant refuse de vérifier l'état de l'installation avant sa remise en service. Douze jours après la sortie du Syndrome chinois, un accident se produit dans la centrale nucléaire de Three Mile Island (États-Unis, Pennsylvanie). Cette coïncidence contribue largement au succès du film. 
34. Inagaki revient sur la fureur de Yoshida lorsqu'il apprend, le 14 mars, que l'essence alimentant les autopompes est épuisée : «Je n'oublierai jamais cet après-midi-là. J'avais mal à l'estomac comme si un bloc de plomb y était resté. Nous avions accepté plusieurs idées et tenté de toutes les essayer. Mais l'éventage de l'enceinte de confinement du réacteur 2 n'avait absolument pas avancé. Dans ce contexte, nous manquions d'essence pour l'autopompe afin d'injecter de l'eau dans le réacteur 2. Nous étions en train de lâcher devant l'évolution de la crise. Quand l'essence était épuisée, Yoshida était comme fou et il allait vraiment mal. Il a quitté la table ronde pendant un certain temps. C'est la situation extrême » (Kobayashi, 2019, p. 129).

35. Les conséquences du "scénario du pire", prenant en compte la perte totale de contrôle de l'ensemble des réacteurs de Fukushima Daiichi et Daini, ont été imaginées par des experts consultés par Naoto Kan durant la crise nucléaire : "Tchernobyl a été un accident très grave, mais il n'impliquait qu'un seul réacteur. À Fukushima, il y avait un, deux, trois réacteurs qui avaient fusionnés, et sur le site complet de Fukushima, il y avait en tout dix réacteurs, Daiichi et Daini compris. Que faire si on n'arrivait pas à contrôler ces dix réacteurs? Si on en arrivait là, les rejets de substances radioactives s'élèveraient à plusieurs centaines de fois la quantité émise lors de Tchernobyl, et je me disais que le danger portait sur une zone extrêmement vaste, couvrant jusqu'à Tôkyô. J'ai demandé une simulation à des experts. Non pas à la NISA, ni à la NSC, mais à la Commission de l'Énergie Atomique (JAEC), et plus particulièrement à son Président $M$. Kondô. Dans le rapport qu'il m'a remis, il a imaginé le scénario du pire, qui préconisait une évacuation dans un rayon de 250 kilomètres autour de la centrale, ce qui inclut la métropole de Tôkyô. Cela signifiait évacuer 50 millions de personnes qui vivent dans cette zone !» (Kan, 2015).

36. Né en 1940, Tsunehisa Katsumata est diplômé de l'Université de Tôkyô en économie. Il est embauché à TEPCO en 1963. Il est Directeur général de l'entreprise (et non Président comme la retranscription de l'audition l'indique) lorsque se produit l'accident nucléaire de Fukushima Daiichi. Il a également été le Président de la Federation of Electric Power Companies of Japan.

37. L'agacement de Naoto Kan est déjà mentionné dans l'audition du 29 juillet 2011.

38. Haruki Madarame se souvient du discours de Naoto Kan : «Il est impossible de retirer les employés de Fukushima Daiichi. Gérez la crise au péril de vos vies. Les cadres qui ont plus de soixante ans peuvent mourir pour cela. Moi aussi j'irai. Si vous détruisez le Japon, il est impossible pour vous de continuer à diriger les affaires. TEPCO sera en faillite à $100 \%$ " (Kobayashi, 2019, p. 139). L'auditoire, selon le Président de la NSC, est consterné par de tels propos : «Kan a haussé le ton en montrant ouvertement sa colère. Sa manière de parler a provoqué inmanquablement la répugnance des auditeurs. Notamment, les employés sur les sites étaient dans une colère noire" (Kobayashi, 2019, p. 139). Inagaki le confirme également : "ce discours nous a complètement choqués. Jusqu'à ce moment, je n'avais jamais entendu un discours aussi choquant " (Kobayashi, 2019, p. 140). L'injonction de Naoto Kan semble renouer avec des rites sacrificiels appartenant au passé, comme le souligne Kenichi Shimomura (secrétaire assistant au Kantei pour la communication publique) dans $D e$ Paris à Fukushima, les secrets d'une catastrophe, un reportage diffusé sur France 2 le 12 février 2017 : «Le Premier ministre a demandé à des Japonais de se sacrifier. Ce n'était jamais arrivé depuis les avions kamikazes de la Seconde Guerre mondiale ». Kan considère toutefois que le contexte de situation d'urgence nucléaire justifie pleinement une telle requête: "le Japon est un pays où, en bien ou en mal, on a ordonné à tellement de personnes de mourir pour la patrie durant la dernière guerre, que depuis, sur la base de cette expérience, on ne demande plus, ou plutôt on pense qu'il ne faut pas exiger ce genre de sacrifice. Dans le principe, 
je suis une personne qui a une réflexion assez proche de celle-ci. Seulement, quand un tel accident survient, qu'un accident survient dans une centrale nucléaire qui se trouve au Japon et qui fabriquait de l'électricité pour les Japonais, je pense que vis-à-vis des habitants, et même vis$\grave{a}$-vis du monde, nous avons une responsabilité qui fait que cela peut représenter un cas où il faut agir, même au prix de la vie» (Kan, 2012).

39. Naoto Kan donne sa version de l'épisode: "Il y a eu moult discussions à ce propos depuis, mais ce que j'ai voulu dire c'est que je comprenais le mal qu'ils se donnaient. Que je comprenais l'énormité de la tâche à laquelle ils étaient attelés. Que je comprenais les difficultés qu'ils rencontraient. Mais qu'il fallait qu'ils résistent coûte que coûte. Que la survie du Japon en dépendait. Je ne sais plus si j'en ai vraiment parlé en ces termes, vous devriez retrouver les termes exacts quelque part, ça a été noté. À ce moment-là, c'était le réacteur 2 qui était dans une situation critique. Alors je leur ai dit 'si vous abandonnez le réacteur 2, que vont devenir les réacteurs 1, 3, 4, 6 et, au-delà, Fukushima Daini ?'. Si on les abandonnait là, dans quelques mois on allait se retrouver dans une situation où toutes les centrales nucléaires, tous les déchets allaient rejeter des éléments radioactifs. Que ce serait une addition de dix, vingt réacteurs, qui représenterait chacun le double ou le triple de Tchernobyl. Que le Japon, en tant qu'État, ne pourrait plus exister. Tant qu'on n'aurait pas maitrisé cette situation au prix de nos vies, on ne pouvait pas se retirer et ignorer ça sans rien faire. Si on faisait une chose pareille, les pays étrangers risquaient de vouloir venir prendre les choses en main eux-mêmes. Je leur ai dit : 'Vous êtes les principaux concernés. S'il vous plaît, donnez votre vie. Vous ne pouvez pas échapper à votre destin. La transmission des informations est lente et imprécise. Elle est même erronée. S'il vous plaît, ne vous recroquevillez pas sur vous-mêmes. Faites monter les informations nécessaires. S'agissant même de ce que vous avez à l'instant sous vos yeux, il est important d'agir en prévoyant ce qui va se passer dans cinq heures, dix heures, une journée ou une semaine. L'argent n'a pas d'importance. TEPCO doit le faire, il n'y a pas d'autre choix. Il ne peut pas y avoir de retrait au moment où le Japon risque de disparaitre'. J'ai aussi demandé que le Président du conseil, le PDG se résignent. Il suffisait que ceux de plus de soixante ans aillent sur le terrain. Que moi-même, j'étais décidé à agir en conséquence. Qu'un retrait était inenvisageable et que si TEPCO se retirait l'entreprise coulerait à coup sûr. La dernière phrase était peut-être un peu provocante, mais c'est ce genre de choses que j'ai dites. Ce n'est pas que je me rappelle de la totalité de ce que j'ai dit, mais il y avait quelqu'un qui avait pris des notes. Toujours est-il qu'après ça, je n'ai jamais plus entendu parler de retrait » (Kan, 2012).

40. Le 14 mars à 19h28, après une discussion avec Ikeda (vice-ministre du METI) et Uchibori (vice-gouverneur de Fukushima), Komori (directeur de la division nucléaire de TEPCO) s'adresse depuis le centre hors site aux cadres supérieurs de l'exploitant situés au siège: "Si la situation continue à se détériorer, nous devrons envisager de retirer les employés de Fukushima Daiichi. Allons-nous discuter du cas où nous devrons prendre cette décision? " (Kobayashi, 2019, p. 130). À 19h55, Takahashi (fellow TEPCO) demande à Mutô à quelle heure tous les employés quitteront la centrale nucléaire. À 20h16, il demande de nouveau si le personnel de Daiichi va s'abriter à Daini. À 20h20, Shimizu (Président de TEPCO) lui répond que la décision finale n'a pas encore été prise et qu'il doit auparavant consulter un interlocuteur. Ces échanges sont à l'origine des reproches formulés à l'égard de TEPCO, accusé de vouloir abandonner la centrale et de renoncer à gérer la crise nucléaire. Komori nie toutefois que les travailleurs de Fukushima Daiichi aient eu l'intention de s'enfuir : «Avant Yoshida, j'étais le directeur de Fukushima Daiichi. Je sais donc que les employés, dont Yoshida, étaient courageux et avaient tendance à aller jusqu'au bout. Mais en cas d'urgence, nous devions leur ordonner d'évacuer provisoirement pour sauver leur vie tout d'abord, ensuite pour qu'ils puissent y retourner et relancer la gestion de la crise 
après que la situation s'améliore. Donc j'ai proposé au siège de définir le cas d'urgence » (Kobayashi, 2019, p. 130). Au même moment, Shimizu téléphone à plusieurs reprises au Gouvernement. Selon le rapport de la NAIIC (2012), il aurait appelé onze fois Kaieda (ministre du METI) entre 18 h00 le 14 mars et 3 hoo le 15 mars. Vers 20h00, Kaieda déclare à Edano (Secrétaire général du Gouvernement) et Terata (Conseiller spécial du Premier ministre), "Shimizu m'a téléphoné et m'a dit qu'il voulait retirer les employés de Fukushima Daiichi. Pour ma part je lui ai répondu : 'Continuez à gérer la crise sur le site, s'il vous plait' » (Kobayashi, 2019, p. 130). Le 15 mars, après minuit, les membres du Gouvernement et leurs conseillers techniques se réunissent dans la salle de séjour du Premier ministre, qui se repose dans une autre pièce. Hosono (Conseiller spécial de Naoto Kan) déclare à Edano qu'il est indispensable de connaître la véritable intention du personnel de Fukushima Daiichi. Il lui propose par conséquent de contacter directement Yoshida pour s'en informer. Edano accepte et interroge Yoshida, qui lui confirme sa volonté de rester et de continuer la lutte. Edano ne comprend pas la situation : "Je me suis demandé: 'Pourquoi le siège nous demande de lui permettre de retirer le personnel de Fukushima Daiichi bien que Yoshida se décide à continuer la gestion de crise ?' " (Kobayashi, 2019, p. 132). Les autres participants de la réunion sont tout aussi perplexes. Les ministres consultent les textes de loi, mais aucun ne permet au Gouvernement d'exiger à une entreprise de mettre en péril la vie de ses employés pour gérer une situation de crise. Face à l'indécision des acteurs politiques et techniques, Fukuyama (vice-Secrétaire du Gouvernement) propose de réveiller le Premier ministre. La situation est exposée à 3 hoo à Naoto Kan. Celui-ci rejette catégoriquement la possibilité d'un retrait du personnel de Fukushima Daiichi. Kan convoque alors Shimizu, qui arrive au Kantei à 4h17. Le Premier ministre annonce au Président de TEPCO qu'il n'autorise pas le retrait. Shimizu acquiesce et accepte également la proposition de Kan d'établir une cellule conjointe entre le Gouvernement et TEPCO. Kan se rend ensuite au siège de TEPCO pour assister à la première réunion de la cellule conjointe et demander aux équipes sur site de ne pas abandonner la centrale - bien que cela n'ait jamais été leur intention.

41. Les rapports ont parfois été tendus entre Yoshida et les acteurs extérieurs durant la crise nucléaire. Le directeur de Fukushima Daiichi a toutefois entretenu des relations cordiales et respectueuses avec Hosono, qu'il appelle toujours dans ses auditions « Hosono-san » - san signifiant «monsieur » en japonais. Takeyuki Inagaki explique les raisons de la confiance que lui a accordée Yoshida : "Je n'ai pas discuté avec Yoshida sur ce sujet, mais Monsieur Hosono a envoyé un bateau de l'Agence de la sécurité maritime à un port proche de la centrale nucléaire afin que les employés puissent faire provision d'énergie et il a convaincu les hôtels près de Fukushima Daiichi d'offrir des repas chauds aux employés. Du fait qu'il ait fait des efforts pour améliorer l'environnement du travail des employés, je pense que Yoshida a fait confiance à Monsieur Hosono " (Kobayashi, 2019, p. 159).

42. Cf. audition du 22 juillet 2011.

43. Pour rappel, des tests ont été effectués pour démarrer l'IC avant la mise en service du réacteur 1 de Fukushima Daiichi et, selon certains témoins, quelques fois après. Les opérateurs ayant effectué ces manœuvres avaient cependant quitté Daiichi depuis de nombreuses années lorsque s'est produit l'accident nucléaire.

44. La centrale nucléaire de Tsuruga est située dans la préfecture de Fukui, dans la région de Chûbu. Elle est exploitée par la Japan Atomic Power Company. Tsuruga 1, réacteur à eau bouillante d'une puissance de 357 MWe (constructeur GE), est mis en 
service en mars 1970. Il est en démantèlement depuis 2015. Tsuruga 2, réacteur à eau pressurisée d'une puissance de 1160 MWe (constructeur Mitsubishi), est mis en service en février 1987. Il est à l'arrêt depuis le 11 mars 2011.

45. Ce type de notation correspond aux jeux de barres qui permettent d'alimenter la centrale à partir des lignes à haute tension. « $2 \mathrm{C}$ » correspond à un des deux jeux de barres de $6,9 \mathrm{kV}$ de la tranche 2 de Fukushima Daiichi. Ce jeu de barre a la particularité d'être également connecté à un des générateurs diesel d'urgence qui prendrait le relai en cas de problème dans la liaison avec le réseau électrique externe.

46. Naoto Kan obtient son premier mandat de représentant du Japon à la Diète en juillet 1980. De tempérament très coléreux, il est surnommé par les parlementaires «Ira Kan ».

47. La prise en charge des travailleurs légèrement blessés est tout d'abord difficile, car plusieurs hôpitaux sont fermés du fait de l'évacuation des populations, tandis que d'autres ne sont pas préparés à accueillir des patients éventuellement contaminés (AIEA, 2015a). Tant que des soins médicaux primaires ne peuvent être prodigués à Fukushima Daiichi, ces blessés sont envoyés vers un hôpital situé à proximité. 17 heures après le séisme, une équipe d'assistance médicale pour les situations d'urgence nucléaire ou radiologique est envoyée au centre hors site afin de procéder à des évaluations de contamination des opérateurs et à leur décontamination. Le 19 mars, des soins primaires commencent à être fournis dans le bâtiment antisismique de Daiichi par des médecins du travail. Deux centres de triage sont ouverts par la suite, le premier sur site et le second au J-Village. Le $1^{\text {er }}$ juillet 2011, un service de soins d'urgence, composés d'un personnel qualifié pour soigner les blessés en situation d'urgence nucléaire ou radiologique en provenance de tout le Japon, est créé à Daiichi.

48. Ville de la préfecture de Fukushima située à une quarantaine de kilomètres de Fukushima Daiichi.

49. Pour rappel, Fukushima Daiichi se trouve à environ 250 kilomètres de Tôkyô.

50. Bourg de la préfecture de Fukushima, situé à une cinquantaine de kilomètres de Fukushima Daiichi.

51. Ville de la préfecture de Fukushima, située à une quarantaine de kilomètres d'Ôno et à environ soixante-dix kilomètres de Fukushima Daiichi.

52. Marque japonaise de prêt-à-porter à prix réduit.

53. Le Tôkyô Denryoku Hospital est un hôpital appartenant à TEPCO et situé dans le quartier Shinjuku de Tôkyô. Cet établissement de sept étages, construit en 1979 et d'une capacité de 113 lits, est utilisé exclusivement pour soigner les employés de l'exploitant et les membres de leur famille. En juillet 2012, TEPCO annonce la mise en vente de l'hôpital. Il a été vendu en janvier 2014.

54. Fondé en 1961, le NIRS est un institut de recherche sur les rayonnements (et notamment leurs effets sur le corps humain) situé dans la ville de Chiba. En avril 2016, il fusionne avec certains laboratoires de l'Agence Japonaise de l'Énergie Atomique pour former les National Institutes for Quantum and Radiological Science and Technology. 


\section{Compte rendu de l'audition du 9 août 2011 (II)}

1 [À traiter avec la plus grande attention]

14 août 2011

\section{Compte rendu d'audition}

2 Kôta Okada

Membre du secrétariat de la Commission d'enquête sur l'accident de la centrale nucléaire de Fukushima de Tôkyô Electric Power Company (Investigation Commitee on the Accident at the Fukushima Nuclear Power Station of Tôkyô Electric Power Company)

Ci-dessous le compte rendu de l'audition du 9 août 2011, menée dans le cadre de l'enquête sur l'accident de la centrale nucléaire de Fukushima de Tôkyô Electric Power Company.

1. Auditionné, date d'audition, lieu d'audition, enquêteurs.

1. Auditionné

Masao Yoshida, Directeur de la centrale nucléaire Fukushima Daiichi de Tôkyô Electric Power Company.

2. Date d'audition

9 août 2011 de 16 ho0 à 17 h00.

3. Lieu d'audition

J-Village.

4. Enquêteur

Kôta Okada. 
5. Enregistrement par enregistrement numérique

Oui

$\square$ Non

2. Contenu de l'audition

Mesures concernant l'eau contaminée

Voir le compte rendu

3. Mentions spéciales

Pas de mention spéciale.

QUESTION : J'ai cru comprendre que vous étiez habituellement au premier étage du bâtiment antisismique, dans la grande salle où se trouve le moniteur, pour prendre les décisions concernant l'ensemble de la centrale de Fukushima Daiichi et celles pouvant influer sur l'extérieur de la centrale. Est-ce exact' ${ }^{\text {? }}$ ?

Réponse : Oui. C'est tout à fait ça.

Q: Quelles sont les personnes avec qui vous êtes en contact direct au sein du Gouvernement et du siège social ?

$\mathbf{R}$ : Nous avons des téléconférences quotidiennes avec le siège. J'y échange avec le chef du groupe "réhabilitation ", le chef du groupe "liaison avec les ministères ", l'Administrateur Komori, le Fellow Takekuro et le vice-Directeur général Mutô ${ }^{2}$. Il m'arrive aussi de leur parler directement au téléphone.

$\mathbf{Q}$ : Considériez-vous personnellement, Monsieur le Directeur, que pour pouvoir utiliser le bâtiment principal du centre de traitement des déchets radioactifs (désigné dorénavant comme "le centre de traitement») comme lieu de stockage de l'eau hautement contaminée, le meilleur choix aurait été de rejeter l'eau de mer qui se trouvait dans l'océan?

$\mathbf{R}$ : Étant donné que nous ne disposions d'aucun réservoir capable de recevoir un aussi gros volume sur le site, j'ai pensé qu'il n'y avait que la solution du transfert vers le centre de traitement. J'ai effectivement pensé que le meilleur moyen était de rejeter l'eau de mer qui s'y trouvait déjà, à l'océan³

$\mathbf{Q}$ : Concernant la réponse à l'inondation de la salle de distribution électrique de la tranche 6 (citée dorénavant comme «la salle MC (Metal-Clad Switch Gear) »), pensiez-vous que le rejet à la mer était la meilleure solution pour évacuer l'eau qui stagnait dans le bâtiment voisin de traitement des déchets, cause de cette inondation? Également pour l'évacuation de l'eau des tunnels de maintenance pour faire baisser le niveau des nappes souterraines, elles-mêmes cause des infiltrations dans le bâtiment voisin?

$\mathbf{R}$ : Effectivement, au début du mois d'avril, nous n'avions pas d'autre réservoir où transférer l'eau contaminée. Pour assurer l'intégrité de la tranche, je pensais qu'il était un moindre mal de rejeter cette eau dans l'océan.

$\mathbf{Q}$ : Pour pouvoir baisser le niveau des nappes souterraines, il aurait fallu continuer à rejeter l'eau par les tunnels de service 4 ...

$\mathbf{R}$ : L'eau des tunnels de service n'était pas d'un niveau de radioactivité élevé et j'avais estimé qu'il était tout à fait acceptable de continuer à la rejeter. Il me semblait qu'ainsi on pourrait globalement réduire le volume d'eau infiltrée dans les bâtiments et que ce serait la meilleure solution.

Mais il s'est avéré que la radioactivité de l'eau des tunnels de service dépassait le niveau officiel autorisé pour un rejet en mer et la NISA nous a interdit de rejeter l'eau des tunnels dans l'océan. Ordre auquel nous nous sommes soumis. Mais s'il est 
impossible de rejeter cette eau à la mer, les eaux souterraines montent, les infiltrations dans les bâtiments s'intensifient, les eaux stagnantes dans les bâtiments augmentent d'autant et il y a toujours plus d'eau à traiter. J'ai pensé, dès le début, que si c'était pour se retrouver dans une situation où il $\mathrm{y}$ a de plus en plus d'eau contaminée à traiter, il valait mieux rejeter l'eau relativement faiblement contaminée pour s'occuper sérieusement du traitement de l'eau fortement polluée.

$\mathbf{Q}$ : Pour ce rejet dans la mer, vous aviez besoin de négocier avec l'État et autres organismes. Qu'en pensiez-vous?

R : Naturellement j'ai pensé que ce serait nécessaire. Mais, pour moi, l'important était de transmettre, en tant que directeur et voix de la centrale, que pour assurer l'intégrité de la tranche, ce rejet était indispensable. Il fallait que le siège, aussi bien que les organismes concernés, partagent ce point de vue et travaillent de concert à la résolution du problème.

Q : D'après ce que j'ai pu apprendre par les différentes auditions que j'ai menées, j'ai cru comprendre que le Conseiller Hosono aurait déclaré le $7^{\text {er }}$ avril qu'» un rejet dans la mer n'était pas envisageable ", qu'il se serait opposé au projet et que l'idée avait été abandonnée...

$\mathbf{R}:$ Je ne m'en souviens pas très bien. C'est une époque où il a fallu faire face à toutes sortes de problèmes en même temps. Quand je vous entends évoquer l'épisode, je me dis que peut-être bien que cela s'était passé comme ça. En tout cas, je me souviens très bien que la réaction du siège aussi bien que de la NISA, avait été d'interdire le rejet et qu'ils m'en avaient fait part.

$\mathbf{Q}$ : Quand ils vous ont dit cela, quelle a été votre réaction, à vous?

$\mathbf{R}$ : Je me suis demandé comment nous allions faire. Le niveau d'eau hautement radioactive allait continuer à monter. Je me suis demandé si on allait continuer à la laisser couler en l'état. Le fait que ni la NISA ni les membres du siège ne partageaient ma préoccupation m'a mis très en colère. On envoyait continuellement de l'eau dans les réacteurs. Elle allait déborder quelque part à un moment ou à un autre. Il était évident qu'il fallait trouver un moyen de traiter cette eau. Et ils se détournaient du problème. Je vous assure que cette histoire m'a longtemps agacé au plus haut point.

$\mathbf{Q}$ : Quand je lis les comptes rendus des réunions, il me semble que, à la suite de la découverte de la fuite d'eau hautement contaminée le 2 avril, l'attitude face au rejet d'eau dans l'océan avait changé le 3 avril et qu'il y avait eu quelques avis admettant que le rejet était peut-être inévitable. Qu'en pensez-vous?

$\mathbf{R}$ : Personnellement, je ne me souviens pas que cela ait changé quoi que ce soit aussi bien à la NISA qu'au cabinet du Premier ministre. Au moment de la réunion de la cellule de crise Gouvernement-TEPCO, le 4 avril au matin, la fuite à partir de la tranche 2 n'était pas encore colmatée et on m'a ordonné d'arrêter cette fuite coûte que coûte ${ }^{5}$. Sans fournir l'ombre d'une idée de la manière de traiter cette eau, d'un endroit où la stocker, ils se contentaient de dire au terrain de se débrouiller. Je crois que j'ai réagi assez violemment, je crois même que je leur ai crié dessus qu'il leur appartenait aussi de réfléchir au problème. Je pense que c'est à partir de ce moment qu'ils ont commencé à y réfléchir sérieusement.

$\mathbf{Q}$ : À cette réunion de la cellule de crise Gouvernement-TEPCO du 4 avril au matin, il me semble que vous n'avez pas exprimé explicitement votre souhait de rejeter dans l'océan. Est-ce exact?

$\mathbf{R}$ : Effectivement, je n'ai pas parlé de rejet à la mer. Le but de mes déclarations au matin du 4 avril était de demander qu'on nous fasse des propositions concrètes 
concernant le traitement de l'eau et son lieu de stockage. Seulement, il était évident que le seul lieu de stockage possible était le bâtiment du centre de traitement. Étant donné que nous ne disposions pas de réservoir où transférer l'eau de mer qui y était déjà stockée, si on voulait l'évacuer, il n'y avait que l'évacuation vers l'océan.

$\mathbf{Q}$ : Le fait que vous ayez adopté un ton assez vif le 4 avril au matin, est-il lié au fait qu'on venait de découvrir le matin même que le niveau d'eau était monté dans la tranche 3 ? Le fait de recevoir cette information vous a-t-il poussé à penser qu'il n'existait plus d'alternative au rejet dans l'océan?

R : Effectivement, oui. Puisqu'il n'y avait plus d'endroit où transférer cette eau, j'ai pensé qu'il ne restait plus que le rejet à la mer pour vider le centre de traitement.

$\mathbf{Q}$ : Concernant le rejet par les tunnels de service, est-ce que le fait qu'on ait découvert dans la nuit du 3 avril l'inondation de certaines tranchées vous a influencé ?

$\mathbf{R}$ : Je pense que oui. C'est un phénomène qui est distinct du problème de l'eau fortement contaminée, mais si on perdait le fonctionnement des réseaux électriques à la suite d'une inondation, nous aurions perdu les moyens de refroidissement et cela nous aurait menés à une situation extrêmement inquiétante. Il fallait à tout prix sortir cette eau des bâtiments.

Q: À la suite de cette réunion de la cellule de crise du 4 avril, le siège a organisé des concertations. Avez-vous été tenu au courant des différentes étapes des formalités?

$\mathbf{R}$ : Oui, le siège m'a contacté pour me dire qu'ils avaient envoyé un rapport circonstancié au Gouvernement, que la NSC s'était prononcée, etc. Je me rappelle que, dans la matinée, ils m'avaient fait savoir qu'un tel rapport serait nécessaire.

Q: Concernant le rejet de l'eau des tunnels de service des tranches 5 et 6 , vous avez obtenu l'autorisation de rejeter 1500 t seulement. Qu'en avez-vous pensé quand vous l'avez su?

$\mathbf{R}$ : Pour ma part, je désirais pouvoir évacuer l'eau des tunnels de manière continue. Je me suis donc demandé d'où sortait ce chiffre. Quand j'ai posé la question au siège, ils m'ont répondu que cela avait été calculé en fonction de l'impact environnemental, qu'ils avaient déjà communiqué ce chiffre à la NISA et qu'il était trop tard pour revenir dessus. Le contact, que j'avais eu dans la matinée au sujet du rapport à faire au Gouvernement, m'avait affirmé que le siège se chargeait d'en assurer la rédaction, mais il n'y a eu aucune concertation avec la centrale pour discuter du contenu avant la remise du rapport à la NISA.

Q: J'ai entendu dire que le siège pensait que lorsque le rejet à la mer serait autorisé et exécuté une première fois, il pourrait réitérer l'opération.

$\mathbf{R}$ : Nous, nous ne pensions pas de même. Si le rapport mentionnait $1500 \mathrm{t}$, nous pensions bien qu'un seul et unique rejet de $1500 \mathrm{t}$ serait autorisé. En me remémorant les divers échanges qu'on avait déjà eus avec le Gouvernement, j'ai trouvé que le jugement du siège n'était pas du tout réaliste. Cela fait bien trente ans que je fréquente le Gouvernement et je savais pertinemment qu'un chiffre qui avait été fourni à la NISA ne pouvait être dépassé sans d'énormes difficultés.

$\mathbf{Q}$ : On m'a également raconté qu'au cas où vous vous seriez trouvé dans une situation réellement grave du fait de l'inondation de la tranche 6 , vous étiez prêt à rejeter l'eau dans l'océan sans attendre l'autorisation du Gouvernement. Est-ce vrai ?

$\mathbf{R}$ : Dans un cas d'extrême urgence, la décision revient non pas à la centrale mais au siège. Mais si celui-ci ne réagissait pas, effectivement, j'étais prêt à mettre ma place en jeu pour effectuer ce qu'il fallait pour la protection de la tranche. 
$\mathbf{Q}$ : Finalement, avec un rejet plafonné à $1 \mathrm{mSv} / \mathrm{an}^{6}$, il a été décidé de rejeter la totalité de l'eau du centre de traitement et de rejeter l'eau des tunnels en fonction de ce qui restait des 1500 t autorisées. Côté centrale, étiez-vous d'accord avec cet ordre des priorités?

$\mathbf{R}$ : Non. Nous pensions que, bien que de nature différente, le fait de vider le centre de traitement et le rejet de l'eau des tunnels étaient aussi importants l'un que l'autre.

Si on ne faisait rien, l'eau fortement contaminée allait augmenter de plus en plus et déborder. La fuite d'eau contaminée de la tranche 2 a été un tel traumatisme pour moi que j'en rêve encore. Empêcher la fuite d'eau hautement radioactive était la priorité des priorités.

Mais, en même temps, si l'inondation de la tranche 6 conduisait à la perte des réseaux électriques, nous n'aurions plus eu de moyens de refroidissement et cela aurait pu nous mener, au pire, jusqu'à la fusion du cœur. Bien que d'un ordre différent, c'était aussi un fait extrêmement important.

Q: À la date du 4 avril, aviez-vous intuitivement une idée du niveau d'eau et de la marge dont vous disposiez avant le débordement?

$\mathbf{R}$ : Oui, tout à fait. Même si on avait vidé l'eau du centre de traitement, avant de pouvoir y transférer de l'eau fortement contaminée, je savais qu'il fallait procéder à des travaux d'étanchéité, qui allaient nous prendre une à deux semaines. En tenant compte du taux d'augmentation des niveaux d'eau des réacteurs 2 et 3, j'avais déterminé la période où il y aurait les plus fortes chances pour que cela déborde. Si je prenais en considération, en plus, la durée des travaux, je savais qu'il fallait que le centre de traitement soit vidé au moins début avril. Je suis ingénieur. Avant d'arriver à une conclusion, j'élabore des hypothèses. J'ai bien réfléchi au volume d'eau qui allait s'accumuler et aux conséquences qu'il convenait d'en tirer.

$\mathbf{Q}$ : Une des raisons qui ont poussé au rejet dans l'océan semble avoir été l'impact de la découverte de la fuite d'eau contaminée au niveau de la tranche 2. Le fait que cette fuite ait été colmatée, le 6 avril, n'a-t-il pas fait que le rejet soit devenu un peu moins urgent?

$\mathbf{R}$ : Non, pas du tout. Le fait d'arrêter la fuite et le traitement de l'eau à long terme sont des sujets totalement différents. Il est bien entendu important d'arrêter la fuite de manière sûre. Mais, d'un autre côté, nous continuions à envoyer de l'eau dans les réacteurs, augmentant la quantité d'eau contaminée. Sachant cela, il fallait réfléchir à la manière de traiter cette eau contaminée.

$\mathbf{Q}$ : La première fois que vous avez évoqué la possibilité de rejeter de l'eau à la mer, était-ce lors de la réunion du 28 mars?

R: J'ai rencontré le ministre Kaieda, le conseiller Hosono et Monsieur Yanase ${ }^{7}$ de la NISA le 27 mars, au siège social. Lors de cette rencontre, j'ai insisté sur le fait que dorénavant le traitement des eaux allait être la priorité des priorités.

Ensuite nous avons eu une réunion au siège avec le groupe " traitement des eaux » le 28 mars, où nous sommes tombés d'accord sur le fait qu'il convenait de transférer l'eau avant de la traiter. Nous avons examiné différentes possibilités pour le lieu de transfert. Nous avons même entendu des idées totalement irresponsables du genre " il suffit de creuser le sol et d'y stocker l'eau ». Au fil de la discussion, nous en sommes venus à la conclusion que seuls le bâtiment turbine de la tranche 4 et le centre de traitement permettaient un stockage d'un volume conséquent. Mais avant d'arriver à la réalisation concrète de l'opération de transfert, nous devions étudier plus en détail l'itinéraire à emprunter. Savoir où conduisait tel conduit, s'il fallait passer d'un côté ou de l'autre. Nous avons donc décidé d'étudier de toute urgence à la fois l'itinéraire 
vers le bâtiment turbine de la tranche 4 et l'itinéraire vers le centre de traitement. Ces études ont démarré le 28.

S'agissant du bâtiment turbine de la tranche 4 , dès ce moment, il avait été question de la possibilité d'en faire une solution de substitution ${ }^{8}$, si le rejet à la mer n'était pas autorisé.

$\mathbf{Q}$ : Je m'éloigne un peu de la vérification des faits, mais il me semble que le sentiment d'urgence du terrain n'était pas suffisamment partagé par le siège, la NISA ou le cabinet du Premier ministre.

R : Oui, notre sentiment d'urgence. Pourtant lors de cette réunion du 27 mars, j'avais très clairement et directement attiré l'attention du ministre Kaieda et du conseiller Hosono sur le fait que le traitement des eaux était primordial et que, sans une solution durable de ce côté-là, la stabilisation des tranches n'était pas possible. Le 28 aussi, j'ai directement fait part de mes inquiétudes à Monsieur Takekuro et aux membres du groupe «traitement des eaux » présents à la réunion. Je ne sais pas si tout cela a été bien " partagé ", mais, pour ma part, j'ai la conviction d'avoir transmis ce qui devait l'être.

L'indignation de constater qu'ils n'avaient rien compris malgré toutes les sonnettes d'alarme que j'avais tirées, "est-ce qu'ils réfléchissaient sérieusement au sort des réacteurs?", a été aussi une des causes de mon explosion à la réunion du 4 avril.

Je pense que le seul à avoir véritablement écouté nos arguments et à avoir eu une vision correcte des problèmes a été Monsieur Takekuro.

$\mathbf{Q}$ : Est-ce vous qui avez provoqué la rencontre du 27 mars avec le ministre Kaieda et le conseiller Hosono pour transmettre les inquiétudes du terrain?

$\mathbf{R}:$ Il était déjà programmé que je fasse un déplacement à Tôkyô à partir du 25 mars. Je crois bien que c'était XXXXX qui m'a dit que le ministre Kaieda et le conseiller Hosono désiraient me voir. D'après lui, ils souhaitaient me remercier pour tout le travail éprouvant fourni depuis l'accident et discuter de l'avenir.

$\mathbf{Q}$ : Était-ce la première fois que vous voyiez le ministre Kaieda et le conseiller Hosono ?

$\mathbf{R}$ : C'était la première fois que je les rencontrais en personne. Mais j'avais déjà eu plusieurs fois l'occasion de parler avec eux au téléphone. Il m'est aussi arrivé de parler avec le Premier ministre Kan.

(Fin de l'audition)

\section{NOTES}

1. Cette audition et la suivante évoquent la question de l'eau contaminée sur le site de Fukushima Daiichi, et non la gestion de l'accident nucléaire entre le 11 et le 15 mars 2011. 
2. Mutô est vice-Président et non vice-Directeur général de TEPCO.

3. Le refroidissement des réacteurs 1 à 3 en circuit ouvert provoque une accumulation de grandes quantités d'eau contaminée dans les enceintes de confinement et les salles des turbines, saturant les capacités de stockage sur le site. En avril et en mai 2011, TEPCO mène d'une part différents travaux pour limiter la dispersion de radioactivité sous forme liquide. L'exploitant procède d'autre part au déversement en mer d'une partie de l'eau faiblement contaminée pour libérer en urgence des espaces de stockage (ASN et IRSN, 2011).

4. Ces tunnels ont été construits en réponse à l'inondation du site survenue en octobre 1991, comme Yoshida le précise dans l'audition du 8 août 2011.

5. La fuite d'eau hautement radioactive, qui se déversait en continu dans l'océan Pacifique, est finalement colmatée le soir du 5 avril 2011. Les opérateurs ont solutionné le problème en injectant dans le sol du silicate de sodium, un agent chimique dont la particularité est de se solidifier au contact de l'eau (AFP, 2011a).

6. Il s'agit de la limite d'exposition pour le public hors radioactivité naturelle et applications médicales.

7. Confusion de Yoshida : Tadao Yanase est alors le chef de service du ministre du METI Banri Kaieda, et non un fonctionnaire de la NISA.

8. En remplacement du centre de traitement. 


\section{Compte rendu de l'audition du 13 octobre 2011}

1 [À traiter avec la plus grande attention]

16 octobre 2011

\section{Compte rendu d'audition}

2 Kôta Okada

Membre du secrétariat de la Commmission d'enquête sur l'accident de la centrale nucléaire de Fukushima de Tôkyô Electric Power Company (Investigation Commitee on the Accident at the Fukushima Nuclear Power Station of Tôkyô Electric Power Company)

Ci-dessous le compte rendu de l'audition du 13 octobre 2011, menée dans le cadre de l'enquête sur l'accident de la centrale nucléaire de Fukushima de Tôkyô Electric Power Company.

1. Auditionné, date d'audition, lieu d'audition, enquêteurs.

1. Auditionné

Masao Yoshida, Directeur de la centrale nucléaire Fukushima Daiichi de Tôkyô Electric Power Company.

2. Date d'audition

13 octobre 2011 de 16 h00 à 17 h00.

3. Lieu d'audition Bâtiment antisismique de la centrale nucléaire de Fukushima Daiichi.

4. Enquêteurs

Tomomitsu Takashima, Kôta Okada. 
5. Enregistrement par enregistreur numérique

Oui

$\square$ Non

2. Contenu de l'audition

Prévision avant le 24 mars de l'existence d'eau fortement contaminée

Intentions et toile de fond des déclarations à la cellule de crise Gouvernement-TEPCO du 4

avril

Voir le compte rendu

3. Mentions spéciales

Pas de mention spéciale.

\section{Compte rendu de l'audition}

\subsection{Prévision avant le 24 mars de l'existence d'eau fortement contaminée'}

Comme cause de la stagnation d'eau dans les différents bâtiments, on peut penser à l'eau apportée par le tsunami. Toutefois il convient de nuancer. Pour moi, un gros volume d'eau avait pénétré dans le bâtiment turbine lors de l'arrivée du tsunami, alors que le bâtiment réacteur avait été relativement épargné. Ceci est étayé par le fait qu'avant d'en arriver à la situation actuelle, du personnel est entré dans certaines parties du bâtiment réacteur, pour inspecter la pompe RCIC, par exemple, a fait rapport de quelques flaques d'eau isolées, mais rien qui empêche l'entrée dans le bâtiment luimême.

5 L'injection d'eau dans les réacteurs a commencé après la survenue du tsunami. Étant donné le volume d'eau injectée, je pensais naturellement qu'une partie de celle-ci devait bien s'échapper de l'enceinte de confinement. Je pensais que l'eau ainsi échappée se déversait vers les tores. Le bâtiment réacteur étant bien isolé, j'escomptais que l'eau ainsi déversée dans les tores allait rester un certain temps à l'intérieur du bâtiment. Mais j'avais conscience aussi qu'à un moment ou un autre, elle finirait par déborder du bâtiment et que la possibilité que, même avant cela, elle puisse s'écouler par des fissures était grande. Seulement, j'étais dans l'incapacité de prévoir le moment précis où cela allait advenir.

6 Il me semble que c'était en 1991, il y a eu des fuites d'eau sur la ligne d'eau de mer du réacteur $1^{2}$. Je me rappelle qu'à ce moment-là de l'eau, qui était passée par des interstices de câbles électriques ou de tuyaux qui perçaient le mur de part en part, avait inondé divers endroits. Ces interstices avaient été obstrués par des joints d'étanchéité ou d'autres moyens, mais j'avais conscience qu'au-delà d'une certaine pression de l'eau, il y avait des risques que ces joints puissent céder et laisser passer l'eau.

7 Seulement, avant le 24 mars, il y avait tellement d'autres choses à faire que je n'ai pas pu prendre le temps de réfléchir et prévoir le moment où l'eau allait s'échapper et en quelle quantité. Cependant, comme j'étais conscient des risques d'écoulement, dès les premiers temps, j'avais pensé qu'il fallait réfléchir au stockage et au traitement des eaux contaminées. C'est pourquoi quand j'ai su le 24 mars que de l'eau fortement contaminée avait été détectée, je n'ai pas été très surpris: «ce qui devait arriver était arrivé». Par contre, j'ai trouvé que cela arrivait plus tôt que je ne l'aurais pensé. 


\subsection{Intentions et toile de fond des déclarations à la cellule de crise Gouvernement- TEPCO du 4 avril}

8 Concernant la découverte de l'inondation des tranchées jouxtant la salle HPCSDG (High Pressure Core Spray System Diesel Generator) ${ }^{3}$ au sous-sol du réacteur 6, j'étais au courant que l'alarme signalant une inondation avait sonné dans la nuit du 3 avril. Mais je n'ai su qu'on y avait trouvé de l'eau stagnante que juste avant la réunion du 4 avril à $8 \mathrm{~h}$, qui a eu lieu au sein de la centrale de Fukushima Daiichi. J'ai donc demandé à XXXXX de nous en faire un rapport plus détaillé au cours de cette réunion. Mais, à ce stade, on ne m'avait pas encore informé de l'origine de cette inondation.

9 Cependant je me doutais qu'il s'agissait de l'eau souterraine. De base, la centrale de Fukushima Daiichi se situe à un endroit où la nappe souterraine est peu profonde et, même en temps normal, nous avions souvent eu des inondations au niveau des bâtiments turbine. C'est globalement une localisation facilement inondable par l'eau souterraine. La partie où se dressent les tranches 5 et 6 est particulièrement sensible, car ce sont d'anciens marais, avec des voies d'eau souterraine bien marquées. Depuis le début, nous avions eu des soucis d'infiltration. Si j'entre dans les détails géologiques, il y a des parties boueuses entre des couches de grès. L'eau circule par ces couches gréseuses pour rejoindre la mer. Mais si, et c'est notamment vrai en période de saison des pluies, l'eau ne s'évacue pas assez vite par cette voie, le niveau de la nappe souterraine monte, augmentant d'autant la pression sur les bâtiments de la centrale. Dans ces cas-là, l'eau peut s'infiltrer par les murs ou passer par de fines craquelures et provoquer une inondation. C'est pourquoi nous avions l'habitude de faire des rondes pour contrôler régulièrement ces infiltrations dans les bâtiments réacteur et turbine au moment de la saison des pluies. Lorsque nous constations la présence d'eau stagnante, nous colmations les murs, mais l'eau avait tendance à se frayer une autre voie pour entrer. C'est ainsi que, depuis la construction, l'infiltration d'eau souterraine était un des gros soucis de la centrale Daiichi. Quiconque a travaillé sur le site est au courant du phénomène. Moi-même, ayant été à quatre reprises en fonction à Fukushima Daiichi ${ }^{4}$, dont une fois en tant que sous-chef des réacteurs 5 et $6, j$ 'étais tout à fait conscient du problème.

Du coup, pour moi, il était évident que l'eau souterraine allait inonder les bâtiments, étant donné que, depuis la survenue du tsunami, nous n'avions pas pu évacuer l'eau par les tunnels de maintenance. J'ai donc tout de suite eu la conviction que la présence d'eau stagnante dans les tranchées des salles RW (Radioactive Waste) 5 et HPCSDG du réacteur 6 était due à l'infiltration des eaux souterraines. De plus, j'ai pensé que si le niveau de la nappe souterraine restait élevé, l'eau pouvait aussi s'infiltrer dans d'autres endroits, provoquant des dégâts sur d'autres installations, mettant à terme en cause l'intégrité même de l'ensemble des tranches 5 et 6 . C'est pourquoi j'ai fait ces déclarations à l'occasion de la réunion de la cellule de crise Gouvernement-TEPCO du 4 avril ${ }^{6}$.

11 Parmi celles-ci, celle évoquant les risques menaçant l'intégrité des tranches 5 et 6 , ne visait pas uniquement les problèmes des seules salles MC ou HPCSDG, mais plutôt la dégradation d'autres installations à l'intérieur des bâtiments, qui auraient pu être touchées par les inondations que n'aurait pas manqué de provoquer la hausse du niveau de la nappe souterraine. Lorsque le niveau de la nappe monte, le colmatage individuel des différentes infiltrations ne représente en rien une solution pérenne. Ce 
qui signifie que d'autres endroits des différents bâtiments couraient le danger d'être inondés. Pour éloigner ce danger, il n'y avait que la solution de l'évacuation de l'eau par les tunnels de maintenance.

12 En fait, après cette réunion, les études et consultations menées au niveau du siège social ont avancé plus vite que je ne m'y attendais et, déjà, dans la journée du 4 avril, la décision a été prise d'exécuter des rejets à la mer. Ce qui a représenté pour moi un grand soulagement, en tant que gestionnaire de la centrale. En fait, lorsque j'ai fait part de mes problèmes à la réunion, je ne m'attendais pas du tout à ce que ces problèmes trouvent une solution dans la journée. Je pensais qu'il faudrait consulter différentes instances et qu'une décision ne serait prise qu'au mieux dans les trois ou quatre jours. Pour ce qui est du temps nécessaire au rejet à la mer, j'avais déjà fait un calcul approximatif du volume d'eau stockée dans le centre de traitement des déchets radioactifs. Sachant que nous disposions de dix pompes d'une puissance de $20 \mathrm{t} / \mathrm{h}$, ce qui fait au total $200 \mathrm{t} / \mathrm{h}$, je savais qu'il nous faudrait grosso modo cinq jours pour l'évacuer. Seulement, comme je ne savais pas combien de temps prendraient les travaux d'étanchéité du bâtiment de traitement des déchets, je désirais démarrer au plus vite le rejet à la mer. Je m'étais dit que si la décision se prenait dans les trois ou quatre jours, je pouvais encore tenir le coup en transvasant l'eau fortement radioactive dans les installations existantes encore vides.

\subsection{Concertation avant le rejet à la mer}

13 Concernant la concertation avec les autorités nationales ou locales et les États limitrophes, nous avions l'habitude, depuis longtemps, lorsqu'un rejet de matières radioactives était envisagé hors de la centrale, que celui-ci dépasse ou non le seuil autorisé, de les prévenir systématiquement avant information à la presse. Si bien que je pensais que sans que je n'en dise rien, le siège s'était occupé de cette partie du travail. Lorsque je parle à la téléconférence de 17 h00, précédant le rejet à la mer, de la nécessité de contacter l'Agence de la sécurité maritime ${ }^{7}$ et les Forces d'autodéfense, c'est en pensant à ce que nous avions l'habitude de faire. Je voulais juste m'assurer que cela avait été fait.

\subsection{Sur la réunion du 27 mars}

Depuis la découverte, le 24 mars, d'eau fortement contaminée, j'avais toujours eu le souci que celle-ci ne s'échappe. C'est pourquoi, lors de la réunion du 27 mars avec le Ministre Kaieda, le Conseiller Hosono et Monsieur Yanase de la NISA, au siège, j'ai beaucoup insisté sur le fait que, dorénavant, le problème de cette eau fortement contaminée était primordial, et que si on ne commençait pas immédiatement à réfléchir à la manière de stocker et de traiter cette eau, qui allait continuer à affluer, nous allions vers d'énormes difficultés. J'ai notamment expliqué qu'il fallait vider l'eau stockée dans le centre de traitement des déchets pour que ces réservoirs puissent recevoir l'eau fortement contaminée, que pour résoudre le problème de l'inondation du sous-sol des tranches 5 et 6 , il convenait d'assurer l'évacuation continue de l'eau par les tunnels d'entretien. Que nous ne disposions pas à ce moment-là de réservoirs pour stocker ces eaux faiblement contaminées, qu'à moins de trouver un endroit préexistant où les transférer, il n'y avait que la solution du rejet à la mer. Je leur ai demandé de se prononcer clairement sur le sujet. 
(Fin de l'audition)

\section{Annexes}

\section{Annexe 1}

\section{Compte rendu de la réunion de la cellule de crise Gouvernement-TEPCO du 4 avril}

\section{YOSHIDA, DIRECTEUR DE FUKUSHIMA DAIICHI}

Si nous en avons fini avec le réacteur 2, je voudrais attirer votre attention sur des examens à entamer sans délais. Le niveau d'eau de la tranche 3 a augmenté de $150 \mathrm{~mm}$ dans la seule journée d'hier. II s'agit de l'augmentation en vingt-quatre heures. Ce matin, ce qui était, vingt-et-une heures auparavant, à 2550, c'est-à-dire à $2 \mathrm{~m} 55 \mathrm{~cm}$ de l'OP' ${ }^{8}$, se trouve à 2,70 $\mathrm{m}$. Ce qui signifie une hausse d'environ $15 \mathrm{~cm}$.

Nous en avons discuté à la centrale et une des explications possibles, actuellement, serait que l'eau du centre de traitement des déchets que nous avons vidée dans le sous-sol du bâtiment turbine de la tranche 4, avec la hausse du niveau d'eau, soit passée de la tranche 4 à la tranche 3 par des passages non prévus. Nous sommes en train de vérifier les plans. II semblerait que cette possibilité soit très probable. II existe en effet des réseaux électriques qui relient les tranches 3 et 4, et qui permettraient à l'eau de circuler.

L'autre cause possible serait l'ouverture partielle d'une porte étanche située dans la salle de distribution électrique de la tranche 4. Juste après le séisme, pour pouvoir mettre en marche le SLC du réacteur 3, nous avons dû faire passer un câble de cette salle de distribution vers la tranche 3 et nous avons ouvert partiellement cette porte. D'après mes archives, cela devait se situer le 12 ou le 13 mars. Nous allons vérifier les élévations pour voir si le passage de l'eau serait possible par cette voie.

Compte tenu de ces éléments, j'ai donné l'ordre d'arrêter provisoirement l'envoi d'eau du centre de traitement vers le bâtiment turbine de la tranche 4. Ce ne sera qu'après avoir bien étudié les différentes possibilités que je viens d'évoquer et avoir pris les mesures nécessaires que nous déciderons si nous reprenons le transfert de l'eau du centre de traitement à la tranche 4.

Encore une autre possibilité qui ne concernerait pas que la tranche 3, mais aussi les tranches 5 et 6 au plus haut point, serait que de l'eau souterraine entre à l'intérieur des bâtiments. Or les pompes des tunnels de maintenance sont à l'arrêt depuis longtemps. Habituellement, lorsque l'eau monte à un certain niveau dans les tunnels, les pompes se mettent automatiquement en marche pour l'évacuer. Les pompes sont à l'arrêt parce qu'on considère qu'on ne remplit pas les conditions de rejet. Ce qui se passe quand ces pompes ne fonctionnent pas, c'est qu'il y a possibilité que de l'eau souterraine s'infiltre dans les bâtiments. Voyez le cas des tranches 5 et 6 . Ce ne sont pas des tranches où on injecte de l'eau dans le réacteur ni ailleurs. Pourtant, le niveau d'eau monte à certains endroits. Ce ne peut être que le fait de la nappe souterraine. Déjà, cette eau a commencé à inonder la salle du générateur du HPCP et d'autres salles primordiales pour l'électricité. Nous en sommes à un stade où ces infiltrations peuvent menacer l'intégrité même des tranches 5 et 6 . Comme solution, vous me suggérez de stocker cette eau comme de l'eau de pluie. Mais nous n'avons plus le temps de construire maintenant un bassin pour ça. Nous sommes pieds et poings liés et vous nous dites de faire au mieux. Mais nous, à la centrale, nous ne sommes vraiment pas en état de "faire au mieux». Je vous demande instamment de prendre une décision. C'est une question qui concerne étroitement l'intégrité à venir des installations, y compris des tranches 5 et 6 . Nos deux gros problèmes, dans l'immédiat, sont la tranche 2 et le traitement des eaux. Je voudrais insister sur le fait que sans décision concernant ces 
deux problèmes, il ne nous est pas possible, à la centrale, de continuer à avancer. C'est, pour moi, ma plus grande préoccupation.

\section{Takekuro, fellow du siège}

J'ai bien compris. Je pense qu'il nous faut prendre une décision importante concernant surtout le deuxième point, le problème de l'évacuation d'eau par les tunnels d'entretien. Je voudrais que nous en discutions tout de suite après cette réunion. Le groupe "sûreté » du siège, ainsi que les membres des groupes " construction ", " réhabilitation nucléaire ", je vous demanderai de rester après cette réunion.

\section{GROUPE « RÉHABILITATION NUCLÉAIRE 》 DU SIÈgE}

Concernant le cas des tunnels d'entretien, nous avions en effet perçu le problème comme important et avons formé un groupe d'étude sous l'autorité de la commission de coordination de l'eau. Le groupe pourra réfléchir à des solutions.

\section{Yoshida}

On n'a pas besoin d'un groupe de réflexion. Il y a urgence. C'est maintenant qu'il faut faire quelque chose. Réfléchis bien à tout ça!

\section{GROUPE « RÉHABILITATION NUCLÉAIRE " DU SIÈgE}

Oui, Monsieur. Excusez-moi.

\section{Takekuro}

Nous allons nous charger du problème. J'ai bien conscience que nous n'en sommes plus au stade d'une réflexion collective erratique. Il $\mathrm{y}$ a autre chose qui me préoccupe, c'est la possibilité que les bâtiments turbine soient reliés. Si c'est le cas, cela signifie qu'on ne pourra plus évacuer l'eau souterraine du centre de traitement. Il va falloir que nous réfléchissions à d'autres solutions que de l'envoyer à la tranche 4. Je pense que le siège va aussi prendre en charge ce problème.

\section{YOSHIDA}

Je vous remercie. Nous allons déjà faire ce que nous pourrons, à la centrale, concernant ce passage entre les deux tranches. Mais cela nous soulagera que vous envisagiez d'autres solutions.

\section{Takekuro}

D'accord.

\section{YOSHIDA}

Je vous ai exposé ce qui me semblait le plus urgent. Maintenant, chaque groupe « réhabilitation » va faire son rapport. Je leur laisse la parole.

\section{Annexe 2}

Le 4 avril, 17 h05

\section{TAKEKURO}

Nous avons consulté les différents organismes concernés. Pour commencer, le rejet d'eau du centre de traitement des déchets radioactifs se fera à partir de 7 h00, après-midi.

\section{Yoshida}

7h00 ou 19 h00?

\section{TAKEKURO}


$19 h 00$.

Yoshida

D'accord, 19 hoo.

\section{TAKEKURO}

Ensuite, le rejet par les tunnels d'entretien de la tranche 6 se fera à 21 h00, 9 h00 du soir.

\section{Yoshida}

Oui. À 21 hoo.

\section{TAKEKURO}

Voilà les horaires que nous avons établis. Groupe «communication » et groupe «liaison avec les ministères », veuillez commencer les démarches nécessaires pour que nous puissions tenir ces horaires à coup sûr. Vous me tiendrez au courant étape par étape.

\section{Groupe " liaison avec les ministères "}

C'est compris pour le groupe « liaison avec les ministères ».

\section{GROUPE « COMMUNICATION »}

C'est compris pour le groupe « communication».

\section{Yoshida}

De notre côté, à la centrale, nous allons vérifier que tout est prêt pour les rejets aux horaires prévus. Juste un dernier point. Comme il s'agit de rejets vers la mer, je pense qu'il est nécessaire de prévenir en plus l'Agence de sécurité maritime et les Forces d'autodéfense, par exemple. Si vous pouviez vous en charger au niveau du siège, je vous en serais reconnaissant.

\section{TAKEKURO}

Oui. Le groupe «liaison avec les ministères » va s'en occuper, en même temps que de l'information aux autres organismes concernés. J'aimerais que vous, de votre côté, vous vérifiiez si tout est prêt sur place une demie heure avant l'horaire prévu et que vous me préveniez.

\section{Yoshida}

D'accord. Je vous recontacterai à $18 \mathrm{~h} 30$ pour le centre de traitement et à $20 \mathrm{~h} 30$ pour la tranche 6.

\section{TAKEKURO}

Merci. Je compte sur vous ${ }^{9}$.

(Fin)

\section{NOTES}

1. Contrairement aux transcriptions des autres auditions, les réponses de Yoshida sont ici présentées dans des rubriques thématiques. 
2. Incident survenu en octobre 1991, déjà mentionné dans les auditions du 22 juillet et du 8 août 2011.

3. Système d'aspersion du cœur à haute pression. Il s'agit d'un ensemble de sauvegarde entièrement indépendant conçu pour le refroidissement d'urgence du réacteur. $\mathrm{Ce}$ système est alimenté par l'électricité externe mais possède des générateurs diesel de secours en cas de panne.

4. Comme Yoshida le mentionne au début de l'audition du 22 juillet 2011, il a été en fonction à Fukushima Daiichi de 1986 à 1988 (sous-chef du service "maintenance » des réacteurs 5 et 6), de 1993 à 1995 (chef du service «maintenance » des réacteurs 1 et 2), de 2005 à 2007 (directeur d'unité de l'exploitation et de la maintenance des réacteurs 1 à 4), et de juin 2010 à novembre 2011 (directeur de la centrale nucléaire).

5. Salles où sont entreposés les déchets radioactifs.

6. Cf. l'annexe 1 de l'audition du 13 octobre 2011.

7. Fondée au Japon en 1948, the Maritime Safety Agency devient the Japan Coast Guard (JCG) en avril 2000. Cette organisation civile a pour mission d'assurer la sécurité et la sûreté en mer (application du droit maritime et sécurité nationale; recherche, sauvetage et intervention en cas de catastrophe; levés hydrographiques; gestion du trafic maritime).

8. OP désigne Onahama Peil, autrement dit le niveau de construction du port d'Onahama, situé à 50 kilomètres au sud de Daiichi. Les chiffres désignent le dépassement en mètres de ce niveau, pris comme référence.

9. Le rejet de l'eau faiblement contaminée commence le 4 avril à 19 h00, comme le précise l'annexe 2 de l'audition du 13 octobre 2011. Cette opération inquiète toutefois grandement l'opinion internationale, et en particulier les pays voisins du Japon, du fait d'une possible contamination en amont de la chaîne alimentaire marine, comme le précise cet article paru dans Libération : "Afin de rassurer la population, le Gouvernement a fixé un taux limite de radioactivité pour les produits de la mer, similaire à celui établi pour les légumes. Au-delà de 2000 becquerels $/ \mathrm{kg}$ pour l'iode 131 et de 500 becquerels pour le césium 137, les poissons seront considérés comme impropres à la consommation. Les rejets massifs d'eau polluée dans l'océan risquent de peser encore sur les exportations de produits frais du Japon. L'Inde a décrété mardi une interdiction totale des importations de produits alimentaires japonais, pour une durée de trois mois éventuellement renouvelable. Il s'agit du premier pays à appliquer une telle décision, alors que la Chine, Taïwan, Singapour, la Russie et les États-Unis ont limité leurs interdictions aux produits venant de certaines régions du Japon. L'Union européenne, qui a introduit depuis le 24 mars des contrôles à l'entrée des produits venant de ces régions, a décidé d'abaisser le niveau de radioactivité autorisé en s'alignant sur les normes du Japon, déjà très sévères. L'UE appliquait jusqu'ici les plafonds de radioactivité fixés en 1987, après Tchernobyl» (AFP, 2011b). Des réservoirs de stockage sont aménagés les mois suivants et s'accumulent au fil des ans sur le site. Le 9 août 2019, TEPCO déclare que la limite de stockage de l'eau contaminée sera atteinte à l'été 2022. Actuellement, plus d'un million de tonnes d'eau contaminée est conservée dans les réservoirs. Plusieurs solutions sont envisagées pour remédier à la saturation du site : le stockage durable, l'évaporation, le rejet en mer de l'eau après traitement. Si l'AIEA et la NRA sont favorables au rejet, Greenpeace se prononce plutôt en faveur d'un stockage à long terme. En octobre 2020, les médias annoncent que le Gouvernement s'apprête à déclarer officiellement sa décision d'autoriser le rejet dans l'océan des eaux 
contaminées (Mesmer, 2020). L'opération, qui nécessite des travaux et une évaluation de l'autorité de sûreté nucléaire, ne devrait pas commencer avant 2022. 


\section{Compte rendu de l'audition du 6 novembre 2011 (I)}

1 [À traiter avec la plus grande attention]

30 novembre 2011

\section{Compte rendu d'audition}

2 Akira Matsumoto

Membre du secrétariat de la Commission d'enquête sur l'accident de la centrale nucléaire de Fukushima de Tôkyô Electric Power Company (Investigation Commitee on the Accident at the Fukushima Nuclear Power Station of Tôkyô Electric Power Company)

Ci-dessous le compte rendu de l'audition du 6 novembre 2011, menée dans le cadre de l'enquête sur l'accident de la centrale nucléaire de Fukushima de Tôkyô Electric Power Company.

1. Auditionné, date d'audition, lieu d'audition, enquêteurs.

1. Auditionné

Masao Yoshida, Directeur de la centrale nucléaire Fukushima Daiichi de Tôkyô Electric Power Company.

2. Date d'audition

6 novembre 2011 de 11 h00 à $16 \mathrm{~h} 20$

(Pauses de 13 h00 à $13 \mathrm{~h} 30$, puis de $15 \mathrm{~h} 10$ à $15 \mathrm{~h} 20$ )

3. Lieu d'audition

Salle de réunion $\mathrm{A}$

Foyer masculin de la Japan Football Association Academy de Fukushima ( $1^{\mathrm{er}}$ étage)

J-Village

8-Utsukushimori Yamadaoka, Naraha, Futaba District, Fukushima 
4. Enquêteurs

Tsunemasa Katô, Akira Matsumoto, Yûki Okada.

5. Enregistrement par enregistreur numérique

$\square$ Oui

$\square$ Non

2. Contenu de l'audition

Situation au moment de l'accident et réponses à l'accident

Voir le compte rendu

3. Mentions spéciales

Pas de mention spéciale.

QUESTION : Je sais que vous vous êtes prêté à des auditions cet été. Pour ma part, j'ai été nommé tardivement en août et j'étudie principalement ce qui concerne le tsunami et la gestion de l'accident. Concernant le tsunami, j'envisage d'interroger les membres du groupe "études génie civil », Monsieur XXXXX, qui est actuellement XXXXX, et Monsieur XXXXX. D'autre part, j'ai déjà eu l'occasion de parler avec Monsieur Mutô. En tenant compte du fait que vous aviez été en charge par le passé du département "gestion des installations nucléaires ${ }^{1}$, je voudrais d'abord vous poser aujourd'hui quelques questions au sujet du tsunami. Puis nous élargirons un peu le sujet pour parler de la manière dont on peut se préparer pour protéger des installations nucléaires des catastrophes naturelles.

Nous allons commencer par le tsunami. Lors d'auditions précédentes, l'enquêteur Katô vous a déjà un peu demandé vos sentiments concernant le tsunami. Depuis, nous avons réussi à obtenir des pièces à conviction de la part de TEPCO et je voudrais, aujourd'hui, repasser sur tout cela avec vous.

Comme cela remonte un peu dans le temps, puisqu'on a commencé à se préoccuper des tsunamis vers 2008, j'ai préparé ce document. J'ai tracé un signe rouge ici, pour marquer le fait que, jusqu'en juin 2010, il s'agissait, chez TEPCO, d'une organisation à trois, avec Messieurs Takekuro, Mutô et vous-même. Je voudrais tout d'abord savoir à quel moment vous, personnellement, avez entendu parler de tsunami à propos des centrales nucléaires de Fukushima, lorsque vous étiez à la tête du département « gestion des installations ».

Réponse : D'abord, de par ma formation, je suis un mécanicien. Je n'étais donc pas du tout au fait des théories concernant la formation des séismes, etc.

$\mathbf{Q}:$ Moi, non plus. J'ai appris beaucoup de choses.

$\mathbf{R}$ : Durant toute ma carrière, j'ai plutôt été du côté de ceux qui se chargent de maintenance, de production ou de conduite. Donc, lorsque j'ai été nommé à ce poste de directeur du département " gestion des installations » le $1^{\mathrm{er}}$ avril 2007, cela faisait bien longtemps que je ne m'étais pas occupé de «construction». De plus, le département "gestion des installations nucléaires» venait d'être créé. Au sein du siège social, le traditionnel département «construction » venait d'être étendu pour superviser aussi les grands travaux de transformation des tranches déjà en exploitation, donnant naissance à ce fameux département «gestion des installations ». J'en ai été le premier directeur, nommé le $1^{\mathrm{er}}$ avril 2007.

$\mathbf{Q}$ : Katô ${ }^{2}$ vous avait, en effet, demandé de dérouler votre carrière dans une des auditions précédentes ${ }^{3}$, mais permettez-moi de revenir encore sur certains détails. Vous dites bien le $1^{\text {er }}$ avril 2007 ?

$\mathbf{R}$ : Oui. Cela faisait vraiment longtemps que je ne m'étais pas mêlé de construction. J'avais donc sous mes ordres des constructeurs, des ingénieurs en génie civil, des gens que je n'avais pas beaucoup fréquentés. Comme je n'étais pas un expert du domaine, 
j'avais demandé qu'on me fasse un rapport détaillé du contexte et de tous les travaux en cours. Mais, ce qui en ressort, c'est qu'à l'époque, l'intérêt n'était pas très marqué vis-à-vis des séismes et des tsunamis. Je veux dire que ni l'entreprise en elle-même, ni même la société japonaise n'y portaient une attention particulière.

Là où les choses ont changé, c'est le 16 juillet avec le séisme au large de Chûetsu. Là, c'était une secousse qui dépassait de plusieurs fois ce qui avait été prévu. Et nous, en tant que département de "gestion des installations nucléaires", nous étions en première ligne pour faire face au problème. C'est pourquoi nous avons ouvert un Centre anti-séisme en novembre 2007, où on a regroupé des ingénieurs " construction ", " génie civil » et, même, " mécanique » pour travailler en priorité à la restauration de Niigata ${ }^{4}$, tout en menant des études et en gérant la crise.

Tout naturellement, nous avons pensé que la survenue d'un séisme dépassant les prévisions, comme à Niigata, était possible sur tout le Japon et qu'une révision globale s'imposait. C'est ainsi que nous avons entamé des études pour voir si les chiffres retenus jusque-là pour les secousses étaient corrects ou pas. Concernant Fukushima Daiichi, par exemple, nous avons effectué de nouvelles études sur la faille de Futaba ${ }^{5}$ et d'autres failles à proximité, ainsi que des études sur la partie immergée, sur le relief sous-marin. Évidemment, tout ça, après le 16 juillet et le séisme au large de Chûetsu.

Q: En 2007, donc.

R : Je dois reconnaître qu'après le 16 juillet 2007, les séismes sont devenus un sujet extrêmement important. Tout de même, il convient de noter deux choses à ce propos. On s'est beaucoup focalisé sur les secousses telluriques à cette époque. Si on considérait qu'une secousse bien plus importante que celle qui avait été prévue pouvait survenir, il fallait renforcer, par exemple, les bâtiments, la tuyauterie et les instruments. Pour cela, il convenait de se pencher plus particulièrement sur l'analyse de ce qui venait d'arriver. Bien sûr, nous avons logiquement pensé aux tsunamis aussi. Mais les tsunamis, au Japon, sont considérés comme des épiphénomènes des séismes. Pour pouvoir en évaluer l'importance, il fallait d'abord déterminer les secousses telluriques. Ce qui fait que, bien que séisme et tsunami aient été tous les deux au centre de l'intérêt général, au début, on s'est d'abord focalisé sur les secousses.

Après les discussions autour des secousses, nous en sommes, bien entendu, venus à l'estimation des tsunamis. Heureusement, dans le cas de Kashiwazaki, sa situation sur la mer du Japon, contrairement à la côte Pacifique, faisait que les tsunamis restaient modestes. Du coup, notre conclusion a été que les problèmes de tsunamis concernaient essentiellement Fukushima Daiichi et Daini, comme on pouvait s'y attendre. Il est vrai que durant la première année, nous étions concentrés sur Kashiwazaki et qu'on ne parlait guère que de tremblements de terre. Je ne me rappelle plus à quel moment précis, mais j'ai le souvenir que c'est au cours de l'année 2008 que nous avons commencé à parler de la nécessité d'évaluer précisément l'ampleur des tsunamis en tant que phénomènes consécutifs aux séismes.

$\mathbf{Q}$ : Justement, je suis allé m'entretenir avec un certain nombre de personnes à ce propos. Cela vous aidera peut-être à rafraîchir votre mémoire. II semblerait en effet que les préoccupations, au sein de TEPCO, autour des tsunamis concernant Fukushima Daiichi ont commencé à poindre vers cette période. Pour preuve, je ne sais pas si vous y aviez jeté un coup d'œil, le sujet est évoqué dans une note, "Mémo explicatif sur l'estimation des 
tsunamis à Fukushima Daiichi et Daini ». Selon ce document, Messieurs Mutô, XXXXX, le directeur du Centre de l'époque et vous-même, ainsi que le GM de l'ingénierie civile et Monsieur XXXXX, l'actuel GM, vous seriez réunis le 10 juin 2008 pour discuter de la question. II semblerait bien qu'il s'agisse d'une des premières occasions où auraient été évoquées ces préoccupations concernant Fukushima.

On imagine assez bien le contenu de vos discussions. En juillet 2002, les Headquarters for Earthquake Research Promotion ${ }^{6}$ ont rendu public un rapport intitulé Estimations à long terme de l'activité sismique au large de la côte Sanriku à la péninsule de Bôsô? ${ }^{7}$. Partant de l'étude du séisme de Sanriku de l'ère Meiji ${ }^{8}$, ce rapport avançait la possibilité qu'il puisse y avoir un séisme de même envergure n'importe où dans une zone partant de la partie nord au large de Sanriku jusqu'aux proximités de la fosse au large de la péninsule de Bôsô. À la suite de cela, et avant que le groupe d'études ingénierie civile n'en fasse lui-même rapport en juin 2008, et avant même le séisme au large de Chûetsu, il y a eu une refonte des normes officielles des mesures antisismiques en 2006, qui a donné lieu à un ordre de révision des installations existantes.

C'est là que se situe l'anecdote du ministre qui, ému par la survenue du séisme au large de Chûetsu, a voulu faire accélérer les choses. Vous êtes donc dans les travaux de mise en conformité aux nouvelles normes antisismiques et vous vous demandez comment vous allez traiter le problème des tsunamis. Le groupe «ingénierie civile » a de nombreuses discussions avec les experts de la JSCE et c'est là qu'ils reçoivent l'avis du Professeur Imamura ${ }^{9}$ vers la fin février 2008. Pensant que l'hypothèse d'un grand séisme au large du département de Fukushima le long de la fosse sous-marine ne pouvant être exclue, il préconise d'en tenir compte comme possible point de formation des tsunamis et affirme qu'il ne serait pas raisonnable d'ignorer les évaluations à long terme fournies par les Headquarters for Earthquake Research Promotion. Lorsque vos équipes ont effectué les calculs en suivant les préconisations de la JSCE concernant les points de formation des tsunamis, ils ont obtenu des résultats comme O.P. 13 ou 10, c'est-à-dire des chiffres de l'ordre de $10 \mathrm{~m}$, voire plus. Vous estimez que ceci doit être porté à la connaissance des dirigeants. Ce qui se concrétisera par cette réunion du 10 juin. J'ai entouré ici le 6 et le 7 , parce que le problème avait été abordé pour la première fois en juin et que Monsieur Mutô, à la suite de cette première présentation, avait demandé des études complémentaires que vous avez effectuées pour le 31 juillet. Ensuite, au vu de ces études, Monsieur Mutô a décidé de l'orientation à prendre et il pense que vous avez vous-même assisté à cette séance. Est-ce que tout ceci vous évoque quelque chose? Les choses se sont-elles déroulées à peu près comme ça pour vous ?

$\mathbf{R}$ : Oui, je pense que oui.

$\mathbf{Q}:$ Vous en rappelez-vous grosso modo?

$\mathbf{R}$ : Je ne suis pas sûr des détails de dates, mais dans les grandes lignes, c'est bien ça.

$\mathbf{Q}$ : Très bien. Si je reprends les choses chronologiquement, d'après Messieurs XXXXX et $X X X X X$, avant d'alerter brutalement tout le monde, ils sont venus vous trouver en juin. Vous, vous avez estimé que le problème était suffisamment grave pour que tout le monde soit mis au courant. D'où la réunion. Vous souvenez-vous de quelque chose en particulier?

$\mathbf{R}$ : Comme je vous l'ai dit tout à l'heure, je n'étais pas très au fait de ces problèmes. Du coup, je demandais souvent l'avis de XXXXX ou de XXXXX, qui était un homme particulièrement sérieux. Depuis le 6 juillet $2007^{10}$, je fréquentais quand même assidument les hommes du génie civil et de la construction, donc, même si je ne me souviens pas des dates et même si au départ il était surtout question de Kashiwazaki, il est certain que, surtout à partir de 2008, j'étais au courant de l'avis du Professeur Imamura et de l'avancement des discussions. 
Q : D'accord. Bien sûr, les chiffres ne sont que des chiffres. Ce sont des chiffres virtuels. Mais quand avez-vous eu vent, pour la première fois, de ces chiffres concernant la hauteur des tsunamis?

$\mathbf{R}$ : Probablement, juste avant.

Q: Juste avant la réunion? D'accord. Vos hommes m'ont dit qu'ils étaient venus vous consulter une ou deux semaines avant la réunion et je me demandais si vous étiez déjà au courant depuis plus longtemps. Mais vous pensez que c'était à peu près à ce moment-là.

$\mathbf{R}$ : Oui, je le pense bien.

$\mathbf{Q}:$ Très bien.

$\mathbf{R}$ : Ce que je vais vous raconter n'est sûrement pas dans des comptes rendus ou autres archives, mais aussi bien XXXXX que moi fumons. Il y a un aquarium, au premier étage du siège social, où les fumeurs sont parqués. Souvent, quand je fumais là, XXXXX venait me raconter ce qu'il faisait, les discussions qu'il avait avec le Professeur Imamura, par exemple. J'étais donc au courant de la situation. La communication était plutôt bonne. Seulement, le temps d'analyser et de faire les calculs, c'est très long. Le Professeur Imamura avait émis son avis en février. En démarrant les études à ce moment-là, il ne me semblait pas exagéré que les calculs exigent environ quatre mois et que les résultats chiffrés ne tombent qu'en juin.

$\mathbf{Q}$ : Donc, si je résume, il y a d'abord l'avis du Professeur Imamura, vos troupes vous en informent et proposent de faire des calculs, vous acceptez. C'est à peu près ça?

$\mathbf{R}$ : Oui, tout à fait.

Q: D'accord. Quand vous avez su ce que disait le Professeur Imamura, quelle a été votre réaction? N'avez-vous pas été choqué qu'il puisse évoquer l'éventualité d'un tsunami qui pouvait se former n'importe où?

R : Bien sûr, ce genre d'éventualité entraîne de grosses responsabilités pour une centrale nucléaire, mais si je vous dis le fonds de ma pensée, pour moi, le problème des tsunamis concerne aussi l'État et les collectivités locales. Qu'allaient-ils faire ? Il ne suffisait pas que seul TEPCO prît des mesures. Il est évident qu'en tant que centrale nucléaire, nous nous devions de prendre des mesures pour la protéger. Mais si des tsunamis devaient se former partout, au large de toutes les côtes Pacifique de tout le Japon, les mesures en vigueur étaient toutes dépassées. Alors je me rappelle m'être demandé ce que les autorités allaient faire, y compris pour le problème des centrales nucléaires.

$\mathbf{Q}$ : Vous voulez dire que, ayant été mis au courant de cette possibilité, vous vous êtes demandé si l'État ou les autorités locales allaient bouger, ou bien si cette hypothèse allait se révéler ne pas être suffisamment forte pour convaincre l'État de bouger. C'est bien ça?

R : Oui.

$\mathbf{Q}:$ Et Monsieur XXXXX, qu'en pensait-il ?

$\mathbf{R}$ : Il était d'accord avec moi. Ça n'était pas seulement le problème de Fukushima Daiichi et Daini, ça concernait tout le Japon. L'orientation des études du Professeur Imamura faisait qu'il était focalisé sur les centrales nucléaires. Cela ne nous dérangeait pas plus que ça. S’il s'intéressait aux centrales, qu'il s'y intéresse. Seulement, il était important pour nous de vérifier la pertinence de sa théorie. Ça, c'est une chose qui devait être faite sérieusement. S'il s'avérait qu'elle tenait la route, c'était alors une histoire qui aurait concerné bien plus de monde que nous. Il aurait fallu retourner la copie aux Headquarters, la Commission de gestion de crise du 
Premier ministre aurait dû prendre des décisions, etc. Nous étions d'accord pour dire que des études étaient nécessaires, mais qu'il était primordial aussi d'évaluer avec soin la pertinence de cette théorie.

$\mathbf{Q}$ : OK, j'ai bien compris. Et vous vous êtes dit que tant qu'à faire des études, il fallait des études chiffrées. Quand vous avez eu ces chiffres en main, la première fois que vous les avez entendus, qu'en avez-vous pensé ?

$\mathbf{R}$ : C'était «ouah!». Vous savez, quand je suis arrivé chez TEPCO, la référence du tsunami le plus important était le tsunami du Chili ${ }^{11}$. Comme je l'ai dit, j'ai toujours travaillé du côté de ceux qui reçoivent les données des séismes et des tsunamis et conçoivent des machines qui y résistent, de ceux qui reçoivent les conditions à respecter et travaillent en fonction, et non pas du côté de ceux qui définissent ces conditions, alors il était très difficile pour moi d'avoir une idée, même grossière, des chiffres qu'on pouvait atteindre. D'ailleurs c'est bien pour ça que j'écoutais toujours très consciencieusement ce que me disaient XXXXX et XXXXX.

Q: J'ai interviewé plusieurs personnes à ce propos, y compris Monsieur Mutô, et je crois que leur première réaction à tous a été l'incrédulité.

$\mathbf{R}$ : Je vous crois.

$\mathbf{Q}$ : Vous aussi, votre première réaction a été l'incrédulité ?

$\mathbf{R}$ : Oui. Tout à l'heure, je ne vous ai pas suffisamment bien expliqué. Quand je suis arrivé chez TEPCO, vers 1979, j'ai tout de suite été envoyé à la construction de Fukushima Daini et la référence était le tsunami du Chili. On disait « $3 \mathrm{~m}$ maximum ».

$\mathbf{Q}$ : De l'ordre de $3 \mathrm{~m}$, donc.

R : Oui. On a construit Fukushima Daini, avec en tête ce chiffre de $3 \mathrm{~m}$. Et, depuis, pendant près de trente ans, pour moi, un tsunami, c'était ça.

Q : En 2002, vous devenez chef de service en ingénierie civil. Et là, la référence est encore à $5 \mathrm{~m}$.

$\mathbf{R}$ : Oui, c'est à peu près ce qu'on nous disait à l'époque. Alors, $10 \mathrm{~m}$ ou plus, cela me paraissait totalement incongru. Est-ce qu'un tsunami de cette ampleur pouvait vraiment venir ? Voilà, ce que j'ai ressenti $1^{12}$.

$\mathbf{Q}$ : Quand j'ai rencontré Monsieur Mutô, il m'a parlé très franchement, si j'ose dire, en tout cas très simplement. En l'occurrence, il m'a dit qu'il pensait qu'un phénomène d'une telle ampleur avait beaucoup de chances de ne pas advenir, mais, qu'en en ayant entendu parler, il se devait de prendre les mesures nécessaires. Sa position était très claire. Et vous ? Étiezvous sur la même longueur d'ondes?

$\mathbf{R}$ : Oui, tout à fait.

$\mathbf{Q}:$ Très bien. Bien qu'incrédule...

$\mathbf{R}$ : Je crois que tout le monde l'était.

Q: J'aimerais bien avoir une idée de l'ambiance qui régnait dans la salle à l'annonce de ces chiffres. Est-ce que c'était la panique ? Ou bien, les gens ne voulaient pas y croire?

$\mathbf{R}$ : Probablement parce que j'avais été mis au courant de l'affaire juste avant la réunion, je n'ai pas été très surpris. L'incrédulité, je l'avais éprouvée au moment où j'ai eu connaissance des chiffres. Et c'est sûr que c'est dans ce même état que j'ai abordé la réunion. J'avais effectivement remarqué que, durant les exposés de XXXXX et de XXXXX, Mutô affichait une expression d'incrédulité également. Ça devait être l'ambiance générale. 
$\mathbf{Q}$ : Avant que le sujet ne soit abordé à cette réunion du 10 juin, aviez-vous pensé utile de prévenir Monsieur Mutô à l'avance pour qu'il soit moins surpris ? L'aviez-vous fait ?

$\mathbf{R}$ : Je ne me rappelle plus. C'était une époque où on se voyait tous les jours pour parler de Kashiwazaki. Vous me donnez la date du 10 juin 2008, mais c'est un point dans le temps.

$\mathbf{Q}$ : Oui, j'ai bien conscience que votre préoccupation principale était Kashiwazaki-Kariwa.

$\mathbf{R}$ : D'après mes souvenirs, c'était le 20 ou le 21 mai qu'on nous avait communiqué le $\mathrm{S}_{\mathrm{s}}$ de Kashiwazaki, c'est-à-dire la nouvelle référence de secousse sismique pour Kashiwazaki. Il s'agissait d'une secousse très importante, de l'ordre de 1000 gal. Il fallait que nous rendions publics ces chiffres et que nous communiquions sur le fait que nous allions renforcer les installations pour résister à cette secousse. Quatrevingt-dix-neuf pour cent de mon travail consistait à travailler dessus.

$\mathbf{Q}$ : Vous étiez donc focalisé sur cette histoire et, au milieu de tout ça, vous aviez de temps en temps quelques nouvelles des tsunamis. C'était à peu près comme ça que vous l'avez vécu à l'époque?

R: Oui. 1000 gal, ça représentait de fameux travaux de renforcement. Comment allions-nous faire ? C'était le gros de mon travail, le courant principal, et j'avoue que, face à ça, les histoires de tsunamis n'étaient que des bras secondaires du fleuve.

$\mathbf{Q}$ : Je pense que c'était tout à fait légitime. Vous étiez encore au lendemain des incidents de Kashiwazaki, vous étiez mobilisés pour sa réouverture. Que Kashiwazaki ait représenté l'essentiel de vos préoccupations me semble naturel. Je me doutais bien que dans ces circonstances ces histoires de tsunami avaient dû entraîner des réactions d'incrédulité. Vous aussi, avez-vous ce genre de souvenir, comme les autres?

R : Oui.

$\mathbf{Q}$ : Très bien. Donc, si je reviens à ce papier, Monsieur XXXXX vous y informe de la hauteur du tsunami. Incidemment, je trouve que Monsieur XXXXX est un homme exceptionnel, le genre de collaborateur extrêmement précieux dans une entreprise. II avait vraiment fait les choses à fond.

$\mathbf{R}:$ Oh, on ne trouve pas souvent des gens de cette qualité.

$\mathbf{Q}$ : Il fournit donc toutes sortes d'explications. II fait remarquer, notamment, que la manière dont la hauteur du tsunami a été calculée était l'exploitation, faute de mieux, d'un modèle préexistant mal adapté au cas présent. Dans ces notes, ses collaborateurs et lui se sont aussi demandé comment se répartirait l'énergie du tsunami si vous construisiez une digue. Vous en souvenez-vous?

$\mathbf{R}$ : Oui, tout à fait. Je ne sais pas si la discussion prend place à ce moment précis ou pas, mais je lui ai demandé ce qu'on pouvait bien faire face à une vague de plus de 10 $m$ et une des solutions qu'il avait évoquées était la construction d'une digue au large. Là-dessus, je me rappelle lui avoir demandé encore ce que cela allait faire si on construisait cette digue.

$\mathbf{Q}$ : D'après les personnes que j'ai interrogées, c'est à ce moment-là que quelqu'un, on ne sait pas qui en a parlé le premier, a émis l'opinion que, si vous construisiez une telle digue, vous et la centrale seriez à l'abri, mais que la vague se propageant alentour, il y aurait beaucoup de dégâts dans le voisinage et que, finalement, l'idée n'avait pas que des côtés positifs.

R: Oh, c'est probablement moi qui en ai parlé. L'estimation de cette vague ne touchait pas que la centrale. Elle devait se refléter dans les mesures de protection de 
la localité dans son ensemble. Il n'était pas question de se contenter de protéger la seule centrale.

$\mathbf{Q}:$ Une vision d'ensemble. Cela devait s'inscrire dans une vision d'ensemble.

$\mathbf{R}$ : Il était essentiel d'avoir cette vision, en être conscient, sinon nous allions vers des solutions bancales. On ne pouvait pas se permettre de construire cette digue et de se rendre compte après coup que, du fait de cette digue, la vague allait être d'autant plus haute de part et d'autre.

$\mathbf{Q}:$ Vous ne pouviez pas ne sauver que vous-mêmes.

$\mathbf{R}$ : Non. C'est pour ça que, s'agissant aussi bien de la longueur de la digue que d'autre chose, il fallait se poser la question de savoir si on pouvait se contenter de prévoir la longueur qui allait nous sauver, nous seuls.

$\mathbf{Q}$ : Et la réponse était évidemment non. Quand j'ai interrogé Monsieur Mutô, il avait l'air de penser que c'était lui qui avait parlé le premier de cette préoccupation. C'est un peu comme I'histoire de la poule et de l'œuf. En tout cas, tout le monde se souvient de cette discussion et d'être tombé d'accord. Vous, vous avez le souvenir d'avoir parlé le premier.

R : Oui.

$\mathbf{Q}$ : Très bien. Après l'exposé de la situation, j'imagine que vous avez beaucoup échangé au cours de la réunion. Toujours est-il que Monsieur Mutô vous donne quatre devoirs. Tout d'abord, il demande la rédaction d'explications détaillées concernant l'étude de ce risque, car la décision de prendre ou non des mesures contre les tsunamis en dépend. Ensuite, il commande une étude rapide de la manière d'abaisser un peu la hauteur de la vague de reflux, dans le cas d'un tsunami de $4 \mathrm{~m}$. II vous demande aussi de vous renseigner sur les autorisations nécessaires à la construction d'une digue et, en parallèle, d'étudier les dispositions à adopter concernant les différents appareils. Vous rappelez-vous Monsieur Mutô énoncer ces différentes demandes, que vous aviez peut-être vous-même suggérées en partie?

$\mathbf{R}$ : Oui. Je ne sais pas si tout venait de Mutô ou si j'y avais aussi mis mon grain de sel, mais je me rappelle qu'on avait évoqué ces problèmes au cours de cette réunion. Les détails m'échappent un peu, mais de toute façon, ce genre de réaction, la demande d'informations complémentaires, me semble tout à fait naturel quand on apprend une chose si inattendue.

Q : D'accord. Maintenant venons-en au 31 juillet. II ne reste pas de compte rendu de cette réunion, mais j'ai le papier qui a servi à rendre les devoirs.

D'autre part, il existe aussi un e-mail que Monsieur XXXXX a envoyé ce jour à d'autres exploitants, à 11h01. C'est un mail où il explique la position de TEPCO face à ce problème, le contenu, grosso modo, de ce que l'entreprise avait l'intention de faire. Monsieur XXXXX dit que le matin du 31, tôt, avant la réunion, il est venu en discuter avec vous. Je suppose qu'il était venu pour s'entretenir avec vous de ce qu'il allait présenter au cours de la réunion concernant les devoirs qui avaient été donnés à la séance de juin.

Je sais qu'à cette époque, vous aviez de nombreuses réunions au sujet de Kashiwazaki, qui était votre préoccupation principale à tous, et que vous aviez, notamment, des séances régulières le vendredi à la NISA. Vous est-il arrivé de demander à Monsieur Mutô, lors d'une de ces réunions, l'orientation qu'il souhaitait donner à la résolution de ce problème?

$\mathbf{R}$ : Non, je ne me souviens pas de ça. Vous avez parlé des séances à la NISA, mais, en dehors de ça, nous avions, à l'intérieur de TEPCO, des réunions une fois par mois, le samedi ou le dimanche, où nous nous retrouvions autour du Directeur général pour discuter des suites du séisme au large de Chûetsu. Là, naturellement, nous parlions 
gros sous. Vous allez rire si je dis "gros sous". Mais il est un fait que, dans un cas comme ça, le budget est un sujet de la première importance.

\section{$\mathbf{Q}$ : Bien sûr, c'est important.}

$\mathbf{R}$ : Je me rappelle qu'à chaque réunion, je devais commenter le résumé des différentes lignes prévues.

Q: : 'était pour Kashiwazaki ?

$\mathbf{R}$ : Kashiwazaki était au centre, bien sûr, mais si on étendait les mesures de renforcement, Fukushima Daiichi et Daini étaient aussi concernés. Le plus important, c'était les mesures de protection contre les séismes. Il fallait prévoir un budget pour ces travaux. Ensuite, il fallait prévoir également de quoi faire face aux tsunamis, en tant que phénomènes consécutifs aux séismes. Tout ça, on en a parlé.

$\mathbf{Q}:$ Tout ça, en juin?

$\mathbf{R}$ : Peut-être pas. Je ne me rappelle pas exactement à quel moment. Mais j'en ai fait des documents concernant cet argent! Tant, pour Kashiwazaki. Tant, pour les travaux de renforcement contre les séismes. À Kashiwazaki, étant donné qu'il y avait eu des dégâts causés par le séisme, il y avait des pertes exceptionnelles qui se montaient à bien plus de deux cent milliards de yens ${ }^{13}$. Il fallait ajouter à ça, le montant des travaux de renforcement, le montant des études, etc. Tout ça allait influer sur la gestion future de l'entreprise. Il fallait prévoir tout cet argent. Évidemment, pour Fukushima Daiichi et Daini, nous devions effectuer aussi des travaux de renforcement pour lesquels il fallait avoir une idée du budget. Mais pour cela, il fallait commander au préalable des analyses, des études. Autre chose qui n'entrait pas forcément dans les travaux de renforcement, mais qui s'est révélé être vital au moment de l'accident, c'est le bâtiment antisismique. C'est le moment où on envisageait justement sa construction sur Fukushima Daiichi, également un bâtiment équivalent pour Fukushima Daini. J'ai donc fourni lors de ces séances des documents pouvant donner une vision d'ensemble des frais à engager. Et tout naturellement, pour pouvoir donner une vision d'ensemble, il aurait fallu aussi inscrire le budget pour la réponse aux tsunamis. Seulement, comme on ne savait sur quelle hauteur de vague se baser, je me rappelle avoir dit aux dirigeants, très tôt, que le budget antitsunami de Fukushima Daiichi et Daini était encore à l'étude et qu'il n'apparaissait pas directement dans les prévisions que j'évoquais, mais qu'il était à prévoir. Ce dont je ne me rappelle pas très bien, c'est si je l'ai précisé tout de suite après cette première réunion, ou un peu plus tard.

$\mathbf{Q}$ : Vous voulez dire que vous l'avez expliqué à Monsieur Mutô?

$\mathbf{R}$ : Non. Je parle des documents de ces réunions matinales. Pardon. Il ne faut pas que j'utilise l'expression « réunions matinales ", c'est un peu ambigu. En fait, il y avait ce qu'on pourrait appeler des « réunions concernant les suites du séisme au large de Chûetsu ». Elles étaient propres à TEPCO et distinctes du conseil d'administration ou du conseil d'orientation. Elles se tenaient une à deux fois par mois. Y assistaient, entre autres, le Directeur général, le Président, XXXXX, les responsables du département "nucléaire ", Takekuro, Mutô et moi-même. Les séances tournaient principalement autour de la situation de Kashiwazaki, bien sûr.

$\mathbf{Q}$ : Vous faisiez des rapports et le point de la situation.

$\mathbf{R}$ : C'est ça. On sollicitait les commentaires des dirigeants. C'est lors de ces réunions qu'on a d'abord parlé, par exemple, de l'arrivée en mai de la nouvelle référence de 
secousse sismique pour Kashiwazaki, qu'on a évoqué notre intention de rendre publics notre méthode de calcul et les nouveaux chiffres. Ensuite, selon les besoins, tout cela était soumis au conseil d'administration ou au conseil d'orientation. C'étaient les étapes que nous respections dans notre entreprise.

$\mathbf{Q}$ : Pouvez-vous me répéter le nom de cette réunion? La « réunion concernant les suites du séisme au large de Chûetsu »?

$\mathbf{R}$ : C'est une réunion qui n'avait pas d'appellation officielle.

$\mathbf{Q}$ : D'accord. Parce que c'était une réunion complètement interne ?

$\mathbf{R}$ : En fait, c'est une réunion qui a été voulue par le Président, si j'ose dire...

$\mathbf{Q}$ : Qui était Président à l'époque? Et Directeur général ? Pardon, je n'ai pas assez travaillé sur le sujet.

R : A partir de juillet, c'était Katsumata... Non, pardon. Je ne me rappelle plus quand Shimizu a été Président ${ }^{14}$. En tout cas, au moment du séisme au large de Chûetsu, Katsumata était Directeur général et Tamura, Président. Alors, c'est en 2008 que Shimizu est devenu Directeur général et Katsumata, Président. Ou alors, en 2009 ? Je ne me rappelle plus ${ }^{15}$. En tout cas, c'est Monsieur Katsumata. C'est lui qui a voulu ces réunions, parce qu'il trouvait que le temps était limité en conseil d'administration, qu'on ne pouvait pas y discuter de détails, que même les points de situation qu'on y faisait n'allaient pas au fond des choses. Il voulait des réunions préliminaires pour préparer ces conseils. C'est pour ça que ces réunions ont débuté très vite après le séisme au large de Chûetsu.

$\mathbf{Q}$ : Ce sont des réunions qui ont été installées à l'initiative des dirigeants pour écouter ce que la base avait à dire sans avoir les échelons intermédiaires?

$\mathbf{R}$ : Ce n'est pas tant qu'ils ne voulaient pas des échelons intermédiaires. Voyez-vous, c'était le département «nucléaire " qui était aux commandes. Les dirigeants souhaitaient avoir des rencontres régulières avec ceux du " nucléaire ». Je pense qu'il y a eu deux choses. La première était qu'il fallait qu'ils entendent à la base ce que nous, les techniciens, avions à dire de la situation, et que, dans un deuxième temps, on se mette d'accord sur la manière dont nous allions communiquer avec les autorités locales. S'il s'agissait de Niigata, comment expliquer les choses au gouverneur du département de Niigata ${ }^{16}$, aux responsables de la municipalité de Kashiwazaki, sans parler du maire de Kariwa ${ }^{17}$. En poussant les choses plus loin, finalement, comment communiquer à l'ensemble de la population locale la position de TEPCO à un moment donné. Au cours de ces réunions, nous décidions grosso modo de la manière dont nous allions procéder, puis nous en rediscutions partiellement au conseil d'administration.

$\mathbf{Q}$ : Alors, vous rappelez-vous à quel moment ces réunions ont débuté?

$\mathbf{R}$ : Tout de suite après le séisme de juillet 2007. Au début, tout le monde était sollicité de toutes parts, les choses n'étaient pas vraiment organisées, mais au bout d'un mois environ, on a commencé à tenir régulièrement ces réunions. En fait, avant, on se réunissait quasiment tous les jours.

Q: Je comprends, vous étiez un peu débordés par la situation. Ensuite, vous avez eu une réunion par mois. En 2008, vous en étiez à une réunion par mois?

$\mathbf{R}$ : Oui, probablement une fois par mois. Mais parfois, quand la situation l'exigeait, comme par exemple au moment de cette histoire de nouvelle référence pour la secousse sismique, qui était un élément déterminant pour la suite, il me semble qu'on 
se réunissait plus souvent. Il faudrait demander au secrétariat, mais, depuis le début, c'était une organisation assez souple de ce point de vue-là.

Q: Très bien. Donc, sur souhait des dirigeants, vous organisez ces réunions. Vous, ou Monsieur Mutô, vous y parlez de l'argent à prévoir pour ces travaux de renforcement antisismique. Vous insistez sur le fait que les nouvelles normes étant ambitieuses, les travaux en seront d'autant plus coûteux. Vous travaillez à leur faire prendre conscience de l'ampleur du budget.

R : Voilà, c'est tout à fait ça. Et nous y avons toujours parlé, en parallèle, des travaux à effectuer sur Fukushima Daiichi et Daini.

Q: Quand vous dites que vous y avez parlé des travaux de renforcement antisismique à mener en parallèle sur Fukushima Daiichi et Daini, cela signifie que vous y aviez évoqué une idée du budget à prévoir pour ces travaux ?

$\mathbf{R}:$ Oui, une idée grossière du budget.

Q: Pourriez-vous me donner justement une idée de ce que vous prévoyiez pour Kashiwazaki-Kariwa?

$\mathbf{R}$ : Comme on a ajusté après coup les pertes exceptionnelles, je n'ai plus le chiffre exact en tête, mais il me semble que c'était quelque chose comme deux-centquarante-trois milliards de yens ${ }^{18}$. La somme a peut-être été rectifiée après coup à cause de la réévaluation des pertes exceptionnelles. Toujours est-il que c'était de cet ordre. Mais ça, c'était juste la remise en état. Maintenant qu'il fallait tenir compte de la nouvelle norme, il fallait payer des études. Des études géologiques sous-marines, des études géologiques sur terre, ensuite une analyse des secousses telluriques. Sur la base de ces résultats, il fallait mettre aux normes les différentes installations, les bâtiments. Alors, au début, on avait chiffré à la louche dix milliards pour chaque tranche. Sept tranches, donc soixante-dix milliards. C'était à peu près ce qu'on avait prévu.

Q: Là, ça comprend à la fois ce qui était prévu pour la remise en état, plus ce qu'il fallait pour la mise aux nouvelles normes.

$\mathbf{R}$ : Oui, les investissements sur les installations.

Q: Oui, d'accord. La remise en état et les nouveaux investissements.

$\mathbf{R}$ : On a donc inscrit la remise en état et les nouveaux investissements. Mais il fallait aussi prévoir les différentes études et analyses, qui sont aussi très coûteuses. Je crois qu'on avait compté à ce titre cinquante milliards pour l'ensemble de Kashiwazaki. Plus les frais pour les travaux de renforcement.

$\mathbf{Q}$ : Donc, frais d'études, cinquante milliards. En plus, dix milliards de travaux sur chaque tranche.

$\mathbf{R}$ : Oui, ça devait être de cet ordre. Il y avait donc la remise en état, les études et les analyses. En plus de ça, et c'est là que l'histoire devient un peu compliquée, à l'époque nous communiquions beaucoup sur le thème des «centrales nucléaires, solides face aux catastrophes ». Cela ne concernait pas forcément les travaux de renforcement des installations nucléaires vis-à-vis des séismes, mais il s'agissait, dans ce cadre, de construire un bâtiment antisismique à Kashiwazaki, par exemple. Ou bien d'améliorer les moyens de lutte contre l'incendie. Vous vous rappelez que lors du séisme, des transformateurs avaient pris feu à Kashiwazaki ${ }^{19}$ et que ça avait fait grand bruit. Je ne me rappelle plus de la somme exacte, mais il y avait aussi eu un budget prévu pour ces travaux. Je pense qu'en tout, avec la remise en état et tout ça, ça devait représenter quelque chose comme quatre-cent et quelques dizaines de 
milliards pour Kashiwazaki, puisqu'on s'était fait la réflexion que cela représentait le prix d'une tranche nucléaire. Oui, quatre-cent et quelques dizaines de milliards.

Pour Fukushima Daiichi et Daini, il n'y avait pas de remise en état, mais il fallait prévoir des travaux de renforcement contre les séismes qui exigeaient aussi des études. Nous avions aussi prévu d'y construire des bâtiments antisismiques, dans le cadre de ces « centrales nucléaires, solides face aux catastrophes». Tous ces travaux étaient budgétés de manière grossière au début, puis affinés au fur et à mesure. À chaque étape, on faisait des rapports. Bien entendu, dans le cas de Fukushima Daiichi et Daini, contrairement à Kashiwazaki, à un certain moment, il a été question des réponses à prévoir en cas de tsunami. Cette ligne n'était pas chiffrée, car nous ne savions pas si la réponse allait consister à construire une digue ou autre chose, de plus, les études sur les différents points de formation des tsunamis n'étaient pas encore suffisamment avancées. Donc pas de chiffres, mais à chaque fois, l'attention des dirigeants étaient attirée sur le fait que ces travaux étaient aussi à prévoir.

$\mathbf{Q}$ : Très bien. Et les travaux de renforcement prévus pour Fukushima Daiichi et Daini se chiffraient grosso modo à combien?

$\mathbf{R}$ : Depuis ce 11 mars, ma mémoire fonctionne en pointillé. J'ai la réputation d'être près de mes sous et, en général, je me souviens plutôt bien des sommes en jeu, mais là, cette partie de mes souvenirs s'est littéralement volatilisée, c'est vous dire le choc que j'ai subi. Il me semble tout de même qu'on s'était dit qu'il ne fallait pas autant d'argent pour les dix tranches de Fukushima que pour les sept de Kashiwazaki, en tout cas pour ce qui était des travaux de renforcement contre les séismes. Il me semble que ça n'atteignait pas dix milliards par tranche, mais en tenant compte des travaux pour les "centrales nucléaires, solides face aux catastrophes", on avait prévu un peu plus de cent milliards pour les dix tranches. Six à Fukushima Daiichi et quatre à Fukushima Daini, dix tranches en tout.

$\mathbf{Q}$ : D'accord. Je voudrais alors revenir à cette fameuse réunion de juillet. C'est une chose que je fais avec chaque personne que j'interroge, je voudrais vérifier avec vous quelle a été l'orientation qui a été choisie, vérifier également la signification de cette ligne budgétaire à part. Vous avez rendu en quelque sorte vos devoirs à Monsieur Mutô au cours de cette réunion du 31 juillet. Dans ces documents, on trouve pas mal d'explications concernant ces devoirs. Vous aviez mené différentes recherches, réflexions. Concernant la digue, vous faites remarquer que sa construction risque d'être très onéreuse.

$\mathbf{R}$ : Oui. Ces feuilles concernent les différentes autorisations nécessaires pour sa construction, le budget prévu est ici.

Q: Vous vous demandez aussi quelle est l'attitude des autres exploitants. Par exemple, vous citez des explications émanant du chef de service génie civil de la centrale d'Onagawa. Malheureusement on n'a pas les décisions qui ont été prises durant la réunion, à la suite de ces différents exposés, car ces papiers ont été juste rédigés comme support aux explications qui allaient y être fournies. Mais si je me réfère à l'e-mail que le Group Manager XXXXX a envoyé à tout le monde après la séance, on peut lire que, " concernant les estimations à long terme des Headquarters for Earthquake Research Promotion, celles-ci n'étant pas encore déclinées dans une méthode d'évaluation des mesures anti-tsunamis déterminée, il ne semble pas nécessaire de les refléter immédiatement sur les installations ».

« Il est un fait que les Headquarters ont pointé du doigt des évènements pouvant se produire au large de Sanriku ou de Bôsô. Toutefois, on ne peut pas considérer que ces dernières estimations aient donné lieu à la détermination de méthodes d'évaluation pouvant être considérées comme contraignantes dans la conception des centrales nucléaires ». "Dès lors, on se dirigera vers la définition de règles à appliquer aux réponses aux séismes et aux tsunamis sur la côte Pacifique, 
au sein de l'Electric Technology Research Association ${ }^{20}$. En attendant, on se basera, pour les mesures antisismiques, à la référence tsunami de la JSCE». "Nous essaierons d'obtenir l'adhésion des spécialistes et des membres de la JSCE à cette idée ».

Ce n'est pas que vous ne faites rien. Si je traduis ça dans un style plus familier, vous vous dites "c'est pas vrai!", "il ne va y avoir un aussi gros tsunami! », " mais maintenant que j'en ai entendu parler, par prudence, je vais demander à la JSCE d'étudier la question ", "s'ils me disent qu'un tsunami de cette importance est possible, on fera ce qu'il faut », " mais à ce stade, où on ne sait même pas si c'est vraiment plausible, on ne va pas commencer à bouger! ", "d'ailleurs, y a-t-il même besoin de bouger à ce stade?», "en attendant, on va continuer comme on a fait jusqu'à présent ». Vous retrouvez-vous dans cette suite de réflexions?

R : Il n'y a pas d'erreur, c'était tout à fait ça.

$\mathbf{Q}$ : L'orientation que vous prenez ici est une décision propre à Monsieur Mutô ou est-elle venue de discussions que vous avez pu avoir avec lui ?

$\mathbf{R}$ : Vous savez, c'est une décision collégiale à la japonaise. Quelqu'un a émis l'idée, les autres ont été d'accord. La décision s'est prise à partir de discussions entre nous.

$\mathbf{Q}$ : Aviez-vous déjà arrêté grosso modo la direction à prendre quelques jours avant cette fameuse réunion du 31 juillet?

$\mathbf{R}$ : Nous étions d'accord pour dire que l'argument n'était pas suffisant pour que nous passions à l'action.

$\mathbf{Q}$ : C'est une question que je pose à chacune des personnes que j'interroge à ce sujet, d'après vous, quelle était la raison qui vous a poussé à penser que les chiffres, tout à fait virtuels, qui ont été obtenus en se fondant sur les estimations à long terme des Headquarters n'étaient pas suffisants pour vous faire passer à l'action?

R : C'est parce qu'il n'y avait pas de zone de formation de tsunami au large du département de Fukushima. Évidemment, je n'y connaissais rien en la matière. Mais si on écoutait XXXXX ou XXXXX, les Headquarters avançaient leur théorie en supposant des zones de formation de tsunamis tout le long de la côte Pacifique. Or, du point de vue des probabilités, d'après les théories en vigueur, les chances qu'il y ait de tels tsunamis étaient relativement fortes au large des départements d'Iwate et Miyagi, car il y a toujours eu des zones de formation. Idem, pour les côtes de la région de Bôsô. Quant à la portion au large du département de Fukushima, pour mes hommes, c'était une zone vierge. On n'avait aucune indication de formation de tsunami là, dans aucune archive. Ils ne comprenaient pas pourquoi tout à coup il fallait y voir une zone de formation de tsunami. Bien sûr, si on supposait de force une zone de formation là, cela pouvait donner ces chiffres. Mais était-ce vraiment plausible? Qu'en pensent les spécialistes ? Voilà ce qu'ils disaient.

Les Headquarters affirmaient ça. Mais ils ont tendance à rendre leurs conclusions sans beaucoup affiner, vous ne trouvez pas? Ce que nous voulons dire, c'est que, si l'on prenait l'exemple des côtes de Tôkai ${ }^{21}$, est-ce que l'État ou les autorités locales ont-ils bougé pour tenir compte des conclusions des Headquarters? La réponse est clairement non. C'est la même chose ici. Est-ce que l'État et les autorités locales ont-ils fait procéder à des analyses, ont-ils calculé la hauteur du tsunami, ont-ils bougé pour répondre au plus vite aux préconisations des Headquarters? Là encore, la réponse est clairement non. D'une certaine manière, les savants sont irresponsables. Ils peuvent toujours affirmer que tel scénario est possible. Mais ont-ils suffisamment affiné leur théorie pour que nous puissions nous appuyer dessus pour faire nos aménagements? Là encore, non. Parce qu'ils se contentent de désigner des possibilités. 
Finalement, j'en suis venu à la conclusion que pour pouvoir refléter tout ça dans nos aménagements, il nous fallait une base plus solide, base que j'ai cherchée auprès de la JSCE. Je ne sais pas si c'était ce qu'il convenait de faire ou pas. Mais XXXXX et XXXXX m'ont dit que dans le monde des constructeurs, il était de tradition que tout ce qui concerne les séismes soit soumis à l'avis de la JSCE. Alors je leur ai dit qu'il n'y avait qu'à solliciter la JSCE, dans les règles. Moi-même, personnellement, je n'avais presque pas de contact avec la JSCE. Mais puisque mes hommes pensaient que c'était la procédure à suivre, je leur ai dit de la solliciter au plus vite.

$\mathbf{Q}$ : Votre idée était donc de demander, dans les règles, à la JSCE de se pencher sur la question et d'attendre leurs conclusions. Monsieur Mutô m'a dit que, franchement, il ne pensait pas qu'un tel tsunami pouvait venir. Vous aussi, étiez-vous dans le même état d'esprit?

R : Oui.

Q: Oui, mais ayant entendu parler de la possibilité d'un tsunami géant, par prudence, vous aviez sollicité un organisme extérieur faisant autorité, pour des études approfondies. S'il s'avérait qu'un tel tsunami pouvait venir, vous étiez prêt à faire les choses correctement. C'était à peu près ça, votre attitude?

R : Oui, c'est ça. De toute façon, une chose est sûre, si les experts disaient qu'un tel tsunami pouvait venir, je savais qu'il fallait faire le nécessaire. L'action s'imposait. Je ne sais pas si la réponse appropriée était de construire une digue ou pas, c'était encore une question à laquelle il fallait réfléchir. Mais " construire une digue ", c'est une image facile à comprendre. Donc, s'il fallait construire une digue, il fallait avoir une idée de la somme à investir. Vous savez comme moi qu'avec les gestionnaires on ne peut pas leur dire d'engager de grosses sommes du jour au lendemain. Donc il fallait prévoir cette somme, d'où la ligne de budget.

$\mathbf{Q}$ : Mais si j'étais à la place du gestionnaire, il faut que j'opère des arbitrages. Si je dois engager de grosses sommes, il faut aussi que je dispose d'éléments pour convaincre les actionnaires. Je pense que ce sont des choses qui dépassent le niveau des Group Managers, etc., sans vouloir les déprécier. C'était donc vous ou Monsieur Mutô qui exposiez tout ça dans les hautes sphères, je suppose.

R: Déjà, rien que Kashiwazaki nous coûtait quatre-cent milliards, plus les cent milliards de mise aux normes des autres centrales, on nous accusait déjà de ne pas regarder à la dépense. Alors, s'il fallait en plus prévoir la construction d'une digue, il était nécessaire d'en parler explicitement, bien en amont. Une fois la décision de la construction prise, il fallait pouvoir la financer. Il était important d'alerter tous les dirigeants du problème. Seulement, comme vous l'avez dit, nous n'avions pas de chiffres, nous ne disposions pas d'éléments suffisants pour pouvoir répondre aux questions des actionnaires, leur fournir des explications satisfaisantes.

$\mathbf{Q}$ : Donc, vous en parlez, pour éviter des situations où on pourrait vous répondre qu'on n'en avait pas entendu parler. Bien que portée à part, la ligne budgétaire est présente, histoire de rappeler aux dirigeants que certaines mesures pourraient s'imposer selon les circonstances.

$\mathbf{R}$ : Oui, la possibilité que ces investissements deviennent nécessaires était réelle.

Q: Mais, pour les montants concrets, vous attendiez les études de la JSCE.

$\mathbf{R}$ : Sans ces conclusions, on ne pouvait pas définir les sommes. D'accord, on va construire une digue. Mais une digue de combien de mètres ? C'était le problème. 
$\mathbf{Q}$ : Oui, mais si on va encore plus loin, on pouvait aussi se poser la question de savoir s'il y avait vraiment nécessité de construire une digue, non? D'accord, on va construire une digue. De quelle importance? Selon la réponse, les plans vont être modifiés, etc. Alors vous commencez par attirer l'attention des dirigeants sur le problème, tout en promettant des détails, une fois les conclusions de la JSCE rendues. Compte tenu de la situation, où les frais engagés pour Kashiwazaki et les mises générales aux normes antisismiques sont énormes, vous avez conscience que l'entreprise ne peut pas engager de l'argent sans avoir des arguments solides, mais vous promettez d'évaluer les choses dans les règles. Il est vrai qu'à ce stade vous n'avez pas les arguments rationnels pour convaincre les dirigeants, les gestionnaires d'engager des investissements. D'autant moins pour convaincre les actionnaires. Vous pensez bien que vous pouvez difficilement leur dire, «je n'en sais rien moi-même, mais il vaut mieux investir dans une digue». Avez-vous parlé de tout ça à Monsieur Mutô ou à Monsieur Takekuro ? En avez-vous parlé aussi aux réunions autour de Kashiwazaki?

$\mathbf{R}$ : Je ne me rappelle pas si j'ai utilisé ces documents pour en parler. Mais vous savez, un gestionnaire, quand il voit qu'il y a une ligne à part, pose toujours la question de savoir ce que cela représente. On m'a demandé, comme de juste, si ça allait être cher. J'ai donc expliqué que, si on suivait les normes actuelles de la JSCE, la vague devait atteindre un peu plus de $5 \times$ m, mais qu'on avait sollicité la JSCE pour reconsidération. J'ai expliqué le contexte de cette demande, le fait que certains savants avaient montré la possibilité d'une plus grosse vague, que nous avions chargé la JSCE de mesurer la pertinence de cette théorie, de voir si une vague d'une telle ampleur était possible, que si elle était possible, quelle hauteur elle pouvait atteindre. Je leur ai aussi dit que si les conclusions de la JSCE étaient positives, il faudrait engager de grosses sommes, que la construction d'une digue, par exemple, pouvait être onéreuse et que c'était en prévision d'une telle situation que la ligne avait été inscrite.

$\mathbf{Q}$ : Je pense que ce que vous venez de me dire soulève deux questions. D'une part, il y a les explications que vous avez fournies à Monsieur Takekuro. Ensuite, il y a celles que vous avez fournies au Président et au Directeur général, lors des réunions autour de Kashiwazaki.

$\mathbf{R}$ : XXXXX ou des conseillers responsables de projets assistaient aussi aux réunions.

$\mathbf{Q}$ : Je voudrais d'abord savoir comment vous avez expliqué ça à Monsieur Takekuro.

$\mathbf{R}$ : Vous savez, Takekuro, il a la manie de convoquer les gens par téléphone. Plutôt que d'organiser des réunions, il aime faire venir les gens dans son bureau et discuter avec eux. Ce qui complique les choses, c'est qu'il $\mathrm{y}$ a eu des fois où il $\mathrm{y}$ avait aussi Mutô, des fois où il n'y était pas et où on a parlé en tête à tête. Avez-vous déjà interrogé Takekuro?

Q: Ce n'est pas encore programmé, mais j'envisage de le voir pour l'interroger plus spécialement sur la question du tsunami.

R : L'avez-vous déjà interrogé sur d'autres thèmes? De base, c'est quelqu'un qui s'attache beaucoup aux détails. Quand il commence à être préoccupé, il devient obsédé. Alors, par exemple, si je lui explique le problème de la secousse du séisme au large de Chûetsu, il va me rappeler au téléphone pour me demander de lui expliquer tel détail.

$\mathbf{Q}:$ II ne peut pas s'en détacher.

$\mathbf{R}$ : Non, il ne peut pas s'en détacher. Alors, vous pensez bien que je n'arrêtais pas d'être convoqué. Du coup, je ne sais plus à quel moment précis je lui en ai parlé, mais, tout à fait naturellement, on en est arrivé à discuter du problème du tsunami. Je me rappelle lui avoir expliqué ça en détails. Qu'il y avait l'histoire des Headquarters, l'avis 
du Professeur Imamura, les études commandées à la JSCE. Qu'il y avait des risques qu'il puisse y avoir un très gros tsunami, qu'on ne savait pas encore très bien, que j'avais confié l'étude du problème à la JSCE. Je me souviens qu'il avait été d'accord avec cette solution.

$\mathbf{Q}$ : Vous vous souvenez donc d'avoir fourni vous-même les explications à Monsieur Takekuro.

R : Je pense qu'il n'a pas eu d'autre interlocuteur que moi à ce moment-là. Parce que c'était moi qui m'occupais à la fois de la gestion de ce qui se passait à Niigata et des deux centrales de Fukushima. Bien sûr, il y avait aussi XXXXX et ses hommes. Je sais que quand il s'agissait de détails techniques, c'étaient eux qui étaient convoqués. Je pense même que XXXXX ou XXXXX sont allés plusieurs fois expliquer certaines choses en direct à Takekuro. Mais quand il s'agissait de l'orientation de l'ensemble, la direction à prendre, c'était moi qui étais convoqué.

$\mathbf{Q}$ : Monsieur Mutô n'était pas inclus dans ces discussions?

$\mathbf{R}$ : Si, il y avait des cas où il était inclus, d'autre pas. Nous étions tous très occupés. Mutô, de son côté, s'occupait des experts.

$\mathbf{Q}$ : Oui, Monsieur Mutô lui-même m'a expliqué que cela faisait partie de ses fonctions.

$\mathbf{R}$ : Mutô regardait l'ensemble, y compris les histoires d'argent. Il m'arrivait donc d'aller m'expliquer auprès de Mutô, d'autres fois auprès de Takekuro. Il y avait des fois où nous étions tous les trois. Plusieurs schémas étaient donc possibles. En tout cas, dans le cas de Takekuro, dès qu'il avait un moment, il appelait en me demandant de venir dans son bureau. Si j'étais libre, j'allais dans son bureau faire un rapport de la situation. On fonctionnait comme ça à la base. Comme je connaissais bien le caractère de Takekuro, moi-même, de mon côté, dès qu'il y avait une chose qui me préoccupait, je faisais un saut dans son bureau pour en parler avec lui. Je passais par sa secrétaire qui me faisait entrer s'il était libre. Je ne restais pas forcément plus de dix minutes dans le bureau. Mais je l'ai souvent fait. Il faut dire que les choses se bousculaient. On était concentré sur la gestion des suites du séisme de Chûetsu et le redémarrage de la centrale de Niigata.

$\mathbf{Q}:$ Oui, cétait votre préoccupation principale.

$\mathbf{R}$ : Oui, c'est ce qui était au centre de presque toutes nos discussions.

$\mathbf{Q}$ : Au milieu de tout ça, Fukushima ne se présentait encore que dans le cadre des mises aux normes antisismiques. Un projet secondaire qui apparaissait de temps à autre dans vos discussions. C'était ça, le schéma, n'est-ce pas? Oui, Fukushima, à ce stade-là, ne risquait pas d'occuper le devant de la scène. D'accord.

Ce que vous avez dit à Monsieur Takekuro, à ce moment-là, allait grosso modo dans le sens de ce que vous aviez décidé avec Monsieur Mutô ?

$\mathbf{R}$ : Oui, tout le monde était au courant du scénario.

$\mathbf{a}$ : Très bien. Du point de vue timing, je pense qu'il vous est difficile de me donner concrètement une date. Si je me reporte à moi-même, je suis plutôt du genre à ne pas garder les choses trop longtemps pour moi seul. Alors, à votre place, je n'aurais pas tardé à en parler. Par exemple, si la conversation avait eu lieu le 31 juillet avec Monsieur Mutô, je pense que dès début août, j'en aurais parlé à Monsieur Takekuro. C'est un peu comme ça que cela s'est passé ?

$\mathbf{R}:$ :ffectivement. 
Q : D'accord. Vous vous souvenez donc d'avoir mis vous-même Monsieur Takekuro au courant du problème en présence de Monsieur XXXXX, même si vous n'êtes pas sûr que Monsieur Mutô ait assisté à la scène ou pas, n'est-ce pas ?

R : Oui.

$\mathbf{Q}$ : Lorsque vous expliquez la situation à Monsieur Takekuro, vous lui parlez de la position que vous avez définie avec Monsieur Mutô, vous parlez également du budget, que vous allez porter sur une ligne à part, puisque vous ne pourrez préciser les montants qu'une fois que la JSCE aura rendu ses conclusions. Comment avez-vous présenté les choses ? « /l y a tant, pour Kashiwazaki. Pour les normes antisismiques de Fukushima aussi, il faudra un certain budget. Tout ça est énorme. En plus de ça, c'est encore flou et ça ne vaut peut-être pas la peine de chiffrer, mais il y aura une ligne à part pour le tsunami ».

$\mathbf{R}$ : Non, je n'ai pas l'intention de lui dire «ça ne vaut pas la peine de chiffrer ça ». C'est le contraire. Ce que je veux lui dire c'est « si nécessaire, on aura besoin de ce budget ». Si je pensais que ça n'était pas nécessaire, je n'avais qu'à l'ignorer, ne pas refléter le problème dans le budget. Mais j'étais très inquiet que la JSCE n'arrive à la conclusion que le Professeur Imamura n'avait pas tort.

$\mathbf{Q}$ : Vous voulez dire que la JSCE pouvait donner tort ou raison à sa théorie, que les deux étaient possibles?

$\mathbf{R}$ : Oui, tout à fait possibles. Le message que je voulais faire passer à la sphère des dirigeants, c'est que, du point de vue de la gestion, il fallait qu'ils soient conscients qu'il y avait de fortes probabilités que cet argent devienne nécessaire. Mis à part le problème de savoir ce qu'on allait faire concrètement pour répondre au tsunami.

$\mathbf{Q}$ : Et donc, comme étape suivante, avez-vous le souvenir d'en avoir parlé au Président et au Directeur général lors d'une réunion sur les suites du séisme de Chûetsu?

$\mathbf{R}$ : Oui, bien sûr. Évidemment, je ne leur ai pas donné de détails comme cette histoire de point de formation du tsunami, mais, en gros, comme je vous l'ai dit tout à l'heure...

$\mathbf{Q}$ : Justement, jusqu'à quel point êtes-vous entré dans les détails ?

$\mathbf{R}$ : À la base, ce sont des gestionnaires. Alors, quand ils voient une ligne budgétaire à part, ils demandent, et c'est normal, « pourquoi cette ligne?». Et la question suivante est «combien d'argent faut-il prévoir pour cette ligne?». Je ne me rappelle pas jusqu'à quel point j'ai détaillé les choses. Mais, grosso modo, ce que je leur ai dit, c'était que du côté de la mer du Japon, c'est-à-dire pour Kashiwazaki, il n'y avait pas de grandes inquiétudes à avoir concernant les tsunamis, mais que pour la côte Pacifique, différentes théories étaient en présence actuellement et certaines faisaient allusion à des tsunamis de grande ampleur. Que si on faisait des calculs sur ces bases, on ne pouvait pas nier la formation d'un tsunami qui dépasserait (et je pense ne pas avoir fourni en l'occurrence un chiffre concret) les $5 \mathrm{~m}$ et quelques qui nous servaient de référence à ce moment-là. Là-dessus, j'ai enchaîné en disant que nous avions sollicité la JSCE, ou que nous allions le faire, là, je ne sais plus très bien, pour qu'elle évalue la validité de ces théories. Suivant les conclusions qui seraient rendues, et si elle concluait à l'arrivée possible d'un très gros tsunami, il nous faudrait prévoir une réponse, qui risquait de se chiffrer à bien plus que cinq cents millions ou un milliard.

$\mathbf{Q}$ : Vous rappelez-vous si vous avez donné ces explications vous-même?

$\mathbf{R}$ : Au cours de ces réunions, c'est moi qui étais en charge des aspects techniques. Il arrivait que, de temps en temps, après avoir introduit les grandes lignes, je fasse intervenir XXXXX ou XXXXX, pour donner les détails. Mais, en gros, c'est moi qui 
parlais toujours gros sous et c'est dans ce cadre-là que j'ai abordé la question du budget pour la réponse aux tsunamis.

$\mathbf{Q}$ : D'accord. À votre avis, à quel moment se situe cette réunion? Assez vite après avoir informé Monsieur Takekuro, je suppose?

$\mathbf{R}$ : Oui. De toute manière, on n'avait aucune idée du moment où on allait avoir besoin de cet argent. Mais, suivant les conclusions de la JSCE, il pouvait arriver qu'on en ait besoin assez rapidement. Si on regarde les délais concernant les travaux de remise aux normes sismiques de Fukushima Daiichi, à partir de 2007, les études et analyses avaient pris beaucoup de retard. Le fabricant manquait de personnel. Si on tenait compte de tous les paramètres sur toutes les tranches, les travaux ne devaient se terminer que vers 2012 ou 2013. Il aurait très bien pu arriver qu'entretemps on ait été amené à entamer des travaux pour le tsunami.

$\mathbf{Q}$ : Donc, vous expliquez à Monsieur Katsumata, on ne sait pas très bien s'il était Président ou Directeur général à l'époque, que, l'essentiel des efforts étant tournés vers Kashiwazaki, les chiffres des mises aux normes antisismiques de Fukushima sont de tant et vous discutez de cette fameuse ligne à part avec lui. Vous précisez que de nouvelles théories concernant les tsunamis sont apparues et que vous avez demandé à la JSCE de se pencher sur la question. Est-ce qu'à ce point de vos explications, a-t-il demandé à ce qu'on fasse ne serait-ce qu'une estimation de la hauteur de ce tsunami?

$\mathbf{R}$ : Je ne m'en souviens pas très bien. Je ne me rappelle pas si j'ai parlé de « $10 \mathrm{~m}$ » ou « de plus de $10 \mathrm{~m}$ ». De toute manière, si on prévoit une somme de l'ordre de plusieurs dizaines de milliards, c'est qu'on doit construire une digue sérieuse, qui suppose une vague d'une dizaine de mètres. Sinon on n'atteindrait pas une telle somme. Mais je ne suis pas sûr d'avoir donné un chiffre rigide comme " $10 \mathrm{~m}$ ", ou bien est-ce que je me suis contenté de parler de la possibilité d'une vague qui dépasse la référence en usage de $5 \mathrm{~m}$ et quelques? Même si je n'avais pas prononcé le chiffre " 10 ", si la vague devait dépasser les $5 \mathrm{~m}$ et quelques, de toute façon, il fallait prévoir de l'argent.

$\mathbf{Q}$ : Donc, au moment où vous parlez, vous présupposez que la vague va dépasser ces $5 \mathrm{~m}$ et quelques.

R : Oui, bien sûr. Elle va les dépasser.

$\mathbf{Q}$ : Ce «dépassement », c'est un dépassement de quel genre, " un grand dépassement», « un assez grand dépassement » ou » pas tant que ça »?

$\mathbf{R}$ : Par la suite, nos calculs ont montré que la vague pouvait monter à $6 \mathrm{~m}$ et quelques et on a rehaussé les pompes.

$\mathbf{Q}:$ C'est ce que vous avez fait pour la tranche 6.

$\mathbf{R}$ : Pour des travaux de ce genre, du point de vue des frais, on reste dans les centaines de millions ou même juste cent millions. Mais quand il s'agit de construire une digue, on atteint plus de cent fois, voire mille fois cette somme. Alors il fallait que les dirigeants gardent bien cette idée en tête. Évidemment, je ne sais plus si j'ai précisément parlé d'un milliard, mais il me semble avoir dit «certaines théories montrent la possibilité d'une vague qui dépasse largement les $6 \mathrm{~m}$ ». Décidément, mes souvenirs m'échappent. Mais, croyez-moi, quand on est en train de parler de quatre cent ou cinq cent milliards, on ne demande pas l'autorisation de dépenser deux cent millions. 
$\mathbf{Q}$ : Ce qui signifie que même si cette ligne ne comportait pas de chiffres, vous avez donné oralement quelques exemples de sommes à prévoir? Vous avez fait allusion à quelques dizaines de milliards, par exemple?

R : Vous savez, s'il s'était agi d'un budget de deux cents ou trois cents millions, il n'y avait pas besoin d'une ligne à part.

$\mathbf{Q}$ : Il n'y avait même pas besoin de l'inscrire. D'accord.

$\mathbf{R}$ : Inscrire cette ligne à part, cela signifiait que d'une manière ou l'autre, que ce soit la construction d'une digue, ou le réaménagement complet du port, une très grosse somme allait devenir nécessaire. Et ça, c'était une mesure nécessaire et logique dans ces circonstances. L'opinion est focalisée sur la hauteur du tsunami, «combien de mètres ? ", mais à partir du moment où il allait dépasser les $6 \mathrm{~m}$, il était évident que cela allait donner lieu à des travaux d'envergure. Mon but, quand j'ai expliqué ce budget prévisionnel, était que cette idée soit bien ancrée dans la tête des dirigeants et cette ligne était là pour servir d'aide-mémoire, en quelque sorte.

$\mathbf{Q}$ : Très bien. Et lorsque vous avez exposé ce budget, quelle a été la réaction du Président et du Directeur général?

$\mathbf{R}$ : Je ne me rappelle pas très bien, mais Monsieur Katsumata a dit « d'accord, et quelle est la probabilité que cela arrive?». Monsieur Katsumata est quelqu'un de très rationnel. Quand on lui explique des choses, il demande souvent quelles sont les probabilités. Disons que cela fait partie de son caractère. Je lui ai donc dit que diverses thèses avaient été émises par les scientifiques, qu'elles différaient selon les chercheurs et que j'avais demandé qu'on les évalue. Et la discussion s'est terminée là. Elle n'est pas allée plus loin.

$\mathbf{Q}$ : Et vous n'avez pas spécialement reçu d'ordres concernant ce sujet.

$\mathbf{R}:$ Non.

$\mathbf{Q}$ : Si je résume, du haut en bas de l'échelle, y compris les dirigeants, vous étiez tous sur la même longueur d'onde. Vous étiez tous incrédules, mais d'accord pour confier cette étude à un organisme compétent et prêts à prendre les mesures qui s'imposaient si nécessaire.

$\mathbf{R}$ : Oui, je crois que tout le monde était sur la même ligne.

$\mathbf{Q}$ : Très bien. Il y a encore une question que je voudrais vous poser. Ce n'est pas que je le pense personnellement, mais dans l'opinion publique et chez certains membres de la commission, il y a l'idée que s'il arrive quoi que ce soit à une centrale nucléaire, les conséquences sont très graves et que, sachant cela, vous auriez dû faire un tant soit peu quelque chose sans attendre les conclusions de la JSCE. Concernant la remarque des Headquarters, comme vous l'avez dit vous-même, la JSCE pouvait aussi bien conclure à la possibilité d'un tel tsunami ou exclure cette possibilité. Dire «les mesures actuelles sont suffisantes » ou «attendez-vous à une vague plus puissante ». Si vous aviez agi en devançant ces conclusions et en prévoyant une vague plus grosse...

$\mathbf{R}$ : Vous voulez dire en réagissant juste avant le tsunami?

$\mathbf{Q}$ : Non, pas une histoire aussi dramatique, mais si vous aviez démarré des travaux même incomplets, sans attendre les conclusions de la JSCE. Je pense vous en reparler tout à I'heure, mais après votre nomination ici, il y a eu un groupe de travail sur la réponse aux tsunamis qui a été constitué. Est-ce que, dans ces séances, il n'a jamais été question de faire quelque chose, même si vous ne saviez pas si ce tsunami allait vraiment venir ou pas, et même si ces travaux devaient plus tard s'avérer inutiles?

$\mathbf{R}$ : Là, je pense que vous faites allusion à ce qu'a dit un professeur de l'Université de Kyôto $^{22}$. Mais c'est la réaction d'un savant. Vous savez aussi bien que moi que s'il n'y a pas de bases précises au départ, on ne peut rien concevoir. Sachant ça, dire qu'il 
faudrait faire ne serait-ce qu'un peu... C'est quoi ce "peu»? Je pense bien que c'est un certain prof de l'Université de Kyôto. Je ne sais plus si ce n'était pas l'ancien recteur. Ce vieux, je voudrais lui dire, "toi aussi, tu le sais bien que ça ne se passe pas comme ça ». C'est vraiment une élucubration de savant. Pour quelqu'un qui est sur le terrain et qui fabrique des choses en vrai, s'il ne reçoit pas les directives de base, il ne peut rien concevoir. Si ces contraintes sont de l'ordre de 10 , très bien, va pour 10 , si c'est de l'ordre de 13, c'est bien aussi. Mais si on ne nous indique pas concrètement le modèle et la forme de la vague, nous, on ne peut rien dessiner. Alors, nous dire d'en faire un tant soit peu, j'ai envie de demander c'est quoi ce "peu», à quoi ça correspond?

Mais, nous aussi, de notre côté, on était vigilant et on voulait faire les choses correctement. C'est pour ça que quand on a su pour la tranche 6 , on a relevé les pompes, par exemple. Peut-être les détracteurs vont-ils me dire que ça, ça n'entre pas dans le «peu » dont ils parlent. Je voudrais bien savoir, moi, à quoi correspond ce «peu » de leur point de vue de savants, qu'ils définissent ce «peu». Qu'est-ce que ça recouvre? Du point de vue de ceux qui dessinent les plans dans la réalité, on ne peut pas se lancer dans une conception aussi floue. C'est parce que nous avions besoin d'une base solide que nous avions sollicité la JSCE. La JSCE nous disait $15 \mathrm{~m}$, j'étais persuadé que nous prendrions illico les mesures qui s'imposaient.

$\mathbf{Q}$ : D'accord. Si je résume ce que nous venons de voir, l'histoire du tsunami prend forme en juin 2008, Monsieur Mutô vous demande des compléments d'information que vous soumettez en juillet. À ce stade, de concert avec Monsieur Mutô, vous décidez de l'attitude à adopter face à ce problème. Vous êtes tous passablement sceptiques quant à l'arrivée d'un tel tsunami et vous pensez que le phénomène est peu probable, toutefois, puisque le problème avait été évoqué et qu'il n'était pas question de l'ignorer, vous décidez de demander à la JSCE d'examiner ces thèses en détails. Mais vous ne pensez pas qu'il soit utile à ce moment d'engager des frais ou de construire quelque chose sur la base de ces calculs. Vous êtes prêts à prendre les mesures qui s'imposent en fonction des conclusions de la JSCE. Mais d'ici là, vous décidez de continuer à vous référer aux chiffres de l'évaluation de la JSCE datant de 2002, qui vous servent de base pour les mesures de sécurité. Ces chiffres étaient de l'ordre de $5 \mathrm{~m}$ et quelques, avant de passer à $6 \mathrm{~m}$.

$\mathbf{R}$ : Ça, c'est au milieu de la baie. Nous avions refait des études.

$\mathbf{Q}$ : ॥ me semble que vous aviez fait des calculs en suivant les données publiées par le département «voies maritimes » de l'Agence pour la sécurité maritime qui ont montré un résultat supérieur de 0,2, ce qui a donné une vague de référence de $6 \mathrm{~m}$. Mis à part ce cas particulier, globalement, vous vous basiez sur les chiffres de 2002 et, pour vous, les mesures qui avaient été prises en référence à ces chiffres étaient suffisantes. Si la JSCE livrait des conclusions les mettant en cause, vous étiez prêts à faire le nécessaire. Voilà à peu près l'attitude que vous aviez décidé d'adopter. Ceci a été très vite communiqué à Monsieur Takekuro, avant d'être évoqué à une réunion sur les suites du séisme de Chûetsu devant le Directeur général et le Président, dans le cadre d'une discussion centrée sur Kashiwazaki-Kariwa, sous la forme d'une ligne budgétaire à part. Ladite ligne ayant été remarquée par les dirigeants qui se sont informés sur sa signification. Pour en revenir à cette fameuse ligne, vous l'aviez inscrite parce que vous ne vouliez pas que, si les conclusions de la JSCE confirmassent la possible survenue d'un tel tsunami, les dirigeants pussent vous dire qu'ils n'étaient pas au courant?

$\mathbf{R}$ : Ça, et puis à cause de l'argent. La gestion, c'est une question de gros sous. On avait déjà eu tellement de difficultés avec l'argent. Personnellement, j'avais la réputation d'être le plus dépensier du groupe. 
$\mathbf{Q}$ : C'est parce que vous vous étiez trouvé là par hasard au moment du séisme de Chûetsu.

$\mathbf{R}$ : Oui, bien sûr, je n'ai pas utilisé cet argent pour moi. Mais dès que j'ai été nommé chef de département, j'ai tout de suite réclamé de grosses sommes, alors... De toute manière, pour moi, à la base, il a toujours été primordial que les dirigeants aient une conscience claire de ce qu'ils pourraient être amenés à dépenser.

$\mathbf{Q}$ : Très bien. Donc aussi bien du point de vue de l'argent que des études techniques, les thèses étaient encore trop floues pour que vous puissiez faire quoi que ce soit. De toute façon, vous ne pouviez pas justifier à ce stade le fait d'engager des frais, auprès des gestionnaires aussi bien que des actionnaires. Et c'est une position que vous partagiez tous à la base, aussi bien Messieurs Mutô, Takekuro, le Président, le Directeur général que vousmême.

$\mathbf{R}$ : Oui, c'est ce que nous pensions. Nous n'avons pas ignoré le problème.

$\mathbf{Q}:$ Très bien.

II va bientôt être midi. Si vous permettez, pouvons-nous continuer encore un peu?

$\mathbf{R}$ : Oui, bien sûr. Alors je voudrais aller aux toilettes. Je vais aux toilettes, je fume une cigarette et je reviens tout de suite.

$\mathbf{Q}$ : Pas de problème. Si vous voulez des pauses-cigarette, dites-le-moi.

(Pause)

Q : Tout à l'heure, nous avons parlé de ce qui s'était passé jusqu'en juillet 2008. Je voudrais qu'on s'intéresse maintenant à la suite. Je sais que Messieurs XXXXX et XXXXX sont allés voir différents experts de la JSCE pour expliquer la position de TEPCO. Dans l'ordre, il y a eu en octobre une rencontre avec le professeur Shudô23. Le professeur Shudô appartenant à la JSCE, ils lui avaient demandé de suivre l'affaire de près. Ensuite, il y a eu des contacts avec le professeur Satake ${ }^{24}$ ou Monsieur XXXXX à propos du tsunami de Jôgan. Concernant toutes ces rencontres, vos troupes vous faisaient-elles des rapports à chaque fois?

$\mathbf{R}$ : Oui, elles m'en parlaient toujours.

$\mathbf{Q}$ : Donc, vous étiez tenu au courant. Très bien.

$\mathbf{R}$ : XXXXX a un côté rêveur, mais en même temps c'est l'homme des détails. Quant à XXXXX, il est encore plus précis. Alors, vous pensez bien que, même si j'ai rarement eu l'occasion de jeter un œil sur ce genre de comptes rendus, j'en entendais parler. Parfois à l'occasion d'une rencontre fortuite, ils m'abordaient pour me faire un rapport verbal, ou bien ils venaient me voir exprès pour me dire «j'ai rendu visite au Professeur Imamura hier ", etc. J'avais l'habitude de vérifier, dans ces occasions, si la révision des normes pouvait encore attendre. Et de cette manière, j'étais à chaque fois au courant.

$\mathbf{Q}$ : Très bien. $Y$ a-t-il des choses dont vous vous souvenez particulièrement ou quelque chose qui vous a frappé, lors de ces discussions informelles?

$\mathbf{R}$ : J'ai compris à travers ces conversations que, grosso modo, pour aucun de ces experts il n'y avait urgence à entreprendre des choses. Ce n'était pas une question d'aujourd'hui ou demain. Je me fichais de savoir où on allait positionner le point de formation de la vague. Non, ce n'est pas ce que je veux dire. Disons que comme je n'y connaissais rien en la matière, que je n'étais pas un spécialiste du domaine, je ne me sentais pas en position de commenter ce genre de chose. Ce genre de question, je laissais naturellement aux experts et à des spécialistes comme XXXXX et XXXXX le soin d'y répondre. Donc, ils me disaient, on a vu tel professeur, on a parlé de ci ou de 
ça et ce que j'en ai retiré, pour moi, c'est que, dans l'ensemble, l'ambiance générale n'était pas à l'urgence.

Q : D'accord. Sur ces entrefaites, en octobre 2008, vos hommes rendent visite au Professeur Satake qui leur parle du séisme et du tsunami de Jôgan. II leur donne même une première version du fameux article ${ }^{25}$. C'est là qu'en plus de la question de la prévision des tsunamis à long terme des Headquarters s'ajoute l'histoire du séisme et du tsunami de l'ère Jôgan. Vous rappelez-vous du moment où vous avez pris connaissance de cette histoire?

$\mathbf{R}$ : Je pense que c'était à peu près à ce moment-là. Parce que, voyez-vous, XXXXX, quand il prenait connaissance de ce genre d'affaires, venait immédiatement m'en parler. S'il dit que c'était à ce moment-là, c'est que c'était bien à ce moment-là, car je suis sûr d'en avoir été informé immédiatement après. Vous connaissez le classique chinois Jôgan Seiy $\hat{0}^{26}$ ? Quand j'ai entendu parler pour la première fois du tsunami de Jôgan, je me suis fait la réflexion qu'il existait aussi une ère Jôgan au Japon. Je pensais jusque-là que ça n'avait existé qu'en Chine. Je me suis même demandé à quelle époque ça se situait et j'ai découvert que c'était dans les années huit cent et quelques $^{27}$. Ça a été ma première rencontre avec le tsunami de Jôgan. En fait, plutôt que sur le tsunami, j'avais focalisé sur le vocable « Jôgan ». Je m'en souviens très bien.

$\mathbf{Q}$ : Donc, vous en aviez entendu parler. Et Monsieur XXXXX, vous a-t-il expliqué les histoires sur la hauteur du tsunami?

R : La première chose que j'ai su, c'est que le Professeur Satake s'intéressait à un tsunami qui avait eu lieu dans les années Jôgan, en huit cent et quelques, qu'il était en train de l'étudier pour savoir quelle ampleur avait atteint ce tsunami. J'étais curieux de savoir quel était l'endroit qui avait subi le plus de dégâts. On m'a répondu que ce qui correspond aujourd'hui aux départements de Miyagi et Iwate avait connu une assez grosse vague. C'est ce que j'ai entendu pour commencer. Ensuite, en mettant à part les discussions de savoir où situer le point de formation du tsunami, il a été question de la nécessité d'étudier l'influence que ce tsunami avait eue sur différentes localités. J'ai tout de suite donné mon accord pour ça. Nous étions déjà en train de mener différentes études, on n'allait pas ignorer ce point, il paraissait indispensable de savoir quelle répercussion ce tsunami avait eu sur la région même de Fukushima.

$\mathbf{Q}$ : D'accord, j'ai bien compris. Maintenant je voudrais que nous nous penchions sur un document qui date de décembre 2008, intitulé «Evaluation des tsunamis au niveau de la localisation de Fukushima Daiichi et Daini ». On y évoque le fait que vos troupes ont consulté différents spécialistes, qu'elles ont reçu un exemplaire de la thèse de Monsieur Satake sur le tsunami de Jôgan, que les connaissances le concernant n'étant pas encore établies, elles proposent de les faire examiner au sein de l'Electric Technology Research Association, tout comme l'avis des Headquarters y avait été soumis. Ensuite, ayant consulté Tôhoku Electric Power à propos du tsunami de Jôgan, elles ont appris que les normes de sécurité en vigueur à Onagawa ne permettaient pas de faire face à un tsunami de cette ampleur et qu'il en serait fait rapport.

Par ailleurs, concernant l'avis des Headquarters, un certain Monsieur Abe28, qu'on retrouvera encore plus tard, leur déclare, « libre à vous de tenir compte ou non de l'avis des Headquarters, mais si vous deviez l'ignorer, il faudrait une bonne raison à cela ». Ceci est consigné dans le rapport, et je ne sais pas si c'en est une conséquence directe, mais suit un tableau de chiffres, peut-être les résultats de calculs sur cette base. Ce tableau contient aussi des chiffres concernant le tsunami de Jôgan. Si je tourne la page, je vois les différentes orientations préconisées. Pour le tsunami de Jôgan, ce sera le même traitement que pour l'avis des Headquarters. On parle aussi d'un projet de recherche sur les dépôts du tsunami le long de la côte du département de Fukushima. Ces recherches ont été 
suggérées par ce même Monsieur Abe, qui leur a dit que ce serait peut-être un bon moyen de savoir ce qui s'était réellement passé. Vos troupes décident donc de suivre ses suggestions et programment les recherches. Elles vous soumettent ces propositions et reçoivent votre aval. Voilà ce qui y est raconté. Êtes-vous d'accord avec ça?

$\mathbf{R}$ : Oui, c'est ça.

$\mathbf{Q}$ : Très bien. On voit dans ce rapport des chiffres qui correspondent au tsunami de Jôgan. Vous rappelez-vous si Monsieur XXXXX, à la suite de sa visite auprès du Professeur Satake, vous a proposé de faire ces calculs, puisqu'il disposait d'un modèle de formation de la vague?

$\mathbf{R}$ : Oui, je m'en rappelle. Il m'avait dit qu'il allait essayer de faire les calculs.

Q : D'accord. Vous avez vu les chiffres de Jôgan. Mais, finalement, vous décidez de traiter ce tsunami de la même manière que vous l'aviez fait de l'avis des Headquarters, en juin et juillet. Quel était votre raisonnement à ce moment-là?

$\mathbf{R}$ : Tout d'abord, plus que des histoires de modèles, le problème du tsunami de Jôgan est qu'à partir de l'estimation d'une secousse tellurique du passé, le Professeur Satake était arrivé à des hauteurs de tsunami qui dépassaient ce que nous connaissions jusque-là. C'était une chose à laquelle il fallait réfléchir. Très naturellement, j'ai pensé qu'il fallait inclure ce problème, y compris la question du point de formation de la vague, dans l'ensemble de ce qui avait été avancé par les Headquarters. Cela devait être évalué dans un ensemble. Ce n'était pas un problème à part. Dans l'évaluation qu'allait faire la JSCE, pour moi, le tsunami de Jôgan devait apparaître comme la conséquence de l'une des secousses telluriques du passé, un tsunami parmi d'autres.

$\mathbf{Q}$ : Comment avez-vous reçu la nouvelle que si on adoptait le modèle préconisé par le Professeur Satake, on arrivait à de tels chiffres? Avez-vous été dubitatif quant à la survenue réelle d'un tel tsunami?

$\mathbf{R}$ : C'était quelque chose qui était arrivé en l'an huit-cent et quelques. Vous vous rendez compte? Il y avait de ça mille cent ans ou mille deux cents ans. Évidemment la probabilité que ça se reproduise n'était pas zéro. Pour ma part, je voulais que tout cela soit correctement étudié comme un ensemble. Et si, après études, la probabilité qu'une telle vague vienne était vraiment forte, on aurait pris les mesures en nous basant sur les estimations. En réalité je n'ai pas été très surpris. Vous comprenez, j'avais déjà entendu parler d'une vague de $10 \mathrm{~m}$, alors le tsunami de Jôgan, pour moi, c'était juste one of them ${ }^{29}$.

$\mathbf{Q}$ : Je me mets à votre place, vous vous dites, " on nous parle de vagues de toutes sortes de hauteur, je n'arrive pas trop à y croire, mais puisqu'il y a des gens qui en parlent, on va demander à la JSCE d'examiner ces thèses et de donner ses conclusions ».

$\mathbf{R}$ : C'est surtout que je pensais qu'il valait mieux tout évaluer ensemble.

Q: Ce qui veut dire que vous avez traité le tsunami de Jôgan comme l'avis des Headquarters.

$\mathbf{R}$ : Et là, je me souviens d'une chose. Ce n'est pas quelque chose de très important, mais enfin. Mes hommes me disent qu'ils veulent faire des recherches sur les sédiments laissés par les tsunamis. Alors je leur demande en quoi cela consiste. Ils me disent qu'ils vont forer. Et moi de leur demander à quoi ça sert. XXXXX m'explique qu'ils vont chercher les coquilles et les preuves d'une vie sous-marine que les tsunamis auraient apportées, qui se seraient fossilisées et formeraient une couche détectable. En comparant les couches successives, on arriverait, malgré les déformations de la croûte terrestre, à savoir la hauteur des tsunamis qui ont assailli la côte de Fukushima par le passé. Et il m'explique ça avec une tête tellement sérieuse 
que je n'ai pas pu m'empêcher de me moquer de lui en disant que si les habitants de l'ère Jôgan avaient mangé des coquillages dans leur soupe, comme nous, et qu'ils avaient laissé là leurs détritus, que si des habitants d'un hameau de l'intérieur avaient mangé des coques ou des palourdes et avaient jeté les coquilles près de leurs habitations, cela ferait des preuves plus que fantaisistes que le tsunami était venu jusque-là et qu'il me semblait difficile de tenir ça pour scientifiquement correct.

\section{Q : Et Monsieur XXXXX, qu'a-t-il répondu?}

$\mathbf{R}:$ Oh, il a grogné en disant que, mise à part la question de savoir s'il s'agissait d'un hameau ou pas, il suffisait de forer en plusieurs endroits. Mais je me rappelle m'être acharné en lui soutenant qu'à sa place je n'aurais pas confiance dans de telles recherches.

$\mathbf{Q}$ : Oui, mais vous lui avez quand même donné l'autorisation de les faire.

$\mathbf{R}$ : Bien sûr. J'étais pour qu'ils fassent toutes les recherches possibles. Surtout si elles étaient suggérées par les experts. Depuis le tremblement de terre de Niigata en 2007, j'ai toujours dit à mes troupes qu'il fallait sortir toutes les données possibles. Jusquelà, même les autres compagnies d'électricité ne sortaient pas de manière précise les données sur les secousses telluriques, par exemple. Pour moi, puisqu'il s'agissait de remettre en cause toutes les normes sur lesquelles nous nous étions basées jusque-là, j'ai dit à mes hommes de sortir toutes les données, de les rendre publiques. Il y a bien eu quelques résistances, notamment de la part des hommes des secousses. Mais j'ai insisté pour qu'ils sortent tout. En même temps, je leur ai dit d'écouter tout ce que leur disaient les experts. "Écouter", ça veut dire que s'ils disaient de faire des recherches, entreprendre toutes les recherches, que ce soit en mer ou sur terre. C'est une position dont je n'avais jamais dévié, donc quand on a entendu parler du tsunami de Jôgan et qu'on nous a suggéré de faire des recherches, j'ai engagé mes hommes à les entreprendre immédiatement. Cela demandait de l'argent. Mais je leur ai dit que j'allais me débrouiller. Nous étions à un moment où il fallait revoir toute la sécurité antisismique des centrales du Japon en repartant de zéro, alors il fallait que ce qui devait être fait soit fait correctement. Je leur ai dit de ne surtout pas hésiter quant aux recherches à effectuer. Et comme c'était une constante chez moi, je crois que mes hommes étaient dans le même état d'esprit que moi.

$\mathbf{Q}$ : Très bien. Concernant donc les recherches de dépôts des tsunamis, vous donnez votre autorisation et, pour ce qui est de la thèse du tsunami de Jôgan, vous la traitez de la même manière que l'avis des Headquarters, vous sollicitez la JSCE pour études. Est-ce que vous avez parlé de tout ceci avec Messieurs Mutô et Takekuro ?

$\mathbf{R}$ : Je me rappelle avoir utilisé plusieurs fois l'expression «tsunami de Jôgan ». Je ne sais plus si j'ai évoqué directement sa hauteur, mais je sais que j'en ai effectivement parlé à Takekuro et à Mutô. Je leur ai présenté la chose de la manière suivante. On a eu vent d'une discussion qui ressemble à ce que nous avons eu précédemment à propos des estimations des Headquarters. Actuellement, le Professeur Satake est en train d'étudier de manière plus particulière une de ces vagues, le tsunami de Jôgan. Apparemment c'est une très grosse vague qu'on a connu par le passé. Cela s'est déroulé il y a plus d'un millénaire, cela fait longtemps, mais il a l'air de considérer que la possibilité de la formation d'une telle vague aujourd'hui n'est pas impossible.

$\mathbf{Q}$ : Et cette rencontre se situe quand?

$\mathbf{R}$ : Quand j'ai entendu parler de ça, comme je n'y connaissais rien, l'expression « tsunami de Jôgan » m'avait fait penser au «Jôgan » des Leçons politiques de l'ère Jôgan 
en Chine, c'est l'histoire de tout à l'heure. J'ai demandé en quelle année ça se situait et on m'a répondu que ça ne devait pas être très loin de l'ère chinoise de Jôgan. Mais je ne sais pas si Takekuro et Mutô étaient là au moment de cette conversation.

Q: Je voulais parler du moment où vous aviez mis Messieurs Takekuro et Mutô au courant de l'orientation que vous vouliez donner à cette affaire. Je pense qu'il a pu y avoir plusieurs possibilités. Comme cela ne dépassait pas les limites de ce que vous aviez décidé en juin et juillet concernant le tsunami au niveau de Fukushima Daiichi, peut-être aviez-vous pris la décision seul?

R : Non, ce n'est pas possible. J'ai bien utilisé l'expression "tsunami de Jôgan " devant eux. Je ne sais plus jusqu'à quel point je suis entré dans le détail de la taille de cette vague, mais je suis sûr d'avoir dit quelque chose comme «l'autre jour, XXXXX qui est allé à l'Université de Tôhoku ${ }^{30}$ et y a vu le Professeur Satake est revenu en disant qu'un tsunami dénommé " tsunami de Jôgan » était à l'étude. Il s'agirait d'un très gros tsunami qui serait survenu dans les années Jôgan c'est-à-dire dans les années huit cents et quelques ». Je suis absolument sûr de leur avoir rapporté ce fait en utilisant l'expression « tsunami de Jôgan ».

$\mathbf{Q}$ : Vous l'aviez dit à Monsieur Mutô.

$\mathbf{R}:$ Et à Takekuro.

$\mathbf{Q}$ : Très bien. Vous rappelez-vous avoir fait vous-même votre rapport à Monsieur Takekuro?

$\mathbf{R}$ : On revient à l'histoire de tout à l'heure. C'était une époque où je fréquentais intimement, pas que j'avais vraiment envie d'intimité, Takekuro. On se voyait tout le temps, alors au détour d'une conversation... Par exemple, je lui faisais le rapport de ce qui s'était passé dans la semaine et...

$\mathbf{Q}$ : Le sujet serait apparu dans ce rapport.

R : Oui. Je lui racontais tout. Par exemple, qu'on avait quelques soucis à Kashiwazaki concernant l'estimation des secousses telluriques, ou bien que tel sujet avait été abordé dans telle réunion, etc. Alors, naturellement, dans ce cadre, je parlais aussi des problèmes de tsunamis au niveau de Fukushima Daiichi. Je lui ai dit que XXXXX avait ramené cette histoire, que nous allions nous pencher dessus pour sortir une estimation, qu'on envisageait de faire des recherches sur les dépôts des tsunamis, que cela allait demander un petit budget, etc.

$\mathbf{Q}:$ Et tous les deux étaient d'accord avec vous?

R : Oui.

$\mathbf{Q}$ : Ils étaient d'accord. Ont-ils ajouté des commentaires ?

$\mathbf{R}$ : Non, rien. Peut-être ne leur ai-je pas bien expliqué ? Toujours est-il que j'avais l'impression qu'ils estimaient, tout comme moi, que ce séisme faisait tout simplement partie d'un des séismes du passé dont se préoccupait l'avis des Headquarters.

Q : D'accord. Mais Monsieur Mutô m'a dit qu'une fois que vous aviez discuté des tsunamis au niveau de Fukushima Daiichi et aviez décidé de l'orientation à donner, il n'a plus entendu parler de tsunami pour Fukushima Daiichi.

$\mathbf{R}$ : Ça, ça n'est pas possible.

$\mathbf{Q}:$ Vous pensez qu'il y aurait erreur?

$\mathbf{R}$ : Concernant le tsunami de Jôgan, j'en ai entendu parler après la réunion avec le Président et le Directeur général, qu'on a évoquée tout à l'heure. Tout de suite après en avoir entendu parler, je me rappelle très bien lui avoir dit qu'il y avait une 
nouvelle histoire de tsunami qu'on appelle le tsunami de Jôgan. Pour moi, j'ai le souvenir très net d'avoir abordé le sujet à la fois avec Takekuro et Mutô. Bien sûr, je ne me rappelle pas jusqu'à quel point je suis entré dans les détails de sa hauteur ou de son point de formation. En tout cas, je leur ai dit qu'on parlait beaucoup de ce tsunami de Jôgan, que le Professeur Satake, je pense l'avoir cité, l'étudiait, qu'il convenait de nous y intéresser, car il était d'une certaine ampleur.

$\mathbf{Q}$ : Que vouliez-vous dire par « une certaine ampleur »?

$\mathbf{R}$ : Une hauteur qui dépasse les normes actuelles. Seulement, j'ai dû nuancer qu'elle correspondait à peu près aux conclusions de l'avis des Headquarters, voire plus petite.

$\mathbf{Q}:$ A ce moment-là, la réaction de tout le monde a été d'adjoindre ce nouvel élément aux études déjà en cours.

$\mathbf{R}$ : Oui. De le rajouter aux études et, je ne me rappelle pas si c'était une suggestion du Professeur Abe, d'organiser des recherches sur les dépôts. De toute manière, on avait décidé d'entreprendre ces recherches de sédiments, il fallait de l'argent pour ça. S'il fallait prévoir des frais de recherche, nécessairement, je devais fournir des explications à Mutô et à Takekuro. Et dans ces explications, j'étais bien obligé d'aborder l'histoire du tsunami de Jôgan pour justifier ces frais. On ne peut pas engager des frais de recherches sans fournir d'explications. Alors dans ces explications, j'ai forcément abordé le sujet du tsunami de Jôgan.

$\mathbf{Q}$ : Si je comprends bien, lorsque vous voyiez Messieurs Mutô et Takekuro, vous discutiez souvent gros sous, puisque c'est le nerf de la guerre. Dans ces discussions, vous aviez été forcément amené à parler de l'argent dont vous alliez avoir besoin pour les recherches sur les sédiments. Ce qui a provoqué une explication sur le but de ces recherches, occasion où vous avez fait allusion au tsunami de Jôgan.

$\mathbf{R}$ : Voilà.

$\mathbf{Q}$ : Très bien. Après ça, je suppose que vous en avez aussi parlé au Président et au Directeur général.

$\mathbf{R}$ : C'est là où ma mémoire me fait défaut. Je ne sais plus très bien si j'ai parlé nommément de ces recherches sur les sédiments. Seulement, à chaque réunion, on parlait argent en fin de séance. Par exemple, si les choses avaient évolué depuis la réunion du mois précédent, on reflétait ça. Si on avait sous-estimé un poste, on y remédiait. Si, au contraire, on avait surévalué quelque chose, on se disait qu'on allait pouvoir récupérer un peu d'argent, etc. Le budget était un sujet qu'on évoquait obligatoirement à la fin de chaque séance. Et puisque dans le cadre du budget prévu pour Fukushima Daiichi, il y avait toujours cette ligne inscrite à part, je me rappelle avoir parlé du tsunami de Jôgan et l'avoir nommé. Que ceux qui l'ont entendu l'aient retenu ou non est un autre problème. Mais j'ai tendance à penser que j'ai aussi fait allusion à ces recherches sur les sédiments à ce moment-là.

$\mathbf{Q}$ : Même si ce n'était pas suffisamment important pour être noté noir sur blanc dans le compte rendu.

$\mathbf{R}$ : C'est ça. J'ai dû dire que l'expert avait suggéré ces recherches. C'était une époque où il $\mathrm{y}$ avait eu beaucoup de suggestions de la part de beaucoup d'experts. Tel professeur nous disait d'effectuer telle recherche, tel autre de faire des forages supplémentaires. Je passais une bonne partie de mon temps à répondre à ces suggestions. Mais le Président et le Directeur général étaient d'accord pour qu'on réalise toutes les suggestions faites par les experts. Alors je réclamais de l'argent pour ci, de l'argent pour ça. Et dans ce contexte, j’ai bien dû en parler. 
Q: L'occasion pour les dirigeants de constater que différentes personnes disaient différentes choses.

Au fait, comment s'appelait le document où la fameuse ligne était inscrite à part ?

$\mathbf{R}$ : J'ai oublié son nom. C'était un genre de récapitulatif des frais engagés pour la réponse au séisme au large de chûetsu. À chaque fois qu'on organisait ces réunions mensuelles, qu'on pourrait appelées privées, le dimanche matin autour du Président et du Directeur général, je distribuais l'état le plus récent du budget. Et comme tout ce qui touche à l'argent est un peu délicat, je récupérais les documents à la fin de la séance. Mais il y a eu quelques conseils d'administration ou comité d'orientation, je ne sais plus, où on s'est basé sur ces mêmes papiers pour fournir des explications. Vous trouverez là la fameuse ligne.

$\mathbf{Q}:$ À quoi ressemble ce document ? C'est une feuille A4 en paysage?

$\mathbf{R}$ : Chez nous, quand on prépare des documents pour les réunions, on utilise deux feuilles A3 recto-verso, avec quatre slides PowerPoint par face. Ce qui fait 4 X $4=16$ vues.

$\mathbf{Q}:$ Comme des slides PowerPoint. D'accord.

$\mathbf{R}$ : Dans ces documents, on commence toujours par un récapitulatif général, puis ça se divise. Dans ces parties plus détaillées, on explique pourquoi telle ligne a été revue à la baisse, pourquoi telle autre a augmenté à cause de fournisseurs qui se plaignent, etc. Ça nous sert de support.

$\mathbf{Q}$ : Au fait, quand vous rédigiez des documents de ce genre, c'est Monsieur XXXXX qui s'en occupait ? II me semble bien qu'il en avait fait. Concrètement, qui s'en chargeait ?

$\mathbf{R}:$ C'était XXXXX.

$\mathbf{Q}$ : Donc, Monsieur XXXXX. Depuis le début?

$\mathbf{R}$ : Oui. XXXXX était GM au centre antisismique. Quand on a inauguré ce centre, je l'ai fait venir du département « construction » où il travaillait. Je ne sais plus si c'était en 2008 ou 2007. Je sais que c'était dans les tout débuts. Vous comprenez, pour faire tourner un centre de ce genre, il me fallait quelques personnalités efficaces sur lesquelles je puisse m'appuyer. Alors j'avais un peu fait le forcing auprès de son patron pour qu'il me le donne. Je lui avais confié non seulement les sous, mais l'organisation générale. Ce qui fait que tout passait par lui. Évidemment, les sous aussi.

$\mathbf{Q}$ : Oui, il était très au fait des différents mouvements du budget, quand je l'ai interrogé. C'était donc bien lui.

Nous voici donc arrivés à la fin de l'année 2008. Comme vous venez de me le raconter, vous avez fait allusion oralement, devant le Président et le Directeur général, au tsunami de Jôgan, dans le cadre des explications à propos du budget lors des réunions concernant les suites du séisme de Chûetsu. C'était vers le 18 décembre, c'est ça?

R : Oui, il me semble bien.

$\mathbf{Q}$ : Vous aviez donc déjà fourni ces explications vers cette époque. Très bien.

Il est tout juste midi et demi. Je vous propose de nous arrêter pour déjeuner. Merci beaucoup. 
Q: Ce matin nous avons évoqué ce qui s'était passé jusque vers décembre 2008. Je voudrais maintenant que nous voyions ce qui s'est passé ensuite.

Nous allons aborder vos rapports avec la NISA. Le 21 juillet 2008, l'évaluation de votre rapport de mi-parcours concernant la tranche 5 de Fukushima Daiichi, tranche choisie comme représentative des autres tranches, est rendue par la NISA. Précédant le rendu de cette évaluation, il y a eu, en juin et en juillet, des discussions au sein d'un groupe mixte de travail NISA-TEPCO.

$\mathbf{R}$ : Nous avions remis notre rapport en mars, il me semble.

$\mathbf{Q}:$ Oui, c'était en mars.

$\mathbf{R}:$ Et nous avons attendu l'évaluation de la NISA.

$\mathbf{Q}$ : Il y a donc eu plusieurs réunions de ce groupe mixte de travail en juin et en juillet, et, à un moment donné, Monsieur Okamura ${ }^{31}$ s'inquiète de savoir si vous avez tenu compte du tsunami de Jôgan dans votre rapport. Avez-vous eu vent de ce mouvement?

$\mathbf{R}$ : Oui, tout à fait. Je n'assistais pas personnellement à ces réunions, mais des hommes comme XXXXX y allaient pour fournir les explications.

$\mathbf{Q}$ : Effectivement, c'est Monsieur XXXXX qui a répondu à cette question.

$\mathbf{R}$ : Oui, quand il s'agissait de secousses sismiques, c'était toujours XXXXX.

$\mathbf{Q}$ : Justement à propos de secousses sismiques, l'épisode de Jôgan ne se résume pas à un tsunami. II a été précédé d'un séisme dont il a été question dans ces discussions, avant d'en arriver au problème du tsunami. Vous souvenez-vous de ce qui s'est passé à cette époque?

$\mathbf{R}$ : Si je résume, tout d'abord, les analystes de chez Tôshiba et Hitachi étaient totalement absorbés par l'examen des sept tranches de Kashiwazaki ${ }^{32}$. Bien sûr, en parallèle, ils étaient censés s'occuper de la secousse de référence pour Fukushima Daiichi. Ils avaient entamé des études à partir de 2008 et étaient arrivés à un modèle de secousse. Mais ils manquaient cruellement de personnel pour passer à l'étape suivante qui consistait à appliquer ce modèle à l'ensemble des bâtiments de Fukushima, détecter effectivement les faiblesses, proposer des solutions de renforcement. Face à cette situation, ce que nous avons décidé de faire, c'est, au lieu d'effectuer une étude systématique de toutes les installations de toutes les tranches, de choisir respectivement le réacteur 4 de Fukushima Daini et le réacteur 5 de Fukushima Daiichi, comme exemples représentatifs, et de soumettre leurs principales installations ayant trait à "l'arrêt, au refroidissement et au confinement " à la nouvelle secousse de référence. C'est le rapport de ces tests qui a été présenté en mars à l'approbation de la NISA. Pour nous, naturellement, il était important de voir quelles mesures étaient approuvées et lesquelles remises en cause, nous étions donc très sensibles à ce qui se passait dans ces commissions. J'étais tenu au courant de toutes les discussions à chaque séance. Dans ce contexte, je me rappelle très bien qu'il avait été question du tsunami de Jôgan, même si je ne suis pas capable de vous dire à la quantième séance cela s'est passé.

$\mathbf{Q}$ : II en a donc été question. Vous souvenez-vous maintenant de la manière dont cette question a été traitée?

$\mathbf{R}$ : Si je suis mes seuls souvenirs, les avis étaient partagés parmi les experts. De plus, l'estimation des tsunamis n'avait pas encore été faite à ce stade-là. L'attitude de la NISA était, pour permettre l'évaluation générale, d'ordonner de terminer d'abord correctement la procédure en cours de l'estimation des secousses, avant d'aborder les phénomènes connexes comme non seulement les tsunamis, mais les glissements de 
terrains. Ce qui est sûr, c'est qu'au stade de ce rapport daté de mars, nous n'en étions pas encore à l'estimation des tsunamis. Bien sûr, au cours des discussions il a été question des tsunamis, mais ils ne constituaient pas encore un sujet de rapport en soi. Nous pensions logiquement qu'ils feraient partie du rapport final, avec toutes les nouvelles connaissances qui auraient été acquises d'ici-là, lorsque nous aurions fait rapport de tous les résultats de toutes les révisions de toutes les installations. Nous partions du principe qu'ils feraient partie du rapport final, mais nous pensions qu'il suffisait qu'ils apparaissent à ce moment-là seulement.

$\mathbf{Q}$ : D'accord. J'ai compris. Je ne sais pas si c'est que la NISA a voulu rendre les choses plus claires, mais dans l'évaluation qu'ils ont donnée de ce rapport intermédiaire, ils ont écrit à propos de l'incident de Jôgan : «étant donné que les différentes études concernant le tsunami et les sédiments déposés par celui-ci à l'occasion du séisme de l'ère Jôgan (896 après J.-C.) sont encore en cours, la NISA pense qu'il serait judicieux que l'exploitant prenne les dispositions adéquates suivant l'état d'avancement des études concernant l'estimation des tsunamis et des secousses sismiques». Vous souvenez-vous du contexte dans lequel cette phrase a été introduite? Par exemple, Messieurs XXXXX ou XXXXX vous en ont-ils parlé ?

$\mathbf{R}$ : Vous savez, la NISA est ce genre d'organisme ministériel qui ne décide rien par lui-même. Elle se réfugie derrière le bouclier des avis d'experts et évite de prendre toute responsabilité. Ce qui donne ce genre de phrase. On l'a évoqué entre nous pour dire que c'était comme d'habitude.

$\mathbf{Q}:$ Il suffisait de se soumettre à l'avis de la JSCE.

$\mathbf{R}$ : Il semble bien que c'était ça.

$\mathbf{Q}$ : Très bien. Si je vous ai parlé de cet épisode c'était pour vous remémorer ce qui s'était passé en juin et en juillet 2009, avant d'évoquer avec vous ce papier, intitulé «Estimation des tsunamis pour Fukushima Daiichi et Daini au 7 août ». Ce sont Messieurs XXXXX et XXXXX qui m'ont confié ceci, en me disant que cela datait du 7 août 2009. On y lit ceci. «Concernant le séisme et le tsunami de Jôgan, il est prévu de confronter les résultats avec ceux de la JNES (Japan Nuclear Energy Safety Organization) ${ }^{33}$. D'autre part, avons reçu une question d'un membre de la NISA, en date du 5 août, demandant où TEPCO en était de ses réflexions sur le tsunami de Jôgan. Avons répondu que la question était à l'étude ». Effectivement, il semblerait qu'ils aient reçu une demande de rapport sur ce sujet de la part d'un Monsieur Nakura ${ }^{34}$ le 5 août. Afin de lui répondre, des hommes du groupe «études Génie civil » sont venus vous trouver avec ce papier, m'a-t-on expliqué. C'était pour vous demander si vous étiez d'accord pour qu'ils disent que, concernant les normes, TEPCO allait continuer à appliquer celles en vigueur, que, cependant, cela ne signifiait pas qu'il ne faisait rien, puisqu'il avait chargé la JSCE d'étudier la question des tsunamis. Pour ce qui était des calculs qui avaient déjà été faits, même si les résultats n'étaient que provisoires, ils voulaient savoir si vous donniez votre accord pour qu'ils soient communiqués. Vous rappelez-vous d'avoir eu ce genre de conversation avec eux?

$\mathbf{R}$ : Je ne me rappelle pas avoir vu ce papier dans cette optique. Il est certain que dans le cadre de la révision des normes, la question du tsunami avait été soulevée et je me rappelle très bien qu'effectivement on avait eu des demandes du Ministère pour qu'on éclaircisse les choses. Mais ce papier en particulier, dans ce contexte, à ce moment-là... Ça ne me dit rien aujourd'hui.

Q : D'accord. J'ai évoqué ce papier, parce qu'ensuite, par deux fois, des gens de TEPCO sont allés fournir des explications à la NISA. Ils y sont allés deux fois, une fois en août, une autre fois en septembre. Ils y sont allés deux fois et il y a une raison à ça. Ils y sont allés une première fois en août pour expliquer que...

$\mathbf{R}$ : C'était à Monsieur Nakura, n'est-ce pas ? 
$\mathbf{Q}$ : Oui, ils ont expliqué à Monsieur Nakura que la norme retenue était toujours les 5,7 en vigueur. Et c'est là qu'on retombe sur l'histoire du papier de tout à l'heure, puisqu'il pose la question des réflexions de TEPCO sur le tsunami de Jôgan. Mais avant d'en arriver au sujet de Jôgan, vos troupes parlent de l'estimation qui est alors à la base de la norme pour les tsunamis au niveau de Fukushima Daiichi et des mesures prévues pour y répondre.

$\mathbf{R}$ : Ils ont donné une explication générale.

$\mathbf{Q}$ : Oui, une explication générale. C'est à ce moment-là que I'homme de la NISA demande si TEPCO avait déjà effectué des calculs «pour voir » et, devant la réponse positive de vos troupes, demande à les examiner. D'où la deuxième séance, en septembre, avec les chiffres. Et c'est là que je reviens à la déclaration de Messieurs XXXXX et XXXXX, d'après euX, lorsqu'ils sont allés vous consulter en août avec ce papier, vous leur auriez dit qu'il était inutile de donner spontanément ces chiffres, si on ne leur demandait rien, qu'ils n'étaient à dévoiler que si une demande claire était formulée de la part de l'interlocuteur. Du coup, ils se sont contentés d'une première explication très générale qui a donné lieu à la fameuse demande. Face à cette requête, ils repassent chercher votre avis et vous leur dites qu'il n'y a rien à cacher du moment qu'il demande à voir. Vous en rappelez-vous ?

$\mathbf{R}$ : Pas du tout.

$\mathbf{a}:$ Ah, bon?

$\mathbf{R}$ : De mon point de vue, comme je vous l'ai dit tout à l'heure, il suffisait de divulguer. Pour moi, les informations, que ce soit les informations concernant le séisme de Niigata ou d'autres choses, toutes les informations devaient être rendues publiques dans la limite de ce qu'on savait. Seulement, j'avais des réticences à révéler des informations qui pouvaient être ambiguës. Là où on avait des résultats sûrs, comme dans le cas des références de secousses sismiques, j'étais contre le fait de cacher des choses, j'étais pour qu'on publie tout sans faire de rétention. Mais, s'agissant d'études en cours, j'étais peu enclin à rendre publiques des choses dont l'interprétation pouvait être incertaine. Ça a toujours été ma position, alors, même si je ne me souviens pas exactement des termes que j'ai utilisés, j'ai dû leur dire de bien expliciter ce dont on était sûr sur le moment, ce qui a dû induire leurs explications très générales sur les normes appliquées à l'époque. Ça n'est qu'une hypothèse, bien sûr. Parce que je n'en ai pas le moindre souvenir. Mais, me connaissant, c'est le genre de discours que j'ai dû leur tenir.

$\mathbf{Q}$ : Vous voulez dire qu'il n'est pas impossible que cela se soit passé de cette manière?

$\mathbf{R}$ : C'est ça. C'est tout à fait possible. Premièrement, je leur ai sûrement recommandé de bien montrer ce que nous faisions effectivement dans la réalité, et, dans un second temps, puisqu'on exigeait de nous ces chiffres, je leur ai peut-être dit que ce n'était pas un secret et qu'à condition de les considérer comme pouvant être sujets à modifications dans le futur, on pouvait donner les résultats des calculs actuels. Ça a dû sûrement se passer comme ça. En tout cas avec cette nuance-là.

$\mathbf{Q}$ : Très bien. Donc, si je résume, ce papier daté du 7 août décrit la situation à ce momentlà. S'agissant du tsunami de Jôgan, il a été exclu des révisions en cours, car on attend, par exemple, les résultats des recherches de sédiments déposés par le tsunami, qui devront ensuite être interprétés par l'Electric Technology Research Association, la JSCE, etc. À la suite de la demande d'un de ses membres, cette situation est explicitée auprès de la NISA. Si XXXXX et XXXXX prétendent que ce papier a servi de cette manière à ce moment-là, c'est une éventualité tout à fait plausible. Etes-vous d'accord?

R : Oui. 
$\mathbf{Q}$ : Si je comprends bien, vous n'avez pas grand souvenir de ce qui s'est passé autour de ça. Vous rappelez-vous, par exemple, avoir eu un compte rendu de la première séance d'explication à la NISA?

R : Non, pas du tout, concernant cette histoire à ce moment-là.

$\mathbf{Q}$ : C'était pourtant la première fois que vous évoquiez l'attitude de TEPCO sur cette question à l'extérieur. Ne vous êtes-vous pas demandé quelle allait être la réaction de la NISA?

$\mathbf{R}$ : Peut-être ma mémoire me joue des tours, mais il me semble bien qu'à cette époque, même la NSC commençait à s'intéresser à la question du tsunami de Jôgan. Alors, qu'un certain éclaircissement de notre attitude s'impose face à la NISA était tout à fait logique. Bien sûr, par la suite, nous avons eu plusieurs fois l'occasion de nous expliquer sur ce sujet devant la NISA et je ne sais plus très bien en quels termes nous l'avons fait la première fois et à quel moment. Pour moi, ils sont tout simplement allés expliquer notre position. C'est comme ça que je m'en souviens. Il n'y avait pas de rétention ou non d'informations. Nous avons répondu à une demande d'informations en apportant les seules certitudes dont nous disposions à ce momentlà.

$\mathbf{Q}$ : Ce qui signifie que vous n'étiez pas particulièrement curieux de voir la réaction des gens de la NISA.

$\mathbf{R}$ : Non, cela ne m'intéressait pas du tout.

$\mathbf{Q}:$ D'accord.

$\mathbf{R}$ : De toute façon, ce sont des gens qui ne réfléchissent pas par eux-mêmes, alors...

$\mathbf{Q}$ : Effectivement. Au moment de la rencontre du mois d'août, seul Monsieur Nakura semble avoir été présent. Mais au mois de septembre, bien qu'il nie y avoir assisté, le directeur Kobayashi35 lui-même était là et vos gens ont présenté cette carte et un modèle de vague.

$\mathbf{R}$ : Tiens, je ne les avais pas vus.

$\mathbf{Q}:$ Ah, bon?

$\mathbf{R}$ : Ou je les ai vus très rapidement et j'ai dû donner mon accord pour qu'ils les utilisent lors de cette réunion. Ce n'est pas quelque chose qui m'a marqué.

$\mathbf{Q}$ : Parce que ce n'était pas la première fois que vous aviez en main ces informations ?

$\mathbf{R}$ : Non, ce n'était pas la première fois. Me connaissant, j'ai dû probablement dire quelque chose comme "puisqu'il veulent des explications, allez leur expliquer une bonne fois pour toutes et qu'ils nous foutent la paix ».

Q: D'accord. Là non plus, vous ne guettiez pas spécialement leur réaction face aux explications?

$\mathbf{R}$ : Ma foi, non. Il me semble qu'ils les ont acceptées assez naturellement. En tout cas, je n'ai pas entendu dire qu'ils s'en étaient particulièrement émus.

$\mathbf{Q}:$ Ils n'en ont pas fait tout un plat.

Nous allons maintenant passer à ce qui s'est passé le 16 février 2010. Ce jour, le gouverneur de Fukushima ${ }^{36}$ fait une déclaration à propos de l'utilisation du $\mathrm{MOX}^{37}$ à Fukushima Daiichi. Je crois que c'est une affaire qui était en marche depuis pas mal de temps, mais ce jour-là, pour la première fois, il a soumis les conditions, au nombre de trois, permettant d'envisager l'utilisation éventuelle du MOX. Juste après cette déclaration, il y a eu une réunion matinale à la NISA, où le sujet principal était la situation à Kashiwazaki, mais je sais, par différents témoignages, qu'il y a été beaucoup question aussi de 
Fukushima Daiichi. Vous rappelez-vous si, dans ce contexte, il a été de nouveau question du tsunami de Jôgan?

$\mathbf{R}:$ Je n'en ai pas le souvenir.

$\mathbf{Q}$ : Ah, bon. Je voudrais maintenant vous montrer un e-mail daté du $1^{\text {er }}$ avril 2010, envoyé par Monsieur XXXXX à Messieurs XXXXX et XXXXX.

$\mathbf{R}:$ Ah, oui, à propos des recherches.

Q: Oui.

$\mathbf{R}$ : Les recherches sur les sédiments apportés par le tsunami de Jôgan.

$\mathbf{Q}$ : Le $1^{\text {er }}$ avril était un jeudi et le lendemain, vendredi, vous aviez une de ces réunions matinales. II semblerait que vous, Monsieur Yoshida, ayez été sollicité pour fournir des explications sur ces recherches et que vous ayez donné l'ordre à vos hommes de vous préparer un document avec un calendrier des recherches à venir et une explication succincte du contenu des recherches. La veille du vendredi, donc, vos subordonnés vous soumettent le document par mail. Je suppose que le lendemain, 2 avril, vous l'avez utilisé lors de la séance à la NISA. En avez-vous souvenir?

$\mathbf{R}$ : Pas du tout. Je n'ai pas souvenir d'avoir commenté ce document. Si je reviens un peu sur ce qui s'était passé, il y a d'abord eu le rapport concernant les mises aux normes antisismiques. Ça, ça avait été présenté au mois de mars de l'année précédente. C'en sont suivies différentes discussions et la conclusion comme quoi ces mises aux normes étaient pertinentes a dû être émise quelque part vers...

$\mathbf{Q}$ : En juillet 2009 .

R : Voilà, c'était bien vers juillet ou août. Donc, dans un premier temps, la résistance aux secousses sismiques du réacteur, de la tranche et des systèmes "arrêt, refroidissement, confinement " de la tranche représentative, dont nous venions de fournir les études dans un rapport intermédiaire, avait été reconnue. Il était bien sûr convenu que nous poursuivions la même procédure pour chaque tranche. Mais l'achèvement de ces études et leur rapport risquaient de ne se faire, pour les dernières tranches, qu'en 2013. Ce dont la NISA avait été prévenue. Mais, au milieu de tout ça, va faire brutalement irruption une toute autre histoire, celle de l'utilisation du MOX. Je ne sais pas qui, chez nous, avait bougé, mais, pour nous, techniciens, c'est une histoire qui nous est tombé dessus sans crier gare. En janvier ou en février, on nous a avertis que les choses allaient bouger très vite autour de l'utilisation du MOX à la tranche 3 de Fukushima Daiichi. Effectivement, il y avait eu des mouvements pour l'introduction du MOX, mais c'était il y avait de ça plus de dix ans ${ }^{38}$. Depuis, les choses avaient évolué, notamment sur le front des mesures antisismiques. Nous étions complètement perplexes, nous ne savions pas du tout comment les choses allaient progresser. Là-dessus, tout s'est compliqué. Cette fois-ci, c'était le département luimême qui prenait le contrôle des opérations, si je puis dire, puisque c'est le gouverneur en personne qui nous présentait ses conditions pour autoriser l'utilisation du MOX. Il y avait trois conditions. Les mesures antisismiques...

$\mathbf{Q}$ : L'adaptation au vieillissement, les mesures antisismiques et le caractère sain du combustible 39 .

R : Voilà. Tout à coup, on nous a dit de démontrer l'adaptation au vieillissement, la fiabilité des mesures antisismiques et le caractère sain du combustible. Pour nous, l'affaire de l'utilisation du MOX était une préoccupation secondaire. Nous étions pressés d'avancer sur les travaux concernant les mesures antisismiques. Et là, on nous imposait tout à coup de nous intéresser à l'utilisation du MOX. Pour «les 
mesures antisismiques ", nous étions dedans. Mais on savait pertinemment que, les fabricants manquant cruellement de personnel, les résultats des études pour l'ensemble des différentes tranches ne seraient pas disponibles avant 2013, pour les derniers, comme on en avait avisé la NISA. S'il fallait fournir quelque chose pour 2010, il fallait nous rabattre sur les résultats concernant la tranche 5 de Fukushima Daiichi, qui nous avait servi de modèle pour notre rapport de mi-parcours. Les tranches 3 et 5, à Fukushima Daiichi, sont des tranches sœurs. Alors...

$\mathbf{Q}$ : Alors, il me semble que vous aviez fourni un rapport intermédiaire sur la tranche 3 , après celui de la tranche 5 .

$\mathbf{R}$ : En fait, avant ça, nous, partie technique, nous penchions pour donner les résultats de la tranche 5, puisqu'elle avait été choisie en tant que tranche représentative. On les aurait fournis en précisant bien qu'ils étaient là pour servir de référence. Malheureusement, les choses se sont encore compliquées et on a exigé que nous fassions une évaluation de la tranche 3 , à part. Du coup, nous nous y sommes mis sans tarder.

Pour "l'adaptation au vieillissement des installations", nous avons estimé qu'il suffisait de fournir une explication détaillée des mesures que nous prenions déjà dans ce sens.

Pour "le caractère sain du combustible", nous pouvions toujours prouver que des contrôles étaient effectués périodiquement. Nous pouvions fournir également moult études scientifiques démontrant rationnellement que la méthode était sûre. S'ils voulaient voir le combustible pour de vrai, il n'y avait pas de problème. Ils pouvaient venir quand ils voulaient. Voilà ce que nous pensions.

Le problème était de savoir jusqu'où on allait entrer dans les détails des mesures antisismiques. J'ai été très clair à ce moment-là, j'ai dit à tout le monde que les seules données dont nous disposions étaient celles de la tranche 5, évoquées dans le rapport intermédiaire, que la meilleure option était de montrer ces chiffres et que j'espérais qu'on obtiendrait l'accord du Département de cette façon. Mais, comme je vous l'ai dit tout à l'heure, on n'a pas voulu de ma solution et il a fallu travailler à l'évaluation de la tranche 3 qui devait accueillir le combustible MOX, en nous basant sur les résultats de la tranche 5 .

Seulement, la NISA, non plus, n'était pas chaude pour cet examen. Elle nous faisait remarquer qu'elle ne voyait pas de raison d'effectuer un examen séparé de la tranche 3. La situation n'arrêtait pas de se compliquer. Moi, je ne voulais pas faire les études, la NISA ne voulait pas les évaluer. Et pourtant, il y avait des gens qui voulaient nous obliger à le faire. Le département « implantation locale». Pour faire court, ce sont des gens qui sont totalement aux ordres des localités où sont implantées les installations. Ils nous faisaient faire tellement de choses inutiles qu'on était toujours à couteaux tirés avec eux. Alors je leur ai dit, "si vous exigez qu'on fasse les études, on va les faire, mais la NISA est contre le fait de faire un cas particulier de la tranche 3 de Fukushima Daiichi et je n'ai pas l'ombre d'une influence sur elle pour la faire changer d'avis ».

On était en contact assez étroit avec la NISA, je voyais notamment Monsieur Moriyama $^{40}$. Mais, cette fois-ci, c'est l'Agence pour l'énergie (Agency for Natural Resources and Energy) ${ }^{41}$ qui a fait pression sur la NISA, dans le but de faire avancer l'utilisation du MOX dans les centrales. Évidemment, ça n'est pas allé tout seul entre la NISA et l'Agence pour l'énergie. Ils se sont disputés longuement, nos stupides 
fonctionnaires, "je veux, je ne veux pas, tu feras, je ne ferai pas ». Et moi, j'étais pris entre deux feux, une position tout à fait inconfortable. Finalement, il a été décidé de donner satisfaction à «l'implantation locale ». C'est pourquoi, dans la précipitation, on a passé commande au constructeur pour des analyses vers février ou mars.

Je ne me souviens pas de la date exacte, mais on était parti pour refaire les mêmes études que pour la tranche 5. Je me rappelle qu'on évoquait l'affaire lors des réunions du vendredi matin avec Monsieur Moriyama ou Monsieur Noguchi ${ }^{42}$, qui était son chef, à l'époque. Eux aussi traînaient des pieds. Une fois, ils me disaient qu'ils n'avaient pas l'intention d'examiner un cas isolé, une autre, quand la pression se faisait plus forte, qu'ils allaient peut-être être obligés de l'examiner. Voilà à peu près où on en était.

En fait, on était tellement obnubilé par cette affaire que je ne me rappelle pas du tout avoir discuté du tsunami de Jôgan dans ce contexte. Mais si on m'avait posé la question de notre position face à ce problème, je pense que j'y aurais tout naturellement répondu. Ce n'était pas quelque chose qu'on voulait cacher.

Q: En fait, vous aviez rendu un rapport sur les tranches 1,2, 3 et 4, une première fois le 19 juin 2009, un rapport concernant donc les tranches autres que la 5, qui avait servi de modèle. En plus, il y avait eu des corrections datant du 19 avril. Pour la tranche 5, le rapport intermédiaire datait de la fin mars 2008.

$\mathbf{R}$ : Oui, ça, je m'en rappelle. Par la suite...

Q: Par la suite, il y a donc eu ce rapport concernant les autres tranches en juin 2009. Après ça, juste avant que vous ne priiez, contre votre gré, ces messieurs de la NISA de bien vouloir se pencher sur le rapport, il y a eu quelques corrections, qui datent d'avril 2010. Avez-vous des choses qui vous reviennent sur cette période?

R : J'ai du mal à me rappeler l'ordre suivant lequel les choses se sont enchaînées, mais je me souviens de manière tout à fait sûre qu'on a rendu les rapports pour la tranche 5 de Fukushima Daiichi et la tranche 4 de Fukushima Daini en mars 2008. Pour les rapports intermédiaires concernant les autres tranches, je pensais qu'on avait mis jusqu'à l'année suivante pour les fournir, mais on avait donc réussi à les sortir cette année-là?

Q : Non, l'année suivante, en 2009.

$\mathbf{R}$ : Ah, je me disais bien. Je m'étais trompé d'une année. Et donc, il nous avait fallu une année pour les rédiger. Là-dessus, nous avons sollicité la NISA pour examen. Seulement, les rapports avaient été mis au placard. Au ministère. Mais oui, je me souviens maintenant.

Pour la tranche 5 de Fukushima Daiichi et la tranche 4 de Daini, l'évaluation avait été faite en commission et nous avons eu un avis favorable. Un an plus tard, quand nous avons déposé les rapports concernant les autres tranches, la NISA était débordée par l'examen de toutes les autres centrales. Du coup, la mise au placard a duré longtemps.

$\mathbf{Q}:$ Je m'en doute.

R : Voilà. C'est comme ça que ça s'est passé. Quand on lui a demandé d'examiner, seul, le dossier de la tranche 3 de Fukushima Daiichi, la NISA nous a répondu qu'il n'était pas question de faire passer Fukushima Daiichi en priorité, juste à cause de l'utilisation du MOX, que toutes les centrales étaient pressées. Pour être franc, elle avait peur qu'on puisse lui reprocher une collusion avec un exploitant. C'est pour ça 
qu'elle a refusé longtemps d'examiner le dossier. Ça veut dire qu'on n'avait toujours pas leur aval.

Q: La NISA voulait signifier par ce refus qu'il vous suffisait d'utiliser le dossier qui avait déjà été avalisé ?

$\mathbf{R}$ : Oui, tout à fait. Mais nous, nous avions besoin de l'évaluation spécifique de la tranche 3. Alors j'ai passé un certain temps à négocier l'examen, pour que la NISA nous donne ses conclusions au plus vite. Mais oui! Je m'en souviens très bien maintenant. C'est à ce moment-là qu'on s'est aperçu qu'il y avait des erreurs dans les données qui avaient été utilisées dans le rapport de 2009. Il fallait les rectifier. Nous en avons parlé à la NISA. Il y a eu discussion pour savoir si on n'attendrait pas les rapports finaux pour faire ces rectifications. Mais en même temps, si on voulait un examen séparé dès maintenant pour la tranche 3 , il fallait que les corrections soient faites. Nous avons donc rédigé un rectificatif que nous avons soumis aussi à la NISA. Voilà comment ça s'est passé.

$\mathbf{Q}:$ Très bien.

$\mathbf{R}$ : Cela vous convient?

a : Oui.

$\mathbf{R}:$ Je vais aux toilettes et je reviens.

\section{(Pause)}

Q: Ce matin, nous avons évoqué les évènements qui se sont déroulés autour des mois de juin et juillet 2008, puis vers décembre 2008, avec le tsunami de Jôgan, qui a remis en question les défenses anti-tsunami. J'ai cru comprendre qu'à chaque fois qu'un élément nouveau se présentait, vous en faisiez tout de suite part à vos supérieurs. Nous venons de voir ce qui s'est passé avec la NISA entre août et septembre 2009, mais vous ne semblez pas en avoir gardé grand souvenir. Je sais que vous étiez plus occupé par les problèmes de Kashiwazaki, mais dans le courant de l'année 2009, par exemple, avez-vous eu des échanges à propos du problème des tsunamis à Fukushima Daiichi, avec vos supérieurs directs, Messieurs Mutô et Takekuro, ou même avec le Président ou le Directeur général ?

$\mathbf{R}$ : Non, pas du tout. Vous savez, à l'époque, la question était encore à l'étude à l'Electric Technology Research Association et ailleurs. S'il y avait eu de nouvelles avancées, j'en aurais bien évidemment averti mes supérieurs. Mais là, on était encore en pleine étude et je ne me rappelle pas avoir fait de rapports supplémentaires sur le sujet.

$\mathbf{Q}:$ À la limite, on ne vous en a pas demandé non plus.

$\mathbf{R}:$ Non.

Q : Très bien. Une fois que vous vous êtes retrouvés en 2010, vous avez eu ces histoires avec l'utilisation du MOX, mais vous ne vous souvenez pas avoir remarqué qu'on ait plus particulièrement parlé du tsunami de Jôgan ou de tsunami tout court à Fukushima Daiichi, dans ce contexte.

$\mathbf{R}$ : Non plus.

$\mathbf{Q}$ : Vous n'avez pas non plus souvenir de vous être entretenu avec vos supérieurs à ce propos. C'est bien ça?

$\mathbf{R}$ : C'est ça. S'il y a eu discussion, ça n'était pas tant autour du tsunami de Jôgan que dans le cadre d'une réflexion plus large. Et ceci était une démarche normale aussi bien pour nous que pour le ministère et les autres exploitants. On discute d'abord du 
bien-fondé de l'évaluation de la secousse sismique. On donne donc la priorité à l'étude des failles, à l'estimation du tremblement qui peut secouer la centrale. Une fois que ces études ont été faites et validées, on effectue, comme vous dites, l'estimation de phénomènes consécutifs à cette secousse, comme le tsunami. C'est la démarche habituelle. À cette étape-là des études, l'intérêt est focalisé sur la secousse sismique et les travaux à entreprendre pour y répondre. L'essentiel se trouve être la discussion sur le séisme. Le tsunami est laissé de côté, comme un phénomène dont on rendra compte dans le rapport final, en pensant, justement, qu'entretemps les experts de la JSCE auront rendu leurs conclusions. Si, à ce moment-là, des travaux s'imposaient, on les aurait inclus dans ce rapport final. C'est à peu de choses près le parcours que nous avons suivi. Sans oublier qu'à cette époque-là, le ministère luimême était encore essentiellement focalisé sur les séismes.

Q: J'ai bien compris. Je vous remercie pour ces explications.

R : S'agissant du ministère, je suppose qu'ils vous ont dit, pour se dédouaner, qu'ils s'intéressaient déjà aux tsunamis.

$\mathbf{Q}$ : Oui, on dirait bien. Mais il y a un tel décalage entre leurs paroles et leurs actes que nous ne sommes pas dupes.

Ce dont je voudrais vous parler maintenant concerne vos rapports avec Monsieur Takekuro. En juin 2010, tout juste avant que vous ne quittiez vos fonctions au siège, a eu lieu, comme tous les mois de juin, la réunion annuelle des actionnaires pour l'exercice 2009. Dans les documents conservés par Monsieur Takekuro à cette occasion, il y a plusieurs allusions au tsunami de Jôgan. II y a des questions concernant l'estimation publiée par le département de Fukushima des hauteurs de tsunami, on y trouve même l'expression «tsunami géant d'après de nouvelles découvertes ». Je sais que vous étiez vraiment sur le départ et que vous avez dû brasser pas mal d'affaires à cette période, mais vous souvenezvous avoir eu des échanges à ce propos avec Monsieur Takekuro?

$\mathbf{R}$ : Il faut que je vous explique comment se prépare une réunion des actionnaires dans notre entreprise. Tous les ans, on prévoit des questionnaires types avec les réponses adéquates à l'intention des dirigeants qui vont être amenés à intervenir. Ce travail est entamé bien avant la réunion. Cette année-là aussi, on a dû débuter probablement en février ou en mars. On commence par faire une liste de questions qui pourraient être posées. On affine ensuite avec les questions qui nous parviennent de différents actionnaires avant la réunion. Cet ajustement se fait juste avant la réunion. En fait, on se base sur les questions-réponses qui ont été prévues pour la réunion des actionnaires de l'année précédente. Comme il s'est passé des choses durant l'année écoulée, on essaie de refléter ces progrès et ces changements. C'est le travail que nous effectuons en février ou en mars. On rajoute donc des questions à la version de l'année précédente. Les services concernés fournissent les réponses. On soumet le tout à Takekuro, par exemple. S'il y a des choses qu'il ne comprend pas, on lui explique. Souvent, on est obligé de rajouter des questions, on discute longuement des réponses à apporter. C'est un travail très fastidieux. Mais en tant que directeur concerné, j'étais bien obligé d'avoir une idée de l'ensemble et de donner des ordres aux uns et aux autres pour que chacun prépare sa part de réponse. C'était un travail sans fin, à faire remanier les passages difficiles à comprendre, à se demander si on n'allait pas aussi nous poser telle question, à suggérer d'alléger certaines réponses, à éliminer certaines rubriques, jusqu'à la date fatidique. Donc, en tant que directeur responsable, j'étais tout naturellement au courant de tout ce qui s'y trouvait. 
$\mathbf{Q}$ : Durant l'élaboration de ces questions-réponses, vous avez eu l'occasion d'expliquer les choses à Monsieur Takekuro.

$\mathbf{R}$ : Bien sûr. Ce sont des documents qui ont été élaborés en vue de la réunion des actionnaires, mais surtout spécialement à l'intention et à l'usage de Takekuro.

$\mathbf{Q}$ : Très bien. J'ai bien compris.

$\mathbf{R}:$ C'est assez facile à imaginer, vous ne trouvez pas ?

$\mathbf{Q}$ : Oui, on visualise facilement et c'est intéressant. Nous, qui travaillons pour l'État, ne connaissons pas très bien ce qui se fait concrètement dans ces cas-là. Vous m'apprenez des choses. Donc, le tsunami de Jôgan est évoqué ici à la suite de la publication de modèles de point de formation du tsunami basés sur les résultats de recherche sur les sédiments, je pense qu'il s'agit de ce qu'on appelle la thèse Satake. Il y est question de deux modèles qui pourraient expliquer la répartition des dépôts sédimentaires, mais aucun n'est préféré. Votre attitude à vous est de dire que vous restez attentifs aux études et aux progrès à venir et que vous êtes prêts à entreprendre des travaux si nécessaire. Je suppose que Monsieur Takekuro était tout à fait au courant de tout ça dès le départ et que ce texte a été rédigé en conséquence.

R : Oui.

$\mathbf{Q}$ : Il n'est pas possible qu'il ait eu connaissance du problème pour la première fois en lisant ce texte?

$\mathbf{R}$ : Non. Ce n'est pas possible.

$\mathbf{Q}$ : Très bien.

Maintenant la question va concerner la période où vous aviez déjà quitté votre poste au siège. Ce que je voudrais savoir, c'est si, avant de partir, vous aviez semé les graines qui ont abouti à ce qui s'est passé par la suite. Dans le cadre du Centre de gestion du séisme au large de Chûetsu, a été constitué, en août 2010, un groupe de travail sur les réponses à apporter aux tsunamis au large de Fukushima. Êtes-vous à l'origine de ce groupe? Avezvous tenu des discours du genre "je m'en vais, mais je vous confie cette mission indispensable »? En avez-vous souvenir?

$\mathbf{R}$ : Je n'ai pas le souvenir d'avoir ordonné la création de ce groupe de travail.

$\mathbf{Q}$ : Ah, bon. C'est que les nouveaux qui sont arrivés après vous ont dû se mettre au travail, en pensant que si les conclusions devaient être rendues en 2012, il était temps de commencer à cogiter sur ce problème. Avant de quitter votre poste, n'aviez-vous pas ordonné de commencer à y réfléchir?

$\mathbf{R}$ : Je n'ai pas ordonné concrètement la formation d'un groupe de travail, mais ce que nous ressentions tous, à cette époque, c'était une certaine hâte à entamer ce travail. Le ministère n'avait pas besoin de nous presser. Nous-mêmes étions pressés de faire avancer les choses. Du coup, nous avions botté les fesses aux fabricants, qui, prétextant le manque de personnel, nous donnaient des échéances à l'horizon 2015. En leur mettant la pression et en suggérant ceci et cela, nous avions réussi à réduire l'écart. Quand je suis parti, on parlait de fin 2012, au pire mars 2013. Nous étions pressés. De toute manière, la date de remise du rapport était fixée. Ce rapport devait non seulement concerner les calculs des structures antisismiques, mais comprendre en plus tous les phénomènes consécutifs au séisme. Alors j'avais toujours dit qu'il fallait œuvrer en ayant en tête ce défi. Le rapport final ne consistait pas à se contenter de rendre compte des calculs de résistance au séisme, il fallait prendre en charge tous les épiphénomènes. Et cela ne pouvait pas se faire en un clin d'œil. Il fallait le préparer bien en amont. C'est une chose que j'avais toujours dite. 
Maintenant, je ne me souviens pas avoir donné des directives particulières pour la constitution de ce groupe de travail.

$\mathbf{Q}$ : Toutes les personnes que j'ai interrogées m'ont dit qu'elles avaient commencé ce travail, tout en sachant qu'il n'y avait aucune visibilité sur les conclusions qui seraient livrées par la JSCE, qu'elles-mêmes se demandaient encore si vraiment un tsunami de cette ampleur pouvait vraiment venir, qu'elles se disaient bien que même si cela restait théorique et une gymnastique de l'esprit, elles devaient s'atteler à ce problème. Vous aussi, vous aviez donné vos directives en pensant que ces réflexions, qui pouvaient ne rester qu'une gymnastique du cerveau, étaient pourtant indispensables?

$\mathbf{R}$ : Oui, ce qui devait être fait devait l'être.

$\mathbf{Q}$ : Très bien. Même si vous n'aviez pas évoqué concrètement la formation d'un groupe de travail, vous aviez bien formulé votre ligne de conduite. Et à la suite de cela, on peut penser que les choses se sont concrétisées et ont évolué après votre départ.

$\mathbf{R}$ : Je suppose.

$\mathbf{Q}:$ C'est la première fois que vous entendez parler de ce groupe de travail ?

$\mathbf{R}$ : Oui, la première fois.

$\mathbf{Q}$ : Ah, bon. En fait, j'en ai aussi parlé à Monsieur XXXXX.

R : Oui, XXXXX était mon successeur.

$\mathbf{Q}$ : De chef de centre, il a repris votre poste. Quand je lui ai parlé de ce groupe de travail, il n'était pas non plus au courant. Du coup, j'ai pensé que c'était peut-être Monsieur XXXXX, qui avait l'air très motivé, qui avait pris l'initiative. De toute manière, cela faisait déjà longtemps que vous étiez tous conscients que la réflexion sur les tsunamis devait se faire. Ce n'est pas une idée qui a germé tout à coup à cette période.

$\mathbf{R}:$ Non.

$\mathbf{Q}:$ En tant que théorie générale.

$\mathbf{R}$ : Pas en tant que théorie générale, mais en tant qu'étude particulière.

$\mathbf{Q}$ : Très bien. Bien que cela ne préjuge pas des mesures définitives, il semblerait qu'à cette époque vous penchiez pour des solutions associant la résistance des installations au séisme, en travaillant sur l'étanchéité des moteurs de pompes, qui n'aurait bien entendu pas tenu seule face à la force d'un tsunami, à des mesures architecturales, en construisant par exemple un bâtiment ou une digue, soit pour dévier la vague, soit pour la contenir. J'ai l'impression que chaque service, que ce soit les études de Génie civil ou les constructions antisismiques, envisageait des solutions chacun de son côté, mais qu'il y avait peu d'occasions de concertation entre eux pour savoir ce que chacun faisait.

$\mathbf{R}$ : Jusqu'à ce moment-là, vous voulez dire?

Q : Oui.

$\mathbf{R}$ : En fait, tout revient toujours à cette histoire de hauteur du tsunami. Parce que rien ne peut se décider sans indication de sa hauteur. Pour ma part, je me dis aujourd'hui que même si on avait pris des dispositions pour contrer un tsunami de 10 $\mathrm{m}$ de haut, avec ce que nous avons connu, qui est monté à $15 \mathrm{~m}$, on n'aurait pas pu faire face. Même une vague de 7 à $8 \mathrm{~m}$ aurait fait monter le niveau d'eau. Même dans ce cas-là, les pompes, les moteurs auraient été inondés. Mais ce qui est le plus important avec les tsunamis, c'est qu'il y a des flux et des reflux. La vague arrive, puis se retire en emportant toute l'eau. Si les installations ont résisté à cette séquence, elles restent opérationnelles quand l'eau reviendra. C'est ça le plus important, il faut et il suffit que les installations soient opérationnelles quand l'eau revient. Pour cela, il faut que les pompes à eau de mer et les moteurs soient étanches et que l'alimentation 
électrique suive. Même si les installations sont à l'arrêt dans l'intervalle, au bout de la deuxième ou troisième vague, l'eau va revenir à la normale. Et si, à ce moment-là, les installations sont capables de fonctionner, on pourra faire face. C'est le genre de choses auxquelles les ingénieurs «moteurs » et «instruments » doivent réfléchir sérieusement.

Bien sûr, s'agissant de la vague que nous avons connue, qui a fait sauter toutes les pompes, d'autres mesures complètement différentes s'imposaient. Ce qui prouve bien qu'il est très difficile de discuter de réponses sans avoir de précisions sur le tsunami en question. Si on envisage un tsunami de $6 \mathrm{~m}$ et quelques, même s'il atteint $7 \mathrm{~m}$, je pense qu'on pourrait s'en sortir en améliorant l'étanchéité et des choses de ce genre. Mais si c'est un tsunami de $15 \mathrm{~m}$, quoi qu'on fasse, sa force sera telle qu'il emportera tout. Même si on avait voulu s'en protéger, il y a des hauteurs où toute protection perd son sens. En fait, on a beau envisager des solutions, tant qu'on n'a pas d'indication nous précisant la hauteur du tsunami de référence pour nous servir de modèle, elles ne peuvent être des solutions sérieuses.

$\mathbf{Q}$ : Je pense que dans les ministères la situation est pire. Tout est cloisonné verticalement. C'est comme si on était chacun dans un piège à poulpe, sans vue d'ensemble. C'est pourquoi je me suis demandé si ce n'était pas une prise de conscience du besoin d'adopter un point de vue plus large, par-delà les cloisonnements, qui avait incité à la création de ce groupe de travail.

$\mathbf{R}$ : Effectivement, c'est ce qui a motivé le fait de regrouper dans un seul lieu les différents services concernés par les réponses antisismiques. Dans notre entreprise aussi, la tendance au cloisonnement vertical est très forte. Cela obstrue la circulation horizontale des informations. On a donc regroupé les gens des différents services, " mécaniciens ", « électriciens ", " génie civil », « construction », à la suite du séisme de 2007. Mais, comme vous dites, la tendance au cloisonnement vertical était encore forte, chacun voulant rester dans son pré carré et c'est là que j'ai voulu casser ce penchant naturel en obligeant les gens de spécialités différentes à travailler ensemble, notamment autour de la question du tsunami. Mais, comme je vous l'ai dit, pour un ingénieur "machines», il est difficile, voire impossible, de concevoir quelque chose sans savoir à quelle hauteur de tsunami sa machine doit résister. En attendant, je leur ai dit de réfléchir à des moteurs et des pompes qui continuent à fonctionner même inondés.

$\mathbf{Q}$ : C'est ce que vous leur avez concrètement dit?

$\mathbf{R}:$ Oui, tout à fait.

$\mathbf{Q}$ : En effet, si on regarde les archives, le problème de l'étanchéité est un de ceux qui ont été examinés les premiers et auxquels vous aviez le plus travaillé.

R : A ce moment-là, assez tôt donc, j'avais suggéré que les ingénieurs "machine » travaillent à ce qu'ils pouvaient faire de ce côté-là. Évidemment, nous ne savions pas qu'il pourrait y avoir un tsunami tel qu'il emporte tout, jusqu'à la dernière pompe.

$\mathbf{Q}$ : Pourquoi, au moment où les ingénieurs «machine» s'activent sur ces problèmes d'étanchéité des équipements, les ingénieurs « construction » ou « travaux » ne bougent-ils pas autant?

$\mathbf{R}:$ Je ne pense pas qu'ils ne voulaient pas bouger. On revient à l'histoire de tout à l'heure. Il leur était tout simplement difficile de passer à la conception. 
$\mathbf{Q}$ : Vous voulez dire que sans connaître les contraintes de départ, ils ne pouvaient pas bouger?

R : Oui. Sans cela, ils ne pouvaient pas bouger. Ce que je veux dire, c'est que quelle que soit la conception qui serait adoptée in fine, il y avait des risques que les machines prennent l'eau. Qu'on conçoive pour une vague de $5 \mathrm{~m}$ et quelques ou $6 \mathrm{~m}$, si la vague atteint $6,5 \mathrm{~m}$ ou $7 \mathrm{~m}$, quelle que soit la force du séisme, tout ça va être inondé et devenir inutilisable. J'avais donc commencé par charger les ingénieurs " machine » de réfléchir à la manière de maintenir les machines fonctionnelles même inondées. Ça, c'est du ressort des ingénieurs « machine ».

$\mathbf{a}$ : Vous voulez dire que, même si on n'a pas déterminé la hauteur de la vague, il est possible de concevoir des machines qui continuent à fonctionner même si elles ont été inondées.

$\mathbf{R}:$ C'est tout à fait ça.

$\mathbf{Q}$ : Mais, concernant les ingénieurs «construction » ou «travaux », tant qu'on ne détermine pas la hauteur de la vague, il ne leur est pas possible de bouger. Leur cas est différent. C'est ça?

$\mathbf{R}$ : Oui, exactement.

$\mathbf{Q}$ : Très bien. J'ai bien compris.

Si nous continuons, nous allons arriver à cette année et aux évènements du 11 mars. Mais avant ça, le 7 mars, le Group Manager XXXXX et quelques autres personnes sont allés à la NISA pour expliquer l'état d'avancement des travaux concernant les tsunamis à Fukushima Daiichi. Etiez-vous au courant?

R : Non, je ne l'ai su qu'après les évènements. Sur le moment, je n'en ai rien su.

$\mathbf{Q}$ : Vous ne le saviez pas du tout. D'accord.

Et là, vous êtes muté, vous arrivez à Fukushima. Toutes ces histoires concernant la centrale deviennent tout à coup très concrètes, puisque tout est là sous vos yeux. Quel était votre ressenti au moment où vous êtes arrivé ? Vous vous êtes dit «allez, on y va! »?

$\mathbf{R}:$ J'y allais à reculons.

$\mathbf{Q}:$ Vraiment ? C'est ce que vous ressentiez?

$\mathbf{R}$ : Vous comprenez, ils voulaient passer au MOX. Franchement, ça me cassait les pieds. Tout le secteur énergie est à la même enseigne, mais on est toujours coincé entre les autorités locales, la NISA et d'autres organismes, on est broyé. Encore, s'il s'agissait de discussions techniques. Mais non. C'est " je veux faire ci », "je veux faire ça ", "je vous ordonne de faire ci». Les techniciens se trouvent complètement noyés sous des discussions totalement stériles. Dans cette ambiance, ils voulaient introduire le MOX. Je me disais que j'allais au-devant d'une montagne de tracas à me retrouver à la tête de cette centrale, et je n'ai pas été déçu. Il fallait organiser des tas de réunions d'information avec les locaux, le département, tout ça. En plus, c'était la quatrième fois que j'étais nommé à Fukushima Daiichi. J'avais conscience du vieillissement de la centrale. Différents travaux de rénovation s'imposaient. Le menu concernant ces travaux était nettement plus chargé que pour d'autres centrales. Il fallait les assumer. Ça aussi, c'était casse pied. L'ambiance générale n'était donc pas à l'enthousiasme.

$\mathbf{Q}$ : D'accord. Encore une fois une question qui s'impose rétrospectivement au vu des évènements qui ont suivi : au moment de votre arrivée à Fukushima, avez-vous donné des 
directives particulières concernant les tsunamis, puisque lors de votre passage au siège il en avait été question?

$\mathbf{R}$ : Là, je faisais totalement confiance au département " gestion des installations » du siège.

$\mathbf{Q}$ : Vous leur laissiez l'initiative, quitte à entreprendre des travaux si nécessaire. C'est ça?

R : Oui, s'il s'avérait qu'il fallait faire des travaux, j'étais prêt à les faire. Mais, vous savez, une fois arrivé à Fukushima, il y avait cette histoire de MOX qui m'attendait. J'ai été littéralement happé par les réunions, les explications à fournir, tout ça, et je n'ai plus eu le temps de me préoccuper des phénomènes consécutifs au séisme. En plus, en cette période cruciale, il y a eu plusieurs incidents à la centrale. Déjà, le 20 juin, là c'était encore Komori qui était le directeur de la centrale, on était tous les deux à la préfecture de Fukushima en visite de courtoisie, quand, au beau milieu des discussions, le réacteur 2 s'est mis en SCRAM. Officiellement, je n'étais pas encore le directeur, alors j'ai souhaité bon courage à Komori. Ensuite, une fois que je me suis retrouvé à sa place, avec cette histoire de MOX, il a fallu organiser des visites sur le site, comme si on n'avait que ça à faire. Au milieu de tout ça, vers début septembre, cette fois-ci, il y a eu l'incident de l'erreur de raccordement du réacteur 5 , c'était une erreur de conduite. Le pilote avait confondu le RCIC du réacteur 5 et du réacteur 6 . L'incident s'est su et évidemment ça a eu des effets sur le MOX. Etait-il possible d'introduire un nouveau combustible au sein d'une centrale qui n'arrête pas de cumuler les incidents? J'aurais bien voulu dire "dans ce cas-là, abandonnons le projet!». Mais, forcément, je ne le pouvais pas. Au contraire, j'étais obligé d'aller présenter mes plus plates excuses un peu partout, y compris au département, en me faisant traiter de tous les noms. Et ça a continué comme ça. Je me coltinais toutes les corvées. J'étais littéralement moulu par le MOX. Même au moment de la mise en service du réacteur 3 , il y a eu quelques incidents qui ont fait beaucoup de bruit. Franchement, j'en avais marre et j'avais envie de démissionner au plus vite. D'ailleurs, j'aurais dû démissionner à ce moment-là.

$\mathbf{Q}:$ Vraiment?

$\mathbf{R}$ : Vous voyez qu'avec tout ça je n'étais absolument pas en état de me préoccuper de phénomènes connexes.

\section{$\mathbf{Q}$ : Effectivement, vous n'étiez pas en état.}

$\mathbf{R}$ : De plus, si vous permettez, en tant que responsable de la centrale, j'étais pressé d'entreprendre au plus vite les travaux de renforcement antisismique plutôt que de m'intéresser aux phénomènes connexes. Car c'était là ma mission. L'examen des phénomènes connexes et leurs calculs étaient du domaine des services du siège. Mais les travaux étaient de ma responsabilité. Je n'arrêtais pas de me demander comment faire pour achever ces travaux au plus vite. Fallait-il revoir la conception? Que pouvait-on faire? Du coup, je n'avais pas l'œil sur les épiphénomènes. Eux étaient du ressort du siège.

$\mathbf{Q}$ : Très bien. Donc, après votre arrivée à Fukushima, vous n'avez plus eu beaucoup de rapport avec ces histoires de tsunami.

Mais au moment où vous avez quitté le siège, avez-vous laissé des consignes spéciales concernant Fukushima Daiichi à votre successeur?

$\mathbf{R}$ : Avec XXXXX, nous travaillions ensemble sur les activités du Centre de gestion du séisme de Chûetsu. Je n'ai donc pas eu à lui laisser de consignes particulières. 
$\mathbf{Q}:$ : Il était déjà suffisamment au courant et n'avait pas besoin de consignes.

R : Oui, je lui ai juste dit de continuer. Par contre, en dehors de ça, il y avait quelques gros dossiers sur lesquels je me suis plus attardé. Il y avait de nouvelles implantations à gérer. Un des gros dossiers était celui de l'implantation des réacteurs 7 et 8 à Fukushima Daiichi ${ }^{43}$. Ensuite, il y avait aussi le dossier "Higashidôri », où on allait commencer la construction du réacteur $1^{44}$. Il fallait aussi gérer le projet du réacteur 2 qui allait suivre. Là, j'ai pris mon temps. Mais le Centre de gestion, il le connaissait déjà bien. Il suffisait qu'il continue.

$\mathbf{Q}$ : D'accord. Pour en revenir à ce que vous m'avez raconté à propos du RCIC des réacteurs 5 et 6 , que s'était-il passé ?

$\mathbf{R}:$ Je ne me rappelle plus bien lequel était lequel. Est-ce le réacteur 6 qui était à l'arrêt et le 5 en fonctionnement?

$\mathbf{Q}:$ Cette histoire se situe quand?

R : L'année dernière. Je venais d'arriver. Ça a dû se passer début septembre. Vous savez que les réacteurs 5 et 6 sont voisins. Le RCIC est un système de refroidissement qui intervient quand on isole le cœur du réacteur. Chaque réacteur dispose du système. Donc, aussi bien le réacteur 5 que le 6 dispose du sien. Je ne me rappelle plus lequel était en fonctionnement et lequel était à l'arrêt. Disons que c'était le réacteur 5 en fonctionnement et le 6 à l'arrêt pour vérification périodique.

$\mathbf{Q}$ : Vous voulez dire qu'il y en avait un en fonctionnement et l'autre à l'arrêt.

$\mathbf{R}$ : Oui. Pour toute tranche en exploitation, on effectue une fois par mois ce qu'on appelle un test de surveillance sur le RCIC. On le fait fonctionner pour de vrai. On ne l'introduit pas dans la cuve pour de bon, mais on fait des calculs à partir de là où on l'introduit dans la cuve, sur la test line ${ }^{45}$.

$\mathbf{Q}:$ La test line?

$\mathbf{R}$ : Oui, la test line. On fait donc ce test où on le fait tourner pour de bon. Or, quand on a effectué ce test de surveillance sur le RCIC, il n'a pas fonctionné. Cette situation, " échec de la mise en œuvre du RCIC lors du contrôle des systèmes ECCS ", fait partie des situations hors LCO (Limiting Condition of Operation) ${ }^{46}$ qui imposent une déclaration en bonne et due forme auprès des autorités compétentes. Donc, déclaration, rapport. Après recherches, on s'est aperçu que l'un des câbles, sur le circuit, n'était pas raccordé au tableau. La raison en était que lors de l'inspection du réacteur 6, il y a eu une phase où il fallait détacher ce câble. Or les tableaux des deux réacteurs se ressemblent et ils se situent dans le même secteur, bien que ce soit à des étages différents. Du coup, l'agent parti détacher le câble du réacteur 6 s'est trompé, a confondu le tableau du 5 et du 6, et, au lieu de détacher le câble du réacteur au repos, a détaché celui de la tranche en exploitation. Et personne ne s'en était rendu compte. Parce que la surveillance sur la tranche en exploitation ne se fait qu'une fois par mois. Il n'y a pas eu d'autre contrôle entretemps. Du coup, ce qui était bon au contrôle du mois d'août, n'était plus bon en septembre. Le câble n'était pas relié. Et il n'était pas relié parce qu'un agent avait effectué la manœuvre prévue sur le réacteur 6, à l'arrêt en inspection programmée en août, sur le réacteur 5 . Vous imaginez bien l'accueil que j'ai reçu, un passage à tabac... Des reproches, comme il en pleuvait.

$\mathbf{Q}:$ C'est une des entreprises partenaires qui avait fait l'erreur?

$\mathbf{R}$ : Non, pas du tout. C'est une manœuvre qui était destinée à être exécutée par un de nos agents de conduite. Du coup, il n'y avait pas d'autre fautif que TEPCO. On ne 
pouvait accuser personne d'autre. Alors, évidemment, tout le monde s'est déchaîné contre nous, disant qu'on n'était pas assez impliqué, alors qu'on allait introduire le MOX. Injures et insultes.

Q: D'accord. Je crois que j'en ai à peu près fini avec le tsunami.

Je voudrais maintenant vous interroger plus généralement sur la gestion de crise. II y a eu, au sein de TEPCO un document datant de 1994 rapportant des réflexions à ce sujet suivi du fameux Rapport sur les dispositions pour la gestion des accidents de 2002. Je sais que vous en avez déjà parlé avec Katô47 . Je voudrais revenir dessus en donnant un éclairage plus spécifique du fait que l'accident de mars a pour cause une catastrophe naturelle. Savoir quelles étaient les mesures prévues face à un accident grave résultant d'une catastrophe naturelle. Après ça, nous ferons une pause.

Avant de venir vous voir pour cette audition, j'ai rencontré beaucoup de personnes, à commencer par Monsieur Mutô, Monsieur XXXXX, le personnel du Centre de gestion du séisme de Chûetsu, pratiquement toutes les personnes du grade de GM. Aujourd'hui, vous occupez les fonctions de directeur de la centrale de Fukushima Daiichi, mais je voudrais vous interroger en tant qu'ancien directeur du département " gestion des installations ». À toutes les personnes auditionnées, j'ai posé la question suivante: quelles étaient les mesures prises par TEPCO pour protéger les installations nucléaires des catastrophes naturelles? Tout le monde m'a répondu d'une même voix qu'il existait d'abord des directives pour l'évaluation d'une construction sécurisée vis-à-vis des catastrophes naturelles, que, concernant plus spécifiquement les séismes et les phénomènes connexes, il y avait des directives pour l'évaluation d'une conception antisismique, que TEPCO tenait compte de ces indications et construisait en s'y conformant scrupuleusement. Concrètement, on imaginait toutes sortes de catastrophes naturelles et on vérifiait que les installations nucléaires étaient construites de manière à résister largement à ces scénarios. Concernant les tranches déjà existantes, des révisions étaient en cours pour voir si ces installations étaient capables de résister à ces catastrophes, notamment leur résistance aux séismes était testée. S'il s'avérait que des changements de normes s'imposaient, les travaux nécessaires étaient effectués afin de protéger les installations nucléaires des catastrophes naturelles. Voilà ce que tout le monde m'a dit. Êtes-vous d'accord avec ça ?

$\mathbf{R}:$ Oui, tout à fait.

Q: Ma deuxième question portait sur l'imprévu, ce qui s'est effectivement passé en mars. Je leur ai demandé s'ils n'avaient pas pensé aux réponses à apporter à des accidents graves qui dépassent les différents scénarios qui avaient pu être imaginés et s'ils ne s'y étaient pas préparés. Et tout le monde m'a répondu que non. En fait, ils m'ont dit que s'il fallait imaginer une catastrophe de si grande envergure, il aurait fallu carrément changer les conditions de construction. Monsieur Mutô était aussi de cet avis. Et vous, qu'en pensezvous?

$\mathbf{R}$ : À la base, je pense comme eux. Seulement, j'ai roulé ma bosse un peu partout. En effet, j'ai été à la tête du département " gestion des installations ». J'ai aussi été à la tête de la "construction». Le poste de directeur du département "gestion des installations » est dans le prolongement du travail d'un directeur de la « construction des installations nucléaires ", qui consiste à la base à réfléchir aux plans. Réfléchir aux plans, cela signifie, comment remplir au mieux les conditions que vous avez évoquées tout à l'heure. Ça, c'est le travail de la «construction ». À côté de ça, pour ma part, j'ai encore plus fréquenté des postes où on s'occupe de conduite, de sûreté. J'ai une plus longue expérience de tranches effectivement en fonctionnement. Du coup, j'ai peut-être une attitude un peu différente. Je pense qu'on ne peut jamais prévoir parfaitement ce qui va arriver. Rappelez-vous l'histoire du gars qui s'était trompé entre le réacteur 5 et le réacteur 6 . Mais lorsqu'on fait effectivement tourner la machine, on se débrouille pour que la tranche ne se trouve pas en danger. C'est 
pour ça qu'on fait des tests de surveillance. Même si le RCIC ne fonctionne pas, il peut être soutenu par le HPCI. Ça, c'est prévu à la base dans la conception. Mais mon ressenti personnel est quand même que tout peut arriver en ce bas monde. Je ne le dis pas maintenant parce que j'ai vécu le traumatisme du tsunami. Même bien avant, je le pensais. Les gens qui ont toujours été dans la construction vous répondront toujours « directives et compagnie ». C'est la bonne réponse, la réponse modèle. Mais moi, personnellement, malgré tout, j'ai toujours eu le sentiment qu'il y avait beaucoup de choses de par le monde qui ne marchent pas comme on l'avait prévu.

$\mathbf{Q}$ : La réponse que vous venez de me donner est somme toute assez proche dans le fond de celle que j'ai évoquée tout à l'heure.

$\mathbf{R}$ : C'est normal. C'est la manière dont le monde du nucléaire a toujours raisonné et je n'ai pas l'intention de lui tourner le dos. Je pense que tous ceux qui vous ont répondu de cette façon ont raison. Seulement, quand on va réellement sur le terrain et qu'on l'observe, on se rend compte qu'il y a aussi des choses qu'on ne peut pas évacuer comme ça d'un trait. Il est vrai que jusqu'à présent ces cas étaient assez rares, mais quand on a fait l'expérience d'incidents, on devient moins péremptoire. J'en ai parlé lors d'une audition précédente à Monsieur Katô, mais l'incident qu'on a connu en 1991 sur la tranche 1 de Fukushima Daiichi, par exemple, est troublant ${ }^{48}$. Cette inondation, qui l'avait imaginée ? Vous vous rendez compte qu'avec cette inondation, pratiquement tout le réseau de refroidissement était mort? Le générateur diesel aussi avait pris l'eau et ne fonctionnait plus. Je continue à penser même aujourd'hui que c'était un incident très grave, un des incidents les plus dangereux survenus au Japon, mis à part ce qui s'est passé cette année. Or, le traitement qu'on en a fait ne le laisse pas soupçonner. Pour ma part, j'ai mesuré à cette occasion toute la menace que pouvait représenter l'eau. L'eau est redoutable. Alors je sentais bien, par exemple, qu'il restait encore des choses à faire pour se prémunir contre les inondations. Mais sur des tranches déjà anciennes c'est compliqué parce qu'il faut défaire ce qui a déjà été fait. Bien sûr, nous avons fait des choses, entrepris des travaux de réfection. Mais mon ressenti est qu'il est difficile de remédier à tout à la perfection, de plus ça demande beaucoup d'argent. Alors en théorie, on sait ce qu'il en est, mais quand il s'agit de mettre tout ça en œuvre concrètement, les choses sont compliquées. Voilà ce que j'en pense.

$\mathbf{Q}$ : Très bien. Encore une question que je pose à tout le monde. En supposant que cet axe représente le temps et celui-ci les conditions de construction, par exemple, au début, au moment où vous soumettez le projet au permis de construire, vous présupposez toutes sortes d'incidents naturels et vous définissez les conditions à remplir pour vous prémunir contre eux. Vous vous situez ici. Ce faisant, une nouvelle donnée A est connue. Concrètement, dans votre cas, une révision des techniques d'évaluation des tsunamis par la JSCE était attendue pour octobre 2012. II est plus que probable que, à la suite de cette révision, les conditions se seraient durcies. Même si vous ne pouviez y répondre dans l'immédiat, des travaux seraient devenus indispensables. Au moment où vous auriez achevé ces travaux, vous seriez situé à ce point-là. Durant ce laps de temps, entre le moment où les nouvelles normes sont rendues publiques et l'achèvement de tous les travaux de mise aux normes, quelles mesures prenez-vous?

R : Votre schéma reflète une situation idéale. Si on suppose qu'il va y avoir une séance plénière de la JSCE en octobre 2012, c'est que la conclusion de l'assemblée va pencher vers A ou B. Ce sera $10 \mathrm{~m}$, ou bien va-t-elle se contenter de 5 ou $6 \mathrm{~m}$, ou bien encore va-t-elle monter à $15 \mathrm{~m}$ ? Probablement, environ un an avant que le verdict ne soit rendu, on commencera à deviner, à travers les discussions, la direction que 
l'assemblée va prendre. Cela veut dire que peut-être déjà à la fin de cette année ou au début de l'année prochaine on aurait vu vers quelle conclusion se dirigeait la JSCE. Donc, ce point sur votre schéma n'est pas atteint parce que la JSCE a rendu ses conclusions, mais il est atteint probablement avant même qu'elles ne soient rendues, parce que nous aurions été obligés de prendre les devants. Je pense que cela fait partie de notre savoir-faire, à nous autres, exploitants, de devancer ces décisions et d'être prêts à y répondre dès leur publication. On dit que la JSCE va rendre ses conclusions, mais avant il y a beaucoup de discussions. On connaît la manière dont elle fonctionne. Vers la fin, tout en sachant très bien que finalement ce sera tel verdict, les experts se consultent et finissent de se mettre d'accord. S'il s'avère que c'est quand même $15 \mathrm{~m}$, on commencera dès 2011 à se préparer à cette éventualité. Ce n'est pas pour revenir à l'histoire de tout à l'heure, mais on commencera à parler budget, à entamer des études, à mettre les dirigeants au parfum.

Mais ce n'est pas pour autant que, comme vous dites, les travaux seront achevés au moment où on connaîtra les nouvelles normes. Et vous voulez sans doute savoir ce que nous faisons à ce moment-là. C'est une question difficile. Si l'avis des experts est que, clairement, dans un avenir proche, c'est-à-dire dans les quelques années qui viennent, la probabilité qu'un tsunami de cette ampleur survienne est extrêmement élevée, tout ça dépend aussi de la PSA (Probabilistic Safety Assessment) ${ }^{49}$, mais enfin si cette probabilité est très forte, il faudra y faire face. Si ça n'est pas possible, je pense que par précaution on arrêterait les réacteurs.

Q: Monsieur Mutô m'a aussi dit que ce serait une décision à prendre en fonction des probabilités. II a dit, en l'occurrence, que si les conclusions de la JSCE étaient de nature à remettre en question tout ce qui avait été fait, il arrêterait les réacteurs. Mais que s'il s'agissait juste d'améliorer ceci ou cela, que ce qui avait été fait s'avérait finalement à peu près acceptable, il ne les arrêterait peut-être pas. À quelques nuances près, c'est ce qu'il m'a dit.

$\mathbf{R}$ : Ça ne m'étonne pas. Par exemple, si on nous dit que la hauteur de référence est de $6,5 \mathrm{~m}$, il y a des tranches pour qui c'est le cas, et que la vague ne dépasse pas cette limite, on pourrait s'en sortir par des manœuvres de conduite et, à ce moment-là, on n'arrêterait pas le réacteur. Mais si dix experts sur dix nous disent qu'il va y avoir un tsunami qui va largement dépasser les normes prévues et que ce tsunami est imminent, c'est sûr qu'on va arrêter les réacteurs. Mais je comprends qu'il puisse hésiter, parce que lui est un gestionnaire et que je ne suis pas sûr qu'il accepte de bon cœur qu'on ne produise plus d'électricité. C'est un gros problème. Je ne suis pas à sa place, mais, tout de même, si on sait de manière très évidente qu'un tel tsunami va venir, on ne peut qu'arrêter les réacteurs.

$\mathbf{Q}$ : Très bien. Vous allez peut-être trouver ça un peu naïf, mais comme moyen de combler ce vide, est-ce qu'on ne pourrait pas considérer des mesures de gestion des accidents, du genre des B.5.b50, pour le cas où, par exemple, il arriverait des catastrophes naturelles, même dans un cas comme celui-là où tout se retrouverait à l'arrêt? Je constate que vous avez eu beaucoup de mal à disposer d'une troupe suffisante pour faire face à l'accident. Est-ce qu'on ne pourrait pas imaginer en amont la manière de répondre à différentes situations, s'entraîner en conséquence? Est-ce que cela pourrait être une façon de combler ce vide? Qu'en pensez-vous ?

$\mathbf{R}$ : Je pense que vous avez raison. Ce que nous faisions, mis à part le cas du tsunami qui est un exemple limite, c'était d'imaginer ce qui pouvait arriver sous toutes sortes de conditions, prévoir les actions à mener, les noter dans des manuels, pour certaines 
choses, ajouter ou modifier des installations. Tout ça, c'est du domaine de la pratique de l'exploitation. Évidemment, ce qui est le plus efficace, face à des changements de normes, c'est de tout reconstruire à partir de zéro. Mais si on ne peut pas le faire, il faut essayer de compenser dans la pratique de l'exploitation.

Pour en revenir aux tsunamis, s'il y a un tsunami, la tranche va s'arrêter. Même si ce n'est pas une vague de $15 \mathrm{~m}$, il y a le problème de la prise d'eau de mer. Si elle n'est plus alimentée en eau au moment du reflux, on va arrêter la tranche automatiquement. Mais pour permettre cet arrêt automatique, il faut qu'on trouve une manière de l'arrêter qui ne provoque pas d'inconvénients par ailleurs. C'est après avoir examiné ce problème sous toutes ses coutures qu'on va pouvoir décrire la procédure à suivre, en disant "il faut surveiller ci et arrêter de telle manière ». En fait, dans la réalité, nous avons recours, en dehors des réponses purement architecturales, à ces actions qui s'inscrivent dans la pratique de l'exploitation.

Évidemment, le tsunami de mars, une vague de $15 \mathrm{~m}$, c'était quelque chose qui était au-delà de notre capacité d'imagination. Un manuel de gestion de crise, face à ça, parait ridicule. Je sais que ces derniers temps on se fait tabasser si on utilise l'expression « hors hypothèse ", mais on n'est plus dans un cas où des procédures ou quoi que ce soit de ce genre permettent de contourner le problème. On a dépassé tout ça. On était dans une zone où la réflexion, elle-même, était paralysée.

$\mathbf{Q}$ : Je comprends. Et ma question, que j'ai posée à toutes les personnes que j'ai auditionnées, est la suivante : ayant eu connaissance des révisions des Headquarters et de la thèse du tsunami de Jôgan, avez-vous pensé qu'il fallait combler ce vide? Tous ceux que j'ai interrogés m'ont répondu qu'étant donné que la survenue d'un tel tsunami semblait invraisemblable, ils n'ont pas pensé à en tenir compte. Et vous, quelle a été votre attitude?

$\mathbf{R}$ : Comme je vous le dis depuis tout à l'heure, c'était une histoire à tout changer structurellement. Alors, ce tsunami, était-il vraisemblable?

$\mathbf{Q}$ : Oui, vous vous êtes demandé s'il y avait quelque chose de sûr dans cette histoire.

R : Oui. Tant qu'il n'y avait rien de sûr, il m'était difficile de bouger. Aujourd'hui, après avoir vécu cette expérience terrible, je pense qu'on aurait dû faire quelque chose, mais ça, c'est un regret rétrospectif. Si je me remets dans le contexte d'avant le tsunami, j'attendais d'avoir une théorie approuvée par les instances officielles, j'attendais que les experts montrent la direction à prendre pour bouger. Bien sûr, ça ne signifiait pas que j'allais attendre octobre 2012 pour bouger. Dès qu'on aurait eu le soupçon d'une orientation, on se serait mis en marche dans ce sens. Parce que c'était là notre devoir.

$\mathbf{Q}:$ Très bien. Je vous remercie.

Je vais vous libérer maintenant pour une pause cigarette. Ensuite nous reprendrons sur la gestion des accidents, plus en général. Katô va se joindre à nous pour cette partie de l'audition.

$\mathbf{R}:$ On reprend vers $15 \mathrm{~h}$ ?

Q : Oui, c'est ça, vers $15 \mathrm{~h}$.

$\mathbf{R}$ : Très bien. 
$\mathbf{Q}$ : Je voudrais donc vous interroger plus avant sur la gestion des accidents. Je sais que Katô vous en a un peu parlé pendant l'été. Moi-même, de mon côté, j'ai eu l'occasion d'interroger Monsieur Mutô sur ce qui s'est passé depuis 1992. Je voudrais savoir si vous, durant vos séjours au siège, par exemple, vous aviez eu l'occasion de participer activement à l'élaboration de l'ensemble de ces mesures de gestion de crise au sein de TEPCO.

$\mathbf{R}$ : Non, pas du tout. Comme je vous l'ai dit tout à l'heure, j'étais plutôt du côté de la production, de la conduite, sur le terrain des tranches. Ce qui se passe en amont, comme la conception de la gestion de crise, est du domaine des départements impliqués dans la construction, pour faire court, c'est le job des « Messieurs sûreté ». Ceci est une chose. De plus, pendant quatre ans, entre 1995 et 1999, j'étais détaché à la Federation of Electric Power Companies of Japan. Je n'étais donc pas présent à TEPCO justement dans ces années où l'entreprise était en pleine réflexion sur les accidents sévères. Dans ce sens, je peux dire que ma contribution est vraiment proche de zéro dans cette élaboration.

$\mathbf{Q}$ : Il y a donc ce rapport de 2002 qui détaille la manière dont TEPCO a conçu la gestion des accidents. Quand on se penche dessus, on se rend compte qu'il n'y est fait à aucun moment allusion à une situation où, que la cause en soit une catastrophe naturelle ou pas, d'un seul coup, plusieurs tranches d'une centrale se trouvent victimes d'un accident grave. On dirait que les gens qui s'occupaient de sûreté n'y avaient pas pensé. Une des raisons de ce trou serait le fait que, lorsqu'ils imaginent ce qui pourrait être entrepris face à un certain évènement, ils ne pensent qu'à des incidents internes à la tranche, ils sont focalisés uniquement sur des problèmes spécifiques à chaque tranche. Quand ils réfléchissent aux conséquences d'un incident, par exemple dans le cas d'une perte d'alimentation électrique, on a l'impression qu'ils n'ont jamais envisagé le cas où la tranche qui est censé venir à la rescousse en partageant son électricité puisse être elle aussi à plat. Et vous, personnellement, n'avez-vous jamais pensé qu'il puisse arriver que plusieurs tranches tombent en panne simultanément? Ou était-ce une question qui ne vous a même pas effleuré?

R : C'est bien sûr une question de conception. Et dans la manière de penser la conception, chez nous, à la base, nous avons toujours donné la priorité aux phénomènes internes. Évidemment, dans mon cas personnel, depuis mon arrivée chez TEPCO jusqu'à maintenant, j'ai très peu eu l'occasion de toucher à ce domaine, mais je savais pertinemment que c'était la base de la conception dans le nucléaire. Maintenant, si vous me posez la question de savoir si, moi qui étais sur le terrain, du côté de l'exploitation, je n'avais jamais imaginé qu'une chose pareille puisse se produire, je vous dirais que, malheureusement, je n'y avais pas pensé avant le 11 mars.

$\mathbf{Q}$ : Là encore, c'est une question que j'ai posée à tout le monde, y compris Monsieur Mutô. Personne n'avait envisagé la possibilité que plusieurs tranches puissent tomber en panne simultanément. II ne s'agit ni de vous le reprocher ni quoi que ce soit d'autre, mais j'aimerais que tout ceci puisse servir de leçon pour le futur, à votre avis, qu'est-ce qui a fait que personne n'y a pensé ?

$\mathbf{R}$ : Il y a une première chose. Si on pense à des incidents simultanés, il y en a eu à Kashiwazaki, lors du séisme au large de Chûetsu. Ils sont survenus simultanément. Mais pour nous, les tranches s'étaient arrêtées. Les dégâts étaient importants, mais, tout compte fait, les réacteurs s'étaient arrêtés de manière sûre sans encombre. Du point de vue des "Messieurs sûreté ", quelle que soit l'étape suivante, du moment que le réacteur s'arrête correctement, l'essentiel est fait. Si vous vous mettez à leur place, « il y a eu un séisme très important, mais voyez comme le réacteur a bien réagi ", voilà ce qu'ils se disent. De plus, après contrôle, malgré un séisme dont 
l'ampleur dépassait largement ce qui avait été prévu à la conception, on s'est aperçu que presque toutes les installations, notamment celles liées à la sûreté, étaient indemnes. On peut dire qu'un séisme est capable en une seule secousse d'arrêter toutes les tranches. Mais cette fois-là cela s'est limité à l'arrêt des réacteurs, il n'y a pas eu d'autres incidents qui seraient venus se greffer dessus, comme la perte de toute source de refroidissement, par exemple. Le séisme n'a pas entraîné cela. Du coup, d'avoir vérifié cette fiabilité des installations face à un séisme qui dépassait de plusieurs fois les références qui avaient servi de base à la conception, cela a eu quelque part pour effet, à l'inverse, de rassurer les gens. Ils se sont dit, la conception à la japonaise est bonne, puisqu'elle a tenu le coup face à un séisme de cette ampleur.

$\mathbf{Q}$ : Vous voulez dire que ça les a confortés dans le fait que les mesures antisismiques étaient à la hauteur, les empêchant de penser jusqu'au tsunami qui, finalement, n'est qu'un épiphénomène? Que c'est ça qui les a empêché d'imaginer que plusieurs tranches pouvaient se retrouver simultanément à plat?

R : Oui.

Q: Dans le cas d'une perte d'alimentation électrique, il existe plusieurs systèmes permettant de continuer à assurer «l'arrêt, le refroidissement et le confinement ». Au niveau de Fukushima Daiichi, comment aviez-vous conçu la gestion de ce genre d'incident?

$\mathbf{R}$ : Dans le cas d'une perte d'alimentation électrique, ce qui fait le plus peur c'est la rupture des câbles de transport. Personnellement, j'avais peur qu'il n'arrive quelque chose à la station de transformation de Shin-Fukushima ${ }^{51}$.

$\mathbf{a}$ : Lorsqu'une perte d'alimentation de l'électricité externe survient, si on regarde les procédures de gestion d'accidents, on s'attend à ce que le générateur diesel d'urgence se mette en route. Si la perte d'alimentation est du fait d'une panne interne à une tranche, on s'attend à ce que sa voisine fournisse l'électricité nécessaire. J'ai beau tourner les directives dans tous les sens, ça part toujours du principe que le partage de la source électrique est possible. N'aviez-vous jamais imaginé que, dans un scénario plus noir, même cette seconde source se trouvât inopérante?

$\mathbf{R}$ : Je pense que c'est une question de probabilité. C'est une discussion qui est basée sur l'expérience. Si vous regardez les choses à l'international, il existe entre quatre cents et cinq cents réacteurs nucléaires de par le monde ${ }^{52}$. Même en ne tenant pas compte des réacteurs expérimentaux ${ }^{53}$, si on pense que les réacteurs commerciaux ont commencé à fonctionner vers le milieu des années $1960^{54}$, cela représente un nombre considérable d'années-réacteurs ${ }^{55}$. Si on suppose qu'une moyenne de quatre cents réacteurs fonctionne pendant vingt ans, cela nous fait une expérience d'environ huit mille années-réacteur. Sur cette durée, nous avons vécu toutes sortes d'incidents. Mais jamais d'accident où toutes les sources d'alimentation ont été perdues, comme ce que nous avons connu. Cela avait peut-être ancré en nous une illusion, une fausse impression de sécurité.

$\mathbf{Q}$ : Parce que vous n'aviez jamais connu d'exemple concret de perte totale d'alimentation, vous voulez dire?

$\mathbf{R}$ : Oui, c'est ça.

$\mathbf{Q}$ : Ce qui signifie que, vous aussi, vous pensiez que vous pourriez recourir éventuellement à l'électricité de la tranche voisine.

$\mathbf{R}$ : Oui, je pensais qu'on pourrait acheminer l'électricité de quelque part. 
$\mathbf{Q}$ : Vous pensiez donc que vous trouveriez de l'électricité à partager. Mais, en mars dernier, vous n'avez pas pu.

$\mathbf{R}:$ C'est ça.

$\mathbf{Q}$ : Pensez-vous aujourd'hui que vous auriez dû envisager ce genre de cas ?

R : Bien sûr.

$\mathbf{Q}$ : Si on revient à la situation d'avant le 11 mars, vous supposiez, dans le cas où vous auriez perdu toute alimentation en courant alternatif, que le générateur diesel se mettrait en marche. Combien de temps pensiez-vous que ce générateur devait tenir avant rétablissement du courant alternatif?

$\mathbf{R}$ : C'est une question difficile. Tout dépend de l'évènement. Il n'est pas possible de déterminer comme ça le nombre d'heures. Tout dépend de la restauration. Il suffit qu'une ligne, au moins, soit restaurée, puisqu'on pense qu'on pourra la partager tant qu'on voudra. Ce qui veut dire qu'on n'envisage pas qu'à la fois la ligne venant d'ôkuma et celle venant de Futaba $^{56}$ soient indisponibles en même temps. La tendance qui nous est naturelle est de présupposer qu'au moins une des deux reste opérationnelle. Du coup, le calcul se fait en journées. Si le générateur tenait le coup pendant une ou deux journées, il y aurait eu moyen de rétablir une quelconque alimentation extérieure. Voilà ce qu'on pensait en général, il me semble. En tout cas, c'est ce que je pensais personnellement.

Même au mois de mars, concernant l'alimentation externe, y compris la ligne Tôhoku ${ }^{57}$, je ne me rappelle pas exactement le nombre de jours qu'il a fallu pour la restaurer, mais il me semble qu'elle a été rétablie vers le 20. Ce qui fait environ six jours. Six jours, si on gère bien l'installation et à condition que celle-ci n'ait pas été inondée, c'est une durée jouable. Ce sera difficile, mais on peut encore envisager de faire tenir le générateur jusque-là.

$\mathbf{Q}$ : En mars, vous avez aussi eu des déboires concernant le courant continu, mis à part le réacteur 3 , puisque les accumulateurs ont aussi été inondés. Je suppose que c'était, là encore, une situation que vous n'aviez pas envisagée. J'imagine que, au cas où vous auriez perdu toute source de courant continu, bien qu'il y ait à chaque fois le réseau $A$ et le réseau $B$, vous pensiez pouvoir, d'une manière ou d'une autre, récupérer du courant alternatif chez le voisin, que vous auriez converti en courant continu pour faire marcher les instruments.

$\mathbf{R}$ : Cela n'aurait pas été immédiat, mais je pensais qu'en prenant un peu de temps, on aurait été capable de bricoler quelque chose. Mais j'ai toujours pensé que la perte du courant continu, qui fait l'objet d'une déclaration suivant l'article 10 et l'article 15, était quelque chose qui pouvait survenir avec une assez forte probabilité.

$\mathbf{Q}$ : Et pourquoi ça?

$\mathbf{R}$ : Si on y réfléchit, le courant continu est alimenté par des batteries. Une batterie, ça n'a qu'une capacité limitée. Lorsqu'on aura vraiment épuisé ce qui était stocké, comment va-t-on la remplir à nouveau ? Ce genre de questions m'a toujours inquiété. Je ne me faisais pas de souci pour le courant alternatif. On en dispose à profusion. Mais le courant continu? Personnellement, je pensais que la perte de courant continu pouvait être une des premières raisons d'une déclaration de type article 15.

$\mathbf{Q}$ : Et vous le pensiez depuis longtemps?

$\mathbf{R}$ : Lorsque les articles 10 et 15 ont été promulgués, j'ai examiné la liste des évènements qui allaient susciter les déclarations et je me suis demandé ce qui pouvait advenir le plus facilement chez nous. En fixant cette liste, je me suis dit à moi-même 
que l'alimentation en courant continu pouvait être notre point faible. Mais ça n'était qu'une impression personnelle.

Q: Vous pensiez que s'il y avait interruption de courant alternatif, le courant continu pouvait encore tenir environ huit heures et que ce laps de temps devait suffire pour restaurer le courant alternatif. C'est ça?

R : Oui.

Q : Le rapport de 2002 parle de l'installation de lignes de liaison.

$\mathbf{R}$ : Pour l'électricité.

Q: Oui, pour l'électricité. Je suppose que si le rapport en parle, vous-même et toutes les personnes concernées étiez largement conscients de leur existence. II s'agissait de lignes de moyenne tension tirées en dehors du réseau des autres lignes à haute tension, qui pouvaient être utilisées aussi pour le courant continu, si j'ai bien compris. Le fait que ce réseau soit indépendant est-il dû au fait que si on le reliait aux autres, on risquait d'être victime de la panne du voisin par ricochet?

R : Oui, certainement.

$\mathbf{Q}$ : Et savez-vous comment ça fonctionne?

R : Là, je ne sais pas ce qui se passe concrètement pour le courant continu. Je sais que ces lignes de liaison existent, mais comment marche ce réseau ? Je suis navré, je ne peux pas vous renseigner.

Q: Très bien. Toutes ces mesures, comme le partage de l'électricité, ce sont des manœuvres qui, à la base, se font à partir de la salle de commande en poussant sur un bouton, n'est-ce pas?

R : Oui.

Q: Parlons maintenant des groupes électrogènes de secours. II y en a plusieurs qui ont survécu au tsunami, à la tranche 3, 6, 2 et 4. Ce sont tous des générateurs refroidis par air. Aviez-vous une intention particulière en choisissant des générateurs refroidis par air? Aviez-vous participé à ce choix ?

$\mathbf{R}$ : Non, je n'ai pas participé à ce choix.

Q : Ah, bon. Alors c'était déjà comme ça quand vous êtes arrivé ?

$\mathbf{R}$ : Oui. C'est une amélioration qui a été apportée il y a pas mal de temps. C'était au moment où j'étais à la Federation of Electric Power Compagnies, puis à Fukushima Daini $^{58}$, à un moment où je suis resté éloigné de Fukushima Daiichi pendant assez longtemps que les travaux ont été réalisés. Du coup, je n'ai pas assisté moi-même aux travaux. Seulement, par le passé, du temps où j'étais chef de la maintenance à Fukushima Daiichi ${ }^{59}$, j'avais trouvé que le nombre de générateurs de secours était un peu faible, du coup, j'ai été très content de savoir qu'on avait augmenté leur nombre à trois.

Q: Parfait. Lors de l'accident, vous aviez perdu toute source d'électricité, vous vous êtes retrouvé en situation de SBO, et vous avez eu beaucoup de mal à faire revenir l'électricité, à effectuer les manœuvres sur les vannes SR ou à réaliser un éventage. Aujourd'hui, à la lumière de ce que vous avez vécu, y a-t-il des dispositifs dont vous auriez aimé bénéficier pour vous faciliter la tâche?

$\mathbf{R}$ : C'est une question qu'on me pose souvent. Je vous avouerai que je ne suis pas encore arrivé moi-même à une conclusion. Il y a plein de choses dont j'aurais aimé disposer. Mais quand je commence à me demander à quoi donner la priorité ou à réfléchir si c'est véritablement faisable, j'ai du mal à répondre. Si on met à part la question de la faisabilité, c'est quelque chose à quoi je pense depuis très longtemps, il 
faudrait quelque chose qui soit capable de produire suffisamment d'électricité pour couvrir les besoins de toutes les tranches, un petit système de générateur turbine au gaz, par exemple, qui constituerait une sorte de centrale à l'intérieur de la centrale, tirer des câbles de là, ce qui permettrait de gagner pas mal de temps. Alors, bien sûr, il y a toujours cette histoire de résistance au séisme. Comme, cette fois-ci, on a été victime à la fois d'un séisme et d'un tsunami, on a un peu tendance à penser que quoi qu'on fasse, quoi qu'on prévoie, si ce quelque chose ne résiste pas au séisme, on ne pourra rien en attendre, ce qui rend la réponse à cette question d'autant plus difficile. Mais si je vous fais une réponse totalement naïve, en ne tenant compte que de mes désirs, je dirais que ce serait bien s'il y avait, à côté des tranches, une sorte de générateur turbine au gaz de plusieurs dizaines, voire plusieurs centaines de kW, particulièrement résistant aux séismes.

$\mathbf{Q}$ : Durant les évènements de mars, les camions générateurs ont joué un rôle important. Mais j'imagine qu'il ne suffit pas de disposer de ces camions. Car, par ailleurs, vous avez effectivement eu de sérieux problèmes au niveau des panneaux de distribution, entre autres. Est-ce qu'il aurait été utile d'avoir des batteries de rechange pour l'usage des compresseurs à air, par exemple? Dans ce cas-là, vous auriez eu directement du courant continu.

R : Bien sûr. Dans ce sens, ce serait bien d'avoir ces choses en réserve. Seulement, dans la pratique, si on imagine qu'on dispose de la série complète de batteries, ça signifie qu'on va devoir recharger tous les jours la série complète en prévision d'un incident dont on ne peut par définition prédire l'avènement. Alors du point de vue de la protection, bien sûr, ce serait très bien de disposer de ces batteries, mais si on regarde tout ça du point de vue pratique, $y$ compris cette recharge quotidienne, si on n'avait pas vécu ce que nous avons connu en mars, eh bien, on se dirait que tout ça est inutile. On finirait par se demander pourquoi on recharge jour après jour des batteries dont on ne sait pas quand elles vont être utiles. Depuis sept mois, je n'arrête pas de me dire que si j'avais moi-même fait cette proposition...

$\mathbf{Q}$ : Vous voulez dire que vous ne l'avez pas réellement proposé, mais que si vous l'aviez fait...

$\mathbf{R}$ : Je joue en moi-même à la fois le rôle de celui qui propose et de celui qui doit prendre la décision, et je me dis qu'aujourd'hui, avec ce que nous avons vécu, je serais content de disposer de ces batteries, mais que si j'avais proposé cette mesure à l'époque, en pensant non seulement à ce que cela aurait coûté, mais aussi à la maind'œuvre que cela aurait demandé, est-ce que j'aurais poussé à mettre en place un dispositif aussi lourd à gérer jour après jour, pour quelque chose dont on ne savait même pas quand il allait servir?

$\mathbf{Q}$ : Que pensez-vous du compresseur portable que vous avez utilisé au moment d'ouvrir les vannes $A O$ ?

R : Je pense que c'est nécessaire. Là aussi, c'est ce que je pense aujourd'hui. Ce que je voudrais dire aux autres exploitants, c'est que les nouvelles directives de la NISA insistent beaucoup sur la prévision des alimentations électriques de rechange, mais n'exigent pas de compresseurs. Pourtant, c'est quelque chose d'indispensable. C'est pour ça qu'il faudrait, mise à part la discussion de savoir si le jeu complet de batteries de réserve est nécessaire ou pas, affiner un peu ces orientations. Calculer combien de batteries en réserve au minimum, combien de compresseurs, en déterminant les cas où ils doivent intervenir. Ce travail est indispensable. Sinon, les exploitants restent 
très exposés. Sans oublier l'entraînement pour que le personnel soit capable d'utiliser ces outils.

$\mathbf{Q}$ : Évidemment, avant le 11 mars, vous ne disposiez pas de tout ça en réserve et les divers entraînements et simulations ne comportaient pas l'utilisation effective de ces outils. Si vous me dites que tout cela était au-delà de toutes les hypothèses envisagées, la discussion s'arrête là. Mais, quand j'interroge les personnalités haut placées, les dirigeants, j'essaie de comprendre pourquoi vous en êtes arrivés à cet état de choses, pourquoi vous n'êtes pas allés plus loin. Qu'en pensez-vous personnellement?

$\mathbf{R}$ : Je pense que c'est parce qu'on était persuadés que cela n'arriverait pas.

$\mathbf{Q}$ : Vous pensiez qu'un accident qui implique toute la centrale n'arriverait pas.

$\mathbf{R}$ : Ce n'est pas pour revenir à l'histoire de tout à l'heure, mais si on regarde à l'international, d'après l'ensemble de l'expérience que nous avons des centrales nucléaires, il n'était jamais arrivé qu'on perde toute alimentation électrique externe, que même à l'intérieur on perde tout. Sachant cela, on en avait déduit que cela n'arriverait pas. Vous pouvez critiquer en disant que nous étions naïfs et tout ce que vous voulez.

$\mathbf{Q}:$ Là n'est pas mon propos. Je suis tourné vers l'avenir.

$\mathbf{R}$ : En tout cas, c'est ce que je pense, moi.

$\mathbf{Q}$ : Je voudrais maintenant que nous parlions des camions de pompiers. Le problème de la perte d'alimentation électrique est directement lié au phénomène du tsunami, un tsunami géant que vous n'aviez pas prévu. Mais la problématique des camions de pompiers me semble être un peu différente. Pour résumer, dès 2002, parmi les nouvelles procédures de gestion d'accident mises en place, on note l'utilisation du réseau incendie (réseau FP) comme moyen substitutif d'injection. Même avant le séisme de Chûetsu, des bouches à incendie avaient été installées. Puis, à la suite de ce séisme, des camions de pompiers et de nouvelles bouches d'incendie ont été prévus. Je pense que l'utilisation du réseau incendie est un moyen «substitutif » d'injection, parce que la vocation première de ce réseau est la lutte contre le feu. Quand on pense "mesures incendie», on pense réseau FP, parce qu'il est là au départ pour éteindre les incendies. Sur ce réseau sont installées des pompes électriques pour faire circuler l'eau qu'on va faire venir du réservoir à eau filtrée. Que fait-on quand on n'a plus d'électricité ? Là, le DDFP prend le relais. In fine, le DDFP va envoyer l'eau et on pourra éteindre l'incendie. Donc tout va bien. Vous prévoyez les choses jusque-là. Au pire, si le DDFP lui-même devient inopérant, on peut encore envoyer de l'eau par les bouches à incendie en utilisant les camions de pompiers pour éteindre le feu. Est-ce que vous aviez pensé jusque-là ?

$\mathbf{R}$ : Je pense qu'à ce moment-là on n'en avait pas conscience de manière aussi claire. Parce qu'on n'avait pas encore prévu les camions de pompiers.

$\mathbf{Q}$ : Que voulez-vous dire par «à ce moment-là » ?

$\mathbf{R}$ : Parce que, quand on a relié les camions au réseau FP par l'extérieur, on n'avait pas beaucoup de camions. Les camions de pompiers n'ont été achetés qu'après le séisme au large de Chûetsu.

$\mathbf{Q}:$ Je suis en train de vous interroger à propos de ce qui s'est passé après ce séisme. Je vous parle de mesures incendie, parce que si on abordait directement le problème de l'injection substitutive cela nous mènerait trop loin. C'est pour ça que je vous parle d'abord de ce pour quoi ces bouches ont été installées au départ. II y a donc des bouches. Par ces bouches, on peut envoyer de l'eau de l'extérieur. Dans quel but ces bouches ont-elles été installées? C'est pour pouvoir relier les camions de pompiers au réseau incendie. Ce qui 
signifie que le jour où on a besoin d'éteindre un incendie à l'intérieur de la tranche à l'aide du réseau FP, même s'il y a défaillance du DDFP, on peut envoyer de l'eau via les camions.

$\mathbf{R}$ : C'est le scénario depuis le séisme au large de Chûetsu. Avant, on ne disposait même pas de camions de pompiers.

$\mathbf{Q}$ : Je vous parle de ce qui se passe depuis le séisme. Après ce fameux séisme, aussi bien au siège de TEPCO qu'à la centrale de Fukushima Daiichi, vous étiez conscients qu'au cas où le feu se déclarerait dans une des tranches, même si le DDFP était hors service, vous pouviez envoyer de l'eau en utilisant les camions de pompiers. D'autre part, vous saviez tous, puisque c'était inscrit noir sur blanc dans les manuels de gestion des accidents, que ce réseau FP avait pour autre vocation de devenir un moyen substitutif d'injection d'eau dans le réacteur, en cas de besoin. Sachant cela, il me semble qu'il aurait été possible de penser aussi loin dans ce second cas que dans le premier et d'arriver à la conclusion qu'on pouvait tout aussi bien envoyer l'eau via les camions pour l'injection dans le réacteur et formuler cette possibilité en toutes lettres parmi les procédures de gestion de crise. Or, il n'en a pas été ainsi. Pourquoi, à votre avis?

$\mathbf{R}$ : La paralysie intellectuelle, encore une fois. On ne réfléchissait plus. C'est-à-dire que notre réflexion s'était arrêtée aux pompes diesel. Si on en disposait, ça suffisait.

$\mathbf{a}$ : Effectivement, tout le monde me répond qu'il n'avait pas tellement pensé que les pompes diesel pouvaient tomber en panne. Mais d'un autre côté, concernant les réacteurs 1 et 2 , il existe des documents fabriqués par le constructeur GE avec des illustrations où on voit des camions de pompiers en train d'injecter de l'eau. Vous me dites donc que vous n'aviez pas pensé à ce que vous feriez si les pompes diesel tombaient en panne. Vous m'auriez dit, " je n'ai pas réfléchi à la suite de ce que je ferais, si les pompes tombaient en panne en voulant éteindre l'incendie » et "je n'ai pas non plus réfléchi à la suite, si elles tombaient en panne en voulant injecter dans le réacteur », j'aurais compris. Mais vous me dites, "si c'est un incendie, je penserais à injecter l'eau en reliant un camion de pompiers à une bouche d'incendie », "mais pour l'utilisation du FP pour injecter l'eau dans le réacteur, je ne pense pas jusqu'au camion de pompiers ». Pourquoi ça?

$\mathbf{R}$ : C'est un problème de conception, ce qui n'est pas mon fort. J'imagine que les gens ont raisonné de cette manière. La probabilité qu'on perde les moyens d'injecter dans le réacteur est très faible. Or, le réseau $\mathrm{FP}$, dans notre imaginaire, est quelque chose de très fragile. Par exemple, du point de vue de la résistance aux séismes, c'est une installation de classe $C$. Dans notre tête, c'est une installation sur laquelle on ne peut pas compter s'il arrive quelque chose. Mais s'il y a incendie, il n'y a que le réseau FP pour l'éteindre. C'est pour cette raison qu'on réfléchit aux mesures incendie de manière aussi poussée, jusqu'à l'utilisation des camions de pompiers. Par contre, pour l'injection d'eau dans le réacteur, avant d'en arriver à recourir au réseau $\mathrm{FP}$, on a des tas de possibilités. On a l'ECCS. Si le réseau habituel de refroidissement est encore vivant, on peut aussi y avoir recours. On peut aussi faire marcher l'ECCS avec le générateur diesel. Enfin, vous voyez, il y a des tas d'autres solutions, pour ce qui est d'injecter de l'eau dans le réacteur. Le réseau FP n'est juste qu'une possibilité parmi des tas d'autres. En plus, comme je viens de vous le dire, la résistance au séisme de ce réseau est particulièrement médiocre. C'est une installation de catégorie $C$ qui court à travers tout le bâtiment. Effectivement, il existe comme possible source d'injection, mais je pense qu'on ne lui attribuait pas une grande importance en tant que telle.

C'est donc bien d'avoir conçu, lors d'accidents graves, la possibilité de faire appel au $\mathrm{MUW}^{60}$ ou au FP, mais si celui qui l'a conçu avait réellement eu la volonté d'y avoir recours en toute dernière instance, il aurait redessiné le tout en classe A pour que ça résiste aux séismes. Regardez, tous les autres systèmes ont été conçus en classe $A$. Je me demande à quel genre de phénomène il pensait, quand il a préconisé l'utilisation 
des systèmes les plus faibles, comme le FP ou le MUW, qu'on n'a pas utilisé en mars, lorsque tous les autres systèmes conçus en classe A, avec double possibilité d'alimentation électrique, auraient été hors d'usage. Je n'arrive pas à comprendre ça.

$\mathbf{Q}$ : Le problème de la résistance aux séismes était un autre des problèmes dont je voulais discuter avec vous. Je voulais justement vous demander votre avis sur le fait qu'un réseau qui avait subsidiairement une mission si importante ait pu être conçu et rester en classe C. Ou faut-il interpréter ça comme le fait que, bien qu'ayant apporté les modifications pour que le réseau FP puisse servir le cas échéant à l'injection dans le réacteur, en fait, le concepteur n'a jamais envisagé sérieusement de l'utiliser de cette manière?

$\mathbf{R}$ : C'est ce que j'imagine.

$\mathbf{Q}$ : Vous l'imaginez. Savez-vous le genre de discussions qu'il a pu y avoir au siège à ce sujet?

$\mathbf{R}:$ Je ne sais pas quelles ont été les discussions au moment où tout cela a été décidé, mais quand nous avons eu accès pour la première fois à ces nouvelles instructions, que nous avons su qu'ils avaient effectué des travaux pour que le FP et le MUW puissent servir en dernier recours à l'injection, nous autres, de l'exploitation, nous sommes dit que, s'il y avait un gros séisme, ces réseaux seraient inexploitables avant même d'être en situation d'être utilisés pour l'injection dans le réacteur. Voilà ce que nous avons pensé.

Q : Alors, au moment de l'accident du 11 mars, justement face à un accident extrêmement grave, à quel moment avez-vous pensé personnellement que vous alliez utiliser ce réseau FP?

$\mathbf{R}$ : Très vite, $\mathrm{j}$ 'ai eu recours au réseau FP. Avec le tsunami, nous avions perdu toute source externe d'électricité. Rien ne marchait. Que restait-il pour injecter l'eau? Il n'y avait que le réseau FP. Bien sûr, nous disposions encore de l'IC, du HPCI et du RCIC, mais ensuite? Quand tous ces systèmes se seraient arrêtés, que resterait-il pour continuer à injecter dans le réacteur? Il y avait de fortes probabilités pour que ce soit le réseau $\mathrm{FP}$.

$\mathbf{Q}:$ Si je résume, avant le 11 mars, personne n'avait même imaginé arriver à une situation où il ne resterait plus que le réseau FP d'opérationnel, d'autant plus que les moyens d'injecter dans le réacteur étaient multiples, donc personne ne pensait sérieusement à une injection avec comme source d'eau des camions de pompiers.

R: C'est ça. Très probablement, avant le 11 mars, personne n'y avait songé sérieusement.

Q : Alors que, s'il s'agissait d'éteindre un incendie, comme il n'y avait que la ligne FP, on avait songé aux camions.

$\mathbf{R}$ : Oui. Le réseau qui couvre tous les bâtiments avec des tuyaux partout, c'est le réseau FP. Sa mission principale étant d'éteindre le feu, dans ce sens, il fallait songer à multiplier les sources d'approvisionnement en eau, donc des camions de pompiers. Mais ils n'avaient pas pensé une seconde que ces camions puissent aussi servir pour l'injection dans le réacteur.

$\mathbf{Q}$ : Je n'ai nullement l'intention de vous contredire, mais tout le monde me dit à ce propos qu'il pensait bien qu'à la toute fin on recourrait aux camions de pompiers pour l'injection, qu'ils le savaient dans leur tête.

$\mathbf{R}$ : Je pense que beaucoup le disent après coup, au vu de ce qui s'est passé, vous ne croyez pas? 


\section{$\mathbf{Q}:$ Et vous, vous y pensiez déjà ?}

$\mathbf{R}$ : Tout à l'heure vous avez limité la discussion à ce qui s'est passé après le séisme au large de Chûetsu, mais dès avant ça, s'ils pensaient vraiment qu'on allait utiliser le réseau FP dans le cadre des procédures de gestion de crise, j'ai envie de dire à ces idiots qui ont conçu ces procédures, qu'il fallait alors prévoir des camions de pompiers et les mettre à notre disposition dès ce moment-là. S'il fallait vraiment en dernier recours faire appel au réseau FP, il fallait revoir l'installation du point de vue antisismique, et s'il fallait vraiment utiliser des camions de pompiers en dernière instance, il fallait en acheter et en posséder en propre depuis longtemps. Vous vous rendez compte que jusqu'au séisme de Chûetsu, on n'en possédait aucun ? Et encore, c'est parce que le transformateur a pris feu à la suite du séisme qu'ils ont acheté des camions de pompiers, ce n'était pas en vue de les utiliser pour l'injection dans le réacteur. Ils n'avaient pas fait le lien. Après coup, on se demande pourquoi on n'y avait pas pensé, ça paraît bête. Mais si on se reporte en arrière, il n'y avait de lien dans la tête de personne. Les gens prétendent qu'ils y avaient pensé parce qu'ils ont vu ce que nous avons fait. C'est facile, après coup.

$\mathbf{Q}$ : N'avez-vous jamais entendu évoquer, ne serait-ce qu'oralement, la possibilité d'utiliser les camions de pompiers comme moyen substitutif d'injection lors de réunions de sécurité ou échanges de points de vue au siège, par exemple?

$\mathbf{R}$ : Comme j'ai très peu travaillé dans ce domaine, je n'ai pas eu l'occasion d'assister à ce genre de réunion. Mais il me semble bien que je n'ai jamais entendu parler de ça avant le 11 mars. Je veux parler de l'utilisation des camions de pompiers pour injecter dans le réacteur.

$\mathbf{Q}$ : Pensez-vous, au regard de ce qui s'est passé, qu'il aurait été utile que cette utilisation des camions de pompiers comme source substitutive d'alimentation pour l'injection du réacteur ait été décrite noir sur blanc dans les procédures à adopter face à un accident grave?

R : Aujourd'hui, oui. Seulement, personnellement, je pense que c'était quand même un pari. On n'a pas su jusqu'à la toute fin si on allait pouvoir injecter par la voie du réseau FP. Pour pouvoir utiliser cette voie, il fallait dépressuriser le réacteur. Ce qui signifie que le niveau d'eau allait baisser. Et là, on allait injecter l'eau via le réseau FP. Mais si le réseau avait été coupé quelque part à cause du séisme ? Dans ce cas-là, on aurait beau envoyer l'eau, rien ne serait arrivé jusqu'au réacteur.

\section{$\mathbf{Q}$ : Mais vous aviez bon espoir que le réseau, dans le bâtiment, ait résisté.}

$\mathbf{R}$ : Bien sûr que j'avais bon espoir. Après le séisme de Chûetsu, je suis allé voir à Kashiwazaki. J'avais examiné minutieusement les bâtiments turbine, les bâtiments réacteur et tout le réseau FP. Une partie s'était déformée, mais il n'y avait de rupture nulle part. Ce qui fait que dans un sens, au moment de l'accident de Fukushima, j'étais assez confiant. Mais on ne sait jamais avec la réalité. Avec un tel séisme, on ne pouvait pas savoir ce qui s'était réellement passé à l'intérieur des bâtiments. À la fin, c'est un pari. On parie que le réseau FP est intact. De toute façon, il n'y avait que ça comme solution, alors on a injecté via le réseau FP. C'est tout.

$\mathbf{Q}$ : Durant les auditions de l'été, il a été question de vos rapports avec les entreprises partenaires comme Nanmei Kôsan. Le contrat que vous aviez avec eux concernait justement la lutte contre le feu et ne comportait évidemment pas la coopération à l'injection d'eau dans le réacteur, coopération que vous avez eu du mal à obtenir, ce qui a un peu retardé le démarrage des opérations avec les camions de pompiers, me semble-t-il. 
Pensez-vous que si tout ça avait été noté noir sur blanc dès le départ, cela aurait simplifié les choses?

$\mathbf{R}$ : Si on y repense aujourd'hui, oui. Mais ce que je veux dire, c'est que les gens qui ont conçu les procédures pour la gestion des accidents n'avaient pas du tout pensé jusque-là. Ils ont tendance à faire beaucoup de bruit après coup, mais je n'ai aucune confiance dans les gars de la sûreté chez nous.

$\mathbf{Q}$ : Bien. Après les camions de pompiers, je voudrais passer maintenant aux manuels. Le sujet a déjà été évoqué cet été. Vous aviez déclaré qu'il existait différents manuels de gestion des accidents, que vous ne pensiez pas forcément qu'aucun n'était utile, que vous en aviez consulté quelques pages, mais que la situation s'aggravant au-delà de ce qui avait été présupposé, vous n'étiez plus en mesure d'appliquer ces procédures en tant que telles. Y a-t-il des chapitres que vous avez concrètement consultés?

$\mathbf{R}$ : Non, aucun.

$\mathbf{Q}:$ Vous n'avez pas ouvert ces manuels?

R : Moi, non.

$\mathbf{Q}$ : Même s'agissant de l'injection?

$\mathbf{R}$ : Le gros de l'histoire, si vous voulez, c'est savoir, in fine, par où on va injecter. Le fait de connaître pour chaque tranche les manœuvres à faire, c'est le travail d'un spécialiste, le chef de quart. Vous comprenez, les lignes à ouvrir, pour le réacteur 1 et le réacteur 2, ne sont pas les mêmes, parce que ces réacteurs ont été construits sur des plans totalement différents. Mais les pilotes savent ce qu'il faut faire et c'est leur travail. Certes je suis le directeur de la centrale, mais est-ce que cela implique que je connaisse tous les manuels de conduite? Je ne le pense pas. Il y a la sûreté et beaucoup d'autres domaines que je dois coordonner. Je dois avoir une vision d'ensemble. Le travail de conduite, c'est le chef de quart qui doit en prendre la responsabilité. Donc, concernant les manuels, je les laissais faire et je ne m'en mêlais pas.

$\mathbf{Q}$ : Quand on examine le rapport de 2002, il semble qu'il y ait quatre thèmes principaux que sont «les installations », "l'organisation », «les manuels » et "l'entraînement-formation ». Dans la rubrique "organisation», sont décrits l'organisme de soutien, dont vous étiez effectivement le chef durant la crise, et les rapports entre cet organisme et les pilotes de quart. Le principe de base est bien celui que vous venez d'évoquer, mais, par ailleurs, le rapport précise qu'en cas de difficulté ou bien pour le choix de la stratégie à adopter, par exemple, l'organisme de soutien est aussi censé intervenir activement. Je pense que vous étiez concrètement dans une situation où vous ne pouviez pas tout laisser au jugement des pilotes. Lors de discussions pour le choix d'une stratégie au sein de la cellule de crise, vousmême...?

$\mathbf{R}$ : Je ne descendais pas jusqu'au détail des manuels. Tout ce qui concernait directement les manœuvres était du ressort des pilotes sous les ordres du chef de quart. Mais bien sûr, nous étions là en soutien. C'est là la raison d'être de cette cellule de crise. Si, par exemple, des difficultés apparaissaient pour une manœuvre de conduite, nous faisions bien entendu ce qu'il fallait pour réparer ou modifier les choses pour la rendre possible. Si des évaluations de sûreté étaient nécessaires, du genre "le niveau d'eau de ce réacteur semble avoir baissé, où en est-on pour de bon?", le groupe «technique » faisaient l'estimation. Ainsi de suite, chacun dans son domaine était en soutien et l'ensemble de l'organisation était derrière les pilotes. Mon rôle, dans tout ça, était de donner des ordres pour que cette aide fonctionne efficacement, 
mais ce n'était ni le moment ni le lieu pour entrer dans les détails de manuels ou de discuter de leur bien-fondé sur tel ou tel point.

Q : Peut-être y a-t-il eu ce genre de discussions au sein des différents groupes, mais votre position était de porter un jugement final sur ce qui était proposé par la base. Ai-je bien compris?

Je suis désolé de revenir encore sur une histoire qui fait intervenir les camions de pompiers, mais je voudrais qu'on parle du fait que vous avez utilisé la mer comme source d'alimentation. Tout le monde sait que les centrales nucléaires ont besoin d'eau pour assurer la partie « refroidissement » de l'« arrêt, refroidissement et confinement ». D'aucuns m'ont dit qu'ils avaient toujours pensé qu'ils pourraient, dans un scénario catastrophe, utiliser l'eau de la mer qui s'étendait sous leurs yeux. Lors de l'accident de mars, quand vous avez voulu concrètement utiliser cette eau pour l'injection, vous avez connu pas mal de difficultés pour la pomper. Cela signifie que, tout en ayant en tête qu'on puisse recourir à l'eau de mer pour l'injection dans les installations, personne n'avait pensé à prévoir un endroit où on pouvait pomper cette eau ?

$\mathbf{R}:$ En tout cas, pas avant les évènements.

$\mathbf{Q}:$ Et pourquoi, à votre avis?

$\mathbf{R}$ : Parce que, si on dispose de l'électricité, les sources d'alimentation en eau sont multiples. Il y a le CST (Condensate Storage Tank) ${ }^{61}$, il y aussi de l'eau dans le système ECCS ou le système RHRS ${ }^{62}$, si ces systèmes sont viables. La conception de base est de refroidir les réacteurs en utilisant ces sources. Personne n'avait pensé qu'on en arriverait pour de vrai à injecter de l'eau de mer. On a tendance à penser, même si on a perdu l'alimentation électrique, que c'est une difficulté temporaire. On a les générateurs diesel de secours, on peut restaurer l'électricité externe. De toute manière, si on récupère l'électricité, on pourra injecter d'une manière ou d'une autre. Voilà ce qu'ils se disaient. Est-ce que les gars de la sûreté pensaient vraiment au tréfonds d'eux-mêmes qu'on devrait faire face un jour à une telle catastrophe ? Ontils vraiment conçu les choses en y songeant? Franchement, j'ai des doutes.

Q: Vous voulez dire qu'intellectuellement, dans leur tête, ils avaient conscience qu'il pourrait y avoir des cas où il faudrait injecter de l'eau de mer, mais qu'ils ne pensaient pas qu'on en arriverait à envoyer de l'eau de mer dans la réalité?

R : Oui. S'ils y avaient vraiment pensé, ils auraient dû prévoir une ligne à part pour permettre de pomper l'eau à partir de la mer. Utiliser l'eau de mer qui était restée dans le bassin de la tranche 3, ce genre de choses, ce sont toutes des initiatives que nous avons prises sur le terrain grâce à notre ingéniosité. Aucun de ceux qui ont conçu les manuels de gestion de crises n'y avaient pensé. Si vous permettez l'expression, je dirais qu'ils ne se sont occupés que de la surface, qu'ils ne l'ont fait que pour la forme. Bien sûr, moi-même, même si je n'ai pas directement participé à leur élaboration, je suis ces recommandations pour diriger la centrale et j'assume le fait d'en être le directeur, je sais que je n'ai pas été à la hauteur puisque je n'y avais pas songé auparavant et j'en suis honteux, mais je dois dire que personne parmi ceux qui, les premiers, ont conçu les réponses à ces accidents, n'a eu le courage d'envisager les choses jusque-là, d'imaginer le pire des scénarios.

$\mathbf{Q}$ : Tout à l'heure nous avons un peu parlé des classes antisismiques, que si on désire utiliser le réseau FP comme moyen d'injection, il faudrait le revoir en classe $S^{63}$. Au vu de votre expérience sur le terrain et pour en tirer les leçons pour l'avenir, y a-t-il d'autres 
installations que vous souhaiteriez voir améliorées du point de vue de la résistance aux séismes?

$\mathbf{R}$ : Ce que je voudrais dire, après avoir vu Kashiwazaki et après avoir vu le comportement des tranches à Fukushima, c'est que, à l'inverse de ce qu'on pourrait croire, de base, les installations sont solides. Après avoir subi un séisme de cette importance, le réseau FP n'avait pas rompu. À l'intérieur des bâtiments. Si on isole le problème de la résistance aux séismes, mon avis personnel est que l'expérience de mars nous apprend paradoxalement que nous avons su y répondre. Personne ne le dit franchement, mais pourvu que les bâtiments soient solides, la tuyauterie résiste. Si les bâtiments sont solides, la tuyauterie qui y est appuyée tient le coup. Même si les bâtiments se déforment un peu sous l'action du séisme, les tuyaux s'allongent ou se courbent. Et si les soudures ont été faites correctement, ils ne se cassent pas. Il n'y a pas de rupture. S'il n'y a pas rupture, l'eau peut continuer à circuler. Donc, du point de vue de la résistance aux séismes...

$\mathbf{Q}$ : Ce réseau s'est révélé assez solide.

$\mathbf{R}:$ Oui, tout à fait.

$\mathbf{Q}$ : Donc vous ne voyez pas d'endroits qu'il conviendrait de renforcer particulièrement?

$\mathbf{R}$ : Au contraire, je le trouve suffisant, puisque sur l'ensemble des tranches, le réseau FP a fonctionné correctement.

$\mathbf{Q}$ : Très bien. Nous allons encore changer de sujet. Je lis ici qu'il est fortement recommandé aux organismes de soutien à la gestion des accidents d'entretenir correctement les appareils qui pourraient être utilisés. II me semble que vous aviez eu pas mal de problèmes avec les équipements de communication. Cet été, vous avez parlé du cas des radios $\mathrm{VHF}^{64}$ dont les ondes n'étaient pas assez puissantes. Y a-t-il d'autres choses, dans le domaine de la télécommunication ou ailleurs, n'aviez-vous pas eu aussi des soucis avec les SPDS, qui vous ont posé particulièrement problème lorsque vous avez voulu les utiliser?

R: Oui, ce qui nous a le plus handicapé, ce sont les équipements de télécommunication. Particulièrement entre le terrain et la salle de contrôle, puis entre la salle de contrôle et la cellule de crise. Tout d'abord, entre le terrain et la salle de contrôle il n'y avait rien.

$\mathbf{Q}$ : Parce que vous ne pouviez plus utiliser le PHS ?

$\mathbf{R}$ : C'est ça. On ne pouvait pas utiliser le PHS. On ne pouvait pas utiliser le Paging ${ }^{65}$, non plus. Habituellement, on communique par Paging. On annonce, " on va faire telle ou telle manœuvre ", avant de l'effectuer. Mais on ne pouvait pas l'utiliser. De plus, entre la salle de contrôle et la cellule de crise, nous disposions peut-être tout juste de deux lignes. Comme on n'en avait pas beaucoup, il nous a fallu énormément de temps, ne serait-ce que pour avoir une idée de la situation. Là, il faut absolument faire quelque chose. Je ne sais pas quel genre d'appareil il faut prévoir pour faire face à un tel accident, je n'y connais rien en matière de télécommunication, mais il est indispensable d'y réfléchir.

$\mathbf{Q}$ : Je suppose que le PHS devait remplir un rôle important. Mais quand j'ai interrogé la personne en charge de la télécommunication, elle m'a dit que lorsqu'il n'y avait plus de courant alternatif, les appareils ne pouvaient tenir que pendant une heure. Étant donné le 
rôle crucial que devaient tenir ces appareils, est-ce que vous n'aviez pas pensé à les améliorer pour les faire tenir plus longtemps, par exemple?

$\mathbf{R}$ : Il semblerait que non. Personne ne pensait que le PHS serait appelé à fonctionner dans des conditions aussi dramatiques.

$\mathbf{Q}$ : Au moment où vous ne pouvez plus utiliser le PHS, vous basculez sur les radios VHF. Aviez-vous fait des essais sur ces appareils, amélioré leur installation, la puissance d'émission, aviez-vous fait des entraînements sur le terrain, où vous auriez pu constater que les bâtiments pouvaient constituer de véritables obstacles pour les ondes, bien plus que ce qu'on aurait pu croire?

R : Malheureusement, non. Lors des entraînements, nous n'avions pas utilisé le VHF. Parce que nous avions le PHS. Là, on n'a pas été prévoyant. C'est quelque chose que je voudrais dire à tous les exploitants, les mesures d'amélioration préconisées par la NISA ne sont pas du tout suffisantes, il faut réfléchir de manière systématique et bien au-delà de ce qui est cité, y compris le genre de problèmes que nous sommes en train d'évoquer. Le problème de la communication est vraiment crucial. Il faut renforcer les installations. Si vous voulez mon avis, concernant l'alimentation électrique et l'alimentation en eau, les directives de la NISA datant de mars et avril sont extrêmement exigeantes. Mais il faut entrer beaucoup plus dans le détail des appareils qui tournent autour, comme le compresseur dont on a parlé tout à l'heure, pour tirer correctement toutes les leçons de cet accident.

$\mathbf{Q}$ : Vous dites que lors des entraînements, vous n'aviez jamais prévu de situation où le PHS serait inopérant. Pourquoi?

$\mathbf{R}$ : Parce que tout le monde pense que quelque part l'électricité reste vivante. Quoi qu'on dise, chacun pense qu'on pourra aller chercher l'électricité quelque part. Personne n'a jamais pensé qu'on puisse être totalement privé d'électricité.

$\mathbf{Q}$ : Et là, tout s'arrête.

D'après la personne de la formation que j'ai interrogée, en matière de gestion des accidents, tout ce qui est formalisé sur le papier est testé au moment des entraînements. Mais ce qui n'est pas écrit dans les manuels, comme l'utilisation du VHF, n'est pas repris dans les divers entraînements que vous effectuez. Aviez-vous conçu des mesures supplémentaires, basées sur votre expérience du terrain et propres à Fukushima Daiichi, pour combler des trous que vous auriez éventuellement détectés dans ces manuels?

R: Malheureusement, non. Cela ne faisait même pas un an que j'étais arrivé à Fukushima Daiichi en tant que directeur de la centrale. Durant ce laps de temps, l'entraînement que nous avions effectué juste avant l'accident a dû être la seule occasion où on a évoqué le problème des accidents graves. Avant ça, j'avais été chef d'exploitation à Fukushima pendant un an et neuf mois. C'était de 2005 à 2007. Vers la fin de cette période, nous avons connu toute une série d'incidents. Des incidents très ordinaires, mais en grand nombre, auxquels il a fallu répondre. Mais je n'ai pas le souvenir d'avoir tenté d'introduire des mesures spéciales concernant les accidents sévères pour autant. Après la publication du rapport de 2002, je pense que je me suis contenté de vérifier que la formation des pilotes, par exemple, suivait bien le cadre défini par le manuel.

Nous n'avons pas pris volontairement des mesures supplémentaires, mais tous les opérateurs de cette centrale, aussi bien pilotes qu'agents de sûreté, ont une très grande expérience des incidents, puisque la centrale en a connu de multiples, ce qui les a formés à une très grande capacité d'adaptation et une très grande souplesse. Je 
peux dire que de ce point de vue-là, ils étaient bien mieux armés que dans les autres centrales.

Q: Il y a bien sûr les aptitudes des pilotes, mais il y a aussi l'aptitude de ceux qui sont audessus à gérer des incidents non prévus. Quand j'ai interrogé ces personnes, j'ai toujours eu l'impression qu'à la base elles tentaient de réagir à ces évènements en poussant sur des boutons depuis la salle de contrôle. J'ai compris petit à petit que rien n'avait été entrepris dans le cadre des entraînements, par exemple, pour aller au-delà, sortir de la salle de contrôle, aller directement sur le terrain, pour imaginer des solutions lorsqu'on ne pouvait plus résoudre le problème en poussant sur des boutons. Croyez-vous que, de la même manière, rien n'a été fait pour aller plus loin dans le domaine des appareils, des essais pour fabriquer des outils adaptés, des compresseurs, etc. ?

$\mathbf{R}$ : Je pense qu'avant le 11 mars, nos préoccupations n'allaient pas jusque-là. On gérait l'incident en présupposant que si on poussait sur tel bouton à la salle de contrôle, la manœuvre se ferait comme prévu. Et ce n'était pas le seul fait de TEPCO. Toutes les centrales au Japon étaient gérées comme ça.

Q: Vous nous avez raconté tout à l'heure l'épisode de l'inondation de 1991. Vous nous avez dit que vous aviez eu vraiment peur, que vous considériez cet incident comme extrêmement grave. À cette occasion, vous aviez constaté qu'une fois inondés, la restauration des générateurs diesel était très ardue. Après cette expérience, n'avez-vous pas pensé que si vous vous retrouviez dans une situation où vous auriez perdu toute alimentation électrique, la restauration serait d'autant plus difficile et qu'il faudrait faire quelque chose en prévision de ce genre d'évènements? Je sais bien que c'est encore une fois une réflexion qu'on se fait après coup, mais pouvez-vous y répondre?

R: Si on va dans ce sens, le fait qu'après l'expérience de cette inondation on ait installé trois générateurs refroidis à l'air supplémentaires était très encourageant, en tout cas pour moi. En 1991, tous les générateurs du bâtiment turbine avaient pris l'eau. Et leur réparation avait coûté très cher. Le fait même qu'on disposait de générateurs supplémentaires, installés dans des locaux différents, puisqu'on avait rajouté un générateur à chaque couple de réacteurs, était encourageant en soi.

$\mathbf{Q}$ : Vous me direz qu'une fois que les plans sont faits, il est difficile de revenir dessus, mais si on regarde le générateur de secours du bâtiment service commun, on a pris soin d'installer un modèle refroidi à l'air, mais le tableau électrique qui lui correspond se trouve en sous-sol et, moralité, ça n'a pas pu fonctionner. Pourtant ça forme un tout. Est-ce que ça veut dire qu'on n'avait pas réfléchi jusque-là avant le 11 mars?

R : Exactement. On n'avait pas imaginé que l'eau puisse arriver jusque-là. Même s'il y avait eu de l'eau, on imaginait une situation comme celle de l'inondation de la tranche 1. Un tuyau enterré a rompu. On n'arrive pas à arrêter l'eau. À la base, en matière d'inondation, on n'avait que l'idée qu'il suffisait de déterminer l'endroit de la fuite, l'isoler en fermant les vannes en amont et en aval pour arrêter l'eau. Plus que de tsunami, il est question, ici, de la manière dont nous concevions l'inondation et de la réponse à y apporter. Et elle était limitée. Mais je ne m'en rends compte qu'aujourd'hui.

$\mathbf{Q}$ : Cet été, vous avez évoqué, à propos des lignes téléphoniques d'urgence qui vous reliaient aux autorités locales, le fait que, bien que vous pensiez que le système fonctionnait, certains interlocuteurs n'avaient pas décroché. Quelle était véritablement la situation?

R : Il faudrait que vous demandiez ça à ceux qui s'en occupaient. On ne m'avait pas mis au courant de qui avait décroché et qui n'avait pas décroché. Après coup, j’ai entendu dire que les informations n'étaient pas parvenues à Namie ${ }^{66}$, etc. Mais, sur le 
moment, le 11 mars, je m'étais contenté d'ordonner de faire circuler l'information. Nous croulions littéralement sous les demandes d'informations, alors je n'avais pas une connaissance exacte de qui était au courant ou pas.

Q: D'accord. Je suppose que si j'interroge Monsieur XXXXX de l'administration, il saura quelque chose.

R : Là, c'est en relation avec les autorités, alors c'est le travail du groupe " communication ». Je pense que c'est le Group Manager XXXXX qui s'en était occupé.

$\mathbf{Q}$ : À propos de l'installation du VHF, aviez-vous donné des indications particulières en pensant qu'elle pourrait être cruciale en cas d'accident?

$\mathbf{R}$ : Vous voulez dire avant le 11 mars?

Q: Oui, avant

$\mathbf{R}$ : Non, rien de spécial, avant.

Q : Encore une chose que je voudrais vérifier. Je suppose que les manuels de procédures, les AMG, EOP, SOP, AOP et autres, étaient naturellement disposés dans le bâtiment antisismique. Mais pour ce qui était des documents plus techniques, j'ai entendu dire que vous deviez aller les consulter dans le bâtiment administratif. Y a-t-il eu des mouvements pour les entreposer dans le bâtiment antisismique?

$\mathbf{R}$ : Le bâtiment antisismique a été achevé en juillet de l'année dernière. On y a effectivement disposé un minimum de documents. Je ne sais pas si c'était un minimum, mais on y a mis ce qui devait y être. La suite, ça devait être un sujet à débattre. Dans la réalité, ce dont on a eu besoin, c'était des plans très détaillés pour savoir comment était la vanne ou où passait le réseau, etc. S'il avait fallu avoir tout sous la main, à la limite, il aurait fallu apporter l'ensemble des documents de la bibliothèque qui se trouvait dans le bâtiment administratif.

$\mathbf{Q}$ : Cela représente un certain volume.

$\mathbf{R}$ : Oui, une masse énorme. Je pense que d'après les plans, il aurait été difficile de tout apporter. Et puis, il s'agit d'un bâtiment à vocation antisismique. S'il n'y avait pas eu toute cette radioactivité, nous présupposions que nous irions chercher les documents dans le bâtiment administratif.

$\mathbf{Q}$ : Très bien. Encore un point. C'est à propos des camions de pompiers. J'ai bien l'intention d'aller interroger les personnes en charge de la protection incendie pour les détails, mais il me semble que vous aviez eu du mal à trouver les bouches d'incendie et des choses comme ça. Je suppose que lorsque vous programmez des entraînements, vous, en tant que directeur de la centrale, vous avez votre mot à dire. Avez-vous donné des directives pour que l'entraînement aille jusqu'au geste même de relier les tuyaux aux bouches ou qu'on vérifie que, tout au moins les équipes de protection incendie et les pompiers maison, aient connaissance de la localisation des cuves d'eau?

R : Oui, l'entraînement incendie. Sans aller jusqu'à l'injection d'eau dans le réacteur, s'il s'agit du simple entraînement à la lutte contre le feu, je dirais que, depuis que j'étais arrivé comme directeur, je n'ai pas le souvenir d'avoir donné ce genre de directives.

$\mathbf{Q}$ : Vous faisiez confiance au personnel qui était chargé du sujet.

$\mathbf{R}$ : Oui. En fait, après le séisme de Chûetsu, on avait rajouté beaucoup de cuves. Au moment où elles ont été installées, en tirant les leçons de Kashiwazaki, le département " protection incendie » du siège avait organisé beaucoup de séances de formation en activant les responsables « protection incendie » sur les sites, y compris 
à Fukushima. Du coup, je pensais, au contraire, que sur ce sujet tout le monde était tout à fait au point.

Q: D'accord. Nous avons parlé tout à l'heure de l'organisme de soutien. Cette entité est composée de différents groupes, le groupe «production», le groupe «restauration», etc. Après avoir expérimenté cette organisation, avez-vous remarqué certains méfaits de cette partition verticale?

R : L'information, c'est une sorte de guerre. Il faut que chacun fasse correctement ce qu'il a à faire. En même temps, il faut se coordonner les uns avec les autres pour bouger ensemble. Il faut les deux. Il y a des endroits où il faut que ce soit vertical. Je ne pense pas du tout que la partition verticale soit mauvaise. S'il n'y avait pas de personnes qui, respectant une organisation verticale, gardent bien leur position et remplissent leur mission, les choses dans la réalité ne bougeraient pas. Seulement, il faut aussi des gens qui ont une vision un peu plus large, qui ajustent les choses pour que ça marche. Si tout le monde regardait les choses d'en haut pour avoir une vision d'ensemble, leurs mains ne travailleraient plus pendant ce temps. C'est pour ça qu'il faut, à la fois, des gens qui se disent, "je suis pompier, je suis là pour lutter contre l'incendie » ou bien "je dois réparer tel réseau », font leur travail en ne regardant que ça, des gens qui se sentent clairement investis de leur mission, puis, d'un autre côté, des gens qui regardent ces deux hommes et peuvent coordonner leur action. Il faut assurer cette répartition des rôles pour que le tout marche. La tendance générale est à se laisser absorber par son propre domaine. Même les chefs de groupe ne voient plus que le travail de leurs équipes. Du coup, il me revenait à moi de regarder ça. J'étais obligé de dire à chacun, « coordonne ceci et cela ». C'était un travail fastidieux.

$\mathbf{Q}$ : Ce qui veut dire que quand vous avez donné l'ordre d'étudier la possibilité d'utiliser les camions de pompiers comme moyen alternatif d'injection, à qui en avez-vous donné l'ordre?

$\mathbf{R}:$ La « protection incendie ». XXXXX.

$\mathbf{Q}:$ À quel moment lui en avez-vous parlé ?

$\mathbf{R}$ : Le 11, assez tôt. Après avoir constaté la perte totale de l'alimentation électrique, j'ai demandé qu'on réfléchisse à la manière d'assurer l'alimentation en eau, y compris dans le cas où on utiliserait les camions pour injecter. Bien sûr, j'en ai aussi parlé à la "sûreté », mais toutes les manœuvres sur les camions de pompiers étant du ressort de la " protection incendie », bien que je ne sache plus exactement à quel moment, je suis sûr d'en avoir parlé à la " protection incendie ».

Q: Vous avez émis un rapport suivant l'article 15, le 11 à 16h36, pour signaler la perte d'alimentation électrique. Était-ce avant ou après ?

$\mathbf{R}$ : Je me rappelle du déroulement dans son ensemble, mais pas des détails. Si on laisse de côté ce problème de timing, tout d'abord, quand on a perdu toute l'électricité, j'étais très embêté. C'était une situation complètement "hors hypothèse ". Je me demandais ce qu'on allait faire. On n'avait pas l'électricité pour injecter l'eau. On avait encore le RCIC, mais que faire après? Que faire si l'eau n'entrait pas avec la pompe diesel ? Dans quel état était le réseau FP ? J'en discutais avec XXXXX et d'autres, quand quelqu'un a dit « il y a aussi les camions de pompiers ». Et c'est là-dessus que j'ai donné l'ordre qu'on prépare aussi les camions de pompiers. Mais je ne sais pas ce qui se situe en premier, cette discussion ou l'avis. 
$\mathbf{Q}$ : Vous avez donné l'ordre d'injecter via le réseau FP en utilisant les camions de pompiers, vers 17h12. L'ordre s'adressait à Monsieur XXXXX?

$\mathbf{R}:$ Il ne peut y avoir que lui, pour la lutte contre le feu.

$\mathbf{Q}$ : Concrètement, vous lui avez dit, " on va injecter dans le réacteur via le réseau FP, étudie la question »? Vous rappelez-vous en quels termes vous lui avez parlé ?

$\mathbf{R}$ : Je pense que j'ai commencé par lui dire, « on va injecter via le réseau $F P$, on va utiliser le réseau de la pompe diesel, si ça ne marche pas avec la pompe, on va passer aux camions de pompiers, penses-tu qu'on puisse les raccorder au réseau? ". Je ne lui ai pas dit "injecte », mais «est-ce que les camions de pompiers sont encore viables?», "as-tu de l'eau?", " regarde si on peut injecter par cette voie-là ».

$\mathbf{Q}:$ Et qu'a dit Monsieur XXXXX?

$\mathbf{R}$ : Je pense qu'il a dit, «je vais étudier la question ».

$\mathbf{Q}$ : Par la suite, que vous a-t-il dit?

$\mathbf{R}$ : Nous possédions trois camions de pompiers. L'un était inutilisable. Un autre était stationné du côté des réacteurs 5 et 6 , mais les voies d'accès avaient subi de gros dégâts, conséquence, on ne pouvait pas le rapprocher. Finalement, un seul était disponible. Je lui ai ordonné de trouver d'autres camions, d'en faire venir de Fukushima Daini, par exemple.

$\mathbf{Q}:$ C'est ce dont vous vous souvenez.

$\mathbf{R}$ : Oui. Il m'a dit que seul un camion était disponible, alors je lui ai répondu de commencer à faire ce qu'il pouvait avec ce seul camion.

$\mathbf{Q}^{67}$ : Le temps presse. Nous allons nous arrêter là.

$\mathbf{Q}$ : Alors, nous allons faire une pause, si vous voulez fumer. Quelle heure est-il ?

$\mathbf{a}:$ Il est 16h10.

$\mathbf{Q}$ : Je vous remercie beaucoup, Monsieur Yoshida.

(Fin de l'audition)

\section{NOTES}

1. Yoshida est nommé directeur du département " gestion des installations nucléaires » de TEPCO le $1^{\text {er }}$ avril 2007 jusqu'au 28 juin 2010, date à laquelle il est nommé directeur de Fukushima Daiichi.

2. Enquêteur de l'ICANPS présent lors des auditions du 22 juillet, du 29 juillet, du 8 août et du 9 août 2011.

3. Cf. début de l'audition du 22 juillet 2011.

4. Yoshida fait allusion au séisme de Niigataken Chûetsu-oki du 16 juillet 2007, déjà évoqué dans les auditions du 22 juillet, du 8 août et du 9 août 2011. 
5. La centrale nucléaire de Fukushima Daiichi est localisée à 8 kilomètres de la faille de Futaba, d'une longueur d'environ 80 kilomètres. Les montagnes d'Abukuma, situées à l'ouest de la faille, sont composées de roches plutoniques comme le granite. La partie est de la faille est composée de roches sédimentaires.

6. Le séisme de Kobe (magnitude 7,2), survenu le 17 janvier 1995, fait 6434 morts et détruit plus de 100000 bâtiments. Il met en lumière un certain nombre de problèmes concernant les mesures nationales de prévention des catastrophes. En réponse, la loi sur les mesures spéciales de prévention des catastrophes sismiques est promulguée en juillet 1995. Conformément à cette loi, sont fondés les Headquarters for Earthquake Research Promotion, une organisation gouvernementale spéciale d'abord rattachée au Cabinet du Premier ministre, puis au ministère de l'Éducation, de la Culture, des Sports, des Sciences et de la Technologie. Sa mission est de promouvoir la recherche sur les tremblements de terre, afin de renforcer les mesures de prévention et réduire les dommages causés par ce type d'évènement.

7. Péninsule englobant la préfecture de Chiba et formant le bord oriental de la baie de Tôkyô.

8. Le séisme de Sanriku (magnitude 8,5) a lieu le 15 juin 1896 à $19 \mathrm{~h} 32$ (heure locale) durant l'ère Meiji (1868-1912). Son épicentre se situe à environ 166 kilomètres au large de la côte de la préfecture d'Iwate. Le tremblement de terre provoque deux tsunamis, qui causent au moins 22000 morts et détruisent environ 9000 maisons.

9. Spécialiste des tsunamis, actuel Directeur de l'IRIDes (International Research Institute of Disaster Science, Tôhoku University).

10. Erreur de date. Yoshida veut en fait évoquer le séisme de Niigataken du 16 juillet 2007.

11. Tsunami provoqué par le séisme de Valdivia, survenu au Chili le 22 mai 1960 et déjà évoqué par Yoshida dans l'audition du 8 août 2011.

12. Finalement, l'amplitude du tsunami qui ravagera le 11 mars 2011 le site de Fukushima Daiichi sera estimée entre 11,5 et 15,5 mètres.

13. Environ 822 millions d'euros.

14. Masataka Shimizu devient Président de TEPCO en juin 2008. Il le reste jusqu'en 2011.

15. Tsunehisa Katsumata est Président de TEPCO de 2002 à 2008 et Directeur général de 2008 à 2012. Shigemi Tamura, né en 1938 et diplômé de l'Université de Tôhoku en ingénierie, est Directeur général de TEPCO de 2002 à 2008.

16. Hirohiko Izumida, né en septembre 1962, est le gouverneur de la préfecture de Niigata d'octobre 2004 à octobre 2016.

17. Les deux communes de la préfecture de Niigata où se trouve la centrale nucléaire de Kashiwazaki-Kariwa.

18. Environ 2 milliards d'euros.

19. Référence à l'incendie qui se déclare à Kashiwazaki-Kariwa le 16 juillet 2007 à la suite du séisme de Niigataken Chûetsu-Oki.

20. L'Association de Recherche en Technologie Électrique (Electric Technology Research Association - ETRA) est fondée à Tokyo en 1933. Elle se compose de neuf comités spéciaux. L'objectif de cette association est de mener des études et des recherches concernant les questions électrotechniques. Ses résultats sont mis à la 
disposition de ses membres. La coordination et la présidence de l'ETRA sont principalement assurées par l'Université de Tôkyô et TEPCO.

21. Région de l'île de Honshû, située entre le Kansai et le Kantô. Elle est composée des préfectures de Mie, Gifu, Shizuoka et Aichi.

22. Université japonaise fondée en 1897 et située dans la préfecture de Kyoto. L'Université de Kyoto compte environ 22000 étudiants. C'est l'une des plus anciennes universités du Japon.

23. Nobuo Shudô est responsable des évaluations des tsunamis au sein de la Japan Society of Civil Engineers et professeur honoraire à l'Université de Tôhoku. Shudô est la première personne à être auditionnée par les enquêteurs de l'ICANPS. Son audition a lieu le 6 juillet 2011 (cf. tableau des auditions).

24. Kenji Satake, chercheur à l'Université de Tôkyô et auteur de « Numerical Simulation of the AD 869 Jôgan Tsunami in Ishinomaki and Sendai Plains » (2008).

25. L'article du Professeur Satake (2008) est cité dans le rapport de l'ICANPS (2012), de la NRC américaine (2014) et de l'AIEA (AIEA, 2015b) - la NAIIC (2012) ne cite pas nommément l'article mais y fait indirectement référence - afin de souligner que, malgré sa connaissance de cette étude, la direction de TEPCO n'avait pas pris de mesures pour mieux protéger Fukushima Daiichi contre le risque d'un tel tsunami. Tsunehisa Katsumata (pour rappel, Président de TEPCO de 2002 à 2008 et Directeur général de 2008 à 2012), Sakae Mutô (vice-Président en mars 2011) et Ichiro Takekuro (vice-Président jusqu'en 2010 et fellow en mars 2011), accusés d'avoir ignoré ce risque, sont poursuivis en 2015 pour « négligence professionnelle ayant entraîné la mort et des blessures ", et notamment le décès de quarante-quatre patients d'un hôpital voisin de la centrale pendant leur évacuation. Le procès a lieu en décembre 2018. Le procureur réclame une peine de cinq ans de prison ferme. Le verdict est rendu en septembre 2019 et les trois dirigeants sont finalement acquittés (AFP, 2019).

26. Zhenguan Zhengyao (Leçons politiques de l'ère Zhenguan) de Wu Jing.

27. En Chine de 626 à 649 , au Japon de 859 à 877.

28. Ikuo Abe, chercheur à l'Institut International de Recherche en Science des Désastres.

29. «Un parmi d'autres ».

30. Université japonaise fondée en 1907 et située à Sendai dans la préfecture de Miyagi. L'Université de Tôhoku compte environ 18000 étudiants.

31. Yukinobu Okamura, né en 1955, est sismologue au National Institute of Advanced Industrial Science and Technology (AIST).

32. Tôshiba a construit les réacteurs 1 à 3 de la centrale de Kashiwazaki et Hitachi les réacteurs 4 et 5 . Les réacteurs 6 et 7 ont été construits par Tôshiba, Hitachi et General Electric.

33. La JNES a pour mission d'établir les bases permettant d'assurer la sûreté nucléaire en menant des analyses de sûreté et en effectuant l'inspection des installations nucléaires au Japon en soutien de la NISA. Cette organisation est créée en 2003 et fusionne avec la NRA en 2013.

34. Shigeki Nakura est un inspecteur de la NISA.

35. L'enquêteur semble faire référence à Takashi Kobayashi, Directeur de 2009 à 2011 du Bureau d'énergie thermique d'Higashi. TEPCO exploite la centrale thermique 
d'Higashi-ôhgishima, construite sur une île artificielle située au large du port de Kawasaki dans la préfecture de Kanagawa. Cette centrale, composée de deux unités (mises en service respectivement en septembre 1987 et en mars 1991), utilise du Gaz Naturel Liquéfié (GNL) comme carburant. En avril 2015, est fondée la société JERA, détenue à parts égales par TEPCO Fuel \& Power (filiale de TEPCO) et Chûbu Electric Power, qui gère depuis la totalité des centrales thermiques des deux exploitants.

36. À cette date, le gouverneur de la préfecture de Fukushima est Yuhei Satô.

37. Combustible nucléaire à base d'un mélange d'oxydes d'uranium appauvri et de plutonium.

38. Un communiqué d'Areva du 30 juin 1999 annonce qu'un transport vers le Japon de combustibles MOX, fabriqués en Europe, est prévu pour la fin de l'année. Les assemblages concernés sont destinés aux réacteurs nucléaires de Fukushima Daiichi et de Takahama, une centrale nucléaire située dans la préfecture de Fukui et exploitée par Kansai Electric Power Company (KEPCO). Eisaku Satô, gouverneur de Fukushima de 1988 à 2006, est dans un premier temps favorable à l'utilisation du MOX. Mais à la suite du scandale liés aux rapports d'inspection falsifiés de TEPCO en 2002 (cf. audition du 22 juillet 2011), le gouverneur retire son soutien, et l'exploitant décide de suspendre l'utilisation du MOX dans ses réacteurs (Pelletier, 2012). En 2006, Eisaku Satô est accusé de corruption dans la construction d'un barrage et il est contraint de démissionner. Le 16 février 2010, Yûhei Satô, gouverneur depuis novembre 2006, soumet - comme le précise plus haut l'enquêteur de l'ICANPS - plusieurs conditions pour envisager l'utilisation du MOX à Fukushima Daiichi. Quelques mois plus tard, en septembre 2010, le réacteur 3 fonctionne avec $30 \%$ de MOX fourni par Areva.

39. Même si ce n'est pas prévu par la loi, les exploitants souhaitant utiliser du combustible MOX dans un de leurs réacteurs demandent généralement l'autorisation du gouverneur. Néanmoins, nos recherches ne permettent pas de spécifier quelles sont exactement les exigences auxquelles TEPCO a dû répondre pour obtenir ladite autorisation à Fukushima Daiichi.

40. Yoshinori Moriyama occupe en 2009 un poste de responsable de service à la NISA chargé des risques d'accident sur des installations nucléaires. Lorsque l'accident de Fukushima se produit le 11 mars 2011, il travaille au ministère de l'Éducation, de la Culture, des Sports, des Sciences et de la Technologie. Il aide toutefois la NISA à répondre à la crise nucléaire.

41. Agence fondée en 1973, placée sous la tutelle du METI. Sa mission est de définir les politiques énergétiques et de gestion des ressources naturelles du Japon.

42. Il semble que Yoshida mentionne Tetsuo Noguchi, alors chef d'inspecteurs au sein de la NISA.

43. Dans un communiqué de presse daté du 20 mai 2011, TEPCO annonce sa décision de démanteler les tranches 1 à 4 de Fukushima Daiichi et de renoncer au projet de construction des tranches 7 et 8 . L'exploitant devait commencer la construction de ces deux tranches en avril 2012 pour la première et 2013 pour la seconde. Leur mise en service était prévue respectivement en octobre 2016 et octobre 2017.

44. En janvier 2011, TEPCO débute la construction d'une centrale nucléaire à Higashidôri, dans la préfecture d'Aomori (Tôhoku). Le chantier est situé près d'une centrale voisine, portant le même nom et exploitée par la Tôhoku Electric Power Company. La centrale de TEPCO devait être composée de deux réacteurs nucléaires. Le 
$\mathrm{n}^{\circ} 1$ devait initialement démarrer en mars 2017, mais l'accident de Fukushima Daiichi contraint TEPCO à arrêter les travaux et à suspendre le projet. L'exploitant le relance en juillet 2018. Mais du fait des conséquences financières de l'accident de Fukushima Daiichi, TEPCO ne peut plus mener seul la construction de la centrale. Il envisage donc de partager les coûts de construction avec d'autres entreprises japonaises, telles que la Tôhoku Electric Power Company, la Chubu Electric Power Company et la Japan Atomic Power Company. La décision concernant cette éventuelle collaboration ne sera prise qu'après la publication d'une série d'études sur le risque sismique auquel le site de construction est exposé.

45. «Ligne d'essai ».

46. Conditions limites d'exploitation sûre; elles désignent des situations où des systèmes de sûreté sont à leur niveau minimal de performance. Préalablement identifiées, elles sont mentionnées dans les Spécifications Techniques.

47. Cf. audition du 8 août 2011. Pour rappel, il s'agit d'un texte rédigé en mai 2002 par TEPCO décrivant les nouvelles dispositions à suivre en cas d'accident.

48. L'incident d'octobre 1991 est évoqué avec l'enquêteur Tsunemasa Katô dans les auditions du 22 juillet et du 8 août 2011.

49. Études (ou Évaluations) Probabilistes de Sûreté. Il s'agit d'une approche très répandue dans le domaine de l'industrie nucléaire, qui repose sur une conception déterministe et quantitative de la sûreté. Celle-ci doit permettre d'identifier les évènements susceptibles de menacer la sûreté, de calculer la probabilité de leur occurrence et de mesurer leurs conséquences.

50. Exigences de la Nuclear Regulatory Commission (NRC) américaine portant sur la perte d'alimentations électriques et les stratégies de prévention d'un accident.

51. La station de transformation de Shin-Fukushima est localisée dans le bourg de Tomioka, situé à environ 8 kilomètres de Fukushima Daiichi. La centrale nucléaire disposait d'une source d'alimentation électrique externe, fournie par la Tôhoku Electric Power Company et acheminée par une ligne de $66 \mathrm{kV}$ depuis la station de ShinFukushima. Le séisme du 11 mars 2011 a toutefois coupé cette ligne et Fukushima Daiichi a ainsi perdu cette source d'alimentation externe avant même la survenue du tsunami.

52. Fin 2011, le parc nucléaire mondial compte 435 unités connectées au réseau, 61 unités en construction et 138 unités arrêtées (CEA, 2012). Fin 2018, 447 unités sont connectées au réseau, 53 unités sont en construction et 176 unités sont arrêtées (CEA, 2019).

53. Les réacteurs expérimentaux (ou de recherche) sont des installations nucléaires dans lesquelles une réaction en chaîne est créée et entretenue pour obtenir un flux de neutrons, afin de répondre à un usage d'expérimentation. Les premiers réacteurs expérimentaux sont construits aux États-Unis, en URSS, en France, en Grande-Bretagne et au Canada. Concernant le démarrage des réacteurs commerciaux dans les années soixante, Yoshida a certainement à l'esprit les réacteurs japonais, en particulier le premier, Tôkai-1, construit dans la centrale nucléaire de Tôkai. Sa divergence a lieu en 1965 et il est mis en service en 1966. Il est à l'arrêt définitif depuis 1998.

54. Les premiers réacteurs nucléaires à produire de l'électricité sont EBR-1 (États-Unis, Idaho) le 20 décembre 1951, la centrale d'Obninsk (Union soviétique, Russie) le 27 juin 1954, le réacteur G1 dans le centre du CEA Marcoule (Gard) le 7 janvier 1956, la centrale 
de Calder Hall (Royaume-Uni, Angleterre) le 27 août 1956. General Electric commence à construire en mai 1956 un premier réacteur commercial, de type REB, dénommé Dresden-1 (centrale nucléaire de Dresden, États-Unis, Illinois). En juin 1960, Dresden-1 atteint sa pleine puissance. Sa réalisation lance le début de la commercialisation des REB, aux États-Unis puis à l'international, par des constructeurs américains, japonais et européens (Grenêche, 2016).

55. Il s'agit du produit du nombre de réacteurs par le nombre d'années pendant lesquels ils ont été exploités. Une année-réacteur correspond ainsi à une année pendant laquelle un réacteur a fonctionné. Dix années-réacteurs peuvent renvoyer à l'exploitation de cinq réacteurs pendant deux ans; ou de deux réacteurs pendant cinq ans. L'utilisation de ce produit reflète les années d'expérience acquise dans l'exploitation des réacteurs nucléaires. Il est également utilisé pour illustrer les probabilités d'occurrence d'évènements sur la centrale, notamment les accidents systémiques.

56. Lignes électriques en provenance d'ôkuma et de Futaba, deux bourgs mitoyens de Fukushima Daiichi.

57. Ligne à part fournie par Tôhoku Electric Power, prévue pour alimenter la centrale en cas d'incident.

58. Yoshida est muté à la FEPC en juillet 1995 et y reste jusqu'en 1999. Il est ensuite nommé directeur du département "production » de Fukushima Daini en 1999, poste qu'il conserve jusqu'en 2002 (cf. début de l'audition du 22 juillet 2011).

59. Il occupe le poste de chef du service « maintenance » des réacteurs 1 et 2 de 1993 à 1995 (cf. début de l'audition du 22 juillet 2011).

60. Mentionné dans les auditions précédentes par le sigle «MUWC " (Make-Up Water Condensate system).

61. Réservoir de stockage de condensats.

62. Mentionné précédemment par le sigle «RHR ( (Residual Heat Removal system).

63. Pour rappel, les installations de classe $S$ sont les installations conçues pour être les plus résistantes aux séismes, notamment du fait de leur importance pour la sûreté nucléaire.

64. Une radio VHF (Very High Frequency) émet sur une bande à très hautes fréquences, s'étendant de $30 \mathrm{MHz}$ à $300 \mathrm{MHz}$.

65. Le Paging est un système de radiomessagerie unidirectionnel : le destinataire reçoit le message et le numéro d'envoi sur son terminal, mais ne peut pas y répondre.

66. Bourg de la préfecture de Fukushima, situé à une dizaine de kilomètres de Fukushima Daiichi.

67. Plusieurs enquêteurs étaient présents lors de cette audition. 


\section{Compte rendu de l'audition du 6 novembre 2011 (II)}

1 [À traiter avec la plus grande attention]

25 novembre 2011

\section{Compte rendu d'audition}

2 Tsunemasa Katô

Membre du secrétariat de la Commission d'enquête sur l'accident des centrales nucléaires de Fukushima de Tôkyô Electric Power Company (Investigation Commitee on the Accident at the Fukushima Nuclear Power Stations of Tôkyô Electric Power Company)

Ci-dessous le compte rendu de l'audition du 6 novembre 2011, menée dans le cadre de l'enquête sur l'accident des centrales nucléaires de Fukushima de Tôkyô Electric Power Company.

1. Auditionné, date d'audition, lieu d'audition, enquêteurs.

1. Auditionné

Masao Yoshida, Directeur de la centrale nucléaire Fukushima Daiichi de Tôkyô Electric Power Company.

2. Date d'audition

6 novembre 2011 de $16 \mathrm{~h} 27$ à 19 h02 (pause de $17 \mathrm{~h} 50$ à 17h55)

3. Lieu d'audition

Salle de réunion $\mathrm{A}$

Foyer masculin de la Japan Football Association Academy de Fukushima ( $1^{\mathrm{er}}$ étage)

J-Village

8-Utsukushimori Yamadaoka, Naraha, Futaba District, Fukushima 
4. Enquêteurs

Tsunemasa Katô, Hiroko Okuzawa.

5. Enregistrement par enregistreur numérique

Oui

$\square$ Non

2. Contenu de l'audition

Situation au moment de l'accident et réponses à l'accident

Voir le compte rendu

3. Mentions spéciales

Pas de mention spéciale.

QUESTION : Pour ma part, je voudrais approfondir un certain nombre de points que nous avons déjà abordés. De plus, depuis la dernière fois, j'ai eu l'occasion d'auditionner Monsieur Takekuro, d'autres personnes de TEPCO, ainsi que les personnes qui se sont retrouvées autour du Premier ministre durant la gestion de l'accident, comme le directeur de la NISA'. En conséquence, j'aimerais croiser votre témoignage avec certaines de leurs déclarations.

Si vous permettez, revenons au moment où vous aviez perdu pratiquement toute votre alimentation électrique après la survenue du tsunami. Des tests ont été pratiqués par TEPCO sur le système IC le $7^{\mathrm{er}}$ avril pour savoir comment étaient positionnées les vannes d'isolation. À quel degré étaient-elles ouvertes? Etaient-elles totalement ouvertes? Totalement fermées? Ou bien, étaient-elles à moitié ouvertes ? C'est ce qu'on a cherché à vérifier en mettant le circuit sous tension, etc.

Les résultats obtenus n'étaient pas en nette contradiction avec ce que les membres de l'équipe de quart, par exemple, avaient déclaré lors de leurs auditions. II existe deux réseaux sur ce système: le réseau $A$ et le réseau $B$. Sur le réseau $B$, toutes les vannes situées à l'extérieur de l'enceinte de confinement étaient fermées. Le système de sécurité avait probablement fonctionné. De plus, le réseau $B$ ne devait pas être utilisé et on avait fermé en conséquence la troisième vanne. L'un dans l'autre, il était tout à fait logique que ce soit fermé. Seulement, à l'intérieur, la vanne était à moitié ouverte. Bien qu'elle ait été à moitié ouverte, quand on observe le volume d'eau dans le réservoir, il n'avait pas beaucoup diminué. Dans le réseau A également, il restait encore environ $65 \%$ du volume, ce qui laisse supposer que même si l'IC avait effectivement fonctionné, les vannes ne devaient pas être très ouvertes. Ces constatations vont tout à fait dans le sens de ce que j'ai entendu lors des conversations que j'ai eues avec les uns et les autres.

Après ces recherches, vous-même êtes allé sur place le 18 octobre dernier et avez constaté de visu l'eau qui restait dans le réservoir, je crois qu'on commence à comprendre peu à peu certaines choses. Une des choses que le grand public a découvertes à cette occasion, j'imagine, est le système de sécurité. Quand on y regarde aujourd'hui, d'un côté, on peut dire que ce système a bien fonctionné, mais que, de l'autre, il y a eu ce problème de la vanne à moitié ouverte. Je pense que la vanne est restée dans cette position parce qu'avant qu'elle ne se ferme tout à fait, l'alimentation électrique, je suppose que c'est du courant alternatif à l'intérieur de l'enceinte, a été coupée. Du coup, on peut présumer que la fermeture ne s'est pas faite complètement et que la vanne est restée à moitié ouverte dans la position dans laquelle elle se trouvait au moment où l'électricité a été interrompue. Dans quelle mesure, les agents de la TEPCO, ceux qui étaient directement impliqués dans le domaine du nucléaire, savaient-ils que ces vannes se fermaient quand le système de sécurité était enclenché ? Je suppose que cette connaissance diffère selon la fonction de chacun.

Réponse : Si on limite la discussion à l'IC, je dirais que seules les équipes de quart des réacteurs 1 et 2 étaient au courant. Les autres n'en savaient presque rien. Parce que l'IC n'est installé que sur le réacteur 1 . À partir du réacteur 2 , on a le système RCIC. 
Quant aux $\mathrm{ABR}^{2}$, ils disposent d'un système totalement différent. Vous savez, l'IC est un système très spécial. Pour être franc, je n'en sais pas moi-même grand-chose.

$\mathbf{Q}$ : Dans la conception de base des réacteurs nucléaires, quand il y a une rupture sur le réseau d'un système directement relié à l'enceinte ou à la cuve, comme le $\mathrm{RCIC}$ ou le $\mathrm{HPCl}$, ces vannes se ferment d'un coup, automatiquement. C'est ce qu'on m'a expliqué au siège social. Ce qui s'est donc passé, c'est que même en ayant perdu l'électricité, structurellement, ça s'est fermé d'un coup. Le réseau de détection des ruptures est toujours sous tension. C'est lorsque celle-ci n'est plus détectée qu'il interprète qu'il y a rupture.

$\mathbf{R}$ : Concernant le fonctionnement des vannes d'isolation de l'enceinte de confinement, nous en avons tous une certaine idée. Mais s'agissant plus spécialement du système IC, il faut reconnaître que nos connaissances sont particulièrement pauvres. La réalité, c'est qu'il n'y a guère que les personnes qui ont conçu le réacteur 1 de Fukushima Daiichi et les agents qui ont effectivement eu à le conduire, qui ont une compréhension fine des manœuvres de l'IC.

$\mathbf{Q}$ : J'insiste sur ce point, car, comme vous vous en êtes sans doute rendu compte, un ou des importuns, on ne sait pas qui exactement, a laissé échapper des informations que j'avais collectées pour le compte de notre commission. Je suppose que vous avez dû les voir dans la presse. II est tout à fait naturel que ceux qui étaient de quart au moment de l'accident aient raisonné à leur manière et je le comprends très bien. Parmi les témoignages, il y a une personne, de l'équipe de quart justement, qui m'a confié que le 11 mars à 18h25, elle avait fermé la vanne 3 . Or il se trouve que personne, ni au siège, ni à la cellule de crise, n'en avait conscience. II y a visiblement eu là un quiproquo. Même en ne sachant pas exactement ce qui se ferme sur le réseau quand le système de sécurité se met en marche, n'y a-t-il pas eu quelqu'un pour s'inquiéter de savoir si, par hasard, une vanne isolant l'enceinte de confinement ne serait pas fermée? Quelqu'un du siège aurait pu y penser, puisque, à mon avis, étant loin du terrain et n'ayant pas à résoudre de problèmes immédiats, ils avaient le temps de réfléchir. Pour moi, c'est le type même de tâches qui leur incombe, vous ne trouvez pas ? Enfin, ça aurait bien sûr pu venir aussi de la centrale.

$\mathbf{R}$ : Non, personne n'y a pensé.

$\mathbf{Q}:$ Vraiment rien?

$\mathbf{R}$ : Non, il n'y a eu aucune suggestion de la part du siège concernant les manœuvres de l'IC.

$\mathbf{Q}$ : Si on regarde en arrière aujourd'hui... Enfin, c'est ce qu'on voit à l'observation de toutes les analyses qui ont été menées depuis. D'ailleurs, chez TEPCO, vous avez commencé les analyses assez vite après l'accident. Bref, si on formule l'hypothèse que I'IC n'avait pas fonctionné pleinement, il convient de vérifier certaines choses qui pourraient étayer cette thèse, dans le cadre de ces analyses. Or, le jour de l'accident, vers 16h45, vous aviez réussi à voir le niveau d'eau du réacteur 1 pendant un court moment. Je pense qu'il s'agissait du niveau d'eau dans la grande largeur. Et là, l'eau était à environ $-90 \mathrm{~cm}$. Le niveau va rester visible durant un peu plus d'une dizaine de minutes. À la fin de ce laps de temps, l'eau n'est plus qu'à environ - $150 \mathrm{~cm}$, ce qui signifie que le niveau baissait. II baissait même très vite. Le groupe "technique » réalise une estimation à ce moment-là, j'ai moi-même refait le calcul, et arrive à la conclusion que si l'eau continue à baisser à ce rythme de $60 \mathrm{~cm}$ toutes les dizaines ou douzaines de minutes, le TAF serait atteint dans les soixante minutes.

$\mathbf{R}:$ Je n'en avais pas entendu parler.

$\mathbf{Q}$ : Au moment de l'accident, vous voulez dire? Vous ne l'aviez pas su?

$\mathbf{R}$ : Pas du tout. 
$\mathbf{Q}$ : Mais imaginez que vous l'ayez su, si vous pensiez que l'IC fonctionnait à plein régime, vous vous seriez demandé pourquoi ces chiffres, non?

R : Oui, je l'aurais pensé. Mais je n'ai pas le souvenir que le niveau d'eau baissait. En tout cas, à ce moment-là.

$\mathbf{Q}$ : À peu près au même moment, à 17h12, comme on l'a rapidement évoqué précédemment, vous donnez l'ordre d'étudier les moyens substitutifs d'injection d'eau. Cela correspond quasiment au moment où cette baisse importante était constatée. Aviez-vous pensé, quand vous avez formulé cet ordre, que vous ne pouviez pas faire entièrement confiance à I'IC ?

\section{$\mathbf{R}:$ Pas du tout.}

$\mathbf{Q}:$ : I n'y avait pas de lien entre les deux?

$\mathbf{R}$ : Non. En tout cas, pas au début. Comme je vous l'ai déjà dit en août, concernant l'IC, bien qu'aujourd'hui on ait des discussions sur la position des vannes d'isolation, j'avais été informé par les agents de production que le système s'était mis en route au moment du séisme et, pour moi, une fois mis en route, il avait continué à fonctionner normalement par la suite.

Pour ce qui est de ces histoires de niveau d'eau, je ne sais qui avait fait ces calculs, mais, très probablement, il ne s'en est pas ouvert devant tout le monde dans la cellule de crise.

$\mathbf{Q}$ : D'après des notes du groupe « communication » de Fukushima Daiichi, on lit, juste après la ligne concernant 16h55, «Niveau d'eau visible. Niveau actuellement en baisse. Prévoir le moment où le TAF sera atteint. Groupe 'technique'».

\section{$\mathbf{R}$ : Vous voulez dire que j'ai dit ça?}

$\mathbf{Q}$ : Non, on ne sait pas qui a parlé. Ça n'a pas été enregistré dans les vidéos des téléconférences. Alors je suis obligé d'essayer d'obtenir des informations en regardant des notes, des mémos, etc. Si on regarde la suite, à 17h15, on a: "Groupe «technique». Réacteur 1, baisse du niveau d'eau. - $150 \mathrm{~cm}$ à la dernière observation. Si la baisse continue, TAF atteint dans une heure ». Ce qui correspond à ce que je viens de vous détailler. En fait, une personne de Kashiwazaki-Kariwa a également noté les mêmes chiffres. Ce qui laisse supposer qu'il les a sus par la téléconférence.

$\mathbf{R}$ : Oui, on a dû en parler en téléconférence.

$\mathbf{Q}$ : Je suppose. Plutôt que vous, je pense que c'est le chef du groupe «technique » qui a dû en parler, histoire de partager l'information. J'imagine que, de votre point de vue, c'était un moment où la situation se dégradait de plus en plus, où vous étiez assailli de nouvelles plus inquiétantes les unes que les autres. Dans une telle situation, demander au seul directeur de la centrale de maîtriser toutes les informations me paraît surhumain. C'est pour ça qu'il y a des groupes opérationnels, qui ont été définis. Chaque groupe se saisit de l'information qui concerne sa mission et en tire les conséquences. Si tout ne peut pas être fait au sein de la centrale, le siège peut remplir ce rôle. De fait, de temps en temps, le directeur de Kashiwazaki ou celui de Fukushima Daini intervenait pour émettre des suggestions basées sur leur expérience. Sachant que le niveau d'eau baissait dans le réacteur 1, quelqu'un aurait pu se demander pourquoi et émettre I'hypothèse que peut-être l'IC ne fonctionnait pas bien.

$\mathbf{R}$ : Si je l'avais su, j'aurais demandé, en effet, qu'on examine cette possibilité.

$\mathbf{Q}$ : Mais vous n'en avez pas souvenir.

$\mathbf{R}$ : Non, pas du tout. 
$\mathbf{Q}$ : Vous ne vous rappelez même pas que quelqu'un ait pu vous le suggérer?

$\mathbf{R}$ : Non. Comme je vous l'ai dit en août, pour ma part, je ne me suis pas inquiété du sort de l'IC jusqu'au moment où on a vu la pression de la cuve pour la première fois, ou bien le moment où la pression de l'enceinte de confinement était montée, je ne sais plus très bien. Bien sûr, c'est un point que je regrette. J'aurais dû redemander comment se comportait l'IC avant cela. Malheureusement, il ne me reste aucune trace de ça dans ma mémoire. Je ne me rappelle pas avoir donné de directives concernant l'IC.

$\mathbf{Q}$ : Plus tard, le groupe « information» a noté à propos de la tranche 1, « hausse des chiffres de l'APD à proximité de la double porte du bâtiment réacteur". D'après l'agent qui était de quart, la hausse n'était pas très importante. En fait, il était justement parti vérifier le niveau d'eau du réservoir du condenseur, quand il s'est rendu compte que la radioactivité près de la double porte était plus élevée qu'à l'ordinaire. Du coup, il avait rebroussé chemin. D'après les conclusions des analystes, si l'IC n'avait pas fonctionné correctement, le TAF aurait été justement atteint vers 18h00. La radioactivité aurait donc augmenté vers cette heure-là. En aviez-vous eu conscience à l'époque, ou pas?

\section{$\mathbf{R}$ : Je ne l'ai su que plus tard.}

$\mathbf{Q}$ : Dans les faits, vous interdisez formellement l'accès à ce bâtiment un peu avant 22h00. À cette heure-là, la radioactivité est forte et il n'est plus question d'entrer à l'intérieur. De la constatation de l'agent à votre interdiction, la radioactivité va augmenter, mais pas forcément de manière brutale. Elle a dû monter petit à petit. Pendant tout ce temps, vous pensiez que l'IC fonctionnait normalement.

R : Oui.

$\mathbf{Q}$ : Avez-vous le souvenir de quelqu'un attirant votre attention sur le fait que l'IC aurait pu avoir des problèmes?

$\mathbf{R}:$ Non.

$\mathbf{Q}$ : Ensuite, vers 18h25, au moment où l'agent prétend avoir fermé la vanne d'isolation, ou plutôt peu après, vous rappelez-vous avoir étudié la possibilité de ravitailler ce fameux réservoir du condenseur, dont le niveau d'eau avait probablement baissé, en passant par la ligne FP en utilisant la pompe diesel ?

$\mathbf{R}$ : Là, vous parlez du réservoir du condenseur ${ }^{3}$ ?

$\mathbf{Q}$ : Oui, je veux dire le réservoir de l'IC. Vous avez bien un réservoir qui refroidit l'IC ?

$\mathbf{R}$ : Ah, pas l'échangeur de chaleur, mais le réservoir qui alimente la ligne de l'IC ?

$\mathbf{Q}$ : Vous avez le réservoir du condenseur ici, avec l'eau qui circule là. En fait, vous avez deux réseaux, le $A$ et le $B$.

$\mathbf{R}$ : Vous voulez donc parler de l'échangeur de chaleur.

$\mathbf{Q}$ : Oui, l'échangeur de chaleur, où doit se trouver de l'eau.

$\mathbf{R}$ : Pour le refroidissement.

$\mathbf{Q}$ : Il semblerait qu'il y a moyen de l'alimenter par le réseau FP. N'avez-vous pas le souvenir d'en avoir discuté ?

R : Là, plutôt que d'envoyer de l'eau dans l'échangeur de chaleur du condenseur, je pensais en fait à utiliser le DDFP pour injecter en fin de compte de l'eau dans le réacteur. Je crois qu'il y a eu un malentendu, là.

$\mathbf{Q}$ : Effectivement, il y a des gens qui ont pensé comme vous et puis d'autres, on le voit bien dans ces quelques mémos, qui ont pensé au condenseur de l'IC. Les réactions étaient divisées. D'après ce que j'ai pu vérifier, les gens qui étaient de quart s'activaient bien à élaborer une ligne pour injecter dans le réacteur, comme vous pensiez le faire. II y a donc eu 
deux interprétations complètement différentes. Vous rappelez-vous avoir évoqué l'autre possibilité, en avoir parlé à la table de la cellule de crise ou en avoir reçu rapport du groupe " production », de Monsieur XXXXX, par exemple?

$\mathbf{R}$ : Non, pas du tout. Pour moi, je partais du principe que l'IC et les RCIC de chaque tranche étaient en fonctionnement. Mais je savais aussi qu'il allait arriver un moment où ils allaient s'arrêter. Il y avait donc nécessité d'élaborer un circuit pour injecter dans le réacteur en passant par le réseau FP. De plus, s'il fallait pour ça utiliser la pompe DD, il fallait prévoir ce circuit en conséquence. Tous mes ordres allaient dans ce sens. Et en plus de ça, il fallait aussi prévoir le cas où la solution de la pompe DD ne fonctionnerait pas. C'est pour ça qu'on s'intéressait à la question des camions de pompiers. Bien entendu, j'en avais déjà avisé le chef du groupe «protection incendie $»$.

$\mathbf{Q}$ : Tout ce que vous me racontez là, c'est ce qui se passait vers $17 \mathrm{~h} 12$ ou dans ses environs?

$\mathbf{R}$ : Tout à fait. Je n'ai donc pas du tout le souvenir, concernant la tranche 1, d'avoir donné des directives particulières concernant sa conduite. Ma position de base, à ce moment-là, était de faire préparer un circuit FP pour l'injection dans le réacteur. Je n'avais absolument pas en tête l'idée d'envoyer de l'eau dans le réservoir de l'IC.

$\mathbf{Q}$ : Mais, supposons qu'on envisage d'envoyer de l'eau dans le réservoir de l'IC, si on pense que cela s'impose, c'est qu'on pense aussi que la vapeur qui arrive du réacteur n'est peutêtre plus suffisamment refroidie. Si on le pense, cela veut dire que la vapeur s'en retourne telle quelle vers le réacteur et que celui-ci n'est peut-être plus non plus suffisamment refroidi. Ce qui amène à soupçonner que l'IC ne fonctionne pas bien. Si, là-dessus, le siège s'était enquis par exemple du fonctionnement de l'IC, les agents auraient répondu qu'ils avaient fermé une vanne et on aurait pu imaginer que cela aurait résolu le quiproquo. Mais ce n'est pas comme ça que les choses se sont passées.

$\mathbf{R}$ : Non. Moi, je raisonnais comme je vous l'ai dit et je ne sais pas ce que XXXXX ou un autre avait compris des ordres que j'avais donnés, mais je les avais émis dans ce sens. De plus, aucune information concernant la conduite de l'IC n'est remontée du terrain. Vous m'avez parlé tout à l'heure de l'histoire du groupe "communication ", c'est quelque chose qui a totalement échappé à ma mémoire. Pourquoi ? Peut-être est-ce tombé au moment où j'étais en communication avec le siège ? Mais de toute manière, s'agissant d'une information de cette importance, le chef de groupe aurait dû insister beaucoup plus.

$\mathbf{Q}$ : Je le pense aussi. Parce que celui qui a formulé ces chiffres avait bien conscience de tout ce que cela impliquait.

$\mathbf{R}$ : Quelqu'un aurait dû l'entendre et en tirer les conséquences.

$\mathbf{Q}$ : Oui, parce que cela aurait changé les dispositions ultérieures concernant la conduite. Vous savez que l'agent de quart qui a noté ces chiffres les a suivis minute par minute? Comme il ne pouvait pas les enregistrer, il les a écrits à la main directement sur le tableau de commandes. On les voit encore.

$\mathbf{R}$ : Dans la salle de contrôle?

$\mathbf{Q}$ : Oui, dans la salle de contrôle. Il était de quart et comme il n'avait plus d'électricité, pour ne pas les oublier, il les a notés en vrac. Je l'ai vu sur la photo. Son écriture était très déformée. Du point de vue horaire, ça correspond tout à fait. On peut supposer que l'agent de quart, en voyant ces chiffres, s'est dit qu'il fallait qu'il aille vérifier le niveau d'eau dans le réservoir du condenseur. Mais il n'a pas pu y accéder à cause de la montée de la radioactivité. II a dû rebrousser chemin. Là, il a dû se dire logiquement que quelque chose n'allait peut-être pas dans le fonctionnement de l'IC. Ensuite, quand il a ouvert vers 18h18, il 
a constaté que la quantité de vapeur était faible. II a refermé en se disant que cela n'allait pas bien. C'est probablement ce qui s'est passé et cela paraît logique, si on a tous les éléments en main.

Pour en revenir aux moyens substitutifs d'injection que vous envisagiez, vous auriez pu disposer du SLC, si l'électricité avait été rétablie, mais cela n'était pas possible sur le moment. Du coup, vous pensez à la DDFP. Mais si la pompe DD ne marchait pas... Et vous arrivez à la solution des camions de pompiers. Si vous adoptiez cette solution, il devenait nécessaire de faire baisser la pression pour que l'eau puisse entrer. Dans ce cas, si l'IC fonctionnait correctement, la tactique d'une dépressurisation suffisante durant l'action de I'IC, suivie d'une injection à basse pression aurait été possible. Mais si ce n'était pas le cas, il fallait imaginer une autre manière de dépressuriser, si on voulait que l'eau entre, puisque les systèmes à haute pression n'étaient pas disponibles. Le HPCl n'était pas disponible, au niveau de la tranche 1. Si même le SLC n'était pas utilisable tout de suite, il fallait impérativement dépressuriser.

II se trouve que, quand vous avez mesuré la pression vers 2 h45, par hasard, celle-ci était déjà brutalement tombée à $0,8^{4}$. Je ne sais pas s'il faut dire que vous avez eu de la chance ou que vous avez joué de malchance, toujours est-il que vous avez tout de suite trouvé les conditions pour pouvoir envoyer de l'eau. Mais imaginez que la pression n'était pas descendue, des manœuvres de dépressurisation se seraient imposées. Habituellement, il vous aurait sans doute suffi de pousser sur un bouton sur le tableau de la salle de contrôle pour ouvrir la vanne SR. Mais, cette fois-ci, vous ne le pouviez pas. Aviez-vous conscience dès ce moment que l'ouverture de cette vanne ne pourrait pas se faire en vous contentant de manœuvrer à partir de la salle de commande?

$\mathbf{R}$ : À ce moment-là, je n'avais pratiquement aucune conscience de ce qui se passait sur le terrain. Par exemple, je n'avais pas véritablement conscience qu'on ne pourrait plus manœuvrer cette vanne de manière habituelle. C'est le genre de choses qu'on a découvert au fur et à mesure. Nous étions mobilisés sur les tranches 3 et 2. Et, effectivement, je voyais toutes les difficultés qu'on rencontrait à exécuter la moindre manœuvre. Malgré tout, je n'arrivais pas encore à imaginer toute la répercussion que pouvait avoir la perte de l'alimentation électrique ou de l'alimentation en air sur la manipulation de chacun des appareils sur le terrain. Dans quel état se sont trouvés les différents appareils, au moment où on a perdu toute source d'alimentation électrique? Cela dépendait sûrement des endroits, mais même ce genre d'informations ne se transmettait du terrain que de manière très limitée. On n'arrivait pas à imaginer à quel point ils étaient démunis sur place. Comme je vous l'ai déjà dit, je me suis révolté contre les gars du siège, je leur ai reproché de ne rien voir, d'être dans leur bulle. Mais je suis sûr que les agents de quart, à commencer par leur chef, ont dû trouver que le directeur du site n'était pas mieux. C'est une chose que je regrette amèrement. Les communications n'étaient décidément pas bonnes et, durant peut-être la première demi-journée, je n'ai pas pu m'imaginer concrètement l'état dans lequel se trouvaient les agents sur le terrain. Me rendre compte, par exemple, que la perte d'électricité rendait impossibles les manœuvres sur les grosses vannes ou les vannes MO (Motor Operated), imaginer que pour pouvoir lire les instruments de mesure, il fallait apporter des batteries, puisqu'il fallait du courant continu, étaient encore des choses possibles, mais, au-delà, pour les opérations plus subtiles, au début, je n'avais pas conscience à quel point les choses étaient devenues compliquées.

Si je me remémore les premiers temps, je pense que, avec le siège, nous étions très focalisés sur les problèmes de déclaration. Avait-on effectué la déclaration suivant l'article 10 et l'article 15 ? Tout ça constituait notre préoccupation principale et, 
s'agissant de la conduite des réacteurs, je me rends compte aujourd'hui que j'avais laissé ça plus ou moins à l'appréciation des équipes de quart. De fait, les rapports concernant la conduite étaient rares et j'avais tendance à me reposer sur mes agents, en me disant qu'ils faisaient ce qu'il fallait. À cette époque, je me contentais de ce qu'ils me disaient et je ne m'étais pas préoccupé de leur réclamer plus de détails sur chaque réacteur.

$\mathbf{Q}$ : Effectivement, d'après ce que j'ai pu en voir, pour les premières réponses, les équipes de quart ont probablement effectué le maximum de tout ce qui était en leur pouvoir. Même sans attendre vos ordres, ils avaient commencé à effectuer les modifications pour adapter le réseau FP en circuit d'injection vers le réacteur, modifications qui vont leur prendre plusieurs heures. Pendant ce temps, ils avaient vérifié que la pompe diesel était en état de fonctionner correctement. Ayant constaté qu'elle était prête, en attendant l'achèvement du circuit, ils n'ont pas oublié de maintenir le bouton en position arrêt, pour que la pompe ne se retrouve pas en panne sèche au moment opportun.

II y avait bien sûr le problème des instruments de mesure qui restaient illisibles, mais il y a une chose qui manque d'une manière cruelle au tableau, c'est le problème de la dépressurisation. En principe, dans la situation où ils se trouvaient, il aurait fallu dépressuriser s'ils voulaient faire entrer de l'eau. Or, du point de vue des agents de quart, si on supposait que I'IC ne fonctionnait pas bien, il aurait fallu imaginer une autre manière de dépressuriser. Mais c'est une situation pour laquelle les équipes de quart ne disposaient pas de solution immédiate. Fallait-il agir comme vous l'avez fait pour le réacteur 3 ? Voilà une question à laquelle le groupe « réhabilitation » aurait dû répondre, mais il ne reste pas trace de cette réflexion. Ce qui me laisse supposer que l'échange d'informations autour du fonctionnement de l'IC ne s'est pas bien fait et que, pour cette raison, ils se sont retrouvés à pousser sur le bouton d'arrêt de la pompe durant plusieurs heures.

Voilà donc quelle était la situation au réacteur 1. II me semble qu'au réacteur 2 aussi, au même moment, il y a eu un temps où vous ne saviez plus si le RCIC fonctionnait ou pas. Au fil des entretiens que j'ai pu avoir, la plupart des personnes interrogées m'ont dit que, pour elles, il était acquis que l'IC fonctionnait d'une manière ou d'une autre et qu'elles étaient plus préoccupées par la situation du RCIC, d'autant plus qu'on ne voyait pas le niveau d'eau. Étiez-vous, vous aussi, dans cet état d'esprit au début?

\section{$\mathbf{R}:$ Oui, tout à fait.}

Q : Finalement, le RCIC fonctionnait. Mais, contrairement au RCIC du réacteur 3 qui avait été piloté de manière contrôlée, car la tranche 3 avait bénéficié d'une petite source de courant continu, rescapée de l'accident, qui avait permis aux agents de moduler le débit pour pouvoir espérer faire durer son fonctionnement le plus longtemps possible, celui du réacteur 2 avait été laissé livré à lui-même. C'est-à-dire qu'il avait continué à fonctionner sans contrôle. La vapeur produite continuait à faire tourner les turbines, indéfiniment. Aviezvous eu connaissance de ces faits ou les avez-vous appris plus tard?

$\mathbf{R}$ : Sur le moment, je n'étais pas du tout au courant que les RCIC de la tranche 3 et de la tranche 2 avaient eu des comportements différents.

$\mathbf{Q}:$ : Ah, bon. Après avoir interrogé beaucoup de monde et à la réflexion, il me semble que les choses se sont passées de cette manière. C'est un fait qui est vaguement évoqué dans la chronologie que TEPCO est en train d'établir. II semblerait qu'à un moment donné, la source d'alimentation en eau (du RCIC de la tranche 2) a été modifiée du réservoir du condenseur à la piscine de la chambre de dépressurisation. Aviez-vous eu connaissance de la baisse du niveau d'eau du réservoir du condenseur et du passage à la piscine de la chambre de dépressurisation?

\section{$\mathbf{R}:$ Ce changement, je n'en ai pas souvenir.}

$\mathbf{Q}$ : Après ce changement, on se retrouve avec un système qui, alimenté par l'eau de la chambre de dépressurisation, tourne indéfiniment. C'est comme ça qu'on aboutit, il me 
semble, au fameux 14 mars vers 12h30, où la température et la pression de la chambre s'étant élevées, la discussion s'installe avec Monsieur Madarame pour savoir s'il faut dépressuriser ou pas. Si vous vous êtes trouvés dans cette situation, c'est, je suppose, que les agents n'avaient plus d'autre solution. Mais avant ça, vers le 12 ou le 13 mars, ne saviezvous pas que le RCIC tournait sans aucun contrôle?

$\mathbf{R}$ : Je ne comprends pas trop ce que vous appelez « contrôle », mais, à la limite, ce sur quoi j'étais totalement focalisé, c'était de savoir si le RCIC fonctionnait ou pas. J'avoue que l'idée ne m'était pas venue de me préoccuper de son mode même de fonctionnement. De plus, il semble logique qu'à un moment ou un autre on change de source d'alimentation en eau, puisqu'on sait pertinemment que le réservoir du condenseur se viderait assez vite. Maintenant, savoir à quel moment précis ils sont passés d'une source à l'autre et à quel moment j'en ai été informé, je n'en garde aucun souvenir. C'est tout à fait déplorable, mais force est de constater qu'à l'intérieur de la cellule de crise, ce genre d'informations, concernant l'état de fonctionnement des différents systèmes, n'était pas partagé. Bien sûr, j'aurais dû donner des directives plus spécifiques sur chaque installation et c'est moi qui n'aie pas été à la hauteur. Si vous voulez, au tout début, j'étais plus occupé à répondre aux sollicitations extérieures et à tenter d'avoir une vision d'ensemble, c'est-à-dire que j'avais donné la priorité à la collecte d'informations concernant chaque groupe de travail, y compris au niveau des réacteurs 5 et 6 , pour me faire une idée de la situation et au fait de faire passer ces informations au siège. C'est peut-être paradoxal, mais à la limite, la conduite était entièrement confiée aux mains des experts qu'étaient les pilotes. Après, je me préoccupais de savoir en gros si tel système marchait ou pas. "Le RCIC est-il en marche ou pas? ». Mais je ne suis pas entré jusqu'aux détails de la façon dont il marchait.

Q: J'imagine bien que vous aviez fort à faire avec l'extérieur et je pense qu'on ne peut décemment pas demander au directeur de la centrale d'être partout. Dans le genre de cas que nous évoquons, il appartenait en principe au groupe "production » de cerner la situation et d'apporter une solution, non?

R : Oui.

$\mathbf{Q}$ : Encore une fois, c'est une chose que j'ai reconstituée après coup. Comme le niveau d'eau dans le réservoir du condenseur baissait très vite, l'équipe de quart s'est dit qu'il y avait peut-être des problèmes sur le réseau. Les hommes ont jugé qu'il était peut-être plus prudent de fermer la vanne et de conserver un certain niveau d'eau. Je pense que c'est un raisonnement qui se tient. C'est là qu'ils ont pensé judicieux de faire fonctionner le système en passant par la chambre de dépressurisation. La manœuvre a été faite à l'aube du 12 mars et ainsi le RCIC a continué à tourner de cette manière.

Maintenant, si on veut faire fonctionner le RCIC le plus longtemps possible, on sait pertinemment que cette manœuvre va faire monter la température et la pression. Si on pouvait encore suivre la température et la pression de la chambre de décompression, mais vous n'étiez pas en état de surveiller ces données. Alors, sachant que ce mode de fonctionnement allait entraîner une hausse de la température et de la pression, que cette hausse était dangereuse, il aurait fallu ne pas placer trop d'espoir dans l'effet du RCIC et réfléchir à une solution de rechange. À froid, après coup, c'est ce qu'on penserait. Seulement, à l'époque, vous vous êtes certes inquiété du fonctionnement ou non du RCIC, mais vous manquiez d'informations concernant concrètement son mode de fonctionnement.

R : C'est ça. De plus, si vous me permettez, j'étais obnubilé par l'idée qu'il fallait injecter à tout prix. Ma préoccupation principale était, quel que soit le réseau qu'on allait utiliser, de trouver impérativement de l'eau, et en suffisance, de trouver des 
camions de pompiers, puisque nous n'en disposions que d'un sur place. Toutes ces négociations avec le siège pour obtenir ces deux choses avaient mobilisé mon énergie et j'ai l'impression que les détails des problèmes de conduite ne sont pas arrivés avec suffisamment d'acuité jusqu'à moi.

$\mathbf{Q}$ : Concernant justement l'organisation de l'injection du réacteur 1 à partir du 11 mars, I'IC est en marche. Or l'IC n'est pas un système qui peut fonctionner pendant des heures et des heures. On dit en général qu'il peut fonctionner une huitaine d'heures. C'est bien ça? Alors, il convenait de trouver une solution de rechange avant de changer de date. D'autre part, vous aviez conscience que si l'IC s'arrêtait, la pression allait encore augmenter. Donc il était important de trouver une solution pendant qu'il fonctionnait. À quoi avez-vous pensé ?

R : Oh, il n'y avait guère que la solution de la pompe diesel en faisant baisser la pression. Du côté de l'électricité, toutes les solutions demandaient du temps. Même en faisant venir un camion générateur. On avait bien le MCC (Motor Control Center) ${ }^{5}$ de la tranche 2 qui avait survécu, mais le temps de concevoir une passerelle, tout ça allait demander beaucoup de travail. Ça, le terrain m'avait prévenu. Oui, les informations en provenance du groupe «réhabilitation» remontaient bien. Par contre, je ne me rappelle presque rien de celles du groupe "production ». Alors que les histoires des pompiers de chez XXXXX m'ont vraiment marqué... Comme je vous l'ai dit tout à l'heure, il ne reste que les quelques discussions autour de « ça marche ou ça ne marche pas? " avec le groupe "production ", et rien concernant les détails de conduite. Il n'en reste rien parce que j'étais occupé par ailleurs et que je ne les ai pas entendus? Je n'en sais trop rien. Toujours est-il que ces informations n'ont pas été reflétées dans les décisions.

$\mathbf{Q}$ : D'une part, il y avait la pompe diesel. D'autre part, les pompiers. Dans les faits, les pompiers ont joué un grand rôle. Que je regarde la chronologie de TEPCO ou que j'interroge des employés, tout le monde me dit que les gens de Nanmei ont été très actifs. Le 12 mars, vers $3 \mathrm{~h} 00$, ils sont à la recherche des bouches d'incendie, qu'ils trouvent. Vers $4 \mathrm{h00}$, ils effectuent ainsi une injection d'une vingtaine de minutes, qu'ils sont contraints d'arrêter car la radioactivité augmente. Ils reviennent se mettre à l'abri dans le bâtiment antisismique. Plus tard, ils se joignent aux pompiers TEPCO pour des injections plus durables. Quand on regarde l'enchaînement des faits, on a l'impression qu'un temps assez long s'écoule entre le moment où vous donnez l'ordre d'envisager l'intervention des pompiers et son exécution. Est-ce dû au fait que vous aviez donné la priorité à l'utilisation de la pompe diesel et que vous ne vous tournez vers la solution des pompiers que lorsque vous constatez les difficultés à mettre en œuvre la pompe diesel ?

R : Non, tout s'était fait en parallèle. Depuis le début, ma position de base a toujours été qu'il ne fallait jamais tomber dans une situation où une solution ayant échoué, on se retrouve sans rien. Il fallait donc réfléchir à tout dès le départ, y compris le cas où la solution de la pompe diesel ne marcherait pas. Il ne s'agissait pas d'envisager B, après avoir exécuté $A$. Dans des moments pareils, il faut réfléchir en parallèle à la fois à $\mathrm{A}$ et $\mathrm{B}$. Ça, c'est une chose que j'ai apprise en gérant des montagnes d'incidents. Si on ne réfléchit pas simultanément en parallèle, on risque de ne pas être prêt à temps. Ceci dit, une fois que j'avais lancé le processus de réflexion, après avoir sollicité XXXXX, les pompiers maison et les gens de Nanmei, je ne sais pas quel a été l'enchaînement de leurs actions dans le concret. Je ne sais pas exactement ce qu'ils ont fait jusqu'à ce qu'ils trouvent ces bouches d'incendie et effectuent l'injection. Je n'en ai pas le moindre souvenir.

$\mathbf{Q}$ : II semblerait que le 12 mars à 1 h48, l'équipe de quart se soit rendu compte que la pompe diesel ne fonctionnait pas et que l'information ait été relayée vers la cellule de crise 
à 2 h03. Ensuite, pour la remettre en route, ils vont l'approvisionner en carburant, en batterie, etc. Mais ils constatent, vers les 3 ou 4h00, qu'elle ne veut toujours pas démarrer. Et comme c'est justement le moment où les choses ont commencé à bouger du côté des pompiers, on a l'impression que vous êtes passé d'une solution à l'autre. Mais, en fait, tout était mené en parallèle, pour vous.

$\mathbf{R}$ : En tout cas, dans mes ordres, ça l'était.

$\mathbf{Q}$ : Et durant ce laps de temps, vous ne saviez pas ce que faisaient les pompiers.

$\mathbf{R}$ : Non, je ne savais pas. Mais imaginez. Le téléphone ne passait pas vers les pompiers non plus. Là où était stationné le camion de pompiers. Alors, si ça se trouve, on avait perdu beaucoup de temps rien qu'à communiquer et à faire partir le camion. Qui sait? C'est bien sûr une partie que je ne supervisais pas moi-même, alors je ne peux que constater l'heure où ils sont partis. Dans cette confusion, il est probable qu'on avait perdu du temps à chercher la personne à qui il fallait faire passer les ordres. Cela n'est que supposition de ma part. Il faudrait vous renseigner auprès de XXXXX ou des pompiers. J'avoue que je n'ai pas suivi les mouvements jusque-là.

$\mathbf{Q}$ : C'était à un moment où vous vous occupiez également de l'éventage. Du coup, il est probable que vous ayez confié le suivi de l'injection à des subordonnés pour vous consacrer à l'éventage, qui imposait des négociations avec l'État. Justement, d'après les archives, vous donnez l'ordre d'éventage à 0h06. Cet ordre est émis à la suite de la constatation que la pression de la chambre sèche du réacteur 1 est à 600 ?

$\mathbf{R}: 0,68$ ou quelque chose comme ça. C'était préoccupant.

$\mathbf{Q}$ : Vous réitérez l'ordre une dizaine de minutes après. Et cette fois-ci, l'ordre concerne non seulement le réacteur 1 , mais aussi le 2 .

$\mathbf{R}$ : En fait, on a d'abord réussi à voir la pression de la chambre sèche du réacteur 1 . J'ai alors donné prioritairement l'ordre d'éventer le réacteur 1. À ce moment-là, on ne voyait pas la pression de l'enceinte de confinement du réacteur 2 . On ne savait pas non plus si le RCIC fonctionnait ou pas. Et, bien entendu, on ne voyait pas non plus le niveau d'eau. C'est là où je pense avoir reçu un coup de fil du siège me suggérant d'éventer non seulement le réacteur 1, mais aussi le 2, puisque, à défaut d'observations, il y avait de fortes probabilités que la pression soit également montée dans l'enceinte de confinement du réacteur 2. Je ne sais plus qui, exactement, m'a téléphoné. Il me semble qu'il y avait eu des pressions de la part du Gouvernement. En tout cas, Monsieur Madarame était très inquiet au sujet du réacteur 2 . On me suggérait donc de procéder simultanément à l'éventage du réacteur 1 et du réacteur 2. Je n'y étais pas opposé, bien que j'aie toujours conservé un petit espoir que le RCIC du 2 ait été en marche. Je ne me rappelle plus si j'ai effectivement donné ces ordres simultanément, mais je les ai donnés.

$\mathbf{a}$ : Cet espoir était-il fondé sur le fait que la veille, vers $21 \mathrm{~h} 00$, presque $22 \mathrm{~h} 00$, vous aviez vu que l'eau dans le réacteur 2 se trouvait à TAF $+3,400 \mathrm{~mm}$ ? Avec un tel niveau, il est vrai qu'on aurait envie de croire que le RCIC fonctionne. Malgré cela, vous donnez l'ordre d'éventer le réacteur 2. Était-ce par précaution, parce que vous n'aviez pas pu vérifier concrètement le fonctionnement du RCIC ? De toute façon, votre priorité était le réacteur 1.

$\mathbf{R}$ : Pour le réacteur 1 , on avait constaté de manière certaine une hausse de la pression. Il était impératif d'intervenir.

$\mathbf{Q}$ : Évidemment, il devenait impératif d'intervenir sur le réacteur 1. Seulement, si je me souviens bien, c'est aussi à ce moment-là que la radioactivité avait brutalement augmenté du côté du réacteur 1 . Vous aviez déjà formellement interdit l'accès au bâtiment. Pourtant, faute d'électricité, les manœuvres ne pouvaient se faire que manuellement sur le terrain. Si 
je consulte le déroulé que le groupe « production » avait rédigé à l'époque, ils s'étaient rendu compte que la petite vanne de la chambre de dépressurisation, par bonheur ou par malheur, on n'en sait trop rien, pouvait être ouverte à la main. Cela supposait que des membres de l'équipe de quart aillent dans un des tores et fassent la manœuvre à la main. Or l'endroit était inaccessible. D'un autre côté, la situation du réacteur 2 n'était pas aussi dégradée. N'avez-vous jamais envisagé de commencer l'opération par le réacteur 2 ?

\section{$\mathbf{R}:$ Je n'en ai pas souvenir.}

$\mathbf{Q}$ : Non? En fait, pour vous dire ça, je me reporte à une note du groupe «information » datant du 12 mars à 2 h34. II est écrit : "les préparatifs pour le réacteur 1 vont prendre du temps ». Je suppose que cela fait allusion à la radioactivité et d'autres obstacles. Ensuite on lit: "La radioactivité est moins forte au réacteur 2. Les manœuvres sur le terrain y sont possibles. Donner la priorité à l'éventage du réacteur 2 ».

$\mathbf{R}$ : Il n'y a sans doute que moi pour dire des choses pareilles.

$\mathbf{Q}:$ Il ne se pourrait pas que ce soient des directives du siège?

$\mathbf{R}$ : Je ne sais pas. Je ne me souviens pas.

$\mathbf{Q}$ : C'est l'histoire de cette conférence de presse où Monsieur Komori s'est emberlificoté et où il ne savait plus si l'éventage du réacteur 1 allait précéder celui du réacteur 2 ou l'inverse ${ }^{6}$. Ce qui s'est passé, si j'ai bien compris, c'est que justement à 2 h55 le fonctionnement du RCIC avait pu être constaté. Du coup, l'urgence était revenue au réacteur 1. La nouvelle a d'abord été captée par la NISA qui en avait conclu que l'éventage concernerait le réacteur 1. Juste avant la conférence de presse, Monsieur Komori et le directeur de la NISA se sont retrouvés dans le bureau du ministre Kaieda. Le directeur a voulu vérifier auprès de Monsieur Komori que l'éventage commencerait bien par le réacteur 1. À quoi Monsieur Komori a répondu qu'il croyait que ce serait le réacteur 2. Ce qui a semé la confusion. Ils ne savaient plus ni l'un ni l'autre sur quel réacteur le premier éventage allait être effectué. Ils décident, d'un commun accord, d'annoncer lors de la conférence de presse qu'il y aura bien éventage, mais sans préciser sur quel réacteur. Mais, comme il fallait s'y attendre, la presse pose la question du réacteur concerné. Le directeur parle du réacteur 1 et Monsieur Komori du réacteur 2. La confusion est totale. La presse s'est emparée du sujet et a fait monter la mayonnaise. Tout a été consigné et se trouve dans les archives.

À la suite de cet évènement, je me suis demandé pourquoi Monsieur Komori avait parlé du réacteur 2. Normalement, dans les conditions qui étaient celles du 12 mars, il me semblait naturel de penser à procéder à l'éventage du réacteur 1. C'est vers 1 h30 que Monsieur Komori s'est présenté au ministère de l'économie et de l'industrie. En me mettant à sa place à cette heure-là, il me semble qu'il ne peut pas s'agir du réacteur 2. Quand j'ai demandé à Monsieur Komori pourquoi il avait parlé du réacteur 2, il m'a répondu que c'était parce qu'on n'en voyait pas le niveau d'eau. Mais je lui ai fait remarquer que, ça, c'était jusqu'à 21 h00 et qu'ils avaient eu une idée du niveau vers les 22h00. Ça l'a troublé. II ne savait plus très bien pourquoi il avait dit ça. Depuis, j'y ai beaucoup réfléchi et je suis arrivé à une hypothèse. Estce que, devant l'impossibilité d'intervenir physiquement sur le réacteur 1, l'équipe de quart n'aurait pas pensé commencer par ce qui leur était encore accessible, c'est-à-dire l'opération sur le réacteur 2, au lieu de rester là dans l'inaction ? C'est pure conjecture de ma part, mais n'auriez-vous pas entendu parler de ce genre de chose? N'en auriez-vous pas le souvenir?

$\mathbf{R}$ : Concernant les difficultés rencontrées autour du réacteur 1 , voici ce dont je me souviens. Je n'ai plus les détails en tête, mais, pour moi, si la pression de l'enceinte de confinement était de $0,6 \mathrm{Mpa}$, il fallait, évidemment, procéder à l'éventage du réacteur 1 . Mon idée était qu'il fallait procéder à l'éventage du réacteur 1 et je n'en ai jamais dévié. Sur ces entrefaites viennent se greffer les inquiétudes de Monsieur Madarame concernant le réacteur 2. Je sais que je ne lui ai pas parlé directement. J'ai eu au bout du fil quelqu'un à qui il en avait parlé, sans doute Komori. J'ai répondu que, pour ma part, j'étais encore confiant pour le réacteur 2. Mais cette personne m'a 
rabroué en me disant qu'on ne voyait pas le niveau d'eau et qu'il ne fallait pas le laisser dans cet état. J'ai donc proposé d'envisager aussi un éventage sur le réacteur 2. Mais la pression du réacteur 1 montait. C'était vers oh00 quelque chose. Rappelez-moi l'heure exacte?

Q : C'était à 23 h50.

R : La pression augmentait à cette heure-là et, dans ma tête, la priorité était le réacteur 1. Là-dessus, cette histoire du réacteur 2 est arrivée et j'avoue que j'ai trouvé ça quelque peu perturbant. Alors, si ça se trouve, Komori est peut-être parti à la conférence de presse dans cet état de confusion. C'est possible. Mais pour moi les choses étaient claires. J'ai donné l'ordre de commencer par le réacteur 1. Comme vous le faisiez remarquer, la situation du réacteur 1 sur le terrain était critique. Il semblait difficile d'agir du côté de la "production ». J'avais donc chargé le groupe «réhabilitation» de voir s'il ne pouvait pas, par exemple, actionner à distance la petite vanne en apportant une source électrique quelconque. $\mathrm{Si}$, dans ces circonstances, quelqu'un m'avait dit, « on peut exécuter l'opération sur le réacteur 2 », il n'est pas impossible que j'aie dit, «tentons le coup sur le réacteur 2 », tout en continuant à donner la priorité au réacteur 1 et à chercher une solution pour lui. Je ne suis pas du tout certain de l'avoir dit, parce que la mémoire me manque. Mais si je l'avais dit, cela n'aurait en aucun cas voulu dire « on laisse tomber le réacteur 1 », mais bien « on le fait tout en continuant à chercher une solution pour le réacteur 1 ».

$\mathbf{Q}$ : Si je me mets à la place de l'équipe de quart, ce qu'il y a à faire pour le réacteur 1 est déjà clair, idem pour le réacteur 2. S'agissant du réacteur 1, la situation ne permet pas d'agir. J'ai du temps. Alors je me dis que, puisque de toute manière tôt ou tard il faudra éventer les deux réacteurs, je peux commencer par celui qui est encore accessible. Voilà une supposition qui peut être plausible. Vous savez que certains, dans l'opinion publique, se demandent ce que faisaient les agents de l'équipe de quart entre 0h06 et 9h00. Ils leur reprochent de n'avoir rien fait pendant ce laps de temps. Une des grandes raisons pour lesquelles je suis revenu vous voir aujourd'hui est de découvrir ce qui s'est passé durant ces heures, remplir ce vide. Car il est évident que l'équipe de quart n'est pas restée là à ne rien faire. S'il devait y avoir des reproches vis-à-vis de cette équipe à cause d'un défaut d'enquête de notre part, nous ne pourrions nous le pardonner. Je voudrais donc éclaircir au maximum ce qui s'est passé durant ce temps. L'équipe de quart, qui était dans la salle de commande et qui avait la responsabilité à la fois de la conduite du réacteur 1 et du réacteur 2 , aurait très bien pu réagir de la sorte en ayant reçu l'ordre de se préparer à l'éventage des deux réacteurs. II faudra, bien entendu, que j'aie confirmation de la part de l'équipe, mais cela me semble une hypothèse possible.

Ensuite, concernant l'IC, comme on l'a vu précédemment, la pression de la chambre sèche atteignait environ $600 \mathrm{MPa}$.

\section{R : Plutôt $600 \mathrm{kPa}$.}

Q : Oui, $600 \mathrm{kPa}$. Là, il s'agit d'un chiffre qui a été clairement observé. En l'apprenant, vous vous êtes sûrement dit que c'était fini pour l'IC. Mais avant?

$\mathbf{R}$ : Ce n'était pas vers les 17 h00, mais plutôt après 20h00, quand on s'est rendu compte que la radioactivité avait augmenté, que ce soit à cause de l'IC ou pas, que j'ai eu confirmation que l'état du réacteur était devenu très critique. Je m'en doutais avant même de prendre connaissance des données concernant la pression de l'enceinte de confinement. Je m'en doutais et j'avais déjà un sentiment d'urgence à ce moment-là. Par contre je ne sais pas si j'éprouvais ce sentiment de danger, alors que je pensais l'IC en fonctionnement, ou pas. Je vous l'ai déjà dit plusieurs fois, comme 
on ne voyait pas les paramètres, il n'était pas véritablement possible de porter des jugements.

$\mathbf{Q}$ : Je lis sur cette note: «27h50, ordre du directeur de ne pas pénétrer dans le bâtiment réacteur de la tranche 1 ». Se trouver face à une radioactivité qui ne permet plus de pénétrer dans le bâtiment signifie qu'il se passe quelque chose au niveau du réacteur. Je ne vois pas d'autre raison que ça. D'autre part, un peu avant, à $21 \mathrm{~h} 19$, vous avez pu voir le niveau d'eau du réacteur 1. À ce moment-là, l'eau est à TAF +200 mm. La marge n'est pas grande, mais le combustible n'est pas dénoyé. Tout le monde a dû s'en réjouir. Seize minutes plus tard, à 21h35, le niveau remonte même à TAF $+450 \mathrm{~mm}$. Ce n'était pas beaucoup, mais c'était là encore un élément plutôt positif. Or vingt minutes plus tard, vous interdisez l'accès au bâtiment réacteur. Que s'est-il passé ?

$\mathbf{R}$ : C'est une décision qui a été prise indépendamment des mouvements du réacteur. Une personne qui était allée au bâtiment réacteur est revenue en disant qu'il y régnait une sorte de brouillard et que la radioactivité y avait fortement augmenté. Je crois me souvenir que c'était quelqu'un du groupe "sûreté ", sans doute XXXXX. Effectivement, il y avait contradiction entre ce que racontait celui qui était allé concrètement sur le terrain et ce que nous venons d'évoquer à propos du niveau d'eau dans le réacteur. Il y avait contradiction, mais si la radioactivité était si forte, il fallait penser à la sécurité des équipes, il ne fallait pas que les agents s'en approchent. Voyez-vous, c'était une radioactivité qui était bien au-delà de ce dont nous avions l'habitude en temps normal. Moi-même, j'en suis resté quasi incrédule. Lorsqu'on procède à des contrôles programmés, on parle d'une radioactivité élevée quand on détecte $300 \mu \mathrm{Sv} / \mathrm{h}, 0,3 \mathrm{mSv} / \mathrm{h}$. Alors, quand cette radioactivité atteint 10 ou 100, c'est une situation qu'on ne peut même pas imaginer. Il ne fallait pas que les gens s'en approchent. La première chose à faire était d'interdire formellement l'accès à ce bâtiment, compte tenu du danger. Je suis bien conscient que cette décision n'est pas du tout en rapport avec le comportement de la tranche.

"Qu'est-ce qui se passe? On m'a pourtant dit que l'IC fonctionnait. Pourquoi la radioactivité a-t-elle tellement augmenté? Il faut bien se rendre à l'évidence que, IC ou pas, il se passe quelque chose de grave ». Voilà ce que j'ai pensé. Mais malgré cette interdiction de pénétrer dans le bâtiment réacteur à cause de la radioactivité, il fallait exécuter l'éventage, et ce sans électricité. Je ne me rappelle plus à quelle heure, mais, à un moment, des agents sont allés ouvrir la vanne MO du premier étage du bâtiment réacteur. Ils avaient mis leur vie en jeu. Il y a des moments où il faut $\mathrm{y}$ aller malgré tout. Et ils y sont allés. Je savais que plus tard, il arriverait des moments où il me faudrait encore envoyer des hommes malgré le danger. C'est comme ça que ce jourlà, à l'aube, j'ai demandé à des agents de tenter une manœuvre du côté de la chambre de dépressurisation, avec consigne de ne pas insister si ça résistait.

$\mathbf{Q}$ : Aujourd'hui, nous pouvons relire les évènements en nous basant sur le comportement de I'IC, mais si je me mets à votre place lors de l'accident, vous réagissiez au coup par coup aux phénomènes qui se déroulaient sous vos yeux. S'il y avait danger, vous interdisiez l'accès à ce bâtiment. Face aux différentes informations qui vous arrivaient, vous réagissiez au cas par cas. Vous n'arriviez pas à relier les différentes informations entre elles et à avoir une vision d'ensemble. Si même vous, directeur Yoshida, vous n'arriviez pas à le faire, cela veut dire qu'un homme de capacité moyenne, face à une situation similaire, n'y arrivera jamais s'il n'y a pas été entraîné préalablement. C'est encore une idée qui me vient parce que je me base sur cette expérience que vous avez vécue. II me semble qu'une formation préalable à la gestion de ce type de situation s'impose de plus en plus. Parce que, face à 
une telle situation, on n'est pas capable de mettre tout de suite en pratique les connaissances qu'on peut posséder en théorie.

$\mathbf{R}$ : Non, c'est vrai. Je voudrais ajouter une chose. Les gars de la production chez nous sont de vrais professionnels. Cela fait longtemps que je les fréquente. Ce sont des gens qui ont toujours travaillé directement sur les tranches. Ils possèdent une conscience professionnelle aiguë. Du coup, ils ont une très forte tendance à vouloir résoudre les problèmes par eux-mêmes ${ }^{7}$. C'est, en quelque sorte, une attitude rassurante pour un directeur de centrale. Parce que, sur d'autres sites, j'ai aussi rencontré des équipes qui, au moindre incident, viennent tout de suite demander de l'aide. Je me rappelle, lorsque j'étais directeur de la production à Fukushima Daini, m'être demandé pourquoi le chef de quart y était aussi fragile. Je veux dire qu'au moindre incident il abdiquait et demandait que la sûreté intervienne. Et il n'était pas le seul. Bien sûr, tout le monde dans la production n'est pas comme lui, mais il y en a un certain nombre. Comparé à eux, les gars de la production à Fukushima Daiichi, que ce soit les chefs d'équipe ou les autres, sont des gens qui essaient toujours de résoudre les problèmes par eux-mêmes. Mais je me demande jusqu'à quel point, dans ce cas précis, cette attitude ne s'est pas retournée contre eux. C'est peut-être une des raisons pour lesquelles les informations concernant leurs manœuvres ne sont pas suffisamment remontées jusqu'à la cellule de crise. À force d'avoir le sens des responsabilités, ils se sont peut-être trop focalisés à vouloir trouver une solution, juste par leurs propres moyens. C'est une chose que j'ai pensée quand j'ai appris les résultats des enquêtes post-accident. C'est très possible.

$\mathbf{Q}$ : Effectivement, l'équipe de quart s'était débrouillée seule, sans même attendre les ordres, jusqu'à la préparation de la pompe DD du réacteur 1.

$\mathbf{R}$ : N'est-ce pas? Ils n'attendaient pas les ordres. Ils prenaient des initiatives par eux-mêmes.

Q: En effet. L'équipe du réacteur 3 aussi régulait le débit du RCIC et du $\mathrm{HPCl}$ en utilisant une ligne de retour vers le réservoir du condenseur qu'ils avaient établie pour l'occasion. Tout ça s'était fait sur l'initiative de l'équipe de quart.

$\mathbf{R}$ : Ce sont des astuces dictées par l'expérience. Ce sont des choses dont je n'ai pas eu connaissance sur le moment. Peut-être en avais-je reçu des rapports, mais ça ne s'était pas imprimé dans ma mémoire. C'est plus tard qu'on me l'a raconté et que j'ai découvert avec émotion l'étendue de leur action.

Q: Par la suite, vous allez commencer à injecter de l'eau de mer tout en préparant l'éventage. Mais avant d'introduire de l'eau de mer, vous aviez injecté de l'eau douce. Si on suit les chronologies après le début de l'injection d'eau douce, il y a une chose que je ne m'explique pas. Tout le monde ne parle que d'« injection continue». Mais dans les faits, au début, l'injection ne se fait que par toutes petites quantités, du genre 1000 I, c'est-à-dire 1 $\mathrm{m}^{3}$ par-ci, $1 \mathrm{~m}^{3}$ par-là. Par exemple, je lis ici: "Exécution de l'injection par voiture de pompiers de 7 h52 à 7 h55. $1 \mathrm{~m}^{3} » . \mathrm{Y}$ avait-il une raison pour que vous effectuiez des injections limitées?

$\mathbf{R}$ : Non, aucune.

$\mathbf{Q}:$ Ah, bon. Tout en effectuant l'injection, ne deviez-vous pas contrôler le niveau d'eau, par exemple?

$\mathbf{R}$ : Non, pas du tout. Le principe de base, à ce moment-là, était d'envoyer de l'eau de manière massive, d'en injecter autant que possible. Si effectivement il y avait une limite, elle était certainement due soit à des problèmes rencontrés par le camion de 
pompiers, soit à des problèmes d'approvisionnement en eau. Pour ce qui est des ordres, ils étaient clairement d'injecter autant d'eau que possible.

$\mathbf{Q}$ : Moi aussi, au début, je croyais que l'injection se faisait en continu à un débit soutenu. Mais ce n'est pas ce qui s'est passé dans les faits. Vous disposiez, par exemple, d'un réservoir d'eau prévu pour les incendies, qui avait une capacité d'environ 40 t. Vous commencez par injecter de manière discontinue par petits bouts dans les réacteurs 1 et 2 . Par la suite, l'injection devient continue et, au total, vous arrivez à quelque chose comme 80 t injectés. Cette manière de procéder reflète-t-elle des directives de votre part?

$\mathbf{R}$ : Non, je n'ai pas ordonné spécifiquement d'opérer de cette manière.

$\mathbf{Q}$ : Donc, vous n'aviez pas donné d'ordre dans ce sens.

$\mathbf{R}$ : Non. Je pense que ce sont des obstacles rencontrés sur le terrain qui ont donné ce résultat.

Q: Je crois qu'il y avait du personnel de chez Nanmei qui a dû participer à ces manœuvres. Peut-être était-ce une manière habituelle de procéder pour eux? Si je comprends bien, on ne peut rien en conclure pour l'instant.

À partir de là les choses se compliquent car il y a, à la fois, l'éventage et l'injection. Vers 8 h00, à peu près au moment où vous raccompagnez Monsieur Kan qui repart, il est question d'éventer vers 9h00. II me semble que je vous ai déjà posé la question précédemment, mais je voudrais savoir : cet horaire signifiait que vous aviez encore besoin d'une heure?

$\mathbf{R}$ : Je crois me souvenir que, dans nos échanges, Monsieur Kan avait demandé qu'on procède au plus vite à l'éventage. Moi, je lui ai répondu que nous nous y préparions. Alors il m'a demandé si l'éventage serait possible vers 9 h00 et je lui ai répondu que nous ferions notre possible pour que ce soit à 9h00. D'où l'idée de 9 h00.

$\mathbf{Q}$ : Alors c'est dans le cours de la conversation que vous en êtes venus à évoquer cet horaire, parce que cela vous laissait environ une heure et que vous pensiez que vous pourriez être prêts pour cette heure-là. C'est comme ça que vous vous étiez fixé 9h00 comme objectif. D'accord. Cet horaire n'avait pas plus de signification que ça, alors.

Ensuite, après l'explosion de la tranche 1, vous reprenez les travaux. L'explosion survient à $15 \mathrm{~h} 36$ et il faut bien que vous repreniez vos travaux à un moment ou un autre. Or vous ne savez pas comment va se comporter la tranche, mais vous ne pouvez pas non plus abandonner vos travaux. Je lis ici : "Départ de l'équipe "pompe d'injection d'eau de mer » à 17 h26 pour étudier l'état de la pompe à eau de mer et l'état du bâtiment ». On peut considérer que c'est à peu près à cette heure que vous reprenez les travaux ?

\section{$\mathbf{R}:$ L'explosion s'était produite à $15 \mathrm{~h} 36$.}

Q: Oui. Vers les $15 \mathrm{~h} 36$.

$\mathbf{R}$ : À ce moment-là, notre première réaction a été de vérifier la situation du personnel, de tout le personnel. Il y avait d'ailleurs quelques blessés, mais nous n'avions pas de morts, ce qui m'a bien soulagé. Ensuite nous nous sommes préoccupés de l'état du terrain. Évidemment, il y avait des dégâts du côté du camion de pompiers. Nous avons ainsi perdu un camion de pompiers. Il fallait reconstruire une ligne d'injection.

Q: Oui, je crois que tous les tuyaux du camion avaient été endommagés.

$\mathbf{R}$ : Oui, il y a eu ces histoires de dommages, etc. Nous étions en pleine vérification de l'état des lieux. Máais pendant ce temps-là, l'injection était interrompue. Il était impératif de reprendre cette injection. Il me semble que j'avais déjà donné l'ordre de passer à l'eau de mer, n'est-ce pas ? 
$\mathbf{Q}$ : Oui, vous en aviez donné l'ordre bien avant et vous étiez en plein préparatifs pour cette injection.

$\mathbf{R}$ : Nous avions préparé le terrain pour cette injection d'eau de mer et la ligne était prête. Il ne restait plus qu'à envoyer l'eau quand l'explosion s'est produite, interrompant de fait toute l'opération. Il fallait impérativement reprendre cette injection d'eau de mer et pour cela il fallait avoir une idée de la situation sur le terrain. Je crois que cette note se situe exactement à ce moment précis.

$\mathbf{Q}$ : Là, on parle de l'équipe "pompe d'injection d'eau de mer». Cela veut dire que des personnes chargées de l'injection d'eau de mer sont parties avec des surveillants de la radioactivité pour constater l'état du terrain et, si possible, continuer leur travail, n'est-ce pas?

$\mathbf{R}$ : Oui, tout à fait.

$\mathbf{Q}$ : Finalement vous allez réussir à injecter de l'eau de mer de manière continue à partir de $19 h 04$.

Je voudrais maintenant passer au réacteur 3. Sur cette tranche aussi, l'équipe de quart avait réfléchi au moyen de faire durer le plus longtemps possible le RCIC. Mais, malheureusement, le 12 mars, avant l'explosion (de la tranche 1), vers 11h36, pour une raison ou une autre, le $\mathrm{RCIC}$ s'arrête. L'équipe tente de le redémarrer, mais échoue à ce moment-là. Ce faisant, le niveau d'eau baisse rapidement et atteint le niveau L2 ${ }^{8}$, mettant automatiquement le $\mathrm{HPCl}$ en marche. II semblerait que, dans le but, encore une fois, de faire durer le $\mathrm{HPCl}$ le plus longtemps possible, l'équipe avait ouvert au préalable la vanne commandant la ligne de retour vers le réservoir du condenseur pour pouvoir limiter le débit. En fait, l'équipe tente d'utiliser le $\mathrm{HPCl}$, non pas en mode "gros débit immédiat » comme d'habitude, mais en "petit débit contrôlé », un peu comme un RCIC.

\section{$\mathbf{R}$ : Qui était le chef d'équipe?}

$\mathbf{Q}$ : Monsieur XXXXX et Monsieur XXXXX. Monsieur XXXXX a l'air de s'être particulièrement investi dans la conduite du HPCl. Seulement, il éprouvait une certaine crainte du fait qu'il utilisait le système dans une situation de basse pression, d'une manière différente de celle pour laquelle il avait été prévu. De fait, le système marchait avec un nombre de rotations bien inférieur à ce qui était prévu structurellement. Tout en surveillant le nombre de rotations, il pensait bien qu'il serait difficile de maintenir le système en marche de cette manière pendant longtemps. Etiez-vous au courant de cette conduite?

\section{$\mathbf{R}$ : Non, pas du tout.}

$\mathbf{Q}$ : Dans ces circonstances, l'équipe de quart travaillait toujours avec, quelque part, l'idée que l'opération serait difficile, qu'elle ne pourrait pas durer indéfiniment. En suivant la chronologie, je suis tombé sur cette note datée du 13 mars à 3 h55. "2h44, arrêt automatique du HPCl du réacteur 3. Pression basse dans la cuve, tentative d'injecter avec le DDFP, mais remontée de la pression jusqu'à $4 \mathrm{MPa}$ après arrêt du HPCl, échec de l'injection »9. D'autre part, lorsque j'ai interrogé les personnes du siège, elles m'ont toutes dit qu'elles étaient persuadées que l'arrêt du HPCI du réacteur 3 était un arrêt automatique. Or, d'après Monsieur XXXXX, le $\mathrm{HPCl}$ a été arrêté manuellement. Etiez-vous au courant de cette histoire?

\section{R : Non.}

Q: Non? Comme je vous l'ai dit à l'instant, l'équipe faisait marcher le système d'une manière inhabituelle. Dans la nuit du 12 au 13, le niveau d'eau de la cuve n'était plus visible. Comme elle ne peut plus contrôler ce paramètre, l'équipe continue avec l'espoir que, comme pour le RCIC du réacteur 2, la différence entre la pression de sortie du HPCl et la pression de la cuve permette encore à l'eau de circuler. Voyant que le nombre de rotations était extrêmement réduit, elle commence à franchement s'inquiéter, d'autant plus que la pression avait aussi baissé. 
D'autre part, dans le cas du réacteur 3, la lampe signalant l'état de la vanne SR était encore allumée sur le panneau de contrôle. L'équipe pense que si elle pousse sur le bouton, la vanne SR va s'ouvrir. Tout en manœuvrant le passage de l'aspersion de la chambre humide, qu'elle était en train d'effectuer, à l'injection par la ligne de la DDFP, elle arrête manuellement le HPCl. Elle tente de faire baisser la pression en activant la vanne SR, mais la baisse ne survient pas. Face à cette situation, elle essaie de faire redémarrer le $\mathrm{HPCl}$, sans succès. Comme la raison de l'arrêt du RCIC était inconnue, elle tente également de le faire redémarrer, là encore sans succès. Ce faisant, la pression de la cuve s'envole jusqu'à 4,1 MPa. Ce qui justifie ce rapport. Mais entre temps, je ne sais pas si l'erreur s'est glissée au départ ou à la réception, l'arrêt manuel est devenu un arrêt automatique dans le rapport. Aviez-vous entendu parler de cette histoire?

$\mathbf{R}$ : Non, pas du tout.

$\mathbf{Q}$ : Vous, vous pensiez que l'équipe faisait fonctionner le HPCl de la manière habituelle.

R : Oui.

$\mathbf{Q}$ : Si on se remet en situation, à ce moment-là vous savez que le RCIC s'est arrêté. Vous savez aussi que le $\mathrm{HPCl}$ est en marche. Je crois qu'on peut comprendre que ce genre d'histoire puisse advenir à la lumière de ce que vous m'avez dit. Si l'équipe de quart pense qu'il lui appartient de trouver une solution, qu'il lui faut d'abord tenter seule toutes les solutions qui lui viennent à l'esprit, tout cela est tout à fait plausible. Seulement, entre ce que pense la cellule de crise et ce que pense l'équipe de quart... Je voudrais vous interroger plus spécialement à propos de l'eau dans le réacteur 3. II y a eu l'explosion de la tranche 1. Vous avez dû reconstruire une ligne pour injecter l'eau de mer dans le réacteur 1, opération achevée à 19h04. Ensuite, vers 20 h00 ou 21h00, que pensiez-vous du réacteur 3, où le $\mathrm{HPCl}$ fonctionnait depuis un certain temps?

$\mathbf{R}$ : Bien sûr, je pensais qu'il était impératif de trouver de l'eau, une source d'alimentation, pour le réacteur 3. Mais il me semble que c'était justement le moment où plusieurs camions de pompiers commençaient enfin à arriver.

$\mathbf{Q}$ : Oui, vous aviez le camion en provenance de Kashiwazaki en réserve. II y en avait trois autres qui servaient pour le réacteur 1 .

$\mathbf{R}$ : C'est ça. J'avais donné l'ordre de réfléchir à la manière de répartir les camions entre l'injection des réacteurs 1 et 3 .

$\mathbf{Q}$ : Que se passait-il dans votre tête le 12 vers $20 \mathrm{h00}$, alors que vous abordez la nuit, en regardant le travail effectué par vos hommes sur le terrain?

$\mathbf{R}$ : Vous voulez dire une fois après l'explosion? Une fois qu'on a recommencé à injecter de l'eau de mer dans le réacteur 1 ?

$\mathbf{Q}$ : Vous injectez de l'eau de mer dans le réacteur 1. Ensuite vous vous attelez de nouveau à la restauration de l'alimentation électrique, qui a été également touchée par l'explosion. Une opération que vous prévoyez semée d'embûches. Ce sont des travaux que vous aviez entamés très tôt après l'accident. Vous aviez dû mobiliser une quarantaine de personnes pour transporter les câbles. C'est dire les difficultés qui vous attendaient encore. Mais vous saviez que si vous arriviez à rétablir l'électricité, vous pourriez réhabiliter le SLC, comme vous en aviez eu l'idée pour le réacteur 1, ou d'autres systèmes. En effet, avec de l'électricité, même la manipulation des vannes de rejet aurait pu se faire à partir des panneaux de contrôle, facilitant grandement l'opération. C'était sûrement une motivation forte. Seulement, si on se remémore les difficultés que vous aviez eues avec le réacteur 1, il n'était pas réaliste de penser que c'était quelque chose qui pouvait se faire en deux ou trois heures. Tout en encourageant les électriciens du groupe « réhabilitation » à poursuivre leurs efforts, vous réfléchissez en parallèle à une autre solution, pour ne pas, comme vous l'avez déjà dit, vous retrouver le bec dans l'eau, si la première solution s'avérait inopérante. Pour 
vous, à ce moment-là, si on exclut la solution du rétablissement de l'électricité pour l'utilisation du SLC, que restait-il ? Les voitures de pompiers?

$\mathbf{R}$ : Oui, juste les voitures de pompiers.

$\mathbf{Q}$ : N'avez-vous pas pensé à la DDFP?

$\mathbf{R}$ : Concernant le réacteur 3 , l'équipe de quart m'avait déjà prévenu que la DDFP n'était pas utilisable. Il me semble qu'elle avait été submergée et, donc, inopérante. Bien que nous ayons eu beaucoup de difficultés à mettre en route cette solution sur le réacteur 1 , le fait est que nous avions quand même réussi à y injecter de l'eau de cette façon. Alors, pour moi, il ne restait que la solution de l'injection avec les voitures de pompiers. C'est pourquoi j'avais demandé qu'on rassemble le plus de voitures possible, de tout le Japon. Voilà ce que j'avais en tête.

$\mathbf{Q}$ : Tout en sachant qu'il est urgent de rétablir l'électricité afin de pouvoir utiliser tous les systèmes disponibles en état de marche, d'un autre côté, vous pensez qu'il n'y a, à court terme, que la solution des voitures de pompiers.

$\mathbf{R}$ : C'est ça. Il n'y avait que les voitures de pompiers.

$\mathbf{Q}$ : Alors, si on regarde quelle était la situation de ce côté-là à la centrale, en fait vous n'aviez que quelques camions sur le site. D'après ce que j'ai pu savoir jusqu'à présent, il y avait cinq camions en état de marche. Trois étaient utilisés au réacteur 1. En ce qui concerne les deux restant, I'un, en provenance de Kashiwazaki via Fukushima Daini, était en attente, l'autre se trouvait sur les hauteurs au niveau des tranches 5 et 6 .

$\mathbf{R}$ : On ne pouvait pas récupérer celui-là, parce que la route était défoncée.

$\mathbf{Q}:$ Vous le saviez, ça?

$\mathbf{R}$ : Oui, je le savais. C'est pour ça que j'avais insisté, très tôt, auprès du siège pour qu'il nous envoie toutes les voitures de pompiers qu'il pourrait trouver. L'autre souci, c'était le carburant. On était presque en panne sèche. Si le gasoil venait à manquer, cela aurait été la fin de tout. Donc, j'ai réclamé à cor et à cri du gasoil, de l'essence, de l'eau et des camions de pompiers, en priorité. Il y avait aussi les batteries. Tout ça, en toute urgence.

$\mathbf{Q}$ : En fait, c'est au petit matin du 13 mars que les choses vont commencer à bouger. Par exemple, le 13, entre 6 h00 et 7h00, quatre camions vont arriver d'un coup, suivis par d'autres. C'est aussi là que se situe cette histoire de batterie. Vous ne disposiez pas de batterie qui puisse servir tout de suite. Du coup, vous aviez démonté les batteries des voitures qui étaient stationnées là pour les utiliser dans des manœuvres de dépressurisation, je crois. Avant d'en arriver là, vous aviez beaucoup cherché sur le site de la centrale?

$\mathbf{R}$ : Évidemment, quand on a eu besoin de batteries, les hommes du groupe " réhabilitation » avaient discuté pour savoir s'il y en avait en réserve et où. Ce n'est pas moi qui en ai eu l'idée, mais au cours de ces discussions, quelqu'un du groupe a dit que des batteries de voiture pouvaient faire l'affaire. Et, effectivement, j'ai eu des rapports indiquant qu'ils avaient démonté des batteries de voiture pour les utiliser. Ce n'est donc pas moi qui leur ai dit d'utiliser des batteries de voiture. C'est encore une fois une de ces astuces qui est venue du terrain. Je pense que c'était quelqu'un du groupe « réhabilitation ».

Dites, Monsieur Katô, est-ce qu'on ne s'arrêterait pas cinq minutes pour fumer ?

$\mathbf{Q}$ : D'accord. Faisons une petite pause. 
$\mathbf{Q}$ : Si je reviens à cette chronologie de TEPCO, à un moment donné vous apprenez que ça s'est arrêté du côté du réacteur 3. Vous l'apprenez et ensuite vous vous dites que vous devez vous dépêcher d'injecter de l'eau. Est-ce que, dès le début, vous vous faites la réflexion qu'il ne reste que la solution de l'injection avec les voitures de pompiers?

R : Oui.

$\mathbf{Q}$ : Seulement, à ce moment-là, le groupe «génie civil» des restaurateurs est en train d'installer des sacs de terre, d'aplanir les différences de niveaux, d'évacuer des gravats pour permettre l'injection. En étiez-vous conscient?

$\mathbf{R}$ : Oui, je le savais. Je savais que les accès étaient bouchés par les gravats, qu'on ne pouvait pas aller du bâtiment administratif vers le bâtiment réacteur. Bien sûr, je ne me suis pas déplacé moi-même sur le terrain et je ne connaissais pas la situation en détails. Mais j'avais conscience que la circulation était extrêmement difficile. J'avais donc demandé aux gars du " génie civil » de faire le nécessaire, en utilisant toutes les machines dont ils disposaient, pour assurer un chemin aux voitures de pompiers. Il fallait aussi réduire les différences de niveaux avec des planches ou autre chose pour permettre la circulation. Tout cela était mené en parallèle.

$\mathbf{Q}$ : Vous réussissez tant bien que mal à dégager un des camions, vous tirez une ligne partant du bassin Reversing Valve Pit de la tranche 3, comme pour le réacteur 1, et vous injectez de l'eau de mer. On en a déjà un peu parlé précédemment, mais c'est une fois que vous aviez mis en fonction cette ligne que vous avez reçu ce coup de fil de la résidence du Premier ministre vous demandant, si possible, d'injecter de l'eau douce. Vous rappelez-vous qui était votre interlocuteur au téléphone? II me semble que vous aviez parlé à Monsieur XXXXX. En avez-vous souvenir?

$\mathbf{R}$ : En effet, XXXXX servait de relais à la résidence du Premier ministre. Il était là-bas avec Takekuro et c'est sans doute lui qui a téléphoné. Mais je n'ai aucun souvenir du contenu de la conversation. Ce pan manque dans ma mémoire. Si vous me dites que c'était XXXXX, je vous répondrai que, probablement, c'était lui.

$\mathbf{Q}$ : Monsieur Takekuro est rentré une première fois de la résidence au siège de TEPCO le 12 dans la nuit. C'est là qu'il a prononcé son grand discours ${ }^{10}$.

\section{$\mathbf{R}$ : Oui, un grand sermon.}

$\mathbf{Q}$ : Oui, il a fait son sermon. Maintenant qu'il a entendu l'enregistrement, il le regrette, d'ailleurs. Il se demande s'il n'en a pas fait un peu trop. Toujours est-il qu'après son discours, il est rentré chez lui et cela s'est passé pendant qu'il était chez lui. II est retourné à la résidence le lendemain vers 9h00. Entretemps, il ne restait plus que Monsieur XXXXX à la résidence et il y aurait organisé une sorte de séance de travail. D'après ce qu'on sait, le Premier ministre n'y aurait pratiquement pas assisté. Mais il y avait Messieurs Hosono et Kaieda, qui étaient venus s'informer. Monsieur Madarame et d'autres s'y sont rajoutés. Ils ont discuté de différents problèmes, notamment le problème de savoir si, une fois qu'on aurait réussi à injecter de l'eau dans le réacteur 1, s'il ne conviendrait pas d'injecter à la fois dans le 3 et le 2, en même temps. Ils ont tourné le problème dans tous les sens. Comme à chaque fois qu'ils se demandaient quelle était la situation sur le terrain, ou qu'ils manquaient d'informations sur tel ou tel point, ils avaient pris l'habitude de vous téléphoner à vous, directeur de la centrale, par l'intermédiaire de Monsieur Takekuro, Monsieur XXXXX ou, même, Monsieur Hosono, ils en sont aussi venus à vous appeler lors des discussions au cours de cette séance de travail. C'est comme ça que vous avez eu Monsieur Madarame au bout du fil.

$\mathbf{R}$ : Vous parlez de ce qui s'est passé autour du réacteur 2.

$\mathbf{Q}:$ Oui, du réacteur 2. En fait, en cours de discussion, Monsieur Madarame s'était échauffé. II voulait absolument que les choses se fassent à sa façon. Et c'est dans ce contexte que vous avez reçu ce fameux coup de fil, dont vous vous seriez bien passé, je suppose. En 
vérité, ce n'était nullement une décision qui avait été prise sous l'autorité du Premier ministre et dont il vous faisait part. Pas du tout.

\section{$\mathbf{R}$ : Ah, oui. C'était une simple séance de travail !}

$\mathbf{Q}$ : Oui. Maintenant que j'ai interrogé personnellement toutes les personnes présentes, toutes me disent que cela s'est passé comme ça. Ce n'était pas un ordre émanant du commandant en chef.

\section{$\mathbf{R}$ : Mais enfin ! Comment est gouverné ce pays ? Quel pays bizarre !}

$\mathbf{Q}$ : Monsieur Takekuro m'a dit la même chose, même son de cloche du côté des gens de la NISA, que ce soit des membres ou le directeur adjoint, tous ceux qui ont assisté à la scène me disent la même chose. Ce qui m'amène à penser qu'il ne devrait pas y avoir d'erreur. Cela veut dire aussi que ces gens n'avaient pas réfléchi une seconde aux répercussions que leurs paroles pourraient avoir sur le terrain, que ce qu'ils disaient pourrait avoir un tel poids pour ceux qui y étaient. Là où, avec une certaine innocence, ils désiraient s'informer pour parfaire leurs connaissances, leurs paroles ont été interprétées, par le siège, comme le reflet d'une direction que le Premier ministre souhaitait faire prendre à l'action menée sur le terrain. Comme le siège n'était pas au courant des débats qui se déroulaient à la résidence du Premier ministre, il a interprété ces phrases comme des ordres émanant du Premier ministre, relayés par ses proches. II est évident que ces maladresses ont provoqué la confusion pour l'exploitant, que ce soit au siège ou sur le terrain.

Nous venons donc de parler du réacteur 3. Maintenant nous allons nous intéresser au 2. D'après ce que vous m'avez dit tout à l'heure, le $\mathrm{RCIC}$ du réacteur 2 était en marche. Je pense que, dans votre tête, l'idéal était de profiter de l'action de l'IC pour le réacteur 1, du $\mathrm{RCIC}$ pour le réacteur 2 et des $\mathrm{RCIC}$ et $\mathrm{HPCl}$ pour le 3 , de ces systèmes prévus structurellement pour le refroidissement ou l'injection, pour faire suffisamment baisser la pression avant d'injecter l'eau. Ai-je raison?

\section{R : Oui.}

$\mathbf{Q}$ : C'était le scénario idéal, si c'était possible. Finalement, que ce soit le réacteur 2 ou le réacteur 3, vous avez finalement été obligé de vous diriger vers une ouverture volontaire des vannes SR pour cette dépressurisation. Si j'ai bien compris, cette manœuvre est une opération inhabituelle. Était-ce une manœuvre qu'il valait mieux éviter, si c'était possible?

R: Qu'il s'agisse du RCIC ou du HPCI, j'ai manqué d'informations pour dire s'ils avaient été arrêtés manuellement ou pas, mais ce que je savais, c'est que ces systèmes avaient tenu longtemps, bien plus longtemps qu'on aurait osé l'espérer. Mais ce qui était tout aussi sûr, c'est qu'ils allaient finir par s'arrêter. Il n'était pas question de dépendre d'un système qui allait s'arrêter d'un moment à l'autre. Il fallait prévoir autre chose et la seule solution était de dépressuriser et d'injecter. Bien sûr, jusquelà, nous avions aussi l'espoir de rétablir l'électricité et de ressusciter l'un ou l'autre système, mais cela paraissait de plus en plus irréaliste. Au fond de moi, je savais que la dernière solution consisterait à dépressuriser et à injecter avec les camions de pompiers.

$\mathbf{Q}$ : Si je m'intéresse à ce qui s'est passé le 13 mars en suivant ce que vous venez de me dire, je constate en effet que vous passez la matinée à vous occuper du réacteur 3 dans ce sens. Vous préparez l'éventage, vous exécutez la manœuvre de dépressurisation. Dès avant cela, vous constituez évidemment la ligne d'injection, avec cette fameuse histoire du passage de l'eau de mer à l'eau douce. Tout est prêt, les batteries de voitures ont été rassemblées. Bien que cela vous prenne du temps, vous réussissez à ouvrir la vanne SR. Vous commencez à injecter. Ceci, pour la matinée. Puis, aux alentours de midi, l'eau douce s'épuise. Vous vous empressez de passer à l'eau de mer. C'est un peu après 13 h00 que vous assurez l'injection à l'eau de mer.

Pour ce qui est du réacteur 2, il semblerait que vous ayez transporté des batteries dans la salle de contrôle pour préparer l'ouverture de la vanne SR. Ensuite, jusqu'en fin d'après-midi, 
vous élaborez la ligne d'injection. C'est-à-dire que vous créez les conditions pour pouvoir injecter à tout moment. Toujours concernant le réacteur 2, vous aviez déjà donné l'ordre de préparer la ligne d'éventage le 12 mars en fin d'après-midi.

En fait, vers la fin de la journée du 13 mars, tous ces préparatifs étaient terminés, mais vous aviez un souci. Nous en avons parlé précédemment lorsqu'on a discuté des actions prévues en cas de gestion d'accidents. Vous preniez de l'eau dans le Reversing Valve Pit, mais cette eau était en train de s'épuiser. À l'aube du 14, vous n'arriviez plus à la pomper. Du coup, l'injection était arrêtée. Notamment pour le réacteur 2, vous aviez élaboré une ligne d'injection, celle-ci était prête, mais faute d'eau, vous n'aviez pas pu l'exploiter. Bien entendu, vos équipes, dès l'après-midi du 13, avaient commencé à explorer d'autres solutions. N'y avait-il pas, par exemple, possibilité de pomper directement l'eau dans la mer? Mais rien ne marchait vraiment et je peux supposer qu'au soir du 13 la situation était grave, puisque, faute d'eau, vous ne pouviez plus recourir à la solution du Reversing Valve Pit, la seule dont vous disposiez. En même temps, j'aurais tendance à me dire quelque part que vous aviez fait tout ce qui était en votre pouvoir.

$\mathbf{R}$ : Si je peux me permettre, je dirais qu'entretemps, nous avions mis la main sur plusieurs pompes submersibles pour amener l'eau directement de la mer au bassin. Nous avions fait des tentatives pour remplir ce bassin de cette façon, tout en continuant nos efforts pour trouver de l'eau de toutes les manières, de toutes les sources possibles. Mais la priorité était de remplir ce bassin. Il me semble me souvenir qu'à un moment donné cela avait marché. On m'avait dit que l'eau arrivait et que le niveau était en train de monter. La radioactivité était très forte à cet endroit, alors on ne pouvait pas y stationner pour surveiller le remplissage. Je ne sais plus à quelle fréquence on allait voir. Quelqu'un en est revenu en disant que la pompe, qui devait fonctionner, était arrêtée et qu'il n'y avait plus suffisamment d'eau. Je pense qu'il y avait aussi eu confusion entre le groupe "incendie » et le groupe « sûreté ». Ils ne savaient plus qui devait aller sur place pour vérifier le niveau d'eau et qui devait se charger du refroidissement de celle-ci. Du coup, je ne l'ai moimême su que bien après en les interrogeant, cela a donné lieu à des scènes où les gars de l'incendie étant revenus du terrain en ayant juste vérifié l'état de la ligne, se sont vus reprocher de ne pas avoir aussi regardé le niveau d'eau. Je crois bien me souvenir que ces échanges se situaient le 13.

$\mathbf{Q}$ : Effectivement, c'est une situation qu'on pourrait expliquer un peu comme tout à l'heure. Pour tout ce qui est prévu et inscrit précisément dans les règles de la gestion d'accident, la distribution des rôles est déjà faite pour chaque circonstance. On sait, pour chaque cas, qui fait quoi. Tout est déjà décidé. Mais pour ce qui sort de ce cadre, toutes les actions imaginées ingénieusement sur place par les équipes, la répartition des rôles n'a pas été faite. On ne sait pas qui doit faire quoi et jusqu'où. Pour ceux qui sont sur le terrain et qui inventent des solutions au fur et à mesure, il y a sans doute un fort sentiment de satisfaction à se dire qu'on a réussi à faire tout ça. Cependant, si c'est une action qui doit s'inscrire dans la durée, il n'y a évidemment pas eu de recommandation pour charger un tel ou un tel de continuer à s'en occuper. Cela peut mener à une certaine confusion et cela me semble, en quelque sorte, inévitable. Tout cela prouve aussi l'importance d'avoir défini des règles à l'avance.

Si je reviens au réacteur 2, finalement le 14 au matin des camions arrivent en renfort en provenance de centrales thermiques du sud du Kantô, de Minami-Yokohama et de Chiba ${ }^{11}$. Là, vous les positionnez directement du côté nord des bâtiments pour l'injection.

$\mathbf{R}$ : Oui. Sur le quai de déchargement.

$\mathbf{Q}$ : Si on examine les lignes que vous aviez élaborées à ce moment-là, vous étiez en train de tirer une nouvelle ligne pour alimenter le fameux bassin de la tranche 3. Avant cela, vous aviez prévu avec une certaine avance une ligne d'injection vers le réacteur 2 , alors que le 
RCIC fonctionnait encore. Bien que cette ligne eût été prête, vous ne l'aviez pas utilisée ce matin-là. Vous donnez l'impression d'avoir été focalisé sur le réacteur 3 et uniquement sur lui.

$\mathbf{R}$ : C'est normal. La pression avait baissé dans le réacteur 3, le niveau d'eau était bas, mais on n'avait pas réalimenté le réacteur en eau. Il fallait absolument injecter de l'eau dans le réacteur 3. Pendant ce temps, du côté du réacteur 2 , le RCIC était toujours en marche. Bien sûr, si on avait pu injecter dans les deux réacteurs en même temps, cela aurait été merveilleux. Mais à défaut de mieux, il fallait d'abord injecter en priorité dans le 3. Il fallait tenir compte de la radioactivité. Si on avait disposé de plus de personnel, si on avait eu dix mille personnes dont on aurait pu disposer à volonté, on aurait pu faire beaucoup plus de choses. Mais puisque le nombre d'agents sur le site était limité, il fallait bien se donner des priorités dans nos opérations. Là, le réacteur 3 était en position vraiment dangereuse, alors il fallait injecter en premier lieu dans le réacteur 1 et le réacteur 3. Une fois qu'on aurait injecté un certain volume dans le réacteur 3 , je pensais bien basculer l'injection vers le réacteur 2 , c'està-dire injecter en parallèle dans les deux réacteurs. Dans ma tête, il était clair qu'une fois la situation stabilisée pour le réacteur 3, j'allais m'occuper du réacteur 2 . Malheureusement c'est là que se produit l'explosion. Moi aussi, je voulais injecter dans le réacteur 2 au plus vite. Loin de moi l'idée de temporiser pour ça. Simplement, le volume d'eau étant limité et les réacteurs 1 et 3 en posture plus périlleuse, j'ai préféré utiliser le peu dont je disposais pour eux.

$\mathbf{Q}$ : Pour le non-initié que je suis, il y a une question, sans doute naïve, que je me pose. Regardez, c'est un schéma de la disposition des camions à ce moment-là. Un de ces schémas dont TEPCO est si fier. Celui-ci décrit justement leur position au moment de l'explosion. Si on regarde ça, on voit que les choses s'organisent autour du Reversing Valve Pit de la tranche 3. II y a là la ligne qui entre directement dans la tranche 3, une autre qui traverse la voie et s'en va vers la tranche 2 , une autre qui alimente le bassin, puis un relais de camions pour acheminer l'eau vers la tranche 1. Une organisation, donc, où on injecte à partir du bassin de la tranche 3, en élaborant une ligne pour le réapprovisionnement du bassin lui-même. Là, les lignes continues représentent les réseaux qui étaient effectivement en fonctionnement. On voit que la ligne d'injection du réacteur 1, par exemple, était en attente. L'amateur que je suis a envie de dire que, malgré une certaine radioactivité, puisque l'eau va couler à flot de la mer, il n'y a qu'à tout envoyer à la fois, injecter simultanément dans les trois réacteurs. Est-ce une idée simpliste?

$\mathbf{R}$ : Tout d'abord, à ce moment-là, le réacteur 2 n'avait pas encore été dépressurisé.

$\mathbf{Q}$ : Effectivement, vous avez raison. Si on ne dépressurise pas, l'eau ne va pas entrer.

$\mathbf{R}$ : C'est pour ça que la ligne d'injection devait être en attente.

$\mathbf{Q}$ : Oui, mais dans la salle de contrôle, les agents étaient prêts. Ils avaient rassemblé dix batteries et ils étaient prêts à dépressuriser dès que vous en auriez donné l'ordre. Cependant il n'y avait de l'eau que dans les réacteurs 3 et 1.

$\mathbf{R}$ : C'est parce que, compte tenu de la quantité d'eau qui alimentait le bassin et le niveau de celui-ci, si on avait injecté dans tous les réacteurs, on serait arrivé à un moment où l'eau ne serait plus entrée.

$\mathbf{Q}$ : Vous voulez dire que vous auriez consommé plus d'eau que vous n'auriez pu en apporter?

$\mathbf{R}$ : C'est ça. On en aurait trop consommé. Et là, on se fait la réflexion que le réacteur 1 a déjà reçu individuellement une certaine quantité d'eau. Il avait reçu pas mal d'eau de mer. L'injection venait juste de commencer pour le réacteur 3, quand on a manqué d'eau douce. De plus, cela ne faisait pas longtemps qu'on était passé à l'eau du bassin, 
quand celui-ci s'est vidé. C'était sans doute celui qui contenait le moins d'eau. Il avait été dépressurisé et le combustible était partiellement à l'air. Or il n'avait pas reçu beaucoup d'eau. Par conséquent, il fallait commencer par lui pour l'injection. Une fois qu'il aurait reçu un certain volume d'eau, on aurait pu envisager d'injecter aussi dans le réacteur 1 . Puis, on aurait dépressurisé le 2 . Voilà le genre de réflexions qu'on se fait normalement.

Parallèlement, il y avait le problème de la capacité du bassin. Même si l'eau coulait à flot, en comparant le volume d'eau qui arrivait et le volume d'eau qu'on injectait, l'équilibre entre les deux aurait été brisé si on avait injecté dans les trois réacteurs simultanément. Il serait très vite arrivé un moment où le niveau d'eau dans le bassin aurait tellement baissé qu'il n'aurait plus été possible d'y pomper l'eau. C'est une préoccupation qui ne m'a pas quitté. On n'avait pas un aussi joli schéma à l'époque, mais j'avais à peu près en tête les dispositions des lieux. «En attendant mieux, on va injecter à partir du Reversing Valve Pit de la tranche 3. Comme le volume d'eau est limité, on va d'abord s'occuper du réacteur 3. On garde le réacteur 1 pour la suite, puisqu'il a déjà reçu une bonne quantité d'eau. On privilégie le réacteur 3 . Le réacteur 2 est en attente? Alors, quand on aura un peu injecté dans le réacteur 3, on va dépressuriser le 2. Tenez-vous prêts ». Voilà quelles ont été mes réactions à ce moment-là.

Q: Tout à l'heure, nous avons évoqué la radioactivité. Le 14 mars vers 7 h00 ou 8h00, elle augmente et vous mettez vos équipes à l'abri. En fait, dès l'aube du 14, la pression de la chambre sèche avait atteint $500 \mathrm{kPa}$, tout comme celle ...

$\mathbf{R}:$ Du réacteur 1.

$\mathbf{Q}$ : Vous aviez jugé que le comportement du réacteur devenait imprévisible.

$\mathbf{R}$ : Oui, c'est pour ça que j'ai ordonné que tout le monde quittât le terrain.

$\mathbf{Q}$ : Vous en aviez donc donné l'ordre. Et c'est dans ces conditions que vos troupes devaient opérer. Je comprends, effectivement, que vous ayez été obligé d'établir des priorités et commencer les manœuvres par ce qui était possible.

Tout au début de l'accident, vers les 11, 12, 13 ou 14 mars, vous aviez envisagé de réparer le circuit à l'acide borique pour vous en servir comme moyen substitutif d'injection, n'est-ce pas?

$\mathbf{R}$ : En ce qui concerne le réacteur 1 , oui.

$\mathbf{Q}$ : Pour le réacteur 1. Et le 3 ?

$\mathbf{R}$ : Nous l'avions aussi envisagé pour le réacteur 3, mais, finalement, nous n'avions pas d'alimentation électrique pour le faire fonctionner. Pour le réacteur 1 , nous avions réussi à avancer dans les préparatifs et nous étions prêts à injecter. Seulement, il ne faut pas oublier que le volume d'eau du système SLC est assez modeste. Je crois que ça doit représenter $80 \mathrm{t}$ en additionnant les deux. Tout de même, c'était mieux que rien. Et puis, l'avantage, c'est qu'elle contient de l'acide borique qui est très efficace pour éviter les accidents de criticité. Donc, si le SLC était disponible, il fallait l'utiliser. Pour être franc, j'aurais voulu disposer d'un moyen qui assure une injection continue. Mais en attendant il fallait injecter tout ce qui était injectable. C'est pourquoi j'ai donné des directives pour que les gars de la « production » y travaillent en priorité. 
$\mathbf{Q}$ : Du côté du réacteur 1, vous aviez déjà injecté assez durablement de l'eau douce. Jusque vers les 14h00, il me semble. Une fois que l'eau douce a été épuisée, vous êtes tout de suite passé à l'eau de mer. Pensiez-vous, tout en injectant l'eau de mer, injecter aussi par le SLC ?

R : Oui. Nous injections de l'eau douce. En fait, la ligne qui permettait de basculer de l'eau douce à l'eau de mer n'était pas encore prête. C'était juste avant l'explosion. Jusque-là, j'avais exigé qu'on injectât tout ce qu'on trouvait comme eau, qu'on se dépêche aussi de préparer le SLC. Ordre donc d'injecter l'eau douce. Et juste au moment où on m'a annoncé que le SLC était prêt et que je m'apprêtais à donner l'ordre de le mettre en marche, ça a explosé. Alors, ce n'était pas une histoire de priorité. Il fallait exécuter tout ce qui était réalisable, injecter tout ce qui était injectable.

Q: Pour en revenir à l'acide borique, vous l'avez introduit une fois que vous aviez commencé à injecter de l'eau de mer à partir du bassin du réacteur 3 , n'est-ce pas ?

R: Oui, nous l'avons fait. Mais là, c'est une autre histoire. Le SLC est un système à part, avec son réseau propre. Nous pensions bien, sans aucun doute possible, que le combustible avait commencé à fondre. Mais nous ne savions pas dans quelles proportions. Aujourd'hui les analystes prétendent différentes choses. Des informations qu'ils n'ont même pas la politesse de faire remonter jusqu'ici, sur le site. Ils viennent après coup, font des révélations dont ils ne nous informent même pas. Il ne faut pas se moquer du monde! Enfin, laissons ça de côté. De toute façon, sur le moment, nous avons eu des discussions, au sein de la centrale, pour savoir s'il était efficace ou non d'introduire de l'acide borique.

$\mathbf{Q}$ : Ces discussions n'ont pas eu lieu, à la suite de la suggestion de quelqu'un.

$\mathbf{R}$ : Moi, je pensais dès le début que s'il y avait moyen d'introduire de l'acide borique, il fallait le faire.

$\mathbf{Q}$ : Concernant l'acide borique lui-même, en aviez-vous en réserve, que ce soit à l'usage du SLC ou autre ? Ou bien avez-vous dû en réclamer?

$\mathbf{R}$ : Dès le départ, chaque tranche avait sa réserve d'acide borique pour alimenter le SLC. De plus, les entrepôts, à Fukushima Daiichi, ont toujours eu la réputation de conserver toutes sortes de choses. C'est une histoire qui remonte un peu, mais vous souvenez-vous de l'accident de la Japan Nuclear Fuels Conversion ${ }^{12}$ ? Ils ont eu besoin d'acide borique. Ils en cherchaient sur tout le territoire. À l'époque j'étais chef du département "production" à Fukushima Daini. On m'a demandé si je disposais d'acide borique à $2 \mathrm{~F}$. Malheureusement nous n'avions tout juste que la réserve pour nos tranches. À ce moment-là, on a interrogé $1 \mathrm{~F}$, qui nous a répondu qu'ils en avaient des stocks anciens en grande quantité qui s'étaient accumulés au fil du temps dans les entrepôts. Ils s'en sont servis à l'époque. Comme quoi notre centrale disposait encore d'un stock non négligeable au moment de l'accident. De plus, quand j'ai demandé s'il n'y en avait pas d'autre à proximité, quelqu'un m'a répondu qu'on pouvait en trouver à ôkuma, dans une usine ou un entrepôt, je ne sais plus. On en a apporté de là aussi. Donc, l'eau seule ne suffisait pas. Pour stabiliser le réacteur, il fallait absorber les neutrons et pour ça il valait mieux injecter de l'acide borique. Nous en avons discuté avec le chef de quart et le groupe "technique ». "Ce serait mieux d'envoyer de l'acide borique ». "Oui, il vaut mieux ça que rien du tout ». "Alors, on y va ». C'est comme ça qu'on a mis de l'acide borique même dans l'eau de mer destinée au réacteur 1. 
$\mathbf{Q}:$ Parce que vous n'en aviez pas mis dans l'eau douce?

$\mathbf{R}$ : Là, il y a eu un décalage avec la préparation de l'acide borique. Puisqu'il fallait faire fondre l'acide dans l'eau, j'aurais bien aimé le faire avec de l'eau douce, mais nous n'en avons pas eu le temps. Sans attendre, nous avons donc injecté juste de l'eau douce.

$\mathbf{Q}$ : Quand vous dites «mettre dans l'eau de mer », ça signifie que vous en mettez comme ça, en vrac dans le bassin?

R : Oui.

$\mathbf{Q}$ : J'entre un peu dans les détails, si vous permettez. Vous poursuivez donc l'injection dans le réacteur 3, le 14 mars. Mais, au fur et à mesure, la teneur de l'eau en acide borique va diminuer. En avez-vous rajouté ?

$\mathbf{R}$ : Si on suit l'injection avec les schémas de tout à l'heure, on commence par l'injection du réacteur 1.

$\mathbf{Q}$ : C'est là où vous avez injecté de l'eau de mer dans le réacteur 1.

R : Ici. C'est cette ligne-là que nous avons utilisée.

$\mathbf{Q}:$ La ligne traverse et arrive là. C'est ici que... ?

$\mathbf{R}$ : On introduisait l'acide borique ici et on injectait comme ça. Quand on a commencé à injecter dans le réacteur 3 , on partait tout d'abord du réservoir d'eau prévu pour l'incendie. Ce genre de réservoir est fermé, ce qui veut dire qu'il n'y avait pas d'endroit où faire fondre la poudre. Du coup, on ne pouvait pas mettre de l'acide borique. Comme c'était fermé, on prenait juste l'eau qui se trouvait dans le réservoir, telle qu'elle était, pour l'injection. On n'a pas mis d'acide borique, tout simplement parce que ce n'était pas un bassin ouvert et qu'on ne pouvait pas faire dissoudre la poudre. Il n'y avait pas d'autres raisons que ça. C'est à partir du moment où on a enfin commencé à se fournir d'eau dans le Reversing Valve Pit que, l'introduction de l'acide borique devenant possible, j'en ai donné l'ordre. J'en ai le souvenir. Par contre, je ne me souviens pas aujourd'hui, si cet ordre a tout de suite été suivi d'effet. Le réacteur 2 , quant à lui, était toujours en attente. Après l'explosion, vous vous souvenez sans doute qu'on a changé de ligne. On a établi une ligne d'injection qui ne passait plus par le fameux bassin. Finalement, pour l'injection du réacteur 2, on avait bien élaboré dans un premier temps un circuit qui passait par le bassin, mais on ne l'a jamais utilisé. L'eau injectée dans le réacteur 2 n'est jamais passée par ce bassin. On a tout de suite utilisé une ligne qui amenait directement l'eau, de la mer vers le réacteur. Et là, il n'y avait plus d'endroit pour ajouter l'acide borique.

$\mathbf{Q}$ : Vous voulez dire que vous n'avez pas introduit d'acide borique dans le réacteur 2 ?

$\mathbf{R}$ : On n'a pas pu. C'était dommage, mais on ne pouvait pas. Au début, quand on pensait que l'eau allait transiter par le bassin du réacteur 3, on avait bien l'intention de le faire. Mais après l'explosion, cela n'a plus été possible.

$\mathbf{Q}:$ Parce qu'on ne peut pas mettre de l'acide borique là ?

$\mathbf{R}$ : Non, on ne peut pas. C'est la mer.

$\mathbf{Q}:$ Ah, oui. Cela ne servirait à rien de mettre de l'acide borique dans la mer.

$\mathbf{R}$ : Au début, quand on s'est aperçu qu'on allait pomper directement l'eau de la mer, on a bien envisagé la possibilité de mettre un réservoir ou quelque chose du genre, ici, en dessous. Mais ce n'était pas possible. Alors nous avons renoncé. 
$\mathbf{Q}$ : Ce qui veut dire que le réacteur 2 n'a pas reçu d'acide borique et que les réacteurs 1 et 3 en ont reçu quand l'eau injectée transitait par le bassin Reversing Valve Pit.

J'ai encore quelques questions de détails à vous poser. Si vous n'avez pas de réponse, ce n'est pas grave. Quand on regarde ces documents, on voit que pour l'éventage l'ouverture des valves MO était de $15 \%$ pour la tranche 3 et de $25 \%$ pour la tranche 2 . Or les préparatifs pour ces deux éventages se sont faits quasi simultanément d'après mes souvenirs. Y a-t-il une raison à cette différence?

\section{$\mathbf{R}$ : Je ne m'en souviens pas.}

$\mathbf{Q}$ : Vous ne savez pas. Alors vous souvenez-vous s'il y avait un ordre dans ces ouvertures, d'abord ouverture de la vanne AO suivie de l'ouverture de la vanne MO, ou le contraire?

$\mathbf{R}$ : Je ne sais pas. Pour moi, je donnais l'ordre de préparer l'éventage. Pour ne pas nous retrouver dans la situation de la tranche $1, \mathrm{j}$ 'avais donné l'ordre de rassembler, avant que la radioactivité n'augmente, les conditions pour un éventage à n'importe quel moment. On m'a fait savoir que la vanne MO s'était ouverte, mais je n'étais pas au courant du taux d'ouverture.

$\mathbf{Q}:$ C'était la «production » qui était chargée de réfléchir à la procédure?

$\mathbf{R}:$ La « production » et la « réhabilitation ».

$\mathbf{Q}$ : Oui, au besoin, il fallait que le groupe « réhabilitation » intervînt aussi.

II y a eu l'explosion de la tranche 3. Des équipes d'analystes de TEPCO en ont mesuré le choc sur la tranche 2. Y a-t-il un endroit où on mesure la secousse, enfin, l'onde de choc, en mettant le réacteur 2 au centre de cercles concentriques? Ils ont analysé ça et ont rendu les résultats publics.

$\mathbf{R}$ : Vous voulez dire au moment où il y a eu l'explosion de la tranche 4 et qu'on se posait des questions sur la tranche 2 ?

$\mathbf{Q}$ : Oui. Ils disent que si on met le réacteur 2 au centre, on constate une onde de choc et que l'origine de ce bruit serait la tranche 4. J'ai regardé les auditions qui ont été faites récemment des agents qui étaient sur place. C'était au moment où les équipes de quart des tranches 3 et 4 devaient se relayer. La relève devait se faire à 6h00. Ceux qui devaient prendre le relais ont dit que, juste avant, ils étaient arrivés normalement en voiture prendre leur quart. Au moment où ils allaient se passer les consignes, ils ont entendu une déflagration en provenance de la tranche 4.

$\mathbf{R}$ : Oui, ils m'ont dit qu'ils avaient senti le vent de l'explosion leur arriver dans le dos.

$\mathbf{Q}$ : Quand on entend ce genre de témoignage, on se dit que la déflagration était due à l'explosion de la tranche 4. En tout cas, ensuite, les agents ont eu beaucoup de mal à rentrer. Ils ont dû marcher au milieu d'innombrables débris, ce qui fait qu'ils ont mis du temps à revenir, retardant d'autant l'arrivée des informations à la cellule de crise.

$\mathbf{R}$ : Si je vous décris la situation de la cellule de crise à ce moment-là, on attendait Monsieur Kan. Enfin, pas à la cellule de crise, mais au siège. Il devait venir au siège pour nous sermonner ou quelque chose de ce genre. Toujours est-il que de bon matin nous étions reliés par téléconférence avec le siège et nous l'attendions. Moi-même, je n'ai pas entendu la déflagration.

$\mathbf{Q}:$ Vous ne l'aviez pas entendue.

$\mathbf{R}$ : Moi-même, dans le bâtiment antisismique, je n'ai pas entendu ce grand bruit. Mais c'est le moment où on m'a informé, je crois que c'était des agents de conduite, coup sur coup que la pression de la chambre humide du réacteur 2 était tombée à 0 et qu'on avait entendu un grand bruit. Je reçois ces deux informations. Je ne savais pas si le bruit provenait de la tranche 2 ou d'ailleurs. Mais il est certain que confronté à ces deux nouvelles, un grand bruit et la pression de la chambre humide du 2 à 0 , j'ai 
pensé au scénario le plus dangereux. À ce moment-là, je n'ai pas encore les informations en provenance de la tranche 4 . Je soupçonne donc que la pression de la chambre humide est tombée à 0 , parce que l'enceinte de confinement du réacteur $2 \mathrm{a}$ été détruite. Mais, en même temps, il y avait encore de la pression dans la chambre sèche. Ce qui a fait dire au siège que la situation n'était peut-être pas si sérieuse. Mais ça, c'est quelque chose qu'on pense quand on est physiquement loin. Si on est sur place, qu'on entend un grand bruit et que la pression de la chambre humide est à 0 , il est normal de penser que la situation est périlleuse. De toute façon, je considérais que le manomètre de la chambre sèche n'était pas fiable depuis bien longtemps. Alors j'ai dit qu'il fallait envisager de mettre les gens à l'abri, mis à part le minimum de personnel nécessaire.

$\mathrm{Au}$ bout de vingt à trente minutes, des agents sont revenus de la tranche 4. Ils me disent que la tranche 4 tombe en lambeaux. J'envoie prendre des photos des bâtiments. En effet, tout était sens dessus dessous. Je ne me rappelle plus qui était le chef de quart. XXXXX ou le chef XXXXX est revenu le premier. Je lui ai demandé comment était la situation. Il m'a répondu qu'il y avait eu une onde de choc. Il l'avait sentie au moment où il entrait dans le bâtiment de service des tranches 3 et 4 . Il avait senti le vent de l'explosion et, au moment de quitter les lieux, il s'était retourné et avait vu que la tranche 4 tombait en lambeaux. Je ne me rappelle plus si XXXXX et XXXXX, que j'ai interrogés plus tard, m'ont dit la même chose. Mais comme le moment où ils ont senti ce souffle et le moment où la pression de la chambre humide a chuté à 0 était sensiblement simultanés, il m'était difficile, à moi, de trancher entre les deux scénarios. De toute manière, puisque la pression de sa chambre humide était nulle, le réacteur 2 était en situation très précaire. Si on supposait qu'il y avait des failles, cela signifiait que des éléments radioactifs s'en échappaient. La situation était grave. J'ai jugé qu'il fallait mettre à l'abri le plus de monde possible. C'est comme ça que j'ai pris la décision d'évacuer.

$\mathbf{Q}$ : Justement cette "évacuation », vous y pensiez dès la veille au soir, quand le réacteur 2 était en mauvaise posture. C'est à ce moment-là qu'il y a eu différentes informations contradictoires à ce sujet dans les médias, il me semble ${ }^{13}$.

$\mathbf{R}$ : Par rapport à tous ces remue-ménages autour de cette évacuation, j'ai envie de dire qu'il n'y a pas de quoi fouetter un chat. Est-ce que nous avons pris la fuite? Visiblement, non. Quand est-ce qu'on a pris la fuite ? Il faudrait me le dire. Je ne sais pas à quel jeu se prête le siège ou le cabinet du Premier ministre, mais regardez, estce que le terrain a fui ? Vous voyez bien que le terrain n'a pas fui. C'est la seule chose que je voudrais clamer haut et fort. Au lieu de ça, on discute futilement pour savoir si on a dit de fuir ou pas, ça c'est le Premier ministre lui-même qui le prétend. Je n'ai pas peur de le dire : quel crétin! Je n'ai jamais dit qu'il fallait fuir. Certes, la situation était déjà très dangereuse, mais si elle empirait, ma position a toujours été d'évacuer, à la toute fin, tout en maintenant sur le site de la centrale le minimum de personnel pour assurer l'injection d'eau, par exemple. Moi-même, bien entendu, je serais resté $^{14}$. Mais il y avait aussi beaucoup d'autres personnes comme les administratifs, que je préférais évacuer vers un lieu plus sûr. 
Q: Oui, c'est ce que vous m'aviez déjà dit précédemment, lorsque nous nous étions vus. Pensez-vous que les officiels du siège, y compris le Directeur général Shimizu, partageaient votre avis?

$\mathbf{R}$ : Ici, à la centrale, on ne savait même pas que le Directeur général avait été appelé à la résidence du Premier ministre.

$\mathbf{Q}$ : II semblerait qu'il ait été convoqué. J'ai eu l'occasion, depuis, de m'entretenir avec le directeur Shimizu. Et j'ai pu constater qu'il pensait la même chose que vous. II m'a dit qu'il ne pouvait pas imaginer qu'on ait même pu évoquer un retrait général en laissant tout en plan. D'ailleurs, il en avait parlé à l'époque directement avec Monsieur Kan qui lui avait dit: "Ah, c'était donc ça. Je trouve que nous ne nous comprenons pas bien mutuellement. II faudrait travailler ensemble ». C'est comme ça que la fameuse cellule conjointe a été fondée avec la bénédiction de Monsieur Shimizu qui avait trouvé que c'était une bonne idée. En fait, Monsieur Shimizu ne comprend pas pourquoi Monsieur Kan est revenu sur cette histoire de retrait général. De votre côté, vous souvenez-vous s'il y a eu des suggestions du siège pour un retrait général ?

$\mathbf{R}$ : Non, il n'y en a pas eu du tout.

$\mathbf{Q}$ : Vous n'auriez pas eu un ordre de retrait du siège que vous auriez ignoré en pensant que vous ne pouviez pas vous y soumettre?

$\mathbf{R}$ : Non. Si je me replace dans le contexte, il y a eu un moment où l'eau n'arrivait pas à entrer dans le réacteur 2. Parce que la vanne SR ne voulait pas s'ouvrir. Là, j'étais très énervé. Ensuite, on a dépressurisé. On avait réussi à dépressuriser, mais cette fois le camion de pompiers n'avait plus de carburant. Le temps de le réapprovisionner, on n'a pas pu injecter l'eau. Là, chaque seconde qui passait m'oppressait. J'étais suspendu au temps en me demandant si l'eau allait vraiment entrer. Si elle n'entrait pas, nous allions vers la catastrophe. Comme je vous l'ai dit l'autre jour, si elle n'entrait pas, le combustible allait fondre. La radioactivité serait montée en flèche. Personne n'aurait pu approcher des réacteurs 1 et 3 pour assurer leur injection. Si cette situation devait se prolonger, les combustibles des réacteurs 1 et 3 auraient suivi le même sort. Si on ne réussissait donc pas à injecter l'eau dans le réacteur 2, la situation allait devenir catastrophique.

Je voyais même plus loin. Si cette situation elle-même perdurait, les éléments radioactifs auraient atteint Fukushima Daini. On n'aurait plus eu la possibilité de travailler sur les quatre tranches de Fukushima Daini non plus. Si on ne pouvait plus assurer leur injection et tout le reste, je savais pertinemment ce qui allait se passer. Toutes ces pensées tournaient dans ma tête. Il y avait des possibilités pour que cela nous arrive. Mais, sur le moment, on ne pouvait pas encore savoir. Il fallait juste attendre en priant que l'eau entre.

Mais même si on avait été confronté au pire, il fallait que les techniciens, les gens capables d'injecter l'eau, de manœuvrer, c'est-à-dire les pilotes, le groupe "réhabilitation », le groupe "incendie » et, bien sûr, moi-même restions à notre poste. Seulement, il y avait des gens qui n'avaient rien à voir avec tout ça. Du personnel des entreprises partenaires, des administratifs de chez nous. Il fallait mettre ces personnes en lieu sûr, puisqu'il y avait danger d'irradiation. Je ne sais plus à qui, mais je me rappelle bien avoir parlé au téléphone du fait que la situation devenait très dangereuse et que j'envisageais, suivant les circonstances, de faire évacuer tout le personnel administratif. Ensuite, j'ai téléphoné à Monsieur Hosono et lui ai dit: "La tranche est dans un état très périlleux. Les choses peuvent basculer d'un instant à l'autre. La seule solution est d'injecter de l'eau. On ne sait pas si on va y arriver ou 
pas. C'est un pari. Mais nous ferons tout pour que ça marche. Simplement, je pense qu'il est nécessaire d'évacuer les personnes qui n'ont rien à faire ici. Nous sommes en train de préparer leur départ $»$.

$\mathbf{Q}$ : Donc, même vis-à-vis de Monsieur Hosono, vous aviez évoqué votre désir, suivant les circonstances, d'évacuer les gens qui n'avaient rien à faire à la centrale.

$\mathbf{R}$ : Oui. Je lui ai décrit la situation, je lui ai expliqué qu'il faudrait continuer à observer de près l'évolution de la tranche, que nous allions le faire, mais que je pensais également à la nécessité de faire évacuer certains. De fait, certaines personnes des entreprises partenaires qui stationnaient dans le couloir du bâtiment antisismique, à qui j'ai dit que si elles n'avaient plus de travail à assurer sur place, elles pouvaient se retirer, sont parties d'elles-mêmes.

Q: Ça, c'est ce qui s'est passé dans la nuit du 14 au 15 mars, avant l'aube.

$\mathbf{R}$ : Oui, c'était au moment où on ne savait pas si l'eau allait entrer dans le réacteur 2 ou pas.

$\mathbf{Q}:$ Ce qui veut dire qu'il n'a jamais été question d'abandonner les opérations nécessaires au contrôle des tranches. Dans les faits, le 15, vous allez être confrontés à la fois aux menaces des réacteurs 2 et 4 , pourtant au moins une cinquantaine de personnes vont se maintenir sur place. Si je comprends bien, seul le personnel minimum indispensable au contrôle des tranches est resté sur place autour de vous et tous ceux qui n'avaient pas de tâches particulières à effectuer dans l'immédiat sont partis provisoirement.

R : Oui, je leur ai demandé de se mettre à l'abri, que ce soit à Fukushima Daini ou ailleurs.

Q: Suivant l'évolution des tranches, vous en rappeliez un, puis deux, pour étoffer les équipes.

$\mathbf{R}:$ C'est ça.

$\mathbf{Q}$ : Du point de vue du langage, avez-vous utilisé l'expression « retraite générale »?

$\mathbf{R}:$ «Retraite générale »? Non, pour sûr, non.

$\mathbf{Q}:$ Vous ne l'avez donc pas utilisée.

$\mathbf{R}$ : Non, je ne l'ai pas utilisée. Je ne sais pas qui a utilisé ce genre d'expression. Peutêtre Kan? En tout cas, il ne me viendrait même pas à l'esprit d'utiliser cette expression. Au contraire, je voudrais protester. Ces abrutis à la télévision parlent de « retraite générale ». J'aimerais leur demander qui n'a jamais parlé de «retraite générale ».

$\mathbf{Q}$ : Votre directeur général m'a dit qu'il n'utilisait pas l'expression «retraite générale », qu'il disait «mise à l'abri ». "Ce que recouvre l'expression «mise à l'abri » pour moi, ça ne peut évidemment pas être un abandon total. Si c'était le cas, cela signifierait, comme l'a évoqué Monsieur Kan, la destruction à terme de tout le Japon, y compris Tôkyô. Alors, je n'y ai même pas pensé. La 'mise à l'abri', pour moi, veut dire un déplacement provisoire du personnel non indispensable au contrôle des tranches nucléaires d'un lieu à un autre, tout en laissant suffisamment de compétences sur place. C'est comme ça que je comprends l'expression et que je l'ai utilisée ». Voilà ce qu'il m'a dit.

Vous aussi, vous dites donc à ce stade que, suivant les circonstances, c'est-à-dire si la situation s'aggravait, vous pourriez être amené à maintenir seul le personnel indispensable et évacuer les autres. Vous ne vous rappelez plus si vous en avez parlé personnellement au directeur de la NISA ou à Monsieur Kaieda, mais vous êtes sûr que la NISA était au courant de vos réflexions. Lorsque j'en ai parlé au directeur Terasaka de la NISA, il m'a confirmé en effet qu'il partageait tout à fait vos points de vue. II n'a jamais pensé qu'il y aurait 
évacuation générale, que tout le monde puisse partir. Ça, c'est ce qui se passait vers les 7 h00 ou 8h00 du soir. Or, les hommes politiques avaient compris une toute autre histoire.

$\mathbf{R}$ : Écoutez, je ne veux pas savoir. Un pays de crétins gouverné par des crétins d'hommes politiques, voilà où nous vivons. Je n'ai qu'une envie, les plaquer tous, tant qu'ils sont. Vous ne voyez pas un bon moyen de les plaquer tous, là ?

$\mathbf{Q}$ : Probablement, ils ont compris «retraite générale » à la place de «mise à l'abri » et comme « retraite générale » évoquait l'idée d'abandon, ils ont trouvé cela inadmissible et se sont monté le bourrichon. C'est comme le jeu du téléphone arabe. J'imagine facilement comment cela s'est passé. Je pense qu'il en sera question aux réunions plénières de la commission. Pouvez-vous m'en dire un mot?

R: Je voudrais que vous leur disiez une chose, une seule. Dites-leur que le directeur voudrait savoir si quelqu'un n'a jamais découvert une seule personne ayant pris la fuite durant ces évènements. Moi, ma réponse est non. Puisque personne n'a fui, je ne tiens pas à savoir qui dit quoi.

$\mathbf{Q}:$ II ne le dit plus trop ces temps-ci, mais il fut une époque où Monsieur Kan se vantait d'avoir empêché lui-même la fuite de TEPCO'15.

$\mathbf{R}$ : Déclaration qu'il a faite dès qu'il a démissionné. Vous croyez sérieusement que ce type a le droit de faire ce genre de déclaration? Lui aussi fait l'objet des auditions de la commission, non? Qu'un type comme lui puisse, dès qu'il a démissionné, déballer sa théorie personnelle à la télévision me semble le comble de la déloyauté. Moi, je ne suis pas loin de penser que la commission devrait même porter plainte.

$\mathbf{Q}$ : Alors que c'est lui-même qui a voulu cette commission.

$\mathbf{R}$ : Dire qu'il prétendait être lui-même un prévenu parmi d'autres. J'ai envie de dire à cet imbécile qu'un prévenu ferait bien de ne pas parler à tort et à travers. Qu'en pensez-vous? Vous ne voudriez pas noter ça dans votre rapport?

$\mathbf{Q}$ : Nous en avons presque fini. Encore quelques questions. Vous vous rappelez que les opérations sur le réacteur 2 ont été très délicates, comme, d'ailleurs, les opérations sur la tranche 1 après son explosion. Là, concernant la tranche 2, il devait être difficile de l'approcher après l'explosion de la tranche 3 à cause de la radioactivité. Si je m'intéresse au moment de la reprise des travaux en suivant les documents dont nous disposons, le 14 mars...

$\mathbf{R}:$ Ça avait explosé à 11 h02, il me semble ${ }^{16}$.

$\mathbf{Q}$ : Ici, ils ont noté «11h01». Après l'explosion, les premières mentions concernant la tranche 2 apparaissent à 13h05. II est écrit: "objectifs pour 14h30: préparer le réseau d'approvisionnement en eau pour la tranche 2, réparer le bassin Reversing Valve Pit de la tranche 3 et terminer les manœuvres d'éventage de la tranche 2 par la vanne SR». Nous sommes à peu près deux heures après l'explosion. Avant ça, il y a aussi l'évocation d'une réparation urgente à effectuer sur la ligne d'éventage de l'enceinte de confinement du réacteur 2, qui aurait été déplacée par l'explosion. D'après les notes laissées à la salle de contrôle des réacteurs 1 et 2, il s'agirait d'un circuit, qui avait été prévu pour charger la vanne magnétique de la vanne $\mathrm{AO}$ en vue de l'éventage, que l'explosion avait déplacé. Cette opération s'est visiblement faite avant. Mais compte tenu du fait qu'elle se faisait à l'intérieur de la salle de contrôle, elle était nettement moins risquée. Donc, si on fait abstraction de cette opération, il semblerait que les travaux autour du réacteur 2 ont repris pour de bon vers les 13h00. Est-ce votre ressenti?

$\mathbf{R}$ : Oui, tout à fait. Dans un cas comme celui-là, le plus important est de s'assurer que tout le monde va bien. Ce qui m'a tout de suite préoccupé était le sort des hommes des forces d'autodéfense. Nous-mêmes avions eu des blessés, bien que, fort heureusement, nous n'ayons pas eu de morts à déplorer. Eux aussi avaient eu des 
blessés. Mais nous n'étions sûrs de rien. Car ils s'étaient enfuis tous seuls, sans prévenir personne, sans faire aucun rapport. Au début, on ne savait pas s'ils avaient eu des morts ou des blessés. Nous, de notre côté, on devait rendre des comptes, alors il a fallu partir à la chasse aux renseignements. Tout ça m'a bien occupé au début. Une fois qu'on avait réglé le problème des blessés, on est revenu aux actions sur le terrain. Là aussi, il fallait impérativement continuer à injecter l'eau. Je ne me rappelle pas exactement de l'heure, mais j'ai le sentiment d'avoir recommencé à donner des ordres dans ce sens assez vite.

C'est là que les hommes qui étaient partis évaluer la situation sur le terrain ont commencé à revenir. Les nouvelles étaient mauvaises. "Ce sont des montagnes de gravats 》. "Les camions de pompiers ont été endommagés 》. "Comme ils étaient stationnés devant le bassin de la tranche 3, les camions, même ceux qui venaient d'arriver, ont besoin de réparation ». "Les voies d'accès sont impraticables à cause des débris ». Dans la cellule de crise, ils étaient tous désemparés, amorphes. Ils n'arrivaient plus à réagir. Je me suis dit que je ne pouvais pas laisser s'installer cet état de choses. Je les ai tous réunis autour de moi. Je leur ai dit: "Je sais que la situation est très grave. Nous avons eu des blessés. Mais il faut injecter de l'eau coûte que coûte. Si on ne le fait pas, le pire pourrait arriver. Je voudrais qu'on reparte avec pour objectif de restaurer les lignes d'injection. D'après les rapports que je viens de recevoir, le terrain est jonché de débris et il n'y a pas de voie d'accès pour les camions de pompiers. Le bassin de la tranche 3 est inutilisable à cause des morceaux de béton qui sont tombés dedans. Je voudrais que le groupe 'génie civil' mette tous ses engins en service pour déblayer le terrain. Les autres, attelez-vous à la restauration des lignes d'injection. On va y arriver ». Après ça, ils étaient tellement galvanisés que tout le monde voulait retourner sur le terrain. Ça a été la confusion.

Je me souviens que XXXXX était resté très calme. Il a dit: «ça ne sert à rien de se précipiter tous ensemble. On va se gêner. On n'arrivera à rien». Il fallait revoir la répartition des tâches, déterminer le rôle de chaque groupe. Ceux qui allaient courir pour aller vérifier les besoins sur place, ceux qui allaient commander et diriger les engins. Le groupe "incendie» devait restaurer la ligne d'injection, remplacer certains tuyaux par de nouveaux, réparer les parties abîmées. L'objectif était de pouvoir reprendre l'injection vers $14 \mathrm{~h} 30$. Je leur ai imposé un horaire, car cela leur donnait un but précis. Il y avait en plus l'histoire du TAF, n'est-ce pas?

Q: Oui, il y avait eu une réévaluation et le TAF devait être atteint à 15h30. Précédemment, vous pensiez qu'avec la baisse de niveau dans le réacteur 2, le TAF serait atteint à 16h00.

$\mathbf{R}$ : Oui, c'est à cause de ça que j'avais fixé l'objectif à 14h30. J'avais dit aux hommes que nous devions réussir à injecter avant cette échéance coûte que coûte.

$\mathbf{Q}$ : Ensuite, si on continue à dérouler les évènements, en début d'après-midi, il y a ce fameux coup de fil de Monsieur Madarame qui va déclencher ces discussions autour de la priorité à accorder à l'éventage (de la chambre de dépressurisation) ou à la dépressurisation pour l'injection dans le réacteur, sans attendre l'achèvement de la ligne d'éventage ${ }^{17}$. Tout cela a été enregistré par visioconférence. Au moment où Monsieur Madarame vous a contacté, vous étiez encore en train de préparer l'éventage?

$\mathbf{R}$ : Les préparatifs avaient déjà commencé avant le coup de fil. On avait apporté l'alimentation électrique dans la salle de contrôle et tout était prêt pour l'éventage. Seulement, les gars de la production avaient des doutes. Même si on éventait, ils avaient peur que la vapeur ne condensât pas à cause de la température élevée de la chambre de dépressurisation. Leur idée était de procéder auparavant à un 
refroidissement de l'enceinte de confinement. J'avais donné l'ordre d'étudier la proposition, qui paraissait bonne si elle était réalisable. Mais j'avais demandé aussi qu'on fût prêt à tout instant à ouvrir la vanne SR.

Mais là encore, les choses étaient compliquées. Les hommes se disputaient pour savoir qui allait ouvrir cette vanne SR. Étaient-ce aux pilotes ou aux agents de sûreté de le faire? Les gars de la production protestaient en demandant pourquoi c'était à eux de le faire. Il a fallu que je les tance et que je leur ordonne d'y aller. Vous voyez que la situation était difficile et que c'est dans ce contexte qu'il a fallu relever tous ces défis.

Alors ma consigne était la suivante : "Quoi que dise Monsieur Madarame, faites ce qu'il y a à faire». Monsieur Madarame ne pouvait pas savoir que la température de la chambre de dépressurisation était montée. Il ne voyait que le niveau d'eau et, se contentant de cela, il décrétait qu'il fallait se dépêcher d'éventer parce que celui-ci commençait à baisser. L'histoire était aussi simple que cela. Je suppose qu'il m'avait passé ce coup de fil de son "club des gentils amis », mais, personnellement, je m'en serais bien passé.

$\mathbf{Q}$ : Si je continue à suivre la chronologie du 14 mars, concernant le réacteur 2 , on lit: «Rapport aux ministères et autres organismes du début de la manœuvre de dépressurisation de la cuve et du début de l'injection d'eau de mer par le réseau incendie à $16 \mathrm{~h} 34$ ». Avant cela, il y a la mise en route des camions de pompiers pour l'injection d'eau de mer dans le réacteur. Je suppose que vous avez commencé à faire tourner le moteur des camions pour permettre l'injection à tout moment avant de dépressuriser. II y a donc dépressurisation. Pour cette opération, vous aviez déjà commencé à raccorder les batteries, etc., dès le 13 mars, raccords qui avaient été détruits par l'explosion et que vous avez dû refaire.

Je vois dans ces notes que, parallèlement, vous continuez à préparer votre éventage. On lit par ici : «manœuvre d'ouverture de la petite vanne de la chambre de dépressurisation ». La ligne a l'air d'avoir été établie, mais par la suite vous éprouvez visiblement beaucoup de difficultés à maintenir la vanne dans la position ouverte, ce qui va vous demander de longs efforts. Finalement, vous vous tournez vers la solution de l'éventage de la chambre sèche en travaillant à l'ouverture de la vanne de la ligne DW18. De ce côté aussi, il semblerait, d'après les dires du siège social, à la suite des analyses effectuées ultérieurement, qu'il y ait eu des problèmes de magnétisation. De toute manière, vous rencontrez de nombreuses difficultés à élaborer ces lignes jusqu'en fin d'après-midi. Je lis, par exemple, ici: «constatation de la fermeture (de la vanne), à la suite du décrochement de la ligne de magnétisation ». Cela signifie qu'il faut raccrocher la ligne de magnétisation qui s'est défaite, réparer, magnétiser de nouveau, tout en envoyant de la pression par le compresseur, n'estce pas? Pour la première fois, on voit ici concrètement des manœuvres pour ouvrir la vanne. Est-ce que cela équivaut à dire que, jusque-là, tout votre temps était consacré à restaurer la ligne?

R: Là, c'est un moment où on avait encore pas mal de discussions autour de l'éventage et de l'injection. Bien entendu, nous travaillions aux deux. Il me semble que c'est justement le moment où j'ai houspillé assez durement XXXXX. Il disait qu'il voulait éventer, qu'il allait le faire et il n'arrêtait pas de me répéter qu'il y travaillait. Il disait bien qu'il y travaillait, mais il ne me disait rien de la situation sur le terrain. Quand je lui demandais quand il pensait en avoir fini, il me répondait : «j’y travaille». À la fin, j'en ai eu marre et je l'ai pressé de me dire à quelle heure ça allait être prêt et ce qu'il était en train de faire. Je pense avoir été assez brutal. Ça a dû être filmé dans la vidéo. Vous comprenez, je ne savais rien. "Qu'est-ce que tu es en train de faire? À quelle heure ce sera prêt ? " «Probablement vers telle heure ». "Comment ça, probablement? Espèce d'abruti, décris-moi la situation à cet instant. Quand auras-tu terminé ?» Après force 
échanges, il a fini par m'avouer qu'il n'en savait rien. Et pendant tout ce temps, je n'ai moi-même pas eu la moindre idée des manœuvres qu'il tentait. Je me suis contenté de lui répéter de se dépêcher. À y réfléchir maintenant, il devait rencontrer des problèmes de pression, assez ou pas assez de pression pour actionner la petite ou la grande vanne.

$\mathbf{Q}$ : Le terrain continue donc à s'ingénier à élaborer une ligne d'éventage, mais les détails du travail ne remontent pas jusqu'à vous. C'est bien ça?

$\mathbf{R}$ : Je ne sais pas si c'est que les détails ne remontaient pas jusqu'à moi, mais XXXXX se contentait de me répéter tout du long que la vanne n'allait pas tarder à s'ouvrir.

$\mathbf{Q}$ : C'est comme ces livraisons de soba ${ }^{19}$ qui n'arrivent jamais.

$\mathbf{R}$ : Tout à fait. C'est pour ça que j'étais littéralement hors de moi. Sur ces entrefaites, le vieux Madarame m'appelle pour me dire d'injecter plutôt que d'éventer, toutes ces histoires sans aucun intérêt. Ça m'a encore plus tapé sur le système. Voilà dans quel état j'étais à ce moment-là.

$\mathbf{Q}$ : Finalement, en fin d'après-midi, à partir de 18h00, la baisse de la pression a l'air de se confirmer. Elle atteint 0,63 MPa à 19h03. À ce niveau de pression, l'eau peut entrer avec les camions de pompiers. Puis, la pression de la cuve remonte progressivement et l'eau n'y entre plus. Cycles que vous recommencez un certain nombre de fois.

$\mathbf{R}:$ Ce qui nous amenait à rouvrir, etc.

$\mathbf{Q}$ : Cette situation va durer toute la nuit.

Je vous promets que ce sera la toute dernière question. Je me suis interrogé sur les camions de pompiers qu'utilise TEPCO. Quand on regarde les normes des pompes préconisées par l'agence nationale contre l'incendie, on voit « 0,85 MPa ». Vous voyez ? Sur la feuille à part, où on a les standards des A-1 et des A-220. Je me suis informé et on m'a dit que TEPCO utilisait des A-2. Vous, en tant que directeur de centrale, étiez-vous au courant de la pression de sortie de ces pompes?

$\mathbf{R}$ : Bien sûr. Le tout n'est pas de savoir si la pompe fait exactement 0,85 MPa. Le réseau FP, structurellement, fonctionne avec $1 \mathrm{MPa}$, ce qui fait du $10 \mathrm{kPa}$. Le standard de la pompe est officiellement de 0,85 MPa. Mais dans la réalité nous disposons d'une marge. Nous pensons donc grosso modo $10 \mathrm{kPa}$. Ce qui veut dire que si la pression descend en dessous de $1 \mathrm{MPa}$, l'eau devrait entrer. Ça, c'est en gros notre raisonnement. Bien entendu, il faut non seulement tenir compte de la pression de sortie, mais il y a aussi des pertes de charge. Ce qui nous amène à penser que si la pression de la cuve est à $1 \mathrm{MPa}$, ça ne va pas marcher et qu'il faut attendre que la pression descende vers 0,6 MPa. À la louche, c'est cet ordre de grandeur.

$\mathbf{Q}$ : Et en comparaison, que se passe-t-il avec la DDFP?

$\mathbf{R}$ : C'est la même chose avec la pompe DD. On est peut-être à 1,5 MPa.

$\mathbf{Q}$ : Concernant les pompes $\mathrm{DD}$, quand on regarde les notes des équipes de quart, on voit qu'elles ont assuré un suivi de leur pression de sortie. Je ne sais pas si c'est dû au fait que la pression était différente selon les tranches ou que les variations étaient liées à leur état de marche. Toujours est-il que pour la tranche 1, la pression de sortie est de 0,69 MPa. La pompe de la tranche 2 n'a pas été mise en marche, contrairement à celle de la tranche 3 qui a tout le temps fonctionné. Sa pression varie selon les moments. Parfois elle est à 0,3 $\mathrm{MPa}$, d'autres à $0,6 \mathrm{MPa}$. Entre la tranche 1 et la tranche 3 , je dirais que la pression se balade en gros entre 0,3 et 0,6 MPa. Ce qui donne l'impression que ces pompes ne sont pas aussi puissantes que les pompes des voitures de pompiers. Qu'en pensez-vous?

$\mathbf{R}$ : Dès le départ, j'avais une confiance modérée dans les pompes DD. Premièrement, les pompes DD se sont révélées fragiles, elles sont très vite tombées en panne. 
Deuxièmement, comme vous venez de le faire remarquer, leur pression de sortie n'était pas très fiable. C'est pourquoi j'ai commencé très tôt à réclamer des voitures de pompiers.

$\mathbf{Q}:$ Si on regarde la fiabilité et la durabilité, elles présentent en effet des faiblesses.

$\mathbf{R}$ : Ce ne sont pas des diesels de grande qualité.

Q : D'accord. Nous allons nous arrêter là pour aujourd'hui. Toute cette semaine, jusqu'à vendredi, je vais vous emprunter le précieux personnel de $1 \mathrm{~F}$ pour des entretiens. Je sais que vous êtes lancés dans des travaux importants sur le terrain. S'il arrivait que vous ne pouviez pas libérer un tel ou un tel à cause des travaux, contactez-moi via Messieurs. XXXXX ou XXXXXX. Je m'adapterai sans problème. J'ai conscience que sur le terrain il peut survenir toutes sortes d'évènements imprévus et que le terrain jouit d'une priorité absolue. Cela me semble être tout à fait normal. N'hésitez donc pas à me le faire savoir. Je suis prêt à réaménager les entretiens et vous remercie de votre coopération.

$\mathbf{R}$ : La situation est stabilisée pour le moment. Même s'il advenait quelque chose, personne n'est irremplaçable. De plus, ce sont surtout les occupants du bâtiment antisismique qui sont convoqués cette fois-ci. Actuellement, le gros de notre travail consiste à surveiller les tranches plutôt que de travailler directement dessus. Il faut que nous soyons là pour donner l'alerte s'il se produisait quelque chose. Mais il me semble qu'il y a peu de chance qu'on soit confronté à une situation de ce type.

$\mathbf{Q}:$ Le mieux serait qu'il ne se passe rien.

$\mathbf{R}$ : Je pense qu'il ne se passera rien, alors je compte sur vous pour mener des entretiens constructifs.

$\mathbf{Q}:$ Je ferai de mon mieux. Je vous remercie beaucoup.

(Fin de l'audition)

\section{NOTES}

1. Dès le début de la crise nucléaire, plusieurs experts se sont rendus au Kantei pour conseiller Kan: Terasaka (pour rappel, directeur de la NISA), Madarame (Président de la NSC) et Takekuro (Fellow de TEPCO). Kan n'est toutefois pas satisfait par les réponses données par ces trois officiels: "aucun de ces trois personnages ne disposait des informations nécessaires pour pouvoir évaluer la situation et prendre des décisions. Par exemple, M. Takekuro m'a répondu qu'il ne savait pas pourquoi l'ouverture des vannes avait pris du retard. La NISA n'avait aucune idée de la situation réelle sur place. M. Madarame avait des connaissances car c'est un expert en la matière, mais sur la situation réelle sur place, il ne pouvait la juger sans consulter TEPCO. En d'autres termes, les trois personnes venues au Kantei ne disposaient pas des informations dont j'avais besoin. Parallèlement, j'ai cherché à avoir l'avis de spécialistes du nucléaire, autres que ceux qui avaient des positions officielles et qui me devaient des rapports. En fait, ces experts indépendants m'ont parlé plus librement que les trois officiels dont je viens de vous parler » (Kan, 2015). 
2. Référence aux ABWR (Advanced Boiling Water Reactor), des réacteurs à eau bouillante de troisième génération. Ce type de réacteur a été conçu par GE, Hitachi et Tôshiba. Les premiers ABWR ont été mis en service dans la centrale nucléaire de KashiwazakiKariwa en 1996 (réacteur n6) et en 1997 (réacteur n7), exploitée par TEPCO.

3. Il s'agit du CST (Condensate Storage Tank).

4. Mesure en MPa.

5. Centre de contrôle des moteurs.

6. L'enquêteur évoque la conférence de presse donnée le 12 mars à 3h00 du matin sur la question de l'éventage des réacteurs (cf. audition du 22 juillet 2011).

7. Hikida précise les raisons de la grande autonomie des pilotes : « Fukushima Daiichi est la vielle centrale nucléaire et les réacteurs tombaient souvent en panne au début. Chaque fois que les problèmes se sont produits, les pilotes des réacteurs se sont rendus sur les sites et ont discuté sur les points à améliorer. Avec le temps ils ont donc développé une très forte tendance à vouloir résoudre les problèmes eux-mêmes » (Kobayashi, 2019, p. 118).

8. Le niveau d'eau dans la cuve d'un REB est constamment mesuré et certains seuils (notés du plus bas au plus haut entre L1 et L8) sont définis. Ainsi, à L4, l'eau est à un niveau normal. À L3, le SCRAM s'active. À L2, les systèmes de refroidissement d'urgence démarrent. À L1, la dépressurisation automatique de la cuve du réacteur commence, pour permettre l'injection d'eau via tous les systèmes de refroidissement. En revanche, en cas de niveau d'eau L5 ou plus, l'alimentation de la cuve est limitée voire arrêtée, le réacteur et la turbine s'arrêtant automatiquement au niveau L8.

9. La cellule de gestion de crise reçoit l'information mais ne comprend pas immédiatement les raisons de l'échec de l'injection. Pour Inagaki, cette incompréhension s'explique du fait de l'épuisement des membres de la cellule: "Combien de temps pouvons-nous continuer à travailler sans jamais dormir? La réponse est 36 heures. C'est la limite pour tous les hommes, car ils perdent leurs facultés de jugement. C'est vrai, quand la salle de commande des réacteurs 3 et 4 a transmis à la cellule de crise qu'il était impossible d'injecter de l'eau dans le réacteur 3 à $3 h 55 d u 13$ mars, 36 heures avaient passé depuis le grand séisme. Les cadres de la table ronde, dont Yoshida somnolaient. Nous n'avons pas pu comprendre tout de suite la situation » (Kobayashi, 2019, p. 135).

10. Discours prononcé par Takekuro le 12 mars à $22 \mathrm{~h} 59$, retranscrit dans le compte rendu de l'audition du 9 août 2011 (I).

11. Ces deux centrales thermiques, exploitées par TEPCO, sont situées respectivement dans les préfectures de Kanagawa et de Chiba, dans la région du Kantô.

12. Pour rappel, Yoshida évoque l'accident de criticité survenu le 30 septembre 1999 dans une usine de traitement de l'Uranium du complexe de Tôkai-mura, exploitée par la Japan Nuclear Fuels Conversion Company.

13. L'affaire du retrait général sur lequel revient l'enquêteur a déjà été évoquée par Yoshida dans l'audition du 9 août 2011 (I).

14. Yoshida est résolu à rester jusqu'au bout, mais ne se voit pas mourir seul. Il confie ainsi aux enquêteurs de la NAIIC qu'au matin du 15 mars, il a regardé les cadres assis à la table ronde et leur a demandé : "Qui se permet de mourir avec moi ? Est-ce qu'une dizaine d'employés avec lesquels je travaille depuis longtemps iraient jusqu'au bout avec moi ?» (Kobayashi, 2019, p. 136). Kadota reconstitue la scène d'après les entretiens menés avec Yoshida: «M. Yoshida m'a aussi raconté ce moment à l'aube du 15 mars 2011, 
quand la pression dans l'enceinte du réacteur $n^{\circ} 2$ a atteint son comble. Il a alors choisi les hommes 'qui mourraient avec [lui]' et les a tous regardés droit dans les yeux. De la salle de contrôle au second étage du bâtiment antisismique d'où il contrôlait les opérations, M. Yoshida s'est levé de son siège et s'est soudain laissé tomber sur les genoux par terre. Il a baissé la tête et est entré en méditation zen, comme plongé dans une intense réflexion. M. Yoshida m'a raconté ce qui s'était passé en lui à ce moment-là : 'À ce moment-là, il n'y avait pas d'autre moyen que d'envoyer directement de l'eau de mer pour éviter l'emballement du réacteur. Quels seront les hommes qui vont le faire? C'était à moi de les choisir. Ce qui signifiait aussi leur demander de mourir avec moi. Leur visage m'est passé l'un après l'autre devant mes yeux, et je pensais, lui, il mourra avec moi... lui aussi... Le premier qui m'est apparu, c'est mon chef d'équipe, qui avait le même âge que moi. Il n'avait pas fait d'études au-delà du lycée, et nous avions vécu ensemble un bon paquet de choses. Oui, ce gars-là, il voudra bien mourir avec moi,j'ai pensé'. Rien d'étonnant à ce que ce soit le visage d'un homme avec qui il avait vécu de nombreux évènements de sa jeunesse qui lui soit venu à l'esprit à ce moment-là. 'Oui, des hommes de mon âge ou presque, des hommes que j'avais longtemps côtoyés me sont venus à l'esprit. Je les envoie à la mort, j'ai pensé. Mais au point où nous en étions, il n'y avait rien d'autre à faire qu'à continuer d'arroser, et il fallait se résoudre aux dernières extrémités. Toutes ces choses me sont passées par la tête, assis sur les genoux...' " (Kadota, 2013). Masuda, le directeur de Fukushima Daini, explique l'attitude de Yoshida: " J'avais en tête, dès le début de la crise, ceux sur qui je pouvais compter pour agir, rien qu'en les regardant dans les yeux. Sur ce point-là, je comprends l'attitude de Yoshida. Moi-même j'étais prêt à y aller si le pire arrivait. Peut-être que je me trompe sur l'attitude de Yoshida, mais à l'époque, c'est comme ça que je l'ai interprétée. Et quand on est prêt à se battre jusqu'au bout, on imagine ceux qu'on veut avoir à ses côtés. Yoshida a dû faire de même. Il a dû imaginer, parmi ceux qui travaillent avec lui, tous ceux qui pouvaient l'aider et quand on regarde la liste de ceux qui sont effectivement restés sur place, ce sont des gens qui ont suivi Yoshida comme un seul homme. Yoshida a dû se dire : 'Laissez-moi terminer le travail avec eux!'» (Kobayashi, 2019, p. 136).

15. Naoto Kan continue de l'affirmer par la suite dans ses interventions publiques ou ses entretiens accordés aux journalistes. La thèse selon laquelle il aurait empêché, grâce à son intervention, l'abandon de la centrale est également défendue par Futoshi Sato dans son long-métrage Fukushima, le couvercle du soleil (2018), dont l'ancien Premier ministre a été le conseiller technique (Portelli, 2019).

16. L'explosion survenue dans le bâtiment réacteur 3 et ses conséquences ont déjà été abordées dans l'audition du 29 juillet 2011.

17. Coup de fil de Madarame - en proie à une crise de panique - évoqué par Yoshida dans l'audition du 9 août 2011 (I).

18. Précédemment mentionnée par le terme « Drywell ».

19. Nouilles de sarrasin.

20. L'Agence japonaise de gestion des incendies et des désastres classe les différentes pompes à incendies selon leurs spécifications techniques. Ainsi, les pompes de classe A-1 ont une pression de sortie de $0,85 \mathrm{MPa}$ et un débit minimum de $2,8 \mathrm{~m}^{3} / \mathrm{min}$. Les pompes de classe A-2 ont, quant à elles, une pression de $0,85 \mathrm{MPa}$ et un débit minimum de $2,0 \mathrm{~m}^{3} / \mathrm{min}$. 


\section{Photographies}

Centrale nucléaire de Fukushima Daiichi (avant le 11 mars 2011) (c) TEPCO.

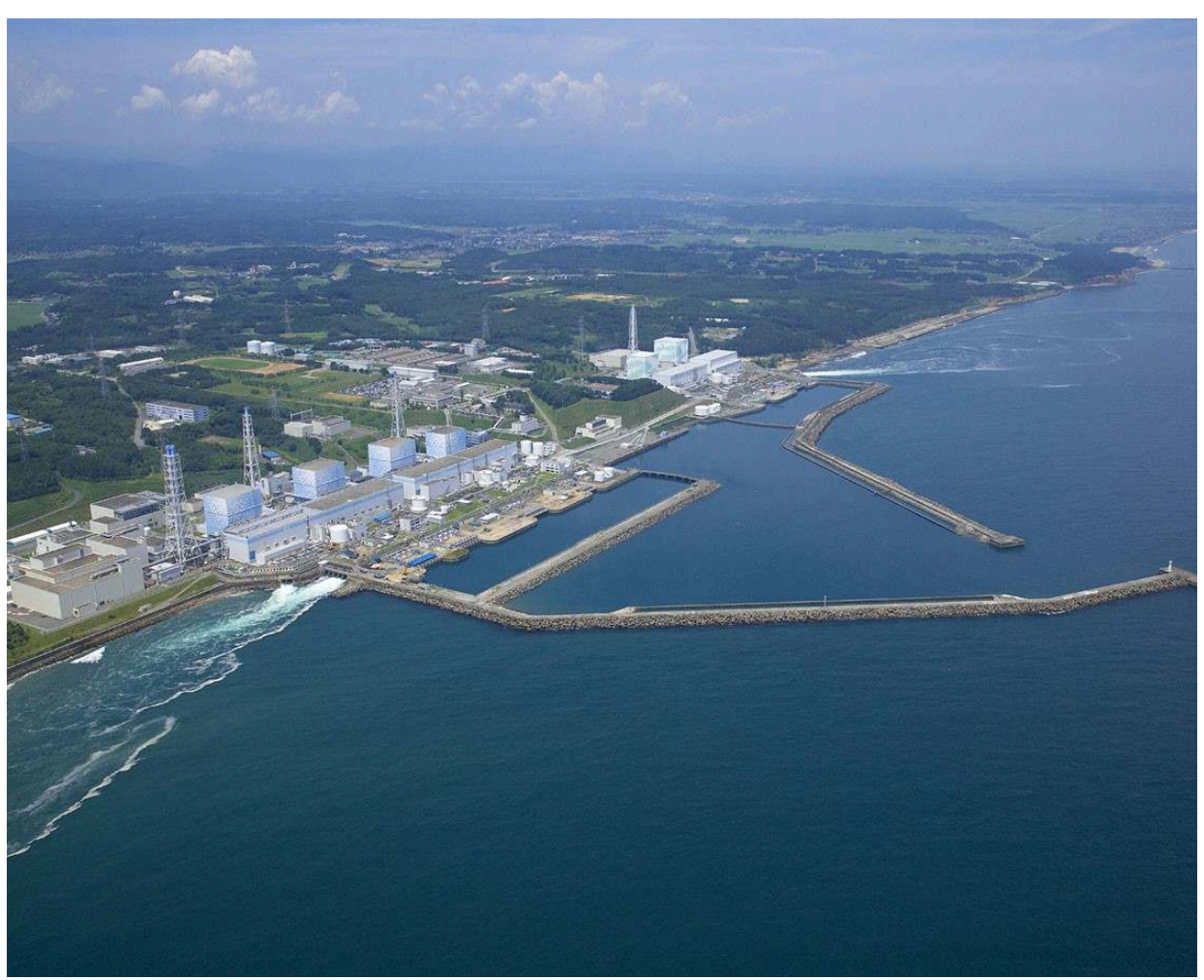


Centrale nucléaire de Fukushima Daini (avant le 11 mars 2011) (C TEPCO.

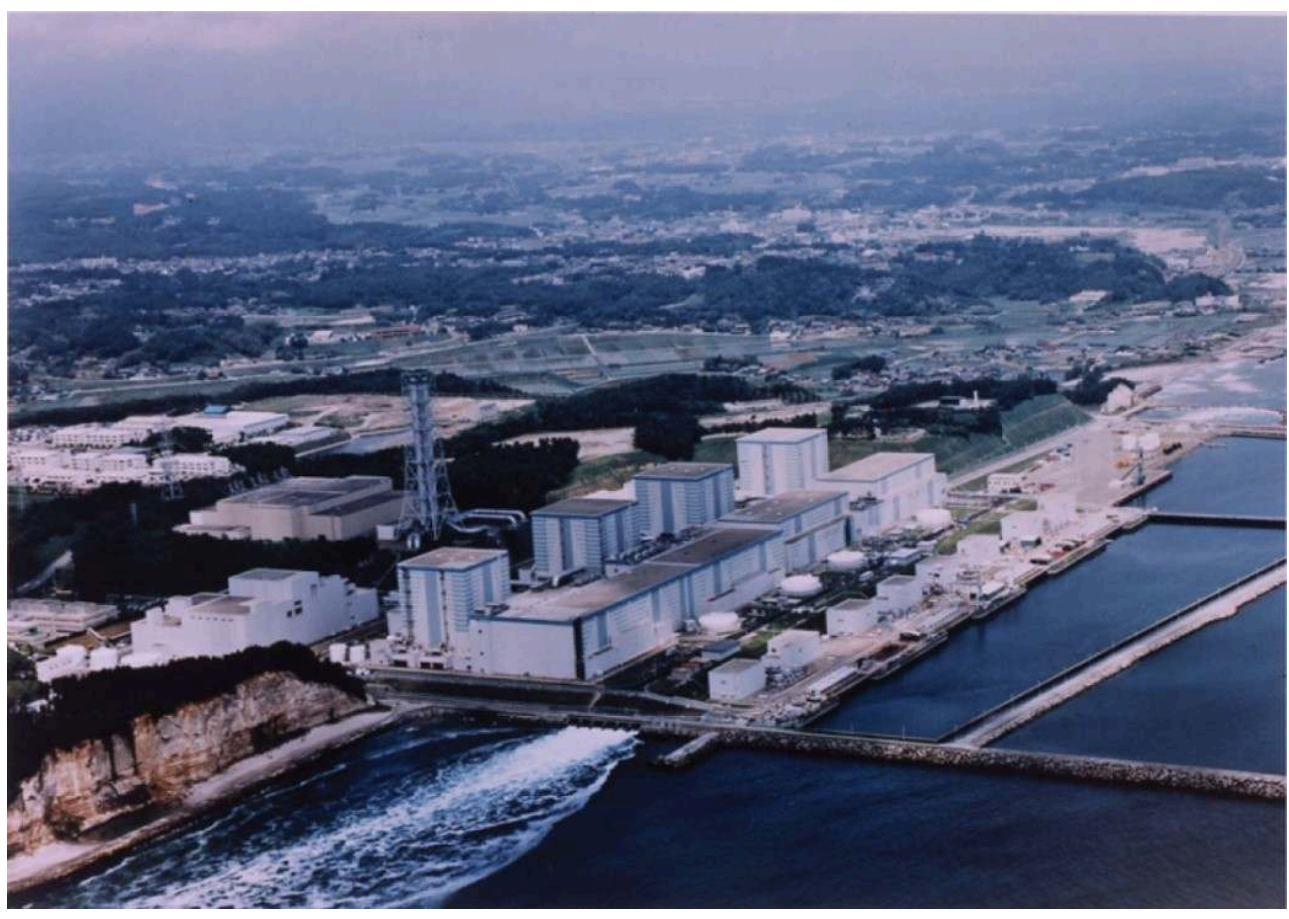

Centrale nucléaire de Kashiwazaki-Kariwa (avant le 11 mars 2011) () TEPCO.

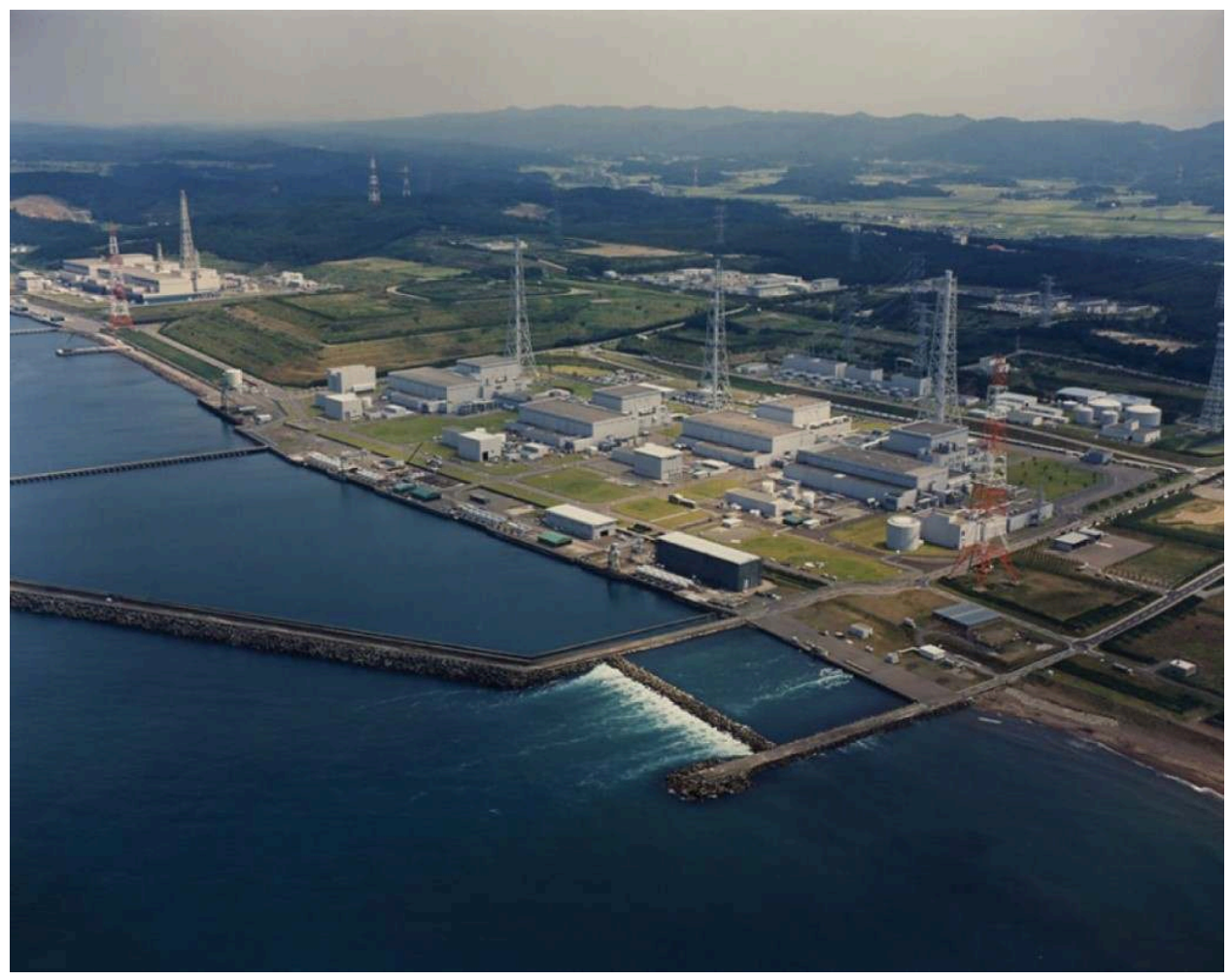


Arrivée du tsunami à Fukushima Daiichi, côté est de l'installation de stockage de déchets solides radioactifs (11 mars 2011) (C) TEPCO.

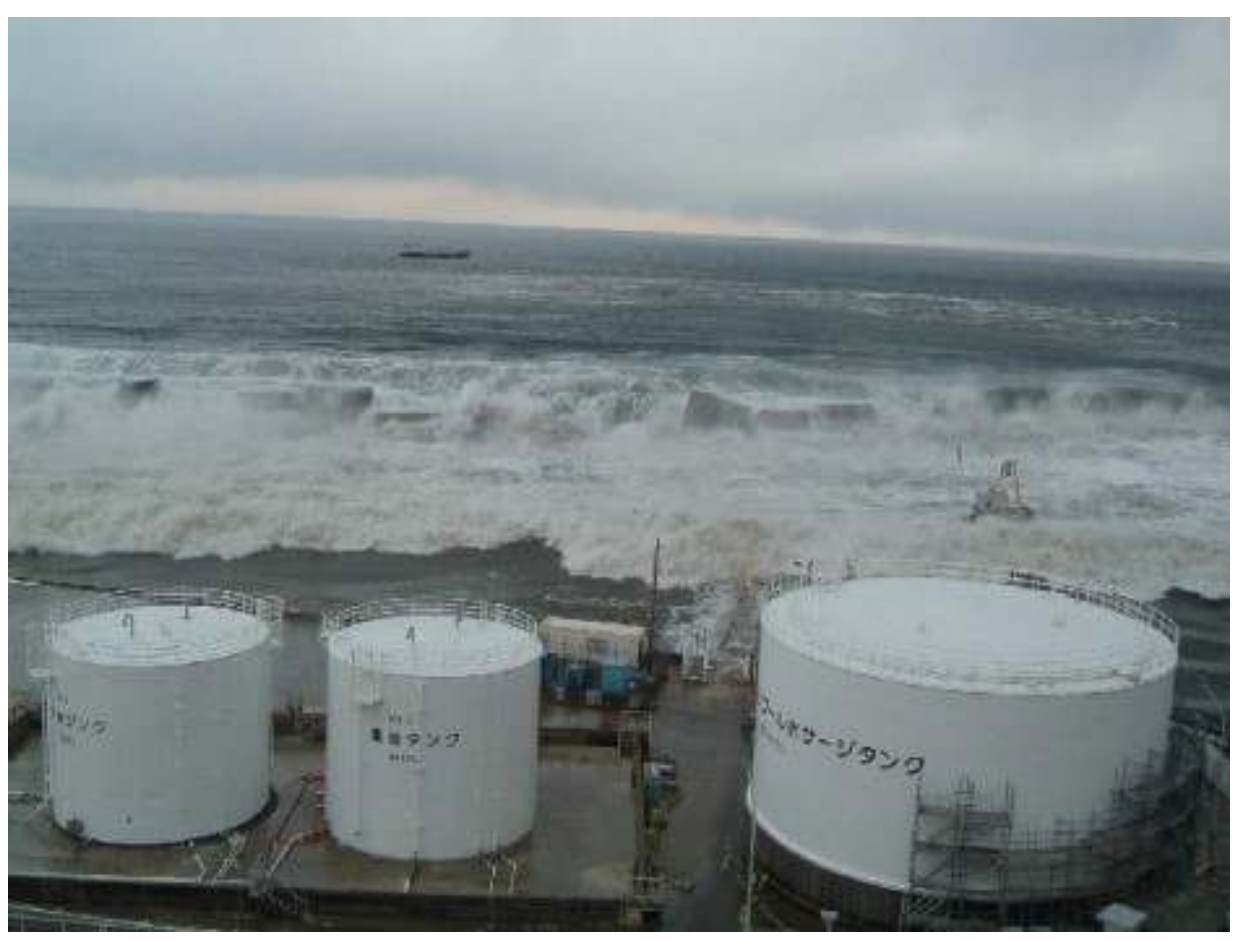

Avant le tsunami, point F de Fukushima Daiichi (11 mars 2011) (c) TEPCO.

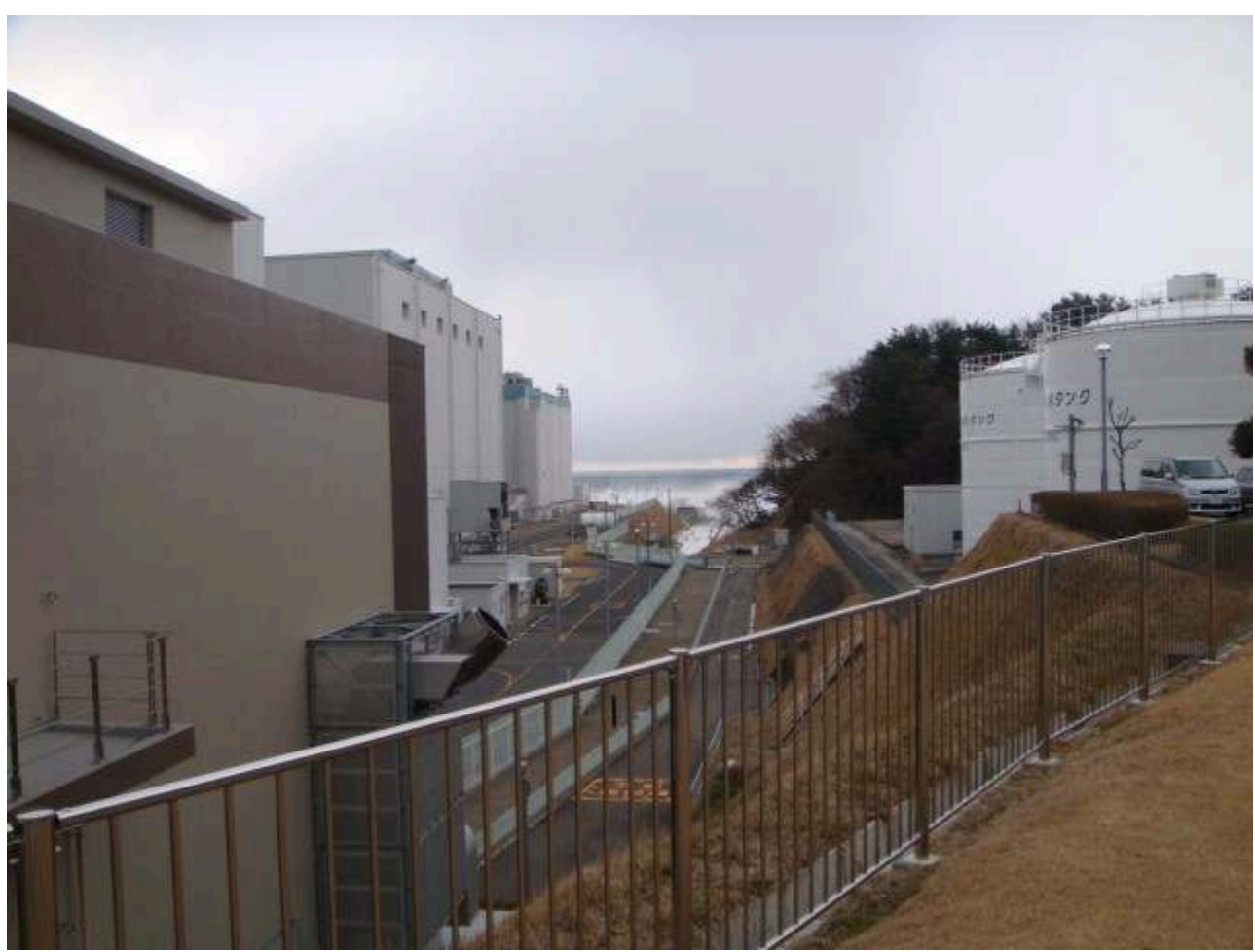


Après le tsunami, point $F$ de Fukushima Daiichi (11 mars 2011) @ TEPCO.

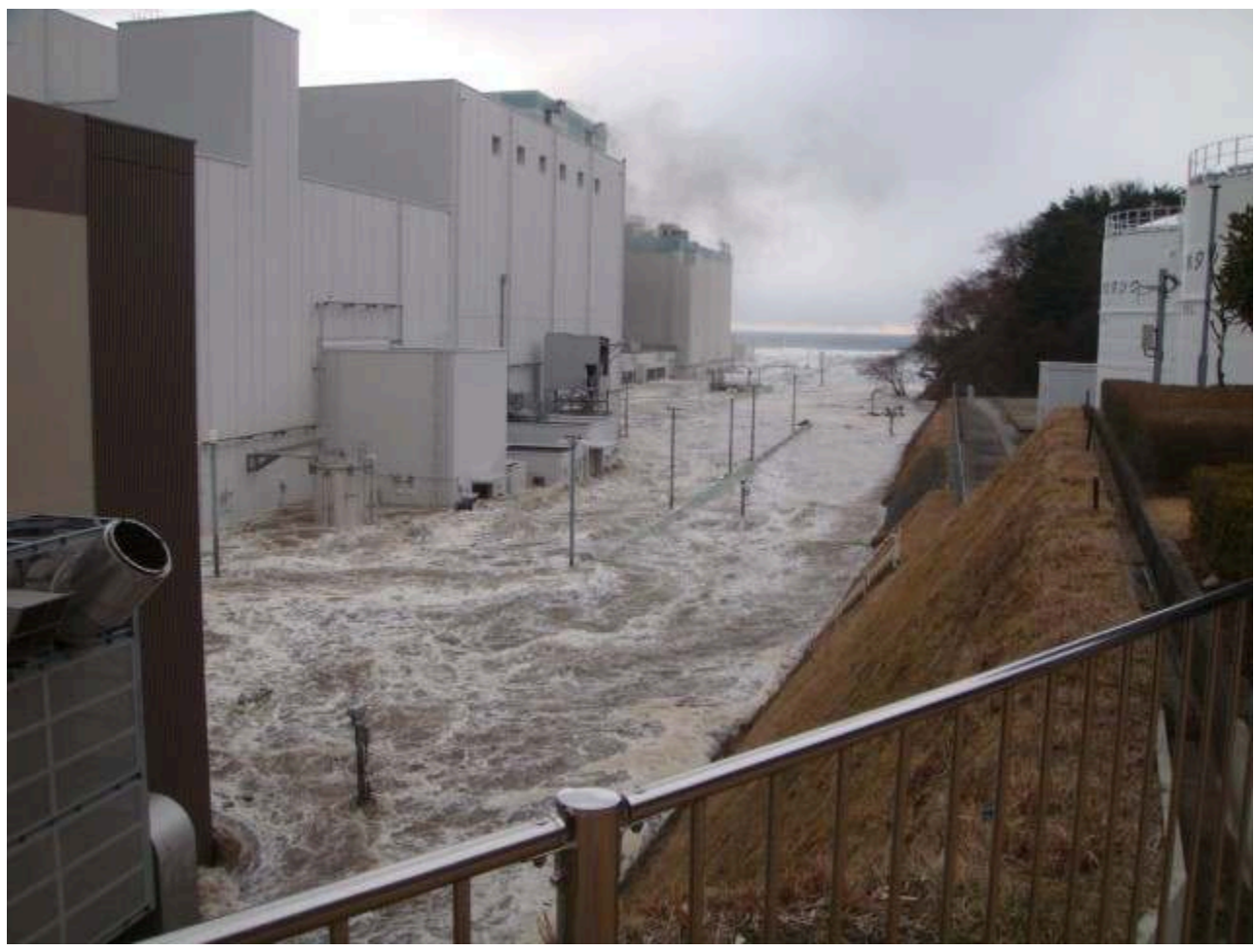

Eau de mer s'engouffrant dans les installations de Fukushima Daiichi (11 mars 2011) () TEPCO.

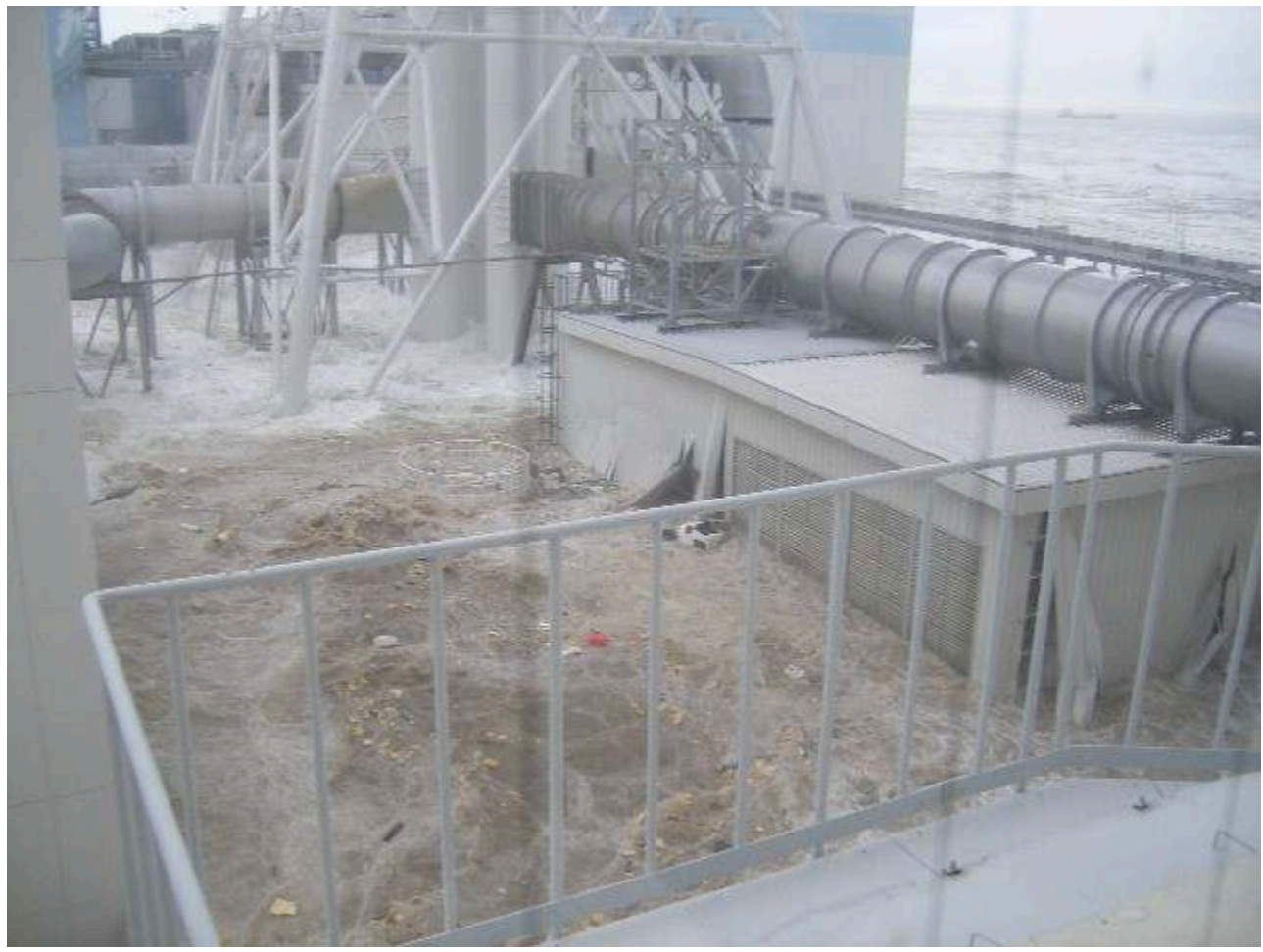


Inondation de Fukushima Daiichi, côté nord du bâtiment antisismique (11 mars 2011) @ TEPCO.

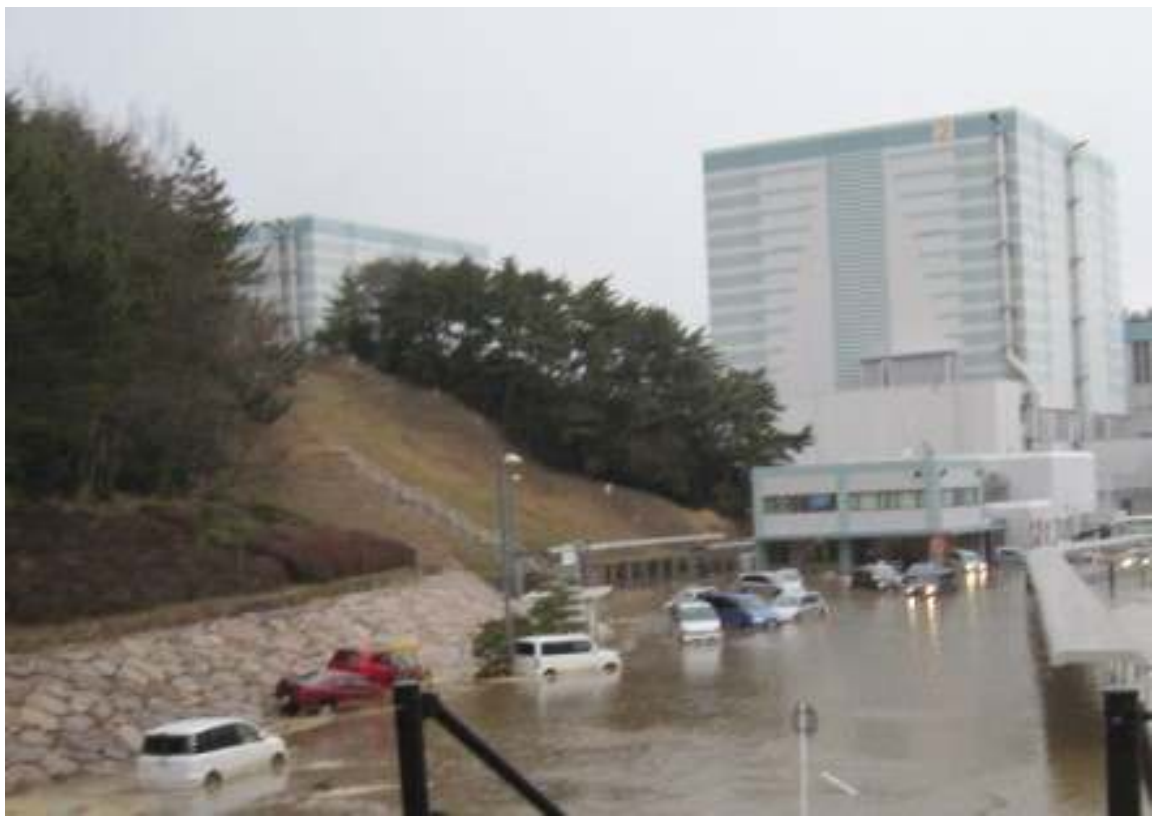

Bâtiment réacteur 1 de Fukushima Daiichi, après l'explosion survenue le 12 mars à $15 \mathrm{~h} 36$ (12 mars 2011) (C) TEPCO.

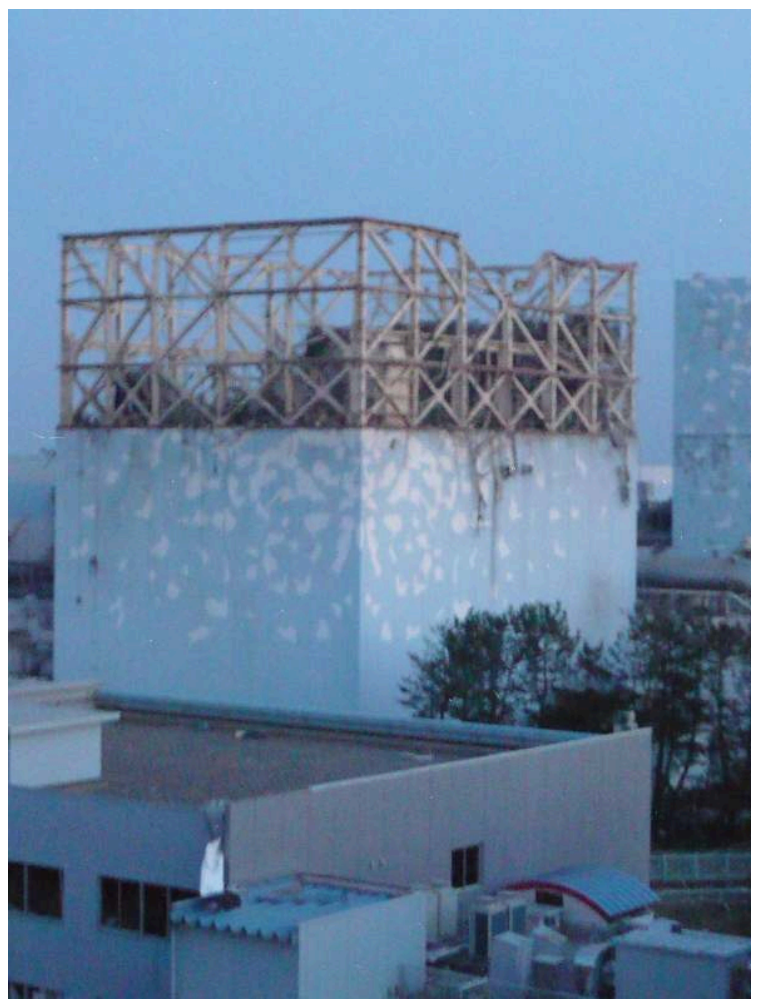


Bâtiment réacteur 3 de Fukushima Daiichi, après l'explosion survenue le 14 mars à 11 h01 (16 mars 2011) () TEPCO.

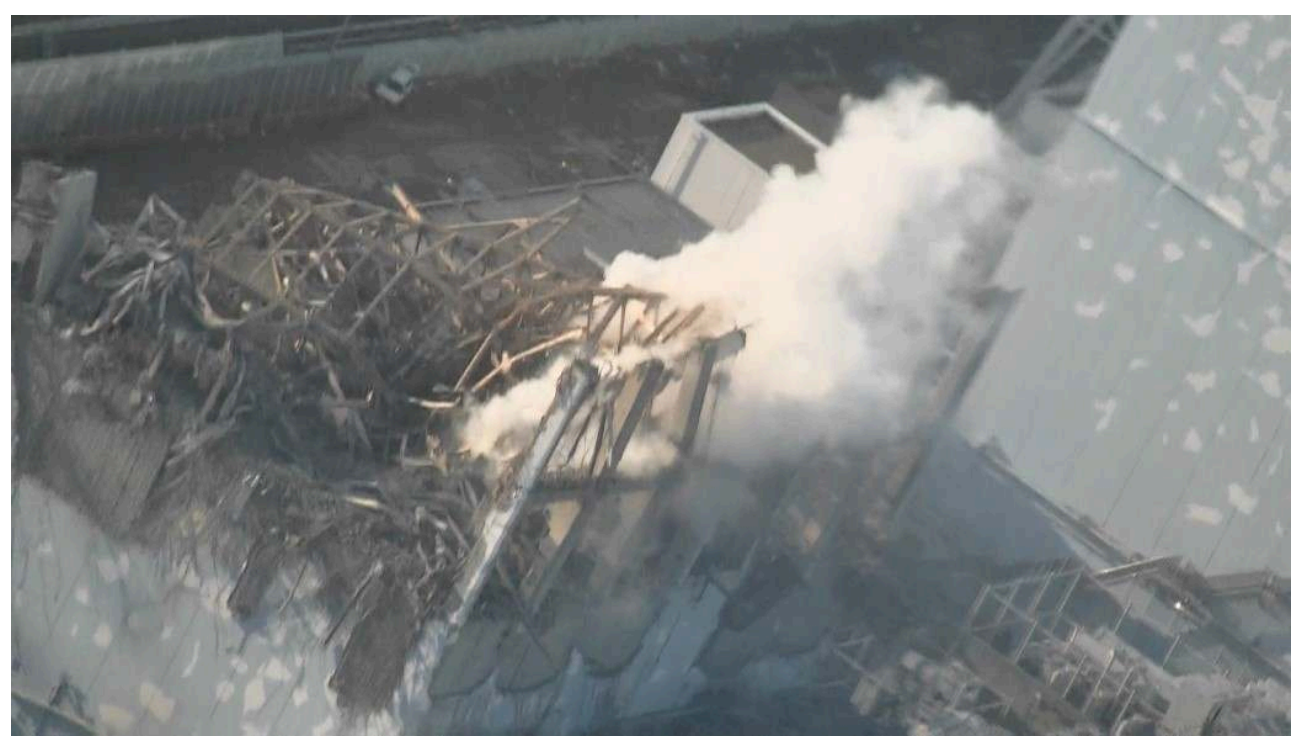

Bâtiment réacteur 4 de Fukushima Daiichi, après l'explosion survenue le 15 mars à 6 h10 (16 mars 2011) () TEPCO.

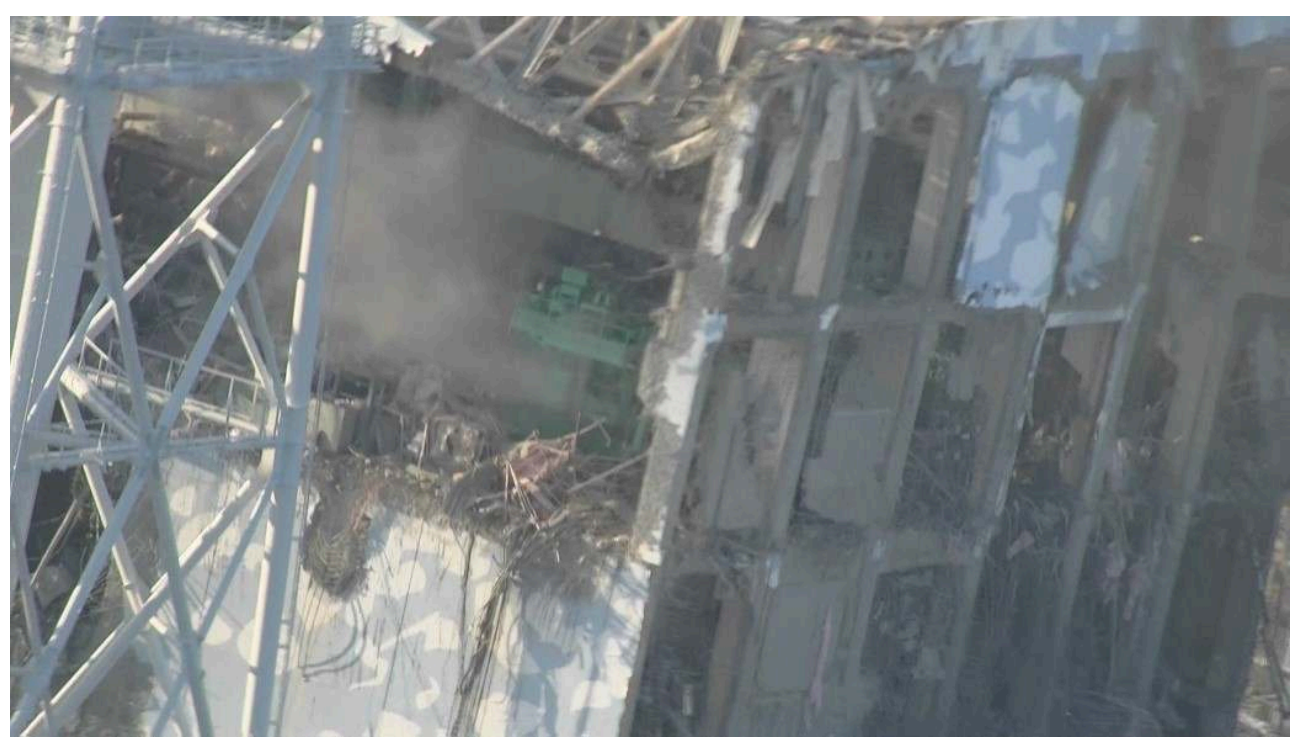


Injection d'eau par les camions de pompiers sur le site de Fukushima Daiichi (16 mars 2011) @ TEPCO.

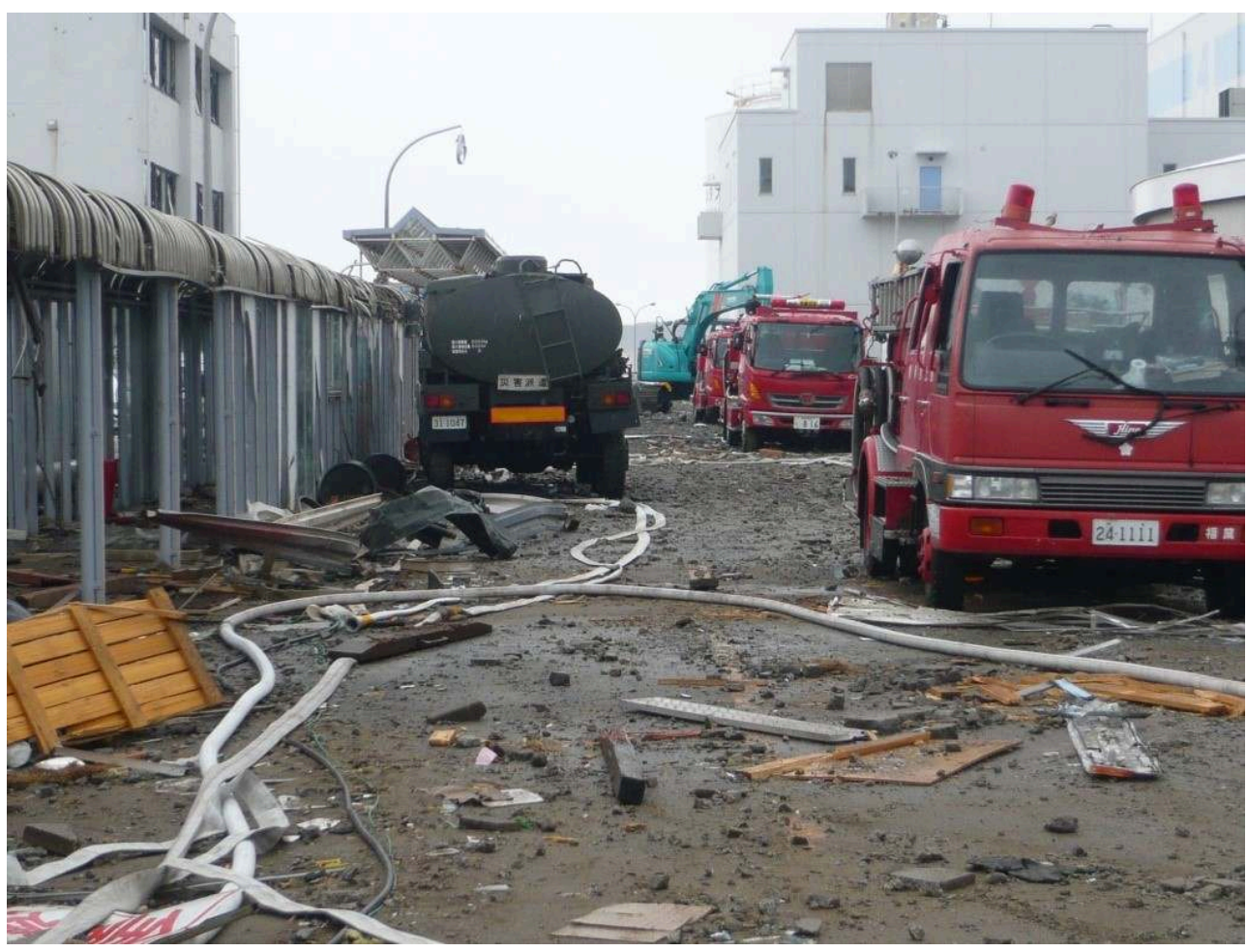

Inondation de la salle des équipements électriques de la tranche 6 de Fukushima Daiichi (17 mars 2011) (C) TEPCO.

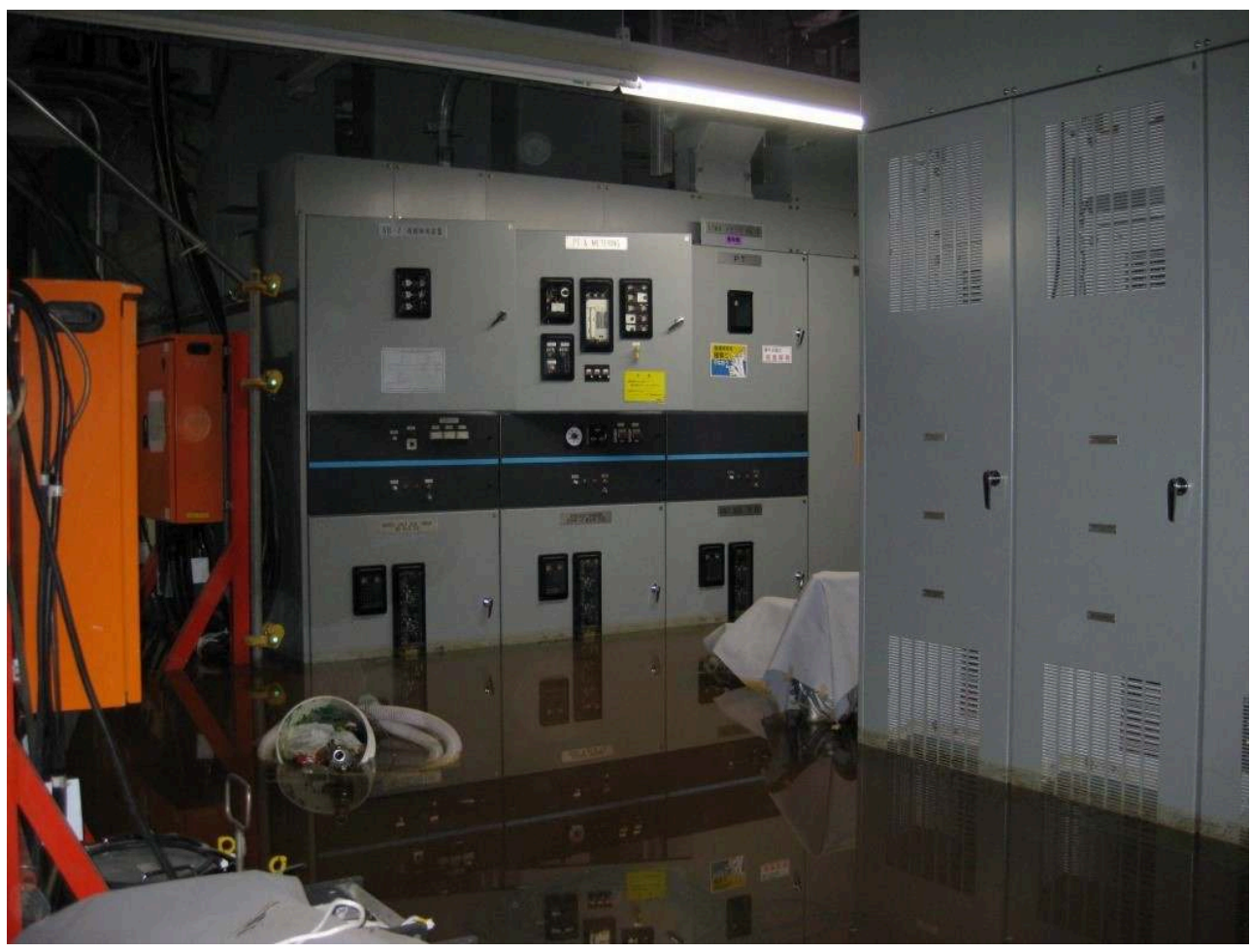


Travaux de connexion de la ligne au réseau électrique externe (ligne d'Okuma) sur le site de Fukushima Daiichi (18 mars 2011) ( ) TEPCO.

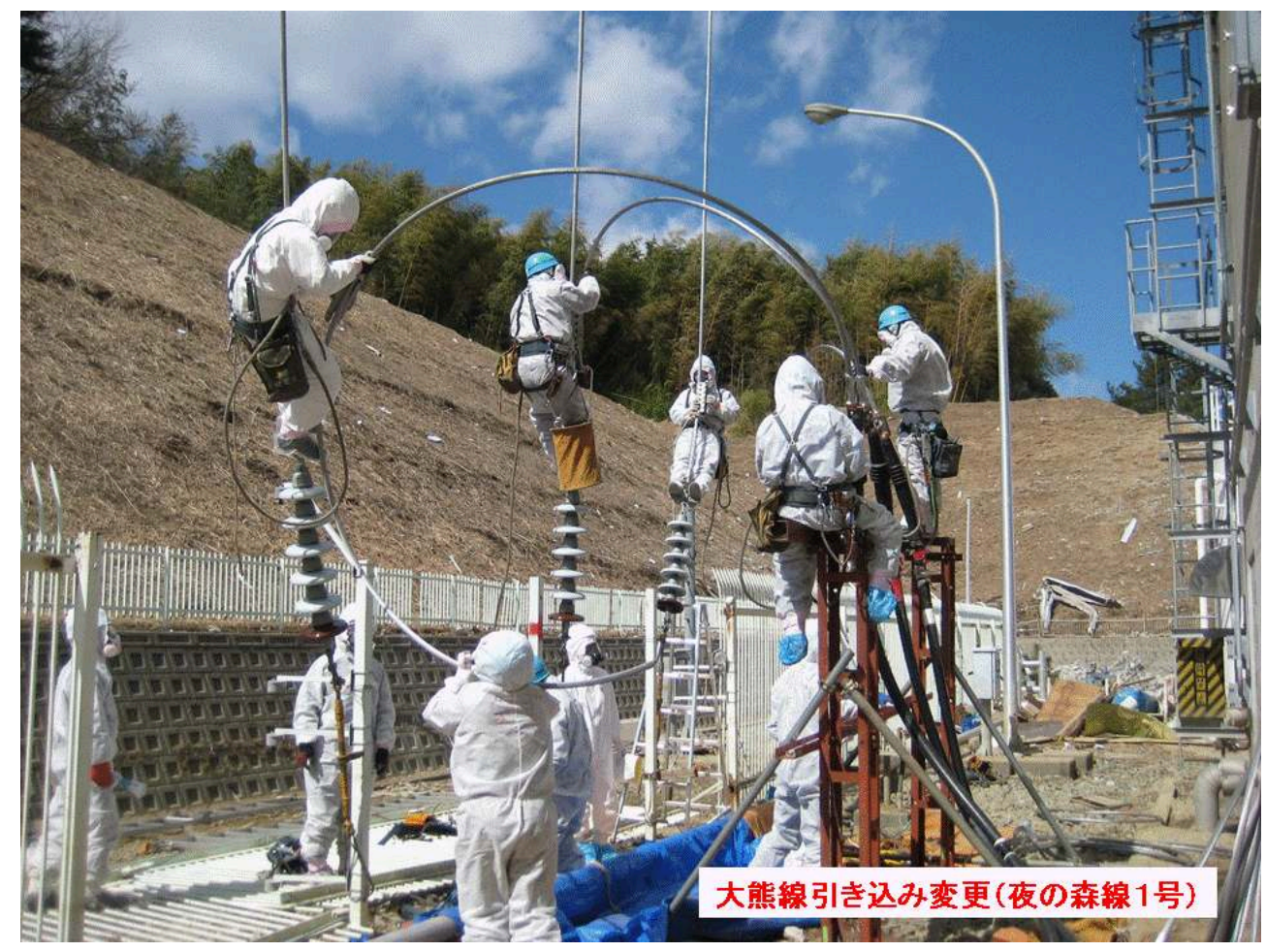

Travaux de réglage de la pompe submersible sur le site de Fukushima Daiichi (17 mars 2011) ( ) TEPCO.

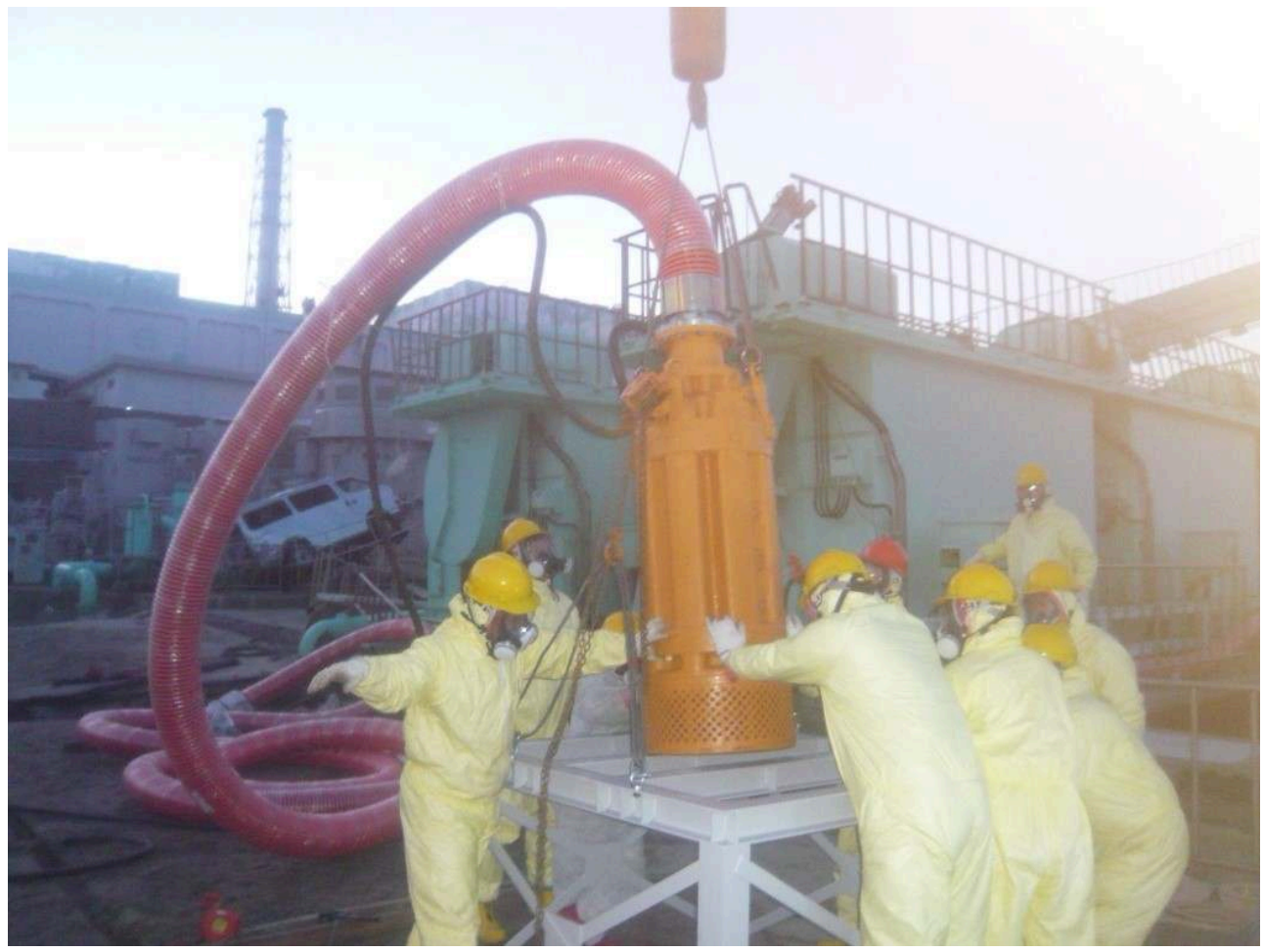


Inondation de la tranche 3 de Fukushima Daiichi (19 mars 2011) @ TEPCO.

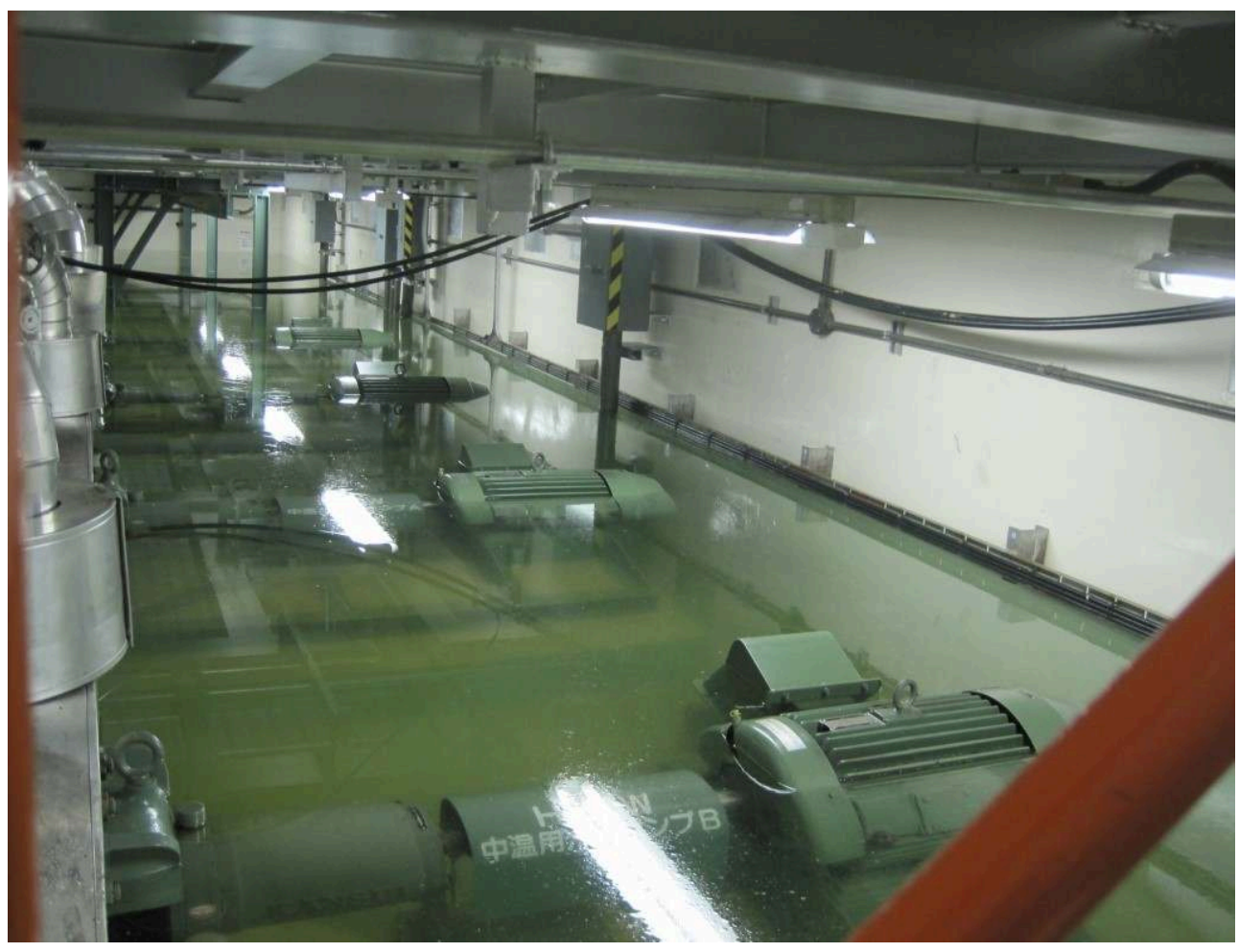

Inondation de la tranche 3 de Fukushima Daiichi (19 mars 2011) (c) TEPCO.

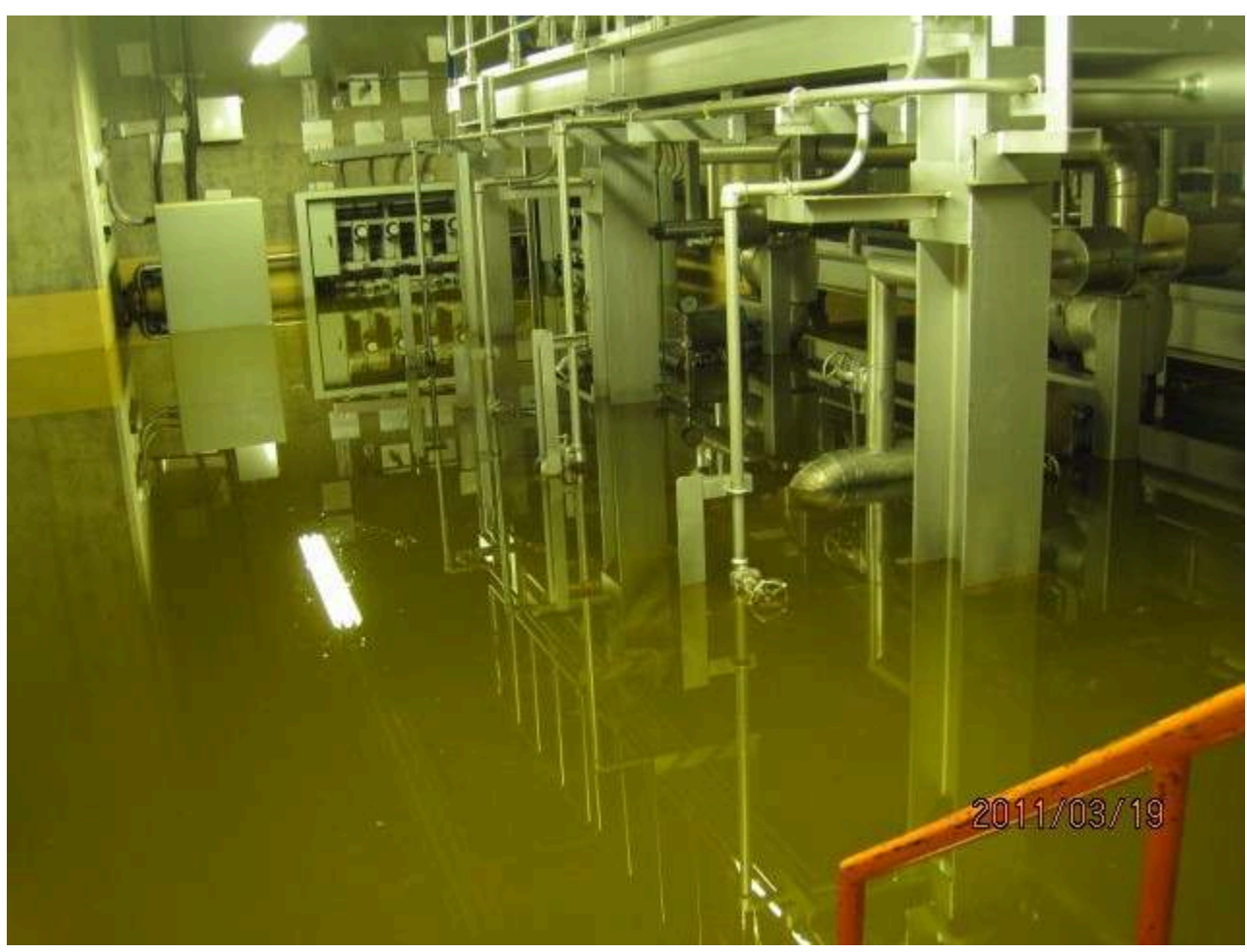


Ravages causés par le tsunami près de la tranche 4 de Fukushima Daiichi (21 mars 2011) (C) TEPCO.

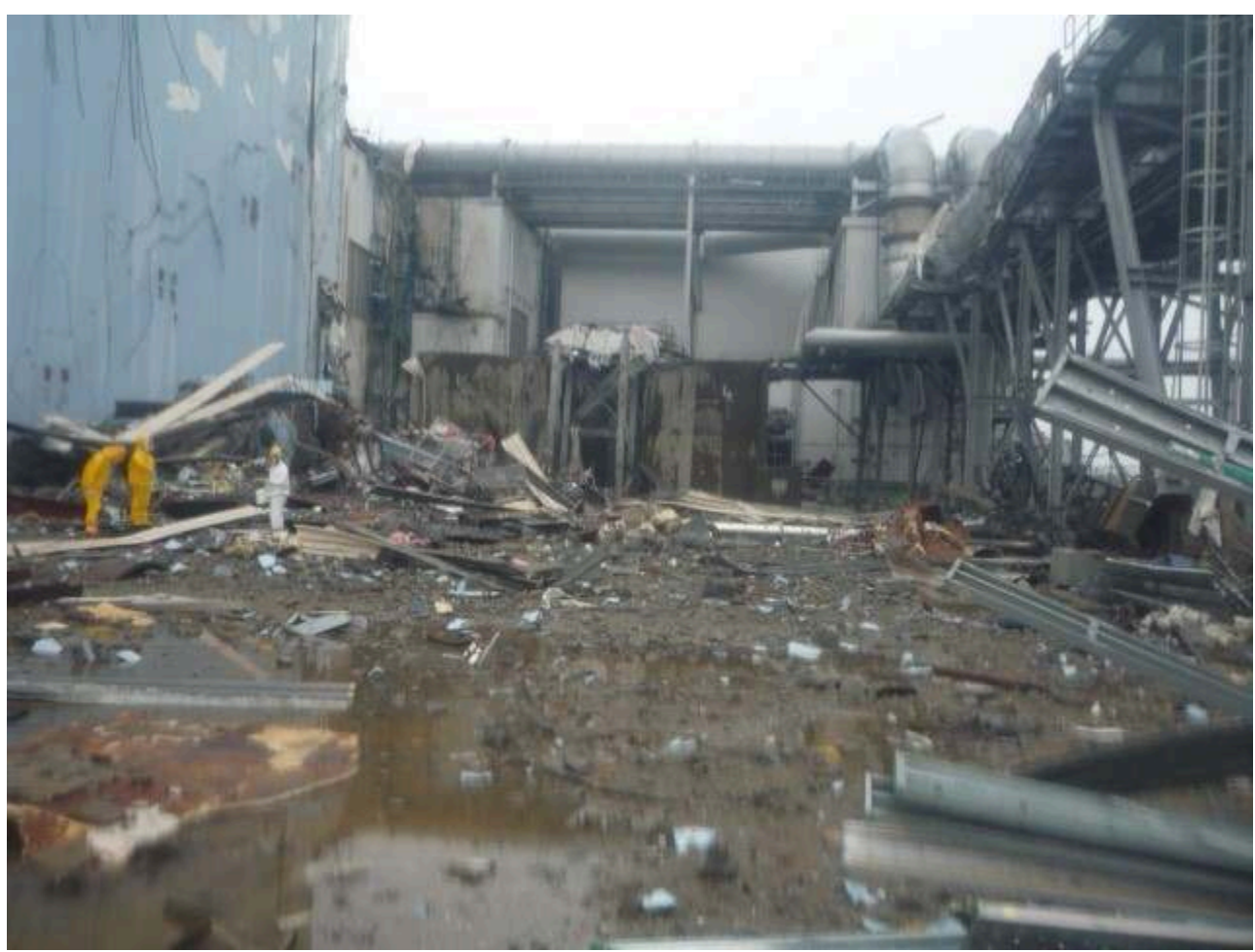

Pompage d'eau à l'aide d'une voiture de pompe à béton, tranche 4 de Fukushima Daiichi (22 mars 2011) (C) TEPCO.

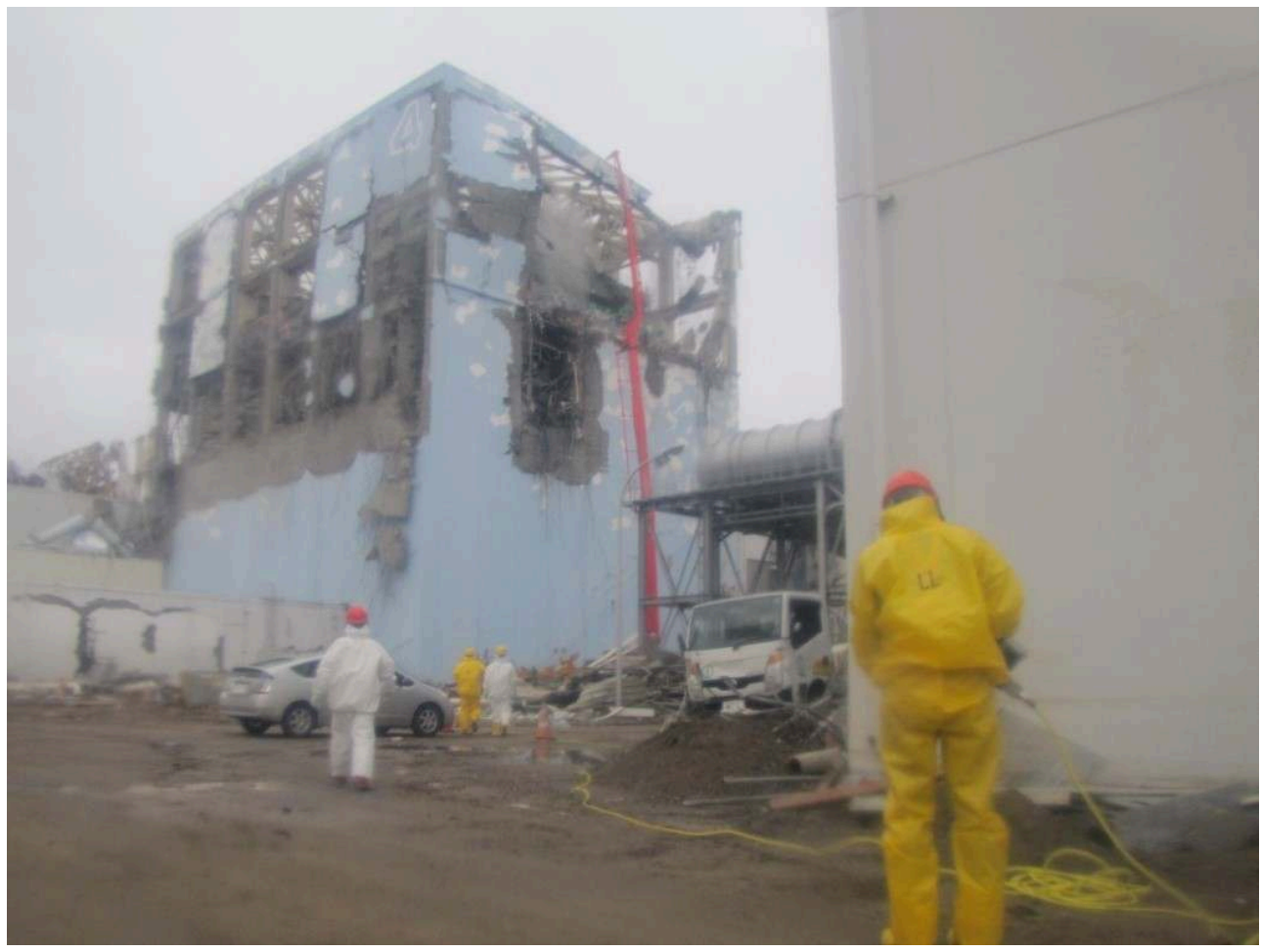


Extérieur de la tranche 2 de Fukushima Daiichi, salle des ressources électriques (23 mars 2011) () TEPCO.

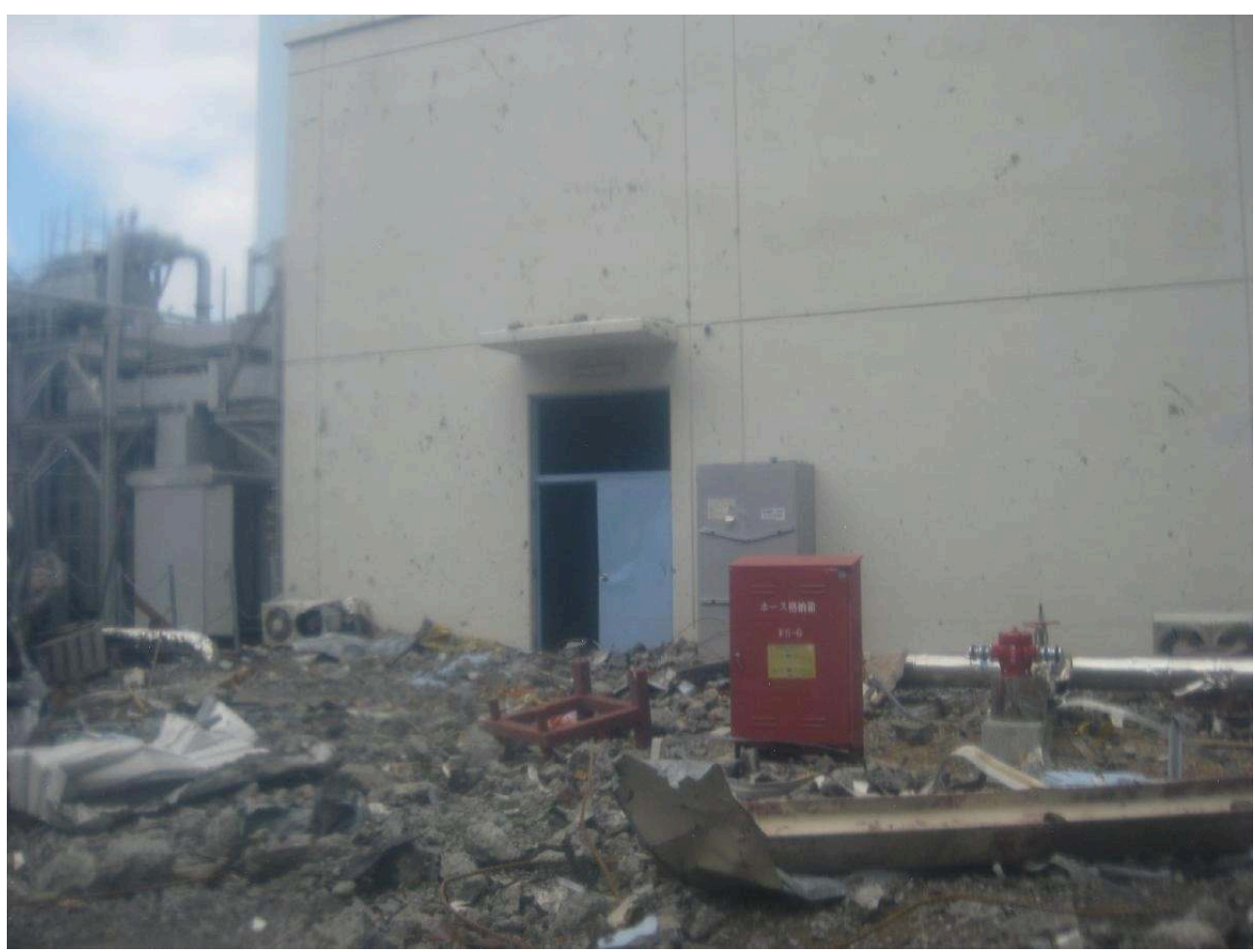

Intérieur de la tranche 2 de Fukushima Daiichi, salle des ressources électriques (23 mars 2011) ( TEPCO.

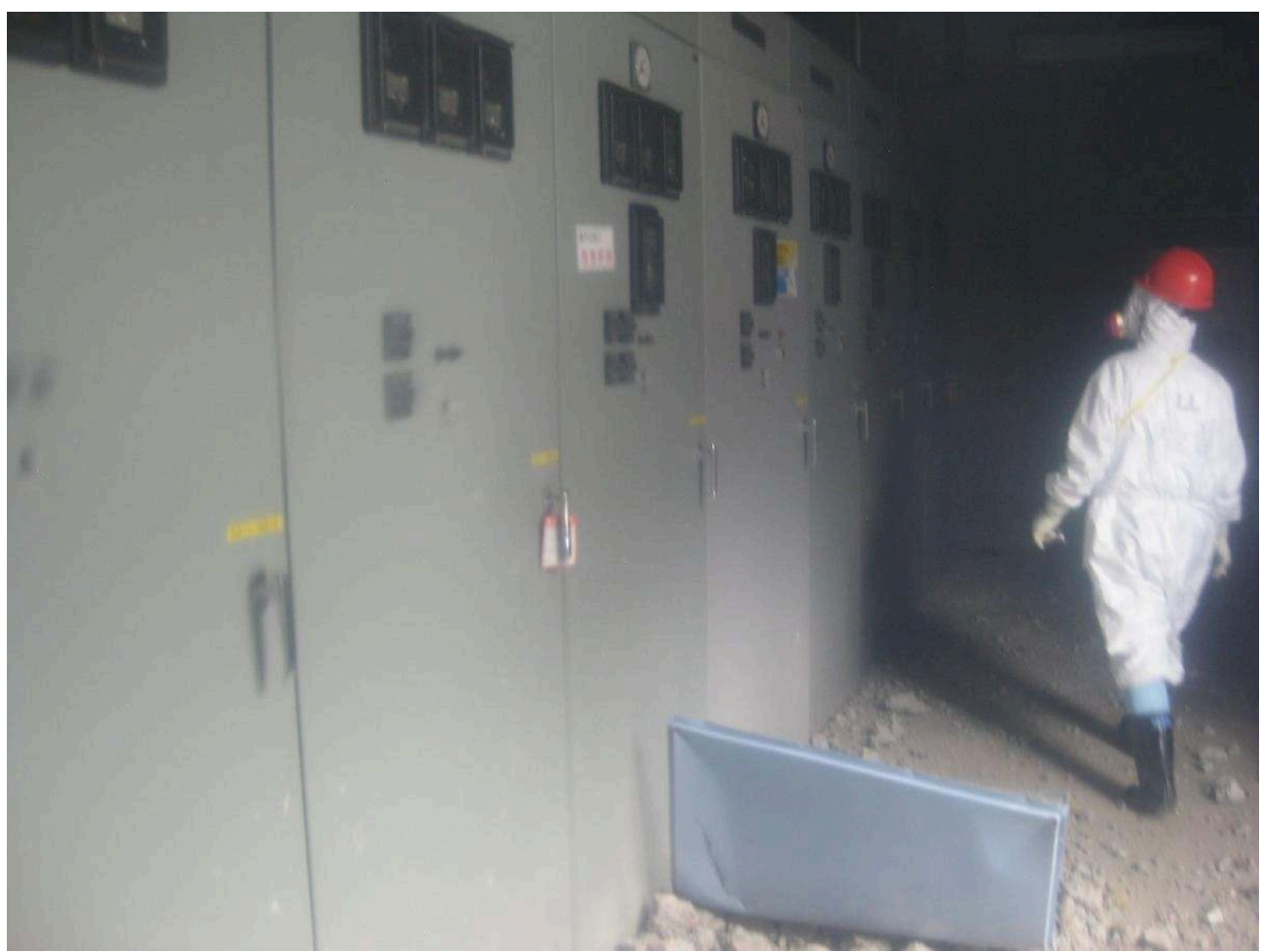


Intérieur de la tranche 2 de Fukushima Daiichi, salle des ressources électriques (23 mars 2011) () TEPCO.

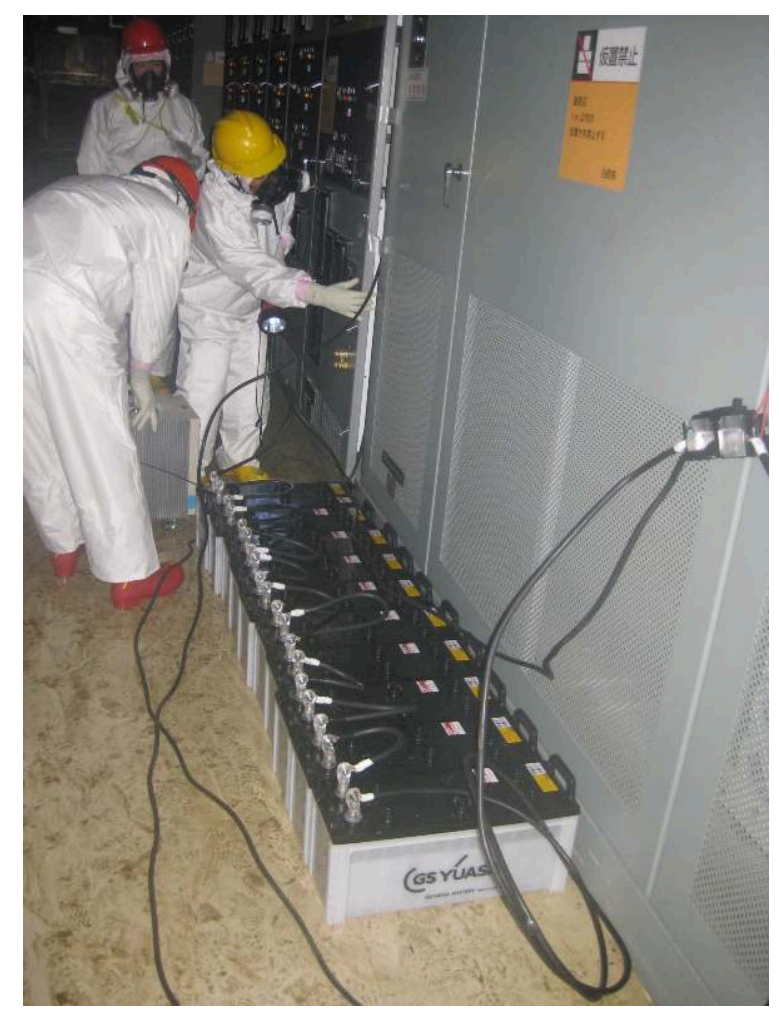

Lecture à la lumière des lampes torches dans une installation de Fukushima Daiichi (23 mars 2011) (C) TEPCO.

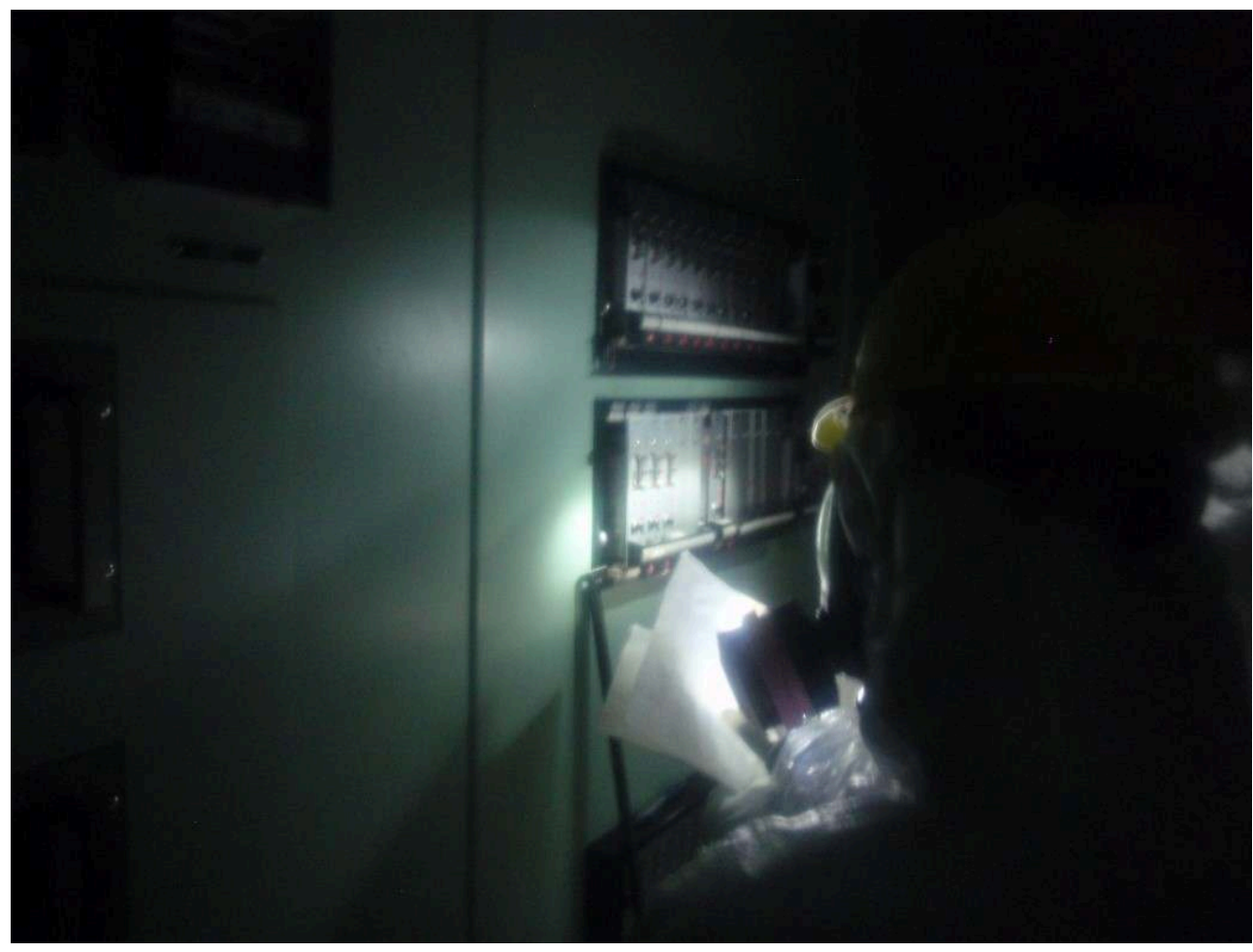


Salle de contrôle de la tranche 2 de Fukushima Daiichi (26 mars 2011) (c) TEPCO.

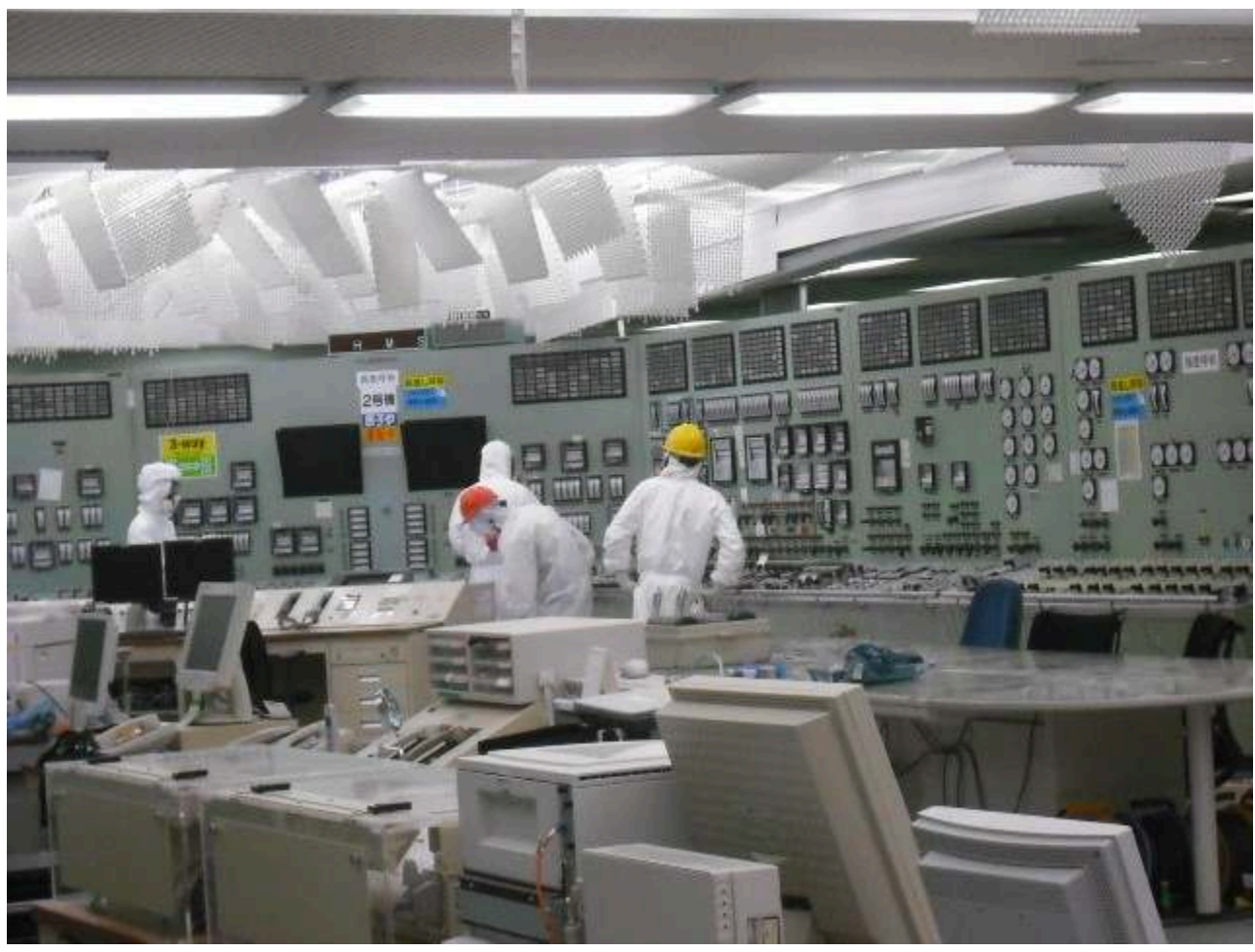

Réunion dans la salle de gestion de crise, bâtiment antisismique de Fukushima Daiichi. Masao Yoshida (habillé en noir) est assis à droite (1 ${ }^{\mathrm{er}}$ avril 2011) @) TEPCO.

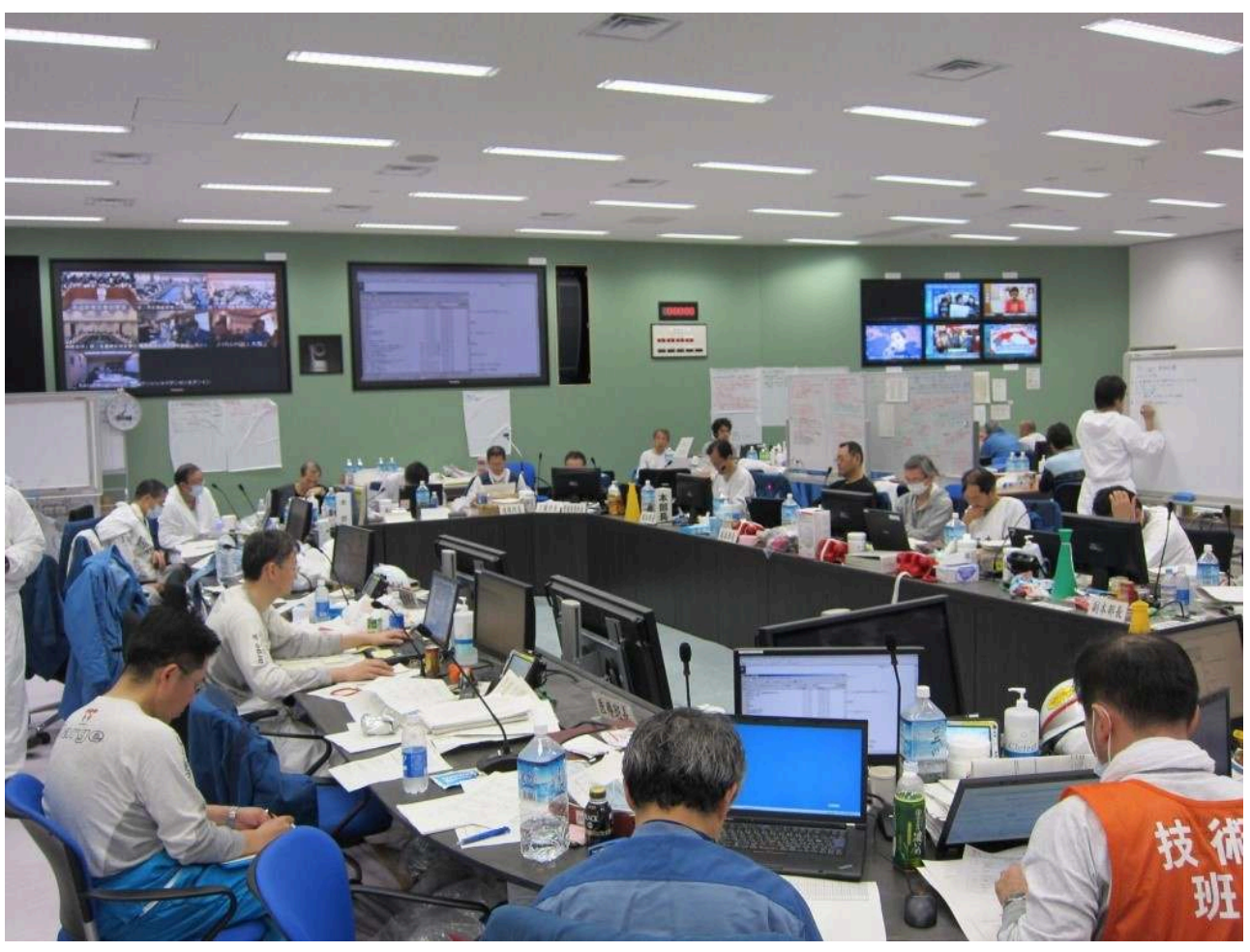


Robot travaillant dans le bâtiment réacteur 3 de Fukushima Daiichi (17 avril 2011) @ TEPCO.

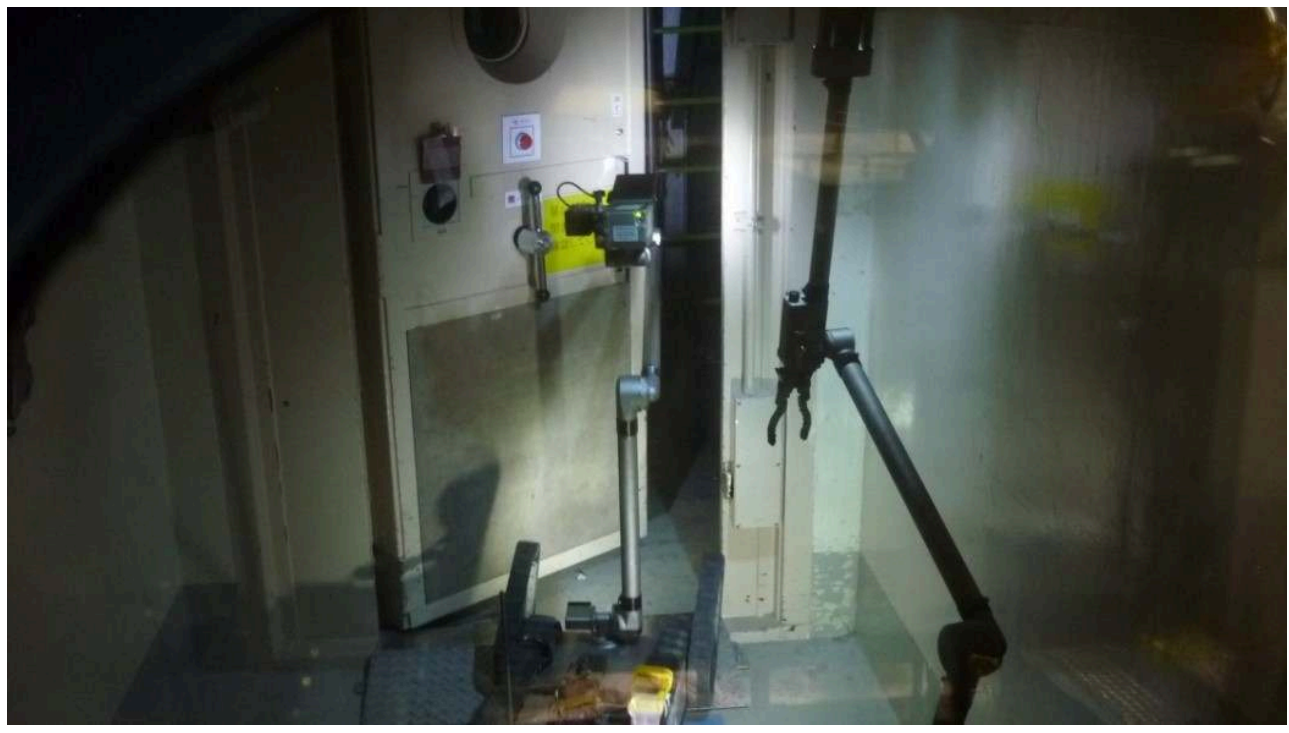

Naoto Kan, Premier ministre du Japon du 8 juin 2010 au 2 septembre 2011, informé de l'état du lycée d'Ishinomaki (préfecture de Miyagi) par le lieutenant-général Yuji Kuno, commandant de la 6 e division des forces d'autodéfense (10 avril 2011). Source : DVIDSHUB - CC By SA.

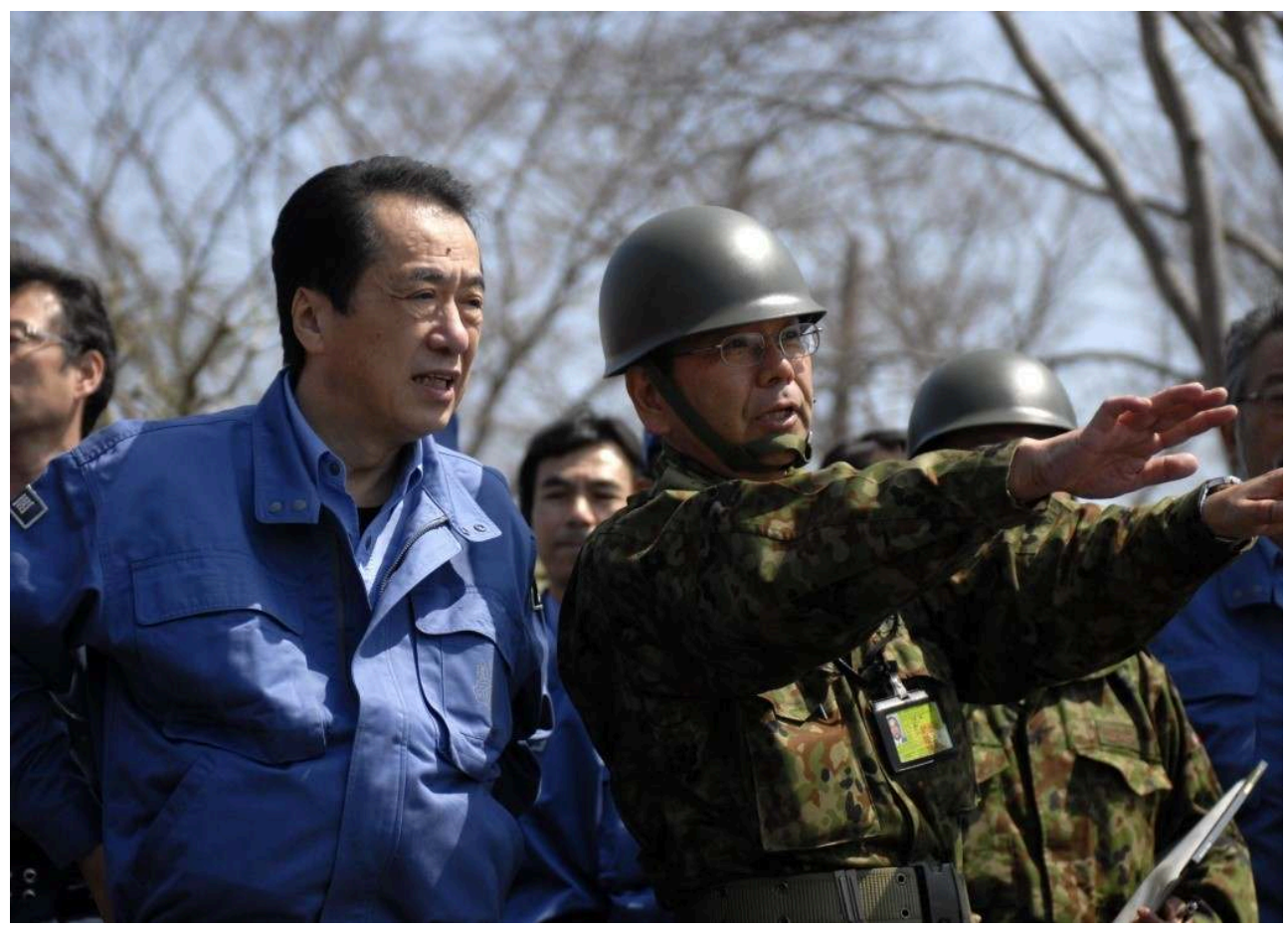


Banri Kaieda, ministre de l'Économie, du Commerce et de l'Industrie et Goshi Hosono, conseiller spécial du Premier ministre à Fukushima Daiichi (15 avril 2011) @ TEPCO.

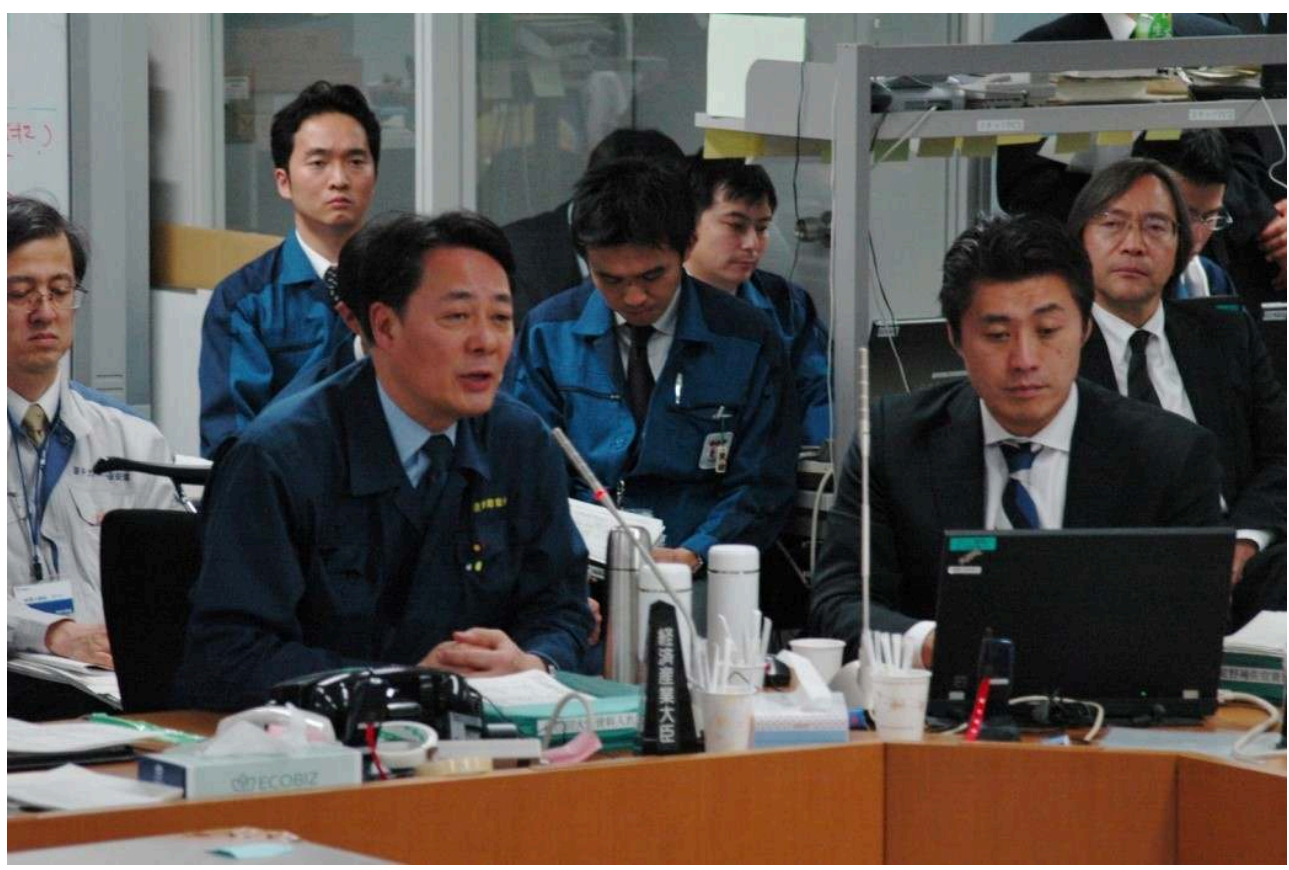

Masao Yoshida, assemblée générale, salle de gestion de crise de Fukushima Daiichi (30 mai 2011) (c) TEPCO.

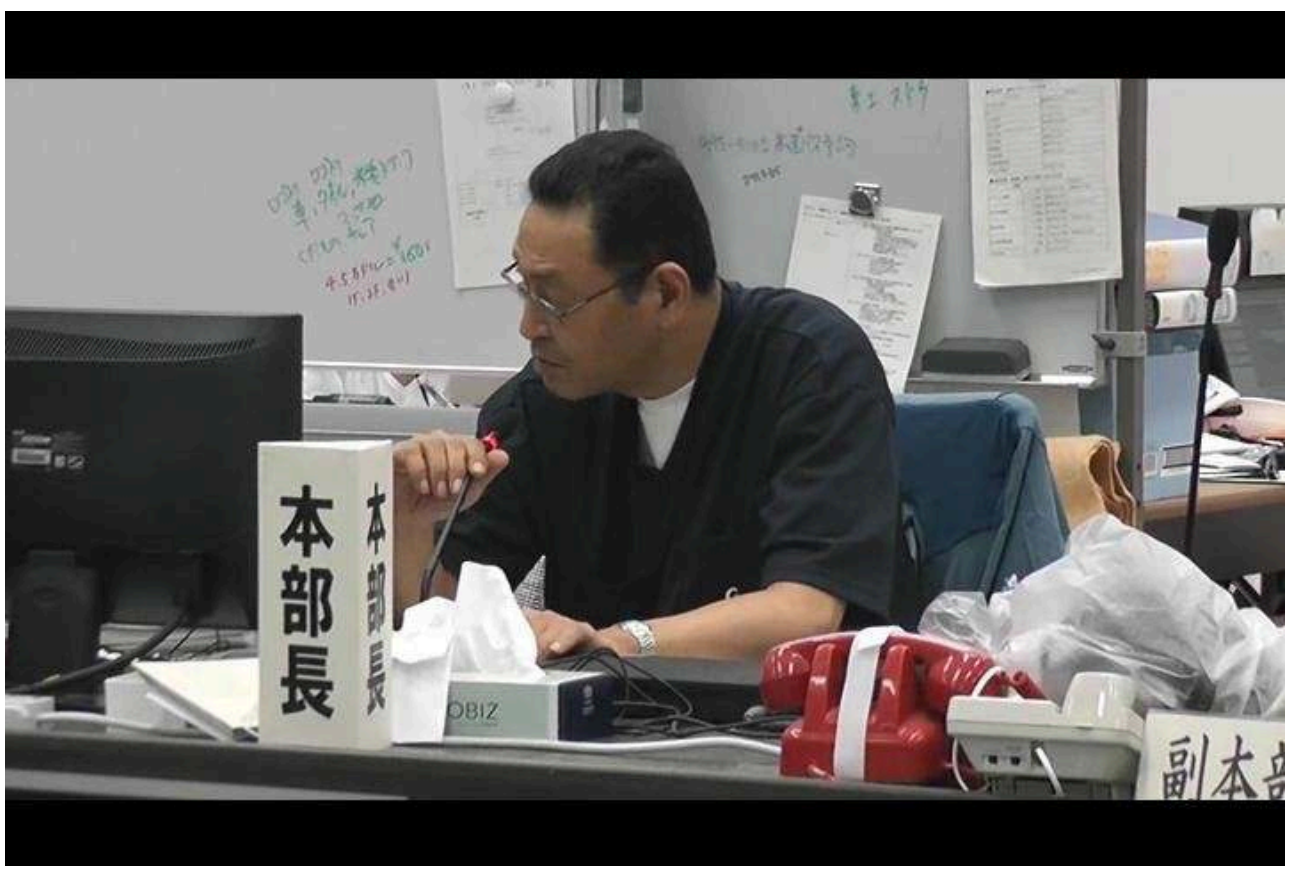


Masao Yoshida reçoit Yukiya Amano, Directeur général de l'Agence Internationale de l'Énergie Atomique (AIEA) dans la centrale de Fukushima Daiichi (25 juillet 2011) () TEPCO.

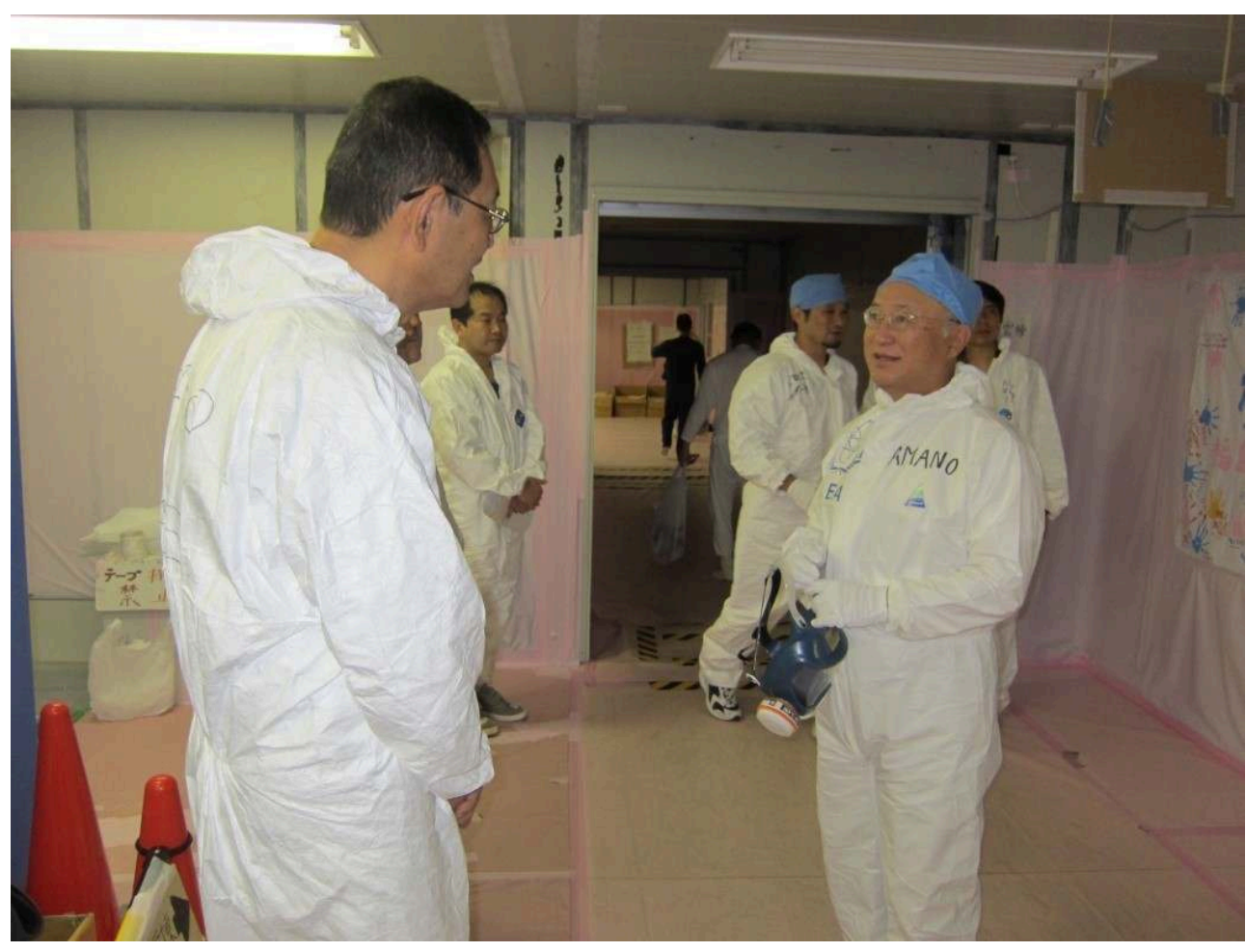

Stock d'équipements de protection en Tyvek dans le complexe sportif nommé le « J-Village » (7 octobre 2011) (c) TEPCO.

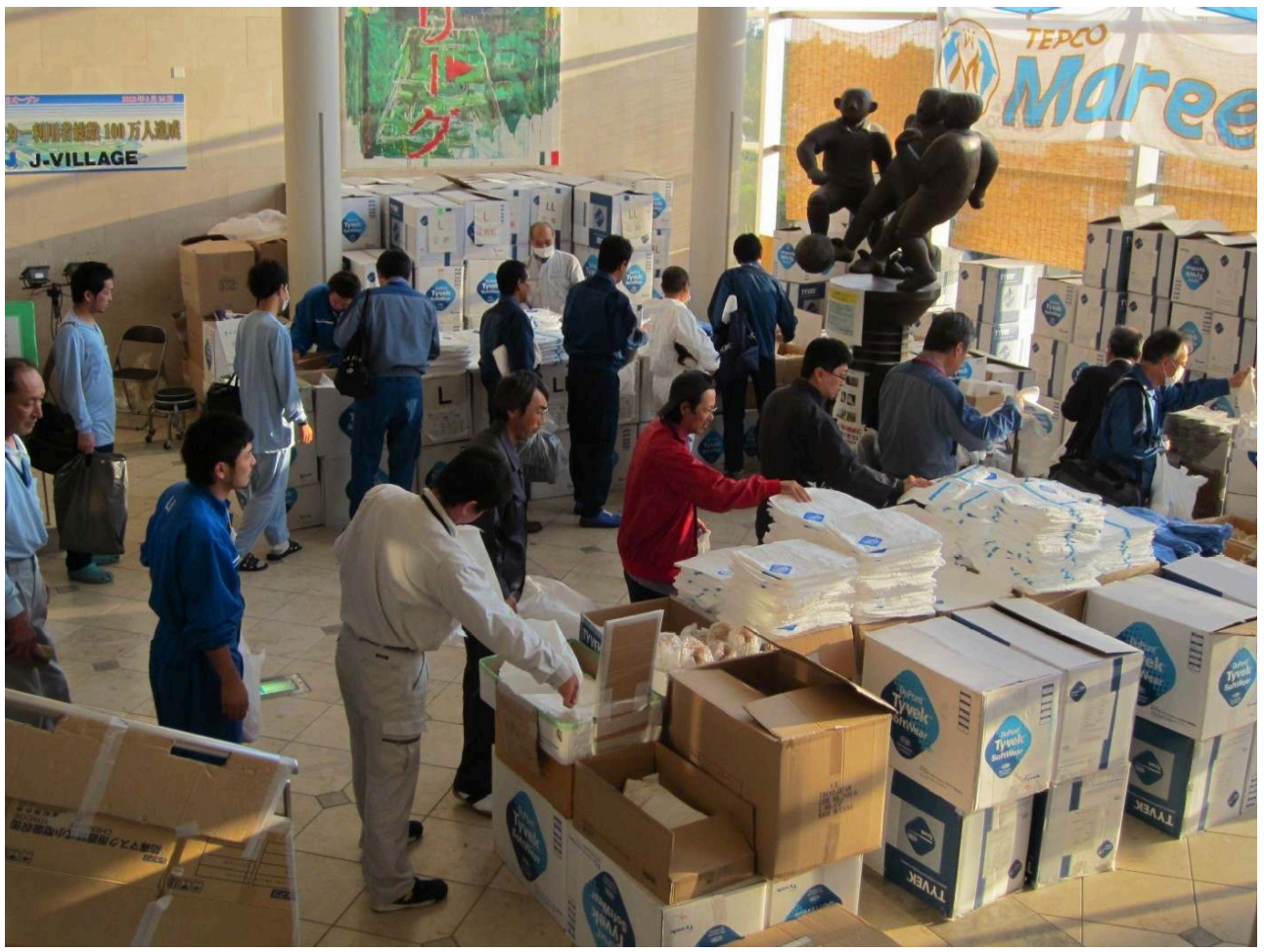


Réservoirs d'eau contaminée, zone G3 de Fukushima Daiichi (3 septembre 2013) (c) TEPCO.

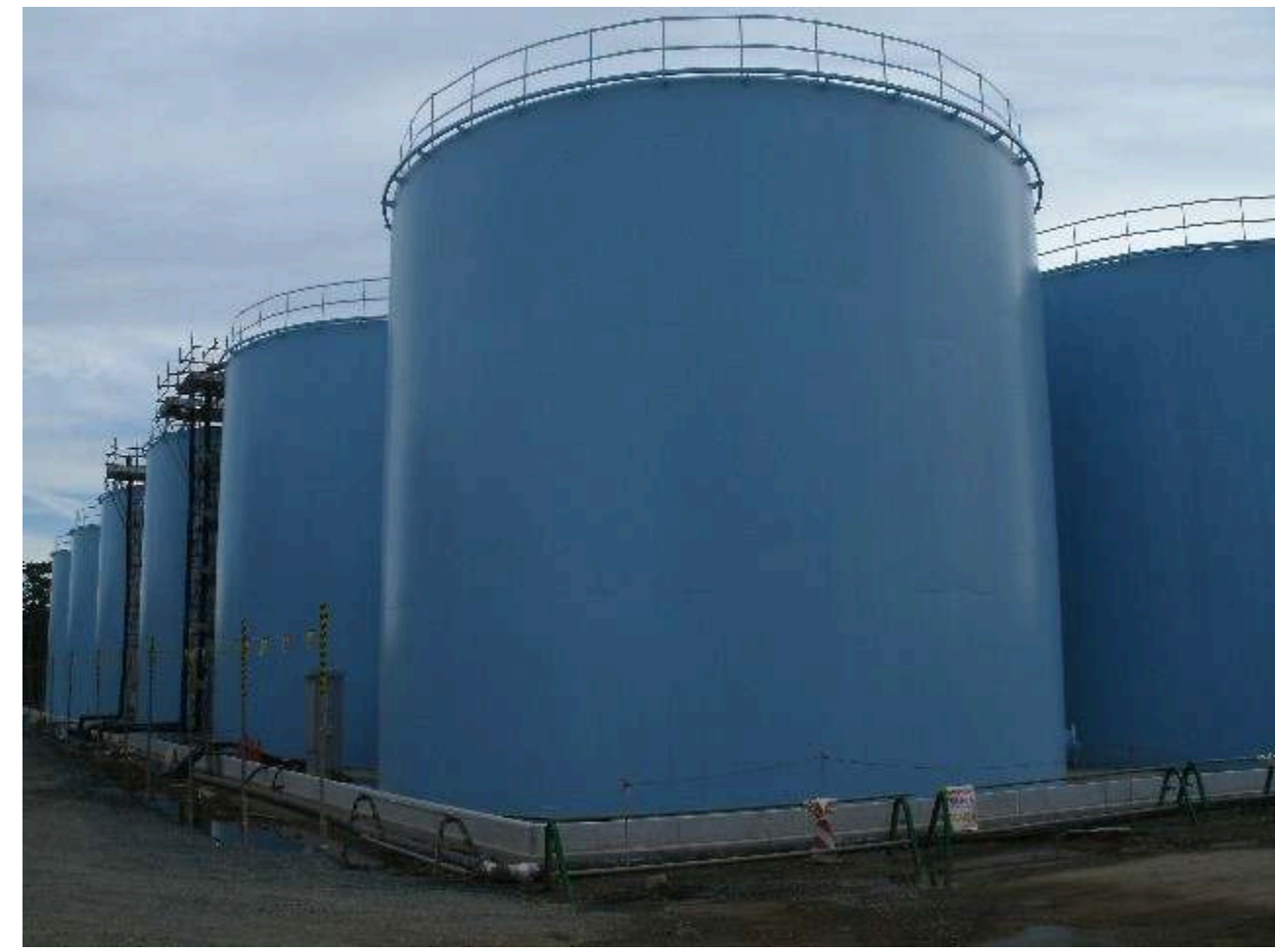

Inspecteurs de l'AIEA devant une aire de stockage d'eau contaminée de Fukushima Daiichi (17 février 2015) ㅇ TEPCO.

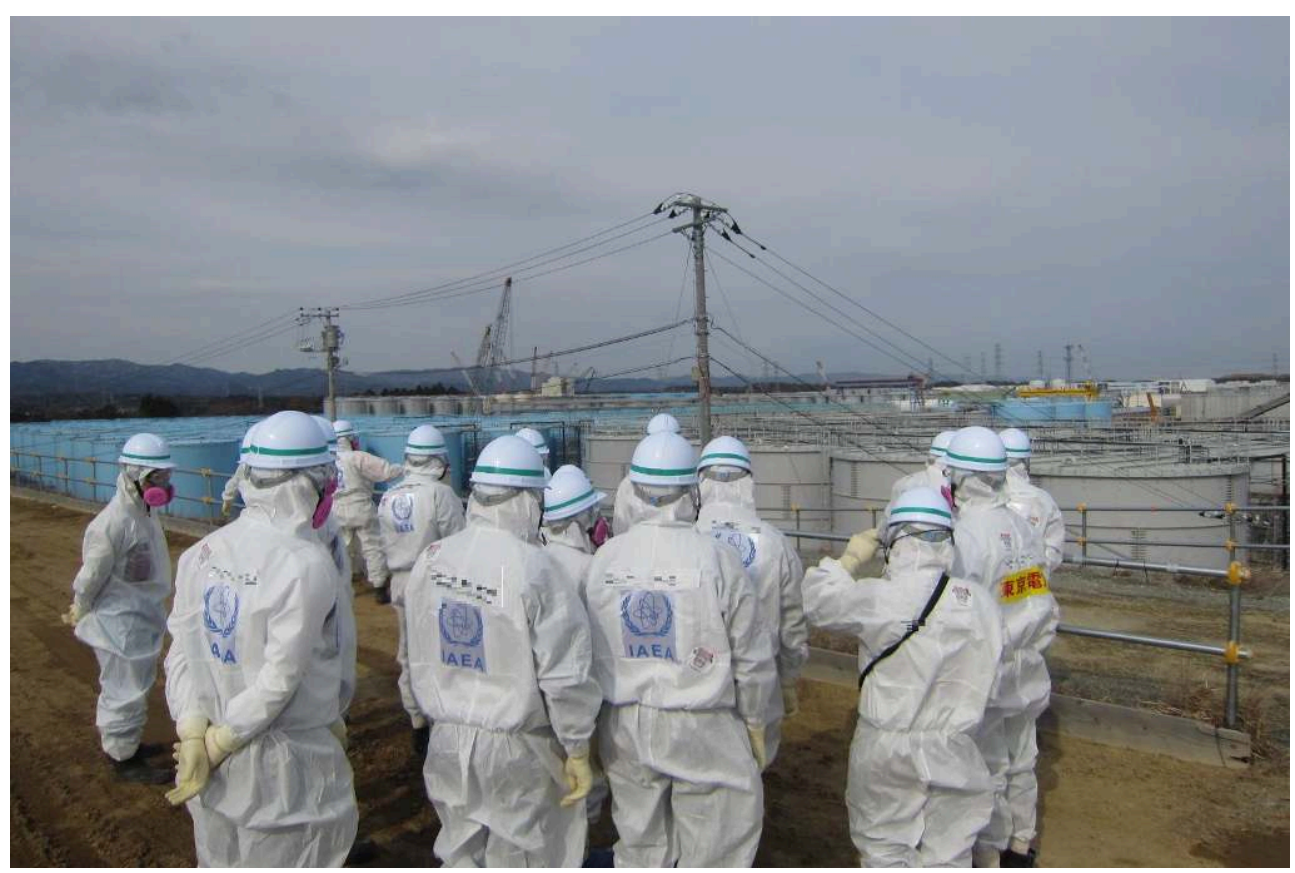


Visite de Fukushima Daiichi par Shinzo Abe, Premier ministre du Japon du 26 décembre 2012 au 16 septembre 2020 ; à gauche, la tranche 3 avec son nouveau dôme finalisé en février 2018 (14 avril 2019) (C) TEPCO.

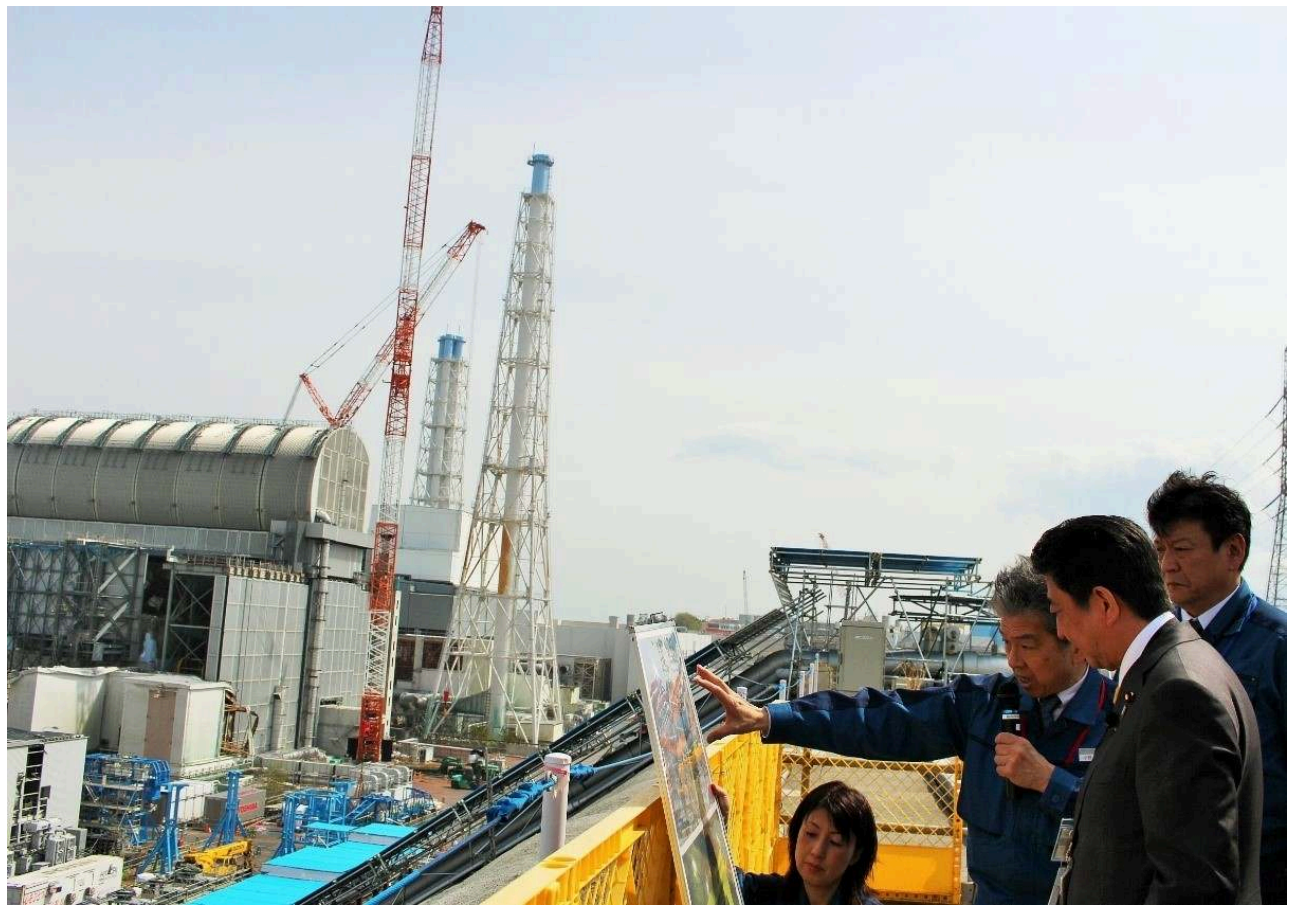




\section{L'accident de Fukushima Daiichi}

1 La centrale nucléaire de Fukushima Daiichi, exploitée par la Tokyo Electric Power Company (TEPCO) se situe dans le district de Futaba (préfecture de Fukushima), à environ 250 kilomètres au nord de Tôkyô (cf. Fig. 1). Elle est équipée de six réacteurs à eau bouillante (cf. Fig. 2 et 3), répartis sur près de $3,5 \mathrm{~km}^{2}$. Le réacteur 1 , mis en service en 1971, a été conçu à partir des plans de modèle BWR/3 et de confinement Mark I de General Electric. Les autres, mis en service entre 1974 et 1979, ont été conçus par différents constructeurs (General Electric, Hitachi et Tôshiba). Les réacteurs 2 à 5 sont de type BWR/4 et de confinement Mark I (cf. Fig. 4), tandis que le 6 est de type BWR/5 et de confinement Mark II (cf. Fig. 5).

2 Le 11 mars 2011, environ 6400 personnes se trouvent sur le site de Fukushima Daiichi. Les réacteurs 1, 2 et 3 sont en fonctionnement, alors que les réacteurs 4, 5 et 6 sont en arrêt programmé.

3 À 14h46, un séisme de magnitude 9 se produit au large de la centrale. Le système d'arrêt d'urgence des réacteurs 1 à 3 est activé automatiquement. Il est toutefois nécessaire d'assurer leur refroidissement pendant plusieurs jours pour éviter que les combustibles n'entrent en fusion.

4 Les employés de TEPCO qui ne sont pas chargés de piloter les réacteurs et les soustraitants encore sur place se réfugient dans le bâtiment antisismique, qui abrite la cellule de gestion de crise. Celle-ci est dirigée par Masao Yoshida.

5 L'Agence Météorologique du Japon lance des alertes au tsunami. Les vagues les plus hautes, dont l'amplitude est estimée entre 11,5 et 15,5 mètres, atteignent la côte à 15h37. Situées à environ 10 mètres au-dessus de la mer, les installations de Fukushima Daiichi sont inondées. La dévastation du site rend difficile l'accès à la centrale ainsi que le déplacement des travailleurs. La dégradation des moyens de télécommunication entrave les échanges d'informations entre la cellule de crises et les équipes présentes sur le terrain.

6 La catastrophe naturelle occasionne la perte du courant alternatif des réacteurs 1 à 5 et celle du courant continu des réacteurs 1, 2 et 4 . Les opérateurs perdent en conséquence l'éclairage, ainsi que les instruments de mesure, de contrôle et de commande. Ils se retrouvent alors dans l'incapacité de contrôler et de surveiller l'état des réacteurs et des systèmes de sauvegarde. Le panneau de distribution électrique et les batteries de 
secours du réacteur 3 résistent cependant à l'inondation, ce qui permet de maintenir l'alimentation en courant continu de l'installation.

7 À cause du manque de ressources électriques, la cellule de crise ne parvient pas à vérifier si les valves de l'IC (Isolation Condenser) du réacteur 1 et du RCIC (Reactor Core Isolation Condenser) du réacteur 2 sont bien ouvertes. Elle redoute dès lors un problème de refroidissement de ces deux réacteurs.

8 Conformément au cadre légal, l'exploitant fait part au Gouvernement de la situation d'urgence nucléaire à $16 \mathrm{~h} 36$.

9 À 17h10, Yoshida demande à ses équipes de concevoir deux solutions alternatives pour injecter de l'eau dans le cœur des réacteurs. La première repose sur l'emploi de pompes à incendie à moteur diesel et la seconde sur l'utilisation des camions de pompiers - une manœuvre non spécifiée dans les procédures de gestion d'accident.

10 À 18 h00, les opérateurs s'aperçoivent que deux valves du système IC du réacteur 1 sont fermées. Ils les actionnent à $18 \mathrm{~h} 18$ pour essayer de les ouvrir. Selon des indices d'ordre visuel et auditif, ils pensent que le refroidissement d'urgence est alors activé. Ils préviennent la cellule de crise mais referment à $18 \mathrm{~h} 25$ l'une des deux vannes d'alimentation du cœur. Cette fois, ils n'informent pas la cellule de crise de leur manœuvre. Par la suite, ces mêmes opérateurs concluent que l'IC ne fonctionne pas. Ils demandent alors des batteries pour effectuer les opérations de dépressurisation, nécessaires à l'injection d'eau à basse pression. Mais leur interlocuteur au sein de la cellule de crise ne comprend pas l'urgence de la situation et la demande n'est pas prise en compte.

11 À $21 \mathrm{~h} 40$, les agents envoyés sur le terrain arrivent à déterminer le niveau d'eau dans la cuve du réacteur 2, qui dépasse de 3,40 mètres la hauteur du combustible. Rassuré, l'exploitant communique l'information au Gouvernement. Plus tard, des mesures de pression dans la cuve et dans l'enceinte de confinement révèlent des valeurs normales. Vingt minutes plus tard, les opérateurs obtiennent un générateur portable, qui leur permet de rétablir l'éclairage dans les salles de commande des réacteurs 3 et 4 . Ils parviennent à vérifier que le RCIC du réacteur 3 fonctionne correctement et que le HPCI (High Pressure Cooling Injection System) est bien disponible.

$12 \mathrm{Au}$ même moment, un travailleur découvre que les doses de radiation autour du bâtiment réacteur 1 sont anormalement élevées. À 23h50, la cellule de crise comprend que le système de refroidissement d'urgence ne fonctionne pas. Le combustible du réacteur 1 est probablement déjà endommagé et des matières radioactives ainsi que de l'hydrogène ont vraisemblablement fui dans l'enceinte de confinement. L'équipe en charge du réacteur 1 prépare les mesures d'éventage de l'enceinte de confinement, la pression dans la cuve ayant dépassé sa valeur de dimensionnement. Les procédures d'urgence sont toutefois impossibles à appliquer car l'alimentation électrique est coupée. Les opérateurs doivent alors ouvrir manuellement les vannes, après les avoir localisées. L'injection d'eau douce dans le réacteur 1 débute le 12 mars vers $5 \mathrm{~h} 46$.

13 Le Premier ministre Naoto Kan décide de visiter le site pour comprendre la situation. Il arrive à 7h00, rencontre Yoshida et repart de Fukushima Daiichi à 8h04.

14 À $11 \mathrm{~h} 36$, le RCIC du réacteur 3 tombe en panne pour une raison indéterminée. Les opérateurs ne parviennent pas à le redémarrer. Le niveau d'eau commence à baisser dans la cuve et le système HPCI prend automatiquement le relais à $12 \mathrm{~h} 35$. 
15 À $14 \mathrm{~h} 30$, les opérateurs parviennent finalement à manipuler les vannes du réacteur 1 et pensent avoir réussi son éventage. Mais à $15 \mathrm{~h} 36$, le bâtiment réacteur 1 est soufflé par une explosion, certainement due à une accumulation d'hydrogène. Cinq travailleurs sont blessés, tandis que les débris endommagent des raccordements aux pompes d'injection d'eau installés auparavant sur le réacteur 2 .

16 L'injection d'eau de mer dans le bâtiment réacteur 1, effectuée à l'aide de camions incendie, débute à 19 h04.

17 À partir de 20h36, les travailleurs ne sont plus capables de surveiller le niveau d'eau dans la cuve du réacteur 3 du fait de l'épuisement de certaines batteries. Préoccupés par la capacité du HPCI à fonctionner dans la durée, ils décident de mettre en place une autre solution pour injecter de l'eau dans le réacteur. Celle-ci implique l'arrêt du HPCI, la dépressurisation de la cuve par les vannes SRV (Safety Relief Valves) et l'utilisation du système diesel de protection incendie pour assurer l'injection. Ces opérations débutent le 13 mars à $2 \mathrm{~h} 45$, mais les agents ne parviennent pas à ouvrir les vannes SRV. Le redémarrage du HPCI échoue également, probablement à cause de l'épuisement des batteries ou de la pression trop élevée dans l'enceinte. Les opérateurs raccordent des batteries de voiture au tableau électrique du réacteur 3. La pression baisse brusquement vers 9 h00, vraisemblablement en raison d'une activation du système automatique de dépressurisation provoquée par un faux signal. De l'eau douce peut dès lors être injectée à partir de $9 \mathrm{~h} 25$, en utilisant le système de protection incendie.

18 Vers 11 h00, la cellule de crise estime que le système RCIC du réacteur 2 risque de tomber en panne. Des opérations de dépressurisation sont menées pour injecter de l'eau douce, comme pour le réacteur 3. Les réserves d'eau utilisées pour refroidir les réacteurs sont toutefois épuisées à $12 \mathrm{~h} 20$. Les travailleurs commencent à injecter de l'eau de mer à partir de 13h12. Mais cette solution ne semble pas être efficace pour le réacteur 3 , certainement à cause d'une pression d'injection insuffisante et d'une impossibilité de maintenir ouverte la vanne de dépressurisation.

19 Le 14 mars à 11 h01, une explosion se produit dans le bâtiment réacteur 3, sans doute à la suite d'une accumulation d'hydrogène. Les tuyauteries et les pompes utilisées pour l'injection de l'eau dans les réacteurs 2 et 3 sont détruites. Sept travailleurs sont blessés et le personnel sur le terrain est évacué. Plus de cinq heures sont nécessaires pour rétablir l'injection d'eau de mer dans le réacteur 3. Les secousses entravent la conduite des travaux.

20 À $13 \mathrm{~h} 30$, le RCIC du réacteur 2 cesse de fonctionner. La vapeur est évacuée via les vannes SRV, opérées mécaniquement de la cuve vers le wetwell. Le niveau d'eau dans la cuve du réacteur commence alors à baisser.

21 Durant toute la journée du 14 mars, les vannes SRV du réacteur 2 sont actionnées manuellement afin de faire baisser la pression dans le réacteur et permettre l'injection d'eau dans la cuve. Tous les camions de pompiers sont alors utilisés pour refroidir les réacteurs 1 et 3. L'eau de mer est injectée de façon discontinue du fait de nombreux problèmes logistiques.

22 Ce n'est qu'à partir de 23 ho0 que l'injection d'eau de mer débute dans le réacteur 2. Les opérateurs ne parviennent cependant pas à éventer l'enceinte de confinement durant la nuit du 14 au 15 mars.

23 Le 15 mars, une explosion se produit à 6 h10 dans le bâtiment réacteur 2 . À 9 h38, une explosion survient cette fois dans le bâtiment réacteur 4 . Il est possible que cette 
explosion soit due à de l'hydrogène provenant du bâtiment réacteur 3 , via un conduit de ventilation commun. Yoshida décide l'évacuation de la centrale pour mettre à l'abri les employés. Il reste en salle de crise avec soixante-neuf collaborateurs (appelés dans les médias étrangers les « Fukushima 50 »). Ils sont rejoints, dans les jours suivants, par des opérateurs supplémentaires. La situation des réacteurs 5 et 6 est moins critique. Elle est gérée de manière relativement autonome par les opérateurs de la salle de commande, car l'un des générateurs diesel de secours du réacteur 6 a résisté au tsunami, ce qui permet d'alimenter l'installation en courant alternatif.

À partir du 13 mars, l'équipe de pilotage parvient à fournir l'eau nécessaire pour évacuer la chaleur résiduelle de manière fiable et continue. Le générateur diesel est relié au réacteur 5 le 12 mars, vers 5 h00. Ce raccordement permet de lire les paramètres du réacteur depuis la salle de commande. Au même moment, l'équipe établit un plan pour dépressuriser le réacteur et l'applique dans l'heure suivante. Il s'agit de forcer les vannes d'éventage de la cuve du réacteur en utilisant les tuyauteries d'approvisionnement en azote depuis l'extérieur du bâtiment. Les opérateurs décident ensuite d'ouvrir manuellement les vannes SRV restantes. L'absence d'éclairage et les secousses incessantes rendent l'opération particulièrement dangereuse. Le 14 mars, à 5 h00, l'équipe est capable de contrôler la pression du réacteur et d'injecter autant d'eau que de vapeur dégagée par les SRV.

À partir du 16 mars, les opérateurs réussissent à réparer le RHRS (Residual Heat Removal System - système d'évacuation de la chaleur résiduelle), en installant des pompes d'eau de mer et des générateurs diesel portables.

À partir du 21 mars, le rétablissement progressif des sources d'alimentation électrique permet aux équipes d'assurer peu à peu le refroidissement continu de tous les réacteurs. TEPCO se charge depuis de mener les opérations de décontamination et de démantèlement du site, programmées sur plusieurs décennies.

Le 16 décembre 2011, Yoshihiko Noda, le Premier ministre du Japon, annonce l'arrêt à froid des réacteurs de Fukushima Daiichi.

L'exploitant poursuit ses efforts, depuis fin mars 2011, pour maitriser les rejets radioactifs dans l'environnement. Des réservoirs de stockage de l'eau contaminée sont aménagés sur le site et s'accumulent au fil des années. En août 2019, TEPCO déclare que la limite de stockage sera atteinte à l'été 2022. Plusieurs solutions sont envisagées pour remédier à la saturation du site, parmi lesquelles la possibilité très controversée du rejet en mer de l'eau après traitement. En octobre 2020, les médias annoncent que le Gouvernement s'apprête à déclarer officiellement sa décision d'autoriser le rejet dans l'océan des eaux contaminées. L'opération ne devrait pas débuter avant 2022. 


\section{Tableau des auditions ${ }^{1}$}

\begin{tabular}{|c|c|c|c|c|}
\hline \multicolumn{2}{|c|}{ Personnes auditionnées } & \multirow{2}{*}{$\begin{array}{l}\text { Dates } \\
\text { d'audition }\end{array}$} & \multirow[b]{2}{*}{ Sujets abordés } & \multirow{2}{*}{$\begin{array}{l}\text { Volume du } \\
\text { compte } \\
\text { rendu }\end{array}$} \\
\hline Noms & $\begin{array}{l}\text { Fonctions mentionnées } \\
\text { dans le compte rendu }\end{array}$ & & & \\
\hline $\begin{array}{l}\text { Motohisa } \\
\text { IKEDA }\end{array}$ & $\begin{array}{l}\text { Ancien vice-ministre de } \\
\text { l'Économie et de } \\
\text { l'Industrie }\end{array}$ & $9 / 2 / 2012$ & $\begin{array}{l}\text { Mesures prises face à } \\
\text { l'accident }\end{array}$ & 37 pages \\
\hline Yukio EDANO & $\begin{array}{l}\text { Ministre de l'Économie et } \\
\text { de l'Industrie (Secrétaire } \\
\text { Général du Gouvernement } \\
\text { au moment de l'accident) }\end{array}$ & $25 / 3 / 2012$ & $\begin{array}{l}\text { Mesures prises face à } \\
\text { l'accident }\end{array}$ & 63 pages \\
\hline \multirow{2}{*}{ Banri KAIEDA } & \multirow{2}{*}{$\begin{array}{l}\text { Député DPJ (Democratic } \\
\text { Party of Japan), ancien } \\
\text { ministre de l'Économie et } \\
\text { de l'Industrie }\end{array}$} & $8 / 2 / 2012$ & $\begin{array}{l}\text { Mesures prises face à } \\
\text { l'accident }\end{array}$ & 34 pages \\
\hline & & $13 / 2 / 2012$ & $\begin{array}{l}\text { Mesures prises face à } \\
\text { l'accident }\end{array}$ & 31 pages \\
\hline Naoto KAN & $\begin{array}{l}\text { Député (Premier ministre } \\
\text { au moment de l'accident) }\end{array}$ & $3 / 4 / 2012$ & $\begin{array}{l}\text { Mesures prises face à } \\
\text { l'accident }\end{array}$ & 64 pages \\
\hline $\begin{array}{l}\text { Toshimi } \\
\text { KITAZAWA }\end{array}$ & $\begin{array}{l}\text { Sénateur DPJ (ministre de } \\
\text { la Défense au moment de } \\
\text { l'accident) }\end{array}$ & $31 / 5 / 2012$ & $\begin{array}{l}\text { Mesures prises face à } \\
\text { l'accident }\end{array}$ & 11 pages \\
\hline \multirow[b]{2}{*}{$\begin{array}{l}\text { Shunsuke } \\
\text { KONDÔ }\end{array}$} & \multirow[b]{2}{*}{$\begin{array}{l}\text { Président de la Japan } \\
\text { Atomic } \\
\text { Commission (JAEC) }\end{array}$} & $1 / 2 / 2012$ & Le scénario du pire & 13 pages \\
\hline & & $1 / 2 / 2012$ & 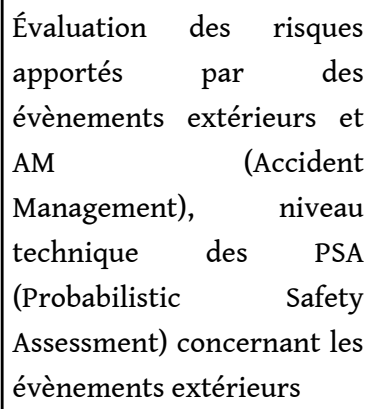 & 47 pages \\
\hline
\end{tabular}




\begin{tabular}{|c|c|c|c|c|}
\hline & & $16 / 4 / 2012$ & $\begin{array}{l}\text { 1. Scénario en cas } \\
\text { d'imprévu, concertation } \\
\text { nippo-américaine } 2 \text {. PSA, } \\
\text { risques apportés par les } \\
\text { évènements extérieurs et } \\
\text { AM }\end{array}$ & 60 pages \\
\hline \multirow[t]{2}{*}{$\begin{array}{l}\text { Nobuo } \\
\text { SHUDÔ }\end{array}$} & \multirow[t]{2}{*}{$\begin{array}{l}\text { Professeur honoraire à } \\
\text { l'Université de Tôhoku }\end{array}$} & $6 / 7 / 2011$ & 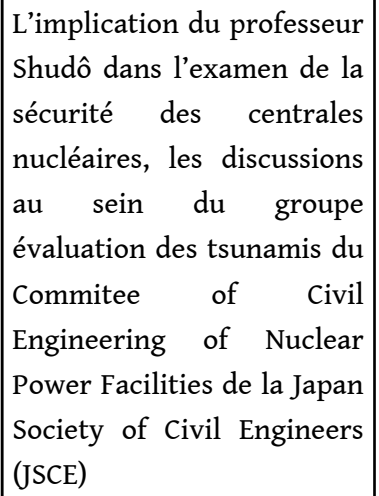 & 7 pages \\
\hline & & $29 / 9 / 2011$ & $\begin{array}{l}\text { À propos de l'article paru le } \\
\text { 17/8/2011 dans l'édition du } \\
\text { soir de Mainichi Shinbun et } \\
\text { les mesures concernant les } \\
\text { tsunamis dans les centrales } \\
\text { nucléaires }\end{array}$ & 5 pages \\
\hline $\begin{array}{l}\text { Atsuyuki } \\
\text { SUZUKI }\end{array}$ & $\begin{array}{l}\text { Président de la Japan } \\
\text { Atomic Energy Agency } \\
\text { (JAEA), ancien Président } \\
\text { de la Nuclear Safety } \\
\text { Commission of Japan }\end{array}$ & $8 / 3 / 2012$ & $\begin{array}{l}\text { Problèmes ressentis à } \\
\text { propos de la Nuclear Safety } \\
\text { Commission of Japan quand } \\
\text { il la présidait et les mesures } \\
\text { prises }\end{array}$ & 16 pages \\
\hline \multirow{2}{*}{$\begin{array}{l}\text { Hiroshi } \\
\text { SUZUKI }\end{array}$} & \multirow{2}{*}{$\begin{array}{l}\text { Sénateur DPJ (vice- } \\
\text { ministre de l'Éducation et } \\
\text { des Sciences au moment } \\
\text { de l'accident) }\end{array}$} & $23 / 2 / 2012$ & $\begin{array}{l}\text { À propos du monitoring et } \\
\text { du System for prediction of } \\
\text { Environmental Emergency } \\
\text { Dose Information (SPEEDI) }\end{array}$ & 33 pages \\
\hline & & $7 / 3 / 2012$ & $\mid \begin{array}{llr}\text { Monitoring, } & \text { SPEEDI } & \text { et } \\
\text { normes } & \text { pour } & \text { l'utilisation } \\
\text { des } & \text { cours } & \text { des } \\
\text { établissements scolaires }\end{array}$ & 32 pages \\
\hline $\begin{array}{l}\text { Yoshiaki } \\
\text { TAKAKI }\end{array}$ & $\begin{array}{l}\text { Député (ancien ministre } \\
\text { de l'Éducation et des } \\
\text { Sciences) }\end{array}$ & $31 / 1 / 2012$ & $\mid \begin{array}{llr}\text { Monitoring, } & \text { SPEEDI } & \text { et } \\
\text { normes } & \text { pour } & \text { l'utilisation } \\
\text { des } & \text { cours } & \text { des } \\
\text { établissements scolaires }\end{array}$ & 32 pages \\
\hline $\begin{array}{l}\text { Kansei } \\
\text { NAKANO }\end{array}$ & Député DPJ & $8 / 5 / 2012$ & $\begin{array}{llr}\text { Concertation du } 11 \text { mars au } \\
\text { 5e étage de la résidence du } \\
\text { Premier } \quad \text { ministre } & \text { et } \\
\text { relèvement des limites } & \text { de } \\
\text { dose radioactive } & \text { en } \\
\text { situation d'urgence } & \end{array}$ & 2 pages \\
\hline
\end{tabular}




\begin{tabular}{|c|c|c|c|c|}
\hline $\begin{array}{l}\text { Akihisa } \\
\text { NAGASHIMA }\end{array}$ & Député DPJ & $2 / 2 / 2012$ & $\begin{array}{l}\text { Concertation nippo- } \\
\text { américaine, question du } \\
\text { relèvement des limites de } \\
\text { dose radioactive à } 500 \mathrm{mSv}\end{array}$ & $\begin{array}{l}58 \text { pages, } \\
\text { dont une } \\
\text { trentaine } \\
\text { censurée }\end{array}$ \\
\hline \multirow{2}{*}{$\begin{array}{l}\text { Tetsurô } \\
\text { FUKUYAMA }\end{array}$} & \multirow{2}{*}{$\begin{array}{l}\text { Sénateur DPJ (secrétaire } \\
\text { général adjoint du } \\
\text { Gouvernement au moment } \\
\text { de l'accident) }\end{array}$} & $15 / 2 / 2012$ & $\begin{array}{l}\text { Mesures prises face à } \\
\text { l'accident }\end{array}$ & 73 pages \\
\hline & & $28 / 2 / 2012$ & $\begin{array}{l}\text { Mesures prises face à } \\
\text { l'accident }\end{array}$ & 40 pages \\
\hline $\begin{array}{l}\text { Toshio } \\
\text { FUJISHIRO }\end{array}$ & $\begin{array}{l}\text { Conseiller auprès de la } \\
\text { Research organisation for } \\
\text { Information Science and } \\
\text { Technology (RIST) }\end{array}$ & $29 / 3 / 2012$ & $\begin{array}{l}\text { Discussions ayant eu lieu } \\
\text { lors de l'amendement des } \\
\text { mesures de prévention au } \\
\text { sein du groupe de travail de } \\
\text { la Nuclear Safety } \\
\text { Commission en } 2006\end{array}$ & 24 pages \\
\hline \multirow[t]{2}{*}{$\begin{array}{l}\text { Gôshi } \\
\text { HOSONO }\end{array}$} & $\begin{array}{l}\text { Ministre } \\
\text { l'Environnement/ministre } \\
\text { chargé de l'Apaisement } \\
\text { après l'accident de la } \\
\text { centrale nucléaire et de la } \\
\text { prévention d'un nouvel } \\
\text { accident/ministre d'État } \\
\text { chargé de la } \\
\text { nucléaire }\end{array}$ & $14 / 12 / 2011$ & $\begin{array}{l}\text { Mesures prises face à } \\
\text { l'accident }\end{array}$ & 73 pages \\
\hline & $\begin{array}{l}\text { Ministre } \\
\text { l'Environnement } \\
\text { (conseiller du Premier } \\
\text { ministre au moment de } \\
\text { l'accident) }\end{array}$ & $31 / 5 / 2012$ & $\begin{array}{l}\text { Scénario de l'imprévu, état } \\
\text { de la Nuclear Regulation } \\
\text { Authority }\end{array}$ & 7 pages \\
\hline $\begin{array}{l}\text { Shôjirô } \\
\text { MATSURA }\end{array}$ & \begin{tabular}{|lrrr} 
Président du comité & de \\
conseil de & la & Nuclear \\
Safety & & \multicolumn{2}{c}{ Research } \\
Association & (NSRA) et \\
Président & du & Nuclear \\
Material & Control & Center \\
(NMCC) & &
\end{tabular} & $2 / 8 / 2011$ & $\begin{array}{l}\text { Accident Management, } \\
\text { utilisation des informations } \\
\text { sur les risques }\end{array}$ & $\begin{array}{lr}28 & \text { pages, } \\
\text { dont } & 18 \\
\text { censurées }\end{array}$ \\
\hline MATSUMOTO & 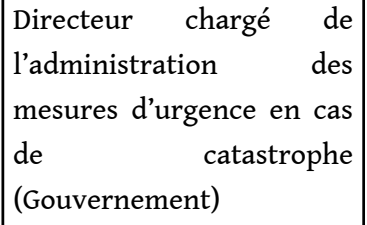 & $16 / 1 / 2012$ & $\begin{array}{l}\text { Transfert d'une partie des } \\
\text { pouvoirs du directeur de } \\
\text { l'unité de gestion de la } \\
\text { catastrophe nucléaire }\end{array}$ & 12 pages \\
\hline & $\begin{array}{l}\text { Directeur exécutif pour la } \\
\text { Nuclear Disaster Response, } \\
\text { Nuclear and Industrial } \\
\text { Safety Agency (NISA) }\end{array}$ & $13 / 9 / 2011$ & $\begin{array}{l}\text { L'eau contaminée et la } \\
\text { communication au sein de } \\
\text { la cellule de crise } \\
\text { Gouvernement-TEPCO }\end{array}$ & 8 pages \\
\hline
\end{tabular}

MORIYAMA 


\begin{tabular}{|c|c|c|c|c|}
\hline & $\begin{array}{l}\text { Directeur exécutif pour la } \\
\text { Nuclear Disaster Response, } \\
\text { (NISA) du ministère de } \\
\text { l'économie et de } \\
\text { l'industrie }\end{array}$ & $18 / 10 / 2011$ & $\begin{array}{l}\text { Évaluation du risque de } \\
\text { tsunami au sein de la NISA }\end{array}$ & 6 pages \\
\hline \multirow{11}{*}{$\begin{array}{l}\text { Masao } \\
\text { YOSHIDA }\end{array}$} & \multirow{11}{*}{$\begin{array}{l}\text { Directeur de la centrale } \\
\text { nucléaire de Fukushima } \\
\text { Daiichi de TEPCO }\end{array}$} & $22 / 7 / 2011$ & $\begin{array}{l}\text { L'accident et les actions } \\
\text { entreprises }\end{array}$ & 59 pages \\
\hline & & $29 / 7 / 2011$ & $\begin{array}{l}\text { L'accident et les actions } \\
\text { entreprises }\end{array}$ & 61 pages \\
\hline & & \multirow{5}{*}{$\begin{array}{l}8 / 8 / 2011 \\
9 / 8 / 2011\end{array}$} & $\begin{array}{l}\text { L'accident et les actions } \\
\text { entreprises } 1\end{array}$ & 37 pages \\
\hline & & & $\begin{array}{l}\text { L'accident et les actions } \\
\text { entreprises } 2\end{array}$ & 31 pages \\
\hline & & & $\begin{array}{l}\text { L'accident et les actions } \\
\text { entreprises } 3\end{array}$ & 35 pages \\
\hline & & & $\begin{array}{l}\text { L'accident et les actions } \\
\text { entreprises } 4\end{array}$ & 68 pages \\
\hline & & & $\begin{array}{l}\text { L'accident et les actions } \\
\text { entreprises (Document) }\end{array}$ & 48 pages \\
\hline & & $9 / 8 / 2011$ & $\begin{array}{l}\text { Mesures concernant l'eau } \\
\text { contaminée }\end{array}$ & 4 pages \\
\hline & & $13 / 10 / 2011$ & $\begin{array}{l}\text { Prévision avant le } 24 \text { mars } \\
\text { de l'existence d'eau } \\
\text { fortement contaminée } \\
\text { intentions et toile de fond } \\
\text { de sa déclaration à la } \\
\text { cellule de crise } \\
\text { Gouvernement-TEPCO du } 4 \\
\text { avril }\end{array}$ & 7 pages \\
\hline & & $6 / 11 / 2011$ & $\begin{array}{l}\text { L'accident et les actions } \\
\text { entreprises }\end{array}$ & 66 pages \\
\hline & & $6 / 11 / 2011$ & $\begin{array}{l}\text { L'accident et les actions } \\
\text { entreprises }\end{array}$ & 37 pages \\
\hline
\end{tabular}

\section{NOTES}

1. Auditions en japonais menées par les enquêteurs de l'ICANPS et mises en ligne sur le site internet du Gouvernement le 9 septembre 2014. 


\section{Glossaire des termes techniques}

1F-1 : tranche 1 de Fukushima Daiichi.

1F-2 : tranche 2 de Fukushima Daiichi.

1F-3 : tranche 3 de Fukushima Daiichi.

ABWR (Advanced Boiling Water Reactor) : Réacteur à Eau Bouillante (REB) de troisième génération. Il a été conçu par GE, Hitachi et Tôshiba. Les premiers ABWR ont été mis en service dans la centrale nucléaire de Kashimazaki-Kariwa (réacteurs $n^{\circ} 6$ et $n^{\circ} 7$ ), exploitée par TEPCO.

5 AC (Atmospheric Control) system: système de contrôle atmosphérique. Lors du fonctionnement du REB, l'enceinte de confinement est remplie d'azote, notamment pour éviter les combustions d'hydrogène. Il est cependant possible de remplir l'enceinte d'air normal, notamment en cas de nécessité d'interruption du REB et d'introduction de personnel. Le système de contrôle atmosphérique permet de remplir l'enceinte de confinement du gaz souhaité et de le maintenir à la composition désirée. Il permet également de filtrer le gaz évacué lors des interruptions planifiées. À Fukushima Daiichi, un conduit reliant le système de contrôle atmosphérique à la cheminée (via le SGTS) a été conçu pour offrir un circuit d'éventage renforcé. Toutefois, le système ACS n'est pas disponible en cas d'accident et le conduit d'éventage n'a donc pas pu être utilisé.

6 Acide borique : composé chimique utilisé comme substance neutrophage.

7 ADS (Automatic Depressurization System) : système de dépressurisation automatique du réacteur.

AMG (Accident Management Guidelines) : guide pour la gestion des accidents.

Années-réacteurs: il s'agit du produit du nombre de réacteurs par le nombre d'années pendant lesquels ils ont été exploités. Une année-réacteur correspond ainsi à une année pendant laquelle un réacteur a fonctionné. Dix années-réacteurs peuvent renvoyer à l'exploitation de cinq réacteurs pendant deux ans; ou de deux réacteurs pendant cinq ans. L'utilisation de ce produit reflète les années d'expérience acquise dans l'exploitation des réacteurs nucléaires. Il est également utilisé pour illustrer les probabilités d'occurrence d'évènements sur la centrale, notamment les accidents systémiques. 
AOP (Abnormal Operating Procedures) : procédures d'exploitation en cas de situation anormale. d'insertion des barres de contrôle des réacteurs de Fukushima Daiichi est à commande hydraulique. L'injection ou le pompage d'eau exerce une pression sur le mécanisme qui permet d'ajuster la position des barres de contrôle dans le cœur du réacteur. En cas d'urgence, c'est grâce à un accumulateur à azote que l'insertion est effectuée. Si ce dernier dysfonctionne, le mécanisme d'injection alternative des barres permet leur insertion grâce à de l'eau pressurisée qui provient directement de la cuve du réacteur. Ce mécanisme n'est efficace que si la pression dans la cuve est suffisamment élevée.

Arrêt à froid : situation d'un réacteur nucléaire à l'arrêt dans lequel le fluide de refroidissement est soumis à une pression quasi-atmosphérique, et dont la température est inférieure à sa température d'ébullition.

Arrêt d'urgence (SCRAM) : arrêt soudain d'un réacteur nucléaire par l'opérateur, manuellement ou automatiquement, habituellement par insertion de barres de contrôle.

rres de contrôle: tubes mobiles contenant une matière neutrophage dont la position permet de contrôler, par absorption de neutrons, le nombre de fissions dans le cœur.

Bâtiment réacteur : bâtiment qui contient, entre autres, l'enceinte de confinement et la piscine de désactivation du combustible usé. noyaux radioactifs, soit du nombre de désintégrations qui s'y produisent par seconde. Un becquerel correspond à une désintégration par seconde.

Blow Out Panel : panneau de décompression.

Booster : une pompe booster permet d'augmenter la pression d'arrivée d'eau dans les pompes avant l'injection.

BTC (B/Training Center) : sigle utilisé dans le témoignage de Yoshida, qui correspond certainement à l'entreprise BWR Operator Training Center corporation, spécialisée dans la formation et la certification du personnel intervenant dans les centrales nucléaires au Japon. Co-détenue par TEPCO, cette entreprise propose des entraînements aux exploitants de REB, notamment grâce à des simulateurs qui reproduisent des salles de commandes de réacteurs japonais.

BWR (Boiling Water Reactor) : voir réacteur à eau bouillante (REB).

Caloporteur : fluide circulant dans le cœur d'un réacteur nucléaire afin d'en évacuer la chaleur produite par les réactions.

CAMS (Containment Atmosphere Monitoring System): le système de contrôle de l'atmosphère dans le confinement (CAMS) est un système qui permet de déterminer la composition du gaz (et notamment la concentration d'hydrogène et d'oxygène), ainsi 
que de mesurer le rayonnement gamma dans l'enceinte de confinement (wetwell et drywell). À Fukushima Daiichi, les dispositifs de mesure d'oxygène et d'hydrogène (qui sont particulièrement énergivores) n'ont pas pu être utilisés après la perte des ressources électriques. pour refroidir certains générateurs diesel de secours et d'autres composants importants pour la sûreté, ainsi que pour servir de source froide aux systèmes d'évacuation de la chaleur résiduelle. Lors d'un tsunami, l'eau se retire des plages dans un premier temps avant de déferler sous la forme d'une importante vague. Ce retrait inquiète Yoshida car il pouvait causer une rotation à sec ou l'aspiration d'air dans les pompes, ce qui pourrait rendre difficile leur redémarrage. Notons que la panne simultanée de tous les circuits de refroidissement à l'eau de mer relatifs à la sécurité est désignée par un accident de perte de la source froide ultime (Loss of Ultimate Heat Sink; LUHS).

Circuits A et B : le niveau d'eau dans la cuve du réacteur est une information primordiale. Un réacteur dispose de plusieurs systèmes de mesure du niveau d'eau avec des plages de mesures qui se chevauchent. Ces systèmes sont réalisés quasiment à l'identique en deux occurrences : le circuit A et le circuit B.

Cœur du réacteur: partie d'un réacteur nucléaire comprenant les assemblages de combustible nucléaire, agencée pour permettre une réaction en chaîne. 
Combustible nucléaire : matière contenant des nucléides dont la fission dans un réacteur nucléaire permet d'y entretenir une réaction en chaîne.

Condenseur : appareil utilisé pour transformer la vapeur en eau grâce à un échangeur thermique permettant la circulation d'un réfrigérant.

Conducteur d'une salle de commande : voir pilote de tranche.

Containment Head Spray: système d'arrosage de la chambre sèche, ou drywell, similaire au système d'arrosage du wetwell (cf. le Containment Spray Mode). Il s'agit cependant d'un système moins important pour la sûreté du réacteur, puisque sa fonction est uniquement de normaliser rapidement la situation dans le réacteur après son arrêt d'urgence.

Containment Spray Mode : mode d'aspersion de l'enceinte du confinement. Dans le cas d'un accident de perte de réfrigérant primaire (Loss of Coolant Accident ; LOCA), le système RHR d'évacuation de la chaleur résiduelle des réacteurs 2 à 6 de Fukushima Daiichi peut basculer entre deux modes: le mode d'aspersion de l'enceinte de confinement (Containment Spray) et l'injection d'eau à basse pression dans le cœur du réacteur (Low Pressure Core Injection). Le mode d'aspersion du confinement permet, via un réseau de buses, de contrôler la température et la pression dans le wetwell et le drywell. Notons que dans le cas du réacteur 1 , le système d'aspersion du confinement et le système d'injection d'eau dans la cuve sont distincts.

Contamination interne : exposition interne aux particules radioactives, lorsque des éléments radioactifs ont pénétré à l'intérieur de l'organisme par inhalation ou par ingestion d'aliments contaminés.

Cooling Line: circuit de refroidissement, qui correspond aux tuyaux reliant la chambre de dépressurisation à la cuve.

Corium : amas de combustibles et d'éléments de structure du cœur d'un réacteur nucléaire fondus et mélangés, pouvant se former en cas d'accident grave.

Crayon de combustible nucléaire : ensemble de pastilles de combustible nucléaire empilées dans des tubes de métal (gaines en alliage de zirconium) fermés aux extrémités.

CRD (Control Rod Drive) : mécanisme de contrôle des barres de commandes. De l'eau peut être injectée dans le réacteur à travers les tubes guides des barres.

Criticité : le risque de criticité est défini comme le risque de démarrage d'une réaction nucléaire en chaîne involontaire et incontrôlée lorsqu'une masse de matière fissile trop importante est rassemblée au même endroit.

CS (Core Spray System) : système d'aspersion du cœur.

CST (Condensate Storage Tank) : réservoir de stockage de condensats.

Cuve du réacteur: récipient métallique étanche contenant le cœur d'un réacteur nucléaire, le modérateur et le caloporteur.

CUW (Clean-Up Water system): système de nettoyage de l'eau. Dans un REB en fonctionnement, l'eau est introduite directement dans la cuve du réacteur dans un état liquide, puis elle s'évapore vers la turbine. Les impuretés peuvent alors s'accumuler dans la cuve du réacteur. Le système CUW permet d'extraire l'eau de la cuve, de la filtrer et de la réinjecter ailleurs, le plus souvent directement dans la cuve. Plusieurs 
manuels de gestion des accidents prévoient l'utilisation de ce système pour injecter de l'eau et refroidir la cuve en dernier recours.

elle peut également servir dans la lutte contre les incendies ou être injectée dans les

DDFP (Diesel Drive Fire Protection) : pompe diesel de protection incendie.

Dénoyage : le dénoyage du cœur d'un réacteur nucléaire signifie qu'il n'est plus noyé dans de l'eau. Si le cœur reste découvert, la température des crayons du combustible augmente et entraîne sa dégradation de manière irrémédiable, voire sa fusion.

DG (Diesel Generator) : générateur diesel de secours.

Disque de rupture : dispositif à usage unique de protection contre les surpressions. Lorsque le système protégé atteint une pression prédéterminée, la membrane du disque de rupture éclate instantanément. Afin d'éviter la fuite de matériaux radioactifs de l'enceinte de confinement des réacteurs vers l'atmosphère, les lignes d'éventage sont scellées grâce à des disques de rupture. Dans les réacteurs de Fukushima Daiichi, ces disques sont conçus pour rompre à la pression nominale de l'enceinte de confinement (soit 5 bars). Cependant, si ces disques de rupture permettent d'éviter l'ouverture accidentelle des vannes d'isolation du confinement, ils limitent également la possibilité d'éventage de l'enceinte du confinement à basse pression et peuvent dès lors s'avérer contre-productifs.

Dose absorbée : quantité d'énergie déposée par unité de masse par un rayonnement ionisant.

Drywell Cooler : le drywell contient la cuve du réacteur et l'essentiel des conduits de réfrigération. Bien qu'ils soient isolés thermiquement, ces composants peuvent transmettre de la chaleur au drywell. Le refroidisseur du drywell est un système dont la fonction est d'éviter que l'air dans l'enceinte de confinement ne chauffe trop. Il consiste en de grands ventilateurs qui poussent l'air vers des échangeurs de chaleur refroidis à l'eau.

\section{Drywell Spray : voir Containment Head Spray.}

Drywell : partie haute de l'enceinte de confinement d'un réacteur à eau bouillante. Dit également chambre sèche.

DS (Dryer Separator pit) : le bassin DS correspond à la piscine du sécheur-séparateur. Le sécheur et le séparateur se situent au-dessus du cœur et filtrent la vapeur qui s'en dégage avant qu'elle ne soit dirigée vers la turbine. Étant donné que ces dispositifs sont hautement contaminés par la radioactivité, ils doivent être stockés sous un important volume d'eau pendant les opérations qui nécessitent d'accéder au reste de la cuve. Pour cette raison, il existe une piscine située à côté du puits de cuve du réacteur, destinée à l'entreposage des composants internes de la cuve du réacteur, et souvent désignée comme la piscine du dryer separator (cf. la " piscine d'entreposage du dispositif de séchage et de séparation de vapeur » de la Fig. 5). Lors du fonctionnement du réacteur, cette piscine est séchée et couverte de briques en béton.

EAO : Enseignement Assisté par Ordinateur.

Eau borée : liquide de refroidissement à base d'eau et de bore, utilisé comme ralentisseur ou absorbeur de neutrons lents afin de contrôler la réaction en chaîne et éviter l'emballement du réacteur nucléaire. 
cuves de réacteurs. La centrale de Fukushima Daiichi dispose de grands réservoirs d'eau filtrée directement reliés aux différentes tranches. Les tuyauteries qui permettent cette relation ont cependant été rompues pendant le séisme. L'eau filtrée n'est donc pas facilement accessible pour être injectée durant la lutte contre l'accident.

ECCS (Emergency Core Cooling System): équipements d'un réacteur nucléaire (pompes, valves, réservoirs, échangeurs de chaleur et tuyauterie) spécifiquement conçus pour dissiper la chaleur résiduelle des combustibles en cas de défaillance du système normal de refroidissement du réacteur. du réacteur nucléaire s'échauffe puis commence à fondre. Il s'agit d'un accident très grave, puisqu'il peut potentiellement causer la dispersion de matières hautement radioactives si le cœur en fusion parvient à traverser le confinement.

Gal $\left(\mathrm{m}^{2} / \mathrm{s}\right)$ : unité d'accélération $\left(=0.01 \mathrm{~m} / \mathrm{s}^{2}\right)$, exprimant l'accélération de la pesanteur en gravimétrie et en géodésie.

Gamma (rayonnement) : rayonnement ionisant composé de photons de haute énergie. Bien qu'il soit moins ionisant, le rayonnement gamma $(\gamma)$ pénètre davantage dans l'organisme que les rayonnements alpha $(\alpha)$ et bêta $(\beta)$. Il est donc très dangereux et peut endommager la moelle osseuse et les organes internes.

77 Gaz noble : les gaz nobles, ou gaz rares, sont des éléments chimiques qui appartiennent au groupe 18 du tableau périodique des éléments. Ce groupe de gaz comprend l'hélium, le néon, l'argon, le krypton, le xénon, le radon.

78 Girafe : pompe à béton embarquée.

79 GM (Group Manager) : dans l'organisation de TEPCO, le GM est placé hiérarchiquement en-dessous du directeur de département.

HPCI (High Pressure Cooling Injection) system : le système d'injection d'eau à haute pression est un système de refroidissement de secours indépendant qui doit permettre de maintenir l'eau dans le réacteur à un niveau suffisant en cas de problème, sans requérir sa dépressurisation au préalable. L'eau injectée par le HPCI provient du 
réservoir tampon ou du wetwell. Il est actionné par une turbopompe alimentée par la vapeur de la cuve.

HPCSDG (High Pressure Core Spray System Diesel Generator) : système d'aspersion du cœur à haute pression. Il s'agit d'un ensemble de sauvegarde entièrement indépendant conçu pour le refroidissement d'urgence du réacteur. Ce système est alimenté par l'électricité externe mais possède des générateurs diesel de secours en cas de panne.

HVS (Hardened Venting System): système d'éventage en situation accidentelle composé de vannes et de soupapes résistantes pour dépressuriser l'enceinte de confinement.

IC (Isolation Condenser) : système de secours de certains réacteurs à eau bouillante. Il permet de refroidir le cœur lorsque l'évacuation de la puissance ne peut plus se faire par le condenseur principal. Ce système condense la vapeur d'eau produite dans un échangeur thermique, puis la réinjecte par gravité dans la cuve.

Iode : élément chimique qui appartient au groupe 17 (famille des halogènes) du tableau périodique des éléments.

Irradiation : exposition externe d'un corps ou d'une substance à des rayonnements ionisants.

Joint torique : un joint torique, ou un 0-Ring, est un joint d'étanchéité en forme de beignet fin, qui, lorsqu'il est compressé, permet d'éviter les fuites de fluides au niveau des liaisons entre deux conduits. Dans un REB, les mécanismes de déplacement des barres de contrôle se logent dans des tubes qui se trouvent en dessous de la cuve du réacteur. Ces tubes sont raccordés à la cuve.

LCO (Limiting Condition of Operation) : conditions limites d'exploitation sûre; elles désignent des situations où des systèmes de sûreté sont à leur niveau minimal de performance. Préalablement identifiées, elles sont mentionnées dans les Spécifications Techniques.

88 LPCI (Low Pressure Coolant Injection) system : système de refroidissement de secours permettant l'injection d'eau à basse pression en cas d'incident. Il s'agit d'un mode du système d'évacuation de la chaleur résiduelle RHR.

89 M/C (Metal-Clad Switch Gear) : appareillage blindé de commutation électrique. Les M/C sont des appareillages électriques (interrupteurs, fusibles, branchements, transformateurs de mesure, câblage de commande, etc.) installés en intérieur, dans des enveloppes métalliques séparées et adéquatement mises à la terre. Ils ont pour objectif de préserver les composants électriques d'agressions externes (éclairs, saleté, animaux...), ainsi que de protéger chaque armoire de l'autre, notamment en cas de défaillance ou d'incendie. Dans les centrales nucléaires, chaque système est opéré grâce à un switch gear, qui peut inclure une ou plusieurs armoires métalliques.

MCC (Motor Control Center) : centre de contrôle des moteurs.

MO (Motor Operated) valve : vanne activée par un moteur (électrique). Modérateur : matière composée d'atomes légers, permettant de ralentir les neutrons issus de la fission nucléaire afin de permettre leur absorption par les noyaux fissiles. MOX : combustible nucléaire à base d'un mélange d'oxydes d'uranium appauvri et de plutonium. 
MSIV (Main Steam Isolation Valve) : vanne d'isolement du circuit principal de vapeur En cas de SCRAM, ces vannes se ferment pour limiter les risques de perte de refroidissement ou de rejets radioactifs.

MUWC (Make-Up Water Condensate) system : système d'appoint en eau à partir d'un réservoir de condensation. En général, cette eau provient initialement du réacteur et est filtrée.

Neutrophage: substance caractérisée par une grande probabilité d'absorption des neutrons, comme l'acide borique.

Onahama Peil (OP) : désigne le niveau de construction du port d'Onahama, situé à 50 kilomètres au sud de Fukushima Daiichi. Lorsqu'ils apparaissent après le sigle "OP », les chiffres désignent le dépassement en mètres de ce niveau, pris comme référence.

P\&ID (Piping \& Instrumentation Diagram) : schéma tuyauterie et instrumentation - il décrit une unité de production avec tous les appareils et équipements, toute la tuyauterie et la robinetterie ainsi que toute l'instrumentation.

P/C (Power Center): les Power Centers sont des jeux de barres, c'est-à-dire des installations qui permettent de conduire l'électricité depuis une ligne d'alimentation, vers un ou plusieurs circuit(s) électrique(s).

Paging : système de radiomessagerie unidirectionnel ; le destinataire reçoit le message et le numéro d'envoi sur son terminal, mais ne peut pas y répondre.

Pascal (Pa) : le pascal et l'atmosphère normale sont deux unités de pression, telles que $1 \mathrm{~atm}=101325 \mathrm{~Pa}=1 \mathrm{~N} / \mathrm{m}^{2}$. L'atmosphère normale est égale à la pression d'une hauteur de mercure de $760 \mathrm{~mm}$ à $0^{\circ} \mathrm{C}$, à gravité normale. $1 \mathrm{MPa}=1000 \mathrm{kPa}=1000000 \mathrm{~Pa}$.

2 PHS (Personal Handy Phone System): le PHS est une norme de téléphonie mobile japonaise.

Pilote de tranche : opérateur chargé de la conduite d'un réacteur nucléaire.

Piscine d'entraînement: les centrales nucléaires disposent souvent de centres d'entraînement pour leurs employés. Certaines disposent même de piscines pour reproduire les piscines d'entreposage de combustible irradié.

Piscine de dépressurisation : voir chambre de condensation.

Piscine de désactivation du combustible usé: bassin rempli d'eau destiné à l'entreposage de combustible nucléaire usé, permettant la protection des personnes et l'évacuation de la chaleur.

7 PSA (Probabilistic Safety Assessment) : Études (ou évaluations) Probabilistes de Sûreté. Il s'agit d'une approche très répandue dans le domaine de l'industrie nucléaire, qui repose sur une conception déterministe et quantitative de la sûreté. Celle-ci doit permettre d'identifier les évènements susceptibles de menacer la sûreté, de calculer la probabilité de leur occurrence et de mesurer leurs conséquences.

Puissance résiduelle (chaleur résiduelle): puissance dégagée dans un réacteur nucléaire à l'arrêt ou dans un assemblage combustible irradié.

Radioactivité : émission d'une quantité d'énergie sous forme de rayonnements et/ou de particules accompagnant la désintégration d'un élément instable ou d'un noyau d'atome bombardé. 
110 Rayonnement ionisant : processus de transmission d'énergie sous forme d'ondes ou de particules capable d'arracher des électrons à des atomes ou des molécules. Ces derniers sont alors dits ionisés.

111 RCIC (Reactor Core Isolation Condenser): système comportant une turbopompe alimentée par la vapeur produite dans la cuve. Il permet d'alimenter la cuve du réacteur en eau à partir d'un réservoir ou du wetwell en cas de problème de refroidissement.

112 RCUW (Reactor Water Cleanup) system : système permettant de nettoyer les systèmes de refroidissement du réacteur de certains radionucléides. Il peut également servir d'appoint à ces systèmes de refroidissement.

113 RCW (Reactor building Cooling Water) System : système d'eau de refroidissement du bâtiment réacteur. Il s'agit de conduits qui fournissent de l'eau pour le refroidissement d'équipements auxiliaires du bâtiment réacteur en temps normal, ainsi que pour les systèmes importants pour la sûreté en cas de besoin.

114 Réacteur expérimental : installation nucléaire où une réaction en chaîne est créée et entretenue pour obtenir un flux de neutrons, afin de répondre à un usage d'expérimentation. Les premiers réacteurs expérimentaux sont construits aux ÉtatsUnis, en URSS, en France, en Grande-Bretagne et au Canada.

115 Réacteur nucléaire : dispositif permettant de produire de la chaleur à travers une réaction en chaîne contrôlée dans une cuve. Un fluide caloporteur évacue cette chaleur dans une turbine qui entraîne un alternateur produisant de l'électricité.

116 Réaction nucléaire: phénomène physique subatomique où des noyaux se transforment sous l'impulsion de particules ou de rayonnements. Cette transformation est accompagnée d'un dégagement d'énergie. Une réaction nucléaire peut être spontanée (naturelle) ou provoquée artificiellement, comme c'est le cas dans un réacteur nucléaire.

117 REB (Réacteur à eau bouillante) : réacteur nucléaire modéré et refroidi par de l'eau ordinaire, portée à ébullition dans le cœur.

118 Réseau d'aspersion de l'enceinte: série de pompes et de diffuseurs permettant d'arroser l'enceinte de confinement afin de faire baisser sa pression.

Reversing Valve Pit : fosse du robinet inverseur.

RHR (Residual Heat Removal) system : ensemble des systèmes d'évacuation de la chaleur résiduelle dans un réacteur nucléaire à l'arrêt.

Rotor : partie mobile rotative (par rapport à la partie immobile, nommée stator) de l'ensemble turbine-générateur. En cas de problème affectant la turbine (vibrations fortes, rotation trop rapide, manque de lubrification, etc.), cette dernière est arrêtée grâce aux vannes d'arrêt de la turbine. Ces vannes permettent de rediriger la vapeur directement vers le condensateur.

RPS (Reactor Protection System) : en cas de problème significatif sur un REB, ce dernier possède un système de protection qui déclenche une réponse automatisée pour protéger l'intégrité du réacteur: celle-ci consiste dans l'initiation du SCRAM et la dépressurisation de la cuve du réacteur (via les vannes SRV).

RPT (Recirculation Pump Trip): arrêt des pompes de recirculation. Lors du fonctionnement normal des REB, les pompes de recirculation permettent de contrôler 
la puissance du réacteur à travers le débit d'eau à l'intérieur de la cuve. En effet, à l'arrêt des pompes de recirculation, des bulles d'eau se forment dans le cœur du réacteur et affectent la densité du modérateur. Ainsi, l'arrêt de ces pompes suffit pour baisser la puissance du réacteur jusqu'à $30 \%$ de sa valeur nominale.

Salle RW (Radioactive Waste) : salle où sont entreposés les déchets radioactifs.

SBO (Station Black-Out): perte des alimentations électriques de la centrale nucléaire.

SC (Suppression Chamber) : voir chambre de condensation.

SCRAM : voir arrêt d'urgence. L'origine du terme n'est pas bien claire. La signification donnée varie entre Safety Control Rod Axe Man, Super-Critical Reactor Axe Man ou Start Cutting Right Away, Man. D'autres suggèrent encore le verbe " to scram ", soit déguerpir, en tant que réaction à avoir en cas de problème.

SGTS (Standby Gas Treatment System) : système auxiliaire de traitement de gaz, filtrant et déchargeant l'air de l'enceinte de confinement vers l'atmosphère. Il permet également de maintenir une pression basse dans l'enceinte de confinement afin de limiter les rejets radioactifs.

Sievert (Sv) : unité légale d'équivalent de dose qui permet de rendre compte de l'effet biologique produit par une dose absorbée donnée sur un organisme vivant. Le Sv/h (ou par seconde) correspond à la dose équivalente absorbée par cet organisme par unité de temps.

SLC (Standby Liquid Control) system : système auxiliaire de contrôle des liquides, capable d'injecter de l'eau borée dans un réacteur nucléaire à haute pression.

SOP (Severe Accident Operating Procedures) : procédures d'exploitation dans le cadre d'un accident sévère.

SPDS (Safety Parameter Display System) : système d'affichage des paramètres de sûreté.

SPEEDI (System for Prediction of Environmental Emergency Dose Information) : réseau de détecteurs et de calculateurs développé au Japon dans le but de prédire en temps réel les conséquences potentielles d'une urgence nucléaire sur l'environnement.

Spray Line : circuit d'aspersion, qui correspond aux tuyaux reliant la chambre de dépressurisation au réseau de buses d'arrosage.

Sprinklers : le sprinkler est un arroseur, utilisé notamment en cas d'incendie. Le bâtiment réacteur contient des sprinklers alimentés par des pompes, afin de permettre d'éteindre des incendies et limiter les dégâts. Le réseau de sprinklers n'atteint cependant pas l'enceinte de confinement du réacteur, qui est généralement inerte et contient peu de matériels inflammables.

SRV (Safety Relief Valve): système de dépressurisation de la cuve. Les soupapes s'ouvrent à haute pression et déversent la vapeur dans le drywell ou l'acheminent vers le wetwell où elle est condensée.

Stack Monitor: système de contrôle des cheminées. Il s'agit d'une station d'observation qui permet de mesurer la direction et la vitesse du vent, les précipitations, etc. En situation d'urgence, ces mesures permettent de déterminer le meilleur moment de réaliser un éventage, en fonction de l'impact potentiel des rejets radioactifs.

Suppression Chamber Spray : mode d'aspersion de la chambre de condensation. 
Suppression pool : voir chambre de condensation.

Surgénérateur : réacteur nucléaire pouvant produire plus de matière fissile qu'il n'en consomme.

Syndrome chinois : expression utilisée pour la première fois en 1971 par Ralph Lapp (1971, p. 11). Elle renvoie à l'hypothèse selon laquelle les éléments en fusion d'un réacteur nucléaire situé en Amérique du Nord pourraient s'enfoncer dans la terre jusqu'en Chine. Elle est popularisée par Le syndrome chinois (The China Syndrome), réalisé en 1979 par James Bridges. Il s'agit du film le plus célèbre sur l'industrie nucléaire. Une journaliste (Jane Fonda) et son cadreur (Michael Douglas) réalisent un reportage dans la centrale de Ventana aux États-Unis. Une secousse sismique cause un incident, filmé clandestinement par le cadreur. La fusion du cœur du réacteur est évitée, mais l'exploitant refuse de vérifier l'état de l'installation avant sa remise en service. Douze jours après la sortie du Syndrome chinois, un accident se produit dans la centrale nucléaire de Three Mile Island (États-Unis, Pennsylvanie). Cette coïncidence contribue largement au succès du film.

TAF (Top of Active Fuel) : le haut du combustible est la référence pour la mesure du niveau d'eau dans la cuve du réacteur. La valeur qui le suit est toujours donnée au millimètre.

Tore : chambre de condensation, ou wetwell dans les REB qui possèdent une enceinte de confinement de type Mark I (tranches 1 à 5 de Fukushima Daiichi).

Tranche nucléaire : unité de production électrique comportant une chaudière et un groupe turbo-alternateur. Elle se caractérise par le type du réacteur et la puissance du groupe turbo-alternateur.

Turbine principale : la turbine principale désigne la grande turbine qui entraîne le générateur pour produire de l'électricité. Hormis celle-ci, chaque tranche possède des turbopompes dans le bâtiment turbine. Celles-ci sont constituées de pompes entraînées par de petites turbines. Leur rôle est de pomper la vapeur condensée du bâtiment turbine et de la déverser dans le réacteur. À Fukushima Daiichi, ces turbopompes ont été arrêtées au moment du SCRAM.

46 Tyvek: tenue de protection pour les agents. Le Tyvek est une marque déposée de l'entreprise américaine du Pont de Nemours.

VHF : une radio VHF (Very High Frequency) émet sur une bande à très hautes fréquences, s'étendant de $30 \mathrm{MHz}$ à $300 \mathrm{MHz}$.

8 Vibration axiale: les trop fortes vibrations font partie des signaux qui peuvent déclencher l'arrêt automatique de la turbine pour éviter sa détérioration. En particulier, la vibration axiale désigne le déplacement de l'arbre du rotor par rapport à l'horizontale.

Wetwell : voir chambre de condensation. 


\section{Glossaire des lieux, évènements et organisations}

1 1F : voir Fukushima Daiichi.

2 2F : voir Fukushima Daini.

3 Abukumagawa : fleuve d'une longueur de 239 kilomètres, qui traverse les préfectures de Fukushima et de Miyagi.

4 Agence de la sécurité maritime (Maritime Safety Agency) : fondée au Japon en 1948, elle devient the Japan Coast Guard (JCG) en avril 2000. Cette organisation civile a pour mission d'assurer la sécurité et la sûreté en mer (application du droit maritime et sécurité nationale, recherche, sauvetage et intervention en cas de catastrophe, levés hydrographiques, gestion du trafic maritime).

5 Agence Internationale de l'Énergie Atomique (AIEA) : fondée en juillet 1957, elle a pour objectif de promouvoir des technologies nucléaires sûres, sécurisées et pacifiques. Son siège est situé à Vienne (Autriche). L'AIEA possède également deux bureaux régionaux (Toronto, Tôkyô) et deux bureaux de liaison (New York, Genève), et administre des laboratoires spécialisés dans le nucléaire (Vienne et Monaco).

6 Agence Météorologique du Japon : fondée en 1875, elle est chargée de relever les données terrestres et maritimes du temps, de lancer et d'exploiter des satellites météorologiques, ainsi que de détecter les séismes dans le Pacifique ouest. Son siège est à Tôkyô.

7 Agence nationale de pompiers (National Fire Agency) : agence placée sous la tutelle du ministère de l'Intérieur japonais. Elle est fondée le $1^{\mathrm{er}}$ mars 1995 afin d'améliorer la lutte contre les incendies, l'efficacité des secours et des services médicaux en cas de catastrophe.

8 Agence pour les ressources naturelles et l'énergie (Agency for Natural Resources and Energy) : agence fondée en 1973, placée sous la tutelle du Ministère de l'Économie, du Commerce et de l'Industrie (METI). Sa mission est de définir les politiques énergétiques et de gestion des ressources naturelles du Japon.

9 Bâtiment antisismique de Fukushima Daiichi : mis en service en juillet 2010 à Fukushima Daiichi, sa conception a tiré profit du retour d'expérience de l'incident de 
Kashiwazaki-Kariwa (16 juillet 2007). Le bâtiment est équipé d'une alimentation électrique de secours, de dispositifs de ventilation filtrée et d'un blindage assurant une protection contre la radioactivité.

10 Browns Ferry : centrale nucléaire située aux États-Unis, en Alabama, et exploitée par l'entreprise américaine Tenessee Valley Authority. Elle se compose de trois REB, construits par GE. Le réacteur Browns Ferry-1 est mis en service en 1973, Browns Ferry-2 en 1974 et Browns Ferry 3 en 1976. La centrale est connue pour avoir subi un grave incendie le 22 mars 1975.

11 Cellule conjointe de gestion de crise entre le Gouvernement et TEPCO : cellule créée par Naoto Kan le 15 mars 2011, remplaçant la cellule de crise du Kantei et la cellule de crise de TEPCO. Située au siège de TEPCO, elle est chargée d'assurer une intervention intégrée entre l'organisme public d'intervention et l'exploitant, et de veiller à ce que les informations soient communiquées en temps voulu à l'échelon national. Kan est le Haut Responsable de la cellule conjointe et ses adjoints sont Shimizu et Kaieda. Hosono en est le Secrétaire général. La première réunion de la cellule a lieu le 15 mars, à $4 \mathrm{~h} 30 \mathrm{du}$ matin. En anglais, cette cellule conjointe est dénommée «Integrated Headquarter for Response to the Incident at the Fukushima NPS ».

12 Cellule de gestion de crise de Fukushima Daiichi : une cellule de crise est formée à Fukushima Daiichi juste après le séisme du Tôhoku. Elle est dirigée par Masao Yoshida. La salle où se réunie la cellule de crise se situe dans le bâtiment antisismique de Daiichi. Cette cellule se compose de douze groupes d'opération d'urgence (groupe de surveillance, de communication publique, d'annonce, d'informations, de réhabilitation, de production électrique, de technique, de maintenance, d'aide médicale, de bien-être, d'affaires générales, de ressources), situés autour de la table où sont assis Yoshida, Fukura et Yoshizawa (les vice-directeurs de la centrale), ainsi que les chefs des douze groupes. En anglais, cette cellule de crise est dénommée «ERC » (« Fukushima Daiichi Emergency Response Center »).

13 Cellule de gestion de crise de TEPCO : cellule de gestion de crise située au siège social de TEPCO, à Tôkyô, et créée le 11 mars 2011 à 15h06. Elle est composée de neuf équipes d'opération d'urgence et regroupe jusqu'à deux cents personnes. Elle est dirigée par Masataka Shimizu, le président directeur général de TEPCO. À partir du 15 mars, elle est remplacée par la Cellule conjointe de gestion de crise entre le Gouvernement et TEPCO.

14 Cellule de gestion de crise du Gouvernement : le 11 mars 2011, à 15h14, Naoto Kan établit le centre d'intervention d'urgence pour les catastrophes au Kantei. À 16h36, le Gouvernement crée le Bureau d'intervention d'urgence pour l'accident nucléaire. À 19h03, l'état d'urgence nucléaire est décrété et le Centre de conduite de l'intervention en cas d'urgence nucléaire est créé. En anglais, cette cellule est dénommée "NERHQ » (Nuclear Emergency Response Headquarters). À partir du 15 mars, le NERHQ est remplacé par la Cellule conjointe de gestion de crise entre le Gouvernement et TEPCO.

15 Cellule de soutien de Fukushima : bureau de TEPCO situé dans la ville de Fukushima, au siège préfectoral de réponse à la catastrophe. Cette cellule, reliée au réseau de téléconférence de l'exploitant, a pour mission de fournir des informations sur l'état des 
centrales nucléaires à la préfecture, qui se charge ensuite d'organiser des points de presse.

16 Centre de crise hors site (ou Off-site Center) : centre de crise situé à ôkuma à environ 5 kilomètres de Fukushima Daiichi, où sont installées la cellule du Gouvernement (dirigée par Motohisa Ikeda, vice-ministre du METI) dénommée en anglais «Local Nuclear Emergency Response Headquarters » (NERHQ local), la cellule de TEPCO (dirigée par Sakae Mutô, vice-président de TEPCO), et la cellule de la préfecture (dirigée par Masao Uchibori, vice-gouverneur de la préfecture de Fukushima). Ce centre est notamment chargé de la gestion de l'évacuation de la population. À cause de l'augmentation de la radioactivité à ôkuma, le centre hors site est évacué le 15 mars 2011. Il est transféré dans la salle publique de la préfecture de Fukushima.

17 Centre de distribution d'Onahama : dans la nuit du 12 mars 2011, TEPCO décide d'utiliser le Coal Center d'Onahama, une filiale de l'exploitant, comme base locale de distribution. Ce centre est situé à moins d'une cinquantaine de kilomètres de Fukushima Daiichi et à une trentaine de kilomètres du J-Village. Il est exploité par une équipe de douze contractuels, chargés notamment de transporter de l'essence, du matériel de contrôle des rayonnements, des générateurs, des pompes ou des batteries à destination de Daiichi.

Chûetsu : nom d'une région de la préfecture de Niigata.

Coal Center d'Onahama : voir centre de distribution d'Onahama.

Côte Sanriku : située au nord-est de l'île de Honshû, elle traverse les préfectures de Miyagi, d'Iwate et d'Aomari.

21 Diète : Parlement japonais. Créée par la Constitution Meiji de 1889, la Diète est composée de la Chambre des représentants (480 membres) et de la Chambre des conseillers (242 membres). Les deux chambres se réunissent dans le Bâtiment de la Diète nationale à Tôkyô.

Electric Technology Research Association (ETRA) : l'Association de Recherche en Technologie Électrique est fondée à Tokyo en 1933. Elle se compose de neuf comités spéciaux. L'objectif de cette association est de mener des études et des recherches concernant les questions électrotechniques. Ses résultats sont mis à la disposition de ses membres. La coordination et la présidence de l'ETRA sont principalement assurées par l'Université de Tôkyô et TEPCO.

Faille de Futaba: la centrale nucléaire de Fukushima Daiichi est localisée à 8 kilomètres de la faille de Futaba, d'une longueur d'environ 80 kilomètres. Les montagnes d'Abukuma, situées à l'ouest de la faille, sont composées de roches plutoniques comme le granite. La partie est de la faille est composée de roches sédimentaires. Fukui (université de) : université nationale située dans la ville de Fukui (préfecture de Fukui, région de Chûbu). Elle est fondée en 1949.

24 Federation of Electric Power Companies of Japan (FEPC): Fédération des compagnies d'électricité du Japon. Cette fédération des dix fournisseurs d'électricité du Japon a été fondée en 1952 pour faciliter leur communication et leur coopération. Elle regroupe Hokkaido Electric Power Co., Hokuriku Electric Power Co., Chubu Electric Power Co., The Chugoku Electric Power Co., Okinawa Electric Power Co., Kyushu Electric Power Co., Shikoku Electric Power Co., Kansai Electric Power Co., Tohokû Electric Power Co. et TEPCO. 

1954, les forces japonaises d'autodéfense constituent l'armée du Japon. Leur statut est déterminé par l'article 9 de la Constitution, votée en 1946 alors que le pays est occupé par les États-Unis, vainqueurs de la Guerre du Pacifique. Cet article stipule que le peuple japonais renonce à la guerre, à la menace ou à l'usage de la force comme moyen de règlement des conflits internationaux. Les lois sur la paix et la sécurité, adoptées par la Diète en septembre 2015, permettent désormais aux forces d'autodéfense d'intervenir militairement pour aider un pays allié dans un conflit, sans que le territoire japonais ne soit directement menacé.

Fukushima (ville de) : capitale de la préfecture de Fukushima. La ville est située à un peu moins de cent kilomètres de Fukushima Daiichi.

Fukushima Daiichi (1F) : centrale nucléaire située dans la préfecture de Fukushima, entre les communes de Futaba et d'ôkuma, à environ 250 kilomètres au nord de Tôkyô. Elle est exploitée par TEPCO. La construction du premier Réacteur à Eau Bouillante (REB) de la centrale est supervisée par la firme américaine General Electric (GE). Il est conçu à partir des plans du modèle BWR/3 et il est de confinement Mark I. Il est mis en service en mars 1971 et sa puissance brute est 460 MWe. Différentes entreprises construisent les réacteurs suivants: GE pour le réacteur 2, Tôshiba pour le 3 et le 5, Hitachi pour le 4. Les réacteurs 2 à 5 sont de type BWR/4 et de confinement Mark I. Le réacteur 2 est mis en service en juillet 1974, le 3 en mars 1976, le 4 en octobre 1978, le 5 en avril 1978. Ces réacteurs affichent chacun une puissance brute de $784 \mathrm{MWe}$. Le réacteur 6 est quant à lui construit par GE-Tôshiba. Il est de type BWR/5 et de confinement Mark II. Il est mis en service en octobre 1979 et sa puissance brute est de 1 100 MWe. Ces six réacteurs sont répartis face à l'océan en deux ensembles, séparés de quelques centaines de mètres : d'un côté les réacteurs 1 à 4 , de l'autre le 5 et le 6 . Le site occupe une surface d'environ $3,5 \mathrm{~km}^{2}$ et s'élève entre 6 et 10 mètres au-dessus du niveau de la mer. Au moment du séisme du 11 mars 2011, environ 6400 personnes travaillent sur le site de Fukushima Daiichi. La centrale nucléaire est à l'arrêt depuis le 11 mars 2011.

Fukushima Daini (2F) : centrale nucléaire exploitée par TEPCO, située dans la préfecture de Fukushima, à une douzaine de kilomètres de Fukushima Daiichi. Ses quatre REB ont été mis en service entre 1982 et 1987. Fukushima Daini est dirigée par Naohiro Masuda lorsque survient le séisme du Tôhoku le 11 mars 2011. Fukushima Daini est à l'arrêt depuis cette date.

Fukushima (préfecture de) : préfecture située dans la région du Tôhoku. Sa capitale est la ville de Fukushima.

30 Fukushima 50 : le 15 mars 2011, Yoshida s'adresse aux chefs des douze groupes de la cellule de crise de Fukushima Daiichi et leur demande de procéder à la sélection des opérateurs qui doivent rester sur le site. Ces travailleurs sont appelés par les médias étrangers les «Fukushima 50 » - en réalité, ils ne sont pas cinquante mais soixanteneuf. Le reste du personnel se met à l'abri en se rendant à Fukushima Daini. Dans les jours suivants, les Fukushima 50 sont rejoints par des opérateurs supplémentaires.

31 General Electric (GE) : entreprise fondée en 1892 à la suite de la fusion d'Edison General Electric Company et de Thomson-Houston Electric Company. Elle a supervisé la construction des réacteurs 1 et 2 de Fukushima Daiichi et co-construit le réacteur 6 avec l'entreprise Tôshiba. 

l'étude et de la cartographie du territoire japonais. Elle remonte historiquement à 1869. Elle est réorganisée à plusieurs reprises et obtient son nom actuel en avril 2010. La GSI dépend du ministère du Territoire, des Infrastructures, des Transports et du Tourisme. Son siège est situé à Tsukuba (préfecture d'Ibaraki).

azama Corporation : entreprise japonaise de construction créée en 1889, dont le siège est situé à Tôkyô. C'est l'une des dix plus grandes sociétés de construction du Japon.

$7,2)$, survenu 17 janvi 7,2), survenu le 17 janvier 1995, fait 6434 personnes et détruit plus de 100000 bâtiments. Il met en lumière un certain nombre de problèmes concernant les mesures nationales de prévention des catastrophes. En réponse, la loi sur les mesures spéciales de prévention des catastrophes sismiques est promulguée en juillet 1995. Conformément à cette loi, sont fondés les Headquarters for Earthquake Research Promotion, une organisation gouvernementale spéciale d'abord rattachée au Cabinet du Premier Ministre, puis au ministère de l'Éducation, de la Culture, des Sports, des Sciences et de la Technologie. Sa mission est de promouvoir la recherche sur les tremblements de terre, afin de renforcer les mesures de prévention et réduire les dommages causés par ce type d'évènement.

Higashidôri-TEPCO : en janvier 2011, TEPCO débute la construction d'une centrale nucléaire à Higashidôri dans la préfecture d'Aomori (Tôhoku). Le chantier est situé près d'une centrale voisine, portant le même nom et exploitée par la Tôhoku Electric Power Company. La centrale de TEPCO devait être composée de deux réacteurs nucléaires. Le $\mathrm{n}^{\circ} 1$ devait initialement démarrer en mars 2017, mais l'accident de Fukushima Daiichi contraint TEPCO à arrêter les travaux et suspendre le projet. L'exploitant le relance en juillet 2018. Mais du fait des conséquences financières de l'accident de Fukushima Daiichi, TEPCO ne peut plus mener seul la construction de la centrale. Il envisage donc de partager les coûts de construction avec d'autres entreprises japonaises, telles que la Tôhoku Electric Power Company, la Chubu Electric Power Company et la Japan Atomic Power Company. La décision concernant cette éventuelle collaboration ne sera prise qu'après la publication d'une série d'études sur le risque sismique auquel le site de construction est exposé.

Hirono : centrale thermique au fioul exploitée par TEPCO, située à une trentaine de kilomètres de Fukushima Daiichi.

Hitachi : entreprise japonaise fondée en 1910, dont le siège est situé à Chiyoda-ku (Tôkyô). Elle propose des produits et des services dans les domaines de l'équipement médical, scientifique et de laboratoire, l'informatique et les télécommunications, les machines de construction, les matériaux et composants hautement fonctionnels, les produits d'affichage, les services financiers, les systèmes d'infrastructures, les systèmes industriels. Hitachi a supervisé la construction du réacteur 4 de Fukushima Daiichi.

Honshû : plus grande île du Japon, regroupant vingt-six préfectures, dont celle de Fukushima.

Hôpital TEPCO : le Tôkyô Denryoku Hospital est un hôpital appartenant à TEPCO et situé dans le quartier Shinjuku de Tôkyô. Cet établissement de sept étages, construit en 1979 et d'une capacité de 113 lits, est utilisé exclusivement pour soigner les employés 
de l'exploitant et les membres de leur famille. En juillet 2012, TEPCO annonce la mise en vente de l'hôpital. Il a été vendu en janvier 2014. les bases permettant d'assurer la sûreté nucléaire en menant des analyses de sûreté et en effectuant l'inspection des installations nucléaires au Japon. Cette organisation est créée en 2003. d'équipements de protection; conception, construction et inspection de maintenance des systèmes et des équipements de protection; prêt et vente de systèmes et 
d'équipements de protection; surveillance des installations; sécurité des transports liés au combustible nucléaire ; éducation et formation du personnel de protection).

51 Japan Society of Civil Engineers (JSCE): Société japonaise des ingénieurs civils, fondée en 1914. Elle est chargée de contribuer à l'avancement de la culture scientifique en promouvant le domaine du génie civil et l'expansion de ses activités. Au fil des années, les membres de la JSCE ont considérablement augmenté, passant des 443 membres initiaux à environ 39000 membres actuellement.

J-Village (Japan Football Association's National Training Center) : centre de formation de footballeurs situé à une vingtaine de kilomètres au sud de Fukushima Daiichi, reconverti en base opérationnelle pour lutter contre l'accident nucléaire.

Kantei : complexe immobilier situé dans le quartier administratif de Nagata à Tôkyô. Le Kantei abrite les bureaux et la résidence du Premier ministre du Japon.

54 Kantô : région située sur l'île de Honshû au sud du Tôhoku. Elle regroupe les préfectures de Chiba, Gunma, Ibaraki, Kanagawa, Saitama, Tochigi et Tôkyô.

Kashiwazaki-Kariwa (accident de) : le 16 juillet 2007 a lieu le séisme de Niigataken Chûetsu-Oki, dont l'épicentre est situé à environ 10 kilomètres de la centrale nucléaire de Kashiwazaki-Kariwa, exploitée par TEPCO. Les trois réacteurs en fonctionnement et un réacteur en cours de démarrage sont mis à l'arrêt automatiquement durant le séisme (les trois autres réacteurs étaient à l'arrêt pour inspection). Un incendie se déclare sur un transformateur électrique à l'extérieur du bâtiment du réacteur 3 , une fuite d'eau est constatée sur le réacteur 6 , des centaines de fûts contenant des déchets de faible activité sont renversés et certains perdent leur couvercle. De la matière radioactive se disperse dans l'atmosphère et dans la mer du Japon.

56 Kashiwazaki-Kariwa : centrale nucléaire exploitée par TEPCO, située entre les communes de Kashiwasaki et de Kariwa, dans la préfecture de Niigata. Elle regroupe sept réacteurs nucléaires et représente l'unité de production électronucléaire la plus importante au monde (environ $8200 \mathrm{MWe}$ ). La centrale est à l'arrêt depuis le séisme et le tsunami du 11 mars 2011.

57 Kôriyama: ville de la préfecture de Fukushima, située à une quarantaine de kilomètres d'ôno et à environ soixante-dix kilomètres de Fukushima Daiichi.

58 Kyushu Electric Power : compagnie d'électricité japonaise, dont le siège se situe à Fukuoka (préfecture de Fukuoka). Créée en 1951, elle exploite de nombreuses centrales de production électrique, dont la centrale nucléaire de Genkai (préfecture de Saga, située sur l'̂lle de Kyûshû). Les quatre réacteurs à eau pressurisée de Genkai ont été mis en service entre 1975 et 1997.

Metropolitan Police Department (MPD) : Département de la Police Métropolitaine de Tôkyô (Keishichô en japonais). Créé en janvier 1874, le MPD est dirigé par un surintendant-général, nommé par la Commission Nationale de la Sécurité Publique, avec l'approbation de la Commission Métropolitaine de Sécurité Publique et l'approbation du Premier ministre.

60 Minami-Yokohama : centrale thermique exploitée par TEPCO à Yokohama, dans la préfecture de Kanagawa (région du Kantô). 
61 Ministry of Economy, Trade and Industry (METI) : ministère de l'Économie, du Commerce et de l'Industrie japonais. Il est institué en 2001, en replacement du MITI (Ministry of International Trade and Industry) qui avait été fondé en 1949. Elle partage le site qu'elle occupe avec la centrale nucléaire de Fitzpatrick, alors exploitée par Entergy. Ces deux centrales sont désormais exploitées par Exelon (Nine Mile Point depuis 2012 et Fitzpatrick depuis 2017). Nine Mile Point se compose de deux REB, construits par GE. Le réacteur 1 est mis en service en 1969 et le 2 en 1987. Le réacteur 1 est le plus ancien qui soit encore en service aux États-Unis.

70 Nippon Hôsô Kyôkai (NHK) : entreprise qui regroupe les stations de radio et de télévision du service public japonais. Sa première station de radio est lancée en 1925 et sa première station de télévision en 1953.

71 Nuclear and Industrial Safety Agency (NISA): agence de sûreté nucléaire et industrielle créée au Japon en 2001. Cette agence est chargée de superviser le respect de la réglementation dans le domaine de la sûreté nucléaire. Elle est placée sous la tutelle du METI. En septembre 2012, elle fusionne avec la Nuclear Safety Commission (NSC) pour devenir la Nuclear Safety Commission (NRA).

72 Nuclear Regulation Authority (NRA) : Autorité de Règlement Nucléaire du Japon, créée en septembre 2012. Elle résulte de la fusion de la NSC et de la NISA. La NRA est placée sous la tutelle du ministère de l'Environnement.

73 Nuclear Safety Commission (NSC) : commission de sûreté nucléaire fondée en 1978 à la suite d'une réforme de la législation sur le nucléaire civil, comme autorité indépendante de surveillance et de vérification des inspections menées par les agences de régulation telle la NISA. Elle est placée sous la tutelle du Cabinet du Premier 
ministre. En mars 2011, la NSC est présidée par Haruki Madarame. Elle fusionne en 2012 avec la NISA pour devenir l'Autorité de Réglementation Nucléaire (NRA).

Tôhoku), exploitée par la Tôhoku Electric Power Company. Elle est composée de trois REB, mis en service entre 1984 et 2002. Il s'agit de la centrale la plus proche (80 kilomètres) de l'épicentre du séisme du 11 mars 2011. Le site est submergé par des vagues de 13 mètres de hauteur, mais les principaux bâtiments, construits à 14,8 mètres au-dessus de la mer, ne sont pas inondés. Si le tsunami endommage malgré tout de nombreux équipements, une ligne électrique et six générateurs diesel continuent de fonctionner. Les opérateurs ont donc pu garder le contrôle des trois réacteurs et les arrêter à froid.

Ordres d'évacuation de la population : le 11 mars 2011, à 20h50, la préfecture de Fukushima donne l'ordre aux habitants situés dans un périmètre de 2 kilomètres autour de Fukushima Daiichi d'évacuer la zone. Le Gouvernement donne ensuite six ordres d'évacuation des populations, élargissant le périmètre selon l'évolution des évènements survenus à Fukushima Daiichi et Daini. Le premier ordre du Gouvernement est donné le 11 mars à $21 \mathrm{~h} 23$ (évacuation de la zone autour de Daiichi à 3 kilomètres), le deuxième est donné le 12 mars à $4 \mathrm{~h} 45$ (élargissement de la zone autour de Daiichi à 10 kilomètres), le troisième à $7 \mathrm{~h} 45$ (évacuation de la zone autour de Daini à 3 kilomètres), le quatrième à $17 \mathrm{~h} 39$ (élargissement de la zone autour de Daini à 10 kilomètres), le cinquième à $18 \mathrm{~h} 25$ (élargissement de la zone autour de Daiichi à 20 kilomètres), le sixième le 15 mars à 11 h00 (élargissement de la zone autour de Daiichi à 30 kilomètres). Environ 150000 personnes sont évacuées. Durant les premiers jours de l'accident, environ 78000 personnes vivant dans un rayon de 20 kilomètres autour de la centrale et 62000 personnes vivant entre 20 et 30 kilomètres sont évacuées. En avril 2011, le Gouvernement recommande l'évacuation d'environ 10000 personnes supplémentaire vivant plus au nord-ouest de Fukushima Daiichi.

79 Péninsule de Bôsô : péninsule englobant la préfecture de Chiba et formant le bord oriental de la baie de Tôkyô.

Power reactor and Nuclear fuel development Corporation (PNC) : société pour le développement des réacteurs de puissance et des combustibles nucléaires au Japon, fondée en octobre 1967. La PNC est dissoute en octobre 1998 et devient la Japan Nuclear Cycle Development Institute (JNC).

81 Préfecture : le Japon est composé de quarante-sept préfectures, circonscriptions administratives et territoriales créées en 1871. Résidence du Premier ministre : voir Kantei.

Sakashita : barrage construit en 1973 dans la préfecture de Fukushima. Il est utilisé pour la production hydroélectrique et l'alimentation en eau de la région. D'une hauteur 
de 43 mètres et d'une longueur de crête de 231 mètres, son volume de retenue est de $2840000 \mathrm{~m}^{3}$.

Santa Maria de Garona: centrale nucléaire située dans la province de Burgos (Espagne, Castille-et-Leon) et mise en service en 1971. Elle se compose d'un seul REB, de conception GE. Ce réacteur nucléaire est arrêté en décembre 2012.

Séisme de l'ère Jôgan: le 13 juillet 869, durant l'ère Jôgan (859-877), un séisme dévastateur se produit au large de l'actuelle préfecture d'Iwate. Il provoque un tsunami détruisant la ville de Tagajo (dans l'actuelle préfecture de Miyagi). La catastrophe fait un millier de morts.

Séisme de la côte Pacifique du Tôhoku : le séisme de la côte Pacifique du Tôhoku se produit le 11 mars 2011, à $14 \mathrm{~h} 46$ (heure locale). Son épicentre se situe dans l'océan Pacifique à 370 kilomètres au nord nord-est de Tôkyô. La durée de la secousse enregistrée à Tôkyô est de plus de deux minutes. La puissance du tremblement de terre provoque un tsunami dévastateur. Le bilan des victimes de la catastrophe s'élève à 15897 morts, 2534 disparus et 6152 blessés. Parmi les séismes les plus importants recensés dans le monde depuis le XVIII ${ }^{e}$ siècle, le séisme du Tôhoku (magnitude 9) se situe au quatrième rang, après celui du 22 mai 1960 de Valdivia au Chili $(9,5)$, du 27 mars 1964 en Alaska $(9,2)$, et celui du 26 décembre 2004 à Sumatra $(9,1)$.

Séisme de Niigataken Chûetsu-Oki : le 16 juillet 2007, à 10 h13 (heure locale), un séisme de magnitude 6,6 se produit dans la préfecture de Niigata (région du Chûbu). Il fait 11 morts et plus de 1000 blessés, tandis que 25000 foyers sont privés d'électricité.

Séisme de Sanriku : ce séisme (magnitude 8,5) a lieu le 15 juin 1896 à 19 h32 (heure locale) durant l'ère Meiji (1868-1912). Son épicentre se situe à environ 166 kilomètres au large de la côte de la préfecture d'Iwate. Le tremblement de terre provoque deux tsunamis, qui causent au moins 22000 morts et détruit environ 9000 maisons.

Séisme de Valdivia : le 22 mai 1960, un séisme de magnitude 9,5 - la plus élevée à avoir été enregistrée - se produit au Chili. Son épicentre se situe dans le sud du pays, près de la ville de Valdivia, à environ 700 kilomètres de Santiago du Chili. Selon l'Institut d'Études Géologiques des États-Unis (United States Geological Survey, USGS), le tremblement de terre cause la mort d'environ 1655 personnes, et le tsunami qu'il provoque fait 61 victimes à Hawaï, 138 au Japon et 32 aux Philippines.

90 Séisme du Chili de 2010 : le 27 février 2010, un séisme de magnitude 8,8 se produit au Chili. Son épicentre se situe dans l'océan Pacifique, à plus de 6 kilomètres au large des côtes et à 325 kilomètres au sud-ouest de Santiago du Chili. Un tsunami se forme à la suite du tremblement de terre. La catastrophe fait 525 morts et disparus.

Shimamura : marque japonaise de prêt-à-porter à prix réduit.

Shin-Fukushima : la station de transformation de Shin-Fukushima est localisée dans le bourg de Tomioka, situé à environ 8 kilomètres de Fukushima Daiichi. La centrale nucléaire disposait d'une source d'alimentation électrique externe, fournie par la Tôhoku Electric Power Company et acheminée par une ligne de $66 \mathrm{kV}$ depuis la station de Shin-Fukushima. Le séisme du 11 mars 2011 a toutefois coupé cette ligne et Fukushima Daiichi a ainsi perdu cette source d'alimentation externe avant même la survenue du tsunami.

Sôma : ville située dans la préfecture de Fukushima à une cinquantaine de kilomètres de Fukushima Daiichi. 

puissance de 357 MWe (constructeur GE), est mis en service en mars 1970. Il est en démantèlement depuis 2015. Tsuruga 2 , réacteur à eau pressurisée d'une puissance de 1160 MWe (constructeur Mitsubishi), est mis en service en février 1987. Il est à l'arrêt depuis le 11 mars 2011.

Tchernobyl (accident de) : le réacteur $\mathrm{n}^{\circ} 4$ de la centrale nucléaire de Tchernobyl (Union soviétique, Ukraine) est détruit le 26 avril 1986, à 1h23, à la suite d'un essai technique durant lequel les opérateurs transgressent les procédures de sûreté. L'explosion du réacteur entraîne l'émission dans l'atmosphère d'une grande quantité de produits radioactifs qui contaminent de vastes territoires en Europe. L'accident de Tchernobyl est classé au niveau 7 sur l'échelle INES. Il s'agit du niveau le plus élevé, qui correspond à un accident entraînant des rejets majeurs dont les effets sont considérables sur la santé et l'environnement.

Tochigi : préfecture située dans la région du Kantô.

Tôhoku Electric Power Company : compagnie d'électricité japonaise, dont le siège social se situe à Sendai (préfecture de Miyagi). Créée en 1951, elle exploite deux-centsneufs centrales hydroélectriques, douze centrales thermiques, quatre centrales géothermiques, quatre centrales solaires, et deux centrales nucléaires (centrale d'Onagawa mise en service en 1984, et centrale d'Higashidôri mise en service en 2005).

Tôhoku : région située au nord-est de l'île de Honshū. Elle regroupe les préfectures d'Akita, d'Aomori, d'Iwate, de Miyagi, d'Yamagata et de Fukushima.

Tôkai : région de l'île de Honshû, située entre le Kansai et le Kantô. Elle est composée des préfectures de Mie, Gifu, Shizuoka et Aichi.

Tôkai-mura : le 30 septembre 1999, un accident de criticité survient dans l'usine de fabrication de combustibles nucléaires de la Japan Nuclear Fuels Conversion Company (JCO), située dans le complexe de Tôkai-mura, situé dans la préfecture d'Ibaraki. Ce complexe regroupe une centrale, une usine de traitement de combustible usagé, un centre de recherche, un accélérateur de particules et plusieurs usines). L'accident provoque la mort de deux employés. Premier accident important dans l'histoire du nucléaire civil au Japon, il a été classé au niveau 4 sur l'échelle INES.

Tôkyô Denryoku Hospital : voir hôpital TEPCO.

Tôkyô Electric Power Company (TEPCO) : la compagnie d'électricité de Tôkyô est fondée en 1951. Son siège social est à Tôkyô. L'entreprise exploite 17 réacteurs nucléaires, répartis sur les centrales nucléaires de Fukushima Daiichi (6), de Fukushima Daini (4) et de Kashiwazaki-Kariwa (7). Elle exploite également centsoixante installations hydrauliques et vingt-cinq centrales thermiques.

Tôkyô Records Management Corporation: filiale de TEPCO, spécialisée dans la production, le stockage et la gestion de documents d'archives.

Tôshiba : entreprise japonaise fondée en 1875 , dont le siège est situé à Tôkyô. Ses activités couvrent le secteur de l'électronique, de la téléphonie, de l'informatique, de l'audiovisuel et du nucléaire. Tôshiba a supervisé la construction des réacteurs 3 et 5 de Fukushima Daiichi, et a co-construit le réacteur 6 avec GE.

Tsuruga : centrale nucléaire située dans la préfecture de Fukui, dans la région de Chûbu. Elle est exploitée par la Japan Atomic Power Company. Tsuruga 1, REB d'une 
105 Université de Kyoto : université japonaise fondée en 1897 et située dans la préfecture de Kyoto. L'Université de Kyoto compte environ 22000 étudiants. C'est l'une des plus anciennes universités du Japon.

106 Université de Tôhoku : université japonaise fondée en 1907 et située à Sendai dans la préfecture de Miyagi. L'Université de Tôhoku compte environ 18000 étudiants. 


\section{Fonctions des personnages}

1 ABE Ikuo : chercheur à l'Institut International de Recherche en Science des Désastres.

EDANO Yukio : né en 1964, il est Secrétaire général du Cabinet de Naoto Kan entre janvier et septembre 2011. Ce poste correspond grosso modo à celui de «Secrétaire général du Gouvernement » en France, mais il a un statut de ministre d'État et assure le rôle aussi de directeur de Cabinet et de porte-parole du Gouvernement. La traduction officielle en anglais est "Chief Cabinet Secretary». Il est assisté de trois Secrétaires généraux adjoints. Edano est député (Chambre des représentants) depuis 1993 et secrétaire général du Parti démocrate japonais de 2014 à sa disparition en 2016. Il préside le Parti démocrate constitutionnel, qu'il lance le 3 octobre 2017.

3 HIBINO Yasushi : ingénieur en électricité et camarade de classe de Naoto Kan à l'Université de technologie de Tôkyô. Il travaille au Nippon Telegraph and Telephone Corporation (NTT) Research Laboratory, avant de devenir vice-Président de la Japan Advanced Institute of Science and Technology (JAIST). Il est nommé conseiller spécial du Gouvernement durant la crise nucléaire.

HIKIDA Shiro : chef de groupe de réhabilitation d'équipement de Fukushima Daiichi.

HOSONO Gôshi : homme politique japonais né en 1971. Il est Conseiller spécial auprès du Premier ministre au moment de la crise nucléaire. Il est ensuite nommé Secrétaire d'État en charge de gestion de l'accident de Fukushima à partir de juin 2011, fonction qu'il cumule avec celle de ministre de l'Environnement de septembre 2011 à octobre 2012 dans le Gouvernement dirigé par Yoshihiko Noda (Premier ministre du 2 septembre 2011 au 26 décembre 2012). Depuis 2014, il est député à la Chambre des représentants.

IDOGAWA Ryuta : pilote du réacteur 2 de Fukushima Daiichi.

7 IKEDA Motohisa : né en 1940, il est vice-ministre du METI lorsque se produit le séisme et le tsunami du 11 mars 2011. Durant la crise nucléaire, il est le directeur de la cellule du Gouvernement située dans le centre de crise hors site à ôkuma.

8 INAGAKI Takeyuki : chef de groupe de réhabilitation d'équipement de Fukushima Daiichi.

9 IZAWA Ikuo : chef de la salle de contrôle des réacteurs 1 et 2 de Fukushima Daiichi. 
10 KAIEDA Banri : né en 1949, est le ministre de l'Économie, du Commerce et de l'Industrie (METI) entre janvier et septembre 2011.

11 KAN Naoto: né le 10 octobre 1945. Après des études scientifiques à l'Université de technologie de Tôkyô, il passe l'examen d'avocat conseil en brevet. Il exerce diverses fonctions politiques avant d'être le Premier ministre du Japon du 8 juin 2010 jusqu'à sa démission le 2 septembre 2011. C'est Yoshihiko Noda, son ministre des finances, qui lui succède au Kantei.

12 KATSUMATA Tsunehisa : né en 1940, il est diplômé de l'Université de Tôkyô en économie. Il est embauché à TEPCO en 1963. Il est le Président de l'entreprise de 2002 à 2008, et Directeur général de 2008 à 2012. Il a également été le Président de la Foundation of Electric Power Companies of Japan (FEPC).

13 KOBAYASHI Takashi : directeur de 2009 à 2011 du Bureau d'énergie thermique d'Higashi.

KOMORI Akio : directeur en 2011 de la division nucléaire de TEPCO. Auparavant, il a été directeur de Fukushima Daiichi. Pendant la crise nucléaire, il est également le directeur adjoint de la cellule de TEPCO située dans le centre de crise hors site à ôkuma.

15 MADARAME Haruki : ingénieur japonais, né en 1948. Après une carrière universitaire au sein de l'Université de Tôkyô et de la Nuclear Professional School, il est nommé Président de la Nuclear Safety Commission (NSC) en 2010 jusqu'à son abolition en 2012.

MASUDA Naohiro : est né en 1958, il étudie à l'Université nationale de Yokohama, où il est diplômé en ingénierie électrique. En 1982, il est engagé à TEPCO. Il occupe son premier poste à Fukushima Daini, alors que la centrale nucléaire est en construction. En 1989, il devient chef d'équipe à Kashiwasaki-Kariwa. Il est ensuite envoyé au siège de TEPCO, puis revient travailler à Daini en 2005 comme chef de l'exploitation et de la maintenance. Il est de nouveau appelé au siège, avant d'être nommé directeur de Daini en juillet 2011. En 2014, il devient Directeur général en charge de la décontamination et du démantèlement de Fukushima Daiichi. Le 14 mars 2018, l'ambassadeur de France au Japon lui remet les insignes de chevalier de l'Ordre national du Mérite.

17 MORIYAMA Yoshinori : il occupe en 2009 un poste de responsable de service à la NISA chargé des risques d'accident sur des installations nucléaires. Lorsque l'accident de Fukushima se produit le 11 mars 2011, il travaille au ministère de l'Éducation, de la Culture, des Sports, des Sciences et de la Technologie. Il aide toutefois la NISA à répondre à la crise nucléaire.

MUTÔ Sakae: né en 1950, il est le vice-Président de TEPCO lorsque se produit l'accident nucléaire de Fukushima Daiichi. Il est également le directeur de la cellule de TEPCO située dans le centre de crise hors site à ôkuma.

NAKURA Shigeki : un inspecteur de la NISA.

NOGUCHI Tetsuo : un chef d'inspecteurs au sein de la NISA.

OKAMURA Yukinobu : né en 1955, il est sismologue au National Institute of Advanced Industrial Science and Technology (AIST).

SATAKE Kenji : chercheur à l'Université de Tôkyô et auteur de « Numerical Simulation of the AD 869 Jogan Tsunami in Ishinomaki and Sendai Plains » (2008). 

est directeur de l'Agency for Natural Resources and Energy. Il est ensuite chargé de la réforme de la sûreté nucléaire, avant de devenir le n 3 de la NRA (en charge de la supervision des mesures d'urgence). Tôkyô, où il obtient un diplôme d'ingénieur en génie nucléaire, il est embauché en 1979 par TEPCO. Il est nommé directeur de Fukushima Daiichi en juin 2010. Le 28 novembre 2011, il quitte son poste pour des raisons de santé. Atteint d'un cancer de l'œsophage, il meurt à Tôkyô le 9 juillet 2013. 


\section{Références}

Ackerman, G., 2006. Tchernobyl, retour sur un désastre, Éditions Gallimard, Paris, 162 p.

AFP, 2019. "Trois ex-dirigeants de Tepco acquittés de la catastrophe de Fukushima ", Libération, 19 septembre 2019 [en ligne].

AFP, 2013. « Mort du directeur de la centrale de Fukushima », Le Temps, 10 juillet 2013 [en ligne].

AFP, 2011a. « Fukushima : la fuite du réacteur 2 a été colmatée », L'Express, 6 avril 2011 [en ligne].

AFP, 2011b. «Fukushima: la fuite d'eau radioactive colmatée... », Libération, 6 avril 2011 [en ligne].

Afrouss, A., 2017. La mise en récit de l'accident de Fukushima Dai Ichi par le directeur de la centrale : le coping en situation extrême, Sous la direction de Franck Guarnieri (Thèse de doctorat), PSL, Thèse préparée à l'Ecole des Mines de Paris, Paris, 183 p.

AIEA, 2015a. « L'accident de Fukushima Daiichi. Rapport du directeur général », Vienne, 261 p.

AIEA, 2015b. " The Fukushima Daiichi Accident. Technical Volume ", n², AIEA, Vienne, 174 p.

Alexievitch, S.A., 2011. « La leçon de Tchernobyl n'a pas été apprise », Libération, 19 mars 2011 [en ligne].

ASN, IRSN, 2011. «L'accident de la centrale nucléaire de Fukushia Daichi », 5 p.

Atomic Energy Society of Japan, 2015. « The Fukushima Daiichi Nuclear Accident: Final Report of the AESJ Investigation Committee », $560 \mathrm{p}$.

Beck, U., 2011. "C'est le mythe du progrès et de la sécurité qui est en train de s'effondrer », Le Monde, 25 mars 2011 [en ligne].

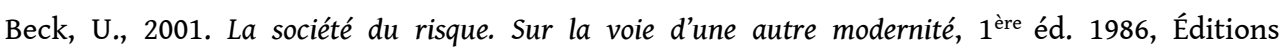
Flammarion, Paris, $521 \mathrm{p}$.

Bouissou, J.-M., 2011. «L'apocalypse japonaise expliquée à l'Occident », Le Monde Diplomatique, avril 2011.

CEA. 2012. « Les centrales nucléaires dans le monde édition », ELECNUC, Gif sur Yvette, 72 p.

CEA. 2019. « Les centrales nucléaires dans le monde édition », ELECNUC, Gif sur Yvette, 74 p.

Coïc, P., 2015. «Les leçons de Fukushima sur les centrales nucléaires françaises : le noyau dur ", Revue Générale Nucléaire, ${ }^{\circ} 2$, mars-avril 2015, 41-43.

Cote, C., 1996. «Viktor Brioukhanov : 'Je ne me sens pas coupable' », Libération, 16 avril 1996 [en ligne]. 
Descamps, F., 2005. L'historien, l'archiviste et le magnétophone: De la constitution de la source orale à son exploitation, Histoire économique et financière - XIX ${ }^{e}-X X^{e}$, Institut de la gestion publique et $\mathrm{du}$ développement économique, Paris [en ligne].

Dulong, R., 2000. « Le témoignage historique : document ou monument? », Hypothèses, vol. 1, n³, 115-119.

Dupuy, J.-P., 2012. «Un paradis habité par des meurtriers sans méchanceté et des victimes sans haine : Hiroshima, Tchernobyl, Fukushima », Ebisu, n47, 49-57.

Dupuy, J.-P., 2004. Pour un catastrophisme éclairé, $1{ }^{\text {ère } e ́ d . ~ 2002, ~ E ́ d i t i o n s ~ d u ~ S e u i l, ~ P a r i s, ~} 224$ p.

Dupuy, J.-P., 1994. « Temps et rationalité », Cahiers d'Économie Politique, n²4-25, 69-104.

Fressoz, J.-B., 2014. «Les leçons de la catastrophe », in Aubry, H., Marcondes, L. (dir.). La culture du risque en question. Des inondations aux débordements nucléaires, La Dispute, Paris, 169-187.

Fressoz, J.-B., 2012. L'apocalypse joyeuse : une histoire du risque technologique, Éditions du Seuil, Paris, $313 \mathrm{p}$.

Japan Times. 2014. «Fukushima No. 1 chief feared nuclear doom for eastern Japan », Japan. Times.

Garrigue-Testard, A., Chevallier, S., 2007. «L'évolution des mentalités dans les années 1990 », in Bouissou, J.-M. (dir.). Le Japon Contemporain, Fayard-CERI, Paris, 467-486.

Gaulène, M., 2020. Fukushima : un accident "Made in Japan". Analyse sémiotique de la causalité au Japon. Sous la direction de Franck Guarnieri et de Sébastien Travadel (Thèse de doctorat), PSL, Thèse préparée à l'Ecole des Mines de Paris, Paris, $257 \mathrm{p}$.

Grenêche, D., 2016. Histoire et techniques des réacteurs nucléaires et de leurs combustibles. EDP Sciences, Les Ulis, $760 \mathrm{p}$.

Guarnieri, F., Travadel, S., 2020. «Fukushima : décider en situation extrême ». Responsabilité et Environnement, $\mathrm{n}^{\circ} 98$, avril 2020, 60-62.

Guarnieri, F., Travadel, S., 2015. "Le temps de l'ingénierie de l'urgence », Guarnieri, F. (dir.). L'accident de Fukushima Daiichi. Le récit du directeur de la centrale. Volume I. L'abîme, Presses des Mines, Paris, 341 p., 23-45.

ICANPS, 2012. « Final Report », 23 juillet 2012, Tôkyô [en ligne].

INPO, 2011. «Special Report on the Nuclear Accident at the Fukushima Daiichi Nuclear Power Station », Atlanta, $97 \mathrm{p}$.

INSAG, 1996. « Defence in depth in nuclear safety », Safety Series, INSAG-10, AIEA, Vienne, 33 p.

INSAG, 1991. «Culture de sûreté », INSAG-4, n75, AIEA, Vienne, 37 p.

IRSN, 2020. « 9 ans après l'accident de la centrale de Fukushima Daiichi : Données clés sur les conséquences environnementales et le retour des populations dans les territoires évacués ", mars 2020, Fontenay-aux-Roses, $6 \mathrm{p}$.

IRSN, 2012a. «Fukushima, un an après. Premières analyses de l'accident et de ses conséquences (Rapport d'expertise en sûreté nucléaire ", IRSN/DG/2012-001, Fontenay-aux-Roses, 12 mars 2012, 189 p.

IRSN, 2012b. «Bilan des conséquences de l'accident de Fukushima sur l'environnement au Japon, un an après l'accident ", 28 février 2012, Fontenay-aux-Roses, 17 p.

IRSN, 2012c. «Synthèse des informations disponibles au Japon concernant la contamination des denrées alimentaires par les radionucléides persistant dans l'environnement après l'accident de Fukushima ", 13 juillet 2012, Fontenay-aux-Roses, $18 \mathrm{p}$.

Kadota, R., 2014. On the Brink: The Inside Story of Fukushima Daiichi. Kurodahan Press, Fukoka, 287 p. Kadota, R., 2013. « Fukushima : l'homme qui a sauvé le Japon est mort », Nippon.com [en ligne]. 
Kan, N., 2017. My Nuclear Nightmare: Leading Japan Through the Fukushima Disaster to a Nuclear-free Future, Cornell University Press, Ithaca, NY, $200 \mathrm{p}$.

Kan, N., 2015. «Entretien réalisé par Franck Guarnieri, Sébastien Travadel et Yuki Kobayashi », Maison des députés, Tôkyô, 2 décembre 2015, 25 p.

Kan, N., 2012. "Compte-rendu de l'audition menée par l'ICANPS», Salle de conférence au $2^{\text {e }}$ niveau du Mitsubishi Research Institute Building, 3 avril 2012, 72 p.

Kobayashi, Y., 2019. Étude de la relation entre les leaders politiques et techniques dans la gestion de l'accident de Fukushima Daiichi entre le 11 et 15 mars 2011, Sous la direction de Franck Guarnieri (Thèse de doctorat), PSL, Thèse préparée à l'Ecole des Mines de Paris, Paris, 263 p.

Lapp, R., 1971. « Thoughts on Nuclear Plumbing », N. Y. Times, 12 décembre 1971 [en ligne].

Le Roux, T., 2016. Risques industriels. Savoirs, régulations, politiques d'assistance fin XVII -début XX siècle, Presses Universitaires de Rennes, Rennes, 346 p.

Le Roux, T., 2014. "L'émergence du risque industriel (France, Grande-Bretagne, XVIII ${ }^{\mathrm{e}}-\mathrm{XIX}^{\mathrm{e}}$ siècle) ", Le Mouvement Social, vol. 4, $\mathrm{n}^{\circ} 249,3-20$.

Levi, P., 1989. Les naufragés et les rescapés. Quarante ans après Auschwitz, Éditions Gallimard, Paris, $200 \mathrm{p}$.

Lozerand, E., 2015. «Il n'y a pas d'individu au Japon : critique et archéologie d'un stéréotype », in Galan, C., Giraud, J.-P. (dir.). Individu-s et Démocratie Au Japon, Presses Universitaires du Midi, Toulouse, $332 \mathrm{p}, 19-71$.

Lozerand, E., 2014. «Il n'y a pas d'individu au Japon. Archéologie d'un stéréotype », Ebisu, n51, 273-302.

Lozerand, E., 2010. «La question de l'individu au Japon », in Corcuff P., Le Bart C., de Singly F. (dir.). L'individu Aujourd'hui, Presses Universitaires de Rennes, Rennes, 400 p., 139-149.

Marguet, S., 2012. Les accidents de réacteurs nucléaires, Lavoisier, Paris, 136 p.

McCurry, J., 2013. « Fukushima boss hailed as hero dies », The Guardian, 10 juillet 2013 [en ligne].

McNeill, D., 2013. «Japanese 'hero' who saved Fukushima plant from total meltdown dies of cancer », Independent, 9 juillet 2013 [en ligne].

Mesmer, P., 2020. «Le Japon prêt à rejeter dans l'océan les eaux contaminées de la centrale de Fukushima ", Le Monde, 16 octobre 2020 [en ligne].

Mesmer, P., 2013. «Masao Yoshida, l'homme qui a évité le pire, est mort », Le Monde, 10 juillet 2013 [en ligne].

NAIIC, 2012. "The official report of Executive summary. The Fukushima Nuclear Accident Independent Investigation Commission », The National Diet of Japan, Tôkyô, 88 p.

Nakahara, S., Ichikawa, M., 2013. « Mortality in the 2011 Tsunami in Japan », Journal of Epidemioly, vol. $23, n^{\circ} 1,5$ janvier 2013 [en ligne].

NEA / OECD, 2002. «Chernobyl: Assessment of Radiological and Health Impacts », Nuclear Energy Agency / Organisation for Economic Co-operation and Development, Issy-les-Moulineaux, 155 p.

Nespoulous, L., 2011. « Les Japonais et l'atome », L'Histoire, n³69, novembre 2011, 96-100.

Niel, J.-C., 2013. « L'après-Fukushima : la résilience des centrales nucléaires doit être renforcée », Responsabilité et Environnement, vol. 4, n², 27-31.

NRC, 2014. « Lessons Learned from the Fukushima Nuclear Accident for Improving Safety of U.S. Nuclear Plants ", National Research Council, Washington, DC, 366 p.

Oé, K., 2015. « Il y a eu Hiroshima, il y a Fukushima, une troisième tragédie est envisageable », L'Obs, 26 juin 2015 [en ligne]. 
OECD/NEA, 2013. «The Fukushima Daiichi Nuclear Power Plant Accident: OECD/NEA Nuclear Safety Response and Lessons Learnt ", $\mathrm{n}^{\circ} 7161$, Paris, $66 \mathrm{p}$.

Okadaand, Y., Adelman, J., 2013. « Masao Yoshida, manager of the Fukushima atomic plant, dies at $58 »$, Whashington Post, 9 juillet 2013 [en ligne].

Orofiamma, R., 2002. «Le travail de la narration dans le récit de vie », in Niewiadomski, C., de Villiers, G. (dir.). Souci et Soin de Soi, Liens et Frontières Entre Histoire de Vie, Psychothérapie et Psychanalyse, Histoire de Vie et Formation, L'Harmattan, Paris, 163-191.

Pelletier, P., 2012. « La guerre de Fukushima », Hérodote, vol. 3, n¹46-147, 277-307.

Peschanski, D., 1992. « Effets pervers », Cahiers de L'IHTP, 45-55.

Pirlot, B., 2007. «Après la catastrophe : mémoire, transmission et vérité dans les témoignages de rescapés des camps de concentration et d'extermination nazis ", Civilisations, n56, 21-41.

Portelli, A., 2019. « Fukushima, le couvercle du soleil », Jeune Cinéma, n³94, mai 2019, 88-89.

Portelli, A., 2018. « Le syndrome chinois », Jeune Cinéma, n³91, décembre 2018, 28-29.

Reason, J., 2013. L'erreur humaine, Presses des Mines, Paris, 403 p.

Renoux, P., 2015. «En moins de 12 heures, la FARN peut envoyer simultanément une soixantaine de professionnels du nucléaire sur n'importe quelle centrale d'EDF », Revue Générale Nucléaire, $\mathrm{n}^{\circ} 2$, mars-avril 2015, 39-40.

RGN, 2019. «Le mix énergétique du Japon, situation actuelle et perspectives ", Revue Générale Nucléaire, 12 mars 2019 [en ligne].

Ricoeur, P., 1990. Soi-même comme un autre, Éditions du Seuil, Paris, 424 p.

Satake, K., 2008. « Numerical simulation of the AD 869 Jogan tsunami in Ishinomaki and Sendai plains ", Annual Report of Active Fault and Paleoearthquake Researches, n8, 71-89.

Sirenelli, J.-F., 2002. «L'événement-monde », Vingtième Siècle, vol. 4, n76, 35-38.

Tabuchi, H., 2013. « Masao Yoshida, Nuclear Engineer and Chief at Fukushima Plant, Dies at 58 », N. Y. Times, 9 juillet 2013 [en ligne].

Taleb, N. N., 2012. Le Cygne Noir. La puissance de l'imprévisible, Les Belles Lettres, Paris, 496 p.

TEPCO, 2012. « Fukushima Nuclear Accident Analysis Report », 20 juin 2012, 487 p.

The Asahi Shimbun, 2014. The Yoshida Testimony, mai 2014 [en ligne].

Topçu, S., 2013. La France nucléaire. L'art de gouverner une technologie contestée, Éditions du Seuil, Paris, $349 \mathrm{p}$.

Travadel, S., Guarnieri, F., 2019. «Pourquoi faire le récit de l'accident de Fukushima?», La Pensée Écologique, vol. 3, n¹ [en ligne].

Travadel, S., Zawieja, P., Guarnieri, F., 2019. «Situation extrême », in Vandevelde-Rougale, A., Fugier, P. (dir.), Dictionnaire de Sociologie Clinique. ERES, Toulouse, 702 p., 589-591.

Werth, N., 2006. «Tchernobyl : enquête sur une catastrophe annoncée », L'Histoire, n³08, avril 2006 [en ligne].

Yatabe, K., 2002. "La société japonaise et la modernité », in Dourille-Feer, E. (dir.) Japon, Le Renouveau, La Documentation Française, Paris, 190 p., 71-132.

Yoshida, R., 2013. «Did late No. 1 plant head avert or facilitate nuclear crisis ? ", Japan Times, 11 juillet 2013 [en ligne].

Zonabend, F., 2014. La presqu'île au nucléaire. Three Mile Island, Tchernobyl, Fukushima... et après?, Odile Jacob, Paris, 240 p. 